\title{
Supplemental Information Related to Risk Assessment for the Off-Site Transportation of Low-Level Mixed Waste for the U.S. Department of Energy Waste Management Programmatic Environmental Impact Statement
}

by F.A. Monette, B.M. Biwer, D.J. LePoire, M.A. Lazaro, A.A. Antonopoulos, H.M. Hartmann, A.J. Policastro, and S.Y. Chen

Environmental Assessment Division, Argonne National Laboratory, 9700 South Cass Avenue, Argonne, Illinois 60439

\section{DISCLAIMER}

This report was prepared as an account of work sponsored by an agency of the United States Government. Neither the United States Government nor any agency thereof, nor any of their employees, makes any warranty, express or implied, or assumes any legal liability or responsibility for the accuracy, completeness, or usefulness of any information, apparatus, product, or process disclosed, or represents that its use would not infringe privately owned rights. Reference herein to any specific commercial product, process, or service by trade name, trademark, manufacturer, or otherwise does not necessarily constitute or imply its endorsement, recommendation, or favoring by the United States Government or any agency thereof. The views and opinions of authors expressed herein do not necessarily state or reflect those of the United States Government or any agency thereof.

December 1996

Work sponsored by United States Department of Energy, Assistant Secretary for Environmental Management 


\section{DISCLAIMER}

Portions of this document may be illegible electronic image products. Images are produced from the best available original document. 


\section{CONTENTS}

NOTATION $\ldots \ldots \ldots \ldots \ldots \ldots \ldots \ldots \ldots \ldots \ldots \ldots \ldots \ldots \ldots \ldots$

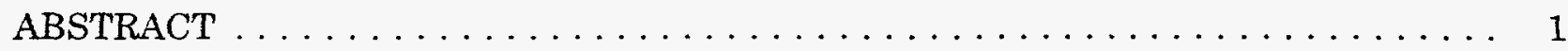

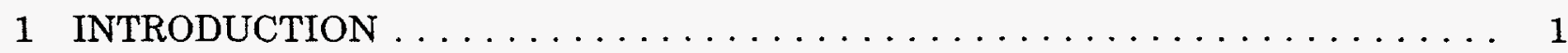

2 LOW-LEVEL MIXED WASTE CHARACTERISTICS, ALTERNATIVES,

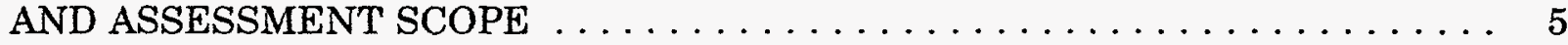

2.1 Definition of Low-Level Mixed Waste $\ldots \ldots \ldots \ldots \ldots \ldots \ldots \ldots$

2.2 Low-Level Mixed Waste Alternatives for the WM PEIS . . . . . . . . . 5

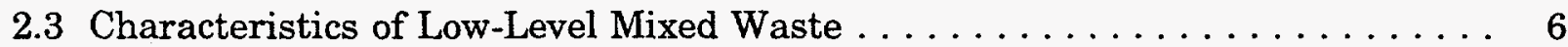

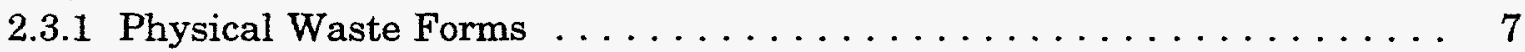

2.3.2 Radiological Profiles ....................... 8

2.3 .3 Chemical Profiles . . . . . . . . . . . . . . . . . . 8

2.4 Quantities and Characteristics of Transported Low-Level Mixed Waste

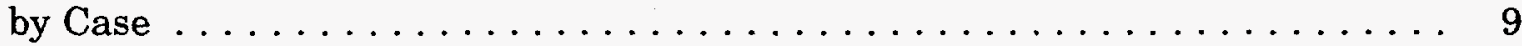

2.5 Scope of the Risk Assessment $\ldots \ldots \ldots \ldots \ldots \ldots \ldots \ldots \ldots \ldots \ldots \ldots$

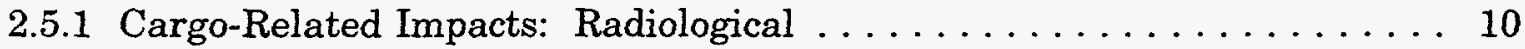

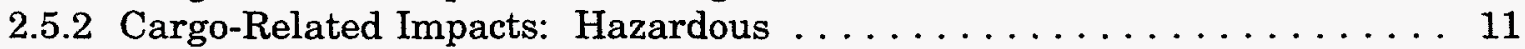

2.5.3 Vehicle-Related Impacts . . . . . . . . . . . . . . . . . . 13

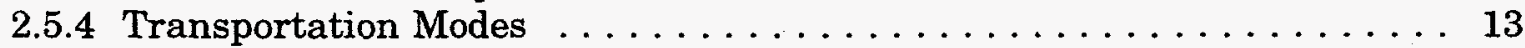

2.5 .5 Receptors . . . . . . . . . . . . . . . . . . 13

3 METHODS FOR CALCULATING TRANSPORTATION-RELATED RISKS $\ldots \ldots \ldots$

3.1 Overview of Risk Assessment Method $\ldots \ldots \ldots \ldots \ldots \ldots \ldots \ldots \ldots$

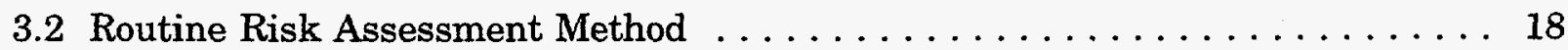

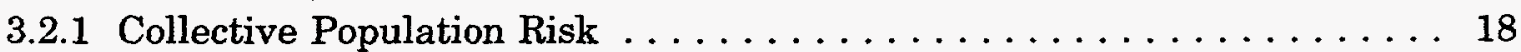

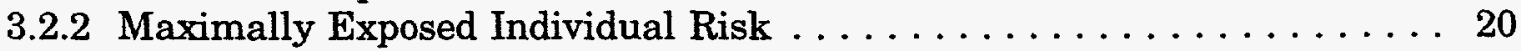

3.2.3 Vehicle-Related Routine Risk . . . . . . . . . . . . . . 20

3.3 Accident Assessment Method . . . . . . . . . . . . . . . . 21

3.3.1 Accident Risk Assessment . . . . . . . . . . . . . . . . . 21

3.3.2 Accident Consequence Assessment . . . . . . . . . . . . . . 23

3.3.2.1 Radiological Accident Consequence Assessment . . . . . . . . 23

3.3.2.2 Hazardous Accident Consequence Assessment . . . . . . . . . . 24

3.3.3 Maximally Exposed Individual Risk . . . . . . . . . . . . . . 26

3.3.3.1 Radiological Maximally Exposed Individual Risk . . . . . . . . 26

3.3.3.2 Hazardous Maximally Exposed Individual Risk . . . . . . . . 28

3.3.4 Vehicle-Related Accident Risk Assessment . . . . . . . . . . . . . . 29

3.4 Representative Route Determination Method . . . . . . . . . . . . . . . . 29

3.4.1 Truck Route Determination . . . . . . . . . . . . . . . . 30

3.4 .2 Rail Route Determination .................... 30 


\section{CONTENTS (Cont.)}

4 RISK ASSESSMENT ASSUMPTIONS AND PARAMETERS . . . . . . . . . 36

4.1 Shipping and Packaging Assumptions for Low-Level Mixed Waste . . . . . 36

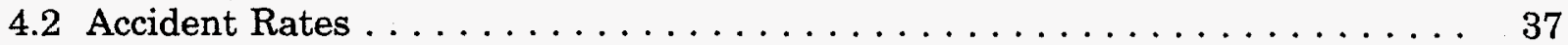

4.3 Characteristics of Accident Releases $\ldots \ldots \ldots \ldots \ldots \ldots \ldots \ldots \ldots$

4.3.1 Accident Severity Categories . . . . . . . . . . . . . . . . . . 41

4.3 .2 Package Release Fractions $\ldots \ldots \ldots \ldots \ldots \ldots \ldots \ldots \ldots \ldots$

4.4 Atmospheric Conditions during Accidents . . . . . . . . . . . . . 45

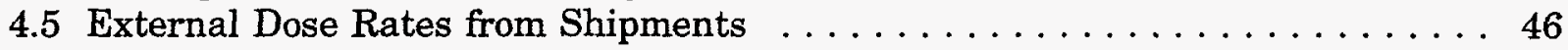

4.6 Radiological Maximally Exposed Individual Exposure Scenarios . . . . . . . . 47

4.7 General Radiological Assessment Input Parameters . . . . . . . . . . . . . 48

$5 \quad$ RISK ASSESSMENT RESULTS $\ldots \ldots \ldots \ldots \ldots \ldots \ldots \ldots \ldots \ldots \ldots \ldots \ldots \ldots \ldots$

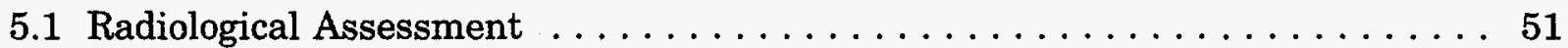

5.1 .1 Collective Population Radiological Risk Results . . . . . . . . . . . 52

5.1.2 Maximally Exposed Individual Assessment: Routine Transport . . . . 53

5.1.3 Radiological Accident Consequence Assessment . . . . . . . . . . 55

5.2 Hazardous Assessment Results . . . . . . . . . . . . . . . 61

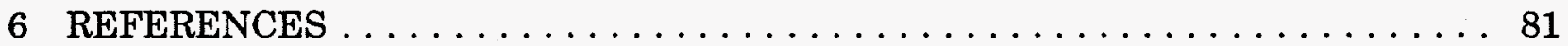

APPENDIX A: Transported Material Summary Files Generated by the

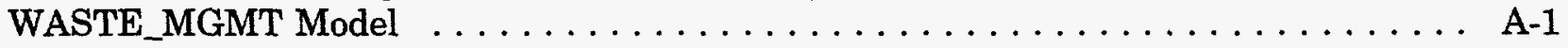

APPENDIX B: Detailed Radiological Risk Assessment Results . . . . . . . . . . . B-1

\section{TABLES}

3.1 Container Breach Rates and Atmospheric Releases Derived from HMIRS Statistical Data for Truck and Railcar Accidents . . . . . . . . . . . . . 25

3.2 Release Fractions for Eight Accident Severity Categories . . . . . . . . . . 26

3.3 Chemical-Specific Values of ICRC and PAEC for LLMW Carcinogenic

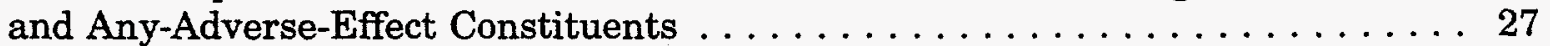

3.4 Truck Route Distances between Major DOE Sites $\ldots \ldots \ldots \ldots \ldots \ldots \ldots$

3.5 Rail Route Distances between Major DOE Sites $\ldots \ldots \ldots \ldots \ldots \ldots \ldots$

4.1 Highway Combination-Truck Accident, Fatality, and Injury Rates by Road Type Based on Reportable Interstate-Carrier Accidents, Reported Fatalities and Injuries, and Estimated Flows, 1986-1988 


\section{TABLES (Cont.)}

4.2 Railroad-Freight-Car Accident Rates, by State, for 1986, 1987, 1988, and 1985-1988 Combined . . . . . . . . . . . . . . . . . . . . . 40

4.3 Fractional Occurrences for Accidents by Severity Category and Population Density Zone ......................... 44

4.4 Estimated Release Fractions for LLMW Packages under Various Accident Severity Categories $\ldots \ldots \ldots \ldots \ldots \ldots \ldots \ldots \ldots \ldots \ldots$

4.5 Aerosolized and Respirable Material Release Fractions for LLMW . . . . . . . . 46

4.6 General RADTRAN Input Parameters for the LLMW Assessment . . . . . . . . 49

5.1 Total Number of Shipments and One-Way Shipping Distances for the WM LLMW Alternatives . . . . . . . . . . . . . . . . . . 54

5.2 Total Routine Dose for the WM LLMW Alternatives $\ldots \ldots \ldots \ldots \ldots \ldots \ldots$

5.3 Total Accident Dose Risk for the WM LLMW Alternatives . . . . . . . . . . 55

5.4 Total Estimated Fatalities for the WM LLMW Alternatives $\ldots \ldots \ldots \ldots \ldots 56$

5.5 Total Estimated Cancer Incidence for the WM LLMW Alternatives . . . . . . . 58

5.6 Total Estimated Genetic Effects for the WM LLMW Alternatives . . . . . . . . . 60

5.7 Estimated Routine Doses and Lifetime Risk of Fatal Cancer to MEIs from Shipments of WM LLMW . . . . . . . . . . . . . . . . . . . 62

5.8 Cumulative Dose and Lifetime Risk to MEI Living along a Site Entrance Route for WM LLMW Shipments $\ldots \ldots \ldots \ldots \ldots \ldots \ldots \ldots 63$

5.9 Estimated Consequences for the Most Severe Accidents Involving Shipments of WM LLMW . . . . . . . . . . . . . . . . . . . 68

5.10 Summary of Cargo-Related Population Risks for WM LLMW Shipments by Highway $\ldots \ldots \ldots \ldots \ldots \ldots \ldots \ldots \ldots \ldots . \ldots \ldots$

5.11 Summary of Cargo-Related Population Risks for WM LLMW

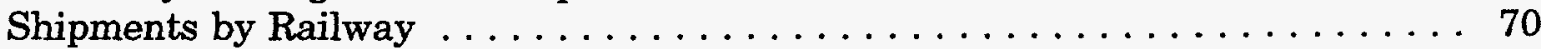

5.12 Lifetime MEI PIC Risk for WM LLMW Transportation $\ldots \ldots \ldots \ldots \ldots \ldots .72$

5.13 MEI Hazard Quotients for PAE End Points for WM LLMW . . . . . . . . . 73

5.14 Lifetime MEI PIC Risk by LLMW Alternative . . . . . . . . . . . . . 74 


\section{TABLES (Cont.)}

5.15 MEI Hazard Quotients for PAE Risk by LLMW Alternative . . . . . . . . . . 75

5.16 Estimated Chemical Exposure Consequences for the Most Severe Accident Involving the Hazardous Component of WM LLMW Shipments . . . . 77

5.17 Estimated Chemical Exposure Consequences Associated with the Most Severe Accident Involving the Radiological Component of WM LLMW Shipments . . . . 79

\section{FIGURES}

2.1 Conceptualization of Threshold Health Effects . . . . . . . . . . . . . . . 12

3.1 Technical Approach for the Radiological Component of the LLMW Transportation Risk Assessment

3.2 Technical Approach for the Hazardous Component of the LLMW Transportation Risk Assessment

4.1 Scheme for NUREG-0170 Classification by Accident Severity Category for Truck Accidents

4.2 Scheme for NUREG-0170 Classification by Accident Severity Category for Rail Accidents 


\section{NOTATION}

The following is a list of the acronyms, initialisms, and abbreviations (including units of measure) used in this document. Some acronyms used only in tables are defined in those tables.

\section{ACRONYMS, INITIALISMS, AND ABBREVIATIONS}

\begin{tabular}{|c|c|}
\hline ANL-E & Argonne National Laboratory-East \\
\hline A-RAMS & ALOHA risk assessment modeling system \\
\hline BNL & Brookhaven National Laboratory \\
\hline CFR & Code of Federal Regulations \\
\hline $\mathrm{CH}$ & contact-handled \\
\hline DOE & U.S. Department of Energy \\
\hline DOT & U.S. Department of Transportation \\
\hline EPA & U.S. Environmental Protection Agency \\
\hline Hanford & Hanford Site \\
\hline HLW & high-level waste \\
\hline $\mathrm{HQ}$ & hazard quotient \\
\hline HW & hazardous waste \\
\hline ICRC & increased carcinogenic risk concentration \\
\hline ICRP & International Commission on Radiological Protection \\
\hline IDB & Integrated Data Base \\
\hline LLMW & low-level mixed waste \\
\hline LLNL & Lawrence Livermore National Laboratory \\
\hline LLW & low-level waste \\
\hline MEI & maximally exposed individual \\
\hline MWIR-2 & Mixed Waste Inventory Report \\
\hline $\mathrm{NRC}$ & U.S. Nuclear Regulatory Commission \\
\hline NTS & Nevada Test Site \\
\hline ORR & Oak Ridge Reservation \\
\hline PAE & potential adverse effect \\
\hline PAEC & potential adverse effect concentration \\
\hline PCB & polychlorinated biphenyl \\
\hline PGDP & Paducah Gaseous Diffusion Plant \\
\hline PIC & potential-increased-cancer \\
\hline PIH & poison inhalation hazard \\
\hline PLC & potential life-threatening concentration \\
\hline PORTS & Portsmouth Gaseous Diffusion Plant \\
\hline RCRA & Resource Conservation and Recovery Act \\
\hline $\mathrm{RfD}$ & reference dose \\
\hline $\mathrm{RH}$ & remote-handled \\
\hline SMAC & Shipment Mobility/Accountability Collection \\
\hline SNL-NM & Sandia National Laboratories (New Mexico) \\
\hline TRU & transuranic \\
\hline TRUW & transuranic waste \\
\hline TSD & treatment, storage, and disposal \\
\hline
\end{tabular}


WM Waste Management

WM PEIS Waste Management Programmatic Environmental Impact Statement

\section{UNITS OF MEASURE}

$\begin{array}{ll}\mathrm{cm} & \text { centimeter(s) } \\ \mathrm{cm}^{2} & \text { square centimeter(s) } \\ \mathrm{cm}^{3} & \text { cubic centimeter(s) } \\ \mathrm{d} & \text { day(s) } \\ \mathrm{ft} & \text { foot (feet) } \\ \mathrm{g} & \text { gram(s) } \\ \mathrm{gal} & \text { gallon(s) } \\ \mathrm{h} & \text { hour(s) } \\ \mathrm{K} & \text { (degree[s]) Kelvin } \\ \mathrm{kg} & \text { kilogram(s) } \\ \mathrm{km} & \text { kilometer(s) } \\ \mathrm{km} & \text { square kilometer(s) } \\ \mathrm{L} & \text { liter(s) } \\ \mathrm{lb} & \text { pound(s) } \\ \mathrm{m} & \text { meter(s) } \\ \mathrm{m} & \text { cubic meter(s) } \\ \mathrm{mg} & \text { milligram(s) } \\ \mathrm{mi} & \text { mile(s) } \\ \mathrm{mi} & \text { square mile(s) } \\ \mathrm{min} & \text { minute(s) } \\ \mathrm{mph} & \text { mile(s) per hour } \\ \mathrm{mrem} & \text { millirem } \\ \mathrm{N} & \text { newton(s) } \\ \mathrm{nCi} & \text { nanocurie(s) } \\ \mathrm{ppm} & \text { part(s) per million } \\ \mathrm{rem} & \text { rem } \\ \mathrm{s} & \text { second(s) } \\ \mathrm{yd} & \text { yard(s) } \\ \mathrm{yr} & \text { year(s) } \\ \mu \mathrm{gg} & \text { microgram(s) } \\ & \end{array}$




\title{
SUPPLEMENTAL INFORMATION RELATED TO RISK ASSESSMENT FOR THE OFF-SITE TRANSPORTATION OF LOW-LEVEL MIXED WASTE FOR THE U.S. DEPARTMENT OF ENERGY WASTE MANAGEMENT PROGRAMMATIC ENVIRONMENTAL IMPACT STATEMENT
}

by

\author{
F.A. Monette, B.M. Biwer, D.J. LePoire, M.A. Lazaro, A.A. Antonopoulos, \\ H.M. Hartmann, A.J. Policastro, and S.Y. Chen
}

\begin{abstract}
This report provides supplemental information to support the human health risk assessment conducted for the transportation of low-level mixed waste (LLMW) in support of the U.S Department of Energy Waste Management Programmatic Environmental Impact Statement (WM PEIS). The assessment considers both the radioactive and chemical hazards associated with LLMW transportation. Detailed descriptions of the transportation health risk assessment methods and results of the assessment are presented in Appendix $E$ of the WM PEIS. This report presents additional information that is not included in Appendix $\mathbf{E}$ but that was needed to conduct the transportation risk assessment for Waste Management (WM) LLMW. Included are definitions of the LLMW alternatives considered in the WM PEIS; data related to the inventory and to the physical, chemical, and radiological characteristics of WM LLMW; an overview of the risk assessment methods; and detailed results of the assessment for each WM LLMW case considered.
\end{abstract}

\section{INTRODUCTION}

This report presents supplemental information to support the human health risk assessment conducted for the transportation of low-level mixed waste (LLMW) for the U.S. Department of Energy (DOE) Waste Management Programmatic Environmental Impact Statement (WM PEIS) (DOE 1996). The assessment considers both the radioactive and chemical hazards associated with LLMW transportation. Detailed descriptions of the transportation health risk assessment methods, major assumptions, and packaging and routing determinations, as well as a summary of the results of the transportation assessment, are presented in Appendix E of the WM PEIS (DOE 1996). This report presents additional information that is not presented in Appendix $\mathrm{E}$ but that was needed to conduct the transportation risk assessment. This report should be read in conjunction with Appendix $E$ to provide a complete description of the transportation risk assessment conducted for LLMW. Similar supporting reports have been prepared for the transportation assessments conducted 
for the WM PEIS alternatives for high-level waste (HLW), low-level waste (LLW), transuranic waste (TRUW), and hazardous waste (HW) (Monette et al. 1996a,b,c; Lazaro et al. 1996).

The WM PEIS assessment of LLMW considers LLMW generated from Waste Management (WM) activities. Wilkins et al. (1996) present a description of the LLMW inventory, characteristics, and treatment options. This analysis does not include the updated LLMW volumes that are presented in Appendix I in the WM PEIS. This present report includes definitions of the LLMW alternatives considered in the WM PEIS and presents data related to the inventory and to the physical, chemical, and radiological characteristics of WM LLMW that are used in the transportation assessment (Section 2). An overview of the methods for risk assessment is provided in Section 3. In addition, supplemental assumptions and parameters for the risk assessment calculations are provided (Section 4); and detailed results of the assessment are presented for each WM LLMW alternative considered (Section 5).

The transportation of LLMW involves a risk to both crew members and members of the public. Part of this risk results from the nature of transportation itself, independent of the radioactive or hazardous characteristics of the cargo; for instance, increased levels of pollution from vehicular exhaust emissions may affect human health. Similarly, accidents during transportation may cause injuries and fatalities. These risks can be viewed as "vehicle-related" risks. On the other hand, the transportation of LLMW may pose an additional risk because of the characteristics and potential hazards of the material itself. These risks are considered to be "cargo-related" risks.

In this report, the risks to human health from both vehicle- and cargo-related causes are assessed for shipments of LLMW. Cargo-related risks from both the radiological and hazardous components of LLMW are assessed. Generally, the approaches are similar for the radioactive and hazardous risk assessment components, except that the physical mechanisms and measures for assessing the cargo-related effects on health are fundamentally different (see WM PEIS Appendix E) (DOE 1996). Differences exist in the assumptions and data used to develop the risk estimators for these two classes of contaminants.

For radioactive materials, the cargo-related impacts on human health during transportation are caused by exposure to ionizing radiation. Exposures to radiation occur during both routine (i.e., incident-free) transportation and during accidents. During routine operations, human exposures can be received from the low-level radiation field in the vicinity of a loaded shipment. The external radiation field must be below limits specified in federal regulations for all shipments of radioactive material. During transportation-related accidents, human exposures may occur following the release and dispersal of radioactive material into the environment via multiple environmental pathways, such as exposure to contaminated ground or air or the ingestion of contaminated food.

The potential exposures to the general population from transportation of radioactive materials, either from routine operations or from postulated accidents, are usually at a low dose, such that the primary adverse health effect is the potential induction of latent cancer (i.e., cancer that occurs after a latency period of several years from the time of exposure). 
The types of cancers induced by radiation are similar to "naturally occurring" cancers and may be expressed at some point in the lifetimes of the exposed individuals. Transportation of radioactive waste is not expected to cause acute (short-term) radiation-induced fatalities or to produce immediately observable effects in exposed individuals.

The effects of low-level radiation are generally characterized by correlating the total human exposure to radiation (i.e., dose) to the number of cases of latent cancer. The correlation of radiation dose and human health effects for low doses has been traditionally based on what is termed the "linear/no-threshold hypothesis," which has been described by various international authorities on protection against radiation. This hypothesis implies, in part, that even small doses of radiation cause some risk of inducing cancer and that doubling the radiation dose would mean doubling the expected numbers of cancers. The data on the health risk from radiation have been derived primarily from human epidemiological studies of past exposures, such as Japanese survivors of the atomic bomb in World War II and persons exposed during medical applications.

In contrast to radioactive materials, hazardous chemicals do not pose cargo-related risks to humans during routine transportation-related operations. Waste transportation operations are generally well regulated with respect to packaging, such that small spills or seepages during routine transport are kept to a minimum and do not result in exposures; for example, containers of liquids are surrounded by absorbent overpacking. Potential cargo-related health risks to humans can occur only if the integrity of a container is compromised during an accident (i.e., a container is breached or spilled). Under such conditions, some toxic chemicals (such as chlorine gas) may cause an immediate health threat to exposed individuals.

For chemical exposures, the severity of the immediate health effects depends strongly on the toxicity and exposure concentration of the specific chemical(s) released. The severity of the immediate (i.e., acute) health effects can range from slight irritation to fatality for the exposed individuals. In this assessment, two end points for acute health effects are assessed: potential life-threatening and potential reversible or irreversible adverse health effects (from slight effects up to and including lethality). A nonlinear or threshold correlation is assumed between the exposure concentration and the toxicity for the evaluation of these acute effects; that is, some low level of exposure can be tolerated without affecting health. In many cases, data on human toxicity relating acute health effects to chemical exposures do not exist. When data on toxicity in humans are not available, chemical risk estimators are derived from levels that are toxic to laboratory animals. The use of animal data to predict toxic concentrations in humans adds uncertainty to the risk estimates.

In addition to acute health effects, the cargo-related risk of excess cases of latent cancer from accidental chemical exposures has been estimated in this report. Similar to the correlation for radionuclides, the correlation of chemical dose with the induction of human cancer has traditionally been based on the linear/no-threshold hypothesis. The treatment of the carcinogenic effects of exposures resulting from accidental chemical releases has added uncertainty because the carcinogenic risk is estimated for short-term (one-hour) exposures. 
Lifetime risks less than $1 \times 10^{-6}$ have been considered negligible and are not estimated. The number of individuals experiencing an increased risk of cancer of $1 \times 10^{-6}$ or greater has been estimated, without attempting to estimate the precise risk for those in the category of greater than $1 \times 10^{-6}$.

For reasons stated previously, health impacts from the radioactive and hazardous components of LLMW are calculated and presented separately in this report. No attempt has been made to add or compare the estimated risks for the two classes of contaminants. To understand and interpret the estimated health impacts presented in this report, readers must keep in mind the fundamental differences between radioactive and chemical contaminants discussed previously. 


\section{LOW-LEVEL MIXED WASTE CHARACTERISTICS, ALTERNATIVES, AND ASSESSMENT SCOPE}

The risks of transporting LLMW have been assessed for seven WM alternatives in the WM PEIS. The transportation risks for each case depend on the amount of waste transported, the physical form of the waste, the specific chemicals and chemical quantities in the waste, and the quantity of radioactivity (curies) in each shipment. This section of the report describes the alternatives considered for WM LLMW in the WM PEIS, presents important LLMW inventory and characterization data used in the transportation assessment, and defines the scope of the human health risk assessment.

\subsection{DEFINITION OF LOW-LEVEL MIXED WASTE}

Low-level mixed waste is material that is both hazardous under the Resource Conservation and Recovery Act (RCRA) and a low-level radioactive waste. Low-level mixed waste contains RCRA-regulated chemicals or special waste types in a form or concentration sufficient to render the waste hazardous under the guidelines of the Code of Federal Regulations (40 CFR Part 261). Although asbestos-contaminated wastes are not hazardous under federal RCRA rules, friable asbestos waste is considered a $H W$ in several states. For the WM PEIS, low-level radioactive waste contaminated with asbestos is considered as LLMW. High-level waste, spent nuclear fuel, and TRUW that may be contaminated with RCRA HW are not LLMW.

Low-level mixed waste is either contact-handled $(\mathrm{CH})$ waste (dose at waste surface $<200 \mathrm{mrem} / \mathrm{h}$ ) or remote-handled $(\mathrm{RH})$ waste (dose at waste surface $>200 \mathrm{mrem} / \mathrm{h}$ ). The handling category determines the level of protective shielding required to safely store and process the material. Low-level mixed waste is also classified as either alpha LLMW (combined activity of transuranic [TRU] radionuclides with half-lives greater than 20 years is between 10 and $100 \mathrm{nCi} / \mathrm{g}$ ) or nonalpha LLMW (TRU activity $<10 \mathrm{nCi} / \mathrm{g}$ ). The alpha status of LLMW is important in determining the choice of waste treatment facilities because, in some states, facilities that process alpha-containing wastes cannot be used for wastes with minimal TRU activity. Mixed TRUW (TRU activity $>100 \mathrm{nCi} / \mathrm{g}$ ) is not addressed in this report. Together, the four radioactivity classifications ( $\mathrm{CH}$ nonalpha LLMW, $\mathrm{CH}$ alpha LLMW, RH nonalpha LLMW, and RH alpha LLMW) determine the handling and routing for treatment or disposal (or both) of all LLMW.

\subsection{LOW-LEVEL MIXED WASTE ALTERNATIVES FOR THE WM PEIS}

For the purposes of the WM PEIS, the DOE is considering a range of alternatives for the configuration of new or expanded WM facilities and the potential consolidation of existing facilities. In general, the WM PEIS alternatives reflect decentralized, regionalized, and centralized approaches. For each type of waste, several options, which are referred to as cases, have been defined for each broad alternative. The individual cases differ in the 
number, location, and types of facilities for treatment, storage, and disposal (TSD) that are being considered. Definitions of WM cases are provided in the following paragraphs.

For LLMW, the alternatives are (1) No Action, (2) Decentralized, (3) Regionalized, and (4) Centralized. The major alternatives are subdivided into seven cases as follows: (1) No Action; (2) Decentralized Alternative (plus one special treatment exception) Case 2a (49 treatment and 16 disposal sites); (3) Regionalized Alternative, including Case 4 (11 treatment plus one special treatment site exception and 12 disposal sites), Case 7 (seven treatment and six disposal sites), Case 10a (seven treatment sites and one disposal site), and Case 15 (four treatment and six disposal sites); and (4) the Centralized Alternative Case 17 (one treatment and disposal site). Each of the alternatives also incorporates Case 26 involving shipment of RH LLMW at about $1 \mathrm{~L} / \mathrm{yr}(0.26 \mathrm{gal} / \mathrm{yr})$, which is negligible in terms of transportation risk.

Transportation risks have been calculated for seven WM LLMW alternatives. The number of disposal sites varies from 16 for decentralized disposal to one for centralized disposal. Options for treatment also vary from decentralized to centralized approaches. In general, sites not having the capability for treatment or disposal ship to the nearest site that has such capability. The alternatives are summarized as follows:

- No Action (Case 1). Treatment and indefinite storage of LLMW generated in the future. No transportation occurs.

- Decentralized (Case 2a). Thirty-seven sites treat LLMW to land disposal restriction (LDR) levels, and 16 sites dispose.

- Regionalized 1 (Case 4). Eleven sites treat LLMW, and 12 sites dispose.

- Regionalized 2 (Case 7). Seven sites treat LLMW, and six sites dispose.

- Regionalized 3 (Case 10a). Seven sites treat LLMW, and one site disposes (Nevada Test Site [NTS]).

- Regionalized 4 (Case 15). Four sites treat LLMW, and six sites dispose.

- Centralized (Case 17). One site treats LLMW (Hanford Site [Hanford]), and one site disposes (Hanford).

\subsection{CHARACTERISTICS OF LOW-LEVEL MIXED WASTE}

To support the WM PEIS analysis of LLMW alternatives, a report has been prepared describing the LLMW inventory and characteristics at each DOE site (Wilkins et al. 1996). In addition, Wilkins et al. (1996) describe the current LLMW TSD capabilities of the DOE complex and present the important assumptions associated with the analysis of LLMW alternatives. The information in the report by Wilkins et al. (1996) was used as the primary source of information for the transportation assessment. 
The report by Wilkins et al. (1996) describes LLMW management alternatives for inventory and operations-generated waste (WM LLMW). The WM LLMW includes the LLMW inventory currently in storage and the LLMW projected to be generated through the year 2013 at 49 sites. For each site, information on the inventory is presented in terms of projected waste volumes and masses. In addition, the physical, chemical, and radiological characteristics of the waste at each site are presented. Information from Wilkins et al. (1996) that is important for the transportation assessment is summarized subsequently.

\subsubsection{Physical Waste Forms}

The LLMW at each site is characterized as a function of the potential options for treatment of the various waste forms in the report by Wilkins et al. (1996). Wilkins et al. (1996) define 32 separate treatment categories for WM LLMW on the basis of the physical characteristics of the waste; however, Wilkins et al. also show how the 32 categories can be compressed into the following eight categories for the purposes of WM PEIS assessment: (1) aqueous liquids, (2) organic liquids, (3) solid process residues, (4) soils, (5) debris, (6) laboratory packs, (7) inherently hazardous material, and (8) monolith waste. For the WM PEIS, all aqueous liquid LLMW is assumed to be treated at the site of generation before transportation. Residuals are then managed as solid LLMW.

For purposes of the transportation assessment, shipments of six different LLMW forms were considered on the basis of the treatability categories defined for LLMW. The following codes identify the LLMW treatability categories considered in the transportation assessment:

- $\mathrm{O}=\mathrm{CH}$ organic liquids, nonalpha - untreated;

- $\mathrm{OA}=\mathrm{CH}$ organic liquids, alpha - untreated;

- $\mathrm{C}=\mathrm{CH}$ heterogeneous solids, nonalpha - untreated;

- $\mathrm{CA}=\mathrm{CH}$ heterogeneous solids, alpha - untreated;

- $\mathrm{CT}=\mathrm{CH}$ heterogeneous solids, nonalpha - treated; and

- $\mathrm{T}=\mathrm{CH}$ heterogeneous solids, alpha - treated.

The previous material categories were considered separately because requirements for treatment differ among them, and different accident release characteristics were used in the transportation assessment (e.g., organic liquids were assumed to be totally aerosolized in severe accidents, versus only about $10 \%$ for heterogeneous solids). In addition, a distinction was drawn between untreated and treated materials because, once treated, all waste would be in a solid physical form. The heterogeneous solids include all treatability categories with the exception of Category 2. No transportation of $\mathrm{RH}$ waste occurs for the LLMW alternatives. 


\subsubsection{Radiological Profiles}

Radiological characteristics of LLMW are described by Wilkins et al. (1996) and are summarized subsequently. For the WM PEIS, the assumption is that radiological profiles for LLMW are dependent on the site but independent of the waste stream. The LLMW radiological profiles developed in the WM PEIS are based on information about the specific mission and operational history that generated radionuclides at the various sites and on the LLW information in the 1991 Integrated Data Base (IDB) (Oak Ridge National Laboratory 1991).

For the WM PEIS, radiological profiles are grouped into five distinct source term categories: (1) fission products, (2) induced activity, (3) uranium or thorium or both (U/Th), (4) TRUW, and (5) tritium. The IDB data provide radiological profiles for the five different LLW source term categories close to the time of waste generation. The IDB data also provide the amount (volume and activity) of each of the five LLW source term categories accumulated at each of the major DOE sites. Because the data have been accumulated over a number of years, the data represent the cumulative result of the various DOE waste-generating activities over those years.

In estimating the radiological profiles for the LLMW in the WM PEIS, the assumption is that, at the time of waste generation, LLMW radiological profiles for each site are the same as those for LLW; however, radioactive decay since the time of generation has been considered for LLMW. Therefore, the radioactive profile of LLMW at the time of treatment will differ considerably from its profile at the time of generation. The appropriate. waste ages, the activity percentages, and the source term radiological profiles are combined and used to generate site-dependent radiological profiles and activity concentrations for LLMW. The individual site radiological profiles are detailed in the report by Wilkins et al. (1996).

\subsubsection{Chemical Profiles}

Site-specific LLMW hazardous-chemical profiles are also provided by Wilkins et al. (1996). The majority of information on the hazardous-chemical composition in LLMW is derived from site-specific (process) operational knowledge.

For the WM PEIS, the chemical profiles (i.e., the composition and concentration of RCRA-hazardous chemical constituents) for CH nonalpha WM LLMW have been developed from (1) compilation of the chemical composition data presented in the Mixed Waste Inventory Report (MWIR-2) (DOE 1994) and (2) an engineering assessment of the industrial processes that generated the respective LLMW streams. Chemical profiles for alpha LLMW are not developed separately because of the relatively small volume (compared with nonalpha LLMW) of this waste. For the same reason, chemical profiles for RH LLMW also assume the composition developed for CH nonalpha LLMW. Polychlorinated biphenyl (PCB) wastes also assume the same generic waste-stream-dependent chemical profiles, with the additional assumption of PCB concentrations of $500 \mathrm{ppm}$ for all PCB-contaminated LLMW. 
Information about historical DOE site operations and industrial processes, as well as MWIR-2 information, suggests that waste streams of a given treatment category from different sites in the DOE complex are similar in chemical composition. Therefore, the chemical profiles for WM-derived LLMW are assumed to be independent of the site that generated the waste and to depend only on the waste-steam treatment category. The chemical profiles developed by Wilkins et al. (1996) focus on 16 distinct chemicals or grouped classes of chemicals that are common to most waste streams. These chemicals and classes include six toxic metals, three inorganic chemicals, and seven classes of organic chemicals that are grouped according to important treatment parameters such as density, solubility, volatility, and degree of chlorination.

\subsection{QUANTITIES AND CHARACTERISTICS OF TRANSPORTED LOW-LEVEL MIXED WASTE BY CASE}

The LLMW inventory and radiological and chemical characterization data described previously are used as the primary input to a computational model called WASTE_MGMT (Kotek et al. 1996). The WASTE_MGMT model has been developed at Argonne National Laboratory-East (ANL-E) to support analyses of risks and costs for all alternatives for each waste type considered in the WM PEIS (Kotek et al. 1996). Input to the model includes not only data on the waste inventory and characterization, but also data on operations for the TSD facilities used for the wastes, and the definitions of the various alternatives. Specific modeling parameters for the LLMW assessment are discussed by Wilkins et al. (1996).

One output of the WASTE_MGMT model consists of the annual quantity, physical form, and radiological characteristics of the waste shipped between sites for each case. The output of the model consists of a set of origin-and-destination pairs that represent shipping linkages among generator, treatment, and storage sites. For each origin-and-destination pair, the model computes the annual volume (cubic meters), weight (kilograms), and chemical and radioactive profiles of the LLMW transported. The effects of potential waste treatment, such as volume reduction or incineration, are considered in the model and are reflected in changes in waste density and activity concentrations. The WASTE_MGMT output files are used directly as input to the transportation risk assessment.

The WASTE_MGMT transportation summary files generated for the LLMW transportation assessment are presented in Appendix A of this report. For each of the WM cases, shipments are summarized separately for heterogeneous solids and for organic liquids. The total quantity of shipments for a case can be found by summing the quantities for these two categories.

Note that the WASTE_MGMT files report the quantity of waste shipped on an annual basis (cubic meters per year or kilograms per year). The WM PEIS considers a 20-year time frame for WM LLMW generation. For most of the WM alternatives considered in the WM PEIS, the assumption was that shipments would occur uniformly over a 10-year period, which would allow an initial 10-year period to build TSD facilities. Sites would essentially store their own waste for the first 10 years and ship all waste over the subsequent 10 years. 
Because of the differences in the time frames noted previously and the uncertainties in the actual timing of future waste shipments, the impacts of transportation for the various cases are best compared by examining the total impacts of transporting a specific inventory of waste, independent of the annual shipping rates. The total amount of LLMW transported for each case can be calculated by multiplying the annual shipment quantities (reported in the WASTE_MGMT files in Appendix A of this report) by 10 years.

\subsection{SCOPE OF THE RISK ASSESSMENT}

The transportation risk assessment is limited to estimating the human health risks incurred during the actual transportation of LLMW by truck or rail. The risks to workers or to the public during the loading, unloading, and handling of waste before or after shipment are considered as part of the normal operations of the facility and are not included in the transportation assessment. Similarly, the transportation risk assessment does not address how increased transportation levels may possibly impact local traffic flow, noise levels, logistics, or infrastructure. The impacts that are addressed are discussed subsequently.

\subsubsection{Cargo-Related Impacts: Radiological}

For radioactive materials, the cargo-related impacts on human health during transportation are caused by exposure to ionizing radiation. For all cases, radiological risks (i.e., risks that result from the radioactive nature of the LLMW) are assessed both for routine (normal) transportation and for accidents. The radiological risk associated with routine transportation results from the potential exposure of people to low levels of external radiation in the vicinity of a loaded shipment. The radiological risk from transportation-related accidents lies in the potential release and dispersal of radioactive material into the environment during an accident and the subsequent exposure of people through multiple exposure pathways (i.e., exposure to contaminated ground or air or the ingestion of contaminated food).

All radiologically related impacts are calculated in terms of dose and associated health effects in the exposed populations. The dose of radiation calculated is the total effective dose equivalent as specified in $10 \mathrm{CFR}$ Part 20, which is the sum of the effective dose equivalent from exposure to external radiation and the 50-year committed effective dose equivalent (International Commission on Radiological Protection [ICRP] 1977) from exposure to internal radiation. Doses of radiation are calculated in units of rem (roentgen-equivalent man) for individuals and in units of person-rem for collective populations.

The potential exposures to the general population from transportation of radioactive materials, whether during routine operations or from postulated accidents, are usually at a low dose, such that the primary adverse health effect is the potential induction of latent cancers (i.e., cancers that occur after a latency period of several years from the time of exposure). The correlation of radiation dose and human health effects for low doses has been traditionally based on what is termed the "linear/no-threshold hypothesis," which has been described by various international authorities on protection against radiation. This 
hypothesis implies, in part, that even small doses of radiation cause some risk of inducing cancer and that doubling the radiation dose would mean doubling the expected numbers of cancers. The types of cancer induced by radiation are similar to "naturally occurring" cancers and can be expressed later in the lifetimes of the exposed individuals.

On the basis of the analyses conducted for this report, LLMW transportation-related operations are not expected to cause acute (short-term) radiation-induced fatalities or to produce immediately observable effects in exposed individuals. Acute radiation-induced fatalities occur at doses well in excess of $100 \mathrm{rem}$ (ICRP 1991), which generally would not occur for a wide range of transportation activities, including routine operations and accidents. ${ }^{1}$ For all severe accident scenarios analyzed, other short-term effects such as temporary sterility and changes in blood chemistry are not expected.

In this report, the radiological impacts are expressed as health risks in terms of the number of estimated latent-cancer fatalities, the incidence of cancer, and the genetic effects in exposed populations for each alternative. The health risk conversion factors (expected latent health effects per dose absorbed) were derived from ICRP publication 60 (ICRP 1991).

\subsubsection{Cargo-Related Impacts: Hazardous}

For hazardous materials, the cargo-related impacts to human health during transportation would be caused by exposure occurring as a result of container failure and chemical release during an accident (i.e., a collision with another vehicle or road obstacle). For all cases, chemical risks (i.e., risks that result from the toxicology of the chemical composition of the hazardous portion of LLMW) are assessed for cargo-related transportation accidents. The chemical risk from transportation-related accidents lies in the potential release, transport, and dispersion of hazardous chemicals into the environment during an accident and the subsequent exposure of people through primarily inhalation exposure. The risks from routine transportation operations, unlike the radiological risks, are due solely to vehicular exhaust emissions (i.e., vehicle-related impacts; see Section 2.5.3).

The risks from exposure to the hazardous component of LLMW during transportation-related accidents can be either acute (result in immediate injury or fatality) or latent (result in cancer that would present itself after a latency period of several years). Both population risks and risks to the maximally exposed individual (MEI) have been evaluated for transportation accidents. The acute health end points, potential lifethreatening effects, and potential adverse effects (PAEs) have been evaluated for the assessment of cargo-related population impacts from transportation accidents. The identification of chemicals in the hazardous component of LLMW having potential lifethreatening effects was based on gaseous and liquid substances designated "poison inhalation hazard" (PIH) chemicals by the U.S. Department of Transportation (DOT) (see Appendix E of the WM PEIS [DOE 1996]). Chemicals selected for the PAE analysis included PIHs and

${ }^{1}$ In general, individual acute whole-body doses in the range of 300-500 rem are expected to cause fatality in 50\% of the exposed individuals within 30-60 days (ICRP 1991). 
gaseous or liquid chemicals with inhalation toxicity values (reference concentrations) available from the U.S. Environmental Protection Agency (EPA 1993a,b).

The acute effects evaluated are assumed to exhibit a threshold nonlinear relationship with exposure; that is, some low level of exposure can be tolerated without inducing a health effect. In order to estimate risks, chemical-specific values for the potential life-threatening concentration (PLC) and potential adverse effect concentration (PAEC) were developed. All individuals exposed at these levels or higher following an accident are included in $H W$ transportation risk estimates. Use of this type of population risk descriptor (i.e., estimate of the number of persons exposed above a specified benchmark level) is recommended under EPA guidance (EPA 1992). Figure 2.1 presents a conceptual diagram of how PLC and PAEC values were derived. Additionally, to address MEIs, the locations of maximum $H W$ concentration were identified for shipments with the largest potential releases of individual HW components.

A latent health end point, the increased risk of cancer, has also been used to assess the cargo-related population impacts from accidents involving carcinogen releases. Traditionally, risk assessment for chemical carcinogens characterizes risk to the MEI (EPA 1989). The MEI is included in the assessment of the hazardous component of risk from LLMW shipments (see Section 3.3.3). Additionally, for assessing risk to the general population, increased carcinogenic risk has been expressed as the number of individuals in the general population with an increased lifetime cancer risk of $1 \times 10^{-6}$ greater, as recommended under EPA guidance for characterization of population risks (EPA 1992). Cancer risks greater than $1 \times 10^{-6}$ have been designated as increased cancer risk concern levels. Overall population risk (in terms of the number of excess cancers expected in the population) has not been calculated for the hazardous component of LLMW as it was for the

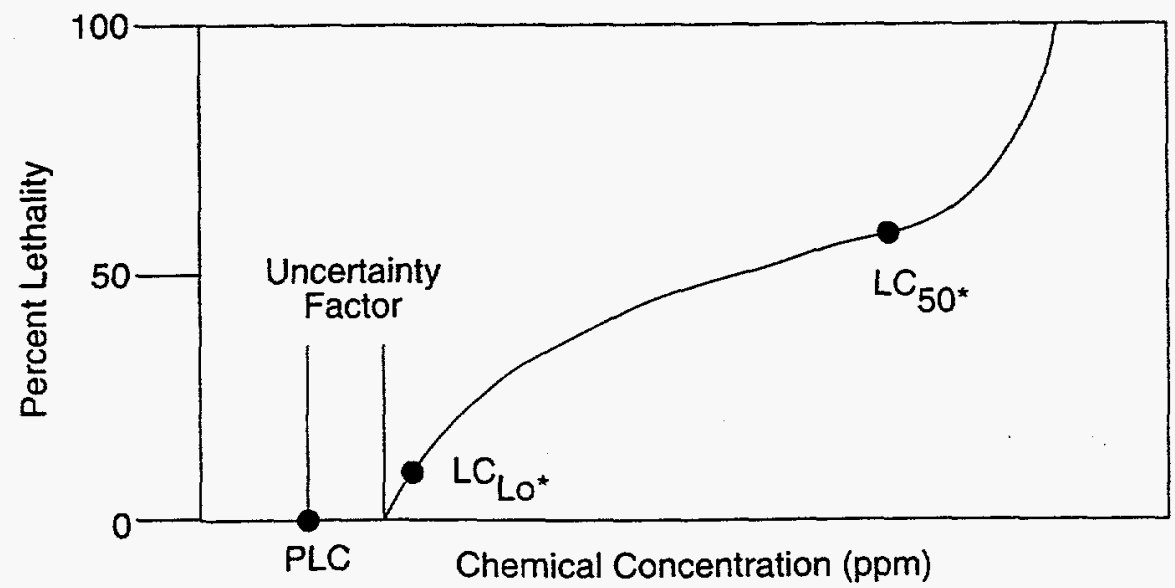

FIGURE 2.1 Conceptualization of Threshold Health Effects $\left(\mathrm{LC}_{50}=\right.$ concentration that is lethal to $50 \%$ of the population; and $L_{C_{L_{0}}}=$ lowest reported lethal concentration). Values for PAEC are derived by using $\mathrm{TC}_{50}$ levels (toxic to $50 \%$ of population) or $T C_{L o}$ levels (lowest toxic levels), instead of $L C_{50}$ and $L C_{L_{0}}$. 
radiological component of the same waste because this calculation would require estimation of average exposure levels in the population, while standardized cancer risk assessment methods address only MEIs. Therefore, characterizing population cancer risks associated with the hazardous component of LLMW as the number of individuals experiencing an increased risk of $1 \times 10^{-6}$ was deemed preferable. Note that the cargo-related population cancer risks presented in this assessment cannot be directly compared with cancer risks for individuals. Instead, population risk has been assessed to account for potentially impacted individuals collectively.

\subsubsection{Vehicle-Related Impacts}

In addition to the cargo-related hazardous and radiological risks posed by transportation-related activities, risks are also assessed for vehicle-related causes for the same routes. These risks are independent of the radioactive nature of the cargo and would be incurred for similar shipments of any commodity. The vehicle-related risks are assessed for both routine conditions and accidents. Vehicle-related risks during routine transportation are caused by potential exposure to increased vehicular exhaust emissions. The routine risks are primarily associated with travel in urban environments. The vehicle-related accident risk refers to the potential for transportation-related accidents that result in fatalities due to physical trauma that are not related to the cargo in the shipment. State-specific rates for transportation-related fatalities are used in the assessment. Vehicle-related risks are presented in terms of estimated fatalities for each alternative.

\subsubsection{Transportation Modes}

Although LLMW could be transported by a variety of modes, all shipments have been assumed to take place by either truck or rail. For each case, risks have been calculated separately for all-truck and all-rail options, although the actual shipping campaigns for a selected alternative may involve a combination of the two modes. Rail shipments are assumed to take place by regular freight-train service; the use of special or dedicated rail service was not considered. Shipments by barge, although feasible for some sites, have not been explicitly considered because this mode of transportation is somewhat limited and has not been established as a major programmatic option for the WM PEIS assessment. Similarly, shipments by aircraft and other modes are not considered.

\subsubsection{Receptors}

In this report, transportation-related risks are calculated and presented separately for workers and for members of the general public. The workers considered are truck and rail crew members involved in transporting LLMW. The general public includes all persons who could be exposed to a shipment while it is moving or stopped en route. Potential risks are estimated for the collective populations of exposed people, as well as for MEIs. The collective population risk is a measure of the radiological and chemical risk posed to society 
as a whole by the alternative being considered. As such, the collective population risk is used as the primary means of comparing various alternatives. 


\section{METHODS FOR CALCULATING TRANSPORTATION-RELATED RISKS}

\subsection{OVERVIEW OF RISK ASSESSMENT METHOD}

The technical approach for conducting the radiological transportation risk assessment was developed following a thorough and critical review of the literature and existing National Environmental Policy Act documentation for federal actions involving transportation of radioactive materials. Consideration was also given to recent DOE commitments arising from both litigation and public awareness. The approach selected uses several computer models and databases to determine the risks for each case.

The approach for the radiological component of the transportation risk assessment is summarized in Figure 3.1. For each case, radiological risks are assessed for both routine transportation and accidents. For the routine assessment, risks are calculated for the collective populations of potentially exposed individuals, as well as for the MEIs. The accident assessment consists of two components: (1) an accident risk assessment, which considers the probabilities and consequences of a range of possible transportation-related accidents, including low-probability accidents that have high consequences, and high-probability accidents that have low consequences; and (2) an accident consequence assessment, which considers only the radiological consequences of the severe transportation-related accidents that are postulated to result in the largest releases of radioactive material.

The approach for the hazardous component of the transportation risk assessment is summarized in Figure 3.2. The approach is similar to the radiological approach; however, no cargo-related impacts are assessed under routine conditions.

The RADTRAN 4 computer code (Neuhauser and Kanipe 1993) is used for the routine and accident risk assessments to estimate the radiological impacts to collective populations. RADTRAN 4 was developed by Sandia National Laboratories (New Mexico) (SNL-NM) to calculate population risks associated with the transportation of radioactive materials by a variety of modes, including truck, rail, air, ship, and barge. The code has been extensively reviewed, updated, and used for transportation risk assessments since it was issued in the late 1970s.

The RADTRAN 4 calculations of population risk take into account both the consequences and the probabilities of potential exposures. The collective population risk is a measure of the total radiological risk posed to society as a whole by the alternative being considered. As such, the collective population risks are used as the primary means of comparing the various cases.

As a complement to the RADTRAN calculations, the RISKIND computer code (Yuan et al. 1993) is used to estimate scenario-specific radiological doses to MEIs for both routine operation and for accidents and to estimate population impacts for the accident consequence assessment. The RISKIND computer code was developed for the DOE Office of 


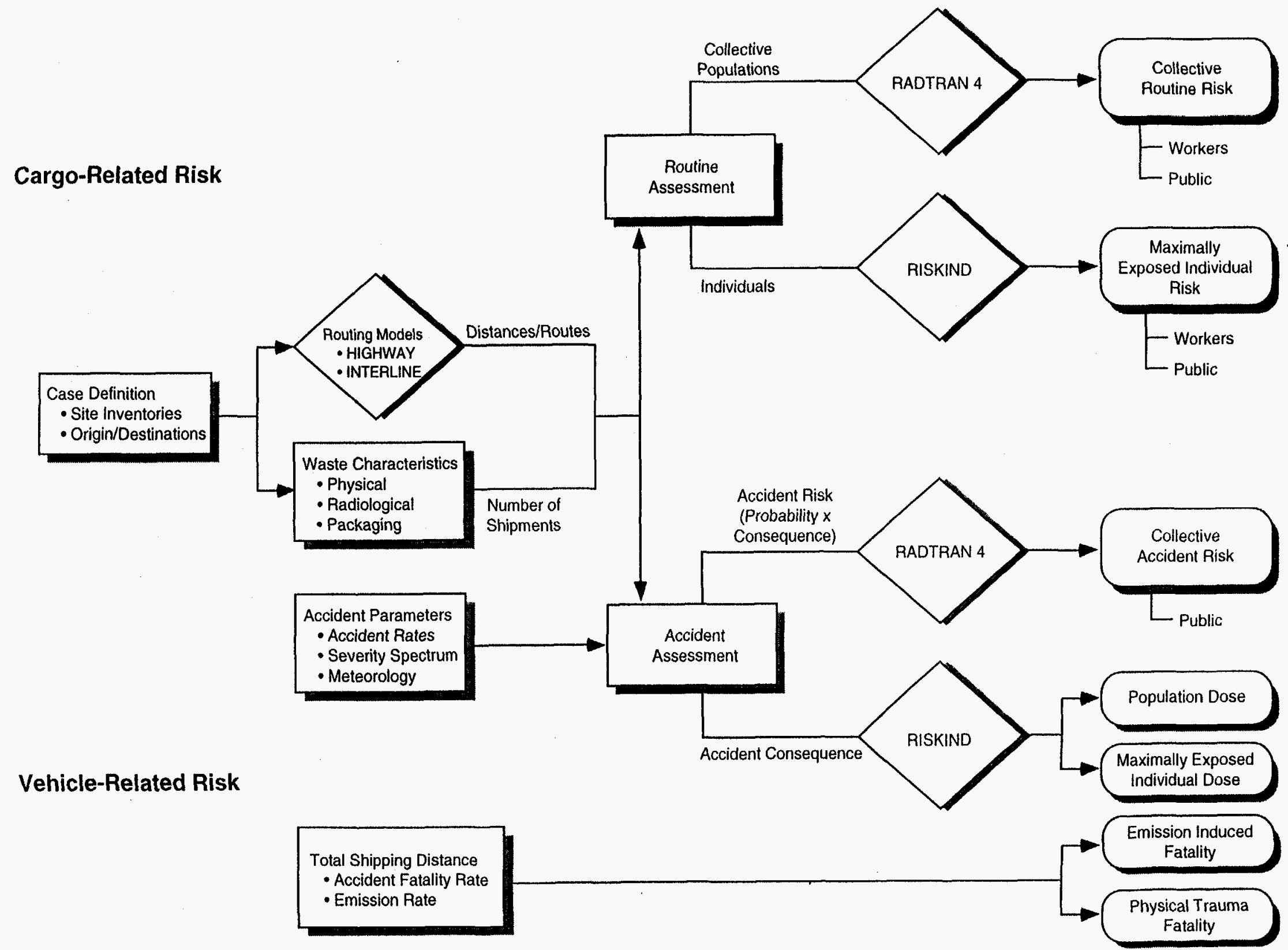

FIGURE 3.1 Technical Approach for the Radiological Component of the LLMW Transportation Risk Assessment 


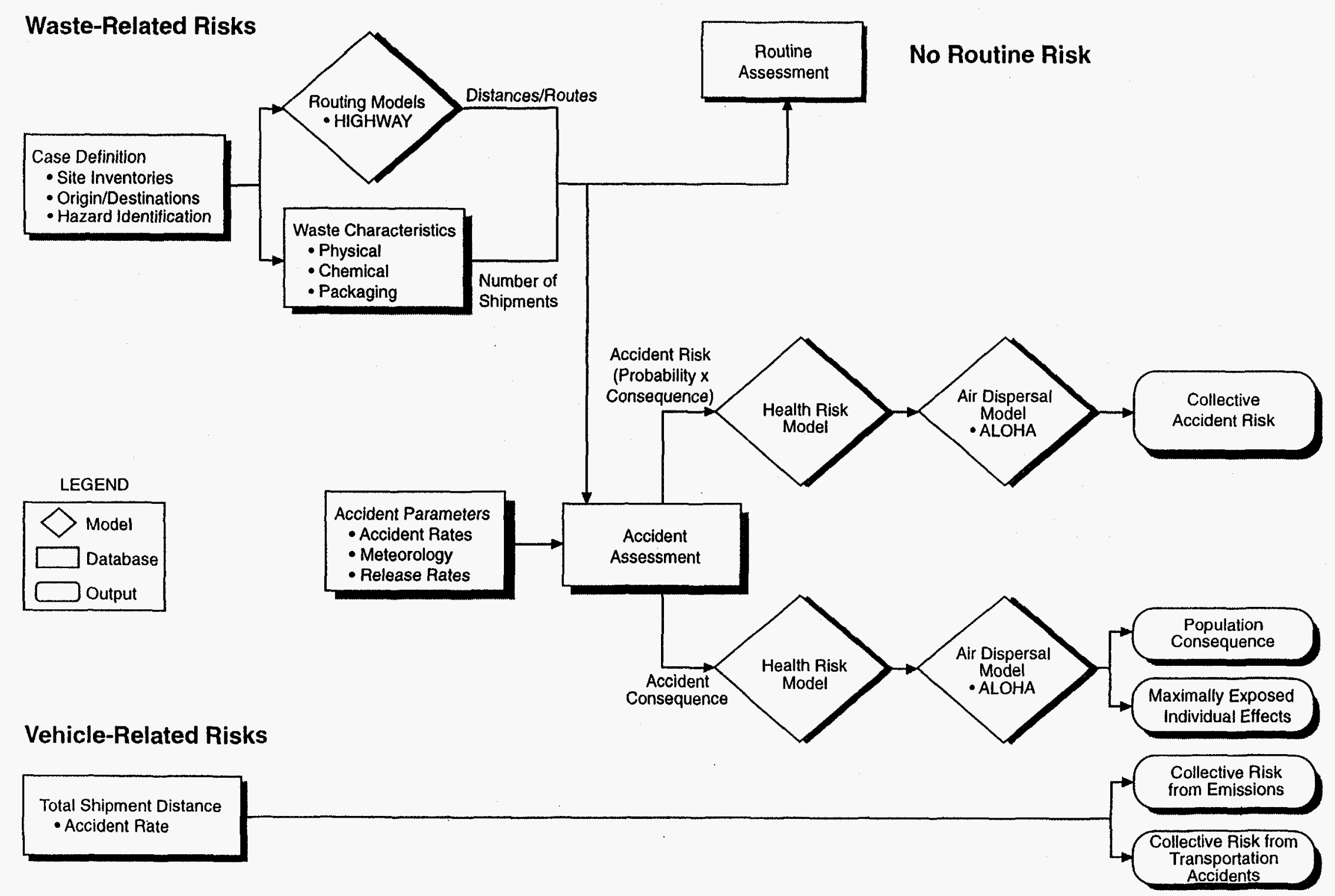

FIGURE 3.2 Technical Approach for the Hazardous Component of the LLMW Transportation Risk Assessment 
Civilian Radioactive Waste Management specifically to analyze radiological consequences to individuals and population subgroups associated with the transportation of spent nuclear fuel. Minor modifications to the code were made for WM PEIS applications to accommodate shipments of all types of radioactive waste.

The RISKIND calculations are conducted for the WM PEIS to supplement the results for collective risk calculated with RADTRAN 4. Whereas the results for collective risk provide a measure of the overall risks of each case, the RISKIND calculations are meant to address areas of specific concern to individuals and subgroups of population. Essentially, the RISKIND analyses are meant to address "what if" questions, such as, "What if I live next to a site access road?" or "What if an accident happens near my town?"

The HW transportation accident consequence assessment relies on the ALOHA ${ }^{\mathrm{TM}}$ model (version 5.1; Reynolds 1992) for both the collective population and individuals. The model is a widely applied code often used by the EPA to assist emergency field personnel in implementing emergency response measures. The ALOHA ${ }^{\mathrm{TM}}$ model has a built-in sourceterm algorithm that is used to compute the rate, quantity, and type of atmospheric release of a hazardous air pollutant, including pool evaporation from a volatile organic liquid spill. The model is able to handle frequently encountered accidental releases from ruptured tanks, drums, and pipes. The model incorporates a chemical data library of physical and chemical properties (such as vapor pressure, boiling point, and molecular weight) for several hundred chemical compounds. These properties, along with container content input, such as the container geometry and rupture characteristics (e.g., hole size), are used by ALOHA ${ }^{\mathrm{TM}}$ to compute chemical release rate and duration. The LLMW consequence assessment for HW assumes organic liquid spills and particulate releases are instantaneous as liquid and solid (as respirable fraction) aerosols. The release fractions are estimated with the approach used for radionuclide releases (described in Section 4.3.2). One additional source term is estimated for contaminated solids (with volatile organic compounds) spilled on the ground. The emission rate of these compounds is estimated with an evaporative gaseous emissions approach described in Section 3.3.2.2. These emission rates are used in the ALOHA ${ }^{\mathrm{TM}}$ code to provide hazard zone exposure estimates.

The risks associated with the consequences estimated with the ALOHA ${ }^{\mathrm{TM}}$ code are computed separately with a risk quantification spreadsheet program. This program, along with the ALOHA model, will be referred to for convenience as the ALOHA risk assessment modeling system (A-RAMS).

\subsection{ROUTINE (INCIDENT-FREE) RISK ASSESSMENT METHOD}

\subsubsection{Collective Population Risk}

The radiological risk associated with routine transportation results from the potential exposure of people to low-level external radiation in the vicinity of loaded shipments. No similar cargo-related risks occur from the hazardous component of LLMW. Because the 
radiological consequences (dose) occur as a direct result of normal operations, the probability of routine consequences is taken to be unity in the RADTRAN 4 code.

For routine transportation, the RADTRAN 4 computer code considers all major groups of potentially exposed persons. The RADTRAN 4 calculations of risk for routine highway and rail transportation include exposures of the following population groups:

1. Persons along the Route (Off-Link Population). Collective doses are calculated for all persons living or working within $0.8 \mathrm{~km}(0.5 \mathrm{mi})$ of each side of a transportation route. The total number of persons within the $1.6-\mathrm{km}$ (1-mi) corridor is calculated separately for each route considered in the assessment.

2. Persons Sharing the Route (On-Link Population). Collective doses are calculated for persons in all vehicles sharing the transportation route. This group includes persons traveling in the same or opposite directions as the shipment, as well as persons in vehicles passing the shipment.

3. Persons at Stops. Collective doses are calculated for people who may be exposed while a shipment is stopped en route. For truck transportation, these stops include stops for refueling, food, and rest. For rail transportation, stops are assumed to occur for purposes of classification.

4. Crew Members. Collective doses are calculated for truck and rail transportation crew members involved in the actual shipment of waste. Workers involved in loading or unloading are not considered.

The doses calculated for the first three population groups are added together to yield the collective dose to the general public; the dose calculated for the fourth group represents the collective dose to workers. The RADTRAN 4 models for routine dose are not intended for estimating specific risks to individuals.

The RADTRAN 4 calculations for routine dose are based on generically expressing the dose rate as a function of distance from a point source (Neuhauser and Kanipe 1993). Associated with the calculation of routine doses for each exposed population group are parameters such as the radiation field strength, the source-receptor distance, the duration of exposure, vehicular speed, stopping time, traffic density, and route characteristics such as population density. The RADTRAN manual contains derivations of the equations and descriptions of these parameters (Neuhauser and Kanipe 1993). The values for many of the most important parameters are presented in Section 4.

The collective routine risks are calculated for each LLMW case as follows. Each case is first defined as a set of origin-destination pairs, as shown in the WASTE_MGMT output files in Appendix A of this report. Representative highway and rail routes are then determined for each unique pair. The number of shipments transported across each linkage is then calculated for both truck and rail modes by dividing the estimated site-specific LLMW 
inventories by the assumed truck and rail shipment capacities. For shipments between each origin-destination pair, RADTRAN 4 is used to calculate the collective risks to workers and the public on the basis of representative radiological and physical properties of the LLMW. The collective risks are then summed over the set of origin-destination pairs to estimate the collective routine risks associated with that case.

\subsubsection{Maximally Exposed Individual Risk}

In addition to the assessment of the routine collective population risk, the risk to MEIs has been estimated for a number of hypothetical exposure scenarios by using RISKIND. The receptors include transportation crew members, departure inspectors, and members of the public exposed during traffic delays, while working at a service station, or while living near a DOE site.

The dose to each MEI considered is calculated with RISKIND for an exposure scenario defined by a given distance, duration, and frequency of exposure specific to that receptor. The distances and durations of exposure are similar to those given in previous transportation risk assessments (DOE 1987b, 1990a) and are presented in Section 4 . The scenarios are not meant to be exhaustive but were selected to provide a range of potential exposure situations.

The RISKIND external dose model considers direct external exposure and exposure from radiation scattered from the ground and air. RISKIND is used to calculate the dose as a function of distance (millirem per hour for stationary exposures and millirem per event for moving shipments) from a shipment on the basis of the dimensions of the shipment. The code approximates the shipment as a cylindrical volume source; and the calculated dose includes contributions from secondary radiation scattering from buildup (scattering by waste contents), cloudshine (scattering by the air), and groundshine (scattering by the ground). The dose rates calculated by using RISKIND have been shown to be comparable with output from existing shielding codes for various waste configurations. As a conservative measure, credit for potential shielding between the cask and the receptor is not considered.

\subsubsection{Vehicle-Related Routine Risk}

Vehicle-related health risks resulting from routine transportation may be associated with the generation of air pollutants by transport vehicles during shipment and are independent of the radioactive nature of the shipment. The health end point assessed under routine transportation conditions is the excess latent mortality due to inhalation of vehicular exhaust emissions. Risk factors for pollutant inhalation in terms of latent mortality have been generated by Rao et al. (1982). These risk factors are $1 \times 10^{-7}$ mortality $/ \mathrm{km}$ $\left(1.6 \times 10^{-7} / \mathrm{mi}\right)$ and $1.3 \times 10^{-7}$ mortality $/ \mathrm{km}\left(2.1 \times 10^{-7} / \mathrm{mi}\right)$ of truck and rail travel in urban areas, respectively. The risk factors are based on regression analyses of the effects of sulfur dioxide and particulate releases from diesel exhaust on mortality rates. Excess latent mortalities are assumed to be equivalent to latent-cancer fatalities. Vehicle-related risks 
from routine transportation are calculated for each case by multiplying the total distance traveled in urban areas by the appropriate risk factor. Similar data are not available for rural and suburban areas.

Risks are summed over the entire route and over all shipments for each LLMW case. This method has been used in several reports to calculate risks from routine transportation of radioactive wastes (DOE 1986, 1987a, 1990a). Lack of information for rural and suburban areas is an obvious data gap, although the risk factors would presumably be lower because of lower total emissions from all sources and lower population densities in rural and suburban areas.

\subsection{ACCIDENT ASSESSMENT METHOD}

\subsubsection{Accident Risk Assessment}

The risk analysis for potential accidents differs fundamentally from the risk analysis for routine transportation because occurrences of accidents are statistical in nature. The accident risk assessment is treated probabilistically in RADTRAN 4 and in the A-RAMS approach used to estimate the hazardous component of risk. Accident risk is defined as the product of the accident consequence (dose or exposure) and the probability of the accident occurring. In this respect, RADTRAN 4 and the A-RAMS approach for the hazardous component estimate the collective accident risk to populations by considering a spectrum of transportation-related accidents. The spectrum of accidents is designed to encompass a range of possible accidents, including low-probability accidents that have high consequences, and high-probability accidents that have low consequences (such as "fender benders"). The results for collective accident risk can be directly compared with the results for routine collective risk because the former results incorporate the probabilities of accident occurrences.

The RADTRAN 4 calculation of collective accident risk employs models that quantify the range of potential accident severities and the responses of transported packages to accidents. The spectrum of accident severity is divided into a number of categories. Each category of severity is assigned a conditional probability of occurrence - that is, the probability that an accident will be of a particular severity if an accident occurs. The more severe the accident, the more remote the chance of such an accident. Release fractions, defined as the fraction of the material in a package that could be released in an accident, are assigned to each accident severity category on the basis of the physical and chemical form of the waste material. The models take into account the mode of transportation and the type of packaging being considered. The accident rates, the definition of accident severity categories, and the release fractions used in this analysis are discussed further in Section 4. The approach for the hazardous component of LLMW (A-RAMS) incorporates the same accident severity categories and release fractions used by RADTRAN 4.

For accidents involving the release of radioactive material, RADTRAN 4 assumes that the material is dispersed in the environment according to standard Gaussian diffusion 
models. For the risk assessment, default data for atmospheric dispersion were used, representing an instantaneous ground-level release and a small-diameter source cloud (Neuhauser and Kanipe 1993). The calculation of the collective population dose following the release and dispersal of radioactive material includes the following exposure pathways:

- External exposure to the passing radioactive cloud,

- External exposure to contaminated ground,

- Internal exposure from inhalation of airborne contaminants, and

- Internal exposure from the ingestion of contaminated food.

For the pathway of ingestion, state-specific food transfer factors, which relate the amount of radioactive material ingested to the amount deposited on the ground, were calculated in accordance with the methods described by the U.S. Nuclear Regulatory Commission (NRC) regulatory guide 1.109 (NRC 1977b) and were used as input to the RADTRAN code. Doses of radiation from the ingestion or inhalation of radionuclides are calculated by using standard dose conversion factors (DOE 1988a,b).

The primary exposure route of concern with respect to accidental release of the hazardous component of LLMW is inhalation. Although direct exposure to hazardous materials via other pathways, such as ingestion or dermal absorption, is also possible, these routes are expected to result in much lower exposure than the inhalation pathway doses. The likelihood of acute effects, such as those evaluated by using PLC and PAEC values, is much less for the ingestion and dermal pathways than for inhalation. Similarly, this assessment addresses inhalation of vapors and gases only; the potential for exposure of the general public via inhalation of particulates is also considered to be much lower than that for inhalation of vapors or gases because the acute toxicity of inhaled particulates is low. Although some particulates are carcinogens (e.g., cadmium salts), low exposure dose and duration make risks low compared with risks from vapors and gases.

The collective accident risk for each case is determined in a manner similar to that described for routine collective risks. Accident risks are first calculated for each unique origin-destination pair and then are summed over all pairs to estimate the total risk for the case. The accident risk assessment uses site-specific and waste-type-specific radiological and physical waste characteristics, as shown in the WASTE_MGMT output files in Appendix A of this report. In addition, the assessment uses route-specific information on population density and accident rates derived for individual states. 


\subsubsection{Accident Consequence Assessment}

\subsubsection{Radiological Accident Consequence Assessment}

The RISKIND code is used to provide a scenario-specific assessment of radiological consequences of severe transportation-related accidents. Whereas the RADTRAN 4 accident risk assessment considers the entire range of accident severities and their related probabilities, the RISKIND accident consequence assessment focuses on accidents that result in the largest release of radioactive material to the environment.

Accident consequences are presented for a shipment of waste that represents the highest potential radiological risk if an accident occurs. This "worst-case" waste is identified by screening the site-specific radiological waste characteristics (i.e., activity concentrations) developed for the WM PEIS, taking into account the physical forms of waste and the relative hazards of individual radionuclides. For the majority of waste shipments, the consequences of severe accidents would be less than those presented for the "worst-case" waste. The accident consequence assessment is intended to provide an estimate of the maximum potential impacts posed by a severe transportation-related accident.

The severe accidents considered in the consequence assessment are characterized by extreme mechanical and thermal forces. In all cases, these accidents result in a release of radioactive material to the environment. The accidents correspond to those within the highest accident severity category, as described previously. These accidents represent lowprobability high-consequence events. The probability of accidents of this magnitude occurring for each case depends on the total shipping distance for the case; however, accidents of this severity are expected to be extremely rare in general.

RISKIND was used for the accident consequence assessment for two reasons. First, the code has the ability to model the complex atmospheric (or site-specific) dispersion present in severe accidents. The atmospheric dispersion is modeled as an instantaneous release by using standard Gaussian puff methods. In addition, because severe accidents routinely involve fires, modeling of the potential radiological consequences takes into account physical phenomena resulting from the fire, such as buoyant plume rise. Second, RISKIND can be used to estimate the dose to MEIs in the vicinity of an accident. The location of the MEI is determined by RISKIND on the basis of the atmospheric conditions assumed at the time of the accident and the thermal characteristics of the release.

The accident consequences are calculated for both local populations and MEIs. The population dose includes the population within $80 \mathrm{~km}(50 \mathrm{mi})$ of the site of the accident. The exposure pathways considered are similar to those discussed previously for the accident risk assessment. Although remedial activities after the accident (e.g., evacuation or ground cleanup) would reduce the consequences of an accident, these activities were not given credit in the consequence assessment. 


\subsubsection{Hazardous Accident Consequence Assessment}

Container releases of hazardous chemicals in LLMW accidents can enter the atmosphere in one or a combination of three modes: (1) as a direct liquid aerosol (liquid spill; no pool); (2) as a volatile organic vapor from contaminated spoils pile (solid waste spill on ground); and (3) as a respirable aerosol fraction (solid spill; direct to atmosphere). The release rate is simulated by a different approach (described subsequently) specific to the physical characteristics of the mode of release.

Hazardous organic chemicals in WM liquid LLMW are assumed to be released directly into the atmosphere following a transportation accident. The release duration is assumed to be one hour. For organic liquid wastes, the amount of liquid spilled is based on statistical data regarding container breach rates and atmospheric release rates for truck and railcar accidents presented in Table 3.1 and is computed from known shipping quantities for each alternative route. The spilled liquids are assumed to form a vapor plume immediately and are modeled as an instantaneous atmospheric release of a liquid aerosol with ALOHA ${ }^{\mathrm{TM}}$.

Transportation accidents with solid wastes involve two release modes. The first mode, the evaporative gaseous emission approach, assumes that the entire cargo-load of the solid wastes would be dumped onto the ground to form a cone-shaped pile no greater than $1.2 \mathrm{~m}(4 \mathrm{ft})$ in height. On the basis of the average density of LLMW solid wastes of $1,250 \mathrm{~kg} / \mathrm{m}^{3}$ and the known shipping quantity per truck or railcar, the following equation is used to estimate the rate of gaseous organic chemical emissions for each alternative route (EPA 1988):

$$
E=D \times C_{s} \times A \times\left(P_{t}^{1.333333}\right) \times\left(M / d_{s c}\right)
$$

where

$$
\begin{aligned}
E & =\text { emission rate of a specific chemical compound }(\mathrm{g} / \mathrm{s}) \\
D & =\text { chemical diffusivity }\left(\mathrm{cm}^{2} / \mathrm{s}\right) \\
C_{s} & =\text { saturation vapor concentration }\left(\mathrm{g} / \mathrm{cm}^{3}\right) \\
M & =\text { chemical fraction in the waste, } \\
P_{t} & =\text { total soil porosity (use default }=0.35) \\
A & =\text { exposure area }\left(\mathrm{cm}^{2}\right), \text { and } \\
d_{s c} & =\text { effective depth of solid LLMW pile }(\mathrm{cm})
\end{aligned}
$$

The second release mode for solid waste accidents involves the direct release of particulates to the atmosphere by using a respirable aerosols emission approach. Because of the size 
TABLE 3.1 Container Breach Rates and Atmospheric Releases Derived from HMIRS Statistical Data for Truck and Railcar Accidents ${ }^{a}$

\begin{tabular}{|c|c|c|c|c|}
\hline $\begin{array}{c}\text { Transportation } \\
\text { Mode }\end{array}$ & $\begin{array}{c}\text { Container } \\
\text { Contents }\end{array}$ & Container Size & $\begin{array}{c}\text { Breach } \\
\operatorname{Rate}^{b}(\%)\end{array}$ & $\begin{array}{c}\text { Quantity } \\
\text { Release (\%) }\end{array}$ \\
\hline \multirow[t]{7}{*}{ Truck } & Liquid/gas & $\begin{array}{l}\text { Package freight containers } \\
\text { - } 0-2 \text { gal in capacity }\end{array}$ & 43.8 & \\
\hline & & - 2-10 gal in capacity & 45.10 & 36.80 \\
\hline & & - $10-50 \mathrm{gal}$ in capacity & 40.70 & 27.10 \\
\hline & & - >50-gal capacity & $35.90^{\mathrm{c}}$ & $19.90^{\mathrm{c}}$ \\
\hline & & Bulk containers ${ }^{d}$ & 100.00 & 116.20 \\
\hline & Solids & Package freight containers & 30.60 & 23.50 \\
\hline & & Bulk containers $^{\mathrm{d}}$ & 100.00 & 32.60 \\
\hline \multirow[t]{4}{*}{ Railcar } & Liquid/gas & Package freight containers & $25.00^{\mathrm{c}}$ & $38.00^{c}$ \\
\hline & & Bulk containers ${ }^{\mathrm{d}}$ & 100.00 & 6.60 \\
\hline & Solids & Package freight containers & 40.90 & 44.15 \\
\hline & & Bulk containers $^{\mathrm{d}}$ & 100.00 & 1.26 \\
\hline
\end{tabular}

a HMIRS = Hazardous Materials Information Reporting System.

b Breach rate refers to the number of shipment containers (expressed as a percentage of the total number of containers shipped) that are ruptured in a transportation accident.

c Statistical data used in hazardous component of LLMW transportation risk assessment.

d Bulk shipments include only one container per shipment.

distribution of the released particles, this method uses the same mass, aerosol, and respirable release fractions in the eight accident severity categories (Table 3.2), consistent with those used for the radiological transportation risk assessment.

For example, if a truck load of WM LLMW solid wastes contains $1,000 \mathrm{~kg}(2,205 \mathrm{lb})$ of benzene, the respirable particulate release of benzene under severity Category III would be $0.5 \mathrm{~kg}$ (i.e., $1,000 \mathrm{~kg} \times 0.1 \times 0.1 \times 0.05$ ). The mass, aerosol, and respirable release fractions are assumed to be zero in severity Category $\mathrm{I}$, indicating no atmospheric $\mathrm{HW}$ releases for a minor transportation-related accident. Thus the population risks for severity Category I will always be zero.

In many of these shipping accidents, a number of chemicals are released to the environment. The issue here is how to account for the inhalation of multiple chemicals for an individual downwind of the release. This additivity of human health impacts is addressed separately for increased cancer risk and the any-adverse-effects end points.

ALOHA $^{\mathrm{TM}}$ was run first for each of the chemicals to determine its individual plume footprint at the specified value for the increased carcinogenic risk concentration (ICRC) or 
TABLE 3.2 Release Fractions for Eight Accident Severity Categories

\begin{tabular}{|c|c|c|c|}
\hline \multirow{2}{*}{$\begin{array}{l}\text { Severity } \\
\text { Category }\end{array}$} & \multicolumn{3}{|c|}{ Release Fractions } \\
\hline & Mass & Aerosol & Respirable \\
\hline I & 0.00 & 0.0 & 0.00 \\
\hline II & 0.01 & 0.1 & 0.05 \\
\hline III & 0.10 & 0.1 & 0.05 \\
\hline IV-VIII & 1.00 & 0.1 & 0.05 \\
\hline
\end{tabular}

for the PAEC (see Table 3.3). By using an iteration method, the "composite" plume footprint for all chemicals of concern is determined such that the following relationship can be reached:

$$
\sum_{i=1}^{n} \frac{C_{n}}{T_{n}}=1,
$$

where

$$
\begin{aligned}
& C_{n}=\text { concentration at "composite" plume footprint for the } n \text {th chemical } \\
& \text { of concern, and } \\
& T_{n}=\text { threshold limit value (level of concern) for } n \text {th chemical. }
\end{aligned}
$$

This method would yield a larger plume area of influence of the chemical mixture than any one of its components.

Because predicting the exact location of a severe transportation-related accident is impossible, separate accident consequences are calculated for accidents occurring in rural, suburban, and urban zones of population density. Moreover, to address the effects of the atmospheric conditions existing at the time of an accident, two different atmospheric conditions are considered. The first case assumes neutral atmospheric conditions, and the second assumes stable conditions. Atmospheric conditions are discussed further in Section 4.

\subsubsection{Maximally Exposed Individual Risk}

\subsubsection{Radiological Maximally Exposed Individual Risk}

RISKIND is used to estimate the radiological dose to MEIs in the vicinity of the most severe transportation accidents. The location of the MEI is determined by RISKIND 
TABLE 3.3 Chemical-Specific Values of ICRC and PAEC for LLMW Carcinogenic and Any-Adverse-Effect Constituents

\begin{tabular}{|c|c|c|c|c|}
\hline $\begin{array}{l}\text { LLMW } \\
\text { Codes }\end{array}$ & Substance & \multicolumn{3}{|c|}{$\begin{array}{l}\text { Health End Point } \\
\text { Concentration (ppm) }\end{array}$} \\
\hline \multicolumn{2}{|c|}{ Carcinogenic Chemical Substances } & & $\operatorname{ICRC}(\mathrm{ppm}$ & \\
\hline $75-09-2$ & Dichloromethane (methylene chloride) & & 380.0 & \\
\hline $\mathrm{Cl}-2-\mathrm{x}$ & Dichloroethane & & 5.8 & \\
\hline $\mathrm{Cl}-4-\mathrm{x}$ & Tetrachloroethene & & 160.0 & \\
\hline HC-soluble $^{\mathrm{a}}$ & $\begin{array}{l}\text { Hydrocarbons: soluble (benzene, } \\
\text { xylene, toluene, etc.) }\end{array}$ & & 23.0 & \\
\hline $\mathrm{As}^{\mathrm{c}}$ & Arsenic & & 0.047 & \\
\hline $\mathrm{Cd}^{\mathrm{c}}$ & Cadmium & & 0.075 & \\
\hline $\mathrm{Cr}^{\mathrm{c}}$ & Chromium & & 0.024 & \\
\hline $\mathrm{Pb}^{\mathrm{d}}$ & Lead & & $\mathrm{d}$ & \\
\hline \multicolumn{2}{|c|}{ Any-Adverse-Effect Chemical Substances } & \multicolumn{3}{|c|}{ PAEC (ppm) } \\
\hline & & $15 \mathrm{~min}$ & $30 \min$ & $60 \mathrm{~min}$ \\
\hline $75-09-2$ & Dichloromethane (methylene chloride) & 147.0 & 73.0 & 37.0 \\
\hline Cl-3-x & 1,1,1-Trichloroethane & 31.0 & 16.0 & 7.8 \\
\hline Cl-F-x & $\begin{array}{l}\text { 1,1,2-Trichloro-1,2,2-trifluoroethane } \\
\text { (Freon 113) }\end{array}$ & 670.0 & 330.0 & 170.0 \\
\hline HC-soluble & $\begin{array}{l}\text { Hydrocarbons: soluble (benzene, } \\
\text { xylene, toluene, etc.) }\end{array}$ & 18.0 & 9.0 & 4.5 \\
\hline HC-insoluble & Hydrocarbons: insoluble & d & $d$ & $d$ \\
\hline $57-12-5^{d}$ & Cyanide & d & d & $\mathrm{d}$ \\
\hline $\mathrm{Ag}^{\mathrm{d}}$ & Silver & d & $\mathrm{d}$ & d \\
\hline $\mathrm{Ba}^{\mathrm{c}}$ & Barium & 0.15 & 0.076 & 0.038 \\
\hline $\mathrm{Hg}^{\mathrm{c}}$ & Mercury & 0.0062 & 0.0031 & 0.0016 \\
\hline $\mathrm{Pb}^{\mathrm{d}}$ & Lead & d & d & $\mathrm{d}$ \\
\hline $\mathrm{Se}^{\mathrm{d}}$ & Selenium & d & d & d \\
\hline
\end{tabular}

a $\mathrm{HC}=$ hydrocarbon.

b The carcinogenic potential of soluble hydrocarbons was calculated by using the ICRC value for benzene.

c Although ICRC or PAEC values are available for the substances, volatilization potential is limited; and the values are therefore not included in risk calculations.

d Inhalation toxicity values are not available for these substances.

e The PAE end point for soluble hydrocarbons was calculated by using the PAEC value for toluene. 
on the basis of the atmospheric conditions assumed at the time of the accident and the thermal characteristics of the release. The MEI is assumed to be present during the entire passage of the radioactive plume. The dose calculation considers inhalation, cloudshine, and groundshine for a period of two hours following the accident. No ingestion dose is considered.

\subsubsection{Hazardous Maximally Exposed Individual Risk}

The MEI for the hazardous component of LLMW was considered to be located at the point of highest chemical concentration accessible to the general public. This location is assumed to be $30 \mathrm{~m}$ (100 ft) from the release point (the closest distance to a residence from the middle of the roadway). Only the shipment accident resulting in the highest chemical concentration is evaluated for the MEI. To evaluate the MEI for each health end point, the primary factors considered were a combination of chemical potency, quantity released, and vapor plume dispersion, as reflected by the chemical concentrations in air predicted by the ALOHA $^{\text {TM }}$ model.

The following formula is used to determine the lifetime MEI carcinogenic risk to adults over an exposure period of 70 years:

$$
M E I \text { carcinogenic risk }=(C A \times I R \times E T \times E F \times E D \times S F) /(B W \times A T) \text {, }
$$

where

$$
\begin{aligned}
& C A=\text { chemical concentration in air }\left(\mathrm{mg} / \mathrm{m}^{3}\right) \text {, } \\
& I R=\text { inhalation rate for adult }\left(0.014 \mathrm{~m}^{3} / \mathrm{min}\right), \\
& E T=\text { exposure time }(\mathrm{min} / \mathrm{d})(\text { same as chemical release duration, which } \\
& \text { is assumed to be } 60 \mathrm{~min} / \mathrm{d} \text { ), } \\
& E F=\text { exposure frequency }(1 \mathrm{~d} / \mathrm{yr}), \\
& E D=\text { exposure duration }(1 \mathrm{yr}) \\
& S F=\text { inhalation slope factor }(\mathrm{mg} / \mathrm{kg}-\mathrm{d})^{-1} \text {, } \\
& B W=\text { average body weight for an adult }(70 \mathrm{~kg}) \text {, and } \\
& A T=\text { averaging time }(70 \mathrm{yr} \times 365 \mathrm{~d} / \mathrm{yr}) .
\end{aligned}
$$

The following formula is used to evaluate the MEI hazard quotient (HQ) for noncarcinogenic substances, on the basis of an average exposure period of 14 days for a 6-year-old child:

$$
H Q=[(C A \times I R \times E T \times E F \times E D) /(B W \times A T)] / R f D
$$


where

$$
\begin{aligned}
& C A=\text { chemical concentration in air }\left(\mathrm{mg} / \mathrm{m}^{3}\right) \text {, } \\
& I R=\text { inhalation rate for 6-year-old child, with moderate activity } \\
& \left(0.033 \mathrm{~m}^{3} / \mathrm{min}\right) \text {, } \\
& E T=\text { exposure time }(\mathrm{min} / \mathrm{d}) \text { (same as chemical release duration, which } \\
& \text { is assumed to be } 60 \mathrm{~min} / \mathrm{d} \text { ), } \\
& E F=\text { exposure frequency }(1 \mathrm{~d} / \mathrm{yr}) \text {, } \\
& E D=\text { exposure duration }(1 \mathrm{yr}) \text {, } \\
& B W=\text { average body weight for a } 6 \text {-year-old child }(21 \mathrm{~kg}) \text {, } \\
& A T=\text { averaging time }(14 \mathrm{~d} / \mathrm{yr} \times 1 \mathrm{yr}) \text {, and } \\
& R f D=\text { reference dose }(\mathrm{mg} / \mathrm{kg} / \mathrm{d}) \text {. }
\end{aligned}
$$

A value for $H Q$ greater than 1 indicates that an adverse effect for the MEI is likely. Note that the level of concern associated with exposure to these compounds does not increase linearly as $H Q$ values exceed 1. In other words, $H Q$ values do not represent a probability or a percentage. As the $\mathrm{HQ}$ value increases above 1 , greater concern exists about potential adverse effects; however, assuming that an $\mathrm{HQ}$ value of 10 indicates that adverse health effects are 10 times more likely to occur than for an $\mathrm{HQ}$ value of 1 is incorrect. Due to uncertainties and conservatism associated with the use of EPA reference dose (RfD) values to evaluate single brief exposures, the assumption may be made that the risk of adverse effects is minimal for substances with HQ values between 1 and 10 .

\subsubsection{Vehicle-Related Accident Risk Assessment}

The vehicle-related accident risk refers to the potential for transportation-related accidents that directly result in fatalities that are not related to the cargo in the shipment. This risk represents fatalities from mechanical causes. State-specific rates for transportation-related fatalities are used in the assessment and are discussed in Section 4. Vehicle-related accident risks are calculated for each case by multiplying the total distance traveled in each state by the appropriate state rate for transportation-related fatalities. In all cases, the vehicle-related accident risks are calculated by using distances for round-trip shipment.

\subsection{REPRESENTATIVE ROUTE DETERMINATION METHOD}

Representative truck and rail routes were determined for all of the pairs of origin and destination sites specified by the case definitions. The routes were selected to be consistent with current routing practices and all applicable routing regulations and 
guidelines; however, because the routes were determined for the purposes of risk assessment, the routes do not necessarily represent the actual routes that would be used to transport LLMW in the future.

The representative truck and rail routes were determined by using the routing models HIGHWAY 3.1 (Johnson et al. 1993a) and INTERLINE 5.0 (Johnson et al. 1993b), respectively. For both truck and rail transportation, the route characteristics that are most important to the radiological risk assessment include the total shipping distance between each origin-destination pair and the fractions of travel in rural, suburban, and urban zones of population density. The route selected determines the total potentially exposed population along a route and the expected frequency of transportation-related accidents. The route distances between major DOE sites are summarized in Tables 3.4 and 3.5 for truck and rail transportation, respectively.

\subsubsection{Truck Route Determination}

The HIGHWAY 3.1 computer program is used for predicting highway routes for transporting radioactive materials within the United States by truck. The HIGHWAY database is a computerized road atlas that currently describes approximately $386,243 \mathrm{~km}$ $(240,000 \mathrm{mi})$ of roads. A complete description of the interstate highway system and of all U.S. highways is included in the database. In addition, most of the principal state highways and a number of local and community highways are also identified. The code is updated periodically to reflect current road conditions and has been benchmarked against reported mileages and observations of commercial trucking firms.

Routes are calculated within the model by minimizing the total impedance between the origin and the destination. The impedance is basically defined as a function of distance and driving time along a particular segment of highway. One of the special features of the HIGHWAY 3.1 model is its ability to calculate routes that maximize the use of interstate highways. This feature allows the user to predict routes for shipment of radioactive materials that conform to the DOT transportation regulations, specifically docket HM-164. The population densities along a route are derived from 1990 data from the U.S. Bureau of the Census. Rural, suburban, and urban areas are characterized according to the following breakdown: rural population densities range from 0 to 54 persons per square kilometer $\left(0-139 / \mathrm{mi}^{2}\right)$; the suburban range is $55-1,284 / \mathrm{km}^{2}\left(140-3,326 / \mathrm{mi}^{2}\right)$; and urban is taken to mean all population densities greater than $1,284 / \mathrm{km}^{2}\left(3,326 / \mathrm{mi}^{2}\right)$.

\subsubsection{Rail Route Determination}

The INTERLINE 5.0 computer program is designed to simulate routing of the U.S. rail system. The INTERLINE database consists of 94 separate subnetworks and 


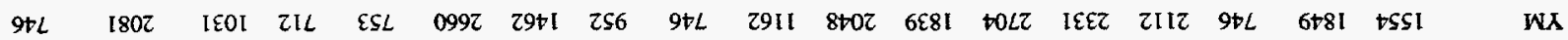

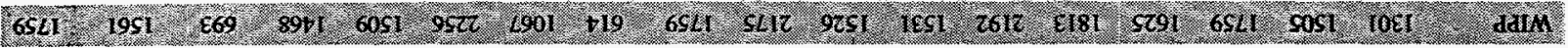

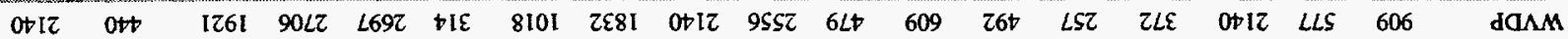

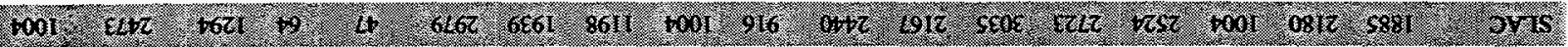
$\begin{array}{lllllllllllllllllll}\text { IIEZ } & 9 S 9 & Z † L I & 0 S L Z & 16 L Z & 196 & L 86 & \text { ES9I } & \text { IIEZ } & L Z L Z & 0 Z 9 & \text { tZ6 } & L 68 & 9 S 9 & 0 Z L & \text { IIEZ } & \text { Z68 } & \text { SLII } & \text { S\&S }\end{array}$

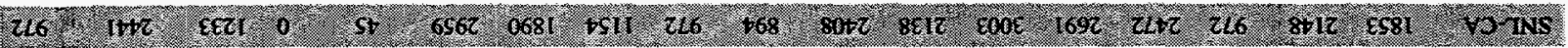

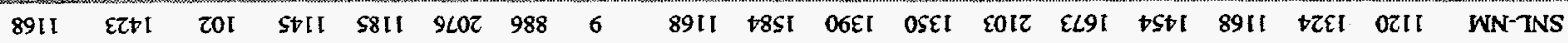

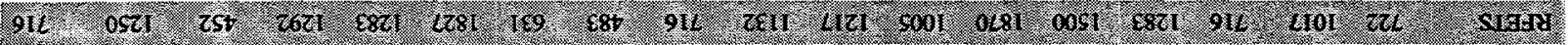

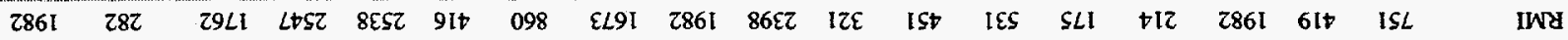

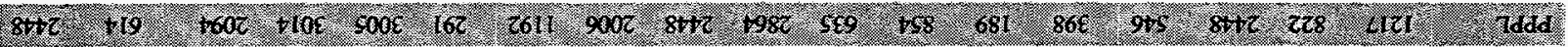

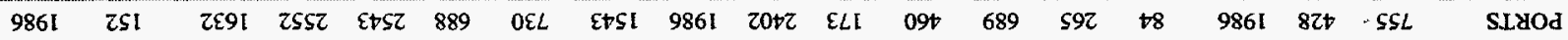

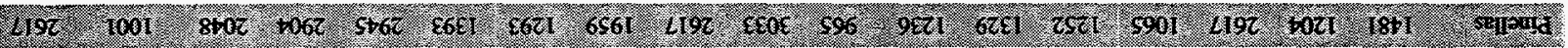

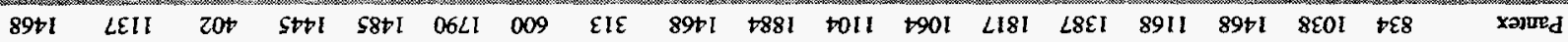

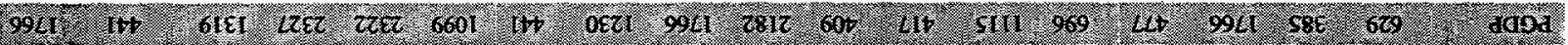

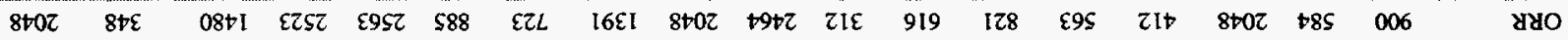

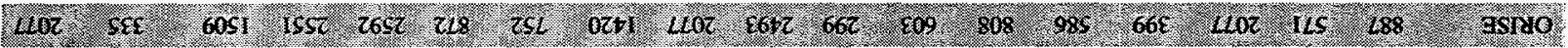

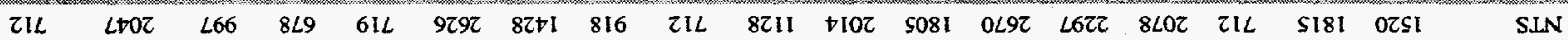

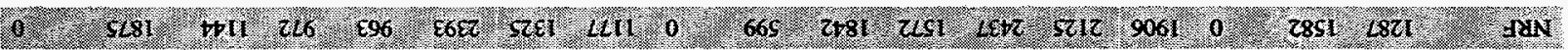

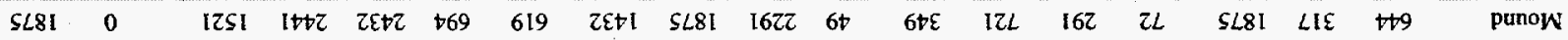

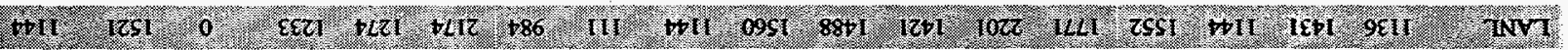

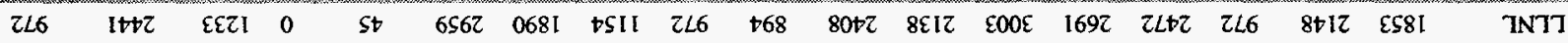

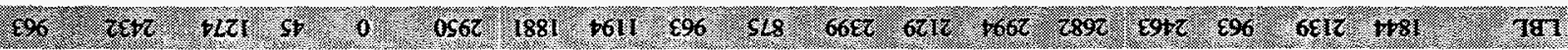

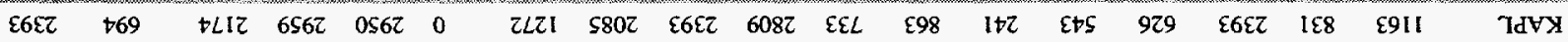

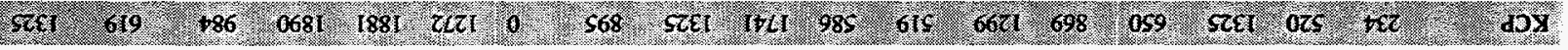

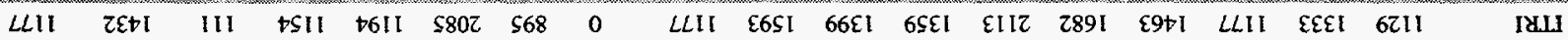

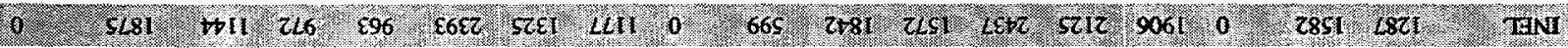

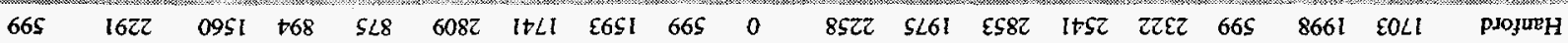

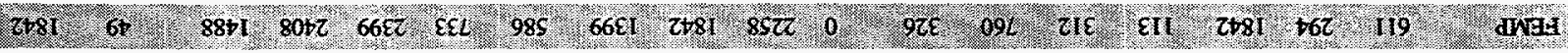

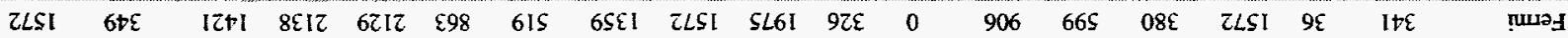

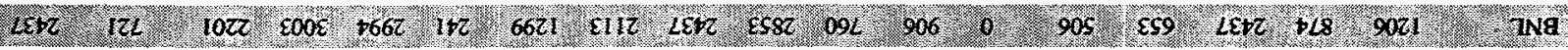

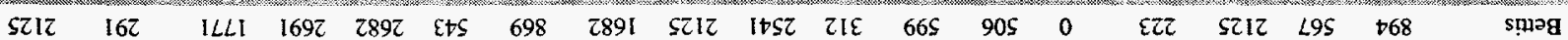

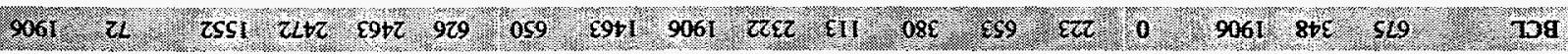
$\begin{array}{lllllllllllllllllll}0 & \$ L 81 & +11 & Z L 6 & \varepsilon 96 & \varepsilon 6 \varepsilon Z & \$ Z E I & L L 11 & 0 & 66 S & Z \$ 81 & Z L S I & L E+Z & \$ Z I Z & 9061 & 0 & Z 8 S I & L 8 Z I & M-7 N V\end{array}$

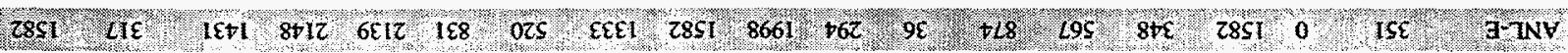

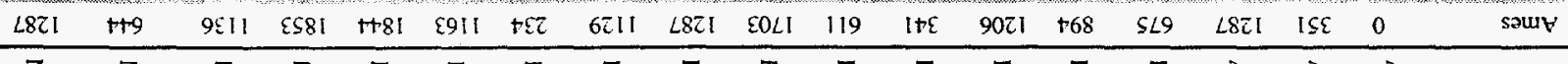

茎




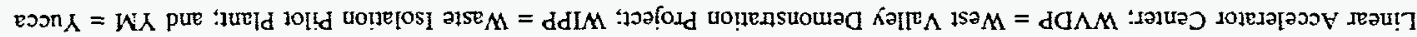

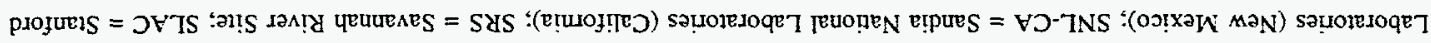

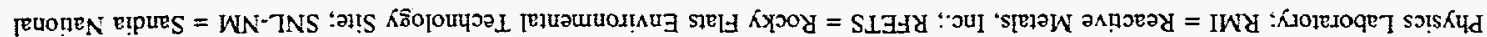

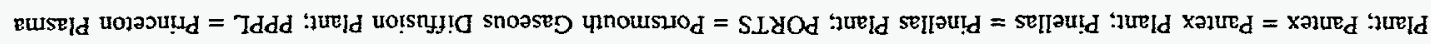

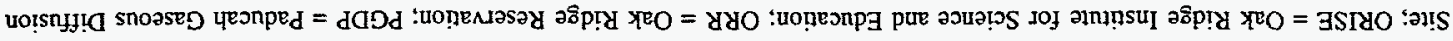

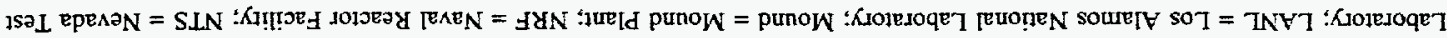

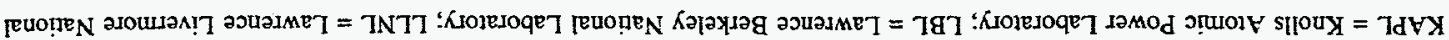

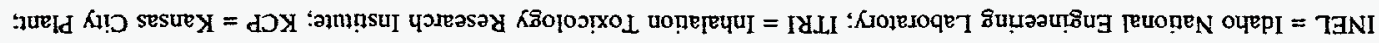

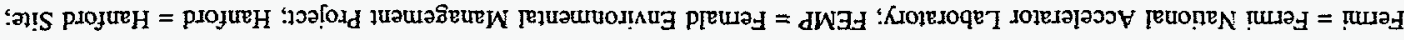

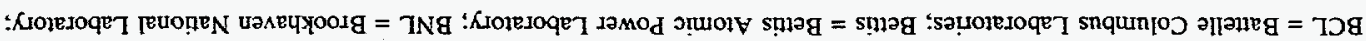

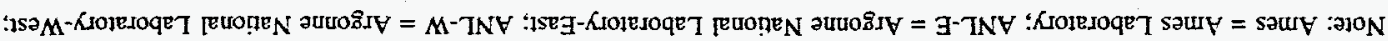

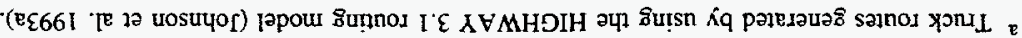

\begin{tabular}{|c|c|c|c|c|c|c|c|c|c|c|c|c|c|c|c|c|}
\hline 0 & $66 \varepsilon I$ & $\operatorname{Lot} z$ & $\varepsilon L L$ & $8 t t z$ & $2 \mathrm{IL}$ & $\varepsilon+6$ & 898 & $8+z z$ & $t S 9 z$ & 2612 & $t S \angle Z$ & $\varepsilon+Z I$ & EO6I & $\$ 812$ & $\pm 1 z z$ & WX \\
\hline 66 & $\theta$ & 0861 & 6851 & besi & 8911 & 809 & L901 & 2081 & 980 & 2891 & 291 & 808 & 857 & 1821 & Itor & $m$ \\
\hline $20 t 2$ & $086 \mathrm{I}$ & 0 & $8 \varepsilon L \tau$ & EZOI & $90 \angle 2$ & $\varepsilon 281$ & $\varepsilon L S I$ & 291 & 680 & $\downarrow \varepsilon t$ & $6 I t I$ & LESI & $5 \div 8$ & $99 L$ & $\mathfrak{E S L}$ & dOAM \\
\hline rets: & 665 & 8212 & 19 & 0288 & to & 8611 & 701 & 648 & 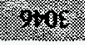 & 4856 & 6962 & 908 & 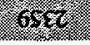 & 189 & 1882 & 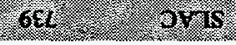 \\
\hline $8 t+2$ & $t Z S I$ & $\varepsilon 201$ & 0282 & 0 & OSLZ & $t+9$ & $819 \mathrm{I}$ & $9 Z L$ & $\angle 9 L$ & $0 t s$ & $0 z 9$ & $8 S E I$ & 895 & $6 L \varepsilon$ & $69 \varepsilon$ & $t+1+z$ \\
\hline 61 & 812 & 300 & 18 & 08 & 0 & s.1. & 2621 & 865 & rlos & $2 \operatorname{seg}$ & 1062 & $5+1$ & 262 & zes & 158 & $\mathrm{~S}_{7 \mathrm{~ns}}$ \\
\hline$\varepsilon \neq 6$ & 509 & $\varepsilon Z 8 I$ & 8611 & $t+91$ & stII & 0 & $t<t$ & +991 & $\angle 661$ & $t E S I$ & $0 S 61$ & toc & $9 z 21$ & $28 \varepsilon 1$ & IItI & WN-TNS \\
\hline 898 & 2001 & $9 \mathrm{si}$ & tzen & 8191 & 266 & tow & 0 & $s(t)$ & 2881 & rogt & 766 & 104 & 601 & sel & f881 & fary \\
\hline $8+z 2$ & 2281 & 291 & $6 L S Z$ & $9 Z L$ & $\angle D S Z$ & $+99 \mathrm{I}$ & SItI & 0 & 825 & $9 L z$ & 1921 & $8 L \varepsilon I$ & $\angle 89$ & 809 & $\$ 6 \varsigma$ & +122 \\
\hline tsez & 9808 & 687 & 9tor & 192 & tiog & 2661 & E681 & $8 z 5$ & 0 & 885 & $2 s 11$ & 0111 & 6001 & $91 \mathrm{~L}$ & $20 L$ & 0202 \\
\hline 2612 & $z \varepsilon 91$ & $t E t$ & $18 S z$ & $0+S$ & $\tau S S Z$ & $t \varepsilon \subseteq I$ & I9\&I & $9 L Z$ & $88 S$ & 0 & $t z 01$ & $8 \pm Z I$ & 0 & $I L \mathcal{E}$ & $85 \varepsilon$ & SILYOd \\
\hline$\sqrt{12}$ & 2941 & $61 \% 1$ & 6962 & 029 & 1062 & 0561 & 7261 & 1921 & 2311 & 7201 & 0. & $y 21$ & 78 & 0 & 268 & sipus \\
\hline$\varepsilon \neq Z I$ & $80 \varepsilon$ & LESI & $90 \mathrm{SI}$ & $8 \subseteq \mathcal{~ I ~}$ & $s t+I$ & toc & $t L L$ & $8 \angle E I$ & OLLI & $8+21$ & 5991 & 0 & $0+6$ & $960 \mathrm{I}$ & $s z I I$ & xazued \\
\hline 605 & 858 & st8 & 6SE & 895 & 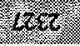 & $9 z 21$ & 2201 & 189 & 61 & 0 & 148 & 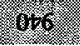 & 0 & ras & 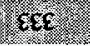 & rapd: \\
\hline$\$ 812$ & โ8Eโ & 992 & $\$ 8 \varsigma Z$ & $6 \angle \mathcal{E}$ & $\varepsilon Z \Omega Z$ & $z 8 E I$ & $t \varsigma \mathfrak{E l}$ & 809 & SIL & ILE & $\$ 89$ & $960 \mathrm{I}$ & tor & 0 & 01 & ช્о \\
\hline 716 & 0171 & ESt & $18 s 2$ & 698 & Iss & $11+1$ & 8 & $s$ & 200 & 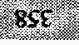 & 9 & 9.11 & . & 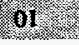 & 0 & 0812 \\
\hline 90 & $59 \varepsilon 1$ & $\varepsilon\llcorner\mathcal{E} Z$ & $6 \varepsilon L$ & $t I t z$ & $8 \angle 9$ & 606 & $9 £ 8$ & $+1 z z$ & $0 z 92$ & 12 & $0 z L z$ & $602 \mathrm{I}$ & 81 & iz & $08 \mathrm{iz}$ & IN \\
\hline 92 & $6 s 4$ & 0716 & tool & TI 182 & 216 & 8911 & 912 & 2861 & 8172 & 9861 & 2198 & 8941 & 994 & $870 z$ & 402 & tran \\
\hline 1802 & [9S1 & $0 t t$ & $\varepsilon<t z$ & $9 \$ 9$ & $1 t+2$ & $\varepsilon z+1$ & OSZI & 282 & $t 19$ & $Z S I$ & 1001 & LEII & 10 & $8+\varepsilon$ & sEE & punow \\
\hline 1Eor & 869 & 1261 & 6621 & $(2+4)$ & isza & 201 & zst & (29LI) & 6607 & 2891 & 8702 & $20 t$ & 610 & $08+1$ & 6091 & WNT \\
\hline ZIL & $89+1$ & $90 \angle Z$ & +9 & $0 S L Z$ & 0 & $s+11$ & 2621 & $L t s z$ & $+10 \varepsilon$ & $255 z$ & t06z & $S t t I$ & LZEZ & $\varepsilon Z \varsigma \tau$ & ISSZ & TN7T \\
\hline 89 & 6051 & 1697 & 6 & 1622 & $s t$ & 8811 & $\varepsilon 8 \mathrm{CI}$ & $8 \mathrm{EST}$ & soos & 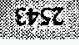 & $6+67$ & 8871 & 202 & Ess & 2652 & 791 \\
\hline 0992 & $9 s z z$ & $t \mid \varepsilon$ & $6 L 62$ & 196 & $6 \subseteq 6 z$ & $9 L 0 Z$ & $\angle 281$ & 915 & $16 z$ & 889 & $\varepsilon 6 \varepsilon I$ & $06 L I$ & $660 \mathrm{I}$ & $\$ 88$ & $z \angle 8$ & $7 d \forall x$ \\
\hline 271 & 1901 & 8101 & $6 \pm 61$ & 286 & 0681 & 988 & 189 & 098 & 2611 & $0 \varepsilon$ & \&6zl & 609 & $1+$ & EzL & $\mathrm{cs}$ & $8 z+1$ \\
\hline$\tau 56$ & \pm 19 & $z \varepsilon 81$ & 8611 & $\varepsilon 59 I$ & $t s 11$ & 6 & $\varepsilon 8 t$ & $\varepsilon \angle 91$ & $900 z$ & $\varepsilon+\mathcal{S} 1$ & $6 S 61$ & $\varepsilon \mathfrak{E} \varepsilon$ & OEZI & $16 \varepsilon !$ & $0 z+1$ & I\&LI \\
\hline 92 & $6 S 4$ & $0+12$ & toot & $118 Z$ & $2 L 6$ & 8911 & $9 \mathrm{IL}$ & $286 \mathrm{I}$ & $8+72$ & 9861 & $219 z$ & 8901 & 9811 & $8+02$ & 402 & $\mathrm{TAN}$ \\
\hline 2911 & SLIZ & $9 s \varsigma z$ & 916 & $\angle Z L Z$ & 568 & $\pm 8 S I$ & $z \varepsilon 11$ & $86 \llbracket z$ & +982 & $z 0+\tau$ & $\mathcal{E} \mathcal{E}$ & $+88 i$ & 2812 & $+9+z$ & $\varepsilon 6+z$ & pjojureH \\
\hline 8102 & 9251 & 624 & $0+2$ & 029 & 8072 & $06 \mathrm{er}$ & art & IIE & $s \in 9$ & $\mathrm{cL}$ & 596 & 7011 & 607 & zIE & 662 & dwal \\
\hline $6 \varepsilon 8 \mathrm{I}$ & IESI & 609 & $\angle 912$ & $t 26$ & $8 \varepsilon 1 Z$ & OSEI & 5001 & ISt & $t 58$ & $09 t$ & $9 \notin Z I$ & +901 & LIt & 919 & $\varepsilon 09$ & guat \\
\hline $102 z$ & 2616 & 267 & SqOS & 268 & Eoos & solz & 0.81 & IES & 681 & 689 & 629 & 2181 & situ & 128 & 808 & $0<92$ \\
\hline IEEZ & El8I & $\angle \Omega \tau$ & $\varepsilon \tau<z$ & $9 \$ 9$ & I 692 & $\varepsilon \angle 9 I$ & $00 S 1$ & $S \angle I$ & $86 \varepsilon$ & $\$ 9 \tau$ & $\tau S Z I$ & $\angle 8 \varepsilon I$ & 969 & 67 & 985 & ร!มวg \\
\hline 2112 & s?91 & $2 L$ & fzse & $0 z 2$ & $z L+2$ & $t s+1$ & $\varepsilon 82 \mathrm{Z}$ & +12 & $96 s$ & 18 & $590 \mathrm{I}$ & 8911 & $\omega t$ & 2lo & 668 & 708 \\
\hline $9+L$ & $6 S \angle I$ & $0+12$ & tool & IIEZ & $z L 6$ & 8911 & $91 L$ & 2861 & $8 t+2$ & 9861 & $\angle 19 \bar{Z}$ & $89+I$ & $99 \angle I$ & $8+0 \bar{c}$ & $\angle L O Z$ & $M-7 N V$ \\
\hline 6681 & sost & LS & 0812 & 268 & $8+12$ & 11 & $\angle 101$ & 615 & 228 & $8 z t$ & pozI & $8 \mathrm{kOl}$ & $\$ 8 \varepsilon$ & 3 & 115 & B-TNV \\
\hline$t \subseteq S I$ & $10 \leq 1$ & 606 & 5881 & $\$ L I I$ & $E S 81$ & $0 \bar{c} 11$ & $i \bar{L}$ & $1 S L$ & $\angle 1 z \mid$ & $S S L$ & $18+1$ & $+\varepsilon 8$ & 629 & 006 & $\angle 88$ & $0 z s 1$ \\
\hline 3 & $\underset{\Xi}{\Xi}$ & $\stackrel{\sum}{\underline{\Sigma}}$ & 3 & 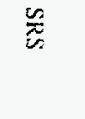 & $\frac{n}{3}$ & $\frac{z}{z}$ & $\frac{\frac{\pi}{2}}{\frac{3}{6}}$ & $\underline{\underline{z}}$ & $\frac{\pi}{\underline{x}}$ & 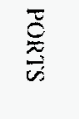 & 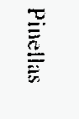 & 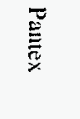 & $\stackrel{\Xi}{\Xi}$ & $\underset{\pi}{\pi}$ & 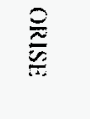 & $\frac{Z}{\dot{z}}$ \\
\hline
\end{tabular}


TABLE 3.5 Rail Route Distances (mi) between Major DOE Sites ${ }^{a}$

\begin{tabular}{|c|c|c|c|c|c|c|c|c|c|c|c|c|c|c|c|c|c|c|}
\hline & $\stackrel{n}{\stackrel{3}{E}}$ & $\stackrel{\overline{\bar{z}}}{\grave{c}}$ & 产 & ¿્. & 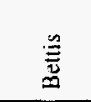 & $\bar{z}$ & 蓄 & $\stackrel{\hat{\overline{\mathbf{s}}}}{\mathbf{s}}$ & $\begin{array}{l}\text { 䮍 } \\
\text { 全 }\end{array}$ & $\underline{\underline{\Delta}}$ & $\bar{q}$ & 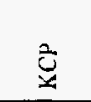 & & $\overrightarrow{\underline{a}}$ & $\overrightarrow{\underline{z}}$ & 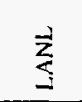 & $\underline{\underline{z}}$ & 苂 \\
\hline mes & & 329 & 1242 & 700 & 823 & 1365 & 291 & 7 & 1788 & 242 & Hor & 275 & 1126 & 1873 & 2018 & 124 & 715 & 242 \\
\hline $\mathbf{A N L}-\mathbf{E}$ & 329 & 0 & 1655 & 401 & 518 & 1066 & 49 & 412 & 2201 & 1655 & 1351 & 439 & 827 & 2549 & 2506 & 1288 & 416 & 655 \\
\hline AI & 1242 & 1655 & 0 & 1942 & 2133 & 2607 & 1533 & 1907 & 658 & 0 & & 238 & 68 & 1102 & 1100 & 9 & 1926 & 0 \\
\hline $\mathrm{BCl}$ & 200 & 401 & 1942 & 0 & 280 & 856 & 427 & 135 & 2488 & 1942 & 1759 & 753 & 615 & 2573 & 2718 & 1696 & 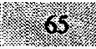 & 1942 \\
\hline Bettis & 823 & 518 & 2133 & 280 & 0 & 772 & 543 & 475 & 2611 & 2133 & 1857 & 943 & 533 & 2696 & 2840 & 1794 & 345 & 2133 \\
\hline $3 \mathrm{NL}$ & 1365 & 1066 & 2607 & 855 & 772 & 0 & 1088 & 984 & 3153 & 2607 & 2414 & 1518 & 239 & 3238 & 3383 & 2351 & 920 & 2607 \\
\hline Ferm & 291 & 49 & 1533 & 427 & 543 & 1088 & 0 & 441 & 1971 & 1533 & 1356 & 45 & 853 & 2343 & 2341 & 1405 & 443 & 1533 \\
\hline FEM & 717 & 412 & 1907. & 135 & 475 & 984 & 41 & 0 & 2505 & 1907 & 1751 & 717 & 745 & 2590 & 273 & 1688 & 69 & 1907 \\
\hline & 1788 & 2201 & 658 & 2488 & 2611 & 3153 & 1971 & 2505 & 0 & 658 & 1793 & 1784 & 2914 & 986 & 973 & 1725 & 2472 & 658 \\
\hline NEL & 1242 & 1655 & 0 & 1942 & 2133 & 2607 & 1533 & 1907 & 658 & 0 & 1247 & 1238 & 2468 & 1102 & 1103 & 1179 & 1926 & 0.0 \\
\hline ITRI & 1187 & 1351 & 1247 & 1759 & 1857 & 2414 & 1356 & 1751 & 1793 & 1247 & & 932 & 217 & 1266 & 12 & 104 & 1767 & 1247 \\
\hline $\mathrm{keP}$ & 275 & 439 & 1238 & 753 & 943 & 1518 & 453 & 771 & 1784 & 1238 & 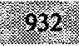 & 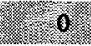 & 1250 & 2016 & 2013 & rean & 708 & 1238 \\
\hline KAP & 1126 & 827 & 2468 & 615 & 533 & 239 & 85 & 745 & 2914 & 2468 & 20 & 125 & & 2999 & 3144 & $2 . .4$ & 680 & 2468 \\
\hline 181 & 1873 & 2549 & 1102 & 2573 & 2696 & 3238 & 2348 & 2590 & 986 & 1102 & 1266 & 2070 & $299 \%$ & 10 & 46 & 1354 & 2 & 2078 \\
\hline & 2018 & 2506 & & & 2840 & 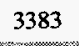 & & & 973 & 0 & & & 3144 & 46 & 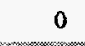 & 1326 & & \\
\hline & 1124 & 1288 & 1179 & 1696 & 1799 & 2351 & 14 & 1688 & 1725 & 9 & 104 & 9 & 2122 & 1354 & 1126 & 0 & & 79 \\
\hline & & & & 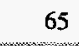 & & & & & & 26 & 176 & & & 2717 & 2695 & & 0 & 26 \\
\hline $\mathrm{NRI}$ & 1242 & 1655 & $\theta$ & 1942 & 2133 & 2607 & 1533 & 1907 & 658 & 0 & 1247 & 1238 & 2468 & 1102 & 1100 & 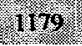 & 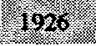 & 28 \\
\hline NTS & 1674 & $23+8$ & 756 & 2374 & 2496 & 3039 & 1997 & 2391 & 1302 & 756 & 1065 & 1670 & 2800 & 860 & 1370 & 1169 & 2386 & 756 \\
\hline gruse & 956 & 651 & 2099 & 366 & 714 & 1221 & 679 & 331 & 2644 & 2099 & 1988 & 81 & 981. & 2890 & 2868 & 1926 & 301 & $009 \%$ \\
\hline ORR & 954 & 649 & 2055 & 393 & 903 & 1152 & 682 & 358 & 2601 & 2055 & 174 & 838 & 957 & 2686 & 2831 & 1686 & 328 & 2055 \\
\hline PGDP & 646 & 390 & 1699 & 881 & 816 & 1346 & 469 & 468 & 245 & 1692 & 1539 & 482 & 1706 & 2490 & 2460 & 1476 & 904 & 1699 \\
\hline Pantex & 809 & 972 & 1141 & 1381 & 1479 & 2035 & 977 & 1373 & 1686 & 1141 & 37 & 4 & 1807 & 1561 & 1534 & 483 & 1396 & 1141 \\
\hline Pincinas & 1623 & 1319 & 2721 & 1151 & 1203 & 1585 & 13.44 & 1116 & 3267 & 2721 & 2079 & 1503 & $13 \%$ & 3278 & 3491 & 2183 & 1086 & 2721 . \\
\hline PORTS & 727 & 422 & 1975 & 31 & 429 & 921 & 431 & 207 & 2515 & 1975 & 176 & onsmo & & 2767 & 2745 & - & . & 1975 \\
\hline PRPI & 1197 & 898 & 2507 & 655 & 400 & 410 & 924 & 9 & 2985 & 2507 & 2248 & 1280 & 214 & 3298 & 3276 & 2186 & 79 & 2507 \\
\hline RMI & 717 & 418 & 2060 & 207 & 136 & 648 & 445 & 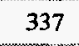 & 250 & 2060 & 1769 & & 408 & 200 & 2829 & 1706 & 272 & 2060 \\
\hline Ers & 782 & 1194 & 738 & 1502 & 1692 & 228 & 1016 & 1466 & 8128 & 738 & 572 & 778 & 2027 & 1320 & 1304 & 504 & 180 & 27 \\
\hline & 1187 & 1351 & 1247 & 1759 & 1857 & 2414 & 1356 & 1751 & 1793 & 1247 & & 932 & 2.7 & 1266 & 1222 & 104 & (1) & 1247 \\
\hline sNerat & 2018 & 2006 & 1100 & 2718 & 2840 & 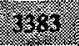 & ser. & 273 & 973 & 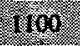 & 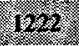 & 2018 & 314 & 46 & & 1326 & $2,8 \%$ & 100 \\
\hline SRS & 1281 & 976 & 2407 & 740 & 947 & 1239 & 1001 & 774 & 2953 & 2407 & 20 & 1161 & 1044 & 3192 & 3183 & $-2-$ & 744 & 2407 \\
\hline & 192 & 2536 & 11 & 294 & 2746 & 3282 & 239 & 2641 & 103 & $166^{\circ}$ & 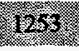 & 207 & 6050 & 56 & 63 & 1357 & 2930 & 11160: \\
\hline WVDP & 881 & 579 & 2123 & & 244 & & & & 2669 & 2123 & 1929 & 1033 & 309 & 2773 & 2898 & 1866 & ons & 2123 \\
\hline (8) & 11. & 1279 & 147 & 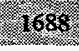 & 83 & 2342 & 8. & 82 & 1393 & 147 & $\sqrt{47}$ & 861 & 4 & 1660 & 1633 & 581 & 1703 & 1447 \\
\hline YM & 1674 & 348 & 756 & 4 & 2496 & 303 & 19 & & 1302 & 756 & 106 & 167 & & 0 & 1370 & 116 & 2386 & 756 \\
\hline
\end{tabular}


TABLE 3.5 (Cont.)

\begin{tabular}{|c|c|c|c|c|c|c|c|c|c|c|c|c|c|c|c|c|c|}
\hline & $\bar{z}$ & $\frac{\sqrt{2}}{\frac{2}{3}}$ & ્ָ & $\overline{\overline{3}}$ & & 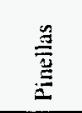 & $\stackrel{\Omega}{\Xi}$ & $\frac{\overrightarrow{\hat{a}}}{\mathrm{a}}$ & $\overline{\bar{\Sigma}}$ & $\frac{\mathscr{2}}{\not{z}}$ & $\sum_{\substack{\bar{z} \\
\dot{\bar{z}}}}$ & 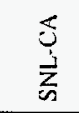 & & $\frac{\dot{s}}{\Delta}$ & $\sum_{3}^{\bar{\xi}}$ & $\hat{\overline{3}}$ & $\sum_{\lambda}$ \\
\hline $\mathrm{Am}$ & 674 & $9=$ & 954 & 646 & 809 & 1623 & 727 & 11 & 717 & 782 & 11 & 2018 & 1281 & 1924 & 881 & 11 & 1674 \\
\hline ANL- & 2348 & 651 & 64 & 390 & 972 & 1319 & 422 & 898 & 418 & 1194 & 1351 & 2506 & 976 & 2536 & 579 & 1279 & 23. \\
\hline ANI & 756 & 2099 & 2055 & 1699 & 1141 & 2721 & 1975 & 2507 & 2060 & 738 & 124 & 1100 & 2407 & 1160 & 2123 & 1497 & 756 \\
\hline $\mathrm{BCl}$ & 2374 & 366 & 393 & 581 & 1381 & IIst & 91 & 655 & 207 & 1502 & 1759 & 2718 & 740 & 2947 & 370 & 1688 & 237 \\
\hline Bettis & 2496 & 714 & 903 & 816 & 1479 & 1293 & 429 & 400 & 136 & 1692 & 1857 & 2840 & 947 & 2746 & 244 & 1785 & \\
\hline PNI & 3039 & 1221 & 1152 & 1346 & 2035 & 1585 & 921 & 410 & 648 & 2266 & 2414 & 3383 & 1239 & 3289 & 549 & 2342 & \\
\hline Fermi & 1997 & 679 & 682 & 469 & 977 & 1344 & 451 & 924 & 445 & 1016 & 1356 & 2341 & 1001 & 2393 & 603 & 1284 & \\
\hline FEM & 2391 & 331 & 358 & 468 & 1373 & 1116 & 207 & 938 & 337 & 1466 & 1751 & 2735 & 774 & 2641 & 631 & 1679 & \\
\hline Hanf & 1302 & 2644 & 2601 & 2245 & 1686 & 3267 & 2515 & 2985 & 2505 & 1284 & 1793 & 973 & 2953 & 1036 & 2669 & 19 & 02 \\
\hline NFI & 756 & 2099 & 2055 & 1699 & 1141 & 2721 & 1975 & 2507 & 2060 & 738 & 1247 & 1100 & 2407 & 1160 & 2123 & 147 & \\
\hline ITRI & 1065 & 1989 & 1749 & 1539 & 379 & 2079 & 1761 & 2248 & 1769 & 572 & a & 1222 & 2315 & 12 & 1929 & 4 & \\
\hline $\mathrm{KCl}$ & 1670 & 881 & 838 & 482 & 53. & 1803 & 758 & 1289 & 842 & 778 & 932 & 2013 & 1161 & 20 & 1033 & 861 & \\
\hline KAP & 2800 & 981 & 957 & 1106 & 1807 & 139 & 68 & 214 & 408 & 2027 & 217 & 3144 & 1044 & & 309 & & \\
\hline IBI & 860 & 2890 & 2686 & 2450 & 1561 & 3778 & $=2767$ & 3298 & 2851 & 20020 & 1266 & 46 & 3192 & hos. & Fes & 120 & \\
\hline LLN & 1370 & 2868 & 2831 & 2469 & 1534 & 3491 & 2745 & 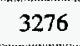 & 2829 & 1394 & 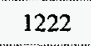 & 0 & 3183 & 0 & 2898 & 16 & \\
\hline LA & 1169 & 1926 & 1686 & 1476 & 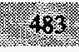 & 2183 & 6000 & 2186 & 1706 & 504 & 104 & 1326 & 2252 & thestos & 1866 & E & \\
\hline & 23 & & & 564 & 1396 & 50 & . & & - & 1485 & (7) & 2695 & & & 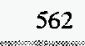 & & \\
\hline NRr & 756 & 2099 & 2055 & 1699 & 114 & 2721 & 1975 & 2507 & 2060 & 738 & 1 & 1100 & 7 & 160 & 3 & & \\
\hline NTS & & 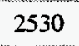 & - & 2131 & 76 & 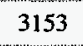 & 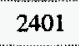 & & 2391 & 987 & & 70 & & 862 & 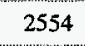 & & 0 \\
\hline 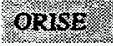 & 2530 & mos & 40 & 602 & 1611 & 2 & 392 & 9176 & 575 & 1658 & 210 & 2868 & 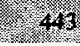 & 3103 & 889 & 1918 & \\
\hline ORR & 2487 & 40 & 0 & 527 & 1371 & 797 & 442 & 760 & 600 & 1586 & 1749 & 2831 & 417 & & 889 & 16 & \\
\hline POO & 2131 & 632 & 527 & 0 & 1103 & 1056 & 495 & 1146 & 698 & 1229 & 1539 & 2469 & 714 & 259 & 861 & 1410 & 2 \\
\hline Pantex & 1376 & 1611 & 1371 & 1103 & 0 & 1825 & 1382 & 1867 & 1387 & 465 & 37 & 1534 & 1937 & 1564 & 1551 & 307 & 13 \\
\hline Thinel & 3153 & 786 & 792 & 1056 & 1825 & 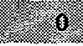 & 1106 & 1207 & 1861 & 2280 & 2079 & 3491 & 485 & 3280 & 1168 & 2019 & 3 \\
\hline PORTS & 2401 & 392 & 442 & 495 & 1382 & 1106 & 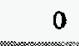 & 838 & 279 & 1535 & 1761 & 2745 & 655 & 20 & 585 & 1689 & \\
\hline PpPL & 2871 & 1176 & 760 & 1145 & 1867 & 1207 & 838 & 0 & 511 & 2066 & 2248 & 3276 & 848 & 3121 & 426 & 2185 & 28 \\
\hline RMI & 2391 & 575 & 600 & 698 & 1387 & 1361 & 279 & 511 & 0 & 1619 & 1700 & 2829 & 920 & 20 & 163 & 270 & \\
\hline FEIS & .987 & 1658 & 1586 & 1220 & 463 & 2280 & 1535 & 3066 & 1619 & 10 & 572 & 1394 & 1938 & 21 & 1782 & 769 & 5 \\
\hline 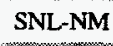 & 1065 & 1989 & 1749 & 1539 & 379 & 2079 & 1761 & 2248 & 1769 & 572 & 0 & 1222 & 2315 & 1235 & 1929 & 477 & 10 \\
\hline $\operatorname{sing} \alpha$ & 1370 & 2868 & 2831 & 2469 & 1584 & $34 \%$ & 2745 & 8276 & 2829 & 1398 & 1222 & 1.0 & 3188 & 60 & 2898 & 1633 & 13 \\
\hline SRS & 2839 & 443 & 417 & 714 & 1937 & 485 & 655 & 848 & 920 & 1938 & 2315 & 3183 & 8 & 3194 & 1223 & 2270 & 2839 \\
\hline Silic & 869 & 3103 & 3031 & 239 & 1164 & $3280^{\circ}$ & 2651 & 3121 & 264 & 1377 & 1253 & 60 & 3109 & 0 & 280 & 1662 & \\
\hline WVDP & 2554 & 889 & 889 & 861 & 1551 & 1568 & 585 & 426 & 163 & 1782 & 1929 & 2898 & 1223 & 2804 & 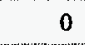 & 1858 & \\
\hline Mir? & 1475 & 1918 & 1678 & 1410 & $30 \%$ & 2619 & 1689 & 2185 & 1708 & 769 & 477 & 163 & 22 & 166 & 20 & sis & (478) \\
\hline YM & 0 & 2530 & 248 & 2131 & 1376 & 3153 & 2401 & 2871 & 2391 & 987 & 1065 & 1370 & 2839 & 862 & 2540 & 1475 & 0 \\
\hline
\end{tabular}

ail routes generated by using the INTERLINE 5.0 routing model (Johnson et al. 1993b).

Note: Ames = Ames Laboratory; ANL-E = Argonne National Laboratory-East; ANL-W = Argonne National Laboratory-West; $\mathrm{BCL}=$ Battelle Columbus Laboratories; Bettis = Bettis Atomic Power Laboratory; BNL = Brookhaven National Laboratory; Fermi = Fermi National Accelerator Laboratory; FEMP = Fernald Environmental Management Project; Hanford = Hanford Site; INEL = Idaho National Engineering Laboratory; ITRI = Inhalation Toxicology Research Institute; KCP = Kansas City Plant; $\mathrm{KAPL}=$ Knoils Atomic Power Laboratory; $\mathrm{LBL}=$ Lawrence Berkeley National Laboratory; $\mathrm{LLNL}=$ Lawrence Livermore National Laboratory; LANL $=$ Los Alamos National Laboratory; Mound $=$ Mound Plant; NRF $=$ Naval Reactor Facility; NTS $=$ Nevada Test Site; ORISE = Oak Ridge Institute for Science and Education; ORR = Oak Ridge Reservation; PGDP = Paducah Gaseous Diffusion Plant; Pantex = Pantex Plant; Pinellas $=$ Pinellas Plant; PORTS = Portsmouth Gaseous Diffusion Plant; PPPL = Princeton Plasma Physics Laboratory; RMI = Reactive Metals, Inc.; RFETS = Rocky Flats Environmental Technology Site; SNL-NM = Sandia National Laboratories (New Mexico); SNL-CA = Sandia National Laboratories (Cajifornia); SRS = Savannah River Site; SLAC = Stanford Linear Accelerator Center; WVDP $=$ West Valley Demonstration Project; WIPP $=$ Waste Isolation Pilot Plant: and YM $=$ Yucca Mountain. 
represents various competing rail companies in the United States. The database used by INTERLINE was originally based on data from the Federal Railroad Administration and reflected the U.S. railroad system in 1974. The database has since been expanded and modified over the past two decades. The code is updated periodically to reflect current track conditions and has been benchmarked against reported mileages and observations of commercial rail firms.

The INTERLINE 5.0 model uses a shortest-route algorithm that finds the path of minimum impedance within an individual subnetwork. A separate routine is used to find paths along the subnetworks. The routes chosen for this study used the standard assumptions in the INTERLINE model that simulate the process of selection that railroads would use to direct shipments of radioactive waste. For sites that do not have direct rail access, the rail siding nearest the site was used for routing purposes. The population densities along a route are derived from 1990 census data. Rural, suburban, and urban areas are characterized according to the following breakdown: rural population densities range from 0 to 54 persons per square kilometer $\left(0-139 / \mathrm{mi}^{2}\right)$, the suburban range for population density is $55-1,284 / \mathrm{km}^{2}\left(140-3,326 / \mathrm{mi}^{2}\right)$, and urban is taken to mean all population densities greater than $1,284 / \mathrm{km}^{2}\left(3,326 / \mathrm{mi}^{2}\right)$. 


\section{RISK ASSESSMENT ASSUMPTIONS AND PARAMETERS}

The majority of parameters and assumptions necessary to conduct the LLMW transportation risk assessment are described in detail in Appendix $E$ of the WM PEIS (DOE 1996). Supplemental information is provided subsequently for a number of the most important assessment parameters.

\subsection{SHIPPING AND PACKAGING ASSUMPTIONS FOR LOW-LEVEL MIXED WASTE}

The type of packaging used to transport radioactive waste is determined primarily by the radiological characteristics of the waste material. For purposes of risk assessment, representative packagings and shipment configurations have been determined for each type of radioactive waste on the basis of average waste characteristics and consistent with currently accepted practice. In practice, packagings are selected on a case-by-case basis and may differ from the representative types presented here.

For all types of waste, the assumptions are that the waste is transported in certified packagings and that exclusive-use vehicles would be used. Highway transportation is assumed to take place by legal-weight heavy-haul combination (tractor-trailer) trucks. Typically, Type A packages are transported on common flatbed or covered trailers; Type B packages are generally shipped on trailers designed specially for the packaging being used. For truck transportation, the maximum payload weight has been taken to be $19,958 \mathrm{~kg}$ $(44,000 \mathrm{lb})$, on the basis of DOT highway weight limitations and an average tractor-trailer weight of $16,330 \mathrm{~kg}(36,000 \mathrm{lb})$.

Rail transportation is assumed to take place by regular freight train service. The use of special or dedicated train service was not considered in the analysis. For rail transportation, average payload weights for boxcars range from 45,360 to $68,040 \mathrm{~kg}$ $(100,000$ to $150,000 \mathrm{lb})$. A median payload weight of $54,431 \mathrm{~kg}(120,000 \mathrm{lb})$ has been assumed for purposes of assessment.

All LLMW is assumed to be transported in Type A or "strong and tight" packaging, such as 208-L (55-gal) drums or standard waste boxes. Suitable Type A or "strong and tight" packagings are readily available from commercial sources. The number of shipments from a given site is calculated on the basis of projected site-specific waste inventory (weight) information and shipment capacity limitations for each transportation mode. The effects of potential waste treatment, such as volume reduction or thermal treatment are reflected in changes in waste density for each case. All shipments are assumed to be at the maximum weight limits for truck and rail shipments. On the basis of typical LLMW densities, roughly eighty 208-L (55-gal) drums would be shipped per truck and 300 per railcar.

Shipments of LLMW would meet any additional characterization and labeling requirements associated with the $\mathrm{HW}$ component. In addition, shipments of liquid waste would meet regulatory requirements specified for liquids (i.e., packages would contain 
adequate absorbent material to absorb twice the liquid volume, or a leak-tight overpack would be used [10 CFR Part 71]).

\subsection{ACCIDENT RATES}

For the calculation of accident risks, rates for vehicular accidents and for fatalities are taken from data provided by Saricks and Kvitek (1994). For each mode of transportation, accident rates are generically defined as the number of accident involvements (or fatalities) in a given year per unit of travel by that mode in that same year. Therefore, the rate is a fractional value, with accident-involvement count as the numerator of the fraction and vehicular activity (total traveled distance) as the denominator. Accident rates are generally determined for a multiyear period. For purposes of assessment, the total number of expected accidents or fatalities is calculated by multiplying the total shipping distance for a specific case by the appropriate accident or fatality rate.

For truck transportation, the rates presented in Saricks and Kvitek (1994) are specifically for heavy combination trucks involved in interstate commerce. Heavy combination trucks are rigs composed of a separable tractor unit containing the engine and one to three freight trailers connected to each other. Heavy combination trucks are typically used for shipments of radioactive waste. Saricks and Kvitek (1994) base their truck accident rates for each state on statistics compiled by the DOT Office of Motor Carriers for 1986 to 1988. Saricks and Kvitek (1994) present counts of accident involvements and of fatalities, estimated kilometers of travel by state, and the corresponding average accident involvement, fatality, and injury rates for the three years investigated. Fatalities are deaths (including crew members) that are attributable to the accident and that occurred at any time within 30 days thereafter. The truck accident rates are summarized in Table 4.1.

Rail accident rates are computed and presented similarly to truck accident rates in Saricks and Kvitek (1994); however, the unit of haulage is considered to be the railcar. Saricks and Kvitek (1994) base their state-specific rail accident involvement and fatality rates on statistics compiled by the Federal Railroad Administration for 1985 to 1988 . Rail accident rates include both main-line accidents and those occurring in rail yards. The rail accident rates are summarized in Table 4.2.

\subsection{CHARACTERISTICS OF ACCIDENT RELEASES}

The transportation accident risk assessment depends on the fraction of material in a package that would be released or spilled to the environment during an accident, commonly referred to as the release fraction. The fraction of material released from a package is a function of the severity of the accident and the waste packaging; for instance, a low-impact accident, such as a "fender-bender," would not be expected to cause any release of material. 
TABLE 4.1 Highway Combination-Truck Accident, Fatality, and Injury Rates by Road Type ${ }^{\text {a }}$ Based on Reportable Interstate-Carrier Accidents, Reported Fatalities and Injuries, and Estimated Flows, 1986-1988 (10 ${ }^{-7}$ accidents and injuries per shipment-kilometer, $10^{-8}$ fatalities per shipment-kilometer)

\begin{tabular}{|c|c|c|c|c|c|c|c|c|c|c|c|c|c|c|c|}
\hline \multirow[b]{2}{*}{ State } & \multicolumn{5}{|c|}{ Accident-Involvement Rates } & \multicolumn{5}{|c|}{ Accident-Fatality Rates } & \multicolumn{5}{|c|}{ Accident-Injury Rates } \\
\hline & FAI-U & FAI-R & FAI & FAP & FAS & FAI-U & FAI-R & FAI & FAP & FAS & FAI-U & FAI-R & FAI & FAP & FAS \\
\hline $\mathrm{AL}$ & 4.68 & 1.26 & 1.85 & 5.16 & 3.96 & 3.29 & 1.84 & 2.09 & 6.34 & 7.84 & 4.66 & 1.24 & 1.83 & 5.62 & 4.22 \\
\hline $\mathrm{AZ}$ & 2.71 & 1.60 & 1.76 & 2.12 & 1.45 & 3.56 & 2.13 & 2.33 & 4.97 & 1.20 & 2.89 & 1.65 & 1.82 & 2.20 & 2.77 \\
\hline $\mathrm{AR}$ & 4.82 & 1.73 & 2.09 & 4.69 & 6.84 & 6.61 & 2.28 & 2.78 & 8.88 & 7.10 & 4.09 & 1.95 & 2.20 & 4.38 & 5.94 \\
\hline $\mathrm{CA}$ & 1.92 & 1.64 & 1.76 & 1.15 & 2.22 & 1.39 & 2.56 & 2.06 & 1.98 & 3.81 & 1.82 & 1.68 & 1.74 & 1.12 & 2.67 \\
\hline $\mathrm{CO}$ & 6.28 & 2.76 & 3.60 & 4.11 & 4.42 & 3.38 & 2.45 & 2.67 & 6.58 & 5.83 & 5.47 & 2.82 & 3.45 & 3.95 & 3.67 \\
\hline CT & 2.67 & $4.60^{\mathrm{c}}$ & 3.23 & 2.56 & 9.09 & 1.01 & $5.11^{\mathrm{c}}$ & 2.20 & 1.17 & 9.09 & 1.71 & $4.61^{\mathrm{c}}$ & 2.55 & 1.89 & 7.09 \\
\hline $\mathrm{DE}$ & 2.56 & .. & 2.56 & 7.35 & 4.81 & 1.66 & -. & 1.66 & $13.50^{c}$ & 7.69 & 3.46 & .. & 3.46 & 7.18 & 3.08 \\
\hline FL & 2.25 & 1.21 & 1.50 & 3.73 & 6.33 & 1.74 & 2.62 & 2.38 & 5.92 & 6.12 & 2.36 & 1.28 & 1.58 & 4.12 & 7.92 \\
\hline GA & 4.87 & 1.65 & 2.28 & 6.15 & 4.04 & 2.37 & 1.86 & 1.96 & 8.30 & 6.74 & 4.37 & 1.75 & 2.26 & 6.43 & 3.71 \\
\hline ID & 1.73 & 2.30 & 2.22 & 4.93 & 2.29 & 0 & 2.06 & 1.78 & 7.46 & 2.54 & 1.18 & 2.12 & 1.99 & 3.54 & 1.69 \\
\hline IL & $8.75^{c}$ & 1.76 & 3.53 & 6.40 & 1.78 & 5.33 & 1.39 & 2.38 & 7.84 & 2.30 & $8.23^{\mathrm{c}}$ & 1.49 & 3.20 & 6.12 & 1.55 \\
\hline IN & 4.58 & 1.92 & 2.43 & 4.72 & 2.80 & 3.51 & 1.22 & 1.66 & 7.66 & 4.02 & 4.36 & 1.81 & 2.30 & 4.42 & 2.32 \\
\hline IA & 3.54 & 1.78 & 2.02 & 4.03 & 1.24 & 2.68 & 1.05 & 1.26 & 6.14 & 0.73 & 3.78 & 1.46 & 1.76 & 3.48 & 0.88 \\
\hline $\mathrm{KS}$ & 4.48 & 2.04 & 2.56 & 5.11 & 1.38 & 5.52 & 1.88 & 2.66 & 9.35 & 1.72 & 3.66 & 1.91 & 2,28 & 4.09 & 1.12 \\
\hline $\mathrm{KY}$ & 5.13 & 1.46 & 1.99 & 5.74 & 8.80 & 3.22 & 1.50 & 1.75 & 6.60 & 6.25 & 5.52 & 1.33 & 1.94 & 5.28 & 8.12 \\
\hline LA & 3.54 & 1.30 & 1.88 & 3.53 & 2.39 & 4.90 & 1.77 & 2.59 & 5.73 & 3.28 & 4.57 & 1.32 & 2.16 & 4.46 & 2.85 \\
\hline $\mathrm{ME}$ & $9.03^{c}$ & 2.44 & 2.93 & 5.44 & 2.28 & 0 & 2.34 & 2.16 & 6.58 & 2.17 & 4.52 & 1.53 & 1.75 & 5.00 & 1.63 \\
\hline MD & 3.08 & 3.95 & 3.46 & 3.56 & 12.40 & 1.62 & 4.03 & 2.69 & 3.66 & 8.99 & 3.41 & 3.98 & $3.66^{\mathrm{c}}$ & 4.32 & 13.40 \\
\hline MA & 1.42 & $6.47^{\mathrm{c}}$ & 2.68 & 3.43 & $46.10^{\mathrm{c}}$ & 1.30 & $6.23^{\mathrm{c}}$ & 2.53 & 3.93 & $52.20^{\mathrm{c}}$ & 1.13 & $4.99^{\mathrm{C}}$ & 2.09 & 3.02 & $43.90^{c}$ \\
\hline MI & 3.16 & 1.59 & 2.12 & 2.68 & 0.81 & 1.52 & 1.23 & 1,33 & 3.96 & 1.22 & 3.04 & 1.29 & 1.87 & 2.61 & 1.38 \\
\hline MN & 2.66 & 2.06 & 2.29 & 4.19 & 2.16 & 2.02 & 1.72 & 1.83 & 7.69 & 7.84 & 2.08 & 1.46 & 1.69 & 3.28 & 1.86 \\
\hline MS & 2.01 & 1.19 & 1.35 & 4.48 & 0.65 & 2.46 & 1.81 & 1,93 & 9.26 & 1.62 & 1.85 & 1.10 & 1.25 & 4.55 & 0.50 \\
\hline MO & 5.18 & 1.78 & 2.61 & 5.36 & 2.49 & 4.30 & 1.23 & 1.99 & 9.68 & 3.25 & 5.53 & 1.63 & 2.59 & 5.06 & 2.23 \\
\hline $\mathrm{MT}$ & $10.00^{\mathrm{C}}$ & 2.52 & 2.89 & 5.38 & 1.02 & 3.12 & 1.44 & 1.52 & 7.97 & 2.04 & 4.69 & 1.79 & 1.93 & 3.95 & 0.20 \\
\hline $\mathrm{NE}$ & 6.97 & 1.77 & 2.09 & 3.62 & 0.99 & 6.58 & 1.10 & 1.43 & 5.75 & 0 & 6.58 & 1.17 & 1.50 & 3.54 & 0.54 \\
\hline NV & 6.33 & 1.57 & 1.97 & 4.35 & 3.17 & $8.89^{\mathrm{c}}$ & 1.14 & 1.79 & 10.50 & 7.94 & 5.67 & 1.58 & 1.93 & 3.72 & 2.54 \\
\hline $\mathrm{NH}$ & 0.22 & 1.39 & 1.18 & 4.36 & 3.33 & 0 & 1.49 & 1.22 & 5.77 & 5.56 & 0 & 1.14 & 0.94 & 4.17 & 2.22 \\
\hline $\mathrm{NJ}$ & 2.77 & $7.65^{\mathrm{c}}$ & $4.24^{\mathrm{c}}$ & 6,80 & 9.69 & 1.56 & $6.56^{\mathrm{c}}$ & 3.06 & 4.57 & 11.50 & 2.69 & $8.00^{\mathrm{c}}$ & $4.28^{\mathrm{c}}$ & 6.86 & 11.30 \\
\hline NM & $9.64^{\mathrm{c}}$ & 1.92 & 2.35 & 4.77 & 12.20 & $9.01^{\mathrm{c}}$ & 1.93 & 2.32 & 6.99 & 5.56 & $8.92^{\mathrm{c}}$ & 1.86 & 2,25 & 4.62 & 10.60 \\
\hline $\mathrm{NY}$ & 5.69 & 2.93 & $3.98^{\mathrm{c}}$ & 3.16 & 9.48 & 2.04 & 1.38 & 1.63 & 4.61 & 10.30 & 4.49 & 2.56 & 3.28 & 2.71 & 10.00 \\
\hline $\mathrm{NC}$ & 5.92 & 2.28 & 2.97 & 5.17 & 6.37 & 5.08 & 2.92 & $3.33^{\mathrm{c}}$ & 6.71 & 11.00 & 6.37 & 2.19 & 2.99 & 5.53 & 6.22 \\
\hline $\mathrm{OH}$ & 3.16 & 2.27 & 2.52 & 4.42 & 11.00 & 1.41 & 1.32 & 1.35 & 6.07 & 9.88 & 2.85 & 2.02 & 2.25 & 4.40 & 1070 \\
\hline OK & 3.76 & 1.47 & 1.91 & 3.61 & 1.73 & 2.70 & 2.06 & 2.18 & 4.88 & 0.71 & 3.34 & 1.36 & 1.74 & 3,05 & 1.59 \\
\hline OR & 3.99 & 2.20 & 2.48 & 4.17 & 1.63 & 2.47 & 1.12 & 1.33 & 5.85 & 4.07 & 3.82 & 1.69 & 2.02 & 2.94 & 0.57 \\
\hline$P A$ & 3.02 & 3.60 & 3.48 & 7.21 & 7.92 & 2.12 & 2.97 & 2.79 & 10.20 & 9.29 & 2.68 & 3.28 & 3.15 & 7.28 & 6.42 \\
\hline RI & 2.27 & 1.98 & 2.16 & 1.37 & 16.70 & 0.71 & 3.70 & 1.80 & 1.71 & $66.70^{c}$ & 3.12 & 2.35 & 2.84 & 0.86 & $23.30^{\mathrm{c}}$ \\
\hline $\mathrm{SC}$ & 3.13 & 1.83 & 1.99 & 6.27 & 2.27 & 2.88 & 2.57 & 2.61 & 8.61 & 3.95 & 2.65 & 2.20 & 2.26 & 6.96 & 2.27 \\
\hline SD & $8.57^{\mathrm{c}}$ & 2.09 & 2.18 & 3.94 & 1.49 & $14.30^{\mathrm{C}}$ & 0.42 & 0.62 & 5.38 & 2.13 & 5.71 & 1.38 & 1.95 & 2.94 & 0 \\
\hline $\mathrm{TN}$ & 7.97 & 1.48 & 2.48 & 5.56 & 6.26 & 5.59 & 1.63 & 2.24 & 6.97 & 12.10 & $7.70^{\mathrm{c}}$ & 1.44 & 2.41 & 5.85 & 4.67 \\
\hline
\end{tabular}


TABLE 4.1 (Cont.)

\begin{tabular}{|c|c|c|c|c|c|c|c|c|c|c|c|c|c|c|c|}
\hline \multirow[b]{2}{*}{ State } & \multicolumn{5}{|c|}{ Accident-Involvement Rates } & \multicolumn{5}{|c|}{ Accident-Fatality Rates } & \multicolumn{5}{|c|}{ Accident-Injury Rates } \\
\hline & FAI-U & FAI-R & FAI & FAP & FAS & FAI-U & FAI-R & FAI & FAP & FAS & FAI-U & FAI-R & FAI & FAP & FAS \\
\hline $\mathrm{TX}$ & 2.74 & 1.56 & 2.00 & 2.78 & 1.09 & 1.93 & 1.97 & 1.95 & 4.77 & 1.84 & 2.53 & 1.42 & 1.83 & 2.53 & 0.92 \\
\hline VT & 0 & 1.38 & 1.33 & 6.30 & 6.80 & 0 & 0.43 & 0.42 & 3.36 & 24.00 & 0 & 1.08 & 1.04 & 6.13 & 4.40 \\
\hline VA & 2.63 & 2.54 & 2.66 & 4.67 & 5.03 & 1.91 & 1.76 & 1.80 & 7.28 & 7.73 & 2.49 & 2.46 & 2.47 & 5.39 & 4.81 \\
\hline WA & 1.61 & 2.50 & 2.10 & 2.62 & 0.73 & 0.80 & 1.47 & 1.17 & 2.54 & 0 & 1.49 & 2.14 & 1.85 & 2.11 & 0.51 \\
\hline WV & 2.95 & 3.10 & 3.07 & $11.70^{c}$ & 7.87 & 1.28 & 1.67 & 1.60 & $17.80^{\mathrm{C}}$ & 1.70 & 2.78 & 2.80 & 2.79 & $9.91^{\mathrm{c}}$ & 7.13 \\
\hline WI & 5.29 & 1.74 & 2.18 & 2.80 & 3.24 & 0.77 & 0.66 & 0.67 & 3.62 & 3.68 & 4.33 & 1.45 & 1.81 & 2.51 & 3.16 \\
\hline WY & 2.98 & 3.42 & 3.40 & 3.41 & 3.70 & 0 & 2.08 & 2.01 & 6.22 & 3.70 & 0 & 2.84 & 2.74 & 1.86 & 2.35 \\
\hline USA & 3.58 & 2.03 & 2.44 & 3.94 & 3.48 & 2.37 & 1.91 & 2.03 & 5.82 & 4.62 & 3.36 & 1.89 & 2.28 & 3.82 & 3.30 \\
\hline$\sigma^{\mathrm{d}}$ & 2,36 & 1.25 & 0.69 & 1.77 & 6.98 & 2.70 & 1.27 & 0.63 & 3.01 & $\begin{array}{r}4.02 \\
11.74\end{array}$ & $\begin{array}{l}.00 \\
1.99\end{array}$ & $\begin{array}{l}1.00 \\
1.22\end{array}$ & 0.69 & $\begin{array}{l}0.72 \\
1.79\end{array}$ & $\begin{array}{l}0.00 \\
7.10\end{array}$ \\
\hline
\end{tabular}

a $\quad \mathrm{FAI}=$ Federally aided interstate

$$
\begin{aligned}
& \mathrm{U}=\text { Urban } \\
& \mathrm{R}=\text { Rural }
\end{aligned}
$$

FAP $=$ Federally aided primary

FAS = Federally aided secondary

b Includes only the 48 contiguous states, plus the totals for the United States.

c Rates are two or more standard deviations greater than the national average rate for that highway category.

d $\sigma=$ standard deviation.

Source: Saricks and Kvitek (1994). 
TABLE 4.2 Railroad-Freight-Car Accident Rates, by State, for 1986, 1987, 1988, and $1985-1988$ Combined $\left(10^{-8}\right.$ accidents per railcar-kilometer $\left.{ }^{\mathrm{a}}\right)$

\begin{tabular}{|c|c|c|c|c|c|c|c|c|}
\hline \multirow[b]{2}{*}{ State $^{b}$} & \multicolumn{2}{|c|}{1986} & \multicolumn{2}{|c|}{1987} & \multicolumn{2}{|c|}{1988} & \multicolumn{2}{|c|}{ 1985-88 } \\
\hline & Total & $\begin{array}{l}\text { Main } \\
\text { Only }\end{array}$ & Total & $\begin{array}{l}\text { Main } \\
\text { Only }\end{array}$ & Total & $\begin{array}{l}\text { Main } \\
\text { Only }\end{array}$ & Total & $\begin{array}{l}\text { Main } \\
\text { Only }\end{array}$ \\
\hline $\mathrm{AL}$ & 3.88 & 2.00 & 4.80 & 3.37 & 4.13 & 2.52 & 4.80 & 2.75 \\
\hline $\mathrm{AZ}$ & 1.40 & 1.05 & 1.30 & 1.08 & 1.44 & 1.18 & 1.75 & 1.30 \\
\hline $\mathrm{AR}$ & 5.05 & 2.59 & 4.27 & 2.32 & 8.34 & 3.94 & 6.78 & 3.54 \\
\hline $\mathrm{CA}$ & 5.10 & 2.64 & 3.30 & 1.98 & 4.32 & 2.30 & 5.10 & 2.51 \\
\hline $\mathrm{CO}$ & 1.44 & 1.01 & 0.86 & 0.40 & 2.55 & 1.51 & 1.73 & 1.02 \\
\hline $\mathrm{CT}$ & 26.47 & 17.65 & 16.67 & 8.34 & 24.95 & 0 & $28.27^{\mathrm{c}}$ & 10.10 \\
\hline $\mathrm{DE}$ & 27.44 & 9.15 & 25.23 & 16.82 & 18.04 & 18.04 & 17.71 & 11.07 \\
\hline $\mathrm{DC}$ & 0 & 0 & 316 & 316 & 0 & 0 & $117.09^{c}$ & $78.06^{c}$ \\
\hline FL & 2.86 & 2.12 & 3.90 & 1.90 & 4.16 & 2.38 & 4.02 & 2.21 \\
\hline GA & 6.44 & 3.08 & 6.77 & 2.81 & 6.18 & 2.69 & 6.44 & 2.84 \\
\hline ID & 5.90 & 5.25 & 6.74 & 4.10 & 9.03 & 3.99 & 7.01 & 4.14 \\
\hline IL & 11.70 & 3.10 & 9.97 & 3.10 & 9.08 & 2.45 & 10.67 & 2.97 \\
\hline IN & 4.18 & 1.85 & 3.71 & 1.48 & 3.80 & 1.44 & 4.64 & 1.93 \\
\hline IA & 17.63 & 7.15 & 12.72 & 6.65 & 11.95 & 7.01 & 14.67 & 7.16 \\
\hline $\mathrm{KS}$ & 3.01 & 1.62 & 2.97 & 1.55 & 4.09 & 1.98 & 3.61 & 1.75 \\
\hline $\mathrm{KY}$ & 4.78 & 2.39 & 3.77 & 1.98 & 3.60 & 1.84 & 4.48 & 2.44 \\
\hline LA & 12.67 & 5.98 & 7.07 & 2.02 & 11.94 & 3.92 & 12.37 & 4.28 \\
\hline $\mathrm{ME}$ & 28.22 & 16.93 & 29.40 & 13.36 & 52.57 & 26.29 & $37.80^{c}$ & $18.53^{c}$ \\
\hline MD & 6.07 & 3.54 & 5.55 & 2.44 & 4.55 & 1.24 & 5.62 & 2.58 \\
\hline MA & 12.33 & 5.87 & 10.06 & 3.71 & 7.31 & 2.81 & 11.65 & 4.97 \\
\hline MI & 18.34 & 8.18 & 15.10 & 4.42 & 15.52 & 7.45 & 16.47 & 7.19 \\
\hline $\mathrm{MN}$ & 8.63 & 2.64 & 6.24 & 3.30 & 7.86 & 3.63 & 8.48 & 3.16 \\
\hline MS & 15.16 & 11.20 & 12.93 & 9.84 & 8.47 & 7.14 & 11.52 & 8.51 \\
\hline MO & 4.59 & 2.12 & 3.60 & 1.87 & 5.05 & 2.87 & 5.28 & 2.56 \\
\hline MT & 1.35 & 1.01 & 1.24 & 0.81 & 2.11 & 1.17 & 1.73 & 1.10 \\
\hline $\mathrm{NE}$ & 4.04 & 2.11 & 4.81 & 2.49 & 4.46 & 2.23 & 4.63 & 2.56 \\
\hline NV & 3.27 & 1.87 & 1.29 & 0.86 & 3.97 & 2.65 & 3.23 & 2.19 \\
\hline $\mathrm{NH}$ & 6.60 & 6.60 & 17.52 & 11.68 & 24.27 & 16.18 & 21.45 & $17.16^{c}$ \\
\hline NJ & 13.86 & 2.64 & 9.44 & 5.31 & 10.10 & 4.75 & 12.38 & 4.82 \\
\hline NM & 0.90 & 0.52 & 0.93 & 0.75 & 0.86 & 0.65 & 0.94 & 0.66 \\
\hline NY & 7.23 & 3.92 & 6.80 & 4.14 & 7.26 & 3.36 & 8.32 & 4.30 \\
\hline $\mathrm{NC}$ & 5.40 & 2.70 & 5.48 & 1.62 & 6.29 & 2.95 & 5.70 & 2.27 \\
\hline ND & 2.18 & 1.48 & 2.43 & 1.86 & 1.49 & 1.12 & 2.41 & 1.80 \\
\hline $\mathrm{OH}$ & 5.35 & 2.50 & 3.55 & 1.58 & 4.04 & 1.92 & 4.73 & 2.12 \\
\hline OK & 5.25 & 3.01 & 3.52 & 1.90 & 3.77 & 1.88 & 4.66 & 2.72 \\
\hline OR & 11.74 & 4.96 & 12.23 & 6.12 & 13.64 & 6.04 & 12.48 & 5.77 \\
\hline PA & 4.86 & 3.73 & 4.22 & 2.37 & 3.54 & 1.95 & 4.38 & 2.69 \\
\hline RI & 0 & 0 & 0 & 0 & 0 & 0 & $105.33^{c}$ & 0 \\
\hline SC & 4.64 & 2.16 & 4.74 & 3.62 & 5.03 & 3.71 & 5.11 & 3.31 \\
\hline $\mathrm{SD}$ & 8.16 & 6.27 & 14.98 & 14.49 & 7.27 & 6.36 & 10.19 & 9.09 \\
\hline $\mathrm{TN}$ & 4.88 & 1.69 & 4.67 & 1.89 & 7.56 & 2.02 & 5.59 & 1.88 \\
\hline TX & 7.03 & 3.36 & 5.29 & 2.50 & 5.92 & 2.45 & 7.12 & 3.16 \\
\hline UT & 3.69 & 1.34 & 1.85 & 0.93 & 11.19 & 4.04 & 5.78 & 2.31 \\
\hline $\mathrm{VT}$ & 5.98 & 2.99 & 7.86 & 5.24 & 24.32 & 18.24 & 15.22 & 11.59 \\
\hline VA & 4.27 & 1.42 & 4.39 & 2.31 & 4.59 & 2.15 & 4.35 & 1.91 \\
\hline
\end{tabular}


TABLE 4.2 (Cont.)

\begin{tabular}{|c|c|c|c|c|c|c|c|c|}
\hline \multirow[b]{2}{*}{ State $^{b}$} & \multicolumn{2}{|c|}{1986} & \multicolumn{2}{|c|}{1987} & \multicolumn{2}{|c|}{1988} & \multicolumn{2}{|c|}{$1985-88$} \\
\hline & Total & $\begin{array}{l}\text { Main } \\
\text { Only }\end{array}$ & Total & $\begin{array}{l}\text { Main } \\
\text { Only }\end{array}$ & Total & $\begin{array}{l}\text { Main } \\
\text { Only }\end{array}$ & Total & $\begin{array}{l}\text { Main } \\
\text { Only }\end{array}$ \\
\hline WA & 3.65 & 1.38 & 2.69 & 1.50 & 3.57 & 1.12 & 3.49 & 1.44 \\
\hline WV & 11.49 & 9.23 & 9.97 & 7.73 & 6.08 & 4.67 & 9.61 & 7.42 \\
\hline WI & 18.79 & 7.52 & 16.75 & 7.75 & 12.57 & 7.21 & 16.53 & 7.66 \\
\hline WY & 2.55 & 1.37 & 2.61 & 1.47 & 2.88 & 1.40 & 3.10 & 1.97 \\
\hline USA & 5.51 & 2.63 & 4.70 & 2.36 & 5.14 & 2.43 & 5.57 & 2.66 \\
\hline$\sigma^{\mathrm{d}}$ & - & - & - & - & - & - & 21.48 & 11.12 \\
\hline
\end{tabular}

a Nationwide average fatality rate, using count of fatalities consistent with combination-truck fatality counts, is $6.50 \times 10^{-10}$ fatalities per railcar-kilometer. Nationwide average injury rate, using count of injuries consistent with combination-truck injury counts, is $7.83 \times 10^{-8}$ injuries per railcar-kilometer.

b Includes the 48 contiguous states, the District of Columbia, and the totals for the United States.

c Rates are one or more standard deviations greater than the national average rate for that category (i.e., all accidents on carrier-owned track or accidents on main lines only).

d $\sigma=$ standard deviation.

Source: Saricks and Kvitek (1994).

Conversely, a very severe accident would be expected to release nearly all of the material in a shipment into the environment. The method used to characterize accident severities and the corresponding release fractions are described subsequently.

\subsubsection{Accident Severity Categories}

A method to characterize the potential severity of transportation-related accidents is described in an NRC report commonly referred to as NUREG-0170 (NRC 1977a). The NRC method divides the spectrum of transportation accident severities into eight categories. Other studies have divided the same accident spectrum into six categories (Wilmot 1981) and into 20 categories (Fischer et al. 1987); however, these studies focused primarily on accidents involving shipments of spent nuclear fuel and thus are not directly applicable to this assessment.

The NUREG-0170 scheme for accident classification is shown in Figures 4.1 and 4.2 for truck and rail transportation, respectively. Severity is described as a function of the magnitudes of the mechanical forces (impact) and thermal forces (fire) to which a package may be subjected during an accident. Because all accidents can be described in these terms, severity is independent of the specific accident sequence. In other words, any sequence of 


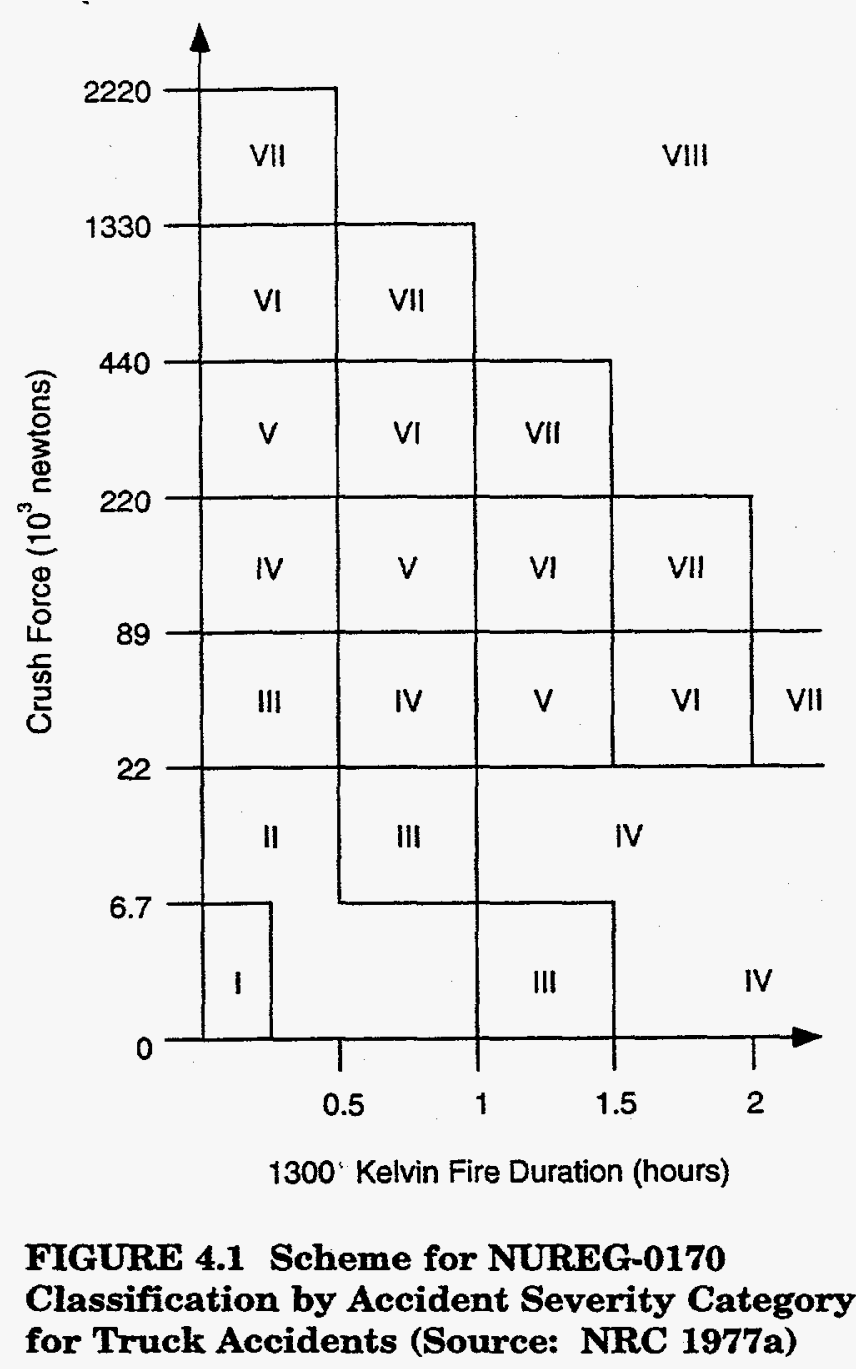

events that results in an accident in which a package is subjected to forces within a certain range of values is assigned to the accident severity category associated with that range. The scheme for accident severity is designed to take into account all credible transportation-related accidents, including accidents with low probability but high consequences and those with high probability but low consequences.

Each severity category represents a set of accident scenarios defined by a combination of mechanical and thermal forces. A conditional probability of occurrence - that is, the probability that if an accident occurs, it is of a particular severity - is assigned to each category. The fractional occurrences for accidents by the accident severity category and the population density zone are shown in Table 4.3.

Category I accidents are the least severe but the most frequent, whereas Category VIII accidents are very severe but very infrequent. To determine the expected 


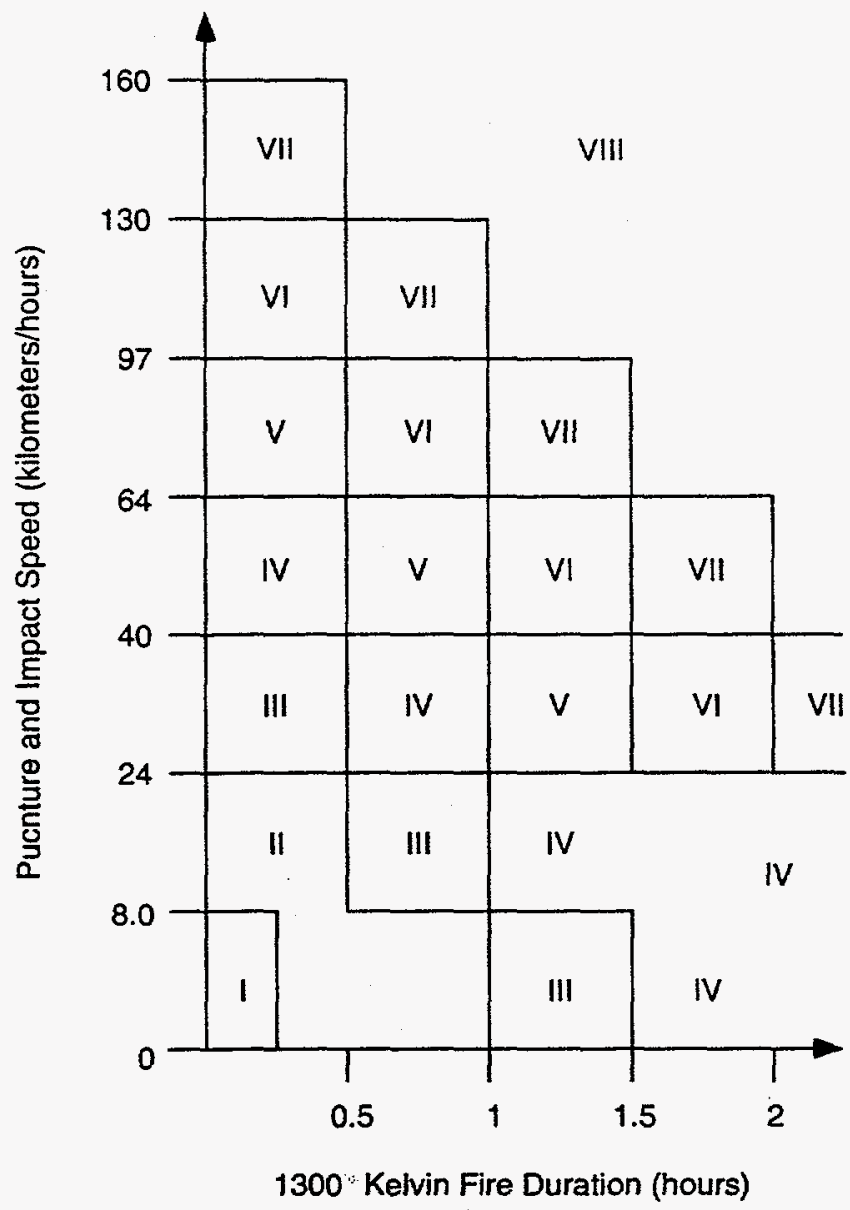

FIGURE 4.2 Scheme for NUREG-0170 Classification by Accident Severity Category for Rail Accidents (Source: NRC 1977a)

frequency of an accident of a given severity, the conditional probability in the category is multiplied by the baseline accident rate. Each population density zone has a distinct baseline accident rate and distribution of accident severities related to differences in average vehicular velocity, traffic density, and other factors, including location (rural, suburban, or urban).

For the accident consequence assessment, the doses were assessed for populations and individuals by assuming an accident of severity Category VIII. This accident severity category represents the most severe accident scenarios that can be postulated that would result in the largest release of radioactive material. Accidents of this severity are extremely rare, occurring approximately once in every 70,000 truck or 100,000 rail accidents involving a shipment of radioactive waste. On the basis of national accident statistics (Saricks and Kvitek 1994), for every $1.6 \mathrm{~km}$ ( $1 \mathrm{mi}$ ) of loaded shipment, the probability of an accident of this severity is $6 \times 10^{-12}$ for shipment by truck and $1 \times 10^{-12}$ for shipment by rail. 
TABLE 4.3 Fractional Occurrences for Accidents by Severity Category and Population Density Zone

\begin{tabular}{lllll}
\hline & \multicolumn{5}{c}{$\begin{array}{c}\text { Fractional Occurrence } \\
\text { by Population Density Zone }\end{array}$} \\
\cline { 3 - 5 } $\begin{array}{c}\text { Severity } \\
\text { Category }\end{array}$ & $\begin{array}{c}\text { Fractional } \\
\text { Occurrence }\end{array}$ & Rural & Suburban & Urban \\
\hline Truck & & & \\
I & $5.5 \mathrm{E}-01$ & $1 \mathrm{E}-01$ & $1 \mathrm{E}-01$ & $8 \mathrm{E}-01$ \\
II & $3.6 \mathrm{E}-01$ & $1 \mathrm{E}-01$ & $1 \mathrm{E}-01$ & $8 \mathrm{E}-01$ \\
III & $7.0 \mathrm{E}-02$ & $3 \mathrm{E}-01$ & $4 \mathrm{E}-01$ & $3 \mathrm{E}-01$ \\
IV & $1.6 \mathrm{E}-02$ & $3 \mathrm{E}-01$ & $4 \mathrm{E}-01$ & $3 \mathrm{E}-01$ \\
V & $2.8 \mathrm{E}-03$ & $5 \mathrm{E}-01$ & $3 \mathrm{E}-01$ & $2 \mathrm{E}-01$ \\
VI & $1.1 \mathrm{E}-03$ & $7 \mathrm{E}-01$ & $2 \mathrm{E}-01$ & $1 \mathrm{E}-01$ \\
VII & $8.5 \mathrm{E}-05$ & $8 \mathrm{E}-01$ & $1 \mathrm{E}-01$ & $1 \mathrm{E}-01$ \\
VIII & $1.5 \mathrm{E}-05$ & $9 \mathrm{E}-01$ & $5 \mathrm{E}-02$ & $5 \mathrm{E}-02$ \\
& & & & \\
Rail & & & & \\
I & $5.0 \mathrm{E}-01$ & $1 \mathrm{E}-01$ & $1 \mathrm{E}-01$ & $8 \mathrm{E}-01$ \\
II & $3.0 \mathrm{E}-01$ & $1 \mathrm{E}-01$ & $1 \mathrm{E}-01$ & $8 \mathrm{E}-01$ \\
III & $1.8 \mathrm{E}-01$ & $3 \mathrm{E}-01$ & $4 \mathrm{E}-01$ & $3 \mathrm{E}-01$ \\
IV & $1.8 \mathrm{E}-02$ & $3 \mathrm{E}-01$ & $4 \mathrm{E}-01$ & $3 \mathrm{E}-01$ \\
V & $1.8 \mathrm{E}-03$ & $5 \mathrm{E}-01$ & $3 \mathrm{E}-01$ & $2 \mathrm{E}-01$ \\
VI & $1.3 \mathrm{E}-04$ & $7 \mathrm{E}-01$ & $2 \mathrm{E}-01$ & $1 \mathrm{E}-01$ \\
VII & $6.0 \mathrm{E}-05$ & $8 \mathrm{E}-01$ & $1 \mathrm{E}-01$ & $1 \mathrm{E}-01$ \\
VIII & $1.0 \mathrm{E}-05$ & $9 \mathrm{E}-01$ & $5 \mathrm{E}-02$ & $5 \mathrm{E}-02$ \\
\hline
\end{tabular}

Source: NRC (1977a).

\subsubsection{Package Release Fractions}

Radiological consequences are calculated by assigning package release fractions to each accident severity category. The release fraction is defined as the fraction of the radioactive material in a package that could be released from the package as the result of an accident of a given severity. Release fractions take into account all mechanisms necessary to create release of radioactive material from a damaged package to the environment. Release fractions vary according to the type of package and the physical form of the waste.

Representative cask release fractions for accidents involving LLMW shipments were taken from NUREG-0170 (NRC 1977a). The recommendations in NUREG-0170 are based on best engineering judgments and have been shown to provide conservative estimates of radioactive material releases following accidents. The release fractions used are those reported in NUREG-0170 for NRC Type A packages. Release fractions for accidents of each severity category are given in Table 4.4. As shown in Table 4.4, the amount of material released from the packaging ranges from zero for minor accidents to $100 \%$ for the most severe accidents. 
Also important for the purposes of risk assessment are the fraction of the released material that can be entrained in an aerosol (part of an airborne radioactive plume) and the fraction of the aerosolized material that is also respirable (of a size that can be inhaled into the lungs). These fractions depend on the physical form of the waste material. As stated previously, the LLMW assessment considered two physical waste forms: heterogeneous solids and organic liquids. The aerosolized and respirable fractions for these two forms are given in Table 4.5.

\subsection{ATMOSPHERIC CONDITIONS DURING ACCIDENTS}

Radioactive and hazardous material released to the atmosphere is transported by the wind. Dispersion or dilution of the radioactive material in the air depends on meteorologic conditions at the time of the accident. Because predicting the specific location of an off-site transportation-related accident is impossible, generic atmospheric conditions were selected for the accident risk and consequence assessments.

For the accident risk assessment, neutral weather conditions (represented by Pasquill stability Class $D$ with a wind speed of $4 \mathrm{~m} / \mathrm{s}$ [9 mph]) were assumed. Because
TABLE 4.4 Estimated Release Fractions for LLMW Packages under Various Accident Severity Categories

\begin{tabular}{lc}
$\begin{array}{l}\text { Severity } \\
\text { Category }\end{array}$ & $\begin{array}{c}\text { Release } \\
\text { Fraction }\end{array}$ \\
\hline $\begin{array}{l}\text { Truck } \\
\text { I }\end{array}$ & $0 \mathrm{E}+00$ \\
II & $1 \mathrm{E}-02$ \\
III & $1 \mathrm{E}-01$ \\
IV & $1.0 \mathrm{E}+00$ \\
V & $1.0 \mathrm{E}+00$ \\
VI & $1.0 \mathrm{E}+00$ \\
VII & $1.0 \mathrm{E}+00$ \\
VIII & $1.0 \mathrm{E}+00$ \\
& \\
Rail & \\
I & $0 \mathrm{E}+00$ \\
II & $1 \mathrm{E}-02$ \\
III & $1 \mathrm{E}-01$ \\
IV & $1.0 \mathrm{E}+00$ \\
V & $1.0 \mathrm{E}+00$ \\
VI & $1.0 \mathrm{E}+00$ \\
VII & $1.0 \mathrm{E}+00$ \\
VIII & $1.0 \mathrm{E}+00$ \\
\hline
\end{tabular}

Source: NRC (1977a). neutral meteorologic conditions constitute the most frequently occurring atmospheric stability conditions in the United States, these conditions are most likely to be present in the event of an accident involving a LLMW shipment. On the basis of observations from National Weather Service surface meteorologic stations at over 300 locations in the United States, on an annual average, neutral conditions (represented by Pasquill Class C) occur about one-half of the time, while stable (Pasquill Classes $\mathbf{E}$ and F) and unstable (Pasquill Classes A through C) conditions occur about one-third and one-sixth of the time, respectively (Doty et al. 1976). The neutral category predominates in all seasons but most frequently in the winter (nearly $60 \%$ of the observations). For the accident consequence assessment, doses were assessed under both neutral and stable (Class $D$ with a wind speed of $4 \mathrm{~m} / \mathrm{s}$ [9 mph] and Class F with 
TABLE 4.5 Aerosolized and Respirable Material Release Fractions for LLMW ${ }^{\mathrm{a}}$

\begin{tabular}{lcc}
$\begin{array}{c}\text { Physical Form } \\
\text { of Waste }\end{array}$ & $\begin{array}{c}\text { Aerosolized } \\
\text { Fraction }\end{array}$ & $\begin{array}{c}\text { Respirable } \\
\text { Fraction }\end{array}$ \\
\hline $\begin{array}{l}\text { Organic liquids } \\
\text { Heterogeneous solids }\end{array}$ & $1 \mathrm{E}+00$ & $1 \mathrm{E}+00$ \\
\hline
\end{tabular}

a The aerosolized fraction is the fraction of the released material that is entrained as an aerosol during an accident. The respirable fraction is the fraction of the aerosolized release that is of a respirable size.

b Considered to behave as a loose powder.

Source: Neuhauser and Kanipe (1993).

a wind speed of $1 \mathrm{~m} / \mathrm{s}$ [2.2 $\mathrm{mph}$ ]) atmospheric conditions. The results calculated for neutral conditions represent the most likely consequences, and the results for stable conditions represent a "worst-case" weather situation (i.e., conditions that result in the least amount of dilution and the highest air concentrations of radioactive material).

\subsection{EXTERNAL DOSE RATES FROM SHIPMENTS}

The dose (and, correspondingly, the risk) to populations during routine transportation is directly proportional to the assumed external dose rate from the shipment. The actual dose rate from the shipment is a complex function of the composition and configuration of shielding and containment materials used in the waste packaging, the geometry of the loaded shipment, and the characteristics of the waste material itself. Representative shipment dose rates have been developed and used in the WM PEIS LLMW transportation analysis. In practice, external dose rates will vary not only from site to site and from waste type to waste type, but also from shipment to shipment at a given site.

The external dose rate for shipments of LLMW was assumed to be the same as for the LLW analysis conducted for the WM PEIS. For WM LLW shipments, the external dose rates from historical waste shipments were investigated by using the Shipment Mobility/Accountability Collection (SMAC) system (R. Morris, 1993, Science Applications International Corp., personal communication to F.A. Monette, ANL-E, Argonne, Ill., Dec. 9). The SMAC database contains information on unclassified commercial freight shipments made by the DOE and its contractors. The information available in the SMAC database is collected from site shipping and receiving documents. Available information for shipments of radioactive materials includes the types of material shipped, the number of packages in each shipment, shipment weights, external dose rates, and package isotopic inventories. Approximately two-thirds of all DOE unclassified shipments are estimated to be reported to the SMAC system. 
Shipment information from the SMAC database was examined for fiscal years 1983 to 1993 (R. Morris, 1993, Science Applications International Corp., personal communication to F.A. Monette, ANL-E, Argonne, Ill., Dec. 9). Information was provided for three general categories of radioactive material: irradiated fuel, "other" highway route controlled quantities, and LLW. (The material categories chosen were dictated by the format in which data are submitted and entered into the SMAC database and are not consistent with the definitions of waste types used in the WM PEIS.) Of the 15,000 LLW shipments recorded in the SMAC database, approximately 2,500 reported external dose rates. The average dose rate reported was approximately $1 \mathrm{mrem} / \mathrm{h}$ measured at $1 \mathrm{~m}(3.3 \mathrm{ft})$ from the surface of a shipment. This value was used for future WM LLMW shipments for the WM PEIS analysis.

\subsection{RADIOLOGICAL MAXIMALLY EXPOSED INDIVIDUAL EXPOSURE SCENARIOS}

The radiological risk to MEIs has been estimated for a number of hypothetical exposure scenarios for LLMW shipments. The receptors include crew members, departure inspectors, and members of the public exposed during traffic obstructions (traffic jam), while working at a service station, or by living near a treatment, storage, or disposal site. The dose and risk to MEIs were calculated for given distances and durations of exposure. The distances and durations of exposure for each receptor are similar to those given in previous transportation assessments (DOE 1987b, 1990a). The scenarios for exposure are not meant to be exhaustive but were selected to provide a range of potential situations for exposure. The assumptions for exposure scenarios are as follows:

- Crew Members. Truck and rail crew members are assumed to be occupational radiation workers and would be monitored by a dosimetry program. Therefore, the maximum allowable dose would be $5 \mathrm{rem} / \mathrm{yr}$. As an administrative procedure, the DOE limits doses to DOE workers to $2 \mathrm{rem} / \mathrm{yr}$ (DOE 1992).

- Inspectors (Truck and Rail). Inspectors are assumed to be either federal or state vehicle inspectors. Inspectors are not assumed to be monitored by a dosimetry program. An average exposure distance of $3 \mathrm{~m}(10 \mathrm{ft})$ and an exposure duration of 30 minutes are assumed.

- Rail-Yard Crew Member. A rail-yard crew member is not assumed to be monitored by a dosimetry program. An average exposure distance of $10 \mathrm{~m}(33 \mathrm{ft})$ and an exposure duration of two hours are assumed.

- Resident (Truck and Rail). A resident is assumed to live $30 \mathrm{~m}$ (98 ft) from a site entrance route (truck or rail). Shipments pass at an average speed of $24 \mathrm{~km} / \mathrm{h}(15 \mathrm{mph})$, and the resident is exposed unshielded. Cumulative doses are assessed for each site on the basis of the number of shipments entering or exiting the site, with the assumption that the resident is present for $100 \%$ of the shipments. 
- Person in Traffic Obstruction (Truck and Rail). A person is assumed to be stopped next to a waste shipment (e.g., because of traffic slowdown). The person is assumed to be exposed unshielded at a distance of $1 \mathrm{~m}$ $(3.3 \mathrm{ft}$ ) for a duration of 30 minutes.

- Person at Truck Service Station. A person is assumed to be exposed at an average distance of $20 \mathrm{~m}$ (66 ft) for a duration of two hours. This receptor could be a worker at a truck stop.

- Resident near a Rail Stop. A resident is assumed to live near a rail classification yard. The resident is assumed to be exposed unshielded at a distance of $200 \mathrm{~m}(656 \mathrm{ft})$ for a duration of 20 hours.

The largest uncertainty in predicting the dose to MEIs during transportation involves determining the frequency of occurrence of exposures. This difficulty results from the uncertainties in future shipment schedules and route selection and from the inherent uncertainty in predicting the frequency of random or chance events; for instance, that an individual may be stopped in traffic next to a shipment of radioactive waste is conceivable; however, predicting how often the same individual would experience this event is difficult. Therefore, for the majority of receptors considered, doses are assessed on a per-event basis. To account for possible multiple exposures, ranges of realistic total doses are discussed qualitatively. One exception is the calculation of the dose to a hypothetical resident living near an entrance route to a storage or disposal site. For these residents, total doses are calculated on the basis of the number of shipments entering or exiting each site for each case.

\subsection{GENERAL RADIOLOGICAL ASSESSMENT INPUT PARAMETERS}

In addition to the specific parameters discussed previously, values for a number of general parameters must be specified within the RADTRAN code to calculate radiological risks. These general parameters define basic characteristics of the shipment and traffic and are specific to the mode of transportation. The user's manual for the RADTRAN code (Neuhauser and Kanipe 1993) contains derivations and descriptions of these parameters. The general RADTRAN input parameters used in the radiological transportation risk assessment are summarized in Table 4.6. 
TABLE 4.6 General RADTRAN Input Parameters for the LLMW Assessment

\begin{tabular}{lcc}
\multicolumn{1}{c}{ Parameter } & Truck & Rail \\
\hline Package type & Type A & Type A \\
Shipment size (length) (m) & 12 & 16 \\
Shipment capacity (kg) & 20,000 & 55,000 \\
No. of crewmen & 2 & 5 \\
Distance from source to crew (m) & 3 & 152 \\
Average vehicle speed (km/h) & 88 & 64 \\
Rural & 40 & 40 \\
Suburban & 24 & 24 \\
Urban & 0.011 & 0.033 \\
Stop time (h/km) & 25 & 100 \\
No. of people exposed while stopped & 2 & 3 \\
No. of people per vehicle sharing route & & \\
Population densities (persons/km ${ }^{2}$ ) & 6 & 6 \\
Rural & 719 & 719 \\
Suburban & 3,861 & 3,861 \\
Urban & & \\
One-way traffic count (vehicles/h) & 470 & 1 \\
Rural & 780 & 5 \\
Suburban & 2,800 & 5 \\
Urban & $\mathrm{a}$ & $\mathrm{a}$ \\
Accident release fractions & $\mathrm{b}$ & $\mathrm{b}$ \\
Accident conditional probabilities & & \\
\hline
\end{tabular}

a See Table 4.4.

b See Table 4.3.

Source: Neuhauser and Kanipe (1993). 


\section{RISK ASSESSMENT RESULTS}

\subsection{RADIOLOGICAL ASSESSMEN'T}

The results of the LLMW transportation radiological risk assessment are summarized in this section for the seven WM cases defined in Section 2.2. Although the method for risk assessment and the important assumptions used for the assessment have been presented in detail in previous sections of this report, a brief overview of the risk assessment process is provided subsequently to aid the reader in interpreting the results of risk assessment.

The impacts of transportation are calculated in three areas: (1) collective population risks during routine conditions and accidents for each case, (2) risks to MEIs during routine conditions for each case, and (3) consequences to individuals and populations after the most severe accidents involving a release of radioactive material. These three areas are detailed in the following paragraphs:

- Collective Population Risk. The collective population risk is a measure of the total risk posed to society as a whole by the case being considered. For the collective population risk assessment, the persons exposed are considered as a group, without specifying individual receptors. The collective population risk is used as the primary means to compare the various cases.

Collective population risks are calculated from both vehicle- and cargorelated causes for routine transportation and accidents. Vehicle-related risks are independent of the cargo in the shipment and include risks from vehicular exhaust emissions and traffic accidents (fatalities caused by physical trauma). Vehicle-related risks are presented in terms of estimated fatalities for each case.

For radioactive material, cargo-related risk refers to the risk posed by the radioactive nature of the material. The collective population risks are calculated for each case by using RADTRAN 4. The RADTRAN 4 calculations for population risk take into account both the consequences and the probabilities of potential exposure-causing events (such as accidents). The accident risk values are referred to as "dose risk" because they incorporate the probabilities of a spectrum of accidents. The collective population risks are presented in terms of the total dose (person-rem) to workers and to members of the public for each case. The collective population risks are also presented in terms of the estimated number of fatal latent-cancer cases, the number of latent-cancer cases (incidence), and the number of severe genetic effects by using the health risk conversion factors from ICRP publication 60 (ICRP 1991). 
- Maximally Exposed Individuals during Routine Conditions. During the routine transportation of radioactive waste, specific individuals may be exposed to radiation in the vicinity of a shipment. The risk to these individuals has been estimated for a number of hypothetical exposurecausing events by using RISKIND. The receptors include transportation crew members, inspectors, and members of the public exposed during traffic delays, while working at a service station, or while living near a DOE site. The assumptions about exposure are given in Section 4.6. The scenarios for exposure are not meant to be exhaustive but were selected to provide a range of potential exposures.

For the majority of the individual receptors considered, doses are assessed and presented on a per-event basis - no attempt is made to estimate the frequency of exposure-causing events, although the range of possible exposures is qualitatively discussed; however, one exception is the calculation of the dose to a hypothetical resident living near the entrance route to a treatment, storage, or disposal site. For these residents, cumulative doses are calculated on the basis of the total number of shipments entering or exiting each site for each case.

- Accident Consequence Assessment. The RISKIND code is used to provide a detailed assessment of the consequences of the most severe transportation-related accidents involving LLMW shipments. Whereas the RADTRAN 4 collective accident risk assessment considers the entire range of accident severities and their related probabilities, the RISKIND accident consequence assessment assumes that an accident of the highest severity category (Category VIII) has occurred. The consequences, in terms of committed dose (rem) and latent-cancer fatalities, are calculated for both exposed populations and individuals in the vicinity of an accident.

\subsubsection{Collective Population Radiological Risk Results}

The results of the collective population risk assessment are summarized in this section. The summarized results present the total transportation impacts that would be incurred across the DOE complex for each case from all LLMW shipments associated with that case. The summarized results do not indicate the quantity of waste that would be shipped between specific sites for a given case; however, detailed results of assessment are presented in Appendix B of this report. The detailed results include route information, the estimated number of annual shipments, and "per-shipment" risk factors (an estimate of the risk for a single shipment between two specific sites) for shipments between all origindestination pairs considered by each case.

The collective risk results for the seven WM LLMW cases are summarized in a series of tables. The collective risk results were calculated with the RADTRAN 4 computer code by 
using the WASTE_MGMT files provided in Appendix A of this report as the primary input. The summarized results present the total radiological impacts from shipping current inventories plus 20 years of WM LLMW generation. As stated previously, the average annual risk (or number of shipments) can be estimated by dividing the summarized results by the duration of the shipping campaigns. Shipments would be distributed uniformly over a 10-year period, with the assumption of a 10-year period to build TSD facilities. Note that LLMW Cases 1 and 26 involve no off-site transportation of waste and have thus been omitted from the following tables.

The total number of shipments and shipment mileages are presented for each of the WM LLMW cases in Table 5.1. The numbers of shipments were calculated by using the inventory data presented in Appendix $\mathrm{A}$ of this report and the assumptions concerning representative shipment sizes presented in Section 4.1. The total incident-free (routine) dose for each case is presented in Table 5.2 in terms of person-rem. The routine doses are presented separately for crew members and for members of the public along the transportation routes; these doses result from exposures to external radiation in the vicinity of a shipment. The total accident dose risk for each case is presented in Table 5.3. The estimates of accident dose risk depend on the specific radionuclides present in each shipment and take into account the probability of accident occurrences and the distribution of accident severities. Finally, impacts are presented in terms of human health risks in Tables 5.4 through 5.6. The health risks are presented for cargo-related (radiological) and vehiclerelated (nonradiological) causes. All impacts are presented in two columns, with the first column assuming all truck shipments and the second assuming all rail shipments.

\subsubsection{Maximally Exposed Individual Assessment: Routine Transport}

Doses to individuals during routine transportation have been estimated for each of the receptor scenarios described in Section 4.6 for WM LLMW shipments. The estimated doses to individuals exposed to WM LLMW shipments are presented in Table 5.7. The doses are calculated by assuming an external dose rate of $1 \mathrm{mrem} / \mathrm{h}$ at $1 \mathrm{~m}(3.3 \mathrm{ft})$ from a shipment and are reported on a per-event basis (rem per event). The total dose for repeated exposures can be estimated by multiplying the per-event dose by the number of exposures.

The potential exists for significant individual exposures if multiple exposure-causing events occur during WM LLMW shipments; for instance, the dose to a person stuck in traffic next to a shipment for 30 minutes is estimated to be $0.5 \mathrm{mrem}$. If the duration of exposure is longer, the dose would rise proportionally. That a person could receive a dose on the order of 2 to 10 mrem while stopped in traffic next to a shipment is conceivable. In addition, a person working at a truck service station could receive an increased dose if trucks use the same stops repeatedly. If a truck-stop worker is present for 100 shipment stops (at the distance and duration given previously), the estimated dose is on the order of 2 mrem. Administrative controls could be instituted to control the location and duration of truck stops if multiple exposures were to happen routinely. The probability of multiple exposures increases as the amount of waste transportation increases. 
TABLE 5.1 Total Number of Shipments and One-Way Shipping Distances for the WM LLMW Alternatives ${ }^{a}$

\begin{tabular}{|c|c|c|c|c|}
\hline \multirow{2}{*}{$\begin{array}{l}\text { WM LLMW } \\
\text { Alternative }\end{array}$} & \multicolumn{2}{|c|}{ No. of Shipments ${ }^{b}$} & \multicolumn{2}{|c|}{ Total Mileage (mi) } \\
\hline & Truck & Rail & Truck & Rail \\
\hline Decentralized & 480 & 350 & $2.46 \mathrm{E}+05$ & $2.31 \mathrm{E}+05$ \\
\hline Regionalized 1 & 1,810 & 1,030 & $5.94 \mathrm{E}+05$ & $4.81 \mathrm{E}+05$ \\
\hline Regionalized 2 & 5,560 & 2,490 & $2.57 \mathrm{E}+06$ & $1.37 \mathrm{E}+06$ \\
\hline Regionalized 3 & 10,990 & 4,540 & $1.49 \mathrm{E}+07$ & $6.76 \mathrm{E}+06$ \\
\hline Regionalized 4 & 4,250 & 2,050 & $2.89 E+06$ & $1.57 \mathrm{E}+06$ \\
\hline Centralized & 7,520 & 3,340 & $1.35 \mathrm{E}+07$ & $6.46 \mathbf{E}+06$ \\
\hline
\end{tabular}

a For each alternative, impacts are calculated and presented separately assuming exclusive truck or exclusive rail shipments. Therefore, the impacts for truck and rail modes should not be added together.

b The number of shipments is calculated by assuming that truck and rail shipments consist of $19,958 \mathrm{~kg}(44,000 \mathrm{lb})$ and $54,431 \mathrm{~kg}(120,000 \mathrm{lb})$ of LLMW, respectively.

TABLE 5.2 Total Routine (Incident-Free) Dose for the WM LLMW Alternatives ${ }^{a}$

\begin{tabular}{|c|c|c|c|c|}
\hline \multirow{3}{*}{$\begin{array}{l}\text { WM LLMW } \\
\text { Alternative }\end{array}$} & \multicolumn{4}{|c|}{ Dose (person-rem) } \\
\hline & \multicolumn{2}{|c|}{ Truck } & \multicolumn{2}{|c|}{ Rail } \\
\hline & Crew $^{b}$ & Public & Crew $^{b}$ & Public ${ }^{c}$ \\
\hline Decentralized & $8.22 \mathrm{E}+00$ & $9.72 \mathrm{E}+00$ & $1.97 \mathrm{E}+00$ & $5.75 \mathrm{E}+00$ \\
\hline Regionalized 1 & $2.04 \mathrm{E}+01$ & $2.31 E+01$ & $4.98 \mathrm{E}+00$ & $1.37 \mathrm{E}+01$ \\
\hline Regionalized 2 & $8.03 E+01$ & $9.26 \mathrm{E}+01$ & $1.29 \mathrm{E}+01$ & $2.91 \mathrm{E}+01$ \\
\hline Regionalized 3 & $4.29 \mathrm{E}+02$ & $5.13 \mathrm{E}+02$ & $4.13 E+01$ & $7.58 \mathrm{E}+01$ \\
\hline Regionalized 4 & $8.41 E+01$ & $9.86 \mathrm{E}+01$ & $1.25 \mathrm{E}+01$ & $2.82 \mathrm{E}+01$ \\
\hline Centralized & $3.74 \mathrm{E}+02$ & $4.47 \mathrm{E}+02$ & $3.66 \mathrm{E}+01$ & $6.93 E+01$ \\
\hline
\end{tabular}

a Routine doses result from exposure to external radiation in the vicinity of a loaded shipment. The external dose rate is assumed to be $1 \mathrm{mrem} / \mathrm{h}$ at $1 \mathrm{~m}(3.3 \mathrm{ft})$ from a WM LLMW shipment. For each alternative, impacts are calculated and presented separately assuming exclusive truck or exclusive rail shipments. Therefore, the impacts for truck and rail modes should not be added together.

b The crew includes truck drivers, rail crew members, and rail workers in marshaling or classification yards.

c Members of the public include persons in vehicles sharing transportation routes, persons living along routes, and persons exposed at shipment stops. 
TABLE 5.3 Total Accident Dose Risk for the WM LLMW Alternatives ${ }^{a, b}$

\begin{tabular}{|c|c|c|}
\hline \multirow{2}{*}{$\begin{array}{l}\text { WM LLMW } \\
\text { Alternative }\end{array}$} & \multicolumn{2}{|c|}{ Dose Risk (person-rem) } \\
\hline & Truck & Rail \\
\hline Decentralized & $1.09 \mathrm{E}+00$ & $3.11 \mathrm{E}-01$ \\
\hline Regionalized 1 & $2.23 \mathrm{E}+00$ & $5.96 \mathrm{E}-01$ \\
\hline Regionalized 2 & $1.54 \mathrm{E}+01$ & $2.18 \mathrm{E}+00$ \\
\hline Regionalized 3 & $2.60 \mathrm{E}+01$ & $4.61 \mathrm{E}+00$ \\
\hline Regionalized 4 & $1.59 \mathrm{E}+01$ & $2.60 \mathrm{E}+00$ \\
\hline Centralized & $1.48 \mathrm{E}+02$ & $2.76 \mathrm{E}+01$ \\
\hline
\end{tabular}

a The calculation of accident dose risk takes into account the dose to the public following accidents involving the release of radioactive material to the environment, the probability of accident occurrences, and the distribution of accident severities.

b For each alternative, impacts are calculated and presented separately assuming exclusive truck or exclusive rail shipments. Therefore, the impacts for truck and rail modes should not be added together.

The potential cumulative dose to a resident living along a site entrance route has been estimated and is summarized in Table 5.8 for each WM LLMW case. The cumulative dose assumes that a resident is present for every shipment either entering or exiting a site and is unshielded at a distance of $30 \mathrm{~m}$ (98 ft) from the roadway. The assessment considers 20 years of shipments for WM LLMW. The lifetime risk of fatality due to cancer has been calculated by multiplying the doses by the ICRP health risk conversion factor of $5 \times 10^{-4}$ fatal cancers per rem for members of the public. The estimated annual dose to a resident would be well below the annual limit of 100 mrem specified for members of the public (DOE 1990b).

\subsubsection{Radiological Accident Consequence Assessment}

The RISKIND (Yuan et al. 1993) code is used to provide a detailed assessment of the consequences of the most severe transportation-related accidents for the WM LLMW shipments. Whereas the RADTRAN 4 accident risk assessment considers the entire range of accident severities and their related probabilities, the RISKIND accident consequence assessment assumes that an accident of the highest credible severity has occurred.

Accident consequences are presented for a shipment of waste that represents the highest potential radiological risk if an accident occurs. This "worst-case" waste is identified by screening the site-specific radiological waste characteristics (i.e., activity concentrations) developed for the WM PEIS, taking into account physical waste forms and the relative 
TABLE 5.4 Total Estimated Fatalities for the WM LLMW Alternatives ${ }^{\text {a,b }}$

\begin{tabular}{|c|c|c|c|}
\hline \multirow[b]{2}{*}{$\begin{array}{l}\text { WM LLMW } \\
\text { Alternative }\end{array}$} & \multirow[b]{2}{*}{ Exposure Group } & \multicolumn{2}{|c|}{ Fatalities } \\
\hline & & Truck & Rail \\
\hline \multirow[t]{7}{*}{ Decentralized } & $\begin{array}{l}\text { Cargo-related } \\
\text { (radiological) }\end{array}$ & & \\
\hline & Normal crew & $3.3 \mathrm{E}-03$ & $8.1 \mathrm{E}-04$ \\
\hline & Normal public & $5.0 \mathrm{E}-03$ & $2.9 \mathrm{E}-03$ \\
\hline & Accident public & $5.4 \mathrm{E}-04$ & $1.6 \mathrm{E}-04$ \\
\hline & $\begin{array}{l}\text { Vehicle-related } \\
\text { (nonradiological) }\end{array}$ & & \\
\hline & Emission & $4.6 \mathrm{E}-03$ & $5.7 \mathrm{E}-03$ \\
\hline & Accident & $1.8 \mathrm{E}-02$ & $5.0 \mathrm{E}-04$ \\
\hline \multirow[t]{7}{*}{ Regionalized 1} & $\begin{array}{l}\text { Cargo-related } \\
\text { (radiological) }\end{array}$ & & \\
\hline & Normal crew & $8.3 \mathrm{E}-03$ & $2.0 \mathrm{E}-03$ \\
\hline & Normal public & $1.2 \mathrm{E}-02$ & $6.9 \mathrm{E}-03$ \\
\hline & Accident public & $1.1 \mathrm{E}-03$ & $3.0 \mathrm{E}-04$ \\
\hline & $\begin{array}{l}\text { Vehicle-related } \\
\text { (nonradiological) }\end{array}$ & & \\
\hline & Emission & $8.5 \mathrm{E}-03$ & $1.3 E-02$ \\
\hline & Accident & $3.8 \mathrm{E}-02$ & $1.0 \mathrm{E}-03$ \\
\hline \multirow[t]{7}{*}{ Regionalized 2} & $\begin{array}{l}\text { Cargo-related } \\
\text { (radiological) }\end{array}$ & & \\
\hline & Normal crew & $3.2 \mathrm{E}-02$ & $5.2 \mathrm{E}-03$ \\
\hline & Normal public & $4.5 \mathrm{E}-02$ & $1.4 \mathrm{E}-02$ \\
\hline & Accident public & $7.6 \mathrm{E}-03$ & $1.1 \mathrm{E}-03$ \\
\hline & $\begin{array}{l}\text { Vehicle-related } \\
\text { (nonradiological) }\end{array}$ & & \\
\hline & Emission & $2.4 \mathrm{E}-02$ & $2.3 \mathrm{E}-02$ \\
\hline & Accident & $1.9 \mathrm{E}-01$ & $2.8 \mathrm{E}-03$ \\
\hline \multirow[t]{7}{*}{ Regionalized 3} & $\begin{array}{l}\text { Cargo-related } \\
\text { (radiological) }\end{array}$ & & \\
\hline & Normal crew & $1.7 \mathrm{E}-01$ & $1.7 \mathrm{E}-02$ \\
\hline & Normal public & $2.6 \mathrm{E}-01$ & $3.8 \mathrm{E}-02$ \\
\hline & Accident public & $1.3 \mathrm{E}-02$ & $2.4 \mathrm{E}-03$ \\
\hline & $\begin{array}{l}\text { Vehicle-related } \\
\text { (nonradiological) }\end{array}$ & & \\
\hline & Emission & $1.0 \mathrm{E}-01$ & $5.5 \mathrm{E}-02$ \\
\hline & Accident & $1.0 \mathrm{E}+00$ & $1.4 \mathrm{E}-02$ \\
\hline
\end{tabular}


TABLE 5.4 (Cont.)

\begin{tabular}{cccc}
\hline & & \multicolumn{2}{c}{ Fatalities } \\
\cline { 3 - 4 } WM LLMW & & & \\
Alternative & Exposure Group & Truck & Rail \\
\hline \multirow{2}{*}{ Regionalized 4 } & Cargo-related & & \\
& (radiological) & & \\
& Normal crew & $3.3 \mathrm{E}-02$ & $5.0 \mathrm{E}-03$ \\
& Normal public & $4.8 \mathrm{E}-02$ & $1.4 \mathrm{E}-02$ \\
& Accident public & $7.8 \mathrm{E}-03$ & $1.3 \mathrm{E}-03$ \\
& Vehicle-related & & \\
& (nonradiological) & & \\
& Emission & $1.5 \mathrm{E}-02$ & $2.4 \mathrm{E}-02$ \\
& Accident & $1.9 \mathrm{E}-01$ & $3.2 \mathrm{E}-03$ \\
& & & \\
& Cargo-related & & \\
(radiological) & & \\
& Normal crew & $1.5 \mathrm{E}-01$ & $1.5 \mathrm{E}-02$ \\
& Normal public & $2.2 \mathrm{E}-01$ & $3.5 \mathrm{E}-02$ \\
& Accident public & $7.4 \mathrm{E}-02$ & $1.4 \mathrm{E}-02$ \\
& Vehicle-related & & \\
& (nonradiological) & & \\
& Emission & $5.4 \mathrm{E}-02$ & $5.3 \mathrm{E}-02$ \\
& Accident & $8.3 \mathrm{E}-01$ & $1.4 \mathrm{E}-02$ \\
\hline
\end{tabular}

a Latent-cancer fatalities are calculated by multiplying dose by the ICRP publication 60 (ICRP 1991) health risk conversion factors of $4 \mathrm{E}-04$ and $5 \mathrm{E}-04$ fatal cancers per person-rem for workers and for the public, respectively.

b For each alternative, impacts are calculated and presented separately assuming exclusive truck or exclusive rail shipments. Therefore, the impacts for truck and rail modes should not be added together.

hazards of individual radionuclides. For the majority of waste shipments, the consequences of severe accidents would be less than those presented for the "worst-case" waste. The accident consequence assessment is intended to provide an estimate of the maximum potential impacts posed by a severe transportation accident involving a given waste type. The assessment is not meant to be used as a basis for comparison among the different alternatives.

As stated previously, the accident consequences were calculated for transportationrelated accidents that result in the maximum release of radioactive material (accident severity Category VIII). For these accidents, the assumptions were that all of the material in the shipment would be released from its packaging, that $10 \%$ would be entrained as an aerosol, and that $5 \%$ of the aerosol would be respirable. 
TABLE 5.5 Total Estimated Cancer Incidence for the WM LLMW Alternatives ${ }^{a, b}$

\begin{tabular}{|c|c|c|c|}
\hline \multirow{2}{*}{$\begin{array}{l}\text { WM LLMW } \\
\text { Alternative }\end{array}$} & \multirow[b]{2}{*}{ Exposure Group } & \multicolumn{2}{|c|}{ Cancer Cases } \\
\hline & & Truck & Rail \\
\hline \multirow[t]{7}{*}{ Decentralized } & $\begin{array}{l}\text { Cargo-related } \\
\text { (radiological) }\end{array}$ & & \\
\hline & Normal crew & $1.2 \mathrm{E}-02$ & $2.9 \mathrm{E}-03$ \\
\hline & Normal public & $1.7 \mathrm{E}-02$ & $1.0 \mathrm{E}-02$ \\
\hline & Accident public & $1.8 \mathrm{E}-03$ & $5.4 \mathrm{E}-04$ \\
\hline & $\begin{array}{l}\text { Vehicle-related } \\
\text { (nonradiological) }\end{array}$ & & \\
\hline & Emission & $4.6 \mathrm{E}-03$ & $5.7 \mathrm{E}-03$ \\
\hline & Accident & $\mathrm{NA}^{\mathrm{c}}$ & NA \\
\hline \multirow[t]{7}{*}{ Regionalized 1} & $\begin{array}{l}\text { Cargo-related } \\
\text { (radiological) }\end{array}$ & & \\
\hline & Normal crew & $2.8 \mathrm{E}-02$ & $7.0 \mathrm{E}-03$ \\
\hline & Normal public & $3.9 \mathrm{E}-02$ & 2.3E-02 \\
\hline & Accident public & $3.8 \mathrm{E}-03$ & $1.0 \mathrm{E}-03$ \\
\hline & $\begin{array}{l}\text { Vehicle-related } \\
\text { (nonradiological) }\end{array}$ & & \\
\hline & Emission & $8.5 E-03$ & $1.3 \mathrm{E}-02$ \\
\hline & Accident & NA & NA \\
\hline \multirow[t]{7}{*}{ Regionalized 2} & $\begin{array}{l}\text { Cargo-related } \\
\text { (radiological) }\end{array}$ & & \\
\hline & Normal crew & $1.1 \mathrm{E}-01$ & $1.8 \mathrm{E}-02$ \\
\hline & Normal public & $1.6 \mathrm{E}-01$ & $4.9 E-02$ \\
\hline & Accident public & $2.6 \mathrm{E}-02$ & $3.8 \mathrm{E}-03$ \\
\hline & $\begin{array}{l}\text { Vehicle-related } \\
\text { (nonradiological) }\end{array}$ & & \\
\hline & Emission & $2.4 \mathrm{E}-02$ & $2.3 \mathrm{E}-02$ \\
\hline & Accident & NA & NA \\
\hline \multirow[t]{7}{*}{ Regionalized 3} & $\begin{array}{l}\text { Cargo-related } \\
\text { (radiological) }\end{array}$ & & \\
\hline & Normal crew & $6.1 \mathrm{E}-01$ & $5.8 \mathrm{E}-02$ \\
\hline & Normal public & $8.7 \mathrm{E}-01$ & $1.3 \mathrm{E}-01$ \\
\hline & Accident public & $4.4 \mathrm{E}-02$ & $7.9 \mathrm{E}-03$ \\
\hline & $\begin{array}{l}\text { Vehicle-related } \\
\text { (nonradiological) }\end{array}$ & & \\
\hline & Emission & $1.0 \mathrm{E}-01$ & $5.5 \mathrm{E}-02$ \\
\hline & Accident & NA & NA \\
\hline
\end{tabular}


TABLE 5.5 (Cont.)

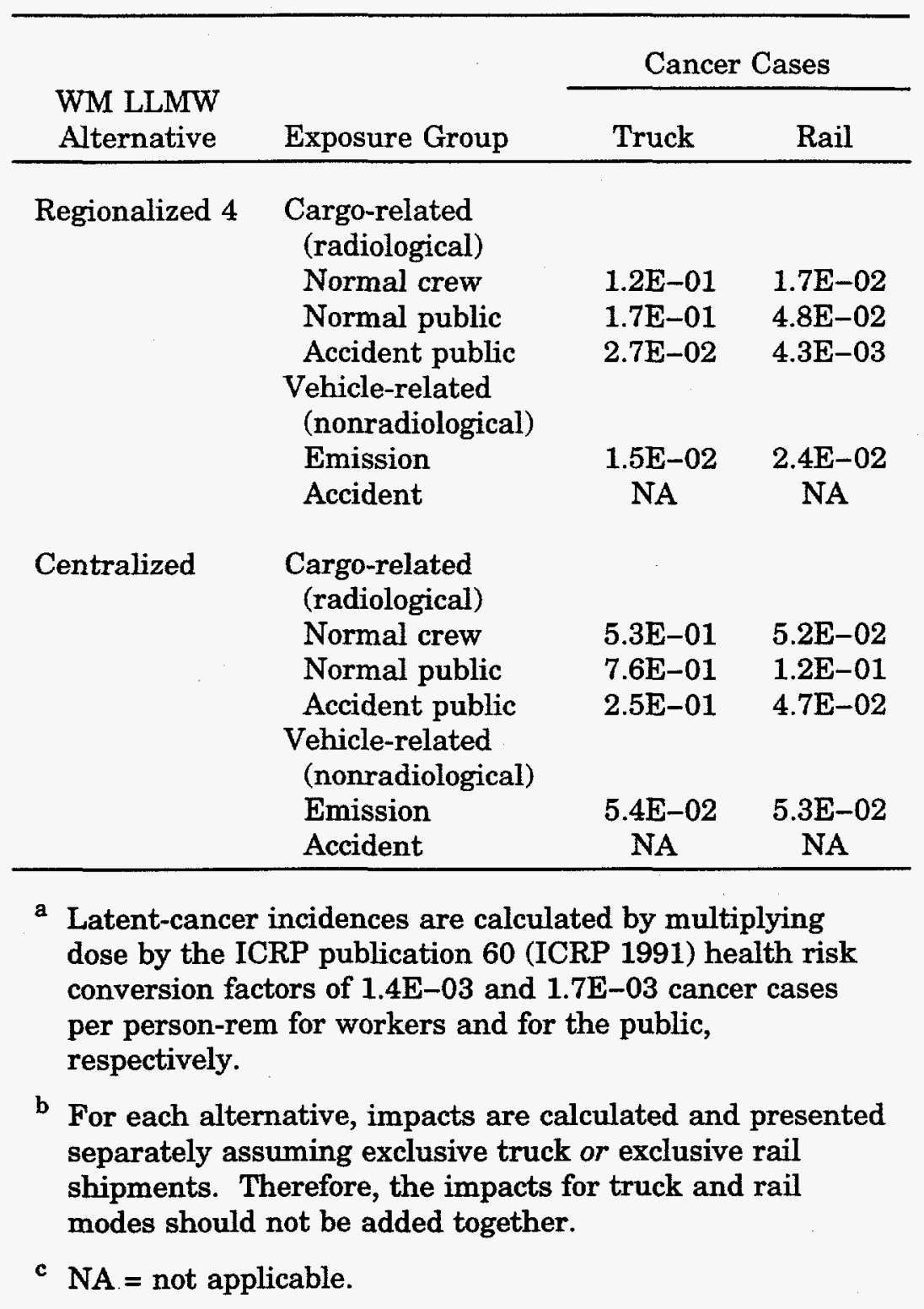

During screening, the heterogeneous solid WM LLMW from the Paducah Gaseous Diffusion Plant (PGDP) was found to result in the highest transportation accident doses. The accident consequence results from RISKIND for WM LLMW shipments are presented in Table 5.9. The population doses are for a uniform population density within an $80-\mathrm{km}$ (50-mi) radius of accidents occurring in rural, suburban, and urban population density zones. The location of the MEI after an accident is determined on the basis of atmospheric conditions and the buoyant characteristics of the released plume. The locations of maximum exposure are approximately $160 \mathrm{~m}(525 \mathrm{ft})$ and $400 \mathrm{~m}(1,312 \mathrm{ft})$ from the accident site for neutral and stable weather conditions, respectively. The dose to the MEI is independent of the location of the accident. The maximum dose to an individual (approximately 5 rem for a rail accident under unfavorable weather conditions) has a potential lifetime fatal-cancer risk of $2 \times 10^{-3}$. 
TABLE 5.6 Total Estimated Genetic Effects for the WM LLMW Alternatives ${ }^{\mathrm{a}, \mathrm{b}}$

\begin{tabular}{|c|c|c|c|}
\hline \multirow{2}{*}{$\begin{array}{l}\text { WM LLMW } \\
\text { Alternative }\end{array}$} & \multirow[b]{2}{*}{ Exposure Group } & \multicolumn{2}{|c|}{ Genetic Effects } \\
\hline & & Truck & Rail \\
\hline \multirow[t]{7}{*}{ Decentralized } & $\begin{array}{l}\text { Cargo-related } \\
\text { (radiological) }\end{array}$ & & \\
\hline & Normal crew & 5.0E-04 & $1.2 \mathrm{E}-04$ \\
\hline & Normal public & $1.0 \mathrm{E}-03$ & $5.8 \mathrm{E}-04$ \\
\hline & Accident public & $7.5 \mathrm{E}-05$ & $2.1 \mathrm{E}-05$ \\
\hline & $\begin{array}{l}\text { Vehicle-related } \\
\text { (nonradiological) }\end{array}$ & & \\
\hline & Emission & $N^{c}$ & NA \\
\hline & Accident & $\mathrm{NA}$ & NA \\
\hline \multirow[t]{7}{*}{ Regionalized 1} & $\begin{array}{l}\text { Cargo-related } \\
\text { (radiological) }\end{array}$ & & \\
\hline & Normal crew & $1.2 \mathrm{E}-03$ & $3.0 \mathrm{E}-04$ \\
\hline & Normal public & $2.3 \mathrm{E}-03$ & $1.4 \mathrm{E}-03$ \\
\hline & Accident public & $1.6 \mathrm{E}-04$ & $4.0 \mathrm{E}-05$ \\
\hline & $\begin{array}{l}\text { Vehicle-related } \\
\text { (nonradiological) }\end{array}$ & & \\
\hline & Emission & NA & NA \\
\hline & Accident & NA & NA \\
\hline \multirow[t]{7}{*}{ Regionalized 2} & $\begin{array}{l}\text { Cargo-related } \\
\text { (radiological) }\end{array}$ & & \\
\hline & Normal crew & $4.8 \mathrm{E}-03$ & $7.7 \mathrm{E}-04$ \\
\hline & Normal public & $9.3 \mathrm{E}-03$ & $2.9 E-03$ \\
\hline & Accident public & $2.6 \mathrm{E}-04$ & $6.1 \mathrm{E}-05$ \\
\hline & $\begin{array}{l}\text { Vehicle-related } \\
\text { (nonradiological) }\end{array}$ & & \\
\hline & Emission & NA & NA \\
\hline & Accident & NA & NA \\
\hline \multirow[t]{7}{*}{ Regionalized 3} & $\begin{array}{l}\text { Cargo-related } \\
\text { (radiological) }\end{array}$ & & \\
\hline & Normal crew & $2.6 \mathrm{E}-02$ & $2.5 E-03$ \\
\hline & Normal public & $5.1 \mathrm{E}-02$ & $7.6 \mathrm{E}-03$ \\
\hline & Accident public & $1.1 \mathrm{E}-03$ & 2.3E-04 \\
\hline & $\begin{array}{l}\text { Vehicle-related } \\
\text { (nonradiological) }\end{array}$ & & \\
\hline & Emission & NA & NA \\
\hline & Accident & NA & NA \\
\hline
\end{tabular}


TABLE 5.6 (Cont.)

\begin{tabular}{cccc}
\hline & & \multicolumn{2}{c}{ Genetic Effects } \\
\cline { 3 - 4 } WM LLMW & & & \\
Alternative & Exposure Group & Truck & Rail \\
\hline \multirow{2}{*}{ Regionalized 4 } & Cargo-related & & \\
& (radiological) & & \\
& Normal crew & $5.0 \mathrm{E}-03$ & $7.4 \mathrm{E}-04$ \\
& Normal public & $9.8 \mathrm{E}-03$ & $2.9 \mathrm{E}-03$ \\
& Accident public & $2.4 \mathrm{E}-04$ & $8.7 \mathrm{E}-05$ \\
& Vehicle-related & & \\
& (nonradiological) & & \\
& Emission & NA & NA \\
& Accident & NA & NA \\
& Cargo-related & & \\
Centralized & (radiological) & & \\
& Normal crew & $2.3 \mathrm{E}-02$ & $2.2 \mathrm{E}-03$ \\
& Normal public & $4.5 \mathrm{E}-02$ & $6.9 \mathrm{E}-03$ \\
& Accident public & $4.5 \mathrm{E}-03$ & $9.0 \mathrm{E}-04$ \\
& Vehicle-related & & \\
& (nonradiological) & & \\
& Emission & NA & NA \\
& Accident & NA & NA \\
\hline
\end{tabular}

a Genetic effects are calculated by multiplying dose by the ICRP publication 60 (ICRP 1991) health risk conversion factors of $6 \mathrm{E}-05$ and $1 \mathrm{E}-04$ adverse genetic effects per person-rem for workers and for the public, respectively.

b For each alternative, impacts are calculated and presented separately assuming exclusive truck or exclusive rail shipments. Therefore, the impacts for truck and rail modes should not be added together.

c $\mathrm{NA}=$ not applicable.

\subsection{HAZARDOUS ASSESSMENT RESULTS}

The results of the transportation risk assessment for the hazardous portion of LLMW are summarized in this section for the seven WM cases defined in Section 2.2. No risks are computed for the WM no action alternative (Case 1) or WM Case 26 because the number of LLMW shipments is zero or too small to produce any significant transportation risk. Cargorelated risks from accident conditions are calculated for the collective population and for the MEI. Vehicle-related risks from routine operations are reported in Section 5.1.1. The method for risk assessment and the assumptions used for the assessment have been presented in detail in Section 3.3 of this report. 
TABLE 5.7 Estimated Routine Doses and Lifetime Risk of Fatal Cancer to MEIs from Shipments of WM LLMW (per Exposure Event) $^{a}$

\begin{tabular}{|c|c|c|c|c|}
\hline \multirow[b]{2}{*}{ Receptor ${ }^{b}$} & \multicolumn{2}{|c|}{ Dose (rem) } & \multicolumn{2}{|c|}{ Lifetime Risk ${ }^{c}$} \\
\hline & Truck & Rail & Truck & Rail \\
\hline \multicolumn{5}{|l|}{ Workers } \\
\hline Crew member & & & & d \\
\hline Inspector & $1.5 \mathrm{E}-04$ & $1.5 \mathrm{E}-04$ & $6 E-08$ & $6 E-08$ \\
\hline Rail-yard crew member & $\mathrm{NA}^{\mathrm{e}}$ & $7.9 \mathrm{E}-05$ & NA & $3 E-08$ \\
\hline \multicolumn{5}{|l|}{ Public } \\
\hline Resident & $1.6 \mathrm{E}-08$ & $1.6 \mathrm{E}-08$ & $8 \mathrm{E}-12$ & $8 \mathrm{E}-12$ \\
\hline Person in traffic jam & $5.0 \mathrm{E}-04$ & $5.0 \mathrm{E}-04$ & $3 \mathrm{E}-07$ & $3 \mathrm{E}-07$ \\
\hline $\begin{array}{l}\text { Person at service } \\
\text { station }\end{array}$ & $2.1 \mathrm{E}-05$ & NA & $1 \mathrm{E}-08$ & NA \\
\hline Resident near rail stop & NA & $1.1 \mathrm{E}-06$ & NA & $6 \mathrm{E}-10$ \\
\hline \multicolumn{5}{|c|}{$\begin{array}{l}\text { Dose rate is assumed to be } 1 \mathrm{mrem} / \mathrm{h} \text { at } 1 \mathrm{~m}(3.3 \mathrm{ft}) \text { from a WM LLMW } \\
\text { shipment. }\end{array}$} \\
\hline \multicolumn{5}{|c|}{ beceptor assumptions are described in Section 4.6 of this report. } \\
\hline \multicolumn{5}{|c|}{$\begin{array}{l}\text { Lifetime risk of fatal cancer is based on ICRP publication } 60 \text { (ICRP } \\
\text { 1991) health risk conversion factors of } 4 \mathrm{E}-04 \text { and } 5 \mathrm{E}-04 \text { fatal cancers } \\
\text { per person-rem for workers and for members of the public, respectively. }\end{array}$} \\
\hline \multicolumn{5}{|c|}{$\begin{array}{l}\text { The DOE administrative control level limits doses to DOE workers to } \\
2 \text { rem/yr. For a representative shipment of } 1,609 \mathrm{~km}(1,000 \mathrm{mi}) \text { and an } \\
\text { average speed of } 80 \mathrm{~km} / \mathrm{h}(50 \mathrm{mph}) \text {, the dose to a driver would be } \\
\text { approximately } 40 \mathrm{mrem} \text { if the dose rate in the crew compartment were } \\
\text { at the regulatory limit of } 2 \mathrm{mrem} / \mathrm{h} \text {. Dose rates are typically much less } \\
\text { than } 2 \mathrm{mrem} / \mathrm{h} \text { in the crew compartment. }\end{array}$} \\
\hline e $\mathrm{NA}=$ not applicable. & & & & \\
\hline
\end{tabular}

The collective cargo-related population risks to the general public for 10 years of off-site WM LLMW transportation, associated with the most frequently occurring meteorologic conditions (i.e., neutral atmospheric stability with a wind speed of $4 \mathrm{~m} / \mathrm{s}$ [9 mph]), are summarized in Table 5.10 for highway shipments and in Table 5.11 for railroad shipments. Transportation accidents involving liquid waste shipments yield much higher population risks than those involving solid waste shipments by at least 4 orders of magnitude under the same LLMW treatment option. Due to the higher payload capacity of a railcar compared with that of a truck, the population risks are generally higher for railway than for highway transportation.

The potential population risks involving liquid waste shipments by trucks and railcars are attributed to the direct release of aerosolized liquid droplets. Truck-accident potential-increased-cancer (PIC) and PAE risks from aerosolized liquid droplets are highest for highway shipments under the centralized alternative, severity Category IV. 
TABLE 5.8 Cumulative Dose and Lifetime Risk to MEI Living Along a Site Entrance Route for WM LLMW Shipments (Current Inventories plus 20 Years of Generation) ${ }^{a}$

\begin{tabular}{|c|c|c|c|c|c|c|}
\hline \multirow{2}{*}{$\begin{array}{c}\text { WM } \\
\text { Alternative } \\
\text { and Site }\end{array}$} & \multicolumn{3}{|c|}{ All Truck } & \multicolumn{3}{|c|}{ All Rail } \\
\hline & $\begin{array}{c}\text { Total } \\
\text { Shipments }\end{array}$ & $\begin{array}{l}\text { Dose } \\
\text { (rem) }\end{array}$ & $\begin{array}{c}\text { Risk } \\
\text { (Fatal Cancer) }^{c} \\
\end{array}$ & $\begin{array}{c}\text { Total } \\
\text { Shipments }\end{array}$ & $\begin{array}{l}\text { Dose } \\
\text { (rem) }\end{array}$ & $\begin{array}{c}\text { Risk } \\
\text { (Fatal Cancer) }^{\mathrm{c}}\end{array}$ \\
\hline \multicolumn{7}{|l|}{ Decentralized } \\
\hline LLNL & 250 & $4.0 \mathrm{E}-06$ & $2.0 E-09$ & 120 & $1.9 \mathrm{E}-06$ & $9.7 \mathrm{E}-10$ \\
\hline ETEC & 110 & $1.8 \mathrm{E}-06$ & $8.9 \mathrm{E}-10$ & 40 & $6.4 \mathrm{E}-07$ & $3.2 \mathrm{E}-10$ \\
\hline NTS & 100 & $1.6 \mathrm{E}-06$ & $8.1 E-10$ & 40 & $6.4 \mathrm{E}-07$ & $3.2 \mathrm{E}-10$ \\
\hline SRS & 90 & $1.4 \mathrm{E}-06$ & $7.2 \mathrm{E}-10$ & 90 & $1.4 \mathrm{E}-06$ & $7.2 \mathrm{E}-10$ \\
\hline PORTS & 90 & $1.4 \mathrm{E}-06$ & $7.2 E-10$ & 90 & $1.4 \mathrm{E}-06$ & $7.2 \mathrm{E}-10$ \\
\hline WVDP & 30 & $4.8 \mathrm{E}-07$ & $2.4 \mathrm{E}-10$ & 30 & $4.8 \mathrm{E}-07$ & $2.4 \mathrm{E}-10$ \\
\hline Mound & 20 & $3.2 \mathrm{E}-07$ & $1.6 \mathrm{E}-10$ & 20 & $3.2 \mathrm{E}-07$ & $1.6 \mathrm{E}-10$ \\
\hline Hanford & 20 & $3.2 \mathrm{E}-07$ & $1.6 \mathrm{E}-10$ & 20 & $3.2 \mathrm{E}-07$ & $1.6 \mathrm{E}-10$ \\
\hline Bettis & 20 & $3.2 \mathrm{E}-07$ & $1.6 \mathrm{E}-10$ & 20 & $3.2 \mathrm{E}-07$ & $1.6 \mathrm{E}-10$ \\
\hline UofMO & 10 & $1.6 \mathrm{E}-07$ & 8.1E-11 & 10 & $1.6 \mathrm{E}-07$ & 8.1E-11 \\
\hline RFETS & 10 & $1.6 \mathrm{E}-07$ & $8.1 E-11$ & 10 & $1.6 \mathrm{E}-07$ & 8.1E-11 \\
\hline $\mathrm{RMI}$ & 10 & $1.6 \mathrm{E}-07$ & $8.1 \mathrm{E}-11$ & 10 & $1.6 \mathrm{E}-07$ & 8.1E-11 \\
\hline Puget So & 10 & $1.6 \mathrm{E}-07$ & 8.1E-11 & 10 & $1.6 \mathrm{E}-07$ & $8.1 E-11$ \\
\hline PPPL & 10 & $1.6 \mathrm{E}-07$ & $8.1 E-11$ & 10 & $1.6 \mathrm{E}-07$ & $8.1 E-11$ \\
\hline Ports Nav & 10 & $1.6 \mathrm{E}-07$ & 8.1E-11 & 10 & $1.6 \mathrm{E}-07$ & $8.1 \mathrm{E}-11$ \\
\hline Pearl H & 10 & $1.6 \mathrm{E}-07$ & $8.1 E-11$ & 10 & $1.6 \mathrm{E}-07$ & $8.1 \mathrm{E}-11$ \\
\hline PGDP & 10 & $1.6 \mathrm{E}-07$ & $8.1 E-11$ & 10 & $1.6 \mathrm{E}-07$ & 8.1E-11 \\
\hline Norfolk & 10 & $1.6 \mathrm{E}-07$ & $8.1 E-11$ & 10 & $1.6 \mathrm{E}-07$ & $8.1 E-11$ \\
\hline Mare Is & 10 & $1.6 \mathrm{E}-07$ & $8.1 E-11$ & 10 & $1.6 \mathrm{E}-07$ & $8.1 E-11$ \\
\hline LBL & 10 & $1.6 \mathrm{E}-07$ & $8.1 \mathrm{E}-11$ & 10 & $1.6 \mathrm{E}-07$ & $8.1 \mathrm{E}-11$ \\
\hline LEHR & 10 & $1.6 \mathrm{E}-07$ & $8.1 \mathrm{E}-11$ & 10 & $1.6 \mathrm{E}-07$ & $8.1 \mathrm{E}-11$ \\
\hline KAPL-N & 10 & $1.6 \mathrm{E}-07$ & $8.1 \mathrm{E}-11$ & 10 & $1.6 \mathrm{E}-07$ & $8.1 \mathrm{E}-11$ \\
\hline KCP & 10 & $1.6 \mathrm{E}-07$ & $8.1 \mathrm{E}-11$ & 10 & $1.6 \mathrm{E}-07$ & $8.1 E-11$ \\
\hline KAPL-W & 10 & $1.6 \mathrm{E}-07$ & 8.1E-11 & 10 & $1.6 \mathrm{E}-07$ & $8.1 \mathrm{E}-11$ \\
\hline KAPL-K & 10 & $1.6 \mathrm{E}-07$ & $8.1 E-11$ & 10 & $1.6 \mathrm{E}-07$ & $8.1 E-11$ \\
\hline GJPO & 10 & $1.6 \mathrm{E}-07$ & $8.1 \mathrm{E}-11$ & 10 & $1.6 \mathrm{E}-07$ & $8.1 \mathrm{E}-11$ \\
\hline GA & 10 & $1.6 \mathrm{E}-07$ & 8.1E-11 & 10 & $1.6 \mathrm{E}-07$ & $8.1 E-11$ \\
\hline Colonie & 10 & $1.6 \mathrm{E}-07$ & 8.1E-11 & 10 & $1.6 \mathrm{E}-07$ & $8.1 \mathrm{E}-11$ \\
\hline Charleston & 10 & $1.6 \mathrm{E}-07$ & $8.1 \mathrm{E}-11$ & 10 & $1.6 \mathrm{E}-07$ & $8.1 E-11$ \\
\hline BCL & 10 & $1.6 \mathrm{E}-07$ & $8.1 E-11$ & 10 & $1.6 \mathrm{E}-07$ & $8.1 E-11$ \\
\hline ANL-E & 10 & $1.6 \mathrm{E}-07$ & $8.1 \mathrm{E}-11$ & 10 & $1.6 \mathrm{E}-07$ & $8.1 \mathrm{E}-11$ \\
\hline Ames & 10 & $1.6 \mathrm{E}-07$ & 8.1E-11 & 10 & $1.6 \mathrm{E}-07$ & $8.1 E-11$ \\
\hline \multicolumn{7}{|l|}{ Regionalized 1} \\
\hline FEMP & 1,060 & $1.7 \mathrm{E}-05$ & 8.5E-09 & 410 & $6.6 \mathrm{E}-06$ & $3.3 \mathrm{E}-09$ \\
\hline PORTS & 820 & $1.3 \mathrm{E}-05$ & $6.6 \mathrm{E}-09$ & 440 & $7.1 \mathrm{E}-06$ & $3.5 \mathrm{E}-09$ \\
\hline ANL-E & 450 & $7.2 \mathrm{E}-06$ & $3.6 \mathrm{E}-09$ & 180 & $2.9 \mathrm{E}-06$ & $1.4 \mathrm{E}-09$ \\
\hline LLNL & 310 & $5.0 \mathrm{E}-06$ & $2.5 \mathrm{E}-09$ & 180 & $2.9 \mathrm{E}-06$ & $1.4 \mathrm{E}-09$ \\
\hline NTS & 120 & $1.9 \mathrm{E}-06$ & $9.7 \mathrm{E}-10$ & 60 & $9.7 \mathrm{E}-07$ & $4.8 \mathrm{E}-10$ \\
\hline SRS & 110 & $1.8 \mathrm{E}-06$ & $8.9 E-10$ & 110 & $1.8 \mathrm{E}-06$ & $8.9 \mathrm{E}-10$ \\
\hline ETEC & 110 & $1.8 \mathrm{E}-06$ & $8.9 E-10$ & 40 & $6.4 \mathrm{E}-07$ & $3.2 \mathrm{E}-10$ \\
\hline Hanford & 40 & $6.4 \mathrm{E}-07$ & $3.2 \mathrm{E}-10$ & 40 & $6.4 \mathrm{E}-07$ & $3.2 \mathrm{E}-10$ \\
\hline WVDP & 30 & $4.8 \mathrm{E}-07$ & $2.4 \mathrm{E}-10$ & 30 & $4.8 \mathrm{E}-07$ & $2.4 E-10$ \\
\hline RFETS & 30 & $4.8 \mathrm{E}-07$ & $2.4 \mathrm{E}-10$ & 30 & $4.8 \mathrm{E}-07$ & $2.4 \mathrm{E}-10$ \\
\hline RMI & 30 & $4.8 \mathrm{E}-07$ & $2.4 \mathrm{E}-10$ & 30 & $4.8 \mathrm{E}-07$ & $2.4 \mathrm{E}-10$ \\
\hline LANL & 30 & $4.8 \mathrm{E}-07$ & $2.4 \mathrm{E}-10$ & 30 & $4.8 \mathrm{E}-07$ & $2.4 \mathrm{E}-10$ \\
\hline LEHR & 30 & $4.8 E-07$ & $2.4 \mathrm{E}-10$ & 30 & $4.8 \mathrm{E}-07$ & $2.4 \mathrm{E}-10$ \\
\hline GJPO & 30 & $4.8 \mathrm{E}-07$ & $2.4 \mathrm{E}-10$ & 30 & $4.8 \mathrm{E}-07$ & $2.4 \mathrm{E}-10$ \\
\hline GA & 30 & $4.8 \mathrm{E}-07$ & $2.4 \mathrm{E}-10$ & 30 & $4.8 \mathrm{E}-07$ & $2.4 \mathrm{E}-10$ \\
\hline Colonie & 30 & $4.8 \mathrm{E}-07$ & $2.4 \mathrm{E}-10$ & 30 & $4.8 \mathrm{E}-07$ & $2.4 \mathrm{E}-10$ \\
\hline Charleston & 30 & $4.8 \mathrm{E}-07$ & $2.4 \mathrm{E}-10$ & 30 & $4.8 \mathrm{E}-07$ & $2.4 \mathrm{E}-10$ \\
\hline Bettis & 30 & $4.8 \mathrm{E}-07$ & $2.4 \mathrm{E}-10$ & 30 & $4.8 \mathrm{E}-07$ & $2.4 \mathrm{E}-10$ \\
\hline
\end{tabular}


TABLE 5.8 (Cont.)

\begin{tabular}{|c|c|c|c|c|c|c|}
\hline \multirow{2}{*}{$\begin{array}{c}\text { WM } \\
\text { Alternative } \\
\text { and Site }\end{array}$} & \multicolumn{3}{|c|}{ All Truck } & \multicolumn{3}{|c|}{ All Rail } \\
\hline & $\begin{array}{c}\text { Total } \\
\text { Shipments }\end{array}$ & $\begin{array}{l}\text { Dose } \\
\text { (rem) }\end{array}$ & $\begin{array}{c}\text { Risk } \\
\text { (Fatal Cancer) }^{\mathrm{c}}\end{array}$ & $\begin{array}{c}\text { Total } \\
\text { Shipments }\end{array}$ & $\begin{array}{l}\text { Dose } \\
\text { (rem) }\end{array}$ & $\begin{array}{c}\text { Risk } \\
\text { (Fatal Cancer) }^{\mathrm{c}}\end{array}$ \\
\hline \multicolumn{7}{|l|}{$\begin{array}{l}\text { Regionalized } 1 \\
\text { (Cont.) }\end{array}$} \\
\hline SNL-NM & 20 & $3.2 \mathrm{E}-07$ & $1.6 \mathrm{E}-10$ & 20 & $3.2 \mathrm{E}-07$ & $1.6 \mathrm{E}-10$ \\
\hline Puget So & 20 & $3.2 \mathrm{E}-07$ & $1.6 \mathrm{E}-10$ & 20 & $3.2 \mathrm{E}-07$ & $1.6 \mathrm{E}-10$ \\
\hline Pearl H & 20 & $3.2 \mathrm{E}-07$ & $1.6 \mathrm{E}-10$ & 20 & $3.2 \mathrm{E}-07$ & $1.6 \mathrm{E}-10$ \\
\hline Mound & 20 & $3.2 \mathrm{E}-07$ & $1.6 \mathrm{E}-10$ & 20 & $3.2 \mathrm{E}-07$ & $1.6 \mathrm{E}-10$ \\
\hline LBL & 20 & $3.2 \mathrm{E}-07$ & $1.6 \mathrm{E}-10$ & 20 & $3.2 \mathrm{E}-07$ & $1.6 \mathrm{E}-10$ \\
\hline KAPL-N & 20 & $3.2 \mathrm{E}-07$ & $1.6 \mathrm{E}-10$ & 20 & $3.2 \mathrm{E}-07$ & $1.6 \mathrm{E}-10$ \\
\hline KAPL-W & 20 & $3.2 \mathrm{E}-07$ & $1.6 \mathrm{E}-10$ & 20 & $3.2 \mathrm{E}-07$ & $1.6 \mathrm{E}-10$ \\
\hline KAPL-K & 20 & $3.2 \mathrm{E}-07$ & $1.6 \mathrm{E}-10$ & 20 & $3.2 \mathrm{E}-07$ & $1.6 \mathrm{E}-10$ \\
\hline BNL & 20 & $3.2 \mathrm{E}-07$ & $1.6 \mathrm{E}-10$ & 20 & $3.2 \mathrm{E}-07$ & $1.6 \mathrm{E}-10$ \\
\hline Ames & 20 & $3.2 \mathrm{E}-07$ & $1.6 \mathrm{E}-10$ & 20 & $3.2 \mathrm{E}-07$ & $1.6 \mathrm{E}-10$ \\
\hline UofMO & 10 & $1.6 \mathrm{E}-07$ & $8.1 \mathrm{E}-11$ & 10 & $1.6 \mathrm{E}-07$ & $8.1 \mathrm{E}-11$ \\
\hline PPPL & 10 & $1.6 \mathrm{E}-07$ & $8.1 E-11$ & 10 & $1.6 \mathrm{E}-07$ & $8.1 \mathrm{E}-11$ \\
\hline Ports Nav & 10 & $1.6 \mathrm{E}-07$ & $8.1 E-11$ & 10 & $1.6 \mathrm{E}-07$ & $8.1 \mathrm{E}-11$ \\
\hline PGDP & 10 & $1.6 \mathrm{E}-07$ & $8.1 \mathrm{E}-11$ & 10 & $1.6 \mathrm{E}-07$ & $8.1 \mathrm{E}-11$ \\
\hline Norfolk & 10 & $1.6 \mathrm{E}-07$ & $8.1 E-11$ & 10 & $1.6 \mathrm{E}-07$ & 8.1E-11 \\
\hline Mare Is & 10 & $1.6 \mathrm{E}-07$ & $8.1 \mathrm{E}-11$ & 10 & $1.6 \mathrm{E}-07$ & $8.1 \mathrm{E}-11$ \\
\hline $\mathrm{KCP}$ & 10 & $1.6 \mathrm{E}-07$ & $8.1 \mathrm{E}-11$ & 10 & $1.6 \mathrm{E}-07$ & $8.1 \mathrm{E}-11$ \\
\hline ITRI & 10 & $1.6 \mathrm{E}-07$ & $8.1 \mathrm{E}-11$ & 10 & $1.6 \mathrm{E}-07$ & $8.1 \mathrm{E}-11$ \\
\hline INEL & 10 & $1.6 \mathrm{E}-07$ & $8.1 \mathrm{E}-11$ & 10 & $1.6 \mathrm{E}-07$ & $8.1 \mathrm{E}-11$ \\
\hline BCL & 10 & $1.6 \mathrm{E}-07$ & $8.1 \mathrm{E}-11$ & 10 & $1.6 \mathrm{E}-07$ & $8.1 \mathrm{E}-11$ \\
\hline \multicolumn{7}{|l|}{ Regionalized 2} \\
\hline LANL & 2,610 & $4.2 \mathrm{E}-05$ & $2.1 \mathrm{E}-08$ & 1,020 & $1.6 \mathrm{E}-05$ & $8.2 \mathrm{E}-09$ \\
\hline RFETS & 2,560 & $4.1 \mathrm{E}-05$ & $2.1 \mathrm{E}-08$ & 980 & $1.6 \mathrm{E}-05$ & $7.9 \mathrm{E}-09$ \\
\hline PORTS & 2,260 & $3.6 \mathrm{E}-05$ & $1.8 \mathrm{E}-08$ & 960 & $1.5 \mathrm{E}-05$ & $7.7 \mathrm{E}-09$ \\
\hline ORR & 1,660 & $2.7 \mathrm{E}-05$ & $1.3 \mathrm{E}-08$ & 650 & $1.0 \mathrm{E}-05$ & $5.2 \mathrm{E}-09$ \\
\hline ANL-E & 450 & $7.2 \mathrm{E}-06$ & $3.6 \mathrm{E}-09$ & 180 & $2.9 \mathrm{E}-06$ & $1.4 \mathrm{E}-09$ \\
\hline INEL & 220 & $3.5 \mathrm{E}-06$ & $1.8 \mathrm{E}-09$ & 120 & $1.9 \mathrm{E}-06$ & $9.7 \mathrm{E}-10$ \\
\hline Hanford & 200 & $3.2 \mathrm{E}-06$ & $1.6 \mathrm{E}-09$ & 150 & $2.4 \mathrm{E}-06$ & $1.2 \mathrm{E}-09$ \\
\hline LLNL & 190 & $3.1 \mathrm{E}-06$ & $1.5 \mathrm{E}-09$ & 90 & $1.4 \mathrm{E}-06$ & $7.2 \mathrm{E}-10$ \\
\hline SRS & 110 & $1.8 \mathrm{E}-06$ & $8.9 \mathrm{E}-10$ & 110 & $1.8 \mathrm{E}-06$ & $8.9 \mathrm{E}-10$ \\
\hline FEMP & 110 & $1.8 \mathrm{E}-06$ & $8.9 \mathrm{E}-10$ & 50 & $8.1 \mathrm{E}-07$ & $4.0 \mathrm{E}-10$ \\
\hline ETEC & 90 & $1.4 \mathrm{E}-06$ & $7.2 \mathrm{E}-10$ & 40 & $6.4 \mathrm{E}-07$ & $3.2 \mathrm{E}-10$ \\
\hline PGDP & 50 & $8.1 E-07$ & $4.0 \mathrm{E}-10$ & 30 & $4.8 \mathrm{E}-07$ & $2.4 \mathrm{E}-10$ \\
\hline Pantex & 40 & $6.4 \mathrm{E}-07$ & $3.2 E-10$ & 30 & $4.8 \mathrm{E}-07$ & $2.4 \mathrm{E}-10$ \\
\hline WVDP & 30 & $4.8 \mathrm{E}-07$ & $2.4 \mathrm{E}-10$ & 30 & $4.8 \mathrm{E}-07$ & $2.4 \mathrm{E}-10$ \\
\hline $\mathrm{RMI}$ & 30 & $4.8 E-07$ & $2.4 \mathrm{E}-10$ & 30 & $4.8 \mathrm{E}-07$ & $2.4 \mathrm{E}-10$ \\
\hline NTS & 30 & $4.8 \mathrm{E}-07$ & $2.4 \mathrm{E}-10$ & 30 & $4.8 \mathrm{E}-07$ & $2.4 \mathrm{E}-10$ \\
\hline LEHR & 30 & $4.8 \mathrm{E}-07$ & $2.4 \mathrm{E}-10$ & 30 & $4.8 \mathrm{E}-07$ & $2.4 \mathrm{E}-10$ \\
\hline GJPO & 30 & $4.8 \mathrm{E}-07$ & $2.4 \mathrm{E}-10$ & 30 & $4.8 \mathrm{E}-07$ & $2.4 \mathrm{E}-10$ \\
\hline GA & 30 & $4.8 \mathrm{E}-07$ & $2.4 \mathrm{E}-10$ & 30 & $4.8 \mathrm{E}-07$ & $2.4 \mathrm{E}-10$ \\
\hline Colonie & 30 & $4.8 \mathrm{E}-07$ & $2.4 \mathrm{E}-10$ & 30 & $4.8 \mathrm{E}-07$ & $2.4 \mathrm{E}-10$ \\
\hline Charleston & 30 & $4.8 \mathrm{E}-07$ & $2.4 \mathrm{E}-10$ & 30 & $4.8 \mathrm{E}-07$ & $2.4 \mathrm{E}-10$ \\
\hline Bettis & 30 & $4.8 \mathrm{E}-07$ & $2.4 \mathrm{E}-10$ & 30 & $4.8 \mathrm{E}-07$ & $2.4 \mathrm{E}-10$ \\
\hline SNL-CA & 20 & $3.2 \mathrm{E}-07$ & $1.6 \mathrm{E}-10$ & 20 & $3.2 \mathrm{E}-07$ & $1.6 \mathrm{E}-10$ \\
\hline SNL-NM & 20 & $3.2 \mathrm{E}-07$ & $1.6 \mathrm{E}-10$ & 20 & $3.2 \mathrm{E}-07$ & $1.6 \mathrm{E}-10$ \\
\hline Puget So & 20 & $3.2 \mathrm{E}-07$ & $1.6 \mathrm{E}-10$ & 20 & $3.2 \mathrm{E}-07$ & $1.6 \mathrm{E}-10$ \\
\hline Peari H & 20 & $3.2 \mathrm{E}-07$ & $1.6 \mathrm{E}-10$ & 20 & $3.2 \mathrm{E}-07$ & $1.6 \mathrm{E}-10$ \\
\hline Mound & 20 & $3.2 \mathrm{E}-07$ & $1.6 \mathrm{E}-10$ & 20 & $3.2 \mathrm{E}-07$ & $1.6 \mathrm{E}-10$ \\
\hline LBL & 20 & $3.2 \mathrm{E}-07$ & $1.6 \mathrm{E}-10$ & 20 & $3.2 \mathrm{E}-07$ & $1.6 \mathrm{E}-10$ \\
\hline KAPL-N & 20 & $3.2 \mathrm{E}-07$ & $1.6 \mathrm{E}-10$ & 20 & $3.2 \mathrm{E}-07$ & $1.6 \mathrm{E}-10$ \\
\hline KAPL-W & 20 & $3.2 \mathrm{E}-07$ & $1.6 \mathrm{E}-10$ & 20 & $3.2 \mathrm{E}-07$ & $1.6 \mathrm{E}-10$ \\
\hline KAPL-K & 20 & $3.2 \mathrm{E}-07$ & $1.6 \mathrm{E}-10$ & 20 & $3.2 \mathrm{E}-07$ & $1.6 \mathrm{E}-10$ \\
\hline BNL & 20 & $3.2 \mathrm{E}-07$ & $1.6 \mathrm{E}-10$ & 20 & $3.2 \mathrm{E}-07$ & $1.6 \mathrm{E}-10$ \\
\hline
\end{tabular}


TABLE 5.8 (Cont.)

\begin{tabular}{|c|c|c|c|c|c|c|}
\hline \multirow{2}{*}{$\begin{array}{c}\text { WM } \\
\text { Alternative } \\
\text { and Site }\end{array}$} & \multicolumn{3}{|c|}{ All Truck } & \multicolumn{3}{|c|}{ All Rail } \\
\hline & $\begin{array}{c}\text { Total } \\
\text { Shipments }\end{array}$ & $\begin{array}{l}\text { Dose } \\
\text { (rem) }\end{array}$ & $\begin{array}{c}\text { Risk } \\
\text { (Fatal Cancer) }^{\mathbf{c}}\end{array}$ & $\begin{array}{c}\text { Total } \\
\text { Shipments }\end{array}$ & $\begin{array}{l}\text { Dose } \\
(\mathrm{rem})\end{array}$ & $\begin{array}{c}\text { Risk } \\
(\text { Fatal Cancer })^{\mathrm{c}}\end{array}$ \\
\hline \multicolumn{7}{|l|}{$\begin{array}{l}\text { Regionalized } 2 \\
\text { (Cont.) }\end{array}$} \\
\hline Ames & 20 & $3.2 \mathrm{E}-07$ & $1.6 \mathrm{E}-10$ & 20 & 3.2E-07 & $1.6 \mathrm{E}-10$ \\
\hline UofMO & 10 & $1.6 \mathrm{E}-07$ & $8.1 E-11$ & 10 & $1.6 \mathrm{E}-07$ & $8.1 E-11$ \\
\hline PPPL & 10 & $1.6 \mathrm{E}-07$ & 8.1E-11 & 10 & $1.6 \mathrm{E}-07$ & $8.1 \mathrm{E}-11$ \\
\hline Ports Nav & 10 & $1.6 \mathrm{E}-07$ & 8.1E-11 & 10 & $1.6 \mathrm{E}-07$ & $8.1 E-11$ \\
\hline Norfolk & 10 & $1.6 \mathrm{E}-07$ & $8.1 \mathrm{E}-11$ & 10 & $1.6 \mathrm{E}-07$ & $8.1 E-11$ \\
\hline Mare Is & 10 & $1.6 \mathrm{E}-07$ & $8.1 \mathrm{E}-11$ & 10 & $1.6 \mathrm{E}-07$ & $8.1 \mathrm{E}-11$ \\
\hline KCP & 10 & $1.6 \mathrm{E}-07$ & $8.1 \mathrm{E}-11$ & 10 & $1.6 E-07$ & $8.1 \mathrm{E}-11$ \\
\hline ITRI & 10 & $1.6 \mathrm{E}-07$ & 8.1E-11 & 10 & $1.6 \mathrm{E}-07$ & $8.1 \mathrm{E}-11$ \\
\hline BCL & 10 & $1.6 \mathrm{E}-07$ & $8.1 E-11$ & 10 & $1.6 \mathrm{E}-07$ & $8.1 E-11$ \\
\hline \multicolumn{7}{|l|}{ Regionalized 3} \\
\hline NTS & 9,650 & $1.6 \mathrm{E}-04$ & $7.8 \mathrm{E}-08$ & 3,700 & $6.0 \mathrm{E}-05$ & $3.0 \mathrm{E}-08$ \\
\hline RFETS & 2,560 & $4.1 \mathrm{E}-05$ & 2.1E-08 & 980 & $1.6 \mathrm{E}-05$ & $7.9 \mathrm{E}-09$ \\
\hline PORTS & 2,260 & $3.6 \mathrm{E}-05$ & $1.8 \mathrm{E}-08$ & 960 & $1.5 \mathrm{E}-05$ & $7.7 \mathrm{E}-09$ \\
\hline ORR & 2,100 & $3.4 \mathrm{E}-05$ & $1.7 \mathrm{E}-08$ & 790 & $1.3 \mathrm{E}-05$ & $6.4 \mathrm{E}-09$ \\
\hline Hanford & 1,690 & $2.7 \mathrm{E}-05$ & $1.4 \mathrm{E}-08$ & 710 & $1.1 \mathrm{E}-05$ & $5.7 \mathrm{E}-09$ \\
\hline INEL & 1,360 & $2.2 \mathrm{E}-05$ & $1.1 \mathrm{E}-08$ & 550 & $8.9 \mathrm{E}-06$ & 4.4E-09 \\
\hline SRS & 710 & $1.1 \mathrm{E}-05$ & $5.7 \mathrm{E}-09$ & 330 & $5.3 \mathrm{E}-06$ & 2.7E-09 \\
\hline ANL-E & 450 & $7.2 \mathrm{E}-06$ & $3.6 \mathrm{E}-09$ & 180 & $2.9 \mathrm{E}-06$ & $1.4 \mathrm{E}-09$ \\
\hline LLNL & 190 & $3.1 \mathrm{E}-06$ & 1.5E-09 & 90 & $1.4 \mathrm{E}-06$ & $7.2 \mathrm{E}-10$ \\
\hline LANL & 180 & $2.9 \mathrm{E}-06$ & $1.4 \mathrm{E}-09$ & 100 & $1.6 \mathrm{E}-06$ & $8.1 \mathrm{E}-10$ \\
\hline FEMP & 110 & $1.8 \mathrm{E}-06$ & $8.9 E-10$ & 50 & 8.1E-07 & $4.0 E-10$ \\
\hline ETEC & 90 & $1.4 \mathrm{E}-06$ & $7.2 \mathrm{E}-10$ & 40 & $6.4 \mathrm{E}-07$ & $3.2 \mathrm{E}-10$ \\
\hline PGDP & 50 & 8.1E-07 & $4.0 \mathrm{E}-10$ & 30 & $4.8 \mathrm{E}-07$ & $2.4 \mathrm{E}-10$ \\
\hline Pantex & 40 & $6.4 \mathrm{E}-07$ & $3.2 \mathrm{E}-10$ & 30 & $4.8 \mathrm{E}-07$ & $2.4 \mathrm{E}-10$ \\
\hline WVDP & 30 & $4.8 \mathrm{E}-07$ & $2.4 \mathrm{E}-10$ & 30 & $4.8 \mathrm{E}-07$ & $2.4 E-10$ \\
\hline RMI & 30 & $4.8 \mathrm{E}-07$ & $2.4 \mathrm{E}-10$ & 30 & $4.8 \mathrm{E}-07$ & $2.4 \mathrm{E}-10$ \\
\hline LEHR & 30 & $4.8 \mathrm{E}-07$ & $2.4 \mathrm{E}-10$ & 30 & $4.8 \mathrm{E}-07$ & $2.4 \mathrm{E}-10$ \\
\hline GJPO & 30 & $4.8 \mathrm{E}-07$ & 2.4E-10 & 30 & $4.8 \mathrm{E}-07$ & $2.4 \mathrm{E}-10$ \\
\hline GA & 30 & 4.8E-07 & $2.4 \mathrm{E}-10$ & 30 & $4.8 \mathrm{E}-07$ & $2.4 \mathrm{E}-10$ \\
\hline Colonie & 30 & $4.8 \mathrm{E}-07$ & $2.4 \mathrm{E}-10$ & 30 & $4.8 \mathrm{E}-07$ & $2.4 \mathrm{E}-10$ \\
\hline Charleston & 30 & $4.8 \mathrm{E}-07$ & $2.4 \mathrm{E}-10$ & 30 & $4.8 \mathrm{E}-07$ & $2.4 \mathrm{E}-10$ \\
\hline Bettis & 30 & $4.8 \mathrm{E}-07$ & $2.4 \mathrm{E}-10$ & 30 & $4.8 \mathrm{E}-07$ & $2.4 \mathrm{E}-10$ \\
\hline SNL-CA & 20 & $3.2 \mathrm{E}-07$ & $1.6 \mathrm{E}-10$ & 20 & $3.2 \mathrm{E}-07$ & $1.6 \mathrm{E}-10$ \\
\hline SNL-NM & 20 & $3.2 \mathrm{E}-07$ & $1.6 \mathrm{E}-10$ & 20 & $3.2 \mathrm{E}-07$ & $1.6 \mathrm{E}-10$ \\
\hline Puget So & 20 & $3.2 \mathrm{E}-07$ & $1.6 \mathrm{E}-10$ & 20 & $3.2 \mathrm{E}-07$ & $1.6 \mathrm{E}-10$ \\
\hline Pearl H & 20 & $3.2 \mathrm{E}-07$ & $1.6 \mathrm{E}-10$ & 20 & $3.2 \mathrm{E}-07$ & $1.6 \mathrm{E}-10$ \\
\hline Mound & 20 & $3.2 \mathrm{E}-07$ & $1.6 \mathrm{E}-10$ & 20 & $3.2 \mathrm{E}-07$ & $1.6 \mathrm{E}-10$ \\
\hline LBL & 20 & $3.2 \mathrm{E}-07$ & $1.6 \mathrm{E}-10$ & 20 & $3.2 \mathrm{E}-07$ & $1.6 \mathrm{E}-10$ \\
\hline KAPL-N & 20 & $3.2 \mathrm{E}-07$ & $1.6 \mathrm{E}-10$ & 20 & $3.2 \mathrm{E}-07$ & $1.6 \mathrm{E}-10$ \\
\hline KAPL-W & 20 & $3.2 \mathrm{E}-07$ & $1.6 \mathrm{E}-10$ & 20 & $3.2 \mathrm{E}-07$ & $1.6 \mathrm{E}-10$ \\
\hline KAPL-K & 20 & $3.2 \mathrm{E}-07$ & $1.6 \mathrm{E}-10$ & 20 & $3.2 \mathrm{E}-07$ & $1.6 \mathrm{E}-10$ \\
\hline $\mathrm{BNL}$ & 20 & $3.2 \mathrm{E}-07$ & $1.6 \mathrm{E}-10$ & 20 & $3.2 \mathrm{E}-07$ & $1.6 \mathrm{E}-10$ \\
\hline Ames & 20 & $3.2 \mathrm{E}-07$ & $1.6 \mathrm{E}-10$ & 20 & $3.2 \mathrm{E}-07$ & $1.6 \mathrm{E}-10$ \\
\hline UofMO & 10 & $1.6 \mathrm{E}-07$ & 8.1E-11 & 10 & $1.6 \mathrm{E}-07$ & $8.1 \mathrm{E}-11$ \\
\hline PPPL & 10 & $1.6 \mathrm{E}-07$ & $8.1 \mathrm{E}-11$ & 10 & $1.6 \mathrm{E}-07$ & $8.1 \mathrm{E}-11$ \\
\hline Ports Nav & 10 & $1.6 \mathrm{E}-07$ & $8.1 \mathrm{E}-11$ & 10 & $1.6 \mathrm{E}-07$ & $8.1 E-11$ \\
\hline Norfolk & 10 & $1.6 \mathrm{E}-07$ & 8.1E-11 & 10 & $1.6 \mathrm{E}-07$ & $8.1 E-11$ \\
\hline Mare Is & 10 & $1.6 \mathrm{E}-07$ & 8.1E-11 & 10 & $1.6 \mathrm{E}-07$ & $8.1 \mathrm{E}-11$ \\
\hline KCP & 10 & $1.6 \mathrm{E}-07$ & $8.1 \mathrm{E}-11$ & 10 & $1.6 \mathrm{E}-07$ & $8.1 \mathrm{E}-11$ \\
\hline ITRI & 10 & $1.6 \mathrm{E}-07$ & 8.1E-11 & 10 & $1.6 \mathrm{E}-07$ & $8.1 E-11$ \\
\hline $\mathrm{BCL}$ & 10 & $1.6 \mathrm{E}-07$ & $8.1 E-11$ & 10 & $1.6 \mathrm{E}-07$ & $8.1 E-11$ \\
\hline
\end{tabular}


TABLE 5.8 (Cont.)

\begin{tabular}{|c|c|c|c|c|c|c|}
\hline \multirow{2}{*}{$\begin{array}{c}\text { WM } \\
\text { Alternative } \\
\text { and Site }\end{array}$} & \multicolumn{3}{|c|}{ All Truck } & \multicolumn{3}{|c|}{ All Rail } \\
\hline & $\begin{array}{c}\text { Total } \\
\text { Shipments }\end{array}$ & $\begin{array}{l}\text { Dose } \\
\text { (rem) }\end{array}$ & $\begin{array}{c}\text { Risk } \\
\text { (Fatal Cancer) }^{\mathrm{c}}\end{array}$ & $\begin{array}{c}\text { Total } \\
\text { Shipments }\end{array}$ & $\begin{array}{c}\text { Dose } \\
\text { (rem) }\end{array}$ & $\begin{array}{c}\text { Risk } \\
\text { (Fatal Cancer) }^{\mathrm{c}}\end{array}$ \\
\hline \multicolumn{7}{|l|}{ Regionalized 4} \\
\hline INEL & 2,450 & $3.9 \mathrm{E}-05$ & $2.0 \mathrm{E}-08$ & 1,040 & $1.7 \mathrm{E}-05$ & $8.5 \mathrm{E}-09$ \\
\hline RFETS & 1,990 & $3.2 \mathrm{E}-05$ & $1.6 \mathrm{E}-08$ & 740 & $1.2 \mathrm{E}-05$ & $6.0 \mathrm{E}-09$ \\
\hline ORR & 1,480 & $2.4 \mathrm{E}-05$ & $1.2 \mathrm{E}-08$ & 740 & $1.2 \mathrm{E}-05$ & $6.0 \mathrm{E}-09$ \\
\hline PORTS & 650 & $1.0 \mathrm{E}-05$ & $5.2 \mathrm{E}-09$ & 260 & $4.2 \mathrm{E}-06$ & $2.1 \mathrm{E}-09$ \\
\hline ANL-E & 450 & $7.2 \mathrm{E}-06$ & $3.6 \mathrm{E}-09$ & 180 & $2.9 \mathrm{E}-06$ & $1.4 \mathrm{E}-09$ \\
\hline Hanford & 210 & $3.4 \mathrm{E}-06$ & $1.7 \mathrm{E}-09$ & 160 & $2.6 \mathrm{E}-06$ & $1.3 \mathrm{E}-09$ \\
\hline LLNL & 190 & $3.1 \mathrm{E}-06$ & $1.5 \mathrm{E}-09$ & 90 & $1.4 \mathrm{E}-06$ & $7.2 \mathrm{E}-10$ \\
\hline LANL & 130 & $2.1 \mathrm{E}-06$ & $1.1 \mathrm{E}-09$ & 80 & $1.3 \mathrm{E}-06$ & $6.5 \mathrm{E}-10$ \\
\hline SRS & 110 & $1.8 \mathrm{E}-06$ & $8.9 \mathrm{E}-10$ & 110 & $1.8 \mathrm{E}-06$ & $8.9 \mathrm{E}-10$ \\
\hline FEMP & 110 & $1.8 \mathrm{E}-06$ & $8.9 \mathrm{E}-10$ & 50 & $8.1 E-07$ & $4.0 \mathrm{E}-10$ \\
\hline ETEC & 90 & $1.4 \mathrm{E}-06$ & $7.2 \mathrm{E}-10$ & 40 & $6.4 \mathrm{E}-07$ & $3.2 \mathrm{E}-10$ \\
\hline PGDP & 50 & $8.1 \mathrm{E}-07$ & $4.0 \mathrm{E}-10$ & 30 & $4.8 \mathrm{E}-07$ & $2.4 \mathrm{E}-10$ \\
\hline Pantex & 40 & $6.4 \mathrm{E}-07$ & $3.2 \mathrm{E}-10$ & 30 & $4.8 \mathrm{E}-07$ & $2.4 \mathrm{E}-10$ \\
\hline WVDP & 30 & $4.8 \mathrm{E}-07$ & $2.4 \mathrm{E}-10$ & 30 & $4.8 \mathrm{E}-07$ & $2.4 \mathrm{E}-10$ \\
\hline RMI & 30 & $4.8 \mathrm{E}-07$ & $2.4 \mathrm{E}-10$ & 30 & $4.8 \mathrm{E}-07$ & $2.4 \mathrm{E}-10$ \\
\hline LEHR & 30 & $4.8 \mathrm{E}-07$ & $2.4 \mathrm{E}-10$ & 30 & $4.8 \mathrm{E}-07$ & $2.4 \mathrm{E}-10$ \\
\hline GJPO & 30 & $4.8 \mathrm{E}-07$ & $2.4 \mathrm{E}-10$ & 30 & $4.8 \mathrm{E}-07$ & $2.4 \mathrm{E}-10$ \\
\hline GA & 30 & $4.8 \mathrm{E}-07$ & $2.4 \mathrm{E}-10$ & 30 & $4.8 \mathrm{E}-07$ & $2.4 \mathrm{E}-10$ \\
\hline Colonie & 30 & $4.8 E-07$ & $2.4 \mathrm{E}-10$ & 30 & $4.8 \mathrm{E}-07$ & $2.4 \mathrm{E}-10$ \\
\hline Charleston & 30 & $4.8 \mathrm{E}-07$ & $2.4 \mathrm{E}-10$ & 30 & $4.8 \mathrm{E}-07$ & $2.4 \mathrm{E}-10$ \\
\hline Bettis & 30 & $4.8 \mathrm{E}-07$ & $2.4 \mathrm{E}-10$ & 30 & $4.8 \mathrm{E}-07$ & $2.4 \mathrm{E}-10$ \\
\hline SNL-CA & 20 & $3.2 \mathrm{E}-07$ & $1.6 \mathrm{E}-10$ & 20 & $3.2 \mathrm{E}-07$ & $1.6 \mathrm{E}-10$ \\
\hline SNL-NM & 20 & $3.2 \mathrm{E}-07$ & $1.6 \mathrm{E}-10$ & 20 & $3.2 \mathrm{E}-07$ & $1.6 \mathrm{E}-10$ \\
\hline Puget So & 20 & $3.2 \mathrm{E}-07$ & $1.6 \mathrm{E}-10$ & 20 & $3.2 \mathrm{E}-07$ & $1.6 \mathrm{E}-10$ \\
\hline Pearl H & 20 & $3.2 \mathrm{E}-07$ & $1.6 \mathrm{E}-10$ & 20 & $3.2 \mathrm{E}-07$ & $1.6 \mathrm{E}-10$ \\
\hline Mound & 20 & $3.2 \mathrm{E}-07$ & $1.6 \mathrm{E}-10$ & 20 & $3.2 \mathrm{E}-07$ & $1.6 \mathrm{E}-10$ \\
\hline LBL & 20 & $3.2 \mathrm{E}-07$ & $1.6 \mathrm{E}-10$ & 20 & $3.2 \mathrm{E}-07$ & $1.6 \mathrm{E}-10$ \\
\hline KAPL-N & 20 & $3.2 \mathrm{E}-07$ & $1.6 \mathrm{E}-10$ & 20 & $3.2 \mathrm{E}-07$ & $1.6 \mathrm{E}-10$ \\
\hline KAPL-W & 20 & $3.2 \mathrm{E}-07$ & $1.6 \mathrm{E}-10$ & 20 & $3.2 \mathrm{E}-07$ & $1.6 \mathrm{E}-10$ \\
\hline KAPL-K & 20 & $3.2 \mathrm{E}-07$ & $1.6 \mathrm{E}-10$ & 20 & $3.2 \mathrm{E}-07$ & $1.6 \mathrm{E}-10$ \\
\hline BNL & 20 & $3.2 \mathrm{E}-07$ & $1.6 \mathrm{E}-10$ & 20 & $3.2 \mathrm{E}-07$ & $1.6 \mathrm{E}-10$ \\
\hline Ames & 20 & $3.2 \mathrm{E}-07$ & $1.6 \mathrm{E}-10$ & 20 & $3.2 \mathrm{E}-07$ & $1.6 \mathrm{E}-10$ \\
\hline NTS & 10 & $1.6 \mathrm{E}-07$ & 8.1E-11 & 10 & $1.6 \mathrm{E}-07$ & $8.1 \mathrm{E}-11$ \\
\hline UofMO & 10 & $1.6 \mathrm{E}-07$ & 8.1E-11 & 10 & $1.6 \mathrm{E}-07$ & $8.1 \mathrm{E}-11$ \\
\hline PPPL & 10 & $1.6 \mathrm{E}-07$ & $8.1 \mathrm{E}-11$ & 10 & $1.6 \mathrm{E}-07$ & $8.1 \mathrm{E}-11$ \\
\hline Ports Nav & 10 & $1.6 \mathrm{E}-07$ & $8.1 \mathrm{E}-11$ & 10 & $1.6 \mathrm{E}-07$ & $8.1 \mathrm{E}-11$ \\
\hline Norfolk & 10 & $1.6 \mathrm{E}-07$ & $8.1 \mathrm{E}-11$ & 10 & $1.6 \mathrm{E}-07$ & $8.1 \mathrm{E}-11$ \\
\hline Mare Is & 10 & $1.6 \mathrm{E}-07$ & $8.1 \mathrm{E}-11$ & 10 & $1.6 \mathrm{E}-07$ & $8.1 \mathrm{E}-11$ \\
\hline $\mathrm{KCP}$ & 10 & $1.6 \mathrm{E}-07$ & $8.1 \mathrm{E}-11$ & 10 & $1.6 \mathrm{E}-07$ & $8.1 \mathrm{E}-11$ \\
\hline ITRI & 10 & $1.6 \mathrm{E}-07$ & $8.1 \mathrm{E}-11$ & 10 & $1.6 \mathrm{E}-07$ & $8.1 \mathrm{E}-11$ \\
\hline BCL & 10 & $1.6 \mathrm{E}-07$ & $8.1 \mathrm{E}-11$ & 10 & $1.6 \mathrm{E}-07$ & $8.1 \mathrm{E}-11$ \\
\hline \multicolumn{7}{|l|}{ Centralized } \\
\hline Hanford & 7,520 & $1.2 \mathrm{E}-04$ & $6.1 \mathrm{E}-08$ & 3,340 & $5.4 \mathrm{E}-05$ & $2.7 \mathrm{E}-08$ \\
\hline RFETS & 1,990 & $3.2 \mathrm{E}-05$ & $1.6 \mathrm{E}-08$ & 740 & $1.2 \mathrm{E}-05$ & $6.0 \mathrm{E}-09$ \\
\hline ORR & 1,970 & $3.2 \mathrm{E}-05$ & $1.6 \mathrm{E}-08$ & 740 & $1.2 \mathrm{E}-05$ & $6.0 \mathrm{E}-09$ \\
\hline INEL & 700 & $1.1 \mathrm{E}-05$ & $5.6 \mathrm{E}-09$ & 290 & $4.7 E-06$ & $2.3 \mathrm{E}-09$ \\
\hline PORTS & 650 & $1.0 \mathrm{E}-05$ & $5.2 \mathrm{E}-09$ & 260 & $4.2 E-06$ & $2.1 \mathrm{E}-09$ \\
\hline SRS & 580 & $9.3 \mathrm{E}-06$ & $4.7 \mathrm{E}-09$ & 240 & $3.9 \mathrm{E}-06$ & $1.9 \mathrm{E}-09$ \\
\hline ANL-E & 450 & $7.2 \mathrm{E}-06$ & $3.6 \mathrm{E}-09$ & 180 & $2.9 \mathrm{E}-06$ & $1.4 \mathrm{E}-09$ \\
\hline LLNL & 190 & $3.1 E-06$ & $1.5 \mathrm{E}-09$ & 90 & $1.4 \mathrm{E}-06$ & $7.2 \mathrm{E}-10$ \\
\hline LANL & 130 & $2.1 \mathrm{E}-06$ & $1.0 \mathrm{E}-09$ & 80 & $1.3 E-06$ & $6.4 \mathrm{E}-10$ \\
\hline FEMP & 110 & $1.8 \mathrm{E}-06$ & $8.9 E-10$ & 50 & $8.1 E-07$ & $4.0 \mathrm{E}-10$ \\
\hline ETEC & 90 & $1.4 \mathrm{E}-06$ & $7.2 \mathrm{E}-10$ & 40 & $6.4 \mathrm{E}-07$ & $3.2 \mathrm{E}-10$ \\
\hline
\end{tabular}


TABLE 5.8 (Cont.)

\begin{tabular}{|c|c|c|c|c|c|c|}
\hline \multirow{2}{*}{$\begin{array}{c}\text { WM } \\
\text { Alternative } \\
\text { and Site }\end{array}$} & \multicolumn{3}{|c|}{ All Truck } & \multicolumn{3}{|c|}{ All Rail } \\
\hline & $\begin{array}{c}\text { Total } \\
\text { Shipments }\end{array}$ & $\begin{array}{l}\text { Dose } \\
\text { (rem) }\end{array}$ & $\begin{array}{c}\text { Risk } \\
{\text { (Fatal Cancer })^{\mathrm{c}}}^{\text {(Fal }}\end{array}$ & $\begin{array}{c}\text { Total } \\
\text { Shipments }\end{array}$ & $\begin{array}{l}\text { Dose } \\
\text { (rem) }\end{array}$ & $\begin{array}{c}\text { Risk } \\
\text { (Fatal Cancer) }\end{array}$ \\
\hline \multicolumn{7}{|l|}{$\begin{array}{l}\text { Centralized } \\
\text { (Cont.) }\end{array}$} \\
\hline PGDP & 50 & 8.1E-07 & $4.0 \mathrm{E}-10$ & 30 & $4.8 \mathrm{E}-07$ & $2.4 \mathrm{E}-10$ \\
\hline Pantex & 40 & $6.4 \mathrm{E}-07$ & $3.2 \mathrm{E}-10$ & 30 & $4.8 \mathrm{E}-07$ & $2.4 \mathrm{E}-10$ \\
\hline WVDP & 30 & 4.8E-07 & $2.4 \mathrm{E}-10$ & 30 & $4.8 \mathrm{E}-07$ & $2.4 \mathrm{E}-10$ \\
\hline RMI & 30 & $4.8 \mathrm{E}-07$ & $2.4 \mathrm{E}-10$ & 30 & $4.8 \mathrm{E}-07$ & $2.4 E-10$ \\
\hline LEHR & 30 & $4.8 \mathrm{E}-07$ & $2.4 E-10$ & 30 & $4.8 E-07$ & $2.4 \mathrm{E}-10$ \\
\hline GJPO & 30 & $4.8 \mathrm{E}-07$ & $2.4 \mathrm{E}-10$ & 30 & $4.8 \mathrm{E}-07$ & $2.4 \mathrm{E}-10$ \\
\hline GA & 30 & $4.8 \mathrm{E}-07$ & $2.4 \mathrm{E}-10$ & 30 & $4.8 \mathrm{E}-07$ & $2.4 \mathrm{E}-10$ \\
\hline Colonie & 30 & $4.8 \mathrm{E}-07$ & $2.4 \mathrm{E}-10$ & 30 & $4.8 \mathrm{E}-07$ & $2.4 E-10$ \\
\hline Charleston & 30 & $4.8 \mathrm{E}-07$ & $2.4 \mathrm{E}-10$ & 30 & $4.8 \mathrm{E}-07$ & $2.4 \mathrm{E}-10$ \\
\hline Bettis & 30 & $4.8 \mathrm{E}-07$ & $2.4 \mathrm{E}-10$ & 30 & $4.8 \mathrm{E}-07$ & $2.4 \mathrm{E}-10$ \\
\hline SNL-CA & 20 & $3.2 \mathrm{E}-07$ & $1.6 \mathrm{E}-10$ & 20 & $3.2 \mathrm{E}-07$ & $1.6 \mathrm{E}-10$ \\
\hline SNL-NM & 20 & $3.2 \mathrm{E}-07$ & $1.6 \mathrm{E}-10$ & 20 & $3.2 \mathrm{E}-07$ & $1.6 \mathrm{E}-10$ \\
\hline Puget So & 20 & $3.2 \mathrm{E}-07$ & $1.6 \mathrm{E}-10$ & 20 & $3.2 \mathrm{E}-07$ & $1.6 \mathrm{E}-10$ \\
\hline Pearl H & 20 & $3.2 \mathrm{E}-07$ & $1.6 \mathrm{E}-10$ & 20 & $3.2 \mathrm{E}-07$ & $1.6 \mathrm{E}-10$ \\
\hline Mound & 20 & $3.2 \mathrm{E}-07$ & $1.6 \mathrm{E}-10$ & 20 & $3.2 E-07$ & $1.6 \mathrm{E}-10$ \\
\hline LBL & 20 & $3.2 \mathrm{E}-07$ & $1.6 \mathrm{E}-10$ & 20 & $3.2 \mathrm{E}-07$ & $1.6 \mathrm{E}-10$ \\
\hline KAPL-N & 20 & $3.2 \mathrm{E}-07$ & $1.6 \mathrm{E}-10$ & 20 & $3.2 \mathrm{E}-07$ & $1.6 \mathrm{E}-10$ \\
\hline KAPL-W & 20 & $3.2 \mathrm{E}-07$ & $1.6 \mathrm{E}-10$ & 20 & $3.2 \mathrm{E}-07$ & $1.6 \mathrm{E}-10$ \\
\hline KAPL-K & 20 & $3.2 \mathrm{E}-07$ & $1.6 \mathrm{E}-10$ & 20 & $3.2 E-07$ & $1.6 \mathrm{E}-10$ \\
\hline BNL & 20 & $3.2 \mathrm{E}-07$ & $1.6 \mathrm{E}-10$ & 20 & $3.2 \mathrm{E}-07$ & $1.6 \mathrm{E}-10$ \\
\hline ANL-W & 20 & $3.2 \mathrm{E}-07$ & $1.6 \mathrm{E}-10$ & 20 & $3.2 \mathrm{E}-07$ & $1.6 \mathrm{E}-10$ \\
\hline Ames & 20 & $3.2 \mathrm{E}-07$ & $1.6 \mathrm{E}-10$ & 20 & $3.2 \mathrm{E}-07$ & $1.6 \mathrm{E}-10$ \\
\hline UofMO & 10 & $1.6 \mathrm{E}-07$ & $8.1 \mathrm{E}-11$ & 10 & $1.6 \mathrm{E}-07$ & $8.1 E-11$ \\
\hline PPPL & 10 & $1.6 \mathrm{E}-07$ & $8.1 \mathrm{E}-11$ & 10 & $1.6 \mathrm{E}-07$ & $8.1 E-11$ \\
\hline Ports Nav & 10 & $1.6 \mathrm{E}-07$ & $8.1 \mathrm{E}-11$ & 10 & $1.6 \mathrm{E}-07$ & $8.1 \mathrm{E}-11$ \\
\hline Norfolk & 10 & $1.6 \mathrm{E}-07$ & $8.1 \mathrm{E}-11$ & 10 & $1.6 \mathrm{E}-07$ & $8.1 E-11$ \\
\hline NTS & 10 & $1.6 \mathrm{E}-07$ & $8.1 \mathrm{E}-11$ & 10 & $1.6 \mathrm{E}-07$ & 8.1E-11 \\
\hline Mare Is & 10 & $1.6 \mathrm{E}-07$ & $8.1 E-11$ & 10 & $1.6 \mathrm{E}-07$ & $8.1 E-11$ \\
\hline KCP & 10 & $1.6 \mathrm{E}-07$ & $8.1 \mathrm{E}-11$ & 10 & $1.6 \mathrm{E}-07$ & $8.1 E-11$ \\
\hline ITRI & 10 & $1.6 \mathrm{E}-07$ & $8.1 \mathrm{E}-11$ & 10 & $1.6 \mathrm{E}-07$ & $8.1 \mathrm{E}-11$ \\
\hline BCL & 10 & $1.6 \mathrm{E}-07$ & $8.1 \mathrm{E}-11$ & 10 & $1.6 \mathrm{E}-07$ & 8.1E-11 \\
\hline
\end{tabular}

a The external dose rate is assumed to be $1 \mathrm{mrem} / \mathrm{h}$ at $1 \mathrm{~m}(3.3 \mathrm{ft})$ for all shipments. The resident is assumed to be present for all shipments that enter or exit the site. Shipments are assumed to pass at a distance of $30 \mathrm{~m}$ ( $98 \mathrm{ft}$ ) and an average speed of $24 \mathrm{~km} / \mathrm{h}(15 \mathrm{mph})$.

b Ames = Ames Laboratory; ANL-W = Argonne National Laboratory-West; BCL = Battelle Columbus Laboratories; Bettis = Bettis Atomic Power Laboratory; Charleston = Charleston Naval Shipyard; Colonie = Colonie; ETEC = Energy Technology Engineering Center; FEMP = Fernald Environmental Management Project; GA = General Atomics; GJPO = Grand Junction Projects Office; ITRI = Inhalation Toxicology Research Institute; KAPL-K = Knolls Atomic Power Laboratory (Kesselring); KAPL-N = Knolls Atomic Power Laboratory (Niskayuna); KAPL-W = Knolls Atomic Power Laboratory (Windsor); KCP = Kansas City Plant; LANL = Los Alamos National Laboratory; LBL = Lawrence Berkeley National Laboratory; LEHR = Laboratory for Energy-Related Health Research; Mare Is = Mare Island Naval Shipyard; Mound = Mound Plant; Norfolk = Norfolk Naval Shipyard; ORR = Oak Ridge Reservation; Pearl H = Pearl Harbor Naval Shipyard; PORTS = Portsmouth Gaseous Diffusion Plant; Ports Nav = Portsmouth Naval Shipyard; PPPL = Princeton Plasma Physics Laboratory; Puget So = Puget Sound Naval Shipyard; RFETS = Rocky Flats Environmental Technology Site; RMI = Reactive Metals, Inc.; SNL-CA = Sandia National Laboratories (California); SRS = Savannah River Site; UofMO = University of Missouri; and WVDP = West Valley Demonstration Project.

c The risk of fatal cancer is calculated by using the ICRP publication 60 (ICRP 1991) health risk conversion factor of 5E-04 fatal cancers per person-rem for members of the public. 
TABLE 5.9 Estimated Consequences for the Most Severe Accidents Involving Shipments of WM LLMW

\begin{tabular}{|c|c|c|c|c|c|c|c|c|}
\hline \multirow[b]{3}{*}{$\begin{array}{l}\text { Mode and } \\
\text { Accident } \\
\text { Location }\end{array}$} & \multicolumn{4}{|c|}{ Neutral Conditions $^{\mathrm{d}}$} & \multicolumn{4}{|c|}{ Stable Conditions ${ }^{f}$} \\
\hline & \multicolumn{2}{|c|}{ Population $^{c}$} & \multicolumn{2}{|c|}{$\mathrm{MEI}^{\mathrm{e}}$} & \multicolumn{2}{|c|}{ Population $^{\mathrm{c}}$} & \multicolumn{2}{|c|}{$\mathrm{MEI}^{\mathrm{e}}$} \\
\hline & $\begin{array}{c}\text { Dose } \\
\text { (person-rem) }\end{array}$ & $\begin{array}{c}\text { Risk } \\
\text { (Cancer } \\
\text { Fatalities) }\end{array}$ & $\begin{array}{l}\text { Dose } \\
\text { (rem) }\end{array}$ & $\begin{array}{c}\text { Risk } \\
\text { (Cancer } \\
\text { Fatality) }\end{array}$ & $\begin{array}{c}\text { Dose } \\
\text { (person-rem) }\end{array}$ & $\begin{array}{c}\text { Risk } \\
\text { (Cancer } \\
\text { Fatalities) }\end{array}$ & $\begin{array}{l}\text { Dose } \\
\text { (rem) }\end{array}$ & $\begin{array}{c}\text { Risk } \\
\text { (Cancer } \\
\text { Fatality) } \\
\end{array}$ \\
\hline \multicolumn{9}{|l|}{ Truck } \\
\hline Urban & $6 \mathrm{E}+02$ & $3 \mathrm{E}-01$ & 5.3E-01 & $2.7 \mathrm{E}-04$ & $4.75 \mathrm{E}+03$ & $2 E+00$ & $1.8 \mathrm{E}+00$ & $9.0 \mathrm{E}-04$ \\
\hline Suburban & $1.1 \mathrm{E}+02$ & $6 \mathrm{E}-02$ & 5.3E-01 & $2.7 \mathrm{E}-04$ & $8.85 E+02$ & $4 \mathrm{E} \sim 01$ & $1.8 \mathrm{E}+00$ & $9.0 \mathrm{E}-04$ \\
\hline Rural & $1.0 \mathrm{E}+00$ & $5 \mathrm{E}-04$ & 5.3E-01 & $2.7 \mathrm{E}-04$ & $7.5 \mathrm{E}+00$ & $4 \mathrm{E}-03$ & $1.8 \mathrm{E}+00$ & $9.0 \mathrm{E}-04$ \\
\hline \multicolumn{9}{|l|}{ Rail } \\
\hline Urban & $1.62 \mathrm{E}+03$ & $8 E-01$ & $1.4 \mathrm{E}+00$ & $7.0 \mathrm{E}-04$ & $1.283 E+04$ & $6 \mathrm{E}+00$ & $4.8 \mathrm{E}+00$ & $2.4 \mathrm{E}-03$ \\
\hline Suburban & $3.0 \mathrm{E}+02$ & $2 \mathrm{E}-01$ & $1.4 \mathrm{E}+00$ & $7.0 \mathrm{E}-04$ & $2.4 \mathrm{E}+03$ & $1 E+00$ & $4.8 \mathrm{E}+00$ & $2.4 \mathrm{E}-03$ \\
\hline Rural & $2.7 \mathrm{E}+00$ & $1 \mathrm{E}-03$ & $1.4 \mathrm{E}+00$ & $7.0 \mathrm{E}-04$ & $2.0 \mathrm{E}+01$ & $1 E-02$ & $4.8 \mathrm{E}+00$ & $2.4 \mathrm{E}-03$ \\
\hline
\end{tabular}

a The most severe accidents correspond to the highest NUREG-0170 accident severity category (Category VIII) (NRC 1977a). Results are reported for WM LLMW from PGDP, which was found to result in the highest potential accident doses. The assumptions were that $100 \%$ of the radioactive material would be released from its packaging in an accident, that $10 \%$ of the release would be entrained in an aerosol, and that $5 \%$ of the aerosolized release would be respirable.

b Buoyant plume rise resulting from fire for a severe accident was included in the exposure model.

c Populations extend at a uniform population density to a radius of $80 \mathrm{~km}(50 \mathrm{mi})$ from the accident site. Population exposure pathways include acute inhalation; acute cloudshine; groundshine; resuspended inhalation; resuspended cloudshine; and ingestion of food, including initially contaminated food (rural only). No decontamination or mitigative actions are taken.

d Neutral weather conditions result in moderate dispersion and dilution of the released plume. Neutral conditions were taken to be Pasquill stability Class D with a wind speed of $4 \mathrm{~m} / \mathrm{s}(9 \mathrm{mph})$. Neutral conditions occur approximately $50 \%$ of the time in the United States.

e The MEI is assumed to be at the location of maximum exposure. The locations of maximum exposure would be $160 \mathrm{~m}(525 \mathrm{ft})$ and $400 \mathrm{~m}(1,312 \mathrm{ft}$ ) from the accident site under neutral and stable atmospheric conditions, respectively. Individual exposure pathways include acute inhalation, acute cloudshine, and groundshine during passage of the plume. No ingested dose is considered.

f Stable weather conditions result in minimal dispersion and dilution of the released plume and are thus unfavorable. Stable conditions were taken to be Pasquill stability Class $F$ with a wind speed of $1 \mathrm{~m} / \mathrm{s}(2.2 \mathrm{mph})$. Stable conditions occur approximately one-third of the time in the United States. 
TABLE 5.10 Summary of Cargo-Related Population Risks ${ }^{\mathfrak{a}}$ for WM (10-Year Period) ${ }^{\mathbf{b}}$ LLMW Shipments by Highway

\begin{tabular}{|c|c|c|c|c|c|c|}
\hline \multirow[b]{2}{*}{ Population Risks } & \multicolumn{6}{|c|}{ LLMW Alternative } \\
\hline & Decentralized & Regionalized 1 & Regionalized 2 & Regionalized 3 & Regionalized 4 & Centralized \\
\hline \multicolumn{7}{|l|}{ Shipment summary } \\
\hline Number of WM shipments & $4.90 \mathrm{E}+02$ & $1.81 \mathrm{E}+03$ & $5.56 \mathrm{E}+03$ & $1.09 \mathrm{E}+04$ & $4.25 \mathrm{E}+03$ & $7.52 \mathrm{E}+03$ \\
\hline WM distance $(\mathbf{k m})^{\mathrm{c}}$ & $3.75 \mathrm{E}+05$ & $9.56 \mathrm{E}+05$ & $4.14 \mathrm{E}+06$ & $2.40 \mathrm{E}+07$ & $4.34 \mathrm{E}+06$ & $2.17 E+07$ \\
\hline \multicolumn{7}{|l|}{ Liquid wastes } \\
\hline \multicolumn{7}{|l|}{ Potential for increased cancer incidence } \\
\hline Severity Categories I-III & $0.00 \mathrm{E}+00$ & $0.00 \mathrm{E}+00$ & $0.00 \mathrm{E}+00$ & $0.00 \mathrm{E}+00$ & $0.00 \mathrm{E}+00$ & $0.00 \mathrm{E}+00$ \\
\hline Severity Category IV $\mathrm{d}^{\mathrm{d}}$ & $1.68 E-07$ & $3.51 E-07$ & $0.68 E-05$ & $0.68 E-05$ & $1.08 E-05$ & $1.95 E-04$ \\
\hline Severity Category V & $1.29 \mathrm{E}-08$ & $2.61 \mathrm{E}-08$ & $4.96 \mathrm{E}-07$ & $4.96 \mathrm{E}-07$ & $0.80 \mathrm{E}-06$ & $1.88 \mathrm{E}-05$ \\
\hline Severity Category VI & $0.65 \mathrm{E}-09$ & $1.23 \mathrm{E}-09$ & $2.28 \mathrm{E}-08$ & $2.28 \mathrm{E}-08$ & $3.72 E-08$ & $0.67 \mathrm{E}-06$ \\
\hline Severity Category VII & $2.03 \mathrm{E}-10$ & $4.06 \mathrm{E}-10$ & $0.73 \mathrm{E}-08$ & $0.73 \mathrm{E}-08$ & $1.14 \mathrm{E}-08$ & $2.05 \mathrm{E}-07$ \\
\hline Severity Category VIII & $2.31 \mathrm{E}-11$ & $4.27 \mathrm{E}-11$ & $0.72 E-09$ & $0.72 \mathrm{E}-09$ & $1.13 \mathrm{E}-09$ & $2.01 \mathrm{E}-08$ \\
\hline \multicolumn{7}{|l|}{ Potential adverse health effects } \\
\hline Severity Categories I-III & $0.00 \mathrm{E}+00$ & $0.00 \mathrm{E}+00$ & $0.00 \mathrm{E}+00$ & $0.00 \mathrm{E}+00$ & $0.00 \mathrm{E}+00$ & $0.00 \mathrm{E}+00$ \\
\hline Severity Category IV $\mathrm{d}$ & $1.04 E-06$ & $2.82 E-06$ & $2.84 E-05$ & $2.84 E-05$ & $4.36 E-0.5$ & $0.90 E-03$ \\
\hline Severity Category V & $0.80 \mathrm{E}-07$ & $2.04 \mathrm{E}-07$ & $2.08 \mathrm{E}-06$ & $2.08 \mathrm{E}-06$ & $3.23 \mathrm{E}-06$ & $0.67 \mathrm{E}-04$ \\
\hline Severity Category VI & $2.02 \mathrm{E}-09$ & $0.99 \mathrm{E}-08$ & $0.96 \mathrm{E}-07$ & $0.96 \mathrm{E}-07$ & $1.51 \mathrm{E}-07$ & 3.13E-06 \\
\hline Severity Category VII & $1.21 \mathrm{E}-09$ & $3.11 \mathrm{E}-09$ & $3.09 \mathrm{E}-08$ & $3.09 \mathrm{E}-08$ & $4.66 \mathrm{E}-08$ & $0.95 \mathrm{E}-06$ \\
\hline Severity Category VIII & $1.33 \mathrm{E}-10$ & $3.26 \mathrm{E}-10$ & $3.05 \mathrm{E}-09$ & $3.05 E-09$ & $4.62 \mathrm{E}-09$ & $0.93 \mathrm{E}-07$ \\
\hline \multicolumn{7}{|l|}{$\begin{array}{l}\text { Solid wastes } \\
\text { (volatile-organic-contaminated soil/debris } \\
\text { evaporative releases) }\end{array}$} \\
\hline Potential for increased cancer incidence & $0.00 E+00$ & $0.00 \mathrm{E}+00$ & $0.00 \mathrm{E}+00$ & $0.00 \mathrm{E}+00$ & $0.00 \mathrm{E}+00$ & $0.00 \mathrm{E}+00$ \\
\hline Potential adverse health effects & $0.00 \mathrm{E}+00$ & $0.00 \mathrm{E}+00$ & $0.00 \mathrm{E}+00$ & $0.00 \mathrm{E}+00$ & $0.00 \mathrm{E}+00$ & $0.00 \mathrm{E}+00$ \\
\hline \multicolumn{7}{|l|}{$\begin{array}{l}\text { Solid wastes } \\
\quad \text { (respirable contaminated aerosol releases) }\end{array}$} \\
\hline Potential for increased cancer incidence & $0.00 \mathrm{E}+00$ & $0.00 \mathrm{E}+00$ & $0.00 \mathrm{E}+00$ & $0.00 \mathrm{E}+00$ & $0.00 \mathrm{E}+00$ & $0.00 \mathrm{E}+00$ \\
\hline Potential adverse health effects & $0.00 \mathrm{E}+00$ & $0.00 \mathrm{E}+00$ & $0.00 \mathrm{E}+00$ & $0.00 \mathrm{E}+00$ & $0.00 \mathrm{E}+00$ & $0.00 \mathrm{E}+00$ \\
\hline \multicolumn{7}{|c|}{$\begin{array}{l}\text { Cargo-related risks refer to the number of people affected and were computed from the product of the probability of accidental release times the number of } \\
\text { people exposed to the health criteria concentration. }\end{array}$} \\
\hline \multicolumn{7}{|c|}{$\begin{array}{l}\text { b Risks and travel distances are for the total shipping duration (10 years for WM LLMW). To obtain the annual values, the risks and distances must be divided } \\
\text { by } 10 \text { for the WM cases. }\end{array}$} \\
\hline \multicolumn{7}{|c|}{$\begin{array}{l}\text { Vehicle-related distances are the highway distances multiplied by } 2 \text { to account for the return of empty trucks with no cargo. As a result, distances may be } \\
\text { overestimated. }\end{array}$} \\
\hline
\end{tabular}


TABLE 5.11 Summary of Cargo-Related Population Risks ${ }^{a}$ for WM (10-Year Period) ${ }^{b}$ LLMW Shipments by Railway

\begin{tabular}{|c|c|c|c|c|c|c|}
\hline \multirow[b]{2}{*}{ Population Risks } & \multicolumn{6}{|c|}{ LLMW Alternative } \\
\hline & Decentralized & Regionalized 1 & Regionalized 2 & Regionalized 3 & Regionalized 4 & Centralized \\
\hline \multicolumn{7}{|l|}{ Shipment summary } \\
\hline Number of WM shipments & $3.60 \mathrm{E}+02$ & $1.03 \mathrm{E}+03$ & $2.49 \mathrm{E}+03$ & $4.54 \mathrm{E}+03$ & $2.05 \mathrm{E}+03$ & $3.34 \mathrm{E}+03$ \\
\hline WM distance $(\mathrm{km})^{\mathrm{c}}$ & $3.44 \mathrm{E}+05$ & $7.74 \mathrm{E}+05$ & $2.20 \mathrm{E}+06$ & $1.09 \mathrm{E}+06$ & $2.45 \mathrm{E}+06$ & $1.04 \mathrm{E}+06$ \\
\hline \multicolumn{7}{|l|}{ Liquid wastes } \\
\hline \multicolumn{7}{|l|}{ Potential for increased cancer incidence } \\
\hline Severity Categories I-III & $0.00 \mathrm{E}+00$ & $0.00 \mathrm{E}+00$ & $0.00 \mathrm{E}+00$ & $0.00 \mathrm{E}+00$ & $0.00 \mathrm{E}+00$ & $0.00 \mathrm{E}+00$ \\
\hline Severity Category $I^{\mathrm{d}}$ & $4.88 E-08$ & $1.16 E-07$ & $2.12 E-06$ & $2.12 E-06$ & $2.44 E-06$ & $2.82 E-05$ \\
\hline Severity Category V & $3.60 \mathrm{E}-09$ & $0.86 \mathrm{E}-08$ & $1.51 \mathrm{E}-07$ & $1.51 \mathrm{E}-07$ & $1.77 \mathrm{E}-07$ & $2.10 \mathrm{E}-06$ \\
\hline Severity Category VI & $1.68 \mathrm{E}-10$ & $3.84 \mathrm{E}-10$ & $0.66 \mathrm{E}-08$ & $0.66 \mathrm{E}-08$ & $0.79 \mathrm{E}-08$ & $0.99 \mathrm{E}-07$ \\
\hline Severity Category VII & $0.58 \mathrm{E}-10$ & $1.34 \mathrm{E}-10$ & $2.46 \mathrm{E}-09$ & $2.46 \mathrm{E}-09$ & $2.96 \mathrm{E}-09$ & $3.58 \mathrm{E}-08$ \\
\hline Severity Category VIII & $0.62 \mathrm{E}-11$ & $1.32 \mathrm{E}-11$ & $2.44 \mathrm{E}-10$ & $2.44 \mathrm{E}-10$ & $3.07 \mathrm{E}-10$ & $3.88 \mathrm{E}-09$ \\
\hline \multicolumn{7}{|l|}{ Potential adverse health effects } \\
\hline Severity Categories I-III & $0.00 \mathrm{E}+00$ & $0.00 \mathrm{E}+00$ & $0.00 \mathrm{E}+00$ & $0.00 \mathrm{E}+00$ & $0.00 \mathrm{E}+00$ & $0.00 \mathrm{E}+00$ \\
\hline Severity Category $I^{\mathrm{d}}$ & $2.70 E-07$ & $0.84 E-06$ & $0.87 E-05$ & $0.87 E-05$ & $1.01 E-05$ & $1.10 E-04$ \\
\hline Severity Category V & $2.00 \mathrm{E}-08$ & $0.61 \mathrm{E}-07$ & $0.67 \mathrm{E}-06$ & $0.67 \mathrm{E}-06$ & $0.73 \mathrm{E}-06$ & $0.82 \mathrm{E}-05$ \\
\hline Severity Category VI & $0.93 \mathrm{E}-09$ & $2.77 \mathrm{E}-09$ & $2.79 \mathrm{E}-08$ & $2.99 \mathrm{E}-08$ & $3.31 \mathrm{E}-08$ & $3.89 \mathrm{E}-07$ \\
\hline Severity Category VII & $3.22 \mathrm{E}-10$ & $0.99 \mathrm{E}-09$ & $1.01 \mathrm{E}-08$ & $1.01 \mathrm{E}-08$ & $1.22 \mathrm{E}-08$ & $1.39 \mathrm{E}-07$ \\
\hline Severity Category VIII & $3.42 \mathrm{E}-11$ & $0.97 \mathrm{E}-10$ & $1.01 \mathrm{E}-09$ & $1.01 \mathrm{E}-09$ & $1.37 \mathrm{E}-09$ & $1.54 \mathrm{E}-08$ \\
\hline \multirow{2}{*}{\multicolumn{7}{|c|}{$\begin{array}{l}\text { Solid wastes } \\
\text { (volatile-organic-contaminated soil/debris } \\
\text { evaporative releases) }\end{array}$}} \\
\hline & & & & & & \\
\hline Potential for increased cancer incidence & $0.00 \mathrm{E}+00$ & $0.00 \mathrm{E}+00$ & $0.00 \mathrm{E}+00$ & $0.00 \mathrm{E}+00$ & $0.00 \mathrm{E}+00$ & $0.00 \mathrm{E}+00$ \\
\hline Potential adverse health effects & $0.00 \mathrm{E}+00$ & $0.00 \mathrm{E}+00$ & $0.00 \mathrm{E}+00$ & $0.00 \mathrm{E}+00$ & $0.00 \mathrm{E}+00$ & $0.00 \mathrm{E}+00$ \\
\hline \multirow{2}{*}{\multicolumn{7}{|c|}{$\begin{array}{l}\text { Solid wastes } \\
\text { (respirable contaminated aerosol } \\
\text { releases) }\end{array}$}} \\
\hline & & & & & & \\
\hline $\begin{array}{l}\text { Potential for increased cancer } \\
\text { incidence }\end{array}$ & $0.00 \mathrm{E}+00$ & $0.00 \mathrm{E}+00$ & $0.00 \mathrm{E}+00$ & $0.00 \mathrm{E}+00$ & $0.00 \mathrm{E}+00$ & $0.00 \mathrm{E}+00$ \\
\hline \multicolumn{7}{|l|}{ Potential adverse health effects } \\
\hline Severity Categories I-III & $0.00 \mathrm{E}+00$ & $0.00 \mathrm{E}+00$ & $0.00 \mathrm{E}+00$ & $0.00 \mathrm{E}+00$ & $0.00 \mathrm{E}+00$ & $0.00 \mathrm{E}+00$ \\
\hline Severity Category IV & $0.00 E+00$ & $0.00 E+00$ & $4.21 E-11$ & $4.21 E-11$ & $0.52 E-10$ & $0.54 E-09$ \\
\hline Severity Category V & $0.00 \mathrm{E}+00$ & $0.00 \mathrm{E}+00$ & $3.12 \mathrm{E}-12$ & $3.12 \mathrm{E}-12$ & $4.08 \mathrm{E}-12$ & $3.98 \mathrm{E}-11$ \\
\hline Severity Category VI & $0.00 \mathrm{E}+00$ & $0.00 \mathrm{E}+00$ & $1.47 \mathrm{E}-13$ & $1.47 \mathrm{E}-13$ & $2,08 \mathrm{E}-13$ & $1.73 \mathrm{E}-12$ \\
\hline Severity Category VII & $0.00 \mathrm{E}+00$ & $0.00 \mathrm{E}+00$ & $4.90 \mathrm{E}-14$ & $4.90 \mathrm{E}-13$ & $0.70 \mathrm{E}-13$ & $0.65 \mathrm{E}-12$ \\
\hline Severity Category VIII & $0.00 \mathrm{E}+00$ & $0.00 \mathrm{E}+00$ & $0.55 \mathrm{E}-14$ & $0.55 \mathrm{E}-14$ & $0.85 \mathrm{E}-14$ & $0.66 \mathrm{E}-13$ \\
\hline
\end{tabular}

a Cargo-related risks refer to the number of people affected and were computed from the product of the probability of accidental release times the number of people exposed to the health criteria concentration.

Risks and travel distances are for the total shipping duration (10 years for WM LLMW). To obtain the annual values, the risks and distances must be divided by 10 for the WM cases.

c Vehicle-related distances are the railway distances multiplied by 2 to account for the return of empty railcars with no cargo. As a result, distances may

d Re overestimated. 
Railcar-accident PIC and PAE risks from aerosolized liquid droplets are also highest for rail shipments under the centralized alternative for the same severity category.

The potential population risks involving solid waste shipments by trucks and railcars are attributed to evaporative organic vapor emissions from a waste spoils-pile ground spill and to the direct release of respirable particulates from an overturned vehicle or a ruptured container (or both). Truck accident risks from evaporative and from respirable particulate releases are found to be zero for all of the WM cases. As for railway shipments, zero population risks are found under all WM cases for evaporative emissions and under the decentralized and regionalized 1 alternatives for respirable particulates. Severity Category V appears to yield slightly higher health-related risk than other severity categories, while zero population risks are found for severity Categories I through III for all LLMW treatment options. Note that the HW component risk of the LLMW shipments is much lower than the transportation risk of the purely $\mathrm{HW}$ shipments (i.e., those with no radiological component).

With regard to MEI risk evaluation, the lifetime increased PIC risk and the PAE HQ are summarized in Tables 5.12 and 5.13. The MEI risks are given for both liquid and solid LLMW transportation accidents by trucks and railcars by using the approach described in Section 3.3.3.2. The risk calculations are based on the maximum ambient concentrations at $30.5 \mathrm{~m}$ (100 ft) from the release point for all shipments for a single truck or railcar accident predicted by the ALOHA ${ }^{\mathrm{TM}}$ model on a chemical-specific basis. As indicated in Table 5.12, the carcinogenic risks (incidence of increased cancers of one in a million or greater) for all chemicals are between $1 \times 10^{-9}$ and $1 \times 10^{-13}$. The HQs, which are shown in Table 5.13, are greater than 1 for all liquid WM LLMW shipments and for solid-waste truck and rail shipments of toluene under accident severity Categories IV through VIII. Thus, accidental release involving any of these shipments would have a potential to result in adverse effects for receptors at the MEI location. Tables 5.14 and 5.15 identify the alternatives producing the largest lifetime increased PIC risks and the PAE HQs to the MEI.

Note that the comparison of the cargo-related population risks among the six LLMW treatment options presented previously is based on the most frequently occurring meteorologic conditions (i.e., neutral atmospheric stability with a wind speed of $4 \mathrm{~m} / \mathrm{s}$ [ $9 \mathrm{mph}$ ]). In general, the severity of a transportation accident is determined by four important factors: prevailing meteorologic conditions, population distribution, chemical content of the container, and atmospheric release rate of the hazardous materials.

The "worst-case" meteorologic conditions associated with a release of hazardous materials into the atmosphere at ground level are light winds in a stable atmosphere (i.e., Pasquill stability Class $F$ with a wind speed of $1 \mathrm{~m} / \mathrm{s}$ [2.2 mph]). These nighttime meteorologic conditions are frequently associated with clear sky and temperature inversion. Population risks would be higher in urban areas than in rural areas due to denser population distributions. The population consequences are also compared for both neutral and stable atmospheric conditions under the most severe hazardous-chemical and radiological accidents. 
TABLE 5.12 Lifetime MEI PIC Risk for WM LLMW Transportation

\begin{tabular}{|c|c|c|c|c|c|c|c|c|c|c|c|}
\hline $\begin{array}{l}\text { Transportation } \\
\text { Modo }\end{array}$ & $\begin{array}{l}\text { Release } \\
\text { Mode }\end{array}$ & $\begin{array}{l}\text { Chemical } \\
\text { Code }\end{array}$ & Chemical Name & $\begin{array}{l}\text { ALOHA } \\
\text { Concentration } \\
\text { (ppm) }\end{array}$ & $\begin{array}{c}\text { Molecular } \\
\text { Weight }\end{array}$ & $\begin{array}{c}\text { ALOHA } \\
\text { Concentration } \\
\left(\mathrm{mg} / \mathrm{m}^{3}\right)\end{array}$ & $\begin{array}{c}\text { Exposure } \\
\text { Time } \\
(\mathrm{min} / \mathrm{d})\end{array}$ & $\begin{array}{l}\text { Inhalation } \\
\text { Air Intake } \\
(\mathrm{m} / \mathrm{kg} / \mathrm{d})\end{array}$ & $\begin{array}{l}\text { Inhalation } \\
\text { Unit Risk } \\
\left(\mu \mathrm{g} / \mathrm{m}^{3}\right)^{-1}\end{array}$ & $\begin{array}{c}\text { Slope } \\
\text { Factor } \\
(\mathrm{mg} / \mathrm{kg} / \mathrm{d})^{-1}\end{array}$ & $\begin{array}{l}\text { Carcinogenic } \\
\text { MEI Risk }\end{array}$ \\
\hline \multirow[t]{20}{*}{ Highway } & Liquid & $75-09-2$ & Dichloromethane & $6.17 \mathrm{E}+01$ & 85 & $2.14 \mathrm{E}+02$ & 60 & $1.01 \mathrm{E}-04$ & $4.70 \mathrm{E}-06$ & $1.65 \mathrm{E}-02$ & $1.7 \mathrm{E}-07$ \\
\hline & aerosol & Cl-2-x & Dichloroethane & $2.72 \mathrm{E}+01$ & 99 & $1.10 \mathrm{E}+02$ & 60 & 5.16E-05 & $2.60 \mathrm{E}-04$ & $9.10 \mathrm{E}-01$ & $4.7 \mathrm{E}-06$ \\
\hline & (direct) & Cl-4-x & Tetrachloroethene & $1.91 \mathrm{E}+02$ & 166 & $1.29 \mathrm{E}+03$ & 60 & $6.08 \mathrm{E}-04$ & $1.70 \mathrm{E}-05$ & $5.95 \mathrm{E}-02$ & $3.6 \mathrm{E}-06$ \\
\hline & & HC-soluble ${ }^{\mathrm{a}}$ & Benzene & $1.09 \mathrm{E}+04$ & 78.1 & 3.47E+04 & 60 & $1.63 \mathrm{E}-02$ & $8.30 \mathrm{E}-05$ & $2.91 \mathrm{E}-01$ & $4.7 \mathrm{E}-04$ \\
\hline & Vapor & $75-09 \cdot 2$ & Dichloromethane & $1.18 \mathrm{E}-02$ & 86 & $4.09 E-02$ & 60 & $1.92 \mathrm{E}-08$ & $4.70 \mathrm{E}-06$ & $1.65 \mathrm{E}-02$ & $3.2 E-11$ \\
\hline & apoils pile & Cl-2-x & Dichloroethane & $9.41 \mathrm{E}-02$ & 99 & $3.80 \mathrm{E}-01$ & 60 & $1.79 E-07$ & $2.60 E-04$ & $9.10 \mathrm{E}-01$ & $1.6 \mathrm{E}-08$ \\
\hline & (Superfund) & Cl-4.x & Tetrachloroethene & $1.26 \mathrm{E}-03$ & 166 & $8.54 \mathrm{E}-03$ & 60 & $4.01 \mathrm{E}-09$ & $1.70 \mathrm{E}-05$ & $5.95 \mathrm{E}-02$ & $2.4 \mathrm{E}-11$ \\
\hline & & HC-soluble & Benzene & $1.80 \mathrm{E}-02$ & 78.1 & 6.74E-02 & 60 & $2.69 \mathrm{E}-08$ & $8.30 \mathrm{E}-06$ & $2.91 \mathrm{E}-01$ & $7.8 \mathrm{E}-10$ \\
\hline & Particulate & $75.09-2$ & Dichloromethane & $1.20 \mathrm{E}-02$ & 85 & $4.16 \mathrm{E}-02$ & 60 & $1.96 \mathrm{E}-08$ & $4.70 \mathrm{E}-06$ & $1.66 \mathrm{E}-02$ & $3.2 \mathrm{E}-11$ \\
\hline & (severity & Cl-2-x & Dichloroethane & $6.97 \mathrm{E}-03$ & 99 & $2.82 \mathrm{E}-02$ & 60 & $1.32 \mathrm{E}-08$ & $2.60 \mathrm{E}-04$ & $9.10 \mathrm{E}-01$ & $1.2 \mathrm{E}-09$ \\
\hline & Category II) & Cl-4-x & Tetrachloroethene & $2.28 \mathrm{E}-02$ & 166 & $1.54 \mathrm{E}-01$ & 60 & $7.26 \mathrm{E}-08$ & $1.70 \mathrm{E}-05$ & 5.95E -02 & $4.3 \mathrm{E}-10$ \\
\hline & & HC-soluble & Benzene & $1.05 \mathrm{E}+01$ & 78.1 & $3.35 \mathrm{E}-01$ & 60 & $1.57 \mathrm{E}-07$ & $8.30 \mathrm{E}-05$ & $2.91 \mathrm{E}-01$ & $4.6 \mathrm{E}-09$ \\
\hline & Particulate & $75.09-2$ & Dichloromethane & $1.20 \mathrm{E}-01$ & 85 & $4.16 \mathrm{E}-01$ & 60 & $1.96 \mathrm{E}-07$ & $4.70 \mathrm{E}-06$ & $1.65 \mathrm{E}-02$ & $3.2 \mathrm{E}-10$ \\
\hline & (severity & Cl-2-x & Dichloroothane & $6.97 \mathrm{E}-02$ & 99 & $2.82 \mathrm{E}-01$ & 60 & $1.32 E-07$ & $2.60 \mathrm{E}-04$ & $9.10 \mathrm{E}-01$ & $1.2 \mathrm{E}-08$ \\
\hline & Category III) & Cl-4-x & Tetrachloroethene & $2.28 E-01$ & 166 & $1.54 E+00$ & 60 & $7.26 \mathrm{E}-07$ & $1.70 \mathrm{E}-0.5$ & $5.96 \mathrm{E}-02$ & $4.3 \mathrm{E}-09$ \\
\hline & & HC-soluble & Benzene & $1.05 \mathrm{E}+00$ & 78.1 & $3.35 \mathrm{E}+00$ & 60 & $1.57 \mathrm{E}-06$ & $8.30 \mathrm{E}-05$ & $2.91 \mathrm{E}-01$ & $4.6 \mathrm{E}-08$ \\
\hline & Particulate & $75-09-2$ & Dichloromethane & $1.20 \mathrm{E}+00$ & 85 & $4.16 \mathrm{E}+00$ & 60 & $1.96 \mathrm{E}-06$ & $4.70 \mathrm{E}-06$ & $1.65 \mathrm{E}-02$ & $3.2 \mathrm{E}-09$ \\
\hline & (severity & Cl-2-x & Dichloroethane & $6.97 \mathrm{E}-01$ & 99 & $2.82 \mathrm{E}+00$ & 60 & $1.32 \mathrm{E}-06$ & $2.60 \mathrm{E}-04$ & $9.10 \mathrm{E}-01$ & $1.2 E-07$ \\
\hline & Categories & Cl-4-x & Tetrachloroethene & $2.28 \mathrm{E}+00$ & 166 & $1.54 E+01$ & 60 & $7.26 \mathrm{E}-06$ & $1.70 \mathrm{E}-05$ & $5.95 \mathrm{E}-02$ & $4.3 \mathrm{E}-08$ \\
\hline & IV-VIII) & HC-soluble & Benzene & $1.05 \mathrm{E}+01$ & 78.1 & $3.35 \mathrm{E}+01$ & 60 & $1.57 \mathrm{E}-05$ & $8.30 \mathrm{E}-05$ & $2.91 \mathrm{E}-01$ & $4.6 \mathrm{E}-07$ \\
\hline \multirow[t]{20}{*}{ Railroad } & Liquid & $75-09-2$ & Dichloromethane & $1.65 \mathrm{E}+02$ & 85 & 5.72E+02 & 60 & $2.69 \mathrm{E}-04$ & $4.70 \mathrm{E}-06$ & $1.65 \mathrm{E}-02$ & $4.4 E-07$ \\
\hline & aerosol & Cl-2-x & Dichloroethane & $5.86 \mathrm{E}+01$ & 99 & $2.36 \mathrm{E}+02$ & 60 & $1.11 E-04$ & $2.60 \mathrm{E}-04$ & $9.10 \mathrm{E}-01$ & $1.0 \mathrm{E}-05$ \\
\hline & (direct) & Cl-4-x & Tetrachloroethene & $9.24 \mathrm{E}+02$ & 166 & $6.26 \mathrm{E}+03$ & 60 & $2.94 \mathrm{E}-03$ & $1.70 \mathrm{E}-05$ & $5.95 \mathrm{E}-02$ & $1.7 \mathrm{E}-05$ \\
\hline & & HC-soluble & Benzene & $1.46 \mathrm{E}+04$ & 78.1 & $4.65 \mathrm{E}+04$ & 60 & $2.19 \mathrm{E}-02$ & $8.30 \mathrm{E}-05$ & $2.91 E-01$ & $6.4 \mathrm{E}-04$ \\
\hline & Vapor & $75 \cdot 09-2$ & Dichloromethane & $1.96 \mathrm{E}-02$ & 85 & $6.80 \mathrm{E}-02$ & 60 & $3.19 \mathrm{E}-08$ & $4.70 \mathrm{E}-06$ & $1.65 \mathrm{E}-02$ & $5.3 \mathrm{E}-11$ \\
\hline & spoils pile & Cl-2-2x & Dichloroethane & $1.67 \mathrm{E}-02$ & 99 & $6.34 \mathrm{E}-02$ & 60 & $2.98 \mathrm{E}-08$ & $2.60 \mathrm{E}-04$ & $9.10 \mathrm{E}-0 \mathrm{I}$ & $2.7 \mathrm{E}-09$ \\
\hline & (Superfund) & Cl-4-x & Tetrachloroethene & 2.53E-03 & 166 & $1.71 \mathrm{E}-02$ & 60 & $8.05 \mathrm{E}-09$ & $1.70 \mathrm{E}-05$ & $5.95 \mathrm{E}-02$ & $4.8 \mathrm{E}-11$ \\
\hline & & HC-soluble & Benzene & 3.31E-02 & 78.1 & $1.06 \mathrm{E}-01$ & 60 & $4.96 \mathrm{E}-08$ & $8.30 \mathrm{E}-05$ & $2.91 \mathrm{E}-01$ & $1.4 \mathrm{E}-09$ \\
\hline & Particulate & $75-09-2$ & Dichloromethane & $3.41 \mathrm{E}-02$ & 85 & $1.18 \mathrm{E}-01$ & 60 & $5.56 \mathrm{E}-08$ & $4.70 \mathrm{E}-06$ & $1.65 \mathrm{E}-02$ & $9.1 \mathrm{E}-11$ \\
\hline & (severity & Cl-2-x & Dichloroethane & $1.98 \mathrm{E}-02$ & 99 & $8.00 \mathrm{E}-02$ & 60 & $3.76 E-08$ & $2.60 \mathrm{E}-04$ & $9.10 \mathrm{E}-01$ & $3.4 \mathrm{E}-09$ \\
\hline & Category II) & Cl-4-x & Tetrachloroethene & $4.50 \mathrm{E}-02$ & 166 & $3.05 \mathrm{E}+01$ & 60 & $1.43 \mathrm{E}-07$ & $1.70 \mathrm{E}-05$ & $5.95 \mathrm{E}-02$ & $8.5 \mathrm{E}-10$ \\
\hline & & HC-soluble & Benzene & $5.25 \mathrm{E}-01$ & 78.1 & $1.67 \mathrm{E}+00$ & 60 & $7.86 \mathrm{E}-07$ & $8.30 \mathrm{E}-05$ & $2.91 \mathrm{E}-01$ & $2.3 \mathrm{E}-08$ \\
\hline & Particulate & $75-09-2$ & Dichloromethane & $3.41 \mathrm{E}-01$ & 85 & $1.18 E+00$ & 60 & 5.56E-07 & $4.70 \mathrm{E}-06$ & $1.65 \mathrm{E}-02$ & $9.1 \mathrm{E}-10$ \\
\hline & (severity & Cl-2-x & Dichloroethane & $1.98 \mathrm{E}-01$ & 99 & $8.00 \mathrm{E}-01$ & 60 & $3.76 \mathrm{E}-07$ & $2.60 \mathrm{E}-04$ & $9.10 \mathrm{E}-01$ & $3.4 \mathrm{E}-08$ \\
\hline & Category III) & Cl. $4-x$ & Tetrachloroethen $\theta$ & $4.50 \mathrm{E}-01$ & 166 & $3.05 \mathrm{E}+00$ & 60 & $1.43 \mathrm{E}-06$ & $1.70 \mathrm{E}-05$ & 5.95E-02 & $8.5 \mathrm{E}-09$ \\
\hline & & HC-soluble & Benzene & $5.25 \mathrm{E}+00$ & 78.1 & $1.67 \mathrm{E}+01$ & 60 & $7.86 \mathrm{E}-06$ & $8.30 \mathrm{E}-05$ & $2.91 E-01$ & $2.3 \mathrm{E}-07$ \\
\hline & Particulate & $75-09-2$ & Dichloromethane & $3.41 \mathrm{E}+00$ & 85 & $1.18 \mathrm{E}+01$ & 60 & $5.56 \mathrm{E}-06$ & $4.70 \mathrm{E}-06$ & $1.65 \mathrm{E}-02$ & 9.1E-09 \\
\hline & (soverity & Cl.2-x & Dichloroethane & $1.98 \mathrm{E}+00$ & 99 & $8.00 \mathrm{E}+00$ & 60 & $3.76 \mathrm{E}-06$ & $2.60 \mathrm{E}-04$ & $9.10 \mathrm{E}-01$ & 3.4E-07 \\
\hline & Categories & Cl-4-x & Tetrachloroethene & $4.50 \mathrm{E}+00$ & 166 & 3.05E +01 & 60 & $1.43 \mathrm{E}-05$ & $1.70 \mathrm{E}-05$ & 5.95E-02 & 8.5E- 08 \\
\hline & IV.viII) & HC-soluble & Benzene & 5.25E+01 & 78.1 & $1.67 \mathrm{E}+02$ & 60 & $7,86 \mathrm{E}-05$ & $8.30 \mathrm{E}-05$ & $2.91 \mathrm{E}-01$ & $2.3 E-06$ \\
\hline
\end{tabular}


TABLE 5.13 MEI Hazard Quotients for PAE End Points for WM LLMW

\begin{tabular}{|c|c|c|c|c|c|c|c|c|c|c|c|}
\hline $\begin{array}{l}\text { TYansportation } \\
\text { Mode }\end{array}$ & $\begin{array}{l}\text { Release } \\
\text { Mode }\end{array}$ & $\begin{array}{c}\text { Chemical } \\
\text { Code }\end{array}$ & Chemical Name & $\begin{array}{c}\text { ALOHA } \\
\text { Concentration } \\
(\mathrm{ppm})\end{array}$ & $\begin{array}{c}\text { Molecular } \\
\text { Weight }\end{array}$ & $\begin{array}{c}\text { ALOHA } \\
\text { Concentration } \\
\left(\mathbf{m g} / \mathrm{m}^{3}\right)\end{array}$ & $\begin{array}{c}\text { Exposure } \\
\text { Time } \\
\text { (min/d) }\end{array}$ & $\begin{array}{l}\text { Inhalation } \\
\text { Air Intake } \\
\text { (m\&/kg/d) }\end{array}$ & $\begin{array}{c}\mathrm{ReC}^{\mathrm{a}} \\
\left(\mathrm{mg} / \mathrm{m}^{3}\right)\end{array}$ & $\begin{array}{c}\mathbf{R N O}^{\mathbf{b}} \\
(\mathrm{mg} / \mathbf{k g} / \mathrm{d})\end{array}$ & HQ \\
\hline \multirow[t]{20}{*}{ Highway } & Liquid spill & $75-09-2$ & Dichloromethane & $6.17 \mathrm{E}+01$ & 85 & $2.14 \mathrm{E}+02$ & 60 & $1.4 E+00$ & $3 E+\infty$ & $8.6 \mathrm{E}+00$ & $1.68 \mathrm{E}+00$ \\
\hline & & Cl-3-z & 1,1,1-Trichlomethane & $2.72 \mathrm{E}+01$ & 133.4 & $1.48 E+02$ & 60 & $10.0 \mathrm{E}-01$ & $1.00 \mathrm{E}+01$ & $2.9 \mathrm{E}+00$ & $3.49 \mathrm{E}+00$ \\
\hline & & $\mathrm{Cl} \cdot \mathrm{F} \cdot \mathbf{x}$ & Freon 113 & $1.91 \mathrm{E}+02$ & 187 & $1.46 \mathrm{E}+03$ & 60 & $9.8 \mathrm{E}+00$ & $3.0 \mathrm{E}+01$ & $8.6 \mathrm{E}+01$ & $1.15 \mathrm{E}+00$ \\
\hline & & HC-soluble ${ }^{\mathrm{C}}$ & Toluene & $1.09 \mathrm{E}+04$ & 92.14 & $4.10 \mathrm{E}+04$ & 60 & $2.8 E+02$ & $4 \mathrm{E}-01$ & $1.1 \mathrm{E}+00$ & $2.42 \mathrm{E}+03$ \\
\hline & Spoils pile & $76-09-2$ & Dichloromethane & $1.18 \mathrm{E}-02$ & 85 & $4.09 \mathrm{E}-02$ & 60 & $2.8 \mathrm{E}-04$ & $3 E+\infty 0$ & $8.6 \mathrm{E}+00$ & $3.22 \mathrm{E}-04$ \\
\hline & vapor & $\mathrm{Cl} \cdot 3-\mathrm{x}$ & 1,1,1-Trichloroethane & $9,41 \mathrm{E}-02$ & 133.4 & $0.12 \mathrm{E}-01$ & 60 & $3.6 \mathrm{E}-03$ & $1,00 \mathrm{E}+01$ & $2.9 E+00$ & $1.21 \mathrm{E}-02$ \\
\hline & (Superfund) & Cl-F-x & Frean 113 & $1.26 \mathrm{E}-03$ & 187 & $9.62 \mathrm{E}-03$ & 60 & $6.5 \mathrm{E}-05$ & $3.0 \mathrm{E}+01$ & $8.6 \mathrm{E}+01$ & $7.56 \mathrm{E}-06$ \\
\hline & & HC-soluble & Toluene & $1.80 \mathrm{E}-02$ & 92.14 & $6.77 \mathrm{E}-02$ & 60 & $4.6 \mathrm{E}-04$ & $4 \mathrm{E}-01$ & $1.1 E+00$ & 3.99E-03 \\
\hline & Particulate & $75-09-2$ & Dichloromethane & $1.20 \mathrm{E}-02$ & 85 & $4.16 \mathrm{E}-02$ & 60 & $2.8 \mathrm{E}-04$ & $3 \mathrm{E}+00$ & $8.6 \mathbf{E}+00$ & 3.27E-04 \\
\hline & (severity & Cl-3-x & 1,1,1-Trichloroethane & $6.97 \mathrm{E}-03$ & 133.4 & $3.80 \mathrm{E}-02$ & 60 & $2.6 \mathrm{E}-04$ & $1.00 \mathrm{E}+0 \mathrm{t}$ & $2.9 \mathrm{E}+00$ & $8.95 \mathrm{E}-04$ \\
\hline & Category 11) & Cl-F.x & Freon 113 & $2.28 \mathrm{E}-02$ & 187 & $1.74 \mathrm{E}-01$ & 60 & $1.2 \mathrm{E}-03$ & $3.0 \mathrm{E}+01$ & $8.6 \mathrm{E}+01$ & $1.37 \mathrm{E}-04$ \\
\hline & & HC-soluble & Toluene & $1.05 \mathrm{E}-01$ & 92.14 & $3.95 \mathrm{E}-01$ & 60 & $2.7 \mathrm{E}-03$ & $4 \mathrm{E}-01$ & $1.1 \mathrm{E}+00$ & 2.33E-02 \\
\hline & Particulate & $75-09-2$ & Dichloromethane & $1.20 \mathrm{E}-01$ & 85 & $4.16 \mathrm{E}-01$ & 60 & $2.8 \mathrm{E}-03$ & $3 E+00$ & $8.6 \mathrm{E}+00$ & $3.27 \mathrm{E}-03$ \\
\hline & (Beverity & $\mathrm{Cl} \cdot 3 \cdot \mathrm{x}$ & 1,1,1-Trichloroethane & $6.97 \mathrm{E}-02$ & 133.4 & $3.80 \mathrm{E}-01$ & 60 & $2.6 \mathrm{E}-03$ & $1.00 \mathrm{E}+01$ & $2.9 \mathrm{E}+\infty 0$ & $8.95 \mathrm{E}-03$ \\
\hline & Category III) & Cl-F-x & Freon 113 & $2.28 \mathrm{E}-01$ & 187 & $1.74 E+00$ & 60 & $1.2 \mathrm{E}-02$ & $3.0 \mathrm{E}+01$ & $8.6 \mathrm{E}+01$ & $1.37 \mathrm{E}-08$ \\
\hline & & HC-soluble & Toluene & $1.08 \mathrm{E}+00$ & 92.14 & $3.95 \mathrm{E}+\infty 0$ & 60 & $2.7 \mathrm{E}-02$ & $4 \mathrm{E}-01$ & $1.1 \mathrm{E}+00$ & $2.33 \mathrm{E}-01$ \\
\hline & Particulate & $75-09-2$ & Dichloromethane & $1.20 \mathrm{E}+\infty 0$ & 85 & $4.16 \mathrm{E}+00$ & 60 & $2.8 \mathrm{E}-02$ & $3 E+00$ & $8.6 \mathrm{E}+00$ & $3.27 \mathrm{E}-02$ \\
\hline & (Beverity & Cl-3-s & 1,1,1-Trichloroethane & $6.97 \mathrm{E}-01$ & 133.4 & $3.80 \mathrm{E}+00$ & 60 & $2.6 \mathrm{E}-02$ & $1.00 \mathrm{E}+01$ & $2.9 \mathrm{E}+00$ & $8.95 \mathrm{E}-02$ \\
\hline & Categories & $\mathbf{C} \cdot \mathbf{F}-\mathbf{x}$ & Freon 113 & $2.28 \mathrm{E}+00$ & 187 & $1.74 \mathrm{E}+01$ & 60 & $1.2 \mathrm{E}-01$ & $3.0 \mathrm{E}+01$ & $8.6 \mathrm{E}+01$ & $1.37 \mathrm{E}-02$ \\
\hline & IV-VIII) & HC-soluble & Toluene & $1.05 \mathrm{E}+01$ & 92.14 & $3.95 \mathrm{E}+01$ & 60 & $2.7 \mathrm{E}-01$ & 4E-01 & $\begin{array}{l}0.0 \mathrm{E}+01 \\
1.1 \mathrm{E}+00\end{array}$ & $2.33 \mathrm{E}+00$ \\
\hline \multirow[t]{20}{*}{ Railroad } & Liquid spill & $75-09-2$ & Dichloromethane & $1.65 \mathrm{E}+02$ & 85 & $5.72 \mathrm{E}+02$ & 60 & $3.9 \mathrm{E}+00$ & $3 E+00$ & $8.6 \mathrm{E}+\infty 0$ & $4.50 \mathrm{E}+00$ \\
\hline & & Cl-3-x & 1,1,1-Trichloroethane & $4.46 \mathrm{E}+03$ & 133.4 & $2.43 \mathrm{E}+04$ & 60 & $1.6 \mathrm{E}+02$ & $1.00 \mathrm{E}+01$ & $2.9 \mathrm{E}+\infty 0$ & $5.72 \mathrm{E}+02$ \\
\hline & & Cl-F-x & Freon 113 & $4.94 \mathrm{E}+00$ & 187 & $3.77 \mathrm{E}+01$ & 60 & $2.5 \mathrm{E}-01$ & $3.0 \mathrm{E}+01$ & $8.6 \mathrm{E}+01$ & $2,96 \mathrm{E}-02$ \\
\hline & & HC-soluble & Toluene & $1.21 \mathrm{E}+04$ & 92.14 & $4.55 \mathrm{E}+04$ & 60 & $3.1 \mathrm{E}+02$ & $4 \mathrm{E}-01$ & $1.1 \mathrm{E}+00$ & $2.68 \mathrm{E}+03$ \\
\hline & Spoile pile & $75-09-2$ & Dichloromethane & $1.96 \mathrm{E}-02$ & 85 & $6.80 \mathrm{E}-02$ & 60 & $4.6 \mathrm{E}-04$ & $3 E+00$ & $8.6 \mathrm{E}+00$ & $5.34 \mathrm{E}-04$ \\
\hline & vapor & $\mathrm{Cl}-3-\mathrm{x}$ & 1,1,1-Trichloroethane & $1.78 \mathrm{E}-02$ & 133.4 & $9.69 \mathrm{E}-02$ & 60 & $6.5 \mathrm{E}-04$ & $1.00 \mathrm{E}+01$ & $2.9 \mathrm{E}+00$ & $2,28 \mathrm{E}-03$ \\
\hline & (Superfund) & Cl-F.x & Freon 113 & $1.87 \mathrm{E}-04$ & 187 & $1.43 \mathrm{E}-03$ & 60 & $9.6 \mathrm{E}-06$ & $3.0 \mathrm{E}+01$ & $8.6 \mathrm{E}+01$ & $1.12 \mathrm{E}-06$ \\
\hline & & HC-soluble & Taluene & $1.18 \mathrm{E}-02$ & 92.14 & 4.44E- -02 & 60 & $3.0 \mathrm{E}-04$ & $4 \mathrm{E}-01$ & $1.1 \mathrm{E}+00$ & $2.62 \mathrm{E}-03$ \\
\hline & Particulate & $75-09-2$ & Dichloromethane & $3.41 \mathrm{E}-02$ & 85 & $1.18 \mathrm{E}-01$ & 60 & $8.0 \mathrm{E}-04$ & $3 \mathrm{E}+00$ & $8.6 \mathrm{E}+00$ & $9.30 \mathrm{E}-04$ \\
\hline & (ooverity & $\mathrm{Cl}-3-\mathbf{x}$ & 1,1,1-Trichloroethane & $\begin{array}{l}7.54 \mathrm{E}-02 \\
7\end{array}$ & 133.4 & 4.11E-01 & 60 & $2.8 \mathrm{E}-03$ & $\begin{array}{r}0 \mathrm{E}+00 \\
1.00 \mathrm{E}+01\end{array}$ & $2.9 \mathrm{E}+00$ & $9.68 \mathrm{E}-03$ \\
\hline & Category II) & Cl-F-x & Freon 113 & $5.07 \mathrm{E}-03$ & 187 & $3.87 \mathrm{E}-02$ & 60 & $2.6 \mathrm{E}-04$ & $3.0 \mathrm{E}+01$ & $8.6 E+01$ & $3.04 \mathrm{E}-05$ \\
\hline & & HC-soluble & Toluene & $2.76 \mathrm{E}-01$ & 92.14 & $1.04 E+00$ & 60 & $7.0 \mathrm{E}-03$ & $4 E-01$ & $1.1 E+00$ & $6.12 \mathrm{E}-02$ \\
\hline & Particulate & $75 \cdot 09 \cdot 2$ & Dichloromethane & $3.41 \mathrm{E}-01$ & 85 & $1.18 \mathrm{E}+00$ & 60 & $8.0 \mathrm{E}-03$ & $3 E+00$ & $8.6 \mathbf{E}+00$ & $9.30 \mathrm{E}-03$ \\
\hline & (soverity & $\mathrm{Cl}-3-\mathrm{x}$ & 1,1,1-Mrichloroethane & $7.54 \mathrm{E}-01$ & 193.4 & $4.11 \mathrm{E}+00$ & 60 & $2.8 \mathrm{E}-02$ & $1.00 E+01$ & $2.9 E+00$ & $9.68 \mathrm{E}-02$ \\
\hline & Category III) & Cl-F-x & Freon 113 & 6.07E-02 & 187 & $3,87 \mathrm{E}-01$ & 60 & $2.6 \mathrm{E}-03$ & $3.0 \mathrm{E}+01$ & $8.6 \mathrm{E}+01$ & $3.04 \mathrm{E}-04$ \\
\hline & & HC-soluble & Toluene & $2.76 \mathrm{E}+00$ & 92.14 & $1.04 \mathrm{E}+01$ & 60 & $7.0 \mathrm{E}-02$ & $4 \mathrm{E}-01$ & $1.1 \mathrm{E}+00$ & 6.12E-01 \\
\hline & Particulato & $75-09 \cdot 2$ & Dichloromethane & $3.41 \mathrm{E}+00$ & 85 & $1.18 \mathrm{E}+01$ & 60 & $8.0 \mathrm{E}-02$ & $3 \mathrm{E}+00$ & $8.6 \mathrm{E}+00$ & $9.30 \mathrm{E}-02$ \\
\hline & (severity & Cl-3-3x & 1,1,1-Trichlorpethane & $7.54 \mathrm{E}+00$ & 133.4 & $4.11 \mathrm{E}+01$ & 60 & $2.8 \mathrm{E}-01$ & $1.00 \mathrm{E}+01$ & $2.9 \mathrm{E}+00$ & $9.68 \mathrm{E}-01$ \\
\hline & Categorieg & $\mathrm{Cl} \cdot \mathrm{F} \cdot \mathbf{x}$ & Freon 113 & $5.07 \mathrm{E}-01$ & 187 & $3.87 \mathrm{E}+00$ & 60 & $2.6 \mathrm{E}-02$ & $3.0 \mathrm{E}+01$ & $8.6 \mathrm{E}+01$ & $3.04 \mathrm{E}-03$ \\
\hline & [V-VIII) & HC-soluble & Toluene & $2.76 \mathrm{E}+01$ & 92.14 & $1.04 \mathrm{E}+02$ & 60 & $7.0 \mathrm{E}-01$ & $4 \mathrm{E}-01$ & $1.1 \mathrm{E}+00$ & $6.12 \mathrm{E}+00$ \\
\hline
\end{tabular}

a $\mathrm{RTC}=\mathrm{x}$ reference concentration.

b $R / D=$ reference dose.

c $\mathrm{HC}=$ hydrocarbon 
TABLE 5.14 Lifetime MEI PIC Risk by LLMW Alternative

\begin{tabular}{|c|c|c|c|c|c|c|c|c|c|}
\hline \multirow{20}{*}{ Highway } & Liquid & Dichloromethane & Centralized & SRS & Hanford & 33 & 17,620 & 24 & $1.65 \mathrm{E}-08$ \\
\hline & aerosol & Dichloroethane & Centralized & SRS & Hanford & 33 & 17,620 & 13 & $4.70 \mathrm{E}-07$ \\
\hline & (direct) & Tetrachloroethene & Centralized & SRS & Hanford & 33 & 17,620 & 308 & $3.62 \mathrm{E}-07$ \\
\hline & & Benzene & Regionalized 4 & PORTS & ORR & 3 & 15,929 & 4,803 & $4.74 \mathrm{E}-05$ \\
\hline & Spoils pile & Dichloromethane & Regionalized 4 & LANL & INEL & 4 & 16,992 & 18 & $3.16 \mathrm{E}-12$ \\
\hline & vapor & Dichloroethane & Regionalized 4 & Pantex & INEL & 2 & 12,559 & 11 & $1.63 \mathrm{E}-09$ \\
\hline & (Superfund) & Tetrachloroethene & Regionalized 4 & Pantex & INEL & 2 & 12,559 & 55 & $2.39 \mathrm{E}-12$ \\
\hline & & Benzene & Centralized & INEL & Hanford & 4 & 15,240 & 137 & $7.83 E-11$ \\
\hline & Particulate & Dichloromethane & Regionalized 4 & LANL & INEL & 4 & 16,992 & 18 & $3.22 \mathrm{E}-12$ \\
\hline & (severity & Dichloroethane & Regionalized 4 & Pantex & INEL & 2 & 12,559 & 11 & $1.20 \mathrm{E}-10$ \\
\hline & Category II) & Tetrachloroethene & Regionalized 4 & Pantex & INEL & 2 & 12,559 & 55 & $4.32 \mathrm{E}-11$ \\
\hline & & Benzene & Centralized & INEL & Hanford & 4 & 15,240 & 137 & $4.57 \mathrm{E}-10$ \\
\hline & Particulate & Dichloromethane & Regionalized 4 & LANL & INEL & 4 & 16,992 & 18 & $3.22 \mathrm{E}-11$ \\
\hline & (severity & Dichloroethane & Regionalized 4 & Pantex & INEL, & 2 & 12,559 & 11 & $1.20 \mathrm{E}-09$ \\
\hline & Category III) & Tetrachloroethene & Regionalized 4 & Pantex & INEL & 2 & 12,559 & 55 & $4.32 \mathrm{E}-10$ \\
\hline & & Benzene & Centralized & INEL & Hanford & 4 & 15,240 & 137 & $4.57 \mathrm{E}-09$ \\
\hline & Particulate & Dichloromethane & Regionalized 4 & LANL & INEL & 4 & 16,992 & 18 & $3.22 \mathrm{E}-10$ \\
\hline & (severity & Dichloroethane & Regionalized 4 & Pantex & INEL & 2 & 12,559 & 11 & $1.20 \mathrm{E}-08$ \\
\hline & Categories & Tetrachloroethene & Regionalized 4 & Pantex & INEL & 2 & 12,559 & 55 & $4.32 \mathrm{E}-09$ \\
\hline & IV-VIII) & Benzene & Centralized & INEL & Hanford & 4 & 15,240 & 137 & $4.57 \mathrm{E}-08$ \\
\hline \multirow[t]{20}{*}{ Railroad } & Liquid & Dichloromethane & Centralized & SRS & Hanford & 13 & 44,727 & 61 & $4.42 \mathrm{E}-08$ \\
\hline & aerosol & Dichloroethane & Centralized & SRS & Hanford & 13 & 44,727 & 32 & $1.01 \mathrm{E}-06$ \\
\hline & (direct) & Tetrachloroethene & Centralized & SRS & Hanford & 13 & $\mathbf{4 4 , 7 2 7}$ & 782 & $1.75 \mathrm{E}-06$ \\
\hline & & Benzene & Regionalized 4 & PORTS & ORR & 2 & 23,894 & 7,204 & $6.35 \mathrm{E}-05$ \\
\hline & Spoils pile & Dichloromethane & Regionalized 4 & LANL & INEL & 2 & 33,983 & 37 & $5.25 \mathrm{E}-12$ \\
\hline & vapor & Dichloroethane & Centralized & Pantex & Hanford & 1 & 25,119 & 22 & $2.71 \mathrm{E}-10$ \\
\hline & (Superfund) & Tetrachloroethene & Centralized & FEMP & Hanford & 3 & 39,356 & 123 & $4.79 \mathrm{E}-12$ \\
\hline & & Benzene & Centralized & FEMP & Hanford & 3 & 39,356 & 313 & $1.44 \mathrm{E}-10$ \\
\hline & Particulate & Dichloromethane & Regionalized 4 & LANL & INEL & 2 & 33,983 & 37 & $9.14 \mathrm{E}-12$ \\
\hline & (severity & Dichloroethane & Centralized & Pantex & Hanford & 1 & 25,119 & 22 & $3.42 \mathrm{E}-10$ \\
\hline & Category II) & Tetrachloroethene & Centralized & FEMP & Hanford & 3 & 39,356 & 123 & 8.52E-11 \\
\hline & & Benzene & Centralized & FEMP & Hanford & 3 & 39,356 & 313 & $2.28 \mathrm{E}-09$ \\
\hline & Particulate & Dichloromethane & Regionalized 4 & LANL & INEL & 2 & 33,983 & 37 & $9.14 \mathrm{E}-11$ \\
\hline & (severity & Dichloroethane & Centralized & Pantex & Hanford & 1 & 25,119 & 22 & 3.42E-09 \\
\hline & Category III) & Tetrachloroethene & Centralized & FEMP & Hanford & 3 & 39,356 & 123 & 8.52E-10 \\
\hline & & Benzene & Centralized & FEMP & Hanford & 3 & 39,356 & 313 & $2.28 \mathrm{E}-08$ \\
\hline & Particulate & Dichloromethane & Regionalized 4 & LANL & INEL, & 2 & 33,983 & 37 & $9.14 \mathrm{E}-10$ \\
\hline & (severity & Dichloroethane & Centralized & Pantex & Hanford & 1 & 25,119 & 22 & $3.42 \mathrm{E}-08$ \\
\hline & Categories & Tetrachloroethene & Centralized & FEMP & Hanford & 3 & $39, \mathbf{a 5 6}$ & 123 & 8.52E- 09 \\
\hline & IV-VIII) & Benzene & Centralized & FEMP & Hanford & 3 & 39,366 & 313 & $2.28 \mathrm{E}-07$ \\
\hline
\end{tabular}

a FEMP $=$ Fernald Environmental Management Project; INEL $=$ Idaho National Engineering Laboratory; LANL $=$ Los Alamos National Laboratory; ORR $=$ Oak Ridge Reservation;

Pantex = Pantex Plant; PORTS $=$ Portsmouth Gaseous Diffusion Plant; and SRS = Savannah River Site. 
TABLE 5.15 MEI Hazard Quotients for PAE Risk by LLMW Alternative

\begin{tabular}{|c|c|c|c|c|c|c|c|c|c|}
\hline $\begin{array}{l}\text { Transportation } \\
\text { Mode }\end{array}$ & $\begin{array}{l}\text { Release } \\
\text { Modo }\end{array}$ & Chemical Name & Alternative & $\begin{array}{l}\text { Origin } \\
\text { Site }^{\mathrm{a}}\end{array}$ & $\begin{array}{c}\text { Treatment } \\
\text { Site }^{\mathrm{a}}\end{array}$ & $\begin{array}{c}\text { Annual } \\
\text { No. of } \\
\text { Shipments }\end{array}$ & $\begin{array}{c}\text { Total } \\
\text { Mass per } \\
\text { Shipment } \\
\text { (kg) }\end{array}$ & $\begin{array}{c}\text { Chemical } \\
\text { Mass per } \\
\text { Shipment } \\
\text { (kg) }\end{array}$ & $\begin{array}{l}\text { Carcinoger } \\
\text { MEI Risk }\end{array}$ \\
\hline \multirow[t]{20}{*}{ Highway } & Liquid & Dichloromethane & Centralized & SRS & Hanford & 33 & 17,620 & 24 & $1.68 \mathrm{E}-01$ \\
\hline & aerosol & 1,1,1-Trichloroethane & Centralized & SRS & Hanford & 33 & 17,620 & 1,384 & $3.49 \mathrm{E}-01$ \\
\hline & (direct) & Freon 113 & Centralized & LANL & Hanford & 4 & 16,496 & 3 & $1.15 \mathrm{E}-01$ \\
\hline & & Toluene & Regionalized 4 & PORTS & ORR & 3 & 15,929 & 4,803 & $2.42 \mathrm{E}+02$ \\
\hline & Spoils pile & Dichloromethane & Regionalized 4 & LANL & INEL & 4 & 16,992 & 18 & 3.22E- 05 \\
\hline & vapor & 1,1,1-Trichloroethane & Centralized & INEL & Hanford & 4 & 15,240 & 87 & $1.21 \mathrm{E}-03$ \\
\hline & (Superfund) & Freon 113 & Centralized & Pantex & Hanford & 2 & 12,559 & 8 & $7.56 \mathrm{E}-07$ \\
\hline & & Toluene & Centralized & INEL & Hanford & 4 & 15,240 & 137 & $3.99 \mathrm{E}-04$ \\
\hline & Particulate & Dichloromethane & Regionalized 4 & LANL & INEL & 4 & 16,992 & 18 & $3.27 \mathrm{E}-05$ \\
\hline & (severity & 1,1,1-Trichloroethane & Centralized & INEL & Hanford & 4 & 15,240 & 87 & $8.95 \mathrm{E}-05$ \\
\hline & Category II) & Freon 113 & Centralized & Pantex & Hanford & 2 & 12,559 & 8 & $1.37 \mathrm{E}-05$ \\
\hline & & Toluene & Centralized & INEL & Hanford & 4 & 15,240 & 137 & $2.33 \mathrm{E}-03$ \\
\hline & Particulate & Dichloromethane & Regionalized 4 & LANL & INEL & 4 & 16,992 & 18 & $3.27 \mathrm{E}-04$ \\
\hline & (severity & 1,1,1-Trichloroethane & Centralized & INEL & Hanford & 4 & 15,240 & 87 & $8.95 \mathrm{E}-04$ \\
\hline & Category III) & Freon 113 & Centralized & Pantex & Hanford & 2 & 12,559 & 8 & $1.37 \mathrm{E}-04$ \\
\hline & & Toluene & Centralized & INEL & Hanford & 4 & 15,240 & 137 & 2.33E-02 \\
\hline & Particulate & Dichloromethane & Regionalized 4 & LANL. & INEL & 4 & 16,992 & 18 & $3.27 \mathrm{E}-03$ \\
\hline & (severity & 1,1,1-Trichloroethane & Centralized & INEL & Hunford & 4 & 15,240 & 87 & $8.95 \mathrm{E}-03$ \\
\hline & Categories & Freon 113 & Centralized & Pantex & Hanford & 2 & 12,559 & 8 & $1.37 \mathrm{E}-03$ \\
\hline & IV-VIII) & Toluene & Centralized & INEL & Hanford & 4 & 15,240 & 137 & $2.33 E-01$ \\
\hline \multirow[t]{20}{*}{ Railroad } & Liquid & Dichloromethane & Regionalized 4 & SRS & Hanford & 13 & 44,727 & 61 & $4.50 \mathrm{E}-01$ \\
\hline & serosol & 1,1,1-Trichloroethane & Centralized & SRS & Hanford & 13 & 44,727 & 3,512 & $5.72 \mathrm{E}+01$ \\
\hline & (direct) & Freon 113 & Centralized & LLNL & Hanford & 2 & 40,799 & 6 & $2.96 \mathrm{E}-03$ \\
\hline & & Toluene & Centralized & PORTS & ORR & 2 & 23,894 & 7,204 & $2.68 \mathrm{E}+02$ \\
\hline & Spoils pile & Dichloromethane & Regionalized 4 & LANL & INEL & 2 & 33,983 & 37 & $5.34 \mathrm{E}-05$ \\
\hline & vapor & 1,1,1-Trichloroethane & Centralized & Pantex & Hanford & 3 & 39,356 & 176 & $2.28 \mathrm{E}-04$ \\
\hline & (Superfund) & Freon 113 & Centralized & FEMP & Hanford & 1 & 25,119 & 16 & $1.12 \mathrm{E}-07$ \\
\hline & & Toluene & Centralized & FEMP & Hanford & 3 & 39,356 & 313 & $2.62 \mathrm{E}-04$ \\
\hline & Particulate & Dichloromethan $\theta$ & Regionalized 4 & LANL & INEL & 2 & 33,983 & 37 & $9.30 \mathrm{E}-05$ \\
\hline & (severity & 1,1,1-Trichloroethane & Centralized & Pantex & Hanford & 3 & 39,356 & 176 & $9.68 \mathrm{E}-04$ \\
\hline & Category II) & Freon 113 & Centralized & FEMP & Hanford & 1 & 25,119 & 16 & $3.04 \mathrm{E}-06$ \\
\hline & & Toluene & Centralized & EEMP & Hanford & 3 & 39,356 & 313 & $6.12 \mathrm{E}-0 \mathrm{~S}$ \\
\hline & Particulate & Dichloromethane & Regionalized 4 & LANL & INEL & 2 & 33,983 & 37 & $9.30 \mathrm{E}-04$ \\
\hline & (severity & 1,1,1-Trichloroethane & Centralized & Pantex & Hanford & 3 & 39,356 & 176 & $9.68 \mathrm{E}-03$ \\
\hline & Category III) & Freon 113 & Centralized & FEMP & Hanford & 1 & 25,119 & 16 & $3.04 \mathrm{E}-05$ \\
\hline & & Toluene & Centralized & FEMP & Hanford & 3 & 39,356 & 313 & $6.12 \mathrm{E}-02$ \\
\hline & Particulate & Dichloromethane & Regionalized 4 & LANL & INEL & 2 & 33,983 & 37 & $9.30 \mathrm{E}-03$ \\
\hline & (severity & 1,1,1-Trichloroethane & Centralized & Pantex & Hanford & 3 & 39,356 & 176 & $9.68 \mathrm{E}-02$ \\
\hline & Categories & Freon 113 & Centralized & FEMP & Hanford & 1 & 25,119 & 16 & $3.04 \mathrm{E}-04$ \\
\hline & (V-VIII) & Toluene & Centralized & FEMP & Hanford & 3 & 39,356 & 313 & $6.12 \mathrm{E}-01$ \\
\hline
\end{tabular}

a FEMP $=$ Fernald Environmental Management Project; INEL $=$ Idaho National Engineering Laboratory; LANL $=$ Los Alamos National Laboratory; LLNL $=$ Lawrence Livermor National Laboratory; ORR = Oak Ridge Reservation; Pantex = Pantex Plant; PORTS = Portsmouth Gaseous Diffusion Plant; and SRS = Savannah River Site. 
The most severe conditions are "worst-case" meteorologic conditions, along with chemical release quantities leading to the largest exposed population area. The degree of consequence imposed by the release quantities would depend on the chemical's inhalation toxicity and the release form (i.e., liquid aerosol, organic vapor, or respirable particle). The consequences from the most severe accidents associated with the shipment of LLMW are discussed subsequently. Note that the reported consequences are not based on the probability of an accident, but the assumption should be that the most severe accident has occurred. The numbers should therefore not be interpreted as risks but as the consequences (i.e., potential increased PIC or PAE incidence) of a "worst-case" release. These estimates should only be used to compare the consequences of a severe release under different meteorologic conditions.

The most severe highway chemical-release accidents are caused by shipments of liquid WM LLMW from the Portsmouth Gaseous Diffusion Plant (PORTS). The most severe railway chemical-release accidents are caused by shipments of liquid WM LLMW from PORTS and from Lawrence Livermore National Laboratory (LLNL). The most severe hazardous-chemical-release accidents are determined from the largest computed exposure areas of all WM shipments under neutral stability conditions. The estimated consequences for the most severe hazardous-chemical release from an accident involving LLMW shipments under both neutral and stable atmospheric conditions are presented in Table 5.16 for the WM alternatives. The largest plume exposure areas to population are less than $0.025 \mathrm{~km}^{2}$ $\left(0.01 \mathrm{mi}^{2}\right)$. Note that the national averaged population distribution statistics for urban, suburban, and rural zones are used for highway and railroad transportation routes. The population risks for urban zones are about 7 times greater than those for suburban zones and are about 200 times greater than those for rural zones. The population risks are generally 3 to 4 times greater for stable conditions than for neutral conditions. The highest hazardous-chemical concentrations at the location of maximum exposure $(30.5 \mathrm{~m}[100 \mathrm{ft}]$ downwind from the accident site) are $8.33 \mathrm{E}+03 \mathrm{mg} / \mathrm{m}^{3}$ or less. The MEI consequences under stable conditions are about 2 to 10 times greater than those under neutral conditions. Detailed chemical-specific MEI calculations are presented in Appendix B of this report (Table B.1 for PIC risk from chemical substances and Table B.2 for PAE risk from chemical substances).

The most severe radiological release accidents are caused by shipments of solid WM LLMW from PGDP (see discussion in Section 5.1.3). Respirable particulate emissions under severity Categories IV through VIII are likely to produce the greatest amount of hazardouschemical releases into the atmosphere. The most severe hazardous-chemical-release accidents are determined from the largest computed exposure areas of all WM shipments under neutral stability conditions. Specifically, these accidents are found to result from shipments of liquid WM LLMW from PORTS. Among the three different atmospheric release modes investigated, the greatest amounts of hazardous-chemical releases into the atmosphere are caused by liquid aerosol releases involving liquid WM LLMW. 
TABLE 5.16 Estimated Chemical Exposure Consequences for the Most Severe Accident Involving the Hazardous Component of WM LL.MW Shipments ${ }^{\mathrm{a}}$

\begin{tabular}{|c|c|c|c|c|c|c|c|c|}
\hline \multirow{3}{*}{$\begin{array}{l}\text { Transportation } \\
\text { Mode and } \\
\text { Population } \\
\text { Density Zone }\end{array}$} & \multicolumn{4}{|c|}{ Neutral Conditions ${ }^{\mathrm{c}}$} & \multicolumn{4}{|c|}{ Stable Conditions ${ }^{e}$} \\
\hline & \multicolumn{2}{|c|}{ Collective Population $^{\mathrm{b}}$} & \multicolumn{2}{|c|}{$\mathrm{MEI}^{\mathrm{d}}$} & \multicolumn{2}{|c|}{ Collective Population ${ }^{b}$} & \multicolumn{2}{|c|}{$\mathrm{MEI}^{\mathrm{d}}$} \\
\hline & $\begin{array}{c}\text { Maximum } \\
\text { Exposure } \\
\text { Area }\left(\mathrm{km}^{2}\right)\end{array}$ & Consequence & $\begin{array}{l}\text { Maximum } \\
\text { Concentration } \\
\left(\mathrm{mg} / \mathrm{m}^{3}\right)\end{array}$ & Consequence & $\begin{array}{c}\text { Maximum } \\
\text { Exposure } \\
\text { Area }\left(\mathrm{km}^{2}\right)\end{array}$ & Consequence & $\begin{array}{l}\text { Maximum } \\
\text { Concentration } \\
\left(\mathrm{mg} / \mathrm{m}^{3}\right)\end{array}$ & Consequence \\
\hline \multicolumn{9}{|c|}{ Potential for Increased Cancer Incidence of $>1 E-06$} \\
\hline \multicolumn{9}{|l|}{ Truck } \\
\hline Urban & $7.75 \mathrm{E}-03$ & $8.40 \mathrm{E}-02$ & $3.48 E+03$ & $4.74 \mathrm{E}-05$ & $2.39 \mathrm{E}-02$ & $2.60 \mathrm{E}-01$ & $8.21 \mathrm{E}+03$ & $1.12 \mathrm{E}-04$ \\
\hline Suburban & $7.75 \mathrm{E}-03$ & $1.30 \mathrm{E}-02$ & $3.48 \mathrm{E}+03$ & $4.74 \mathrm{E}-05$ & $2.39 \mathrm{E}-02$ & $4.10 \mathrm{E}-02$ & $8.21 E+03$ & $1.12 \mathrm{E}-04$ \\
\hline Rural & $7.75 \mathrm{E}-03$ & $4.40 \mathrm{E}-04$ & $3.48 \mathrm{E}+03$ & $4.74 \mathrm{E}-05$ & $2.39 \mathrm{E}-02$ & $1.40 \mathrm{E}-03$ & $8.21 \mathrm{E}+03$ & $1.12 \mathrm{E}-04$ \\
\hline \multicolumn{9}{|l|}{ Rail } \\
\hline Urban & $1.12 \mathrm{E}-02$ & $1.20 \mathrm{E}-01$ & $4.66 \mathrm{E}+03$ & $6.35 \mathrm{E}-05$ & $3.38 \mathrm{E}-02$ & $3.70 \mathrm{E}-01$ & $1.28 \mathrm{E}+04$ & $1.74 \mathrm{E}-04$ \\
\hline Suburban & $1.12 \mathrm{E}-02$ & $1.90 \mathrm{E}-02$ & $4.66 \mathrm{E}+03$ & $6.35 \mathrm{E}-05$ & $3.38 \mathrm{E}-02$ & $5.80 \mathrm{E}-02$ & $1.28 \mathrm{E}+04$ & $1.74 E-04$ \\
\hline Rura! & $1.12 \mathrm{E}-02$ & $6.40 \mathrm{E}-04$ & $4.66 \mathrm{E}+03$ & $6.35 \mathrm{E}-05$ & $3.38 \mathrm{E}-02$ & $1.90 \mathrm{E}-03$ & $1.28 \mathrm{E}+04$ & $1.74 E-04$ \\
\hline \multicolumn{9}{|c|}{ Hazard Quotients for Potential Adverse Health Effects } \\
\hline \multicolumn{9}{|l|}{ Truck } \\
\hline Urban & $3.07 \mathrm{E}-02$ & $3.30 \mathrm{E}-01$ & $3.48 \mathrm{E}+03$ & $2.04 \mathrm{E}+02$ & $1.39 \mathrm{E}-01$ & $1.50 \mathrm{E}+00$ & $8.33 \mathrm{E}+03$ & $4.86 \mathrm{E}+02$ \\
\hline Suburban & $3.07 \mathrm{E}-02$ & $5.30 \mathrm{E}-02$ & $3.48 \mathrm{E}+03$ & $2.04 \mathrm{E}+02$ & $1.39 \mathrm{E}-01$ & $2.40 \mathrm{E}-01$ & $8.33 E+03$ & $4.86 E+02$ \\
\hline Rural & $3.07 \mathrm{E}-02$ & $1.70 \mathrm{E}-03$ & $3.48 \mathrm{E}+03$ & $2.04 \mathrm{E}+02$ & $1.39 \mathrm{E}-01$ & $7.90 \mathrm{E}-03$ & $8.33 E+03$ & $4.86 \mathrm{E}+02$ \\
\hline \multicolumn{9}{|l|}{ Rail } \\
\hline Urban & $4.50 \mathrm{E}-02$ & $4.90 \mathrm{E}-01$ & $4.79 \mathrm{E}+03$ & $2.72 \mathrm{E}+02$ & $2.03 \mathrm{E}-01$ & $2.20 \mathrm{E}+00$ & $1.34 \mathrm{E}+04$ & $7.67 E+02$ \\
\hline Suburban & $4.50 \mathrm{E}-02$ & $7.70 \mathrm{E}-02$ & $4.79 E+03$ & $2.72 \mathrm{E}+02$ & $2.03 \mathrm{E}-01$ & $3.50 \mathrm{E}-01$ & $1.34 \mathrm{E}+04$ & $7.67 \mathrm{E}+02$ \\
\hline Rural & $4.50 \mathrm{E}-02$ & $2.60 \mathrm{E}-03$ & $4.79 \mathrm{E}+03$ & $2.72 \mathrm{E}+02$ & $2.03 \mathrm{E}-01$ & $1.20 \mathrm{E}-02$ & $1.34 E+04$ & $7.67 \mathrm{E}+02$ \\
\hline
\end{tabular}

a The most severe hazardous-chemical release accidents are caused by shipments of liquid WM LLMW originating from PORTS. Results are reported for the largest releases of liquid aerosols directly into the atmosphere following a transportation accident.

b Populations extend at a uniform population density to a radius of $80 \mathrm{~km}$ (50 mi) from the accident site.

c Neutral atmospheric conditions were based on Pasquill stability Class D with a wind speed of $4 \mathrm{~m} / \mathrm{s}(9 \mathrm{mph})$.

d The MEI is determined at the location of maximum exposure, which is assumed to be $30.5 \mathrm{~m}(100 \mathrm{ft})$ from the accident under both neutral and stable atmospheric conditions.

- Stable atmospheric conditions were based on Pasquill stability Class F with a wind speed of $1 \mathrm{~m} / \mathrm{s}(2.2 \mathrm{mph})$ 
The estimated consequences for the most severe radiological release accidents under both neutral and stable atmospheric conditions are presented in Table 5.17. The population consequences are found to be zero for all cases under both neutral and stable atmospheric conditions due to small plume footprints less than $30.5 \mathrm{~m}$ (100 ft) from the accident site. Because MEI consequences are determined at the location of maximum exposure $(30.5 \mathrm{~m}$ [100 ft] from the accident site), independent of population distributions, the estimated risks are the same for urban, suburban, and rural population zones. In general, the MEI consequences under stable conditions are about 3 to 10 times greater than those under neutral conditions. Detailed chemical-specific MEI calculations are presented in Appendix B of this report (Table B.3 for PIC risk from chemical substances and Table B.4 for any PAE risk from chemical substances). 
TABLE 5.17 Estimated Chemical Exposure Consequences Associated with the Most Severe Accident Involving the Radiological Component of WM LLMW Shipments ${ }^{\text {a }}$

\begin{tabular}{|c|c|c|c|c|c|c|c|c|}
\hline \multirow{3}{*}{$\begin{array}{l}\text { Transportation } \\
\text { Mode and } \\
\text { Population } \\
\text { Density Zone }\end{array}$} & \multicolumn{4}{|c|}{ Neutral Conditions ${ }^{c}$} & \multicolumn{4}{|c|}{ Stable Conditions $\mathbf{s}^{\mathbf{e}}$} \\
\hline & \multicolumn{2}{|c|}{ Collective Population ${ }^{b}$} & \multicolumn{2}{|c|}{ MEI $^{d}$} & \multicolumn{2}{|c|}{ Collective Population ${ }^{\mathrm{b}}$} & \multicolumn{2}{|c|}{$\mathrm{MEI}^{\mathrm{d}}$} \\
\hline & $\begin{array}{c}\text { Maximum } \\
\text { Exposure } \\
\text { Area }\left(\mathbf{k m}^{2}\right)\end{array}$ & Consequence & $\begin{array}{c}\text { Maximum } \\
\text { Concentration } \\
\left(\mathbf{m g} / \mathbf{m}^{3}\right)\end{array}$ & Consequence & $\begin{array}{c}\text { Maximum } \\
\text { Exposure } \\
\text { Area }\left(\mathrm{km}^{2}\right)\end{array}$ & Consequence & $\begin{array}{c}\text { Maximum } \\
\text { Concentration } \\
\left(\mathrm{mg} / \mathrm{m}^{3}\right)\end{array}$ & Consequence \\
\hline \multicolumn{9}{|c|}{ Potential for Increased Cancer Incidence of $>1 E-06$} \\
\hline \multicolumn{9}{|l|}{ Truck } \\
\hline Urban & $0.00 \mathrm{E}+00$ & $0.00 \mathrm{E}+00$ & $1,43 E+00$ & $1.78 \mathrm{E}-08$ & $0.00 \mathrm{E}+00$ & $0.00 E+00$ & $4.54 \mathrm{E}+00$ & $5.58 \mathrm{E}-08$ \\
\hline Suburban & $0.00 \mathrm{E}+00$ & $0.00 \mathrm{E}+00$ & $1.43 \mathrm{E}+00$ & $1.78 \mathrm{E}-08$ & $0.00 \mathrm{E}+00$ & $0.00 \mathrm{E}+00$ & $4.54 \mathrm{E}+00$ & $5.58 \mathrm{E}-08$ \\
\hline Rural & $0.00 \mathrm{E}+00$ & $0.00 \mathrm{E}+00$ & $1.43 \mathrm{E}+00$ & $1.78 \mathrm{E}-08$ & $0.00 \mathrm{E}+00$ & $0.00 \mathrm{E}+00$ & $4.54 \mathrm{E}+00$ & $5.58 \mathrm{E}-08$ \\
\hline \multicolumn{9}{|l|}{ Rail } \\
\hline Urban & $0.00 \mathrm{E}+00$ & $0.00 \mathrm{E}+00$ & $1.43 \mathrm{E}+00$ & $1.78 \mathrm{E}-08$ & $0.00 \mathrm{E}+00$ & $0.00 \mathrm{E}+00$ & $4.54 \mathrm{E}+00$ & $5.58 \mathrm{E}-08$ \\
\hline Suburban & $0.00 \mathrm{E}+00$ & $0.00 \mathrm{E}+00$ & $1.43 \mathrm{E}+00$ & $1.78 \mathrm{E}-08$ & $0.00 \mathrm{E}+00$ & $0.00 \mathrm{E}+00$ & $4.54 \mathrm{E}+00$ & $5.58 \mathrm{E}-08$ \\
\hline Rural & $0.00 \mathrm{E}+00$ & $0.00 \mathrm{E}+00$ & $1.43 \mathrm{E}+00$ & $1.78 \mathrm{E}-08$ & $0.00 \mathrm{E}+00$ & $0.00 \mathrm{E}+00$ & $4.54 \mathrm{E}+00$ & $5.58 \mathrm{E}-08$ \\
\hline \multicolumn{9}{|c|}{ Hazard Quotients for Potential Adverse Health Effects } \\
\hline \multicolumn{9}{|l|}{ Truck } \\
\hline Urban & $0.00 \mathrm{E}+00$ & $0.00 \mathrm{E}+00$ & $2.03 \mathrm{E}+00$ & $8.18 \mathrm{E}-02$ & $0.00 \mathrm{E}+00$ & $0.00 \mathrm{E}+00$ & $5.46 \mathrm{E}+00$ & $2.26 \mathrm{E}-01$ \\
\hline Suburban & $0.00 \mathrm{E}+00$ & $0.00 \mathrm{E}+00$ & $2.03 \mathrm{E}+00$ & $8.18 \mathrm{E}-02$ & $0.00 \mathrm{E}+00$ & $0.00 \mathrm{E}+00$ & $5.46 \mathrm{E}+00$ & $2.26 \mathrm{E}-01$ \\
\hline Rural & $0.00 \mathrm{E}+00$ & $0.00 \mathrm{E}+00$ & $2.03 \mathrm{E}+00$ & $8.18 \mathrm{E}-02$ & $0.00 \mathrm{E}+00$ & $0.00 \mathrm{E}+00$ & $5.46 \mathrm{E}+00$ & $2.26 \mathrm{E}-01$ \\
\hline \multicolumn{9}{|l|}{ Rail } \\
\hline Urban & $0.00 \mathrm{E}+00$ & $0.00 \mathrm{E}+00$ & $2.03 \mathrm{E}+00$ & $8.18 \mathrm{E}-02$ & $0.00 \mathrm{E}+00$ & $0.00 \mathrm{E}+00$ & $5.46 \mathrm{E}+00$ & $2.26 \mathrm{E}-01$ \\
\hline Suburban & $0.00 \mathrm{E}+00$ & $0.00 \mathrm{E}+00$ & $2.03 \mathrm{E}+00$ & $8.18 \mathrm{E}-02$ & $0.00 \mathrm{E}+00$ & $0.00 E+00$ & $5.46 \mathrm{E}+00$ & $2.26 \mathrm{E}-01$ \\
\hline Rural & $0.00 E+00$ & $0.00 \mathrm{E}+00$ & $2.03 \mathrm{E}+00$ & $8.18 \mathrm{E}-02$ & $0.00 \mathrm{E}+00$ & $0.00 E+00$ & $5.46 \mathrm{E}+00$ & $2.26 \mathrm{E}-01$ \\
\hline
\end{tabular}

a The most severe hazardous-chemical release accidents are caused by shipments of solid WM LLMW originating from PGDP (see Table 5.9). Results are reported for atmospheric releases of respirable particulate emissions under severity Categories IV through VIII.

b Populations extend at a uniform population density to a radius of $80 \mathrm{~km}$ ( $50 \mathrm{mi}$ ) from the accident site.

c Neutral atmospheric conditions were based on Pasquill stability Class D with a wind speed of $4 \mathrm{~m} / \mathrm{s}(9 \mathrm{mph})$

d The MEI is determined at the location of maximum exposure, which is assumed to be $30.5 \mathrm{~m}$ (100 ft) from the accident under both neutral and stable atmospheric conditions.

- Stable atmospheric conditions were based on Pasquill stability Class $F$ with a wind speed of $1 \mathrm{~m} / \mathrm{s}(2.2 \mathrm{mph})$ 


\section{REFERENCES}

DOE: See U.S. Department of Energy.

Doty, S.R., et al., 1976, A Climatological Analysis of Pasquill Stability Categories Based on "STAR" Summaries, National Oceanic and Atmospheric Administration, National Climatic Center, Asheville, N.C., April.

EPA: See U.S. Environmental Protection Agency.

Fischer, L.E., et al., 1987, Shipping Container Response to Severe Highway and Railway Accident Conditions, NUREG/CR-4829, UCID-20733, prepared by Lawrence Livermore National Laboratory, Livermore, Calif., for U.S. Nuclear Regulatory Commission, Washington, D.C.

ICRP: See International Commission on Radiological Protection.

International Commission on Radiological Protection, 1977, "Recommendations of the International Commission on Radiological Protection," ICRP publication 26, Annals of the ICRP 1(3), Pergamon Press, New York, N.Y.

International Commission on Radiological Protection, 1991, "1990 Recommendations of the International Commission on Radiological Protection," ICRP publication 60, Annals of the ICRP 21(1-3), Pergamon Press, New York, N.Y.

Johnson, P.E., et al., 1993a, HIGHWAY 3.1, An Enhanced Transportation Routing Model: Program Description, Methodology, and Revised User's Manual, ORNL/TM-12124, Oak Ridge National Laboratory, Oak Ridge, Tenn., March.

Johnson, P.E., et al., 1993b, INTERLINE 5.0, An Expanded Railroad Routing Model: Program Description, Methodology, and Revised User's Manual, ORNL/TM-12090, Oak Ridge National Laboratory, Oak Ridge, Tenn., March.

Kotek, T.J., et al., 1996, WASTE_MGMT: A Computer Model for Calculation of Waste Loads, Profiles, and Emissions, ANL/EAD/TM-30, Argonne National Laboratory, Argonne, Ill., Dec.

Lazaro, M.A., et al., 1996, Hazardous Waste Inventory, Characteristics, Generation, and Facility Assessment for Treatment, Storage, and Disposal Alternatives Considered in the U.S. Department of Energy Waste Management Programmatic Environmental Impact Statement, ANL/EAD/TM-25, Argonne National Laboratory, Argonne, Ill., Dec.

Monette, F.A., et al., 1996a, Supplemental Information Related to Risk Assessment for the Off-Site Transportation of Transuranic Waste for the U.S. Department of Energy Waste Management Programmatic Environmental Impact Statement, ANL/EAD/TM-27, Argonne National Laboratory, Argonne, Ill., Dec. 
Monette, F.A., et al., 1996b, Risk Assessment for the Off-Site Transportation of High-Level Waste for the U.S. Department of Energy Waste Management Programmatic Environmental Impact Statement, ANL/EAD/TM-21, Argonne National Laboratory, Argonne, Ill., Dec.

Monette, F.A., et al., 1996c, Supplemental Information Related to Risk Assessment for the Off-Site Transportation of Low-Level Waste for the U.S. Department of Energy Waste Management Programmatic Environmental Impact Statement, ANL/EAD/TM-23, Argonne National Laboratory, Argonne, Ill., Dec.

Neuhauser, K.S., and F.L. Kanipe, 1993, RADTRAN 4: Volume II. Technical Manual, SAND89-2370, Sandia National Laboratories, Albuquerque, N.M., Aug.

NRC: See U.S. Nuclear Regulatory Commission.

Oak Ridge National Laboratory, 1991, Integrated Data Base for 1991: U.S. Spent Fuel and Radioactive Waste Inventories, Projections, and Characteristics, DOE/RW-0006-Rev. 7, Oak Ridge, Tenn.

Rao, R.K., et al., 1982, Non-Radiological Impacts of Transporting Radioactive Material, SAND81-1703, TTC-0236, Sandia National Laboratories, Albuquerque, N.M.

Reynolds, R.M., 1992, ALOHA ${ }^{T M}$ (Areal Locations of Hazardous Atmospheres) 5.0: Theoretical Description, NOAA-TM NOS ORCA-65, National Oceanic and Atmospheric Administration, Seattle, Wash., Aug.

Saricks, C., and T. Kvitek, 1994, Longitudinal Review of State-Level Accident Statistics for Carriers of Interstate Freight, ANL/ESD/TM-68, Argonne National Laboratory, Argonne, Ill., Mar.

U.S. Department of Energy, 1986, Environmental Assessment: Yucca Mountain Site, Nevada Research and Development Area, Nevada, DOE/RW-0073, Office of Civilian Radioactive Waste Management, Washington, D.C., May.

U.S. Department of Energy, 1987a, Final Environmental Impact Statement: Disposal of Hanford Defense High-Level, Transuranic and Tank Wastes, DOE/EIS-0113, Washington, D.C., Dec.

U.S. Department of Energy, 1987b, Analysis of Radiation Doses from Operation of Postulated Commercial Spent Fuel Transportation Systems, DOE-CH/TPO-001, Pacific Northwest Laboratory, Richland, Wash., Nov.

U.S. Department of Energy, 1988a, External Dose Rate Conversion Factors for Calculation of Dose to the Public, DOE/EH-0070, Office of Environment, Safety, and Health, Washington, D.C.

U.S. Department of Energy, 1988b, Internal Dose Conversion Factors for Calculation of Dose to the Public, DOE/EH-0071, Office of Environment, Safety, and Health, Washington, D.C. 
U.S. Department of Energy, 1990a, Supplemental Environmental Impact Statement: Waste Isolation Pilot Plant, DOE/EIS-0026-FS, Washington, D.C., Jan.

U.S. Department of Energy, 1990b, "Radiation Protection of the Public and the Environment," DOE Order 5400.5.

U.S. Department of Energy, 1992, "Public Affairs Policy and Planning Requirements for Emergencies," DOE Order 5500.4A.

U. S. Department of Energy, 1994, Mixed Waste Inventory Report: Final Phase II Mixed Inventory Report Data, EM-352, Washington, D.C., May.

U.S. Department of Energy, 1996, Office of Waste Management Programmatic Environmental Impact Statement for Managing Treatment, Storage, and Disposal of Radioactive and Hazardous Waste, DOE/EIS-0200-F, Office of Waste Management, Washington, D.C.

U.S. Environmental Protection Agency, 1988, Superfund Exposure Assessment Manual, EPA/540/1-68/001, Office of Emergency and Remedial Response, Washington, D.C., April.

U.S. Environmental Protection Agency, 1989, Exposure Factor Handbook, EPA/600/8-89/043, Office of Health and Environmental Assessment, Washington, D.C., July.

U.S. Environmental Protection Agency, 1992, "Guidance of Risk Characterization for Risk Managers and Risk Assessors," memorandum from F. Henry Habicht II, Deputy Administrator to Assistant and Regional Administrators, Feb. 26.

U.S. Environmental Protection Agency, 1993a, Health Effects Assessment Summary Tables (HEAST), Annual FY 1993, OERR 9200.6-303 (92-1), Office of Emergency and Remedial Response, Washington, D.C., March.

U.S. Environmental Protection Agency, 1993b, Integrated Risk Information System (IRIS), database, Office of Emergency and Remedial Response, Washington, D.C. (accessed Oct. 1993).

U.S. Nuclear Regulatory Commission, 1977a, Final Environmental Statement on the Transportation of Radioactive Material by Air and Other Modes, NUREG-0170, Washington, D.C.

U.S. Nuclear Regulatory Commission, 1977b, Calculation of Annual Dose to Man from Routine Releases of Reactor Effluents for the Purpose of Evaluating Compliance with 10 CFR Part 50, Appendix I, Rev. 1, Regulatory Guide 1.109, Washington, D.C.

Wilkins, B.D., et al., 1996, Low-Level Mixed Waste Inventory, Characteristics, Generation, and Facility Assessment for Treatment, Storage, and Disposal Alternatives Considered in the U.S. Department of Energy Waste Management Programmatic Environmental Impact Statement, ANL/EAD/TM-32, Argonne National Laboratory, Argonne, Ml., Dec. 
Wilmot, E.L., 1981, Transportation Accident Scenarios for Commercial Spent Fuel, SAND80-2124, Sandia National Laboratories, Albuquerque, N.M.

Yuan, Y.C., et al., 1993, RISKIND: A Computer Program for Calculating Radiological Consequences and Health Risks from Transportation of Spent Nuclear Fuel, ANL/EAIS-6-Rev. 0, Argonne National Laboratory, Argonne, Ill., Feb. 
APPENDIX A:

TRANSPORTED MATERIAL SUMMARY FILES GENERATED BY THE WASTE_MGMT MODEL 
A-2

$\bullet$

$\bullet$

$\bullet$ 


\section{APPENDIX A:}

\section{TRANSPORTED MATERIAL SUMMARY FILES GENERATED BY THE WASTE_MGMT MODEL}

The WASTE_MGMT model has been developed at Argonne National Laboratory to support analyses of risks and costs for all alternatives for each waste type considered in the Waste Management Programmatic Environmental Impact Statement (WM PEIS) (Kotek et al. 1996). One output of the WASTE_MGMT model consists of the annual quantity, physical form, and radiological and chemical characteristics of the waste shipped between sites for each case. The WASTE_MGMT output files are used directly as input to the transportation risk assessment.

The WASTE_MGMT transportation summary files generated for the low-level mixed waste (LLMW) transportation assessment are presented in this appendix. For each of the seven WM alternatives, shipment summaries are presented separately for heterogeneous solids and organic liquids. The total quantity of shipments for a case can be found by summing the quantities for heterogeneous solids and organic liquids.

The WASTE_MGMT files report the quantity of waste shipped on an annual basis (cubic meters per year or kilograms per year). As stated previously, the WM PEIS considers a 20-year time frame for WM LLMW. For the Waste Management (WM) alternatives considered in the WM PEIS, the assumption was that shipments would occur uniformly over a 10-year period, which would allow an initial 10-year period to build treatment, storage, and disposal facilities. Sites would essentially store their own waste for the first 10 years and ship all waste over the subsequent 10 years.

Because of the differences in the time frames noted and the uncertainties in the actual timing of future waste shipments, the transportation impacts for the various cases are best compared by examining the total impacts of transporting a specific inventory of waste, independent of the annual shipping rates. The total amount of LLMW transported for each case can be calculated by multiplying the annual shipment quantities (reported in the WASTE_MGMT output files in this Appendix A) by 10 years for the WM alternatives. 


\section{A.1 WM LLMW DECENTRALIZED ALTERNATIVE (CASE 2a)}

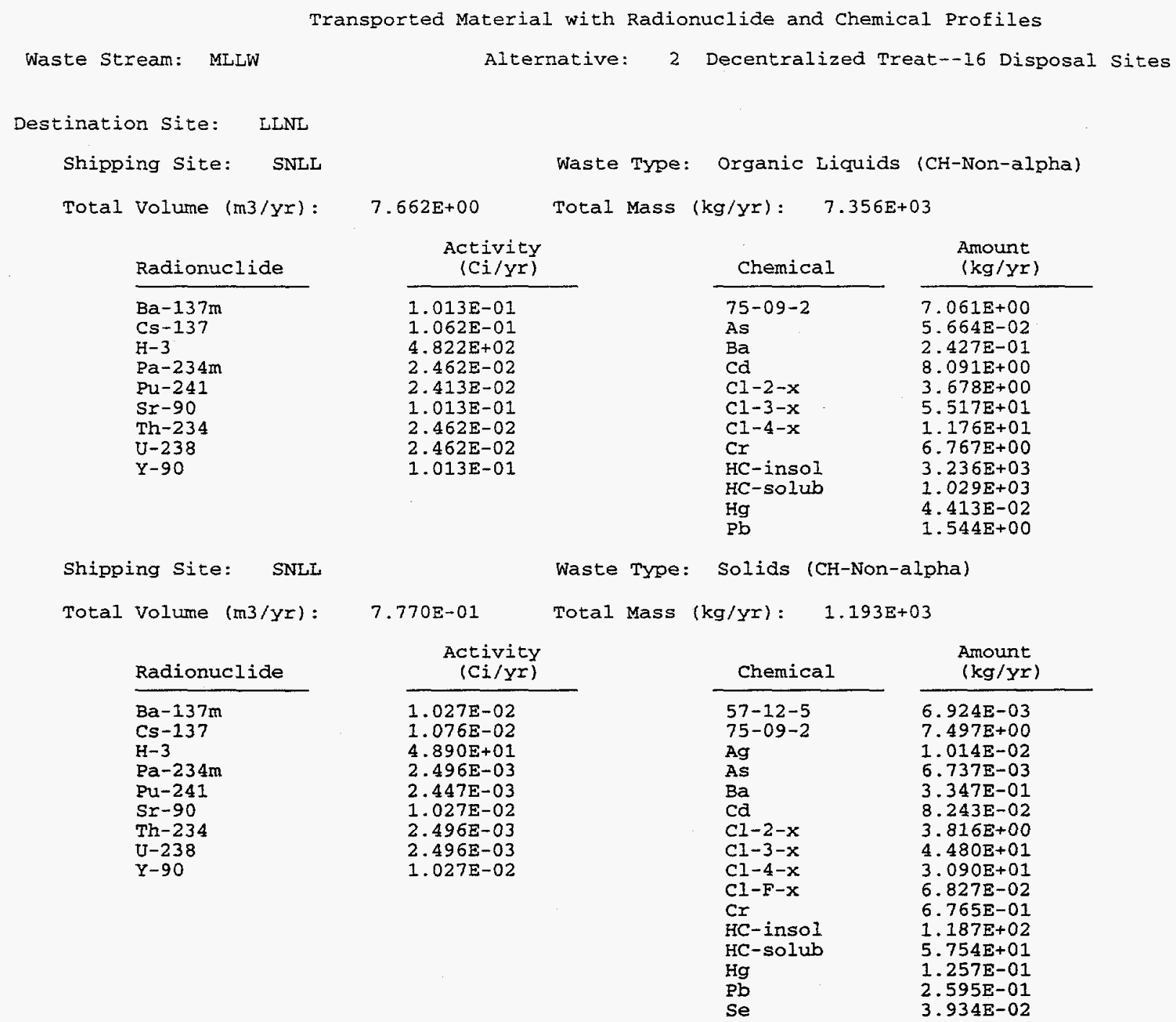


Destination Site: SRS

Shipping Site: BAPL Waste Type: Solids (CH-Alpha)

Total volume (m3/yr):

$8.357 E-01$

Total Mass (kg/yr): $3.493 \mathrm{E}+02$

Radionuclide

Am- 241

$\mathrm{Ba}-137 \mathrm{~m}$

Co-60

Cs -137

Eu-154

$\mathrm{H}-3$

$\mathrm{No}-94$

$\mathrm{Ni}-59$

$\mathrm{Ni}-63$

$\mathrm{Pa}-234 \mathrm{~m}$

Pu-238

$\mathrm{Pu}-239$

$\mathrm{Pu}-240$

Pu-241

$\mathrm{Sm}-151$

$\mathrm{Sr}-90$

Tc -99

Th-234

$\mathrm{U}-238$

$\mathrm{Y}-90$

\begin{tabular}{r}
$\begin{array}{r}\text { Activity } \\
\text { (Ci/yr) }\end{array}$ \\
\hline $6.984 \mathrm{E}-04$ \\
$7.629 \mathrm{E}-01$ \\
$1.021 \mathrm{E}-03$ \\
$8.160 \mathrm{E}-01$ \\
$1.217 \mathrm{E}-03$ \\
$6.053 \mathrm{E}-02$ \\
$6.984 \mathrm{E}-05$ \\
$1.563 \mathrm{E}-03$ \\
$1.606 \mathrm{E}-01$ \\
$4.490 \mathrm{E}-04$ \\
$3.791 \mathrm{E}-02$ \\
$6.286 \mathrm{E}-04$ \\
$2.793 \mathrm{E}-04$ \\
$1.633 \mathrm{E}-02$ \\
$1.167 \mathrm{E}-02$ \\
$7.264 \mathrm{E}-01$ \\
$3.625 \mathrm{E}-04$ \\
$4.490 \mathrm{E}-04$ \\
$4.490 \mathrm{E}-04$ \\
$7.264 \mathrm{E}-01$
\end{tabular}

\begin{tabular}{|c|c|}
\hline Chemical & $\begin{array}{l}\text { Amount } \\
\left(\mathrm{kg} / \mathrm{yr}^{\prime}\right)\end{array}$ \\
\hline $75-09-2$ & $2.095 E-01$ \\
\hline $\mathrm{Ag}$ & 3. $493 \mathrm{E}-03$ \\
\hline $\mathrm{Ba}$ & $1.397 \mathrm{E}-01$ \\
\hline $\mathrm{cd}$ & $6.986 E-04$ \\
\hline CI-2-x & $2.445 \mathrm{E}-01$ \\
\hline$C l-3-x$ & $8.733 \mathrm{E}-01$ \\
\hline$C 1-4-x$ & $4.191 \mathrm{E}-01$ \\
\hline$C 1-F-x$ & $3.493 \mathrm{E}-0 \mathrm{I}$ \\
\hline $\mathrm{Cr}$ & $5.239 \mathrm{E}-03$ \\
\hline $\mathrm{HC}$-insol & $3.493 \mathrm{E}+00$ \\
\hline HC-solub & $1.746 \mathrm{E}-03$ \\
\hline $\mathrm{Hg}$ & $6.986 E-04$ \\
\hline $\mathrm{Pb}$ & 3. $493 \mathrm{E}-02$ \\
\hline se & $1.047 \mathrm{E}-03$ \\
\hline
\end{tabular}

Shipping Site: MOUND

Waste Type: Organic Liquids (CH-Alpha)

Total Volume $(\mathrm{m} 3 / \mathrm{yr})$ :

$1.768 \mathrm{E}+00$

Total Mass $(\mathrm{kg} / \mathrm{yr}): 2.190 \mathrm{E}+03$

Radionuclide

$\mathrm{Ba}-137 \mathrm{~m}$

Cs -137

$\mathrm{H}-3$

$\mathrm{Pa}-234 \mathrm{~m}$

Pu-238

$\mathrm{Pu}-241$

Sr -90

$\mathrm{Th}-234$

$\mathrm{U}-238$

$\mathrm{Y}-90$

\begin{tabular}{c}
$\begin{array}{c}\text { Activity } \\
\text { (Ci/Yr) }\end{array}$ \\
\hline $2.339 \mathrm{E}-02$ \\
$2.450 \mathrm{E}-02$ \\
$1.111 \mathrm{E}+02$ \\
$5.680 \mathrm{E}-03$ \\
$5.569 \mathrm{E}-03$ \\
$1.113 \mathrm{E}-01$ \\
$2.339 \mathrm{E}-02$ \\
$5.680 \mathrm{E}-03$ \\
$5.680 \mathrm{E}-03$ \\
$2.339 \mathrm{E}-02$
\end{tabular}

\begin{tabular}{l} 
Chemical \\
\hline $75-09-2$ \\
As \\
$\mathrm{Ba}$ \\
$\mathrm{Cd}$ \\
$\mathrm{Cl}-2-\mathrm{x}$ \\
$\mathrm{Cl}-3-\mathrm{x}$ \\
$\mathrm{Cl}-4-\mathrm{x}$ \\
$\mathrm{Cl}-\mathrm{F}-\mathrm{x}$ \\
$\mathrm{Cr}$ \\
$\mathrm{HC}-$ insol \\
$\mathrm{HC}-$ solub \\
$\mathrm{Hg}$ \\
$\mathrm{Pb}$
\end{tabular}

\begin{tabular}{c}
$\begin{array}{c}\text { Amount } \\
(\mathrm{kg} / \mathrm{Y} r)\end{array}$ \\
\hline $2.978 \mathrm{E}+00$ \\
$6.322 \mathrm{E}-03$ \\
$2.709 \mathrm{E}-02$ \\
$2.409 \mathrm{E}+00$ \\
$1.574 \mathrm{E}+00$ \\
$1.718 \mathrm{E}+02$ \\
$3.827 \mathrm{E}+01$ \\
$6.845 \mathrm{E}-02$ \\
$2.069 \mathrm{E}+00$ \\
$3.617 \mathrm{E}+02$ \\
$1.237 \mathrm{E}+02$ \\
$1.355 \mathrm{E}-02$ \\
$4.599 \mathrm{E}-01$
\end{tabular}

Shipping Site: MOUND

Waste Type: Solids (CH-Alpha)

Total Volume $(\mathrm{m} 3 / \mathrm{yr})$ :

$5.712 E-03$

Total Mass $(\mathrm{kg} / \mathrm{yr})$ :

$6.176 E+00$

Radionuclide

$\mathrm{Ba}-137 \mathrm{~m}$

Cs -137

$\mathrm{H}-3$

$\mathrm{Pa}-234 \mathrm{~m}$

Pu-238

Pu-241

Sr -90

Th-234

$\mathrm{U}-238$

$\mathrm{Y}-90$

\begin{tabular}{c}
$\begin{array}{r}\text { Activity } \\
\text { (Ci/yr) }\end{array}$ \\
\hline $7.557 \mathrm{E}-05$ \\
$7.917 \mathrm{E}-05$ \\
$3.591 \mathrm{E}-01$ \\
$1.835 \mathrm{E}-05$ \\
$1.799 \mathrm{E}-05$ \\
$3.598 \mathrm{E}-04$ \\
$7.557 \mathrm{E}-05$ \\
$1.835 \mathrm{E}-05$ \\
$1.835 \mathrm{E}-05$ \\
$7.557 \mathrm{E}-05$
\end{tabular}

\begin{tabular}{l} 
Chemical \\
\hline $75-09-2$ \\
Ag \\
Ba \\
Cd \\
C1-2-x \\
Cl-3-x \\
CI-4-x \\
CI-F-x \\
Cr \\
HC-insol \\
HC-solub \\
Hg \\
Pb \\
Se
\end{tabular}

\begin{tabular}{c}
$\begin{array}{c}\text { Amount } \\
(\mathrm{kg} / \mathrm{y} r)\end{array}$ \\
\hline $1.926 \mathrm{E}-02$ \\
$1.191 \mathrm{E}-04$ \\
$2.561 \mathrm{E}-04$ \\
$2.102 \mathrm{E}-05$ \\
$9.886 \mathrm{E}-03$ \\
$9.601 \mathrm{E}-02$ \\
$7.627 \mathrm{E}-02$ \\
$6.403 \mathrm{E}-04$ \\
$4.674 \mathrm{E}-05$ \\
$2.423 \mathrm{E}-01$ \\
$1.132 \mathrm{E}-01$ \\
$1.951 \mathrm{E}-05$ \\
$9.361 \mathrm{E}-05$ \\
$1.921 \mathrm{E}-06$
\end{tabular}


Shipping site: UMC

Total Volume $(\mathrm{m} 3 / \mathrm{yr})$ :

Radionuclide

$\mathrm{Am}-241$

$\mathrm{Cm}-244$

$\mathrm{Pa}-234 \mathrm{~m}$

Pu-238

Pu-239

$\mathrm{Pu}-240$

Pu-241

Th-234

$\mathrm{U}-238$

Shipping Site: WVDP

Total Volume (m3/yr):

Radionuclide

\begin{tabular}{l}
\hline $\mathrm{Am}-241$ \\
$\mathrm{Ba}-137 \mathrm{~m}$ \\
$\mathrm{Co}-60$ \\
$\mathrm{Cs}-137$ \\
$\mathrm{Eu}-154$ \\
$\mathrm{H}-3$ \\
$\mathrm{Nb}-94$ \\
$\mathrm{Ni}-59$ \\
$\mathrm{Ni}-63$ \\
$\mathrm{~Pa}-234 \mathrm{~m}$ \\
$\mathrm{Pu}-238$ \\
$\mathrm{Pu}-239$ \\
$\mathrm{Pu}-240$ \\
$\mathrm{Pu}-241$ \\
$\mathrm{Sm}-151$ \\
$\mathrm{Sr}-90$ \\
$\mathrm{TC}-99$ \\
$\mathrm{Th}-234$ \\
$\mathrm{U}-238$ \\
$\mathrm{Y}-90$
\end{tabular}

Shipping site: WVDP

Total Volume $(\mathrm{m} 3 / \mathrm{yr})$ :

Radionuclide

\begin{tabular}{l}
\hline $\mathrm{Am}-241$ \\
$\mathrm{Ba}-137 \mathrm{~m}$ \\
$\mathrm{Co}-60$ \\
$\mathrm{Cs}-137$ \\
$\mathrm{Eu}-154$ \\
$\mathrm{H}-3$ \\
$\mathrm{Nb}-94$ \\
$\mathrm{Ni}-59$ \\
$\mathrm{Ni}-63$ \\
$\mathrm{~Pa}-234 \mathrm{~m}$ \\
$\mathrm{Pu}-238$ \\
$\mathrm{Pu}-239$ \\
$\mathrm{Pu}-240$ \\
$\mathrm{Pu}-241$ \\
$\mathrm{Sm}-151$ \\
$\mathrm{Sr}-90$ \\
$\mathrm{~T} C-99$ \\
$\mathrm{Th}-234$ \\
$\mathrm{U}-238$ \\
$\mathrm{Y}-90$
\end{tabular}

Waste Type: Solids (CH-Alpha)

Total Mass $(\mathrm{kg} / \mathrm{yr}): 5.809 \mathrm{E}+01$

$3.944 E \div 02$

\begin{tabular}{l} 
Chemical \\
\hline $57-12-5$ \\
$75-09-2$ \\
$\mathrm{Ag}$ \\
$\mathrm{As}$ \\
$\mathrm{Ba}$ \\
$\mathrm{Cd}$ \\
$\mathrm{Cl}-2-\mathrm{x}$ \\
$\mathrm{Cl}-3-\mathrm{x}$ \\
$\mathrm{Cl}-4-\mathrm{x}$ \\
$\mathrm{C} 1-\mathrm{E}-\mathrm{x}$ \\
$\mathrm{Cr}$ \\
$\mathrm{HC}-$ insol \\
$\mathrm{HC}-$ solub \\
$\mathrm{Hg}$ \\
$\mathrm{Pb}$ \\
$\mathrm{Se}$
\end{tabular}

$$
\begin{aligned}
& \begin{array}{c}
\text { Amount } \\
(\mathrm{kg} / \mathrm{yr})
\end{array} \\
& \hline 9.876 \mathrm{E}-03 \\
& 1.161 \mathrm{E}-02 \\
& 1.742 \mathrm{E}-03 \\
& 5.809 \mathrm{E}-04 \\
& 1.161 \mathrm{E}-01 \\
& 2.904 \mathrm{E}-04 \\
& 1.161 \mathrm{E}-02 \\
& 3.253 \mathrm{E}-02 \\
& 2.033 \mathrm{E}-02 \\
& 5.809 \mathrm{E}-03 \\
& 1.161 \mathrm{E}-01 \\
& 5.809 \mathrm{E}-02 \\
& 2.904 \mathrm{E}-03 \\
& 5.809 \mathrm{E}-03 \\
& 2.904 \mathrm{E}-01 \\
& 1.742 \mathrm{E}-03
\end{aligned}
$$

$1.743 \mathrm{E}-07$

$2.358 \mathrm{E}-07$

$8.511 \mathrm{E}-07$

9.108E-05

$9.331 \mathrm{E}-06$

3. $260 \mathrm{E}-05$

$8.894 \mathrm{E}-04$

8.511E 0 ?

8.511E-07
Waste Type: Organic Liquids (CH-Alpha)

$1.773 E+00$

Total Mass $(\mathrm{kg} / \mathrm{yr})$ :

$1.897 \mathrm{E}+03$

\begin{tabular}{c}
$\begin{array}{c}\text { Activity } \\
(\mathrm{Ci} / \mathrm{Y})\end{array}$ \\
\hline $1.482 \mathrm{E}-03$ \\
$1.619 \mathrm{E}+00$ \\
$2.167 \mathrm{E}-03$ \\
$1.731 \mathrm{E}+00$ \\
$2.583 \mathrm{E}-03$ \\
$1.284 \mathrm{E}-01$ \\
$1.482 \mathrm{E}-04$ \\
$3.317 \mathrm{E}-03$ \\
$3.409 \mathrm{E}-01$ \\
$9.529 \mathrm{E}-04$ \\
$8.047 \mathrm{E}-02$ \\
$1.334 \mathrm{E}-03$ \\
$5.929 \mathrm{E}-04$ \\
$3.465 \mathrm{E}-02$ \\
$2.477 \mathrm{E}-02$ \\
$1.541 \mathrm{E}+00$ \\
$7.694 \mathrm{E}-04$ \\
$9.529 \mathrm{E}-04$ \\
$9.529 \mathrm{E}-04$ \\
$1.541 \mathrm{E}+00$
\end{tabular}

\begin{tabular}{l} 
Chemical \\
\hline $57-12-5$ \\
$75-09-2$ \\
Ag \\
As \\
Ba \\
Cd \\
CI-2-x \\
Cl-3-x \\
CI-4-x \\
Cl-F-x \\
Cr \\
HC-insol \\
HC-solub \\
Hg \\
Pb \\
Se
\end{tabular}

\begin{tabular}{c}
$\begin{array}{c}\text { Amount } \\
(\mathrm{kg} / \mathrm{Yr})\end{array}$ \\
\hline $1.600 \mathrm{E}-01$ \\
$2.840 \mathrm{E}-01$ \\
$7.866 \mathrm{E}-02$ \\
$9.986 \mathrm{E}-03$ \\
$6.783 \mathrm{E}-02$ \\
$1.250 \mathrm{E}-01$ \\
$4.212 \mathrm{E}-01$ \\
$2.491 \mathrm{E}+01$ \\
$3.373 \mathrm{E}+00$ \\
$2.744 \mathrm{E}-01$ \\
$2.914 \mathrm{E}-01$ \\
$1.686 \mathrm{E}+01$ \\
$3.228 \mathrm{E}+02$ \\
$4.473 \mathrm{E}-01$ \\
$9.175 \mathrm{E}-02$ \\
$6.881 \mathrm{E}-02$
\end{tabular}

Waste Type: Solids (CH-Alpha)

Total Mass (kg/yr): $1.355 \mathrm{E}+03$

\section{$8.473 E-01$}

\begin{tabular}{l} 
Chemical \\
\hline $57-12-5$ \\
$75-09-2$ \\
Ag \\
As \\
Ba \\
Cd \\
Cl-2-x \\
Cl-3-x \\
Cl-4-x \\
Cl-F-x \\
Cr \\
HC-insol \\
HC-solub \\
Hg \\
Pb \\
Se
\end{tabular}

Amount

1. $069 \mathrm{E}-03$

2. $958 \mathrm{E}-01$

1. $709 \mathrm{E}-02$

1. $081 \mathrm{E}-02$

3. $475 \mathrm{E}-01$

1. $558 \mathrm{E}-01$

2. $803 \mathrm{E}-01$

1. $573 \mathrm{E}+01$

2. $998 \mathrm{E}+00$

1. $176 \mathrm{E}-01$

5. $922 \mathrm{E}-01$

5. $230 \mathrm{E}+01$

2. $619 \mathrm{E}+01$

1. $760 \mathrm{E}-01$

2. $784 \mathrm{E}-01$
$4.680 \mathrm{E}-02$

$3.662 E-04$

$4.561 \mathrm{E}-04$

4. 561E-04

7. $339 \mathrm{E}-01$ 


\section{A.2 WM LLMW REGIONALIZED 1 ALTERNATIVE (CASE 4)}

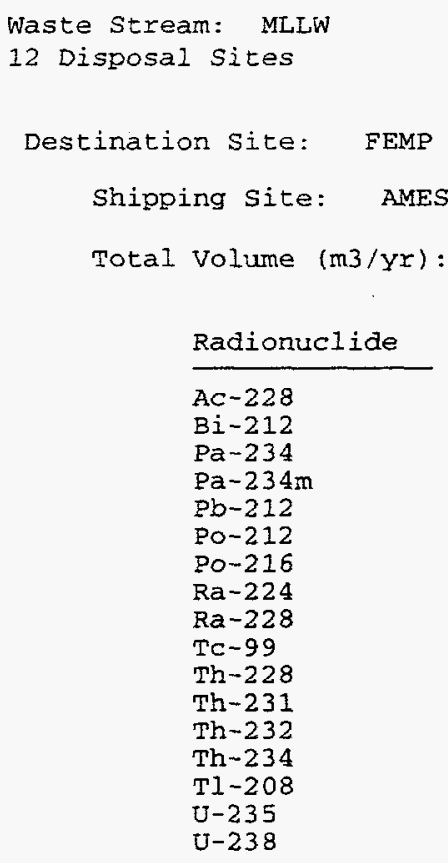

Alternative: 4 Regional 11 Treatment Sites -

Waste Type: Solids (CH-Non-alpha)

1.008E-01 Total Mass $(\mathrm{kg} / \mathrm{Yr}): 1.367 \mathrm{E}+02$

\begin{tabular}{c}
$\begin{array}{r}\text { ACtivity } \\
(C i / y r)\end{array}$ \\
\hline $2.165 E-07$ \\
$3.642 E-08$ \\
$2.752 E-08$ \\
$2.673 E-04$ \\
$3.642 E-08$ \\
$2.347 E-08$ \\
$3.642 E-08$ \\
$3.614 E-08$ \\
$2.148 E-07$ \\
$3.995 E-06$ \\
$3.642 E-08$ \\
$2.088 E-07$ \\
$2.198 E-06$ \\
$2.673 E-04$ \\
$1.376 E-08$ \\
$2.080 E-07$ \\
$2.673 E-04$
\end{tabular}

\begin{tabular}{l} 
Chemical \\
\hline $57-12-5$ \\
$75-09-2$ \\
Ag \\
As \\
Ba \\
Cd \\
Cl-2-x \\
Cl-3-x \\
Cl-4-x \\
C1-F-x \\
Cr \\
HC-insol \\
HC-solub \\
$\mathrm{Hg}$ \\
$\mathrm{Pb}$ \\
$\mathrm{Se}$
\end{tabular}

\begin{tabular}{c}
$\begin{array}{c}\text { Amount } \\
(\mathrm{kg} / \mathrm{Y} r)\end{array}$ \\
\hline $7.878 \mathrm{E}-05$ \\
$6.039 \mathrm{E}-04$ \\
$6.841 \mathrm{E}-04$ \\
$3.071 \mathrm{E}-06$ \\
$1.626 \mathrm{E}-04$ \\
$6.901 \mathrm{E}-04$ \\
$8.012 \mathrm{E}-04$ \\
$2.826 \mathrm{E}-03$ \\
$3.618 \mathrm{E}-03$ \\
$3.019 \mathrm{E}-04$ \\
$9.937 \mathrm{E}-04$ \\
$8.754 \mathrm{E}-05$ \\
$4.115 \mathrm{1}-02$ \\
$6.756 \mathrm{E}-04$ \\
$8.168 \mathrm{E}-04$ \\
$4.877 \mathrm{E}-06$
\end{tabular}

Waste Type: Solids (CH-Non-alpha)

4.838E+02 Total Mass $(\mathrm{kg} / \mathrm{yr}): \quad 8.170 \mathrm{E}+05$

\begin{tabular}{c}
$\begin{array}{c}\text { Activity } \\
(\mathrm{Ci} / \mathrm{y})\end{array}$ \\
\hline $2.380 \mathrm{E}-02$ \\
$3.504 \mathrm{E}+01$ \\
$1.304 \mathrm{E}+01$ \\
$3.747 \mathrm{E}+01$ \\
$1.388 \mathrm{E}-01$ \\
$4.761 \mathrm{E}-02$ \\
$2.699 \mathrm{E}+00$ \\
$9.919 \mathrm{E}-02$ \\
$2.104 \mathrm{E}+00$ \\
$2.214 \mathrm{E}+02$ \\
$4.796 \mathrm{E}+00$ \\
$1.474 \mathrm{E}+00$ \\
$2.380 \mathrm{E}-02$ \\
$3.571 \mathrm{E}-02$ \\
$1.561 \mathrm{E}+00$ \\
$4.519 \mathrm{E}-01$ \\
$3.313 \mathrm{E}+01$ \\
$3.963 \mathrm{E}-01$ \\
$3.967 \mathrm{E}-02$ \\
$4.796 \mathrm{E}+00$ \\
$4.796 \mathrm{E}+00$ \\
$3.313 \mathrm{E}+01$
\end{tabular}

\begin{tabular}{c}
$\begin{array}{c}\text { Amount } \\
(\mathrm{kg} / \mathrm{y} r)\end{array}$ \\
\hline $2.002 \mathrm{E}+01$ \\
$2.778 \mathrm{E}+01$ \\
$1.623 \mathrm{E}+01$ \\
$6.714 \mathrm{E}+00$ \\
$2.781 \mathrm{E}+02$ \\
$9.989 \mathrm{E}+00$ \\
$1.822 \mathrm{E}+01$ \\
$3.658 \mathrm{E}+02$ \\
$4.608 \mathrm{E}+01$ \\
$1.759 \mathrm{E}+01$ \\
$3.334 \mathrm{E}+02$ \\
$1.482 \mathrm{E}+03$ \\
$7.360 \mathrm{E}+02$ \\
$1.110 \mathrm{E}+02$ \\
$4.704 \mathrm{E}+02$ \\
$4.660 \mathrm{E}+01$
\end{tabular}


Destination site: HANF

\begin{tabular}{l} 
Shipping Site: PHNS \\
Total Volume (m3/yr): \\
Radionuclide \\
\hline Ba-137m \\
Co-60 \\
Cs-134 \\
Cs-137 \\
Eu-154 \\
Eu-155 \\
Fe-55 \\
H-3 \\
Mn-54 \\
Nb-94 \\
Ni-59 \\
Ni -63 \\
Pa-234m \\
Pm-147 \\
Pu-238 \\
Pu-241 \\
Sb-125 \\
Sm-151 \\
Sr -90 \\
Te-125m \\
Th-234 \\
U-238 \\
Y -90 \\
M
\end{tabular}

Shipping Site: PHNS
$1.736 \mathrm{E}-03$

Activity (Ci/Yr)

$1.152 \mathrm{E}-03$

$1.877 \mathrm{E}-02$

$1.103 \mathrm{E}-05$

$1.227 \mathrm{E}-03$

$1.493 E-05$

$7.141 \mathrm{E}-06$

$2.454 \mathrm{E}-03$

$5.346 \mathrm{E}-03$

$7.791 \mathrm{E}-06$

$1.168 \mathrm{E}-05$

$2.454 \mathrm{E}-04$

$3.299 \mathrm{E}-02$

4. $609 \mathrm{E}-05$

$1.337 \mathrm{E}-04$

$3.830 \mathrm{E}-05$

$3.116 \mathrm{E}-05$

$5.194 \mathrm{E}-06$

$1.038 \mathrm{E}-05$

$1.155 \mathrm{E}-03$

$1.298 \mathrm{E}-06$

$4.609 \mathrm{E}-05$

$4.609 \mathrm{E}-05$

$1.155 \mathrm{E}-03$

Waste Type: Organic Iiquids (CH-Non-alpha)

Total Mass (kg/yr): $1.979 \mathrm{E}+00$

\begin{tabular}{|c|c|}
\hline Chemical & $\begin{array}{l}\text { Amount } \\
(\mathrm{kg} / \mathrm{yr})\end{array}$ \\
\hline $75-09-2$ & $2.572 E-04$ \\
\hline $\mathrm{Ag}$ & $8.707 \mathrm{E}-05$ \\
\hline As & $2.176 E-05$ \\
\hline $\mathrm{Ba}$ & $1.484 \mathrm{E}-04$ \\
\hline Cl-2-x & $7.322 E-04$ \\
\hline$c 1-3-x$ & $2.73 \mathrm{IE}-02$ \\
\hline C $1-4-x$ & $1.543 E-03$ \\
\hline $\mathrm{Cl}-\mathrm{F}-\mathrm{x}$ & $5.937 E-04$ \\
\hline $\mathrm{HC}$-insol & $2.968 \mathrm{E}-03$ \\
\hline HC-solub & $7.718 \mathrm{E}-04$ \\
\hline $\mathrm{Hg}$ & $4.353 \mathrm{E}-04$ \\
\hline $\mathrm{Pb}$ & $5.343 E-05$ \\
\hline $\mathrm{Se}$ & $7.322 \mathrm{E}-05$ \\
\hline
\end{tabular}

Waste Type: Solids (CH-Non-alpha)

Total Volume $(\mathrm{m} 3 / \mathrm{yr})$ :

$2.962 \mathrm{E}-01$

Total Mass $(\mathrm{kg} / \mathrm{yr}): 4.406 \mathrm{E}+02$

Radionuclide

$\mathrm{Ba}-137 \mathrm{~m}$

Co- 60

Cs -134

Cs -137

Eu-154

Eu-155

Fe -55

Hi-3

$\mathrm{Mn}-54$

$\mathrm{Nb}-94$

$\mathrm{Ni}-59$

$\mathrm{Ni}-63$

Pa-234m

Pm-147

Pu-238

Pu-241

Sb-125

Sm-151

Sr-90

Te-125m

Th-234

$\mathrm{U}-238$

$\mathrm{Y}-90$

\begin{tabular}{c}
$\begin{array}{c}\text { Activity } \\
\text { (Ci/yr) }\end{array}$ \\
\hline $1.983 \mathrm{E}-01$ \\
$3.204 \mathrm{E}+00$ \\
$1.883 \mathrm{E}-03$ \\
$2.094 \mathrm{E}-01$ \\
$2.548 \mathrm{E}-03$ \\
$1.218 \mathrm{E}-03$ \\
$4.188 \mathrm{E}-01$ \\
$9.124 \mathrm{E}-01$ \\
$1.329 \mathrm{E}-03$ \\
$1.994 \mathrm{E}-03$ \\
$4.188 \mathrm{E}-02$ \\
$5.631 \mathrm{E}+00$ \\
$7.867 \mathrm{E}-03$ \\
$2.282 \mathrm{E}-02$ \\
$6.538 \mathrm{E}-03$ \\
$5.319 \mathrm{E}-03$ \\
$8.865 \mathrm{E}-04$ \\
$1.773 \mathrm{E}-03$ \\
$1.972 \mathrm{E}-01$ \\
$2.216 \mathrm{E}-04$ \\
$7.867 \mathrm{E}-03$ \\
$7.867 \mathrm{E}-03$ \\
$1.972 \mathrm{E}-01$
\end{tabular}

\begin{tabular}{l} 
Chemical \\
\hline $57-12-5$ \\
$75-09-2$ \\
$\mathrm{Ag}$ \\
$\mathrm{As}$ \\
$\mathrm{Ba}$ \\
$\mathrm{Cd}$ \\
$\mathrm{Cl}-2-\mathrm{x}$ \\
$\mathrm{Cl}-3-\mathrm{x}$ \\
$\mathrm{Cl}-4-\mathrm{x}$ \\
$\mathrm{Cl}-\mathrm{F}-\mathrm{x}$ \\
$\mathrm{Cr}$ \\
$\mathrm{HC}-$ insol \\
$\mathrm{HC}-$ solub \\
$\mathrm{Hg}$ \\
$\mathrm{Pb}$ \\
$\mathrm{Se}$
\end{tabular}

\begin{tabular}{c}
$\begin{array}{c}\text { Amount } \\
(\mathrm{kg} / \mathrm{yr})\end{array}$ \\
\hline $4.073 \mathrm{E}-03$ \\
$5.831 \mathrm{E}-02$ \\
$2.691 \mathrm{E}-03$ \\
$1.603 \mathrm{E}-03$ \\
$1.038 \mathrm{E}-01$ \\
$2.472 \mathrm{E}-02$ \\
$6.160 \mathrm{E}-02$ \\
$2.088 \mathrm{E}+00$ \\
$4.422 \mathrm{E}-01$ \\
$6.398 \mathrm{E}-02$ \\
$7.805 \mathrm{E}-01$ \\
$6.908 \mathrm{E}+00$ \\
$3.289 \mathrm{E}+00$ \\
$2.617 \mathrm{E}-02$ \\
$1.563 \mathrm{E}-01$ \\
$6.731 \mathrm{E}-03$
\end{tabular}


Shipping Site: PSNS

Total volume (m3/yr):

Radionuclide

$\mathrm{Ba}-137 \mathrm{~m}$

Co-60

Cs -134

Cs -137

Eu-154

Eu-155

$\mathrm{Fe}-55$

$\mathrm{H}-3$

$\mathrm{Mn}-54$

$\mathrm{Nb}-94$

$\mathrm{Ni}-59$

$\mathrm{Ni}-63$

$\mathrm{Pa}-234 \mathrm{~m}$

Pm-147

Pu-238

$\mathrm{Pu}-241$

$\mathrm{Sb}-125$

Sm-151

$\mathrm{S} r-90$

$\mathrm{Te}-125 \mathrm{~m}$

$\mathrm{Th}-234$

$\mathrm{U}-238$

$\mathrm{Y}-90$

Shipping site: PSNS Total Volume (m3/yr):

Radionuclide

$\mathrm{Ba}-137 \mathrm{~m}$

Co- 60

Cs-134

Cs -137

$\mathrm{Eu}-154$

Eu-155

$\mathrm{Fe}-55$

$\mathrm{FI}-3$

$\mathrm{Mn}-54$

$\mathrm{Nb}-94$

$\mathrm{Ni}-59$

$N i-63$

$\mathrm{Pa}-234 \mathrm{~m}$

Pm-147

$\mathrm{Pu}-238$

$\mathrm{Pu}-241$

Sb-125

$\mathrm{Sm}-151$

S $r-90$

Te-125m

Th-234

$\mathrm{U}-238$

$Y-90$
$3.990 \mathrm{E}-02$

Waste Type: Organic Licuids

(CH-Non-alpha)

Total Mass (kg/yr): $5.986 \mathrm{E}+01$

\begin{tabular}{c}
$\begin{array}{c}\text { Activity } \\
(C i / y r)\end{array}$ \\
\hline $2.671 \mathrm{E}-02$ \\
$4.315 \mathrm{E}-01$ \\
$2.537 \mathrm{E}-04$ \\
$2.820 \mathrm{E}-02$ \\
$3.432 \mathrm{E}-04$ \\
$1.641 \mathrm{E}-04$ \\
$5.641 \mathrm{E}-02$ \\
$1.228 \mathrm{E}-01$ \\
$1.791 \mathrm{E}-04$ \\
$2.686 \mathrm{E}-04$ \\
$5.641 \mathrm{E}-03$ \\
$7.585 \mathrm{E}-01$ \\
$1.059 \mathrm{E}-03$ \\
$3.074 \mathrm{E}-03$ \\
$8.806 \mathrm{E}-04$ \\
$7.164 \mathrm{E}-04$ \\
$1.194 \mathrm{E}-04$ \\
$2.388 \mathrm{E}-04$ \\
$2.656 \mathrm{E}-02$ \\
$2.985 \mathrm{E}-05$ \\
$1.059 \mathrm{E}-03$ \\
$1.059 \mathrm{E}-03$ \\
$2.656 \mathrm{E}-02$
\end{tabular}

\begin{tabular}{l} 
Chemical \\
\hline $75-09-2$ \\
Cd \\
$\mathrm{Cl}-2-\mathrm{x}$ \\
$\mathrm{Cl}-3-\mathrm{x}$ \\
$\mathrm{Cl}-4-\mathrm{x}$ \\
$\mathrm{Cl}-\mathrm{F}-\mathrm{x}$ \\
$\mathrm{Cr}$ \\
$\mathrm{HC}-$ insol \\
$\mathrm{HC}-$ Solub \\
$\mathrm{Hg}$ \\
$\mathrm{Pb}$
\end{tabular}

\begin{tabular}{c}
$\begin{array}{c}\text { Amount } \\
(\mathrm{kg} / \mathrm{Yr})\end{array}$ \\
\hline $9.577 \mathrm{E}-02$ \\
$6.584 \mathrm{E}-02$ \\
$5.088 \mathrm{E}-02$ \\
$7.243 \mathrm{E}+00$ \\
$1.616 \mathrm{E}+00$ \\
$2.993 \mathrm{E}-03$ \\
$5.746 \mathrm{E}-02$ \\
$1.975 \mathrm{E}-02$ \\
$3.831 \mathrm{E}-01$ \\
$3.771 \mathrm{E}-04$ \\
$1.257 \mathrm{E}-02$
\end{tabular}

Waste Type: Solids (CH-Non-alpha)

$6.586 \mathrm{E}+00$

Total Mass (kg/yr): $9.518 \mathrm{E}+03$

\begin{tabular}{c}
$\begin{array}{c}\text { ACtivity } \\
\text { (Ci } / y r)\end{array}$ \\
\hline $4.409 \mathrm{E}+00$ \\
$7.122 \mathrm{E}+01$ \\
$4.187 \mathrm{E}-02$ \\
$4.655 \mathrm{E}+00$ \\
$5.665 \mathrm{E}-02$ \\
$2.709 \mathrm{E}-02$ \\
$9.311 \mathrm{E}+00$ \\
$2.028 \mathrm{E}+01$ \\
$2.956 \mathrm{E}-02$ \\
$4.434 \mathrm{E}-02$ \\
$9.311 \mathrm{E}-01$ \\
$1.251 \mathrm{E}+02$ \\
$1.749 \mathrm{E}-01$ \\
$5.074 \mathrm{E}-01$ \\
$1.453 \mathrm{E}-01$ \\
$1.182 \mathrm{E}-01$ \\
$1.970 \mathrm{E}-02$ \\
$3.941 \mathrm{E}-02$ \\
$4.384 \mathrm{E}+00$ \\
$4.926 \mathrm{E}-03$ \\
$1.749 \mathrm{E}-01$ \\
$1.749 \mathrm{E}-01$ \\
$4.384 \mathrm{E}+00$
\end{tabular}

\begin{tabular}{c}
$\begin{array}{c}\text { Amount } \\
(\mathrm{kg} / Y r)\end{array}$ \\
\hline $7.312 \mathrm{E}-01$ \\
$1.279 \mathrm{E}+00$ \\
$1.357 \mathrm{E}-01$ \\
$4.301 \mathrm{E}-02$ \\
$8.869 \mathrm{E}+00$ \\
$1.229 \mathrm{E}-01$ \\
$1.346 \mathrm{E}+00$ \\
$4.520 \mathrm{E}+00$ \\
$2.989 \mathrm{E}+00$ \\
$1.385 \mathrm{E}+00$ \\
$2.225 \mathrm{E}+01$ \\
$6.852 \mathrm{E}+00$ \\
$2.684 \mathrm{E}-01$ \\
$4.633 \mathrm{E}-01$ \\
$2.158 \mathrm{E}+01$ \\
$1.310 \mathrm{E}-01$
\end{tabular}


Destination Site: INEL

Shipping site: NTS

Waste Type: Solids (CH-Non-alpha)

Total Volume (m3/yr):

$6.621 E-03$

Total Mass (kg/yr):

$8.886 E+00$

Radionuclide

Activity

$\mathrm{Ba}-137 \mathrm{~m}$

Cs -137

$\mathrm{H}-3$

$\mathrm{Pa}-234 \mathrm{~m}$

Pu-241

Sr -90

Th-234

$\mathrm{U}-238$

(Ci/yr)

$8.760 \mathrm{E}-05$

$9.177 \Xi-05$

4.167E-01

$2.127 \mathrm{E}-05$

$2.085 \mathrm{E}-05$

8.760E-05

$2.127 E-05$

$2.127 \mathrm{E}-05$

8. $760 \mathrm{E}-05$

\begin{tabular}{|c|c|}
\hline Chemical & $\begin{array}{l}\text { Amount } \\
(\mathrm{kg} / \mathrm{yr})\end{array}$ \\
\hline $75-09-2$ & $5.331 \Sigma-03$ \\
\hline $\mathrm{Ag}$ & $9.774 \mathrm{E}-05$ \\
\hline $\mathrm{cd}$ & $5.331 \mathrm{E}-04$ \\
\hline$C l-2-x$ & $5.331 E-03$ \\
\hline$c 1-3-x$ & $2.221 \mathrm{E}-02$ \\
\hline$C 1-4-x$ & $1.688 \mathrm{E}-01$ \\
\hline$C l-F-x$ & $2.665 E-03$ \\
\hline $\mathrm{Cr}$ & $1.777 \mathrm{E}-04$ \\
\hline $\mathrm{HC}$-insol & $4.443 \mathrm{E}-02$ \\
\hline HC-solub & $2.221 \mathrm{E}-02$ \\
\hline $\mathrm{Hg}$ & $3.465 E-04$ \\
\hline $\mathrm{Pb}$ & $3.199 \mathrm{E}-04$ \\
\hline $\mathrm{Se}$ & $8.886 E-06$ \\
\hline
\end{tabular}


Destination Site: LANL

\begin{tabular}{l} 
Shipping Site: ITRI \\
Total Volume (m3/Yr): \\
Radionuclide \\
\hline Ba-137m \\
Co-60 \\
Cs -137 \\
Eu-154 \\
Fe-55 \\
H -3 \\
Ni-59 \\
Ni-63 \\
Pm-147 \\
Pu-238 \\
Pu-241 \\
Sm-151 \\
Sr-90 \\
Y-90
\end{tabular}

Shipping Site: SNLA

Total Volume $(\mathrm{m} 3 / \mathrm{yr})$ :

Radionuclide

$\mathrm{Ba}-137 \mathrm{~m}$
$\mathrm{Co}-60$
$\mathrm{Cs}-137$
$\mathrm{Eu}-154$
$\mathrm{Eu}-155$
$\mathrm{Fe}-55$
$\mathrm{H}-3$
$\mathrm{Nb}-94$
$\mathrm{Ni}-59$
$\mathrm{Ni}-63$
$\mathrm{~Pa}-234 \mathrm{~m}$
$\mathrm{Pu}-238$
$\mathrm{Pu}-240$
$\mathrm{Pu}-241$
$\mathrm{Sm}-151$
$\mathrm{Sr}-90$
$\mathrm{TC}-99$
$\mathrm{Th}-234$
$\mathrm{U}-238$
$\mathrm{Y}-90$

Shipping site: SNLA

Total Volume (m3/Yr):

Radionuclide

$\mathrm{Ba}-137 \mathrm{~m}$
$\mathrm{Co}-60$
$\mathrm{Cs}-137$
$\mathrm{Eu}-154$
$\mathrm{Eu}-155$
$\mathrm{Fe}-55$
$\mathrm{H}-3$
$\mathrm{Nb}-94$
$\mathrm{Ni}-59$
$\mathrm{Ni}-63$
$\mathrm{~Pa}-234 \mathrm{~m}$
$\mathrm{Pu}-238$
$\mathrm{Pu}-240$
$\mathrm{Pu}-241$
$\mathrm{Sm}-151$
$\mathrm{Sr}-90$
$\mathrm{TC}-99$
$\mathrm{Th}-234$
$\mathrm{U}-238$
$\mathrm{Y}-90$

$1.028 \mathrm{E}-01$

Total Mass (kg/yr): $1.007 E+02$

\begin{tabular}{l} 
Chemical \\
\hline $57-12-5$ \\
$\mathrm{Ag}$ \\
$\mathrm{Cd}$ \\
$\mathrm{Cl}-3-\mathrm{x}$ \\
$\mathrm{C} 1-4-\mathrm{x}$ \\
$\mathrm{Cr}$ \\
$\mathrm{HC}-$ insol \\
$\mathrm{HC}-$ solub \\
$\mathrm{Hg}$ \\
$\mathrm{Pb}$ \\
$\mathrm{Se}$
\end{tabular}

\begin{tabular}{c}
$\begin{array}{c}\text { Amount } \\
(\mathrm{kg} / \mathrm{yr})\end{array}$ \\
\hline $1.813 \mathrm{E}-02$ \\
$4.433 \mathrm{E}-03$ \\
$4.937 \mathrm{E}-04$ \\
$8.061 \mathrm{E}-02$ \\
$4.534 \mathrm{E}-03$ \\
$2.116 \mathrm{E}-02$ \\
$1.108 \mathrm{E}+00$ \\
$3.627 \mathrm{E}+01$ \\
$2.821 \mathrm{E}-02$ \\
$5.038 \mathrm{E}-03$ \\
$4.030 \mathrm{E}-03$
\end{tabular}

Waste Type: Organic Liquids (CH-Non-alpha)

$5.487 \mathrm{E}-04$

Tatal Mass $(\mathrm{kg} / \mathrm{yr}): 5.793 \mathrm{E}-01$

\begin{tabular}{l} 
Chemical \\
\hline $57-12-5$ \\
$75-09-2$ \\
$\mathrm{Ag}$ \\
$\mathrm{As}$ \\
$\mathrm{Ba}$ \\
$\mathrm{Cd}$ \\
$\mathrm{Cl}-2-\mathrm{x}$ \\
$\mathrm{Cl}-3-\mathrm{x}$ \\
$\mathrm{Cl}-4-\mathrm{x}$ \\
$\mathrm{Cl}-\mathrm{F}-\mathrm{x}$ \\
$\mathrm{Cr}$ \\
$\mathrm{HC}-\mathrm{insO}$ \\
$\mathrm{HC}-\mathrm{SOI}$ I \\
$\mathrm{Hg}$ \\
$\mathrm{Pb}$ \\
$\mathrm{Se}$
\end{tabular}

\begin{tabular}{c}
$\begin{array}{c}\text { Amount } \\
(\mathrm{kg} / \mathrm{Yr})\end{array}$ \\
\hline $5.085 \mathrm{E}-05$ \\
$3.859 \mathrm{E}-05$ \\
$2.549 \mathrm{E}-05$ \\
$3.265 \mathrm{E}-06$ \\
$2.226 \mathrm{E}-05$ \\
$1.384 \mathrm{E}-06$ \\
$1.098 \mathrm{E}-04$ \\
$4.322 \mathrm{E}-03$ \\
$2.442 \mathrm{E}-04$ \\
$8.905 \mathrm{E}-05$ \\
$5.933 \mathrm{E}-05$ \\
$3.553 \mathrm{E}-03$ \\
$1.018 \mathrm{E}-01$ \\
$1.444 \mathrm{E}-04$ \\
$2.214 \mathrm{E}-05$ \\
$2.228 \mathrm{E}-05$
\end{tabular}

Waste Type: Solids (CH-Non-alpha)

Total Mass $(\mathrm{kg} / \mathrm{Yr}): 2.300 \mathrm{E}+03$
$2.849 \mathrm{E}+00$

\begin{tabular}{l} 
Chemica1 \\
\hline $57-12-5$ \\
$75-09-2$ \\
$\mathrm{Ag}$ \\
$\mathrm{AS}$ \\
$\mathrm{Ba}$ \\
$\mathrm{Cd}$ \\
$\mathrm{C} 1-2-\mathrm{x}$ \\
$\mathrm{C} 1-3-\mathrm{x}$ \\
$\mathrm{Cl}-4-\mathrm{x}$ \\
$\mathrm{C} 1-\mathrm{F}-\mathrm{x}$ \\
$\mathrm{Cr}$ \\
$\mathrm{HC}-\mathrm{insol}$ \\
$\mathrm{HC}-\mathrm{SO}$ Iub \\
$\mathrm{Hg}$ \\
$\mathrm{Pb}$ \\
$\mathrm{Se}$
\end{tabular}


Destination site: LINL

Shipping Site: GATOMIC

Waste Type: Organic Liquids (CH-Non-alpha)

Total Volume $(\mathrm{m} 3 / \mathrm{yr})$ :

$7.077 \mathrm{E}-02$

Total Mass $(\mathrm{kg} / \mathrm{yr})$ :

$7.476 \mathrm{E}+01$

Radionuclide

Ac- 228

$\mathrm{Bi}-212$

$\mathrm{Pa}-234$

$\mathrm{Pa}-234 \mathrm{~m}$

$\mathrm{Pb}-212$

Po-212

Po-216

$\mathrm{Ra}-224$

Ra-228

TC-99

Th-228

Th -231

Th-232

Th-234

T1-208

U-235

$\mathrm{U}-238$

Shipping site: GATOMIC

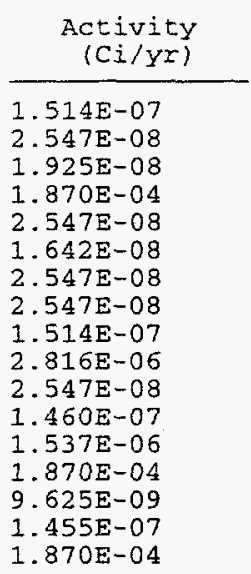

\begin{tabular}{l} 
Chemical \\
\hline $57-12-5$ \\
$75-09-2$ \\
Ag \\
As \\
Ba \\
Cd \\
Cl-2-x \\
Cl-3-x \\
Cl-4-x \\
Cl-F-x \\
Cr \\
HC-insol \\
HC-solub \\
Hg \\
Pb \\
Se
\end{tabular}

Waste Type: Solids (CH-Non-alpha)

Total Mass (kg/Yr): $8.419 \mathrm{E}+02$

$5.632 \mathrm{E}-01$

\begin{tabular}{l} 
Chemical \\
\hline $57-12-5$ \\
$75-09-2$ \\
$\mathrm{Ag}$ \\
$\mathrm{As}$ \\
$\mathrm{Ba}$ \\
$\mathrm{Cd}$ \\
$\mathrm{Cl}-2-\mathrm{x}$ \\
$\mathrm{Cl}-3-\mathrm{x}$ \\
$\mathrm{Cl}-4-\mathrm{x}$ \\
$\mathrm{CI}-\mathrm{F}-\mathrm{x}$ \\
$\mathrm{Cr}$ \\
$\mathrm{HC}-$ insol \\
$\mathrm{HC}-$ solub \\
$\mathrm{Hg}$ \\
$\mathrm{Pb}$ \\
Se
\end{tabular}

\begin{tabular}{c}
$\begin{array}{c}\text { Amount } \\
(\mathrm{kg} / \mathrm{yr})\end{array}$ \\
\hline $9.298 \mathrm{E}-02$ \\
$1.998 \mathrm{E}-01$ \\
$2.502 \mathrm{E}-02$ \\
$9.721 \mathrm{E}-03$ \\
$1.175 \mathrm{E}+00$ \\
$4.463 \mathrm{E}-02$ \\
$1.741 \mathrm{E}-01$ \\
$3.347 \mathrm{E}+00$ \\
$8.150 \mathrm{E}-01$ \\
$1.017 \mathrm{E}-01$ \\
$1.315 \mathrm{E}+00$ \\
$1.041 \mathrm{E}+01$ \\
$6.899 \mathrm{E}+00$ \\
$1.942 \mathrm{E}-01$ \\
$2.810 \mathrm{E}+00$ \\
$7.067 \mathrm{E}-02$
\end{tabular}

Waste Type: Organic Liquids (CH-Non-alpha)

Shipping Site: LBL

Total Volume $(\mathrm{m} 3 / \mathrm{yr})$ :

$3.230 E-01$

Total Mass $(\mathrm{kg} / \mathrm{yr}):$

$3.100 E+02$

Radionuclide

Ba-137m

Cs -137

$\mathrm{H}-3$

$\mathrm{Pa}-234 \mathrm{~m}$

Pu-241

Sr-90

Th-234

U-238

Y-90

\begin{tabular}{l} 
Chemical \\
\hline $75-09-2$ \\
As \\
$\mathrm{Ba}$ \\
$\mathrm{Cd}$ \\
$\mathrm{Cl}-2-\mathrm{x}$ \\
$\mathrm{Cl}-3-\mathrm{x}$ \\
$\mathrm{Cl}-4-\mathrm{x}$ \\
$\mathrm{Cr}$ \\
$\mathrm{HC}-$ insol \\
$\mathrm{HC}-501 \mathrm{~b}$ \\
$\mathrm{Hg}$ \\
$\mathrm{Pb}$
\end{tabular}

Amount

$6.543 E-03$

$5.261 E-03$

3. $280 \mathrm{E}-03$

$2.867 \mathrm{E}-03$

4. $175 \mathrm{E}-04$

1. $428 \mathrm{E}-02$

$3.523 \mathrm{E}-02$

$1.146 \mathrm{E}-02$

31E-01

. $311 \mathrm{E}+01$

1. $858 E-02$

.894E-0

\begin{tabular}{r}
$\begin{array}{r}\text { Activity } \\
\text { (Ci/yr) }\end{array}$ \\
\hline $1.218 E-06$ \\
$2.049 E-07$ \\
$1.548 E-07$ \\
$1.504 E-03$ \\
$2.049 E-07$ \\
$1.320 E-07$ \\
$2.049 E-07$ \\
$1.996 E-07$ \\
$1.186 E-06$ \\
$2.207 E-05$ \\
$2.049 E-07$ \\
$1.175 E-06$ \\
$1.237 E-05$ \\
$1.504 E-03$ \\
$7.742 E-08$ \\
$1.170 E-06$ \\
$1.504 E-03$
\end{tabular}




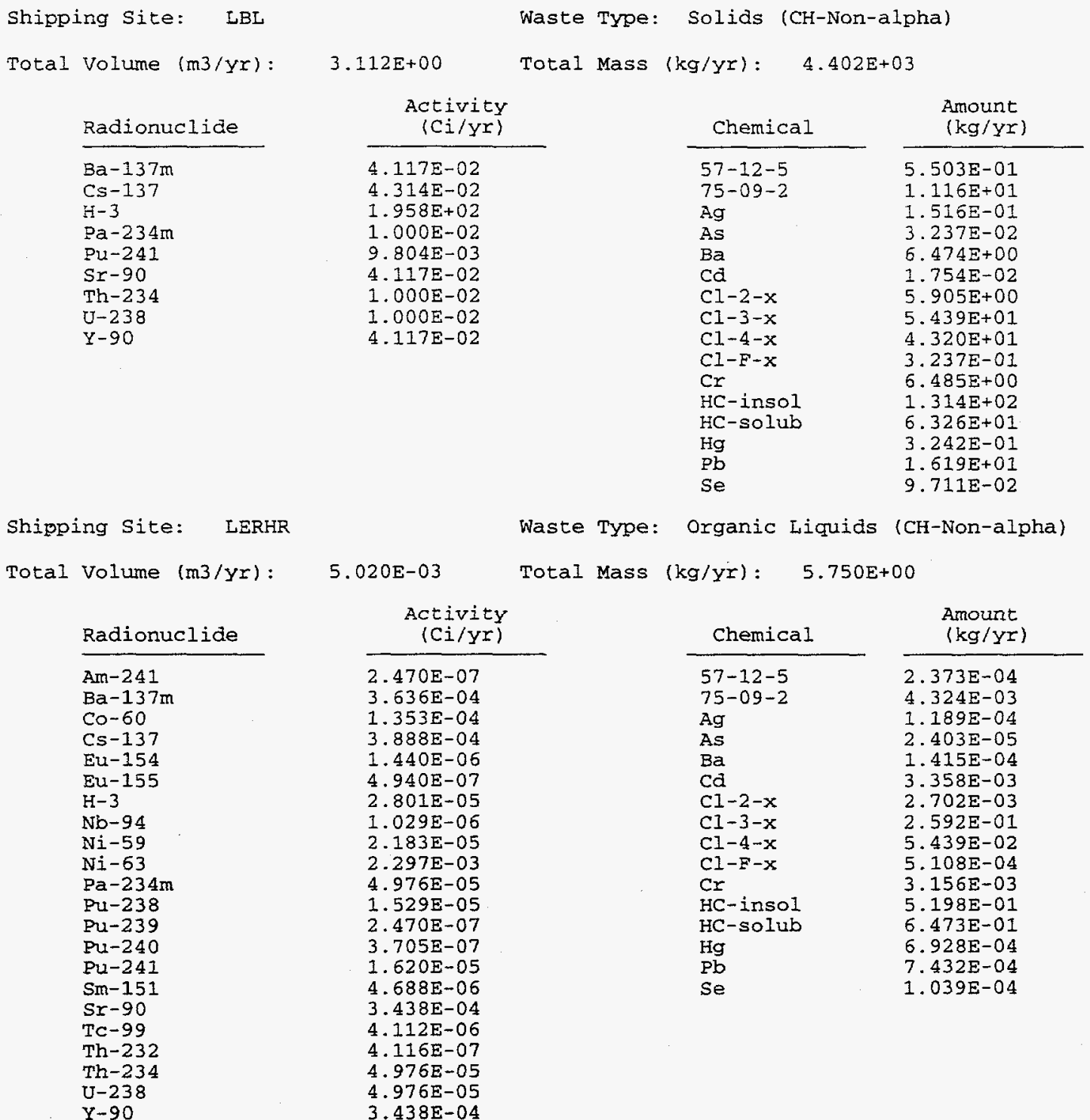

Shipping site: LERHR

Waste Type: Solids (CH-Non-alpha)

Total Volume $(\mathrm{m} 3 / \mathrm{yr})$ :

3. $442 \mathrm{E}-01$

Total Mass $(\mathrm{kg} / \mathrm{yr})$ :

$2.492 \mathrm{E}+02$

Radionuclide

Am- 241

$\mathrm{Ba}-137 \mathrm{~m}$

Co-60

$\mathrm{Cs}-137$

Eu-154

Eu-155

$\mathrm{H}-3$

$\mathrm{Nb}-94$

$\mathrm{Ni}-59$

$\mathrm{Ni}-63$

$\mathrm{Pa}-234 \mathrm{~m}$

Pu -238

Pu-239

Pu-240

Pu-24I

$\mathrm{Sm}-151$

Sr -90

Tc-99

Th-232

Th-234

$\mathrm{U}-238$

$Y-90$

\section{Activity \\ (Ci/Yr)}

1.701E-05

2. $505 E-02$

$9.323 \mathrm{E}-03$

$2.641 \mathrm{E}-02$

$9.928 \mathrm{E}-05$

3. $403 \mathrm{E}-05$

1. $930 \mathrm{E}-03$

7. $091 \mathrm{E}-05$

1. $504 \mathrm{E}-03$

1. $583 \mathrm{E}-01$

3. $428 \mathrm{E}-03$

1. $054 \mathrm{E}-03$

1. 701E-05

2. 552E-05

1. $116 \mathrm{E}-03$

3. $230 \mathrm{E}-04$

2. $335 \mathrm{E}-02$

$2.793 \mathrm{E}-04$

$2.836 E-05$

$3.428 \mathrm{E}-03$

$3.428 E-03$

$2.335 E-02$

\begin{tabular}{l} 
Chemical \\
\hline $57-12-5$ \\
$75-09-2$ \\
Ag \\
As \\
$\mathrm{Ba}$ \\
$\mathrm{Cd}$ \\
$\mathrm{Cl}-2-\mathrm{x}$ \\
$\mathrm{Cl}-3-\mathrm{x}$ \\
$\mathrm{Cl}-4-\mathrm{x}$ \\
$\mathrm{Cl}-\mathrm{F}-\mathrm{x}$ \\
$\mathrm{Cr}$ \\
$\mathrm{HC}-\mathrm{insol}$ \\
$\mathrm{HC}-\mathrm{solub}$ \\
$\mathrm{Hg}$ \\
$\mathrm{Pb}$ \\
$\mathrm{Se}$
\end{tabular}

\begin{tabular}{c}
$\begin{array}{c}\text { Amount } \\
(\mathrm{kg} / \mathrm{Yr})\end{array}$ \\
\hline $4.707 \mathrm{E}-04$ \\
$1.438 \mathrm{E}-01$ \\
$2.547 \mathrm{E}-03$ \\
$1.464 \mathrm{E}-05$ \\
$3.843 \mathrm{E}-02$ \\
$8.851 \mathrm{E}-03$ \\
$1.556 \mathrm{E}-01$ \\
$6.005 \mathrm{E}-01$ \\
$2.831 \mathrm{E}+00$ \\
$1.391 \mathrm{E}-01$ \\
$4.652 \mathrm{E}-03$ \\
$1.675 \mathrm{E}+00$ \\
$3.579 \mathrm{E}-01$ \\
$5.762 \mathrm{E}-03$ \\
$1.542 \mathrm{E}-02$ \\
$4.361 \mathrm{E}-04$
\end{tabular}


Shipping Site: MINS Waste Type: Solids (CH-Non-alpha)

Total Volume $(\mathrm{m} 3 / \mathrm{yr})$ :

$3.647 \mathrm{E}+00$

Total Mass $(\mathrm{kg} / \mathrm{yr}): \quad 3.545 \mathrm{E}+03$

Radionuclide

$\mathrm{Ba}-137 \mathrm{~m}$

Co- 60

Cs-134

Cs -137

Eu-154

Eu-155

$\mathrm{Fe}-55$

$\mathrm{H}-3$

$\mathrm{Mn}-54$

$\mathrm{Nb}-94$

$\mathrm{Ni}-59$

$\mathrm{Ni}-63$

$\mathrm{Pa}-234 \mathrm{~m}$

$\mathrm{Pm}-147$

Pu-238

$\mathrm{Pu}-241$

$\mathrm{Sb}-125$

Sm-151

$\mathrm{Sr}-90$

Te-125m

$\mathrm{Th}-234$

$\mathrm{U}-238$

$Y-90$

Shipping Site: SNLL

Total Volume $(\mathrm{m} 3 / \mathrm{yr})$ :

Radionuclide

$\mathrm{Ba}-137 \mathrm{~m}$

Cs-137

$\mathrm{H}-3$

$\mathrm{Pa}-234 \mathrm{~m}$

$\mathrm{Pu}-241$

Sr -90

Th-234

$\mathrm{U}-238$

$\mathrm{Y}-90$

Shipping Site: SNLL Total Volume $(\mathrm{m} 3 / \mathrm{yr})$ :

Radionuclide

$\mathrm{Ba}-137 \mathrm{~m}$

Cs -137

$\mathrm{H}-3$

$\mathrm{Pa}-234 \mathrm{~m}$

Pu-241

Sr -90

Th-234

$\mathrm{U}-238$

$Y-90$

\begin{tabular}{l}
$\begin{array}{c}\text { Activity } \\
(\text { Ci } / y x)\end{array}$ \\
\hline $2.441 E+00$ \\
$3.943 E+01$ \\
$2.318 E-02$ \\
$2.578 E+00$ \\
$3.137 E-02$ \\
$1.500 E-02$ \\
$5.156 E+00$ \\
$1.123 E+01$ \\
$1.636 E-02$ \\
$2.455 E-02$ \\
$5.156 E-01$ \\
$6.932 E+01$ \\
$9.684 E-02$ \\
$2.809 E-01$ \\
$8.047 E-02$ \\
$6.547 E-02$ \\
$1.091 E-02$ \\
$2.182 E-02$ \\
$2.427 E+00$ \\
$2.728 E-03$ \\
$9.684 E-02$ \\
$9.684 E-02$ \\
$2.427 E+00$
\end{tabular}

\begin{tabular}{l} 
Chemical \\
\hline $75-09-2$ \\
Ag \\
As \\
$\mathrm{Ba}$ \\
$\mathrm{Cd}$ \\
$\mathrm{Cl}-2-\mathrm{x}$ \\
$\mathrm{Cl}-3-\mathrm{x}$ \\
$\mathrm{CI}-4-\mathrm{x}$ \\
$\mathrm{Cl}-\mathrm{F}-\mathrm{x}$ \\
$\mathrm{Cr}$ \\
$\mathrm{HC}-\mathrm{insol}$ \\
$\mathrm{HC}-\mathrm{SO} \mathrm{ub}$ \\
$\mathrm{Hg}$ \\
$\mathrm{Pb}$ \\
$\mathrm{Se}$
\end{tabular}

Amount

$(\mathrm{kg} / \mathrm{Yr})$

$5.544 \mathrm{E}-01$

1. $457 \mathrm{E}-02$

7. $197 E-03$

2. $427 \mathrm{E}+00$

1. $150 \mathrm{E}-01$

5. $975 \mathrm{E}-01$

1. $190 \mathrm{E}+01$

2. $652 \mathrm{E}+00$

6. $891 \mathrm{E}-01$

7. $414 \mathrm{E}-01$

4. $047 \mathrm{E}+01$

1. $726 \mathrm{E}+01$

5. $165 \mathrm{E}-01$

4. $557 \mathrm{E}-01$

3. $325 E-02$

Waste Type: Organic Liquids (CH-Non-alpha)

$7.662 E+00$

Total Mass $(\mathrm{kg} / \mathrm{yr}): \quad 7.356 \mathrm{E}+03$

\begin{tabular}{c}
$\begin{array}{c}\text { Activity } \\
(\mathrm{Ci} / \mathrm{Yr})\end{array}$ \\
\hline $1.013 \mathrm{E}-01$ \\
$1.062 \mathrm{E}-01$ \\
$4.822 \mathrm{E}+02$ \\
$2.462 \mathrm{E}-02$ \\
$2.413 \mathrm{E}-02$ \\
$1.013 \mathrm{E}-01$ \\
$2.462 \mathrm{E}-02$ \\
$2.462 \mathrm{E}-02$ \\
$1.013 \mathrm{E}-01$
\end{tabular}

\begin{tabular}{l} 
Chemical \\
\hline $75-09-2$ \\
As \\
$\mathrm{Ba}$ \\
$\mathrm{Cd}$ \\
$\mathrm{Cl}-2-\mathrm{x}$ \\
$\mathrm{Cl}-3-\mathrm{x}$ \\
$\mathrm{Cl}-4-\mathrm{x}$ \\
$\mathrm{Cr}$ \\
$\mathrm{HC}-$ insol \\
$\mathrm{HC}-\mathrm{solub}$ \\
$\mathrm{Hg}$ \\
$\mathrm{Pb}$
\end{tabular}

\begin{tabular}{c}
$\begin{array}{c}\text { Amount } \\
(\mathrm{kg} / \mathrm{Yr})\end{array}$ \\
\hline $7.061 \mathrm{E}+00$ \\
$5.664 \mathrm{E}-02$ \\
$2.427 \mathrm{E}-01$ \\
$8.091 \mathrm{E}+00$ \\
$3.678 \mathrm{E}+00$ \\
$5.517 \mathrm{E}+01$ \\
$1.176 \mathrm{E}+01$ \\
$6.767 \mathrm{E}+00$ \\
$3.236 \mathrm{E}+03$ \\
$1.029 \mathrm{E}+03$ \\
$4.413 \mathrm{E}-02$ \\
$1.544 \mathrm{E}+00$
\end{tabular}

Waste Type: Solids (CH-Non-alpha)

$7.770 \mathrm{E}-01$

Total Mass $(\mathrm{kg} / \mathrm{yr})$ :

$1.193 E+03$

\begin{tabular}{c}
$\begin{array}{c}\text { Activity } \\
(\mathrm{Ci} / \mathrm{Y})\end{array}$ \\
\hline $1.027 \mathrm{E}-02$ \\
$1.076 \mathrm{E}-02$ \\
$4.890 \mathrm{E}+01$ \\
$2.496 \mathrm{E}-03$ \\
$2.447 \mathrm{E}-03$ \\
$1.027 \mathrm{E}-02$ \\
$2.496 \mathrm{E}-03$ \\
$2.496 \mathrm{E}-03$ \\
$1.027 \mathrm{E}-02$
\end{tabular}

\begin{tabular}{c}
$\begin{array}{c}\text { Amount } \\
(\mathrm{kg} / \mathrm{YI})\end{array}$ \\
\hline $6.924 \mathrm{E}-03$ \\
$7.497 \mathrm{E}+00$ \\
$1.014 \mathrm{E}-02$ \\
$6.737 \mathrm{E}-03$ \\
$3.347 \mathrm{E}-01$ \\
$8.243 \mathrm{E}-02$ \\
$3.816 \mathrm{E}+00$ \\
$4.480 \mathrm{E}+01$ \\
$3.090 \mathrm{E}+01$ \\
$6.827 \mathrm{E}-02$ \\
$6.765 \mathrm{E}-01$ \\
$1.187 \mathrm{E}+02$ \\
$5.754 \mathrm{E}+01$ \\
$1.257 \mathrm{E}-01$ \\
$2.595 \mathrm{E}-01$ \\
$3.934 \mathrm{E}-02$
\end{tabular}


Destination site: NTS

\begin{tabular}{lcc} 
Shipping Site: LLNL & & Wast \\
Total Volume (m3/yr): & $1.852 \mathrm{E}+00$ & Total \\
& $\begin{array}{c}\text { Activity } \\
\text { (Ci/yr) }\end{array}$ \\
Radionuclide & $\frac{2.681 \mathrm{E}-06}{6.052 \mathrm{E}-05}$ \\
\hline Ba-137m & $6.065 \mathrm{E}-01$ \\
$\mathrm{Cs}-137$ & $6.512 \mathrm{E}-07$ \\
$\mathrm{H}-3$ & $6.384 \mathrm{E}-07$ \\
$\mathrm{~Pa}-234 \mathrm{~m}$ & $1.276 \mathrm{E}-05$ \\
$\mathrm{Pu}-238$ & $5.777 \mathrm{E}-05$ \\
$\mathrm{Pu}-241$ & $6.512 \mathrm{E}-07$ \\
Sr-90 & $6.512 \mathrm{E}-07$ \\
Th-234 & $5.777 \mathrm{E}-05$ \\
$\mathrm{U}-238$ &
\end{tabular}

Waste Type: Organic Liquids ( $\mathrm{CH}-\mathrm{Alpha})$

Total Mass $(\mathrm{kg} / \mathrm{yr}): 1.852 \mathrm{E}+03$

\begin{tabular}{|c|c|}
\hline Chemical & $\begin{array}{l}\text { Amount } \\
(\mathrm{kg} / \mathrm{yr})\end{array}$ \\
\hline $57-12-5$ & $2.012 E-09$ \\
\hline $75-09-2$ & $6.870 \mathrm{E}-10$ \\
\hline $\mathrm{Ag}$ & $9.364 \mathrm{E}-06$ \\
\hline As & 1. $110 \mathrm{E}-06$ \\
\hline $\mathrm{Ba}$ & $7.574 \mathrm{E}-06$ \\
\hline $\mathrm{cd}$ & 5. $479 \mathrm{E}-07$ \\
\hline$C 1-2-x$ & $1.954 \mathrm{E}-09$ \\
\hline Cl-3-x & $7.751 \mathrm{E}-08$ \\
\hline$C 1-4-x$ & $4.380 E-09$ \\
\hline$C l-F-x$ & $1.584 \mathrm{E}-09$ \\
\hline $\mathrm{Cr}$ & $2.348 E-05$ \\
\hline HC-insol & $7.182 E-08$ \\
\hline HC-solub & $4.020 E-01$ \\
\hline $\mathrm{Hg}$ & $5.352 E-05$ \\
\hline $\mathrm{Pb}$ & $8.317 \Xi-06$ \\
\hline se & $8.209 E-06$ \\
\hline
\end{tabular}

Destination Site: PADUCAH

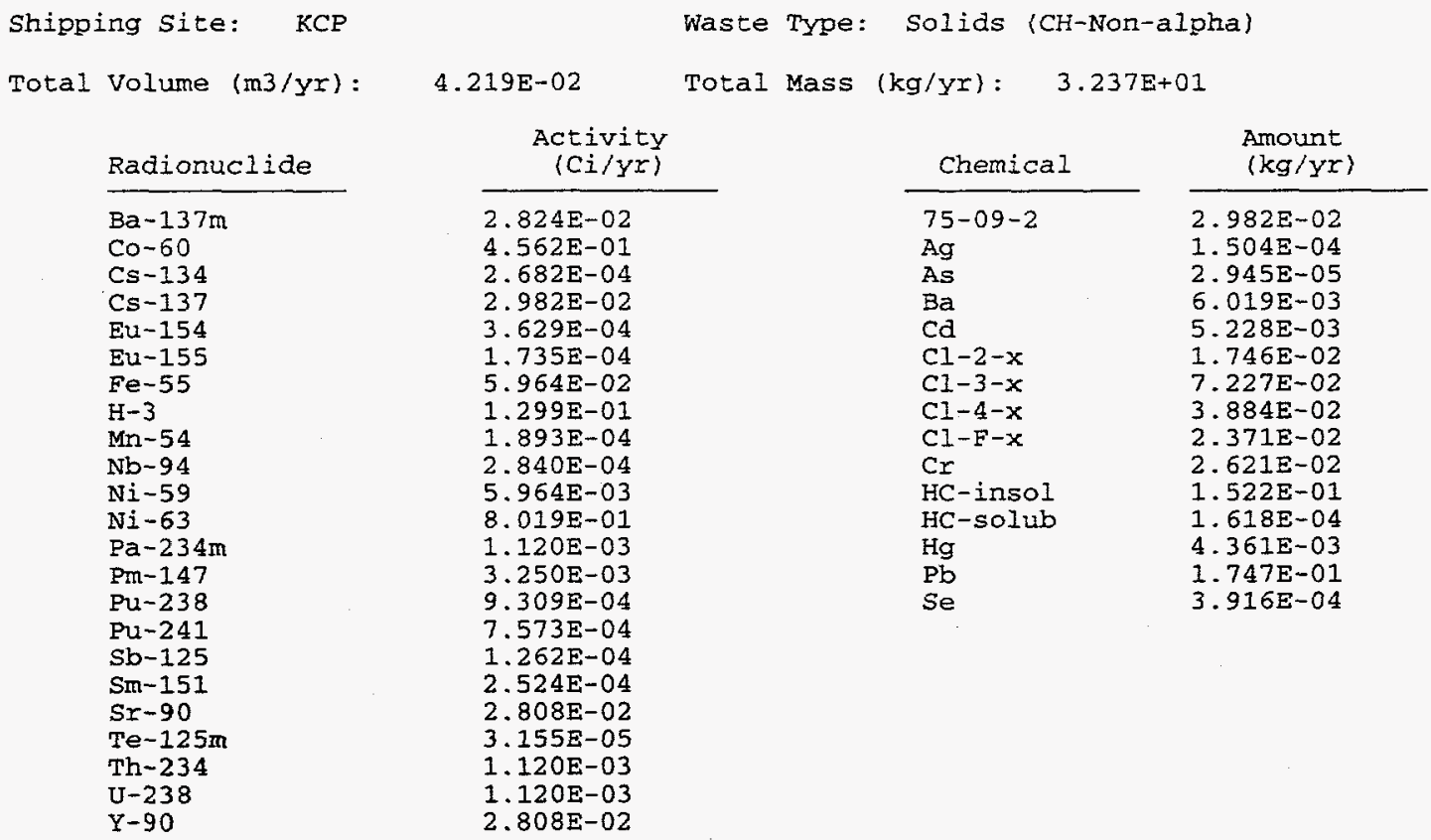


Destination Site: PORTS

Shipping site: BAPL Waste Type: Organic Liquids (CH-Non-alpha)

Total Volume $(\mathrm{m} 3 / \mathrm{yr})$ :

$6.410 \mathrm{E}-02$

Total Mass $(\mathrm{kg} / \mathrm{yr}): 6.163 \mathrm{E}+01$

Radionuclide

An-241

$\mathrm{Ba}-137 \mathrm{~m}$

Co-60

Cs -137

Eu1-154

H-3

$\mathrm{Nb}-94$

$\mathrm{Ni}-59$

Ni-63

$\mathrm{Pa}-234 \mathrm{~m}$

Pu-238

Pu-239

Pu-240

Pu-241

Sin-151

$\mathrm{Sr}-90$

TC -99

Th-234

$\mathrm{U}-238$

$\mathrm{Y}-90$

Shipping site: BAPL

Total Volume $(\mathrm{m} 3 / \mathrm{yr})$ :

Radionuclide

Am-241

$\mathrm{Ba}-137 \mathrm{~m}$

Co- 60

Cs -137

Eu-154

H-3

$\mathrm{Nb}-94$

$\mathrm{Ni}-59$

$\mathrm{Ni}-63$

$\mathrm{Pa}-234 \mathrm{~m}$

Pu-238

Pu-239

Pu-240

Pu-241

$\mathrm{Sm}-151$

Sr -90

Tc-99

Th-234

U-238

$\mathrm{Y}-90$

Shipping site:

BATELLE

\begin{tabular}{c}
$\begin{array}{c}\text { Activity } \\
\text { (Ci/yr) }\end{array}$ \\
\hline $5.332 E-05$ \\
$5.866 \mathrm{E}-02$ \\
$7.908 \mathrm{E}-05$ \\
$6.272 \mathrm{E}-02$ \\
$9.439 \mathrm{E}-05$ \\
$4.694 \mathrm{E}-03$ \\
$5.357 \mathrm{E}-06$ \\
$1.209 \mathrm{E}-04$ \\
$1.242 \mathrm{E}-02$ \\
$3.469 \mathrm{E}-05$ \\
$2.806 \mathrm{E}-03$ \\
$3.801 \mathrm{E}-05$ \\
$2.168 \mathrm{E}-05$ \\
$6.225 \mathrm{E}-04$ \\
$9.031 \mathrm{E}-04$ \\
$5.586 \mathrm{E}-02$ \\
$2.806 \mathrm{E}-05$ \\
$3.469 \mathrm{E}-05$ \\
$3.469 \mathrm{E}-05$ \\
$5.586 \mathrm{E}-02$
\end{tabular}

\begin{tabular}{l} 
Chemical \\
\hline $75-09-2$ \\
As \\
Ba \\
Cd \\
Cl-2-x \\
Cl-3-x \\
Cl-4-x \\
Cl-F-x \\
CI \\
HC-insol \\
HC-solub \\
Hg \\
$\mathrm{Pb}$
\end{tabular}

\begin{tabular}{c}
$\begin{array}{c}\text { Amount } \\
(\mathrm{kg} / \mathrm{yr})\end{array}$ \\
\hline $5.934 \mathrm{E}-02$ \\
$4.724 \mathrm{E}-04$ \\
$2.024 \mathrm{E}-03$ \\
$6.779 \mathrm{E}-02$ \\
$3.091 \mathrm{E}-02$ \\
$4.931 \mathrm{E}-01$ \\
$1.055 \mathrm{E}-01$ \\
$1.360 \mathrm{E}-05$ \\
$5.671 \mathrm{E}-02$ \\
$2.699 \mathrm{E}+01$ \\
$8.592 \mathrm{E}+00$ \\
$3.698 \mathrm{E}-04$ \\
$1.294 \mathrm{E}-02$
\end{tabular}

Waste Type: Solids (CH-Non-alpha)

Total Mass (kg/Yr): $6.010 \mathrm{E}+02$
$6.105 E-01$

\begin{tabular}{l} 
Chemical \\
\hline $57-12-5$ \\
$75-09-2$ \\
Ag \\
As \\
Ba \\
Cd \\
Cl-2-x \\
Cl-3-x \\
Cl-4-x \\
Cl-F-x \\
Cr \\
HC-insol \\
HC-solub \\
Hg \\
Pb \\
Se
\end{tabular}

\begin{tabular}{c}
$\begin{array}{c}\text { Amount } \\
(\mathrm{kg} / \mathrm{yr})\end{array}$ \\
\hline $1.703 \mathrm{E}-03$ \\
$6.266 \mathrm{E}-02$ \\
$6.515 \mathrm{E}-03$ \\
$1.598 \mathrm{E}-03$ \\
$6.259 \mathrm{E}-02$ \\
$1.698 \mathrm{E}-02$ \\
$7.067 \mathrm{E}-02$ \\
$4.679 \mathrm{E}-01$ \\
$4.156 \mathrm{E}-01$ \\
$8.727 \mathrm{E}-02$ \\
$4.102 \mathrm{E}-02$ \\
$2.697 \mathrm{E}+00$ \\
$2.666 \mathrm{E}+00$ \\
$1.514 \mathrm{E}-02$ \\
$7.746 \mathrm{E}-02$ \\
$2.393 \mathrm{E}-03$
\end{tabular}

\section{Activity
(Ci/yr)}

$5.078 \mathrm{E}-04$

5. $587 \mathrm{E}-01$

7. $532 \mathrm{E}-04$

5. $974 \mathrm{E}-01$

8. $990 \mathrm{E}-04$

$4.471 E-02$

5. 102E-05

1. $151 \mathrm{E}-03$

1. $183 \mathrm{E}-01$

3. $304 \mathrm{E}-04$

2. $672 \mathrm{E}-02$

3. $620 \mathrm{E}-04$

2. $065 \mathrm{E}-04$

5. $929 \mathrm{E}-03$

8. $601 \mathrm{E}-03$

5. $321 \mathrm{E}-01$

2. $672 \mathrm{E}-04$

3. $304 \mathrm{E}-04$

3. $304 \mathrm{E}-04$

5. 321E-01

Total Volume $(\mathrm{m} 3 / \mathrm{yr})$ :

$1.500 \mathrm{E}-03$

Waste Type: Solids (CH-Non-alpha)

Total Mass $(\mathrm{kg} / \mathrm{Yr}): 1.977 \mathrm{E}+00$

Radionuclide

Ac -228

$\mathrm{Bi}-212$

$\mathrm{Pa}-234$

$\mathrm{Pa}-234 \mathrm{~m}$

$\mathrm{Pb}-212$

PO-212

Po-216

$\mathrm{Ra}-224$

Ra-228

TC-99

Th-228

Th-231

Th-232

Th-234

TI -208

$\mathrm{U}-235$

$\mathrm{U}-238$

\begin{tabular}{c}
$\begin{array}{c}\text { Activity } \\
\text { (Ci/yr) }\end{array}$ \\
\hline $3.211 \mathrm{E}-09$ \\
$5.400 \mathrm{E}-10$ \\
$4.080 \mathrm{E}-10$ \\
$3.964 \mathrm{E}-06$ \\
$5.400 \mathrm{E}-10$ \\
$3.480 \mathrm{E}-10$ \\
$5.400 \mathrm{E}-10$ \\
$5.400 \mathrm{E}-10$ \\
$3.211 \mathrm{E}-09$ \\
$5.971 \mathrm{E}-08$ \\
$5.400 \mathrm{E}-10$ \\
$3.097 \mathrm{E}-09$ \\
$3.260 \mathrm{E}-08$ \\
$3.954 \mathrm{E}-06$ \\
$2.040 \mathrm{E}-10$ \\
$3.085 \mathrm{E}-09$ \\
$3.964 \mathrm{E}-06$
\end{tabular}

\begin{tabular}{l} 
Chemical \\
\hline $75-09-2$ \\
C1-2-x \\
C1-3-x \\
C1-4-x \\
HC-ins 01 \\
HC-solub
\end{tabular}

\begin{tabular}{c}
$\begin{array}{c}\text { Amount } \\
(\mathrm{kg} / \mathrm{Yr})\end{array}$ \\
\hline $3.955 \mathrm{E}-02$ \\
$1.977 \mathrm{E}-02$ \\
$1.977 \mathrm{E}-01$ \\
$1.582 \mathrm{E}-01$ \\
$4.943 \mathrm{E}-01$ \\
$2.373 \mathrm{E}-01$
\end{tabular}


Shipping Site: BNL

Total Volume $(\mathrm{m} 3 / \mathrm{yr})$ :

Radionuclide

Am-241

$\mathrm{Ba}-137 \mathrm{~m}$

Co-50

Cs -137

Eu-154

Eu-155

$\mathrm{H}-3$

$\mathrm{Nb}-94$

$\mathrm{Ni}-59$

$\mathrm{Ni}-53$

$\mathrm{Pa}-234 \mathrm{~m}$

$\mathrm{Pu}-238$

Pu-239

Pu-240

Pu-241

$\mathrm{Sm}-151$

$\mathrm{Sr}-90$

TC-99

Th -232

$\mathrm{Th}-234$

$\mathrm{U}-238$

$\mathrm{Y}-90$

Shipping Site: BNL

Total Volume (m3/yr):

Radionuclide

Am-241

$\mathrm{Ba}-137 \mathrm{~m}$

Co- 50

Cs -137

Eu-154

Eu-155

$\mathrm{H}-3$

$\mathrm{Nb}-94$

Ni-59

$\mathrm{Ni}-63$

Pa-234m

Pu-238

Pu-239

Pu-240

Pu-241

Sm-151

Sr -90

TC-99

Th-232

$\mathrm{Th}-234$

U-238

$\mathrm{Y}-90$

Shipping site: COLONIE

Total Volume (m3/yr):

Radionuclide

Ac -228

$\mathrm{Bi}-212$

$\mathrm{Pa}-234$

$\mathrm{Pa}-234 \mathrm{~m}$

$\mathrm{Pb}-212$

Po-212

PO-216

$\mathrm{Ra}-224$

$\mathrm{Ra}-228$

TC-99

Th-228

$\mathrm{Th}-231$

Th-232

Th-234

TI-208

$\mathrm{U}-235$

$\mathrm{U}-238$
1. $544 E+00$

Total Mass (kg/yr):

1. $749 \mathrm{E}+03$

\begin{tabular}{|c|c|}
\hline Chemical & $\begin{array}{l}\text { Amount } \\
\text { (kg/yr) }\end{array}$ \\
\hline $57-12-5$ & $8.298 E-02$ \\
\hline $75-09-2$ & 1. $156 \mathrm{E}+00$ \\
\hline $\mathrm{Ag}$ & $4.159 E-02$ \\
\hline AS & $7.649 \mathrm{E}-03$ \\
\hline $\mathrm{Ba}$ & $4.627 E-02$ \\
\hline $\mathrm{Cd}$ & $8.868 \mathrm{E}-01$ \\
\hline$C l-2-x$ & $7.572 \mathrm{E}-0 \mathrm{I}$ \\
\hline$C I-3-x$ & $7.014 E+01$ \\
\hline$C I-4-x$ & $1.445 \mathrm{E}+01$ \\
\hline$C I-F-x$ & $1.704 \mathrm{E}-01$ \\
\hline $\mathrm{Cr}$ & $8.567 \mathrm{E}-01$ \\
\hline $\mathrm{HC}$-insol & $1.386 \mathrm{E}+02$ \\
\hline $\mathrm{HC}$-solub & $2.115 \mathrm{E}+02$ \\
\hline $\mathrm{Hg}$ & $2.406 \mathrm{E}-01$ \\
\hline $\mathrm{Pb}$ & $2.050 z-01$ \\
\hline $\mathrm{Se}$ & $3.636 \mathrm{E}-02$ \\
\hline
\end{tabular}

Waste Type: Solids (CH-Non-alpha)

Total Mass (kg/yx): $1.190 \mathrm{E}+04$

\begin{tabular}{l} 
Chemical \\
\hline $57-12-5$ \\
$75-09-2$ \\
Ag \\
As \\
Ba \\
Cd \\
Cl-2-x \\
Cl-3-x \\
CI-4-x \\
Cl-F-x \\
Cr \\
HC-insol \\
HC-solub \\
Hg \\
Pb \\
Se
\end{tabular}

\begin{tabular}{c}
$\begin{array}{c}\text { Amount } \\
(\mathrm{kg} / \mathrm{Y})\end{array}$ \\
\hline $1.617 \mathrm{E}+00$ \\
$8.722 \mathrm{E}-01$ \\
$7.571 \mathrm{E}-01$ \\
$2.698 \mathrm{E}-01$ \\
$2.157 \mathrm{E}+01$ \\
$4.530 \mathrm{E}-01$ \\
$8.722 \mathrm{E}-01$ \\
$2.419 \mathrm{E}+01$ \\
$3.963 \mathrm{E}-01$ \\
$1.019 \mathrm{E}+00$ \\
$2.698 \mathrm{E}+01$ \\
$1.091 \mathrm{E}+02$ \\
$5.459 \mathrm{E}+01$ \\
$8.531 \mathrm{E}+00$ \\
$2.912 \mathrm{E}+01$ \\
$3.668 \mathrm{E}+00$
\end{tabular}

$8.327 \mathrm{E}-03$
$6.105 \mathrm{E}-01$

7. $303 \mathrm{E}-03$

7. $310 \mathrm{E}-04$

8. $837 \mathrm{E}-02$

$8.837 \mathrm{E}-02$

$6.105 \mathrm{E}-01$

Waste Type: Organic Liquids (CH-Non-alpha)

Total Mass (kg/yr): $1.026 \mathrm{E}+03$
$9.054 E-01$

\begin{tabular}{|c|c|}
\hline $\begin{array}{c}\text { Activity } \\
\text { (Ci/yr) }\end{array}$ & Chemical \\
\hline $\begin{array}{l}1.937 \mathrm{E}-06 \\
3.259 \mathrm{E}-07 \\
2.462 \mathrm{E}-07 \\
2.392 \mathrm{E}-03 \\
3.259 \mathrm{E}-07 \\
2.100 \mathrm{E}-07 \\
3.259 \mathrm{E}-07 \\
3.259 \mathrm{E}-07 \\
1.937 \mathrm{E}-06 \\
3.603 \mathrm{E}-05 \\
3.259 \mathrm{E}-07 \\
1.868 \mathrm{E}-06 \\
1.967 \mathrm{E}-05 \\
2.392 \mathrm{E}-03 \\
1.231 \mathrm{E}-07 \\
1.861 \mathrm{E}-06 \\
2.392 \mathrm{E}-03\end{array}$ & $\begin{array}{l}57-12-5 \\
75-09-2 \\
\mathrm{Ag} \\
\mathrm{As} \\
\mathrm{Ba} \\
\mathrm{Cd} \\
\mathrm{Cl}-2-\mathrm{x} \\
\mathrm{Cl}-3-\mathrm{x} \\
\mathrm{Cl}-4-\mathrm{x} \\
\mathrm{Cl}-\mathrm{F}-\mathrm{x} \\
\mathrm{Cr} \\
\mathrm{HC}-\mathrm{insol} \\
\mathrm{HC}-\text { solub } \\
\mathrm{Hg} \\
\mathrm{Pb} \\
\mathrm{Se}\end{array}$ \\
\hline
\end{tabular}

Amount

$(\mathrm{kg} / \mathrm{yr})$

5. $151 \mathrm{E}-02$

7. $241 \mathrm{E}-01$

2. 274E-02

3. $999 \mathrm{E}-03$

2. 356E-02

5. $620 \mathrm{E}-01$

4. $522 E-01$

4. $355 E+01$

9. $136 \mathrm{E}+00$

$8.522 \mathrm{E}-02$

$5.418 \mathrm{E}-01$

8. $707 \mathrm{E}+01$

1. $317 \mathrm{E}+02$

1. 340E-01

1.275E-01

1. $998 \mathrm{E}-02$ 
A-18

\begin{tabular}{l} 
Shipping site: COLON \\
Total Volume (m3/yr): \\
Radionuclide \\
\hline Ac-228 \\
Bi-212 \\
Pa-234 \\
Pa-234m \\
Pb-212 \\
Po-212 \\
Po-216 \\
Ra-224 \\
Ra-228 \\
Tc-99 \\
Th-228 \\
Th-231 \\
Th-232 \\
Th-234 \\
Tl-208 \\
$\mathrm{U}-235$ \\
$\mathrm{U}-238$
\end{tabular}

Shipping Site: KAPL

Total volume $(\mathrm{m} 3 / \mathrm{yr})$ :

Radionuclide

\begin{tabular}{l}
\hline $\mathrm{Ba}-137 \mathrm{~m}$ \\
$\mathrm{Co}-60$ \\
$\mathrm{Cs}-134$ \\
$\mathrm{Cs}-137$ \\
$\mathrm{Eu}-154$ \\
$\mathrm{Eu}-155$ \\
$\mathrm{Fe}-55$ \\
$\mathrm{H}-3$ \\
$\mathrm{Mn}-54$ \\
$\mathrm{Nb}-94$ \\
$\mathrm{Ni}-59$ \\
$\mathrm{Ni}-63$ \\
$\mathrm{~Pa}-234 \mathrm{~m}$ \\
$\mathrm{Pm}-147$ \\
$\mathrm{Pu}-238$ \\
$\mathrm{Pu}-241$ \\
$\mathrm{Sb}-125$ \\
$\mathrm{Sm}-151$ \\
$\mathrm{Sr}-90$ \\
$\mathrm{Te}-125 \mathrm{~m}$ \\
$\mathrm{Th}-234$ \\
$\mathrm{U}-238$ \\
$\mathrm{Y}-90$
\end{tabular}

Waste Type: Solids (CH-Non-alpha)

1.235E-02 Total Mass $(\mathrm{kg} / \mathrm{yr}): 1.606 \mathrm{E}+01$

\begin{tabular}{lcc} 
Chemical & & $\begin{array}{c}\text { Amount } \\
(\mathrm{kg} / \mathrm{YI})\end{array}$ \\
\cline { 1 - 1 } $57-12-5$ & $7.229 \mathrm{E}-04$ \\
$75-09-2$ & & $8.484 \mathrm{E}-04$ \\
$\mathrm{Ag}$ & $2.570 \mathrm{E}-05$ \\
$\mathrm{As}$ & $2.249 \mathrm{E}-05$ \\
$\mathrm{Ba}$ & $1.606 \mathrm{E}-05$ \\
$\mathrm{Cd}$ & $1.429 \mathrm{E}-04$ \\
$\mathrm{Cl}-2-\mathrm{x}$ & $4.242 \mathrm{E}-03$ \\
$\mathrm{Cl}-3-\mathrm{x}$ & $5.345 \mathrm{E}-03$ \\
$\mathrm{Cl}-4-\mathrm{x}$ & $5.006 \mathrm{E}-03$ \\
$\mathrm{Cl}-\mathrm{F}-\mathrm{x}$ & $4.242 \mathrm{E}-04$ \\
$\mathrm{Cr}$ & $5.462 \mathrm{E}-04$ \\
$\mathrm{HC}-$ insol & $9.333 \mathrm{E}-04$ \\
$\mathrm{HC}-\mathrm{SOlub}$ & $8.032 \mathrm{E}-04$ \\
$\mathrm{Hg}$ & $3.695 \mathrm{E}-06$ \\
$\mathrm{~Pb}$ & $1.044 \mathrm{E}-03$ \\
$\mathrm{Se}$ & $8.032 \mathrm{E}-06$
\end{tabular}

Waste Type: Organic Liquids ( $\mathrm{CH}-\mathrm{Non}-\mathrm{alpha})$

Total Mass (kg/yr): $7.065 \mathrm{E}+02$

$6.167 \Xi-01$
Activity

$\frac{(\mathrm{Ci} / \mathrm{yr})}{\mathrm{sin}}$

4. $129 \mathrm{E}-01$

3. $921 \mathrm{E}-03$

$4.359 \mathrm{E}-01$

$5.305 \mathrm{E}-03$

$2.537 \mathrm{E}-03$

8.719E-01

$1.899 \mathrm{E}+00$

$2.768 \mathrm{E}-03$

$4.152 \mathrm{E}-03$

$8.719 \mathrm{E}-02$

$1.172 \mathrm{E}+01$

1. $637 \mathrm{E}-02$

$4.752 \mathrm{E}-02$

1. $361 \mathrm{E}-02$

1. $107 \mathrm{E}-02$

1. $845 \mathrm{E}-03$

$3.690 \mathrm{E}-03$

$4.106 \mathrm{E}-01$

4. $613 \mathrm{E}-04$

1. $637 \mathrm{E}-02$

1. $637 \mathrm{E}-02$

4.106E-01

\begin{tabular}{l} 
Chemical \\
\hline $57-12-5$ \\
$75-09-2$ \\
Ag \\
As \\
Ba \\
Cd \\
Cl-2-x \\
Cl-3-x \\
Cl-4-x \\
Cl-F-x \\
Cr \\
HC-insol \\
HC-solub \\
Hg \\
Pb \\
Se
\end{tabular}

Amount

(kg/Yr)

2. $915 \mathrm{E}-02$

5. 312E-01

1. 461E-02

2. $952 \mathrm{E}-03$

1. $739 \mathrm{E}-02$

$4.125 \mathrm{E}-01$

3. $320 E-01$

3. $184 \mathrm{E}+01$

$6.682 \mathrm{E}+00$

$6.275 \mathrm{E}-02$

3. $877 \mathrm{E}-01$

6. $386 \mathrm{E}+01$

$7.952 \mathrm{E}+01$

8. 511E-02

$9.130 \mathrm{E}-02$

1. $277 \mathrm{E}-02$ 
Shipping Site: KAPL

Total Volume $(\mathrm{m} 3 / \mathrm{yr})$ :

Radionuclide

$\mathrm{Ba}-137 \mathrm{~m}$

Co-60

$\mathrm{Cs}-134$

Cs-137

Eu-154

Eu-155

$\mathrm{Fe}-55$

$\mathrm{H}-3$

Mn-54

$\mathrm{Nb}-94$

$\mathrm{Ni}-59$

$\mathrm{Ni}-63$

$\mathrm{Pa}-234 \mathrm{~m}$

Pm-147

Pu-238

Pu-241

Sb-125

$\mathrm{Sm}-151$

$\mathrm{Sr}-90$

$\mathrm{Te}-125 \mathrm{~m}$

$\mathrm{Th}-234$

U -238

$Y-90$

Shipping Site: KKS

Total Volume $(\mathrm{m} 3 / \mathrm{yr})$ :

Radionuclide

Ba-137m

Co-60

Cs-134

Cs -137

Eu-154

Eu-155

$\mathrm{Fe}-55$

H-3

$M n-54$

$\mathrm{Nb}-94$

Ni-59

$\mathrm{Ni}-63$

$\mathrm{Pa}-234 \mathrm{~m}$

Pm-147

Pu-238

Pu-241

Sb-125

sm-151

Sr-90

$\mathrm{Te}-125 \mathrm{~m}$

Th-234

$\mathrm{U}-238$

$\mathrm{Y}-90$

Waste Type: Solids (CH-Non-alpha)

Total Mass $(\mathrm{kg} / \mathrm{yr}): 5.162 \mathrm{E}+03$

$3.572 E+00$

Activity (Ci/yr)

$2.391 \mathrm{E}+00$

$3.862 \mathrm{E}+01$

2. $271 \mathrm{E}-02$

$2.525 \mathrm{E}+00$

$3.072 \mathrm{E}-02$

$1.469 \mathrm{E}-02$

$5.050 \mathrm{E}+00$

$1.100 \mathrm{E}+01$

1. $603 \mathrm{E}-02$

2. $404 \mathrm{E}-02$

$5.050 \mathrm{E}-01$

$6.790 \mathrm{E}+01$

$9.486 \mathrm{E}-02$

$2.752 \mathrm{E}-01$

$7.882 \mathrm{E}-02$

$6.413 \mathrm{E}-02$

$1.068 \mathrm{E}-02$

$2.137 \mathrm{E}-02$

$2.378 \mathrm{E}+00$

$2.672 \mathrm{E}-03$

$9.486 \mathrm{E}-02$

$9.486 \mathrm{E}-02$

$2.378 E+00$

\begin{tabular}{|c|c|}
\hline Chemical & $\begin{array}{l}\text { Amount } \\
(\mathrm{kg} / \mathrm{yr})\end{array}$ \\
\hline $\begin{array}{l}57-12-5 \\
75-09-2\end{array}$ & $\begin{array}{l}1.840 \mathrm{E}-02 \\
2.577 \mathrm{E}+00\end{array}$ \\
\hline $\mathrm{Ag}$ & $6.267 \mathrm{E}-02$ \\
\hline As & $2.265 \mathrm{E}-02$ \\
\hline $\mathrm{Ba}$ & $5.050 \mathrm{E}-01$ \\
\hline $\mathrm{cd}$ & $2.428 \mathrm{E}-02$ \\
\hline$C I-2-x$ & $1.454 \mathrm{E}+00$ \\
\hline Cl-3-x & $1.328 \mathrm{E}+01$ \\
\hline CI-4-x & 1. $024 \mathrm{E}+01$ \\
\hline $\mathrm{CI}-\mathrm{F}-\mathrm{x}$ & $4.115 \mathrm{E}-01$ \\
\hline $\mathrm{Cr}$ & $4.769 \mathrm{E}-01$ \\
\hline $\mathrm{HC}-$ insol & $3.511 \mathrm{E}+01$ \\
\hline HC-solub & $1.516 \mathrm{E}+01$ \\
\hline $\mathrm{Hg}$ & $6.065 \mathrm{E}-02$ \\
\hline $\mathrm{Pb}$ & $7.229 E-01$ \\
\hline $\mathrm{Se}$ & 1. $698 \mathrm{E}-02$ \\
\hline
\end{tabular}

Waste Type: Organic Liquids (CH-Non-alpha)

4.733E-01 Total Mass (kg/Yr): $5.422 \mathrm{E}+02$

\begin{tabular}{c}
$\begin{array}{c}\text { Activity } \\
(\mathrm{Ci} / \mathrm{Yr})\end{array}$ \\
\hline $3.168 \mathrm{E}-01$ \\
$5.118 \mathrm{E}+00$ \\
$3.009 \mathrm{E}-03$ \\
$3.345 \mathrm{E}-01$ \\
$4.071 \mathrm{E}-03$ \\
$1.947 \mathrm{E}-03$ \\
$6.691 \mathrm{E}-01$ \\
$1.457 \mathrm{E}+00$ \\
$2.124 \mathrm{E}-03$ \\
$3.186 \mathrm{E}-03$ \\
$6.691 \mathrm{E}-02$ \\
$8.997 \mathrm{E}+00$ \\
$1.256 \mathrm{E}-02$ \\
$3.646 \mathrm{E}-02$ \\
$1.044 \mathrm{E}-02$ \\
$8.497 \mathrm{E}-03$ \\
$1.416 \mathrm{E}-03$ \\
$2.832 \mathrm{E}-03$ \\
$3.151 \mathrm{E}-01$ \\
$3.540 \mathrm{E}-04$ \\
$1.256 \mathrm{E}-02$ \\
$1.256 \mathrm{E}-02$ \\
$3.151 \mathrm{E}-01$
\end{tabular}

\begin{tabular}{l} 
Chemical \\
\hline $57-12-5$ \\
$75-09-2$ \\
Ag \\
As \\
$\mathrm{Ba}$ \\
$\mathrm{Cd}$ \\
$\mathrm{Cl}-2-\mathrm{x}$ \\
$\mathrm{Cl}-3-\mathrm{x}$ \\
$\mathrm{Cl}-4-\mathrm{x}$ \\
$\mathrm{Cl}-\mathrm{F}-\mathrm{x}$ \\
$\mathrm{Cr}$ \\
$\mathrm{HC}-\mathrm{insol}$ \\
$\mathrm{HC}-\mathrm{solub}$ \\
$\mathrm{Hg}$ \\
$\mathrm{Pb}$ \\
$\mathrm{Se}$
\end{tabular}

Amount

$(\mathrm{kg} / \mathrm{yr})$

2. $237 \mathrm{E}-02$

$4.077 \mathrm{E}-01$

1. $121 \mathrm{E}-02$

$2.266 \mathrm{E}-03$

1. $335 \mathrm{E}-02$

3. $166 \mathrm{E}-01$

2. $548 \mathrm{E}-01$

2. $443 \mathrm{E}+01$

$5.128 \mathrm{E}+00$

4. $816 \mathrm{E}-02$

$2.976 \mathrm{E}-01$

4. $901 \mathrm{E}+01$

$6.103 \mathrm{E}+01$

$6.532 \mathrm{E}-02$

7.007E-02

$9.805 \mathrm{E}-03$ 


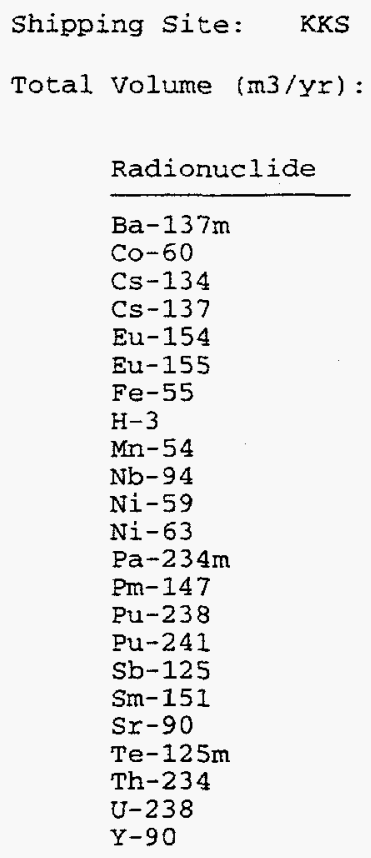

Shipping Site: KWS

Total Volume $(\mathrm{m} 3 / \mathrm{Yr})$ :

Radionuclide

$\mathrm{Ba}-137 \mathrm{~m}$

Co- 50

Cs -134

Cs -137

Eu-154

Eu-155

$\mathrm{Fe}-55$

H-3

$\mathrm{Mn}-54$

$\mathrm{Nb}-94$

Ni-59

$\mathrm{Ni}-63$

$\mathrm{Pa}-234 \mathrm{~m}$

Pm-147

Pu-238

Pu-241

$\mathrm{Sb}-125$

Sm-151

$\mathrm{Sr}-90$

$\mathrm{Te}-125 \mathrm{~m}$

$\mathrm{Th}-234$

$\mathrm{U}-238$

$Y-90$
Waste Type: Solids (CH-Non-alpha)

Total Mass (kg/yr): $6.620 \mathrm{E}+03$

$$
\begin{gathered}
\text { Activity } \\
\text { (Ci/yr) }
\end{gathered}
$$

\begin{tabular}{|c|c|}
\hline Chemical & $\begin{array}{l}\text { Amount } \\
(\mathrm{kg} / \mathrm{Yr})\end{array}$ \\
\hline $\begin{array}{l}57-12-5 \\
75-09-2\end{array}$ & $\begin{array}{l}1.282 E-01 \\
2.231 E+00\end{array}$ \\
\hline Ag & $8.783 E-02$ \\
\hline AS & $3.299 \pm-02$ \\
\hline $\mathrm{Ba}$ & 1. $662 \mathrm{E}+00$ \\
\hline $\mathrm{cd}$ & $5.269 E-02$ \\
\hline$C I-2-x$ & 1. $281 E+00$ \\
\hline$C I-3-x$ & $1.108 \mathrm{E}+01$ \\
\hline$C l-4-x$ & 1. $215 E+01$ \\
\hline$C 1-F-x$ & $2.090 \mathrm{E}-01$ \\
\hline $\mathrm{Cr}$ & I. $846 E+00$ \\
\hline HC-insol & $2.753 E+01$ \\
\hline $\mathrm{HC}-\mathrm{solub}$ & $1.411 \mathrm{E}+0 \mathrm{I}$ \\
\hline $\mathrm{Hg}$ & 1. $438 E-01$ \\
\hline $\mathrm{Pb}$ & $4.165 \mathrm{E}+00$ \\
\hline $\mathrm{Se}$ & $3.790 E-02$ \\
\hline
\end{tabular}

Waste Type: Organic Liquids (CH-Non-alpha)

$6.454 \mathrm{E}-02$

Total Mass $(\mathrm{kg} / \mathrm{yr}): \quad 7.394 \mathrm{E}+01$

\begin{tabular}{|c|c|}
\hline Chemical & $\begin{array}{l}\text { Amount } \\
(\mathrm{kg} / \mathrm{yr})\end{array}$ \\
\hline $57-12-5$ & $3.051 \mathrm{E}-03$ \\
\hline $75-09-2$ & $5.559 \Xi-02$ \\
\hline $\mathrm{Ag}$ & $1.529 E-03$ \\
\hline As & $3.090 \mathrm{E}-04$ \\
\hline $\mathrm{Ba}$ & $1.820 \mathrm{E}-03$ \\
\hline$\overline{c d}$ & $4.317 \mathrm{E}-02$ \\
\hline Cl $-2-x$ & $3.474 E-02$ \\
\hline$c 1-3-x$ & $3.332 \mathrm{E}+00$ \\
\hline$c 1-4-x$ & $6.993 E-01$ \\
\hline $\mathrm{Cl}-\mathrm{F}-\mathrm{x}$ & $6.567 \bar{E}-03$ \\
\hline $\mathrm{Cr}$ & $4.058 E-02$ \\
\hline $\mathrm{HC}$-insol & $6.683 E+00$ \\
\hline HC-solub & $8.322 \mathrm{E}+00$ \\
\hline $\mathrm{Hg}$ & $8.907 \mathrm{E}-03$ \\
\hline $\mathrm{Pb}$ & $9.555 \mathrm{E}-03$ \\
\hline $\mathrm{Se}$ & $1.337 \mathrm{E}-03$ \\
\hline
\end{tabular}

$$
\begin{aligned}
& \text { Activity } \\
& \text { (Ci/yr) }
\end{aligned}
$$

$4.321 \varepsilon-02$

$6.979 \mathrm{E}-01$

4. $103 \mathrm{E}-04$

4. 562E-02

5. 552E-04

$2.655 \mathrm{E}-04$

9.125E-02

$1.987 \mathrm{E}-01$

$2.896 \mathrm{E}-04$

$4.345 \mathrm{E}-04$

$9.125 \mathrm{E}-03$

1. $226 \mathrm{E}+00$

1. 714E-03

$4.973 \mathrm{E}-03$

1. $424 \mathrm{E}-03$

1. $158 \mathrm{E}-03$

1. $931 \mathrm{E}-04$

$3.862 \mathrm{E}-04$

4. 297E-02

4. 828E-05

1. $714 \mathrm{E}-03$

1. $714 \mathrm{E}-03$

4. $297 \mathrm{E}-02$ 
Shipping Size: KWS Waste Type: Solids (CH-Non-alpha)

Total Volume $(\mathrm{m} 3 / \mathrm{yr})$ :

$1.262 \mathrm{E}+00$

Total Mass $(\mathrm{kg} / \mathrm{yr})$ :

$1.580 \mathrm{E}+03$

Radionuclide

$\mathrm{Ba}-137 \mathrm{~m}$

Co- 60

Cs -134

Cs -137

Eu-154

Eu-155

$\mathrm{Fe}-55$

$\mathrm{H}-3$

$\mathrm{Mn}-54$

$\mathrm{Nb}-94$

$\mathrm{Ni}-59$

$\mathrm{Ni}-63$

$\mathrm{Pa}-234 \mathrm{~m}$

Pm-147

Pu -238

$\mathrm{Pu}-241$

$\mathrm{Sb}-125$

Sm-151

$\mathrm{Sr}-90$

$\mathrm{Te}-125 \mathrm{~m}$

Th-234

$\mathrm{U}-238$

Y-90

Shipping Site: PNS

Total volume $(\mathrm{m} 3 / \mathrm{yr})$ :

Radionuclide

$\mathrm{Ba}-137 \mathrm{~m}$

Co-60

Cs -134

Cs-137

Eu-154

Eu-155

$\mathrm{Fe}-55$

$\mathrm{H}-3$

$\mathrm{Mn}-54$

$\mathrm{Nb}-94$

$\mathrm{Ni}-59$

$\mathrm{Ni}-63$

$\mathrm{Pa}-234 \mathrm{~m}$

Pm-147

Pu-238

Pu-241

$\mathrm{Sb}-125$

$\mathrm{Sm}-151$

Sr -90

Te-125m

Th-234

$\mathrm{U}-238$

$\mathrm{Y}-90$

Shipping site: PPPL

Total volume $(\mathrm{m} 3 / \mathrm{yr})$ :

Radionuclide

$\mathrm{Ba}-137 \mathrm{~m}$

Cs -137

$\mathrm{H}-3$

$\mathrm{Pa}-234 \mathrm{~m}$

Pu-241

$\mathrm{Sr}-90$

Th-234

$\mathrm{U}-238$

$Y-90$

\begin{tabular}{c}
$\begin{array}{c}\text { Activity } \\
\text { (Ci/yr) }\end{array}$ \\
\hline $8.449 \mathrm{E}-01$ \\
$1.364 \mathrm{E}+01$ \\
$8.024 \mathrm{E}-03$ \\
$8.921 \mathrm{E}-01$ \\
$1.085 \mathrm{E}-02$ \\
$5.192 \mathrm{E}-03$ \\
$1.784 \mathrm{E}+00$ \\
$3.886 \mathrm{E}+00$ \\
$5.664 \mathrm{E}-03$ \\
$8.496 \mathrm{E}-03$ \\
$1.784 \mathrm{E}-01$ \\
$2.399 \mathrm{E}+01$ \\
$3.351 \mathrm{E}-02$ \\
$9.724 \mathrm{E}-02$ \\
$2.785 \mathrm{E}-02$ \\
$2.265 \mathrm{E}-02$ \\
$3.776 \mathrm{E}-03$ \\
$7.552 \mathrm{E}-03$ \\
$8.402 \mathrm{E}-01$ \\
$9.440 \mathrm{E}-04$ \\
$3.351 \mathrm{E}-02$ \\
$3.351 \mathrm{E}-02$ \\
$8.402 \mathrm{E}-01$
\end{tabular}

Chemical

Amount

$57-12-5$

$75-09-2 \quad 3.801 \mathrm{E}-01$

Ag

As

$\mathrm{Ba}$

Cd

C $1-2-x$

Cl $-3-x$

C1 $1-4-x$

$\mathrm{C} 1-\mathrm{F}-\mathrm{X}$

$\mathrm{Cr}$

$\mathrm{HC}$-insol

$\mathrm{HC}-\mathrm{solub}$

$\mathrm{Hg}$

$\mathrm{Pb}$

$\mathrm{Se}$

$2.572 \mathrm{E}-02$

$8.795 \mathrm{E}-03$

$1.035 \mathrm{E}+00$

$1.157 \mathrm{E}-02$

$3.221 E-01$

$1.699 \mathrm{E}+00$

$1.988 \mathrm{E}+00$

$2.323 \mathrm{E}-01$

$1.010 \mathrm{E}+00$

$4.421 \mathrm{E}+00$

$1.600 \mathrm{E}+00$

$6.065 \mathrm{E}-02$

$2.396 \mathrm{E}+00$

1. $789 \mathrm{E}-02$
Waste Type: Solids (CH-Non-alpha)

Total Mass (kg/yr): $9.088 \mathrm{E}+01$

$5.200 E-02$

\begin{tabular}{|c|c|}
\hline Chemical & $\begin{array}{l}\text { Amount } \\
(\mathrm{kg} / \mathrm{yr})\end{array}$ \\
\hline $75-09-2$ & 1. $324 \mathrm{E}-02$ \\
\hline $\mathrm{Ag}$ & $6.570 \mathrm{E}-04$ \\
\hline As & $5.453 \mathrm{E}-04$ \\
\hline $\mathrm{Ba}$ & $1.708 \mathrm{E}-02$ \\
\hline$\overline{c d}$ & $8.438 E-03$ \\
\hline Cl-2-x & 1. $324 \mathrm{E}-02$ \\
\hline$C I-3-x$ & $7.909 \mathrm{E}-01$ \\
\hline$C I-4-x$ & $1.483 \mathrm{E}-01$ \\
\hline$C I-F-x$ & $7.499 \mathrm{E}-03$ \\
\hline Cr & $1.051 \mathrm{E}-01$ \\
\hline HC-insol & $2.629 E+00$ \\
\hline HC-solub & $1.314 \mathrm{E}+00$ \\
\hline $\mathrm{Hg}$ & $9.046 \bar{E}-03$ \\
\hline $\mathrm{Pb}$ & $1.319 \mathrm{E}-02$ \\
\hline $\mathrm{Se}$ & $2.365 E-03$ \\
\hline
\end{tabular}

1. $147 E-03$

$9.335 \mathrm{E}-04$

$1.555 \mathrm{E}-04$

3. $111 \mathrm{E}-04$

3. $462 \mathrm{E}-02$

3. $889 \mathrm{E}-05$

1.380E-03

1. $380 \mathrm{E}-03$

3. $462 \mathrm{E}-02$

$2.365 E-03$

Waste Type: Solids (CH-Non-alpha)

$5.456 \Xi-04$

Total Mass (kg/yr): 7.191E-01

\begin{tabular}{c}
$\begin{array}{c}\text { Activity } \\
\text { (Ci/yr) }\end{array}$ \\
\hline $7.218 \mathrm{E}-06$ \\
$7.562 \mathrm{E}-06$ \\
$3.433 \mathrm{E}-02$ \\
$1.753 \mathrm{E}-06$ \\
$1.718 \mathrm{E}-06$ \\
$7.218 \mathrm{E}-06$ \\
$1.753 \mathrm{E}-06$ \\
$1.753 \mathrm{E}-06$ \\
$7.218 \mathrm{E}-06$
\end{tabular}

\begin{tabular}{l} 
Chemical \\
\hline $75-09-2$ \\
Cl-2-x \\
Cl-3-x \\
Cl-4-x \\
HC-insol \\
HC-solub
\end{tabular}

Amount

(kg/yr)

1. $438 \mathrm{E}-02$

7. $190 \mathrm{E}-03$

$7.190 \mathrm{E}-02$

$5.752 \mathrm{E}-02$

1.797E-0I

8. $629 \mathrm{E}-02$ 
Shipping Site: RMI

Total Volume $(\mathrm{m} 3 / \mathrm{yr})$ :

Radionuclide

Ac -228

$\mathrm{Bi}-212$

$\mathrm{Pa}-234$

$\mathrm{Pa}-234 \mathrm{~m}$

$\mathrm{Pb}-212$

Po- 212

Po-216

$\mathrm{Ra}-224$

Ra- 228

TC-99

Th-228

Th-231

Th -232

Th-234

T1-208

$\mathrm{U}-235$

$\mathrm{U}-238$

Shipping site: RMI

Total Volume (m3/yr):

Radionuclide

$\mathrm{Ac}-228$
$\mathrm{Bi}-212$
$\mathrm{~Pa}-234$
$\mathrm{~Pa}-234 \mathrm{~m}$
$\mathrm{~Pb}-212$
$\mathrm{PO}-212$
$\mathrm{PO}-216$
$\mathrm{Ra}-224$
$\mathrm{Ra}-228$
$\mathrm{~T} \mathrm{C}-99$
$\mathrm{Th}-228$
$\mathrm{Th}-231$
$\mathrm{Th}-232$
$\mathrm{Th}-234$
$\mathrm{~T} \mathrm{I}-208$
$\mathrm{U}-235$
$\mathrm{U}-238$

Waste Type: Organic Liquids ( $\mathrm{CH}-\mathrm{Non}-\mathrm{alpha})$

$6.677 \mathrm{E}-01$

Total Mass $(\mathrm{kg} / \mathrm{yr})$ :

$9.741 \mathrm{E}+02$

\begin{tabular}{|c|c|}
\hline Chemical & $\begin{array}{l}\text { Amount } \\
(\mathrm{kg} / \mathrm{yr})\end{array}$ \\
\hline $75-09-2$ & $1.527 \mathrm{E}+00$ \\
\hline As & $3.769 \mathrm{E}-04$ \\
\hline $\mathrm{Ba}$ & $1.615 \bar{E}-03$ \\
\hline $\mathrm{cd}$ & $1.071 \mathrm{E}+00$ \\
\hline$c 1-2-x$ & $8.108 \mathrm{E}-01$ \\
\hline$C I-3-x$ & $1.123 E+02$ \\
\hline C] $-4-x$ & $2.505 E+01$ \\
\hline $\mathrm{Cl}-\mathrm{F}-\mathrm{x}$ & $4.625 \mathrm{E}-02$ \\
\hline $\mathrm{Cr}$ & $9.331 \mathrm{E}-01$ \\
\hline HC-insol & $2.184 \mathrm{E}+01$ \\
\hline HC-solub & $1.277 \mathrm{E}+01$ \\
\hline $\mathrm{Hg}$ & $6.122 \mathrm{E}-03$ \\
\hline $\mathrm{Pb}$ & $2.045 \mathrm{E}-01$ \\
\hline
\end{tabular}

Waste Type: Solids (CH-Non-alpha)

$1.091 \mathrm{E}+00 \quad$ Total Mass $(\mathrm{kg} / \mathrm{Yr}): 8.323 \mathrm{E}+02$

\section{Activity}

(Ci/yr)

$2.370 \mathrm{E}-06$

$3.988 \mathrm{E}-07$

$3.013 \mathrm{E}-07$

$2.927 E-03$

$3.988 \mathrm{E}-07$

$2.570 \mathrm{E}-07$

$3.988 \mathrm{E}-07$

$3.815 \mathrm{E}-07$

$2.267 \mathrm{E}-06$

$4.218 \mathrm{E}-05$

$3.988 \mathrm{E}-07$

$2.286 \mathrm{E}-06$

$2.407 \mathrm{E}-05$

$2.927 \mathrm{E}-03$

$1.506 \mathrm{E}-07$

2. $277 \mathrm{E}-06$

$2.927 \mathrm{E}-03$

\begin{tabular}{l} 
Chemical \\
\hline $57-12-5$ \\
$75-09-2$ \\
$\mathrm{Ag}$ \\
$\mathrm{As}$ \\
$\mathrm{Ba}$ \\
$\mathrm{Cd}$ \\
$\mathrm{Cl}-2-\mathrm{x}$ \\
$\mathrm{Cl}-3-\mathrm{x}$ \\
$\mathrm{Cl}-4-\mathrm{x}$ \\
$\mathrm{Cl}-\mathrm{F}-\mathrm{x}$ \\
$\mathrm{Cr}$ \\
$\mathrm{HC}-\mathrm{insol}$ \\
$\mathrm{HC}-\mathrm{Solub}$ \\
$\mathrm{Hg}$ \\
$\mathrm{Pb}$ \\
$\mathrm{Se}$
\end{tabular}

Amount

(kg/yr)

4. $647 \mathrm{E}-03$

2. $315 \mathrm{E}-01$

$6.651 \mathrm{E}-03$

1. $974 \mathrm{E}-03$

$5.734 \mathrm{E}-01$

1. $836 \mathrm{E}-02$

2. $862 \mathrm{E}-01$

2. $561 \mathrm{E}+00$

7. $371 \mathrm{E}-01$

3. $452 \mathrm{E}-01$

2. $638 \mathrm{E}-01$

$8.888 \mathrm{E}+00$

$2.825 \mathrm{E}+00$

2. $678 \mathrm{E}-02$

8. $498 \mathrm{E}-02$

$6.070 \mathrm{E}-03$ 
Destination Site: RFP

\begin{tabular}{|c|c|c|c|c|c|c|}
\hline Shipp. & ing site: GJCT & & Waste & Type: & Organic Liquids & (CH-Non-alpha) \\
\hline Total & Volume $(\mathrm{m} 3 / \mathrm{yr})$ : & $1.290 \mathrm{E}-02$ & Total & Mass & $(\mathrm{kg} / \mathrm{yr}):$ & 01 \\
\hline & Radionuclide & $\begin{array}{c}\text { Activity } \\
\text { (Ci/yr) }\end{array}$ & & & Chemical & $\begin{array}{l}\text { Amount } \\
(\mathrm{kg} / \mathrm{Yr})\end{array}$ \\
\hline & $\begin{array}{l}\mathrm{Ac}-228 \\
\mathrm{Bi}-212 \\
\mathrm{~Pa}-234 \\
\mathrm{~Pa}-234 \mathrm{~m} \\
\mathrm{~Pb}-212 \\
\mathrm{~Pa}-212 \\
\mathrm{Po}-216 \\
\mathrm{Ra}-224 \\
\mathrm{Ra}-228 \\
\mathrm{TC}-99 \\
\mathrm{Th}-228 \\
\mathrm{Th}-231 \\
\mathrm{Th}-232 \\
\mathrm{Th}-234 \\
\mathrm{~T}-208 \\
\mathrm{U}-235 \\
\mathrm{U}-238\end{array}$ & $\begin{array}{l}2.762 \mathrm{E}-08 \\
4.647 \mathrm{E}-09 \\
3.511 \mathrm{E}-09 \\
3.411 \mathrm{E}-05 \\
4.647 \mathrm{E}-09 \\
2.995 \mathrm{E}-09 \\
4.647 \mathrm{E}-09 \\
4.647 \mathrm{E}-09 \\
2.762 \mathrm{E}-08 \\
5.138 \mathrm{E}-07 \\
4.647 \mathrm{E}-09 \\
2.664 \mathrm{E}-08 \\
2.805 \mathrm{E}-07 \\
3.411 \mathrm{E}-05 \\
1.756 \mathrm{E}-09 \\
2.654 \mathrm{E}-08 \\
3.411 \mathrm{E}-05\end{array}$ & & & $\begin{array}{l}57-12-5 \\
75-09-2 \\
\mathrm{Ag} \\
\mathrm{As} \\
\mathrm{Ba} \\
\mathrm{Cd} \\
\mathrm{Cl}-2-\mathrm{x} \\
\mathrm{Cl}-3-\mathrm{x} \\
\mathrm{Cl}-4-\mathrm{x} \\
\mathrm{Cl}-\mathrm{F}-\mathrm{x} \\
\mathrm{Cr} \\
\mathrm{HC}-\text { insol } \\
\mathrm{HC}-\text { solub } \\
\mathrm{Hg} \\
\mathrm{Pb} \\
\mathrm{Se}\end{array}$ & $\begin{array}{l}6.102 E-04 \\
1.111 E-02 \\
3.059 E-04 \\
6.180 E-05 \\
3.641 E-04 \\
8.635 E-03 \\
6.949 E-03 \\
6.665 E-01 \\
1.398 E-01 \\
1.313 E-03 \\
8.116 E-03 \\
1.336 E+00 \\
1.664 E+00 \\
1.781 E-03 \\
1.911 E-03 \\
2.674 E-04\end{array}$ \\
\hline Shipp: & ing Site: & & Waste & Type: & Solids (CH-Non-a & Ipha) \\
\hline Total & Volume $(\mathrm{m} 3 / \mathrm{yr})$ : & $2.953 \mathrm{E}-02$ & Total & Mass & $(\mathrm{kg} / \mathrm{yr}):$ & 01 \\
\hline & Radionuclide & $\begin{array}{c}\text { Activity } \\
\text { (Ci/yr) }\end{array}$ & & & Chemical & $\begin{array}{l}\text { Amount } \\
(\mathrm{kg} / \mathrm{Yr})\end{array}$ \\
\hline & $\begin{array}{l}\mathrm{Ac}-228 \\
\mathrm{Bi}-212 \\
\mathrm{~Pa}-234 \\
\mathrm{~Pa}-234 \mathrm{~m} \\
\mathrm{~Pb}-212 \\
\mathrm{PO}-212 \\
\mathrm{PO}-216 \\
\mathrm{Ra}-224 \\
\mathrm{Ra}-228 \\
\mathrm{~T} \mathrm{C}-99 \\
\mathrm{Th}-228 \\
\mathrm{Th}-231 \\
\mathrm{Th}-232 \\
\mathrm{Th}-234 \\
\mathrm{~T} 1-208 \\
\mathrm{U}-235 \\
\mathrm{U}-238\end{array}$ & $\begin{array}{l}5.356 \mathrm{E}-08 \\
1.069 \mathrm{E}-08 \\
8.080 \mathrm{E}-09 \\
7.849 \mathrm{E}-05 \\
1.069 \mathrm{E}-08 \\
6.891 \mathrm{E}-09 \\
1.069 \mathrm{E}-08 \\
1.054 \mathrm{E}-08 \\
6.268 \mathrm{E}-08 \\
1.165 \mathrm{E}-06 \\
1.069 \mathrm{E}-08 \\
6.131 \mathrm{E}-08 \\
6.454 \mathrm{E}-07 \\
7.849 \mathrm{E}-05 \\
4.040 \mathrm{E}-09 \\
6.107 \mathrm{E}-08 \\
7.849 \mathrm{E}-05\end{array}$ & & & $\begin{array}{l}57-12-5 \\
75-09-2 \\
\mathrm{Ag} \\
\mathrm{AS} \\
\mathrm{Ba} \\
\mathrm{Cd} \\
\mathrm{Cl}-2-\mathrm{x} \\
\mathrm{Cl}-3-\mathrm{x} \\
\mathrm{Cl}-4-\mathrm{x} \\
\mathrm{Cl}-\mathrm{F}-\mathrm{x} \\
\mathrm{Cr} \\
\mathrm{HC}-\text { insol } \\
\mathrm{HC}-\text { solub } \\
\mathrm{Hg} \\
\mathrm{Pb} \\
\mathrm{Se}\end{array}$ & $\begin{array}{l}1.997 E-04 \\
1.051 E-03 \\
6.108 E-04 \\
2.981 E-04 \\
3.480 E-03 \\
4.003 E-04 \\
1.051 E-03 \\
2.387 E-02 \\
7.830 E-03 \\
5.619 E-04 \\
4.507 E-03 \\
7.674 E-02 \\
8.522 E-02 \\
1.306 E-03 \\
5.304 E-03 \\
4.666 E-04\end{array}$ \\
\hline
\end{tabular}


Destination Site: SRS

\begin{tabular}{|c|c|c|c|c|c|}
\hline \multicolumn{3}{|c|}{ Shipping Site: BAPL } & \multirow{2}{*}{$\begin{array}{l}\text { Waste Type: } \\
\text { Total Mass }\end{array}$} & \multicolumn{2}{|l|}{ Solids (CH-Alpha) } \\
\hline Total & Volume $(\mathrm{m} 3 / \mathrm{yr})$ : & $8.357 \mathrm{E}-01$ & & $(\mathrm{~kg} / \mathrm{yr}):$ & 02 \\
\hline & Radionuclide & $\begin{array}{l}\text { Activity } \\
(\text { Ci/yr) }\end{array}$ & \multicolumn{2}{|r|}{ Chemical } & $\begin{array}{l}\text { Amount } \\
(\mathrm{kg} / \mathrm{Yr})\end{array}$ \\
\hline & $\begin{array}{l}\mathrm{Am}-241 \\
\mathrm{Ba}-137 \mathrm{~m} \\
\mathrm{Co}-60 \\
\mathrm{Cs}-137 \\
\mathrm{Eu}-154 \\
\mathrm{H}-3 \\
\mathrm{Nb}-94 \\
\mathrm{Ni}-59 \\
\mathrm{Ni}-63 \\
\mathrm{~Pa}-234 \mathrm{~m} \\
\mathrm{Pu}-238 \\
\mathrm{Pu}-239 \\
\mathrm{Pu}-240 \\
\mathrm{Pu}-241 \\
\mathrm{Sm}-151 \\
\mathrm{Sr}-90 \\
\mathrm{TC}-99 \\
\mathrm{Th}-234 \\
\mathrm{U}-238 \\
\mathrm{Y}-90\end{array}$ & $\begin{array}{l}6.984 \mathrm{E}-04 \\
7.629 \mathrm{E}-01 \\
1.021 \mathrm{E}-03 \\
8.160 \mathrm{E}-01 \\
1.217 \mathrm{E}-03 \\
6.053 \mathrm{E}-02 \\
6.984 \mathrm{E}-05 \\
1.563 \mathrm{E}-03 \\
1.606 \mathrm{E}-01 \\
4.490 \mathrm{E}-04 \\
3.791 \mathrm{E}-02 \\
6.286 \mathrm{E}-04 \\
2.793 \mathrm{E}-04 \\
1.633 \mathrm{E}-02 \\
1.167 \mathrm{E}-02 \\
7.264 \mathrm{E}-01 \\
3.625 \mathrm{E}-04 \\
4.490 \mathrm{E}-04 \\
4.490 \mathrm{E}-04 \\
7.264 \mathrm{E}-01\end{array}$ & & $\begin{array}{l}75-09-2 \\
\mathrm{Ag} \\
\mathrm{Ba} \\
\mathrm{Cd} \\
\mathrm{CI}-2-\mathrm{x} \\
\mathrm{Cl}-3-\mathrm{x} \\
\mathrm{Cl}-4-\mathrm{x} \\
\mathrm{Cl}-\mathrm{F}-\mathrm{x} \\
\mathrm{Cr} \\
\mathrm{HC}-\text { insol } \\
\mathrm{HC}-\text { solub } \\
\mathrm{Hg} \\
\mathrm{Pb} \\
\mathrm{Se}\end{array}$ & $\begin{array}{l}2.095 \mathrm{E}-01 \\
3.493 \mathrm{E}-03 \\
1.397 \mathrm{E}-01 \\
6.986 \mathrm{E}-04 \\
2.445 \mathrm{E}-01 \\
8.733 \mathrm{E}-01 \\
4.191 \mathrm{E}-01 \\
3.493 \mathrm{E}-01 \\
5.239 \mathrm{E}-03 \\
3.493 \mathrm{E}+00 \\
1.746 \mathrm{E}-03 \\
6.986 \mathrm{E}-04 \\
3.493 \mathrm{E}-02 \\
1.047 \mathrm{E}-03\end{array}$ \\
\hline \multirow{4}{*}{$\begin{array}{l}\text { Shipp } \\
\text { TotaI }\end{array}$} & ing site: & & \multirow{4}{*}{$\begin{array}{l}\text { Waste Type: } \\
\text { Total Mass }\end{array}$} & Organic Iiquids & (CH-Non-alpha) \\
\hline & Volume $(\mathrm{m} 3 / \mathrm{yr})$ : & $9.054 \mathrm{E}-01$ & & $(\mathrm{~kg} / \mathrm{yr}):$ & 03 \\
\hline & Radionuclide & $\begin{array}{l}\text { Activity } \\
(\text { Ci/yr) }\end{array}$ & & Chemical & $\begin{array}{l}\text { Amount } \\
(\mathrm{kg} / \mathrm{yr})\end{array}$ \\
\hline & $\begin{array}{l}\mathrm{Ba}-137 \mathrm{~m} \\
\mathrm{Co}-60 \\
\mathrm{Cs}-134 \\
\mathrm{Cs}-137 \\
\mathrm{Eu}-154 \\
\mathrm{Eu}-155 \\
\mathrm{Fe}-55 \\
\mathrm{H}-3 \\
\mathrm{Mn}-54 \\
\mathrm{Nb}-94 \\
\mathrm{Ni}-59 \\
\mathrm{Ni}-63 \\
\mathrm{~Pa}-234 \mathrm{~m} \\
\mathrm{Pm}-147 \\
\mathrm{Pu}-238 \\
\mathrm{Pu}-241 \\
\mathrm{Sb}-125 \\
\mathrm{Sm}-151 \\
\mathrm{Sr}-90 \\
\mathrm{Te}-125 \mathrm{~m} \\
\mathrm{Th}-234 \\
\mathrm{U}-238 \\
\mathrm{Y}-90\end{array}$ & $\begin{array}{l}6.061 \mathrm{E}-01 \\
9.790 \mathrm{E}+00 \\
5.756 \mathrm{E}-03 \\
6.400 \mathrm{E}-01 \\
7.788 \mathrm{E}-03 \\
3.724 \mathrm{E}-03 \\
1.280 \mathrm{E}+00 \\
2.788 \mathrm{E}+00 \\
4.063 \mathrm{E}-03 \\
6.095 \mathrm{E}-03 \\
1.280 \mathrm{E}-01 \\
1.720 \mathrm{E}+01 \\
2.404 \mathrm{E}-02 \\
6.975 \mathrm{E}-02 \\
1.997 \mathrm{E}-02 \\
1.625 \mathrm{E}-02 \\
2.709 \mathrm{E}-03 \\
5.418 \mathrm{E}-03 \\
6.027 \mathrm{E}-01 \\
6.772 \mathrm{E}-04 \\
2.404 \mathrm{E}-02 \\
2.404 \mathrm{E}-02 \\
6.027 \mathrm{E}-01\end{array}$ & & $\begin{array}{l}57-12-5 \\
75-09-2 \\
\mathrm{Ag} \\
\mathrm{As} \\
\mathrm{Ba} \\
\mathrm{Cd} \\
\mathrm{Cl}-2-\mathrm{x} \\
\mathrm{Cl}-3-\mathrm{x} \\
\mathrm{Cl}-4-\mathrm{x} \\
\mathrm{Cl}-\mathrm{F}-\mathrm{x} \\
\mathrm{Cr} \\
\mathrm{HC}-\text { insol } \\
\mathrm{HC}-\text { solub } \\
\mathrm{Hg} \\
\mathrm{Pb} \\
\mathrm{Se}\end{array}$ & $\begin{array}{l}5.151 \mathrm{E}-02 \\
7.241 \mathrm{E}-01 \\
2.274 \mathrm{E}-02 \\
3.999 \mathrm{E}-03 \\
2.356 \mathrm{E}-02 \\
5.620 \mathrm{E}-01 \\
4.522 \mathrm{E}-01 \\
4.355 \mathrm{E}+01 \\
9.136 \mathrm{E}+00 \\
8.522 \mathrm{E}-02 \\
5.418 \mathrm{E}-01 \\
8.707 \mathrm{E}+01 \\
1.317 \mathrm{E}+02 \\
1.340 \mathrm{E}-01 \\
1.275 \mathrm{E}-01 \\
1.998 \mathrm{E}-02\end{array}$ \\
\hline
\end{tabular}




\begin{tabular}{l} 
Shipping Site: CNS \\
Total Volume (m3/Yr) \\
Radionuclide \\
\hline Ba-137m \\
Co-60 \\
Cs-134 \\
Cs-137 \\
Eu-154 \\
Eu-155 \\
Fe-55 \\
$\mathrm{H}-3$ \\
$\mathrm{Mn}-54$ \\
$\mathrm{Nb}-94$ \\
$\mathrm{Ni}-59$ \\
$\mathrm{Ni}-63$ \\
$\mathrm{~Pa}-234 \mathrm{~m}$ \\
$\mathrm{Pm}-147$ \\
$\mathrm{Pu}-238$ \\
$\mathrm{Pu}-241$ \\
$\mathrm{Sb}-125$ \\
$\mathrm{Sm}-151$ \\
$\mathrm{Sr}-90$ \\
$\mathrm{Te}-125 \mathrm{~m}$ \\
$\mathrm{Th}-234$ \\
$\mathrm{U}-238$ \\
$\mathrm{Y}-90$
\end{tabular}

Shipping site: MOUND
$9.030 \mathrm{E}-02$

$$
\text { Activity }
$$

$\frac{1 \mathrm{Ci} / \mathrm{y}}{6.218 \mathrm{E}-02}$

1. $004 \mathrm{E}+00$

$6.038 \mathrm{E}-02$

$7.990 \mathrm{E}-04$

3. $821 \mathrm{E}-04$

1. $313 \mathrm{E}-01$

$2.860 \mathrm{E}-01$

$4.169 \mathrm{E}-04$

$6.253 \mathrm{E}-04$

1. $313 \mathrm{E}-02$

$1.765 \mathrm{E}+00$

$2.466 \mathrm{E}-03$

7. $156 \mathrm{E}-03$

$2.049 \mathrm{E}-03$

1. $667 \mathrm{E}-03$

$2.779 \mathrm{E}-04$

$5.558 \mathrm{E}-04$

$5.686 \mathrm{E}-02$

$6.948 \mathrm{E}-05$

$2.466 \mathrm{E}-03$

$2.466 \mathrm{E}-03$

$5.686 \mathrm{E}-02$

Waste Type: Solids (CH-Non-alpha)

Total Mass $(\mathrm{kg} / \mathrm{Yr}): 6.772 \mathrm{E}+01$

\begin{tabular}{lll}
\multicolumn{1}{c}{ Chemical } & & $\begin{array}{c}\text { Amount } \\
(\mathrm{kg} / \mathrm{yr})\end{array}$ \\
\cline { 1 - 1 } $57-12-5$ & $2.357 \mathrm{E}-03$ \\
$75-09-2$ & $2.142 \mathrm{E}-02$ \\
$\mathrm{Ag}$ & $7.345 \mathrm{E}-04$ \\
$\mathrm{AS}$ & $2.549 \mathrm{E}-04$ \\
$\mathrm{Ba}$ & $3.376 \mathrm{E}-02$ \\
$\mathrm{Cd}$ & $2.213 \mathrm{E}-03$ \\
$\mathrm{C} 1-2-\mathrm{x}$ & $2.738 \mathrm{E}-02$ \\
$\mathrm{Cl}-3-\mathrm{x}$ & $2.718 \mathrm{E}-01$ \\
$\mathrm{C} 1-4-\mathrm{x}$ & $7.524 \mathrm{E}-02$ \\
$\mathrm{Cl}-\mathrm{F}-\mathrm{x}$ & $2.847 \mathrm{E}-02$ \\
$\mathrm{Cr}$ & $2.755 \mathrm{E}-02$ \\
$\mathrm{HC}-$ insol & $9.141 \mathrm{E}-01$ \\
$\mathrm{HC}-\mathrm{SOlub}$ & $3.299 \mathrm{E}-01$ \\
$\mathrm{Hg}$ & $3.234 \mathrm{E}-03$ \\
$\mathrm{~Pb}$ & $5.495 \mathrm{E}-02$ \\
$\mathrm{Se}$ & $9.647 \mathrm{E}-04$
\end{tabular}

Waste Type: Organic Liquids ( $\mathrm{CH}-\mathrm{Alpha}$

Total Volume $(\mathrm{m} 3 / \mathrm{yr})$ :

$1.768 \mathrm{E}+00$

Total Mass (kg/yr): $2.190 \mathrm{E}+03$

Radionuclide

$\mathrm{Ba}-137 \mathrm{~m}$

Cs -137

$\mathrm{H}-3$

$\mathrm{Pa}-234 \mathrm{~m}$

Pu-238

Pu-241

Sr -90

$\mathrm{Th}-234$

$\mathrm{U}-238$

$\mathrm{Y}-90$

Shipping Site: MOUND

Total Volume $(\mathrm{m} 3 / \mathrm{yr})$ :

Radionuclide

$\mathrm{Ba}-137 \mathrm{~m}$

Cs -137

$\mathrm{H}-3$

$\mathrm{Pa}-234 \mathrm{~m}$

Pu-238

Pu-241

$\mathrm{Sr}-90$

$\mathrm{Th}-234$

$\mathrm{U}-238$

$\mathrm{Y}-90$

\begin{tabular}{c}
$\begin{array}{c}\text { Activity } \\
(\text { Ci/yr) }\end{array}$ \\
\hline $2.339 \mathrm{E}-02$ \\
$2.450 \mathrm{E}-02$ \\
$1.111 \mathrm{E}+02$ \\
$5.680 \mathrm{E}-03$ \\
$5.569 \mathrm{E}-03$ \\
$1.113 \mathrm{E}-01$ \\
$2.339 \mathrm{E}-02$ \\
$5.680 \mathrm{E}-03$ \\
$5.680 \mathrm{E}-03$ \\
$2.339 \mathrm{E}-02$
\end{tabular}

\begin{tabular}{lc} 
Chemical & $\begin{array}{c}\text { Amount } \\
(\mathrm{kg} / \mathrm{yr})\end{array}$ \\
\cline { 1 - 1 } $75-09-2$ & $2.978 \mathrm{E}+00$ \\
$\mathrm{As}$ & $6.322 \mathrm{E}-03$ \\
$\mathrm{Ba}$ & $2.709 \mathrm{E}-02$ \\
$\mathrm{Cd}$ & $2.409 \mathrm{E}+00$ \\
$\mathrm{Cl}-2-\mathrm{x}$ & $1.574 \mathrm{E}+00$ \\
$\mathrm{Cl}-3-\mathrm{x}$ & $1.718 \mathrm{E}+02$ \\
$\mathrm{Cl}-4-\mathrm{x}$ & $3.827 \mathrm{E}+01$ \\
$\mathrm{C} 1-\mathrm{F}-\mathrm{x}$ & $6.845 \mathrm{E}-02$ \\
$\mathrm{Cr}$ & $2.069 \mathrm{E}+00$ \\
$\mathrm{HC}-$ insol & $3.617 \mathrm{E}+02$ \\
HC-solub & $1.237 \mathrm{E}+02$ \\
$\mathrm{Hg}$ & $1.355 \mathrm{E}-02$ \\
$\mathrm{~Pb}$ & $4.599 \mathrm{E}-01$
\end{tabular}

Waste Type: Solids (CH-Alpha)

Total Mass $(\mathrm{kg} / \mathrm{yr}): 6.176 \mathrm{E}+00$

\begin{tabular}{|c|c|}
\hline Chemical & $\begin{array}{l}\text { Amount } \\
\left(\mathrm{kg} / \mathrm{y}^{\mathrm{r}}\right)\end{array}$ \\
\hline $75-09-2$ & $1.926 \mathrm{E}-02$ \\
\hline $\mathrm{Ag}$ & $1.191 \mathrm{E}-04$ \\
\hline $\mathrm{Ba}$ & $2.561 \mathrm{E}-04$ \\
\hline $\mathrm{Cd}$ & $2.102 \mathrm{E}-05$ \\
\hline CI $-2-x$ & $9.886 \mathrm{E}-03$ \\
\hline CI $-3-x$ & $9.601 \mathrm{E}-02$ \\
\hline$C I-4-x$ & $7.627 \mathrm{E}-02$ \\
\hline $\mathrm{Cl}-\mathrm{F}-\mathrm{x}$ & $6.403 E-04$ \\
\hline $\mathrm{Cr}$ & $4.674 E-05$ \\
\hline HC-insol & $2.423 E-01$ \\
\hline HC-solub & $1.132 \mathrm{E}-01$ \\
\hline $\mathrm{Hg}$ & $1.951 E-05$ \\
\hline $\mathrm{Pb}$ & $9.361 \mathrm{E}-05$ \\
\hline $\mathrm{Se}$ & $1.921 \mathrm{E}-06$ \\
\hline
\end{tabular}




\begin{tabular}{l} 
Shipping Site: NNS \\
Total Volume (m3/yr): \\
Radionuclide \\
\hline Ba-137m \\
Co-60 \\
Cs-134 \\
Cs-137 \\
Eu-154 \\
Eu-155 \\
Fe-55 \\
H-3 \\
Mn-54 \\
Nb-94 \\
Ni-59 \\
Ni-63 \\
Pa-234m \\
Pm-147 \\
Pu-238 \\
Pu-241 \\
Sb-125 \\
Sm-151 \\
Sr-90 \\
Te-125m \\
Th-234 \\
U-238 \\
Y-90 \\
T
\end{tabular}

Shipping site: UMC

Total Volume (m3/yr):

Radionuclide

$\mathrm{Am}-241$

$\mathrm{Cm}-244$

$\mathrm{Pa}-234 \mathrm{~m}$

Pu-238

Pu-239

Pu-240

$\mathrm{Pu}-241$

Th-234

$\mathrm{v}-238$

Shipping Site: WVDP

Total Volume (m3/yr):

Radionuclide

Am-241

$\mathrm{Ba}-137 \mathrm{~m}$

Co-60

Cs -137

Eu-154

$\mathrm{H}-3$

$\mathrm{Nb}-94$

$\mathrm{Ni}-59$

$\mathrm{Ni}-63$

$\mathrm{Pa}-234 \mathrm{~m}$

$\mathrm{Pu}-238$

Pu-239

Pu-240

Pu-24I

$\mathrm{sm}-151$

$\mathrm{Sr}-90$

TC-99

Th-234

$\mathrm{U}-238$

$\mathrm{Y}-90$
3. $560 \pm-01$

Total Mass $(\mathrm{kg} / \mathrm{Yr}): 3.925 \mathrm{E}+02$

\begin{tabular}{|c|c|}
\hline Chemical & $\begin{array}{l}\text { Amount } \\
(\mathrm{kg} / \mathrm{yr})\end{array}$ \\
\hline $75-09-2$ & $1.041 \mathrm{E}-01$ \\
\hline $\mathrm{Ag}$ & $3.925 \mathrm{E}-03$ \\
\hline As & $2.726 \mathrm{E}-03$ \\
\hline $\mathrm{Ba}$ & $1.110 \mathrm{E}-01$ \\
\hline $\mathrm{cd}$ & $3.955 E-02$ \\
\hline$C 1-2-x$ & $1.105 \mathrm{E}-01$ \\
\hline C1-3-x & $4.102 \mathrm{E}+00$ \\
\hline$C l-4-x$ & $7.996 \mathrm{E}-01$ \\
\hline$C 1-F-x$ & $9.360 \mathrm{E}-02$ \\
\hline $\mathrm{Cr}$ & $1.488 \mathrm{E}-01$ \\
\hline $\mathrm{HC}$-insol & $1.378 \mathrm{E}+01$ \\
\hline HC-solub & $6.570 E+00$ \\
\hline $\mathrm{Hg}$ & $4.448 E-02$ \\
\hline $\mathrm{Pb}$ & $7.211 \mathrm{E}-02$ \\
\hline $\mathrm{Se}$ & $1.201 \mathrm{E}-02$ \\
\hline
\end{tabular}

Waste Type: Solids (CH-Alpha)

Total Mass $(\mathrm{kg} / \mathrm{Yr}): \quad 5.809 \mathrm{E}+01$

$3.944 \mathrm{E}-02$

Activity

\begin{tabular}{c} 
(Ci/yr) \\
\hline $.743 \mathrm{E}-07$ \\
$2.358 \mathrm{E}-07$ \\
$8.511 \mathrm{E}-07$ \\
$.108 \mathrm{E}-05$ \\
$.331 \mathrm{E}-06$ \\
$3.260 \mathrm{E}-05$ \\
$8.894 \mathrm{E}-04$ \\
$8.511 \mathrm{E}-07$ \\
$8.511 \mathrm{E}-07$
\end{tabular}

\begin{tabular}{l} 
Chemical \\
\hline $57-12-5$ \\
$75-09-2$ \\
$\mathrm{Ag}$ \\
$\mathrm{As}$ \\
$\mathrm{Ba}$ \\
$\mathrm{Cd}$ \\
$\mathrm{Cl}-2-\mathrm{x}$ \\
$\mathrm{Cl}-3-\mathrm{x}$ \\
$\mathrm{Cl}-4-\mathrm{x}$ \\
$\mathrm{Cl}-\mathrm{F}-\mathrm{x}$ \\
$\mathrm{Cr}$ \\
$\mathrm{HC}-$ insol \\
$\mathrm{HC}-$ Solub \\
$\mathrm{Hg}$ \\
$\mathrm{Pb}$ \\
$\mathrm{Se}$
\end{tabular}

\begin{tabular}{c}
$\begin{array}{c}\text { Amount } \\
(\mathrm{kg} / \mathrm{yr})\end{array}$ \\
\hline $9.876 \mathrm{E}-03$ \\
$1.161 \mathrm{E}-02$ \\
$1.742 \mathrm{E}-03$ \\
$5.809 \mathrm{E}-04$ \\
$1.161 \mathrm{E}-01$ \\
$2.904 \mathrm{E}-04$ \\
$1.161 \mathrm{E}-02$ \\
$3.253 \mathrm{E}-02$ \\
$2.033 \mathrm{E}-02$ \\
$5.809 \mathrm{E}-03$ \\
$1.161 \mathrm{E}-01$ \\
$5.809 \mathrm{E}-02$ \\
$2.904 \mathrm{E}-03$ \\
$5.809 \mathrm{E}-03$ \\
$2.904 \mathrm{E}-01$ \\
$1.742 \mathrm{E}-03$
\end{tabular}

Waste Type: Organic Liquids (CH-Alpha)

Total Mass $(\mathrm{kg} / \mathrm{Yr}): 1.897 E+03$

\begin{tabular}{l} 
ChemicaI \\
\hline $57-12-5$ \\
$75-09-2$ \\
Ag \\
As \\
Ba \\
Cd \\
Cl-2-x \\
CI-3-x \\
Cl-4-x \\
CI-F-x \\
CI \\
HC-insol \\
HC-solub \\
Hg \\
Pb \\
Se
\end{tabular}

\begin{tabular}{c}
$\begin{array}{c}\text { Amount } \\
(\mathrm{kg} / \mathrm{yr})\end{array}$ \\
\hline $1.600 \mathrm{E}-01$ \\
$2.840 \mathrm{E}-01$ \\
$7.866 \mathrm{E}-02$ \\
$9.986 \mathrm{E}-03$ \\
$6.783 \mathrm{E}-02$ \\
$1.250 \mathrm{E}-01$ \\
$4.212 \mathrm{E}-01$ \\
$2.491 \mathrm{E}+01$ \\
$3.373 \mathrm{E}+00$ \\
$2.744 \mathrm{E}-01$ \\
$2.914 \mathrm{E}-01$ \\
$1.686 \mathrm{E}+01$ \\
$3.228 \mathrm{E}+02$ \\
$4.473 \mathrm{E}-01$ \\
$9.175 \mathrm{E}-02$ \\
$6.881 \mathrm{E}-02$
\end{tabular}


Shipping site: WVDP

Total Volume $(\mathrm{m} 3 / \mathrm{yr})$ :

Radionuclide

Am-241

$\mathrm{Ba}-137 \mathrm{~m}$

Co-60

Cs -137

Eu -1.54

$\mathrm{H}-3$

$\mathrm{Nb}-94$

$\mathrm{Ni}-59$

$\mathrm{Ni}-63$

$\mathrm{Pa}-234 \mathrm{~m}$

$\mathrm{Pu}-238$

Pu-239

Pu-240

$\mathrm{Pu}-241$

$\mathrm{Sm}-151$

Sr-90

TC- 99

Th-234

$\mathrm{U}-238$

$Y-90$
Waste Type: Solids (CH-Alpha)
$8.473 E-01$
Total Mass (kg/yr):
$1.355 \mathrm{E}+03$

\begin{tabular}{c}
$\begin{array}{c}\text { Activity } \\
\text { (Ci/yr) }\end{array}$ \\
\hline $7.096 \mathrm{E}-04$ \\
$7.751 \mathrm{E}-01$ \\
$1.037 \mathrm{E}-03$ \\
$8.243 \mathrm{E}-01$ \\
$1.236 \mathrm{E}-03$ \\
$6.150 \mathrm{E}-02$ \\
$7.096 \mathrm{E}-05$ \\
$1.588 \mathrm{E}-03$ \\
$1.632 \mathrm{E}-01$ \\
$4.561 \mathrm{E}-04$ \\
$3.852 \mathrm{E}-02$ \\
$6.386 \mathrm{E}-04$ \\
$2.838 \mathrm{E}-04$ \\
$1.659 \mathrm{E}-02$ \\
$1.186 \mathrm{E}-02$ \\
$7.339 \mathrm{E}-01$ \\
$3.662 \mathrm{E}-04$ \\
$4.561 \mathrm{E}-04$ \\
$4.561 \mathrm{E}-04$ \\
$7.339 \mathrm{E}-01$
\end{tabular}

\begin{tabular}{c}
$\begin{array}{c}\text { Amount } \\
(\mathrm{kg} / \mathrm{yr})\end{array}$ \\
\hline $1.069 \mathrm{E}-03$ \\
$2.958 \mathrm{E}-01$ \\
$1.709 \mathrm{E}-02$ \\
$1.081 \mathrm{E}-02$ \\
$3.475 \mathrm{E}-01$ \\
$1.558 \mathrm{E}-01$ \\
$2.803 \mathrm{E}-01$ \\
$1.573 \mathrm{E}+01$ \\
$2.998 \mathrm{E}+00$ \\
$1.176 \mathrm{E}-01$ \\
$5.922 \mathrm{E}-01$ \\
$5.230 \mathrm{E}+01$ \\
$2.619 \mathrm{E}+01$ \\
$1.760 \mathrm{E}-01$ \\
$2.784 \mathrm{E}-01$ \\
$4.680 \mathrm{E}-02$
\end{tabular}




\section{A.3 WM LLMW REGIONALIZED 2 ALTERNATIVE (CASE 7)}

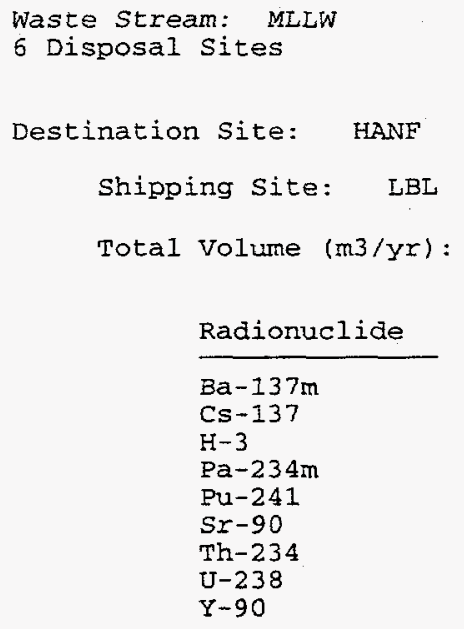

Alternative: 7 Regional 7 Treatment Sites Waste Type: Organic Liquids (CH-Non-alpha)

$3.230 \mathrm{E}-01$ Total Mass $(\mathrm{kg} / \mathrm{yr}): 3.100 \mathrm{E}+02$

\begin{tabular}{c}
$\begin{array}{c}\text { Activity } \\
\text { (Ci/yr) }\end{array}$ \\
\hline $4.273 \mathrm{E}-03$ \\
$4.476 \mathrm{E}-03$ \\
$2.032 \mathrm{E}+01$ \\
$1.037 \mathrm{E}-03$ \\
$1.017 \mathrm{E}-03$ \\
$4.273 \mathrm{E}-03$ \\
$1.037 \mathrm{E}-03$ \\
$1.037 \mathrm{E}-03$ \\
$4.273 \mathrm{E}-03$
\end{tabular}

Waste Type: Solids (CH-Non-alpha)

Total Mass $(\mathrm{kg} / \mathrm{yr}): 4.402 \mathrm{E}+03$

\begin{tabular}{|c|c|}
\hline Chemical & $\begin{array}{l}\text { Amount } \\
(\mathrm{kg} / \mathrm{Yr})\end{array}$ \\
\hline $57-12-5$ & $5.503 \mathrm{E}-01$ \\
\hline $75-09-2$ & $1.116 \mathrm{E}+01$ \\
\hline $\mathrm{Ag}$ & $1.516 \mathrm{E}-01$ \\
\hline As & $3.237 E-02$ \\
\hline $\mathrm{Ba}$ & $6.474 \mathrm{E}+00$ \\
\hline $\mathrm{cd}$ & $1.754 \mathrm{E}-02$ \\
\hline$C 1-2-x$ & $5.905 E+00$ \\
\hline$c 1-3-x$ & $5.439 \mathrm{E}+01$ \\
\hline$c 1-4-x$ & $4.320 \mathrm{E}+01$ \\
\hline$C l-F-x$ & $3.237 \mathrm{E}-01$ \\
\hline $\mathrm{Cr}$ & $6.485 \mathrm{E}+00$ \\
\hline HC-insol & $1.314 \mathrm{E}+02$ \\
\hline HC-solub & $6.326 \mathrm{E}+01$ \\
\hline $\mathrm{Hg}$ & $3.242 \mathrm{E}-01$ \\
\hline $\mathrm{Pb}$ & $1.619 E+0 I$ \\
\hline $\mathrm{Se}$ & $9.711 \mathrm{E}-02$ \\
\hline
\end{tabular}


Shipping Site: LERHR

Total Volume $(\mathrm{m} 3 / \mathrm{yr})$ :

Radionuclide

Am-241

$\mathrm{Ba}-137 \mathrm{~m}$

$\mathrm{Co}-60$

Cs -137

Eu-154

Eu-155

$\mathrm{H}-3$

$\mathrm{Nb}-94$

$\mathrm{Ni}-59$

$\mathrm{Ni}-63$

$\mathrm{Pa}-234 \mathrm{~m}$

$\mathrm{Pu}-238$

Pu-239

Pu-240

Pu-241

$\mathrm{Sm}-151$

$\mathrm{Sr}-90$

TC-99

Th -232

Th-234

$\mathrm{U}-238$

$\mathrm{Y}-90$

Shipping Site: LERHR

Total Volume (m3/yr):

Radionuclide

Am-241

$\mathrm{Ba}-137 \mathrm{~m}$

Co-60

Cs -137

Eu-154

Eu-155

$\mathrm{H}-3$

$\mathrm{Nb}-94$

Ni-59

Ni-63

$\mathrm{Pa}-234 \mathrm{~m}$

Pu-238

$\mathrm{Pu}-239$

Pu-240.

Pu-241

$\mathrm{Sm}-151$

Sr-90

TC-99

Th-232

Th-234

$\mathrm{U}-238$

$Y-90$
$5.020 E-03$

Waste Type: Organic Liquids (CH-Non-alpha)

Total Mass (kg/Yr): $5.750 \mathrm{E}+00$

\begin{tabular}{c}
$\begin{array}{r}\text { Activity } \\
\text { (Ci/yI) }\end{array}$ \\
\hline $2.470 E-07$ \\
$3.536 E-04$ \\
$1.353 E-04$ \\
$3.888 E-04$ \\
$1.440 E-06$ \\
$4.940 E-07$ \\
$2.801 E-05$ \\
$1.029 E-06$ \\
$2.183 E-05$ \\
$2.297 E-03$ \\
$4.976 E-05$ \\
$1.529 E-05$ \\
$2.470 E-07$ \\
$3.705 E-07$ \\
$1.620 E-05$ \\
$4.688 E-06$ \\
$3.438 E-04$ \\
$4.112 E-06$ \\
$4.116 E-07$ \\
$4.976 E-05$ \\
$4.976 E-05$ \\
$3.438 E-04$
\end{tabular}

\begin{tabular}{l} 
Chemical \\
\hline $57-12-5$ \\
$75-09-2$ \\
$\mathrm{Ag}$ \\
$\mathrm{AS}$ \\
$\mathrm{Ba}$ \\
$\mathrm{Cd}$ \\
$\mathrm{Cl}-2-\mathrm{x}$ \\
$\mathrm{Cl}-3-\mathrm{x}$ \\
$\mathrm{Cl}-4-\mathrm{x}$ \\
$\mathrm{Cl}-\mathrm{F}-\mathrm{x}$ \\
$\mathrm{Cr}$ \\
$\mathrm{HC}-\mathrm{insO}$ \\
$\mathrm{HC}-\mathrm{SOl}$ - \\
$\mathrm{Hg}$ \\
$\mathrm{Pb}$ \\
$\mathrm{Se}$
\end{tabular}

\begin{tabular}{c}
$\begin{array}{c}\text { Amount } \\
(\mathrm{kg} / \mathrm{yr})\end{array}$ \\
\hline $2.373 \mathrm{E}-04$ \\
$4.324 \mathrm{E}-03$ \\
$1.189 \mathrm{E}-04$ \\
$2.403 \mathrm{E}-05$ \\
$1.415 \mathrm{E}-04$ \\
$3.358 \mathrm{E}-03$ \\
$2.702 \mathrm{E}-03$ \\
$2.592 \mathrm{E}-01$ \\
$5.439 \mathrm{E}-02$ \\
$5.108 \mathrm{E}-04$ \\
$3.156 \mathrm{E}-03$ \\
$5.198 \mathrm{E}-01$ \\
$6.473 \mathrm{E}-01$ \\
$6.928 \mathrm{E}-04$ \\
$7.432 \mathrm{E}-04$ \\
$1.039 \mathrm{E}-04$
\end{tabular}

Waste Type: Solids (CH-Non-alpha)

Total Mass $(\mathrm{kg} / \mathrm{Yr}): 2.492 \mathrm{E}+02$

\section{$3.442 \mathrm{E}-01$}

\begin{tabular}{l} 
Chemical \\
\hline $57-12-5$ \\
$75-09-2$ \\
$\mathrm{Ag}$ \\
$\mathrm{As}$ \\
$\mathrm{Ba}$ \\
$\mathrm{Cd}$ \\
$\mathrm{Cl}-2-\mathrm{x}$ \\
$\mathrm{Cl}-3-\mathrm{x}$ \\
$\mathrm{Cl}-4-\mathrm{x}$ \\
$\mathrm{Cl}-\mathrm{F}-\mathrm{x}$ \\
$\mathrm{Cr}$ \\
$\mathrm{HC}-$ insol \\
$\mathrm{HC}-\mathrm{Solub}$ \\
$\mathrm{Hg}$ \\
$\mathrm{Pb}$ \\
$\mathrm{Se}$
\end{tabular}

\begin{tabular}{c}
$\begin{array}{c}\text { Amount } \\
(\mathrm{kg} / \mathrm{Yr})\end{array}$ \\
\hline $4.707 \mathrm{E}-04$ \\
$1.438 \mathrm{E}-01$ \\
$2.547 \mathrm{E}-03$ \\
$1.464 \mathrm{E}-05$ \\
$3.843 \mathrm{E}-02$ \\
$8.851 \mathrm{E}-03$ \\
$1.556 \mathrm{E}-01$ \\
$6.005 \mathrm{E}-01$ \\
$2.831 \mathrm{E}+00$ \\
$1.391 \mathrm{E}-01$ \\
$4.652 \mathrm{E}-03$ \\
$1.675 \mathrm{E}+00$ \\
$3.579 \mathrm{E}-01$ \\
$5.762 \mathrm{E}-03$ \\
$1.542 \mathrm{E}-02$ \\
$4.361 \mathrm{E}-04$
\end{tabular}




\begin{tabular}{l} 
Shipping Site: LLNL \\
Total Volume (m3/Yr): \\
Radionuclide \\
\hline Ba-137m \\
Cs -137 \\
H-3 \\
Pa-234m \\
Pu-241 \\
Sr -90 \\
Th-234 \\
U-238 \\
Y -90
\end{tabular}

Shipping Site: LLNL Total Volume (m3/yr):

Radionuclide

$\mathrm{Ba}-137 \mathrm{~m}$

Cs-137

$\mathrm{H}-3$

$\mathrm{Pa}-234 \mathrm{~m}$

$\mathrm{Pu}-241$

$\mathrm{Sr}-90$

Th-234

$\mathrm{U}-238$

$Y-90$

\begin{tabular}{c}
$7.727 \mathrm{E}+01$ \\
$\begin{array}{r}\text { Activity } \\
(\mathrm{Ci} / \mathrm{yr})\end{array}$ \\
\hline $1.022 \mathrm{E}+00$ \\
$1.071 \mathrm{E}+00$ \\
$4.863 \mathrm{E}+03$ \\
$2.482 \mathrm{E}-01$ \\
$2.434 \mathrm{E}-01$ \\
$1.022 \mathrm{E}+00$ \\
$2.482 \mathrm{E}-01$ \\
$2.482 \mathrm{E}-01$ \\
$1.022 \mathrm{E}+00$
\end{tabular}

Waste Type: Organic Liquids (CH-Non-alpha) Total Mass $(\mathrm{kg} / \mathrm{Yr})=8.159 \mathrm{E}+04$

\begin{tabular}{lcc} 
Chemical & $\begin{array}{c}\text { Amount } \\
(\mathrm{kg} / \mathrm{yr})\end{array}$ \\
\cline { 1 - 1 } $57-12-5$ & $7.162 \mathrm{E}+00$ \\
$75-09-2$ & $5.434 \mathrm{E}+00$ \\
$\mathrm{Ag}$ & $3.590 \mathrm{E}+00$ \\
$\mathrm{As}$ & $4.598 \mathrm{E}-01$ \\
$\mathrm{Ba}$ & $3.135 \mathrm{E}+00$ \\
$\mathrm{Cd}$ & $1.949 \mathrm{E}-01$ \\
$\mathrm{Cl}-2-\mathrm{x}$ & $1.546 \mathrm{E}+01$ \\
$\mathrm{Cl}-3-\mathrm{x}$ & $6.087 \mathrm{E}+02$ \\
$\mathrm{Cl}-4-\mathrm{X}$ & $3.439 \mathrm{E}+01$ \\
$\mathrm{Cl}-\mathrm{F}-\mathrm{x}$ & $1.254 \mathrm{E}+01$ \\
$\mathrm{Cr}$ & $8.355 \mathrm{E}+00$ \\
$\mathrm{HC}-$ insol & $5.003 \mathrm{E}+02$ \\
$\mathrm{HC}-$ solub & $1.434 \mathrm{E}+04$ \\
$\mathrm{Hg}$ & $2.033 \mathrm{E}+01$ \\
$\mathrm{~Pb}$ & $3.118 \mathrm{E}+00$ \\
$\mathrm{Se}$ & $3.138 \mathrm{E}+00$
\end{tabular}

Waste Type: Solids (CH-Non-alpha)

4.978E+01 Total Mass (kg/yr): $6.833 \mathrm{E}+04$

\begin{tabular}{c}
$\begin{array}{c}\text { Activity } \\
(\mathrm{Ci} / \mathrm{yr})\end{array}$ \\
\hline $6.586 \mathrm{E}-01$ \\
$6.899 \mathrm{E}-01$ \\
$3.132 \mathrm{E}+03$ \\
$1.599 \mathrm{E}-01$ \\
$1.568 \mathrm{E}-01$ \\
$6.586 \mathrm{E}-01$ \\
$1.599 \mathrm{E}-01$ \\
$1.599 \mathrm{E}-01$ \\
$6.586 \mathrm{E}-01$
\end{tabular}

\begin{tabular}{l} 
Chemical \\
\hline $57-12-5$ \\
$75-09-2$ \\
Ag \\
As \\
Ba \\
Cd \\
Cl-2-x \\
C1-3-x \\
C1-4-x \\
Cl-F-x \\
Cr \\
HC-insol \\
HC-Solub \\
Hg \\
Pb \\
Se
\end{tabular}

\begin{tabular}{l}
$\begin{array}{c}\text { Amount } \\
(\mathrm{kg} / \mathrm{Yr})\end{array}$ \\
\hline $8.172 \mathrm{E}+00$ \\
$8.995 \mathrm{E}+00$ \\
$3.852 \mathrm{E}+00$ \\
$1.401 \mathrm{E}+00$ \\
$1.097 \mathrm{E}+02$ \\
$2.705 \mathrm{E}+00$ \\
$7.372 \mathrm{E}+00$ \\
$1.900 \mathrm{E}+02$ \\
$3.100 \mathrm{E}+01$ \\
$5.418 \mathrm{E}+00$ \\
$1.262 \mathrm{E}+02$ \\
$7.525 \mathrm{E}+02$ \\
$3.756 \mathrm{E}+02$ \\
$4.188 \mathrm{E}+01$ \\
$1.529 \mathrm{E}+02$ \\
$1.787 \mathrm{E}+01$
\end{tabular}

Shipping Site: MINS Waste Type: Solids (CH-Non-alpha)

Total Volume $(\mathrm{m} 3 / \mathrm{y} r)$ : 3. $647 \mathrm{E}+00$ Total Mass (kg/yr): $3.545 \mathrm{E}+03$

\begin{tabular}{l} 
Radionuclide \\
\hline $\mathrm{Ba}-137 \mathrm{~m}$ \\
$\mathrm{Co}-60$ \\
$\mathrm{Cs}-134$ \\
$\mathrm{Cs}-137$ \\
$\mathrm{Eu}-154$ \\
$\mathrm{Eu}-155$ \\
$\mathrm{Fe}-55$ \\
$\mathrm{H}-3$ \\
$\mathrm{Mn}-54$ \\
$\mathrm{Nb}-94$ \\
$\mathrm{Ni}-59$ \\
$\mathrm{Ni}-63$ \\
$\mathrm{~Pa}-234 \mathrm{~m}$ \\
$\mathrm{Pm}-147$ \\
$\mathrm{Pu}-238$ \\
$\mathrm{Pu}-241$ \\
$\mathrm{Sb}-125$ \\
$\mathrm{Sm}-151$ \\
$\mathrm{Sr}-90$ \\
$\mathrm{Te}-125 \mathrm{~m}$ \\
$\mathrm{Th}-234$ \\
$\mathrm{U}-238$ \\
$\mathrm{Y}-90$
\end{tabular}

\begin{tabular}{r}
$\begin{array}{r}\text { Activity } \\
(\mathrm{Ci} / \mathrm{Y})\end{array}$ \\
\hline $2.441 \mathrm{E}+00$ \\
$3.943 \mathrm{E}+01$ \\
$2.318 \mathrm{E}-02$ \\
$2.578 \mathrm{E}+00$ \\
$3.137 \mathrm{E}-02$ \\
$1.500 \mathrm{E}-02$ \\
$5.156 \mathrm{E}+00$ \\
$1.123 \mathrm{E}+01$ \\
$1.636 \mathrm{E}-02$ \\
$2.455 \mathrm{E}-02$ \\
$5.156 \mathrm{E}-01$ \\
$6.932 \mathrm{E}+01$ \\
$9.684 \mathrm{E}-02$ \\
$2.809 \mathrm{E}-01$ \\
$8.047 \mathrm{E}-02$ \\
$6.547 \mathrm{E}-02$ \\
$1.091 \mathrm{E}-02$ \\
$2.182 \mathrm{E}-02$ \\
$2.427 \mathrm{E}+00$ \\
$2.728 \mathrm{E}-03$ \\
$9.684 \mathrm{E}-02$ \\
$9.684 \mathrm{E}-02$ \\
$2.427 \mathrm{E}+00$
\end{tabular}

\begin{tabular}{c}
$\begin{array}{c}\text { Amount } \\
(\mathrm{kg} / \mathrm{yr})\end{array}$ \\
\hline $5.544 \mathrm{E}-01$ \\
$1.457 \mathrm{E}-02$ \\
$7.197 \mathrm{E}-03$ \\
$2.427 \mathrm{E}+00$ \\
$1.150 \mathrm{E}-01$ \\
$5.975 \mathrm{E}-01$ \\
$1.190 \mathrm{E}+01$ \\
$2.652 \mathrm{E}+00$ \\
$6.891 \mathrm{E}-01$ \\
$7.414 \mathrm{E}-01$ \\
$4.047 \mathrm{E}+01$ \\
$1.726 \mathrm{E}+01$ \\
$5.165 \mathrm{E}-01$ \\
$4.557 \mathrm{E}-01$ \\
$3.325 \mathrm{E}-02$
\end{tabular}

\begin{tabular}{l} 
Chemical \\
\hline $75-09-2$ \\
$\mathrm{Ag}$ \\
$\mathrm{As}$ \\
$\mathrm{Ba}$ \\
$\mathrm{Cd}$ \\
$\mathrm{Cl}-2-\mathrm{x}$ \\
$\mathrm{Cl}-3-\mathrm{x}$ \\
$\mathrm{Cl}-4-\mathrm{x}$ \\
$\mathrm{CI}-\mathrm{F}-\mathrm{x}$ \\
$\mathrm{Cr}$ \\
$\mathrm{HC}-$ insol \\
$\mathrm{HC}-$ Solub \\
$\mathrm{Hg}$ \\
$\mathrm{Pb}$ \\
$\mathrm{Se}$
\end{tabular}


$A-31$

Shipping Site: PHNS Waste Type: Organic Liquids (CH-Non-alpha)

Total Volume $(\mathrm{m} 3 / \mathrm{yr})$ :

$1.736 \mathrm{E}-03$

Total Mass (kg/yr):

$1.979 E+00$

Radionuclide

$\mathrm{Ba}-137 \mathrm{~m}$

$\mathrm{Co}-60$

Cs- 134

Cs-137

Eu-154

Eu-155

Fe -55

$\mathrm{H}-3$

Mn -54

$\mathrm{Nb}-94$

$\mathrm{Ni}-59$

$\mathrm{Ni}-63$

$\mathrm{Pa}-234 \mathrm{~m}$

$\mathrm{Pm}-147$

Pu-238

Pu-241

$\mathrm{Sb}-125$

$\mathrm{Sm}-151$

S $r-90$

$\mathrm{Te}-125 \mathrm{~m}$

Th-234

$\mathrm{U}-238$

$Y-90$

Shipping Site: PHNS

Total volume (m3/yr):

Radionuclide

$\mathrm{Ba}-137 \mathrm{~m}$

Co-60

Cs-134

Cs -137

Eu-154

Eu-155

$\mathrm{Fe}-55$

H-3

$\mathrm{Mn}-54$

$\mathrm{Nb}-94$

$\mathrm{Ni}-59$

$\mathrm{Ni}-63$

$\mathrm{Pa}-234 \mathrm{~m}$

Pm-147

Pu-238

Pu-241

Sb-125

Sm-151

Sr -90

$\mathrm{Te}-125 \mathrm{~m}$

Th-234

$\mathrm{U}-238$

$Y-90$

\begin{tabular}{c}
$\begin{array}{c}\text { Activity } \\
\text { (Ci/yr) }\end{array}$ \\
\hline $1.162 \mathrm{E}-03$ \\
$1.877 \mathrm{E}-02$ \\
$1.103 \mathrm{E}-05$ \\
$1.227 \mathrm{E}-03$ \\
$1.493 \mathrm{E}-05$ \\
$7.141 \mathrm{E}-06$ \\
$2.454 \mathrm{E}-03$ \\
$5.346 \mathrm{E}-03$ \\
$7.791 \mathrm{E}-06$ \\
$1.168 \mathrm{E}-05$ \\
$2.454 \mathrm{E}-04$ \\
$3.299 \mathrm{E}-02$ \\
$4.609 \mathrm{E}-05$ \\
$1.337 \mathrm{E}-04$ \\
$3.830 \mathrm{E}-05$ \\
$3.116 \mathrm{E}-05$ \\
$5.194 \mathrm{E}-06$ \\
$1.038 \mathrm{E}-05$ \\
$1.155 \mathrm{E}-03$ \\
$1.298 \mathrm{E}-06$ \\
$4.609 \mathrm{E}-05$ \\
$4.609 \mathrm{E}-05$ \\
$1.155 \mathrm{E}-03$
\end{tabular}

\begin{tabular}{l} 
Chemical \\
\hline $75-09-2$ \\
$\mathrm{Ag}$ \\
$\mathrm{AS}$ \\
$\mathrm{Ba}$ \\
$\mathrm{Cl}-2-\mathrm{x}$ \\
$\mathrm{Cl}-3-\mathrm{x}$ \\
$\mathrm{Cl}-4-\mathrm{x}$ \\
$\mathrm{Cl}-\mathrm{F}-\mathrm{x}$ \\
$\mathrm{HC}-$ insol \\
$\mathrm{HC}-$ solub \\
$\mathrm{Hg}$ \\
$\mathrm{Pb}$ \\
$\mathrm{Se}$
\end{tabular}

\begin{tabular}{c}
$\begin{array}{c}\text { Amount } \\
(\mathrm{kg} / \mathrm{yr})\end{array}$ \\
\hline $2.572 \mathrm{E}-04$ \\
$8.707 \mathrm{E}-05$ \\
$2.176 \mathrm{E}-05$ \\
$1.484 \mathrm{E}-04$ \\
$7.322 \mathrm{E}-04$ \\
$2.731 \mathrm{E}-02$ \\
$1.543 \mathrm{E}-03$ \\
$5.937 \mathrm{E}-04$ \\
$2.968 \mathrm{E}-03$ \\
$7.718 \mathrm{E}-04$ \\
$4.353 \mathrm{E}-04$ \\
$5.343 \mathrm{E}-05$ \\
$7.322 \mathrm{E}-05$
\end{tabular}

Waste Type: Solids (CH-Non-alpha)

$2.962 \mathrm{E}-01$

Total Mass (kg/yr):

$4.406 \mathrm{E}+02$

\begin{tabular}{c}
$\begin{array}{c}\text { Activity } \\
\text { (Ci/yr) }\end{array}$ \\
\hline $1.983 \mathrm{E}-01$ \\
$3.204 \mathrm{E}+00$ \\
$1.883 \mathrm{E}-03$ \\
$2.094 \mathrm{E}-01$ \\
$2.548 \mathrm{E}-03$ \\
$1.218 \mathrm{E}-03$ \\
$4.188 \mathrm{E}-01$ \\
$9.124 \mathrm{E}-01$ \\
$1.329 \mathrm{E}-03$ \\
$1.994 \mathrm{E}-03$ \\
$4.188 \mathrm{E}-02$ \\
$5.631 \mathrm{E}+00$ \\
$7.867 \mathrm{E}-03$ \\
$2.282 \mathrm{E}-02$ \\
$6.538 \mathrm{E}-03$ \\
$5.319 \mathrm{E}-03$ \\
$8.865 \mathrm{E}-04$ \\
$1.773 \mathrm{E}-03$ \\
$1.972 \mathrm{E}-01$ \\
$2.216 \mathrm{E}-04$ \\
$7.867 \mathrm{E}-03$ \\
$7.867 \mathrm{E}-03$ \\
$1.972 \mathrm{E}-01$
\end{tabular}

\begin{tabular}{c}
$\begin{array}{c}\text { Amount } \\
(\mathrm{kg} / \mathrm{yr})\end{array}$ \\
\hline $4.073 \mathrm{E}-03$ \\
$5.831 \mathrm{E}-02$ \\
$2.691 \mathrm{E}-03$ \\
$1.603 \mathrm{E}-03$ \\
$1.038 \mathrm{E}-01$ \\
$2.472 \mathrm{E}-02$ \\
$6.160 \mathrm{E}-02$ \\
$2.088 \mathrm{E}+00$ \\
$4.422 \mathrm{E}-01$ \\
$6.398 \mathrm{E}-02$ \\
$7.805 \mathrm{E}-01$ \\
$6.908 \mathrm{E}+00$ \\
$3.289 \mathrm{E}+00$ \\
$2.617 \mathrm{E}-02$ \\
$1.563 \mathrm{E}-01$ \\
$6.731 \mathrm{E}-03$
\end{tabular}


Shipping Site: PSNS Waste Type: Organic Liquids (CH-Non-alpha) Total Volume $(\mathrm{m} 3 / \mathrm{yr})$ : $3.990 \mathrm{E}-02$ Total Mass $(\mathrm{kg} / \mathrm{yr}): 5.986 \mathrm{E}+01$

Radionuclide

$\mathrm{Ba}-137 \mathrm{~m}$

Co- 60

Cs -134

Cs -137

Eu-154

Eu-155

$\mathrm{Fe}-55$

$\mathrm{H}-3$

$\mathrm{Mn}-54$

$\mathrm{Nb}-94$

$\mathrm{Ni}-59$

$\mathrm{Ni}-63$

$\mathrm{Pa}-234 \mathrm{~m}$

$\mathrm{Pm}-147$

Pu-238

Pu-241

Sb-125

Sm-151

Sr -90

Te-125m

Th-234

$\mathrm{U}-238$

$\mathrm{Y}-90$

Shipping site: PSNS

Total Volume $(\mathrm{m} 3 / \mathrm{yr})$ :

Radionuclide

$\mathrm{Ba}-137 \mathrm{~m}$

Co-60

Cs -134

Cs -137

Eu-154

Eu-155

Fe-55

$\mathrm{H}-3$

Mn -54

$\mathrm{Nb}-94$

$\mathrm{Ni}-59$

$\mathrm{Ni}-63$

$\mathrm{Pa}-234 \mathrm{~m}$

$\mathrm{Pm}-147$

$\mathrm{Pu}-238$

Pu -241

$\mathrm{Sb}-125$

$\mathrm{Sm}-151$

Sr -90

$\mathrm{Te}-125 \mathrm{~m}$

Th-234

$\mathrm{U}-238$

$\mathrm{Y}-90$

Shipping Site: SNLL

Total Volume (m3/yr):

Radionuclide

Ba $-137 m$

Cs -137

$\mathrm{H}-3$

$\mathrm{Pa}-234 \mathrm{~m}$

$\mathrm{Pu}-241$

Sr -90

Th -234

$\mathrm{U}-238$

$\mathrm{Y}-90$

\section{Activity
(Ci/yr)}

2. $671 \mathrm{E}-02$

4. $315 E-01$

$2.537 \mathrm{E}-04$

2. $820 E-02$

3. $432 \mathrm{E}-04$

1. $641 \mathrm{E}-04$

5. $641 E-02$

1.228E-01

$1.791 \mathrm{E}-04$

2. $686 \mathrm{E}-04$

5. $641 \mathrm{E}-03$

7. $585 \mathrm{E}-01$

1. $059 \mathrm{E}-03$

3. $074 \mathrm{E}-03$

8. 806E-04

$7.164 \mathrm{E}-04$

1. $194 \mathrm{E}-04$

2. $388 \mathrm{E}-04$

$2.656 \mathrm{E}-02$

$2.985 E-05$

1. $059 \mathrm{E}-03$

$1.059 \mathrm{E}-03$

$2.656 \mathrm{E}-02$

\begin{tabular}{|c|c|}
\hline Chemical & $\begin{array}{l}\text { Amount } \\
\text { (kg/yr) }\end{array}$ \\
\hline $75-09-2$ & $9.577 \mathrm{E}-02$ \\
\hline $\mathrm{cd}$ & $6.584 E-02$ \\
\hline$C 1-2-x$ & $5.088 \mathrm{E}-02$ \\
\hline$C I-3-x$ & 7. $243 E+00$ \\
\hline$C 1-4-x$ & $1.616 E+00$ \\
\hline$C l-F-x$ & $2.993 E-03$ \\
\hline $\mathrm{Cr}$ & $5.746 \mathrm{E}-02$ \\
\hline HC-insol & $1.975 \mathrm{E}-02$ \\
\hline HC-solub & $3.831 \mathrm{E}-01$ \\
\hline $\mathrm{Hg}$ & $3.771 \mathrm{E}-04$ \\
\hline $\mathrm{Pb}$ & $1.257 E-02$ \\
\hline
\end{tabular}

Waste Type: Solids (CH-Non-alpha)

$6.586 \mathrm{E}+00 \quad$ Total Mass $(\mathrm{kg} / \mathrm{yr}): 9.518 \mathrm{E}+03$

\begin{tabular}{c}
$\begin{array}{c}\text { Activity } \\
(C i / y r)\end{array}$ \\
\hline $4.409 \mathrm{E}+00$ \\
$7.122 \mathrm{E}+01$ \\
$4.187 \mathrm{E}-02$ \\
$4.655 \mathrm{E}+00$ \\
$5.665 \mathrm{E}-02$ \\
$2.709 \mathrm{E}-02$ \\
$9.311 \mathrm{E}+00$ \\
$2.028 \mathrm{E}+01$ \\
$2.956 \mathrm{E}-02$ \\
$4.434 \mathrm{E}-02$ \\
$9.311 \mathrm{E}-01$ \\
$1.251 \mathrm{E}+02$ \\
$1.749 \mathrm{E}-01$ \\
$5.074 \mathrm{E}-01$ \\
$1.453 \mathrm{E}-01$ \\
$1.182 \mathrm{E}-01$ \\
$1.970 \mathrm{E}-02$ \\
$3.941 \mathrm{E}-02$ \\
$4.384 \mathrm{E}+00$ \\
$4.926 \mathrm{E}-03$ \\
$1.749 \mathrm{E}-01$ \\
$1.749 \mathrm{E}-01$ \\
$4.384 \mathrm{E}+00$
\end{tabular}

\begin{tabular}{|c|c|}
\hline Chemical & $\begin{array}{l}\text { Amount } \\
\left(\mathrm{kg} / \mathrm{y}^{\mathrm{r}}\right)\end{array}$ \\
\hline $\begin{array}{l}57-12-5 \\
75-09-2\end{array}$ & 7. $312 \mathrm{E}-01$ \\
\hline $\begin{array}{l}75-09-2 \\
\mathrm{Ag}\end{array}$ & $\begin{array}{l}1.279 \mathrm{E}+00 \\
157 \mathrm{E}-01\end{array}$ \\
\hline As & $4.301 \mathrm{E}-02$ \\
\hline $\mathrm{Ba}$ & $8.869 E+00$ \\
\hline $\mathrm{cd}$ & $1.229 \mathrm{E}-01$ \\
\hline$C l-2-x$ & $1.346 \mathrm{E}+00$ \\
\hline C1-3-x & $4.520 \mathrm{E}+00$ \\
\hline$C 1-4-x$ & $2.989 \mathrm{E}+00$ \\
\hline$C I-F-x$ & $1.385 \mathrm{E}+00$ \\
\hline $\mathrm{Cr}$ & $2.225 E+01$ \\
\hline $\mathrm{HC}$-insol & $6.852 \mathrm{E}+00$ \\
\hline $\mathrm{HC}-\mathrm{solub}$ & $2.684 \mathrm{E}-01$ \\
\hline $\mathrm{Hg}$ & $4.633 E-01$ \\
\hline $\mathrm{Pb}$ & $2.158 \mathrm{E}+01$ \\
\hline & $1.310 \mathrm{E}-01$ \\
\hline
\end{tabular}

Waste Type: Organic Liquids ( $\mathrm{CH}-\mathrm{Non}-a 1 p h a)$

$7.662 \mathrm{E}+00$

Total Mass $(\mathrm{kg} / \mathrm{yr}): \quad 7.356 \mathrm{E}+03$

\begin{tabular}{c}
$\begin{array}{c}\text { Activity } \\
(C i / y r)\end{array}$ \\
\hline $1.013 \mathrm{E}-01$ \\
$1.062 \mathrm{E}-01$ \\
$4.822 \mathrm{E}+02$ \\
$2.462 \mathrm{E}-02$ \\
$2.413 \mathrm{E}-02$ \\
$1.013 \mathrm{E}-01$ \\
$2.462 \mathrm{E}-02$ \\
$2.462 \mathrm{E}-02$ \\
$1.013 \mathrm{E}-01$
\end{tabular}

\begin{tabular}{l} 
Chemical \\
\hline $75-09-2$ \\
As \\
$\mathrm{Ba}$ \\
$\mathrm{Cd}$ \\
$\mathrm{Cl}-2-\mathrm{x}$ \\
$\mathrm{Cl}-3-\mathrm{x}$ \\
$\mathrm{Cl}-4-\mathrm{x}$ \\
$\mathrm{Cr}$ \\
$\mathrm{HC}-$ insol \\
$\mathrm{HC}-$ solub \\
$\mathrm{Hg}$ \\
$\mathrm{Pb}$
\end{tabular}

\begin{tabular}{c}
$\begin{array}{c}\text { Amount } \\
(\mathrm{kg} / \mathrm{yr})\end{array}$ \\
\hline $7.061 \mathrm{E}+00$ \\
$5.664 \mathrm{E}-02$ \\
$2.427 \mathrm{E}-01$ \\
$8.091 \mathrm{E}+00$ \\
$3.678 \mathrm{E}+00$ \\
$5.517 \mathrm{E}+01$ \\
$1.176 \mathrm{E}+01$ \\
$6.767 \mathrm{E}+00$ \\
$3.236 \mathrm{E}+03$ \\
$1.029 \mathrm{E}+03$ \\
$4.413 \mathrm{E}-02$ \\
$1.544 \mathrm{E}+00$
\end{tabular}


A-33

Shipping Site: SNLL Waste Type: Solids (CH-Non-alpha)

Total Volume $(\mathrm{m} 3 / \mathrm{yr})$ :

$7.770 \mathrm{E}-01$

Total Mass $(\mathrm{kg} / \mathrm{yr}): 1.193 \mathrm{E}+03$

Radionuclide

Activity

$\mathrm{Ba}-137 \mathrm{~m}$

Cs -137

$\mathrm{H}-3$

$\mathrm{Pa}-234 \mathrm{~m}$

Pu-241

Sr -90

Th-234

$U-238$

(Ci/yr)

\begin{tabular}{l} 
Chemical \\
\hline $57-12-5$ \\
$75-09-2$ \\
$\mathrm{Ag}$ \\
$\mathrm{As}$ \\
$\mathrm{Ba}$ \\
$\mathrm{Cd}$ \\
$\mathrm{C} 1-2-\mathrm{x}$ \\
$\mathrm{C} 1-3-\mathrm{x}$ \\
$\mathrm{C} 1-4-\mathrm{x}$ \\
$\mathrm{C} 1-\mathrm{F}-\mathrm{x}$ \\
$\mathrm{Cr}$ \\
$\mathrm{HC}-$ insol \\
$\mathrm{HC}-$ Solub \\
$\mathrm{Hg}$ \\
$\mathrm{Pb}$ \\
$\mathrm{Se}$
\end{tabular}

Amount

$1.027 \mathrm{E}-02$

$1.076 \mathrm{E}-02$

$4.890 \mathrm{E}+01$

$2.496 \mathrm{E}-03$

$2.447 \mathrm{E}-03$

$1.027 \mathrm{E}-02$

$2.496 \mathrm{E}-03$

$2.496 \mathrm{E}-03$

1. $027 \mathrm{E}-02$

\begin{tabular}{c}
$\begin{array}{c}\text { Amount } \\
(\mathrm{kg} / \mathrm{Yr})\end{array}$ \\
\hline $6.924 \mathrm{E}-03$ \\
$7.497 \mathrm{E}+00$ \\
$1.014 \mathrm{E}-02$ \\
$6.737 \mathrm{E}-03$ \\
$3.347 \mathrm{E}-01$ \\
$8.243 \mathrm{E}-02$ \\
$3.816 \mathrm{E}+00$ \\
$4.480 \mathrm{E}+01$ \\
$3.090 \mathrm{E}+01$ \\
$6.827 \mathrm{E}-02$ \\
$6.765 \mathrm{E}-01$ \\
$1.187 \mathrm{E}+02$ \\
$5.754 \mathrm{E}+01$ \\
$1.257 \mathrm{E}-01$ \\
$2.595 \mathrm{E}-01$ \\
$3.934 \mathrm{E}-02$
\end{tabular}


Destination Site: INEL

Shipping Site: ETEC Waste Type: Organic Liquids (CH-Non-alpha) Total volume $(\mathrm{m} 3 / \mathrm{yr})$ : $6.767 \mathrm{E}-03$ Total Mass $(\mathrm{kg} / \mathrm{yr}): 7.145 \mathrm{E}+00$

\begin{tabular}{l} 
Radionuc \\
\hline $\mathrm{Am}-241$ \\
$\mathrm{Ba}-137 \mathrm{~m}$ \\
$\mathrm{Co}-60$ \\
$\mathrm{Cs}-137$ \\
$\mathrm{Eu}-154$ \\
$\mathrm{Eu}-155$ \\
$\mathrm{H}-3$ \\
$\mathrm{Nb}-94$ \\
$\mathrm{Ni}-59$ \\
$\mathrm{Ni}-63$ \\
$\mathrm{~Pa}-234 \mathrm{~m}$ \\
$\mathrm{Pu}-238$ \\
$\mathrm{Pu}-239$ \\
$\mathrm{Pu}-240$ \\
$\mathrm{Pu}-241$ \\
$\mathrm{Sm}-151$ \\
$\mathrm{Sr}-90$ \\
$\mathrm{TC}-99$ \\
$\mathrm{Th}-232$ \\
$\mathrm{Th}-234$ \\
$\mathrm{U}-238$ \\
$\mathrm{Y}-90$
\end{tabular}

Shipping Site: ETEC Total Volume $(\mathrm{m} 3 / \mathrm{yr})$;

Radionuclide

\begin{tabular}{l}
\hline $\mathrm{Am}-241$ \\
$\mathrm{Ba}-137 \mathrm{~m}$ \\
$\mathrm{Co}-60$ \\
$\mathrm{Cs}-137$ \\
$\mathrm{Eu}-154$ \\
$\mathrm{Eu}-155$ \\
$\mathrm{H}-3$ \\
$\mathrm{Nb}-94$ \\
$\mathrm{Ni}-59$ \\
$\mathrm{Ni}-63$ \\
$\mathrm{~Pa}-234 \mathrm{~m}$ \\
$\mathrm{Pu}-238$ \\
$\mathrm{Pu}-239$ \\
$\mathrm{Pu}-240$ \\
$\mathrm{Pu}-241$ \\
$\mathrm{Sm}-151$ \\
$\mathrm{Sr}-90$ \\
$\mathrm{TC}-99$ \\
$\mathrm{Th}-232$ \\
$\mathrm{Th}-234$ \\
$\mathrm{U}-238$ \\
$\mathrm{Y}-90$
\end{tabular}

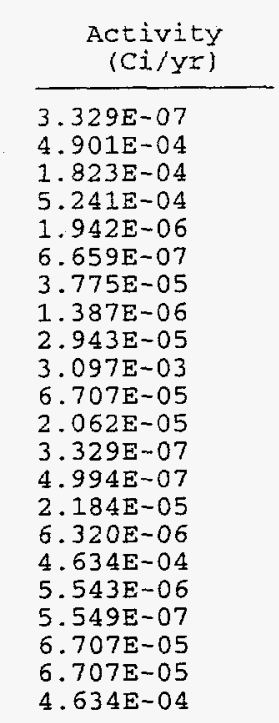

\begin{tabular}{l} 
Chemical \\
\hline $57-12-5$ \\
$75-09-2$ \\
$\mathrm{Ag}$ \\
$\mathrm{As}$ \\
$\mathrm{Ba}$ \\
$\mathrm{Cd}$ \\
$\mathrm{Cl}-2-\mathrm{x}$ \\
$\mathrm{Cl}-3-\mathrm{x}$ \\
$\mathrm{Cl}-4-\mathrm{x}$ \\
$\mathrm{Cl}-\mathrm{F}-\mathrm{x}$ \\
$\mathrm{Cr}$ \\
$\mathrm{HC}-\mathrm{insol}$ \\
$\mathrm{HC}-\mathrm{solub}$ \\
$\mathrm{Hg}$ \\
$\mathrm{Pb}$ \\
$\mathrm{Se}$
\end{tabular}

\begin{tabular}{c}
$\begin{array}{c}\text { Amount } \\
(\mathrm{kg} / \mathrm{yr})\end{array}$ \\
\hline $6.272 \mathrm{E}-04$ \\
$4.759 \mathrm{E}-04$ \\
$3.144 \mathrm{E}-04$ \\
$4.027 \mathrm{E}-05$ \\
$2.745 \mathrm{E}-04$ \\
$1.707 \mathrm{E}-05$ \\
$1.354 \mathrm{E}-03$ \\
$5.331 \mathrm{E}-02$ \\
$3.012 \mathrm{E}-03$ \\
$1.098 \mathrm{E}-03$ \\
$7.317 \mathrm{E}-04$ \\
$4.382 \mathrm{E}-02$ \\
$1.255 \mathrm{E}+00$ \\
$1.781 \mathrm{E}-03$ \\
$2.730 \mathrm{E}-04$ \\
$2.748 \mathrm{E}-04$
\end{tabular}

Waste Type: Solids (CH-Non-alpha)
$7.380 E+01$
Total Mass (kg/yr):
1. $326 \mathrm{E}+05$

\begin{tabular}{c}
$\begin{array}{c}\text { ACtivity } \\
\text { (Ci/YY) }\end{array}$ \\
\hline $3.631 \mathrm{E}-03$ \\
$5.346 \mathrm{E}+00$ \\
$1.989 \mathrm{E}+00$ \\
$5.716 \mathrm{E}+00$ \\
$2.118 \mathrm{E}-02$ \\
$7.262 \mathrm{E}-03$ \\
$4.118 \mathrm{E}-01$ \\
$1.513 \mathrm{E}-02$ \\
$3.210 \mathrm{E}-01$ \\
$3.377 \mathrm{E}+01$ \\
$7.316 \mathrm{E}-01$ \\
$2.249 \mathrm{E}-01$ \\
$3.631 \mathrm{E}-03$ \\
$5.447 \mathrm{E}-03$ \\
$2.382 \mathrm{E}-01$ \\
$6.893 \mathrm{E}-02$ \\
$5.054 \mathrm{E}+00$ \\
$6.046 \mathrm{E}-02$ \\
$6.052 \mathrm{E}-03$ \\
$7.316 \mathrm{E}-01$ \\
$7.316 \mathrm{E}-01$ \\
$5.054 \mathrm{E}+00$
\end{tabular}

\begin{tabular}{c}
$\begin{array}{c}\text { Amount } \\
(\mathrm{kg} / \mathrm{Yr})\end{array}$ \\
\hline $4.222 \mathrm{E}-02$ \\
$1.466 \mathrm{E}-01$ \\
$1.331 \mathrm{E}+00$ \\
$6.654 \mathrm{E}-01$ \\
$2.587 \mathrm{E}+00$ \\
$4.415 \mathrm{E}-01$ \\
$1.504 \mathrm{E}-01$ \\
$5.990 \mathrm{E}+00$ \\
$1.869 \mathrm{E}+00$ \\
$9.652 \mathrm{E}-02$ \\
$3.303 \mathrm{E}+00$ \\
$1.648 \mathrm{E}+01$ \\
$8.724 \mathrm{E}+00$ \\
$7.346 \mathrm{E}-01$ \\
$6.598 \mathrm{E}+00$ \\
$2.188 \mathrm{E}-01$
\end{tabular}




\section{A.35}

Shipping site: GATOMIC

Waste Type: Organic Liquids (CH-Non-alpha)

Total Volume $(\mathrm{m} 3 / \mathrm{yr}\rangle:$

$7.077 \mathrm{E}-02$

Total Mass $(\mathrm{kg} / \mathrm{yr}): \quad 7.476 \mathrm{E}+01$

Radionuclide

Ac -228

Bi -212

$\mathrm{Pa}-234$

$\mathrm{Pa}-234 \mathrm{~m}$

$\mathrm{Pb}-212$

Po- 212

Po- 216

$\mathrm{Ra}-224$

$\mathrm{Ra}-228$

TC- -99

Th-228

Th-231

Th -232

Th-234

T1-208

$\mathrm{U}-235$

$\mathrm{U}-238$

Shipping Site: GATOMIC

\begin{tabular}{c}
$\begin{array}{c}\text { Activity } \\
(\mathrm{Ci} / \mathrm{yr})\end{array}$ \\
\hline $1.514 \mathrm{E}-07$ \\
$2.547 \mathrm{E}-08$ \\
$1.925 \mathrm{E}-08$ \\
$1.870 \mathrm{E}-04$ \\
$2.547 \mathrm{E}-08$ \\
$1.642 \mathrm{E}-08$ \\
$2.547 \mathrm{E}-08$ \\
$2.547 \mathrm{E}-08$ \\
$1.514 \mathrm{E}-07$ \\
$2.816 \mathrm{E}-06$ \\
$2.547 \mathrm{E}-08$ \\
$1.460 \mathrm{E}-07$ \\
$1.537 \mathrm{E}-06$ \\
$1.870 \mathrm{E}-04$ \\
$9.625 \mathrm{E}-09$ \\
$1.455 \mathrm{E}-07$ \\
$1.870 \mathrm{E}-04$
\end{tabular}

\begin{tabular}{l} 
Chemical \\
\hline $57-12-5$ \\
$75-09-2$ \\
$\mathrm{Ag}$ \\
$\mathrm{AS}$ \\
$\mathrm{Ba}$ \\
$\mathrm{Cd}$ \\
$\mathrm{Cl}-2-\mathrm{x}$ \\
$\mathrm{C} 1-3-\mathrm{x}$ \\
$\mathrm{Cl}-4-\mathrm{x}$ \\
$\mathrm{C} 1-\mathrm{F}-\mathrm{x}$ \\
$\mathrm{Cr}$ \\
$\mathrm{HC}-$ insol \\
$\mathrm{HC}-$ solub \\
$\mathrm{Hg}$ \\
$\mathrm{Pb}$ \\
$\mathrm{Se}$
\end{tabular}

\begin{tabular}{c}
$\begin{array}{c}\text { Amount } \\
(\mathrm{kg} / \mathrm{Y} x)\end{array}$ \\
\hline $6.543 E-03$ \\
$5.261 \mathrm{E}-03$ \\
$3.280 \mathrm{E}-03$ \\
$4.207 \mathrm{E}-04$ \\
$2.867 \mathrm{E}-03$ \\
$4.175 \mathrm{E}-04$ \\
$1.428 \mathrm{E}-02$ \\
$5.732 \mathrm{E}-01$ \\
$3.523 \mathrm{E}-02$ \\
$1.146 \mathrm{E}-02$ \\
$7.839 \mathrm{E}-03$ \\
$4.931 \mathrm{E}-01$ \\
$1.311 \mathrm{E}+01$ \\
$1.858 \mathrm{E}-02$ \\
$2.894 \mathrm{E}-03$ \\
$2.867 \mathrm{E}-03$
\end{tabular}

Waste Type: Solids (CH-Non-alpha)

Total Mass $(\mathrm{kg} / \mathrm{yr}): \quad 8.419 \mathrm{E}+02$

\begin{tabular}{l} 
Chemica \\
\hline $57-12-5$ \\
$75-09-2$ \\
$\mathrm{Ag}$ \\
$\mathrm{As}$ \\
$\mathrm{Ba}$ \\
$\mathrm{Cd}$ \\
$\mathrm{C} 1-2-\mathrm{x}$ \\
$\mathrm{Cl}-3-\mathrm{x}$ \\
$\mathrm{C} 1-4-\mathrm{x}$ \\
$\mathrm{C} 1-\mathrm{F}-\mathrm{x}$ \\
$\mathrm{Cr}$ \\
$\mathrm{HC}-$ insol \\
$\mathrm{HC}-\mathrm{solub}$ \\
$\mathrm{Hg}$ \\
$\mathrm{Pb}$ \\
$\mathrm{Se}$
\end{tabular}

\begin{tabular}{c}
$\begin{array}{c}\text { Amount } \\
(\mathrm{kg} / \mathrm{Yr})\end{array}$ \\
\hline $9.298 E-02$ \\
$1.998 E-01$ \\
$2.502 E-02$ \\
$9.721 E-03$ \\
$1.175 E+00$ \\
$4.463 E-02$ \\
$1.741 E-01$ \\
$3.347 E+00$ \\
$8.150 E-01$ \\
$1.017 E-01$ \\
$1.315 E+00$ \\
$1.041 E+01$ \\
$6.899 E+00$ \\
$1.942 E-01$ \\
$2.810 E+00$ \\
$7.067 E-02$
\end{tabular}

Shipping site: LLNL Total volume (m3/yr):

Radionuclide

$\mathrm{Ba}-137 \mathrm{~m}$

Cs -137

$\mathrm{H}-3$

$\mathrm{Pa}-234 \mathrm{~m}$

Pu-238

Pu-241

$\mathrm{Sr}-90$

Th-234

$\mathrm{U}-238$

$Y-90$

\begin{tabular}{c}
$\begin{array}{c}\text { Activity } \\
\text { (Ci/yr) }\end{array}$ \\
\hline $1.218 \mathrm{E}-06$ \\
$2.049 \mathrm{E}-07$ \\
$1.548 \mathrm{E}-07$ \\
$1.504 \mathrm{E}-03$ \\
$2.049 \mathrm{E}-07$ \\
$1.320 \mathrm{E}-07$ \\
$2.049 \mathrm{E}-07$ \\
$1.996 \mathrm{E}-07$ \\
$1.186 \mathrm{E}-06$ \\
$2.207 \mathrm{E}-05$ \\
$2.049 \mathrm{E}-07$ \\
$1.175 \mathrm{E}-06$ \\
$1.237 \mathrm{E}-05$ \\
$1.504 \mathrm{E}-03$ \\
$7.742 \mathrm{E}-08$ \\
$1.170 \mathrm{E}-06$ \\
$1.504 \mathrm{E}-03$
\end{tabular}

Waste Type: Organic Liquids ( $\mathrm{CH}-\mathrm{Alpha}$

$5.737 E+01$

Total Mass $(\mathrm{kg} / \mathrm{yr})$ :

$6.269 \mathrm{E}+04$

\begin{tabular}{c}
$\begin{array}{c}\text { Activity } \\
\text { (Ci/yr) }\end{array}$ \\
\hline $7.591 \mathrm{E}-01$ \\
$7.952 \mathrm{E}-01$ \\
$3.607 \mathrm{E}+03$ \\
$1.843 \mathrm{E}-01$ \\
$1.807 \mathrm{E}-01$ \\
$3.514 \mathrm{E}+00$ \\
$7.591 \mathrm{E}-01$ \\
$1.843 \mathrm{E}-01$ \\
$1.843 \mathrm{E}-01$ \\
$7.591 \mathrm{E}-01$
\end{tabular}

\begin{tabular}{|c|c|}
\hline Chemical & $\begin{array}{l}\text { Amount } \\
(\mathrm{kg} / \mathrm{Yr})\end{array}$ \\
\hline $\begin{array}{l}57-12-5 \\
75-09-2\end{array}$ & $\begin{array}{l}4.251 E+00 \\
2.252 E+01\end{array}$ \\
\hline $\mathrm{Ag}$ & $2.130 \mathrm{E}+00$ \\
\hline As & 3.14IE-01 \\
\hline $\mathrm{Ba}$ & $2.037 \mathrm{E}+00$ \\
\hline $\mathrm{Cd}$ & $1.580 \mathrm{E}+01$ \\
\hline Cl-2-x & $1.943 \mathrm{E}+01$ \\
\hline Cl-3-x & $1.480 \mathrm{E}+03$ \\
\hline$C 1-4-x$ & $2.696 \mathrm{E}+02$ \\
\hline$C l-F-x$ & $7.889 \mathrm{E}+00$ \\
\hline $\mathrm{Cr}$ & $1.843 \mathrm{E}+01$ \\
\hline $\mathrm{HC}$-insol & $2.552 \mathrm{E}+03$ \\
\hline HC-solub & $9.317 \mathrm{E}+03$ \\
\hline $\mathrm{Hg}$ & $1.216 \mathrm{E}+01$ \\
\hline $\mathrm{Pb}$ & $4.845 \mathrm{E}+00$ \\
\hline $\mathrm{Se}$ & $1.862 \mathrm{E}+00$ \\
\hline
\end{tabular}




Shipping Site: LLNL
Total Volume ( $\mathrm{m} 3 / \mathrm{Yr}$ ) :

Radionuclide
Ba-137m
Cs-137
$\mathrm{H}-3$
$\mathrm{~Pa}-234 \mathrm{~m}$
$\mathrm{Pu}-238$
$\mathrm{Pu}-241$
$\mathrm{Sr}-90$
$\mathrm{Th}-234$
$\mathrm{U}-238$
$\mathrm{Y}-90$

Shipping Site: NTS

Total volume (m3/yr):

Radionuclide

$\mathrm{Ba}-137 \mathrm{~m}$

$\mathrm{Cs}-137$

$\mathrm{H}-3$

$\mathrm{Pa}-234 \mathrm{~m}$

Pu-241

$\mathrm{Sr}-90$

Th-234

$\mathrm{U}-238$

$\mathrm{Y}-90$
Waste Type: Solids (CH-Alpha)

Total Mass (kg/yr): $1.017 \mathrm{E}+05$

\begin{tabular}{c}
$\begin{array}{c}\text { Activity } \\
\text { (Ci/yr) }\end{array}$ \\
\hline $6.919 E-01$ \\
$7.249 E-01$ \\
$3.288 E+03$ \\
$1.680 E-01$ \\
$1.647 E-01$ \\
$3.295 E+00$ \\
$6.919 E-01$ \\
$1.680 E-01$ \\
$1.680 E-01$ \\
$6.919 E-01$
\end{tabular}

\begin{tabular}{ll} 
Chemical & $\begin{array}{c}\text { Amount } \\
(\mathrm{kg} / \mathrm{Yr})\end{array}$ \\
\hline $57-12-5$ & $4.138 \mathrm{E}+00$ \\
$75-09-2$ & $1.192 \mathrm{E}+01$ \\
$\mathrm{Ag}$ & $9.746 \mathrm{E}-01$ \\
$\mathrm{As}$ & $3.439 \mathrm{E}-01$ \\
$\mathrm{Ba}$ & $5.008 \mathrm{E}+01$ \\
$\mathrm{Cd}$ & $3.649 \mathrm{E}+00$ \\
$\mathrm{Cl}-2-\mathrm{x}$ & $7.183 \mathrm{E}+00$ \\
$\mathrm{Cl}-3-\mathrm{x}$ & $4.229 \mathrm{E}+01$ \\
$\mathrm{Cl}-4-\mathrm{x}$ & $2.492 \mathrm{E}+01$ \\
$\mathrm{Cl}-\mathrm{F}-\mathrm{x}$ & $1.009 \mathrm{E}+01$ \\
$\mathrm{Cr}$ & $2.709 \mathrm{E}+02$ \\
$\mathrm{HC}-$ insol & $7.977 \mathrm{E}+01$ \\
$\mathrm{HC}-\mathrm{solub}$ & $2.905 \mathrm{E}+01$ \\
$\mathrm{Hg}$ & $8.247 \mathrm{E}+00$ \\
$\mathrm{~Pb}$ & $1.721 \mathrm{E}+02$ \\
$\mathrm{Se}$ & $2.561 \mathrm{E}+00$
\end{tabular}

Waste Type: Solids (CH-Non-alpha)
Total Mass (kg/Yr): $8.886 E+00$
$6.621 E-03$

Activity (Ci/yr)

$8.760 \mathrm{E}-05$

$9.177 \mathrm{E}-05$

4.167E-01

$2.127 \mathrm{E}-05$

$2.085 \mathrm{E}-05$

$8.760 \mathrm{E}-05$

$2.127 \mathrm{E}-05$

$2.127 \mathrm{E}-05$

8.760E-05

\begin{tabular}{l} 
Chemical \\
\hline $75-09-2$ \\
Ag \\
Cd \\
Cl-2-x \\
Cl-3-x \\
Cl-4-x \\
Cl-F-x \\
Cr \\
$\mathrm{HC}-$ insol \\
$\mathrm{HC}-$ solub \\
$\mathrm{Hg}$ \\
$\mathrm{Pb}$ \\
$\mathrm{Se}$
\end{tabular}


Destination site: LANL

Shipping site: ITRI Waste Type: Organic Liquids (CH-Non-alpha)

Total Volume $(\mathrm{m} 3 / \mathrm{yr})$ :

$1.028 \mathrm{E}-01$

Total Mass (kg/yr):

$1.007 E+02$

Radionuclide

$\mathrm{Ba}-137 \mathrm{~m}$

Co- 60

Cs -137

Eu-154

Fe -55

$\mathrm{H}-3$

$\mathrm{Ni}-59$

$\mathrm{Ni}-63$

Pm-147

$\mathrm{Pu}-238$

Pu-241

Sm-151

Sr -90

$Y-90$

Shipping site: PANT

Total Volume (m3/yr):

Radionuclide

$\mathrm{Ba}-137 \mathrm{~m}$

Co-60

Cs -137

Eu-154

$\mathrm{Fe}-55$

$\mathrm{H}-3$

$\mathrm{Ni}-59$

$\mathrm{Ni}-63$

Pm-147

Pu-238

Pu-241

Sm-151

Sr -90

$Y-90$

Shipping site:

PANT

Total Volume (m3/yr):

Radionuclide

$\mathrm{Ba}-137 \mathrm{~m}$

Co- 60

Cs -137

Eu-154

$F \in-55$

$\mathrm{H}-3$

Ni-59

Ni-63

Pm-147

Pu-238

Pu-241

$\mathrm{sm}-151$

Sr -90

$Y-90$

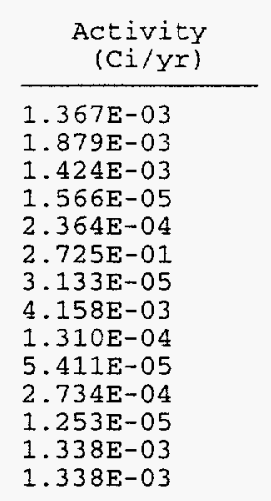

\begin{tabular}{l} 
Chemical \\
\hline $57-12-5$ \\
$\mathrm{Ag}$ \\
$\mathrm{Cd}$ \\
$\mathrm{Cl}-3-\mathrm{x}$ \\
$\mathrm{Cl}-4-\mathrm{x}$ \\
$\mathrm{Cr}$ \\
$\mathrm{HC}-$ insol \\
$\mathrm{HC}-$ solub \\
$\mathrm{Hg}$ \\
$\mathrm{Pb}$ \\
$\mathrm{Se}$
\end{tabular}

\begin{tabular}{c}
$\begin{array}{c}\text { Amount } \\
(\mathrm{kg} / \mathrm{y})\end{array}$ \\
\hline $1.813 \mathrm{E}-02$ \\
$4.433 \mathrm{E}-03$ \\
$4.937 \mathrm{E}-04$ \\
$8.061 \mathrm{E}-02$ \\
$4.534 \mathrm{E}-03$ \\
$2.116 \mathrm{E}-02$ \\
$1.108 \mathrm{E}+00$ \\
$3.627 \mathrm{E}+01$ \\
$2.821 \mathrm{E}-02$ \\
$5.038 \mathrm{E}-03$ \\
$4.030 \mathrm{E}-03$
\end{tabular}

Waste Type: Organic Liquids (CH-Non-alpha)

$1.461 E+00$

Total Mass (kg/yr):

$2.110 E+03$

\begin{tabular}{l}
$\begin{array}{r}\text { Activity } \\
\text { (Ci/yr) }\end{array}$ \\
\hline $1.942 \mathrm{E}-02$ \\
$2.671 \mathrm{E}-02$ \\
$2.023 \mathrm{E}-02$ \\
$2.225 \mathrm{E}-04$ \\
$3.359 \mathrm{E}-03$ \\
$3.872 \mathrm{E}+00$ \\
$4.451 \mathrm{E}-04$ \\
$5.908 \mathrm{E}-02$ \\
$1.861 \mathrm{E}-03$ \\
$7.689 \mathrm{E}-04$ \\
$3.885 \mathrm{E}-03$ \\
$1.780 \mathrm{E}-04$ \\
$1.902 \mathrm{E}-02$ \\
$1.902 \mathrm{E}-02$
\end{tabular}

\begin{tabular}{l} 
Chemica \\
\hline $75-09-2$ \\
$\mathrm{As}$ \\
$\mathrm{Ba}$ \\
$\mathrm{Cd}$ \\
$\mathrm{Cl}-2-\mathrm{x}$ \\
$\mathrm{Cl}-3-\mathrm{x}$ \\
$\mathrm{Cl}-4-\mathrm{x}$ \\
$\mathrm{Cl}-\mathrm{F}-\mathrm{x}$ \\
$\mathrm{Cr}$ \\
$\mathrm{HC}-$ insol \\
$\mathrm{HC}-\mathrm{Solub}$ \\
$\mathrm{Hg}$ \\
$\mathrm{Pb}$
\end{tabular}

\begin{tabular}{c}
$\begin{array}{c}\text { Amount } \\
(\mathrm{kg} / \mathrm{yr})\end{array}$ \\
\hline $3.284 \mathrm{E}+00$ \\
$1.112 \mathrm{E}-03$ \\
$4.766 \mathrm{E}-03$ \\
$2.321 \mathrm{E}+00$ \\
$1.743 \mathrm{E}+00$ \\
$2.389 \mathrm{E}+02$ \\
$5.331 \mathrm{E}+01$ \\
$9.829 \mathrm{E}-02$ \\
$2.020 \mathrm{E}+00$ \\
$6.419 \mathrm{E}+01$ \\
$3.280 \mathrm{E}+01$ \\
$1.325 \mathrm{E}-02$ \\
$4.431 \mathrm{E}-01$
\end{tabular}

Waste Type: Solids (CH-Non-alpha)

3. $957 E+01$

Total Mass (kg/yr): $2.511 E+04$

\begin{tabular}{l}
$\begin{array}{c}\text { Activity } \\
\text { (Ci/yr) }\end{array}$ \\
\hline $5.276 \mathrm{E}-01$ \\
$7.254 \mathrm{E}-01$ \\
$5.454 \mathrm{E}-01$ \\
$6.045 \mathrm{E}-03$ \\
$9.123 \mathrm{E}-02$ \\
$1.051 \mathrm{E}+02$ \\
$1.209 \mathrm{E}-02$ \\
$1.604 \mathrm{E}+00$ \\
$5.056 \mathrm{E}-02$ \\
$2.088 \mathrm{E}-02$ \\
$1.055 \mathrm{E}-01$ \\
$4.836 \mathrm{E}-03$ \\
$5.126 \mathrm{E}-01$ \\
$5.126 \mathrm{E}-01$
\end{tabular}

\begin{tabular}{ll} 
Chemical & \multicolumn{1}{c}{$\begin{array}{c}\text { Amount } \\
(\mathrm{kg} / \mathrm{Yr})\end{array}$} \\
\cline { 1 - 1 } $57-12-5$ & $2.480 \mathrm{E}-01$ \\
$75-09-2$ & $3.448 \mathrm{E}+01$ \\
$\mathrm{Ag}$ & $1.998 \mathrm{E}-01$ \\
$\mathrm{As}$ & $3.903 \mathrm{E}-02$ \\
$\mathrm{Ba}$ & $1.047 \mathrm{E}+01$ \\
$\mathrm{Cd}$ & $1.602 \mathrm{E}+00$ \\
$\mathrm{Cl}-2-\mathrm{x}$ & $2.240 \mathrm{E}+01$ \\
$\mathrm{Cl}-3-\mathrm{x}$ & $1.712 \mathrm{E}+02$ \\
$\mathrm{Cl}-4-\mathrm{x}$ & $1.094 \mathrm{E}+02$ \\
$\mathrm{Cl}-\mathrm{F}-\mathrm{x}$ & $1.631 \mathrm{E}+01$ \\
$\mathrm{Cr}$ & $1.027 \mathrm{E}+01$ \\
$\mathrm{HC}-\mathrm{ins01}$ & $4.777 \mathrm{E}+02$ \\
$\mathrm{HC}-\mathrm{SOlub}$ & $1.648 \mathrm{E}+02$ \\
$\mathrm{Hg}$ & $1.896 \mathrm{E}+00$ \\
$\mathrm{~Pb}$ & $5.162 \mathrm{E}+01$ \\
$\mathrm{Se}$ & $2.443 \mathrm{E}-01$
\end{tabular}




\section{A-38}

Shipping site: SNIA Waste Type: Organic Liquids (CH-Non-alpha)

Total Volume (m3/yr):

$5.487 \mathrm{E}-04$

Total Mass (kg/yr):

$5.793 \mathrm{E}-01$

Radionuclide

$\mathrm{Ba}-137 \mathrm{~m}$

$\mathrm{CO}-60$

Cs -137

$\mathrm{Eu}-154$

Eu-155

$\mathrm{Fe}-55$

$\mathrm{H}-3$

$\mathrm{Nb}-94$

$\mathrm{Ni}-59$

$\mathrm{Ni}-63$

$\mathrm{Pa}-234 \mathrm{~m}$

$\mathrm{Pu}-238$

Pu-240

Pu-241

Sm-15I

Sr-90

Tc-99

Th-234

U-238

$Y-90$

shipping site: SNLA

Total Volume (m3/yr):

Radionuclide

$\mathrm{Ba}-137 \mathrm{~m}$

$\mathrm{Co}-60$

Cs -137

Eu-154

Eu-155

Fe-55

$\mathrm{H}-3$

$\mathrm{Nb}-94$

Ni-59

$\mathrm{Ni}-63$

$\mathrm{Pa}-234 \mathrm{~m}$

Pu-238

$\mathrm{Pu}-240$

Pu-241

Sm-151

Sx-90

Tc-99

Th-234

$\mathrm{U}-238$

$Y-90$

\section{Activity}

6. $627 \mathrm{E}-05$

9. $630 \mathrm{E}-05$

$7.006 \mathrm{E}-05$

3. $516 \mathrm{E}-07$

8. $115 \mathrm{E}-08$

1. $920 \mathrm{E}-06$

1. $223 \mathrm{E}-03$

$4.598 \mathrm{E}-07$

$9.089 \mathrm{E}-06$

1. $099 \mathrm{E}-03$

3. $246 \mathrm{E}-07$

2. 380E-06

1. $893 \mathrm{E}-06$

1. $893 \mathrm{E}-06$

7. $574 \mathrm{E}-07$

$6.519 \mathrm{E}-05$

2. $705 E-08$

3. $246 \mathrm{E}-07$

3. $246 \mathrm{E}-07$

$6.519 \mathrm{E}-05$

\begin{tabular}{l} 
Chemical \\
\hline $57-12-5$ \\
$75-09-2$ \\
Ag \\
As \\
Ba \\
Cd \\
Cl-2-x \\
Cl-3-x \\
Cl-4-x \\
Cl-F-x \\
Cr \\
HC-insol \\
HC-solub \\
Hg \\
Pb \\
Se
\end{tabular}

\begin{tabular}{c}
$\begin{array}{c}\text { Amount } \\
(\mathrm{kg} / \mathrm{Yr})\end{array}$ \\
\hline $5.085 E-05$ \\
$3.859 E-05$ \\
$2.549 E-05$ \\
$3.265 E-06$ \\
$2.226 E-05$ \\
$1.384 E-06$ \\
$1.098 E-04$ \\
$4.322 E-03$ \\
$2.442 E-04$ \\
$8.905 E-05$ \\
$5.933 E-05$ \\
$3.553 E-03$ \\
$1.018 E-01$ \\
$1.444 E-04$ \\
$2.214 E-05$ \\
$2.228 E-05$
\end{tabular}

waste Type: Solids (CH-Non-alpha)

Total Mass (kg/yr): $2.300 \mathrm{E}+03$

$2.849 E+00$

\begin{tabular}{l} 
Chemical \\
\hline $57-12-5$ \\
$75-09-2$ \\
Ag \\
As \\
Ba \\
Cd \\
CI-2-x \\
C1-3-x \\
C1-4-x \\
C1-F-x \\
Cr \\
$\mathrm{HC}-$ insol \\
HC-solub \\
$\mathrm{Hg}$ \\
$\mathrm{Pb}$ \\
$\mathrm{Se}$
\end{tabular}

Amount

Activity (ci/yr)

3. $441 \mathrm{E}-01$

5. $000 \mathrm{E}-01$

3. $637 \mathrm{E}-01$

1. $825 \mathrm{E}-03$

4. $213 E-04$

$9.972 \mathrm{E}-03$

$6.351 \mathrm{E}+00$

2. $387 \mathrm{E}-03$

4. $719 \mathrm{E}-02$

5. $706 \mathrm{E}+00$

1. $685 \mathrm{E}-03$

1. $236 E-02$

$9.832 \mathrm{E}-03$

$9.832 \mathrm{E}-03$

$3.932 \mathrm{E}-03$

3. $385 \mathrm{E}-01$

1. $404 \mathrm{E}-04$

1. $685 \mathrm{E}-03$

1. $685 \mathrm{E}-03$

3. $385 \mathrm{E}-01$ 
Destination Site: ORNL

shipping site: PADUCAH

Waste Type: Organic Liquids (CH-Non-alpha)

Total Volume $(\mathrm{m} 3 / \mathrm{yr})$ :

$2.970 \mathrm{E}+01$

Total Mass $(\mathrm{kg} / \mathrm{yr}): \quad 3.589 \mathrm{E}+04$

\begin{tabular}{l} 
Radionuclide \\
\hline $\mathrm{Ac}-228$ \\
$\mathrm{Bi}-212$ \\
$\mathrm{~Pa}-234$ \\
$\mathrm{~Pa}-234 \mathrm{~m}$ \\
$\mathrm{~Pb}-212$ \\
$\mathrm{Po}-212$ \\
$\mathrm{Po}-215$ \\
$\mathrm{Ra}-224$ \\
$\mathrm{Ra}-228$ \\
$\mathrm{TC}-99$ \\
$\mathrm{Th}-228$ \\
$\mathrm{Th}-231$ \\
$\mathrm{Th}-232$ \\
$\mathrm{Th}-234$ \\
$\mathrm{~T} 1-208$ \\
$\mathrm{U}-235$ \\
$\mathrm{U}-238$
\end{tabular}

\begin{tabular}{c}
$\begin{array}{c}\text { ACtivity } \\
\text { (Ci/Yr) }\end{array}$ \\
\hline $1.796 E-02$ \\
$2.967 E-03$ \\
$2.486 E-03$ \\
$2.420 E+01$ \\
$2.967 E-03$ \\
$1.965 E-03$ \\
$2.967 E-03$ \\
$2.967 E-03$ \\
$1.796 E-02$ \\
$7.291 E+00$ \\
$2.967 E-03$ \\
$1.892 E-02$ \\
$1.990 E-01$ \\
$2.420 E+01$ \\
$1.163 E-03$ \\
$1.876 E-02$ \\
$2.420 E+01$
\end{tabular}

\begin{tabular}{l} 
Chemical \\
\hline $57-12-5$ \\
$75-09-2$ \\
Ag \\
As \\
Ba \\
Cd \\
Cl-2-x \\
Cl-3-x \\
Cl-4-x \\
Cl-F-x \\
Cr \\
HC-insol \\
HC-solub \\
Hg \\
$\mathrm{Pb}$ \\
Se
\end{tabular}

\begin{tabular}{c}
$\begin{array}{c}\text { Amount } \\
(\mathrm{kg} / \mathrm{yr})\end{array}$ \\
\hline $4.590 \mathrm{E}-01$ \\
$4.205 \mathrm{E}+01$ \\
$2.300 \mathrm{E}-01$ \\
$1.180 \mathrm{E}-01$ \\
$5.803 \mathrm{E}-01$ \\
$3.374 \mathrm{E}+01$ \\
$2.303 \mathrm{E}+01$ \\
$2.444 \mathrm{E}+03$ \\
$5.381 \mathrm{E}+02$ \\
$1.762 \mathrm{E}+00$ \\
$2.951 \mathrm{E}+01$ \\
$5.097 \mathrm{E}+03$ \\
$2.651 \mathrm{E}+03$ \\
$1.493 \mathrm{E}+00$ \\
$6.639 \mathrm{E}+00$ \\
$2.011 \mathrm{E}-01$
\end{tabular}

Shipping Site: PADUCAH

Waste Type: Solids (CH-Non-alpha)

Total Volume (m3/yr):

$4.740 E+00$

Total Mass (kg/yr): $6.808 E+03$

Radionuclide

Ac- 228

$\mathrm{B} i-212$

$\mathrm{Pa}-234$

pa-234m

$\mathrm{Pb}-212$

Po- 212

Po-216

$\mathrm{Ra}-224$

$\mathrm{Ra}-228$

TC-99

Th-228

Th-231

Th-232

Th-234

Tl-208

$\mathrm{U}-235$

$\mathrm{U}-238$

\begin{tabular}{c}
$\begin{array}{c}\text { Activity } \\
\text { (Ci/yr) }\end{array}$ \\
\hline $2.890 \mathrm{E}-03$ \\
$4.773 \mathrm{E}-04$ \\
$3.999 \mathrm{E}-04$ \\
$3.894 \mathrm{E}+00$ \\
$4.773 \mathrm{E}-04$ \\
$3.161 \mathrm{E}-04$ \\
$4.773 \mathrm{E}-04$ \\
$4.664 \mathrm{E}-04$ \\
$2.824 \mathrm{E}-03$ \\
$1.146 \mathrm{E}+00$ \\
$4.773 \mathrm{E}-04$ \\
$3.044 \mathrm{E}-03$ \\
$3.202 \mathrm{E}-02$ \\
$3.894 \mathrm{E}+00$ \\
$1.870 \mathrm{E}-04$ \\
$3.019 \mathrm{E}-03$ \\
$3.894 \mathrm{E}+00$
\end{tabular}

\begin{tabular}{l} 
Chemical \\
\hline $57-12-5$ \\
$75-09-2$ \\
Ag \\
As \\
$\mathrm{Ba}$ \\
$\mathrm{Cd}$ \\
$\mathrm{Cl}-2-\mathrm{x}$ \\
$\mathrm{Cl}-3-\mathrm{x}$ \\
$\mathrm{Cl}-4-\mathrm{x}$ \\
$\mathrm{Cl}-\mathrm{F}-\mathrm{x}$ \\
$\mathrm{Cr}$ \\
$\mathrm{HC}-\mathrm{insol}$ \\
$\mathrm{HC}-\mathrm{sol}$ lub \\
$\mathrm{Hg}$ \\
$\mathrm{Pb}$ \\
$\mathrm{Se}$
\end{tabular}

\begin{tabular}{c}
$\begin{array}{c}\text { Amount } \\
(\mathrm{kg} / \mathrm{yr})\end{array}$ \\
\hline $1.016 \mathrm{E}+00$ \\
$1.710 \mathrm{E}+00$ \\
$2.502 \mathrm{E}-01$ \\
$8.712 \mathrm{E}-02$ \\
$1.228 \mathrm{E}+01$ \\
$1.504 \mathrm{E}-01$ \\
$1.444 \mathrm{E}+00$ \\
$1.250 \mathrm{E}+01$ \\
$4.496 \mathrm{E}+00$ \\
$6.729 \mathrm{E}-01$ \\
$1.293 \mathrm{E}+01$ \\
$3.436 \mathrm{E}+01$ \\
$1.740 \mathrm{E}+01$ \\
$1.732 \mathrm{E}+00$ \\
$2.789 \mathrm{E}+01$ \\
$6.716 \mathrm{E}-01$
\end{tabular}


Destination Site: PORTS

Shipping Site: AMES Waste Type: Solids (CH-Non-alpha)

Total Volume $(\mathrm{m} 3 / \mathrm{yr})$ :

$8.585 \mathrm{E}-03$

Total Mass $(\mathrm{kg} / \mathrm{yr}): \quad 1.162 \mathrm{E}+01$

Radionuclide

Ac-228

Bi-212

Pa-234

$\mathrm{Pa}-234 \mathrm{~m}$

$\mathrm{Pb}-212$

Po- 212

Po- 216

$\mathrm{Ra}-224$

$\mathrm{Ra}-228$

TC-99

Th-228

$\mathrm{Th}-231$

Th -232

Th-234

TI-208

$\mathrm{U}-235$

$\mathrm{U}-238$

Shipping Site: ANL-E

Total Volume $(\mathrm{m} 3 / \mathrm{yx})$ :

Radionuclide

Am-241

$\mathrm{Ba}-137 \mathrm{~m}$

Co- 60

Cs -137

Eu-154

Eu-155

$\mathrm{H}-3$

$\mathrm{Nb}-94$

$\mathrm{Ni}-59$

$\mathrm{Ni}-63$

$\mathrm{Pa}-234 \mathrm{~m}$

Pu-238

$\mathrm{Pu}-239$

Pu-240

Pu-241

$\mathrm{Sm}-151$

$\mathrm{S} x-90$

TC-99

Th-232

Th-234

$\mathrm{U}-238$

$\mathrm{Y}-90$

Shipping Site:

BAPL

Total Volume $(\mathrm{m} 3 / \mathrm{yr})$ :

Radionuclide

Am-241

$\mathrm{Ba}-137 \mathrm{~m}$

Co-60

Cs -137

Eu-154

$\mathrm{H}-3$

$\mathrm{Nb}-94$

Ni-59

$\mathrm{Ni}-63$

$\mathrm{Pa}-234 \mathrm{~m}$

Pu-238

Pu-239

Pu-240

Pu-24I

$\mathrm{Sm}-151$

Sr -90

TC-99

Th-234

$\mathrm{U}-238$

$\mathrm{Y}-90$

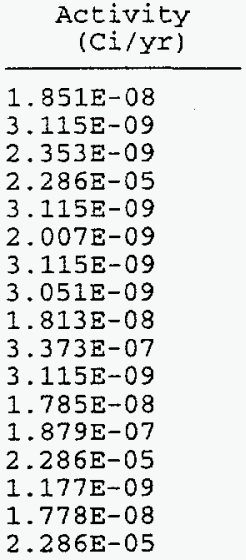

\begin{tabular}{l} 
Chemical \\
\hline $57-12-5$ \\
$75-09-2$ \\
Ag \\
As \\
Ba \\
Cd \\
CI-2-x \\
CI-3-x \\
CI-4-x \\
CI-F-x \\
Cr \\
$\mathrm{HC}-$ insol \\
$\mathrm{HC}-$ solub \\
$\mathrm{Hg}$ \\
$\mathrm{Pb}$ \\
$\mathrm{Se}$
\end{tabular}

\begin{tabular}{c}
$\begin{array}{c}\text { Amount } \\
(\mathrm{kg} / \mathrm{yr})\end{array}$ \\
\hline $1.759 \mathrm{E}-05$ \\
$1.485 \mathrm{E}-04$ \\
$5.841 \mathrm{E}-05$ \\
$7.025 \mathrm{E}-07$ \\
$4.061 \mathrm{E}-05$ \\
$5.957 \mathrm{E}-05$ \\
$1.879 \mathrm{E}-04$ \\
$4.658 \mathrm{E}-04$ \\
$8.901 \mathrm{E}-04$ \\
$7.426 \mathrm{E}-05$ \\
$1.342 \mathrm{E}-04$ \\
$1.917 \mathrm{E}-05$ \\
$1.017 \mathrm{E}-02$ \\
$5.635 \mathrm{E}-05$ \\
$8.863 \mathrm{E}-05$ \\
$1.196 \mathrm{E}-06$
\end{tabular}

Waste Type: Solids (CH-Non-alpha)

$4.838 \mathrm{E}+02$

Total Mass (kg/yr): $8.170 \mathrm{E}+05$

\begin{tabular}{c}
$\begin{array}{c}\text { Activity } \\
(\mathrm{Ci} / \mathrm{Yr})\end{array}$ \\
\hline $2.380 \mathrm{E}-02$ \\
$3.504 \mathrm{E}+01$ \\
$1.304 \mathrm{E}+01$ \\
$3.747 \mathrm{E}+01$ \\
$1.388 \mathrm{E}-01$ \\
$4.7 .61 \mathrm{E}-02$ \\
$2.699 \mathrm{E}+00$ \\
$9.919 \mathrm{E}-02$ \\
$2.104 \mathrm{E}+00$ \\
$2.214 \mathrm{E}+02$ \\
$4.796 \mathrm{E}+00$ \\
$1.474 \mathrm{E}+00$ \\
$2.380 \mathrm{E}-02$ \\
$3.571 \mathrm{E}-02$ \\
$1.561 \mathrm{E}+00$ \\
$4.519 \mathrm{E}-01$ \\
$3.313 \mathrm{E}+01$ \\
$3.963 \mathrm{E}-01$ \\
$3.967 \mathrm{E}-02$ \\
$4.796 \mathrm{E}+00$ \\
$4.796 \mathrm{E}+00$ \\
$3.313 \mathrm{E}+01$
\end{tabular}

\begin{tabular}{l} 
Chemical \\
\hline $57-12-5$ \\
$75-09-2$ \\
Ag \\
As \\
Ba \\
Cd \\
Cl-2-x \\
Cl-3-x \\
Cl-4-x \\
CI-F-x \\
Cr \\
HC-insol \\
HC-solub \\
Hg \\
$\mathrm{Pb}$ \\
Se
\end{tabular}

\begin{tabular}{c}
$\begin{array}{c}\text { Amount } \\
(\mathrm{kg} / \mathrm{Yr})\end{array}$ \\
\hline $2.002 \mathrm{E}+01$ \\
$2.778 \mathrm{E}+01$ \\
$1.623 \mathrm{E}+01$ \\
$6.714 \mathrm{E}+00$ \\
$2.781 \mathrm{E}+02$ \\
$9.989 \mathrm{E}+00$ \\
$1.822 \mathrm{E}+01$ \\
$3.658 \mathrm{E}+02$ \\
$4.608 \mathrm{E}+01$ \\
$1.759 \mathrm{E}+01$ \\
$3.334 \mathrm{E}+02$ \\
$1.482 \mathrm{E}+03$ \\
$7.360 \mathrm{E}+02$ \\
$1.110 \mathrm{E}+02$ \\
$4.704 \mathrm{E}+02$ \\
$4.660 \mathrm{E}+01$
\end{tabular}

Waste Type: Organic Liquids (CH-Non-alpha)

Total Mass (kg/Yr): $6.163 \mathrm{E}+01$

$6.410 E-02$

\begin{tabular}{l} 
Chemical \\
\hline $75-09-2$ \\
$\mathrm{As}$ \\
$\mathrm{Ba}$ \\
$\mathrm{Cd}$ \\
$\mathrm{Cl}-2-\mathrm{x}$ \\
$\mathrm{Cl}-3-\mathrm{x}$ \\
$\mathrm{Cl}-4-\mathrm{x}$ \\
$\mathrm{Cl}-\mathrm{F}-\mathrm{x}$ \\
$\mathrm{Cr}$ \\
$\mathrm{HC}-\mathrm{insol}$ \\
$\mathrm{HC}-$ solub \\
$\mathrm{Hg}$ \\
$\mathrm{Pb}$
\end{tabular}

\begin{tabular}{c}
$\begin{array}{c}\text { Amount } \\
(\mathrm{kg} / \mathrm{Yr})\end{array}$ \\
\hline $5.934 E-02$ \\
$4.724 E-04$ \\
$2.024 E-03$ \\
$6.779 E-02$ \\
$3.091 E-02$ \\
$4.931 E-01$ \\
$1.055 E-01$ \\
$1.360 E-05$ \\
$5.671 E-02$ \\
$2.699 E+01$ \\
$8.592 E+00$ \\
$3.698 E-04$ \\
$1.294 E-02$
\end{tabular}

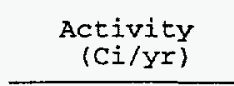

$5.332 \mathrm{E}-05$

$5.866 \mathrm{E}-02$

$7.908 \mathrm{E}-05$

$6.272 \mathrm{E}-02$

9. $439 \mathrm{E}-05$

$4.694 \mathrm{E}-03$

$5.357 \mathrm{E}-06$

1. $209 \mathrm{E}-04$

1. $242 \mathrm{E}-02$

3. $469 \mathrm{E}-05$

$2.806 \mathrm{E}-03$

3. 801E-05

$2.168 \mathrm{E}-05$

6. 225E-04

$9.031 \mathrm{E}-04$

$5.586 \mathrm{E}-02$

$2.806 \mathrm{E}-05$

3. $469 \mathrm{E}-05$

3. $469 \mathrm{E}-05$

$5.586 \mathrm{E}-02$ 
Shipping Site: BAPL Waste Type: Solids (CH-Non-alpha)

Total Volume (m3/yr):

$6.105 E-01$

Total Mass (kg/yr):

$6.010 E+02$

\begin{tabular}{l} 
Radionuclide \\
\hline $\mathrm{Am}-241$ \\
$\mathrm{Ba}-137 \mathrm{~m}$ \\
$\mathrm{Co}-60$ \\
$\mathrm{Cs}-137$ \\
$\mathrm{Eu}-154$ \\
$\mathrm{H}-3$ \\
$\mathrm{Nb}-94$ \\
$\mathrm{Ni}-59$ \\
$\mathrm{Ni}-63$ \\
$\mathrm{~Pa}-234 \mathrm{~m}$ \\
$\mathrm{Pu}-238$ \\
$\mathrm{Pu}-239$ \\
$\mathrm{Pu}-240$ \\
$\mathrm{Pu}-241$ \\
$\mathrm{Sm}-151$ \\
$\mathrm{Sr}-90$ \\
$\mathrm{TC}-99$ \\
$\mathrm{Th}-234$ \\
$\mathrm{U}-238$ \\
$\mathrm{Y}-90$
\end{tabular}

Shipping site:

BATELLE

Total Volume $(\mathrm{m} 3 / \mathrm{yr})$ :

\begin{tabular}{l} 
Radionuclide \\
\hline $\mathrm{Ac}-228$ \\
$\mathrm{~B}$ - 212 \\
$\mathrm{~Pa}-234$ \\
$\mathrm{~Pa}-234 \mathrm{~m}$ \\
$\mathrm{~Pb}-212$ \\
$\mathrm{Po}-212$ \\
$\mathrm{Po}-216$ \\
$\mathrm{Ra}-224$ \\
$\mathrm{Ra}-228$ \\
$\mathrm{TC}-99$ \\
$\mathrm{Th}-228$ \\
$\mathrm{Th}-231$ \\
$\mathrm{Th}-232$ \\
$\mathrm{Th}-234$ \\
$\mathrm{~T}-208$ \\
$\mathrm{U}-235$ \\
$\mathrm{U}-238$
\end{tabular}

Shipping Site: BNL

Total Volume (m3/yr):

Radionuclide

Ara-241

$\mathrm{Ba}-137 \mathrm{~m}$

Co- 60

Cs -137

Eu-154

Eu-155

$\mathrm{H}-3$

$\mathrm{Nb}-94$

$\mathrm{Ni}-59$

$\mathrm{Ni}-63$

$\mathrm{Pa}-234 \mathrm{~m}$

Pu -238

Pu-239

Pu-240

Pu-241

$\mathrm{Sm}-151$

$\mathrm{Sr}-90$

TC- 99

Th-232

Th-234

$\mathrm{U}-238$

$\mathrm{Y}-90$

\begin{tabular}{c}
$\begin{array}{c}\text { Activity } \\
\text { (Ci/yr) }\end{array}$ \\
\hline $5.078 \mathrm{E}-04$ \\
$5.587 \mathrm{E}-01$ \\
$7.532 \mathrm{E}-04$ \\
$5.974 \mathrm{E}-01$ \\
$8.990 \mathrm{E}-04$ \\
$4.471 \mathrm{E}-02$ \\
$5.102 \mathrm{E}-05$ \\
$1.151 \mathrm{E}-03$ \\
$1.183 \mathrm{E}-01$ \\
$3.304 \mathrm{E}-04$ \\
$2.672 \mathrm{E}-02$ \\
$3.620 \mathrm{E}-04$ \\
$2.065 \mathrm{E}-04$ \\
$5.929 \mathrm{E}-03$ \\
$8.601 \mathrm{E}-03$ \\
$5.321 \mathrm{E}-01$ \\
$2.672 \mathrm{E}-04$ \\
$3.304 \mathrm{E}-04$ \\
$3.304 \mathrm{E}-04$ \\
$5.321 \mathrm{E}-01$
\end{tabular}

\begin{tabular}{|c|c|}
\hline Chemical & $\begin{array}{l}\text { Amount } \\
(\mathrm{kg} / \mathrm{Y})\end{array}$ \\
\hline $57-12-5$ & $1.703 \mathrm{E}-03$ \\
\hline $75-09-2$ & $6.266 \mathrm{E}-02$ \\
\hline $\mathrm{Ag}$ & $6.515 \mathrm{E}-03$ \\
\hline As & $1.598 \mathrm{E}-03$ \\
\hline $\mathrm{Ba}$ & $6.259 \mathrm{E}-02$ \\
\hline $\mathrm{ca}$ & 1. $698 \mathrm{E}-02$ \\
\hline$C I-2-x$ & $7.067 \mathrm{E}-02$ \\
\hline$C 1-3-x$ & $4.679 \varepsilon-01$ \\
\hline Cl-4-x & $4.156 \mathrm{E}-01$ \\
\hline $\mathrm{Cl}-\mathrm{F}-\mathrm{x}$ & $8.727 \mathrm{E}-02$ \\
\hline $\mathrm{Cr}$ & $4.102 \mathrm{E}-02$ \\
\hline $\mathrm{HC}-$ insol & $2.697 \mathrm{E}+00$ \\
\hline HC-solub & $2.666 E+00$ \\
\hline $\mathrm{Hg}$ & $1.514 E-02$ \\
\hline $\mathrm{Pb}$ & $7.746 \mathrm{E}-02$ \\
\hline & \\
\hline
\end{tabular}

Waste Type: Solids (CH-Non-alpha)

$1.500 E-03$

Total Mass $(\mathrm{kg} / \mathrm{yr}): 1.977 \mathrm{E}+00$

\begin{tabular}{c}
$\begin{array}{c}\text { ACtivity } \\
\text { (Ci/yr) }\end{array}$ \\
\hline $3.211 \mathrm{E}-09$ \\
$5.400 \mathrm{E}-10$ \\
$4.080 \mathrm{E}-10$ \\
$3.964 \mathrm{E}-06$ \\
$5.400 \mathrm{E}-10$ \\
$3.480 \mathrm{E}-10$ \\
$5.400 \mathrm{E}-10$ \\
$5.400 \mathrm{E}-10$ \\
$3.211 \mathrm{E}-09$ \\
$5.971 \mathrm{E}-08$ \\
$5.400 \mathrm{E}-10$ \\
$3.097 \mathrm{E}-09$ \\
$3.260 \mathrm{E}-08$ \\
$3.964 \mathrm{E}-06$ \\
$2.040 \mathrm{E}-10$ \\
$3.085 \mathrm{E}-09$ \\
$3.964 \mathrm{E}-06$
\end{tabular}

\begin{tabular}{|c|c|}
\hline Chemical & $\begin{array}{l}\text { Amount } \\
\langle\mathrm{kg} / \mathrm{Yr}\rangle\end{array}$ \\
\hline $\begin{array}{l}75-09-2 \\
\text { C1-2-x } \\
\text { Cl-3-x } \\
\text { Cl-4-x } \\
\text { HC-insol } \\
\text { HC-solub }\end{array}$ & $\begin{array}{l}3.955 E-02 \\
1.977 E-02 \\
1.977 E-01 \\
1.582 E-01 \\
4.943 E-01 \\
2.373 E-01\end{array}$ \\
\hline
\end{tabular}

Waste Type: Organic Liquids (CH-Non-alpha)

1. $544 \mathrm{E}+00$

Total Mass $(\mathrm{kg} / \mathrm{yr}): 1.749 \mathrm{E}+03$

\begin{tabular}{c}
$\begin{array}{c}\text { Activity } \\
\text { (Ci/yr) }\end{array}$ \\
\hline $7.598 \mathrm{E}-05$ \\
$1.118 \mathrm{E}-01$ \\
$4.162 \mathrm{E}-02$ \\
$1.196 \mathrm{E}-01$ \\
$4.432 \mathrm{E}-04$ \\
$1.519 \mathrm{E}-04$ \\
$8.617 \mathrm{E}-03$ \\
$3.166 \mathrm{E}-04$ \\
$6.718 \mathrm{E}-03$ \\
$7.068 \mathrm{E}-01$ \\
$1.530 \mathrm{E}-02$ \\
$4.706 \mathrm{E}-03$ \\
$7.598 \mathrm{E}-05$ \\
$1.139 \mathrm{E}-04$ \\
$4.984 \mathrm{E}-03$ \\
$1.442 \mathrm{E}-03$ \\
$1.057 \mathrm{E}-01$ \\
$1.265 \mathrm{E}-03$ \\
$1.266 \mathrm{E}-04$ \\
$1.530 \mathrm{E}-02$ \\
$1.530 \mathrm{E}-02$ \\
$1.057 \mathrm{E}-01$
\end{tabular}

\begin{tabular}{c}
$\begin{array}{c}\text { Amount } \\
(\mathrm{kg} / \mathrm{Yr})\end{array}$ \\
\hline $8.298 \mathrm{E}-02$ \\
$1.156 \mathrm{E}+00$ \\
$4.159 \mathrm{E}-02$ \\
$7.649 \mathrm{E}-03$ \\
$4.627 \mathrm{E}-02$ \\
$8.868 \mathrm{E}-01$ \\
$7.572 \mathrm{E}-01$ \\
$7.014 \mathrm{E}+01$ \\
$1.445 \mathrm{E}+01$ \\
$1.704 \mathrm{E}-01$ \\
$8.567 \mathrm{E}-01$ \\
$1.386 \mathrm{E}+02$ \\
$2.115 \mathrm{E}+02$ \\
$2.406 \mathrm{E}-01$ \\
$2.050 \mathrm{E}-01$ \\
$3.636 \mathrm{E}-02$
\end{tabular}


Shipping Site: BNL Waste Type: Solids (CH-Non-alpha)

Total Volume $(\mathrm{m} 3 / \mathrm{yr})$ :

8. $915 E+00$

Total Mass $(\mathrm{kg} / \mathrm{Yr}): \quad 1.190 \mathrm{E}+04$

Radionuclide

$$
\begin{aligned}
& \text { Activity } \\
& \text { (Ci/yr) }
\end{aligned}
$$

Am-241

$\mathrm{Ba}-137 \mathrm{~m}$

Co- 60

Cs -137

Eu-154

Eu-155

$\mathrm{H}-3$

$\mathrm{Nb}-94$

Ni-59

$\mathrm{Ni}-63$

$\mathrm{Pa}-234 \mathrm{~m}$

$\mathrm{Pu}-238$

Pu-239

Pu-240

$\mathrm{Pu}-241$

$\mathrm{Sm}-151$

$\operatorname{Sr}-90$

$\mathrm{Tc}-99$

Th-232

Th-234

$\mathrm{U}-238$

$Y-90$

Shipping site:

COLONIE

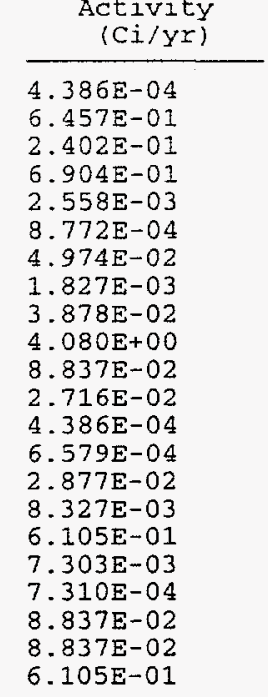

Waste Type: Organic Liquids (CH-Non-alpha)

\begin{tabular}{c}
$\begin{array}{c}\text { Amount } \\
(\mathrm{kg} / \mathrm{yr})\end{array}$ \\
\hline $1.617 \mathrm{E}+00$ \\
$8.722 \mathrm{E}-01$ \\
$7.571 \mathrm{E}-01$ \\
$2.698 \mathrm{E}-01$ \\
$2.157 \mathrm{E}+01$ \\
$4.530 \mathrm{E}-01$ \\
$8.722 \mathrm{E}-01$ \\
$2.419 \mathrm{E}+01$ \\
$3.963 \mathrm{E}-01$ \\
$1.019 \mathrm{E}+00$ \\
$2.698 \mathrm{E}+01$ \\
$1.091 \mathrm{E}+02$ \\
$5.459 \mathrm{E}+01$ \\
$8.531 \mathrm{E}+00$ \\
$2.912 \mathrm{E}+01$ \\
$3.668 \mathrm{E}+00$
\end{tabular}

Amount

Chemical
$57-12-5$
$75-09-2$
$\mathrm{Ag}$
$\mathrm{As}$
$\mathrm{Ba}$
$\mathrm{Cd}$
$\mathrm{Cl}-2-\mathrm{x}$
$\mathrm{Cl}-3-\mathrm{x}$
$\mathrm{Cl}-4-\mathrm{x}$
$\mathrm{C} 1-\mathrm{F}-\mathrm{x}$
$\mathrm{Cr}$
$\mathrm{HC}-$ insol
$\mathrm{HC}-$ solub
$\mathrm{Hg}$
$\mathrm{Pb}$
$\mathrm{Se}$

$9.054 \mathrm{E}-01$
Total Mass $(\mathrm{kg} / \mathrm{yr}): 1.026 \mathrm{E}+03$

\begin{tabular}{l} 
Chemical \\
\hline $57-12-5$ \\
$75-09-2$ \\
Ag \\
As \\
Ba \\
Cd \\
CI-2-x \\
CI-3-x \\
Cl-4-x \\
CI-F-x \\
Cr \\
HC-insol \\
HC-solub \\
Hg \\
Pb \\
Se
\end{tabular}

\begin{tabular}{l} 
Radionuclide \\
\hline $\mathrm{AC}-228$ \\
$\mathrm{Bi}-212$ \\
$\mathrm{~Pa}-234$ \\
$\mathrm{~Pa}-234 \mathrm{~m}$ \\
$\mathrm{~Pb}-212$ \\
$\mathrm{Po}-212$ \\
$\mathrm{Po}-216$ \\
$\mathrm{Ra}-224$ \\
$\mathrm{Ra}-228$ \\
$\mathrm{TC}-99$ \\
$\mathrm{Th}-228$ \\
$\mathrm{Th}-231$ \\
$\mathrm{Th}-232$ \\
$\mathrm{Th}-234$ \\
$\mathrm{~T} 1-208$ \\
$\mathrm{U}-235$ \\
$\mathrm{U}-238$
\end{tabular}

\begin{tabular}{c}
$\begin{array}{c}\text { Amount } \\
(\mathrm{kg} / \mathrm{Yr})\end{array}$ \\
\hline $5.151 \mathrm{E}-02$ \\
$7.241 \mathrm{E}-01$ \\
$2.274 \mathrm{E}-02$ \\
$3.999 \mathrm{E}-03$ \\
$2.356 \mathrm{E}-02$ \\
$5.620 \mathrm{E}-01$ \\
$4.522 \mathrm{E}-01$ \\
$4.355 \mathrm{E}+01$ \\
$9.136 \mathrm{E}+00$ \\
$8.522 \mathrm{E}-02$ \\
$5.418 \mathrm{E}-01$ \\
$8.707 \mathrm{E}+01$ \\
$1.317 \mathrm{E}+02$ \\
$1.340 \mathrm{E}-01$ \\
$1.275 \mathrm{E}-01$ \\
$1.998 \mathrm{E}-02$
\end{tabular}


Shipping site: COLONIE

Waste Type: Solids (CH-Non-alpha)

Total Volume $(\mathrm{m} 3 / \mathrm{yr})$ :

1. $235 \mathrm{E}-02$

Total Mass $(\mathrm{kg} / \mathrm{yr})$ :

$1.606 \mathrm{E}+01$

\begin{tabular}{l} 
Radionuclide \\
\hline Ac-228 \\
$\mathrm{Bi}-212$ \\
$\mathrm{~Pa}-234$ \\
$\mathrm{~Pa}-234 \mathrm{~m}$ \\
$\mathrm{~Pb}-212$ \\
$\mathrm{PO}-212$ \\
$\mathrm{PO}-216$ \\
$\mathrm{Ra}-224$ \\
$\mathrm{Ra}-228$ \\
$\mathrm{~T}-99$ \\
$\mathrm{Th}-228$ \\
$\mathrm{Th}-231$ \\
$\mathrm{Th}-232$ \\
$\mathrm{Th}-234$ \\
$\mathrm{~T} 1-208$ \\
$\mathrm{U}-235$ \\
$\mathrm{U}-238$
\end{tabular}

Shipping site: FEMP

Total Volume $(m 3 / y r)$ :

Radionuclide

AC-228

$\mathrm{Bi}-212$

$\mathrm{Pa}-234$

$\mathrm{Pa}-234 \mathrm{~m}$

$\mathrm{Pb}-212$

Po-212

P० -216

Ra-224

Ra-228

Tc-99

Th-228

Th-231

Th-232

Th-234

T1-208

$\mathrm{U}-235$

$\mathrm{U}-238$

Shipping site: FEMP Total Volume $(\mathrm{m} 3 / \mathrm{yr})$ :

Radionuclide

AC -228

Bi-212

$\mathrm{Pa}-234$

$\mathrm{Pa}-234 \mathrm{~m}$

$\mathrm{Pb}-212$

Po-212

Po-216

Ra-224

Ra-228

Tc-99

Th-228

Th-231

Th-232

Th-234

TI-208

$\mathrm{U}-235$

$\mathrm{U}-238$

\begin{tabular}{r}
$\begin{array}{c}\text { Activity } \\
\text { (Ci/Yr) }\end{array}$ \\
\hline $3.198 \mathrm{E}-08$ \\
$5.380 \mathrm{E}-09$ \\
$4.065 \mathrm{E}-09$ \\
$3.949 \mathrm{E}-05$ \\
$5.380 \mathrm{E}-09$ \\
$3.467 \mathrm{E}-09$ \\
$5.380 \mathrm{E}-09$ \\
$2.690 \mathrm{E}-09$ \\
$1.599 \mathrm{E}-08$ \\
$2.974 \mathrm{E}-07$ \\
$5.380 \mathrm{E}-09$ \\
$3.084 \mathrm{E}-08$ \\
$3.247 \mathrm{E}-07$ \\
$3.949 \mathrm{E}-05$ \\
$2.032 \mathrm{E}-09$ \\
$3.072 \mathrm{E}-08$ \\
$3.949 \mathrm{E}-05$
\end{tabular}

\begin{tabular}{l} 
Chemical \\
\hline $57-12-5$ \\
$75-09-2$ \\
$\mathrm{Ag}$ \\
$\mathrm{As}$ \\
$\mathrm{Ba}$ \\
$\mathrm{Cd}$ \\
$\mathrm{Cl}-2-\mathrm{x}$ \\
$\mathrm{C} 1-3-\mathrm{x}$ \\
$\mathrm{Cl}-4-\mathrm{x}$ \\
$\mathrm{CI}-\mathrm{F}-\mathrm{x}$ \\
$\mathrm{Cr}$ \\
$\mathrm{HC}-$ insol \\
$\mathrm{HC}-$ Solub \\
$\mathrm{Hg}$ \\
$\mathrm{Pb}$ \\
$\mathrm{Se}$
\end{tabular}

\begin{tabular}{c}
$\begin{array}{c}\text { Amount } \\
\langle\mathrm{kg} / \mathrm{Y} Y\end{array}$ \\
\hline $7.229 \mathrm{E}-04$ \\
$8.484 \mathrm{E}-04$ \\
$2.570 \mathrm{E}-05$ \\
$2.249 \mathrm{E}-05$ \\
$1.606 \mathrm{E}-05$ \\
$1.429 \mathrm{E}-04$ \\
$4.242 \mathrm{E}-03$ \\
$5.345 \mathrm{E}-03$ \\
$5.006 \mathrm{E}-03$ \\
$4.242 \mathrm{E}-04$ \\
$5.462 \mathrm{E}-04$ \\
$9.333 \mathrm{E}-04$ \\
$8.032 \mathrm{E}-04$ \\
$3.695 \mathrm{E}-06$ \\
$1.044 \mathrm{E}-03$ \\
$8.032 \mathrm{E}-06$
\end{tabular}

Waste Type: Organic Liquids (CH-Non-alpha)

$2.034 E+01$

Total Mass $(\mathrm{kg} / \mathrm{yr})$ :

$2.423 E+04$

\begin{tabular}{c}
$\begin{array}{c}\text { Activity } \\
\text { (Ci/yr) }\end{array}$ \\
\hline $4.353 \mathrm{E}-05$ \\
$7.324 \mathrm{E}-06$ \\
$5.533 \mathrm{E}-06$ \\
$5.376 \mathrm{E}-02$ \\
$7.324 \mathrm{E}-06$ \\
$4.720 \mathrm{E}-06$ \\
$7.324 \mathrm{E}-06$ \\
$7.324 \mathrm{E}-06$ \\
$4.353 \mathrm{E}-05$ \\
$8.097 \mathrm{E}-04$ \\
$7.324 \mathrm{E}-06$ \\
$4.199 \mathrm{E}-05$ \\
$4.420 \mathrm{E}-04$ \\
$5.376 \mathrm{E}-02$ \\
$2.766 \mathrm{E}-06$ \\
$4.182 \mathrm{E}-05$ \\
$5.376 \mathrm{E}-02$
\end{tabular}

\begin{tabular}{l} 
Chemical \\
\hline $57-12-5$ \\
$75-09-2$ \\
$\mathrm{Ag}$ \\
$\mathrm{As}$ \\
$\mathrm{Ba}$ \\
$\mathrm{Cd}$ \\
$\mathrm{Cl}-2-\mathrm{x}$ \\
$\mathrm{CI}-3-\mathrm{x}$ \\
$\mathrm{Cl}-4-\mathrm{x}$ \\
$\mathrm{CI}-\mathrm{F}-\mathrm{x}$ \\
$\mathrm{Cr}$ \\
$\mathrm{HC}-$ insol \\
$\mathrm{HC}-\mathrm{solub}$ \\
$\mathrm{Hg}$ \\
$\mathrm{Pb}$ \\
$\mathrm{Se}$
\end{tabular}

Amount

(kg/Yr)

4. $910 \mathrm{E}-01$

$2.872 E+01$

1. $270 \mathrm{E}-01$

6. $728 \mathrm{E}-02$

2. $928 \mathrm{E}-01$

2. $349 E+01$

1. $522 \mathrm{E}+01$

1. $621 \mathrm{E}+03$

3. $603 \mathrm{E}+02$

6. $895 \mathrm{E}-01$

2. $072 \mathrm{E}+01$

$3.779 E+03$

2. $255 E+03$

9. $307 \mathrm{E}-01$

4. $623 \mathrm{E}+00$

1. $150 \mathrm{E}-01$

Waste Type: Solids (CH-Non-alpha)

$7.909 E+01$

Total Mass (kg/yr):

$1.180 \mathrm{E}+05$

$\begin{array}{r}\text { Activi } \\ \text { (Ci/Y } / Y)\end{array}$
$1.696 \mathrm{E}-04$
$2.854 \mathrm{E}-05$
$2.156 \mathrm{E}-05$
$2.095 \mathrm{E}-01$
$2.854 \mathrm{E}-05$
$1.839 \mathrm{E}-05$
$2.854 \mathrm{E}-05$
$2.837 \mathrm{E}-05$
$1.686 \mathrm{E}-04$
$3.136 \mathrm{E}-03$
$2.854 \mathrm{E}-05$
$1.636 \mathrm{E}-04$
$1.722 \mathrm{E}-03$
$2.095 \mathrm{E}-01$
$1.078 \mathrm{E}-05$
$1.630 \mathrm{E}-04$
$2.095 \mathrm{E}-01$

Chemical

57-12-5

75-09-2

Ag

As

$\mathrm{Ba}$

$\mathrm{Cd}$

C $1-2-x$

CI $1-3-x$

Cl-4-x

Cl-F-X

Cr

HC-insol

HC-solub

$\mathrm{Hg}$

$\mathrm{Pb}$

$\mathrm{Se}$
Amount

(kg/yr)

$5.948 \mathrm{E}+00$

$2.117 E+01$

3. $226 \mathrm{E}+00$

$1.249 \mathrm{E}+00$

$1.085 E+02$

6. $815 \mathrm{E}+00$

2. $073 \mathrm{E}+01$

$5.283 \mathrm{E}+02$

$3.692 E+02$

1. $313 \mathrm{E}+01$

1. $531 \mathrm{E}+02$

1. $786 \mathrm{E}+03$

9. $390 \mathrm{E}+02$

3. $514 \mathrm{E}+01$

1. $240 \mathrm{E}+02$

1. $366 \mathrm{E}+01$ 
Shipping Site: KAPL

Total Volume (m3/yr):

Radionuclide

$\mathrm{Ba}-137 \mathrm{~m}$

$\mathrm{CO}-6 \mathrm{O}$

Cs -134

$\mathrm{Cs}-137$

Eu-154

Eu-155

Fe-55

$\mathrm{H}-3$

$\mathrm{Mn}-54$

$\mathrm{Nb}-94$

$\mathrm{Ni}-59$

$\mathrm{Ni}-63$

$\mathrm{Pa}-234 \mathrm{~m}$

Pm-147

Pu -238

Pu-241

$\mathrm{Sb}-125$

$\mathrm{Sm}-151$

Sr -90

$\mathrm{Te}-125 \mathrm{~m}$

$\mathrm{Th}-234$

$\mathrm{U}-238$

$\mathrm{Y}-90$

Shipping site: KAPL

Total Volume (m3/yr):

Radionuclide

$\mathrm{Ba}-137 \mathrm{~m}$

Co- 60

Cs -134

Cs-137

Eu-154

Eu-155

$\mathrm{Fe}-55$

$\mathrm{H}-3$

$\mathrm{Mn}-54$

$\mathrm{Nb}-94$

$\mathrm{Ni}-59$

$\mathrm{Ni}-63$

Pa-234m

Pm-147

$\mathrm{Pu}-238$

$\mathrm{Pu}-241$

$\mathrm{Sb}-125$

Sm-151

Sr -90

Te-125m

Th-234

$\mathrm{U}-238$

$\mathrm{Y}-90$

Waste Type: Organic Liguids (CH-Non-alpha)

$6.167 E-01$

Total Mass (kg/yr):

$7.065 E+02$

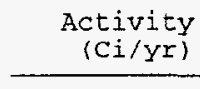

4. $129 \mathrm{E}-01$

$6.669 \mathrm{E}+00$

3. $921 \mathrm{E}-03$

4. 359E-01

$5.305 E-03$

$2.537 \mathrm{E}-03$

$8.719 \mathrm{E}-01$

1. $899 \mathrm{E}+00$

$2.768 \mathrm{E}-03$

4. $152 \mathrm{E}-03$

$8.719 \mathrm{E}-02$

1. $172 \mathrm{E}+01$

$1.637 \mathrm{E}-02$

$4.752 \mathrm{E}-02$

1. $361 \mathrm{E}-02$

1. $107 \mathrm{E}-02$

1. $845 \mathrm{E}-03$

3. $690 \mathrm{E}-03$

4. $106 \mathrm{E}-01$

4. $613 \mathrm{E}-04$

1. $637 \mathrm{E}-02$

1. $637 \mathrm{E}-02$

4. $106 \mathrm{E}-01$

$\begin{array}{lc}\text { Chemical } & \begin{array}{c}\text { Amount } \\ (\mathrm{kg} / \mathrm{yr})\end{array} \\ 57-12-5 & 2.915 \mathrm{E}-02 \\ 75-09-2 & 5.312 \mathrm{E}-01 \\ \mathrm{Ag} & 1.461 \mathrm{E}-02 \\ \mathrm{As} & 2.952 \mathrm{E}-03 \\ \mathrm{Ba} & 1.739 \mathrm{E}-02 \\ \mathrm{Cd} & 4.125 \mathrm{E}-01 \\ \mathrm{Cl}-2-\mathrm{x} & 3.320 \mathrm{E}-01 \\ \mathrm{Cl}-3-\mathrm{x} & 3.184 \mathrm{E}+01 \\ \mathrm{Cl}-4-\mathrm{x} & 6.682 \mathrm{E}+00 \\ \mathrm{Cl}-\mathrm{F}-\mathrm{x} & 6.275 \mathrm{E}-02 \\ \mathrm{Cr} & 3.877 \mathrm{E}-01 \\ \mathrm{HC}-\mathrm{insO} 1 & 6.386 \mathrm{E}+01 \\ \mathrm{HC}-\mathrm{s} 0 \mathrm{Iub} & 7.952 \mathrm{E}+01 \\ \mathrm{Hg} & 8.511 \mathrm{E}-02 \\ \mathrm{~Pb} & 9.130 \mathrm{E}-02 \\ \mathrm{Se} & 1.277 \mathrm{E}-02\end{array}$

Waste Type: Solids (CH-Non-alpha)

$3.572 \mathrm{E}+00$ Total Mass $(\mathrm{kg} / \mathrm{yr}): 5.162 \mathrm{E}+03$

\begin{tabular}{|c|c|c|}
\hline $\begin{array}{c}\text { Activity } \\
\text { (ci/yr) }\end{array}$ & Chemical & $\begin{array}{l}\text { Amount } \\
(\mathrm{kg} / \mathrm{yr})\end{array}$ \\
\hline $\begin{array}{l}2.391 \mathrm{E}+00 \\
3.862 \mathrm{E}+01 \\
2.271 \mathrm{E}-02 \\
2.525 \mathrm{E}+00 \\
3.072 \mathrm{E}-02 \\
1.469 \mathrm{E}-02 \\
5.050 \mathrm{E}+00 \\
1.100 \mathrm{E}+01 \\
1.603 \mathrm{E}-02 \\
2.404 \mathrm{E}-02 \\
5.050 \mathrm{E}-01 \\
6.790 \mathrm{E}+01 \\
9.486 \mathrm{E}-02 \\
2.752 \mathrm{E}-01 \\
7.882 \mathrm{E}-02 \\
6.413 \mathrm{E}-02\end{array}$ & $\begin{array}{l}57-12-5 \\
75-09-2 \\
\mathrm{Ag} \\
\mathrm{As} \\
\mathrm{Ba} \\
\mathrm{Cd} \\
\mathrm{Cl}-2-\mathrm{x} \\
\mathrm{Cl}-3-\mathrm{x} \\
\mathrm{Cl}-4-\mathrm{x} \\
\mathrm{Cl}-\mathrm{F}-\mathrm{x} \\
\mathrm{Cr} \\
\mathrm{HC}-\text { insol } \\
\mathrm{HC}-\text { solub } \\
\mathrm{Hg} \\
\mathrm{Pb} \\
\mathrm{Se}\end{array}$ & $\begin{array}{l}1.840 \mathrm{E}-02 \\
2.577 \mathrm{E}+00 \\
6.267 \mathrm{E}-02 \\
2.265 \mathrm{E}-02 \\
5.050 \mathrm{E}-01 \\
2.428 \mathrm{E}-02 \\
1.464 \mathrm{E}+00 \\
1.328 \mathrm{E}+01 \\
1.024 \mathrm{E}+01 \\
4.115 \mathrm{E}-01 \\
4.769 \mathrm{E}-01 \\
3.511 \mathrm{E}+01 \\
1.516 \mathrm{E}+01 \\
6.065 \mathrm{E}-02 \\
7.229 \mathrm{E}-01 \\
1.698 \mathrm{E}-02\end{array}$ \\
\hline
\end{tabular}




\begin{tabular}{l} 
Shipping Site: KKS \\
Total Volume (m3/Yr): \\
Radionuclide \\
\hline Ba-137m \\
Co-50 \\
Cs -134 \\
Cs -137 \\
Eu-154 \\
Eu-155 \\
Fe-55 \\
H-3 \\
Mn-54 \\
Nb-94 \\
Ni -59 \\
Ni-53 \\
Pa-234m \\
Pm-147 \\
Pu-238 \\
Pu-241 \\
Sb-125 \\
Sm-151 \\
Sr-90 \\
Te-125m \\
Th-234 \\
U-238 \\
Y-90 \\
\end{tabular}

Shipping Site: KKS

Total Volume (m3/yr):

Radionuclide

$\mathrm{Ba}-137 \mathrm{~m}$

Co-60

Cs-134

Cs -137

Eu-154

Eu-155

$\mathrm{Fe}-55$

H -3

$\mathrm{Mn}-54$

$\mathrm{Nb}-94$

$\mathrm{Ni}-59$

$\mathrm{Ni}-63$

$\mathrm{Pa}-234 \mathrm{~m}$

$\mathrm{Pm}-147$

Pu-238

Pu-24I

$\mathrm{Sb}-125$

Sm-151

$\mathrm{Sr}-90$

$\mathrm{Te}-125 \mathrm{~m}$

Th-234

$\mathrm{U}-238$

$\mathrm{Y}-90$
Waste Type: Organic Liquids (CH-Non-alpha)

$4.733 \mathrm{E}-01$

Total Mass $(\mathrm{kg} / \mathrm{yr})$ :

$5.422 \mathrm{E}+02$

\begin{tabular}{|c|c|}
\hline Chemical & $\begin{array}{l}\text { Amount } \\
(\mathrm{kg} / \mathrm{yr})\end{array}$ \\
\hline $57-12-5$ & $2.237 \mathrm{E}-02$ \\
\hline $75-09-2$ & $4.077 \mathrm{E}-01$ \\
\hline $\mathrm{Ag}$ & $1.121 E-02$ \\
\hline As & $2.266 \mathrm{E}-03$ \\
\hline $\mathrm{Ba}$ & $1.335 \mathrm{E}-02$ \\
\hline $\mathrm{Cd}$ & $3.166 \mathrm{E}-01$ \\
\hline$c l-2-x$ & $2.548 \mathrm{E}-01$ \\
\hline c1-3-x & $2.443 \mathrm{E}+01$ \\
\hline$C l-4-x$ & $5.128 \mathrm{E}+00$ \\
\hline $\mathrm{Cl}-\mathrm{F}-\mathrm{x}$ & $4.816 \mathrm{E}-02$ \\
\hline $\mathrm{Cr}$ & $2.976 \mathrm{E}-01$ \\
\hline HC-insol & $4.90 I E+01$ \\
\hline HC-solub & $6.103 E+01$ \\
\hline $\mathrm{Hg}$ & $6.532 \mathrm{E}-02$ \\
\hline $\mathrm{Pb}$ & $7.007 \mathrm{E}-02$ \\
\hline $\mathrm{Se}$ & $9.805 E-03$ \\
\hline
\end{tabular}

Waste Type: Solids (CH-Non-alpha)

$3.928 E+00$

Activity
(Ci/yr)

2. $630 \mathrm{E}+00$

$4.248 \mathrm{E}+01$

$2.497 \mathrm{E}-02$

$2.776 \mathrm{E}+00$

$3.379 \mathrm{E}-02$

$1.616 \mathrm{E}-02$

$5.553 \mathrm{E}+00$

$1.209 \mathrm{E}+01$

1. $763 \mathrm{E}-02$

$2.644 \mathrm{E}-02$

$5.553 \mathrm{E}-01$

$7.467 \mathrm{E}+01$

$1.043 \mathrm{E}-01$

3. $026 \mathrm{E}-01$

$8.668 \mathrm{E}-02$

$7.052 \mathrm{E}-02$

$1.175 \mathrm{E}-02$

$2.350 \mathrm{E}-02$

2. $615 \mathrm{E}+00$

$2.938 \mathrm{E}-03$

1. $043 E-01$

$1.043 \mathrm{E}-01$

2. $615 \mathrm{E}+00$
Total Mass $(\mathrm{kg} / \mathrm{yr}): 6.620 \mathrm{E}+03$

\begin{tabular}{|c|c|}
\hline Chemical & $\begin{array}{l}\text { Amount } \\
(\mathrm{kg} / \mathrm{yr})\end{array}$ \\
\hline $57-12-5$ & I. $282 \mathrm{E}-01$ \\
\hline & $2.231 \mathrm{E}+00$ \\
\hline $\mathrm{Ag}$ & $8.783 \mathrm{E}-02$ \\
\hline As & $3.299 \mathrm{E}-02$ \\
\hline $\mathrm{Ba}$ & $1.662 \mathrm{E}+00$ \\
\hline $\mathrm{Cd}$ & $5.269 \mathrm{E}-02$ \\
\hline$C 1-2-x$ & $1.281 \mathrm{E}+00$ \\
\hline$C 1-3-x$ & $1.108 \mathrm{E}+01$ \\
\hline$C 1-4-x$ & $1.215 E+01$ \\
\hline$C l-F-x$ & $2.090 \mathrm{E}-01$ \\
\hline $\mathrm{Cr}$ & $1.846 \mathrm{E}+00$ \\
\hline HC-insol & $2.753 E+01$ \\
\hline HC-solub & $1.411 \mathrm{E}+01$ \\
\hline $\mathrm{Hg}$ & $1.438 \mathrm{E}-01$ \\
\hline $\mathrm{Pb}$ & $4.165 E+00$ \\
\hline $\mathrm{Se}$ & $3.790 \mathrm{E}-02$ \\
\hline
\end{tabular}

\begin{tabular}{|c|c|}
\hline Chemical & $\begin{array}{l}\text { Amount } \\
(\mathrm{kg} / \mathrm{yr})\end{array}$ \\
\hline $\begin{array}{l}57-12-5 \\
75-09-2\end{array}$ & $\begin{array}{l}1.282 \mathrm{E}-01 \\
2.231 \mathrm{E}+00\end{array}$ \\
\hline $\mathrm{Ag}$ & $8.783 \mathrm{E}-02$ \\
\hline As & $3.299 \mathrm{E}-02$ \\
\hline $\mathrm{Ba}$ & $1.662 \mathrm{E}+00$ \\
\hline $\mathrm{cd}$ & $5.269 \mathrm{E}-02$ \\
\hline$C 1-2-x$ & $1.281 \mathrm{E}+00$ \\
\hline$c 1-3-x$ & $1.108 \mathrm{E}+01$ \\
\hline$C 1-4-x$ & $1.215 E+01$ \\
\hline$C l-F-x$ & $2.090 \mathrm{E}-01$ \\
\hline $\mathrm{Cr}$ & $1.846 \mathrm{E}+00$ \\
\hline HC-insol & $2.753 E+01$ \\
\hline HC-solub & $1.411 \mathrm{E}+01$ \\
\hline $\mathrm{Hg}$ & $1.438 \mathrm{E}-01$ \\
\hline $\mathrm{Pb}$ & $4.165 E+00$ \\
\hline $\mathrm{se}$ & $3.790 \mathrm{E}-02$ \\
\hline
\end{tabular}


Shipping Site: KWS Waste Type: Organic Liquids ( $\mathrm{CH}-\mathrm{Non}-a l p h a)$

Total Volume $(\mathrm{m} 3 / \mathrm{yr})$ :

6. $454 \mathrm{E}-02$

Total Mass $(\mathrm{kg} / \mathrm{yr}): \quad 7.394 \mathrm{E}+01$

\begin{tabular}{l} 
Radionuclide \\
\hline Ba-137m \\
$\mathrm{Co}-60$ \\
$\mathrm{Cs}-134$ \\
$\mathrm{Cs}-137$ \\
$\mathrm{Eu}-154$ \\
$\mathrm{Eu}-155$ \\
$\mathrm{Fe}-55$ \\
$\mathrm{H}-3$ \\
$\mathrm{Mn}-54$ \\
$\mathrm{Nb}-94$ \\
$\mathrm{Ni}-59$ \\
$\mathrm{Ni}-63$ \\
$\mathrm{~Pa}-234 \mathrm{~m}$ \\
$\mathrm{Pm}-147$ \\
$\mathrm{Pu}-238$ \\
$\mathrm{Pu}-241$ \\
$\mathrm{Sb}-125$ \\
$\mathrm{Sm}-151$ \\
$\mathrm{Sr}-90$ \\
$\mathrm{Te}-125 \mathrm{~m}$ \\
$\mathrm{Th}-234$ \\
$\mathrm{U}-238$ \\
$\mathrm{Y}-90$
\end{tabular}

Shipping site:

\begin{tabular}{c}
$\begin{array}{c}\text { Activity } \\
(C i / y r)\end{array}$ \\
\hline $4.321 \mathrm{E}-02$ \\
$6.979 \mathrm{E}-01$ \\
$4.103 \mathrm{E}-04$ \\
$4.562 \mathrm{E}-02$ \\
$5.552 \mathrm{E}-04$ \\
$2.655 \mathrm{E}-04$ \\
$9.125 \mathrm{E}-02$ \\
$1.987 \mathrm{E}-01$ \\
$2.896 \mathrm{E}-04$ \\
$4.345 \mathrm{E}-04$ \\
$9.125 \mathrm{E}-03$ \\
$1.226 \mathrm{E}+00$ \\
$1.714 \mathrm{E}-03$ \\
$4.973 \mathrm{E}-03$ \\
$1.424 \mathrm{E}-03$ \\
$1.158 \mathrm{E}-03$ \\
$1.931 \mathrm{E}-04$ \\
$3.862 \mathrm{E}-04$ \\
$4.297 \mathrm{E}-02$ \\
$4.828 \mathrm{E}-05$ \\
$1.714 \mathrm{E}-03$ \\
$1.714 \mathrm{E}-03$ \\
$4.297 \mathrm{E}-02$
\end{tabular}

\begin{tabular}{l} 
Chemical \\
\hline $57-12-5$ \\
$75-09-2$ \\
Ag \\
As \\
Ba \\
Cd \\
CI-2-x \\
Cl-3-x \\
Cl-4-x \\
C1-F-x \\
Cr \\
HC-insol \\
HC-solub \\
Hg \\
Pb \\
Se
\end{tabular}

Total Volume (m3/yr):

Radionuclide

$\mathrm{Ba}-137 \mathrm{~m}$

Co- 60

$\mathrm{Cs}-134$

Cs -137

Eu-154

Eu-155

$\mathrm{Fe}-55$

$\mathrm{H}-3$

$\mathrm{Mn}-54$

$\mathrm{Nb}-94$

Ni-59

$\mathrm{Ni}-63$

$\mathrm{Pa}-234 \mathrm{~m}$

Pm -147

Pu-238

Pu-241

$\mathrm{Sb}-125$

$\mathrm{Sm}-151$

Sr -90

$\mathrm{Te}-125 \mathrm{~m}$

Th-234

$\mathrm{U}-238$

$Y-90$
Waste Type: Solids (CH-Non-alpha)

Total Mass (kg/yr): $1.580 \mathrm{E}+03$

\begin{tabular}{l}
$\begin{array}{c}\text { Amount } \\
(\mathrm{kg} / \mathrm{Yr})\end{array}$ \\
\hline $3.051 \mathrm{E}-03$ \\
$5.559 \mathrm{E}-02$ \\
$1.529 \mathrm{E}-03$ \\
$3.090 \mathrm{E}-04$ \\
$1.820 \mathrm{E}-03$ \\
$4.317 \mathrm{E}-02$ \\
$3.474 \mathrm{E}-02$ \\
$3.332 \mathrm{E}+00$ \\
$6.993 \mathrm{E}-01$ \\
$6.567 \mathrm{E}-03$ \\
$4.058 \mathrm{E}-02$ \\
$6.683 \mathrm{E}+00$ \\
$8.322 \mathrm{E}+00$ \\
$8.907 \mathrm{E}-03$ \\
$9.555 \mathrm{E}-03$ \\
$1.337 \mathrm{E}-03$
\end{tabular}

1. $262 \mathrm{E}+00$

8. $449 \mathrm{E}-01$

1. $364 E+01$

$8.024 \mathrm{E}-03$

$8.921 \mathrm{E}-01$

1. $085 \mathrm{E}-02$

$5.192 \mathrm{E}-03$

1. $784 \mathrm{E}+00$

3. $886 \mathrm{E}+00$

5. $664 \mathrm{E}-03$

8. $496 \mathrm{E}-03$

$1.784 \mathrm{E}-01$

$2.399 E+01$

3. $351 \mathrm{E}-02$

$9.724 \mathrm{E}-02$

2.785E-02

2. $265 \mathrm{E}-02$

3. $776 \mathrm{E}-03$

7. $552 \mathrm{E}-03$

8. $402 E-01$

9. $440 \mathrm{E}-04$

3. $351 \mathrm{E}-02$

3. $351 E-02$

8. $402 \mathrm{E}-01$

\begin{tabular}{|c|c|}
\hline Chemical & $\begin{array}{l}\text { Amount } \\
\text { (kg/yr) }\end{array}$ \\
\hline $\begin{array}{l}57-12-5 \\
75-09-2\end{array}$ & $\begin{array}{l}8.005 E-02 \\
3.801 E-01\end{array}$ \\
\hline $\mathrm{Ag}$ & $2.572 \mathrm{E}-02$ \\
\hline As & $8.795 \mathrm{E}-03$ \\
\hline $\mathrm{Ba}$ & $1.035 \mathrm{E}+00$ \\
\hline $\mathrm{Cd}$ & $1.157 \mathrm{E}-02$ \\
\hline$C 1-2-x$ & $3.221 \mathrm{E}-01$ \\
\hline$c 1-3-x$ & $1.699 \mathrm{E}+00$ \\
\hline CI-4-x & $1.988 \mathrm{E}+00$ \\
\hline$C 1-F-x$ & $2.323 E-01$ \\
\hline $\mathrm{Cr}$ & $1.010 E+00$ \\
\hline HC-insol & $4.421 E+00$ \\
\hline HC-soIub & 1. $600 \mathrm{E}+00$ \\
\hline $\mathrm{Hg}$ & $6.065 \mathrm{E}-02$ \\
\hline $\mathrm{Pb}$ & $2.396 \mathrm{E}+00$ \\
\hline Se & 1. $789 \mathrm{E}-02$ \\
\hline
\end{tabular}




\begin{tabular}{l} 
Shipping Site: PNS \\
Total Volume (m3/yr): \\
Radionuclide \\
\hline Ba-137m \\
Co-60 \\
Cs -134 \\
Cs-137 \\
Eu-154 \\
Eu-155 \\
Fe-55 \\
H-3 \\
Mn-54 \\
Nb-94 \\
Ni-59 \\
Ni -63 \\
Pa-234m \\
Pm-147 \\
Pu-238 \\
Pu-241 \\
Sb-125 \\
Sm-15I \\
Sr -90 \\
Te-125m \\
Th-234 \\
U-238 \\
Y-90 \\
P
\end{tabular}

Shipping Site: PPPL

Total Volume (m3/yr):

Radionuclide

$\mathrm{Ba}-137 \mathrm{~m}$

Cs -137

$\mathrm{H}-3$

$\mathrm{Pa}-234 \mathrm{~m}$

Pu-241

$\mathrm{Sr}-90$

Th-234

$\mathrm{U}-238$

$Y-90$

Shipping Site: RMI

Total Volume $(\mathrm{m} 3 / \mathrm{yr})$ :

Radionuclide

Ac -228

$\mathrm{Bi}-212$

$\mathrm{Pa}-234$

$\mathrm{Pa}-234 \mathrm{~m}$

$\mathrm{Pb}-212$

Po- 212

Po-216

$\mathrm{Ra}-224$

$\mathrm{Ra}-228$

TC-99

Th -228

Th-231

Th-232

$\mathrm{Th}-234$

T1-208

$\mathrm{U}-235$

$\mathrm{U}-238$
Waste Type: Solids (CH-Non-alpha)

Total Mass (kg/vr): $9.088 \mathrm{E}+01$

$5.200 E-02$

\begin{tabular}{l} 
Chemical \\
\hline $75-09-2$ \\
Ag \\
As \\
Ba \\
Cd \\
C1-2-x \\
Cl-3-x \\
Cl-4-x \\
Cl-F-x \\
Cr \\
HC-insol \\
HC-solub \\
$\mathrm{Hg}$ \\
$\mathrm{Pb}$ \\
$\mathrm{Se}$
\end{tabular}

\begin{tabular}{c}
$\begin{array}{c}\text { Amount } \\
(\mathrm{kg} / \mathrm{Yr})\end{array}$ \\
\hline $1.324 \mathrm{E}-02$ \\
$6.570 \mathrm{E}-04$ \\
$5.453 \mathrm{E}-04$ \\
$1.708 \mathrm{E}-02$ \\
$8.438 \mathrm{E}-03$ \\
$1.324 \mathrm{E}-02$ \\
$7.909 \mathrm{E}-01$ \\
$1.483 \mathrm{E}-01$ \\
$7.499 \mathrm{E}-03$ \\
$1.051 \mathrm{E}-01$ \\
$2.629 \mathrm{E}+00$ \\
$1.314 \mathrm{E}+00$ \\
$9.046 \mathrm{E}-03$ \\
$1.319 \mathrm{E}-02$ \\
$2.365 \mathrm{E}-03$
\end{tabular}

$9.335 \mathrm{E}-04$

$1.555 \mathrm{E}-04$

$3.111 \mathrm{E}-04$

$3.462 E-02$

$3.889 \mathrm{E}-05$

$1.380 \mathrm{E}-03$

$1.380 \mathrm{E}-03$

$3.462 \mathrm{E}-02$

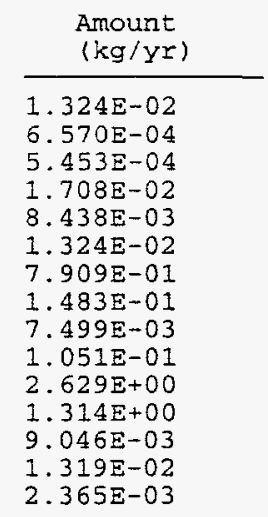

Waste Type: Solids (CH-Non-alpha)

$5.456 \mathrm{E}-04$

Total Mass (kg/Yr): 7.191E-01

\begin{tabular}{|c|c|}
\hline Chemical & $\begin{array}{l}\text { Amount } \\
(\mathrm{kg} / \mathrm{Yr})\end{array}$ \\
\hline $75-09-2$ & 1. $438 \mathrm{E}-02$ \\
\hline$C I-2-x$ & $7.190 \mathrm{E}-03$ \\
\hline Cl-3-x & $7.190 \mathrm{E}-02$ \\
\hline$C l-4-x$ & $5.752 E-02$ \\
\hline HC-insol & $1.797 \mathrm{E}-01$ \\
\hline HC-solub & $8.629 \mathrm{E}-02$ \\
\hline
\end{tabular}

HC-solub

Waste Type: Organic Liquids ( $\mathrm{CH}-\mathrm{Non}-\mathrm{alpha})$

6.677E-01 Total Mass $(\mathrm{kg} / \mathrm{yr}): 9.741 \mathrm{E}+02$

\begin{tabular}{c}
$\begin{array}{c}\text { Activity } \\
\text { (Ci/yr) }\end{array}$ \\
\hline $1.429 \mathrm{E}-06$ \\
$2.403 \mathrm{E}-07$ \\
$1.816 \mathrm{E}-07$ \\
$1.764 \mathrm{E}-03$ \\
$2.403 \mathrm{E}-07$ \\
$1.549 \mathrm{E}-07$ \\
$2.403 \mathrm{E}-07$ \\
$2.403 \mathrm{E}-07$ \\
$1.429 \mathrm{E}-06$ \\
$2.657 \mathrm{E}-05$ \\
$2.403 \mathrm{E}-07$ \\
$1.378 \mathrm{E}-06$ \\
$1.450 \mathrm{E}-05$ \\
$1.764 \mathrm{E}-03$ \\
$9.081 \mathrm{E}-08$ \\
$1.372 \mathrm{E}-06$ \\
$1.764 \mathrm{E}-03$
\end{tabular}

\begin{tabular}{l} 
Chemical \\
\hline $75-09-2$ \\
As \\
$\mathrm{Ba}$ \\
$\mathrm{Cd}$ \\
$\mathrm{Cl}-2-\mathrm{x}$ \\
$\mathrm{Cl}-3-\mathrm{x}$ \\
$\mathrm{Cl}-4-\mathrm{x}$ \\
$\mathrm{Cl}-\mathrm{F}-\mathrm{x}$ \\
$\mathrm{Cr}$ \\
$\mathrm{HC}-$ insol \\
$\mathrm{HC}-$ SOlub \\
Hg \\
$\mathrm{Pb}$
\end{tabular}

Amount

$(\mathrm{kg} / \mathrm{Yr})$

$1.527 E+00$

$3.769 \mathrm{E}-04$

$1.615 \mathrm{E}-03$

1. $071 \mathrm{E}+00$

8. $108 \mathrm{E}-01$

1. $123 \mathrm{E}+02$

2. $505 \mathrm{E}+01$

4. $625 \mathrm{E}-02$

9. $331 \mathrm{E}-01$

$2.184 \mathrm{E}+01$

$1.277 E+01$

$6.122 \mathrm{E}-03$

2. $045 \mathrm{E}-01$ 
A-48

Shipping site: RMI

Total volume $(\mathrm{m} 3 / \mathrm{yr})$ :

Radionuclide

AC -228

$\mathrm{Bi}-212$

$\mathrm{Pa}-234$

$\mathrm{Pa}-234 \mathrm{~m}$

$\mathrm{Pb}-212$

Po-212

Po-216

Ra-224

$\mathrm{Ra}-228$

Tc-99

Th-228

Th-231

Th-232

Th-234

TI-208

U-235

$\mathrm{U}-238$
Waste Type: Solids (CH-Non-alpha)

$1.091 \mathrm{E}+00$

Total Mass $(\mathrm{kg} / \mathrm{yr}): \quad 8.323 \mathrm{E}+02$

\begin{tabular}{l} 
Chemical \\
\hline $57-12-5$ \\
$75-09-2$ \\
Ag \\
As \\
$\mathrm{Ba}$ \\
$\mathrm{Cd}$ \\
$\mathrm{Cl}-2-\mathrm{x}$ \\
$\mathrm{Cl}-3-\mathrm{x}$ \\
$\mathrm{Cl}-4-\mathrm{x}$ \\
$\mathrm{Cl}-\mathrm{F}-\mathrm{x}$ \\
$\mathrm{Cr}$ \\
$\mathrm{HC}-\mathrm{insol}$ \\
$\mathrm{HC}-\mathrm{solub}$ \\
$\mathrm{Hg}$ \\
$\mathrm{Pb}$ \\
$\mathrm{Se}$
\end{tabular}

Amount

2. $315 \mathrm{E}-01$

$6.651 \mathrm{E}-03$

1. $974 \mathrm{E}-03$

$5.734 \mathrm{E}-01$

1. $836 \mathrm{E}-02$

2. $862 \mathrm{E}-01$

2. $561 \mathrm{E}+00$

$7.371 \mathrm{E}-01$

3. $452 \mathrm{E}-01$

2. $638 \mathrm{E}-01$

8. $888 \mathrm{E}+00$

2. $825 E+00$

2. $678 \mathrm{E}-02$

8. $498 \mathrm{E}-02$

6. $070 \mathrm{E}-03$ 
Destination site: RFP

\begin{tabular}{l} 
Shipping Site: GJCT \\
Total Volume (m3/yr): \\
Radionuclide \\
\hline AC-228 \\
Bi-212 \\
Pa-234 \\
Pa-234m \\
Pb-212 \\
Po-212 \\
Po-216 \\
Ra-224 \\
Ra-228 \\
TC-99 \\
Th-228 \\
Th-231 \\
Th-232 \\
Th-234 \\
T1-208 \\
U-235 \\
U-238
\end{tabular}

Shipping Site:

GJCT
$1.290 \mathrm{E}-02$
Waste Type: Organic Liquids (CH-Non-alpha)

Total Mass $(\mathrm{kg} / \mathrm{yr}): 1.478 \mathrm{E}+01$
Radionuclide

Ac -228

$\mathrm{Bi}-212$

$\mathrm{Pa}-234$

$\mathrm{Pa}-234 \mathrm{~m}$

$\mathrm{Pb}-212$

Po-212

PO-216

$\mathrm{Ra}-224$

$\mathrm{Ra}-228$

TC-99

Th-228

Th-231

Th-232

Th-234

T1-208

$\mathrm{U}-235$

$\mathrm{U}-238$

shipping site: KCP

Total Volume (m3/yr):

Radionuclide

$\mathrm{Ba}-137 \mathrm{~m}$

Co-60

Cs -134

Cs -137

Eu-154

Eu-155

$\mathrm{Fe}-55$

$\mathrm{H}-3$

Mn-54

$\mathrm{Nb}-94$

$\mathrm{Ni}-59$

Ni-63

$\mathrm{Pa}-234 \mathrm{~m}$

$\mathrm{Pm}-147$

Pu-238

Pu-241

$\mathrm{Sb}-125$

$\mathrm{Sm}-151$

$\mathrm{Sr}-90$

$\mathrm{Te}-125 \mathrm{~m}$

Th-234

$\mathrm{U}-238$

$\mathrm{Y}-90$

\begin{tabular}{r}
$\begin{array}{r}\text { Activity } \\
(\text { Ci/yr })\end{array}$ \\
\hline $2.762 \mathrm{E}-08$ \\
$4.647 \mathrm{E}-09$ \\
$3.511 \mathrm{E}-09$ \\
$3.411 \mathrm{E}-05$ \\
$4.647 \mathrm{E}-09$ \\
$2.995 \mathrm{E}-09$ \\
$4.647 \mathrm{E}-09$ \\
$4.647 \mathrm{E}-09$ \\
$2.762 \mathrm{E}-08$ \\
$5.138 \mathrm{E}-07$ \\
$4.647 \mathrm{E}-09$ \\
$2.664 \mathrm{E}-08$ \\
$2.805 \mathrm{E}-07$ \\
$3.411 \mathrm{E}-05$ \\
$1.756 \mathrm{E}-09$ \\
$2.654 \mathrm{E}-08$ \\
$3.411 \mathrm{E}-05$
\end{tabular}

\begin{tabular}{l} 
Chemical \\
\hline $57-12-5$ \\
$75-09-2$ \\
$\mathrm{Ag}$ \\
$\mathrm{As}$ \\
$\mathrm{Ba}$ \\
$\mathrm{Cd}$ \\
$\mathrm{Cl}-2-\mathrm{x}$ \\
$\mathrm{Cl}-3-\mathrm{x}$ \\
$\mathrm{Cl}-4-\mathrm{x}$ \\
$\mathrm{Cl}-\mathrm{F}-\mathrm{x}$ \\
$\mathrm{Cr}$ \\
$\mathrm{HC}-$ insol \\
$\mathrm{HC}-\mathrm{solub}$ \\
$\mathrm{Hg}$ \\
$\mathrm{Pb}$ \\
$\mathrm{Se}$
\end{tabular}
Waste Type: Solids (CH-Non-alpha)

Total Mass $(\mathrm{kg} / \mathrm{Yr}): 5.478 \mathrm{E}+01$
$2.953 E-02$

\begin{tabular}{c}
$\begin{array}{c}\text { Activity } \\
\text { (Ci/yr) }\end{array}$ \\
\hline $6.356 \mathrm{E}-08$ \\
$1.069 \mathrm{E}-08$ \\
$8.080 \mathrm{E}-09$ \\
$7.849 \mathrm{E}-05$ \\
$1.069 \mathrm{E}-08$ \\
$6.891 \mathrm{E}-09$ \\
$1.069 \mathrm{E}-08$ \\
$1.054 \mathrm{E}-08$ \\
$6.268 \mathrm{E}-08$ \\
$1.165 \mathrm{E}-06$ \\
$1.069 \mathrm{E}-08$ \\
$6.131 \mathrm{E}-08$ \\
$6.454 \mathrm{E}-07$ \\
$7.849 \mathrm{E}-05$ \\
$4.040 \mathrm{E}-09$ \\
$6.107 \mathrm{E}-08$ \\
$7.849 \mathrm{E}-05$
\end{tabular}

Waste Type: Solids (CH-Non-alpha)

Total Mass $(\mathrm{kg} / \mathrm{yr}): 3.237 \mathrm{E}+01$

$4.219 \mathrm{E}-02$

Chemical

Amount

$57-12-5$

$75-09-2$

Ag

As

$\mathrm{Ba}$

C1-2-x

Cl-3-x

$\mathrm{C} 1-4-\mathrm{x}$

Cl-F-x

$\mathrm{Cr}$

HC-insol

$\mathrm{HC}-\mathrm{SO}$ Iub

$\mathrm{Hg}$

$\mathrm{Pb}$

Se

\begin{tabular}{c}
$\begin{array}{c}\text { Amount } \\
(\mathrm{kg} / \mathrm{Yr})\end{array}$ \\
\hline $6.102 \mathrm{E}-04$ \\
$1.111 \mathrm{E}-02$ \\
$3.059 \mathrm{E}-04$ \\
$6.180 \mathrm{E}-05$ \\
$3.641 \mathrm{E}-04$ \\
$8.635 \mathrm{E}-03$ \\
$6.949 \mathrm{E}-03$ \\
$6.665 \mathrm{E}-01$ \\
$1.398 \mathrm{0}-01$ \\
$1.313 \mathrm{E}-03$ \\
$8.116 \mathrm{E}-03$ \\
$1.336 \mathrm{E}+00$ \\
$1.664 \mathrm{E}+00$ \\
$1.781 \mathrm{E}-03$ \\
$1.911 \mathrm{0}-03$ \\
$2.674 \mathrm{E}-04$
\end{tabular}

Amount

1

\begin{tabular}{l} 
Amount \\
$(\mathrm{kg} / \mathrm{yr})$ \\
\hline
\end{tabular}

1. $997 \mathrm{E}-04$

1. $051 \mathrm{E}-03$

6. 108E-04

2. $981 E-04$

3. $480 E-03$

4. $003 \mathrm{E}-04$

1. $051 E-03$

2. $387 \mathrm{E}-02$

$7.830 \mathrm{E}-03$

$5.619 \mathrm{E}-04$

4. $507 \mathrm{E}-03$

7. $674 \mathrm{E}-02$

8. $522 \mathrm{E}-02$

1. $306 \mathrm{E}-03$

$5.304 \mathrm{E}-03$

$4.666 \mathrm{E}-04$ 
Destination Site: SRS

Shipping site: BAPL

Total Volume (m3/yr):

Radionuclide

Am- 241

$\mathrm{Ba}-137 \mathrm{~m}$

Co- 60

Cs -137

Eu-154

$\mathrm{H}-3$

$\mathrm{Nb}-94$

$\mathrm{Ni}-59$

$\mathrm{Ni}-63$

$\mathrm{Pa}-234 \mathrm{~m}$

Pu -238

$\mathrm{Pu}-239$

Pu-240

Pu-241

$\mathrm{Sm}-151$

Sr -90

TC- 99

Th-234

$\mathrm{U}-238$

$\mathrm{Y}-90$

Shipping site: CNS

Total Volume $(\mathrm{m} 3 / \mathrm{yr})$ :

Radionuclide

$\mathrm{Ba}-137 \mathrm{~m}$

Co-60

Cs-134

Cs -137

Eu-154

Eu-155

$\mathrm{Fe}-55$

$\mathrm{H}-3$

$\mathrm{Mn}-54$

$\mathrm{Nb}-94$

$\mathrm{Ni}-59$

$\mathrm{Ni}-63$

$\mathrm{Pa}-234 \mathrm{~m}$

$\mathrm{Pm}-147$

Pu-238

Pu-241

Sb-125

$\mathrm{Sm}-151$

Sr-90

Te-125m

Th-234

$\mathrm{U}-238$

$\mathrm{Y}-90$
Waste Type: Solids (CH-Alpha)

Total Mass (kg/yr): $\quad 3.493 E+02$

8.357E-01 Total Mass (kg/Yr): $3.493 E+02$

\begin{tabular}{c}
$\begin{array}{c}\text { Activity } \\
(\mathrm{Ci} / \mathrm{Y})\end{array}$ \\
\hline $6.984 \mathrm{E}-04$ \\
$7.629 \mathrm{E}-01$ \\
$1.021 \mathrm{E}-03$ \\
$8.160 \mathrm{E}-01$ \\
$1.217 \mathrm{E}-03$ \\
$6.053 \mathrm{E}-02$ \\
$6.984 \mathrm{E}-05$ \\
$1.563 \mathrm{E}-03$ \\
$1.606 \mathrm{E}-01$ \\
$4.490 \mathrm{E}-04$ \\
$3.791 \mathrm{E}-02$ \\
$6.286 \mathrm{E}-04$ \\
$2.793 \mathrm{E}-04$ \\
$1.633 \mathrm{E}-02$ \\
$1.167 \mathrm{E}-02$ \\
$7.264 \mathrm{E}-01$ \\
$3.625 \mathrm{E}-04$ \\
$4.490 \mathrm{E}-04$ \\
$4.490 \mathrm{E}-04$ \\
$7.264 \mathrm{E}-01$
\end{tabular}

\begin{tabular}{|c|c|}
\hline Chemical & $\begin{array}{l}\text { Amount } \\
(\mathrm{kg} / \mathrm{yr})\end{array}$ \\
\hline $75-09-2$ & $2.095 \mathrm{E}-01$ \\
\hline $\mathrm{Ag}$ & $3.4938-03$ \\
\hline $\mathrm{Ba}$ & $1.397 \mathrm{E}-01$ \\
\hline $\mathrm{Cd}$ & $6.986 \mathrm{E}-04$ \\
\hline Cl-2-x & $2.445 E-01$ \\
\hline$C 1-3-x$ & $8.733 E-01$ \\
\hline$C 1-4-x$ & $4.191 \mathrm{E}-01$ \\
\hline $\mathrm{Cl}-\mathrm{F}-\mathrm{X}$ & $3.493 E-01$ \\
\hline $\mathrm{Cr}$ & $5.239 \mathrm{E}-03$ \\
\hline HC-insol & $3.493 E+00$ \\
\hline $\mathrm{HC}-$ solub & $1.746 \mathrm{E}-03$ \\
\hline $\mathrm{Hg}$ & $6.986 \mathrm{E}-04$ \\
\hline $\mathrm{Pb}$ & $3.493 \mathrm{E}-02$ \\
\hline $\mathrm{Se}$ & $1.047 \mathrm{E}-03$ \\
\hline
\end{tabular}

Waste Type: Organic Liquids (CH-Non-alpha)

$9.054 \mathrm{E}-01$

Total Mass (kg/yr): $1.026 \mathrm{E}+03$

\begin{tabular}{r}
$\begin{array}{r}\text { Activity } \\
\text { (Ci/yr) }\end{array}$ \\
\hline $6.061 \mathrm{E}-01$ \\
$9.790 \mathrm{E}+00$ \\
$5.756 \mathrm{E}-03$ \\
$6.400 \mathrm{E}-01$ \\
$7.788 \mathrm{E}-03$ \\
$3.724 \mathrm{E}-03$ \\
$1.280 \mathrm{E}+00$ \\
$2.788 \mathrm{E}+00$ \\
$4.063 \mathrm{E}-03$ \\
$6.095 \mathrm{E}-03$ \\
$1.280 \mathrm{E}-01$ \\
$1.720 \mathrm{E}+01$ \\
$2.404 \mathrm{E}-02$ \\
$6.975 \mathrm{E}-02$ \\
$1.997 \mathrm{E}-02$ \\
$1.625 \mathrm{E}-02$ \\
$2.709 \mathrm{E}-03$ \\
$5.418 \mathrm{E}-03$ \\
$6.027 \mathrm{E}-01$ \\
$6.772 \mathrm{E}-04$ \\
$2.404 \mathrm{E}-02$ \\
$2.404 \mathrm{E}-02$ \\
$6.027 \mathrm{E}-01$
\end{tabular}

\begin{tabular}{c}
$\begin{array}{c}\text { Amount } \\
(\mathrm{kg} / \mathrm{yr})\end{array}$ \\
\hline $5.151 \mathrm{E}-02$ \\
$7.241 \mathrm{E}-01$ \\
$2.274 \mathrm{E}-02$ \\
$3.999 \mathrm{E}-03$ \\
$2.356 \mathrm{E}-02$ \\
$5.620 \mathrm{E}-01$ \\
$4.522 \mathrm{E}-01$ \\
$4.355 \mathrm{E}+01$ \\
$9.136 \mathrm{E}+00$ \\
$8.522 \mathrm{E}-02$ \\
$5.418 \mathrm{E}-01$ \\
$8.707 \mathrm{E}+01$ \\
$1.317 \mathrm{E}+02$ \\
$1.340 \mathrm{E}-01$ \\
$1.275 \mathrm{E}-01$ \\
$1.998 \mathrm{E}-02$
\end{tabular}


$A-51$

Shipping Site: CNS waste Type: Solids (CH-Non-alpha)

Total Volume $(\mathrm{m} 3 / \mathrm{yr})$

$9.030 \mathrm{E}-02$

Total Mass $(\mathrm{kg} / \mathrm{yr})$ :

$6.772 E+01$

Radionuclide

$\mathrm{Ba}-137 \mathrm{~m}$

Co-50

Cs -134

Cs -137

Eu-154

Eu-155

Fe-55

$\mathrm{H}-3$

$\mathrm{Mn}-54$

$\mathrm{Nb}-94$

$\mathrm{Ni}-59$

$\mathrm{Ni}-63$

$\mathrm{Pa}-234 \mathrm{~m}$

$\mathrm{Pm}-147$

Pu-238

Pu-241

$\mathrm{Sb}-125$

$\mathrm{Sm}-151$

Sr -90

$\mathrm{Te}-125 \mathrm{~m}$

Th-234

$\mathrm{U}-238$

$Y-90$

\begin{tabular}{c}
$\begin{array}{c}\text { Activity } \\
\text { (Ci/yr) }\end{array}$ \\
\hline $6.218 \mathrm{E}-02$ \\
$1.004 \mathrm{E}+00$ \\
$5.431 \mathrm{E}-04$ \\
$6.038 \mathrm{E}-02$ \\
$7.990 \mathrm{E}-04$ \\
$3.821 \mathrm{E}-04$ \\
$1.313 \mathrm{E}-01$ \\
$2.860 \mathrm{E}-01$ \\
$4.169 \mathrm{E}-04$ \\
$6.253 \mathrm{E}-04$ \\
$1.313 \mathrm{E}-02$ \\
$1.765 \mathrm{E}+00$ \\
$2.466 \mathrm{E}-03$ \\
$7.156 \mathrm{E}-03$ \\
$2.049 \mathrm{E}-03$ \\
$1.667 \mathrm{E}-03$ \\
$2.779 \mathrm{E}-04$ \\
$5.558 \mathrm{E}-04$ \\
$5.686 \mathrm{E}-02$ \\
$6.948 \mathrm{E}-05$ \\
$2.466 \mathrm{E}-03$ \\
$2.466 \mathrm{E}-03$ \\
$5.686 \mathrm{E}-02$
\end{tabular}

\begin{tabular}{l}
\multicolumn{1}{c}{ Chemical } \\
\hline $57-12-5$ \\
$75-09-2$ \\
$\mathrm{Ag}$ \\
$\mathrm{As}$ \\
$\mathrm{Ba}$ \\
$\mathrm{Cd}$ \\
$\mathrm{Cl}-2-\mathrm{x}$ \\
$\mathrm{Cl}-3-\mathrm{x}$ \\
$\mathrm{Cl}-4-\mathrm{x}$ \\
$\mathrm{Cl}-\mathrm{F}-\mathrm{x}$ \\
$\mathrm{Cr}$ \\
$\mathrm{HC}-$ insol \\
$\mathrm{HC}-\mathrm{SO}$ lub \\
$\mathrm{Hg}$ \\
$\mathrm{Pb}$ \\
$\mathrm{Se}$
\end{tabular}

\begin{tabular}{c}
$\begin{array}{c}\text { Amount } \\
(\mathrm{kg} / \mathrm{Y})\end{array}$ \\
\hline $2.357 \mathrm{E}-03$ \\
$2.142 \mathrm{E}-02$ \\
$7.345 \mathrm{E}-04$ \\
$2.549 \mathrm{E}-04$ \\
$3.376 \mathrm{E}-02$ \\
$2.213 \mathrm{E}-03$ \\
$2.738 \mathrm{E}-02$ \\
$2.718 \mathrm{E}-01$ \\
$7.524 \mathrm{E}-02$ \\
$2.847 \mathrm{E}-02$ \\
$2.755 \mathrm{E}-02$ \\
$9.141 \mathrm{E}-01$ \\
$3.299 \mathrm{E}-01$ \\
$3.234 \mathrm{E}-03$ \\
$5.496 \mathrm{E}-02$ \\
$9.647 \mathrm{E}-04$
\end{tabular}

Shipping Site: MOUND

Waste Type: Organic Iiquids (CH-Alpha)

Total Volume $(\mathrm{m} 3 / \mathrm{yr})$ :

$1.768 E+00$

Total Mass $(\mathrm{kg} / \mathrm{Yr}): 2.190 \mathrm{E}+03$

\begin{tabular}{l} 
Radionuclide \\
\hline $\mathrm{Ba}-137 \mathrm{~m}$ \\
$\mathrm{Cs}-137$ \\
$\mathrm{H}-3$ \\
$\mathrm{~Pa}-234 \mathrm{~m}$ \\
$\mathrm{Pu}-238$ \\
$\mathrm{Pu}-241$ \\
$\mathrm{Sr}-90$ \\
$\mathrm{Th}-234$ \\
$\mathrm{U}-238$ \\
$\mathrm{Y}-90$
\end{tabular}

\begin{tabular}{c}
$\begin{array}{c}\text { Activity } \\
(C i / y r)\end{array}$ \\
\hline $2.339 \mathrm{E}-02$ \\
$2.450 \mathrm{E}-02$ \\
$1.111 \mathrm{E}+02$ \\
$5.680 \mathrm{E}-03$ \\
$5.569 \mathrm{E}-03$ \\
$1.113 \mathrm{E}-01$ \\
$2.339 \mathrm{E}-02$ \\
$5.680 \mathrm{E}-03$ \\
$5.680 \mathrm{E}-03$ \\
$2.339 \mathrm{E}-02$
\end{tabular}

\begin{tabular}{l} 
Chemical \\
\hline $75-09-2$ \\
$\mathrm{As}$ \\
$\mathrm{Ba}$ \\
$\mathrm{Cd}$ \\
$\mathrm{Cl}-2-\mathrm{x}$ \\
$\mathrm{Cl}-3-\mathrm{x}$ \\
$\mathrm{Cl}-4-\mathrm{x}$ \\
$\mathrm{CI}-\mathrm{F}-\mathrm{x}$ \\
$\mathrm{Cr}$ \\
$\mathrm{HC}-$ insol \\
$\mathrm{HC}-\mathrm{Solub}$ \\
$\mathrm{Hg}$ \\
$\mathrm{Pb}$
\end{tabular}

\begin{tabular}{c}
$\begin{array}{c}\text { Amount } \\
(\mathrm{kg} / \mathrm{Yr})\end{array}$ \\
\hline $2.978 \mathrm{E}+00$ \\
$6.322 \mathrm{E}-03$ \\
$2.709 \mathrm{E}-02$ \\
$2.409 \mathrm{E}+00$ \\
$1.574 \mathrm{E}+00$ \\
$1.718 \mathrm{E}+02$ \\
$3.827 \mathrm{E}+01$ \\
$6.845 \mathrm{E}-02$ \\
$2.069 \mathrm{E}+00$ \\
$3.617 \mathrm{E}+02$ \\
$1.237 \mathrm{E}+02$ \\
$1.355 \mathrm{E}-02$ \\
$4.599 \mathrm{E}-01$
\end{tabular}

Shipping Site: MOUND

Waste Type: Solids (CH-Alpha)

Total Volume (m3/yr):

5. 712E-03

Total Mass $(\mathrm{kg} / \mathrm{yx})$ :

$6.176 \mathrm{E}+00$

Radionuclide

\begin{tabular}{c}
$\begin{array}{c}\text { Activity } \\
\text { (Ci/yr) }\end{array}$ \\
\hline $7.557 \mathrm{E}-05$ \\
$7.917 \mathrm{E}-05$ \\
$3.591 \mathrm{E}-01$ \\
$1.835 \mathrm{E}-05$ \\
$1.799 \mathrm{E}-05$ \\
$3.598 \mathrm{E}-04$ \\
$7.557 \mathrm{E}-05$ \\
$1.835 \mathrm{E}-05$ \\
$1.835 \mathrm{E}-05$ \\
$7.557 \mathrm{E}-05$
\end{tabular}

\begin{tabular}{l} 
Chemical \\
\hline $75-09-2$ \\
Ag \\
Ba \\
Cd \\
Cl-2-x \\
Cl-3-x \\
Cl-4-x \\
Cl-F-x \\
Cr \\
HC-insol \\
HC-solub \\
Hg \\
Pb \\
Se
\end{tabular}

\begin{tabular}{c}
$\begin{array}{c}\text { Amount } \\
(\mathrm{kg} / \mathrm{yr})\end{array}$ \\
\hline $1.926 \mathrm{E}-02$ \\
$1.191 \mathrm{E}-04$ \\
$2.561 \mathrm{E}-04$ \\
$2.102 \mathrm{E}-05$ \\
$9.886 \mathrm{E}-03$ \\
$9.601 \mathrm{E}-02$ \\
$7.627 \mathrm{E}-02$ \\
$6.403 \mathrm{E}-04$ \\
$4.674 \mathrm{E}-05$ \\
$2.423 \mathrm{E}-01$ \\
$1.132 \mathrm{E}-01$ \\
$1.951 \mathrm{E}-05$ \\
$9.361 \mathrm{E}-05$ \\
$1.921 \mathrm{E}-06$
\end{tabular}


Shipping Site: NNS

Waste Type: Solids (CH-Non-alpha)

Total Volume $(\mathrm{m} 3 / \mathrm{yr})$

$3.560 \mathrm{E}-01$

Total Mass (kg/yr): $3.925 E+02$

Radionuclide

$\mathrm{Ba}-137 \mathrm{~m}$

$\mathrm{Co}-60$

Cs -134

Cs -137

$\mathrm{Eu}-154$

Eu-155

Fe-55

$\mathrm{H}-3$

$\mathrm{Mn}-54$

$\mathrm{Nb}-94$

$\mathrm{Ni}-59$

$\mathrm{Ni}-63$

$\mathrm{Pa}-234 \mathrm{~m}$

Pm-147

$\mathrm{Pu}-238$

Pu-241

$\mathrm{Sb}-125$

Sin-151

Sr -90

$\mathrm{Te}-125 \mathrm{~m}$

Th -234

$\mathrm{U}-238$

$Y-90$

$\begin{gathered}\text { Activity } \\ (C i / y r)\end{gathered}$
$2.383 E-01$
$3.849 \mathrm{E}+00$
$2.263 \mathrm{E}-03$
$2.516 \mathrm{E}-01$
$3.062 \mathrm{E}-03$
$1.464 \mathrm{E}-03$
$5.032 \mathrm{E}-01$
$1.096 \mathrm{E}+00$
$1.597 \mathrm{E}-03$
$2.396 \mathrm{E}-03$
$5.032 \mathrm{E}-02$
$6.766 \mathrm{E}+00$
$9.453 \mathrm{E}-03$
$2.742 \mathrm{E}-02$
$7.855 \mathrm{E}-03$
$6.390 \mathrm{E}-03$
$1.065 \mathrm{E}-03$
$2.130 \mathrm{E}-03$
$2.369 \mathrm{E}-01$
$2.662 \mathrm{E}-04$
$9.453 \mathrm{E}-03$
$9.453 \mathrm{E}-03$
$2.369 \mathrm{E}-01$

\begin{tabular}{|c|c|}
\hline Chemical & $\begin{array}{l}\text { Amount } \\
(\mathrm{kg} / \mathrm{yr})\end{array}$ \\
\hline $75-09-2$ & $1.041 \mathrm{E}-01$ \\
\hline $\mathrm{Ag}$ & $3.925 E-03$ \\
\hline As & $2.726 \mathrm{E}-03$ \\
\hline $\mathrm{Ba}$ & $1.110 \mathrm{E}-01$ \\
\hline $\mathrm{cd}$ & $3.955 \mathrm{E}-02$ \\
\hline$C 1-2-x$ & $1.105 \mathrm{E}-01$ \\
\hline Cl-3-x & $4.102 \mathrm{E}+00$ \\
\hline Cl-4-x & $7.996 \mathrm{E}-01$ \\
\hline$C l-F-x$ & $9.360 \mathrm{E}-02$ \\
\hline $\mathrm{Cr}$ & $1.488 \mathrm{E}-01$ \\
\hline $\mathrm{HC}$-insol & $1.378 \mathrm{E}+01$ \\
\hline $\mathrm{HC}-\mathrm{solub}$ & $6.570 \mathrm{E}+00$ \\
\hline $\mathrm{Hg}$ & $4.448 \mathrm{E}-02$ \\
\hline $\mathrm{Pb}$ & $7.211 E-02$ \\
\hline Se & $1.201 E-02$ \\
\hline
\end{tabular}

Shipping site: UMC

Total Volume $(\mathrm{m} 3 / \mathrm{Yr})$ :

$3.944 \mathrm{E}-02$

waste Type: Solids (CH-Alpha)

Radionuclide

Am-241

$\mathrm{Cm}-244$

$\mathrm{Pa}-234 \mathrm{~m}$

Pu-238

Pu-239

Pu-240

Pu-241

Th-234

$\mathrm{U}-238$

\begin{tabular}{r}
$\begin{array}{c}\text { Activity } \\
\text { (Ci/yr) }\end{array}$ \\
\hline $1.743 \mathrm{E}-07$ \\
$2.358 \mathrm{E}-07$ \\
$8.511 \mathrm{E}-07$ \\
$9.108 \mathrm{E}-05$ \\
$9.331 \mathrm{E}-06$ \\
$3.260 \mathrm{E}-05$ \\
$8.894 \mathrm{E}-04$ \\
$8.511 \mathrm{E}-07$ \\
$8.511 \mathrm{E}-07$
\end{tabular}

Total Mass (kg/yr): $5.809 \mathrm{E}+01$

\begin{tabular}{|c|c|}
\hline Chemical & $\begin{array}{l}\text { Amount } \\
(\mathrm{kg} / \mathrm{YI})\end{array}$ \\
\hline $\begin{array}{l}57-12-5 \\
75-09-2\end{array}$ & $9.876 \mathrm{E}-03$ \\
\hline $\mathrm{Ag}$ & $1.742 \mathrm{E}-03$ \\
\hline As & $5.809 \mathrm{E}-04$ \\
\hline $\mathrm{Ba}$ & $1.161 \mathrm{E}-01$ \\
\hline$c a$ & $2.904 \mathrm{E}-04$ \\
\hline Cl $-2-x$ & $1.161 \mathrm{E}-02$ \\
\hline$C 1-3-x$ & $3.253 \mathrm{E}-02$ \\
\hline$C 1-4-x$ & $2.033 \mathrm{E}-02$ \\
\hline$C I-F-x$ & $5.809 \mathrm{E}-03$ \\
\hline $\mathrm{Cr}$ & $1.161 \mathrm{E}-01$ \\
\hline $\mathrm{HC}$-insol & $5.809 \mathrm{E}-02$ \\
\hline $\mathrm{HC}$-solub & $2.904 E-03$ \\
\hline $\mathrm{Hg}$ & $5.809 \mathrm{E}-03$ \\
\hline $\mathrm{Pb}$ & $2.904 \mathrm{E}-01$ \\
\hline se & $1.742 \mathrm{E}-03$ \\
\hline
\end{tabular}

Shipping site: WVDP

Waste Type: Organic Liquids ( $\mathrm{CH}$-Alpha)

Total Volume $(\mathrm{m} 3 / \mathrm{yr})$ :

$1.773 \mathrm{E}+00$

Total Mass $(\mathrm{kg} / \mathrm{yr}): 1.897 \mathrm{E}+03$

Radionuclide

Am-241

$\mathrm{Ba}-137 \mathrm{~m}$

Co-60

Cs -137

Eu-154

$\mathrm{H}-3$

$\mathrm{Nb}-94$

$\mathrm{Ni}-59$

$\mathrm{Ni}-63$

$\mathrm{Pa}-234 \mathrm{~m}$

Pu-238

Pu-239

Pu-240

Pu-241

Sm-151

$\mathrm{Sr}-90$

TC-99

Th-234

U-238

$\mathrm{Y}-90$

\begin{tabular}{c}
$\begin{array}{r}\text { Activity } \\
\text { (Ci/yr) }\end{array}$ \\
\hline $1.482 \mathrm{E}-03$ \\
$1.619 \mathrm{E}+00$ \\
$2.167 \mathrm{E}-03$ \\
$1.731 \mathrm{E}+00$ \\
$2.583 \mathrm{E}-03$ \\
$1.284 \mathrm{E}-01$ \\
$1.482 \mathrm{E}-04$ \\
$3.317 \mathrm{E}-03$ \\
$3.409 \mathrm{E}-01$ \\
$9.529 \mathrm{E}-04$ \\
$8.047 \mathrm{E}-02$ \\
$1.334 \mathrm{E}-03$ \\
$5.929 \mathrm{E}-04$ \\
$3.465 \mathrm{E}-02$ \\
$2.477 \mathrm{E}-02$ \\
$1.541 \mathrm{E}+00$ \\
$7.694 \mathrm{E}-04$ \\
$9.529 \mathrm{E}-04$ \\
$9.529 \mathrm{E}-04$ \\
$1.541 \mathrm{E}+00$
\end{tabular}

\begin{tabular}{l} 
Chemical \\
\hline $57-12-5$ \\
$75-09-2$ \\
$\mathrm{Ag}$ \\
$\mathrm{As}$ \\
$\mathrm{Ba}$ \\
$\mathrm{Cd}$ \\
$\mathrm{Cl}-2-\mathrm{x}$ \\
$\mathrm{Cl}-3-\mathrm{x}$ \\
$\mathrm{Cl}-4-\mathrm{x}$ \\
$\mathrm{Cl}-\mathrm{F}-\mathrm{x}$ \\
$\mathrm{Cr}$ \\
$\mathrm{HC}-$ insol \\
$\mathrm{HC}-$ solub \\
$\mathrm{Hg}$ \\
$\mathrm{Pb}$ \\
$\mathrm{Se}$
\end{tabular}

\begin{tabular}{l}
$\begin{array}{c}\text { Amount } \\
(\mathrm{kg} / \mathrm{Yr})\end{array}$ \\
\hline $1.600 \mathrm{E}-01$ \\
$2.840 \mathrm{E}-01$ \\
$7.866 \mathrm{E}-02$ \\
$9.986 \mathrm{E}-03$ \\
$6.783 \mathrm{E}-02$ \\
$1.250 \mathrm{E}-01$ \\
$4.212 \mathrm{E}-01$ \\
$2.491 \mathrm{E}+01$ \\
$3.373 \mathrm{E}+00$ \\
$2.744 \mathrm{E}-01$ \\
$2.914 \mathrm{E}-01$ \\
$1.686 \mathrm{E}+01$ \\
$3.228 \mathrm{E}+02$ \\
$4.473 \mathrm{E}-01$ \\
$9.175 \mathrm{E}-02$ \\
$6.881 \mathrm{E}-02$
\end{tabular}


A-53

Shipping site: WVDP

Waste Type: Solids (CH-Alpha)

Total Volume (m3/Yr):

$8.473 E-01$

Total Mass (kg/yr):

$1.355 E+03$

Radionuclide

\begin{tabular}{c}
$\begin{array}{c}\text { Activity } \\
\text { (Ci/yr) }\end{array}$ \\
\hline $7.096 \mathrm{E}-04$ \\
$7.751 \mathrm{E}-01$ \\
$1.037 \mathrm{E}-03$ \\
$8.243 \mathrm{E}-01$ \\
$1.236 \mathrm{E}-03$ \\
$6.150 \mathrm{E}-02$ \\
$7.096 \mathrm{E}-05$ \\
$1.588 \mathrm{E}-03$ \\
$1.632 \mathrm{E}-01$ \\
$4.561 \mathrm{E}-04$ \\
$3.852 \mathrm{E}-02$ \\
$6.386 \mathrm{E}-04$ \\
$2.838 \mathrm{E}-04$ \\
$1.659 \mathrm{E}-02$ \\
$1.186 \mathrm{E}-02$ \\
$7.339 \mathrm{E}-01$ \\
$3.662 \mathrm{E}-04$ \\
$4.561 \mathrm{E}-04$ \\
$4.561 \mathrm{E}-04$ \\
$7.339 \mathrm{E}-01$
\end{tabular}

Chemical

$57-12-5$

$75-09-2$

$\mathrm{Ag}$

As

$\mathrm{Ba}$

$\mathrm{Cd}$

Cl $1-2-x$

$\mathrm{C} 1-3-\mathrm{x}$

C $1-4-x$

$\mathrm{C} 1-\mathrm{F}-\mathrm{x}$

$\mathrm{Cr}$

$\mathrm{HC}$-insol

$\mathrm{HC}-\mathrm{solub}$

$\mathrm{Hg}$

$\mathrm{Pb}$

$\mathrm{Se}$
Amount

(kg/yr)

1. $069 \mathrm{E}-03$

2. $958 \mathrm{E}-01$

1. $709 \mathrm{E}-02$

1. 081E-02

$3.475 \mathrm{E}-01$

1. $558 \mathrm{E}-01$

2. $803 \mathrm{E}-01$

1. $573 \mathrm{E}+01$

$2.998 \mathrm{E}+00$

1. $176 \mathrm{E}-01$

5. $922 \mathrm{E}-01$

$5.230 \mathrm{E}+01$

2. $619 E+01$

$1.760 \mathrm{E}-01$

$2.784 \mathrm{E}-01$

$4.680 \mathrm{E}-02$

TC-99

Th-234

$\mathrm{U}-238$

$\mathrm{Y}-90$

$7.339 \mathrm{E}-01$ 


\section{A.4 WM LLMW REGIONALIZED 3 ALTERNATIVE (CASE 10a)}

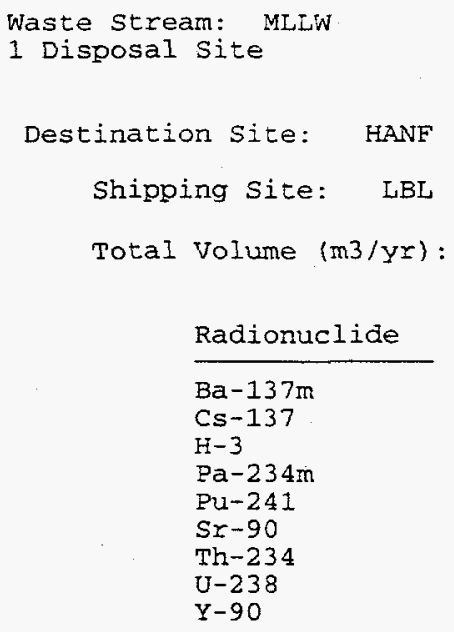

Alternative: 10 Regional 7 Treatment Sites Waste Type: Organic Liquids (CH-Non-alpha)

$3.230 \mathrm{E}-01$

Total Mass $(\mathrm{kg} / \mathrm{y} r): \quad 3.100 \mathrm{E}+02$

\begin{tabular}{r}
$\begin{array}{r}\text { Activity } \\
\text { (Ci/yr) }\end{array}$ \\
\hline $4.273 \mathrm{E}-03$ \\
$4.476 \mathrm{E}-03$ \\
$2.032 \mathrm{E}+01$ \\
$1.037 \mathrm{E}-03$ \\
$1.017 \mathrm{E}-03$ \\
$4.273 \mathrm{E}-03$ \\
$1.037 \mathrm{E}-03$ \\
$1.037 \mathrm{E}-03$ \\
$4.273 \mathrm{E}-03$
\end{tabular}

\begin{tabular}{l} 
Chemical \\
\hline $75-09-2$ \\
$\mathrm{As}$ \\
$\mathrm{Ba}$ \\
$\mathrm{Cd}$ \\
$\mathrm{CI}-2-\mathrm{x}$ \\
$\mathrm{Cl}-3-\mathrm{x}$ \\
$\mathrm{Cl}-4-\mathrm{x}$ \\
$\mathrm{Cr}$ \\
$\mathrm{HC}-$ insol \\
$\mathrm{HC}-$ solub \\
$\mathrm{Hg}$ \\
$\mathrm{Pb}$
\end{tabular}

\begin{tabular}{c}
$\begin{array}{c}\text { Amount } \\
(\mathrm{kg} / \mathrm{yr}\rangle\end{array}$ \\
\hline $2.976 \mathrm{E}-01$ \\
$2.387 \mathrm{E}-03$ \\
$1.023 \mathrm{E}-02$ \\
$3.410 \mathrm{E}-01$ \\
$1.550 \mathrm{E}-01$ \\
$2.325 \mathrm{E}+00$ \\
$4.961 \mathrm{E}-01$ \\
$2.852 \mathrm{E}-01$ \\
$1.364 \mathrm{E}+02$ \\
$4.341 \mathrm{E}+01$ \\
$1.860 \mathrm{E}-03$ \\
$6.511 \mathrm{E}-02$
\end{tabular}

Waste Type: Solids (CH-Non-alpha)

Total Mass (kg/yr): $4.402 \mathrm{E}+03$

\begin{tabular}{l} 
Chemical \\
\hline $57-12-5$ \\
$75-09-2$ \\
Ag \\
As \\
Ba \\
Cd \\
CI-2-x \\
CI-3-x \\
CI-4-x \\
CI-F-x \\
Cr \\
HC-insol \\
HC-solub \\
Hg \\
Pb \\
Se
\end{tabular}

Activity

4. $117 \mathrm{E}-02$

$4.314 \mathrm{E}-02$

1. $958 \mathrm{E}+02$

1. $000 \mathrm{E}-02$

$9.804 \mathrm{E}-03$

4. $117 \mathrm{E}-02$

1. $000 \mathrm{E}-02$

1. $000 \mathrm{E}-02$

4. $117 \mathrm{E}-02$
Amount

\begin{tabular}{c}
$\begin{array}{c}\text { Amount } \\
(\mathrm{kg} / \mathrm{Yr})\end{array}$ \\
\hline $5.503 \mathrm{E}-01$ \\
$1.116 \mathrm{E}+01$ \\
$1.516 \mathrm{E}-01$ \\
$3.237 \mathrm{E}-02$ \\
$6.474 \mathrm{E}+00$ \\
$1.754 \mathrm{E}-02$ \\
$5.905 \mathrm{E}+00$ \\
$5.439 \mathrm{E}+01$ \\
$4.320 \mathrm{E}+01$ \\
$3.237 \mathrm{E}-01$ \\
$6.485 \mathrm{E}+00$ \\
$1.314 \mathrm{E}+02$ \\
$6.326 \mathrm{E}+01$ \\
$3.242 \mathrm{E}-01$ \\
$1.619 \mathrm{E}+01$ \\
$9.711 \mathrm{E}-02$
\end{tabular}




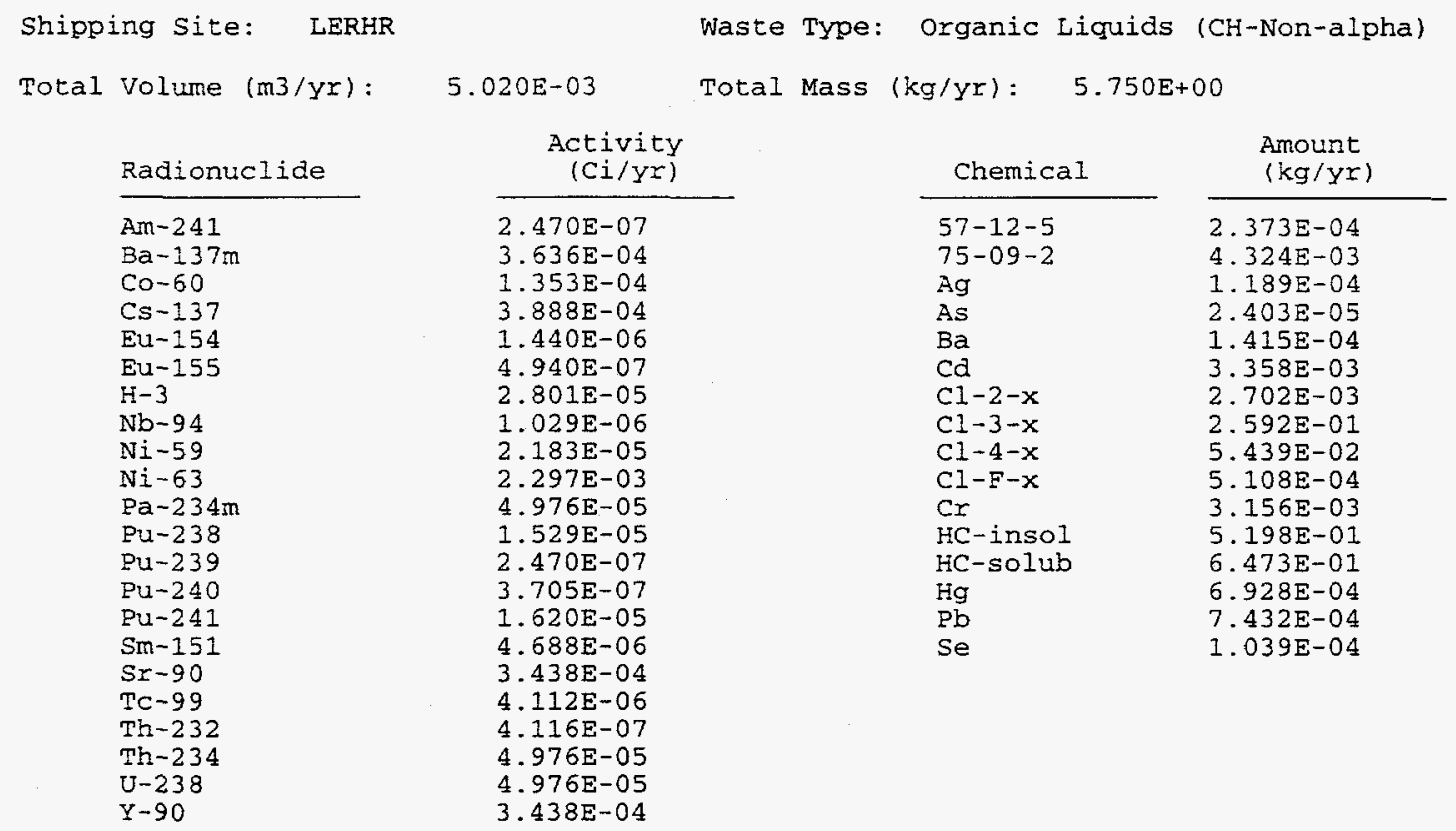

Shipping Site: LERHR Total Volume $(\mathrm{m} 3 / \mathrm{yr})$ :

Radionuclide

Am-241

$\mathrm{Ba}-137 \mathrm{~m}$

Co- 60

Cs-137

Eu-154

Eu-155

$\mathrm{H}-3$

$\mathrm{Nb}-94$

$\mathrm{Ni}-59$

$\mathrm{Ni}-63$

$\mathrm{Pa}-234 \mathrm{~m}$

Pu-238

Pu-239

Pu-2 40

Pu-241

$\mathrm{Sm}-151$

Sr -90

TC-99

Th-232

Th-234

$\mathrm{U}-238$

$Y-90$

Shipping Site: LLNL
$3.442 \mathrm{E}-01$
Waste Type: Solids (CH-Non-alpha)

Total Mass $(\mathrm{kg} / \mathrm{Yr}): 2.492 \mathrm{E}+02$

\begin{tabular}{c}
$\begin{array}{c}\text { Activity } \\
(\mathrm{Ci} / \mathrm{Y})\end{array}$ \\
\hline $1.701 \mathrm{E}-05$ \\
$2.505 \mathrm{E}-02$ \\
$9.323 \mathrm{E}-03$ \\
$2.641 \mathrm{E}-02$ \\
$9.928 \mathrm{E}-05$ \\
$3.403 \mathrm{E}-05$ \\
$1.930 \mathrm{E}-03$ \\
$7.091 \mathrm{E}-05$ \\
$1.504 \mathrm{E}-03$ \\
$1.583 \mathrm{E}-01$ \\
$3.428 \mathrm{E}-03$ \\
$1.054 \mathrm{E}-03$ \\
$1.701 \mathrm{E}-05$ \\
$2.552 \mathrm{E}-05$ \\
$1.116 \mathrm{E}-03$ \\
$3.230 \mathrm{E}-04$ \\
$2.335 \mathrm{E}-02$ \\
$2.793 \mathrm{E}-04$ \\
$2.836 \mathrm{E}-05$ \\
$3.428 \mathrm{E}-03$ \\
$3.428 \mathrm{E}-03$ \\
$2.335 \mathrm{E}-02$
\end{tabular}

\begin{tabular}{|c|c|}
\hline Chemical & $\begin{array}{l}\text { Amount } \\
(\mathrm{kg} / \mathrm{yr})\end{array}$ \\
\hline $\begin{array}{l}57-12-5 \\
75-09-2\end{array}$ & $\begin{array}{l}4.707 \mathrm{E}-04 \\
1.438 \mathrm{E}-01\end{array}$ \\
\hline Ag & $2.547 \mathrm{E}-03$ \\
\hline As & $1.464 \mathrm{E}-05$ \\
\hline $\mathrm{Ba}$ & $3.843 E-02$ \\
\hline $\mathrm{cd}$ & $8.851 E-03$ \\
\hline Cl-2-x & $1.556 \mathrm{E}-01$ \\
\hline$c l-3-x$ & $6.005 E-01$ \\
\hline$c l-4-x$ & $2.831 \mathrm{E}+00$ \\
\hline$C 1-F-x$ & $1.391 \mathrm{E}-01$ \\
\hline $\mathrm{Cr}$ & $4.652 \mathrm{E}-03$ \\
\hline HC-insol & $1.675 \mathrm{E}+00$ \\
\hline HC-solub & $3.579 \mathrm{E}-01$ \\
\hline $\mathrm{Hg}$ & $5.762 \mathrm{E}-03$ \\
\hline $\mathrm{Pb}$ & 1. $542 \mathrm{E}-02$ \\
\hline $\mathrm{Se}$ & $4.361 \varepsilon-04$ \\
\hline
\end{tabular}

Waste Type: Organic Liquids (CH-Non-alpha)

Total Mass $\left(\mathrm{kg} / \mathrm{Yr}^{\mathrm{C}}\right): \quad 8.159 \mathrm{E}+04$
Radionuclide

$\mathrm{Ba}-137 \mathrm{~m}$

Cs -137

$\mathrm{H}-3$

$\mathrm{Pa}-234 \mathrm{~m}$

Pu -241

$\mathrm{Sr}-90$

Th-234

$\mathrm{U}-238$

$\mathrm{Y}-90$
$7.727 \mathrm{E}+01$

\begin{tabular}{c}
$\begin{array}{c}\text { Activity } \\
(C i / y x)\end{array}$ \\
\hline $.022 \mathrm{E}+00$ \\
$.071 \mathrm{E}+00$ \\
$.863 \mathrm{E}+03$ \\
$.482 \mathrm{E}-01$ \\
$.434 \mathrm{E}-01$ \\
$.022 \mathrm{E}+00$ \\
$.482 \mathrm{E}-01$ \\
$.482 \mathrm{E}-01$ \\
$.022 \mathrm{E}+00$
\end{tabular}

\begin{tabular}{|c|c|}
\hline Chemical & $\begin{array}{l}\text { Amount } \\
(\mathrm{kg} / \mathrm{Yr})\end{array}$ \\
\hline $\begin{array}{l}57-12-5 \\
75-09-2\end{array}$ & $\begin{array}{l}7.162 E+00 \\
5.434 E+00\end{array}$ \\
\hline $\mathrm{Ag}$ & $3.590 \mathrm{E}+00$ \\
\hline As & $4.598 \mathrm{E}-01$ \\
\hline $\mathrm{Ba}$ & $3.135 \mathrm{E}+00$ \\
\hline $\mathrm{cd}$ & $1.949 \mathrm{E}-01$ \\
\hline$C 1-2-x$ & $1.546 \mathrm{E}+01$ \\
\hline$C 1-3-x$ & $6.087 \mathrm{E}+02$ \\
\hline$C 1-4-x$ & $3.439 \mathrm{E}+01$ \\
\hline $\mathrm{Cl}-\mathrm{F}-\mathrm{x}$ & $1.254 \mathrm{E}+01$ \\
\hline $\mathrm{Cr}$ & $8.355 \mathrm{E}+00$ \\
\hline HC-insol & $5.003 E+02$ \\
\hline HC-solub & $1.434 \mathrm{E}+04$ \\
\hline $\mathrm{Hg}$ & $2.033 E+01$ \\
\hline $\mathrm{pb}$ & $3.118 \mathrm{E}+00$ \\
\hline se & $3.138 \mathrm{E}+00$ \\
\hline
\end{tabular}


Shipping site: LLNL Total volume (m3/yr):

Radionuclide

$\mathrm{Ba}-137 \mathrm{~m}$

Cs -137

$\mathrm{H}-3$

$\mathrm{Pa}-234 \mathrm{~m}$

$\mathrm{Pu}-241$

$\mathrm{Sr}-90$

Th-234

$\mathrm{U}-238$

$Y-90$
Shipping Site: MINS

Total Volume $(\mathrm{m} 3 / \mathrm{yr})$ :

Radionuclide

$\mathrm{Ba}-137 \mathrm{~m}$

Co-60

Cs -134

Cs -137

Eน-154

Eu-155

$\mathrm{Fe}-55$

$\mathrm{H}-3$

$\mathrm{Mn}-54$

$\mathrm{Nb}-94$

$\mathrm{Ni}-59$

$\mathrm{Ni}-63$

$\mathrm{Pa}-234 \mathrm{~m}$

Pm-147

Pu-238

Pu-241

$\mathrm{Sb}-125$

$\mathrm{Sm}-151$

$\mathrm{Sr}-90$

$\mathrm{Te}-125 \mathrm{~m}$

Th-234

$\mathrm{U}-238$

$\mathrm{Y}-90$

Shipping Site: PHNS

Total Volume (m3/yr):

Radionuclide

$\mathrm{Ba}-137 \mathrm{~m}$

Co-60

Cs -134

Cs -137

Eu-154

Eu-155

$\mathrm{Fe}-55$

$\mathrm{H}-3$

$M n-54$

$\mathrm{Nb}-94$

Ni -59

$\mathrm{Ni}-63$

$\mathrm{Pa}-234 \mathrm{~m}$

Pm-147

Pu -238

Pu-241

$\mathrm{Sb}-125$

$\mathrm{Sm}-151$

$\mathrm{Sr}-90$

$\mathrm{Te}-125 \mathrm{~m}$

$\mathrm{Th}-234$

$\mathrm{U}-238$

$\mathrm{Y}-90$
Waste Type: Solids (CH-Non-alpha)

$4.978 \mathrm{E}+01$

Total Mass (kg/yr): $6.833 \mathrm{E}+04$

\begin{tabular}{lcc}
\multicolumn{1}{c}{ Chemical } & & $\begin{array}{c}\text { Amount } \\
(\mathrm{kg} / \mathrm{Y} Y)\end{array}$ \\
\cline { 1 - 1 } $77-12-5$ & & $8.172 \mathrm{E}+00$ \\
$75-09-2$ & & $8.995 \mathrm{E}+00$ \\
$\mathrm{Ag}$ & $3.852 \mathrm{E}+00$ \\
$\mathrm{As}$ & $1.401 \mathrm{E}+00$ \\
$\mathrm{Ba}$ & $1.097 \mathrm{E}+02$ \\
$\mathrm{Cd}$ & $2.705 \mathrm{E}+00$ \\
$\mathrm{Cl}-2-\mathrm{x}$ & $7.372 \mathrm{E}+00$ \\
$\mathrm{Cl}-3-\mathrm{x}$ & $1.900 \mathrm{E}+02$ \\
$\mathrm{Cl}-4-\mathrm{x}$ & $3.100 \mathrm{E}+01$ \\
$\mathrm{Cl}-\mathrm{F}-\mathrm{x}$ & $5.418 \mathrm{E}+00$ \\
$\mathrm{Cr}$ & $1.262 \mathrm{E}+02$ \\
$\mathrm{HC}-$ insol & $7.525 \mathrm{E}+02$ \\
$\mathrm{HC}-$ Solub & $3.756 \mathrm{E}+02$ \\
$\mathrm{Hg}$ & $4.188 \mathrm{E}+01$ \\
$\mathrm{~Pb}$ & $1.529 \mathrm{E}+02$ \\
$\mathrm{Se}$ & $1.787 \mathrm{E}+01$
\end{tabular}

waste Type: Solids (CH-Non-alpha)

3.647E+00 Total Mass $(\mathrm{kg} / \mathrm{Yr}): \quad 3.545 \mathrm{E}+03$

\begin{tabular}{c}
$\begin{array}{c}\text { Activity } \\
\text { (Ci/yr) }\end{array}$ \\
\hline $2.441 \mathrm{E}+00$ \\
$3.943 \mathrm{E}+01$ \\
$2.318 \mathrm{E}-02$ \\
$2.578 \mathrm{E}+00$ \\
$3.137 \mathrm{E}-02$ \\
$1.500 \mathrm{E}-02$ \\
$5.156 \mathrm{E}+00$ \\
$1.123 \mathrm{E}+01$ \\
$1.636 \mathrm{E}-02$ \\
$2.455 \mathrm{E}-02$ \\
$5.156 \mathrm{E}-01$ \\
$6.932 \mathrm{E}+01$ \\
$9.684 \mathrm{E}-02$ \\
$2.809 \mathrm{E}-01$ \\
$8.047 \mathrm{E}-02$ \\
$5.547 \mathrm{E}-02$ \\
$1.091 \mathrm{E}-02$ \\
$2.182 \mathrm{E}-02$ \\
$2.427 \mathrm{E}+00$ \\
$2.728 \mathrm{E}-03$ \\
$9.684 \mathrm{E}-02$ \\
$9.684 \mathrm{E}-02$ \\
$2.427 \mathrm{E}+00$
\end{tabular}

\begin{tabular}{|c|c|}
\hline Chemical & $\begin{array}{l}\text { Amount } \\
(\mathrm{kg} / \mathrm{y} r)\end{array}$ \\
\hline $75-09-2$ & $5.544 \mathrm{E}-01$ \\
\hline $\mathrm{Ag}$ & $1.457 \mathrm{E}-02$ \\
\hline As & $7.197 \mathrm{E}-03$ \\
\hline $\mathrm{Ba}$ & $2.427 \mathrm{E}+00$ \\
\hline$c d$ & $1.150 \mathrm{E}-01$ \\
\hline Cl-2-x & $5.975 \mathrm{E}-01$ \\
\hline$C 1-3-x$ & $1.190 \mathrm{E}+01$ \\
\hline$C 1-4-x$ & $2.652 E+00$ \\
\hline$C I-F-x$ & $6.891 E-01$ \\
\hline $\mathrm{Cr}$ & $7.414 \mathrm{E}-01$ \\
\hline HC-insol & $4.047 E+01$ \\
\hline HC-solub & $1.726 E+01$ \\
\hline $\mathrm{Hg}$ & $5.165 \mathrm{E}-01$ \\
\hline $\mathrm{Pb}$ & $4.557 \mathrm{E}-01$ \\
\hline $\mathrm{Se}$ & $3.325 \mathrm{E}-02$ \\
\hline
\end{tabular}

Waste Type: Organic Liquids (CH-Non-alpha)

$1.736 \mathrm{E}-03$

Total Mass (kg/yr): $1.979 \mathrm{E}+00$

\begin{tabular}{c}
$\begin{array}{c}\text { Activity } \\
\text { (Ci/yr) }\end{array}$ \\
\hline $1.162 \mathrm{E}-03$ \\
$1.877 \mathrm{E}-02$ \\
$1.103 \mathrm{E}-05$ \\
$1.227 \mathrm{E}-03$ \\
$1.493 \mathrm{E}-05$ \\
$7.141 \mathrm{E}-05$ \\
$2.454 \mathrm{E}-03$ \\
$5.346 \mathrm{E}-03$ \\
$7.791 \mathrm{E}-06$ \\
$1.168 \mathrm{E}-05$ \\
$2.454 \mathrm{E}-04$ \\
$3.299 \mathrm{E}-02$ \\
$4.609 \mathrm{E}-05$ \\
$1.337 \mathrm{E}-04$ \\
$3.830 \mathrm{E}-05$ \\
$3.116 \mathrm{E}-05$ \\
$5.194 \mathrm{E}-06$ \\
$1.038 \mathrm{E}-05$ \\
$1.155 \mathrm{E}-03$ \\
$1.298 \mathrm{E}-06$ \\
$4.609 \mathrm{E}-05$ \\
$4.609 \mathrm{E}-05$ \\
$1.155 \mathrm{E}-03$
\end{tabular}

\begin{tabular}{lcc} 
Chemical & & $\begin{array}{c}\text { Amount } \\
(\mathrm{kg} / \mathrm{y} r)\end{array}$ \\
\cline { 1 - 1 } $75-09-2$ & & $2.572 \mathrm{E}-04$ \\
$\mathrm{Ag}$ & $8.707 \mathrm{E}-05$ \\
$\mathrm{AS}$ & $2.176 \mathrm{E}-05$ \\
$\mathrm{Ba}$ & $1.484 \mathrm{E}-04$ \\
$\mathrm{CI}-2-\mathrm{x}$ & $7.322 \mathrm{E}-04$ \\
$\mathrm{CI}-3-\mathrm{x}$ & $2.731 \mathrm{E}-02$ \\
$\mathrm{CI}-4-\mathrm{x}$ & $1.543 \mathrm{E}-03$ \\
$\mathrm{CI}-\mathrm{F}-\mathrm{x}$ & $5.937 \mathrm{E}-04$ \\
$\mathrm{HC}-$ insol & $2.968 \mathrm{E}-03$ \\
$\mathrm{HC}-$ Solub & $7.718 \mathrm{E}-04$ \\
$\mathrm{Hg}$ & $4.353 \mathrm{E}-04$ \\
$\mathrm{~Pb}$ & $5.343 \mathrm{E}-05$ \\
$\mathrm{Se}$ & $7.322 \mathrm{E}-05$
\end{tabular}




\begin{tabular}{l} 
Shipping Site: PHNS \\
Total Volume (m3/yr): \\
\\
Radionuclide \\
\hline Ba-137m \\
Co-60 \\
Cs -134 \\
Cs-137 \\
Eu-154 \\
Eu-155 \\
Fe-55 \\
$\mathrm{H}-3$ \\
$\mathrm{Mn}-54$ \\
$\mathrm{Nb}-94$ \\
$\mathrm{Ni}-59$ \\
$\mathrm{Ni}-63$ \\
$\mathrm{~Pa}-234 \mathrm{~m}$ \\
$\mathrm{Pm}-147$ \\
$\mathrm{Pu}-238$ \\
$\mathrm{Pu}-241$ \\
$\mathrm{Sb}-125$ \\
$\mathrm{Sm}-151$ \\
$\mathrm{Sr}-90$ \\
$\mathrm{Te}-125 \mathrm{~m}$ \\
$\mathrm{Th}-234$ \\
$\mathrm{U}-238$ \\
$\mathrm{Y}-90$ \\
\end{tabular}

Shipping site: PSNS

Total Volume $(\mathrm{m} 3 / \mathrm{yr})$ :

Radionuclide

$\mathrm{Ba}-137 \mathrm{~m}$

Co- 60

Cs -134

Cs -137

Eu-154

Eu-155

$\mathrm{Fe}-55$

H-3

$\mathrm{Mn}-54$

$\mathrm{Nb}-94$

$\mathrm{Ni}-59$

$\mathrm{Ni}-63$

$\mathrm{Pa}-234 \mathrm{~m}$

Pm-147

Pu-238

Pu-241

$\mathrm{Sb}-125$

Sm-151

Sr-90

$\mathrm{Te}-125 \mathrm{~m}$

Th-234

$\mathrm{U}-238$

$Y-90$

Waste Type: Solids (CH-Non-alpha)

Total Mass $(\mathrm{kg} / \mathrm{Yr}): 4.406 \mathrm{E}+02$

$2.962 E-01$
Chemical

$57-12-5$

$75-09-2$

$\mathrm{Ag}$

As

$\mathrm{Ba}$

$\mathrm{Cd}$

Cl $-2-x$

C1-3-x

C1-4-x

Cl-F-X

$\mathrm{Cr}$

HC-insol

HC-solub

$\mathrm{Hg}$

$\mathrm{Se}$
Amount

$(\mathrm{kg} / \mathrm{Yr})$

4. $073 \mathrm{E}-03$

$5.831 \mathrm{E}-02$

$2.691 \mathrm{E}-03$

$1.603 \mathrm{E}-03$

$1.038 \mathrm{E}-01$

$2.472 \mathrm{E}-02$

$6.160 \mathrm{E}-02$

$2.088 \mathrm{E}+00$

$4.422 \mathrm{E}-01$

$6.398 \mathrm{E}-02$

$7.805 E-01$

$6.908 \mathrm{E}+00$

$3.289 \mathrm{E}+00$

$2.617 \mathrm{E}-02$

$1.563 \mathrm{E}-01$

6.731E-03

$8.865 \mathrm{E}-04$

$1.773 \mathrm{E}-03$

1. $972 \mathrm{E}-01$

$2.216 \mathrm{E}-04$

$7.867 \mathrm{E}-03$

$7.867 \mathrm{E}-03$

$1.972 \mathrm{E}-01$

Waste Type: Organic Liquids (CH-Non-alpha)

$3.990 \mathrm{E}-02$

Total Mass $(\mathrm{kg} / \mathrm{yr})$ :

$5.986 \mathrm{E}+01$

\begin{tabular}{l}
$\begin{array}{r}\text { Activity } \\
\text { (Ci/yr) }\end{array}$ \\
\hline $2.671 \mathrm{E}-02$ \\
$4.315 \mathrm{E}-01$ \\
$2.537 \mathrm{E}-04$ \\
$2.820 \mathrm{E}-02$ \\
$3.432 \mathrm{E}-04$ \\
$1.641 \mathrm{E}-04$ \\
$5.641 \mathrm{E}-02$ \\
$1.228 \mathrm{E}-01$ \\
$1.791 \mathrm{E}-04$ \\
$2.686 \mathrm{E}-04$ \\
$5.641 \mathrm{E}-03$ \\
$7.585 \mathrm{E}-01$ \\
$1.059 \mathrm{E}-03$ \\
$3.074 \mathrm{E}-03$ \\
$8.806 \mathrm{E}-04$ \\
$7.164 \mathrm{E}-04$ \\
$1.194 \mathrm{E}-04$ \\
$2.388 \mathrm{E}-04$ \\
$2.656 \mathrm{E}-02$ \\
$2.985 \mathrm{E}-05$ \\
$1.059 \mathrm{E}-03$ \\
$1.059 \mathrm{E}-03$ \\
$2.656 \mathrm{E}-02$
\end{tabular}

Chemical

$75-09-2$

$\mathrm{cd}$

Cl-2-x

Cl-3-x

$\mathrm{Cl}-4-\mathrm{x}$

Cl-F-x

$\mathrm{Cr}$

HC-insoI

HC-solub

$\mathrm{Hg}$

$\mathrm{pb}$
Amount

(kg/yr)

9. $577 \mathrm{E}-02$

$6.584 \mathrm{E}-02$

$5.088 \mathrm{E}-02$

7. $243 \mathrm{E}+00$

1. $616 \mathrm{E}+00$

2. $993 \mathrm{E}-03$

$5.746 \mathrm{E}-02$

1. $975 \mathrm{E}-02$

3. $831 \mathrm{E}-01$

3. $771 \mathrm{E}-04$

1. $257 \mathrm{E}-02$ 


\begin{tabular}{l} 
Shipping Site: PSNS \\
Total Volume (m3/Yr) : \\
Radionuclide \\
\hline Ba-137m \\
Co-60 \\
Cs-134 \\
Cs -137 \\
Eu-154 \\
Eu-155 \\
Fe-55 \\
H-3 \\
Mn-54 \\
Nb-94 \\
Ni-59 \\
Ni-63 \\
Pa-234m \\
Pm-147 \\
$\mathrm{Pu}-238$ \\
$\mathrm{Pu}-241$ \\
$\mathrm{Sb}-125$ \\
$\mathrm{Sm}-151$ \\
$\mathrm{Sr}-90$ \\
$\mathrm{Te}-125 \mathrm{~m}$ \\
$\mathrm{Th}-234$ \\
$\mathrm{U}-238$ \\
$\mathrm{Y}-90$ \\
$\mathrm{H}$
\end{tabular}

Shipping site: SNLL

Total Volume (m3/yr):

Radionuclide

Ba-137m

Cs-137

$\mathrm{H}-3$

$\mathrm{Pa}-234 \mathrm{~m}$

Pu-241

$\mathrm{Sr}-90$

Th-234

$\mathrm{U}-238$

$\mathrm{Y}-90$

Shipping Site: SNLL

Total Volume (m3/yr):

Radionuclide

$\mathrm{Ba}-137 \mathrm{~m}$

Cs -137

$\mathrm{H}-3$

$\mathrm{Pa}-234 \mathrm{~m}$

Pu-241

$\mathrm{Sr}-90$

Th-234

$\mathrm{U}-238$

$\mathrm{Y}-90$
$6.586 \mathrm{E}+00$

Waste Type: Solids (CH-Non-alpha)

Total Mass $(\mathrm{kg} / \mathrm{yr}): 9.518 \mathrm{E}+03$

\begin{tabular}{|c|c|}
\hline Chemical & $\begin{array}{l}\text { Amount } \\
(\mathrm{kg} / \mathrm{Yr})\end{array}$ \\
\hline $57-12-5$ & 7. $312 E-01$ \\
\hline $75-09-2$ & $1.279 \mathrm{E}+00$ \\
\hline $\mathrm{Ag}$ & $1.357 \mathrm{E}-01$ \\
\hline As & $4.301 \mathrm{E}-02$ \\
\hline $\mathrm{Ba}$ & $8.859 E+00$ \\
\hline $\mathrm{cd}$ & $1.229 \mathrm{E}-01$ \\
\hline$C l-2-x$ & $1.346 \mathrm{E}+00$ \\
\hline Cl-3-x & $4.520 \mathrm{E}+00$ \\
\hline$c 1-4-x$ & $2.989 E+00$ \\
\hline$C l-F-x$ & 1. $385 \mathrm{E}+00$ \\
\hline $\mathrm{Cr}$ & $2.225 E+01$ \\
\hline HC-insol & $6.852 \mathrm{E}+00$ \\
\hline $\mathrm{HC}$-solub & $2.684 \mathrm{E}-01$ \\
\hline $\mathrm{Hg}$ & $4.633 \mathrm{E}-01$ \\
\hline $\mathrm{Pb}$ & $2.158 \mathrm{E}+01$ \\
\hline $\mathrm{Se}$ & $1.310 \mathrm{E}-01$ \\
\hline
\end{tabular}

Waste Type: Organic Liquids (CH-Non-alpha)

$7.662 \mathrm{E}+00$

Total Mass $(\mathrm{kg} / \mathrm{yr}): 7.356 \mathrm{E}+03$

\begin{tabular}{c}
$\begin{array}{c}\text { Activity } \\
\text { (Ci/yr) }\end{array}$ \\
\hline $1.013 \mathrm{E}-01$ \\
$1.062 \mathrm{E}-01$ \\
$4.822 \mathrm{E}+02$ \\
$2.462 \mathrm{E}-02$ \\
$2.413 \mathrm{E}-02$ \\
$1.013 \mathrm{E}-01$ \\
$2.462 \mathrm{E}-02$ \\
$2.462 \mathrm{E}-02$ \\
$1.013 \mathrm{E}-01$
\end{tabular}

\begin{tabular}{l} 
Chemical \\
\hline $75-09-2$ \\
$\mathrm{As}$ \\
$\mathrm{Ba}$ \\
$\mathrm{Cd}$ \\
$\mathrm{Cl}-2-\mathrm{x}$ \\
$\mathrm{Cl}-3-\mathrm{x}$ \\
$\mathrm{Cl}-4-\mathrm{x}$ \\
$\mathrm{Cr}$ \\
$\mathrm{HC}-$ insol \\
$\mathrm{HC}-$ solub \\
$\mathrm{Hg}$ \\
$\mathrm{Pb}$
\end{tabular}

\begin{tabular}{c}
$\begin{array}{c}\text { Amount } \\
(\mathrm{kg} / \mathrm{yr})\end{array}$ \\
\hline $7.061 \mathrm{E}+00$ \\
$5.664 E-02$ \\
$2.427 \mathrm{E}-01$ \\
$8.091 \mathrm{E}+00$ \\
$3.678 \mathrm{E}+00$ \\
$5.517 \mathrm{E}+01$ \\
$1.176 \mathrm{E}+01$ \\
$6.767 \mathrm{E}+00$ \\
$3.236 \mathrm{E}+03$ \\
$1.029 \mathrm{E}+03$ \\
$4.413 \mathrm{E}-02$ \\
$1.544 \mathrm{E}+00$
\end{tabular}

Waste Type: Solids (CH-Non-alpha)

$7.770 \mathrm{E}-01$

Total Mass $(\mathrm{kg} / \mathrm{yr}): 1.193 \mathrm{E}+03$

\begin{tabular}{c}
$\begin{array}{c}\text { Activity } \\
\text { (Ci/yr) }\end{array}$ \\
\hline $1.027 \mathrm{E}-02$ \\
$1.076 \mathrm{E}-02$ \\
$4.890 \mathrm{E}+01$ \\
$2.496 \mathrm{E}-03$ \\
$2.447 \mathrm{E}-03$ \\
$1.027 \mathrm{E}-02$ \\
$2.496 \mathrm{E}-03$ \\
$2.496 \mathrm{E}-03$ \\
$1.027 \mathrm{E}-02$
\end{tabular}

\begin{tabular}{l} 
Chemical \\
\hline $57-12-5$ \\
$75-09-2$ \\
Ag \\
As \\
$\mathrm{Ba}$ \\
$\mathrm{Cd}$ \\
$\mathrm{Cl}-2-\mathrm{x}$ \\
$\mathrm{Cl}-3-\mathrm{x}$ \\
$\mathrm{Cl}-4-\mathrm{x}$ \\
$\mathrm{Cl}-\mathrm{F}-\mathrm{x}$ \\
$\mathrm{Cr}$ \\
$\mathrm{HC}-$ insol \\
$\mathrm{HC}-\mathrm{SOlub}$ \\
$\mathrm{Hg}$ \\
$\mathrm{Pb}$ \\
$\mathrm{Se}$
\end{tabular}


Destination Site: INEL

Shipping Site: ETEC Waste Type: Organic Liquids (CH-Non-alpha)

Total volume $(\mathrm{m} 3 / \mathrm{yr})$ :

$6.767 E-03$

Total Mass (kg/yr):

$7.145 \mathrm{E}+00$

Radionuclide

Am-241

$\mathrm{Ba}-137 \mathrm{~m}$

Co-50

$\mathrm{Cs}-137$

Eu-154

Eu-155

$\mathrm{H}-3$

Nb-94

$\mathrm{Ni}-59$

$\mathrm{Ni}-63$

$\mathrm{Pa}-234 \mathrm{~m}$

Pu-238

$\mathrm{Pu}-239$

Pu-240

Pu-241

$\mathrm{Sm}-151$

$\mathrm{Sr}-90$

Tc-99

Th-232

Th-234

$\mathrm{U}-238$

$Y-90$

Shipping Site: ETEC

Total Volume (m3/yr):

Radionuclide

Am-241

$\mathrm{Ba}-137 \mathrm{~m}$

Co-60

Cs-137

Eu-154

Eu-155

H-3

$\mathrm{Nb}-94$

$\mathrm{Ni}-59$

$\mathrm{Ni}-63$

Pa-234m

Pu-238

Pu-239

$\mathrm{Pu}-240$

Pu-241

$\mathrm{sm}-151$

Sr-90

TC-99

Th-232

Th-234

U-238

Y-90

\begin{tabular}{c}
$\begin{array}{c}\text { Activity } \\
(\text { Ci/Yr) }\end{array}$ \\
\hline $3.329 E-07$ \\
$4.901 E-04$ \\
$1.823 E-04$ \\
$5.241 E-04$ \\
$1.942 E-06$ \\
$6.559 E-07$ \\
$3.775 E-05$ \\
$1.387 E-06$ \\
$2.943 E-05$ \\
$3.097 E-03$ \\
$6.707 E-05$ \\
$2.062 E-05$ \\
$3.329 E-07$ \\
$4.994 E-07$ \\
$2.184 E-05$ \\
$6.320 E-06$ \\
$4.634 E-04$ \\
$5.543 E-06$ \\
$5.549 E-07$ \\
$6.707 E-05$ \\
$6.707 E-05$ \\
$4.634 E-04$
\end{tabular}

\begin{tabular}{l} 
Chemical \\
\hline $57-12-5$ \\
$75-09-2$ \\
$\mathrm{Ag}$ \\
$\mathrm{As}$ \\
$\mathrm{Ba}$ \\
$\mathrm{Cd}$ \\
$\mathrm{Cl}-2-\mathrm{x}$ \\
$\mathrm{Cl}-3-\mathrm{x}$ \\
$\mathrm{C} 1-4-\mathrm{x}$ \\
$\mathrm{Cl}-\mathrm{F}-\mathrm{x}$ \\
$\mathrm{Cr}$ \\
$\mathrm{HC}-\mathrm{insol}$ \\
$\mathrm{HC}-\mathrm{SOl} \mathrm{b}$ \\
$\mathrm{Hg}$ \\
$\mathrm{Pb}$ \\
$\mathrm{Se}$
\end{tabular}

\begin{tabular}{c}
$\begin{array}{c}\text { Amount } \\
(\mathrm{kg} / \mathrm{Yr})\end{array}$ \\
\hline $5.272 \mathrm{E}-04$ \\
$4.759 \mathrm{E}-04$ \\
$3.144 \mathrm{E}-04$ \\
$4.027 \mathrm{E}-05$ \\
$2.745 \mathrm{E}-04$ \\
$1.707 \mathrm{E}-05$ \\
$1.354 \mathrm{E}-03$ \\
$5.331 \mathrm{E}-02$ \\
$3.012 \mathrm{E}-03$ \\
$1.098 \mathrm{E}-03$ \\
$7.317 \mathrm{E}-04$ \\
$4.382 \mathrm{E}-02$ \\
$1.255 \mathrm{E}+00$ \\
$1.781 \mathrm{E}-03$ \\
$2.730 \mathrm{E}-04$ \\
$2.748 \mathrm{E}-04$
\end{tabular}

Waste Type: Solids (CH-Non-alpha)

Total Mass (kg/yr): $1.326 \mathrm{E}+05$

$7.380 E+01$

\begin{tabular}{l} 
Chemical \\
\hline $57-12-5$ \\
$75-09-2$ \\
Ag \\
As \\
Ba \\
Cd \\
Cl-2-x \\
Cl-3-x \\
C1-4-x \\
Cl-F-x \\
Cr \\
HC-insol \\
HC-solub \\
Hg \\
Pb \\
Se
\end{tabular}

\begin{tabular}{c}
$\begin{array}{c}\text { Amount } \\
(\mathrm{kg} / \mathrm{yr})\end{array}$ \\
\hline $4.222 \mathrm{E}-02$ \\
$1.466 \mathrm{E}-01$ \\
$1.331 \mathrm{E}+00$ \\
$6.654 \mathrm{E}-01$ \\
$2.587 \mathrm{E}+00$ \\
$4.415 \mathrm{E}-01$ \\
$1.504 \mathrm{E}-01$ \\
$5.990 \mathrm{E}+00$ \\
$1.869 \mathrm{E}+00$ \\
$9.652 \mathrm{E}-02$ \\
$3.303 \mathrm{E}+00$ \\
$1.648 \mathrm{E}+01$ \\
$8.724 \mathrm{E}+00$ \\
$7.346 \mathrm{E}-01$ \\
$6.598 \mathrm{E}+00$ \\
$2.188 \mathrm{E}-01$
\end{tabular}

$\begin{array}{r}\text { ACtivity } \\ (\mathrm{Ci} / \mathrm{Yr})\end{array}$
$3.631 \mathrm{E}-03$
$5.346 \mathrm{E}+00$
$1.989 \mathrm{E}+00$
$5.716 \mathrm{E}+00$
$2.118 \mathrm{E}-02$
$7.262 \mathrm{E}-03$
$4.118 \mathrm{E}-01$
$1.513 \mathrm{E}-02$
$3.210 \mathrm{E}-01$
$3.377 \mathrm{E}+01$
$7.316 \mathrm{E}-01$
$2.249 \mathrm{E}-01$
$3.631 \mathrm{E}-03$
$5.447 \mathrm{E}-03$
$2.382 \mathrm{E}-01$
$6.893 \mathrm{E}-02$
$5.054 \mathrm{E}+00$
$6.046 \mathrm{E}-02$
$6.052 \mathrm{E}-03$
$7.316 \mathrm{E}-01$
$7.316 \mathrm{E}-01$
$5.054 \mathrm{E}+00$




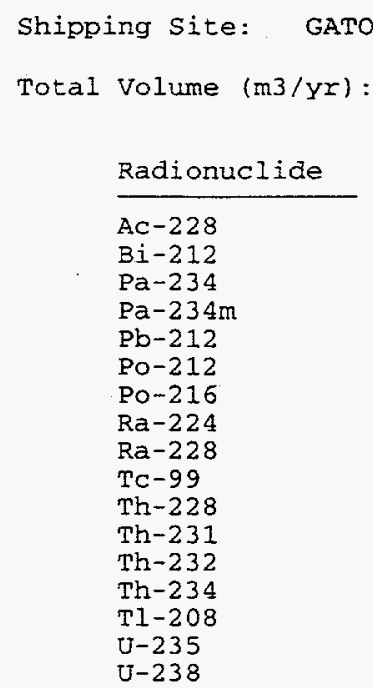

Shipping site:
$7.077 \mathrm{E}-02$

\begin{tabular}{c}
$\begin{array}{c}\text { Activity } \\
\text { (Ci/yr) }\end{array}$ \\
\hline $1.514 \mathrm{E}-07$ \\
$2.547 \mathrm{E}-08$ \\
$1.925 \mathrm{E}-08$ \\
$1.870 \mathrm{E}-04$ \\
$2.547 \mathrm{E}-08$ \\
$1.642 \mathrm{E}-08$ \\
$2.547 \mathrm{E}-08$ \\
$2.547 \mathrm{E}-08$ \\
$1.514 \mathrm{E}-07$ \\
$2.816 \mathrm{E}-06$ \\
$2.547 \mathrm{E}-08$ \\
$1.460 \mathrm{E}-07$ \\
$1.537 \mathrm{E}-06$ \\
$1.870 \mathrm{E}-04$ \\
$9.625 \mathrm{E}-09$ \\
$1.455 \mathrm{E}-07$ \\
$1.870 \mathrm{E}-04$
\end{tabular}

Waste Type: Organic Liquids (CH-Non-alpha)

Total Mass $(\mathrm{kg} / \mathrm{yr}): 7.476 \mathrm{E}+01$

\begin{tabular}{|c|c|}
\hline Chemical & $\begin{array}{l}\text { Amount } \\
(\mathrm{kg} / \mathrm{yr})\end{array}$ \\
\hline $57-12-5$ & $6.543 \mathrm{E}-03$ \\
\hline $75-09-2$ & $5.261 \mathrm{E}-03$ \\
\hline $\mathrm{Ag}$ & $3.280 \mathrm{E}-03$ \\
\hline As & $4.207 E-04$ \\
\hline $\mathrm{Ba}$ & $2.867 \mathrm{E}-03$ \\
\hline $\mathrm{cd}$ & $4.175 E-04$ \\
\hline$c 1-2-x$ & $1.428 \mathrm{E}-02$ \\
\hline C1-3-x & $5.732 \mathrm{E}-01$ \\
\hline Cl- $-4-x$ & $3.523 E-02$ \\
\hline$C l-F-x$ & $1.146 \mathrm{E}-02$ \\
\hline $\mathrm{Cr}$ & $7.839 \mathrm{E}-03$ \\
\hline HC-insol & $4.931 E-01$ \\
\hline $\mathrm{HC}-$ solub & $1.311 E+01$ \\
\hline Hg & $1.858 \mathrm{E}-02$ \\
\hline $\mathrm{Pb}$ & $2.894 E-03$ \\
\hline & \\
\hline
\end{tabular}

Total Volume (m3/yr):

Radionuclide

Ac -228

$\mathrm{Bi}-212$

$\mathrm{Pa}-234$

$\mathrm{Pa}-234 \mathrm{~m}$

$\mathrm{Pb}-212$

Po-212

Po -216

$\mathrm{Ra}-224$

$\mathrm{Ra}-228$

TC-99

Th-228

$\mathrm{Th}-231$

Th-232

Th-234

T1-208

$\mathrm{U}-235$

$\mathrm{U}-238$

Shipping site: NTS

Total volume $(\mathrm{m} 3 / \mathrm{yr})$ :

Radionuclide

$\mathrm{Ba}-137 \mathrm{~m}$

Cs -137

$\mathrm{H}-3$

$\mathrm{Pa}-234 \mathrm{~m}$

Pu-241

$\mathrm{Sr}-90$

Th-234

$\mathrm{U}-238$

$\mathrm{Y}-90$
Waste Type: Solids (CH-Non-alpha)

$.632 \mathrm{E}-01$ Total Mass $(\mathrm{kg} / \mathrm{yr}): 8.419 \mathrm{E}+02$

\begin{tabular}{c}
$\begin{array}{c}\text { Activity } \\
(C i / y r)\end{array}$ \\
\hline $1.218 \mathrm{E}-06$ \\
$2.049 \mathrm{E}-07$ \\
$1.548 \mathrm{E}-07$ \\
$1.504 \mathrm{E}-03$ \\
$2.049 \mathrm{E}-07$ \\
$1.320 \mathrm{E}-07$ \\
$2.049 \mathrm{E}-07$ \\
$1.996 \mathrm{E}-07$ \\
$1.186 \mathrm{E}-06$ \\
$2.207 \mathrm{E}-05$ \\
$2.049 \mathrm{E}-07$ \\
$1.175 \mathrm{E}-06$ \\
$1.237 \mathrm{E}-05$ \\
$1.504 \mathrm{E}-03$ \\
$7.742 \mathrm{E}-08$ \\
$1.170 \mathrm{E}-06$ \\
$1.504 \mathrm{E}-03$
\end{tabular}

\begin{tabular}{|c|c|}
\hline Chemical & $\begin{array}{l}\text { Amount } \\
(\mathrm{kg} / \mathrm{yr})\end{array}$ \\
\hline $\begin{array}{l}57-12-5 \\
75-09-2\end{array}$ & $\begin{array}{l}9.298 \mathrm{E}-02 \\
1.998 \mathrm{E}-01\end{array}$ \\
\hline $\mathrm{Ag}$ & $2.502 \mathrm{E}-02$ \\
\hline As & $9.721 \mathrm{E}-03$ \\
\hline $\mathrm{Ba}$ & $1.175 E+00$ \\
\hline $\mathrm{cd}$ & $4.463 \mathrm{E}-02$ \\
\hline$C 1-2-x$ & $1.741 \mathrm{E}-01$ \\
\hline$C 1-3-x$ & $3.347 E+00$ \\
\hline$C 1-4-x$ & $8.150 E-01$ \\
\hline$C l-F-x$ & $1.017 \mathrm{E}-01$ \\
\hline $\mathrm{Cr}$ & $1.315 \mathrm{E}+00$ \\
\hline $\mathrm{HC}$-insol & $1.041 E+01$ \\
\hline HC-solub & $6.8998+00$ \\
\hline $\mathrm{Hg}$ & $1.942 \mathrm{E}-01$ \\
\hline $\mathrm{Pb}$ & $2.810 E+00$ \\
\hline se & $7.067 \mathrm{E}-02$ \\
\hline
\end{tabular}

Waste Type: Solids (CH-Non-alpha)

.621E-03 Total Mass $(\mathrm{kg} / \mathrm{yr}): \quad 8.886 \mathrm{E}+00$

\begin{tabular}{c}
$\begin{array}{c}\text { Activity } \\
\text { (Ci/yr) }\end{array}$ \\
\hline $8.760 \mathrm{E}-05$ \\
$9.177 \mathrm{E}-05$ \\
$4.167 \mathrm{E}-01$ \\
$2.127 \mathrm{E}-05$ \\
$2.085 \mathrm{E}-05$ \\
$8.760 \mathrm{E}-05$ \\
$2.127 \mathrm{E}-05$ \\
$2.127 \mathrm{E}-05$ \\
$8.760 \mathrm{E}-05$
\end{tabular}

\begin{tabular}{|c|c|}
\hline Chemical & $\begin{array}{l}\text { Amount } \\
\text { (kg/Yr) }\end{array}$ \\
\hline $\begin{array}{l}75-09-2 \\
\text { Ag }\end{array}$ & $\begin{array}{l}5.331 E-03 \\
9.774 E-05\end{array}$ \\
\hline cd & $5.331 E-04$ \\
\hline Cl-2-x & $5.331 E-03$ \\
\hline$C l-3-x$ & $2.221 E-02$ \\
\hline$C 1-4-x$ & $1.688 \mathrm{E}-01$ \\
\hline$C I-F-x$ & $2.665 E-03$ \\
\hline $\mathrm{Cr}$ & $1.777 \mathrm{E}-04$ \\
\hline HC-insol & $4.443 E-02$ \\
\hline HC-solub & $2.221 E-02$ \\
\hline $\mathrm{Hg}$ & $3.465 E-04$ \\
\hline $\mathrm{Pb}$ & $3.199 \mathrm{E}-04$ \\
\hline $\mathrm{se}$ & $8.886 \mathrm{E}-06$ \\
\hline
\end{tabular}


Destination site: LANL

\begin{tabular}{l} 
Shipping Site: ITRI \\
Total Volume (m3/yr): \\
Radionuclide \\
\hline Ba-137m \\
Co-60 \\
Cs -137 \\
Eu-154 \\
Fe-55 \\
H-3 \\
Ni-59 \\
Ni-53 \\
Pm-147 \\
Pu-238 \\
Pu-241 \\
Sm-151 \\
Sr-90 \\
Y-90
\end{tabular}

Shipping site: PANT

Total Volume (m3/yr):

Radionuclide

$\mathrm{Ba}-137 \mathrm{~m}$

Co-60

Cs -137

Eu-154

$\mathrm{Fe}-55$

$\mathrm{H}-3$

$\mathrm{Ni}-59$

$\mathrm{Ni}-63$

Pm-147

Pu-238

Pu-241

Sm-151

Sr -90

$\mathrm{Y}-90$

Shipping Site: PANT

Total Volume $(\mathrm{m} 3 / \mathrm{yr})$ :

Radionuclide

$\mathrm{Ba}-137 \mathrm{~m}$

Co-60

Cs -137

Eu-154

$\mathrm{Fe}-55$

$\mathrm{H}-3$

$\mathrm{Ni}-59$

$\mathrm{Ni}-63$

Pm-147

$\mathrm{Pu}-238$

Pu-241

$\mathrm{Sm}-151$

$\mathrm{Sr}-90$

$\mathrm{Y}-90$
Waste Type: Organic Liquids ( $\mathrm{CH}$-Non-alpha)

1.028E-01 Total Mass (kg/yr): $1.007 \mathrm{E}+02$

\begin{tabular}{c}
$\begin{array}{r}\text { Activity } \\
\text { (Ci/yr) }\end{array}$ \\
\hline $1.367 E-03$ \\
$1.879 \mathrm{E}-03$ \\
$1.424 \mathrm{E}-03$ \\
$1.566 \mathrm{E}-05$ \\
$2.364 \mathrm{E}-04$ \\
$2.725 \mathrm{E}-01$ \\
$3.133 \mathrm{E}-05$ \\
$4.158 \mathrm{E}-03$ \\
$1.310 \mathrm{E}-04$ \\
$5.411 \mathrm{E}-05$ \\
$2.734 \mathrm{E}-04$ \\
$1.253 \mathrm{E}-05$ \\
$1.338 \mathrm{E}-03$ \\
$1.338 \mathrm{E}-03$
\end{tabular}

\begin{tabular}{l} 
Chemical \\
\hline $57-12-5$ \\
$\mathrm{Ag}$ \\
$\mathrm{Cd}$ \\
$\mathrm{Cl}-3-\mathrm{x}$ \\
$\mathrm{Cl}-4-\mathrm{x}$ \\
$\mathrm{Cr}$ \\
$\mathrm{HC}-$ insol \\
$\mathrm{HC}-\mathrm{solub}$ \\
$\mathrm{Hg}$ \\
$\mathrm{Pb}$ \\
$\mathrm{Se}$
\end{tabular}

\begin{tabular}{c}
$\begin{array}{c}\text { Amount } \\
(\mathrm{kg} / \mathrm{Yr})\end{array}$ \\
\hline $1.813 \mathrm{E}-02$ \\
$4.433 \mathrm{E}-03$ \\
$4.937 \mathrm{E}-04$ \\
$8.061 \mathrm{E}-02$ \\
$4.534 \mathrm{E}-03$ \\
$2.116 \mathrm{E}-02$ \\
$1.108 \mathrm{E}+00$ \\
$3.627 \mathrm{E}+01$ \\
$2.821 \mathrm{E}-02$ \\
$5.038 \mathrm{E}-03$ \\
$4.030 \mathrm{E}-03$
\end{tabular}

Waste Type: Organic Liquids (CH-Non-alpha)

1. $461 \mathrm{E}+00$

Total Mass (kg/Yr): $2.110 \mathrm{E}+03$

\begin{tabular}{c}
$\begin{array}{c}\text { Activity } \\
\text { (Ci/yr) }\end{array}$ \\
\hline $1.942 \mathrm{E}-02$ \\
$2.671 \mathrm{E}-02$ \\
$2.023 \mathrm{E}-02$ \\
$2.225 \mathrm{E}-04$ \\
$3.359 \mathrm{E}-03$ \\
$3.872 \mathrm{E}+00$ \\
$4.451 \mathrm{E}-04$ \\
$5.908 \mathrm{E}-02$ \\
$1.861 \mathrm{E}-03$ \\
$7.689 \mathrm{E}-04$ \\
$3.885 \mathrm{E}-03$ \\
$1.780 \mathrm{E}-04$ \\
$1.902 \mathrm{E}-02$ \\
$1.902 \mathrm{E}-02$
\end{tabular}

\begin{tabular}{l} 
Chemical \\
\hline $75-09-2$ \\
As \\
$\mathrm{Ba}$ \\
$\mathrm{Cd}$ \\
$\mathrm{C} 1-2-\mathrm{x}$ \\
$\mathrm{Cl}-3-\mathrm{x}$ \\
$\mathrm{Cl}-4-\mathrm{x}$ \\
$\mathrm{Cl}-\mathrm{F}-\mathrm{x}$ \\
$\mathrm{Cr}$ \\
$\mathrm{HC}-$ insol \\
$\mathrm{HC}-$ solub \\
$\mathrm{Hg}$ \\
$\mathrm{Pb}$
\end{tabular}

\begin{tabular}{c}
$\begin{array}{c}\text { Amount } \\
(\mathrm{kg} / \mathrm{Y} r)\end{array}$ \\
\hline $3.284 \mathrm{E}+00$ \\
$1.112 \mathrm{E}-03$ \\
$4.766 \mathrm{E}-03$ \\
$2.321 \mathrm{E}+00$ \\
$1.743 \mathrm{E}+00$ \\
$2.389 \mathrm{E}+02$ \\
$5.331 \mathrm{E}+01$ \\
$9.829 \mathrm{E}-02$ \\
$2.020 \mathrm{E}+00$ \\
$6.419 \mathrm{E}+01$ \\
$3.280 \mathrm{E}+01$ \\
$1.325 \mathrm{E}-02$ \\
$4.431 \mathrm{E}-01$
\end{tabular}

Waste Type: Solids (CH-Non-alpha)

3.957E+01 Total Mass $(\mathrm{kg} / \mathrm{yr}): 2.511 \mathrm{E}+04$

\begin{tabular}{c}
$\begin{array}{c}\text { Activity } \\
\text { (Ci/yr) }\end{array}$ \\
\hline $5.276 \mathrm{E}-01$ \\
$7.254 \mathrm{E}-01$ \\
$5.454 \mathrm{E}-01$ \\
$6.045 \mathrm{E}-03$ \\
$9.123 \mathrm{E}-02$ \\
$1.051 \mathrm{E}+02$ \\
$1.209 \mathrm{E}-02$ \\
$1.604 \mathrm{E}+00$ \\
$5.056 \mathrm{E}-02$ \\
$2.088 \mathrm{E}-02$ \\
$1.055 \mathrm{E}-01$ \\
$4.836 \mathrm{E}-03$ \\
$5.126 \mathrm{E}-01$ \\
$5.126 \mathrm{E}-01$
\end{tabular}

\begin{tabular}{l} 
Chemical \\
\hline $57-12-5$ \\
$75-09-2$ \\
$\mathrm{Ag}$ \\
$\mathrm{As}$ \\
$\mathrm{Ba}$ \\
$\mathrm{Cd}$ \\
$\mathrm{Cl}-2-\mathrm{x}$ \\
$\mathrm{Cl}-3-\mathrm{x}$ \\
$\mathrm{Cl}-4-\mathrm{x}$ \\
Cl-F-x \\
Cr \\
$\mathrm{HC}-\mathrm{insol}$ \\
$\mathrm{HC}-\mathrm{solub}$ \\
$\mathrm{Hg}$ \\
$\mathrm{Pb}$ \\
$\mathrm{Se}$
\end{tabular}

\begin{tabular}{c}
$\begin{array}{c}\text { Amount } \\
(\mathrm{kg} / \mathrm{Yr})\end{array}$ \\
\hline $2.480 E-01$ \\
$3.448 \mathrm{E}+01$ \\
$1.998 \mathrm{E}-01$ \\
$3.903 \mathrm{E}-02$ \\
$1.047 \mathrm{E}+01$ \\
$1.602 \mathrm{E}+00$ \\
$2.240 \mathrm{E}+01$ \\
$1.712 \mathrm{E}+02$ \\
$1.094 \mathrm{E}+02$ \\
$1.631 \mathrm{E}+01$ \\
$1.027 \mathrm{E}+01$ \\
$4.777 \mathrm{E}+02$ \\
$1.648 \mathrm{E}+02$ \\
$1.896 \mathrm{E}+00$ \\
$5.162 \mathrm{E}+01$ \\
$2.443 \mathrm{E}-01$
\end{tabular}




\begin{tabular}{l} 
Shipping Site: SNLA \\
Total Volume (m3/Yr): \\
\\
Radionuclide \\
\hline Ba-137m \\
Co-50 \\
Cs -137 \\
Eu-154 \\
Eu-155 \\
Fe-55 \\
H-3 \\
Nb-94 \\
Ni-59 \\
Ni-63 \\
Pa-234m \\
Pu-238 \\
Pu-240 \\
Pu-241 \\
Sm-151 \\
Sr-90 \\
Tc -99 \\
Th-234 \\
$\mathrm{U}-238$ \\
$\mathrm{Y}-90$
\end{tabular}

Shipping Site: SNLA

Total Volume $(\mathrm{m} 3 / \mathrm{yr}\rangle$ :

\begin{tabular}{l} 
Radionuclide \\
\hline $\mathrm{Ba}-137 \mathrm{~m}$ \\
$\mathrm{Co}-60$ \\
$\mathrm{Cs}-137$ \\
$\mathrm{Eu}-154$ \\
$\mathrm{Eu}-155$ \\
$\mathrm{Fe}-55$ \\
$\mathrm{H}-3$ \\
$\mathrm{Nb}-94$ \\
$\mathrm{Ni}-59$ \\
$\mathrm{Ni}-63$ \\
$\mathrm{~Pa}-234 \mathrm{~m}$ \\
$\mathrm{Pu}-238$ \\
$\mathrm{Pu}-240$ \\
$\mathrm{Pu}-241$ \\
$\mathrm{Sm}-151$ \\
$\mathrm{Sr}-90$ \\
$\mathrm{TC}-99$ \\
$\mathrm{Th}-234$ \\
$\mathrm{U}-238$ \\
$\mathrm{Y}-90$
\end{tabular}

Waste Type: Organic Liquids (CH-Non-alpha)

$5.487 E-04$

Total Mass $(\mathrm{kg} / \mathrm{yr}): 5.793 \mathrm{E}-01$

\begin{tabular}{|c|c|}
\hline Chemical & $\begin{array}{l}\text { Amount } \\
(\mathrm{kg} / \mathrm{yr})\end{array}$ \\
\hline $\begin{array}{l}57-12-5 \\
75-09-2\end{array}$ & $5.085 \mathrm{E}-05$ \\
\hline $\begin{array}{l}75-09-2 \\
\mathrm{Ag}\end{array}$ & $\begin{array}{l}3.859 \mathrm{E}-05 \\
2.549 \mathrm{E}-05\end{array}$ \\
\hline Ag & $2.549 E-05$ \\
\hline As & $3.265 \mathrm{E}-06$ \\
\hline $\mathrm{Ba}$ & $2.226 \mathrm{E}-05$ \\
\hline $\mathrm{cd}$ & $1.384 \mathrm{E}-06$ \\
\hline$C 1-2-x$ & $1.098 \mathrm{E}-04$ \\
\hline $\mathrm{Cl}-3-\mathrm{x}$ & $4.322 E-03$ \\
\hline$C l-4-x$ & $2.442 E-04$ \\
\hline$C l-F-x$ & $8.905 E-05$ \\
\hline $\mathrm{Cr}$ & $5.933 \mathrm{E}-05$ \\
\hline HC-insol & $3.553 \mathrm{E}-03$ \\
\hline HC-solub & $1.018 \mathrm{E}-01$ \\
\hline $\mathrm{Hg}$ & $1.444 \mathrm{E}-04$ \\
\hline $\mathrm{Pb}$ & $2.214 E-05$ \\
\hline $\mathrm{Se}$ & $2.228 \mathrm{E}-05$ \\
\hline
\end{tabular}

Waste Type: Solids (CH-Non-alpha)

$2.849 E+00$

Total Mass (kg/Yx): $2.300 \mathrm{E}+03$

\begin{tabular}{c}
$\begin{array}{r}\text { Activity } \\
\text { (Ci/yr) }\end{array}$ \\
\hline $3.441 \mathrm{E}-01$ \\
$5.000 \mathrm{E}-01$ \\
$3.637 \mathrm{E}-01$ \\
$1.825 \mathrm{E}-03$ \\
$4.213 \mathrm{E}-04$ \\
$9.972 \mathrm{E}-03$ \\
$6.351 \mathrm{E}+00$ \\
$2.387 \mathrm{E}-03$ \\
$4.719 \mathrm{E}-02$ \\
$5.706 \mathrm{E}+00$ \\
$1.685 \mathrm{E}-03$ \\
$1.236 \mathrm{E}-02$ \\
$9.832 \mathrm{E}-03$ \\
$9.832 \mathrm{E}-03$ \\
$3.932 \mathrm{E}-03$ \\
$3.385 \mathrm{E}-01$ \\
$1.404 \mathrm{E}-04$ \\
$1.685 \mathrm{E}-03$ \\
$1.685 \mathrm{E}-03$ \\
$3.385 \mathrm{E}-01$
\end{tabular}

\begin{tabular}{|c|c|}
\hline Chemical & $\begin{array}{l}\text { Amount } \\
(\mathrm{kg} / \mathrm{yr})\end{array}$ \\
\hline $57-12-5$ & $2.628 \mathrm{E}-01$ \\
\hline $\begin{array}{l}75-09-2 \\
\mathrm{Ag}\end{array}$ & $\begin{array}{l}7.747 \mathrm{E}-01 \\
5\end{array}$ \\
\hline Ag & $5.397 \mathrm{E}-02$ \\
\hline As & $1.546 \mathrm{E}-02$ \\
\hline $\mathrm{Ba}$ & $3.392 \mathrm{E}+00$ \\
\hline $\mathrm{cd}$ & $9.542 \mathrm{E}-03$ \\
\hline$C I-2-x$ & $8.422 \mathrm{E}-01$ \\
\hline CI-3-x & $2.813 E+00$ \\
\hline$C 1-4-x$ & $1.497 \mathrm{E}+00$ \\
\hline$C 1-F-x$ & $9.064 \mathrm{E}-01$ \\
\hline $\mathrm{Cr}$ & $3.104 \mathrm{E}+00$ \\
\hline HC-insol & $7.682 \mathrm{E}+00$ \\
\hline $\mathrm{HC}-$ solub & 1. $619 \mathrm{E}-01$ \\
\hline $\mathrm{Hg}$ & $1.563 \mathrm{E}-01$ \\
\hline $\mathrm{Pb}$ & $7.814 E+00$ \\
\hline $\mathrm{Se}$ & $4.865 \mathrm{E}-02$ \\
\hline
\end{tabular}




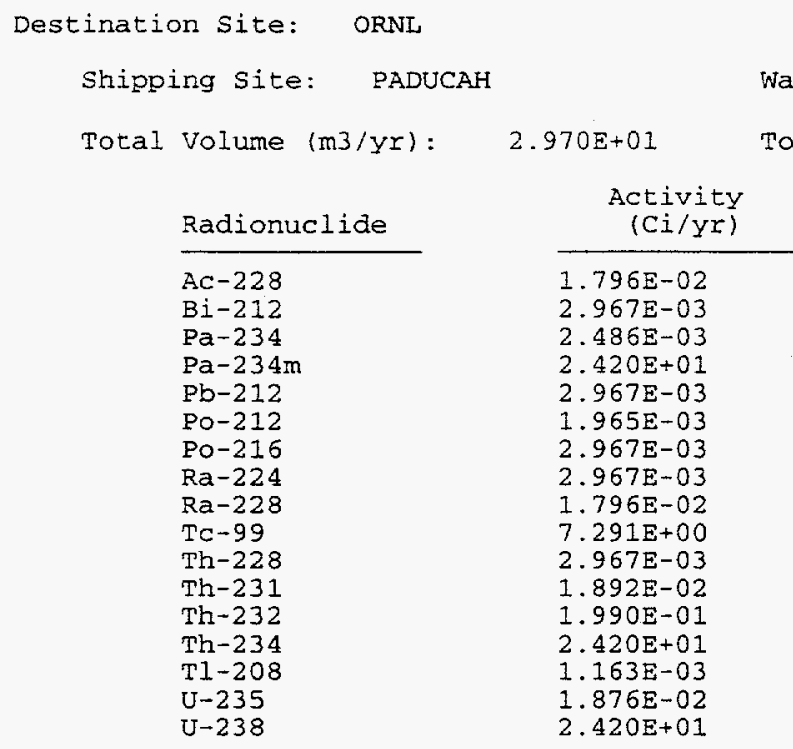

Waste Type: Organic Liquids (CH-Non-alpha)

Total Mass $(\mathrm{kg} / \mathrm{yr}): \quad 3.589 \mathrm{E}+04$

Shipping site: PADUCAH

Waste Type: Solids (CH-Non-alpha)

Total Volume $(\mathrm{m} 3 / \mathrm{yr})$ :

$4.740 E+00$

Total Mass $(\mathrm{kg} / \mathrm{yr}): 6.808 \mathrm{E}+03$

\begin{tabular}{l} 
Radionuclide \\
\hline Ac-228 \\
Bi-212 \\
$\mathrm{Pa}-234$ \\
$\mathrm{~Pa}-234 \mathrm{~m}$ \\
$\mathrm{~Pb}-212$ \\
$\mathrm{PO}-212$ \\
$\mathrm{PO}-216$ \\
$\mathrm{Ra}-224$ \\
$\mathrm{Ra}-228$ \\
$\mathrm{~T}-99$ \\
$\mathrm{Th}-228$ \\
$\mathrm{Th}-231$ \\
$\mathrm{Th}-232$ \\
$\mathrm{Th}-234$ \\
$\mathrm{~T}-208$ \\
$\mathrm{U}-235$ \\
$\mathrm{U}-238$
\end{tabular}

\begin{tabular}{|c|}
\hline $\begin{array}{l}\text { Activit } \\
\text { (Ci/yr }\end{array}$ \\
\hline $\begin{array}{l}2.890 \mathrm{E}-03 \\
4.773 \mathrm{E}-04 \\
3.999 \mathrm{E}-04 \\
3.894 \mathrm{E}+00 \\
4.773 \mathrm{E}-04 \\
3.161 \mathrm{E}-04 \\
4.773 \mathrm{E}-04 \\
4.664 \mathrm{E}-04 \\
2.824 \mathrm{E}-03 \\
1.146 \mathrm{E}+00 \\
4.773 \mathrm{E}-04 \\
3.044 \mathrm{E}-03 \\
3.202 \mathrm{E}-02 \\
3.894 \mathrm{E}+00 \\
1.870 \mathrm{E}-04 \\
3.019 \mathrm{E}-03 \\
3.894 \mathrm{E}+00\end{array}$ \\
\hline
\end{tabular}

\begin{tabular}{llc} 
Chemical & & $\begin{array}{c}\text { Amount } \\
(\mathrm{kg} / \mathrm{Yr})\end{array}$ \\
\cline { 1 - 1 } $57-12-5$ & & $4.590 \mathrm{E}-01$ \\
$75-09-2$ & & $4.205 \mathrm{E}+01$ \\
$\mathrm{Ag}$ & $2.300 \mathrm{E}-01$ \\
$\mathrm{As}$ & $1.180 \mathrm{E}-01$ \\
$\mathrm{Ba}$ & $5.803 \mathrm{E}-01$ \\
$\mathrm{Cd}$ & $3.374 \mathrm{E}+01$ \\
$\mathrm{Cl}-2-\mathrm{x}$ & $2.303 \mathrm{E}+01$ \\
$\mathrm{Cl}-3-\mathrm{x}$ & $2.444 \mathrm{E}+03$ \\
$\mathrm{Cl}-4-\mathrm{x}$ & $5.381 \mathrm{E}+02$ \\
$\mathrm{Cl}-\mathrm{F}-\mathrm{x}$ & $1.762 \mathrm{E}+00$ \\
$\mathrm{Cr}$ & $2.951 \mathrm{E}+01$ \\
$\mathrm{HC}-$ insol & $5.097 \mathrm{E}+03$ \\
$\mathrm{HC}-$ Solub & $2.651 \mathrm{E}+03$ \\
$\mathrm{Hg}$ & $1.493 \mathrm{E}+00$ \\
$\mathrm{~Pb}$ & $6.639 \mathrm{E}+00$ \\
$\mathrm{Se}$ & $2.011 \mathrm{E}-01$
\end{tabular}

PORTS

Shipping Site: AMES Waste Type: Solids (CH-Non-alpha)

Total Volume $(\mathrm{m} 3 / \mathrm{yr})$ :

$8.585 E-03$

Total Mass $(\mathrm{kg} / \mathrm{yr}): 1.162 \mathrm{E}+01$

Radionuclide

Ac-228

$\mathrm{Bi}-212$

$\mathrm{Pa}-234$

$\mathrm{Pa}-234 \mathrm{~m}$

$\mathrm{Pb}-212$

Po-212

Po- 216

$\mathrm{Ra}-224$

$\mathrm{Ra}-228$

TC-99

$\mathrm{Th}-228$

Th-231

Th-232

Th-234

TI-208

$\mathrm{U}-235$

$\mathrm{U}-238$

\begin{tabular}{c}
$\begin{array}{c}\text { Activity } \\
(\text { Ci/yr) }\end{array}$ \\
\hline $1.851 \mathrm{E}-08$ \\
$3.115 \mathrm{E}-09$ \\
$2.353 \mathrm{E}-09$ \\
$2.286 \mathrm{E}-05$ \\
$3.115 \mathrm{E}-09$ \\
$2.007 \mathrm{E}-09$ \\
$3.115 \mathrm{E}-09$ \\
$3.051 \mathrm{E}-09$ \\
$1.813 \mathrm{E}-08$ \\
$3.373 \mathrm{E}-07$ \\
$3.115 \mathrm{E}-09$ \\
$1.785 \mathrm{E}-08$ \\
$1.879 \mathrm{E}-07$ \\
$2.286 \mathrm{E}-05$ \\
$1.177 \mathrm{E}-09$ \\
$1.778 \mathrm{E}-08$ \\
$2.286 \mathrm{E}-05$
\end{tabular}

\begin{tabular}{|c|c|}
\hline Chemical & $\begin{array}{l}\text { Amount } \\
(\mathrm{kg} / \mathrm{y})\end{array}$ \\
\hline $57-12-5$ & $1.016 E+00$ \\
\hline $75-09-2$ & $1.710 \mathrm{E}+00$ \\
\hline $\mathrm{Ag}$ & $2.502 \mathrm{E}-01$ \\
\hline As & $8.712 E-02$ \\
\hline $\mathrm{Ba}$ & $1.228 \mathrm{E}+01$ \\
\hline $\mathrm{cd}$ & $1.504 \mathrm{E}-01$ \\
\hline$C l-2-x$ & $1.444 \mathrm{E}+00$ \\
\hline$c 1-3-x$ & $1.250 \mathrm{E}+01$ \\
\hline$C l-4-x$ & $4.496 \mathrm{E}+00$ \\
\hline$C 1-F-x$ & $6.729 E-01$ \\
\hline Cr & $1.293 \mathrm{E}+01$ \\
\hline HC-insol & $3.436 \mathrm{E} \div 01$ \\
\hline HC-solub & $1.740 E+01$ \\
\hline $\mathrm{Hg}$ & $1.732 \mathrm{E}+00$ \\
\hline $\mathrm{Pb}$ & $2.789 \mathrm{E}+01$ \\
\hline $\mathrm{Se}$ & $6.716 \mathrm{E}-01$ \\
\hline
\end{tabular}

\begin{tabular}{|c|c|}
\hline Chemical & $\begin{array}{l}\text { Amount } \\
(\mathrm{kg} / \mathrm{yr})\end{array}$ \\
\hline $\begin{array}{l}57-12-5 \\
75-09-2\end{array}$ & $\begin{array}{r}1.759 \mathrm{E}-05 \\
485 \mathrm{E}-04\end{array}$ \\
\hline Ag & $5.841 E-05$ \\
\hline As & $7.025 \mathrm{E}-07$ \\
\hline $\mathrm{Ba}$ & $4.061 E-05$ \\
\hline $\mathrm{cd}$ & $5.957 \mathrm{E}-05$ \\
\hline Cl-2-x & $1.879 \mathrm{E}-04$ \\
\hline$C l-3-x$ & $4.658 E-04$ \\
\hline$C 1-4-x$ & $8.901 E-04$ \\
\hline$C l-F-x$ & $7.426 \mathrm{E}-05$ \\
\hline $\mathrm{Cr}$ & $1.342 \mathrm{E}-04$ \\
\hline HC-insol & $1.917 \mathrm{E}-05$ \\
\hline $\mathrm{HC}$-solub & $1.017 \mathrm{E}-02$ \\
\hline $\mathrm{Hg}$ & $5.635 E-05$ \\
\hline $\mathrm{Pb}$ & $8.863 E-05$ \\
\hline $\mathrm{Se}$ & $1.196 \mathrm{E}-06$ \\
\hline
\end{tabular}


Shipping Site: ANL-E Waste Type: Solids (CH-Non-alpha)

Total Volume $(\mathrm{m} 3 / \mathrm{yr})$ :

$4.838 \mathrm{E}+02$

Total Mass (kg/yr):

$8.170 \mathrm{E}+05$

Radionuclide

Am-241

$\mathrm{Ba}-137 \mathrm{~m}$

Co- 60

Cs -137

Eu-154

Eu-155

$\mathrm{H}-3$

$\mathrm{Nb}-94$

$\mathrm{Ni}-59$

$\mathrm{Ni}-63$

Pa-234m

Pu -238

$\mathrm{Pu}-239$

Pu -240

Pu-241

$\mathrm{Sm}-151$

Sr -90

TC-99

$\mathrm{Th}-232$

$\mathrm{Th}-234$

$\mathrm{U}-238$

$\mathrm{Y}-90$

Shipping Site: BAPL

Total Volume $(\mathrm{m} 3 / y r)$ :

Radionuclide

Am-241

$\mathrm{Ba}-137 \mathrm{~m}$

Co-60

Cs -137

Eu-154

$\mathrm{H}-3$

$\mathrm{Nb}-94$

Ni-59

$\mathrm{Ni}-63$

$\mathrm{Pa}-234 \mathrm{~m}$

$\mathrm{Pu}-238$

Pu-239

Pu-240

$\mathrm{Pu}-241$

$\mathrm{Sm}-151$

Sr -90

Tc-99

Th-234

$\mathrm{U}-238$

$\mathrm{Y}-90$

Shipping site: BAPL

Total Volume $(\mathrm{m} 3 / \mathrm{yr})$ :

Radionuclide

Am-241

$\mathrm{Ba}-137 \mathrm{~m}$

Co-60

Cs -137

Eu-154

H- 3

$\mathrm{Nb}-94$

Ni-59

$\mathrm{Ni}-63$

$\mathrm{Pa}-234 \mathrm{~m}$

Pu-238

Pu-239

Pu-240

Pu-241

sm-151

Sr -90

TC-99

Th-234

U-238

$\mathrm{Y}-90$

\begin{tabular}{c}
$\begin{array}{c}\text { Activity } \\
(C i / Y Y)\end{array}$ \\
\hline $2.380 \mathrm{E}-02$ \\
$3.504 \mathrm{E}+01$ \\
$1.304 \mathrm{E}+01$ \\
$3.747 \mathrm{E}+01$ \\
$1.388 \mathrm{E}-01$ \\
$4.761 \mathrm{E}-02$ \\
$2.699 \mathrm{E}+00$ \\
$9.919 \mathrm{E}-02$ \\
$2.104 \mathrm{E}+00$ \\
$2.214 \mathrm{E}+02$ \\
$4.796 \mathrm{E}+00$ \\
$1.474 \mathrm{E}+00$ \\
$2.380 \mathrm{E}-02$ \\
$3.571 \mathrm{E}-02$ \\
$1.561 \mathrm{E}+00$ \\
$4.519 \mathrm{E}-01$ \\
$3.313 \mathrm{E}+01$ \\
$3.963 \mathrm{E}-01$ \\
$3.967 \mathrm{E}-02$ \\
$4.796 \mathrm{E}+00$ \\
$4.796 \mathrm{E}+00$ \\
$3.313 \mathrm{E}+01$
\end{tabular}

\begin{tabular}{l} 
Chemical \\
\hline $57-12-5$ \\
$75-09-2$ \\
Ag \\
As \\
$\mathrm{Ba}$ \\
$\mathrm{Cd}$ \\
$\mathrm{Cl}-2-\mathrm{x}$ \\
$\mathrm{Cl}-3-\mathrm{x}$ \\
$\mathrm{Cl}-4-\mathrm{x}$ \\
$\mathrm{Cl}-\mathrm{F}-\mathrm{x}$ \\
$\mathrm{Cr}$ \\
$\mathrm{HC}-$ insol \\
$\mathrm{HC}-\mathrm{SOl}$ (ub \\
$\mathrm{Hg}$ \\
$\mathrm{Pb}$ \\
$\mathrm{Se}$
\end{tabular}

\begin{tabular}{c}
$\begin{array}{c}\text { Amount } \\
(\mathrm{kg} / \mathrm{yr})\end{array}$ \\
\hline $2.002 \mathrm{E}+01$ \\
$2.778 \mathrm{E}+01$ \\
$1.623 \mathrm{E}+01$ \\
$6.714 \mathrm{E}+00$ \\
$2.781 \mathrm{E}+02$ \\
$9.989 \mathrm{E}+00$ \\
$1.822 \mathrm{E}+01$ \\
$3.658 \mathrm{E}+02$ \\
$4.608 \mathrm{E}+01$ \\
$1.759 \mathrm{E}+01$ \\
$3.334 \mathrm{E}+02$ \\
$1.482 \mathrm{E}+03$ \\
$7.360 \mathrm{E}+02$ \\
$1.110 \mathrm{E}+02$ \\
$4.704 \mathrm{E}+02$ \\
$4.660 \mathrm{E}+01$
\end{tabular}

Waste Type: Organic Liquids ( $\mathrm{CH}$-Non-alpha)

$6.410 E-02$

Total Mass (kg/yr) :

$6.163 \mathrm{E}+01$

\begin{tabular}{c}
$\begin{array}{c}\text { Activity } \\
\text { (Ci/yr) }\end{array}$ \\
\hline $5.332 \mathrm{E}-05$ \\
$5.866 \mathrm{E}-02$ \\
$7.908 \mathrm{E}-05$ \\
$6.272 \mathrm{E}-02$ \\
$9.439 \mathrm{E}-05$ \\
$4.694 \mathrm{E}-03$ \\
$5.357 \mathrm{E}-06$ \\
$1.209 \mathrm{E}-04$ \\
$1.242 \mathrm{E}-02$ \\
$3.469 \mathrm{E}-05$ \\
$2.806 \mathrm{E}-03$ \\
$3.801 \mathrm{E}-05$ \\
$2.168 \mathrm{E}-05$ \\
$6.225 \mathrm{E}-04$ \\
$9.031 \mathrm{E}-04$ \\
$5.586 \mathrm{E}-02$ \\
$2.806 \mathrm{E}-05$ \\
$3.469 \mathrm{E}-05$ \\
$3.469 \mathrm{E}-05$ \\
$5.586 \mathrm{E}-02$
\end{tabular}

\begin{tabular}{l} 
Chemical \\
\hline $75-09-2$ \\
As \\
$\mathrm{Ba}$ \\
$\mathrm{Cd}$ \\
$\mathrm{Cl}-2-\mathrm{x}$ \\
$\mathrm{Cl}-3-\mathrm{x}$ \\
$\mathrm{Cl}-4-\mathrm{x}$ \\
$\mathrm{Cl}-\mathrm{F}-\mathrm{x}$ \\
$\mathrm{Cr}$ \\
$\mathrm{HC}-$ insol \\
$\mathrm{HC}-$ solub \\
$\mathrm{Hg}$ \\
$\mathrm{Pb}$
\end{tabular}

\begin{tabular}{c}
$\begin{array}{c}\text { Amount } \\
(\mathrm{kg} / \mathrm{Yr})\end{array}$ \\
\hline $5.934 \mathrm{E}-02$ \\
$4.724 \mathrm{E}-04$ \\
$2.024 \mathrm{E}-03$ \\
$6.779 \mathrm{E}-02$ \\
$3.091 \mathrm{E}-02$ \\
$4.931 \mathrm{E}-01$ \\
$1.055 \mathrm{E}-01$ \\
$1.360 \mathrm{E}-05$ \\
$5.671 \mathrm{E}-02$ \\
$2.699 \mathrm{E}+01$ \\
$8.592 \mathrm{E}+00$ \\
$3.698 \mathrm{E}-04$ \\
$1.294 \mathrm{E}-02$
\end{tabular}

Waste Type: Solids (CH-Non-alpha)

6.105E-01

Total Mass $(\mathrm{kg} / \mathrm{yr})$ :

$6.010 \mathrm{E}+02$

\begin{tabular}{c}
$\begin{array}{c}\text { Activity } \\
\text { (Ci/yr) }\end{array}$ \\
\hline $5.078 \mathrm{E}-04$ \\
$5.587 \mathrm{E}-01$ \\
$7.532 \mathrm{E}-04$ \\
$5.974 \mathrm{E}-01$ \\
$8.990 \mathrm{E}-04$ \\
$4.471 \mathrm{E}-02$ \\
$5.102 \mathrm{E}-05$ \\
$1.151 \mathrm{E}-03$ \\
$1.183 \mathrm{E}-01$ \\
$3.304 \mathrm{E}-04$ \\
$2.672 \mathrm{E}-02$ \\
$3.620 \mathrm{E}-04$ \\
$2.065 \mathrm{E}-04$ \\
$5.929 \mathrm{E}-03$ \\
$8.601 \mathrm{E}-03$ \\
$5.321 \mathrm{E}-01$ \\
$2.672 \mathrm{E}-04$ \\
$3.304 \mathrm{E}-04$ \\
$3.304 \mathrm{E}-04$ \\
$5.321 \mathrm{E}-01$
\end{tabular}

\begin{tabular}{c}
$\begin{array}{c}\text { Amount } \\
(\mathrm{kg} / \mathrm{yr})\end{array}$ \\
\hline $1.703 \mathrm{E}-03$ \\
$6.266 \mathrm{E}-02$ \\
$6.515 \mathrm{E}-03$ \\
$1.598 \mathrm{E}-03$ \\
$6.259 \mathrm{E}-02$ \\
$1.698 \mathrm{E}-02$ \\
$7.067 \mathrm{E}-02$ \\
$4.679 \mathrm{E}-01$ \\
$4.156 \mathrm{E}-01$ \\
$8.727 \mathrm{E}-02$ \\
$4.102 \mathrm{E}-02$ \\
$2.697 \mathrm{E}+00$ \\
$2.656 \mathrm{E}+00$ \\
$1.514 \mathrm{E}-02$ \\
$7.746 \mathrm{E}-02$ \\
$2.393 \mathrm{E}-03$
\end{tabular}


Shipping Site: BATELLE Waste Type: Solids (CH-Non-alpha)

Total volume $(\mathrm{m} 3 / \mathrm{yr})$ : $1.500 E-03$ Total Mass (kg/Yr): $1.977 \mathrm{E}+00$

Radionuclide

Ac -228

$\mathrm{Bi}-212$

$\mathrm{Pa}-234$

$\mathrm{Pa}-234 \mathrm{~m}$

$\mathrm{Pb}-212$

P० -212

Po- 216

$\mathrm{Ra}-224$

Ra-228

TC- 99

Th-228

Th-231

Th -232

Th-234

TI-208

$\mathrm{U}-235$

$\mathrm{U}-238$

Shipping Site: BNL

Total Volume (m3/yr):

Radionuclide

Am-241

$\mathrm{Ba}-137 \mathrm{~m}$

Co-60

Cs -137

Eu-154

Eu-155

$\mathrm{H}-3$

$\mathrm{Nb}-94$

$\mathrm{Ni}-59$

$\mathrm{Ni}-63$

$\mathrm{Pa}-234 \mathrm{~m}$

Pu-238

$\mathrm{Pu}-239$

Pu-240

Pu-241

$\mathrm{Sm}-151$

$\mathrm{Sr}-90$

TC-99

Th-232

$\mathrm{Th}-234$

$\mathrm{U}-238$

$\mathrm{Y}-90$

Shipping site: BNL

Total Volume $(\mathrm{m} 3 / \mathrm{yr})$ :

Radionuclide

Am-24I

$\mathrm{Ba}-137 \mathrm{~m}$

Co-60

Cs -137

Eu-154

Eu-155

H-3

$\mathrm{Nb}-94$

Ni-59

Ni-63

$\mathrm{Pa}-234 \mathrm{~m}$

Pu-238

Pu-239

Pu-240

Pu-241

Sm-151

$\mathrm{Sr}-90$

TC-99

$\mathrm{Th}-232$

Th-234

$\mathrm{U}-238$

$\mathrm{Y}-90$

\begin{tabular}{c}
$\begin{array}{c}\text { Activity } \\
\text { (Ci/yr) }\end{array}$ \\
\hline $3.211 \mathrm{E}-09$ \\
$5.400 \mathrm{E}-10$ \\
$4.080 \mathrm{E}-10$ \\
$3.964 \mathrm{E}-06$ \\
$5.400 \mathrm{E}-10$ \\
$3.480 \mathrm{E}-10$ \\
$5.400 \mathrm{E}-10$ \\
$5.400 \mathrm{E}-10$ \\
$3.211 \mathrm{E}-09$ \\
$5.971 \mathrm{E}-08$ \\
$5.400 \mathrm{E}-10$ \\
$3.097 \mathrm{E}-09$ \\
$3.260 \mathrm{E}-08$ \\
$3.964 \mathrm{E}-06$ \\
$2.040 \mathrm{E}-10$ \\
$3.085 \mathrm{E}-09$ \\
$3.964 \mathrm{E}-06$
\end{tabular}

\begin{tabular}{lcc}
\multicolumn{1}{c}{ Chemical } & & $\begin{array}{c}\text { Amount } \\
(\mathrm{kg} / \mathrm{Yr})\end{array}$ \\
\cline { 1 - 1 } $75-09-2$ & & $3.955 \mathrm{E}-02$ \\
C1-2-x & & $1.977 \mathrm{E}-02$ \\
$\mathrm{Cl}-3-\mathrm{x}$ & & $1.977 \mathrm{E}-01$ \\
$\mathrm{Cl}-4-\mathrm{x}$ & $1.582 \mathrm{E}-01$ \\
HC-inso1 & & $4.943 \mathrm{E}-01$ \\
HC-SOIub & & $2.373 \mathrm{E}-01$
\end{tabular}

Waste Type: Organic Liquids ( $\mathrm{CH}-\mathrm{Non}-\mathrm{alpha}$

$1.544 \mathrm{E}+00$ Total Mass $(\mathrm{kg} / \mathrm{yr}): 1.749 \mathrm{E}+03$

\begin{tabular}{l}
$\begin{array}{c}\text { Activity } \\
\text { (Ci/yr) }\end{array}$ \\
\hline $7.598 \mathrm{E}-05$ \\
$1.118 \mathrm{E}-01$ \\
$4.162 \mathrm{E}-02$ \\
$1.196 \mathrm{E}-01$ \\
$4.432 \mathrm{E}-04$ \\
$1.519 \mathrm{E}-04$ \\
$8.617 \mathrm{E}-03$ \\
$3.166 \mathrm{E}-04$ \\
$6.718 \mathrm{E}-03$ \\
$7.068 \mathrm{E}-01$ \\
$1.530 \mathrm{E}-02$ \\
$4.706 \mathrm{E}-03$ \\
$7.598 \mathrm{E}-05$ \\
$1.139 \mathrm{E}-04$ \\
$4.984 \mathrm{E}-03$ \\
$1.442 \mathrm{E}-03$ \\
$1.057 \mathrm{E}-01$ \\
$1.265 \mathrm{E}-03$ \\
$1.266 \mathrm{E}-04$ \\
$1.530 \mathrm{E}-02$ \\
$1.530 \mathrm{E}-02$ \\
$1.057 \mathrm{E}-01$
\end{tabular}

\begin{tabular}{|c|c|}
\hline Chemical & $\begin{array}{l}\text { Amount } \\
(\mathrm{kg} / \mathrm{Yr})\end{array}$ \\
\hline $57-12-5$ & $8.298 \mathrm{E}-02$ \\
\hline $75-09-2$ & $1.156 \mathrm{E}+00$ \\
\hline As & $7.649 \mathrm{E}-03$ \\
\hline $\mathrm{Ba}$ & $4.627 \mathrm{E}-02$ \\
\hline $\mathrm{cd}$ & $8.868 \mathrm{E}-01$ \\
\hline$C l-2-x$ & $7.572 \mathrm{E}-01$ \\
\hline Cl-3-x & $7.014 \mathrm{E}+01$ \\
\hline Cl-4-x & $1.445 \mathrm{E}+01$ \\
\hline $\mathrm{Cl}-\mathrm{F}-\mathrm{x}$ & $1.704 \mathrm{E}-01$ \\
\hline $\mathrm{Cr}$ & $8.567 \mathrm{E}-01$ \\
\hline $\mathrm{HC}$-insol & $1.386 \mathrm{E}+02$ \\
\hline HC-solub & $2.115 E+02$ \\
\hline $\mathrm{Hg}$ & $2.406 \mathrm{E}-01$ \\
\hline $\mathrm{Pb}$ & $2.050 \mathrm{E}-01$ \\
\hline $\mathrm{Se}$ & $3.636 \mathrm{E}-02$ \\
\hline
\end{tabular}

Waste Type: Solids (CH-Non-alpha)

$8.915 E+00$

Total Mass $(\mathrm{kg} / \mathrm{yr}): 1.190 \mathrm{E}+04$

\begin{tabular}{c}
$\begin{array}{r}\text { Activity } \\
(C i / Y r)\end{array}$ \\
\hline $4.386 \mathrm{E}-04$ \\
$6.457 \mathrm{E}-01$ \\
$2.402 \mathrm{E}-01$ \\
$6.904 \mathrm{E}-01$ \\
$2.558 \mathrm{E}-03$ \\
$8.772 \mathrm{E}-04$ \\
$4.974 \mathrm{E}-02$ \\
$1.827 \mathrm{E}-03$ \\
$3.878 \mathrm{E}-02$ \\
$4.080 \mathrm{E}+00$ \\
$8.837 \mathrm{E}-02$ \\
$2.716 \mathrm{E}-02$ \\
$4.386 \mathrm{E}-04$ \\
$6.579 \mathrm{E}-04$ \\
$2.877 \mathrm{E}-02$ \\
$8.327 \mathrm{E}-03$ \\
$6.105 \mathrm{E}-01$ \\
$7.303 \mathrm{E}-03$ \\
$7.310 \mathrm{E}-04$ \\
$8.837 \mathrm{E}-02$ \\
$8.837 \mathrm{E}-02$ \\
$6.105 \mathrm{E}-01$
\end{tabular}

\begin{tabular}{c}
$\begin{array}{c}\text { Amount } \\
(\mathrm{kg} / \mathrm{y} r)\end{array}$ \\
\hline $1.617 \mathrm{E}+00$ \\
$8.722 \mathrm{E}-01$ \\
$7.571 \mathrm{E}-01$ \\
$2.698 \mathrm{E}-01$ \\
$2.157 \mathrm{E}+01$ \\
$4.530 \mathrm{E}-01$ \\
$8.722 \mathrm{E}-01$ \\
$2.419 \mathrm{E}+01$ \\
$3.963 \mathrm{E}-01$ \\
$1.019 \mathrm{E}+00$ \\
$2.698 \mathrm{E}+01$ \\
$1.091 \mathrm{E}+02$ \\
$5.459 \mathrm{E}+01$ \\
$8.531 \mathrm{E}+00$ \\
$2.912 \mathrm{E}+01$ \\
$3.668 \mathrm{E}+00$
\end{tabular}


Shipping Site: COLONIE Waste Type: Organic Liquids (CH-Non-alpha)

$9.054 E-01$

Total Mass $(\mathrm{kg} / \mathrm{Yr}): 1.026 \mathrm{E}+03$

Radionuclide

\begin{tabular}{c}
$\begin{array}{c}\text { Activity } \\
(C i / y z)\end{array}$ \\
\hline $1.937 E-06$ \\
$3.259 E-07$ \\
$2.462 E-07$ \\
$2.392 E-03$ \\
$3.259 E-07$ \\
$2.100 E-07$ \\
$3.259 E-07$ \\
$3.259 E-07$ \\
$1.937 E-06$ \\
$3.603 E-05$ \\
$3.259 E-07$ \\
$1.868 E-06$ \\
$1.967 E-05$ \\
$2.392 E-03$ \\
$1.231 E-07$ \\
$1.861 E-06$ \\
$2.392 \mathrm{E}-03$
\end{tabular}

\begin{tabular}{l} 
Chemical \\
\hline $57-12-5$ \\
$75-09-2$ \\
$\mathrm{Ag}$ \\
$\mathrm{As}$ \\
$\mathrm{Ba}$ \\
$\mathrm{Cd}$ \\
$\mathrm{Cl}-2-\mathrm{x}$ \\
$\mathrm{Cl}-3-\mathrm{x}$ \\
$\mathrm{Cl}-4-\mathrm{x}$ \\
$\mathrm{Cl}-\mathrm{F}-\mathrm{x}$ \\
$\mathrm{Cr}$ \\
$\mathrm{HC}-$ insol \\
$\mathrm{HC}-\mathrm{SO}$ lub \\
$\mathrm{Hg}$ \\
$\mathrm{Pb}$ \\
$\mathrm{Se}$
\end{tabular}

\begin{tabular}{c}
$\begin{array}{c}\text { Amount } \\
(\mathrm{kg} / \mathrm{yr})\end{array}$ \\
\hline $5.151 \mathrm{E}-02$ \\
$7.241 \mathrm{E}-01$ \\
$2.274 \mathrm{E}-02$ \\
$3.999 \mathrm{E}-03$ \\
$2.356 \mathrm{E}-02$ \\
$5.620 \mathrm{E}-01$ \\
$4.522 \mathrm{E}-01$ \\
$4.35 \mathrm{E}+01$ \\
$9.136 \mathrm{E}+00$ \\
$8.522 \mathrm{E}-02$ \\
$5.418 \mathrm{E}-01$ \\
$8.707 \mathrm{E}+01$ \\
$1.317 \mathrm{E}+02$ \\
$1.340 \mathrm{E}-01$ \\
$1.275 \mathrm{E}-01$ \\
$1.998 \mathrm{E}-02$
\end{tabular}

Shipping site: COLONIE

Waste Type: Solids (CH-Non-alpha)

Total Volume (m3/yr):

$1.235 E-02$

Total Mass $(\mathrm{kg} / \mathrm{yr}): 1.506 \mathrm{E}+01$

\begin{tabular}{l} 
Radionuclide \\
\hline $\mathrm{AC}-228$ \\
$\mathrm{Bi}-212$ \\
$\mathrm{~Pa}-234$ \\
$\mathrm{~Pa}-234 \mathrm{~m}$ \\
$\mathrm{~Pb}-212$ \\
$\mathrm{Po}-212$ \\
$\mathrm{PO}-216$ \\
$\mathrm{Ra}-224$ \\
$\mathrm{Ra}-228$ \\
$\mathrm{TC}-99$ \\
$\mathrm{Th}-228$ \\
$\mathrm{Th}-231$ \\
$\mathrm{Th}-232$ \\
$\mathrm{Th}-234$ \\
$\mathrm{~T}-208$ \\
$\mathrm{U}-235$ \\
$\mathrm{U}-238$
\end{tabular}

Shipping site: FEMP Total Volume $(\mathrm{m} 3 / \mathrm{Yr})$ :

Radionuclide

Ac- -228

$\mathrm{Bi}-212$

$\mathrm{Pa}-234$

$\mathrm{Pa}-234 \mathrm{~m}$

$\mathrm{Pb}-212$

Po- 212

Po-216

Ra-224

$\mathrm{Ra}-228$

TC-99

Th-228

Th -231

$\mathrm{Th}-232$

Th-234

TI-208

$\mathrm{T}-235$

$\mathrm{U}-238$

\begin{tabular}{c}
$\begin{array}{c}\text { Activity } \\
(\mathrm{Ci} / \mathrm{Y})\end{array}$ \\
\hline $3.198 \mathrm{E}-08$ \\
$5.380 \mathrm{E}-09$ \\
$4.065 \mathrm{E}-09$ \\
$3.949 \mathrm{E}-05$ \\
$5.380 \mathrm{E}-09$ \\
$3.467 \mathrm{E}-09$ \\
$5.380 \mathrm{E}-09$ \\
$2.690 \mathrm{E}-09$ \\
$1.599 \mathrm{E}-08$ \\
$2.974 \mathrm{E}-07$ \\
$5.380 \mathrm{E}-09$ \\
$3.084 \mathrm{E}-08$ \\
$3.247 \mathrm{E}-07$ \\
$3.949 \mathrm{E}-05$ \\
$2.032 \mathrm{E}-09$ \\
$3.072 \mathrm{E}-08$ \\
$3.949 \mathrm{E}-05$
\end{tabular}

\begin{tabular}{l} 
Chemical \\
\hline $57-12-5$ \\
$75-09-2$ \\
$\mathrm{Ag}$ \\
$\mathrm{As}$ \\
$\mathrm{Ba}$ \\
$\mathrm{Cd}$ \\
$\mathrm{Cl}-2-\mathrm{x}$ \\
$\mathrm{Cl}-3-\mathrm{x}$ \\
$\mathrm{Cl}-4-\mathrm{x}$ \\
$\mathrm{CI}-\mathrm{F}-\mathrm{x}$ \\
$\mathrm{Cr}$ \\
$\mathrm{HC}-$ insol \\
$\mathrm{HC}-\mathrm{Solub}$ \\
$\mathrm{Hg}$ \\
$\mathrm{Pb}$ \\
$\mathrm{Se}$
\end{tabular}

\begin{tabular}{c}
$\begin{array}{c}\text { Amount } \\
(\mathrm{kg} / \mathrm{yr})\end{array}$ \\
\hline $7.229 \mathrm{E}-04$ \\
$8.484 \mathrm{E}-04$ \\
$2.570 \mathrm{E}-05$ \\
$2.249 \mathrm{E}-05$ \\
$1.606 \mathrm{E}-05$ \\
$1.429 \mathrm{E}-04$ \\
$4.242 \mathrm{E}-03$ \\
$5.345 \mathrm{E}-03$ \\
$5.006 \mathrm{E}-03$ \\
$4.242 \mathrm{E}-04$ \\
$5.462 \mathrm{E}-04$ \\
$9.333 \mathrm{E}-04$ \\
$8.032 \mathrm{E}-04$ \\
$3.695 \mathrm{E}-06$ \\
$1.044 \mathrm{E}-03$ \\
$8.032 \mathrm{E}-06$
\end{tabular}

Waste Type: Organic Liquids ( $\mathrm{CH}$-Non-alpha)

$2.034 \mathrm{E}+01$

Total Mass (kg/yr):

$2.423 E+04$

\begin{tabular}{c}
$\begin{array}{c}\text { Activity } \\
\text { (Ci/Yr) }\end{array}$ \\
\hline $4.353 \mathrm{E}-05$ \\
$7.324 \mathrm{E}-06$ \\
$5.533 \mathrm{E}-06$ \\
$5.376 \mathrm{E}-02$ \\
$7.324 \mathrm{E}-06$ \\
$4.720 \mathrm{E}-06$ \\
$7.324 \mathrm{E}-06$ \\
$7.324 \mathrm{E}-06$ \\
$4.353 \mathrm{E}-05$ \\
$8.097 \mathrm{E}-04$ \\
$7.324 \mathrm{E}-06$ \\
$4.199 \mathrm{E}-05$ \\
$4.420 \mathrm{E}-04$ \\
$5.376 \mathrm{E}-02$ \\
$2.766 \mathrm{E}-06$ \\
$4.182 \mathrm{E}-05$ \\
$5.376 \mathrm{E}-02$
\end{tabular}

Amount

\begin{tabular}{l} 
Chemical \\
\hline $57-12-5$ \\
$75-09-2$ \\
$\mathrm{Ag}$ \\
$\mathrm{As}$ \\
$\mathrm{Ba}$ \\
$\mathrm{Cd}$ \\
$\mathrm{Cl}-2-\mathrm{x}$ \\
$\mathrm{Cl}-3-\mathrm{x}$ \\
$\mathrm{Cl}-4-\mathrm{x}$ \\
$\mathrm{Cl}-\mathrm{F}-\mathrm{x}$ \\
$\mathrm{Cr}$ \\
$\mathrm{HC}-\mathrm{insol}$ \\
$\mathrm{HC}-\mathrm{SOlub}$ \\
$\mathrm{Hg}$ \\
$\mathrm{Pb}$
\end{tabular}

$(\mathrm{kg} / \mathrm{yr})$

4. $910 \mathrm{E}-01$

$2.872 \mathrm{E}+01$

1.270E-01

6.728E-02

$2.928 \mathrm{E}-01$

2. $349 \mathrm{E}+01$

1. $522 \mathrm{E}+01$

1. $621 \mathrm{E}+03$

3. $603 \mathrm{E}+02$

6. 895E-01

$2.072 \mathrm{E}+01$

3. $779 E+03$

$255 E+03$

9. 307E-01

1. 150E-01
4. $623 \mathrm{E}+00$ 
Shipping Site: FEMP

Total volume $(\mathrm{m} 3 / \mathrm{yr})$ :

Radionuclide

Ac- -228

$\mathrm{Bi}-212$

$\mathrm{Pa}-234$

$\mathrm{Pa}-234 \mathrm{~m}$

$\mathrm{Pb}-212$

Po-212

Po-216

Ra-224

Ra-228

TC -99

Th-228

Th -231

Th -232

Th -234

TI-208

$\mathrm{U}-235$

$\mathrm{U}-238$

Shipping site: KAPL Total Volume $\{\mathrm{m} 3 / \mathrm{yr}\}$ :

Radionuclide

$\mathrm{Ba}-137 \mathrm{~m}$

$\mathrm{Co}-60$

Cs -134

Cs -137

Eu-154

Eu-155

$\mathrm{Fe}-55$

H-3

$\mathrm{Mn}-54$

$\mathrm{Nb}-94$

$\mathrm{Ni}-59$

$\mathrm{Ni}-63$

$\mathrm{Pa}-234 \mathrm{~m}$

$\mathrm{Pm}-147$

Pu-238

Pu-241

$\mathrm{Sb}-125$

$\mathrm{Sm}-151$

Sr -90

$\mathrm{Te}-125 \mathrm{~m}$

Th-234

$\mathrm{U}-238$

$\mathrm{Y}-90$
Waste Type: Solids (CH-Non-alpha)

Total Mass $(\mathrm{kg} / \mathrm{Yr}): 1.180 \mathrm{E}+05$

$.909 E+01$

\begin{tabular}{lc} 
Chemical & $\begin{array}{c}\text { Amount } \\
(\mathrm{kg} / \mathrm{yr})\end{array}$ \\
\cline { 1 - 1 } $57-12-5$ & $5.948 \mathrm{E}+00$ \\
$75-09-2$ & $2.117 \mathrm{E}+01$ \\
$\mathrm{Ag}$ & $3.226 \mathrm{E}+00$ \\
$\mathrm{AS}$ & $1.249 \mathrm{E}+00$ \\
$\mathrm{Ba}$ & $1.085 \mathrm{E}+02$ \\
$\mathrm{Cd}$ & $6.815 \mathrm{E}+00$ \\
$\mathrm{Cl}-2-\mathrm{x}$ & $2.073 \mathrm{E}+01$ \\
$\mathrm{Cl}-3-\mathrm{x}$ & $5.283 \mathrm{E}+02$ \\
$\mathrm{C} 1-4-\mathrm{x}$ & $3.692 \mathrm{E}+02$ \\
$\mathrm{C} 1-\mathrm{F}-\mathrm{x}$ & $1.313 \mathrm{E}+01$ \\
$\mathrm{Cr}$ & $1.531 \mathrm{E}+02$ \\
$\mathrm{HC}-$ insol & $1.786 \mathrm{E}+03$ \\
$\mathrm{HC}-\mathrm{solub}$ & $9.390 \mathrm{E}+02$ \\
$\mathrm{Hg}$ & $3.514 \mathrm{E}+01$ \\
$\mathrm{~Pb}$ & $1.240 \mathrm{E}+02$ \\
$\mathrm{Se}$ & $1.366 \mathrm{E}+01$
\end{tabular}

Waste Type: Organic Liquids ( $\mathrm{CH}-\mathrm{Non}-\mathrm{alpha})$

6.167E-01 Total Mass $(\mathrm{kg} / \mathrm{Yr}): 7.065 \mathrm{E}+02$

\begin{tabular}{|c|c|c|}
\hline $\begin{array}{c}\text { Activity } \\
(\mathrm{Ci} / \mathrm{yr})\end{array}$ & Chemical & $\begin{array}{l}\text { Amount } \\
(\mathrm{kg} / \mathrm{Yr})\end{array}$ \\
\hline $\begin{array}{l}4.129 \mathrm{E}-01 \\
6.669 \mathrm{E}+00 \\
3.921 \mathrm{E}-03 \\
4.359 \mathrm{E}-01 \\
5.305 \mathrm{E}-03 \\
2.537 \mathrm{E}-03 \\
8.719 \mathrm{E}-01 \\
1.899 \mathrm{E}+00 \\
2.768 \mathrm{E}-03 \\
4.152 \mathrm{E}-03 \\
8.719 \mathrm{E}-02 \\
1.172 \mathrm{E}+01 \\
1.637 \mathrm{E}-02 \\
4.752 \mathrm{E}-02 \\
1.361 \mathrm{E}-02 \\
1.107 \mathrm{E}-02 \\
1.845 \mathrm{E}-03 \\
3.690 \mathrm{E}-03 \\
4.106 \mathrm{E}-01 \\
4.613 \mathrm{E}-04 \\
1.637 \mathrm{E}-02 \\
1.637 \mathrm{E}-02 \\
4.106 \mathrm{E}-01\end{array}$ & $\begin{array}{l}57-12-5 \\
75-09-2 \\
\mathrm{Ag} \\
\mathrm{As} \\
\mathrm{Ba} \\
\mathrm{Cd} \\
\mathrm{CI}-2-\mathrm{x} \\
\mathrm{CI}-3-\mathrm{x} \\
\mathrm{CI}-4-\mathrm{x} \\
\mathrm{CI}-\mathrm{F}-\mathrm{x} \\
\mathrm{Cr} \\
\mathrm{HC}-\text { insol } \\
\mathrm{HC}-\text { solub } \\
\mathrm{Hg} \\
\mathrm{Pb} \\
\mathrm{Se}\end{array}$ & $\begin{array}{l}2.915 \mathrm{E}-02 \\
5.312 \mathrm{E}-01 \\
1.461 \mathrm{E}-02 \\
2.952 \mathrm{E}-03 \\
1.739 \mathrm{E}-02 \\
4.125 \mathrm{E}-01 \\
3.320 \mathrm{E}-01 \\
3.184 \mathrm{E}+01 \\
6.682 \mathrm{E}+00 \\
6.275 \mathrm{E}-02 \\
3.877 \mathrm{E}-01 \\
6.386 \mathrm{E}+01 \\
7.952 \mathrm{E}+01 \\
8.511 \mathrm{E}-02 \\
9.130 \mathrm{E}-02 \\
1.277 \mathrm{E}-02\end{array}$ \\
\hline
\end{tabular}


A-68

Shipping Site: KAPL

Waste Type: Solids (CH-Non-alpha)

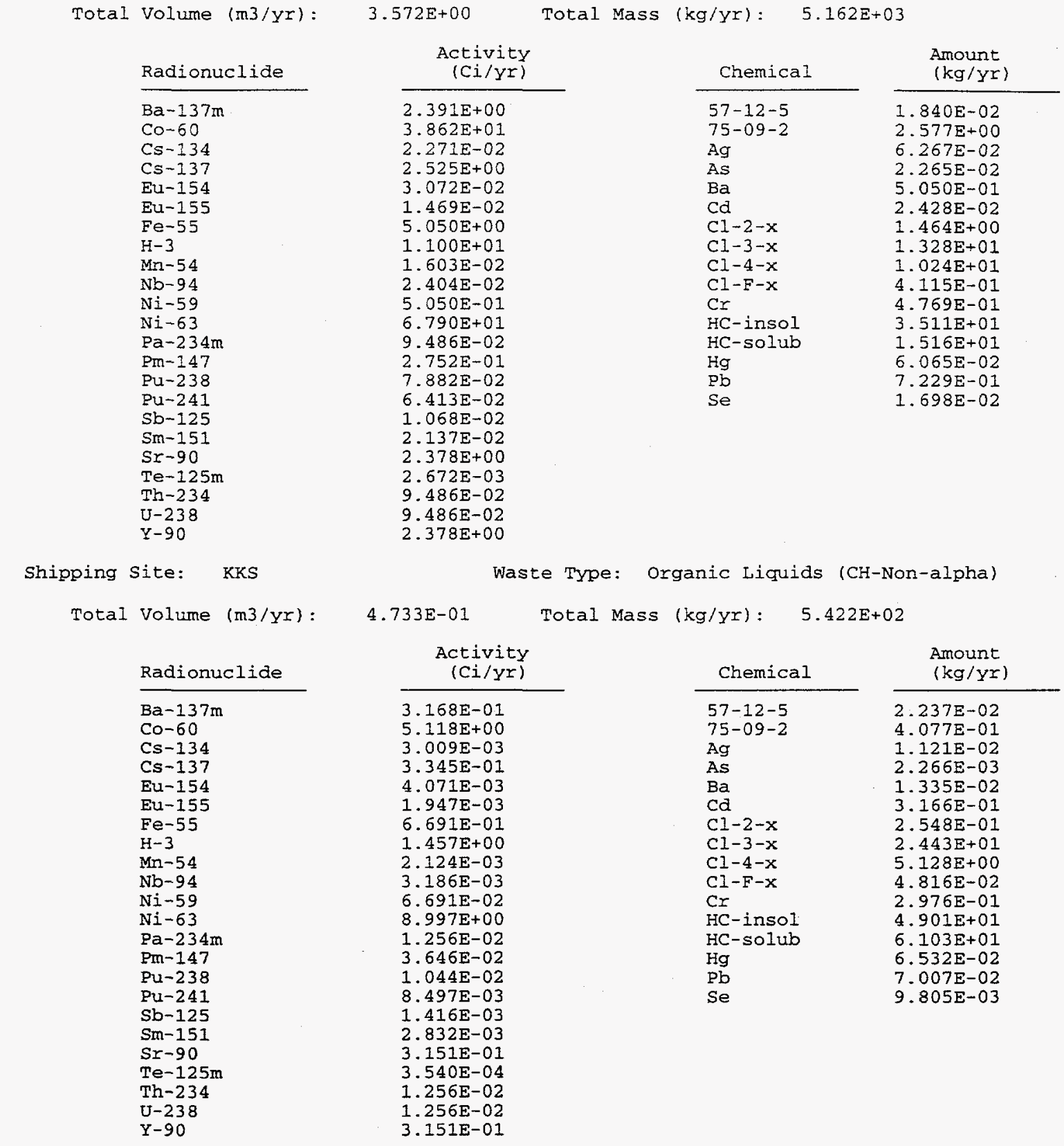

Shipping Site: KKS

$3.572 \mathrm{E}+00 \quad$ Total Mass $(\mathrm{kg} / \mathrm{yr}): 5.162 \mathrm{E}+03$ 
Shipping Site: KKS

Total volume (m3/yr):

Radionuclide

$\mathrm{Ba}-137 \mathrm{~m}$

$\mathrm{CO}-6 \mathrm{O}$

Cs -134

Cs -137

Eu-154

Eu-155

Fe- 55

$\mathrm{H}-3$

$\mathrm{Mn}-54$

$\mathrm{Nb}-94$

$\mathrm{Ni}-59$

$\mathrm{Ni}-63$

$\mathrm{Pa}-234 \mathrm{~m}$

PIn-147

Pu-238

Pu-241

$\mathrm{Sb}-125$

$\mathrm{Sm}-151$

$\mathrm{Sr}-90$

Te-125m

Th-234

$\mathrm{U}-238$

$\mathrm{Y}-90$

Shipping site: KWS

Total Volume (m3/yr):

Radionuclide

$\mathrm{Ba}-137 \mathrm{~m}$

Co -60

Cs -134

$\mathrm{Cs}-137$

$\mathrm{Eu}-154$

Eu-155

Fe-55

$\mathrm{H}-3$

$\mathrm{Mn}-54$

$\mathrm{Nb}-94$

$\mathrm{Ni}-59$

$\mathrm{Ni}-63$

$\mathrm{Pa}-234 \mathrm{~m}$

Pm-147

Pu-238

$\mathrm{Pu}-241$

$\mathrm{Sb}-125$

$\mathrm{Sm}-151$

Sr -90

Te-125m

Th-234

$\mathrm{U}-238$

$\mathrm{Y}-90$
Waste Type: Solids (CH-Non-alpha)

$3.928 \mathrm{E}+00$ Total Mass $(\mathrm{kg} / \mathrm{Yr}): 6.620 \mathrm{E}+03$

\begin{tabular}{c}
$\begin{array}{c}\text { Activity } \\
(\mathrm{Ci} / \mathrm{y})\end{array}$ \\
\hline $2.630 \mathrm{E}+00$ \\
$4.248 \mathrm{E}+01$ \\
$2.497 \mathrm{E}-02$ \\
$2.776 \mathrm{E}+00$ \\
$3.379 \mathrm{E}-02$ \\
$1.616 \mathrm{E}-02$ \\
$5.553 \mathrm{E}+00$ \\
$1.209 \mathrm{E}+01$ \\
$1.763 \mathrm{E}-02$ \\
$2.644 \mathrm{E}-02$ \\
$5.553 \mathrm{E}-01$ \\
$7.467 \mathrm{E}+01$ \\
$1.043 \mathrm{E}-01$ \\
$3.026 \mathrm{E}-01$ \\
$8.668 \mathrm{E}-02$ \\
$7.052 \mathrm{E}-02$ \\
$1.175 \mathrm{E}-02$ \\
$2.350 \mathrm{E}-02$ \\
$2.615 \mathrm{E}+00$ \\
$2.938 \mathrm{E}-03$ \\
$1.043 \mathrm{E}-01$ \\
$1.043 \mathrm{E}-01$ \\
$2.615 \mathrm{E}+00$
\end{tabular}

\begin{tabular}{l} 
Chemical \\
\hline $57-12-5$ \\
$75-09-2$ \\
$\mathrm{Ag}$ \\
$\mathrm{As}$ \\
$\mathrm{Ba}$ \\
$\mathrm{Cd}$ \\
$\mathrm{Cl}-2-\mathrm{x}$ \\
$\mathrm{Cl}-3-\mathrm{x}$ \\
$\mathrm{Cl}-4-\mathrm{x}$ \\
$\mathrm{Cl}-\mathrm{F}-\mathrm{x}$ \\
$\mathrm{Cr}$ \\
$\mathrm{HC}-$ insol \\
$\mathrm{HC}-$ solub \\
$\mathrm{Hg}$ \\
$\mathrm{Pb}$ \\
$\mathrm{Se}$
\end{tabular}

Amount

$(\mathrm{kg} / \mathrm{Yr})$

1. $282 \mathrm{E}-01$

$2.231 \mathrm{E}+00$

$8.783 \mathrm{E}-02$

3. 299E-02

1. $662 \mathrm{E}+00$

$5.269 E-02$

1. $281 \mathrm{E}+00$

1. $108 \mathrm{E}+01$

1. $215 \mathrm{E}+01$

2. $090 \mathrm{E}-01$

1. $846 \mathrm{E}+00$

2. $753 \mathrm{E}+01$

1. $411 \mathrm{E}+01$

1. $438 \mathrm{E}-01$

4. $165 \mathrm{E}+00$

3. $790 \mathrm{E}-02$
Waste Type: Organic Liquids (CH-Non-alpha)

6.454E-02 Total Mass $(\mathrm{kg} / \mathrm{yr}): 7.394 \mathrm{E}+01$

\begin{tabular}{c}
$\begin{array}{r}\text { Activity } \\
\text { (Ci/yr) }\end{array}$ \\
\hline $4.321 \mathrm{E}-02$ \\
$6.979 \mathrm{E}-01$ \\
$4.103 \mathrm{E}-04$ \\
$4.562 \mathrm{E}-02$ \\
$5.552 \mathrm{E}-04$ \\
$2.655 \mathrm{E}-04$ \\
$9.125 \mathrm{E}-02$ \\
$1.987 \mathrm{E}-01$ \\
$2.896 \mathrm{E}-04$ \\
$4.345 \mathrm{E}-04$ \\
$9.125 \mathrm{E}-03$ \\
$1.226 \mathrm{E}+00$ \\
$1.714 \mathrm{E}-03$ \\
$4.973 \mathrm{E}-03$ \\
$1.424 \mathrm{E}-03$ \\
$1.158 \mathrm{E}-03$ \\
$1.931 \mathrm{E}-04$ \\
$3.862 \mathrm{E}-04$ \\
$4.297 \mathrm{E}-02$ \\
$4.828 \mathrm{E}-05$ \\
$1.714 \mathrm{E}-03$ \\
$1.714 \mathrm{E}-03$ \\
$4.297 \mathrm{E}-02$
\end{tabular}

Amount

Chemical

$57-12-5$

$75-09-2$

Ag

As

$\mathrm{Ba}$

$\mathrm{Cl}-2-\mathrm{x}$

Cl $1-3-x$

C1-4-x

Cl $-F-x$

$\mathrm{Cr}$

HC-insol

$\mathrm{HC}$-solub

$\mathrm{Hg}$

$\mathrm{Pb}$

(kg/yr)

3. $051 E-03$

5. 559E-02

1. $529 \mathrm{E}-03$

3. $090 \mathrm{E}-04$

1. $820 \mathrm{E}-03$

4. $317 \mathrm{E}-02$

3. $474 \mathrm{E}-02$

3. $332 E+00$

6. $993 E-01$

6. 567E-03

$4.058 \mathrm{E}-02$

$6.683 \mathrm{E}+00$

8. $322 \mathrm{E}+00$

8.907E-03

9. 555E-03

1. $337 \mathrm{E}-03$ 
Shipping Site: KWS Waste Type: Solids (CH-Non-alpha)

Total Volume $(\mathrm{m} 3 / \mathrm{yr})$ :

$1.262 \mathrm{E}+00$

Total Mass (kg/yr):

$1.580 E+03$

Radionuclide

$\mathrm{Ba}-137 \mathrm{~m}$

Co-60

Cs -134

Cs -137

Eu-154

Eu-155

$\mathrm{Fe}-55$

$\mathrm{H}-3$

$\mathrm{Mn}-54$

$\mathrm{Nb}-94$

$\mathrm{Ni}-59$

$\mathrm{Ni}-63$

$\mathrm{Pa}-234 \mathrm{~m}$

Pm-147

$\mathrm{Pu}-238$

$\mathrm{Pu}-24 \mathrm{I}$

Sb-125

$\mathrm{Sm}-151$

Sr -90

Te-125m

Th-234

$\mathrm{U}-238$

$Y-90$

Shipping Site: PNS

$\frac{\begin{array}{c}\text { Activity } \\ (\text { Ci/yr) }\end{array}}{8.449 \mathrm{E}-01}$

1. $364 \mathrm{E}+01$

8. $024 \mathrm{E}-03$

8. $921 E-01$

$1.085 E-02$

$5.192 E-03$

$1.784 \mathrm{E}+00$

3. $886 \mathrm{E}+00$

5. $664 \mathrm{E}-03$

$8.496 \mathrm{E}-03$

1. $784 \mathrm{E}-01$

2. $399 \mathrm{E}+01$

$3.351 \mathrm{E}-02$

9. $724 \mathrm{E}-02$

$2.785 \mathrm{E}-02$

2. $265 \mathrm{E}-02$

3. $776 \mathrm{E}-03$

7. $552 \mathrm{E}-03$

$8.402 \mathrm{E}-01$

$9.440 \mathrm{E}-04$

$3.351 \mathrm{E}-02$

$3.351 \mathrm{E}-02$

$8.402 \mathrm{E}-01$

\begin{tabular}{|c|c|}
\hline Chemical & $\begin{array}{l}\text { Amount } \\
(\mathrm{kg} / \mathrm{yr})\end{array}$ \\
\hline $\begin{array}{l}57-12-5 \\
75-09-2\end{array}$ & $\begin{array}{l}8.005 E-02 \\
3.801 E-01\end{array}$ \\
\hline $\mathrm{Ag}$ & $2.572 \mathrm{E}-02$ \\
\hline As & $8.795 E-03$ \\
\hline $\mathrm{Ba}$ & $1.035 E+00$ \\
\hline $\mathrm{Cd}$ & $1.157 \mathrm{E}-02$ \\
\hline $\mathrm{Cl}-2-\mathrm{x}$ & $3.221 \mathrm{E}-01$ \\
\hline CI $-3-\mathrm{x}$ & 1. $699 \mathrm{E}+00$ \\
\hline$C 1-4-x$ & $1.988 E+00$ \\
\hline$C 1-F-x$ & $2.323 E-01$ \\
\hline $\mathrm{Cr}$ & 1.010E +00 \\
\hline HC-insol & $4.421 \mathrm{E}+00$ \\
\hline HC-solub & $1.600 \mathrm{E}+00$ \\
\hline $\mathrm{Hg}$ & $6.065 \mathrm{E}-02$ \\
\hline $\mathrm{Pb}$ & $2.396 \mathrm{E}+00$ \\
\hline $\mathrm{Se}$ & 1.789E-02 \\
\hline
\end{tabular}

Total volume (m3/yr):

Radionuclide

Ba-137m

CO-60

Cs -134

Cs -137

$\mathrm{Eu}-154$

Eu-155

$\mathrm{Fe}-55$

$\mathrm{H}-3$

$\mathrm{Mn}-54$

$\mathrm{Nb}-94$

$\mathrm{Ni}-59$

$\mathrm{Ni}-63$

$\mathrm{Pa}-234 \mathrm{~m}$

Pm-147

Pu-238

Pu-241

Sb-125

Sm-151

$S r-90$

Te-125m

Th -234

$\mathrm{U}-238$

$Y-90$

Shipping site: PPPI

Total Mass (kg/yr): $9.088 \mathrm{E}+01$

$5.200 \mathrm{E}-02$

\begin{tabular}{c}
$\begin{array}{c}\text { Activity } \\
\text { (Ci/yr) }\end{array}$ \\
\hline $3.481 \mathrm{E}-02$ \\
$5.623 \mathrm{E}-01$ \\
$3.306 \mathrm{E}-04$ \\
$3.676 \mathrm{E}-02$ \\
$4.473 \mathrm{E}-04$ \\
$2.139 \mathrm{E}-04$ \\
$7.352 \mathrm{E}-02$ \\
$1.601 \mathrm{E}-01$ \\
$2.333 \mathrm{E}-04$ \\
$3.500 \mathrm{E}-04$ \\
$7.352 \mathrm{E}-03$ \\
$9.884 \mathrm{E}-01$ \\
$1.380 \mathrm{E}-03$ \\
$4.006 \mathrm{E}-03$ \\
$1.147 \mathrm{E}-03$ \\
$9.335 \mathrm{E}-04$ \\
$1.555 \mathrm{E}-04$ \\
$3.111 \mathrm{E}-04$ \\
$3.462 \mathrm{E}-02$ \\
$3.889 \mathrm{E}-05$ \\
$1.380 \mathrm{E}-03$ \\
$1.380 \mathrm{E}-03$ \\
$3.462 \mathrm{E}-02$
\end{tabular}

$\frac{\text { Chemical }}{75-09-2} \quad \frac{\begin{array}{c}\text { Amount } \\ (\mathrm{kg} / \mathrm{yr})\end{array}}{1.324 \mathrm{E}-02}$

Ag

$6.570 \mathrm{E}-04$

As $\quad 5.453 \mathrm{E}-04$

$\mathrm{Ba} \quad 1.708 \mathrm{E}-02$

$\mathrm{Cd} \quad 8.438 \mathrm{E}-03$

C1-2-x 1.324E-02

Cl-3-x 7.909E-01

$\mathrm{C} 1-4-\mathrm{x} \quad 1.483 \mathrm{E}-01$

Cl-F-x $\quad 7.499 \mathrm{E}-03$

Cr $1.051 \mathrm{E}-01$

$\mathrm{HC}$-insol $2.629 \mathrm{E}+00$

HC-solub 1.314E+00

$\mathrm{Hg} \quad 9.046 \mathrm{E}-03$

$\mathrm{Pb} \quad 1.319 \mathrm{E}-02$

Se $2.365 \mathrm{E}-03$

Waste Type: Solids (CH-Non-alpha)

Total Volume (m3/yr):

$5.456 \mathrm{E}-04$

Total Mass $(\mathrm{kg} / \mathrm{yr}): \quad 7.191 \mathrm{E}-01$

Radionuclide

$\mathrm{Ba}-137 \mathrm{~m}$

Cs-137

$\mathrm{H}-3$

$\mathrm{Pa}-234 \mathrm{~m}$

Pu-241

Sr -90

Th-234

$\mathrm{U}-238$

$y-90$

\begin{tabular}{c}
$\begin{array}{c}\text { Activity } \\
\text { (Ci/yr) }\end{array}$ \\
\hline $7.218 \mathrm{E}-06$ \\
$7.562 \mathrm{E}-06$ \\
$3.433 \mathrm{E}-02$ \\
$1.753 \mathrm{E}-06$ \\
$1.718 \mathrm{E}-06$ \\
$7.218 \mathrm{E}-06$ \\
$1.753 \mathrm{E}-06$ \\
$1.753 \mathrm{E}-06$ \\
$7.218 \mathrm{E}-06$
\end{tabular}

Chemical

75-09-2

C $1-2-x$

Cl-3-x

C1-4-x

$\mathrm{HC}$-inso 1

HC-solub

\begin{tabular}{|c|}
\hline $\begin{array}{l}\text { Amount } \\
(\mathrm{kg} / \mathrm{y} x)\end{array}$ \\
\hline $\begin{array}{l}1.438 \mathrm{E}-02 \\
7.190 \mathrm{E}-03 \\
7.190 \mathrm{E}-02 \\
5.752 \mathrm{E}-02 \\
1.797 \mathrm{E}-01 \\
8.629 \mathrm{E}-02\end{array}$ \\
\hline
\end{tabular}


Shipping Site: RMI Total Volume $(\mathrm{m} 3 / \mathrm{yr})$ :

Radionuclide

AC -228

$\mathrm{Bi}-212$

Pa-234

$\mathrm{Pa}-234 \mathrm{~m}$

$\mathrm{Pb}-212$

PO-212

Po-216

$\mathrm{Ra}-224$

$\mathrm{Ra}-228$

Tc -99

Th-228

Th-231

Th-232

Th-234

T1-208

$\mathrm{U}-235$

$\mathrm{U}-238$

Shipping Site:

RMI

Total volume $(\mathrm{m} 3 / \mathrm{yr})$ :

Radionuclide

Ac- 228

$\mathrm{Bi}-212$

$\mathrm{Pa}-234$

$\mathrm{Pa}-234 \mathrm{~m}$

$\mathrm{Pb}-212$

Po-212

Po- -216

$\mathrm{Ra}-224$

$\mathrm{Ra}-228$

Tc-99

Th -228

Th-231

Th-232

Th-234

Tl-208

$\mathrm{U}-235$

$\mathrm{U}-238$
Waste Type: Organic Liquids (CH-Non-alpha)

6.677E-01 Total Mass $(\mathrm{kg} / \mathrm{yr}): 9.741 \mathrm{E}+02$

\begin{tabular}{c}
$\begin{array}{c}\text { Activity } \\
\text { (Ci/yr) }\end{array}$ \\
\hline $1.429 \mathrm{E}-06$ \\
$2.403 \mathrm{E}-07$ \\
$1.816 \mathrm{E}-07$ \\
$1.764 \mathrm{E}-03$ \\
$2.403 \mathrm{E}-07$ \\
$1.549 \mathrm{E}-07$ \\
$2.403 \mathrm{E}-07$ \\
$2.403 \mathrm{E}-07$ \\
$1.429 \mathrm{E}-06$ \\
$2.657 \mathrm{E}-05$ \\
$2.403 \mathrm{E}-07$ \\
$1.378 \mathrm{E}-06$ \\
$1.450 \mathrm{E}-05$ \\
$1.754 \mathrm{E}-03$ \\
$9.081 \mathrm{E}-08$ \\
$1.372 \mathrm{E}-05$ \\
$1.764 \mathrm{E}-03$
\end{tabular}

\begin{tabular}{ll} 
Chemical & \multicolumn{1}{c}{$\begin{array}{c}\text { Amount } \\
(\mathrm{kg} / \mathrm{y})\end{array}$} \\
\cline { 1 - 1 } $75-09-2$ & $\frac{1.527 \mathrm{E}+00}{3.769 \mathrm{E}-04}$ \\
$\mathrm{As}$ & $1.615 \mathrm{E}-03$ \\
$\mathrm{Ba}$ & $1.071 \mathrm{E}+00$ \\
$\mathrm{Cd}$ & $8.108 \mathrm{E}-01$ \\
$\mathrm{Cl}-2-\mathrm{x}$ & $1.123 \mathrm{E}+02$ \\
$\mathrm{Cl}-3-\mathrm{x}$ & $2.505 \mathrm{E}+01$ \\
$\mathrm{Cl}-4-\mathrm{x}$ & $4.625 \mathrm{E}-02$ \\
$\mathrm{Cl}-\mathrm{F}-\mathrm{x}$ & $9.331 \mathrm{E}-01$ \\
$\mathrm{Cr}$ & $2.184 \mathrm{E}+01$ \\
$\mathrm{HC}-\mathrm{insol}$ & $1.277 \mathrm{E}+01$ \\
$\mathrm{HC}-$ solub & $6.122 \mathrm{E}-03$ \\
$\mathrm{Hg}$ & $2.045 \mathrm{E}-01$ \\
$\mathrm{~Pb}$ &
\end{tabular}

Waste Type: Solids (CH-Non-alpha)

Total Mass (kg/yr): $8.323 E+02$

$1.091 E+00$

\begin{tabular}{l} 
Chemical \\
\hline $57-12-5$ \\
$75-09-2$ \\
$\mathrm{Ag}$ \\
$\mathrm{As}$ \\
$\mathrm{Ba}$ \\
$\mathrm{Cd}$ \\
$\mathrm{Cl}-2-\mathrm{x}$ \\
$\mathrm{Cl}-3-\mathrm{x}$ \\
$\mathrm{Cl}-4-\mathrm{x}$ \\
$\mathrm{Cl}-\mathrm{F}-\mathrm{x}$ \\
$\mathrm{Cr}$ \\
$\mathrm{HC}-$ insol \\
$\mathrm{HC}-\mathrm{solub}$ \\
$\mathrm{Hg}$ \\
$\mathrm{Pb}$ \\
$\mathrm{Se}$
\end{tabular}

Activity

$2.370 E-06$

3. $988 \mathrm{E}-07$

3. $013 E-07$

2. $927 \mathrm{E}-03$

3. $988 \mathrm{E}-07$

2. $570 \mathrm{E}-07$

$3.988 \mathrm{E}-07$

3. $815 \mathrm{E}-07$

2. 267E-06

4. 218E-05

$3.988 \mathrm{E}-07$

2. $286 \mathrm{E}-06$

$2.407 \mathrm{E}-05$

2. $927 \mathrm{E}-03$

1. $506 \mathrm{E}-07$

2. 277E-06

$2.927 \mathrm{E}-03$
Amount

\begin{tabular}{c}
$\begin{array}{c}\text { Amount } \\
(\mathrm{kg} / \mathrm{Y})\end{array}$ \\
\hline $4.647 \mathrm{E}-03$ \\
$2.315 \mathrm{E}-01$ \\
$6.651 \mathrm{E}-03$ \\
$1.974 \mathrm{E}-03$ \\
$5.734 \mathrm{E}-01$ \\
$1.836 \mathrm{E}-02$ \\
$2.862 \mathrm{E}-01$ \\
$2.561 \mathrm{E}+00$ \\
$7.371 \mathrm{E}-01$ \\
$3.452 \mathrm{E}-01$ \\
$2.638 \mathrm{E}-01$ \\
$8.888 \mathrm{E}+00$ \\
$2.825 \mathrm{E}+00$ \\
$2.678 \mathrm{E}-02$ \\
$8.498 \mathrm{E}-02$ \\
$6.070 \mathrm{E}-03$
\end{tabular}




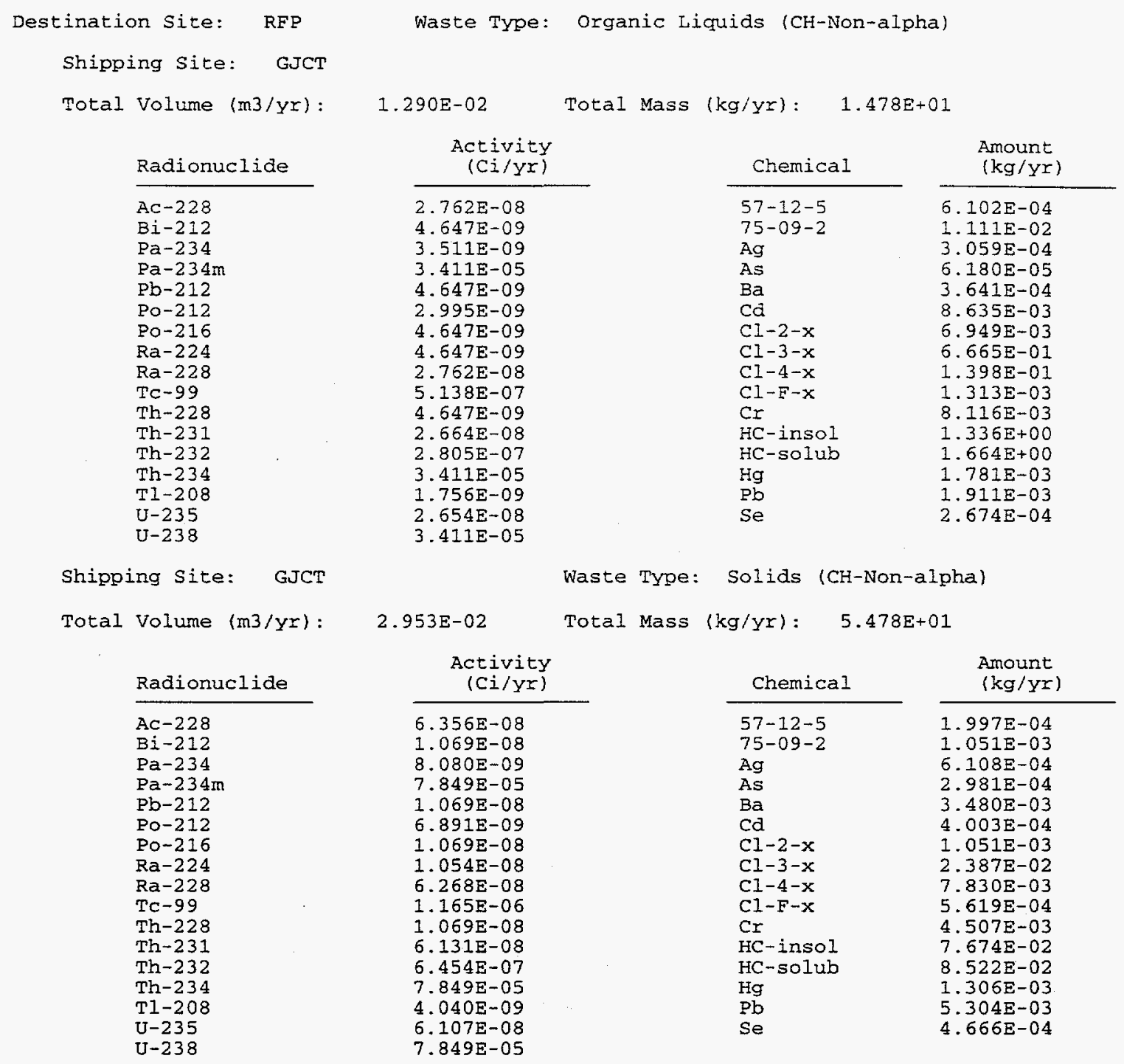




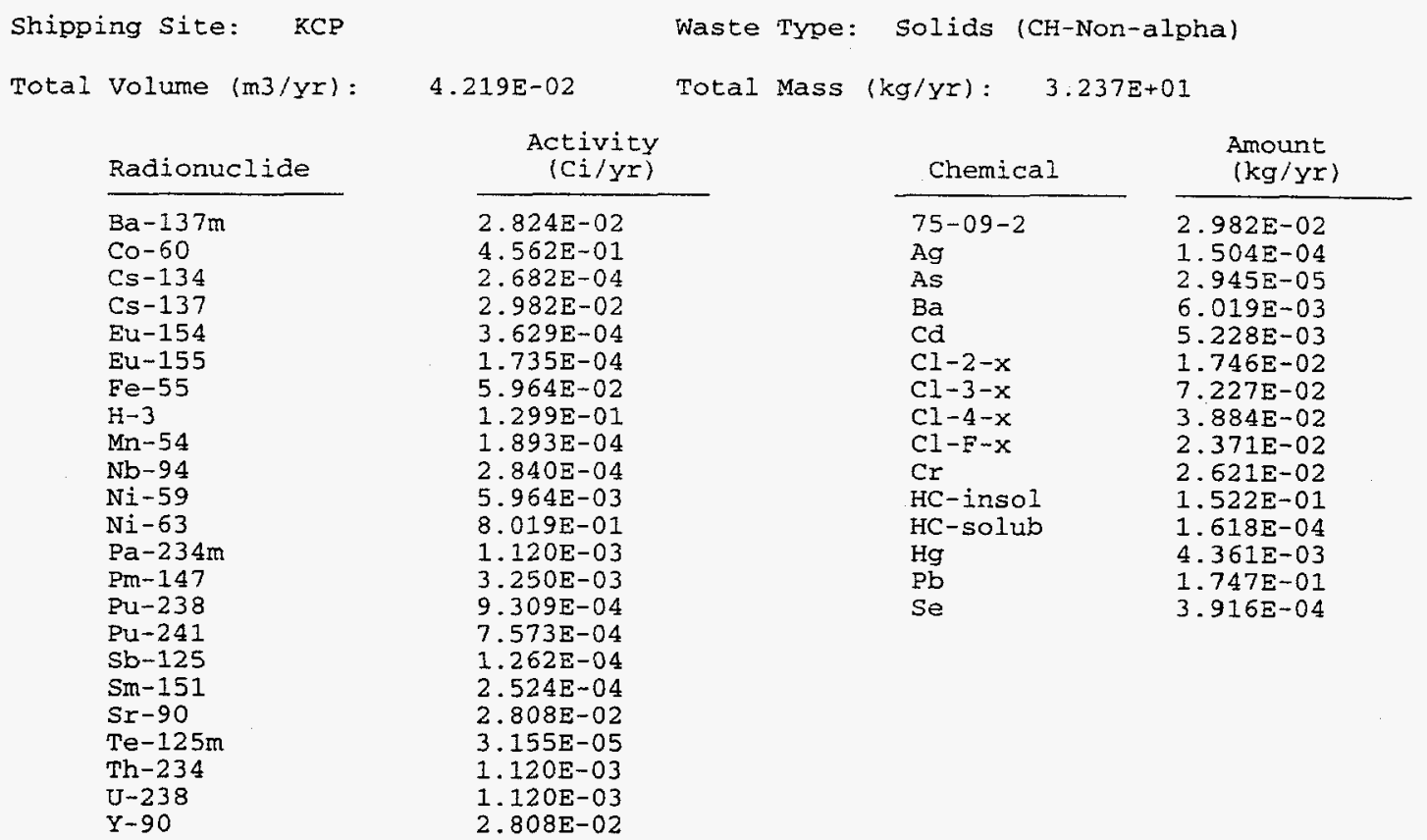


Destination Site: SRS

\begin{tabular}{l} 
Shipping Site: CNS \\
Total Volume (m3/yr): \\
\\
Radionuclice \\
\hline Ba-137m \\
Co-60 \\
Cs-134 \\
Cs-137 \\
Eu-154 \\
Eu-155 \\
Fe-55 \\
H-3 \\
Mn-54 \\
Nb-94 \\
Ni-59 \\
Ni-63 \\
Pa-234m \\
Pm-147 \\
Pu-238 \\
Pu-241 \\
Sb-125 \\
Sm-151 \\
Sr-90 \\
Te-125m \\
Th-234 \\
U-238 \\
Y-90 \\
- 12
\end{tabular}

Shipping Site:

CNS
Waste Type: Organic Liquids ( $\mathrm{CH}$-Non-alpha)

$9.054 E-01$

Total Mass $(\mathrm{kg} / \mathrm{yr})$ :

$1.026 \mathrm{E}+03$

\begin{tabular}{c}
$\begin{array}{c}\text { Activity } \\
\text { (Ci/yr) }\end{array}$ \\
\hline $6.061 \mathrm{E}-01$ \\
$9.790 \mathrm{E}+00$ \\
$5.756 \mathrm{E}-03$ \\
$6.400 \mathrm{E}-01$ \\
$7.788 \mathrm{E}-03$ \\
$3.724 \mathrm{E}-03$ \\
$1.280 \mathrm{E}+00$ \\
$2.788 \mathrm{E}+00$ \\
$4.063 \mathrm{E}-03$ \\
$6.095 \mathrm{E}-03$ \\
$1.280 \mathrm{E}-01$ \\
$1.720 \mathrm{E}+01$ \\
$2.404 \mathrm{E}-02$ \\
$6.975 \mathrm{E}-02$ \\
$1.997 \mathrm{E}-02$ \\
$1.625 \mathrm{E}-02$ \\
$2.709 \mathrm{E}-03$ \\
$5.418 \mathrm{E}-03$ \\
$6.027 \mathrm{E}-01$ \\
$6.772 \mathrm{E}-04$ \\
$2.404 \mathrm{E}-02$ \\
$2.404 \mathrm{E}-02$ \\
$6.027 \mathrm{E}-01$
\end{tabular}

\begin{tabular}{l} 
Chemical \\
\hline $57-12-5$ \\
$75-09-2$ \\
$\mathrm{Ag}$ \\
$\mathrm{As}$ \\
$\mathrm{Ba}$ \\
$\mathrm{Cd}$ \\
$\mathrm{Cl}-2-\mathrm{x}$ \\
$\mathrm{Cl}-3-\mathrm{x}$ \\
$\mathrm{Cl}-4-\mathrm{x}$ \\
$\mathrm{Cl}-\mathrm{F}-\mathrm{x}$ \\
$\mathrm{Cr}$ \\
$\mathrm{HC}-$ insol \\
$\mathrm{HC}-$ Solub \\
$\mathrm{Hg}$ \\
$\mathrm{Pb}$ \\
$\mathrm{Se}$
\end{tabular}

\begin{tabular}{c}
$\begin{array}{c}\text { Amount } \\
(\mathrm{kg} / \mathrm{Yr})\end{array}$ \\
\hline $5.151 \mathrm{E}-02$ \\
$7.241 \mathrm{E}-01$ \\
$2.274 \mathrm{E}-02$ \\
$3.999 \mathrm{E}-03$ \\
$2.356 \mathrm{E}-02$ \\
$5.620 \mathrm{E}-01$ \\
$4.522 \mathrm{E}-01$ \\
$4.355 \mathrm{E}+01$ \\
$9.136 \mathrm{E}+00$ \\
$8.522 \mathrm{E}-02$ \\
$5.418 \mathrm{E}-01$ \\
$8.707 \mathrm{E}+01$ \\
$1.317 \mathrm{E}+02$ \\
$1.340 \mathrm{E}-01$ \\
$1.275 \mathrm{E}-01$ \\
$1.998 \mathrm{E}-02$
\end{tabular}

Waste Type: Solids (CH-Non-alpha)

Total Volume (m3/yr):

$9.030 \mathrm{E}-02$

Total Mass $(\mathrm{kg} / \mathrm{yr})$ :

$6.772 \mathrm{E}+01$

\begin{tabular}{c}
$\begin{array}{c}\text { Activity } \\
\text { (Ci/yr) }\end{array}$ \\
\hline $6.218 \mathrm{E}-02$ \\
$1.004 \mathrm{E}+00$ \\
$5.431 \mathrm{E}-04$ \\
$6.038 \mathrm{E}-02$ \\
$7.990 \mathrm{E}-04$ \\
$3.821 \mathrm{E}-04$ \\
$1.313 \mathrm{E}-01$ \\
$2.860 \mathrm{E}-01$ \\
$4.169 \mathrm{E}-04$ \\
$6.253 \mathrm{E}-04$ \\
$1.313 \mathrm{E}-02$ \\
$1.765 \mathrm{E}+00$ \\
$2.466 \mathrm{E}-03$ \\
$7.156 \mathrm{E}-03$ \\
$2.049 \mathrm{E}-03$ \\
$1.667 \mathrm{E}-03$ \\
$2.779 \mathrm{E}-04$ \\
$5.558 \mathrm{E}-04$ \\
$5.686 \mathrm{E}-02$ \\
$6.948 \mathrm{E}-05$ \\
$2.466 \mathrm{E}-03$ \\
$2.466 \mathrm{E}-03$ \\
$5.686 \mathrm{E}-02$
\end{tabular}

\begin{tabular}{c}
$\begin{array}{c}\text { Amount } \\
(\mathrm{kg} / \mathrm{yr})\end{array}$ \\
\hline $2.357 \mathrm{E}-03$ \\
$2.142 \mathrm{E}-02$ \\
$7.345 \mathrm{E}-04$ \\
$2.549 \mathrm{E}-04$ \\
$3.376 \mathrm{E}-02$ \\
$2.213 \mathrm{E}-03$ \\
$2.738 \mathrm{E}-02$ \\
$2.718 \mathrm{E}-01$ \\
$7.524 \mathrm{E}-02$ \\
$2.847 \mathrm{E}-02$ \\
$2.755 \mathrm{E}-02$ \\
$9.141 \mathrm{E}-01$ \\
$3.299 \mathrm{E}-01$ \\
$3.234 \mathrm{E}-03$ \\
$5.496 \mathrm{E}-02$ \\
$9.647 \mathrm{E}-04$
\end{tabular}


Shipping Site: NNS

Total Volume $(\mathrm{m} 3 / \mathrm{yr})$ :

Radionuclide

$\mathrm{Ba}-137 \mathrm{~m}$

Co-60

Cs -134

Cs -137

Eu-154

Eu-155

$\mathrm{Fe}-55$

$\mathrm{H}-3$

$\mathrm{Mn}-54$

$\mathrm{Nb}-94$

$\mathrm{Ni}-59$

$\mathrm{Ni}-63$

$\mathrm{Pa}-234 \mathrm{~m}$

Pm-147

$\mathrm{Pu}-238$

$\mathrm{Pu}-241$

$\mathrm{Sb}-125$

Sm-151

Sr-90

$\mathrm{Te}-125 \mathrm{~m}$

Th-234

$\mathrm{U}-238$

$\mathrm{Y}-90$
Waste Type: Solids (CH-Non-alpha)

Total Mass (kg/yr): $3.925 \mathrm{E}+02$

. 560E-01

\begin{tabular}{l} 
Chemical \\
\hline $75-09-2$ \\
$\mathrm{Ag}$ \\
$\mathrm{As}$ \\
$\mathrm{Ba}$ \\
$\mathrm{Cd}$ \\
$\mathrm{Cl}-2-\mathrm{x}$ \\
$\mathrm{Cl}-3-\mathrm{x}$ \\
$\mathrm{Cl}-4-\mathrm{x}$ \\
$\mathrm{Cl}-\mathrm{F}-\mathrm{x}$ \\
$\mathrm{Cr}$ \\
$\mathrm{HC}-$ insol \\
$\mathrm{HC}-$ solub \\
$\mathrm{Hg}$ \\
$\mathrm{Pb}$ \\
$\mathrm{Se}$
\end{tabular}

Activity

( $\mathrm{Ci} / \mathrm{Yr}$ )

$2.383 \mathrm{E}-01$

$3.849 \mathrm{E}+00$

$2.263 \mathrm{E}-03$

$2.516 \mathrm{E}-01$

$3.062 \mathrm{E}-03$

$1.464 \mathrm{E}-03$

$5.032 \mathrm{E}-01$

$1.096 \mathrm{E}+00$

$1.597 \mathrm{E}-03$

$2.396 \mathrm{E}-03$

$5.032 \mathrm{E}-02$

$6.766 \mathrm{E}+00$

$9.453 \mathrm{E}-03$

$2.742 \mathrm{E}-02$

$7.855 \mathrm{E}-03$

$6.390 \mathrm{E}-03$

1. $065 \mathrm{E}-03$

$2.130 \mathrm{E}-03$

$2.369 \mathrm{E}-01$

$2.662 \mathrm{E}-04$

$9.453 \mathrm{E}-03$

$9.453 \mathrm{E}-03$

$2.369 \mathrm{E}-01$
Amount

\begin{tabular}{c}
$\begin{array}{c}\text { Amount } \\
(\mathrm{kg} / \mathrm{yr})\end{array}$ \\
\hline $1.041 \mathrm{E}-01$ \\
$3.925 \mathrm{E}-03$ \\
$2.726 \mathrm{E}-03$ \\
$1.110 \mathrm{E}-01$ \\
$3.955 \mathrm{E}-02$ \\
$1.105 \mathrm{E}-01$ \\
$4.102 \mathrm{E}+00$ \\
$7.996 \mathrm{E}-01$ \\
$9.360 \mathrm{E}-02$ \\
$1.488 \mathrm{E}-01$ \\
$1.378 \mathrm{E}+01$ \\
$6.570 \mathrm{E}+00$ \\
$4.448 \mathrm{E}-02$ \\
$7.211 \mathrm{E}-02$ \\
$1.201 \mathrm{E}-02$
\end{tabular}




\section{A.5 WM LLMW REGIONALIZED 4 ALTERNATIVE (CASE 15)}

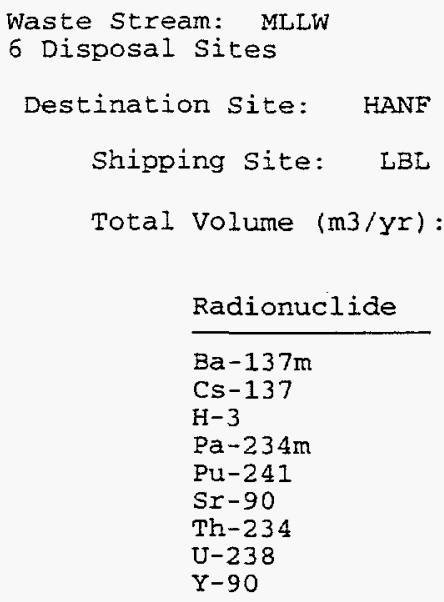

Alternative: 15 Regional 4 Treatment Sites -

Waste Type: Organic Liquids ( $\mathrm{CH}-\mathrm{Non}-\mathrm{alpha})$

3. $230 \mathrm{E}-01$

Total Mass (kg/yr): $3.100 \mathrm{E}+02$

\begin{tabular}{lc} 
Chemical & \multicolumn{1}{c}{$\begin{array}{c}\text { Amount } \\
(\mathrm{kg} / \mathrm{yr})\end{array}$} \\
\cline { 1 - 1 } $75-09-2$ & $2.976 \mathrm{E}-01$ \\
$\mathrm{As}$ & $2.387 \mathrm{E}-03$ \\
$\mathrm{Ba}$ & $1.023 \mathrm{E}-02$ \\
$\mathrm{Cd}$ & $3.410 \mathrm{E}-01$ \\
$\mathrm{Cl}-2-\mathrm{x}$ & $1.550 \mathrm{E}-01$ \\
$\mathrm{Cl}-3-\mathrm{x}$ & $2.325 \mathrm{E}+00$ \\
$\mathrm{Cl}-4-\mathrm{x}$ & $4.961 \mathrm{E}-01$ \\
$\mathrm{Cr}$ & $2.852 \mathrm{E}-01$ \\
$\mathrm{HC}-\mathrm{inso1}$ & $1.364 \mathrm{E}+02$ \\
$\mathrm{HC}-\mathrm{solub}$ & $4.341 \mathrm{E}+01$ \\
$\mathrm{Hg}$ & $1.860 \mathrm{E}-03$ \\
$\mathrm{~Pb}$ & $6.511 \mathrm{E}-02$
\end{tabular}

Waste Type: Solids (CH-Non-alpha)

Total Mass (kg/yr): $4.402 \mathrm{E}+03$

\begin{tabular}{l} 
Chemical \\
\hline $57-12-5$ \\
$75-09-2$ \\
$\mathrm{Ag}$ \\
$\mathrm{As}$ \\
$\mathrm{Ba}$ \\
$\mathrm{Cd}$ \\
$\mathrm{Cl}-2-\mathrm{x}$ \\
$\mathrm{Cl}-3-\mathrm{x}$ \\
$\mathrm{Cl}-4-\mathrm{x}$ \\
$\mathrm{Cl}-\mathrm{F}-\mathrm{x}$ \\
$\mathrm{Cr}$ \\
$\mathrm{HC}-\mathrm{insO} 1$ \\
$\mathrm{HC}-\mathrm{solub}$ \\
$\mathrm{Hg}$ \\
$\mathrm{Pb}$ \\
$\mathrm{Se}$
\end{tabular}

\begin{tabular}{c}
$\begin{array}{c}\text { Amount } \\
(\mathrm{kg} / \mathrm{Yr})\end{array}$ \\
\hline $5.503 \mathrm{E}-01$ \\
$1.116 \mathrm{E}+01$ \\
$1.516 \mathrm{E}-01$ \\
$3.237 \mathrm{E}-02$ \\
$6.474 \mathrm{E}+00$ \\
$1.754 \mathrm{E}-02$ \\
$5.905 \mathrm{E}+00$ \\
$5.439 \mathrm{E}+01$ \\
$4.320 \mathrm{E}+01$ \\
$3.237 \mathrm{E}-01$ \\
$6.485 \mathrm{E}+00$ \\
$1.314 \mathrm{E}+02$ \\
$6.326 \mathrm{E}+01$ \\
$3.242 \mathrm{E}-01$ \\
$1.619 \mathrm{E}+01$ \\
$9.711 \mathrm{E}-02$
\end{tabular}


Shipping site: LERHR

Total Volume (m3/yr):

Radionuclide

\begin{tabular}{l}
\hline Am-241 \\
Ba-137m \\
Co-60 \\
Cs -137 \\
Eu-154 \\
Eu-155 \\
$\mathrm{H}-3$ \\
$\mathrm{Nb}-94$ \\
$\mathrm{Ni}-59$ \\
$\mathrm{Ni}-63$ \\
$\mathrm{~Pa}-234 \mathrm{~m}$ \\
$\mathrm{Pu}-238$ \\
$\mathrm{Pu}-239$ \\
$\mathrm{Pu}-240$ \\
$\mathrm{Pu}-241$ \\
$\mathrm{Sm}-151$ \\
$\mathrm{~S} r-90$ \\
$\mathrm{~T} c-99$ \\
$\mathrm{Th}-232$ \\
$\mathrm{Th}-234$ \\
$\mathrm{U}-238$ \\
$\mathrm{Y}-90$
\end{tabular}

Shipping Site: LERHR

Total Volume (m3/yr):

Radionuclide

\begin{tabular}{l}
\hline $\mathrm{Am}-241$ \\
$\mathrm{Ba}-137 \mathrm{~m}$ \\
$\mathrm{Co}-60$ \\
$\mathrm{Cs}-137$ \\
$\mathrm{Eu}-154$ \\
$\mathrm{Eu}-155$ \\
$\mathrm{H}-3$ \\
$\mathrm{Nb}-94$ \\
$\mathrm{Ni}-59$ \\
$\mathrm{~N}-63$ \\
$\mathrm{~Pa}-234 \mathrm{~m}$ \\
$\mathrm{Pu}-238$ \\
$\mathrm{Pu}-239$ \\
$\mathrm{Pu}-240$ \\
$\mathrm{Pu}-241$ \\
$\mathrm{Sm}-151$ \\
$\mathrm{Sr}-90$ \\
$\mathrm{TC}-99$ \\
$\mathrm{Th}-232$ \\
$\mathrm{Th}-234$ \\
$\mathrm{U}-238$ \\
$\mathrm{Y}-90$
\end{tabular}

shipping site: LINL

Total Volume $(\mathrm{m} 3 / \mathrm{yx})$ :

Radionuclide

$\mathrm{Ba}-137 \mathrm{~m}$

Cs -137

$\mathrm{H}-3$

$\mathrm{Pa}-234 \mathrm{~m}$

Pu-241

Sr -90

$\mathrm{Th}-234$

$\mathrm{U}-238$

$\mathrm{Y}-90$
$5.020 E-03$

Waste Type:

Organic Liquids (CH-Non-alpha)

Total Mass $(\mathrm{kg} / \mathrm{yr}): \quad 5.750 \mathrm{E}+00$

\begin{tabular}{c}
$\begin{array}{c}\text { Activity } \\
\text { (Ci/yr) }\end{array}$ \\
\hline $2.470 E-07$ \\
$3.636 E-04$ \\
$1.353 E-04$ \\
$3.888 E-04$ \\
$1.440 E-06$ \\
$4.940 E-07$ \\
$2.801 E-05$ \\
$1.029 E-06$ \\
$2.183 E-05$ \\
$2.297 E-03$ \\
$4.976 E-05$ \\
$1.529 E-05$ \\
$2.470 E-07$ \\
$3.705 E-07$ \\
$1.620 E-05$ \\
$4.688 E-06$ \\
$3.438 E-04$ \\
$4.112 E-06$ \\
$4.116 E-07$ \\
$4.976 E-05$ \\
$4.976 E-05$ \\
$3.438 E-04$
\end{tabular}

\begin{tabular}{l} 
Chemical \\
\hline $57-12-5$ \\
$75-09-2$ \\
$\mathrm{Ag}$ \\
$\mathrm{As}$ \\
$\mathrm{Ba}$ \\
$\mathrm{Cd}$ \\
$\mathrm{Cl}-2-\mathrm{x}$ \\
$\mathrm{Cl}-3-\mathrm{x}$ \\
$\mathrm{CI}-4-\mathrm{x}$ \\
$\mathrm{Cl}-\mathrm{F}-\mathrm{x}$ \\
$\mathrm{Cr}$ \\
$\mathrm{HC}-\mathrm{insol}$ \\
$\mathrm{HC}-\mathrm{SO} 1 \mathrm{ub}$ \\
$\mathrm{Hg}$ \\
$\mathrm{Pb}$ \\
$\mathrm{Se}$
\end{tabular}

\begin{tabular}{c}
$\begin{array}{c}\text { Amount } \\
(\mathrm{kg} / \mathrm{Y})\end{array}$ \\
\hline $2.373 \mathrm{E}-04$ \\
$4.324 \mathrm{E}-03$ \\
$1.189 \mathrm{E}-04$ \\
$2.403 \mathrm{E}-05$ \\
$1.415 \mathrm{E}-04$ \\
$3.358 \mathrm{E}-03$ \\
$2.702 \mathrm{E}-03$ \\
$2.592 \mathrm{E}-01$ \\
$5.439 \mathrm{E}-02$ \\
$5.108 \mathrm{E}-04$ \\
$3.156 \mathrm{E}-03$ \\
$5.198 \mathrm{E}-01$ \\
$6.473 \mathrm{E}-01$ \\
$6.928 \mathrm{E}-04$ \\
$7.432 \mathrm{E}-04$ \\
$1.039 \mathrm{E}-04$
\end{tabular}

Waste Type: Solids ( $\mathrm{CH}-\mathrm{Non}-\mathrm{alpha})$

3. $442 E-01$

Total Mass $(\mathrm{kg} / \mathrm{yr})$ :

$2.492 E+02$

\begin{tabular}{c}
$\begin{array}{c}\text { Activity } \\
\text { (Ci/yr) }\end{array}$ \\
\hline $1.701 \mathrm{E}-05$ \\
$2.505 \mathrm{E}-02$ \\
$9.323 \mathrm{E}-03$ \\
$2.641 \mathrm{E}-02$ \\
$9.928 \mathrm{E}-05$ \\
$3.403 \mathrm{E}-05$ \\
$1.930 \mathrm{E}-03$ \\
$7.091 \mathrm{E}-05$ \\
$1.504 \mathrm{E}-03$ \\
$1.583 \mathrm{E}-01$ \\
$3.428 \mathrm{E}-03$ \\
$1.054 \mathrm{E}-03$ \\
$1.701 \mathrm{E}-05$ \\
$2.552 \mathrm{E}-05$ \\
$1.116 \mathrm{E}-03$ \\
$3.230 \mathrm{E}-04$ \\
$2.335 \mathrm{E}-02$ \\
$2.793 \mathrm{E}-04$ \\
$2.836 \mathrm{E}-05$ \\
$3.428 \mathrm{E}-03$ \\
$3.428 \mathrm{E}-03$ \\
$2.335 \mathrm{E}-02$
\end{tabular}

\begin{tabular}{l} 
Chemical \\
\hline $57-12-5$ \\
$75-09-2$ \\
Ag \\
As \\
$\mathrm{Ba}$ \\
$\mathrm{Cd}$ \\
$\mathrm{Cl}-2-\mathrm{x}$ \\
$\mathrm{Cl}-3-\mathrm{x}$ \\
$\mathrm{Cl}-4-\mathrm{x}$ \\
$\mathrm{Cl}-\mathrm{F}-\mathrm{x}$ \\
$\mathrm{Cr}$ \\
$\mathrm{HC}-\mathrm{insol}$ \\
$\mathrm{HC}-\mathrm{solub}$ \\
$\mathrm{Hg}$ \\
$\mathrm{Pb}$ \\
$\mathrm{Se}$
\end{tabular}

\begin{tabular}{l}
$\begin{array}{c}\text { Amount } \\
(\mathrm{kg} / \mathrm{yr})\end{array}$ \\
\hline $4.707 \mathrm{E}-04$ \\
$1.438 \mathrm{E}-01$ \\
$2.547 \mathrm{E}-03$ \\
$1.464 \mathrm{E}-05$ \\
$3.843 \mathrm{E}-02$ \\
$8.851 \mathrm{E}-03$ \\
$1.556 \mathrm{E}-01$ \\
$6.005 \mathrm{E}-01$ \\
$2.831 \mathrm{E}+00$ \\
$1.391 \mathrm{E}-01$ \\
$4.652 \mathrm{E}-03$ \\
$1.675 \mathrm{E}+00$ \\
$3.579 \mathrm{E}-01$ \\
$5.762 \mathrm{E}-03$ \\
$1.542 \mathrm{E}-02$ \\
$4.361 \mathrm{E}-04$
\end{tabular}

Waste Type: Organic Liquids (CH-Non-alpha)

$7.727 \mathrm{E}+01$

Total Mass (kg/Yr):

8. $159 E+04$

\begin{tabular}{c}
$\begin{array}{c}\text { Activity } \\
(\mathrm{Ci} / \mathrm{Y})\end{array}$ \\
\hline $1.022 \mathrm{E}+00$ \\
$1.071 \mathrm{E}+00$ \\
$4.863 \mathrm{E}+03$ \\
$2.482 \mathrm{E}-01$ \\
$2.434 \mathrm{E}-01$ \\
$1.022 \mathrm{E}+00$ \\
$2.482 \mathrm{E}-01$ \\
$2.482 \mathrm{E}-01$ \\
$1.022 \mathrm{E}+00$
\end{tabular}

\begin{tabular}{l} 
Chemical \\
\hline $57-12-5$ \\
$75-09-2$ \\
Ag \\
As \\
$\mathrm{Ba}$ \\
$\mathrm{Cd}$ \\
$\mathrm{Cl}-2-\mathrm{x}$ \\
$\mathrm{Cl}-3-\mathrm{x}$ \\
$\mathrm{Cl}-4-\mathrm{x}$ \\
$\mathrm{Cl}-\mathrm{F}-\mathrm{x}$ \\
$\mathrm{Cr}$ \\
$\mathrm{HC}-$ insol \\
$\mathrm{HC}-\mathrm{Solub}$ \\
$\mathrm{Hg}$ \\
$\mathrm{Pb}$ \\
$\mathrm{Se}$
\end{tabular}


Shipping Site: LLNL Total volume $(\mathrm{m} 3 / \mathrm{yr})$ :

Radionuclide

$\mathrm{Ba}-137 \mathrm{~m}$

Cs -137

$\mathrm{H}-3$

$\mathrm{Pa}-234 \mathrm{~m}$

Pu-241

Sr -90

Th-234

$\mathrm{U}-238$

$Y-90$

Shipping Site: MINS

Total Volume (m3/yr):

Radionuclide

$\mathrm{Ba}-137 \mathrm{~m}$

Co-60

Cs -134

Cs-137

Eu-154

Eu-155

$\mathrm{Fe}-55$

$\mathrm{H}-3$

$\mathrm{Mn}-54$

$\mathrm{Nb}-94$

$\mathrm{Ni}-59$

$\mathrm{Ni}-63$

$\mathrm{Pa}-234 \mathrm{~m}$

$\mathrm{Pm}-147$

Pu -238

Pu-24.1

$\mathrm{Sb}-125$

Sm-151

$\mathrm{Sr}-90$

$\mathrm{Te}-125 \mathrm{~m}$

Th-234

$\mathrm{U}-238$

$\mathrm{Y}-90$

Shipping Site: PHNS

Total Volume (m3/yr):

Radionuclide

$\mathrm{Ba}-137 \mathrm{~m}$

Co- 60

$\mathrm{Cs}-134$

Cs -137

Eu-154

Eu-155

$\mathrm{Fe}-55$

$\mathrm{H}-3$

$\mathrm{Mn}-54$

$\mathrm{Nb}-94$

$\mathrm{Ni}-59$

$\mathrm{Ni}-63$

$\mathrm{Pa}-234 \mathrm{~m}$

Pm- 147

Pu-238

Pu-241

Sb-125

$\mathrm{Sm}-151$

$\mathrm{S} r-90$

Te-125m

$\mathrm{Th}-234$

$\mathrm{U}-238$

$\mathrm{Y}-90$
Waste Type: Solids (CH-Non-alpha)

$4.978 \mathrm{E}+01$

Total Mass (kg/yr):

$6.833 \mathrm{E}+04$

\begin{tabular}{l}
$\begin{array}{c}\text { Activity } \\
\text { (Ci/yr) }\end{array}$ \\
\hline $6.586 \mathrm{E}-01$ \\
$6.899 \mathrm{E}-01$ \\
$3.132 \mathrm{E}+03$ \\
$1.599 \mathrm{E}-01$ \\
$1.568 \mathrm{E}-01$ \\
$6.586 \mathrm{E}-01$ \\
$1.599 \mathrm{E}-01$ \\
$1.599 \mathrm{E}-01$ \\
$6.586 \mathrm{E}-01$
\end{tabular}

\begin{tabular}{l} 
Chemical \\
\hline $57-12-5$ \\
$75-09-2$ \\
$\mathrm{Ag}$ \\
$\mathrm{As}$ \\
$\mathrm{Ba}$ \\
$\mathrm{Cd}$ \\
$\mathrm{Cl}-2-\mathrm{x}$ \\
$\mathrm{Cl}-3-\mathrm{x}$ \\
$\mathrm{Cl}-4-\mathrm{x}$ \\
$\mathrm{Cl}-\mathrm{F}-\mathrm{x}$ \\
$\mathrm{Cr}$ \\
$\mathrm{HC}-\mathrm{insol}$ \\
$\mathrm{HC}-$ solub \\
$\mathrm{Hg}$ \\
$\mathrm{Pb}$ \\
$\mathrm{Se}$
\end{tabular}

\begin{tabular}{c}
$\begin{array}{c}\text { Amount } \\
(\mathrm{kg} / \mathrm{Yr})\end{array}$ \\
\hline $8.172 \mathrm{E}+00$ \\
$8.995 \mathrm{E}+00$ \\
$3.852 \mathrm{E}+00$ \\
$1.401 \mathrm{E}+00$ \\
$1.097 \mathrm{E}+02$ \\
$2.705 \mathrm{E}+00$ \\
$7.372 \mathrm{E}+00$ \\
$1.900 \mathrm{E}+02$ \\
$3.100 \mathrm{E}+01$ \\
$5.418 \mathrm{E}+00$ \\
$1.262 \mathrm{E}+02$ \\
$7.525 \mathrm{E}+02$ \\
$3.756 \mathrm{E}+02$ \\
$4.188 \mathrm{E}+01$ \\
$1.529 \mathrm{E}+02$ \\
$1.787 \mathrm{E}+01$
\end{tabular}

Waste Type: Solids (CH-Non-alpha)

Total Mass (kg/yr): $3.545 E+03$

$3.647 \mathrm{E}+00$

\begin{tabular}{l} 
Chemica \\
\hline $75-09-2$ \\
$\mathrm{Ag}$ \\
$\mathrm{As}$ \\
$\mathrm{Ba}$ \\
$\mathrm{Cd}$ \\
$\mathrm{Cl}-2-\mathrm{x}$ \\
$\mathrm{Cl}-3-\mathrm{x}$ \\
$\mathrm{Cl}-4-\mathrm{x}$ \\
$\mathrm{Cl}-\mathrm{F}-\mathrm{x}$ \\
$\mathrm{Cr}$ \\
$\mathrm{HC}-\mathrm{insol}$ \\
$\mathrm{HC}-\mathrm{solub}$ \\
$\mathrm{Hg}$ \\
$\mathrm{Pb}$ \\
$\mathrm{Se}$
\end{tabular}

Amount

\begin{tabular}{c}
$\begin{array}{c}\text { Activity } \\
\text { (Ci/YI\} }\end{array}$ \\
\hline $2.441 \mathrm{E}+00$ \\
$3.943 \mathrm{E}+01$ \\
$2.318 \mathrm{E}-02$ \\
$2.578 \mathrm{E}+00$ \\
$3.137 \mathrm{E}-02$ \\
$1.500 \mathrm{E}-02$ \\
$5.156 \mathrm{E}+00$ \\
$1.123 \mathrm{E}+01$ \\
$1.636 \mathrm{E}-02$ \\
$2.455 \mathrm{E}-02$ \\
$5.156 \mathrm{E}-01$ \\
$6.932 \mathrm{E}+01$ \\
$9.684 \mathrm{E}-02$ \\
$2.809 \mathrm{E}-01$ \\
$8.047 \mathrm{E}-02$ \\
$6.547 \mathrm{E}-02$ \\
$1.091 \mathrm{E}-02$ \\
$2.182 \mathrm{E}-02$ \\
$2.427 \mathrm{E}+00$ \\
$2.728 \mathrm{E}-03$ \\
$9.684 \mathrm{E}-02$ \\
$9.684 \mathrm{E}-02$ \\
$2.427 \mathrm{E}+00$
\end{tabular}

$(\mathrm{kg} / \mathrm{Yr})$

$5.544 \mathrm{E}-01$

1. $457 \mathrm{E}-02$

$7.197 \mathrm{E}-03$

$2.427 \mathrm{E}+00$

1. $150 \mathrm{E}-01$

$5.975 \mathrm{E}-01$

1. $190 \mathrm{E}+01$

$2.652 \mathrm{E}+00$

$6.891 \mathrm{E}-01$

$7.414 \Sigma-01$

4. $047 \mathrm{E}+01$

1. $726 \mathrm{E}+01$

$5.165 \mathrm{E}-01$

$4.557 \mathrm{E}-01$

3. $325 E-02$

Waste Type: Organic Liquids (CH-Non-alpha)

$1.736 \mathrm{E}-03$

Total Mass $(\mathrm{kg} / \mathrm{Yr}): \quad 1.979 \mathrm{E}+00$

\begin{tabular}{c}
$\begin{array}{c}\text { Activity } \\
\text { (Ci/yI) }\end{array}$ \\
\hline $1.162 \mathrm{E}-03$ \\
$1.877 \mathrm{E}-02$ \\
$1.103 \mathrm{E}-05$ \\
$1.227 \mathrm{E}-03$ \\
$1.493 \mathrm{E}-05$ \\
$7.141 \mathrm{E}-06$ \\
$2.454 \mathrm{E}-03$ \\
$5.346 \mathrm{E}-03$ \\
$7.791 \mathrm{E}-06$ \\
$1.168 \mathrm{E}-05$ \\
$2.454 \mathrm{E}-04$ \\
$3.299 \mathrm{E}-02$ \\
$4.609 \mathrm{E}-05$ \\
$1.337 \mathrm{E}-04$ \\
$3.830 \mathrm{E}-05$ \\
$3.116 \mathrm{E}-05$ \\
$5.194 \mathrm{E}-05$ \\
$1.038 \mathrm{E}-05$ \\
$1.155 \mathrm{E}-03$ \\
$1.298 \mathrm{E}-06$ \\
$4.609 \mathrm{E}-05$ \\
$4.609 \mathrm{E}-05$ \\
$1.155 \mathrm{E}-03$ \\
\end{tabular}

\begin{tabular}{|c|c|}
\hline Chemical & $\begin{array}{l}\text { Amount } \\
(\mathrm{kg} / \mathrm{y} r)\end{array}$ \\
\hline $75-09-2$ & $2.572 E-04$ \\
\hline $\mathrm{Ag}$ & $8.707 \mathrm{E}-05$ \\
\hline As & $2.176 \mathrm{E}-05$ \\
\hline $\mathrm{Ba}$ & 1. $484 \mathrm{E}-04$ \\
\hline Cl-2-x & $7.322 \mathrm{E}-04$ \\
\hline Cl-3-x & $2.731 E-02$ \\
\hline Cl-4-x & $1.543 E-03$ \\
\hline $\mathrm{Cl}-\mathrm{F}-\mathrm{x}$ & $5.937 E-04$ \\
\hline HC-insol & $2.968 E-03$ \\
\hline $\mathrm{HC}-$ solub & $7.718 \mathrm{E}-04$ \\
\hline $\mathrm{Hg}$ & $4.353 \mathrm{E}-04$ \\
\hline $\mathrm{Pb}$ & $5.343 E-05$ \\
\hline $\mathrm{Se}$ & $7.322 \mathrm{E}-05$ \\
\hline
\end{tabular}


Shipping Site: PHNS

Total Volume (m3/yr):

Radionuclide

$\mathrm{Ba}-137 \mathrm{~m}$

Co- 60

Cs -134

Cs-137

Eu-154

Eu-155

Fe- 55

$\mathrm{H}-3$

$\mathrm{M} \Omega-54$

$\mathrm{Nb}-94$

$\mathrm{Ni}-59$

$\mathrm{Ni}-63$

$\mathrm{Pa}-234 \mathrm{~m}$

Prn-147

Pu-238

Pu-241

Sb- 325

Sin-151

$\mathrm{Sr}-90$

Te-125m

Th-234

$\mathrm{U}-238$

$Y-90$

Shipping Site: PSNS

Total Volume $(\mathrm{m} 3 / \mathrm{yr})$ :

Radionuclide

$\mathrm{Ba}-137 \mathrm{~m}$

Co- 60

Cs -134

Cs -137

Eu-154

Eu- 155

$F \in-55$

$\mathrm{H}-3$

$\mathrm{Mn}-54$

$\mathrm{Nb}-94$

$\mathrm{Ni}-59$

$\mathrm{Ni}-63$

$\mathrm{Pa}-234 \mathrm{~m}$

Pm-147

Pu-238

Pu-241

$\mathrm{Sb}-125$

Sm-151

Sr -90

Te-125m

Th-234

$\mathrm{U}-238$

$Y-90$

Waste Type: Solids (CH-Non-alpha)

Total Mass (kg/yr): $4.406 \mathrm{E}+02$

$.962 \mathrm{E}-01$

\begin{tabular}{l} 
Chemical \\
\hline $57-12-5$ \\
$75-09-2$ \\
$\mathrm{Ag}$ \\
$\mathrm{As}$ \\
$\mathrm{Ba}$ \\
$\mathrm{Cd}$ \\
$\mathrm{Cl}-2-\mathrm{x}$ \\
$\mathrm{Cl}-3-\mathrm{x}$ \\
$\mathrm{Cl}-4-\mathrm{x}$ \\
$\mathrm{Cl}-\mathrm{F}-\mathrm{x}$ \\
$\mathrm{Cr}$ \\
$\mathrm{HC}-\mathrm{insol}$ \\
$\mathrm{HC}-\mathrm{solub}$ \\
$\mathrm{Hg}$ \\
$\mathrm{Pb}$ \\
$\mathrm{Se}$
\end{tabular}

\begin{tabular}{c}
$\begin{array}{c}\text { Amount } \\
(\mathrm{kg} / \mathrm{Yr})\end{array}$ \\
\hline $4.073 \mathrm{E}-03$ \\
$5.831 \mathrm{E}-02$ \\
$2.691 \mathrm{E}-03$ \\
$1.603 \mathrm{E}-03$ \\
$1.038 \mathrm{E}-01$ \\
$2.472 \mathrm{E}-02$ \\
$6.160 \mathrm{E}-02$ \\
$2.088 \mathrm{E}+00$ \\
$4.422 \mathrm{E}-01$ \\
$6.398 \mathrm{E}-02$ \\
$7.805 \mathrm{E}-01$ \\
$6.908 \mathrm{E}+00$ \\
$3.289 \mathrm{E}+00$ \\
$2.617 \mathrm{E}-02$ \\
$1.563 \mathrm{E}-01$ \\
$6.731 \mathrm{E}-03$
\end{tabular}

$8.865 \mathrm{E}-04$

$1.773 \mathrm{E}-03$

1. $972 \mathrm{E}-01$

2. $216 \mathrm{E}-04$

$7.867 \mathrm{E}-03$

$7.867 \mathrm{E}-03$

1. $972 \mathrm{E}-01$

Waste Type: Organic Liquids (CH-Non-alpha)

$3.990 E-02$

Tota1 Mass (kg/yr):

$5.986 \mathrm{E}+01$

\begin{tabular}{r}
$\begin{array}{r}\text { Activity } \\
\text { (Ci/Yr) }\end{array}$ \\
\hline $2.671 E-02$ \\
$4.315 E-01$ \\
$2.537 E-04$ \\
$2.820 E-02$ \\
$3.432 E-04$ \\
$1.641 E-04$ \\
$5.641 E-02$ \\
$1.228 E-01$ \\
$1.791 E-04$ \\
$2.686 E-04$ \\
$5.641 E-03$ \\
$7.585 E-01$ \\
$1.059 E-03$ \\
$3.074 E-03$ \\
$8.806 E-04$ \\
$7.164 E-04$ \\
$1.194 E-04$ \\
$2.388 E-04$ \\
$2.656 E-02$ \\
$2.985 E-05$ \\
$1.059 E-03$ \\
$1.059 E-03$ \\
$2.656 E-02$ \\
\end{tabular}

Chemical

75-09-2

cd

CI $-2-x$

Cl-3-x

Cl-4-x

$C I-F-x$

Cx

HC-insol

HC-solub

$\mathrm{Hg}$
Amount

( $\mathrm{kg} / \mathrm{yr}$ )

9. $577 \varepsilon-02$

6. 584E-02

5. $088 \mathrm{E}-02$

7. $243 \mathrm{E} \div 00$

1. $616 \mathrm{E}+00$

$2.993 \mathrm{E}-03$

5. 746E-02

1. $975 \mathrm{E}-02$

3. $831 \mathrm{E}-01$

3. $771 \mathrm{E}-04$

1.257E-02 
Shipping Site: PSNS

Total Volume (m3/yr):

Radionuclide

$\mathrm{Ba}-137 \mathrm{~m}$

Co -60

Cs -134

Cs -137

Eu-154

$\mathrm{Eu}-155$

$\mathrm{Fe}-55$

$\mathrm{H}-3$

$\mathrm{Mn}-54$

$\mathrm{Nb}-94$

Ni-59

$\mathrm{Ni}-63$

$\mathrm{Pa}-234 \mathrm{~m}$

$\mathrm{Pm}-147$

$\mathrm{Pu}-238$

Pu-241

$\mathrm{Sb}-125$

$\mathrm{Sm}-151$

Sr -90

Te-125m

Th-234

$\mathrm{U}-238$

$\mathrm{Y}-90$

Shipping site: SNLL

Total Volume $(\mathrm{m} 3 / \mathrm{yr})$ :

Radionuclide

$\mathrm{Ba}-137 \mathrm{~m}$

Cs -137

$\mathrm{H}-3$

$\mathrm{Pa}-234 \mathrm{~m}$

Pu-241

$\mathrm{Sr}-90$

Th-234

$\mathrm{U}-238$

$Y-90$

Shipping Site: SNLL

Total Volume (m3/yr):

Radionuclide

$\mathrm{Ba}-137 \mathrm{~m}$

Cs -137

$\mathrm{H}-3$

$\mathrm{Pa}-234 \mathrm{~m}$

$\mathrm{Pu}-241$

$\mathrm{Sr}-90$

Th-234

$\mathrm{U}-238$

$Y-90$
$6.586 E+00$

Waste Type: Solids (CH-Non-alpha)

Total Mass (kg/yr): $9.518 \mathrm{E}+03$

$\begin{array}{r}\text { Activity } \\ \text { (Ci/yr) } \\ \hline\end{array}$

4. $409 \mathrm{E}+00$

7. $122 \mathrm{E}+01$

4. $655 \mathrm{E}+00$

$5.665 \mathrm{E}-02$

$2.709 \mathrm{E}-02$

9. $311 \mathrm{E}+00$

$2.028 \mathrm{E}+01$

$2.956 \mathrm{E}-02$

4. $434 \mathrm{E}-02$

9. $311 \mathrm{E}-01$

1. $251 \mathrm{E}+02$

1. $749 \mathrm{E}-01$

$5.074 \mathrm{E}-01$

1. $453 \mathrm{E}-01$

1. $182 \mathrm{E}-01$

1. $970 \mathrm{E}-02$

3. $941 \mathrm{E}-02$

$4.384 \mathrm{E}+00$

$4.926 \mathrm{E}-03$

1. $749 \mathrm{E}-01$

1. $749 \mathrm{E}-01$

4. $384 \mathrm{E}+00$
$4.187 \mathrm{E}-02$

$\begin{array}{ll}\text { Chenical } & \begin{array}{c}\text { Amount } \\ (\mathrm{kg} / \mathrm{yr})\end{array} \\ 57-12-5 & \begin{array}{c}7.312 \mathrm{E}-01 \\ 1.279 \mathrm{E}+00\end{array} \\ 75-09-2 & 1.357 \mathrm{E}-01 \\ \mathrm{Ag} & 4.301 \mathrm{E}-02 \\ \mathrm{Ba} & 8.869 \mathrm{E}+00 \\ \mathrm{Cd} & 1.229 \mathrm{E}-01 \\ \mathrm{Cl}-2-\mathrm{x} & 1.346 \mathrm{E}+00 \\ \mathrm{Cl}-3-\mathrm{x} & 4.520 \mathrm{E}+00 \\ \mathrm{Cl}-4-\mathrm{x} & 2.989 \mathrm{E}+00 \\ \mathrm{Cl}-\mathrm{F}-\mathrm{x} & 1.385 \mathrm{E}+00 \\ \mathrm{Cr} & 2.225 \mathrm{E}+01 \\ \mathrm{HC}-\mathrm{insol} & 6.852 \mathrm{E}+00 \\ \mathrm{HC}-\mathrm{solub} & 2.684 \mathrm{E}-01 \\ \mathrm{Hg} & 4.633 \mathrm{E}-01 \\ \mathrm{~Pb} & 2.158 \mathrm{E}+01 \\ \mathrm{Se} & 1.310 \mathrm{E}-01\end{array}$

Waste Type: Organic Liquids ( $\mathrm{CH}$-Non-alpha)

$7.662 \mathrm{E}+00$

Total Mass (kg/yr):

$7.356 \mathrm{E}+03$

\begin{tabular}{r}
$\begin{array}{c}\text { Activity } \\
\text { (Ci/yr) }\end{array}$ \\
\hline $1.013 \mathrm{E}-01$ \\
$1.062 \mathrm{E}-01$ \\
$4.822 \mathrm{E}+02$ \\
$2.462 \mathrm{E}-02$ \\
$2.413 \mathrm{E}-02$ \\
$1.013 \mathrm{E}-01$ \\
$2.462 \mathrm{E}-02$ \\
$2.462 \mathrm{E}-02$ \\
$1.013 \mathrm{E}-01$
\end{tabular}

\begin{tabular}{l} 
Chemical \\
\hline $75-09-2$ \\
As \\
$\mathrm{Ba}$ \\
$\mathrm{Cd}$ \\
$\mathrm{Cl}-2-\mathrm{x}$ \\
$\mathrm{Cl}-3-\mathrm{x}$ \\
$\mathrm{Cl}-4-\mathrm{x}$ \\
$\mathrm{Cr}$ \\
$\mathrm{HC}-$ insol \\
$\mathrm{HC}-$ solub \\
$\mathrm{Hg}$ \\
$\mathrm{Pb}$
\end{tabular}

\begin{tabular}{c}
$\begin{array}{c}\text { Amount } \\
(\mathrm{kg} / \mathrm{yr})\end{array}$ \\
\hline $7.061 \mathrm{E}+00$ \\
$5.664 \mathrm{E}-02$ \\
$2.427 \mathrm{E}-01$ \\
$8.091 \mathrm{E}+00$ \\
$3.678 \mathrm{E}+00$ \\
$5.517 \mathrm{E}+01$ \\
$1.176 \mathrm{E}+01$ \\
$6.767 \mathrm{E}+00$ \\
$3.236 \mathrm{E}+03$ \\
$1.029 \mathrm{E}+03$ \\
$4.413 \mathrm{E}-02$ \\
$1.544 \mathrm{E}+00$
\end{tabular}

Waste Type: Solids (CH-Non-alpha)

Total Mass (kg/yr): $1.193 E+03$

\section{$7.770 \mathrm{E}-01$}

\begin{tabular}{c}
$\begin{array}{c}\text { Activity } \\
\text { (Ci/yr) }\end{array}$ \\
\hline $1.027 \mathrm{E}-02$ \\
$1.076 \mathrm{E}-02$ \\
$4.890 \mathrm{E}+01$ \\
$2.496 \mathrm{E}-03$ \\
$2.447 \mathrm{E}-03$ \\
$1.027 \mathrm{E}-02$ \\
$2.496 \mathrm{E}-03$ \\
$2.496 \mathrm{E}-03$ \\
$1.027 \mathrm{E}-02$
\end{tabular}

\begin{tabular}{l} 
Chemical \\
\hline $57-12-5$ \\
$75-09-2$ \\
Ag \\
As \\
Ba \\
Cd \\
Cl-2-x \\
Cl-3-x \\
Cl-4-x \\
Cl-F-x \\
Cr \\
HC-insol \\
HC-solub \\
Hg \\
Pb \\
Se
\end{tabular}


Destination Site: INEL

\begin{tabular}{l} 
Shipping Site: ETEC \\
Total Volume $(\mathrm{m} 3 / \mathrm{yr})$ : \\
\\
Radionuclide \\
\hline Am-241 \\
Ba-137m \\
Co-60 \\
Cs-137 \\
Eu-154 \\
Eu-155 \\
H-3 \\
Nb-94 \\
Ni-59 \\
Ni-53 \\
Pa-234m \\
Pu-238 \\
Pu-239 \\
Pu-240 \\
Pu-241 \\
Sm-151 \\
Sr-90 \\
Tc-99 \\
Th-232 \\
Th-234 \\
U-238 \\
Y-90
\end{tabular}

Shipping site: ETEC Total volume (m3/yr):

Radionuclide

Am-241

$\mathrm{Ba}-137 \mathrm{~m}$

Co-60

Cs -137

Eu-154

Eu-155

H-3

$\mathrm{Nb}-94$

Ni-59

$\mathrm{Ni}-63$

$\mathrm{Pa}-234 \mathrm{~m}$

Pu-238

Pu-239

Pu-240

Pu-241

$\mathrm{Sm}-151$

S $x-90$

TC-99

Th-232

Th-234

$\mathrm{U}-238$

$Y-90$
Waste Type: Organic Liquids (CH-Non-alpha)

$6.767 E-03$

Total Mass $(\mathrm{kg} / \mathrm{yr})$ :

$7.145 \mathrm{E}+00$

\begin{tabular}{c}
$\begin{array}{c}\text { Activity } \\
(\text { Ci } / y r)\end{array}$ \\
\hline $3.329 \mathrm{E}-07$ \\
$4.901 \mathrm{E}-04$ \\
$1.823 \mathrm{E}-04$ \\
$5.241 \mathrm{E}-04$ \\
$1.942 \mathrm{E}-06$ \\
$6.659 \mathrm{E}-07$ \\
$3.775 \mathrm{E}-05$ \\
$1.387 \mathrm{E}-06$ \\
$2.943 \mathrm{E}-05$ \\
$3.097 \mathrm{E}-03$ \\
$6.707 \mathrm{E}-05$ \\
$2.062 \mathrm{E}-05$ \\
$3.329 \mathrm{E}-07$ \\
$4.994 \mathrm{E}-07$ \\
$2.184 \mathrm{E}-05$ \\
$6.320 \mathrm{E}-06$ \\
$4.634 \mathrm{E}-04$ \\
$5.543 \mathrm{E}-06$ \\
$5.549 \mathrm{E}-07$ \\
$6.707 \mathrm{E}-05$ \\
$6.707 \mathrm{E}-05$ \\
$4.634 \mathrm{E}-04$
\end{tabular}

\begin{tabular}{l} 
Chemical \\
\hline $57-12-5$ \\
$75-09-2$ \\
$\mathrm{Ag}$ \\
$\mathrm{AS}$ \\
$\mathrm{Ba}$ \\
$\mathrm{Cd}$ \\
$\mathrm{Cl}-2-\mathrm{x}$ \\
$\mathrm{Cl}-3-\mathrm{x}$ \\
$\mathrm{Cl}-4-\mathrm{x}$ \\
$\mathrm{Cl}-\mathrm{F}-\mathrm{x}$ \\
$\mathrm{Cr}$ \\
$\mathrm{HC}-$ insol \\
$\mathrm{HC}-$ solub \\
$\mathrm{Hg}$ \\
$\mathrm{Pb}$ \\
$\mathrm{Se}$
\end{tabular}

\begin{tabular}{c}
$\begin{array}{c}\text { Amount } \\
(\mathrm{kg} / \mathrm{y})\end{array}$ \\
\hline $5.272 \mathrm{E}-04$ \\
$4.759 \mathrm{E}-04$ \\
$3.144 \mathrm{E}-04$ \\
$4.027 \mathrm{E}-05$ \\
$2.745 \mathrm{E}-04$ \\
$1.707 \mathrm{E}-05$ \\
$1.354 \mathrm{E}-03$ \\
$5.331 \mathrm{E}-02$ \\
$3.012 \mathrm{E}-03$ \\
$1.098 \mathrm{E}-03$ \\
$7.317 \mathrm{E}-04$ \\
$4.382 \mathrm{E}-02$ \\
$1.255 \mathrm{E}+00$ \\
$1.781 \mathrm{E}-03$ \\
$2.730 \mathrm{E}-04$ \\
$2.748 \mathrm{E}-04$
\end{tabular}

Waste Type: Solids (CH-Non-alpha)

$7.380 E+01$

Total Mass (kg/yr):

$1.326 \mathrm{E}+05$

\begin{tabular}{c}
$\begin{array}{c}\text { Activity } \\
\text { (Ci/yI) }\end{array}$ \\
\hline $3.631 E-03$ \\
$5.346 E+00$ \\
$1.989 E+00$ \\
$5.716 E+00$ \\
$2.118 E-02$ \\
$7.262 E-03$ \\
$4.118 E-01$ \\
$1.513 E-02$ \\
$3.210 E-01$ \\
$3.377 E+01$ \\
$7.316 E-01$ \\
$2.249 E-01$ \\
$3.631 E-03$ \\
$5.447 E-03$ \\
$2.382 E-01$ \\
$5.893 E-02$ \\
$5.054 E+00$ \\
$6.046 E-02$ \\
$6.052 E-03$ \\
$7.316 E-01$ \\
$7.316 E-01$ \\
$5.054 E+00$
\end{tabular}

\begin{tabular}{c}
$\begin{array}{c}\text { Amount } \\
(\mathrm{kg} / \mathrm{y} r)\end{array}$ \\
\hline $4.222 \mathrm{E}-02$ \\
$1.466 \mathrm{E}-01$ \\
$1.331 \mathrm{E}+00$ \\
$6.654 \mathrm{E}-01$ \\
$2.587 \mathrm{E}+00$ \\
$4.415 \mathrm{E}-01$ \\
$1.504 \mathrm{E}-01$ \\
$5.990 \mathrm{E}+00$ \\
$1.869 \mathrm{E}+00$ \\
$9.652 \mathrm{E}-02$ \\
$3.303 \mathrm{E}+00$ \\
$1.648 \mathrm{E}+01$ \\
$8.724 \mathrm{E}+00$ \\
$7.346 \mathrm{E}-01$ \\
$6.598 \mathrm{E}+00$ \\
$2.188 \mathrm{E}-01$
\end{tabular}


A.82

Shipping site: GATOMIC

Waste Type: Organic Liquids (CH-Non-alpha)

Total Volume $(\mathrm{m} 3 / \mathrm{yr})$ :

$7.077 \mathrm{E}-02$

Total Mass $(\mathrm{kg} / \mathrm{yr}): 7.476 \mathrm{E}+01$

Radionuclide

Ac -228

$\mathrm{Bi}-212$

$\mathrm{Pa}-234$

$\mathrm{Pa}-234 \mathrm{~m}$

$\mathrm{Pb}-212$

Po -212

Po- 216

Ra -224

$\mathrm{Ra}-228$

TC -99

Th -228

Th-23I

Th-232

Th-234

T1-208

$\mathrm{U}-235$

$\mathrm{U}-238$

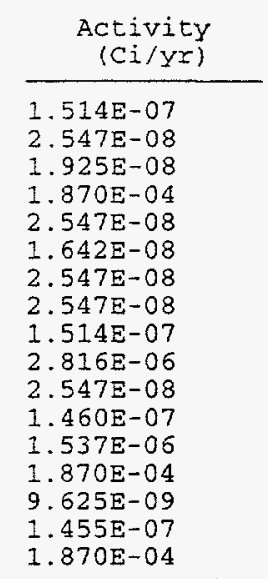

\begin{tabular}{ll} 
Chemical & $\begin{array}{c}\text { Amount } \\
(\mathrm{kg} / \mathrm{Y})\end{array}$ \\
\cline { 1 - 1 } $57-12-5$ & $6.543 \mathrm{E}-03$ \\
$75-09-2$ & $5.261 \mathrm{E}-03$ \\
$\mathrm{Ag}$ & $3.280 \mathrm{E}-03$ \\
$\mathrm{As}$ & $4.207 \mathrm{E}-04$ \\
$\mathrm{Ba}$ & $2.867 \mathrm{E}-03$ \\
$\mathrm{Cd}$ & $4.175 \mathrm{E}-04$ \\
$\mathrm{Cl}-2-\mathrm{x}$ & $1.428 \mathrm{E}-02$ \\
$\mathrm{Cl}-3-\mathrm{x}$ & $5.732 \mathrm{E}-01$ \\
$\mathrm{Cl}-4-\mathrm{x}$ & $3.523 \mathrm{E}-02$ \\
$\mathrm{Cl}-\mathrm{F}-\mathrm{x}$ & $1.146 \mathrm{E}-02$ \\
$\mathrm{Cr}$ & $7.839 \mathrm{E}-03$ \\
$\mathrm{HC}-\mathrm{insol}$ & $4.931 \mathrm{E}-01$ \\
$\mathrm{HC}-\mathrm{solub}$ & $1.311 \mathrm{E}+01$ \\
$\mathrm{Hg}$ & $1.858 \mathrm{E}-02$ \\
$\mathrm{~Pb}$ & $2.894 \mathrm{E}-03$ \\
$\mathrm{Se}$ & $2.867 \mathrm{E}-03$
\end{tabular}

Shipping site: GATOMIC

Waste Type: Solids (CH-Non-alpha)

Total Volume $(\mathrm{m} 3 / \mathrm{yr})$ :

$5.632 \mathrm{E}-01$

Total Mass (kg/yx):

8. $419 E+02$

Radionuclide

Ac -228

$\mathrm{Bi}-212$

$\mathrm{Pa}-234$

$\mathrm{Pa}-234 \mathrm{~m}$

$\mathrm{Pb}-212$

Po-212

Po-216

Ra -224

Ra-228

TC-99

Th-228

Th-23I

Th-232

Th-234

T1-208

$\mathrm{U}-235$

$U-238$

Shipping site: GJCT

Total volume (m3/yr):

Radionuclide

Ac -228

$\mathrm{Bi}-212$

$\mathrm{Pa}-234$

$\mathrm{Pa}-234 \mathrm{~m}$

$\mathrm{Pb}-212$

PO- 212

Po- 216

Ra -224

Ra -228

Tc -99

Th-228

Th-231

Th-232

Th-234

Tl -208

$\mathrm{U}-235$

$U-238$

\begin{tabular}{|c|c|}
\hline Chemical & $\begin{array}{l}\text { Amount } \\
(\mathrm{kg} / \mathrm{Yr})\end{array}$ \\
\hline $\begin{array}{l}57-12-5 \\
75-09-2\end{array}$ & $\begin{array}{l}9.298 \mathrm{E}-02 \\
1.998 \mathrm{E}-01\end{array}$ \\
\hline $\mathrm{Ag}$ & $2.502 \mathrm{E}-02$ \\
\hline As & $9.721 \mathrm{E}-03$ \\
\hline $\mathrm{Ba}$ & 1. $175 E+00$ \\
\hline $\mathrm{Cd}$ & $4.463 E-02$ \\
\hline$C l-2-x$ & $1.741 \mathrm{E}-01$ \\
\hline Cl $-3-x$ & $3.347 \mathrm{E}+00$ \\
\hline CI-4-x & $8.150 E-01$ \\
\hline$C l-F-x$ & $1.017 \mathrm{E}-01$ \\
\hline $\mathrm{Cr}$ & $1.315 \mathrm{E}+00$ \\
\hline HC-insol & $1.041 \mathrm{E}+01$ \\
\hline $\mathrm{HC}$-solub & $6.899 E+00$ \\
\hline $\mathrm{Hg}$ & $1.942 \mathrm{E}-01$ \\
\hline $\mathrm{Pb}$ & $2.810 E+00$ \\
\hline $\mathrm{Se}$ & $7.067 \mathrm{E}-02$ \\
\hline
\end{tabular}

Waste Type: Organic Liquids (CH-Non-alpha)

1.290E-02

Total Mass $(\mathrm{kg} / \mathrm{yr})$ :

$1.478 \mathrm{E}+01$

\begin{tabular}{l}
$\begin{array}{c}\text { Activity } \\
\text { (Ci/yr) }\end{array}$ \\
\hline $2.762 \mathrm{E}-08$ \\
$4.647 \mathrm{E}-09$ \\
$3.511 \mathrm{E}-09$ \\
$3.411 \mathrm{E}-05$ \\
$4.647 \mathrm{E}-09$ \\
$2.995 \mathrm{E}-09$ \\
$4.647 \mathrm{E}-09$ \\
$4.647 \mathrm{E}-09$ \\
$2.762 \mathrm{E}-08$ \\
$5.138 \mathrm{E}-07$ \\
$4.647 \mathrm{E}-09$ \\
$2.664 \mathrm{E}-08$ \\
$2.805 \mathrm{E}-07$ \\
$3.411 \mathrm{E}-05$ \\
$1.756 \mathrm{E}-09$ \\
$2.654 \mathrm{E}-08$ \\
$3.411 \mathrm{E}-05$
\end{tabular}

\begin{tabular}{c}
$\begin{array}{c}\text { Amount } \\
(\mathrm{kg} / \mathrm{y})\end{array}$ \\
\hline $6.102 \mathrm{E}-04$ \\
$1.111 \mathrm{E}-02$ \\
$3.059 \mathrm{E}-04$ \\
$6.180 \mathrm{E}-05$ \\
$3.641 \mathrm{E}-04$ \\
$8.635 \mathrm{E}-03$ \\
$6.949 \mathrm{E}-03$ \\
$6.665 \mathrm{E}-01$ \\
$1.398 \mathrm{E}-01$ \\
$1.313 \mathrm{E}-03$ \\
$8.116 \mathrm{E}-03$ \\
$1.336 \mathrm{E}+00$ \\
$1.664 \mathrm{E}+00$ \\
$1.781 \mathrm{E}-03$ \\
$1.911 \mathrm{E}-03$ \\
$2.674 \mathrm{E}-04$
\end{tabular}




\section{A-83}

Shipping Site: GJCT

Waste Type: Solids (CH-Non-alpha)

Total volume $(\mathrm{m} 3 / \mathrm{yr})$ :

$2.953 \mathrm{E}-02$

Total Mass $(\mathrm{kg} / \mathrm{yr})$ :

5. $478 E+01$

Radionuclide

$\mathrm{AC}-228$

$B i-212$

$\mathrm{Pa}-234$

pa-234m

$\mathrm{Pb}-212$

PO- 212

Po- 216

$\mathrm{Ra}-224$

$\mathrm{Ra}-228$

Tc -99

Th-228

Th-231

$\mathrm{Th}-232$

Th-234

T1-208

II-235

$\mathrm{v}-238$

Shipping site: ITRI

Total Volume $(\mathrm{m} 3 / \mathrm{yr})$ :

Radionuclide

$\mathrm{Ba}-137 \mathrm{~m}$

Co-60

Cs -137

Eu-154

$\mathrm{Fe}-55$

$\mathrm{H}-3$

$\mathrm{Ni}-59$

$\mathrm{Ni}-63$

$\mathrm{Pm}-147$

Pu-238

$\mathrm{Pu}-241$

Sm-151

Sr -90

$Y-90$

Shipping site:

LANL

Total Volume (m3/yr):

Radionuclide

$\mathrm{Ba}-137 \mathrm{~m}$

Co- 60

Cs-137

Eu-154

$\mathrm{Fe}-55$

$\mathrm{H}-3$

$\mathrm{Ni}-59$

$\mathrm{Ni}-63$

Pm-147

Pu-238

Pu-241

$\mathrm{Sm}-151$

Sr -90

$Y-90$

\begin{tabular}{r}
$\begin{array}{r}\text { Activity } \\
(\mathrm{Ci} / \mathrm{y})\end{array}$ \\
\hline $6.356 \mathrm{E}-08$ \\
$1.069 \mathrm{E}-08$ \\
$8.080 \mathrm{E}-09$ \\
$7.849 \mathrm{E}-05$ \\
$1.069 \mathrm{E}-08$ \\
$6.891 \mathrm{E}-09$ \\
$1.069 \mathrm{E}-08$ \\
$1.054 \mathrm{E}-08$ \\
$6.268 \mathrm{E}-08$ \\
$1.165 \mathrm{E}-06$ \\
$1.069 \mathrm{E}-08$ \\
$6.131 \mathrm{E}-08$ \\
$6.454 \mathrm{E}-07$ \\
$7.849 \mathrm{E}-05$ \\
$4.040 \mathrm{E}-09$ \\
$6.107 \mathrm{E}-08$ \\
$7.849 \mathrm{E}-05$
\end{tabular}

Chemical

$57-12-5$

75-09-2

Ag

As

$\mathrm{Ba}$

$\mathrm{ca}$

Cl $-2-x$

C1 $-3-x$

Cl-4-X

Cl $-F-x$

Cr

HC-insol

$\mathrm{HC}$-solub

$\mathrm{Hg}$

$\mathrm{Pb}$
Amount

(kg/yr)

1. $997 \mathrm{E}-04$

$1.051 \mathrm{E}-03$

$6.108 \mathrm{E}-04$

2. $981 E-04$

3. $480 \mathrm{E}-03$

$4.0038-04$

$1.051 \mathrm{E}-03$

2. $387 \mathrm{E}-02$

$7.830 \mathrm{E}-03$

5. $619 \mathrm{E}-04$

$4.507 E-03$

$7.674 \mathrm{E}-02$

8. $522 \mathrm{E}-02$

1. $306 \mathrm{E}-03$

5. 304E-03

$4.666 \mathrm{E}-04$

Waste Type: Organic Liquids (CH-Non-alpha)

1. $028 \mathrm{E}-01$

Total Mass $(\mathrm{kg} / \mathrm{yr})$ :

$1.007 \mathrm{E}+02$

\begin{tabular}{l}
$\begin{array}{c}\text { Activity } \\
\text { (Ci/yr) }\end{array}$ \\
\hline $1.367 \mathrm{E}-03$ \\
$1.879 \mathrm{E}-03$ \\
$1.424 \mathrm{E}-03$ \\
$1.566 \mathrm{E}-05$ \\
$2.364 \mathrm{E}-04$ \\
$2.725 \mathrm{E}-01$ \\
$3.133 \mathrm{E}-05$ \\
$4.158 \mathrm{E}-03$ \\
$1.310 \mathrm{E}-04$ \\
$5.411 \mathrm{E}-05$ \\
$2.734 \mathrm{E}-04$ \\
$1.253 \mathrm{E}-05$ \\
$1.338 \mathrm{E}-03$ \\
$1.338 \mathrm{E}-03$
\end{tabular}

\begin{tabular}{l} 
Chemical \\
\hline $57-12-5$ \\
$\mathrm{Ag}$ \\
$\mathrm{Cd}$ \\
$\mathrm{CI}-3-\mathrm{x}$ \\
$\mathrm{Cl}-4-\mathrm{x}$ \\
$\mathrm{Cr}$ \\
$\mathrm{HC}-$ insol \\
$\mathrm{HC}-501 \mathrm{~b}$ \\
$\mathrm{Hg}$ \\
$\mathrm{Pb}$ \\
$\mathrm{Se}$
\end{tabular}

Amount

$(\mathrm{kg} / \mathrm{yr})$

1. $813 \mathrm{E}-02$

4. $433 \mathrm{E}-03$

4. $937 \mathrm{E}-04$

8. $061 \mathrm{E}-02$

4. 534E-03

2. $116 \mathrm{E}-02$

1. $108 \mathrm{E}+00$

3. $627 \mathrm{E}+01$

2. $821 \mathrm{E}-02$

$5.038 \mathrm{E}-03$

$4.030 \mathrm{E}-03$

Waste Type: Organic Liquids (CH-Non-alpha)

$7.053 E+00$

Total Mass (kg/Yr):

$6.912 E+03$

\begin{tabular}{l}
$\begin{array}{c}\text { Activity } \\
\text { (Ci/yr) }\end{array}$ \\
\hline $9.378 \mathrm{E}-02$ \\
$1.289 \mathrm{E}-01$ \\
$9.769 \mathrm{E}-02$ \\
$1.074 \mathrm{E}-03$ \\
$1.621 \mathrm{E}-02$ \\
$1.869 \mathrm{E}+01$ \\
$2.149 \mathrm{E}-03$ \\
$2.852 \mathrm{E}-01$ \\
$8.987 \mathrm{E}-03$ \\
$3.712 \mathrm{E}-03$ \\
$1.875 \mathrm{E}-02$ \\
$8.597 \mathrm{E}-04$ \\
$9.183 \mathrm{E}-02$ \\
$9.183 \mathrm{E}-02$
\end{tabular}

Amount

( $\mathrm{kg} / \mathrm{yr})$

1. $244 \mathrm{E}+00$

3. $041 \mathrm{E}-01$

3. $387 \mathrm{E}-02$

5. $530 \mathrm{E}+00$

3. $110 \mathrm{E}-01$

1. $451 \mathrm{E}+00$

7. $603 E+01$

2. $488 \mathrm{E}+03$

1. $935 \mathrm{E}+00$

3. $456 \mathrm{E}-01$

2. 765E-01 
Shipping Site: LANL

Waste Type: Solids (CH-Non-alpha)

TotaI Volume $(\mathrm{m} 3 / \mathrm{yr})$ :

1. $292 E+01$

Total Mass $(\mathrm{kg} / \mathrm{yr})$ :

$1.871 E+04$

\begin{tabular}{l} 
Radionuclide \\
\hline Ba-137m \\
Co-60 \\
Cs -137 \\
Eu-154 \\
Fe-55 \\
H-3 \\
$\mathrm{Ni}-59$ \\
$\mathrm{Ni}-63$ \\
$\mathrm{Pm}-147$ \\
$\mathrm{Pu}-238$ \\
$\mathrm{Pu}-241$ \\
$\mathrm{Sm}-151$ \\
$\mathrm{Sr}-90$ \\
$\mathrm{Y}-90$
\end{tabular}

\begin{tabular}{r}
$\begin{array}{c}\text { Activity } \\
\text { (Ci/Yr) }\end{array}$ \\
\hline $1.719 \mathrm{E}-01$ \\
$2.364 \mathrm{E}-01$ \\
$1.787 \mathrm{E}-01$ \\
$1.970 \mathrm{E}-03$ \\
$2.973 \mathrm{E}-02$ \\
$3.428 \mathrm{E}+01$ \\
$3.940 \mathrm{E}-03$ \\
$5.230 \mathrm{E}-01$ \\
$1.647 \mathrm{E}-02$ \\
$6.806 \mathrm{E}-03$ \\
$3.439 \mathrm{E}-02$ \\
$1.576 \mathrm{E}-03$ \\
$1.680 \mathrm{E}-01$ \\
$1.680 \mathrm{E}-01$
\end{tabular}

\begin{tabular}{l} 
Chemical \\
\hline $57-12-5$ \\
$75-09-2$ \\
$\mathrm{Ag}$ \\
$\mathrm{As}$ \\
$\mathrm{Ba}$ \\
$\mathrm{Cd}$ \\
$\mathrm{Cl}-2-\mathrm{x}$ \\
$\mathrm{Cl}-3-\mathrm{x}$ \\
$\mathrm{Cl}-4-\mathrm{x}$ \\
$\mathrm{Cl}-\mathrm{F}-\mathrm{x}$ \\
$\mathrm{Cr}$ \\
$\mathrm{HC}-\mathrm{insol}$ \\
$\mathrm{HC}-\mathrm{solub}$ \\
$\mathrm{Hg}$ \\
$\mathrm{Pb}$ \\
$\mathrm{Se}$
\end{tabular}

\begin{tabular}{c}
$\begin{array}{c}\text { Amount } \\
(\mathrm{kg} / \mathrm{yr})\end{array}$ \\
\hline $1.814 \mathrm{E}-01$ \\
$4.543 \mathrm{E}+00$ \\
$5.519 \mathrm{E}-02$ \\
$2.353 \mathrm{E}-02$ \\
$1.752 \mathrm{E}+01$ \\
$1.078 \mathrm{E}+00$ \\
$1.687 \mathrm{E}+00$ \\
$8.308 \mathrm{E}+00$ \\
$5.036 \mathrm{E}+00$ \\
$1.893 \mathrm{E}+00$ \\
$9.451 \mathrm{E}+00$ \\
$9.627 \mathrm{E}-01$ \\
$3.150 \mathrm{E}+00$ \\
$3.315 \mathrm{E}+00$ \\
$4.093 \mathrm{E}+01$ \\
$1.026 \mathrm{E}-01$
\end{tabular}

Shipping Site: LANL Waste Type: Organic Liquids ( $\mathrm{CH}-\mathrm{Alpha})$

Total volume $(\mathrm{m} 3 / \mathrm{yr})$ :

$6.158 \mathrm{E}+01$

Total Mass (kg/yr):

$6.598 \mathrm{E}+04$

Radionuclide

$\mathrm{Ba}-137 \mathrm{~m}$

Co-60

Cs -137

Eu-154

$\mathrm{Fe}-55$

H-3

$\mathrm{Ni}-59$

$\mathrm{Ni}-63$

Pm-147

Pu-238

$\mathrm{Pu}-239$

Pu-240

$\mathrm{Pu}-241$

$\mathrm{Sm}-151$

$\mathrm{Sr}-90$

$\mathrm{Y}-90$

Shipping Site:

LANL

Total volume $(\mathrm{m} 3 / \mathrm{yr})$ :

Radionuclide

$\mathrm{Ba}-137 \mathrm{~m}$

Co-60

Cs -137

Eu-154

Fe-55

$\mathrm{H}-3$

$\mathrm{Ni}-59$

$\mathrm{N} i-63$

Pm-147

Pu-238

Pu-239

Pu-240

Pu-241

$\mathrm{Sm}-151$

Sr -90

$\mathrm{Y}-90$

\begin{tabular}{c}
$\begin{array}{c}\text { Activity } \\
\text { (Ci/yr) }\end{array}$ \\
\hline $8.711 E-01$ \\
$1.197 \mathrm{E}+00$ \\
$9.073 \mathrm{E}-01$ \\
$9.977 \mathrm{E}-03$ \\
$1.505 \mathrm{E}-01$ \\
$1.736 \mathrm{E}+02$ \\
$1.995 \mathrm{E}-02$ \\
$2.649 \mathrm{E}+00$ \\
$8.351 \mathrm{E}-02$ \\
$1.814 \mathrm{E}-01$ \\
$1.459 \mathrm{E}-02$ \\
$4.896 \mathrm{E}-02$ \\
$3.230 \mathrm{E}+00$ \\
$7.944 \mathrm{E}-03$ \\
$8.530 \mathrm{E}-01$ \\
$8.530 \mathrm{E}-01$
\end{tabular}

\begin{tabular}{l} 
Chemical \\
\hline $57-12-5$ \\
$75-09-2$ \\
Ag \\
As \\
Ba \\
Cd \\
Cl-2-x \\
Cl-3-x \\
Cl-4-x \\
Cl-F-x \\
Cr \\
HC-insol \\
$\mathrm{HC}-$ solub \\
Hg \\
$\mathrm{Pb}$ \\
Se
\end{tabular}

Amount

(kg/yr)

4. $658 \mathrm{E}+00$

5. $213 \mathrm{E}+00$

$2.903 E+00$

4. $411 \mathrm{E}-01$

3. $007 E+00$

1. $268 \mathrm{E}-01$

1. $483 \mathrm{E}+01$

5. $741 \mathrm{E}+02$

3. $244 E+01$

1. $203 E+01$

5. $435 \mathrm{E}+00$

3. $448 \mathrm{E}+02$

$9.332 \mathrm{E}+03$

1. $606 \mathrm{E}+01$

$2.376 \mathrm{E}+00$

2. $519 \mathrm{E}+00$

Waste Type: Solids (CH-Alpha)

$2.801 E+01$

Total Mass (kg/Yr):

$6.796 \mathrm{E}+04$

\begin{tabular}{c}
$\begin{array}{c}\text { Activity } \\
\text { (Ci/yr) }\end{array}$ \\
\hline $3.962 \mathrm{E}-01$ \\
$5.448 \mathrm{E}-01$ \\
$4.126 \mathrm{E}-01$ \\
$4.538 \mathrm{E}-03$ \\
$6.850 \mathrm{E}-02$ \\
$7.900 \mathrm{E}+01$ \\
$9.077 \mathrm{E}-03$ \\
$1.205 \mathrm{E}+00$ \\
$3.799 \mathrm{E}-02$ \\
$8.253 \mathrm{E}-02$ \\
$6.639 \mathrm{E}-03$ \\
$2.227 \mathrm{E}-02$ \\
$1.469 \mathrm{E}+00$ \\
$3.614 \mathrm{E}-03$ \\
$3.879 \mathrm{E}-01$ \\
$3.879 \mathrm{E}-01$
\end{tabular}

\begin{tabular}{l} 
Chemical \\
\hline $57-12-5$ \\
$75-09-2$ \\
Ag \\
As \\
Ba \\
Cd \\
Cl-2-x \\
Cl-3-x \\
Cl-4-x \\
Cl-F-x \\
Cr \\
HC-insol \\
HC-solub \\
Hg \\
Pb \\
Se
\end{tabular}

Amount

( $\mathrm{kg} / \mathrm{yr})$

9. $199 \mathrm{E}-01$

7. $358 \mathrm{E}+01$

5. $600 \mathrm{E}-01$

1. $525 \mathrm{E}-01$

1. $081 \mathrm{E}+01$

1. $740 \mathrm{E}+01$

$2.574 \mathrm{E}+01$

1. $337 \mathrm{E}+02$

8. $345 \mathrm{E}+01$

$2.951 \mathrm{E}+01$

9. $888 \mathrm{E}+01$

$4.851 \mathrm{E}+01$

1. $967 \mathrm{E}+01$

1. $501 \mathrm{E}+01$

$6.059 \mathrm{E}+02$

1. $320 \mathrm{E}+00$ 
Shipping Site: LINL

Total Volume (m3/yr):

\begin{tabular}{l} 
Radionuclide \\
\hline $\mathrm{Ba}-137 \mathrm{~m}$ \\
$\mathrm{Cs}-137$ \\
$\mathrm{H}-3$ \\
$\mathrm{~Pa}-234 \mathrm{~m}$ \\
$\mathrm{Pu}-238$ \\
$\mathrm{Pu}-241$ \\
$\mathrm{~S} r-90$ \\
$\mathrm{Th}-234$ \\
$\mathrm{U}-238$ \\
$\mathrm{Y}-90$
\end{tabular}

Shipping Site: LINL

Total Volume (m3/yr):

Radionuclide

\begin{tabular}{l}
\hline $\mathrm{Ba}-137 \mathrm{~m}$ \\
$\mathrm{Cs}-137$ \\
$\mathrm{H}-3$ \\
$\mathrm{~Pa}-234 \mathrm{~m}$ \\
$\mathrm{Pu}-238$ \\
$\mathrm{Pu}-241$ \\
$\mathrm{Sr}-90$ \\
$\mathrm{Th}-234$ \\
$\mathrm{U}-238$ \\
$\mathrm{Y}-90$
\end{tabular}

Shipping Site: NTS

Total Volume (m3/yr):

Radionuclide

$\mathrm{Ba}-137 \mathrm{~m}$

Cs -137

$\mathrm{H}-3$

$\mathrm{Pa}-234 \mathrm{~m}$

Pu-241

$\mathrm{S} T-90$

Th-234

$\mathrm{U}-238$

$Y-90$
$5.737 E+01$

Waste Type:

Organic Liquids (CH-Alpha)

Total Mass (kg/yr): $6.269 \mathrm{E}+04$

\begin{tabular}{c}
$\begin{array}{c}\text { ACtivity } \\
(C i / y r)\end{array}$ \\
\hline $7.591 E-01$ \\
$7.952 E-01$ \\
$3.607 E+03$ \\
$1.843 E-01$ \\
$1.807 E-01$ \\
$3.614 E+00$ \\
$7.591 E-01$ \\
$1.843 E-01$ \\
$1.843 E-01$ \\
$7.591 E-01$
\end{tabular}

\begin{tabular}{l} 
Chemical \\
\hline $57-12-5$ \\
$75-09-2$ \\
$\mathrm{Ag}$ \\
$\mathrm{As}$ \\
$\mathrm{Ba}$ \\
$\mathrm{Cd}$ \\
$\mathrm{Cl}-2-\mathrm{x}$ \\
$\mathrm{Cl}-3-\mathrm{x}$ \\
$\mathrm{Cl}-4-\mathrm{x}$ \\
$\mathrm{Cl}-\mathrm{F}-\mathrm{x}$ \\
$\mathrm{Cr}$ \\
$\mathrm{HC}-$ insol \\
$\mathrm{HC}-$ Solub \\
$\mathrm{Hg}$ \\
$\mathrm{Pb}$ \\
$\mathrm{Se}$
\end{tabular}

\begin{tabular}{c}
$\begin{array}{c}\text { Amount } \\
(\mathrm{kg} / \mathrm{Yr})\end{array}$ \\
\hline $4.251 \mathrm{E}+00$ \\
$2.262 \mathrm{E}+01$ \\
$2.130 \mathrm{E}+00$ \\
$3.141 \mathrm{E}-01$ \\
$2.037 \mathrm{E}+00$ \\
$1.580 \mathrm{E}+01$ \\
$1.943 \mathrm{E}+01$ \\
$1.480 \mathrm{E}+03$ \\
$2.696 \mathrm{E}+02$ \\
$7.889 \mathrm{E}+00$ \\
$1.843 \mathrm{E}+01$ \\
$2.652 \mathrm{E}+03$ \\
$9.317 \mathrm{E}+03$ \\
$1.216 \mathrm{E}+01$ \\
$4.845 \mathrm{E}+00$ \\
$1.862 \mathrm{E}+00$
\end{tabular}

Waste Type: Solids (CH-Alpha)

Total Mass $(\mathrm{kg} / \mathrm{yr}): \quad 1.017 \mathrm{E}+05$

$5.230 E+01$

\begin{tabular}{l} 
Chemical \\
\hline $57-12-5$ \\
$75-09-2$ \\
Ag \\
AS \\
Ba \\
Cd \\
Cl-2-x \\
Cl-3-x \\
Cl-4-x \\
Cl-F-x \\
Cr \\
HC-insol \\
HC-solub \\
Hg \\
Pb \\
Se
\end{tabular}

Amount

Activity
(Ci/yr)

$6.919 \mathrm{E}-01$

$7.249 E-01$

$3.288 \mathrm{E}+03$

$1.680 E-01$

1. $647 \mathrm{E}-01$

3. $295 \mathrm{E}+00$

$6.919 \mathrm{E}-01$

1. $680 \mathrm{E}-01$

1. $680 \mathrm{E}-01$

$6.919 E-01$

$(\mathrm{kg} / \mathrm{yr})$

4. $138 \mathrm{E}+00$

$1.192 E+01$

$9.746 \mathrm{E}-01$

$3.439 E-01$

$5.008 \mathrm{E}+01$

$3.649 \mathrm{E}+00$

$7.183 E+00$

$4.229 E+01$

2. $492 E+01$

$1.009 \mathrm{E}+01$

$2.709 \mathrm{E}+02$

$7.977 \mathrm{E}+01$

$2.905 E+01$

$8.247 \mathrm{E}+00$

$1.721 \mathrm{E}+02$

$2.561 \mathrm{E}+00$

Waste Type: Solids (CH-Non-alpha)

$6.621 \mathrm{E}-03$

Total Mass (kg/yr):

$8.886 E+00$

\begin{tabular}{c}
$\begin{array}{c}\text { Activity } \\
\text { (Ci/yr) }\end{array}$ \\
\hline $8.760 \mathrm{E}-05$ \\
$9.177 \mathrm{E}-05$ \\
$4.167 \mathrm{E}-01$ \\
$2.127 \mathrm{E}-05$ \\
$2.085 \mathrm{E}-05$ \\
$8.760 \mathrm{E}-05$ \\
$2.127 \mathrm{E}-05$ \\
$2.127 \mathrm{E}-05$ \\
$8.760 \mathrm{E}-05$
\end{tabular}

\begin{tabular}{l} 
Chemical \\
\hline $75-09-2$ \\
Ag \\
Cd \\
CI-2-x \\
CI-3-x \\
Cl-4-x \\
Cl-F-x \\
Cr \\
HC-insol \\
HC-solub \\
Hg \\
Pb \\
Se
\end{tabular}

\begin{tabular}{c}
$\begin{array}{c}\text { Amount } \\
(\mathrm{kg} / \mathrm{Y} r)\end{array}$ \\
\hline $5.331 \mathrm{E}-03$ \\
$9.774 \mathrm{E}-05$ \\
$5.331 \mathrm{E}-04$ \\
$5.331 \mathrm{E}-03$ \\
$2.221 \mathrm{E}-02$ \\
$1.688 \mathrm{E}-01$ \\
$2.665 \mathrm{E}-03$ \\
$1.777 \mathrm{E}-04$ \\
$4.443 \mathrm{E}-02$ \\
$2.221 \mathrm{E}-02$ \\
$3.465 \mathrm{E}-04$ \\
$3.199 \mathrm{E}-04$ \\
$8.886 \mathrm{E}-06$
\end{tabular}


A-86

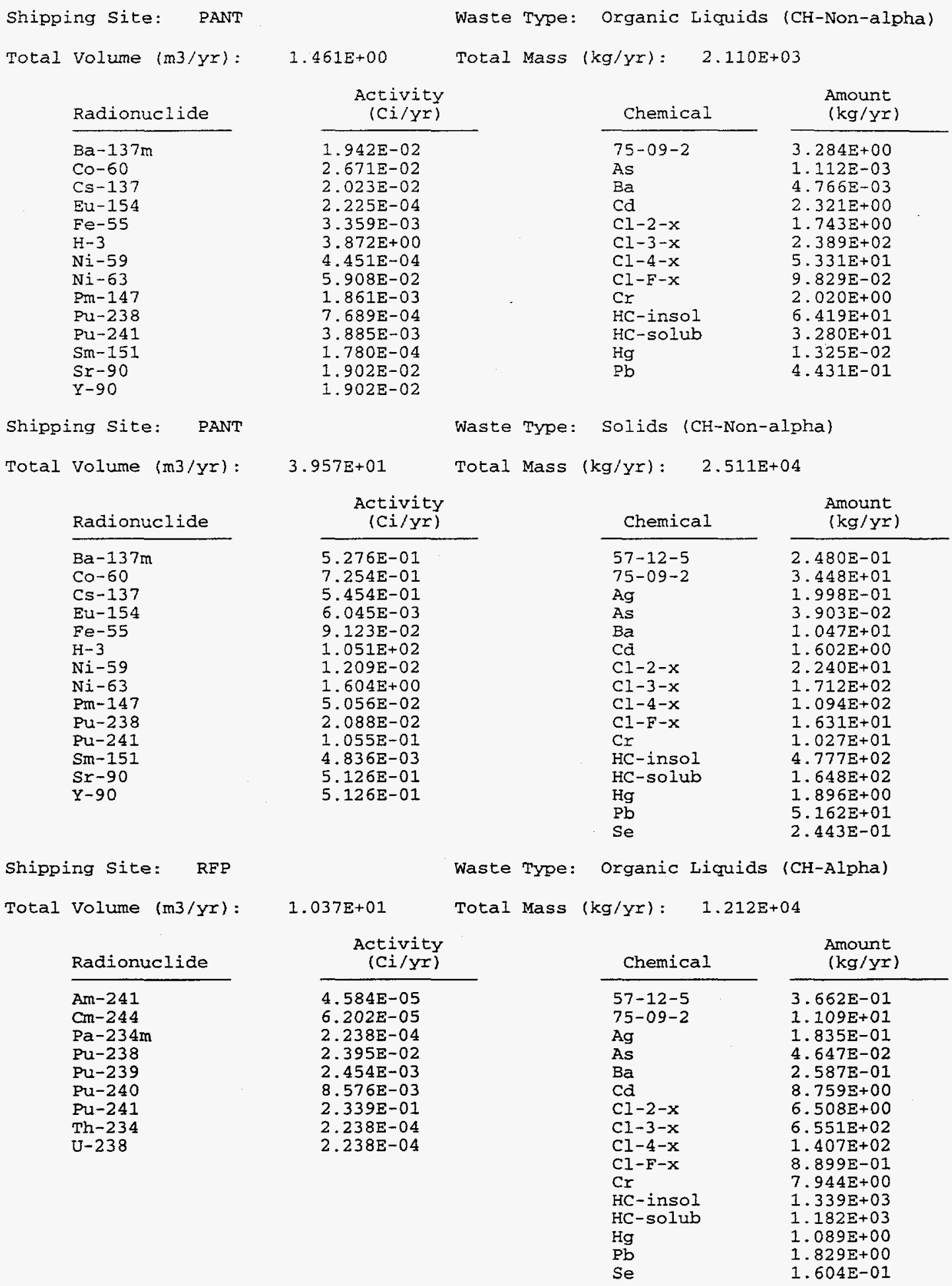




\begin{tabular}{|c|c|c|}
\hline Total & $\begin{array}{l}\text { Volume }(\mathrm{m} 3 / \mathrm{yr}) \text { : } \\
\text { Radionuclide }\end{array}$ & $\begin{array}{l}1.143 E+03 \\
\text { ACtivity } \\
(\mathrm{Ci} / \mathrm{YI})\end{array}$ \\
\hline & $\begin{array}{l}\mathrm{Am}-241 \\
\mathrm{Cm}-244 \\
\mathrm{~Pa}-234 \mathrm{~m} \\
\mathrm{Pu}-238 \\
\mathrm{Pu}-239 \\
\mathrm{Pu}-240 \\
\mathrm{Pu}-241 \\
\mathrm{Th}-234 \\
\mathrm{U}-238\end{array}$ & $\begin{array}{l}5.109 \mathrm{E}-03 \\
6.913 \mathrm{E}-03 \\
2.494 \mathrm{E}-02 \\
2.669 \mathrm{E}+00 \\
2.735 \mathrm{E}-01 \\
9.558 \mathrm{E}-01 \\
2.607 \mathrm{E}+01 \\
2.494 \mathrm{E}-02 \\
2.494 \mathrm{E}-02\end{array}$ \\
\hline
\end{tabular}

Waste Type: Solids (CH-Alpha)

Total Mass $(\mathrm{kg} / \mathrm{yr}): \quad 2.424 \mathrm{E}+06$

Shipping Site: SNLA

Total volume (m3/yr):

Radionuclide

$\mathrm{Ba}-137 \mathrm{~m}$

Co- 60

Cs -137

Eu-154

Eu-155

$\mathrm{Fe}-55$

$\mathrm{H}-3$

$\mathrm{Nb}-94$

$\mathrm{Ni}-59$

$\mathrm{Ni}-63$

$\mathrm{Pa}-234 \mathrm{~m}$

Pu-238

Pu-240

Pu-241

Sm-151

Sr -90

TC -99

Th-234

$\mathrm{U}-238$

$\mathrm{Y}-90$

Shipping Site:

SNLA

Total volume $(\mathrm{m} 3 / \mathrm{yr})$ :

Radionuclide

$\mathrm{Ba}-137 \mathrm{~m}$

Co-60

Cs -137

Eu- 154

Eu-155

Fe-55

$\mathrm{H}-3$

$\mathrm{Nb}-94$

$\mathrm{Ni}-59$

$\mathrm{Ni}-63$

$\mathrm{Pa}-234 \mathrm{~m}$

Pu-238

Pu-240

Pu-241

$\mathrm{Sm}-151$

Sr -90

Tc-99

Th-234

$\mathrm{U}-238$

$\mathrm{Y}-90$ Waste Type: Organic Liquids (CH-Non-alpha) $5.487 \mathrm{E}-04$ Total Mass (kg/yr): 5.793E-0I

\begin{tabular}{r}
$\begin{array}{r}\text { Activity } \\
\text { (Ci/yr) }\end{array}$ \\
\hline $6.627 \mathrm{E}-05$ \\
$9.630 \mathrm{E}-05$ \\
$7.006 \mathrm{E}-05$ \\
$3.516 \mathrm{E}-07$ \\
$8.115 \mathrm{E}-08$ \\
$1.920 \mathrm{E}-06$ \\
$1.223 \mathrm{E}-03$ \\
$4.598 \mathrm{E}-07$ \\
$9.089 \mathrm{E}-06$ \\
$1.099 \mathrm{E}-03$ \\
$3.246 \mathrm{E}-07$ \\
$2.380 \mathrm{E}-06$ \\
$1.893 \mathrm{E}-06$ \\
$1.893 \mathrm{E}-06$ \\
$7.574 \mathrm{E}-07$ \\
$6.519 \mathrm{E}-05$ \\
$2.705 \mathrm{E}-08$ \\
$3.246 \mathrm{E}-07$ \\
$3.246 \mathrm{E}-07$ \\
$6.519 \mathrm{E}-05$
\end{tabular}

Chemical

$57-12-5$

75-09-2

Ag

As

$\mathrm{Ba}$

$\mathrm{Cd}$

Cl $-2-x$

Cl $-3-x$

Cl-4-x

Cl-F-X

Cr

HC-insol

HC-solub

$\mathrm{Hg}$

$\mathrm{Pb}$

$\mathrm{Se}$
Amount

( $\mathrm{kg} / \mathrm{yr})$

1. $417 \mathrm{E}+01$

1. $164 E+02$

3. $781 \mathrm{E}+00$

1. $394 \mathrm{E}+00$

5. $438 E+01$

9. $092 \mathrm{E}+01$

7. $111 \mathrm{E}+02$

7. $915 \mathrm{E}+02$

1. $730 \mathrm{E}+02$

$6.697 \mathrm{E}+03$

1. $167 \mathrm{E}+03$

3. $682 \mathrm{E}+03$

2. $810 E+01$

4. $658 \mathrm{E}+02$

$3.182 \mathrm{E}+00$
1. $522 \mathrm{E}+02$

6. 519E-05

Waste Type: Solids (CH-Non-alpha)

$2.849 E+00$

Total Mass $(\mathrm{kg} / \mathrm{yr})$ :

$2.300 \mathrm{E}+03$

\begin{tabular}{c}
$\begin{array}{c}\text { Activity } \\
\text { (Ci/yr) }\end{array}$ \\
\hline $3.441 E-01$ \\
$5.000 E-01$ \\
$3.637 E-01$ \\
$1.825 E-03$ \\
$4.213 E-04$ \\
$9.972 E-03$ \\
$6.351 E+00$ \\
$2.387 E-03$ \\
$4.719 E-02$ \\
$5.706 E+00$ \\
$1.685 E-03$ \\
$1.236 E-02$ \\
$9.832 E-03$ \\
$9.832 E-03$ \\
$3.932 E-03$ \\
$3.385 E-01$ \\
$1.404 E-04$ \\
$1.685 E-03$ \\
$1.685 E-03$ \\
$3.385 E-01$
\end{tabular}

\begin{tabular}{l} 
Chemical \\
\hline $57-12-5$ \\
$75-09-2$ \\
$\mathrm{Ag}$ \\
$\mathrm{As}$ \\
$\mathrm{Ba}$ \\
$\mathrm{Cd}$ \\
$\mathrm{Cl}-2-\mathrm{x}$ \\
$\mathrm{Cl}-3-\mathrm{x}$ \\
$\mathrm{CI}-4-\mathrm{x}$ \\
$\mathrm{Cl}-\mathrm{F}-\mathrm{x}$ \\
$\mathrm{Cr}$ \\
$\mathrm{HC}-\mathrm{insol}$ \\
$\mathrm{HC}-$ solub \\
$\mathrm{Hg}$ \\
$\mathrm{Pb}$ \\
$\mathrm{Se}$
\end{tabular}

Amount

( $\mathrm{kg} / \mathrm{yr}$ )

2. $628 \mathrm{E}-01$

7. $747 \mathrm{E}-01$

$5.397 \mathrm{E}-02$

1. $546 \mathrm{E}-02$

3. $392 \mathrm{E}+00$

9. $542 \mathrm{E}-03$

8. $422 \mathrm{E}-0 \mathrm{I}$

$2.813 \mathrm{E}+00$

1. $497 \mathrm{E}+00$

9. $064 \mathrm{E}-01$

3. $104 \mathrm{E}+00$

7. $682 \mathrm{E}+00$

1. $619 \mathrm{E}-01$

1. $563 \mathrm{E}-01$

7. $814 \mathrm{E}+00$

$4.865 \mathrm{E}-02$ 
Destination site: ORNL

\begin{tabular}{l} 
Shipping Site: AMES \\
Total Volume (m3/yr): \\
Radionuclide \\
\hline Ac-228 \\
Bi-212 \\
Pa-234 \\
Pa-234m \\
Pb-212 \\
Po-212 \\
Po-216 \\
Ra-224 \\
Ra-228 \\
Tc-99 \\
Th-228 \\
Th-231 \\
Th-232 \\
Th-234 \\
T1-208 \\
U-235 \\
U- 238
\end{tabular}

Shipping Site: ANL-E

Total Volume $(\mathrm{m} 3 / \mathrm{yr})$ :

Radionuclide

\begin{tabular}{l}
\hline $\mathrm{Am}-241$ \\
$\mathrm{Ba}-137 \mathrm{~m}$ \\
$\mathrm{Co}-60$ \\
$\mathrm{Cs}-137$ \\
$\mathrm{Eu}-154$ \\
$\mathrm{Eu}-155$ \\
$\mathrm{H}-3$ \\
$\mathrm{Nb}-94$ \\
$\mathrm{Ni}-59$ \\
$\mathrm{Ni}-63$ \\
$\mathrm{~Pa}-234 \mathrm{~m}$ \\
$\mathrm{Pu}-238$ \\
$\mathrm{Pu}-239$ \\
$\mathrm{Pu}-240$ \\
$\mathrm{Pu}-241$ \\
$\mathrm{Sm}-151$ \\
$\mathrm{Sr}-90$ \\
$\mathrm{TC}-99$ \\
$\mathrm{Th}-232$ \\
$\mathrm{Th}-234$ \\
$\mathrm{U}-238$ \\
$\mathrm{Y}-90$
\end{tabular}

Shipping site: BAPL

Total Volume $(\mathrm{m} 3 / \mathrm{Yr})$ :

\begin{tabular}{l} 
Radionuclide \\
\hline $\mathrm{Am}-241$ \\
$\mathrm{Ba}-137 \mathrm{~m}$ \\
$\mathrm{CO}-60$ \\
$\mathrm{Cs}-137$ \\
$\mathrm{Eu}-154$ \\
$\mathrm{H}-3$ \\
$\mathrm{Nb}-94$ \\
$\mathrm{Ni}-59$ \\
$\mathrm{Ni}-63$ \\
$\mathrm{~Pa}-234 \mathrm{~m}$ \\
$\mathrm{Pu}-238$ \\
$\mathrm{Pu}-239$ \\
$\mathrm{Pu}-240$ \\
$\mathrm{Pu}-241$ \\
$\mathrm{Sm}-151$ \\
$\mathrm{~S}-90$ \\
$\mathrm{TC}-99$ \\
$\mathrm{Th}-234$ \\
$\mathrm{U}-238$ \\
$\mathrm{Y}-90$
\end{tabular}

Waste Type: Solids (CH-Non-alpha)

$8.585 E-03$

Total Mass $(\mathrm{kg} / \mathrm{Yr}): 1.162 \mathrm{E}+01$

\begin{tabular}{|c|c|}
\hline Chemical & $\begin{array}{l}\text { Amount } \\
(\mathrm{kg} / \mathrm{yr})\end{array}$ \\
\hline $57-12-5$ & $1.759 \mathrm{E}-05$ \\
\hline $75-09-2$ & $1.485 E-04$ \\
\hline $\mathrm{Ag}$ & $5.841 E-05$ \\
\hline As & $7.025 \mathrm{E}-07$ \\
\hline $\mathrm{Ba}$ & $4.061 \mathrm{E}-05$ \\
\hline $\mathrm{Cd}$ & $5.957 \mathrm{E}-05$ \\
\hline$C 1-2-x$ & $1.879 \mathrm{E}-04$ \\
\hline$C l-3-x$ & $4.658 E-04$ \\
\hline$C 1-4-x$ & $8.901 \mathrm{E}-04$ \\
\hline$C l-F-x$ & $7.426 \mathrm{E}-05$ \\
\hline $\mathrm{Cr}$ & $1.342 \mathrm{E}-04$ \\
\hline HC-insol & $1.917 \mathrm{E}-05$ \\
\hline HC-solub & $1.017 E-02$ \\
\hline $\mathrm{Hg}$ & $5.635 E-05$ \\
\hline $\mathrm{Pb}$ & $8.863 E-05$ \\
\hline $\mathrm{Se}$ & $1.196 \mathrm{E}-06$ \\
\hline
\end{tabular}

Waste Type: Solids (CH-Non-alpha)

4.838E+02 Total Mass (kg/yr): $8.170 \mathrm{E}+05$

\begin{tabular}{c}
$\begin{array}{r}\text { Activity } \\
\text { (Ci } / \mathrm{Yr})\end{array}$ \\
\hline $2.380 \mathrm{E}-02$ \\
$3.504 \mathrm{E}+01$ \\
$1.304 \mathrm{E}+01$ \\
$3.747 \mathrm{E}+01$ \\
$1.388 \mathrm{E}-01$ \\
$4.761 \mathrm{E}-02$ \\
$2.699 \mathrm{E}+00$ \\
$9.919 \mathrm{E}-02$ \\
$2.104 \mathrm{E}+00$ \\
$2.214 \mathrm{E}+02$ \\
$4.796 \mathrm{E}+00$ \\
$1.474 \mathrm{E}+00$ \\
$2.380 \mathrm{E}-02$ \\
$3.571 \mathrm{E}-02$ \\
$1.561 \mathrm{E}+00$ \\
$4.519 \mathrm{E}-01$ \\
$3.313 \mathrm{E}+01$ \\
$3.963 \mathrm{E}-01$ \\
$3.967 \mathrm{E}-02$ \\
$4.796 \mathrm{E}+00$ \\
$4.796 \mathrm{E}+00$ \\
$3.313 \mathrm{E}+01$
\end{tabular}

Waste Type: Organic Liquids (CH-Non-alpha)

$6.410 E-02$
$6.163 \mathrm{E}+01$

\begin{tabular}{l} 
Chemical \\
\hline $57-12-5$ \\
$75-09-2$ \\
Ag \\
As \\
$\mathrm{Ba}$ \\
$\mathrm{Cd}$ \\
$\mathrm{Cl}-2-\mathrm{x}$ \\
$\mathrm{Cl}-3-\mathrm{x}$ \\
$\mathrm{Cl}-4-\mathrm{x}$ \\
$\mathrm{Cl}-\mathrm{F}-\mathrm{x}$ \\
$\mathrm{Cr}$ \\
$\mathrm{HC}-$ insol \\
$\mathrm{HC}-$ solub \\
$\mathrm{Hg}$ \\
$\mathrm{Pb}$ \\
$\mathrm{Se}$
\end{tabular}

\begin{tabular}{c}
$\begin{array}{c}\text { Amount } \\
(\mathrm{kg} / \mathrm{YY})\end{array}$ \\
\hline $2.002 \mathrm{E}+01$ \\
$2.778 \mathrm{E}+01$ \\
$1.623 \mathrm{E}+01$ \\
$6.714 \mathrm{E}+00$ \\
$2.781 \mathrm{E}+02$ \\
$9.989 \mathrm{E}+00$ \\
$1.822 \mathrm{E}+01$ \\
$3.658 \mathrm{E}+02$ \\
$4.608 \mathrm{E}+01$ \\
$1.759 \mathrm{E}+01$ \\
$3.334 \mathrm{E}+02$ \\
$1.482 \mathrm{E}+03$ \\
$7.360 \mathrm{E}+02$ \\
$1.110 \mathrm{E}+02$ \\
$4.704 \mathrm{E}+02$ \\
$4.660 \mathrm{E}+01$
\end{tabular}

\begin{tabular}{r}
$\begin{array}{r}\text { Activity } \\
(C i / y r)\end{array}$ \\
\hline $5.332 \mathrm{E}-05$ \\
$5.866 \mathrm{E}-02$ \\
$7.908 \mathrm{E}-05$ \\
$6.272 \mathrm{E}-02$ \\
$9.439 \mathrm{E}-05$ \\
$4.694 \mathrm{E}-03$ \\
$5.357 \mathrm{E}-06$ \\
$1.209 \mathrm{E}-04$ \\
$1.242 \mathrm{E}-02$ \\
$3.469 \mathrm{E}-05$ \\
$2.806 \mathrm{E}-03$ \\
$3.801 \mathrm{E}-05$ \\
$2.168 \mathrm{E}-05$ \\
$6.225 \mathrm{E}-04$ \\
$9.031 \mathrm{E}-04$ \\
$5.586 \mathrm{E}-02$ \\
$2.806 \mathrm{E}-05$ \\
$3.469 \mathrm{E}-05$ \\
$3.469 \mathrm{E}-05$ \\
$5.586 \mathrm{E}-02$
\end{tabular}

Amount

(kg/Yr)

$5.934 E-02$

4. $724 E-04$

2. $024 \mathrm{E}-03$

6.779E-02

3. $091 \mathrm{E}-02$

4. $931 \mathrm{E}-01$

$1.055 \mathrm{E}-01$

1. $360 \mathrm{E}-05$

5. $671 \mathrm{E}-02$

2. $699 \mathrm{E}+01$

$8.592 \mathrm{E}+00$

3. $698 \mathrm{E}-04$

1. $294 \mathrm{E}-02$ 
Shipping Site: BAPL

Total Volume $(\mathrm{m} 3 / \mathrm{yr})$ :

Radionuclide

\begin{tabular}{l}
\hline $\mathrm{Am}-241$ \\
$\mathrm{Ba}-137 \mathrm{~m}$ \\
$\mathrm{Co}-60$ \\
$\mathrm{Cs}-137$ \\
$\mathrm{Eu}-154$ \\
$\mathrm{H}-3$ \\
$\mathrm{~N} b-94$ \\
$\mathrm{Ni}-59$ \\
$\mathrm{Ni}-63$ \\
$\mathrm{~Pa}-234 \mathrm{~m}$ \\
$\mathrm{Pu}-238$ \\
$\mathrm{Pu}-239$ \\
$\mathrm{Pu}-240$ \\
$\mathrm{Pu}-241$ \\
$\mathrm{Sm}-151$ \\
$\mathrm{Sr}-90$ \\
$\mathrm{TC}-99$ \\
$\mathrm{Th}-234$ \\
$\mathrm{U}-238$ \\
$\mathrm{Y}-90$
\end{tabular}

Shipping site: BATELLE
$6.105 \mathrm{E}-01$

Waste Type:

Solids

(CH-Non-alpha)

Total Mass (kg/yr):

$6.010 \mathrm{E}+02$

\begin{tabular}{|c|c|}
\hline Chemical & $\begin{array}{l}\text { Amount } \\
\text { (kg/yr) }\end{array}$ \\
\hline $\begin{array}{l}57-12-5 \\
75-09-2\end{array}$ & $\frac{1.703 \mathrm{E}-03}{6}$ \\
\hline $\mathrm{Ag}$ & $6.200 E-02$ \\
\hline As & $1.598 \mathrm{E}-03$ \\
\hline $\mathrm{Ba}$ & $6.259 \mathrm{E}-02$ \\
\hline $\mathrm{cd}$ & $1.698 E-02$ \\
\hline Cl-2-x & $7.067 \mathrm{E}-02$ \\
\hline$C 1-3-x$ & $4.679 E-01$ \\
\hline$C 1-4-x$ & $4.156 \mathrm{E}-01$ \\
\hline$C l-F-x$ & $8.727 \mathrm{E}-02$ \\
\hline $\mathrm{Cr}$ & $4.102 \mathrm{E}-02$ \\
\hline HC-insol & $2.697 E+00$ \\
\hline HC-solub & $2.666 \bar{E}+00$ \\
\hline $\mathrm{Hg}$ & $1.514 E-02$ \\
\hline $\mathrm{Pb}$ & $7.746 \mathrm{E}-02$ \\
\hline Se & $2.393 \mathrm{E}-03$ \\
\hline
\end{tabular}

Waste Type: Solids (CH-Non-alpha)

Total Mass (kg/yr): $1.977 \mathrm{E}+00$

\begin{tabular}{l} 
Radionuclide \\
\hline Ac-228 \\
Bi-212 \\
Pa-234 \\
Pa-234m \\
Po-212 \\
Po-212 \\
Po-216 \\
Ra-224 \\
Ra-228 \\
TC-99 \\
Th-228 \\
Th-231 \\
Th-232 \\
Th-234 \\
Tl-208 \\
U-235 \\
U-238
\end{tabular}

Shipping site: BNL

Total Volume $(\mathrm{m} 3 / \mathrm{yr})$ :

Radionuclide

Am-241

$\mathrm{Ba}-137 \mathrm{~m}$

co-60

Cs -137

Eu-154

Eu-155

$\mathrm{H}-3$

$\mathrm{Nb}-94$

$\mathrm{Ni}-59$

$\mathrm{Ni}-63$

Pa-234m

$\mathrm{Pu}-238$

Pu-239

Pu-240

Pu-241

Sm-151

Sr -90

TC-99

Th-232

Th-234

$\mathrm{U}-238$

Y-90
1. 500E-03

\begin{tabular}{l} 
Chemical \\
\hline $75-09-2$ \\
$C 1-2-x$ \\
$C 1-3-x$ \\
$C 1-4-x$ \\
$H C-$ insol \\
$H C-$ solub
\end{tabular}

\begin{tabular}{c}
$\begin{array}{c}\text { Amount } \\
\text { (kg/yr) }\end{array}$ \\
\hline $3.955 E-02$ \\
$1.977 E-02$ \\
$1.977 E-01$ \\
$1.582 E-01$ \\
$4.943 E-01$ \\
$2.373 E-01$
\end{tabular}

3. $480 \mathrm{E}-10$

5. $400 \mathrm{E}-10$

5. $400 \mathrm{E}-10$

3. $211 \mathrm{E}-09$

$5.971 \mathrm{E}-08$

5. $400 \mathrm{E}-10$

3. $097 \mathrm{E}-09$

3. 260E-08

3. $964 \mathrm{E}-06$

2. 040E-10

3. $085 \mathrm{E}-09$

$3.964 E-06$
Waste Type: Organic Liquids (CH-Non-alpha)

Total Mass (kg/yr): $1.749 \mathrm{E}+03$

\section{$1.544 \mathrm{E}+00$}

\begin{tabular}{l} 
Chemical \\
\hline $57-12-5$ \\
$75-09-2$ \\
$\mathrm{Ag}$ \\
$\mathrm{As}$ \\
$\mathrm{Ba}$ \\
$\mathrm{Cd}$ \\
$\mathrm{Cl}-2-\mathrm{x}$ \\
$\mathrm{Cl}-3-\mathrm{x}$ \\
$\mathrm{Cl}-4-\mathrm{x}$ \\
$\mathrm{Cl}-\mathrm{F}-\mathrm{x}$ \\
$\mathrm{Cr}$ \\
$\mathrm{HC}-$ insol \\
$\mathrm{HC}-\mathrm{SO} 1 \mathrm{ub}$ \\
$\mathrm{Hg}$ \\
$\mathrm{Pb}$ \\
$\mathrm{Se}$
\end{tabular}

\begin{tabular}{c}
$\begin{array}{c}\text { Amount } \\
(\mathrm{kg} / \mathrm{Yr})\end{array}$ \\
\hline $8.298 \mathrm{E}-02$ \\
$1.156 \mathrm{E}+00$ \\
$4.159 \mathrm{E}-02$ \\
$7.649 \mathrm{E}-03$ \\
$4.627 \mathrm{E}-02$ \\
$8.868 \mathrm{E}-01$ \\
$7.572 \mathrm{E}-01$ \\
$7.014 \mathrm{E}+01$ \\
$1.445 \mathrm{E}+01$ \\
$1.704 \mathrm{E}-01$ \\
$8.567 \mathrm{E}-01$ \\
$1.386 \mathrm{E}+02$ \\
$2.115 \mathrm{E}+02$ \\
$2.406 \mathrm{E}-01$ \\
$2.050 \mathrm{E}-01$ \\
$3.636 \mathrm{E}-02$
\end{tabular}




\begin{tabular}{l} 
Shipping Site: BNL \\
Total Volume (m3/Yr): \\
\\
Radionuclide \\
\hline Am-241 \\
Ba-137m \\
Co-60 \\
Cs-137 \\
Eu-154 \\
Eu-155 \\
H-3 \\
Nb-94 \\
Ni-59 \\
Ni-63 \\
$\mathrm{Pa}-234 \mathrm{~m}$ \\
$\mathrm{Pu}-238$ \\
$\mathrm{Pu}-239$ \\
$\mathrm{Pu}-240$ \\
$\mathrm{Pu}-241$ \\
$\mathrm{Sm}-151$ \\
$\mathrm{Sr}-90$ \\
$\mathrm{Tc}-99$ \\
$\mathrm{Th}-232$ \\
$\mathrm{Th}-234$ \\
$\mathrm{U}-233$ \\
$\mathrm{Y}-90$
\end{tabular}

Shipping Site: COLONIE
Waste Type: Solids (CH-Non-alpha)

$8.915 E+00$

Total Mass $(\mathrm{kg} / \mathrm{yr}): 1.190 \mathrm{E}+04$

\begin{tabular}{l} 
Chemical \\
\hline $57-12-5$ \\
$75-09-2$ \\
$\mathrm{Ag}$ \\
$\mathrm{As}$ \\
$\mathrm{Ba}$ \\
$\mathrm{Cd}$ \\
$\mathrm{Cl}-2-\mathrm{x}$ \\
$\mathrm{Cl}-3-\mathrm{x}$ \\
$\mathrm{Cl}-4-\mathrm{x}$ \\
$\mathrm{Cl}-\mathrm{F}-\mathrm{x}$ \\
$\mathrm{Cr}$ \\
$\mathrm{HC}-$ insol \\
$\mathrm{HC}-\mathrm{SOI} \mathrm{ub}$ \\
$\mathrm{Hg}$ \\
$\mathrm{Pb}$ \\
$\mathrm{Se}$
\end{tabular}

\begin{tabular}{c}
$\begin{array}{c}\text { Amount } \\
(\mathrm{kg} / \mathrm{Yr})\end{array}$ \\
\hline $1.617 \mathrm{E}+00$ \\
$8.722 \mathrm{E}-01$ \\
$7.571 \mathrm{E}-01$ \\
$2.698 \mathrm{E}-01$ \\
$2.157 \mathrm{E}+01$ \\
$4.530 \mathrm{E}-01$ \\
$8.722 \mathrm{E}-01$ \\
$2.419 \mathrm{E}+01$ \\
$3.963 \mathrm{E}-01$ \\
$1.019 \mathrm{E}+00$ \\
$2.698 \mathrm{E}+01$ \\
$1.091 \mathrm{E}+02$ \\
$5.459 \mathrm{E}+01$ \\
$8.531 \mathrm{E}+00$ \\
$2.912 \mathrm{E}+01$ \\
$3.668 \mathrm{E}+00$
\end{tabular}

$8.327 \mathrm{E}-03$
$6.105 \mathrm{E}-01$

$7.303 \mathrm{E}-03$

$7.310 \mathrm{E}-04$

$8.837 \mathrm{E}-02$

$8.837 \mathrm{E}-02$

$6.105 \mathrm{E}-01$

Total Volume (m3/yr):

Radionuclide

$\mathrm{AC}-228$
$\mathrm{Bi}-212$
$\mathrm{~Pa}-234$
$\mathrm{~Pa}-234 \mathrm{~m}$
$\mathrm{~Pb}-212$
$\mathrm{Po}-212$
$\mathrm{Po}-216$
$\mathrm{Ra}-224$
$\mathrm{Ra}-228$
$\mathrm{TC}-99$
$\mathrm{Th}-228$
$\mathrm{Th}-231$
$\mathrm{Th}-232$
$\mathrm{Th}-234$
$\mathrm{~T} 1-208$
$\mathrm{U}-235$
$\mathrm{U}-238$

Shipping site:
$9.054 \mathrm{E}-01$
Organic Iiquids ( $\mathrm{CH}-\mathrm{Non}-\mathrm{alpha})$

Total Mass (kg/yr): $1.026 \mathrm{E}+03$

\begin{tabular}{c}
$\begin{array}{r}\text { Activity } \\
\text { (Ci/yr) }\end{array}$ \\
\hline $1.937 E-06$ \\
$3.259 E-07$ \\
$2.462 \mathrm{E}-07$ \\
$2.392 \mathrm{E}-03$ \\
$3.259 \mathrm{E}-07$ \\
$2.100 \mathrm{E}-07$ \\
$3.259 \mathrm{E}-07$ \\
$3.259 \mathrm{E}-07$ \\
$1.937 \mathrm{E}-06$ \\
$3.603 \mathrm{E}-05$ \\
$3.259 \mathrm{E}-07$ \\
$1.868 \mathrm{E}-06$ \\
$1.967 \mathrm{E}-05$ \\
$2.392 \mathrm{E}-03$ \\
$1.231 \mathrm{E}-07$ \\
$1.861 \mathrm{E}-06$ \\
$2.392 \mathrm{E}-03$
\end{tabular}

\begin{tabular}{l} 
Chemical \\
\hline $57-12-5$ \\
$75-09-2$ \\
Ag \\
As \\
Ba \\
Cd \\
Cl-2-x \\
Cl-3-x \\
Cl-4-x \\
Cl-F-x \\
Cr \\
HC-insol \\
HC-solub \\
Hg \\
Pb \\
Se
\end{tabular}

\begin{tabular}{c}
$\begin{array}{c}\text { Amount } \\
(\mathrm{kg} / \mathrm{yr})\end{array}$ \\
\hline $5.151 \mathrm{E}-02$ \\
$7.241 \mathrm{E}-01$ \\
$2.274 \mathrm{E}-02$ \\
$3.999 \mathrm{E}-03$ \\
$2.356 \mathrm{E}-02$ \\
$5.620 \mathrm{E}-01$ \\
$4.522 \mathrm{E}-01$ \\
$4.355 \mathrm{E}+01$ \\
$9.136 \mathrm{E}+00$ \\
$8.522 \mathrm{E}-02$ \\
$5.418 \mathrm{E}-01$ \\
$8.707 \mathrm{E}+01$ \\
$1.317 \mathrm{E}+02$ \\
$1.340 \mathrm{E}-01$ \\
$1.275 \mathrm{E}-01$ \\
$1.998 \mathrm{E}-02$
\end{tabular}

Waste Type: Solids (CH-Non-alpha)

TotaI Mass $(\mathrm{kg} / \mathrm{Yr}): 1.606 \mathrm{E}+01$

1. 235E-02

\begin{tabular}{l} 
Chemical \\
\hline $57-12-5$ \\
$75-09-2$ \\
Ag \\
As \\
Ba \\
Cd \\
Cl-2-x \\
Cl-3-x \\
Cl-4-x \\
Cl-F-x \\
Cr \\
HC-insol \\
HC-solub \\
$\mathrm{Hg}$ \\
$\mathrm{Pb}$ \\
$\mathrm{Se}$
\end{tabular}

Amount

$(\mathrm{kg} / \mathrm{Yr})$

7. $229 \mathrm{E}-04$

8. $484 \mathrm{E}-04$

2. 570E-05

2. $249 \mathrm{E}-05$

1. $606 \mathrm{E}-05$

1. $429 \mathrm{E}-04$

4. $242 \mathrm{E}-03$

$5.345 \mathrm{E}-03$

5. $006 \mathrm{E}-03$

4. $242 E-04$

$5.462 \mathrm{E}-04$

$9.333 E-04$

8. $032 \mathrm{E}-04$

3. $695 \mathrm{E}-06$

1. $044 \mathrm{E}-03$

$8.032 E-06$ 
Shipping site: FEMP

Waste Type: Organic Liquids (CH-Non-alpha)

Total volume $(\mathrm{m} 3 / \mathrm{yr})$ :

$2.034 E+01$

Total Mass (kg/yr):

$2.423 E+04$

Radionuclide

Ac -228

$\mathrm{Bi}-212$

Pa-234

Pa- $234 \mathrm{~m}$

$\mathrm{Pb}-212$

Po- 212

PO- 216

$\mathrm{Ra}-224$

$\mathrm{Ra}-228$

TC -99

$\mathrm{Th}-228$

Th-231

$\mathrm{Th}-232$

Th -234

T1-208

$\mathrm{U}-235$

$\mathrm{U}-238$

Shipping site: FEMP

Total Volume (m3/yr):

Radionuclide

AC -228

$\mathrm{Bi}-212$

$\mathrm{Pa}-234$

$\mathrm{Pa}-234 \mathrm{~m}$

$\mathrm{Pb}-212$

PO- 212

$\mathrm{Po}-216$

$\mathrm{Ra}-224$

$R a-228$

TC-99

Th-228

$\mathrm{Th}-231$

$\mathrm{Th}-232$

$\mathrm{Th}_{1}-234$

Tl-208

$\mathrm{U}-235$

$\mathrm{U}-238$

Shipping Site: KAPL

Total Volume $(\mathrm{m} 3 / y x)$ :

Radionuclide

$\mathrm{Ba}-137 \mathrm{~m}$

$\mathrm{Co}-6 \mathrm{O}$

$\mathrm{C} s-134$

Cs -137

Eu-154

EU-155

Fe- 55

$\mathrm{H}-3$

$\mathrm{Mr}-54$

$\mathrm{Nb}-94$

$\mathrm{Ni}-59$

$\mathrm{Ni}-63$

$\mathrm{Pa}-234 \mathrm{~m}$

Pm-147

Pu-238

Pu-241

Sb-125

Sm-151

Sr -90

Te-125m

Th-234

$\mathrm{U}-238$

$\mathrm{Y}-90$

\begin{tabular}{c}
$\begin{array}{c}\text { Activity } \\
(\mathrm{Ci} / \mathrm{yr})\end{array}$ \\
\hline $4.353 \mathrm{E}-05$ \\
$7.324 \mathrm{E}-06$ \\
$5.533 \mathrm{E}-06$ \\
$5.376 \mathrm{E}-02$ \\
$7.324 \mathrm{E}-06$ \\
$4.720 \mathrm{E}-06$ \\
$7.324 \mathrm{E}-06$ \\
$7.324 \mathrm{E}-06$ \\
$4.353 \mathrm{E}-05$ \\
$8.097 \mathrm{E}-04$ \\
$7.324 \mathrm{E}-06$ \\
$4.199 \mathrm{E}-05$ \\
$4.420 \mathrm{E}-04$ \\
$5.376 \mathrm{E}-02$ \\
$2.766 \mathrm{E}-06$ \\
$4.182 \mathrm{E}-05$ \\
$5.376 \mathrm{E}-02$
\end{tabular}

Chemical

57-12-5

75-09-2

Ag

As

$\mathrm{Ba}$

Cd

Cl $-2-x$

C1-3-x

CI-4-x

CI-F-X

Cr

HC-insol

$\mathrm{HC}-$ solub

$\mathrm{Hg}$

$\mathrm{Pb}$

$\mathrm{Se}$
Amount

( $\mathrm{kg} / \mathrm{yr})$

4. $910 \mathrm{E}-01$

2. $872 E+01$

1. $270 \mathrm{E}-01$

$6.728 \mathrm{E}-02$

2. $928 \mathrm{E}-01$

2. $349 E+01$

1. $522 E+01$

1. $621 \mathrm{E}+03$

3. $603 \mathrm{E}+02$

$6.895 \mathrm{E}-01$

$2.072 \mathrm{E}+01$

3. $779 \mathrm{E}+03$

2. $255 \mathrm{E}+03$

9. $307 \mathrm{E}-01$

$4.623 \mathrm{E}+00$

1. $150 \mathrm{E}-01$
Waste Type: Solids (CH-Non-alpha)

$7.909 E+01$

Total Mass (kg/yr):

$1.180 \mathrm{E}+05$

\begin{tabular}{c}
$\begin{array}{c}\text { Activity } \\
\text { (Ci/yr) }\end{array}$ \\
\hline $1.696 \mathrm{E}-04$ \\
$2.854 \mathrm{E}-05$ \\
$2.156 \mathrm{E}-05$ \\
$2.095 \mathrm{E}-01$ \\
$2.854 \mathrm{E}-05$ \\
$1.839 \mathrm{E}-05$ \\
$2.854 \mathrm{E}-05$ \\
$2.837 \mathrm{E}-05$ \\
$1.686 \mathrm{E}-04$ \\
$3.136 \mathrm{E}-03$ \\
$2.854 \mathrm{E}-05$ \\
$1.636 \mathrm{E}-04$ \\
$1.722 \mathrm{E}-03$ \\
$2.095 \mathrm{E}-01$ \\
$1.078 \mathrm{E}-05$ \\
$1.630 \mathrm{E}-04$ \\
$2.095 \mathrm{E}-01$
\end{tabular}

\begin{tabular}{l} 
Chemical \\
\hline $57-12-5$ \\
$75-09-2$ \\
Ag \\
As \\
$\mathrm{Ba}$ \\
$\mathrm{Cd}$ \\
$\mathrm{Cl}-2-\mathrm{x}$ \\
$\mathrm{Cl}-3-\mathrm{x}$ \\
$\mathrm{Cl}-4-\mathrm{x}$ \\
$\mathrm{Cl}-\mathrm{F}-\mathrm{x}$ \\
$\mathrm{Cr}$ \\
$\mathrm{HC}-\mathrm{ins} \mathrm{Ol}$ \\
$\mathrm{HC}-\mathrm{solub}$ \\
$\mathrm{Hg}$ \\
$\mathrm{Pb}$ \\
$\mathrm{Se}$
\end{tabular}

\begin{tabular}{c}
$\begin{array}{c}\text { Anount } \\
(\mathrm{kg} / \mathrm{Yr})\end{array}$ \\
\hline $5.948 \mathrm{E}+00$ \\
$2.117 \mathrm{E}+01$ \\
$3.226 \mathrm{E}+00$ \\
$1.249 \mathrm{E}+00$ \\
$1.085 \mathrm{E}+02$ \\
$6.815 \mathrm{E}+00$ \\
$2.073 \mathrm{E}+01$ \\
$5.283 \mathrm{E}+02$ \\
$3.692 \mathrm{E}+02$ \\
$1.313 \mathrm{E}+01$ \\
$1.531 \mathrm{E}+02$ \\
$1.786 \mathrm{E}+03$ \\
$9.390 \mathrm{E}+02$ \\
$3.514 \mathrm{E}+01$ \\
$1.240 \mathrm{E}+02$ \\
$1.366 \mathrm{E}+01$
\end{tabular}

Waste Type: Organic Liquids (CH-Non-aIpha)

$6.167 E-01$

Total Mass $(\mathrm{kg} / \mathrm{Yr}): \quad 7.065 E+02$

\begin{tabular}{l}
$\begin{array}{c}\text { Activity } \\
\text { (Ci/yr) }\end{array}$ \\
\hline $4.129 \mathrm{E}-01$ \\
$6.669 \mathrm{E}+00$ \\
$3.921 \mathrm{E}-03$ \\
$4.359 \mathrm{E}-01$ \\
$5.305 \mathrm{E}-03$ \\
$2.537 \mathrm{E}-03$ \\
$8.719 \mathrm{E}-01$ \\
$1.899 \mathrm{E}+00$ \\
$2.768 \mathrm{E}-03$ \\
$4.152 \mathrm{E}-03$ \\
$8.719 \mathrm{E}-02$ \\
$1.172 \mathrm{E}+01$ \\
$1.637 \mathrm{E}-02$ \\
$4.752 \mathrm{E}-02$ \\
$1.361 \mathrm{E}-02$ \\
$1.107 \mathrm{E}-02$ \\
$1.845 \mathrm{E}-03$ \\
$3.690 \mathrm{E}-03$ \\
$4.106 \mathrm{E}-01$ \\
$4.613 \mathrm{E}-04$ \\
$1.637 \mathrm{E}-02$ \\
$1.637 \mathrm{E}-02$ \\
$4.106 \mathrm{E}-01$
\end{tabular}

Amount

2. $915 \mathrm{E}-02$

5. $312 \mathrm{E}-01$

1. $461 \mathrm{E}-02$

2. $952 \mathrm{E}-03$

1. $739 \mathrm{E}-02$

4. $125 \mathrm{E}-01$

3. $320 \mathrm{E}-01$

3. $184 E+01$

$6.682 \mathrm{E}+00$

$6.275 \mathrm{E}-02$

3. $877 \mathrm{E}-01$

6. $386 E+01$

$7.952 \mathrm{E}+01$

8. 511E-02

9.130E-02

1. 277 E-02 (kg/yr) 


\begin{tabular}{l} 
Shipping Site: KAPL \\
Total Volume (m3/Yr) : \\
\\
Radionuclide \\
\hline Ba-137m \\
Co-60 \\
Cs-134 \\
$\mathrm{Cs}-137$ \\
Eu-154 \\
Eu-155 \\
$\mathrm{Fe}-55$ \\
$\mathrm{H}-3$ \\
$\mathrm{Mn}-54$ \\
$\mathrm{Nb}-94$ \\
$\mathrm{Ni}-59$ \\
$\mathrm{Ni}-63$ \\
$\mathrm{~Pa}-234 \mathrm{~m}$ \\
$\mathrm{Pm}-147$ \\
$\mathrm{Pu}-238$ \\
$\mathrm{Pu}-241$ \\
$\mathrm{Sb}-125$ \\
$\mathrm{Sm}-151$ \\
$\mathrm{Sr}-90$ \\
$\mathrm{Te}-125 \mathrm{~m}$ \\
$\mathrm{Th}-234$ \\
$U-238$ \\
$\mathrm{Y}-90$ \\
\end{tabular}

Shipping site: $\mathrm{KCP}$

Total Volume (m3/yr):

Radionuclide

$\mathrm{Ba}-137 \mathrm{~m}$

Co-60

Cs -134

Cs -137

Eu-154

Eu-155

$\mathrm{Fe}-55$

$\mathrm{H}-3$

$\mathrm{Mn}-54$

$\mathrm{Nb}-94$

$\mathrm{Ni}-59$

Ni-53

$\mathrm{Pa}-234 \mathrm{~m}$

Pm-147

$\mathrm{Pu}-238$

Pu-241

Sb-125

Sm-151

Sr-90

$\mathrm{Te}-125 \mathrm{~m}$

Th-234

$\mathrm{U}-238$

$Y-90$
Waste Type: Solids (CH-Non-alpha)

Total Mass ( $\mathrm{kg} / \mathrm{yr}): 5.162 E+03$

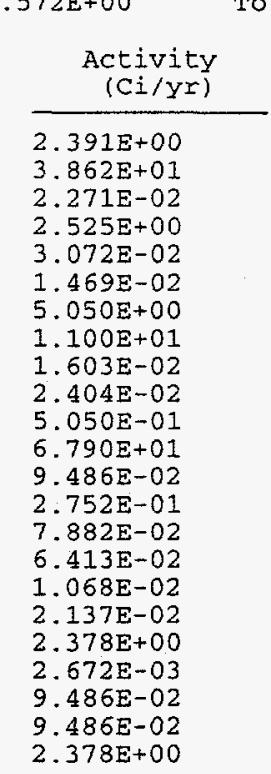

\begin{tabular}{|c|c|}
\hline Chemical & $\begin{array}{l}\text { Anount } \\
(\mathrm{kg} / \mathrm{Yr})\end{array}$ \\
\hline $57-12-5$ & $1.840 E-02$ \\
\hline $75-09-2$ & $2.577 \mathrm{E}+00$ \\
\hline $\mathrm{Ag}$ & $6.267 \mathrm{E}-02$ \\
\hline As & $2.265 E-02$ \\
\hline $\mathrm{Ba}$ & $5.050 E-01$ \\
\hline. $\mathrm{CA}$ & $2.428 E-02$ \\
\hline Cl $-2-x$ & $1.464 \mathrm{E}+00$ \\
\hline$c 1-3-x$ & $1.328 E+01$ \\
\hline C1-4-x & $1.024 \mathrm{E}+01$ \\
\hline $\mathrm{Cl}-\mathrm{F}-\mathrm{x}$ & $4.115 E-01$ \\
\hline $\mathrm{Cr}$ & $4.769 \mathrm{E}-01$ \\
\hline HC-insol & $3.511 E+01$ \\
\hline $\mathrm{HC}-\mathrm{solub}$ & $1.516 \mathrm{E}+01$ \\
\hline $\mathrm{Hg}$ & $6.065 \mathrm{E}-02$ \\
\hline $\mathrm{Pb}$ & $7.229 \mathrm{E}-01$ \\
\hline $\mathrm{Se}$ & $1.698 \mathrm{E}-02$ \\
\hline
\end{tabular}

Waste Type: Solids (CH-Non-alpha)

4.219E-02 Total Mass (kg/Yr): $3.237 E+01$

\begin{tabular}{r}
$\begin{array}{r}\text { Activity } \\
\text { (Ci/yr) }\end{array}$ \\
\hline $2.824 E-02$ \\
$4.562 \mathrm{E}-01$ \\
$2.682 \mathrm{E}-04$ \\
$2.982 \mathrm{E}-02$ \\
$3.629 \mathrm{E}-04$ \\
$1.735 \mathrm{E}-04$ \\
$5.964 \mathrm{E}-02$ \\
$1.299 \mathrm{E}-01$ \\
$1.893 \mathrm{E}-04$ \\
$2.840 \mathrm{E}-04$ \\
$5.964 \mathrm{E}-03$ \\
$8.019 \mathrm{E}-01$ \\
$1.120 \mathrm{E}-03$ \\
$3.250 \mathrm{E}-03$ \\
$9.309 \mathrm{E}-04$ \\
$7.573 \mathrm{E}-04$ \\
$1.262 \mathrm{E}-04$ \\
$2.524 \mathrm{E}-04$ \\
$2.808 \mathrm{E}-02$ \\
$3.155 \mathrm{E}-05$ \\
$1.120 \mathrm{E}-03$ \\
$1.120 \mathrm{E}-03$ \\
$2.808 \mathrm{E}-02$
\end{tabular}

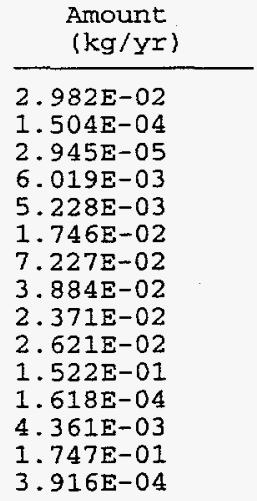


Shipping Site: KKS

Total Volume $(\mathrm{m} 3 / \mathrm{yr})$ :

Radionuclide

$\mathrm{Ba}-137 \mathrm{~m}$

Co-50

Cs -134

Cs -137

Eu-154

Eu-155

$\mathrm{Fe}-55$

$\mathrm{H}-3$

$\mathrm{Mn}-54$

$\mathrm{Nb}-94$

$\mathrm{Ni}-59$

$\mathrm{Ni}-63$

Pa-234m

$\mathrm{Pm}-147$

Pu-238

$\mathrm{Pu}-241$

$\mathrm{Sb}-125$

Sm-151

Sr -90

$\mathrm{Te}-125 \mathrm{~m}$

Th-234

$\mathrm{U}-238$

$\mathrm{Y}-90$

Shipping site:

KKS

Total Volume $(\mathrm{m} 3 / \mathrm{yr})$ :

Radionuclide

$\mathrm{Ba}-137 \mathrm{~m}$

Co- 60

Cs -134

Cs-137

Eu-154

Eu-155

Fe-55

$\mathrm{H}-3$

Mn-54

$\mathrm{Nb}-94$

$\mathrm{Ni}-59$

$\mathrm{Ni}-63$

$\mathrm{Pa}-234 \mathrm{~m}$

Prn-147

Pu-238

Pu-241

Sb- 125

Sm-151

Sr-90

$\mathrm{Te}-125 \mathrm{~m}$

Th-234

$\mathrm{U}-238$

$\mathrm{Y}-90$
Waste Type: Organic Iiquids (CH-Non-alpha)

$4.733 \mathrm{E}-01$

Total Mass (kg/yr):

$5.422 \mathrm{E}+02$

\begin{tabular}{c}
$\begin{array}{r}\text { Activity } \\
\text { (Ci/yr) }\end{array}$ \\
\hline $3.168 \mathrm{E}-01$ \\
$5.118 \mathrm{E}+00$ \\
$3.009 \mathrm{E}-03$ \\
$3.345 \mathrm{E}-01$ \\
$4.071 \mathrm{E}-03$ \\
$1.947 \mathrm{E}-03$ \\
$6.691 \mathrm{E}-01$ \\
$1.457 \mathrm{E}+00$ \\
$2.124 \mathrm{E}-03$ \\
$3.186 \mathrm{E}-03$ \\
$6.691 \mathrm{E}-02$ \\
$8.997 \mathrm{E}+00$ \\
$1.256 \mathrm{E}-02$ \\
$3.646 \mathrm{E}-02$ \\
$1.044 \mathrm{E}-02$ \\
$8.497 \mathrm{E}-03$ \\
$1.416 \mathrm{E}-03$ \\
$2.832 \mathrm{E}-03$ \\
$3.151 \mathrm{E}-01$ \\
$3.540 \mathrm{E}-04$ \\
$1.256 \mathrm{E}-02$ \\
$1.256 \mathrm{E}-02$ \\
$3.151 \mathrm{E}-01$
\end{tabular}

\begin{tabular}{l} 
Chemical \\
\hline $57-12-5$ \\
$75-09-2$ \\
$\mathrm{Ag}$ \\
$\mathrm{As}$ \\
$\mathrm{Ba}$ \\
$\mathrm{Cd}$ \\
$\mathrm{C} 1-2-\mathrm{x}$ \\
$\mathrm{C} 1-3-\mathrm{x}$ \\
$\mathrm{Cl}-4-\mathrm{x}$ \\
$\mathrm{C} 1-\mathrm{F}-\mathrm{x}$ \\
$\mathrm{Cr}$ \\
$\mathrm{HC}-$ insol \\
$\mathrm{HC}-\mathrm{Solub}$ \\
$\mathrm{Hg}$ \\
$\mathrm{Pb}$ \\
$\mathrm{Se}$
\end{tabular}

\begin{tabular}{c}
$\begin{array}{c}\text { Amount } \\
(\mathrm{kg} / \mathrm{yr})\end{array}$ \\
\hline $2.237 \mathrm{E}-02$ \\
$4.077 \mathrm{E}-01$ \\
$1.121 \mathrm{E}-02$ \\
$2.266 \mathrm{E}-03$ \\
$1.335 \mathrm{E}-02$ \\
$3.166 \mathrm{E}-01$ \\
$2.548 \mathrm{E}-01$ \\
$2.443 \mathrm{E}+01$ \\
$5.128 \mathrm{E}+00$ \\
$4.816 \mathrm{E}-02$ \\
$2.976 \mathrm{E}-01$ \\
$4.901 \mathrm{E}+01$ \\
$6.103 \mathrm{E}+01$ \\
$6.532 \mathrm{E}-02$ \\
$7.007 \mathrm{E}-02$ \\
$9.805 \mathrm{E}-03$
\end{tabular}

Waste TYpe: Solids (CH-Non-alpha)

Total Mass $(\mathrm{kg} / \mathrm{yr}): 6.620 \mathrm{E}+03$

\begin{tabular}{l} 
Chemical \\
\hline $57-12-5$ \\
$75-09-2$ \\
Ag \\
As \\
Ba \\
Cd \\
Cl-2-x \\
Cl-3-x \\
Cl-4-x \\
Cl-F-x \\
Cr \\
FC-insol \\
HC-Solub \\
Hg \\
Pb \\
Se
\end{tabular}

\begin{tabular}{c}
$\begin{array}{c}\text { Amount } \\
(\mathrm{kg} / \mathrm{Yr})\end{array}$ \\
\hline $1.282 \mathrm{E}-01$ \\
$2.231 \mathrm{E}+00$ \\
$8.783 \mathrm{E}-02$ \\
$3.299 \mathrm{E}-02$ \\
$1.662 \mathrm{E}+00$ \\
$5.269 \mathrm{E}-02$ \\
$1.281 \mathrm{E}+00$ \\
$1.108 \mathrm{E}+01$ \\
$1.215 \mathrm{E}+01$ \\
$2.090 \mathrm{E}-01$ \\
$1.846 \mathrm{E}+00$ \\
$2.753 \mathrm{E}+01$ \\
$1.411 \mathrm{E}+01$ \\
$1.438 \mathrm{E}-01$ \\
$4.165 \mathrm{E}+00$ \\
$3.790 \mathrm{E}-02$
\end{tabular}


Shipping Site: KWS Waste Type: Organic Liquids (CH-Non-alpha)

Total Volume $(\mathrm{m} 3 / \mathrm{Yr})$ :

$6.454 E-02$

Total Mass $(\mathrm{kg} / \mathrm{yr}): \quad 7.394 \mathrm{E}+01$

Radionuclide

$\mathrm{Ba}-13.7 \mathrm{~m}$

$\mathrm{CO}-60$

$\mathrm{Cs}-134$

Cs -137

Eu-154

$\mathrm{Eu}-155$

$\mathrm{F} \in-55$

$\mathrm{H}-3$

$\mathrm{Mn}-54$

$\mathrm{Nb}-94$

$\mathrm{Ni}-59$

$\mathrm{Ni}-63$

$\mathrm{Pa}-234 \mathrm{~m}$

PIn- 147

$\mathrm{Pu}-238$

Pu-241

$\mathrm{Sb}-125$

$\mathrm{Sm}-151$

$\mathrm{S} x-90$

$\mathrm{T} e-125 \mathrm{~m}$

Th-234

$\mathrm{U}-238$

$Y-90$

Shipping Site:

KWS

Total Volume $(\mathrm{m} 3 / \mathrm{yr})$ :

Radionuclide

$\mathrm{Ba}-137 \mathrm{~m}$

Co -60

$\mathrm{Cs}-134$

Cs-137

Eu-154

Eu-155

Fe -55

$\mathrm{H}-3$

$\mathrm{Mn}-54$

$\mathrm{Nb}-94$

$\mathrm{Ni}-59$

$\mathrm{Ni}-63$

$\mathrm{Pa}-234 \mathrm{~m}$

Pm-147

Pu-238

Pu-241

Sb-125

$\mathrm{Sm}-151$

Sr -90

$\mathrm{Te}-125 \mathrm{~m}$

Th-234

$\mathrm{U}-238$

$Y-90$

\begin{tabular}{r}
$\begin{array}{c}\text { Activity } \\
\text { (Ci/Yr) }\end{array}$ \\
\hline $4.321 E-02$ \\
$6.979 E-01$ \\
$4.103 E-04$ \\
$4.562 E-02$ \\
$5.552 E-04$ \\
$2.655 E-04$ \\
$9.125 E-02$ \\
$1.987 E-01$ \\
$2.896 E-04$ \\
$4.345 E-04$ \\
$9.125 E-03$ \\
$1.226 E+00$ \\
$1.714 E-03$ \\
$4.973 E-03$ \\
$1.424 E-03$ \\
$1.158 E-03$ \\
$1.931 E-04$ \\
$3.862 E-04$ \\
$4.297 E-02$ \\
$4.828 E-05$ \\
$1.714 E-03$ \\
$1.714 E-03$ \\
$4.297 E-02$ \\
\end{tabular}

\begin{tabular}{l} 
Chemical \\
\hline $57-12-5$ \\
$75-09-2$ \\
Ag \\
As \\
Ba \\
Cd \\
CI-2-x \\
CI-3-x \\
CI-4-x \\
Cl-F-x \\
Cr \\
HC-insol \\
HC-solub \\
Hg \\
Pb \\
Se
\end{tabular}

\begin{tabular}{c}
$\begin{array}{c}\text { Amount } \\
(\mathrm{kg} / Y r)\end{array}$ \\
\hline $3.051 E-03$ \\
$5.559 E-02$ \\
$1.529 E-03$ \\
$3.090 E-04$ \\
$1.820 E-03$ \\
$4.317 E-02$ \\
$3.474 E-02$ \\
$3.332 E+00$ \\
$6.993 E-01$ \\
$6.567 E-03$ \\
$4.058 E-02$ \\
$6.683 E+00$ \\
$8.322 E+00$ \\
$8.907 E-03$ \\
$9.555 E-03$ \\
$1.337 E-03$
\end{tabular}

Waste Type: Solids (CH-Non-alpha)

Total Mass $(\mathrm{kg} / \mathrm{yr}): 1.580 \mathrm{E}+03$

\begin{tabular}{l} 
Chemical \\
\hline $57-12-5$ \\
$75-09-2$ \\
$\mathrm{Ag}$ \\
$\mathrm{As}$ \\
$\mathrm{Ba}$ \\
$\mathrm{Cd}$ \\
$\mathrm{Cl}-2-\mathrm{x}$ \\
$\mathrm{Cl}-3-\mathrm{x}$ \\
$\mathrm{Cl}-4-\mathrm{x}$ \\
$\mathrm{Cl}-\mathrm{F}-\mathrm{x}$ \\
$\mathrm{Cr}$ \\
$\mathrm{HC}-\mathrm{inso} 1$ \\
$\mathrm{HC}-\mathrm{solub}$ \\
$\mathrm{Hg}$ \\
$\mathrm{Pb}$ \\
$\mathrm{Se}$
\end{tabular}

\begin{tabular}{|c|}
\hline $\begin{array}{l}\text { Amount } \\
(\mathrm{kg} / \mathrm{y} x)\end{array}$ \\
\hline $\begin{array}{l}8.005 E-02 \\
3.801 E-01 \\
2.572 E-02 \\
8.795 E-03 \\
1.035 E+00 \\
1.157 E-02 \\
3.221 E-01 \\
1.699 E+00 \\
1.988 E+00 \\
2.323 E-01 \\
1.010 E+00 \\
4.421 E+00 \\
1.600 E+00 \\
6.055 E-02 \\
2.396 E+00 \\
1.789 E-02\end{array}$ \\
\hline
\end{tabular}


Shipping Site: PADUCAH

Waste Type: Organic Liquids ( $\mathrm{CH}$-Non-alpha)

Total Volume $(\mathrm{m} 3 / \mathrm{Yr})$ :

$2.970 E+01$

Tatal Mass $(\mathrm{kg} / \mathrm{yr})$ :

$3.589 \mathrm{E}+04$

Radionuclide

AC -228

$\mathrm{Bi}-212$

$\mathrm{Pa}-234$

$\mathrm{Pa}-234 \mathrm{~m}$

$\mathrm{Pb}-212$

Po- 212

Po -216

$\mathrm{Ra}-224$

$\mathrm{Ra}-228$

Tc -99

$\mathrm{Th}-228$

Th-231

Th-232

Th -234

$\mathrm{T} 1-208$

$\mathrm{U}-235$

$\mathrm{U}-238$

Shipping site:

PADUCAH

Total volume (m3/yr):

Radionuclide

AC -228

$\mathrm{Bi}-212$

$\mathrm{Pa}-234$

$\mathrm{Pa}-234 \mathrm{~m}$

$\mathrm{Pb}-212$

PO- 212

Po-216

$\mathrm{Ra}-224$

$\mathrm{Ra}-228$

Tc-99

Th-228

Th-231

Th -232

Th-234

T1-208

$\mathrm{U}-235$

$\mathrm{U}-238$

Shipping Site: PNS

Total Volume (m3/yr):

Radionuclide

$\mathrm{Ba}-137 \mathrm{~m}$

Co- 60

Cs -134

Cs -137

Eu-154

Eu-155

$\mathrm{Fe}-55$

$\mathrm{H}-3$

$\mathrm{Mn}-54$

$\mathrm{Nb}-94$

$\mathrm{Ni}-59$

$\mathrm{Ni}-63$

$\mathrm{Pa}-234 \mathrm{~m}$

Pm-147

$\mathrm{Pu}-238$

Pu-241

$\mathrm{Sb}-125$

$\mathrm{Sm}-151$

Sr -90

Te-125m

$\mathrm{Th}-234$

$\mathrm{U}-238$

$Y-90$

\begin{tabular}{l}
$\begin{array}{c}\text { Activity } \\
(\mathrm{Ci} / \mathrm{Yr})\end{array}$ \\
\hline $1.796 \mathrm{E}-02$ \\
$2.967 \mathrm{E}-03$ \\
$2.486 \mathrm{E}-03$ \\
$2.420 \mathrm{E}+01$ \\
$2.967 \mathrm{E}-03$ \\
$1.965 \mathrm{E}-03$ \\
$2.967 \mathrm{E}-03$ \\
$2.967 \mathrm{E}-03$ \\
$1.796 \mathrm{E}-02$ \\
$7.291 \mathrm{E}+00$ \\
$2.967 \mathrm{E}-03$ \\
$1.892 \mathrm{E}-02$ \\
$1.990 \mathrm{E}-01$ \\
$2.420 \mathrm{E}+01$ \\
$1.163 \mathrm{E}-03$ \\
$1.876 \mathrm{E}-02$ \\
$2.420 \mathrm{E}+01$
\end{tabular}

\begin{tabular}{l} 
Chenical \\
\hline $57-12-5$ \\
$75-09-2$ \\
$\mathrm{Ag}$ \\
$\mathrm{As}$ \\
$\mathrm{Ba}$ \\
$\mathrm{Cd}$ \\
$\mathrm{Cl}-2-\mathrm{x}$ \\
$\mathrm{Cl}-3-\mathrm{x}$ \\
$\mathrm{Cl}-4-\mathrm{x}$ \\
$\mathrm{Cl}-\mathrm{F}-\mathrm{x}$ \\
$\mathrm{Cr}$ \\
$\mathrm{HC}-$ insol \\
$\mathrm{HC}-$ solub \\
$\mathrm{Hg}$ \\
$\mathrm{Pb}$ \\
$\mathrm{Se}$
\end{tabular}

\begin{tabular}{c}
$\begin{array}{c}\text { Amount } \\
(\mathrm{kg} / \mathrm{Yr})\end{array}$ \\
\hline $4.590 \mathrm{E}-01$ \\
$4.205 \mathrm{E}+01$ \\
$2.300 \mathrm{E}-01$ \\
$1.180 \mathrm{E}-01$ \\
$5.803 \mathrm{E}-01$ \\
$3.374 \mathrm{E}+01$ \\
$2.303 \mathrm{E}+01$ \\
$2.444 \mathrm{E}+03$ \\
$5.381 \mathrm{E}+02$ \\
$1.762 \mathrm{E}+00$ \\
$2.951 \mathrm{E}+01$ \\
$5.097 \mathrm{E}+03$ \\
$2.651 \mathrm{E}+03$ \\
$1.493 \mathrm{E}+00$ \\
$6.639 \mathrm{E}+00$ \\
$2.011 \mathrm{E}-01$
\end{tabular}

Waste Type: Solids (CH-Non-alpha)

$4.740 E+00$

Total Mass $(\mathrm{kg} / \mathrm{yr})$ :

$6.808 \mathrm{E}+03$

\begin{tabular}{l} 
Chemical \\
\hline $57-12-5$ \\
$75-09-2$ \\
$\mathrm{Ag}$ \\
$\mathrm{As}$ \\
$\mathrm{Ba}$ \\
$\mathrm{Cd}$ \\
$\mathrm{Cl}-2-\mathrm{x}$ \\
$\mathrm{Cl}-3-\mathrm{x}$ \\
$\mathrm{Cl}-4-\mathrm{x}$ \\
$\mathrm{Cl}-\mathrm{F}-\mathrm{x}$ \\
$\mathrm{Cr}$ \\
$\mathrm{HC}-$ insol \\
$\mathrm{HC}-$ solub \\
$\mathrm{Hg}$ \\
$\mathrm{Pb}$ \\
$\mathrm{Se}$
\end{tabular}

\begin{tabular}{l}
$\begin{array}{c}\text { Amount } \\
(\mathrm{kg} / \mathrm{Yr})\end{array}$ \\
\hline $1.016 \mathrm{E}+00$ \\
$1.710 \mathrm{E}+00$ \\
$2.502 \mathrm{E}-01$ \\
$8.712 \mathrm{E}-02$ \\
$1.228 \mathrm{E}+01$ \\
$1.504 \mathrm{E}-01$ \\
$1.444 \mathrm{E}+00$ \\
$1.250 \mathrm{E}+01$ \\
$4.496 \mathrm{E}+00$ \\
$6.729 \mathrm{E}-01$ \\
$1.293 \mathrm{E}+01$ \\
$3.436 \mathrm{E}+01$ \\
$1.740 \mathrm{E}+01$ \\
$1.732 \mathrm{E}+00$ \\
$2.789 \mathrm{E}+01$ \\
$6.716 \mathrm{E}-01$
\end{tabular}

Waste Type: Solids (CH-Non-alpha)

Total Mass $(\mathrm{kg} / \mathrm{Yr}): 9.088 \mathrm{E}+01$ 
Shipping site: PORTS

Waste Type: Organic Liquids ( $\mathrm{CH}-\mathrm{Non}-\mathrm{alpha})$

Total Volume (m3/yr) :

$4.765 E+01$

Total Mass $(\mathrm{kg} / \mathrm{yr})$ :

$4.778 \mathrm{E}+04$

Radionuclide

AC -228

$\mathrm{Bi}-212$

$\mathrm{Pa}-234$

$\mathrm{Pa}-234 \mathrm{~m}$

$\mathrm{Pb}-212$

Po-212

Po- 216

$\mathrm{Ra}-224$

$\mathrm{Ra}-228$

$\mathrm{Tc}-99$

Th-228

Th-231

Th-232

Th-234

Tl-208

$\mathrm{U}-235$

$\mathrm{u}-238$

Shipping Site:

PORTS

Total Volume ( $\pi 3 / y r)$ :

Radionuclide

AC -228

$\mathrm{Bi}-212$

$\mathrm{Pa}-234$

$\mathrm{Pa}-234 \mathrm{~m}$

$\mathrm{Pb}-212$

Po- 212

Po- 216

$\mathrm{Ra}-224$

$\mathrm{Ra}-228$

TC-99

Th-228

$\mathrm{Th}-231$

Th-232

Th-234

T1-208

$\mathrm{U}-235$

$\mathrm{U}-238$

Shipping site: PPPL

Total Volume (m3/yr):

Radionuclide

$\mathrm{Ba}-137 \mathrm{~m}$

$\mathrm{Cs}-137$

$\mathrm{H}-3$

$\mathrm{Pa}-234 \mathrm{~m}$

Pu-241

Sr -90

$\mathrm{Th}-234$

$\mathrm{U}-238$

$Y-90$

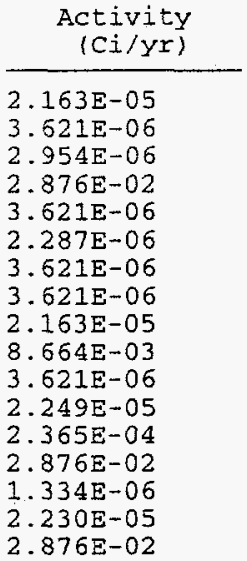

\begin{tabular}{l} 
Chemica 1 \\
\hline $57-12-5$ \\
$75-09-2$ \\
$\mathrm{Ag}$ \\
$\mathrm{As}$ \\
$\mathrm{Ba}$ \\
$\mathrm{Cd}$ \\
$\mathrm{Cl}-2-\mathrm{x}$ \\
$\mathrm{Cl}-3-\mathrm{x}$ \\
$\mathrm{Cl}-4-\mathrm{x}$ \\
$\mathrm{Cl}-\mathrm{F}-\mathrm{x}$ \\
$\mathrm{Cr}$ \\
$\mathrm{HC}-\mathrm{insol}$ \\
$\mathrm{HC}-$ solub \\
$\mathrm{Hg}$ \\
$\mathrm{Pb}$ \\
$\mathrm{Se}$
\end{tabular}

\begin{tabular}{c}
$\begin{array}{c}\text { Amount } \\
(\mathrm{kg} / \mathrm{yr})\end{array}$ \\
\hline $7.201 \mathrm{E}+00$ \\
$1.017 \mathrm{E}+00$ \\
$2.102 \mathrm{E}+00$ \\
$8.553 \mathrm{E}-02$ \\
$5.830 \mathrm{E}-01$ \\
$2.050 \mathrm{E}-01$ \\
$2.879 \mathrm{E}+00$ \\
$1.392 \mathrm{E}+02$ \\
$7.874 \mathrm{E}+00$ \\
$2.331 \mathrm{E}+00$ \\
$8.409 \mathrm{E}+00$ \\
$4.553 \mathrm{E}+02$ \\
$1.440 \mathrm{E}+04$ \\
$1.291 \mathrm{E}+01$ \\
$2.212 \mathrm{E}+00$ \\
$1.887 \mathrm{E}+00$
\end{tabular}

Waste Type: Solids (CH-Non-alpha)

Total Mass $(\mathrm{kg} / \mathrm{yr}): 1.096 \mathrm{E}+06$

$7.763 \mathrm{E}+02$

\begin{tabular}{l} 
Chemical \\
\hline $57-12-5$ \\
$75-09-2$ \\
Ag \\
As \\
Ba \\
Cd \\
Cl-2-x \\
Cl-3-x \\
Cl-4 - \\
Cl-F-x \\
Cr \\
HC-insol \\
HC-solub \\
$\mathrm{Hg}$ \\
Pb \\
Se
\end{tabular}

\begin{tabular}{c}
$\begin{array}{c}\text { Amount } \\
(\mathrm{kg} / \mathrm{Yr})\end{array}$ \\
\hline $1.691 \mathrm{E}+02$ \\
$2.135 \mathrm{E}+02$ \\
$3.471 \mathrm{E}+01$ \\
$1.183 \mathrm{E}+01$ \\
$2.025 \mathrm{E}+03$ \\
$1.587 \mathrm{E}+01$ \\
$2.135 \mathrm{E}+02$ \\
$1.306 \mathrm{E}+03$ \\
$5.694 \mathrm{E}+02$ \\
$1.185 \mathrm{E}+02$ \\
$2.062 \mathrm{E}+03$ \\
$2.947 \mathrm{E}+03$ \\
$1.642 \mathrm{E}+03$ \\
$1.720 \mathrm{E}+02$ \\
$4.847 \mathrm{E}+03$ \\
$6.061 \mathrm{E}+01$
\end{tabular}

Waste Type: Solids (CH-Non-alpha)

Total Mass (kg/yr): 7.191E-01

$5.456 \mathrm{E}-04$

\begin{tabular}{l} 
Chemical \\
\hline $75-09-2$ \\
Cl-2-x \\
Cl-3-x \\
Cl-4-x \\
HC-insol \\
HC-solub
\end{tabular}

\begin{tabular}{c}
$\begin{array}{c}\text { Amount } \\
(\mathrm{kg} / \mathrm{Yr})\end{array}$ \\
\hline $1.438 \mathrm{E}-02$ \\
$7.190 \mathrm{E}-03$ \\
$7.190 \mathrm{E}-02$ \\
$5.752 \mathrm{E}-02$ \\
$1.797 \mathrm{E}-01$ \\
$8.629 \mathrm{E}-02$
\end{tabular}


Shipping site: RMI

Total Volume $(\mathrm{m} 3 / \mathrm{yr})$ :

Radionuclide

Ac -228

$\mathrm{Bi}-212$

$\mathrm{Pa}-234$

$\mathrm{Pa}-234 \mathrm{~m}$

$\mathrm{Pb}-212$

Po-212

Po-216

$\mathrm{Ra}-224$

Ra -228

TC-99

Th-228

Th-23I

$\mathrm{Th}-232$

Th-234

TI-208

$\mathrm{U}-235$

$\mathrm{U}-238$

Shipping site: RMI

Total Volume (m3/yr):

Radionuclide

Ac- 228

$\mathrm{Bi}-212$

$\mathrm{Pa}-234$

$\mathrm{Pa}-234 \mathrm{~m}$

$\mathrm{Pb}-212$

Po-21.2

Po-216

$\mathrm{Ra}-224$

Ra-228

Tc-99

Th-228

Th-231

Th-232

Th-234

T1-208

$\mathrm{U}-235$

$\mathrm{U}-238$
Waste Type: Organic Liquids ( $\mathrm{CH}-\mathrm{Non}-\mathrm{alpha}$ )

$6.677 \mathrm{E}-01$

Total Mass $(\mathrm{kg} / \mathrm{yr}): \quad 9.741 E+02$

\begin{tabular}{|c|c|}
\hline Chemical & $\begin{array}{l}\text { Amount } \\
(\mathrm{kg} / \mathrm{yr})\end{array}$ \\
\hline $75-09-2$ & $1.527 \mathrm{E}+00$ \\
\hline As & $3.769 E-04$ \\
\hline $\mathrm{Ba}$ & 1. $615 E-03$ \\
\hline $\mathrm{cd}$ & $1.071 E+00$ \\
\hline cl-2-x & $8.108 \mathrm{E}-01$ \\
\hline$C 1-3-x$ & $1.123 \mathrm{E}+02$ \\
\hline$C 1-4-x$ & $2.505 E+0 I$ \\
\hline$C l-E-x$ & $4.625 E-02$ \\
\hline $\mathrm{Cr}$ & $9.331 \mathrm{E}-01$ \\
\hline HC-insoI & $2.184 E+01$ \\
\hline $\mathrm{HC}$-solub & $1.277 \mathrm{E}+01$ \\
\hline $\mathrm{Hg}$ & $6.122 E-03$ \\
\hline $\mathrm{Pb}$ & $2.045 E-01$ \\
\hline
\end{tabular}

Waste Type: Solids (CH-Non-alpha)

1.091E+00 Total Mass $(\mathrm{kg} / \mathrm{yr}): \quad 8.323 \mathrm{E}+02$

\begin{tabular}{l}
$\begin{array}{c}\text { Activity } \\
\text { (Ci/yr) }\end{array}$ \\
\hline $2.370 E-06$ \\
$3.988 \mathrm{E}-07$ \\
$3.013 \mathrm{E}-07$ \\
$2.927 \mathrm{E}-03$ \\
$3.988 \mathrm{E}-07$ \\
$2.570 \mathrm{E}-07$ \\
$3.988 \mathrm{E}-07$ \\
$3.815 \mathrm{E}-07$ \\
$2.267 \mathrm{E}-06$ \\
$4.218 \mathrm{E}-05$ \\
$3.988 \mathrm{E}-07$ \\
$2.286 \mathrm{E}-06$ \\
$2.407 \mathrm{E}-05$ \\
$2.927 \mathrm{E}-03$ \\
$1.506 \mathrm{E}-07$ \\
$2.277 \mathrm{E}-06$ \\
$2.927 \mathrm{E}-03$
\end{tabular}

\begin{tabular}{l} 
Chemical \\
\hline $57-12-5$ \\
$75-09-2$ \\
$\mathrm{Ag}$ \\
$\mathrm{As}$ \\
$\mathrm{Ba}$ \\
$\mathrm{Cd}$ \\
$\mathrm{Cl}-2-\mathrm{x}$ \\
$\mathrm{Cl}-3-\mathrm{x}$ \\
$\mathrm{Cl}-4-\mathrm{x}$ \\
$\mathrm{Cl}-\mathrm{F}-\mathrm{x}$ \\
$\mathrm{Cr}$ \\
$\mathrm{HC}-\mathrm{insol}$ \\
$\mathrm{HC}-\mathrm{solub}$ \\
$\mathrm{Hg}$ \\
$\mathrm{Pb}$ \\
$\mathrm{Se}$
\end{tabular}

Amount

( $\mathrm{kg} / \mathrm{yr}$ )

4. $647 \mathrm{E}-03$

$2.315 \mathrm{E}-01$

$6.65 I E-03$

1. $974 \mathrm{E}-03$

$5.734 E-01$

1. $836 \mathrm{E}-02$

$2.862 E-01$

$2.561 \mathrm{E}+00$

7. $371 E-01$

3. $452 \mathrm{E}-01$

$2.638 \mathrm{E}-01$

$8.888 \mathrm{E}+00$

$2.825 \mathrm{E}+0 \mathrm{O}$

$2.678 \mathrm{E}-02$

$8.498 \mathrm{E}-02$

$6.070 E-03$ 
Destination site: SRS

Shipping site: BAPL

Total Volume (m3/yr):

Radionuclide

Am-241

$\mathrm{Ba}-137 \mathrm{~m}$

$\mathrm{Co}-60$

Cs -137

Eu-154

$\mathrm{H}-3$

$\mathrm{Nb}-94$

$\mathrm{Ni}-59$

$\mathrm{Ni}-63$

$\mathrm{Pa}-234 \mathrm{~m}$

$\mathrm{Pu}-238$

Pu-239

$\mathrm{Pu}-240$

Pu-241

Sm-151

Sr -90

Tc-99

Th-234

$\mathrm{U}-238$

$Y-90$

Shipping site:

CNS

Total Volume $(\mathrm{m} 3 / \mathrm{yr})$ :

Radionuclide

Ba-137m

$\mathrm{Co}-60$

$\mathrm{Cs}-134$

$\mathrm{Cs}-137$

Eu-154

Eu-155

Fe- 55

H -3

$\mathrm{Mn}-54$

$\mathrm{Nb}-94$

$\mathrm{Ni}-59$

$\mathrm{Ni}-63$

$\mathrm{Pa}-234 \mathrm{~m}$

Pm-147

$\mathrm{Pu}-238$

Pu-241

$\mathrm{Sb}-125$

Sm-151

Sr -90

Te-125m

Th-234

$\mathrm{U}-238$

$\mathrm{Y}-90$
Waste Type: Solids (CH-Alpha)
8.357E-01 Total Mass (kg/yr): $3.493 \mathrm{E}+02$

\begin{tabular}{l}
$\begin{array}{c}\text { Activity } \\
\text { (Ci/yr) }\end{array}$ \\
\hline $6.984 E-04$ \\
$7.629 \mathrm{E}-01$ \\
$1.021 \mathrm{E}-03$ \\
$8.160 \mathrm{E}-01$ \\
$1.217 \mathrm{E}-03$ \\
$6.053 \mathrm{E}-02$ \\
$6.984 \mathrm{E}-05$ \\
$1.563 \mathrm{E}-03$ \\
$1.606 \mathrm{E}-01$ \\
$4.490 \mathrm{E}-04$ \\
$3.791 \mathrm{E}-02$ \\
$6.286 \mathrm{E}-04$ \\
$2.793 \mathrm{E}-04$ \\
$1.633 \mathrm{E}-02$ \\
$1.167 \mathrm{E}-02$ \\
$7.264 \mathrm{E}-01$ \\
$3.625 \mathrm{E}-04$ \\
$4.490 \mathrm{E}-04$ \\
$4.490 \mathrm{E}-04$ \\
$7.264 \mathrm{E}-01$
\end{tabular}

\begin{tabular}{l} 
Chemical \\
\hline $75-09-2$ \\
$\mathrm{Ag}$ \\
$\mathrm{Ba}$ \\
$\mathrm{Cd}$ \\
$\mathrm{Cl}-2-\mathrm{x}$ \\
$\mathrm{Cl}-3-\mathrm{x}$ \\
$\mathrm{Cl}-4-\mathrm{x}$ \\
$\mathrm{Cl}-\mathrm{F}-\mathrm{x}$ \\
$\mathrm{Cr}$ \\
$\mathrm{HC}-$ insol \\
$\mathrm{HC}-$ solub \\
$\mathrm{Hg}$ \\
$\mathrm{Pb}$ \\
$\mathrm{Se}$
\end{tabular}

\begin{tabular}{c}
$\begin{array}{c}\text { Amount } \\
(\mathrm{kg} / \mathrm{Yr})\end{array}$ \\
\hline $2.095 \mathrm{E}-01$ \\
$3.493 \mathrm{E}-03$ \\
$1.397 \mathrm{E}-01$ \\
$6.986 \mathrm{E}-04$ \\
$2.445 \mathrm{E}-01$ \\
$8.733 \mathrm{E}-01$ \\
$4.191 \mathrm{E}-01$ \\
$3.493 \mathrm{E}-01$ \\
$5.239 \mathrm{E}-03$ \\
$3.493 \mathrm{E}+00$ \\
$1.746 \mathrm{E}-03$ \\
$6.986 \mathrm{E}-04$ \\
$3.493 \mathrm{E}-02$ \\
$1.047 \mathrm{E}-03$
\end{tabular}

Waste Type: Organic Liquids (CH-Non-alpha)

Total Mass $(\mathrm{kg} / \mathrm{Yr}): 1.026 \mathrm{E}+03$

\begin{tabular}{l} 
Chemical \\
\hline $57-12-5$ \\
$75-09-2$ \\
Ag \\
As \\
Ba \\
Cd \\
Cl-2-x \\
C1-3-x \\
C1-4-x \\
CI-F-x \\
Cr \\
HC-insol \\
HC-solub \\
Hg \\
Pb \\
Se
\end{tabular}

\begin{tabular}{c}
$\begin{array}{c}\text { Amount } \\
(\mathrm{kg} / \mathrm{yr})\end{array}$ \\
\hline $5.151 \mathrm{E}-02$ \\
$7.241 \mathrm{E}-01$ \\
$2.274 \mathrm{E}-02$ \\
$3.999 \mathrm{E}-03$ \\
$2.356 \mathrm{E}-02$ \\
$5.620 \mathrm{E}-01$ \\
$4.522 \mathrm{E}-01$ \\
$4.355 \mathrm{E}+01$ \\
$9.136 \mathrm{E}+00$ \\
$8.522 \mathrm{E}-02$ \\
$5.418 \mathrm{E}-01$ \\
$8.707 \mathrm{E}+01$ \\
$1.317 \mathrm{E}+02$ \\
$1.340 \mathrm{E}-01$ \\
$1.275 \mathrm{E}-01$ \\
$1.998 \mathrm{E}-02$
\end{tabular}




\begin{tabular}{l} 
Shipping Site: CNS \\
Total volume (m3/yr) \\
\\
Radionuclide \\
\hline Ba-137m \\
Co-50 \\
Cs -134 \\
Cs -137 \\
Eu-154 \\
Eu-155 \\
Fe-55 \\
H-3 \\
Mn-54 \\
Nb-94 \\
Ni -59 \\
Ni -63 \\
Pa-234m \\
Pm-147 \\
Pu-238 \\
Pu-241 \\
Sb-125 \\
Sm-151 \\
Sr -90 \\
Te-125m \\
Th-234 \\
U-238 \\
Y-90
\end{tabular}

Shipping Site: MOUND

Total volume (m3/yr):

Radionuclide

$\mathrm{Ba}-137 \mathrm{~m}$

Cs-137

$\mathrm{H}-3$

$\mathrm{Pa}-234 \mathrm{~m}$

Pu-238

Pu-241

$\mathrm{Sr}-90$

Th-234

$\mathrm{U}-238$

$\mathrm{Y}-90$

Shipping site: MOUND

Total Volume (m3/yr):

Radionuclide

$\mathrm{Ba}-137 \mathrm{~m}$

Cs -137

$\mathrm{H}-3$

$\mathrm{Pa}-234 \mathrm{~m}$

Pu-238

Pu-241

$\mathrm{Sr}-90$

Th-234

$\mathrm{U}-238$

$\mathrm{Y}-90$
$9.030 E-02$

Total Mass (kg/yr):

(CH-Non-alpha)

$6.772 E+01$

\begin{tabular}{l} 
Chemical \\
\hline $57-12-5$ \\
$75-09-2$ \\
$\mathrm{Ag}$ \\
$\mathrm{As}$ \\
$\mathrm{Ba}$ \\
$\mathrm{Cd}$ \\
$\mathrm{Cl}-2-\mathrm{x}$ \\
$\mathrm{Cl}-3-\mathrm{x}$ \\
$\mathrm{Cl}-4-\mathrm{x}$ \\
$\mathrm{Cl}-\mathrm{F}-\mathrm{x}$ \\
$\mathrm{Cr}$ \\
$\mathrm{HC}-\mathrm{insol}$ \\
$\mathrm{HC}-\mathrm{solub}$ \\
$\mathrm{Hg}$ \\
$\mathrm{Pb}$ \\
$\mathrm{Se}$
\end{tabular}

Waste Type: Organic Iiquids (CH-Alpha)

Total Mass (kg/yr): $2.190 \mathrm{E}+03$

\begin{tabular}{ll} 
Chemical & \multicolumn{1}{c}{$\begin{array}{c}\text { Amount } \\
(\mathrm{kg} / \mathrm{Yr})\end{array}$} \\
\cline { 1 - 1 } $75-09-2$ & $2.978 \mathrm{E}+00$ \\
$\mathrm{As}$ & $6.322 \mathrm{E}-03$ \\
$\mathrm{Ba}$ & $2.709 \mathrm{E}-02$ \\
$\mathrm{Cd}$ & $2.409 \mathrm{E}+00$ \\
$\mathrm{Cl}-2-\mathrm{x}$ & $1.574 \mathrm{E}+00$ \\
$\mathrm{Cl}-3-\mathrm{x}$ & $1.718 \mathrm{E}+02$ \\
$\mathrm{Cl}-4-\mathrm{x}$ & $3.827 \mathrm{E}+01$ \\
$\mathrm{Cl}-\mathrm{F}-\mathrm{x}$ & $6.845 \mathrm{E}-02$ \\
$\mathrm{Cr}$ & $2.069 \mathrm{E}+00$ \\
$\mathrm{HC}-\mathrm{insO1}$ & $3.617 \mathrm{E}+02$ \\
$\mathrm{HC}-\mathrm{SOlub}$ & $3.237 \mathrm{E}+02$ \\
$\mathrm{Hg}$ & $1.355 \mathrm{E}-02$ \\
$\mathrm{~Pb}$ & $4.599 \mathrm{E}-01$
\end{tabular}

Waste Type: Solias (CH-Alpha)

$5.712 \mathrm{E}-03$

Total Mass $(\mathrm{kg} / \mathrm{Yr})$ :

$6.176 E+00$

\begin{tabular}{l} 
Chemical \\
\hline $75-09-2$ \\
$\mathrm{Ag}$ \\
$\mathrm{Ba}$ \\
$\mathrm{Cd}$ \\
$\mathrm{Cl}-2-\mathrm{x}$ \\
$\mathrm{Cl}-3-\mathrm{x}$ \\
$\mathrm{Cl}-4-\mathrm{x}$ \\
$\mathrm{Cl}-\mathrm{F}-\mathrm{x}$ \\
$\mathrm{Cr}$ \\
$\mathrm{HC}-\mathrm{insol}$ \\
$\mathrm{HC}-\mathrm{Solub}$ \\
$\mathrm{Hg}$ \\
$\mathrm{Pb}$ \\
$\mathrm{Se}$
\end{tabular}


Shipping Site: NNS

Total volume (m3/yr):

Radionuclide

$\mathrm{Ba}-137 \mathrm{~m}$

Co-60

Cs -134

Cs -137

Eu-154

$\mathrm{Eu}-155$

$\mathrm{Fe}-55$

$\mathrm{H}-3$

$\mathrm{Mn}-54$

$\mathrm{Nb}-94$

$\mathrm{Ni}-59$

$\mathrm{Ni}-63$

$\mathrm{Pa}-234 \mathrm{~m}$

$\mathrm{Pm}-147$

Pu-238

Pu-241

$\mathrm{Sb}-125$

Sm-151

Sr -90

$\mathrm{Te}-125 \mathrm{~m}$

Th-234

$\mathrm{U}-238$

$Y-90$

Shipping Site: UMC

Total Volume (m3/yr):

Radionuclice

Am-241

$\mathrm{Cm}-244$

$\mathrm{Pa}-234 \mathrm{~m}$

Pu-238

Pu-239

Pu-240

P1-241

Th -234

$\mathrm{U}-238$

Shipping site: WVDP

Total Volume (m3/yr) :

Radionuclide

Am-241

$\mathrm{Ba}-137 \mathrm{~m}$

Co-60

Cs -137

Eu-154

$\mathrm{H}-3$

$\mathrm{Nb}-94$

$\mathrm{Ni}-59$

$\mathrm{Ni}-63$

$\mathrm{Pa}-234 \mathrm{~m}$

Pu-238

Pu-239

Pu-240

Pu-241

$5 \mathrm{Sm}-151$

Sr -90

TC -99

Th-234

$\mathrm{U}-238$

$\mathrm{Y}-90$
Waste Type: Solids (CH-Non-alpha)

Total Mass $(\mathrm{kg} / \mathrm{yr}): \quad 3.925 E+02$

3. $560 E-01$

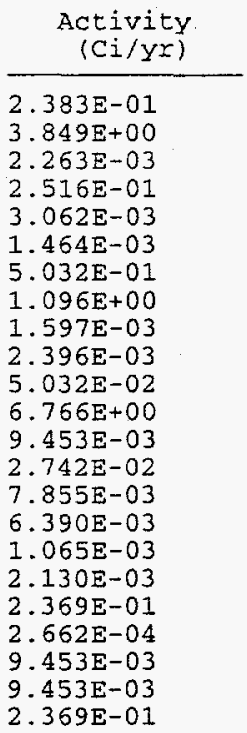

\begin{tabular}{l} 
Chemical \\
\hline $75-09-2$ \\
Ag \\
As \\
Ba \\
Cd \\
Cl-2-x \\
Cl-3-x \\
Cl-4-x \\
Cl-F-x \\
Cr \\
HC-insol \\
HC-Solub \\
$\mathrm{Hg}$ \\
$\mathrm{Pb}$ \\
$\mathrm{Se}$
\end{tabular}

\begin{tabular}{c}
$\begin{array}{c}\text { Amount } \\
(\mathrm{kg} / \mathrm{Yr})\end{array}$ \\
\hline $1.041 \mathrm{E}-01$ \\
$3.925 \mathrm{E}-03$ \\
$2.726 \mathrm{E}-03$ \\
$1.110 \mathrm{E}-01$ \\
$3.955 \mathrm{E}-02$ \\
$1.105 \mathrm{E}-01$ \\
$4.102 \mathrm{E}+00$ \\
$7.996 \mathrm{E}-01$ \\
$9.360 \mathrm{E}-02$ \\
$1.488 \mathrm{E}-01$ \\
$1.378 \mathrm{E}+01$ \\
$6.570 \mathrm{E}+00$ \\
$4.448 \mathrm{E}-02$ \\
$7.211 \mathrm{E}-02$ \\
$1.201 \mathrm{E}-02$
\end{tabular}

Waste Type: Solids (CH-Alpha)

Total Mass $(\mathrm{kg} / \mathrm{Yr}): \quad 5.809 \mathrm{E}+01$

$3.944 E-02$

\begin{tabular}{l} 
Chemical \\
\hline $57-12-5$ \\
$75-09-2$ \\
$\mathrm{Ag}$ \\
$\mathrm{As}$ \\
$\mathrm{Ba}$ \\
$\mathrm{Cd}$ \\
$\mathrm{CI}-2-\mathrm{x}$ \\
$\mathrm{CI}-3-\mathrm{x}$ \\
$\mathrm{Cl}-4-\mathrm{x}$ \\
$\mathrm{CI}-\mathrm{F}-\mathrm{x}$ \\
$\mathrm{Cr}$ \\
$\mathrm{HC}-$ insol \\
$\mathrm{HC}-$ solub \\
$\mathrm{Hg}$ \\
$\mathrm{Pb}$ \\
$\mathrm{Se}$
\end{tabular}

\begin{tabular}{c}
$\begin{array}{c}\text { Amount } \\
(\mathrm{kg} / \mathrm{Yr})\end{array}$ \\
\hline $9.876 \mathrm{E}-03$ \\
$1.161 \mathrm{E}-02$ \\
$1.742 \mathrm{E}-03$ \\
$5.809 \mathrm{E}-04$ \\
$1.161 \mathrm{E}-01$ \\
$2.904 \mathrm{E}-04$ \\
$1.161 \mathrm{E}-02$ \\
$3.253 \mathrm{E}-02$ \\
$2.033 \mathrm{E}-02$ \\
$5.809 \mathrm{E}-03$ \\
$1.161 \mathrm{E}-01$ \\
$5.809 \mathrm{E}-02$ \\
$2.904 \mathrm{E}-03$ \\
$5.809 \mathrm{E}-03$ \\
$2.904 \mathrm{E}-01$ \\
$1.742 \mathrm{E}-03$
\end{tabular}

Waste Type: Organic Liquids (CH-Alpha)

$1.773 \mathrm{E}+00$

Total Mass $(\mathrm{kg} / \mathrm{yr}): \quad 1.897 \mathrm{E}+03$

\begin{tabular}{l}
$\begin{array}{c}\text { Activity } \\
(\mathrm{Ci} / Y r)\end{array}$ \\
\hline $1.482 \mathrm{E}-03$ \\
$1.619 \mathrm{E}+00$ \\
$2.167 \mathrm{E}-03$ \\
$1.731 \mathrm{E}+00$ \\
$2.583 \mathrm{E}-03$ \\
$1.284 \mathrm{E}-01$ \\
$1.482 \mathrm{E}-04$ \\
$3.317 \mathrm{E}-03$ \\
$3.409 \mathrm{E}-01$ \\
$9.529 \mathrm{E}-04$ \\
$8.047 \mathrm{E}-02$ \\
$1.334 \mathrm{E}-03$ \\
$5.929 \mathrm{E}-04$ \\
$3.465 \mathrm{E}-02$ \\
$2.477 \mathrm{E}-02$ \\
$1.541 \mathrm{E}+00$ \\
$7.694 \mathrm{E}-04$ \\
$9.529 \mathrm{E}-04$ \\
$9.529 \mathrm{E}-04$ \\
$1.541 \mathrm{E}+00$
\end{tabular}

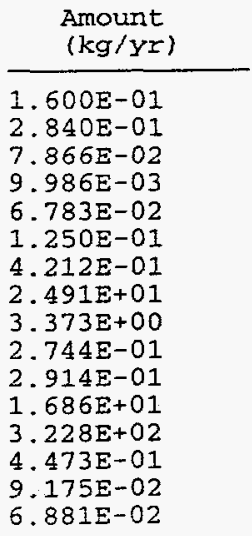




\section{Shipping Site: WVDP}

Total Volume (m3/yr):

Radionuclide

Am-241

$\mathrm{Ba}-137 \mathrm{~m}$

Co-60

Cs -137

Eu-154

$\mathrm{H}-3$

$\mathrm{Nb}-94$

$\mathrm{Ni}-59$

$\mathrm{Ni}-63$

$\mathrm{Pa}-234 \mathrm{~m}$

Pu-238

Pu-239

Pu-240

Pu-241

$\mathrm{sm}-151$

$\mathrm{Sr}-90$

TC-99

$\mathrm{Th}-234$

$\mathrm{U}-238$

$Y-90$

Waste Type: Solids (CH-Alpha)

Total Mass $(\mathrm{kg} / \mathrm{yr}): 1.355 \mathrm{E}+03$

$8.473 E-01$

Activity

$$
\text { (Ci/yr) }
$$

$7.096 \mathrm{E}-04$

$7.751 \mathrm{E}-01$

$1.037 \mathrm{E}-03$

$8.243 \mathrm{E}-01$

1. $236 \mathrm{E}-03$

$6.150 \mathrm{E}-02$

$7.096 \mathrm{E}-05$

$1.588 \mathrm{E}-03$

1. $632 \mathrm{E}-01$

$4.561 E-04$

$3.852 \mathrm{E}-02$

$6.386 \mathrm{E}-04$

$2.838 \mathrm{E}-04$

$1.559 \mathrm{E}-02$

$1.186 \mathrm{E}-02$

$7.339 \mathrm{E}-01$

$3.662 \mathrm{E}-04$

$4.561 \mathrm{E}-04$

$4.561 \mathrm{E}-04$

$7.339 \mathrm{E}-01$

\begin{tabular}{l} 
Chemical \\
\hline $57-12-5$ \\
$75-09-2$ \\
$\mathrm{Ag}$ \\
$\mathrm{As}$ \\
$\mathrm{Ba}$ \\
$\mathrm{Cd}$ \\
$\mathrm{Cl}-2-\mathrm{x}$ \\
$\mathrm{Cl}-3-\mathrm{x}$ \\
$\mathrm{Cl}-4-\mathrm{x}$ \\
$\mathrm{Cl}-\mathrm{F}-\mathrm{x}$ \\
$\mathrm{Cr}$ \\
$\mathrm{HC}-$ inso 1 \\
$\mathrm{HC}-$ solub \\
$\mathrm{Hg}$ \\
$\mathrm{Pb}$ \\
$\mathrm{Se}$
\end{tabular}

\begin{tabular}{c}
$\begin{array}{c}\text { Amount } \\
(\mathrm{kg} / \mathrm{Yr})\end{array}$ \\
\hline $1.069 \mathrm{E}-03$ \\
$2.958 \mathrm{E}-01$ \\
$1.709 \mathrm{E}-02$ \\
$1.081 \mathrm{E}-02$ \\
$3.475 \mathrm{E}-01$ \\
$1.558 \mathrm{E}-01$ \\
$2.803 \mathrm{E}-01$ \\
$1.573 \mathrm{E}+01$ \\
$2.998 \mathrm{E}+00$ \\
$1.176 \mathrm{E}-01$ \\
$5.922 \mathrm{E}-01$ \\
$5.230 \mathrm{E}+01$ \\
$2.619 \mathrm{E}+01$ \\
$1.760 \mathrm{E}-01$ \\
$2.784 \mathrm{E}-01$ \\
$4.680 \mathrm{E}-02$
\end{tabular}




\section{A.6 WM LLMW CENTRALIZED ALTERNATIVE (CASE 17)}

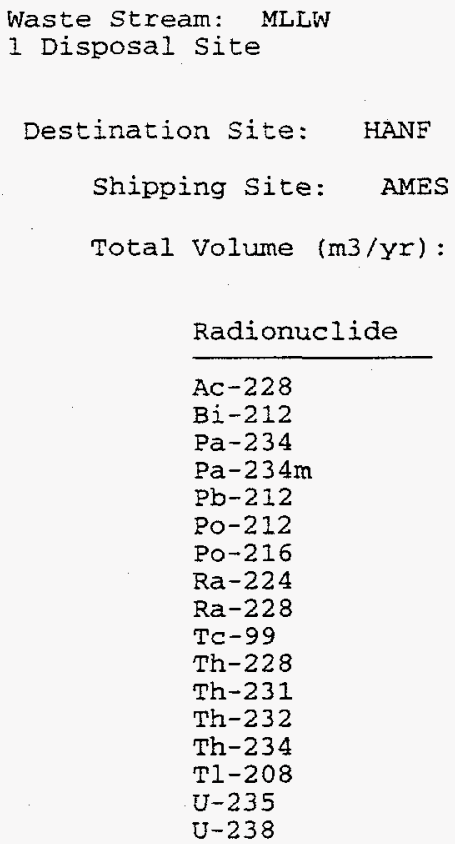

Shipping Site: ANL-E

Total Volume $(m 3 / y r)$ :

Radionuclide

$$
\begin{aligned}
& \mathrm{Am}-241 \\
& \mathrm{Ba}-137 \mathrm{~m} \\
& \mathrm{CO}-60 \\
& \mathrm{Cs}-137 \\
& \mathrm{Eu}-154 \\
& \mathrm{Eu}-155 \\
& \mathrm{H}-3 \\
& \mathrm{~N}-94 \\
& \mathrm{~N} i-59 \\
& \mathrm{~N}-63 \\
& \mathrm{~Pa}-234 \mathrm{~m} \\
& \mathrm{Pu}-238 \\
& \mathrm{Pu}-239 \\
& \mathrm{Pu}-240 \\
& \mathrm{Pu}-241 \\
& \mathrm{Sm}-151 \\
& \mathrm{Sr}-90 \\
& \mathrm{TC}-99 \\
& \mathrm{Th}-232 \\
& \mathrm{Th}-234 \\
& \mathrm{U}-238 \\
& \mathrm{Y}-90
\end{aligned}
$$

\begin{tabular}{|c|c|}
\hline Chemical & $\begin{array}{l}\text { Amount } \\
(\mathrm{kg} / \mathrm{yr})\end{array}$ \\
\hline $\begin{array}{l}57-12-5 \\
75-09-2 \\
\mathrm{Ag} \\
\mathrm{As} \\
\mathrm{Ba} \\
\mathrm{Cd} \\
\mathrm{Cl}-2-\mathrm{x} \\
\mathrm{Cl}-3-\mathrm{x} \\
\mathrm{Cl}-4-\mathrm{x} \\
\mathrm{Cl}-\mathrm{F}-\mathrm{x} \\
\mathrm{Cr} \\
\mathrm{HC}-\text { insol } \\
\mathrm{HC}-\text { solub } \\
\mathrm{Hg} \\
\mathrm{Pb} \\
\mathrm{Se}\end{array}$ & $\begin{array}{l}1.759 E-05 \\
1.485 E-04 \\
5.841 E-05 \\
7.025 E-07 \\
4.061 E-05 \\
5.957 E-05 \\
1.879 E-04 \\
4.658 E-04 \\
8.901 E-04 \\
7.426 E-05 \\
1.342 E-04 \\
1.917 E-05 \\
1.017 E-02 \\
5.535 E-05 \\
8.863 E-05 \\
1.196 E-06\end{array}$ \\
\hline
\end{tabular}

\begin{abstract}
Waste Type: Solids (CH-Non-alpha)
\end{abstract}
8. $585 \mathrm{E}-03$

Total Mass (kg/yr): $1.162 \mathrm{E}+01$
Waste Type: Solids ( $\mathrm{CH}$-Non-alpha)

\subsection{E+02 Total Mass $(\mathrm{kg} / \mathrm{Yr}): \quad 8.170 \mathrm{E}+05$}

\begin{tabular}{c}
$\begin{array}{c}\text { Activity } \\
\text { (Ci/yr) }\end{array}$ \\
\hline $2.380 \mathrm{E}-02$ \\
$3.504 \mathrm{E}+01$ \\
$1.304 \mathrm{E}+01$ \\
$3.747 \mathrm{E}+01$ \\
$1.388 \mathrm{E}-01$ \\
$4.761 \mathrm{E}-02$ \\
$2.699 \mathrm{E}+00$ \\
$9.919 \mathrm{E}-02$ \\
$2.104 \mathrm{E}+00$ \\
$2.214 \mathrm{E}+02$ \\
$4.796 \mathrm{E}+00$ \\
$1.474 \mathrm{E}+00$ \\
$2.380 \mathrm{E}-02$ \\
$3.571 \mathrm{E}-02$ \\
$1.561 \mathrm{E}+00$ \\
$4.519 \mathrm{E}-01$ \\
$3.313 \mathrm{E}+01$ \\
$3.963 \mathrm{E}-01$ \\
$3.967 \mathrm{E}-02$ \\
$4.796 \mathrm{E}+00$ \\
$4.796 \mathrm{E}+00$ \\
$3.313 \mathrm{E}+01$ \\
\end{tabular}

\begin{tabular}{l} 
Chemical \\
\hline $57-12-5$ \\
$75-09-2$ \\
Ag \\
As \\
Ba \\
Cd \\
Cl-2-x \\
Cl-3-x \\
Cl-4-x \\
Cl-F-x \\
Cr \\
HC-insol \\
HC-solub \\
Hg \\
Pb \\
Se
\end{tabular}

\begin{tabular}{c}
$\begin{array}{c}\text { Amount } \\
(\mathrm{kg} / \mathrm{Yr})\end{array}$ \\
\hline $2.002 \mathrm{E}+01$ \\
$2.778 \mathrm{E}+01$ \\
$1.623 \mathrm{E}+01$ \\
$6.714 \mathrm{E}+00$ \\
$2.781 \mathrm{E}+02$ \\
$9.989 \mathrm{E}+00$ \\
$1.822 \mathrm{E}+01$ \\
$3.658 \mathrm{E}+02$ \\
$4.608 \mathrm{E}+01$ \\
$1.759 \mathrm{E}+01$ \\
$3.334 \mathrm{E}+02$ \\
$1.482 \mathrm{E}+03$ \\
$7.360 \mathrm{E}+02$ \\
$1.110 \mathrm{E}+02$ \\
$4.704 \mathrm{E}+02$ \\
$4.660 \mathrm{E}+01$
\end{tabular}


Shipping Site: ANL-W

Total Volume (m3/yr):

Radionuclide

$\mathrm{Ba}-137 \mathrm{~m}$

Co-60

Cs -134

Cs -137

$\mathrm{Eu}-154$

$\mathrm{Eu}-155$

Fe- 55

$\mathrm{H}-3$

$\mathrm{Mn}-54$

$\mathrm{Nb}-94$

$\mathrm{Ni}-59$

$\mathrm{Ni}-63$

$\mathrm{Pa}-234 \mathrm{~m}$

$\mathrm{Pm}-147$

$\mathrm{Pu}-238$

$\mathrm{Pu}-241$

$\mathrm{Sb}-125$

$\mathrm{Sm}-151$

$\mathrm{Sr}-90$

$\mathrm{Te}-125 \mathrm{~m}$

Th-234

$\mathrm{U}-238$

$Y-90$

Shipping site: ANL-W

Total Volume (m3/yr):

Radionuclide

$\mathrm{Ba}-137 \mathrm{~m}$

Co-60

Cs -134

Cs -137

$\mathrm{Eu}-154$

Eu-155

Fe-55

$\mathrm{H}-3$

$\mathrm{Mn}-54$

$\mathrm{Nb}-94$

$\mathrm{Ni}-59$

$\mathrm{Ni}-63$

$\mathrm{Pa}-234 \mathrm{~m}$

Pm-147

Pu-238

$\mathrm{Pu}-241$

$\mathrm{Sb}-125$

Sm-151

Sr -90

$\mathrm{Te}-125 \mathrm{~m}$

Th -234

U-238

$\mathrm{Y}-90$
Waste Type: Organic Licuids (CH-Non-alpha)

Total Mass $(\mathrm{kg} / \mathrm{yr}): 1.643 \mathrm{E}+00$

$.434 \mathrm{E}-03$

\begin{tabular}{l} 
Chemical \\
\hline $57-12-5$ \\
$75-09-2$ \\
Ag \\
As \\
Ba \\
Cd \\
Cl-2-x \\
Cl-3-x \\
Cl-4-x \\
Cl-F-x \\
Cr \\
HC-insol \\
HC-Solub \\
Hg \\
Pb \\
Se
\end{tabular}

\begin{tabular}{c}
$\begin{array}{c}\text { Amount } \\
(\mathrm{kg} / \mathrm{yr})\end{array}$ \\
\hline $6.780 \mathrm{E}-05$ \\
$1.235 \mathrm{E}-03$ \\
$3.399 \mathrm{E}-05$ \\
$6.867 \mathrm{E}-06$ \\
$4.045 \mathrm{E}-05$ \\
$9.595 \mathrm{E}-04$ \\
$7.722 \mathrm{E}-04$ \\
$7.405 \mathrm{E}-02$ \\
$1.554 \mathrm{E}-02$ \\
$1.459 \mathrm{E}-04$ \\
$9.018 \mathrm{E}-04$ \\
$1.485 \mathrm{E}-01$ \\
$1.849 \mathrm{E}-01$ \\
$1.979 \mathrm{E}-04$ \\
$2.123 \mathrm{E}-04$ \\
$2.971 \mathrm{E}-05$
\end{tabular}

Waste Type: Solids (CH-Non-alpha)

Total Mass $(\mathrm{kg} / \mathrm{yr}): \quad 7.134 \mathrm{E}+02$

$.921 \mathrm{E}-01$

\begin{tabular}{l} 
Chemical \\
\hline $57-12-5$ \\
$75-09-2$ \\
$\mathrm{Ag}$ \\
$\mathrm{As}$ \\
$\mathrm{Ba}$ \\
$\mathrm{Cd}$ \\
$\mathrm{Cl}-2-\mathrm{x}$ \\
$\mathrm{Cl}-3-\mathrm{x}$ \\
$\mathrm{Cl}-4-\mathrm{-}$ \\
$\mathrm{Cl}-\mathrm{F}-\mathrm{x}$ \\
$\mathrm{Cr}$ \\
$\mathrm{HC}-$ insol \\
$\mathrm{HC}-\mathrm{solub}$ \\
$\mathrm{Hg}$ \\
$\mathrm{Pb}$ \\
$\mathrm{Se}$
\end{tabular}

\begin{tabular}{c}
$\begin{array}{c}\text { Amount } \\
(\mathrm{kg} / \mathrm{Yr})\end{array}$ \\
\hline $5.489 \mathrm{E}-02$ \\
$1.724 \mathrm{E}-01$ \\
$1.359 \mathrm{E}-02$ \\
$5.853 \mathrm{E}-03$ \\
$7.577 \mathrm{E}-01$ \\
$3.970 \mathrm{E}-02$ \\
$1.798 \mathrm{E}-01$ \\
$4.161 \mathrm{E}+00$ \\
$8.978 \mathrm{E}-01$ \\
$1.350 \mathrm{E}-01$ \\
$7.891 \mathrm{E}-01$ \\
$1.339 \mathrm{E}+01$ \\
$6.340 \mathrm{E}+00$ \\
$7.512 \mathrm{E}-02$ \\
$1.685 \mathrm{E}+00$ \\
$2.129 \mathrm{E}-02$
\end{tabular}


Shipping Site: BAPL

Total Volume $(\mathrm{m} 3 / \mathrm{yr})$ :

Radionuclide

Am-241

$\mathrm{Ba}-137 \mathrm{~m}$

Co- 60

Cs -137

Eu-154

$\mathrm{H}-3$

$\mathrm{Nb}-94$

$\mathrm{Ni}-59$

$\mathrm{Ni}-63$

$\mathrm{Pa}-234 \mathrm{~m}$

Pu-238

$\mathrm{Pu}-239$

$\mathrm{Pu}-240$

Pu-241

$\mathrm{Sm}-151$

Sr -90

Tc-99

Th-234

$\mathrm{U}-238$

$\mathrm{Y}-90$

Shipping Site: BAPL

Total Volume (m3/yr):

Radionuclide

Am -241

$\mathrm{Ba}-137 \mathrm{~m}$

Co-60

Cs-137

Eu-154

$\mathrm{H}-3$

$\mathrm{Nb}-94$

$\mathrm{Ni}-59$

Ni -63

$\mathrm{Pa}-234 \mathrm{~m}$

$\mathrm{Pu}-238$

Pu-239

Pu-240

Pu-241

Sm-151

$\mathrm{Sx}-90$

TC-99

Th-234

$\mathrm{U}-238$

$\mathrm{Y}-90$

Shipping Site: BAPL

Total Volume (m3/yr):

Radionuclide

Am-24I

$\mathrm{Ba}-137 \mathrm{~m}$

Co-60

Cs -137

$\mathrm{Eu}-154$

$\mathrm{H}-3$

$\mathrm{Nb}-94$

$\mathrm{Ni}-59$

$\mathrm{Ni}-63$

$\mathrm{Pa}-234 \mathrm{~m}$

Pu -238

Pu-239

Pu-240

Pu-241

Sm-151

Sr-90

TC-99

Th-234

$\mathrm{U}-238$

$\mathrm{Y}-90$
Waste Type: Organic Liquids (CH-Non-alpha)

$6.410 \mathrm{E}-02$

Total Mass (kg/yr):

$6.163 \mathrm{E}+01$

\begin{tabular}{c}
$\begin{array}{c}\text { Activity } \\
\text { (Ci/yr) }\end{array}$ \\
\hline $5.332 \mathrm{E}-05$ \\
$5.866 \mathrm{E}-02$ \\
$7.908 \mathrm{E}-05$ \\
$6.272 \mathrm{E}-02$ \\
$9.439 \mathrm{E}-05$ \\
$4.594 \mathrm{E}-03$ \\
$5.357 \mathrm{E}-06$ \\
$1.209 \mathrm{E}-04$ \\
$1.242 \mathrm{E}-02$ \\
$3.469 \mathrm{E}-05$ \\
$2.806 \mathrm{E}-03$ \\
$3.801 \mathrm{E}-05$ \\
$2.168 \mathrm{E}-05$ \\
$6.225 \mathrm{E}-04$ \\
$9.031 \mathrm{E}-04$ \\
$5.586 \mathrm{E}-02$ \\
$2.806 \mathrm{E}-05$ \\
$3.469 \mathrm{E}-05$ \\
$3.469 \mathrm{E}-05$ \\
$5.586 \mathrm{E}-02$
\end{tabular}

\begin{tabular}{l} 
Chemical \\
\hline $75-09-2$ \\
As \\
$\mathrm{Ba}$ \\
$\mathrm{Cd}$ \\
$\mathrm{Cl}-2-\mathrm{x}$ \\
$\mathrm{Cl}-3-\mathrm{x}$ \\
$\mathrm{Cl}-4-\mathrm{x}$ \\
$\mathrm{Cl}-\mathrm{F}-\mathrm{x}$ \\
$\mathrm{Cr}$ \\
$\mathrm{HC}-$ insol \\
$\mathrm{HC}-$ solub \\
$\mathrm{Hg}$ \\
$\mathrm{Pb}$
\end{tabular}

\begin{tabular}{c}
$\begin{array}{c}\text { Amount } \\
(\mathrm{kg} / \mathrm{Yr})\end{array}$ \\
\hline $5.934 \mathrm{E}-02$ \\
$4.724 \mathrm{E}-04$ \\
$2.024 \mathrm{E}-03$ \\
$6.779 \mathrm{E}-02$ \\
$3.091 \mathrm{E}-02$ \\
$4.931 \mathrm{E}-01$ \\
$1.055 \mathrm{E}-01$ \\
$1.360 \mathrm{E}-05$ \\
$5.671 \mathrm{E}-02$ \\
$2.699 \mathrm{E}+01$ \\
$8.592 \mathrm{E}+00$ \\
$3.698 \mathrm{E}-04$ \\
$1.294 \mathrm{E}-02$
\end{tabular}

Waste Type: Solids (CH-Non-alpha)

$6.105 E-01$

Total Mass (kg/yr):

$6.010 \mathrm{E}+02$

\begin{tabular}{c}
$\begin{array}{c}\text { Activity } \\
\text { (Ci/yr) }\end{array}$ \\
\hline $5.078 E-04$ \\
$5.587 E-01$ \\
$7.532 E-04$ \\
$5.974 E-01$ \\
$8.990 E-04$ \\
$4.471 E-02$ \\
$5.102 E-05$ \\
$1.151 E-03$ \\
$1.183 E-01$ \\
$3.304 E-04$ \\
$2.672 E-02$ \\
$3.620 E-04$ \\
$2.065 E-04$ \\
$5.929 E-03$ \\
$8.601 E-03$ \\
$5.321 E-01$ \\
$2.672 E-04$ \\
$3.304 E-04$ \\
$3.304 E-04$ \\
$5.321 E-01$
\end{tabular}

\begin{tabular}{l} 
Chemical \\
\hline $57-12-5$ \\
$75-09-2$ \\
Ag \\
As \\
Ba \\
Cd \\
Cl-2-x \\
Cl-3-x \\
Cl-4-x \\
Cl-F-x \\
Cr \\
HC-insol \\
HC-solub \\
Hg \\
$\mathrm{Pb}$ \\
Se
\end{tabular}

\begin{tabular}{c}
$\begin{array}{c}\text { Amount } \\
(\mathrm{kg} / \mathrm{yr})\end{array}$ \\
\hline $1.703 \mathrm{E}-03$ \\
$6.266 \mathrm{E}-02$ \\
$6.515 \mathrm{E}-03$ \\
$1.598 \mathrm{E}-03$ \\
$6.259 \mathrm{E}-02$ \\
$1.698 \mathrm{E}-02$ \\
$7.067 \mathrm{E}-02$ \\
$4.679 \mathrm{E}-01$ \\
$4.156 \mathrm{E}-01$ \\
$8.727 \mathrm{E}-02$ \\
$4.102 \mathrm{E}-02$ \\
$2.697 \mathrm{E}+00$ \\
$2.666 \mathrm{E}+00$ \\
$1.514 \mathrm{E}-02$ \\
$7.746 \mathrm{E}-02$ \\
$2.393 \mathrm{E}-03$
\end{tabular}

Waste Type: Solids (CH-Alpha)

$8.357 E-01$

Total Mass (kg/yr): $\quad 3.493 E+02$

\begin{tabular}{c}
$\begin{array}{c}\text { Activity } \\
\text { (Ci/yr) }\end{array}$ \\
\hline $6.984 \mathrm{E}-04$ \\
$7.629 \mathrm{E}-01$ \\
$1.021 \mathrm{E}-03$ \\
$8.160 \mathrm{E}-01$ \\
$1.217 \mathrm{E}-03$ \\
$6.053 \mathrm{E}-02$ \\
$6.984 \mathrm{E}-05$ \\
$1.563 \mathrm{E}-03$ \\
$1.606 \mathrm{E}-01$ \\
$4.490 \mathrm{E}-04$ \\
$3.791 \mathrm{E}-02$ \\
$6.286 \mathrm{E}-04$ \\
$2.793 \mathrm{E}-04$ \\
$1.633 \mathrm{E}-02$ \\
$1.167 \mathrm{E}-02$ \\
$7.264 \mathrm{E}-01$ \\
$3.625 \mathrm{E}-04$ \\
$4.490 \mathrm{E}-04$ \\
$4.490 \mathrm{E}-04$ \\
$7.264 \mathrm{E}-01$
\end{tabular}

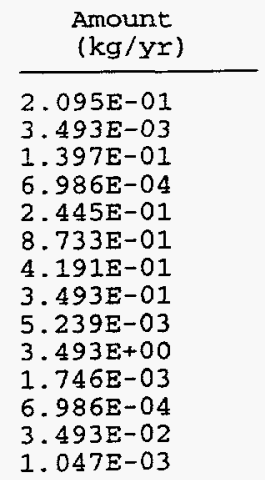


Shipping Site: BATELLE

Waste Type: Solids (CH-Non-alpha)

Total Volume (m3/yr):

$1.500 \mathrm{E}-03$

Total Mass (kg/yr): $1.977 \mathrm{E}+00$

Radionuclide

Ac -228

$\mathrm{Bi}-212$

$\mathrm{Pa}-234$

$\mathrm{Pa}-234 \mathrm{~m}$

$\mathrm{Pb}-212$

Po-212

Po- 216

$\mathrm{Ra}-224$

$\mathrm{Ra}-228$

TC-99

Th-228

Th-231

$\mathrm{Th}-232$

Th-234

TI-208

$\mathrm{U}-235$

$\mathrm{U}-238$

Shipping site:

BNL

Total Volume (m3/yr):

Radionuclide

Am-241

$\mathrm{Ba}-137 \mathrm{~m}$

Co- 60

$\mathrm{Cs}-137$

Eu-154

Eน-155

H-3

$\mathrm{Nb}-94$

$\mathrm{Ni}-59$

$\mathrm{Ni}-63$

$\mathrm{Pa}-234 \mathrm{~m}$

Pu-238

Pu-239

PU-240

Pu-241

$\mathrm{Sm}-151$

Sr -90

TC-99

Th -232

$\mathrm{Th}-234$

U-238

$\mathrm{Y}-90$

Shipping site: BNL

Total Volume (m3/yr) :

Radionuclide

Am-241

$\mathrm{Ba}-137 \mathrm{~m}$

Co-60

Cs -137

Eu-154

Eu- 155

$\mathrm{H}-3$

Nb-94

$\mathrm{Ni}-59$

$\mathrm{Ni}-63$

Pa-234m

Pu-238

Pu-239

Pu-240

Pu-241

$\mathrm{Sm}-151$

$\mathrm{S} x-90$

Tc-99

Th-232

Th-234

$\mathrm{U}-238$

$\mathrm{Y}-90$

\begin{tabular}{r}
$\begin{array}{c}\text { Activity } \\
(\mathrm{Ci} / \mathrm{Y})\end{array}$ \\
\hline $3.211 \mathrm{E}-09$ \\
$5.400 \mathrm{E}-10$ \\
$4.080 \mathrm{E}-10$ \\
$3.964 \mathrm{E}-06$ \\
$5.400 \mathrm{E}-10$ \\
$3.480 \mathrm{E}-10$ \\
$5.400 \mathrm{E}-10$ \\
$5.400 \mathrm{E}-10$ \\
$3.211 \mathrm{E}-09$ \\
$5.971 \mathrm{E}-08$ \\
$5.400 \mathrm{E}-10$ \\
$3.097 \mathrm{E}-09$ \\
$3.260 \mathrm{E}-08$ \\
$3.964 \mathrm{E}-06$ \\
$2.040 \mathrm{E}-10$ \\
$3.085 \mathrm{E}-09$ \\
$3.964 \mathrm{E}-06$
\end{tabular}

\begin{tabular}{lll}
\multicolumn{1}{c}{ Chemical } & & $\begin{array}{c}\text { Amount } \\
(\mathrm{kg} / \mathrm{y} r)\end{array}$ \\
$75-09-2$ & & $3.955 \mathrm{E}-02$ \\
C1-2-x & & $1.977 \mathrm{E}-02$ \\
$\mathrm{C} 1-3-\mathrm{x}$ & & $1.977 \mathrm{E}-01$ \\
$\mathrm{Cl}-4-\mathrm{x}$ & $1.582 \mathrm{E}-01$ \\
HC-insol & & $4.943 \mathrm{E}-01$ \\
HC-solub & $2.373 \mathrm{E}-01$
\end{tabular}

Waste Type: Organic Liquids ( $\mathrm{CH}$-Non-alpha)

$1.544 \mathrm{E}+00 \quad$ Total Mass $(\mathrm{kg} / \mathrm{yr}): \quad 1.749 \mathrm{E}+03$

\begin{tabular}{r}
$\begin{array}{c}\text { Activity } \\
\text { (Ci/yr) }\end{array}$ \\
\hline $7.598 \mathrm{E}-05$ \\
$1.118 \mathrm{E}-01$ \\
$4.162 \mathrm{E}-02$ \\
$1.196 \mathrm{E}-01$ \\
$4.432 \mathrm{E}-04$ \\
$1.519 \mathrm{E}-04$ \\
$8.617 \mathrm{E}-03$ \\
$3.166 \mathrm{E}-04$ \\
$6.718 \mathrm{E}-03$ \\
$7.068 \mathrm{E}-01$ \\
$1.530 \mathrm{E}-02$ \\
$4.706 \mathrm{E}-03$ \\
$7.598 \mathrm{E}-05$ \\
$1.139 \mathrm{E}-04$ \\
$4.984 \mathrm{E}-03$ \\
$1.442 \mathrm{E}-03$ \\
$1.057 \mathrm{E}-01$ \\
$1.265 \mathrm{E}-03$ \\
$1.266 \mathrm{E}-04$ \\
$1.530 \mathrm{E}-02$ \\
$1.530 \mathrm{E}-02$ \\
$1.057 \mathrm{E}-01$ \\
\end{tabular}

\begin{tabular}{l} 
Chemical \\
\hline $57-12-5$ \\
$75-09-2$ \\
Ag \\
As \\
$\mathrm{Ba}$ \\
$\mathrm{Cd}$ \\
$\mathrm{Cl}-2-\mathrm{x}$ \\
$\mathrm{Cl}-3-\mathrm{x}$ \\
$\mathrm{Cl}-4-\mathrm{x}$ \\
$\mathrm{C} 1-\mathrm{F}-\mathrm{x}$ \\
$\mathrm{Cr}$ \\
$\mathrm{HC}-\mathrm{insol}$ \\
$\mathrm{HC}-\mathrm{sol}$ lub \\
$\mathrm{Hg}$ \\
$\mathrm{Pb}$ \\
$\mathrm{Se}$
\end{tabular}

\begin{tabular}{c}
$\begin{array}{c}\text { Anount } \\
(\mathrm{kg} / \mathrm{Yr})\end{array}$ \\
\hline $8.298 \mathrm{E}-02$ \\
$1.156 \mathrm{E}+00$ \\
$4.159 \mathrm{E}-02$ \\
$7.649 \mathrm{E}-03$ \\
$4.627 \mathrm{E}-02$ \\
$8.868 \mathrm{E}-01$ \\
$7.572 \mathrm{E}-01$ \\
$7.014 \mathrm{E}+01$ \\
$1.445 \mathrm{E}+01$ \\
$1.704 \mathrm{E}-01$ \\
$8.567 \mathrm{E}-01$ \\
$1.386 \mathrm{E}+02$ \\
$2.115 \mathrm{E}+02$ \\
$2.406 \mathrm{E}-01$ \\
$2.050 \mathrm{E}-01$ \\
$3.636 \mathrm{E}-02$
\end{tabular}

Waste Type: Solids (CH-Non-alpha)

$8.915 E+00$

Total Mass (kg/yr): $1.190 E+04$

$\begin{array}{r}\text { Activity } \\ \text { (Ci/yr) } \\ \hline\end{array}$

4. 386E-04

$6.457 \mathrm{E}-01$

2. $402 \mathrm{E}-01$

$6.904 E-01$

2. $558 \mathrm{E}-03$

8.772E-04

$4.974 \mathrm{E}-02$

1. $827 \mathrm{E}-03$

$3.878 \mathrm{E}-02$

$4.080 \mathrm{E}+00$

8. $837 \mathrm{E}-02$

2. $716 \mathrm{E}-02$

$4.386 E-04$

$6.579 \mathrm{E}-04$

2. $877 \mathrm{E}-02$

8. 327E-03

6. $105 \mathrm{E}-01$

7. $303 \mathrm{E}-03$

7. 310 $\mathrm{E}-04$

8. $837 \mathrm{E}-02$

8. $837 \mathrm{E}-02$

6. $105 \mathrm{E}-01$

\begin{tabular}{l} 
Chemical \\
\hline $57-12-5$ \\
$75-09-2$ \\
$\mathrm{Ag}$ \\
$\mathrm{As}$ \\
$\mathrm{Ba}$ \\
$\mathrm{Cd}$ \\
$\mathrm{Cl}-2-\mathrm{x}$ \\
$\mathrm{Cl}-3-\mathrm{x}$ \\
$\mathrm{Cl}-4-\mathrm{x}$ \\
$\mathrm{Cl}-\mathrm{F}-\mathrm{x}$ \\
$\mathrm{Cr}$ \\
$\mathrm{HC}-$ insol \\
$\mathrm{HC}-\mathrm{solub}$ \\
$\mathrm{Hg}$ \\
$\mathrm{Pb}$ \\
$\mathrm{Se}$
\end{tabular}

\begin{tabular}{c}
$\begin{array}{c}\text { Amount } \\
(\mathrm{kg} / \mathrm{Yr})\end{array}$ \\
\hline $1.617 \mathrm{E}+00$ \\
$8.722 \mathrm{E}-01$ \\
$7.571 \mathrm{E}-01$ \\
$2.698 \mathrm{E}-01$ \\
$2.157 \mathrm{E}+01$ \\
$4.530 \mathrm{E}-01$ \\
$8.722 \mathrm{E}-01$ \\
$2.419 \mathrm{E}+01$ \\
$3.963 \mathrm{E}-01$ \\
$1.019 \mathrm{E}+00$ \\
$2.698 \mathrm{E}+01$ \\
$1.091 \mathrm{E}+02$ \\
$5.459 \mathrm{E}+01$ \\
$8.531 \mathrm{E}+00$ \\
$2.912 \mathrm{E}+01$ \\
$3.668 \mathrm{E}+00$
\end{tabular}


Shipping Site: CNS

Total Volume $(\mathrm{m} 3 / \mathrm{yr})$ :

Radionuclide

$\mathrm{Ba}-137 \mathrm{~m}$

Co- 60

Cs -134

Cs -137

$\mathrm{Eu}-154$

Eu-155

$\mathrm{Fe}-55$

$\mathrm{H}-3$

$\mathrm{Mn}-54$

$\mathrm{Nb}-94$

$\mathrm{Ni}-59$

$\mathrm{Ni}-63$

Pa-234m

Pm-147

Pu-238

$\mathrm{Pu}-241$

$\mathrm{Sb}-125$

Sm-151

Sr -90

$\mathrm{Te}-125 \mathrm{~m}$

$\mathrm{Th}-234$

$\mathrm{U}-238$

$Y-90$

Shipping site: CNS

Total Volume $(\mathrm{m} 3 / \mathrm{yr})$ :

Radionuclide

$\mathrm{Ba}-137 \mathrm{~m}$

Co- 60

Cs -134

Cs -137

$\mathrm{Eu}-154$

Eu-155

$\mathrm{Fe}-55$

$\mathrm{H}-3$

$\mathrm{Mn}-54$

$\mathrm{Nb}-94$

$\mathrm{Ni}-59$

$\mathrm{Ni}-63$

$\mathrm{Pa}-234 \mathrm{~m}$

Pm-147

Pu-238

Pu-241

$\mathrm{Sb}-125$

Sm-151

Sr -90

Te-125m

Th-234

$\mathrm{U}-238$

$Y-90$
Waste Type: Organic Liquids (CH-Non-alpha)

$9.054 \mathrm{E}-01$

Total Mass (kg/yr):

$1.026 \mathrm{E}+03$

\begin{tabular}{r}
$\begin{array}{r}\text { Activity } \\
(\mathrm{Ci} / \mathrm{yr})\end{array}$ \\
\hline $6.061 \mathrm{E}-01$ \\
$9.790 \mathrm{E}+00$ \\
$5.756 \mathrm{E}-03$ \\
$6.400 \mathrm{E}-01$ \\
$7.788 \mathrm{E}-03$ \\
$3.724 \mathrm{E}-03$ \\
$1.280 \mathrm{E}+00$ \\
$2.788 \mathrm{E}+00$ \\
$4.063 \mathrm{E}-03$ \\
$6.095 \mathrm{E}-03$ \\
$1.280 \mathrm{E}-01$ \\
$1.720 \mathrm{E}+01$ \\
$2.404 \mathrm{E}-02$ \\
$6.975 \mathrm{E}-02$ \\
$1.997 \mathrm{E}-02$ \\
$1.625 \mathrm{E}-02$ \\
$2.709 \mathrm{E}-03$ \\
$5.418 \mathrm{E}-03$ \\
$6.027 \mathrm{E}-01$ \\
$6.772 \mathrm{E}-04$ \\
$2.404 \mathrm{E}-02$ \\
$2.404 \mathrm{E}-02$ \\
$6.027 \mathrm{E}-01$. \\
\end{tabular}

\begin{tabular}{l} 
Chemical \\
\hline $57-12-5$ \\
$75-09-2$ \\
$\mathrm{Ag}$ \\
$\mathrm{As}$ \\
$\mathrm{Ba}$ \\
$\mathrm{Cd}$ \\
$\mathrm{Cl}-2-\mathrm{x}$ \\
$\mathrm{C} 1-3-\mathrm{x}$ \\
$\mathrm{Cl}-4-\mathrm{x}$ \\
$\mathrm{Cl}-\mathrm{F}-\mathrm{x}$ \\
$\mathrm{Cr}$ \\
$\mathrm{HC}-\mathrm{insol}$ \\
$\mathrm{HC}-\mathrm{sol}$ lub \\
$\mathrm{Hg}$ \\
$\mathrm{Pb}$ \\
$\mathrm{Se}$
\end{tabular}

\begin{tabular}{c}
$\begin{array}{c}\text { Amount } \\
(\mathrm{kg} / \mathrm{Y})\end{array}$ \\
\hline $5.151 E-02$ \\
$7.241 E-01$ \\
$2.274 E-02$ \\
$3.999 E-03$ \\
$2.356 E-02$ \\
$5.620 E-01$ \\
$4.522 E-01$ \\
$4.355 E+01$ \\
$9.136 E+00$ \\
$8.522 E-02$ \\
$5.418 E-01$ \\
$8.707 E+01$ \\
$1.317 E+02$ \\
$1.340 E-01$ \\
$1.275 E-01$ \\
$1.998 E-02$
\end{tabular}

Waste Type: Solids (CH-Non-alpha)

$9.030 \mathrm{E}-02$ Total Mass $(\mathrm{kg} / \mathrm{yr}): \quad 6.772 \mathrm{E}+01$

\begin{tabular}{r}
$\begin{array}{r}\text { Activity } \\
(\mathrm{C} / \mathrm{Y} \text { ) }\end{array}$ \\
\hline $6.218 E-02$ \\
$1.004 \mathrm{E}+00$ \\
$5.431 \mathrm{E}-04$ \\
$6.038 \mathrm{E}-02$ \\
$7.990 \mathrm{E}-04$ \\
$3.821 \mathrm{E}-04$ \\
$1.313 \mathrm{E}-01$ \\
$2.860 \mathrm{E}-01$ \\
$4.169 \mathrm{E}-04$ \\
$6.253 \mathrm{E}-04$ \\
$1.313 \mathrm{E}-02$ \\
$1.765 \mathrm{E}+00$ \\
$2.466 \mathrm{E}-03$ \\
$7.156 \mathrm{E}-03$ \\
$2.049 \mathrm{E}-03$ \\
$1.667 \mathrm{E}-03$ \\
$2.779 \mathrm{E}-04$ \\
$5.558 \mathrm{E}-04$ \\
$5.686 \mathrm{E}-02$ \\
$6.948 \mathrm{E}-05$ \\
$2.466 \mathrm{E}-03$ \\
$2.466 \mathrm{E}-03$ \\
$5.686 \mathrm{E}-02$
\end{tabular}

\begin{tabular}{|c|c|}
\hline Chemical & $\begin{array}{l}\text { Amount } \\
(\mathrm{kg} / \mathrm{yr})\end{array}$ \\
\hline $57-12-5$ & $2.357 \mathrm{E}-03$ \\
\hline $75-09-2$ & $2.142 \mathrm{E}-02$ \\
\hline Ag & $7.345 E-04$ \\
\hline As & $2.549 \mathrm{E}-04$ \\
\hline $\mathrm{Ba}$ & $3.376 \bar{E}-02$ \\
\hline Ca & $2.213 \mathrm{E}-03$ \\
\hline Cl-2-x & $2.738 \varepsilon-02$ \\
\hline CI $-3-x$ & $2.718 \mathrm{E}-01$ \\
\hline$C 1-4-x$ & $7.524 \mathrm{E}-02$ \\
\hline $\mathrm{Cl}-\mathrm{F}-\mathrm{x}$ & $2.847 \mathrm{E}-02$ \\
\hline $\mathrm{Cr}$ & $2.755 E-02$ \\
\hline HC-insol & $9.141 \mathrm{E}-01$ \\
\hline HC-solub & $3.299 \mathrm{E}-01$ \\
\hline $\mathrm{Hg}$ & $3.234 E-03$ \\
\hline $\mathrm{Pb}$ & $5.496 \mathrm{E}-02$ \\
\hline $\mathrm{Se}$ & $9.647 \mathrm{E}-04$ \\
\hline
\end{tabular}


Shipping Site: COLONIE.

Waste Type: Organic Liquids (CH-Non-alpha)

Total Volume (m3/yr):

$9.054 \mathrm{E}-01$

Total Mass $(\mathrm{kg} / \mathrm{yr}): \quad 1.026 \mathrm{E}+03$

Radionuclide

Ac -228

$\mathrm{Bi}-212$

$\mathrm{Pa}-234$

$\mathrm{Pa}-234 \mathrm{~m}$

$\mathrm{Pb}-212$

$\mathrm{PO}-212$

Po-216

$\mathrm{Ra}-224$

Ra -228

TC -99

Th-228

Th-231

Th-232

Th-234

TI-208

$\mathrm{U}-235$

$\mathrm{U}-238$

\begin{tabular}{c}
$\begin{array}{c}\text { Activity } \\
(\text { Ci/yr) }\end{array}$ \\
\hline $1.937 E-06$ \\
$3.259 E-07$ \\
$2.462 E-07$ \\
$2.392 E-03$ \\
$3.259 E-07$ \\
$2.100 E-07$ \\
$3.259 E-07$ \\
$3.259 E-07$ \\
$1.937 E-06$ \\
$3.603 E-05$ \\
$3.259 E-07$ \\
$1.868 E-06$ \\
$1.967 E-05$ \\
$2.392 E-03$ \\
$1.231 E-07$ \\
$1.861 E-06$ \\
$2.392 E-03$
\end{tabular}

\begin{tabular}{l} 
Chemical \\
\hline $57-12-5$ \\
$75-09-2$ \\
Ag \\
As \\
Ba \\
Cd \\
Cl-2-x \\
Cl-3-x \\
Cl-4-x \\
Cl-F-x \\
Cr \\
$\mathrm{HC}-$ insol \\
HC-solub \\
$\mathrm{Hg}$ \\
$\mathrm{Pb}$ \\
$\mathrm{Se}$
\end{tabular}

\begin{tabular}{l} 
Shipping Site: CoLO \\
Total Volume (m3/yr) \\
Radionuclide \\
\hline Ac-228 \\
Bi-212 \\
Pa-234 \\
Pa-234m \\
Pb-212 \\
Po-212 \\
Po-216 \\
Ra -224 \\
Ra -228 \\
Tc -99 \\
Th-228 \\
Th-231 \\
Th-232 \\
Th-234 \\
Tl-208 \\
$\mathrm{U}-235$ \\
$\mathrm{U}-238$
\end{tabular}

Shipping site: ETEC Total Volume (m3/yr):

Radionuclide

Am-241

$\mathrm{Ba}-137 \mathrm{~m}$

Co-60

Cs -137

Eu-154

Eu-155

$\mathrm{H}-3$

$\mathrm{Nb}-94$

$\mathrm{Ni}-59$

$\mathrm{Ni}-63$

Pa-234m

Pu-238

Pu-239

PUI-240

Pu-241

Sm-151

Sr -90

TC-99

Th-232

Th-234

$\mathrm{U}-238$

$Y-90$
Waste Type: Solids (CH-Non-alpha)

Total Mass $(\mathrm{kg} / \mathrm{Yr}): \quad 1.606 \mathrm{E}+01$

\begin{tabular}{r}
$\begin{array}{r}\text { Activity } \\
\text { (Ci/yr) }\end{array}$ \\
\hline $3.198 \mathrm{E}-08$ \\
$5.380 \mathrm{E}-09$ \\
$4.065 \mathrm{E}-09$ \\
$3.949 \mathrm{E}-05$ \\
$5.380 \mathrm{E}-09$ \\
$3.467 \mathrm{E}-09$ \\
$5.380 \mathrm{E}-09$ \\
$2.690 \mathrm{E}-09$ \\
$1.599 \mathrm{E}-08$ \\
$2.974 \mathrm{E}-07$ \\
$5.380 \mathrm{E}-09$ \\
$3.084 \mathrm{E}-08$ \\
$3.247 \mathrm{E}-07$ \\
$3.949 \mathrm{E}-05$ \\
$2.032 \mathrm{E}-09$ \\
$3.072 \mathrm{E}-08$ \\
$3.949 \mathrm{E}-05$
\end{tabular}

\begin{tabular}{l} 
Chemical \\
\hline $57-12-5$ \\
$75-09-2$ \\
Ag \\
As \\
$\mathrm{Ba}$ \\
$\mathrm{Cd}$ \\
$\mathrm{Cl}-2-\mathrm{x}$ \\
$\mathrm{CI}-3-\mathrm{x}$ \\
$\mathrm{CI}-4-\mathrm{x}$ \\
$\mathrm{Cl}-\mathrm{F}-\mathrm{x}$ \\
$\mathrm{Cr}$ \\
$\mathrm{HC}-$ insol \\
$\mathrm{HC}-\mathrm{Sol}$ - b \\
$\mathrm{Hg}$ \\
$\mathrm{Pb}$ \\
$\mathrm{Se}$
\end{tabular}

\begin{tabular}{c}
$\begin{array}{c}\text { Amount } \\
(\mathrm{kg} / \mathrm{Yr})\end{array}$ \\
\hline $7.229 \mathrm{E}-04$ \\
$8.484 \mathrm{E}-04$ \\
$2.570 \mathrm{E}-05$ \\
$2.249 \mathrm{E}-05$ \\
$1.506 \mathrm{E}-05$ \\
$1.429 \mathrm{E}-04$ \\
$4.242 \mathrm{E}-03$ \\
$5.345 \mathrm{E}-03$ \\
$5.006 \mathrm{E}-03$ \\
$4.242 \mathrm{E}-04$ \\
$5.462 \mathrm{E}-04$ \\
$9.333 \mathrm{E}-04$ \\
$8.032 \mathrm{E}-04$ \\
$3.695 \mathrm{E}-06$ \\
$1.044 \mathrm{E}-03$ \\
$8.032 \mathrm{E}-06$
\end{tabular}

Waste Type: Organic Liquids. (CH-Non-alpha)

$6.767 E-03$

Total Mass $(\mathrm{kg} / \mathrm{yr}): \quad 7.145 \mathrm{E}+00$

\begin{tabular}{c}
$\begin{array}{r}\text { Activity } \\
(C i / y+)\end{array}$ \\
\hline $3.329 E-07$ \\
$4.901 E-04$ \\
$1.823 E-04$ \\
$5.241 E-04$ \\
$1.942 E-06$ \\
$6.659 E-07$ \\
$3.775 E-05$ \\
$1.387 E-06$ \\
$2.943 E-05$ \\
$3.097 E-03$ \\
$6.707 E-05$ \\
$2.062 E-05$ \\
$3.329 E-07$ \\
$4.994 E-07$ \\
$2.184 E-05$ \\
$6.320 E-06$ \\
$4.634 E-04$ \\
$5.543 E-06$ \\
$5.549 E-07$ \\
$6.707 E-05$ \\
$6.707 E-05$ \\
$4.634 E-04$
\end{tabular}

\begin{tabular}{c}
$\begin{array}{c}\text { Amount } \\
(\mathrm{kg} / \mathrm{Y})\end{array}$ \\
\hline $6.272 \mathrm{E}-04$ \\
$4.759 \mathrm{E}-04$ \\
$3.144 \mathrm{E}-04$ \\
$4.027 \mathrm{E}-05$ \\
$2.745 \mathrm{E}-04$ \\
$1.707 \mathrm{E}-05$ \\
$1.354 \mathrm{E}-03$ \\
$5.331 \mathrm{E}-02$ \\
$3.012 \mathrm{E}-03$ \\
$1.098 \mathrm{E}-03$ \\
$7.317 \mathrm{E}-04$ \\
$4.382 \mathrm{E}-02$ \\
$1.255 \mathrm{E}+00$ \\
$1.781 \mathrm{E}-03$ \\
$2.730 \mathrm{E}-04$ \\
$2.748 \mathrm{E}-04$
\end{tabular}




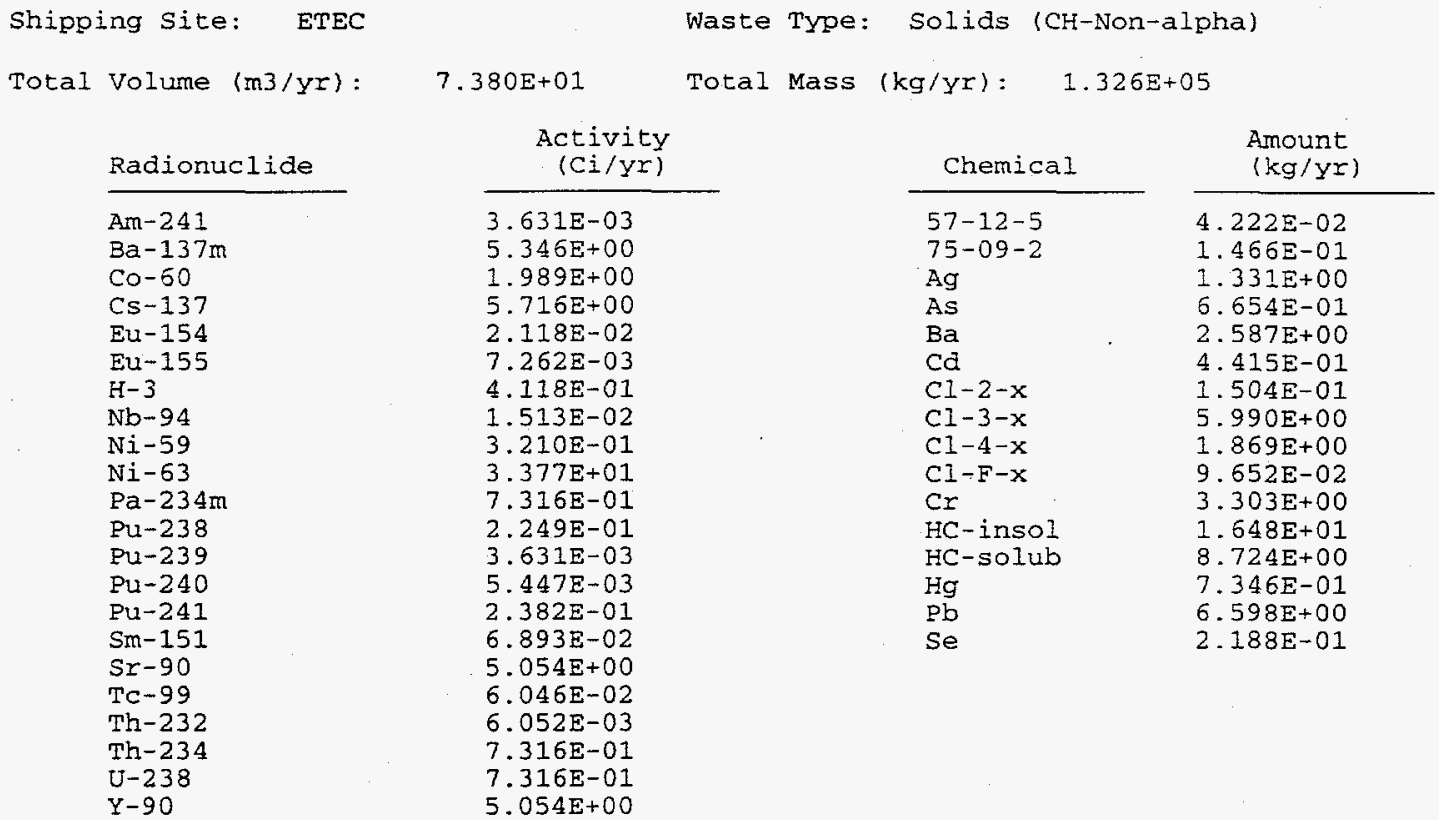

Shipping site: FEMP

Waste Type: Organic Liquids (CH-Non-alpha)

Total Volume $(\mathrm{m} 3 / \mathrm{yr})$ :

$2.034 E+01$

Total Mass (kg/Yr): $2.423 \mathrm{E}+04$

Radionuclide

AC -228

$B i-212$

$\mathrm{Pa}-234$

$\mathrm{Pa}-234 \mathrm{~m}$

$\mathrm{Pb}-212$

PO- 212

Po-216

$\mathrm{Ra}-224$

$\mathrm{Ra}-228$

TC-99

Th-228

Th-231

Th -232

Th-234

T1-208

$\mathrm{U}-235$

U-238

Shipping Site:

FEMP

Total Volume $(\mathrm{m} 3 / \mathrm{yr})$ :

Radionuclide

Ac- 228

$\mathrm{Bi}-212$

$\mathrm{Pa}-234$

$\mathrm{Pa}-234 \mathrm{~m}$

$\mathrm{Pb}-212$

Po-212

ㅇo-216

$\mathrm{Ra}-224$

$\mathrm{Ra}-228$

TC-99

Th-228

Th-231

Th-232

Th-234

T1-208

$\mathrm{U}-235$

$\mathrm{U}-238$

\begin{tabular}{c}
$\begin{array}{r}\text { Activity } \\
\text { (Ci/yr) }\end{array}$ \\
\hline $4.353 \mathrm{E}-05$ \\
$7.324 \mathrm{E}-06$ \\
$5.533 \mathrm{E}-06$ \\
$5.376 \mathrm{E}-02$ \\
$7.324 \mathrm{E}-06$ \\
$4.720 \mathrm{E}-06$ \\
$7.324 \mathrm{E}-06$ \\
$7.324 \mathrm{E}-06$ \\
$4.353 \mathrm{E}-05$ \\
$8.097 \mathrm{E}-04$ \\
$7.324 \mathrm{E}-06$ \\
$4.199 \mathrm{E}-05$ \\
$4.420 \mathrm{E}-04$ \\
$5.376 \mathrm{E}-02$ \\
$2.766 \mathrm{E}-06$ \\
$4.182 \mathrm{E}-05$ \\
$5.376 \mathrm{E}-02$
\end{tabular}

\begin{tabular}{l} 
Chemical \\
\hline $57-12-5$ \\
$75-09-2$ \\
Ag \\
As \\
$\mathrm{Ba}$ \\
$\mathrm{Cd}$ \\
$\mathrm{Cl}-2-\mathrm{x}$ \\
$\mathrm{Cl}-3-\mathrm{x}$ \\
$\mathrm{Cl}-4-\mathrm{x}$ \\
$\mathrm{Cl}-\mathrm{F}-\mathrm{x}$ \\
$\mathrm{Cr}$ \\
$\mathrm{HC}-\mathrm{ins} 1$ \\
HC-solub \\
Hg \\
$\mathrm{Pb}$ \\
$\mathrm{Se}$
\end{tabular}

Waste Type: Solids (CH-Non-alpha)

$7.909 \mathrm{E}+01$

Total Mass $(\mathrm{kg} / \mathrm{yr}): 1.180 \mathrm{E}+05$

\begin{tabular}{c}
$\begin{array}{c}\text { Activity } \\
\text { (Ci/yr) }\end{array}$ \\
\hline $1.696 \mathrm{E}-04$ \\
$2.854 \mathrm{E}-05$ \\
$2.156 \mathrm{E}-05$ \\
$2.095 \mathrm{E}-01$ \\
$2.854 \mathrm{E}-05$ \\
$1.839 \mathrm{E}-05$ \\
$2.854 \mathrm{E}-05$ \\
$2.837 \mathrm{E}-05$ \\
$1.686 \mathrm{E}-04$ \\
$3.136 \mathrm{E}-03$ \\
$2.854 \mathrm{E}-05$ \\
$1.636 \mathrm{E}-04$ \\
$1.722 \mathrm{E}-03$ \\
$2.095 \mathrm{E}-01$ \\
$1.078 \mathrm{E}-05$ \\
$1.630 \mathrm{E}-04$ \\
$2.095 \mathrm{E}-01$
\end{tabular}

\begin{tabular}{l} 
Chemical \\
\hline $57-12-5$ \\
$75-09-2$ \\
Ag \\
As \\
Ba \\
Cd \\
C1-2-x \\
Cl-3-x \\
C1-4-x \\
CI-F-x \\
Cr \\
HC-insol \\
HC-Solub \\
Hg \\
Pb \\
Se
\end{tabular}

\begin{tabular}{c}
$\begin{array}{c}\text { Amount } \\
(\mathrm{kg} / \mathrm{yr})\end{array}$ \\
\hline $5.948 \mathrm{E}+00$ \\
$2.117 \mathrm{E}+01$ \\
$3.226 \mathrm{E}+00$ \\
$1.249 \mathrm{E}+00$ \\
$1.085 \mathrm{E}+02$ \\
$6.815 \mathrm{E}+00$ \\
$2.073 \mathrm{E}+01$ \\
$5.283 \mathrm{E}+02$ \\
$3.692 \mathrm{E}+02$ \\
$1.313 \mathrm{E}+01$ \\
$1.531 \mathrm{E}+02$ \\
$1.786 \mathrm{E}+03$ \\
$9.390 \mathrm{E}+02$ \\
$3.514 \mathrm{E}+01$ \\
$1.240 \mathrm{E}+02$ \\
$1.366 \mathrm{E}+01$
\end{tabular}


Shipping Site: GATOMIC

Total Volume (m3/yr):

Radionuclide

AC -228

$\mathrm{Bi}-212$

$\mathrm{Pa}-234$

$\mathrm{Pa}-234 \mathrm{~m}$

$\mathrm{Pb}-212$

Po-212

Po-216

$\mathrm{Ra}-224$

$\mathrm{Ra}-228$

Tc-99

Th- 228

Th-231

$\mathrm{Th}-232$

Th -234

$\mathrm{T} 1-208$

$\mathrm{U}-235$

$\mathrm{U}-238$

Shipping site: GATOMIC
$7.077 \mathrm{E}-02$

\begin{tabular}{c}
$\begin{array}{c}\text { Activity } \\
\text { (Ci/yr) }\end{array}$ \\
\hline $1.514 E-07$ \\
$2.547 E-08$ \\
$1.925 E-08$ \\
$1.870 E-04$ \\
$2.547 E-08$ \\
$1.642 E-08$ \\
$2.547 E-08$ \\
$2.547 E-08$ \\
$1.514 E-07$ \\
$2.816 E-06$ \\
$2.547 E-08$ \\
$1.460 E-07$ \\
$1.537 E-06$ \\
$1.870 E-04$ \\
$9.625 E-09$ \\
$1.455 E-07$ \\
$1.870 E-04$
\end{tabular}

Waste Type: Organic Liquids (CH-Non-alpha)

Total Mass $(\mathrm{kg} / \mathrm{yr}): \quad 7.476 \mathrm{E}+01$
Total Volume $(\mathrm{m} 3 / \mathrm{yr}\}$ :

Radionuclide

Ac -228

$\mathrm{Bi}-212$

Pa -234

$\mathrm{Pa}-234 \mathrm{~m}$

$\mathrm{Pb}-212$

Po- 212

Po- 216

$\mathrm{Ra}-224$

$\mathrm{Ra}-228$

Tc-99

$\mathrm{Th}-228$

Th-231

Th-232

Th-234

T1-208

$\mathrm{U}-235$

U-238

Shipping Site: GJCT

Total Volume (m3/yr):

Radionuclide

Ac -228

Bi-212

$\mathrm{Pa}-234$

$\mathrm{Pa}-234 \mathrm{~m}$

$\mathrm{Pb}-212$

Po-2 12

Po-216

Ra-224

Ra-228

TC-99

Th-228

Th-231

Th-232

Th-234

T1-208

U-235

$\mathrm{U}-238$
Waste Type: Solids (CH-Non-alpha)

Total Mass $(\mathrm{kg} / \mathrm{y} r): \quad 8.419 \mathrm{E}+02$

\begin{tabular}{r}
$\begin{array}{r}\text { Activity } \\
(\mathrm{Ci} / \mathrm{Y} \text { ) }\end{array}$ \\
\hline $1.218 \mathrm{E}-06$ \\
$2.049 \mathrm{E}-07$ \\
$1.548 \mathrm{E}-07$ \\
$1.504 \mathrm{E}-03$ \\
$2.049 \mathrm{E}-07$ \\
$1.320 \mathrm{E}-07$ \\
$2.049 \mathrm{E}-07$ \\
$1.996 \mathrm{E}-07$ \\
$1.186 \mathrm{E}-06$ \\
$2.207 \mathrm{E}-05$ \\
$2.049 \mathrm{E}-07$ \\
$1.175 \mathrm{E}-06$ \\
$1.237 \mathrm{E}-05$ \\
$1.504 \mathrm{E}-03$ \\
$7.742 \mathrm{E}-08$ \\
$1.170 \mathrm{E}-06$ \\
$1.504 \mathrm{E}-03$
\end{tabular}

\begin{tabular}{l} 
Chemical \\
\hline $57-12-5$ \\
$75-09-2$ \\
$\mathrm{Ag}$ \\
$\mathrm{As}$ \\
$\mathrm{Ba}$ \\
$\mathrm{Cd}$ \\
$\mathrm{Cl}-2-\mathrm{x}$ \\
$\mathrm{Cl}-3-\mathrm{x}$ \\
$\mathrm{Cl}-4-\mathrm{x}$ \\
$\mathrm{Cl}-\mathrm{F}-\mathrm{x}$ \\
$\mathrm{Cr}$ \\
$\mathrm{HC}-\mathrm{insol}$ \\
$\mathrm{HC}-\mathrm{solub}$ \\
$\mathrm{Hg}$ \\
$\mathrm{Pb}$ \\
$\mathrm{Se}$
\end{tabular}

\begin{tabular}{c}
$\begin{array}{c}\text { Amount } \\
(\mathrm{kg} / \mathrm{Yr})\end{array}$ \\
\hline $9.298 \mathrm{E}-02$ \\
$1.998 \mathrm{E}-01$ \\
$2.502 \mathrm{E}-02$ \\
$9.721 \mathrm{E}-03$ \\
$1.175 \mathrm{E}+00$ \\
$4.463 \mathrm{E}-02$ \\
$1.741 \mathrm{E}-01$ \\
$3.347 \mathrm{E}+00$ \\
$8.150 \mathrm{E}-01$ \\
$1.017 \mathrm{E}-01$ \\
$1.315 \mathrm{E}+00$ \\
$1.041 \mathrm{E}+01$ \\
$6.899 \mathrm{E}+00$ \\
$1.942 \mathrm{E}-01$ \\
$2.810 \mathrm{E}+00$ \\
$7.067 \mathrm{E}-02$
\end{tabular}

Waste Type: Organic Liquids ( $\mathrm{CH}-\mathrm{Non}-\mathrm{alpha})$

1.290E-02

Total Mass (kg/yr): $1.478 \mathrm{E}+01$

\begin{tabular}{l} 
Chemical \\
\hline $57-12-5$ \\
$75-09-2$ \\
$\mathrm{Ag}$ \\
$\mathrm{As}$ \\
$\mathrm{Ba}$ \\
$\mathrm{Cd}$ \\
$\mathrm{Cl}-2-\mathrm{x}$ \\
$\mathrm{Cl}-3-\mathrm{x}$ \\
$\mathrm{Cl}-4-\mathrm{x}$ \\
$\mathrm{Cl}-\mathrm{F}-\mathrm{x}$ \\
$\mathrm{Cr}$ \\
$\mathrm{HC}-$ insol \\
$\mathrm{HC}-\mathrm{solub}$ \\
$\mathrm{Hg}$ \\
$\mathrm{Pb}$ \\
$\mathrm{Se}$
\end{tabular}

\begin{tabular}{c}
$\begin{array}{c}\text { Amount } \\
(\mathrm{kg} / \mathrm{yr})\end{array}$ \\
\hline $6.543 \mathrm{E}-03$ \\
$5.261 \mathrm{E}-03$ \\
$3.280 \mathrm{E}-03$ \\
$4.207 \mathrm{E}-04$ \\
$2.867 \mathrm{E}-03$ \\
$4.175 \mathrm{E}-04$ \\
$1.428 \mathrm{E}-02$ \\
$5.732 \mathrm{E}-01$ \\
$3.523 \mathrm{E}-02$ \\
$1.146 \mathrm{E}-02$ \\
$7.839 \mathrm{E}-03$ \\
$4.931 \mathrm{E}-01$ \\
$1.311 \mathrm{E}+01$ \\
$1.858 \mathrm{E}-02$ \\
$2.894 \mathrm{E}-03$ \\
$2.867 \mathrm{E}-03$
\end{tabular}


Shipping Site: GJCT Waste Type: Solids (CH-Non-alpha)

Total Volume $(\mathrm{m} 3 / \mathrm{yr})$ :

2. $953 E-02$

Total Mass (kg/yr): $5.478 E+01$

\begin{tabular}{l} 
Radionucilide \\
\hline AC- -228 \\
$\mathrm{~B} i-212$ \\
$\mathrm{~Pa}-234$ \\
$\mathrm{~Pa}-234 \mathrm{~m}$ \\
$\mathrm{~Pb}-212$ \\
$\mathrm{Po}-212$ \\
$\mathrm{~Pa}-216$ \\
$\mathrm{Ra}-224$ \\
$\mathrm{Ra}-228$ \\
$\mathrm{TC}-99$ \\
$\mathrm{Th}-228$ \\
$\mathrm{Th}-231$ \\
$\mathrm{Th}-232$ \\
$\mathrm{Th}-234$ \\
$\mathrm{~T} 1-208$ \\
$\mathrm{U}-235$ \\
$\mathrm{U}-238$
\end{tabular}

Shipping Site:

INEI

Total Volume $(\mathrm{m} 3 / \mathrm{yr})$ :

Radionuclide

$\mathrm{Ba}-137 \mathrm{~m}$

Co -60

Cs -134

Cs -137

Eu -154

Eu-155

$\mathrm{F} e-55$

$\mathrm{H}-3$

$\mathrm{Mn}-54$

Nb- 94

$\mathrm{Ni}-59$

$\mathrm{Ni}-63$

Pa-234m

Pm-147

Pu-238

Pu-241

$\mathrm{Sb}-125$

$\mathrm{Sm}-151$

Sr -90

$\mathrm{Te}-125 \mathrm{~m}$

Th-234

$\mathrm{U}-238$

$\mathrm{Y}-90$

\begin{tabular}{l}
$\begin{array}{c}\text { Activity } \\
\text { (Ci/yr) }\end{array}$ \\
\hline $6.356 \mathrm{E}-08$ \\
$1.069 \mathrm{E}-08$ \\
$8.080 \mathrm{E}-09$ \\
$7.849 \mathrm{E}-05$ \\
$1.069 \mathrm{E}-08$ \\
$6.891 \mathrm{E}-09$ \\
$1.069 \mathrm{E}-08$ \\
$1.054 \mathrm{E}-08$ \\
$6.268 \mathrm{E}-08$ \\
$1.165 \mathrm{E}-06$ \\
$1.069 \mathrm{E}-08$ \\
$6.131 \mathrm{E}-08$ \\
$6.454 \mathrm{E}-07$ \\
$7.849 \mathrm{E}-05$ \\
$4.040 \mathrm{E}-09$ \\
$6.107 \mathrm{E}-08$ \\
$7.849 \mathrm{E}-05$
\end{tabular}

Waste Type: Organic Liquids ( $\mathrm{CH}-\mathrm{Non}-\mathrm{alpha}$ )

$3.228 \mathrm{E} \div 00$

Total Mass (kg/yr):

\begin{tabular}{r}
$\begin{array}{r}\text { Activity } \\
\text { (Ci/Yr) }\end{array}$ \\
\hline $2.161 \mathrm{E}+00$ \\
$3.491 \mathrm{E}+01$ \\
$2.052 \mathrm{E}-02$ \\
$2.282 \mathrm{E}+00$ \\
$2.777 \mathrm{E}-02$ \\
$1.328 \mathrm{E}-02$ \\
$4.564 \mathrm{E}+00$ \\
$9.942 \mathrm{E}+00$ \\
$1.448 \mathrm{E}-02$ \\
$2.173 \mathrm{E}-02$ \\
$4.564 \mathrm{E}-01$ \\
$6.136 \mathrm{E}+01$ \\
$8.573 \mathrm{E}-02$ \\
$2.487 \mathrm{E}-01$ \\
$7.124 \mathrm{E}-02$ \\
$5.795 \mathrm{E}-02$ \\
$9.659 \mathrm{E}-03$ \\
$1.931 \mathrm{E}-02$ \\
$2.149 \mathrm{E}+00$ \\
$2.414 \mathrm{E}-03$ \\
$8.573 \mathrm{E}-02$ \\
$8.573 \mathrm{E}-02$ \\
$2.149 \mathrm{E}+00$ \\
\end{tabular}

$3.542 E+03$

\begin{tabular}{lcc}
\multicolumn{1}{c}{ Chemical } & $\begin{array}{c}\text { Amount } \\
(\mathrm{kg} / \mathrm{yr})\end{array}$ \\
\cline { 1 - 1 } $57-12-5$ & $1.997 \mathrm{E}-04$ \\
$75-09-2$ & & $1.051 \mathrm{E}-03$ \\
$\mathrm{Ag}$ & $6.108 \mathrm{E}-04$ \\
$\mathrm{AS}$ & $2.981 \mathrm{E}-04$ \\
$\mathrm{Ba}$ & $3.480 \mathrm{E}-03$ \\
$\mathrm{Cd}$ & $4.003 \mathrm{E}-04$ \\
$\mathrm{Cl}-2-\mathrm{x}$ & $1.051 \mathrm{E}-03$ \\
$\mathrm{Cl}-3-\mathrm{x}$ & $2.387 \mathrm{E}-02$ \\
$\mathrm{C} 1-4-\mathrm{x}$ & $7.830 \mathrm{E}-03$ \\
$\mathrm{Cl}-\mathrm{F}-\mathrm{x}$ & $5.619 \mathrm{E}-04$ \\
$\mathrm{Cr}$ & $4.507 \mathrm{E}-03$ \\
$\mathrm{HC}-$ insol & $7.674 \mathrm{E}-02$ \\
$\mathrm{HC}-$ Solub & $8.522 \mathrm{E}-02$ \\
$\mathrm{Hg}$ & $1.306 \mathrm{E}-03$ \\
$\mathrm{~Pb}$ & $5.304 \mathrm{E}-03$ \\
$\mathrm{Se}$ & $4.666 \mathrm{E}-04$
\end{tabular}

\begin{tabular}{l} 
Chemical \\
\hline $57-12-5$ \\
$75-09-2$ \\
$\mathrm{Ag}$ \\
$\mathrm{As}$ \\
$\mathrm{Ba}$ \\
$\mathrm{Cd}$ \\
$\mathrm{CI}-2-\mathrm{x}$ \\
$\mathrm{CI}-3-\mathrm{x}$ \\
$\mathrm{Cl}-4-\mathrm{x}$ \\
$\mathrm{Cl}-\mathrm{F}-\mathrm{x}$ \\
$\mathrm{Cr}$ \\
$\mathrm{HC}-\mathrm{insol}$ \\
$\mathrm{HC}-\mathrm{solub}$ \\
$\mathrm{Hg}$ \\
$\mathrm{Pb}$ \\
$\mathrm{Se}$
\end{tabular}


Shipping Site: INEL

Waste Type: Solids (CH-Non-alpha)

Total Volume $(\mathrm{m} 3 / \mathrm{yr})$ :

$3.913 E+01$

Total Mass $(\mathrm{kg} / \mathrm{yr}): 6.096 \mathrm{E}+04$

Radionuclide

$\mathrm{Ba}-137 \mathrm{~m}$

$\mathrm{Co}-60$

Cs -134

Cs -137

$\mathrm{Eu}-154$

$\mathrm{Eu}-155$

$\mathrm{Fe}-55$

$\mathrm{H}-3$

Mn-54

$\mathrm{Nb}-94$

$\mathrm{Ni}-59$

$\mathrm{Ni}-63$

$\mathrm{Pa}-234 \mathrm{~m}$

Pm-147

Pu-238

Pu-241

$\mathrm{Sb}-125$

$\mathrm{Sm}-151$

$\mathrm{Sr}-90$

Te-125m

Th-234

$\mathrm{U}-238$

$\mathrm{Y}-90$

Shipping Site:

INEL

Total Volume (m3/yr):

Radionuclide

Am-241

$\mathrm{Ba}-137 \mathrm{~m}$

Co-60

Cs-137

Eu-154

$\mathrm{H}-3$

$\mathrm{Nb}-94$

$\mathrm{Ni}-59$

$\mathrm{Ni}-63$

$\mathrm{Pa}-234 \mathrm{~m}$

$\mathrm{Pu}-238$

Pu -240

$\mathrm{Pu}-241$

$\mathrm{Sm}-151$

Sr -90

Th-234

$\mathrm{U}-238$

$\mathrm{Y}-90$

Shipping site:

ITRI

Total Volume (m3/yr):

Rađionuclide

$\mathrm{Ba}-137 \mathrm{~m}$

CO-60

Cs -137

Eu-154

$\mathrm{Fe}-55$

$\mathrm{H}-3$

$\mathrm{Ni}-59$

Ni-63

Pm-147

$\mathrm{Pu}-238$

Pu-241

Sin-151

$\mathrm{Sr}-90$

$\mathrm{Y}-90$

\begin{tabular}{c}
$\begin{array}{r}\text { Activity } \\
\text { (Ci/yr) }\end{array}$ \\
\hline $2.622 \mathrm{E}+01$ \\
$4.236 \mathrm{E}+02$ \\
$2.484 \mathrm{E}-01$ \\
$2.761 \mathrm{E}+01$ \\
$3.370 \mathrm{E}-01$ \\
$1.611 \mathrm{E}-01$ \\
$5.538 \mathrm{E}+01$ \\
$1.206 \mathrm{E}+02$ \\
$1.758 \mathrm{E}-01$ \\
$2.637 \mathrm{E}-01$ \\
$5.538 \mathrm{E}+00$ \\
$7.447 \mathrm{E}+02$ \\
$1.040 \mathrm{E}+00$ \\
$3.018 \mathrm{E}+00$ \\
$8.645 \mathrm{E}-01$ \\
$7.033 \mathrm{E}-01$ \\
$1.172 \mathrm{E}-01$ \\
$2.344 \mathrm{E}-01$ \\
$2.601 \mathrm{E}+01$ \\
$2.930 \mathrm{E}-02$ \\
$1.040 \mathrm{E}+00$ \\
$1.040 \mathrm{E}+00$ \\
$2.601 \mathrm{E}+01$
\end{tabular}

\begin{tabular}{l} 
Chemical \\
\hline $57-12-5$ \\
$75-09-2$ \\
Ag \\
As \\
Ba \\
Cd \\
Cl-2-x \\
Cl-3-x \\
Cl-4-x \\
Cl-F-x \\
Cr \\
HC-insol \\
HC-solub \\
Hg \\
Pb \\
Se
\end{tabular}

\begin{tabular}{c}
$\begin{array}{c}\text { Amount } \\
(\mathrm{kg} / \mathrm{Yr})\end{array}$ \\
\hline $3.846 \mathrm{E}+00$ \\
$1.705 \mathrm{E}+01$ \\
$1.037 \mathrm{E}+00$ \\
$4.884 \mathrm{E}-01$ \\
$5.299 \mathrm{E}+01$ \\
$5.011 \mathrm{E}+00$ \\
$1.273 \mathrm{E}+01$ \\
$3.471 \mathrm{E}+02$ \\
$7.556 \mathrm{E}+01$ \\
$8.352 \mathrm{E}+00$ \\
$6.930 \mathrm{E}+01$ \\
$1.084 \mathrm{E}+03$ \\
$5.495 \mathrm{E}+02$ \\
$8.011 \mathrm{E}+00$ \\
$1.706 \mathrm{E}+02$ \\
$2.072 \mathrm{E}+00$
\end{tabular}

Waste Type: Solids (CH-Alpha)

$6.473 E+02$

Total Mass $(\mathrm{kg} / \mathrm{yr}): 1.178 \mathrm{E}+06$

\begin{tabular}{c}
$\begin{array}{c}\text { Activity } \\
\text { (Ci/yr) }\end{array}$ \\
\hline $2.175 E-01$ \\
$1.914 E+02$ \\
$6.829 E+01$ \\
$2.022 E+02$ \\
$3.262 E-01$ \\
$2.751 E+02$ \\
$4.567 E+00$ \\
$1.044 E+02$ \\
$9.589 E+03$ \\
$1.729 E+01$ \\
$1.011 E+01$ \\
$3.262 E-01$ \\
$8.700 E+00$ \\
$2.936 E+00$ \\
$1.826 E+02$ \\
$1.729 E+01$ \\
$1.729 E+01$ \\
$1.826 E+02$
\end{tabular}

\begin{tabular}{l} 
Chemical \\
\hline $57-12-5$ \\
$75-09-2$ \\
Ag \\
As \\
$\mathrm{Ba}$ \\
$\mathrm{Cd}$ \\
$\mathrm{Cl}-2-\mathrm{x}$ \\
$\mathrm{Cl}-3-\mathrm{x}$ \\
$\mathrm{Cl}-4-\mathrm{-}$ \\
$\mathrm{Cl}-\mathrm{F}-\mathrm{x}$ \\
$\mathrm{Cr}$ \\
$\mathrm{HC}-$ insol \\
$\mathrm{HC}-$ solub \\
$\mathrm{Hg}$ \\
$\mathrm{Pb}$ \\
$\mathrm{Se}$
\end{tabular}

\begin{tabular}{c}
$\begin{array}{c}\text { Amount } \\
(\mathrm{kg} / \mathrm{Y})\end{array}$ \\
\hline $5.736 \mathrm{E}+01$ \\
$6.107 \mathrm{E}+02$ \\
$1.245 \mathrm{E}+01$ \\
$5.295 \mathrm{E}+00$ \\
$7.474 \mathrm{E}+02$ \\
$1.467 \mathrm{E}+02$ \\
$2.715 \mathrm{E}+02$ \\
$2.218 \mathrm{E}+03$ \\
$8.828 \mathrm{E}+02$ \\
$2.953 \mathrm{E}+02$ \\
$2.118 \mathrm{E}+03$ \\
$4.349 \mathrm{E}+03$ \\
$1.889 \mathrm{E}+03$ \\
$1.775 \mathrm{E}+02$ \\
$5.937 \mathrm{E}+03$ \\
$2.939 \mathrm{E}+01$
\end{tabular}

Waste Type: Organic Liquids (CH-Non-alpha)

$1.028 \mathrm{E}-01$ Total Mass $(\mathrm{kg} / \mathrm{yx}): 1.007 \mathrm{E}+02$

\begin{tabular}{c}
$\begin{array}{c}\text { Activity } \\
\text { (Ci/yr) }\end{array}$ \\
\hline $1.367 \mathrm{E}-03$ \\
$1.879 \mathrm{E}-03$ \\
$1.424 \mathrm{E}-03$ \\
$1.566 \mathrm{E}-05$ \\
$2.364 \mathrm{E}-04$ \\
$2.725 \mathrm{E}-01$ \\
$3.133 \mathrm{E}-05$ \\
$4.158 \mathrm{E}-03$ \\
$1.310 \mathrm{E}-04$ \\
$5.411 \mathrm{E}-05$ \\
$2.734 \mathrm{E}-04$ \\
$1.253 \mathrm{E}-05$ \\
$1.338 \mathrm{E}-03$ \\
$1.338 \mathrm{E}-03$
\end{tabular}

Amount

$\frac{\text { Chemical }}{57-12-5}$

Ag

$\mathrm{cd}$

$\mathrm{Cl}-3-\mathrm{x}$

CI $-4-x$

$\mathrm{Cr}$

HC-insol

HC-solub

$\mathrm{Hg}$

$\mathrm{Pb}$

$\mathrm{Se}$ $(\mathrm{kg} / \mathrm{Yr})$

1. $813 \mathrm{E}-02$

$4.433 \mathrm{E}-03$

4. $937 E-04$

$8.061 \mathrm{E}-02$

$4.534 \mathrm{E}-03$

2. $116 \mathrm{E}-02$

1. $108 \mathrm{E}+00$

3. $627 \mathrm{E}+01$

2. 821E-02

$5.038 \mathrm{E}-03$

$4.030 \mathrm{E}-03$ 
Shipping Site: KAPL Total volume $(\mathrm{m} 3 / \mathrm{yr})$ :

Radionuclide

$\mathrm{Ba}-137 \mathrm{~m}$

Co-60

Cs-134

Cs -137

$\mathrm{Eu}-154$

Eu-155

$\mathrm{Fe}-55$

$\mathrm{H}-3$

$\mathrm{Mn}-54$

$\mathrm{Nb}-94$

$\mathrm{Ni}-59$

$\mathrm{Ni}-63$

$\mathrm{Pa}-234 \mathrm{~m}$

Pm-147

Pu -238

$\mathrm{Pu}-241$

$\mathrm{Sb}-125$

$\mathrm{Sm}-151$

$\mathrm{Sr}-90$

$\mathrm{Te}-125 \mathrm{~m}$

$\mathrm{Th}-234$

$\mathrm{U}-238$

$\mathrm{Y}-90$

Shipping Site:

KAPL
$6.167 E-01$

\begin{tabular}{c}
$\begin{array}{c}\text { Activity } \\
\text { (Ci/Yr) }\end{array}$ \\
\hline $4.129 \mathrm{E}-01$ \\
$6.669 \mathrm{E}+00$ \\
$3.921 \mathrm{E}-03$ \\
$4.359 \mathrm{E}-01$ \\
$5.305 \mathrm{E}-03$ \\
$2.537 \mathrm{E}-03$ \\
$8.719 \mathrm{E}-01$ \\
$1.899 \mathrm{E}+00$ \\
$2.768 \mathrm{E}-03$ \\
$4.152 \mathrm{E}-03$ \\
$8.719 \mathrm{E}-02$ \\
$1.172 \mathrm{E}+01$ \\
$1.637 \mathrm{E}-02$ \\
$4.752 \mathrm{E}-02$ \\
$1.361 \mathrm{E}-02$ \\
$1.107 \mathrm{E}-02$ \\
$1.845 \mathrm{E}-03$ \\
$3.690 \mathrm{E}-03$ \\
$4.106 \mathrm{E}-01$ \\
$4.613 \mathrm{E}-04$ \\
$1.637 \mathrm{E}-02$ \\
$1.637 \mathrm{E}-02$ \\
$4.106 \mathrm{E}-01$
\end{tabular}

Waste Type: Organic Liquids ( $\mathrm{CH}-\mathrm{Non}-\mathrm{alpha})$

Total Mass (kg/yr): $7.065 \mathrm{E}+02$

\begin{tabular}{lcc} 
Chemical & & $\begin{array}{c}\text { Amount } \\
(\mathrm{kg} / \mathrm{yr})\end{array}$ \\
\cline { 1 - 1 } $57-12-5$ & & $2.915 \mathrm{E}-02$ \\
$75-09-2$ & & $5.312 \mathrm{E}-01$ \\
$\mathrm{Ag}$ & $1.461 \mathrm{E}-02$ \\
$\mathrm{As}$ & $2.952 \mathrm{E}-03$ \\
$\mathrm{Ba}$ & $1.739 \mathrm{E}-02$ \\
$\mathrm{Cd}$ & $4.125 \mathrm{E}-01$ \\
$\mathrm{Cl}-2-\mathrm{x}$ & $3.320 \mathrm{E}-01$ \\
$\mathrm{Cl}-3-\mathrm{X}$ & $3.184 \mathrm{E}+01$ \\
$\mathrm{Cl}-4-\mathrm{X}$ & $6.682 \mathrm{E}+00$ \\
$\mathrm{Cl}-\mathrm{F}-\mathrm{x}$ & $6.275 \mathrm{E}-02$ \\
$\mathrm{Cr}$ & $3.877 \mathrm{E}-01$ \\
$\mathrm{HC}-\mathrm{inso1}$ & $6.386 \mathrm{E}+01$ \\
$\mathrm{HC}-\mathrm{SOlub}$ & $7.952 \mathrm{E}+01$ \\
$\mathrm{Hg}$ & $8.511 \mathrm{E}-02$ \\
$\mathrm{~Pb}$ & $9.130 \mathrm{E}-02$ \\
$\mathrm{Se}$ & $1.277 \mathrm{E}-02$
\end{tabular}

Waste Type: Solids (CH-Non-alpha)

Total Mass $(\mathrm{kg} / \mathrm{yr}): 5.162 \mathrm{E}+03$

\begin{tabular}{|c|c|}
\hline Chemical & $\begin{array}{l}\text { Amount } \\
\text { (kg/yr) }\end{array}$ \\
\hline $\begin{array}{l}57-12-5 \\
75-09-2\end{array}$ & $\begin{array}{l}1.840 \mathrm{E}-02 \\
2.577 \mathrm{E}+00\end{array}$ \\
\hline Ag & $6.267 E-02$ \\
\hline As & $2.255 E-02$ \\
\hline $\mathrm{Ba}$ & $5.050 E-01$ \\
\hline $\mathrm{cd}$ & $2.428 \mathrm{E}-02$ \\
\hline Cl-2-x & $1.464 \mathrm{E}+00$ \\
\hline Cl-3-x & $1.328 E+01$ \\
\hline Cl-4-x & $1.024 \mathrm{E}+01$ \\
\hline $\mathrm{C} 1-\mathrm{F}-\mathrm{x}$ & $4.115 \mathrm{E}-01$ \\
\hline $\mathrm{Cr}$ & $4.769 \mathrm{E}-01$ \\
\hline HC-insol & $3.511 E+01$ \\
\hline HC-solub & $1.516 \mathrm{E}+01$ \\
\hline $\mathrm{Hg}$ & $6.065 E-02$ \\
\hline $\mathrm{Pb}$ & $7.229 \mathrm{E}-01$ \\
\hline Se & $1.698 \mathrm{E}-02$ \\
\hline
\end{tabular}

\begin{tabular}{l} 
Radionuclide \\
\hline $\mathrm{Ba}-137 \mathrm{~m}$ \\
$\mathrm{Co}-60$ \\
$\mathrm{Cs}-134$ \\
$\mathrm{Cs}-137$ \\
$\mathrm{Eu}-154$ \\
$\mathrm{Eu}-155$ \\
$\mathrm{Fe}-55$ \\
$\mathrm{H}-3$ \\
$\mathrm{Mn}-54$ \\
$\mathrm{Nb}-94$ \\
$\mathrm{Ni}-59$ \\
$\mathrm{Ni}-63$ \\
$\mathrm{~Pa}-234 \mathrm{~m}$ \\
$\mathrm{Pm}-147$ \\
$\mathrm{Pu}-238$ \\
$\mathrm{Pu}-241$ \\
$\mathrm{Sb}-125$ \\
$\mathrm{Sm}-151$ \\
$\mathrm{Sr}-90$ \\
$\mathrm{Te}-125 \mathrm{~m}$ \\
$\mathrm{Th}-234$ \\
$\mathrm{U}-238$ \\
$\mathrm{Y}-90$
\end{tabular}

\begin{tabular}{c}
$\begin{array}{r}\text { Activity } \\
\text { (Ci/yr) }\end{array}$ \\
\hline $2.391 \mathrm{E}+00$ \\
$3.862 \mathrm{E}+01$ \\
$2.271 \mathrm{E}-02$ \\
$2.525 \mathrm{E}+00$ \\
$3.072 \mathrm{E}-02$ \\
$1.469 \mathrm{E}-02$ \\
$5.050 \mathrm{E}+00$ \\
$1.100 \mathrm{E}+01$ \\
$1.603 \mathrm{E}-02$ \\
$2.404 \mathrm{E}-02$ \\
$5.050 \mathrm{E}-01$ \\
$6.790 \mathrm{E}+01$ \\
$9.486 \mathrm{E}-02$ \\
$2.752 \mathrm{E}-01$ \\
$7.882 \mathrm{E}-02$ \\
$6.413 \mathrm{E}-02$ \\
$1.068 \mathrm{E}-02$ \\
$2.137 \mathrm{E}-02$ \\
$2.378 \mathrm{E}+00$ \\
$2.672 \mathrm{E}-03$ \\
$9.486 \mathrm{E}-02$ \\
$9.486 \mathrm{E}-02$ \\
$2.378 \mathrm{E}+00$
\end{tabular}


Shipping Site: $\mathrm{KCP}$ Waste Type: Solids (CH-Non-alpha)

Total volume $(\mathrm{m} 3 / \mathrm{yr})$ :

$4.219 \mathrm{E}-02$

Total Mass $(\mathrm{kg} / \mathrm{yr}): \quad 3.237 \mathrm{E}+01$

\begin{tabular}{l} 
Radionuclide \\
\hline $\mathrm{Ba}-137 \mathrm{~m}$ \\
$\mathrm{Co}-60$ \\
$\mathrm{Cs}-134$ \\
$\mathrm{Cs}-137$ \\
$\mathrm{Eu}-154$ \\
$\mathrm{Eu}-155$ \\
$\mathrm{Fe}-55$ \\
$\mathrm{H}-3$ \\
$\mathrm{Mn}-54$ \\
$\mathrm{Nb}-94$ \\
$\mathrm{Ni}-59$ \\
$\mathrm{Ni}-63$ \\
$\mathrm{~Pa}-234 \mathrm{~m}$ \\
$\mathrm{Pm}-147$ \\
$\mathrm{Pu}-238$ \\
$\mathrm{Pu}-241$ \\
$\mathrm{Sb}-125$ \\
$\mathrm{Sm}-151$ \\
$\mathrm{Sr}-90$ \\
$\mathrm{Te}-125 \mathrm{~m}$ \\
$\mathrm{Th}-234$ \\
$\mathrm{U}-238$ \\
$\mathrm{Y}-90$
\end{tabular}

Shipping site: KKS

Total Volume $(\mathrm{m} 3 / \mathrm{yr})$ :

Radionuclide

$\mathrm{Ba}-137 \mathrm{~m}$

Co-60

Cs -134

Cs -137

$\mathrm{Eu}-154$

Eu-155

$\mathrm{Fe}-55$

$\mathrm{H}-3$

$\mathrm{Mn}-54$

$\mathrm{Nb}-94$

$\mathrm{Ni}-59$

$\mathrm{Ni}-63$

$\mathrm{Pa}-234 \mathrm{~m}$

Pm-147

Pu -238

Pu-241

$\mathrm{Sb}-125$

$\mathrm{Sm}-151$

$\mathrm{Sr}-90$

$\mathrm{Te}-125 \mathrm{~m}$

Th-234

$\mathrm{U}-238$

$\mathrm{Y}-90$

\begin{tabular}{c}
$\begin{array}{c}\text { Activity } \\
(\mathrm{Ci} / \mathrm{y})\end{array}$ \\
\hline $2.824 \mathrm{E}-02$ \\
$4.562 \mathrm{E}-01$ \\
$2.682 \mathrm{E}-04$ \\
$2.982 \mathrm{E}-02$ \\
$3.629 \mathrm{E}-04$ \\
$1.735 \mathrm{E}-04$ \\
$5.964 \mathrm{E}-02$ \\
$1.299 \mathrm{E}-01$ \\
$1.893 \mathrm{E}-04$ \\
$2.840 \mathrm{E}-04$ \\
$5.964 \mathrm{E}-03$ \\
$8.019 \mathrm{E}-01$ \\
$1.120 \mathrm{E}-03$ \\
$3.250 \mathrm{E}-03$ \\
$9.309 \mathrm{E}-04$ \\
$7.573 \mathrm{E}-04$ \\
$1.262 \mathrm{E}-04$ \\
$2.524 \mathrm{E}-04$ \\
$2.808 \mathrm{E}-02$ \\
$3.155 \mathrm{E}-05$ \\
$1.120 \mathrm{E}-03$ \\
$1.120 \mathrm{E}-03$ \\
$2.808 \mathrm{E}-02$
\end{tabular}

\begin{tabular}{l} 
Chemical \\
\hline $75-09-2$ \\
$\mathrm{Ag}$ \\
$\mathrm{AS}$ \\
$\mathrm{Ba}$ \\
$\mathrm{Cd}$ \\
$\mathrm{Cl}-2-\mathrm{x}$ \\
$\mathrm{Cl}-3-\mathrm{x}$ \\
$\mathrm{Cl}-4-\mathrm{x}$ \\
$\mathrm{Cl}-\mathrm{E}-\mathrm{x}$ \\
$\mathrm{Cr}$ \\
$\mathrm{HC}-\mathrm{insO}$ \\
$\mathrm{HC}-\mathrm{solub}$ \\
$\mathrm{Hg}$ \\
$\mathrm{Pb}$ \\
$\mathrm{Se}$
\end{tabular}

Waste Type: Organic Liquids ( $\mathrm{CH}-\mathrm{Non}-\mathrm{alpha})$

$4.733 \mathrm{E}-01$

Total Mass $(\mathrm{kg} / \mathrm{yr}): 5.422 \mathrm{E}+02$

\begin{tabular}{c}
$\begin{array}{c}\text { Activity } \\
\text { (Ci/yr) }\end{array}$ \\
\hline $3.168 E-01$ \\
$5.118 \mathrm{E}+00$ \\
$3.009 \mathrm{E}-03$ \\
$3.345 \mathrm{E}-01$ \\
$4.071 \mathrm{E}-03$ \\
$1.947 \mathrm{E}-03$ \\
$6.691 \mathrm{E}-01$ \\
$1.457 \mathrm{E}+00$ \\
$2.124 \mathrm{E}-03$ \\
$3.186 \mathrm{E}-03$ \\
$6.691 \mathrm{E}-02$ \\
$8.997 \mathrm{E}+00$ \\
$1.256 \mathrm{E}-02$ \\
$3.646 \mathrm{E}-02$ \\
$1.044 \mathrm{E}-02$ \\
$8.497 \mathrm{E}-03$ \\
$1.416 \mathrm{E}-03$ \\
$2.832 \mathrm{E}-03$ \\
$3.151 \mathrm{E}-01$ \\
$3.540 \mathrm{E}-04$ \\
$1.256 \mathrm{E}-02$ \\
$1.256 \mathrm{E}-02$ \\
$3.151 \mathrm{E}-01$
\end{tabular}

\begin{tabular}{c}
$\begin{array}{c}\text { Amount } \\
(\mathrm{kg} / \mathrm{yI})\end{array}$ \\
\hline $2.237 \mathrm{E}-02$ \\
$4.077 \mathrm{E}-01$ \\
$1.121 \mathrm{E}-02$ \\
$2.266 \mathrm{E}-03$ \\
$1.335 \mathrm{E}-02$ \\
$3.166 \mathrm{E}-01$ \\
$2.548 \mathrm{E}-01$ \\
$2.443 \mathrm{E}+01$ \\
$5.128 \mathrm{E}+00$ \\
$4.816 \mathrm{E}-02$ \\
$2.976 \mathrm{E}-01$ \\
$4.901 \mathrm{E}+01$ \\
$6.103 \mathrm{E}+01$ \\
$6.532 \mathrm{E}-02$ \\
$7.007 \mathrm{E}-02$ \\
$9.805 \mathrm{E}-03$
\end{tabular}


A-114

Shipping Site: KKS

Waste Type: Solids (CH-Non-alpha)

\begin{tabular}{l} 
Total Volume (m3/yr): \\
Radionuclide \\
\hline Ba-137m \\
Co-60 \\
Cs -134 \\
Cs -137 \\
Eu-154 \\
Eu-155 \\
Fe-55 \\
H-3 \\
Mn-54 \\
Nb-94 \\
Ni -59 \\
Ni-63 \\
Pa-234m \\
Pm-147 \\
Pu-238 \\
Pu-241 \\
Sb-125 \\
Sm-151 \\
Sr-90 \\
Te-125m \\
Th-234 \\
U-238 \\
Y-90
\end{tabular}

Shipping Site: KWS

Total Volume (m3/yr):

Radionuclide

$\mathrm{Ba}-137 \mathrm{~m}$

Co-60

Cs-134

Cs -137

Eu-154

Eu-155

$\mathrm{Fe}-55$

$\mathrm{H}-3$

$\mathrm{Mn}-54$

$\mathrm{Nb}-94$

$\mathrm{Ni}-59$

$\mathrm{Ni}-63$

$\mathrm{Pa}-234 \mathrm{~m}$

Pin-147

Pu-238

Pu-241

$\mathrm{Sb}-125$

$\mathrm{Sm}-151$

$\mathrm{Sr}-90$

$\mathrm{Te}-125 \mathrm{~m}$

Th -234

$\mathrm{U}-238$

$Y-90$
$3.928 \mathrm{E}+00$

Total Mass $(\mathrm{kg} / \mathrm{yr})$ :

$6.620 \mathrm{E}+03$

\begin{tabular}{l} 
Chemical \\
\hline $57-12-5$ \\
$75-09-2$ \\
Ag \\
As \\
$\mathrm{Ba}$ \\
$\mathrm{Cd}$ \\
$\mathrm{Cl}-2-\mathrm{x}$ \\
$\mathrm{Cl}-3-\mathrm{x}$ \\
$\mathrm{Cl}-4-\mathrm{x}$ \\
$\mathrm{Cl}-\mathrm{F}-\mathrm{x}$ \\
$\mathrm{Cr}$ \\
$\mathrm{HC}-$ insol \\
$\mathrm{HC}-$ solub \\
$\mathrm{Hg}$ \\
$\mathrm{Pb}$ \\
$\mathrm{Se}$
\end{tabular}

\begin{tabular}{l}
$\begin{array}{c}\text { Amount } \\
(\mathrm{kg} / \mathrm{yr})\end{array}$ \\
\hline $1.282 \mathrm{E}-01$ \\
$2.231 \mathrm{E}+00$ \\
$8.783 \mathrm{E}-02$ \\
$3.299 \mathrm{E}-02$ \\
$1.662 \mathrm{E}+00$ \\
$5.269 \mathrm{E}-02$ \\
$1.281 \mathrm{E}+00$ \\
$1.108 \mathrm{E}+01$ \\
$1.215 \mathrm{E}+01$ \\
$2.090 \mathrm{E}-01$ \\
$1.846 \mathrm{E}+00$ \\
$2.753 \mathrm{E}+01$ \\
$1.411 \mathrm{E}+01$ \\
$1.438 \mathrm{E}-01$ \\
$4.165 \mathrm{E}+00$ \\
$3.790 \mathrm{E}-02$
\end{tabular}

Waste Type: Organic Liquids (CH-Non-alpha)

Total Mass $(\mathrm{kg} / \mathrm{Yr}): \quad 7.394 \mathrm{E}+01$

\begin{tabular}{c}
$\begin{array}{c}\text { Activity } \\
\text { (Ci/yr) }\end{array}$ \\
\hline $4.321 \mathrm{E}-02$ \\
$6.979 \mathrm{E}-01$ \\
$4.103 \mathrm{E}-04$ \\
$4.562 \mathrm{E}-02$ \\
$5.552 \mathrm{E}-04$ \\
$2.655 \mathrm{E}-04$ \\
$9.125 \mathrm{E}-02$ \\
$1.987 \mathrm{E}-01$ \\
$2.896 \mathrm{E}-04$ \\
$4.345 \mathrm{E}-04$ \\
$9.125 \mathrm{E}-03$ \\
$1.226 \mathrm{E}+00$ \\
$1.714 \mathrm{E}-03$ \\
$4.973 \mathrm{E}-03$ \\
$1.424 \mathrm{E}-03$ \\
$1.158 \mathrm{E}-03$ \\
$1.931 \mathrm{E}-04$ \\
$3.862 \mathrm{E}-04$ \\
$4.297 \mathrm{E}-02$ \\
$4.828 \mathrm{E}-05$ \\
$1.714 \mathrm{E}-03$ \\
$1.714 \mathrm{E}-03$ \\
$4.297 \mathrm{E}-02$
\end{tabular}

\begin{tabular}{|c|c|}
\hline Chemical & $\begin{array}{l}\text { Amount } \\
(\mathrm{kg} / \mathrm{yr})\end{array}$ \\
\hline $\begin{array}{l}57-12-5 \\
75-09-2\end{array}$ & $\begin{array}{l}3.051 \mathrm{E}-03 \\
5.559 \mathrm{E}-02\end{array}$ \\
\hline Ag & $\begin{array}{l}2.529 \mathrm{E}-02 \\
1.529 \mathrm{E}-03\end{array}$ \\
\hline As & $3.090 \mathrm{E}-04$ \\
\hline $\mathrm{Ba}$ & $1.820 \mathrm{E}-03$ \\
\hline $\mathrm{cd}$ & $4.317 \mathrm{E}-02$ \\
\hline $\mathrm{Cl}-2-x$ & $3.474 \mathrm{E}-02$ \\
\hline Cl-3-x & $3.332 \mathrm{E}+00$ \\
\hline $\mathrm{Cl}-4-\mathrm{x}$ & $6.993 \mathrm{E}-01$ \\
\hline$C I-F-x$ & $6.567 \mathrm{E}-03$ \\
\hline $\mathrm{Cr}$ & 4. $058 \mathrm{E}-02$ \\
\hline HC-insol & $6.683 E+00$ \\
\hline HC-solub & $8.322 \mathrm{E}+00$ \\
\hline $\mathrm{Hg}$ & $8.907 \mathrm{E}-03$ \\
\hline $\mathrm{Pb}$ & $9.555 \mathrm{E}-03$ \\
\hline $\mathrm{Se}$ & $1.337 \mathrm{E}-03$ \\
\hline
\end{tabular}




\begin{tabular}{l} 
Shipping Site: KWS \\
Total Volume (m3/yr): \\
Radionuclide \\
\hline Ba-137m \\
Co-60 \\
Cs-134 \\
Cs-137 \\
Eu-154 \\
Eu-155 \\
Fe-55 \\
H-3 \\
Mn-54 \\
Nb-94 \\
Ni-59 \\
Ni-63 \\
Pa-234m \\
Pm-147 \\
Pu-238 \\
Pu-241 \\
Sb-125 \\
Sm-151 \\
Sr-90 \\
Te-125m \\
Th-234 \\
U-238 \\
Y-90 \\
P
\end{tabular}

Shipping site: LANL

Total Volume (m3/yr):

Radionuclide

$\mathrm{Ba}-137 \mathrm{~m}$

$\mathrm{Ca}-60$

Cs-137

Eu-154

$\mathrm{Fe}-55$

$\mathrm{H}-3$

$\mathrm{Ni}-59$

$\mathrm{Ni}-63$

Pm-147

Pu-238

$\mathrm{Pu}-241$

Sm-15I

$\mathrm{Sr}-90$

$\mathrm{Y}-90$

Shipping site: LANL

Total Volume $(\mathrm{m} 3 / \mathrm{yr})$ :

Radionuclide

$\mathrm{Ba}-137 \mathrm{~m}$

Co-60

Cs-137

Eu-154

$\mathrm{Fe}-55$

$\mathrm{H}-3$

$\mathrm{Ni}-59$

$\mathrm{Ni}-63$

Pm-147

$\mathrm{Pu}-238$

Pu-241

$\mathrm{Sm}-151$

$\mathrm{Sr}-90$

$Y-90$

$$
\begin{gathered}
1.262 \mathrm{E}+00 \\
\begin{array}{r}
\text { Activity } \\
\text { (Ci/ys) }
\end{array} \\
\hline 8.449 \mathrm{E}-01 \\
1.364 \mathrm{E}+01 \\
8.024 \mathrm{E}-03 \\
8.921 \mathrm{E}-01 \\
1.085 \mathrm{E}-02 \\
5.192 \mathrm{E}-03 \\
1.784 \mathrm{E}+00 \\
3.886 \mathrm{E}+00 \\
5.664 \mathrm{E}-03 \\
8.496 \mathrm{E}-03 \\
1.784 \mathrm{E}-01 \\
2.399 \mathrm{E}+01 \\
3.351 \mathrm{E}-02 \\
9.724 \mathrm{E}-02 \\
2.785 \mathrm{E}-02 \\
2.265 \mathrm{E}-02 \\
3.776 \mathrm{E}-03 \\
7.552 \mathrm{E}-03 \\
8.402 \mathrm{E}-01 \\
9.440 \mathrm{E}-04 \\
3.351 \mathrm{E}-02 \\
3.351 \mathrm{E}-02 \\
8.402 \mathrm{E}-01
\end{gathered}
$$

\begin{tabular}{|c|c|}
\hline Chemical & $\begin{array}{l}\text { Amount } \\
(\mathrm{kg} / \mathrm{Y} \mathrm{r})\end{array}$ \\
\hline $57-12-5$ & 1. $814 \mathrm{E}-01$ \\
\hline $75-09-2$ & $4.543 E+00$ \\
\hline $\mathrm{Ag}$ & $6.519 \mathrm{E}-02$ \\
\hline As & $2.353 \mathrm{E}-02$ \\
\hline $\mathrm{Ba}$ & $1.752 \mathrm{E}+01$ \\
\hline $\mathrm{cd}$ & $1.078 \mathrm{E}+00$ \\
\hline$C 1-2-x$ & 1. $687 \mathrm{E}+00$ \\
\hline$C l-3-x$ & $8.308 \mathrm{E}+00$ \\
\hline Cl-4-x & $5.036 \mathrm{E}+00$ \\
\hline$C 1-F-x$ & $1.893 \mathrm{E}+00$ \\
\hline $\mathrm{Cr}$ & $9.451 \mathrm{E}+00$ \\
\hline HC-insol & $9.627 \mathrm{E}-01$ \\
\hline HC-solub & $3.150 \mathrm{E}+00$ \\
\hline $\mathrm{Hg}$ & $3.315 \mathrm{E}+00$ \\
\hline $\mathrm{Pb}$ & $4.093 \mathrm{E}+01$ \\
\hline & $1.026 \mathrm{E}-01$ \\
\hline
\end{tabular}

Waste Type: Solids (CH-Non-alpha)

Total Mass $(\mathrm{kg} / \mathrm{yr}): 1.580 \mathrm{E}+03$

\begin{tabular}{lcc} 
Chemical & & $\begin{array}{c}\text { Amount } \\
(\mathrm{kg} / \mathrm{yr})\end{array}$ \\
\cline { 1 - 1 } $75-12-5$ & & $8.005 \mathrm{E}-02$ \\
$\mathrm{Ag}-09-2$ & $3.801 \mathrm{E}-01$ \\
$\mathrm{As}$ & $2.572 \mathrm{E}-02$ \\
$\mathrm{Ba}$ & $8.795 \mathrm{E}-03$ \\
$\mathrm{Cd}$ & $1.035 \mathrm{E}+00$ \\
$\mathrm{Cl}-2-\mathrm{x}$ & $3.157 \mathrm{E}-02$ \\
$\mathrm{Cl}-3-\mathrm{x}$ & $1.699 \mathrm{E}+01$ \\
$\mathrm{Cl}-4-\mathrm{x}$ & $1.988 \mathrm{E}+00$ \\
$\mathrm{Cl}-\mathrm{F}-\mathrm{x}$ & $2.323 \mathrm{E}-01$ \\
$\mathrm{Cr}$ & $1.010 \mathrm{E}+00$ \\
$\mathrm{HC}-$-insol & $4.421 \mathrm{E}+00$ \\
$\mathrm{HC}-$ Solub & $1.600 \mathrm{E}+00$ \\
$\mathrm{Hg}$ & $5.065 \mathrm{E}-02$ \\
$\mathrm{~Pb}$ & $2.396 \mathrm{E}+00$ \\
$\mathrm{Se}$ & $1.789 \mathrm{E}-02$
\end{tabular}

Waste Type: Organic Liquids (CH-Non-alpha)

$7.053 E+00$

Total Mass $(\mathrm{kg} / \mathrm{yr})$ :

$6.912 \mathrm{E}+03$

\begin{tabular}{c}
$\begin{array}{c}\text { Activity } \\
\text { (Ci/yr) }\end{array}$ \\
\hline $9.378 \mathrm{E}-02$ \\
$1.289 \mathrm{E}-01$ \\
$9.769 \mathrm{E}-02$ \\
$1.074 \mathrm{E}-03$ \\
$1.621 \mathrm{E}-02$ \\
$1.869 \mathrm{E}+01$ \\
$2.149 \mathrm{E}-03$ \\
$2.852 \mathrm{E}-01$ \\
$8.987 \mathrm{E}-03$ \\
$3.712 \mathrm{E}-03$ \\
$1.875 \mathrm{E}-02$ \\
$8.597 \mathrm{E}-04$ \\
$9.183 \mathrm{E}-02$ \\
$9.183 \mathrm{E}-02$
\end{tabular}

\begin{tabular}{l} 
Chemical \\
\hline $57-12-5$ \\
$\mathrm{Ag}$ \\
$\mathrm{Cd}$ \\
$\mathrm{C} 1-3-\mathrm{x}$ \\
$\mathrm{Cl}-4-\mathrm{x}$ \\
$\mathrm{Cr}$ \\
$\mathrm{HC}-$ insol \\
$\mathrm{HC}-\mathrm{solub}$ \\
$\mathrm{Hg}$ \\
$\mathrm{Pb}$ \\
$\mathrm{Se}$
\end{tabular}

\begin{tabular}{c}
$\begin{array}{c}\text { Amount } \\
(\mathrm{kg} / \mathrm{Yr})\end{array}$ \\
\hline $1.244 \mathrm{E}+00$ \\
$3.041 \mathrm{E}-01$ \\
$3.387 \mathrm{E}-02$ \\
$5.530 \mathrm{E}+00$ \\
$3.110 \mathrm{E}-01$ \\
$1.451 \mathrm{E}+00$ \\
$7.603 \mathrm{E}+01$ \\
$2.488 \mathrm{E}+03$ \\
$1.935 \mathrm{E}+00$ \\
$3.456 \mathrm{E}-01$ \\
$2.765 \mathrm{E}-01$
\end{tabular}

Waste Type: Solids (CH-Non-alpha)

1. $292 \mathrm{E}+01$

Total Mass $(\mathrm{kg} / \mathrm{yr}): 1.871 \mathrm{E}+04$

\begin{tabular}{c}
$\begin{array}{c}\text { Activity } \\
\text { (Ci/yr) }\end{array}$ \\
\hline $1.719 \mathrm{E}-01$ \\
$2.364 \mathrm{E}-01$ \\
$1.787 \mathrm{E}-01$ \\
$1.970 \mathrm{E}-03$ \\
$2.973 \mathrm{E}-02$ \\
$3.428 \mathrm{E}+01$ \\
$3.940 \mathrm{E}-03$ \\
$5.230 \mathrm{E}-01$ \\
$1.647 \mathrm{E}-02$ \\
$6.806 \mathrm{E}-03$ \\
$3.439 \mathrm{E}-02$ \\
$1.576 \mathrm{E}-03$ \\
$1.680 \mathrm{E}-01$ \\
$1.680 \mathrm{E}-01$
\end{tabular}


Shipping Site: LANL Waste Type: Organic Liquids (CH-Alpha)

Total Volume $(\mathrm{m} 3 / \mathrm{yr})$ :

$6.158 \mathrm{E}+01$

Total Mass $(\mathrm{kg} / \mathrm{yr})$ :

$6.598 \mathrm{E}+04$

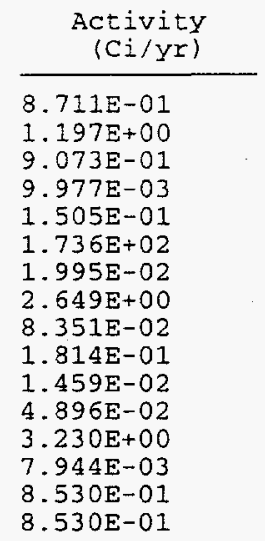

\begin{tabular}{l} 
Chemical \\
\hline $57-12-5$ \\
$75-09-2$ \\
$\mathrm{Ag}$ \\
$\mathrm{As}$ \\
$\mathrm{Ba}$ \\
$\mathrm{Cd}$ \\
$\mathrm{Cl}-2-\mathrm{x}$ \\
$\mathrm{Cl}-3-\mathrm{x}$ \\
$\mathrm{Cl}-4-\mathrm{x}$ \\
$\mathrm{Cl}-\mathrm{F}-\mathrm{x}$ \\
$\mathrm{Cr}$ \\
$\mathrm{HC}-$ insol \\
$\mathrm{HC}-\mathrm{SOlub}$ \\
$\mathrm{Hg}$ \\
$\mathrm{Pb}$ \\
$\mathrm{Se}$
\end{tabular}

Shipping Site: IANL

Waste Type: Solids (CH-Alpha)

Total Volume $(\mathrm{m} 3 / \mathrm{yr})$ :

$2.801 E+01$

Total Mass (kg/yr) :

$6.796 \mathrm{E}+04$

Radionuclide

$\mathrm{Ba}-137 \mathrm{~m}$

Co -60

Cs -137

Eu-154

$\mathrm{Fe}-55$

$\mathrm{H}-3$

Ni-59

$\mathrm{Ni}-63$

$\mathrm{Pm}-147$

$\mathrm{Pu}-238$

$\mathrm{Pu}-239$

Pu -240

$\mathrm{Pu}-241$

$\mathrm{Sm}-151$

Sr -90

$\mathrm{Y}-90$

Shipping Site: LBL

\begin{tabular}{c}
$\begin{array}{c}\text { Activity } \\
(\mathrm{Ci} / \mathrm{Y})\end{array}$ \\
\hline $3.962 \mathrm{E}-01$ \\
$5.448 \mathrm{E}-01$ \\
$4.126 \mathrm{E}-01$ \\
$4.538 \mathrm{E}-03$ \\
$6.850 \mathrm{E}-02$ \\
$7.900 \mathrm{E}+01$ \\
$9.077 \mathrm{E}-03$ \\
$1.205 \mathrm{E}+00$ \\
$3.799 \mathrm{E}-02$ \\
$8.253 \mathrm{E}-02$ \\
$6.639 \mathrm{E}-03$ \\
$2.227 \mathrm{E}-02$ \\
$1.469 \mathrm{E}+00$ \\
$3.614 \mathrm{E}-03$ \\
$3.879 \mathrm{E}-01$ \\
$3.879 \mathrm{E}-01$
\end{tabular}

Chemical
57-12-5
$75-09-2$
$\mathrm{Ag}$
$\mathrm{As}$
$\mathrm{Ba}$
$\mathrm{Cd}$
$\mathrm{CI}-2-\mathrm{x}$
$\mathrm{CI}-3-\mathrm{x}$
$\mathrm{Cl}-4-\mathrm{x}$
$\mathrm{Cl}-\mathrm{F}-\mathrm{x}$
$\mathrm{Cr}$
$\mathrm{HC}-$ insol
$\mathrm{HC}-\mathrm{solub}$
$\mathrm{Hg}$
$\mathrm{Pb}$
$\mathrm{Se}$

\begin{tabular}{c}
$\begin{array}{c}\text { Amount } \\
(\mathrm{kg} / \mathrm{Yr})\end{array}$ \\
\hline $9.199 \mathrm{E}-01$ \\
$7.358 \mathrm{E}+01$ \\
$5.600 \mathrm{E}-01$ \\
$1.525 \mathrm{E}-01$ \\
$1.081 \mathrm{E}+01$ \\
$1.740 \mathrm{E}+01$ \\
$2.574 \mathrm{E}+01$ \\
$1.337 \mathrm{E}+02$ \\
$8.345 \mathrm{E}+01$ \\
$2.951 \mathrm{E}+01$ \\
$9.888 \mathrm{E}+01$ \\
$4.851 \mathrm{E}+01$ \\
$1.967 \mathrm{E}+01$ \\
$1.501 \mathrm{E}+01$ \\
$6.059 \mathrm{E}+02$ \\
$1.320 \mathrm{E}+00$
\end{tabular}

Waste Type: Organic Liquids (CH-Non-alpha)

Total volume $(\mathrm{m} 3 / \mathrm{yr})$ :

$3.230 \mathrm{E}-01$

Total Mass $(\mathrm{kg} / \mathrm{yr}): 3.100 \mathrm{E}+02$

\begin{tabular}{c}
$\begin{array}{c}\text { Activity } \\
\text { (Ci/yr) }\end{array}$ \\
\hline $4.273 \mathrm{E}-03$ \\
$4.476 \mathrm{E}-03$ \\
$2.032 \mathrm{E}+01$ \\
$1.037 \mathrm{E}-03$ \\
$1.017 \mathrm{E}-03$ \\
$4.273 \mathrm{E}-03$ \\
$1.037 \mathrm{E}-03$ \\
$1.037 \mathrm{E}-03$ \\
$4.273 \mathrm{E}-03$
\end{tabular}

\begin{tabular}{l} 
Chemical \\
\hline $75-09-2$ \\
As \\
$\mathrm{Ba}$ \\
$\mathrm{Cd}$ \\
$\mathrm{Cl}-2-\mathrm{x}$ \\
$\mathrm{Cl}-3-\mathrm{x}$ \\
$\mathrm{Cl}-4-\mathrm{x}$ \\
$\mathrm{Cr}$ \\
$\mathrm{HC}-$ insol \\
$\mathrm{HC}-\mathrm{SOlub}$ \\
$\mathrm{Hg}$ \\
$\mathrm{Pb}$
\end{tabular}




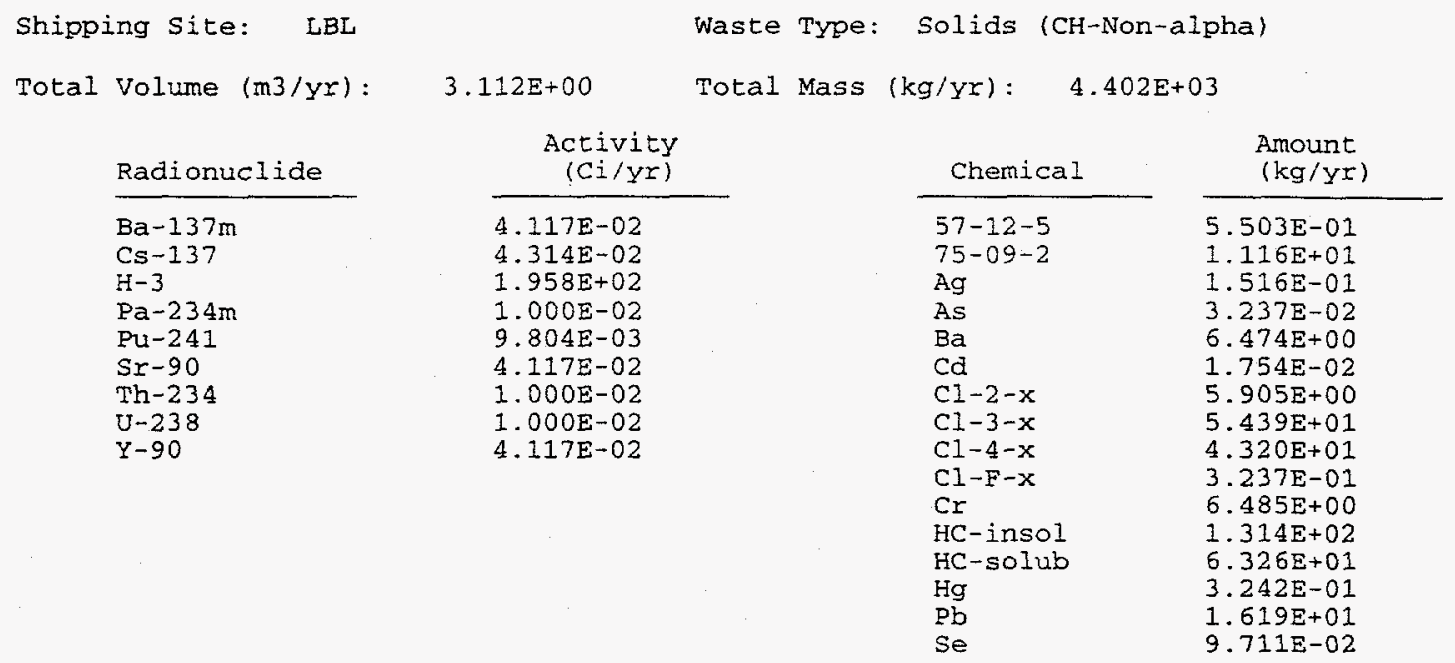

Shipping Site: LERHR Waste Type: Organic Liquids (CH-Non-alpha)

\begin{tabular}{l} 
Total Volume (m3/yr): \\
Radionuclice \\
\hline $\mathrm{Am}-241$ \\
$\mathrm{Ba}-137 \mathrm{~m}$ \\
$\mathrm{Co}-60$ \\
$\mathrm{Cs}-137$ \\
$\mathrm{Eu}-154$ \\
$\mathrm{Eu}-155$ \\
$\mathrm{H}-3$ \\
$\mathrm{Nb}-94$ \\
$\mathrm{Ni}-59$ \\
$\mathrm{Ni}-63$ \\
$\mathrm{~Pa}-234 \mathrm{~m}$ \\
$\mathrm{Pu}-238$ \\
$\mathrm{Pu}-239$ \\
$\mathrm{Pu}-240$ \\
$\mathrm{Pu}-241$ \\
$\mathrm{Sm}-151$ \\
$\mathrm{Sr}-90$ \\
$\mathrm{Tc}-99$ \\
$\mathrm{Th}-232$ \\
$\mathrm{Th}-234$ \\
$\mathrm{U}-238$ \\
$\mathrm{Y}-90$
\end{tabular}

Shipping site: LERHR Total Volume (m3/yr):

Radionuclide

Am-241

$\mathrm{Ba}-137 \mathrm{~m}$

Co-60

Cs-137

Eu-154

$\mathrm{Eu}-155$

$\mathrm{H}-3$

$\mathrm{Nb}-94$

$\mathrm{Ni}-59$

$\mathrm{Ni}-63$

$\mathrm{Pa}-234 \mathrm{~m}$

Pu -238

Pu-239

$\mathrm{Pu}-240$

$\mathrm{Pu}-241$

$\mathrm{Sm}-151$

Sr -90

Tc-99

Th-232

Th-234

$\mathrm{U}-238$

$\mathrm{Y}-90$
$5.020 E-03$

Total Mass (kg/yr):

$5.750 E+00$

\begin{tabular}{c}
$\begin{array}{c}\text { Activity } \\
(C i / y r)\end{array}$ \\
\hline $2.470 \mathrm{E}-07$ \\
$3.636 \mathrm{E}-04$ \\
$1.353 \mathrm{E}-04$ \\
$3.888 \mathrm{E}-04$ \\
$1.440 \mathrm{E}-06$ \\
$4.940 \mathrm{E}-07$ \\
$2.801 \mathrm{E}-05$ \\
$1.029 \mathrm{E}-06$ \\
$2.183 \mathrm{E}-05$ \\
$2.297 \mathrm{E}-03$ \\
$4.976 \mathrm{E}-05$ \\
$1.529 \mathrm{E}-05$ \\
$2.470 \mathrm{E}-07$ \\
$3.705 \mathrm{E}-07$ \\
$1.620 \mathrm{E}-05$ \\
$4.688 \mathrm{E}-06$ \\
$3.438 \mathrm{E}-04$ \\
$4.112 \mathrm{E}-06$ \\
$4.116 \mathrm{E}-07$ \\
$4.976 \mathrm{E}-05$ \\
$4.976 \mathrm{E}-05$ \\
$3.438 \mathrm{E}-04$
\end{tabular}

\begin{tabular}{l} 
Chemical \\
\hline $57-12-5$ \\
$75-09-2$ \\
$\mathrm{Ag}$ \\
$\mathrm{As}$ \\
$\mathrm{Ba}$ \\
$\mathrm{Cd}$ \\
$\mathrm{Cl}-2-\mathrm{x}$ \\
$\mathrm{Cl}-3-\mathrm{x}$ \\
$\mathrm{Cl}-4-\mathrm{x}$ \\
$\mathrm{Cl}-\mathrm{F}-\mathrm{x}$ \\
$\mathrm{Cr}$ \\
$\mathrm{HC}-$ insol \\
$\mathrm{HC}-\mathrm{solub}$ \\
$\mathrm{Hg}$ \\
$\mathrm{Pb}$ \\
$\mathrm{Se}$
\end{tabular}

\begin{tabular}{c}
$\begin{array}{c}\text { Amount } \\
(\mathrm{kg} / \mathrm{yr})\end{array}$ \\
\hline $2.373 \mathrm{E}-04$ \\
$4.324 \mathrm{E}-03$ \\
$1.189 \mathrm{E}-04$ \\
$2.403 \mathrm{E}-05$ \\
$1.415 \mathrm{E}-04$ \\
$3.358 \mathrm{E}-03$ \\
$2.702 \mathrm{E}-03$ \\
$2.592 \mathrm{E}-01$ \\
$5.439 \mathrm{E}-02$ \\
$5.108 \mathrm{E}-04$ \\
$3.156 \mathrm{E}-03$ \\
$5.198 \mathrm{E}-01$ \\
$6.473 \mathrm{E}-01$ \\
$6.928 \mathrm{E}-04$ \\
$7.432 \mathrm{E}-04$ \\
$1.039 \mathrm{E}-04$
\end{tabular}

Waste Type: Solids (CH-Non-alpha)

3. $442 \mathrm{E}-01$

Total Mass (kg/yr):

$2.492 E+02$

$\begin{array}{r}\text { Activity } \\ (C i / Y r)\end{array}$
$1.701 E-05$
$2.505 E-02$
$9.323 E-03$
$2.641 E-02$
$9.928 E-05$
$3.403 E-05$
$1.930 E-03$
$7.091 E-05$
$1.504 E-03$
$1.583 E-01$
$3.428 E-03$
$1.054 E-03$
$1.701 E-05$
$2.552 E-05$
$1.115 E-03$
$3.230 E-04$
$2.335 E-02$
$2.793 E-04$
$2.836 E-05$
$3.428 E-03$
$3.428 E-03$
$2.335 E-02$

Amount $(\mathrm{kg} / \mathrm{YI})$

4.707E-04

1. $438 \mathrm{E}-01$

2. $547 \mathrm{E}-03$

1. $464 \mathrm{E}-05$

3. $843 \mathrm{E}-02$

$8.851 \mathrm{E}-03$

1. $556 \mathrm{E}-01$

6. $005 \mathrm{E}-01$

2. $831 \mathrm{E}+00$

1. 391E-01

4. $652 \mathrm{E}-03$

1. $675 \mathrm{E}+00$

3. 579E-01

$5.762 \mathrm{E}-03$

1. $542 \mathrm{E}-02$

4. $361 \mathrm{E}-04$ 
Shipping Site: LLNL

Total Volume (m3/yr):

Radionuclide

$\mathrm{Ba}-137 \mathrm{~m}$

Cs -137

$\mathrm{H}-3$

$\mathrm{Pa}-234 \mathrm{~m}$

$\mathrm{Pu}-241$

S $r-90$

Th-234

$\mathrm{U}-238$

$Y-90$

Shipping site: ILNL

Total Volume $(\mathrm{m} 3 / \mathrm{yr})$ :

Radionuclide

$\mathrm{Ba}-137 \mathrm{~m}$

Cs -137

$\mathrm{H}-3$

$\mathrm{Pa}-234 \mathrm{~m}$

$\mathrm{Pu}-241$

Sr-90

Th-234

$\mathrm{U}-238$

$\mathrm{Y}-90$

Shipping Site: LLNL

Total volume $(\mathrm{m} 3 / \mathrm{YI}\}$ :

Radionuclide

$\mathrm{Ba}-137 \mathrm{~m}$

Cs -137

$\mathrm{H}-3$

$\mathrm{Pa}-234 \mathrm{~m}$

$\mathrm{Pu}-238$

Pu-241

Sr -90

Th-234

$\mathrm{U}-238$

$Y-90$
$7.727 \mathrm{E}+01$

Total Mass (kg/yr) :

$8.159 E+04$

\begin{tabular}{|c|c|}
\hline Chemical & $\begin{array}{l}\text { Anount } \\
(\mathrm{kg} / \mathrm{yr})\end{array}$ \\
\hline $57-12-5$ & $7.162 \mathrm{E}+00$ \\
\hline $75-09-2$ & $5.434 E+00$ \\
\hline $\mathrm{Ag}$ & $3.590 E+00$ \\
\hline As & $4.598 \mathrm{E}-01$ \\
\hline $\mathrm{Ba}$ & $3.135 E+00$ \\
\hline $\mathrm{cd}$ & $1.949 \mathrm{E}-01$ \\
\hline Cl-2-x & $1.546 \mathrm{E}+01$ \\
\hline$c l-3-x$ & $6.087 \mathrm{E}+02$ \\
\hline$C 1-4-x$ & $3.439 \mathrm{E}+01$ \\
\hline$C 1-F-x$ & $1.254 E+01$ \\
\hline $\mathrm{Cr}$ & $8.355 \mathrm{E}+00$ \\
\hline $\mathrm{HC}$-insol & $5.003 E+02$ \\
\hline $\mathrm{HC}$-solub & $1.434 \mathrm{E}+04$ \\
\hline $\mathrm{Hg}$ & $2.033 E+01$ \\
\hline $\mathrm{Pb}$ & $3.118 E+00$ \\
\hline $\mathrm{Se}$ & $3.138 \mathrm{E}+00$ \\
\hline
\end{tabular}

Waste Type: Solids (CH-Non-alpha)

$4.978 \mathrm{E}+01$ Total Mass $(\mathrm{kg} / \mathrm{yr}): \quad 6.833 \mathrm{E}+04$

\begin{tabular}{c}
$\begin{array}{c}\text { Activity } \\
\text { (Ci/yr) }\end{array}$ \\
\hline $6.586 \mathrm{E}-01$ \\
$6.899 \mathrm{E}-01$ \\
$3.132 \mathrm{E}+03$ \\
$1.599 \mathrm{E}-01$ \\
$1.568 \mathrm{E}-01$ \\
$6.586 \mathrm{E}-01$ \\
$1.599 \mathrm{E}-01$ \\
$1.599 \mathrm{E}-01$ \\
$6.586 \mathrm{E}-01$
\end{tabular}

\begin{tabular}{lc} 
Chemical & $\begin{array}{c}\text { Amount } \\
(\mathrm{kg} / \mathrm{yr})\end{array}$ \\
\cline { 1 - 1 } $57-12-5$ & $8.172 \mathrm{E}+00$ \\
$75-09-2$ & $8.995 \mathrm{E}+00$ \\
$\mathrm{Ag}$ & $3.852 \mathrm{E}+00$ \\
$\mathrm{As}$ & $1.401 \mathrm{E}+00$ \\
$\mathrm{Ba}$ & $1.097 \mathrm{E}+02$ \\
$\mathrm{Cd}$ & $2.705 \mathrm{E}+00$ \\
$\mathrm{Cl}-2-\mathrm{x}$ & $7.372 \mathrm{E}+00$ \\
$\mathrm{Cl}-3-\mathrm{x}$ & $1.900 \mathrm{E}+02$ \\
$\mathrm{Cl}-4-\mathrm{x}$ & $3.100 \mathrm{E}+01$ \\
$\mathrm{Cl}-\mathrm{F}-\mathrm{x}$ & $5.418 \mathrm{E}+00$ \\
$\mathrm{Cr}$ & $1.262 \mathrm{E}+02$ \\
$\mathrm{HC}-\mathrm{insol}$ & $7.525 \mathrm{E}+02$ \\
$\mathrm{HC}-\mathrm{SOlub}$ & $3.756 \mathrm{E}+02$ \\
$\mathrm{Hg}$ & $4.188 \mathrm{E}+01$ \\
$\mathrm{~Pb}$ & $1.529 \mathrm{E}+02$ \\
$\mathrm{Se}$ & $1.787 \mathrm{E}+01$
\end{tabular}

Waste Type: Organic Liquids ( $\mathrm{CH}-\mathrm{Alpha})$

5. $737 \mathrm{E}+01$

Total Mass

$(\mathrm{kg} / \mathrm{yr}):$

$6.269 \mathrm{E}+04$

Chemical
$57-12-5$
$75-09-2$
Ag
As
Ba
Cd
Cl-2-x
Cl-3-x
Cl-4-x
Cl-F-x
Cr
HC-insol
HC-solub
Hg
Pb
Se

\begin{tabular}{c}
$\begin{array}{c}\text { Amount } \\
(\mathrm{kg} / \mathrm{yr})\end{array}$ \\
\hline $4.251 \mathrm{E}+00$ \\
$2.262 \mathrm{E}+01$ \\
$2.130 \mathrm{E}+00$ \\
$3.141 \mathrm{E}-01$ \\
$2.037 \mathrm{E}+00$ \\
$1.580 \mathrm{E}+01$ \\
$1.943 \mathrm{E}+01$ \\
$1.480 \mathrm{E}+03$ \\
$2.696 \mathrm{E}+02$ \\
$7.889 \mathrm{E}+00$ \\
$1.843 \mathrm{E}+01$ \\
$2.652 \mathrm{E}+03$ \\
$9.317 \mathrm{E}+03$ \\
$1.216 \mathrm{E}+01$ \\
$4.845 \mathrm{E}+00$ \\
$1.862 \mathrm{E}+00$
\end{tabular}


Shipping site: LLNL

Total Volume (m3/yr):

Radionuclide

$\mathrm{Ba}-137 \mathrm{~m}$

Cs -137

$\mathrm{H}-3$

$\mathrm{Pa}-234 \mathrm{~m}$

$\mathrm{Pu}-238$

Pu-241

Sr-90

Th-234

$\mathrm{U}-238$

$\mathrm{Y}-90$

Shipping site: MINS

Total Volume $(\mathrm{m} 3 / \mathrm{yr})$ :

Radionuclide

$\mathrm{Ba}-137 \mathrm{~m}$

Co-60

Cs -134

Cs -137

Eu-154

Eu-155

$\mathrm{Fe}-55$

$\mathrm{H}-3$

$\mathrm{Mn}-54$

$\mathrm{Nb}-94$

$\mathrm{Ni}-59$

Ni-63

$\mathrm{Pa}-234 \mathrm{~m}$

Pm-147

Pu-238

Pu-241

$\mathrm{Sb}-125$

$\mathrm{Sm}-151$

Sr-90

$\mathrm{Te}-125 \mathrm{~m}$

Th-234

$\mathrm{U}-238$

$\mathrm{Y}-90$
Waste Type: Solids (CH-Alpha)

Total Mass $(\mathrm{kg} / \mathrm{yr}): 1.017 \mathrm{E}+05$

\begin{tabular}{lc} 
Chemical & $\begin{array}{c}\text { Amount } \\
(\mathrm{kg} / \mathrm{yr})\end{array}$ \\
\cline { 1 - 1 } $57-12-5$ & $4.138 \mathrm{E}+00$ \\
$75-09-2$ & $1.192 \mathrm{E}+01$ \\
$\mathrm{Ag}$ & $9.746 \mathrm{E}-01$ \\
$\mathrm{As}$ & $3.439 \mathrm{E}-01$ \\
$\mathrm{Ba}$ & $5.008 \mathrm{E}+01$ \\
$\mathrm{Cd}$ & $3.649 \mathrm{E}+00$ \\
$\mathrm{Cl}-2-\mathrm{x}$ & $7.183 \mathrm{E}+00$ \\
$\mathrm{Cl}-3-\mathrm{x}$ & $4.229 \mathrm{E}+01$ \\
$\mathrm{Cl}-4-\mathrm{x}$ & $2.492 \mathrm{E}+01$ \\
$\mathrm{Cl}-\mathrm{F}-\mathrm{x}$ & $1.009 \mathrm{E}+01$ \\
$\mathrm{Cr}$ & $2.709 \mathrm{E}+02$ \\
$\mathrm{HC}-\mathrm{insol}$ & $7.977 \mathrm{E}+01$ \\
$\mathrm{HC}-\mathrm{solub}$ & $2.905 \mathrm{E}+01$ \\
$\mathrm{Hg}$ & $8.247 \mathrm{E}+00$ \\
$\mathrm{~Pb}$ & $1.721 \mathrm{E}+02$ \\
$\mathrm{Se}$ & $2.561 \mathrm{E}+00$
\end{tabular}

Waste Type: Solids (CH-Non-alpha)

Total Mass $(\mathrm{kg} / \mathrm{yr}): 3.545 \mathrm{E}+03$

\begin{tabular}{|c|c|}
\hline Chemical & $\begin{array}{l}\text { Amount } \\
(\mathrm{kg} / \mathrm{yr})\end{array}$ \\
\hline $75-09-2$ & $5.544 \mathrm{E}-01$ \\
\hline $\mathrm{Ag}$ & $1.457 \mathrm{E}-02$ \\
\hline As & $7.197 \mathrm{E}-03$ \\
\hline $\mathrm{Ba}$ & $2.427 E+00$ \\
\hline $\mathrm{cd}$ & $1.150 \mathrm{E}-01$ \\
\hline$C I-2-x$ & $5.975 E-01$ \\
\hline Cl $-3-x$ & $1.190 \mathrm{E}+01$ \\
\hline Cl-4-x & $2.652 \mathrm{E}+00$ \\
\hline $\mathrm{C} I-\mathrm{F}-\mathrm{x}$ & $6.891 \mathrm{E}-01$ \\
\hline Cr & $7.414 E-01$ \\
\hline $\mathrm{HC}$-insol & $4.047 \mathrm{E}+01$ \\
\hline HC-solub & $1.726 \mathrm{E}+01$ \\
\hline $\mathrm{Hg}$ & $5.165 \mathrm{E}-01$ \\
\hline $\mathrm{Pb}$ & $4.557 E-01$ \\
\hline $\mathrm{Se}$ & $3.325 \mathrm{E}-02$ \\
\hline
\end{tabular}


Shipping Site: MOUND Waste Type: Organic Liquids (CH-Alpha)

Total Volume (m3/yr):

1. $768 E+00$

Total Mass (kg/yr):

$2.190 E+03$

Radionuclide

$\mathrm{Ba}-137 \mathrm{~m}$

Cs -137

$\mathrm{H}-3$

$\mathrm{Pa}-234 \mathrm{~m}$

Pu-238

Pu-241

Sr -90

Th -234

$\mathrm{U}-238$

$\mathrm{Y}-90$

\begin{tabular}{c}
$\begin{array}{c}\text { Activity } \\
\text { (Ci/Yr) }\end{array}$ \\
\hline $2.339 \mathrm{E}-02$ \\
$2.450 \mathrm{E}-02$ \\
$1.111 \mathrm{E}+02$ \\
$5.680 \mathrm{E}-03$ \\
$5.569 \mathrm{E}-03$ \\
$1.113 \mathrm{E}-01$ \\
$2.339 \mathrm{E}-02$ \\
$5.680 \mathrm{E}-03$ \\
$5.680 \mathrm{E}-03$ \\
$2.339 \mathrm{E}-02$
\end{tabular}

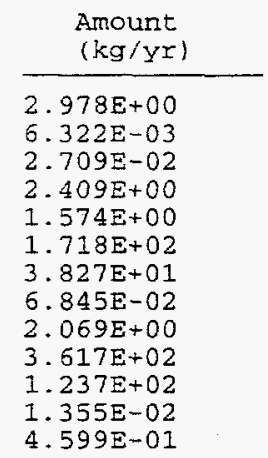

Waste Type: Solids (CH-Alpha)

Total Mass (kg/yr): $6.176 \mathrm{E}+00$

Total Volume $(\mathrm{m} 3 / \mathrm{yr})$ :

$5.712 \mathrm{E}-03$

Radionuclide

$\mathrm{Ba}-137 \mathrm{~m}$

Cs -137

$\mathrm{H}-3$

Pa $-234 \mathrm{~m}$

Pu-238

Pu-241

Sr -90

Th-234

$\mathrm{U}-238$

$Y-90$

\begin{tabular}{r}
$\begin{array}{c}\text { Activity } \\
(\mathrm{Ci} / \mathrm{yr})\end{array}$ \\
\hline $7.557 \mathrm{E}-05$ \\
$7.917 \mathrm{E}-05$ \\
$3.591 \mathrm{E}-01$ \\
$1.835 \mathrm{E}-05$ \\
$1.799 \mathrm{E}-05$ \\
$3.598 \mathrm{E}-04$ \\
$7.557 \mathrm{E}-05$ \\
$1.835 \mathrm{E}-05$ \\
$1.835 \mathrm{E}-05$ \\
$7.557 \mathrm{E}-05$
\end{tabular}

\begin{tabular}{l} 
Chemical \\
\hline $75-09-2$ \\
$\mathrm{Ag}$ \\
$\mathrm{Ba}$ \\
$\mathrm{Cd}$ \\
$\mathrm{Cl}-2-\mathrm{x}$ \\
$\mathrm{Cl}-3-\mathrm{x}$ \\
$\mathrm{Cl}-4-\mathrm{x}$ \\
$\mathrm{Cl}-\mathrm{F}-\mathrm{x}$ \\
$\mathrm{Cr}$ \\
$\mathrm{HC}-$ insol \\
$\mathrm{HC}-\mathrm{SOlub}$ \\
$\mathrm{Hg}$ \\
$\mathrm{Pb}$ \\
$\mathrm{Se}$
\end{tabular}

Shipping Site: NNS

Waste Type: Solids (CH-Non-alpha)

Total Volume (m3/yr):

$3.560 E-01$

Tota1 Mass $(\mathrm{kg} / \mathrm{yr})$ :

$3.925 \mathrm{E}+02$

Radionuclide

\begin{tabular}{l}
\hline $\mathrm{Ba}-137 \mathrm{~m}$ \\
$\mathrm{Co}-60$ \\
$\mathrm{Cs}-134$ \\
$\mathrm{Cs}-137$ \\
$\mathrm{Eu}-154$ \\
$\mathrm{Eu}-155$ \\
$\mathrm{Fe}-55$ \\
$\mathrm{H}-3$ \\
$\mathrm{Mn}-54$ \\
$\mathrm{Nb}-94$ \\
$\mathrm{Ni}-59$ \\
$\mathrm{Ni}-63$ \\
$\mathrm{~Pa}-234 \mathrm{~m}$ \\
$\mathrm{Pm}-147$ \\
$\mathrm{Pu}-238$ \\
$\mathrm{Pu}-241$ \\
$\mathrm{Sb}-125$ \\
$\mathrm{Sm}-151$ \\
$\mathrm{Sr}-90$ \\
$\mathrm{Te}-125 \mathrm{~m}$ \\
$\mathrm{Th}-234$ \\
$\mathrm{U}-238$ \\
$\mathrm{Y}-90$
\end{tabular}

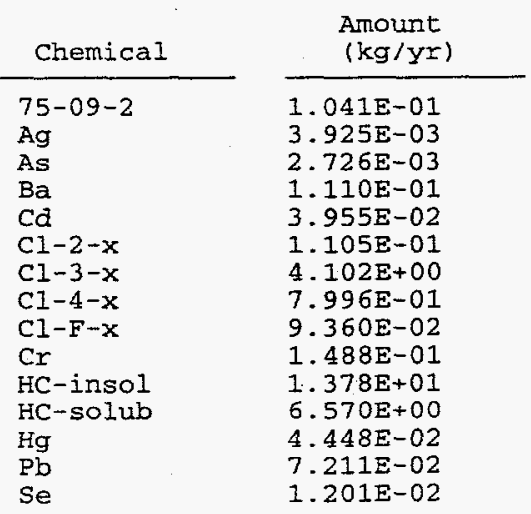


Shipping Site: NTS Waste Type: Solids (CH-Non-alpha)

Total Volume $(\mathrm{m} 3 / \mathrm{yr})$ :

$6.621 E-03$

Total Mass $(\mathrm{kg} / \mathrm{yr})$ :

$8.886 E+00$

Radionuclide

$\mathrm{Ba}-137 \mathrm{~m}$

Cs -137

$\mathrm{H}-3$

$\mathrm{Pa}-234 \mathrm{~m}$

$\mathrm{Pu}-241$

Sr -90

Th- -234

$\mathrm{U}-238$

$\mathrm{Y}-90$
Shipping Site: ORNL

Total Volume $(\mathrm{m} 3 / \mathrm{yr})$ :

Radionuclide

Am-241

$\mathrm{Ba}-137 \mathrm{~m}$

Co-60

Cs -137

Eu-154

Eu-155

H-3

$\mathrm{Nb}-94$

Ni -59

$\mathrm{Ni}-63$

$\mathrm{Pa}-234 \mathrm{~m}$

$\mathrm{Pu}-238$

Pu-239

Pu -240

Pu-241

$\mathrm{Sm}-151$

Sr -90

Tc -99

Th-232

Th-234

$\mathrm{U}-238$

$Y-90$

Shipping Site:

ORNL

Total Volume $(\mathrm{m} 3 / \mathrm{yr})$ :

Radionuclide

Am-241

$\mathrm{Ba}-137 \mathrm{~m}$

Co- 60

Cs -137

Eu-154

Eบ-155

$\mathrm{H}-3$

$\mathrm{Nb}-94$

$\mathrm{Ni}-59$

$\mathrm{Ni}-63$

$\mathrm{Pa}-234 \mathrm{~m}$

Pu-238

Pu-239

Pu $1-240$

Pu-241

$\mathrm{Sm}-151$

Sr -90

TC- 99

Th- 232

Th-234

$\mathrm{U}-238$

$\mathrm{Y}-90$

$1.093 E+03$

Waste Type: Organic Licuids

\begin{tabular}{l} 
Chemical \\
\hline $75-09-2$ \\
$\mathrm{Ag}$ \\
$\mathrm{Cd}$ \\
$\mathrm{Cl}-2-\mathrm{x}$ \\
$\mathrm{C} 1-3-\mathrm{x}$ \\
$\mathrm{Cl}-4-\mathrm{x}$ \\
$\mathrm{Cl}-\mathrm{F}-\mathrm{x}$ \\
$\mathrm{Cr}$ \\
$\mathrm{HC}-$ insol \\
$\mathrm{HC}-\mathrm{solub}$ \\
$\mathrm{Hg}$ \\
$\mathrm{Pb}$ \\
$\mathrm{Se}$
\end{tabular}

\begin{tabular}{c}
$\begin{array}{c}\text { Amount } \\
(\mathrm{kg} / \mathrm{Yr})\end{array}$ \\
\hline $5.331 \mathrm{E}-03$ \\
$9.774 \mathrm{E}-05$ \\
$5.331 \mathrm{E}-04$ \\
$5.331 \mathrm{E}-03$ \\
$2.221 \mathrm{E}-02$ \\
$1.688 \mathrm{E}-01$ \\
$2.665 \mathrm{E}-03$ \\
$1.777 \mathrm{E}-04$ \\
$4.443 \mathrm{E}-02$ \\
$2.221 \mathrm{E}-02$ \\
$3.465 \mathrm{E}-04$ \\
$3.199 \mathrm{E}-04$ \\
$8.886 \mathrm{E}-06$
\end{tabular}

(CH-Non-alpha)

Total Mass $(\mathrm{kg} / \mathrm{yr}): \quad 1.273 \mathrm{E}+06$

\begin{tabular}{c}
$\begin{array}{c}\text { Activity } \\
\text { (Ci/yr) }\end{array}$ \\
\hline $5.381 \mathrm{E}-02$ \\
$7.922 \mathrm{E}+01$ \\
$2.947 \mathrm{E}+01$ \\
$8.470 \mathrm{E}+01$ \\
$3.138 \mathrm{E}-01$ \\
$1.076 \mathrm{E}-01$ \\
$6.102 \mathrm{E}+00$ \\
$2.242 \mathrm{E}-01$ \\
$4.757 \mathrm{E}+00$ \\
$5.005 \mathrm{E}+02$ \\
$1.084 \mathrm{E}+01$ \\
$3.332 \mathrm{E}+00$ \\
$5.381 \mathrm{E}-02$ \\
$8.071 \mathrm{E}-02$ \\
$3.529 \mathrm{E}+00$ \\
$1.021 \mathrm{E}+00$ \\
$7.490 \mathrm{E}+01$ \\
$8.959 \mathrm{E}-01$ \\
$8.968 \mathrm{E}-02$ \\
$1.084 \mathrm{E}+01$ \\
$1.084 \mathrm{E}+01$ \\
$7.490 \mathrm{E}+01$
\end{tabular}

\begin{tabular}{l} 
Chemical \\
\hline $57-12-5$ \\
$75-09-2$ \\
$\mathrm{Ag}$ \\
$\mathrm{As}$ \\
$\mathrm{Ba}$ \\
$\mathrm{Cd}$ \\
$\mathrm{Cl}-2-\mathrm{x}$ \\
$\mathrm{Cl}-3-\mathrm{x}$ \\
$\mathrm{Cl}-4-\mathrm{x}$ \\
$\mathrm{Cl}-\mathrm{F}-\mathrm{x}$ \\
$\mathrm{Cr}$ \\
$\mathrm{HC}-$ insol \\
$\mathrm{HC}-\mathrm{SOI}$ Ib \\
$\mathrm{Hg}$ \\
$\mathrm{Pb}$ \\
$\mathrm{Se}$
\end{tabular}

\begin{tabular}{c}
$\begin{array}{c}\text { Amount } \\
(\mathrm{kg} / \mathrm{yr})\end{array}$ \\
\hline $5.083 \mathrm{E}+01$ \\
$1.025 \mathrm{E}+03$ \\
$2.485 \mathrm{E}+01$ \\
$4.853 \mathrm{E}+00$ \\
$2.866 \mathrm{E}+01$ \\
$7.813 \mathrm{E}+02$ \\
$6.277 \mathrm{E}+02$ \\
$6.415 \mathrm{E}+04$ \\
$1.361 \mathrm{E}+04$ \\
$1.088 \mathrm{E}+02$ \\
$7.309 \mathrm{E}+02$ \\
$1.035 \mathrm{E}+05$ \\
$1.366 \mathrm{E}+05$ \\
$1.456 \mathrm{E}+02$ \\
$1.706 \mathrm{E}+02$ \\
$2.174 \mathrm{E}+01$
\end{tabular}

Waste Type: Solids (CH-Non-alpha)

$1.685 \mathrm{E}+03$

Total Mass $(\mathrm{kg} / \mathrm{yr}): 2.230 \mathrm{E}+06$

\section{Activity (Ci/yr)}

8.298E-02

1. $221 \mathrm{E}+02$

$4.545 E+01$

$1.303 \mathrm{E}+02$

4. $840 \mathrm{E}-01$

$1.659 \mathrm{E}-01$

$9.410 \mathrm{E}+00$

$3.457 \mathrm{E}-01$

$7.337 E+00$

$7.718 E+02$

1. $671 \mathrm{E}+01$

$5.139 \mathrm{E}+00$

8.298E-02

1.244E-01

$5.443 \mathrm{E}+00$

$1.575 \mathrm{E}+00$

1. $152 E+02$

$1.378 \mathrm{E}+00$

$1.383 E-01$

1. $671 E+01$

$1.671 \mathrm{E}+01$

$1.152 \mathrm{E}+02$

\begin{tabular}{l} 
Chemical \\
\hline $57-12-5$ \\
$75-09-2$ \\
$\mathrm{Ag}$ \\
$\mathrm{As}$ \\
$\mathrm{Ba}$ \\
$\mathrm{Cd}$ \\
$\mathrm{Cl}-2-\mathrm{x}$ \\
$\mathrm{CI}-3-\mathrm{x}$ \\
$\mathrm{Cl}-4-\mathrm{x}$ \\
$\mathrm{Cl}-\mathrm{F}-\mathrm{x}$ \\
$\mathrm{Cr}$ \\
$\mathrm{HC}-$ insol \\
$\mathrm{HC}-\mathrm{solub}$ \\
$\mathrm{Hg}$ \\
$\mathrm{Pb}$ \\
$\mathrm{Se}$
\end{tabular}

\begin{tabular}{c}
$\begin{array}{c}\text { Amount } \\
(\mathrm{kg} / \mathrm{yr})\end{array}$ \\
\hline $2.297 \mathrm{E}+02$ \\
$2.058 \mathrm{E}+02$ \\
$1.090 \mathrm{E}+02$ \\
$3.946 \mathrm{E}+01$ \\
$3.128 \mathrm{E}+03$ \\
$6.604 \mathrm{E}+01$ \\
$2.067 \mathrm{E}+02$ \\
$3.997 \mathrm{E}+03$ \\
$4.388 \mathrm{E}+02$ \\
$2.170 \mathrm{E}+02$ \\
$3.510 \mathrm{E}+03$ \\
$1.703 \mathrm{E}+04$ \\
$8.350 \mathrm{E}+03$ \\
$1.152 \mathrm{E}+03$ \\
$4.348 \mathrm{E}+03$ \\
$4.888 \mathrm{E}+02$
\end{tabular}


Shipping Site: PADUCAH

Waste Type: Organic Liquids ( $\mathrm{CH}-\mathrm{Non}-\mathrm{alpha})$

Total Volume $(\mathrm{m} 3 / \mathrm{yr})$ :

$2.970 E+01$

Total Mass $(\mathrm{kg} / \mathrm{yr}): 3.589 \mathrm{E}+04$

Radionuclide

$\mathrm{AC}-228$

$\mathrm{Bi}-212$

$\mathrm{Pa}-234$

$\mathrm{Pa}-234 \mathrm{~m}$

$\mathrm{Pb}-212$

PO-212

Po-216

$\mathrm{Ra}-224$

$\mathrm{Ra}-228$

TC-99

Th-228

Th-231

Th-232

$\mathrm{Th}-234$

T1-208

$U-235$

$\mathrm{U}-238$

Shipping Site: PADUCAH

Total Volume $(\mathrm{m} 3 / \mathrm{yr})$ :

Radionuclide

$\mathrm{AC}-228$

$\mathrm{Bi}-212$

$\mathrm{Pa}-234$

$\mathrm{Pa}-234 \mathrm{~m}$

$\mathrm{Pb}-212$

PO-212

Po-216

$\mathrm{Ra}-224$

Ra-228

TC-99

Th -228

Th-231

Th-232

Th-234

Tl-208

$\mathrm{U}-235$

$\mathrm{U}-238$

Shipping Site:

PANT

Total Volume $(\mathrm{m} 3 / \mathrm{yr})$ :

Radionuclide

$\mathrm{Ba}-137 \mathrm{~m}$
$\mathrm{Co}-60$
$\mathrm{Cs}-137$
$\mathrm{Eu}-154$
$\mathrm{Fe}-55$
$\mathrm{H}-3$
$\mathrm{Ni}-59$
$\mathrm{Ni}-63$
$\mathrm{Pm}-147$
$\mathrm{Pu}-238$
$\mathrm{Pu}-241$
$\mathrm{Sm}-151$
$\mathrm{~S}-90$
$\mathrm{Y}-90$

\begin{tabular}{c}
$\begin{array}{c}\text { Activity } \\
(C i / Y r)\end{array}$ \\
\hline $1.796 \mathrm{E}-02$ \\
$2.967 \mathrm{E}-03$ \\
$2.486 \mathrm{E}-03$ \\
$2.420 \mathrm{E}+01$ \\
$2.967 \mathrm{E}-03$ \\
$1.965 \mathrm{E}-03$ \\
$2.967 \mathrm{E}-03$ \\
$2.967 \mathrm{E}-03$ \\
$1.796 \mathrm{E}-02$ \\
$7.291 \mathrm{E}+00$ \\
$2.967 \mathrm{E}-03$ \\
$1.892 \mathrm{E}-02$ \\
$1.990 \mathrm{E}-01$ \\
$2.420 \mathrm{E}+01$ \\
$1.163 \mathrm{E}-03$ \\
$1.876 \mathrm{E}-02$ \\
$2.420 \mathrm{E}+01$
\end{tabular}

\begin{tabular}{l} 
Chemical \\
\hline $57-12-5$ \\
$75-09-2$ \\
$\mathrm{Ag}$ \\
$\mathrm{As}$ \\
$\mathrm{Ba}$ \\
$\mathrm{Cd}$ \\
$\mathrm{Cl}-2-\mathrm{x}$ \\
$\mathrm{Cl}-3-\mathrm{x}$ \\
$\mathrm{Cl}-4-\mathrm{x}$ \\
$\mathrm{Cl}-\mathrm{F}-\mathrm{x}$ \\
$\mathrm{Cr}$ \\
$\mathrm{HC}-$ insol \\
$\mathrm{HC}-\mathrm{solub}$ \\
$\mathrm{Hg}$ \\
$\mathrm{Pb}$ \\
$\mathrm{Se}$
\end{tabular}

\begin{tabular}{c}
$\begin{array}{c}\text { Amount } \\
(\mathrm{kg} / \mathrm{Y})\end{array}$ \\
\hline $4.590 \mathrm{E}-01$ \\
$4.205 \mathrm{E}+01$ \\
$2.300 \mathrm{E}-01$ \\
$1.180 \mathrm{E}-01$ \\
$5.803 \mathrm{E}-01$ \\
$3.374 \mathrm{E}+01$ \\
$2.303 \mathrm{E}+01$ \\
$2.444 \mathrm{E}+03$ \\
$5.381 \mathrm{E}+02$ \\
$1.762 \mathrm{E}+00$ \\
$2.951 \mathrm{E}+01$ \\
$5.097 \mathrm{E}+03$ \\
$2.651 \mathrm{E}+03$ \\
$1.493 \mathrm{E}+00$ \\
$6.639 \mathrm{E}+00$ \\
$2.011 \mathrm{E}-01$
\end{tabular}

Waste Type: Solids (CH-Non-alpha)

$4.740 E+00$

Total Mass ( $\mathrm{kg} / \mathrm{Yr})$ :

$6.808 \mathrm{E}+03$

\begin{tabular}{l} 
Chemical \\
\hline $57-12-5$ \\
$75-09-2$ \\
$\mathrm{Ag}$ \\
$\mathrm{As}$ \\
$\mathrm{Ba}$ \\
$\mathrm{Cd}$ \\
$\mathrm{Cl}-2-\mathrm{x}$ \\
$\mathrm{Cl}-3-\mathrm{x}$ \\
$\mathrm{Cl}-4-\mathrm{x}$ \\
$\mathrm{Cl}-\mathrm{F}-\mathrm{x}$ \\
$\mathrm{Cr}$ \\
$\mathrm{HC}-\mathrm{inso1}$ \\
$\mathrm{HC}-\mathrm{SOlub}$ \\
$\mathrm{Hg}$ \\
$\mathrm{Pb}$ \\
$\mathrm{Se}$
\end{tabular}

\begin{tabular}{c}
$\begin{array}{c}\text { Amount } \\
(\mathrm{kg} / \mathrm{yr})\end{array}$ \\
\hline $1.016 \mathrm{E}+00$ \\
$1.710 \mathrm{E}+00$ \\
$2.502 \mathrm{E}-01$ \\
$8.712 \mathrm{E}-02$ \\
$1.228 \mathrm{E}+01$ \\
$1.504 \mathrm{E}-01$ \\
$1.444 \mathrm{E}+00$ \\
$1.250 \mathrm{E}+01$ \\
$4.496 \mathrm{E}+00$ \\
$6.729 \mathrm{E}-01$ \\
$1.293 \mathrm{E}+01$ \\
$3.436 \mathrm{E}+01$ \\
$1.740 \mathrm{E}+01$ \\
$1.732 \mathrm{E}+00$ \\
$2.789 \mathrm{E}+01$ \\
$6.716 \mathrm{E}-01$
\end{tabular}

Waste Type: Organic Liquids (CH-Non-alpha)

Total Mass (kg/yr): $2.110 \mathrm{E}+03$

\begin{tabular}{l} 
Chemical \\
\hline $75-09-2$ \\
As \\
$\mathrm{Ba}$ \\
$\mathrm{Cd}$ \\
$\mathrm{Cl}-2-\mathrm{x}$ \\
$\mathrm{Cl}-3-\mathrm{x}$ \\
$\mathrm{Cl}-4-\mathrm{x}$ \\
$\mathrm{Cl}-\mathrm{F}-\mathrm{x}$ \\
$\mathrm{Cr}$ \\
$\mathrm{HC}-$ insol \\
$\mathrm{HC}-$ Solub \\
$\mathrm{Hg}$ \\
$\mathrm{Pb}$
\end{tabular}

\begin{tabular}{c}
$\begin{array}{c}\text { Amount } \\
(\mathrm{kg} / \mathrm{yr})\end{array}$ \\
\hline $3.284 \mathrm{E}+00$ \\
$1.112 \mathrm{E}-03$ \\
$4.766 \mathrm{E}-03$ \\
$2.321 \mathrm{E}+00$ \\
$1.743 \mathrm{E}+00$ \\
$2.389 \mathrm{E}+02$ \\
$5.331 \mathrm{E}+01$ \\
$9.829 \mathrm{E}-02$ \\
$2.020 \mathrm{E}+00$ \\
$6.419 \mathrm{E}+01$ \\
$3.280 \mathrm{E}+01$ \\
$1.325 \mathrm{E}-02$ \\
$4.431 \mathrm{E}-01$
\end{tabular}


Shipping site: PANT Waste Type: Solids (CH-Non-alpha)

Total Volume (m3/yr):

$3.957 E+01$

Total Mass $(\mathrm{kg} / \mathrm{yr})$ :

$2.511 E+04$

Radionuclide

$\mathrm{Ba}-137 \mathrm{~m}$

Co-60

Cs -137

Eu-154

$\mathrm{Fe}-55$

$\mathrm{H}-3$

$\mathrm{Ni}-59$

$\mathrm{Ni}-63$

$\mathrm{Pm}-147$

Pu-238

Pu-241

$\mathrm{Sm}-151$

$\mathrm{Sr}-90$

$Y-90$

Shipping Site: PHNS

Total Volume $(\mathrm{m} 3 / \mathrm{yr})$ :

Radionuclide

$\mathrm{Ba}-137 \mathrm{~m}$

Co-60

Cs -134

Cs -137

Eu-154

Eu-155

$\mathrm{Fe}-55$

$\mathrm{H}-3$

$\mathrm{Mn}-54$

$\mathrm{Nb}-94$

$\mathrm{Ni}-59$

$\mathrm{Ni}-63$

$\mathrm{Pa}-234 \mathrm{~m}$

$\mathrm{Pm}-147$

$\mathrm{Pu}-238$

Pu-241

$\mathrm{Sb}-125$

$\mathrm{Sm}-151$

Sr -90

$\mathrm{Te}-125 \mathrm{~m}$

Th-234

$\mathrm{U}-238$

$\mathrm{Y}-90$

Shipping Site: PHNS

Total Volume (m3/yr):

Radionuclide

$\mathrm{Ba}-137 \mathrm{~m}$

Co-60

Cs -134

Cs -137

Eu-154

Eu-155

$\mathrm{Fe}-55$

F-3

$\mathrm{Mn}-54$

$\mathrm{Nb}-94$

Ni-59

$\mathrm{Ni}-63$

$\mathrm{Pa}-234 \mathrm{~m}$

Pm-147

Pu-238

Pu-241

Sb-125

$\mathrm{Sm}-151$

Sr-90

Te-125m

Th-234

U-238

$\mathrm{Y}-90$

\begin{tabular}{c}
$\begin{array}{r}\text { Activity } \\
\text { (Ci/yr) }\end{array}$ \\
\hline $5.276 \mathrm{E}-01$ \\
$7.254 \mathrm{E}-01$ \\
$5.454 \mathrm{E}-01$ \\
$6.045 \mathrm{E}-03$ \\
$9.123 \mathrm{E}-02$ \\
$1.051 \mathrm{E}+02$ \\
$1.209 \mathrm{E}-02$ \\
$1.604 \mathrm{E}+00$ \\
$5.056 \mathrm{E}-02$ \\
$2.088 \mathrm{E}-02$ \\
$1.055 \mathrm{E}-01$ \\
$4.836 \mathrm{E}-03$ \\
$5.126 \mathrm{E}-01$ \\
$5.126 \mathrm{E}-01$
\end{tabular}

\begin{tabular}{|c|c|}
\hline Chemical & $\begin{array}{l}\text { Amount } \\
(\mathrm{kg} / \mathrm{yr})\end{array}$ \\
\hline $57-12-5$ & $2.480 \mathrm{E}-01$ \\
\hline $75-09-2$ & $3.448 \mathrm{E}+01$ \\
\hline $\mathrm{Ag}$ & $1.998 \mathrm{E}-01$ \\
\hline As & $3.903 E-02$ \\
\hline $\mathrm{Ba}$ & $1.047 \mathrm{E}+01$ \\
\hline $\mathrm{cd}$ & $1.602 \mathrm{E}+0 \mathrm{D}$ \\
\hline$C I-2-x$ & 2. $240 \mathrm{E}+01$ \\
\hline$c 1-3-x$ & $1.712 \mathrm{E}+02$ \\
\hline$C I-4-x$ & $1.094 \mathrm{E}+02$ \\
\hline$C I-F-x$ & $1.631 E+01$ \\
\hline $\mathrm{Cr}$ & $1.027 \mathrm{E}+01$ \\
\hline $\mathrm{HC}$-insol & $4.777 \mathrm{E}+02$ \\
\hline $\mathrm{HC}-\mathrm{solub}$ & $1.648 \mathrm{E}+02$ \\
\hline $\mathrm{Hg}$ & $1.896 \mathrm{E}+00$ \\
\hline $\mathrm{Pb}$ & $5.162 \mathrm{E}+01$ \\
\hline $\mathrm{Se}$ & $2.443 \mathrm{E}-01$ \\
\hline
\end{tabular}

Waste Type: Organic Liquids (CH-Non-alpha)

Total Mass $(\mathrm{kg} / \mathrm{yr}): 1.979 \mathrm{E}+00$
$1.736 \mathrm{E}-03$

$$
\text { Activity }
$$

$1.152 \mathrm{E}-03$

$1.877 \mathrm{E}-02$

1. $103 \mathrm{E}-05$

1.227E-03

1. $493 \mathrm{E}-05$

$7.141 \mathrm{E}-06$

$2.454 \mathrm{E}-03$

$5.346 \mathrm{E}-03$

$7.791 \mathrm{E}-06$

$1.168 \mathrm{E}-05$

$2.454 \mathrm{E}-04$

$3.299 \mathrm{E}-02$

$4.609 E-05$

$1.337 \mathrm{E}-04$

$3.830 \mathrm{E}-05$

$3.116 \mathrm{E}-05$

$5.194 \mathrm{E}-06$

$1.038 \mathrm{E}-05$

$1.155 \mathrm{E}-03$

$1.298 \mathrm{E}-06$

$4.609 \mathrm{E}-05$

$4.609 \mathrm{E}-05$

1. $155 \mathrm{E}-03$

\begin{tabular}{lcc}
\multicolumn{1}{c}{ Chemical } & $\begin{array}{c}\text { Amount } \\
(\mathrm{kg} / \mathrm{yr})\end{array}$ \\
\cline { 1 - 1 } $75-09-2$ & $2.572 \mathrm{E}-04$ \\
$\mathrm{Ag}$ & $8.707 \mathrm{E}-05$ \\
$\mathrm{AS}$ & $2.176 \mathrm{E}-05$ \\
$\mathrm{Ba}$ & $1.484 \mathrm{E}-04$ \\
$\mathrm{CI}-2-\mathrm{x}$ & $7.322 \mathrm{E}-04$ \\
$\mathrm{CI}-3-\mathrm{x}$ & $2.731 \mathrm{E}-02$ \\
$\mathrm{CI}-4-\mathrm{x}$ & $1.543 \mathrm{E}-03$ \\
$\mathrm{C} 1-\mathrm{F}-\mathrm{x}$ & $5.937 \mathrm{E}-04$ \\
$\mathrm{HC}-$ inso1 & $2.968 \mathrm{E}-03$ \\
$\mathrm{HC}-$ Solub & $7.718 \mathrm{E}-04$ \\
$\mathrm{Hg}$ & $4.353 \mathrm{E}-04$ \\
$\mathrm{~Pb}$ & $5.343 \mathrm{E}-05$ \\
$\mathrm{Se}$ & $7.322 \mathrm{E}-05$
\end{tabular}

Waste Type: Solids (CH-Non-alpha)

2.962E-01 Total Mass (kg/yr): 4.406E+02

\begin{tabular}{c}
$\begin{array}{c}\text { Activity } \\
(\mathrm{Ci} / \mathrm{yr})\end{array}$ \\
\hline $1.983 \mathrm{E}-01$ \\
$3.204 \mathrm{E}+00$ \\
$1.883 \mathrm{E}-03$ \\
$2.094 \mathrm{E}-01$ \\
$2.548 \mathrm{E}-03$ \\
$1.218 \mathrm{E}-03$ \\
$4.188 \mathrm{E}-01$ \\
$9.124 \mathrm{E}-01$ \\
$1.329 \mathrm{E}-03$ \\
$1.994 \mathrm{E}-03$ \\
$4.188 \mathrm{E}-02$ \\
$5.631 \mathrm{E}+00$ \\
$7.867 \mathrm{E}-03$ \\
$2.282 \mathrm{E}-02$ \\
$6.538 \mathrm{E}-03$ \\
$5.319 \mathrm{E}-03$ \\
$8.865 \mathrm{E}-04$ \\
$1.773 \mathrm{E}-03$ \\
$1.972 \mathrm{E}-01$ \\
$2.216 \mathrm{E}-04$ \\
$7.867 \mathrm{E}-03$ \\
$7.867 \mathrm{E}-03$ \\
$1.972 \mathrm{E}-01$
\end{tabular}

\begin{tabular}{c}
$\begin{array}{c}\text { Amount } \\
(\mathrm{kg} / \mathrm{Yr})\end{array}$ \\
\hline $4.073 \mathrm{E}-03$ \\
$5.831 \mathrm{E}-02$ \\
$2.691 \mathrm{E}-03$ \\
$1.603 \mathrm{E}-03$ \\
$1.038 \mathrm{E}-01$ \\
$2.472 \mathrm{E}-02$ \\
$6.160 \mathrm{E}-02$ \\
$2.088 \mathrm{E}+00$ \\
$4.422 \mathrm{E}-01$ \\
$6.398 \mathrm{E}-02$ \\
$7.805 \mathrm{E}-01$ \\
$6.908 \mathrm{E}+00$ \\
$3.289 \mathrm{E}+00$ \\
$2.617 \mathrm{E}-02$ \\
$1.563 \mathrm{E}-01$ \\
$6.731 \mathrm{E}-03$
\end{tabular}


Shipping Site: PNS Waste Type: Solids (CH-Non-alpha)

Total volume $(\mathrm{m} 3 / \mathrm{yr})$ :

Total Mass (kg/yr):

$9.088 E+01$

Radionuclide

a $-137 \mathrm{~m}$

Co-60

Cs -134

Cs -137

Eu-154

Eu-155

Fe- 55

$\mathrm{H}-3$

$\mathrm{Mn}-54$

$\mathrm{Nb}-94$

$\mathrm{Ni}-59$

$\mathrm{Ni}-63$

$\mathrm{Pa}-234 \mathrm{~m}$

Pm- 147

Pu-238

Pu-241

$\mathrm{Sb}-125$

Sm-151

$s r-90$

Te-125m

$\mathrm{Th}-234$

$\mathrm{U}-238$

$Y-90$

Shipping Site: PORTS

\begin{tabular}{r}
$\begin{array}{r}\text { Activity } \\
\text { (Ci/yr) }\end{array}$ \\
\hline $3.481 E-02$ \\
$5.623 E-01$ \\
$3.306 E-04$ \\
$3.676 E-02$ \\
$4.473 E-04$ \\
$2.139 E-04$ \\
$7.352 E-02$ \\
$1.601 E-01$ \\
$2.333 E-04$ \\
$3.500 E-04$ \\
$7.352 E-03$ \\
$9.884 E-01$ \\
$1.380 E-03$ \\
$4.006 E-03$ \\
$1.147 E-03$ \\
$9.335 E-04$ \\
$1.555 E-04$ \\
$3.111 E-04$ \\
$3.462 E-02$ \\
$3.889 E-05$ \\
$1.380 E-03$ \\
$1.380 E-03$ \\
$3.462 E-02$ \\
\end{tabular}

\begin{tabular}{|c|c|}
\hline Chemical & $\begin{array}{l}\text { Amount } \\
(\mathrm{kg} / \mathrm{Yr})\end{array}$ \\
\hline $75-09-2$ & $1.324 E-02$ \\
\hline Ag & $6.570 E-04$ \\
\hline As & $5.453 E-04$ \\
\hline $\mathrm{Ba}$ & $1.708 E-02$ \\
\hline $\mathrm{Cd}$ & $8.438 E-03$ \\
\hline Cl-2-x & $1.324 \mathrm{E}-02$ \\
\hline$C 1-3-x$ & $7.909 \mathrm{E}-01$ \\
\hline$C l-4-x$ & $1.483 \mathrm{E}-01$ \\
\hline$C l-F-x$ & $7.499 \mathrm{E}-03$ \\
\hline $\mathrm{Cr}$ & $1.051 \mathrm{E}-01$ \\
\hline $\mathrm{HC}$-insol & $2.629 E+00$ \\
\hline HC-solub & $1.314 \mathrm{E}+00$ \\
\hline $\mathrm{Hg}$ & $9.046 E-03$ \\
\hline $\mathrm{Pb}$ & $1.319 \mathrm{E}-02$ \\
\hline Se & $2.365 E-03$ \\
\hline
\end{tabular}

Waste Type: Organic Liquids (CH-Non-alpha)

Total Volume $(\mathrm{m} 3 / \mathrm{yr})$ :

$4.765 \mathrm{E}+01$

Total Mass $(\mathrm{kg} / \mathrm{yr})$ :

$4.778 \mathrm{E}+04$

Radionuclide

Ac- -228

Bi-212

$\mathrm{Pa}-234$

$\mathrm{Pa}-234 \mathrm{~m}$

$\mathrm{Pb}-212$

Po-212

Po-2 16

$\mathrm{Ra}-224$

Ra-228

TC-99

Th-228

Th-231

Th-232

Th-234

T $1-208$

$\mathrm{U}-235$

$\mathrm{U}-238$

Shipping Site: PORTS

\begin{tabular}{r}
$\begin{array}{r}\text { Activity } \\
\langle C i / y x\rangle\end{array}$ \\
\hline $2.163 E-05$ \\
$3.621 E-06$ \\
$2.954 E-06$ \\
$2.876 E-02$ \\
$3.621 E-06$ \\
$2.287 E-06$ \\
$3.621 E-06$ \\
$3.621 E-06$ \\
$2.163 E-05$ \\
$8.664 E-03$ \\
$3.621 E-06$ \\
$2.249 E-05$ \\
$2.365 E-04$ \\
$2.876 E-02$ \\
$1.334 E-06$ \\
$2.230 E-05$ \\
$2.876 E-02$
\end{tabular}

\begin{tabular}{l} 
Chemical \\
\hline $57-12-5$ \\
$75-09-2$ \\
Ag \\
As \\
Ba \\
Cd \\
Cl-2-x \\
Cl-3-x \\
Cl-4-x \\
Cl-F-x \\
Cr \\
HC-insol \\
HC-solub \\
Hg \\
Pb \\
Se
\end{tabular}

\begin{tabular}{c}
$\begin{array}{c}\text { Amount } \\
(\mathrm{kg} / \mathrm{yr})\end{array}$ \\
\hline $7.201 \mathrm{E}+00$ \\
$1.017 \mathrm{E}+00$ \\
$2.102 \mathrm{E}+00$ \\
$8.553 \mathrm{E}-02$ \\
$5.830 \mathrm{E}-01$ \\
$2.050 \mathrm{E}-01$ \\
$2.879 \mathrm{E}+00$ \\
$1.392 \mathrm{E}+02$ \\
$7.874 \mathrm{E}+00$ \\
$2.331 \mathrm{E}+00$ \\
$8.409 \mathrm{E}+00$ \\
$4.553 \mathrm{E}+02$ \\
$1.440 \mathrm{E}+04$ \\
$1.291 \mathrm{E}+01$ \\
$2.212 \mathrm{E}+00$ \\
$1.887 \mathrm{E}+00$
\end{tabular}

Waste Type: Solids (CH-Non-alpha)

Total volume $(\mathrm{m} 3 / \mathrm{Yr})$ :

$7.763 E+02$

Total Mass $(\mathrm{kg} / \mathrm{Yr})$ :

$1.096 E+06$

\begin{tabular}{|c|c|}
\hline Chemical & $\begin{array}{l}\text { Amount } \\
(\mathrm{kg} / \mathrm{yr})\end{array}$ \\
\hline $57-12-5$ & $1.691 \mathrm{E}+02$ \\
\hline $75-09-2$ & $2.135 \mathrm{E}+02$ \\
\hline $\mathrm{Ag}$ & $3.471 \mathrm{E}+01$ \\
\hline As & $1.183 \mathrm{E}+01$ \\
\hline $\mathrm{Ba}$ & $2.025 E+03$ \\
\hline $\mathrm{cd}$ & $1.587 \mathrm{E}+01$ \\
\hline C1-2-x & $2.135 \mathrm{E}+02$ \\
\hline Cl-3-x & $1.306 \mathrm{E}+03$ \\
\hline Cl-4-x & $5.694 \mathrm{E}+02$ \\
\hline$C l-F-x$ & $1.185 \mathrm{E}+02$ \\
\hline $\mathrm{Cr}$ & $2.062 E+03$ \\
\hline HC-insol & $2.947 \mathrm{E}+03$ \\
\hline HC-solub & $1.642 E+03$ \\
\hline $\mathrm{Hg}$ & $1.720 E+02$ \\
\hline $\mathrm{Pb}$ & $4.847 E+03$ \\
\hline Se & $6.061 E+01$ \\
\hline
\end{tabular}


Shipping site: PPPL Waste Type: Solids (CH-Non-alpha)

Total volume $(\mathrm{m} 3 / \mathrm{yr})$ :

$5.456 \mathrm{E}-04$

Total Mass $(\mathrm{kg} / \mathrm{yr}): \quad 7.191 \mathrm{E}-01$

Radionuclide

Ba $-137 \mathrm{~m}$

Cs -137

$\mathrm{H}-3$

$\mathrm{Pa}-234 \mathrm{~m}$

Pu-241

Sr -90

Th -234

$\mathrm{U}-238$

$Y-90$

Shipping Site: PSNS

Total Volume (m3/yr):

Radionuclide

$\mathrm{Ba}-137 \mathrm{~m}$

$\mathrm{Co}-60$

Cs -134

$\mathrm{Cs}-137$

Eu -154

Eu-155

Fe- 55

$\mathrm{H}-3$

$\mathrm{Mn} \rightarrow 54$

$\mathrm{Nb}-94$

$\mathrm{Ni}-59$

$\mathrm{Ni}-63$

Pa-234m

Pm-147

Pu-238

Pu-241

$\mathrm{Sb}-125$

Sm-151

Sr -90

Te-125m

Th-234

$\mathrm{U}-238$

$Y-90$

Shipping Site: PSNS

Total Volume (m3/yr):

Radionuclide

$\mathrm{Ba}-137 \mathrm{~m}$

Co-60

Cs-134

Cs -137

Eu-154

Eu-155

$\mathrm{Fe}-55$

H-3

$\mathrm{Mn}-54$

$\mathrm{Nb}-94$

$\mathrm{Ni}-59$

$\mathrm{Ni}-63$

$\mathrm{Pa}-234 \mathrm{~m}$

$\mathrm{Pm}-147$

Pu-238

Pu-241

Sb-125

$\mathrm{Sm}-151$

Sr -90

Te-125m

Th-234

$\mathrm{U}-238$

$Y-90$

\begin{tabular}{c}
$\begin{array}{c}\text { Activity } \\
(\text { Ci/yr) }\end{array}$ \\
\hline $7.218 E-06$ \\
$7.562 E-06$ \\
$3.433 E-02$ \\
$1.753 E-06$ \\
$1.718 E-06$ \\
$7.218 E-06$ \\
$1.753 E-06$ \\
$1.753 E-06$ \\
$7.218 E-06$
\end{tabular}

\begin{tabular}{|c|c|}
\hline Chemical & $\begin{array}{l}\text { Amount } \\
(\mathrm{kg} / \mathrm{Yr})\end{array}$ \\
\hline $\begin{array}{l}75-09-2 \\
C 1-2-x \\
C 1-3-x \\
C I-4-x \\
\text { HC-insoI } \\
\text { HC-solub }\end{array}$ & $\begin{array}{l}1.438 \mathrm{E}-02 \\
7.190 \mathrm{E}-03 \\
7.190 \mathrm{E}-02 \\
5.752 \mathrm{E}-02 \\
1.797 \mathrm{E}-01 \\
8.629 \mathrm{E}-02\end{array}$ \\
\hline
\end{tabular}

Waste Type: Organic Liquids (CH-Non-alpha)

3. $990 E-02$

Total Mass (kg/yx): $5.986 \mathrm{E}+01$

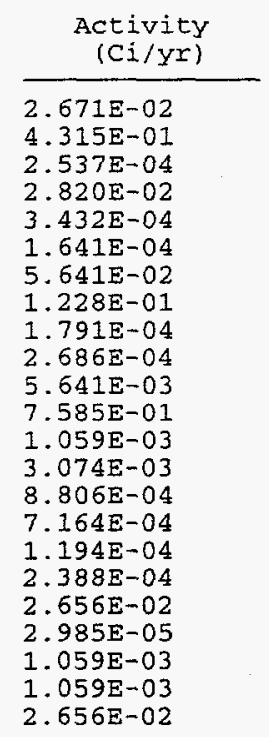

\begin{tabular}{|c|c|}
\hline Chemical & $\begin{array}{l}\text { Amount } \\
(\mathrm{kg} / \mathrm{Yr})\end{array}$ \\
\hline $\begin{array}{l}75-09-2 \\
\mathrm{Cd} \\
\mathrm{Cl}-2-\mathrm{x} \\
\mathrm{Cl}-3-\mathrm{x} \\
\mathrm{Cl}-4-\mathrm{x} \\
\mathrm{Cl}-\mathrm{F}-\mathrm{x} \\
\mathrm{Cr} \\
\mathrm{HC}-\mathrm{insol} \\
\mathrm{HC}-\text { solub } \\
\mathrm{Hg} \\
\mathrm{Pb}\end{array}$ & $\begin{array}{l}9.577 E-02 \\
6.584 E-02 \\
5.088 E-02 \\
7.243 E+00 \\
1.616 E+00 \\
2.993 E-03 \\
5.746 E-02 \\
1.975 E-02 \\
3.831 E-01 \\
3.771 E-04 \\
1.257 E-02\end{array}$ \\
\hline
\end{tabular}

Waste Type: Solids (CH-Non-alpha)

$6.586 \mathrm{E}+00$

Total Mass (kg/yr): $9.518 E+03$

\begin{tabular}{r}
$\begin{array}{r}\text { Activity } \\
\text { (Ci/yr) }\end{array}$ \\
\hline $4.409 \mathrm{E}+00$ \\
$7.122 \mathrm{E}+01$ \\
$4.187 \mathrm{E}-02$ \\
$4.655 \mathrm{E}+00$ \\
$5.665 \mathrm{E}-02$ \\
$2.709 \mathrm{E}-02$ \\
$9.311 \mathrm{E}+00$ \\
$2.028 \mathrm{E}+01$ \\
$2.956 \mathrm{E}-02$ \\
$4.434 \mathrm{E}-02$ \\
$9.311 \mathrm{E}-01$ \\
$1.251 \mathrm{E}+02$ \\
$1.749 \mathrm{E}-01$ \\
$5.074 \mathrm{E}-01$ \\
$1.453 \mathrm{E}-01$ \\
$1.182 \mathrm{E}-01$ \\
$1.970 \mathrm{E}-02$ \\
$3.941 \mathrm{E}-02$ \\
$4.384 \mathrm{E}+00$ \\
$4.926 \mathrm{E}-03$ \\
$1.749 \mathrm{E}-01$ \\
$1.749 \mathrm{E}-01$ \\
$4.384 \mathrm{E}+00$
\end{tabular}


Shipping Site: RFP

Waste Type: Organic Liquids ( $\mathrm{CH}$-Alpha)

Total volume $(\mathrm{m} 3 / \mathrm{yr})$ :

$1.037 E+01$

Total Mass (kg/yr):

$1.212 \mathrm{E}+04$

Radionuclide

Am-241

$\mathrm{Cm}-244$

$\mathrm{Pa}-234 \mathrm{~m}$

Pu-238

Pu-239

Pu-240

Pu-241

Th -234

$\mathrm{U}-238$

Shipping Site: RFP

Total Volume $(\mathrm{m} 3 / \mathrm{yr})$ :

Radionuclide

Am-241

$\mathrm{Cm}-244$

$\mathrm{Pa}-234 \mathrm{~m}$

Pu-238

Pu-239

Pu-240

Pu-241

Th-234

$U-238$

Shipping Site: RMI

Total Volume (m3/yr)

Radionuclide

Ac- 228

$\mathrm{Bi}-212$

$\mathrm{Pa}-234$

$\mathrm{Pa}-234 \mathrm{~m}$

$\mathrm{Pb}-212$

PO-212

Po-216

$\mathrm{Ra}-224$

$\mathrm{Ra}-228$

TC-99

Th-228

Th-231

Th-232

$\mathrm{Th}-234$

T1-208

$\mathrm{U}-235$

$\mathrm{U}-238$

\begin{tabular}{c}
$\begin{array}{c}\text { Activity } \\
\text { (Ci/yr) }\end{array}$ \\
\hline $4.584 \mathrm{E}-05$ \\
$5.202 \mathrm{E}-05$ \\
$2.238 \mathrm{E}-04$ \\
$2.395 \mathrm{E}-02$ \\
$2.454 \mathrm{E}-03$ \\
$8.576 \mathrm{E}-03$ \\
$2.339 \mathrm{E}-01$ \\
$2.238 \mathrm{E}-04$ \\
$2.238 \mathrm{E}-04$
\end{tabular}

Chemical

$57-12-5$

75-09-2

Ag

As

$\mathrm{Ba}$

Ca

Cl $-2-x$

Cl $-3-x$

$\mathrm{Cl}-4-\mathrm{x}$

$\mathrm{C} 1-\mathrm{F}-\mathrm{X}$

$\mathrm{Cr}$

$\mathrm{HC}$-insol

HC-solub

$\mathrm{Hg}$

$\mathrm{Pb}$

$\mathrm{Se}$

\begin{tabular}{c}
$\begin{array}{c}\text { Amount } \\
(\mathrm{kg} / \mathrm{Yr})\end{array}$ \\
\hline $3.662 \mathrm{E}-01$ \\
$1.109 \mathrm{E}+01$ \\
$1.835 \mathrm{E}-01$ \\
$4.647 \mathrm{E}-02$ \\
$2.587 \mathrm{E}-01$ \\
$8.759 \mathrm{E}+00$ \\
$6.508 \mathrm{E}+00$ \\
$5.551 \mathrm{E}+02$ \\
$1.407 \mathrm{E}+02$ \\
$8.899 \mathrm{E}-01$ \\
$7.944 \mathrm{E}+00$ \\
$1.339 \mathrm{E}+03$ \\
$1.182 \mathrm{E}+03$ \\
$1.089 \mathrm{E}+00$ \\
$1.829 \mathrm{E}+00$ \\
$1.604 \mathrm{E}-01$
\end{tabular}

Waste Type: Solids ( $\mathrm{CH}-\mathrm{Alpha})$

Total Mass $(\mathrm{kg} / \mathrm{yr}): 2.424 \mathrm{E}+06$

$.143 \mathrm{E}+03$

\begin{tabular}{l} 
Chemical \\
\hline $57-12-5$ \\
$75-09-2$ \\
$\mathrm{Ag}$ \\
$\mathrm{As}$ \\
$\mathrm{Ba}$ \\
$\mathrm{Cd}$ \\
$\mathrm{Cl}-2-\mathrm{x}$ \\
$\mathrm{Cl}-3-\mathrm{x}$ \\
$\mathrm{Cl}-4-\mathrm{x}$ \\
$\mathrm{Cl}-\mathrm{F}-\mathrm{x}$ \\
$\mathrm{Cr}$ \\
$\mathrm{HC}-\mathrm{inso}$ \\
$\mathrm{HC}-\mathrm{Solub}$ \\
$\mathrm{Hg}$ \\
$\mathrm{Pb}$ \\
$\mathrm{Se}$
\end{tabular}

\begin{tabular}{c}
$\begin{array}{c}\text { Amount } \\
(\mathrm{kg} / \mathrm{yr})\end{array}$ \\
\hline $1.417 \mathrm{E}+01$ \\
$1.164 \mathrm{E}+02$ \\
$3.781 \mathrm{E}+00$ \\
$1.394 \mathrm{E}+00$ \\
$1.522 \mathrm{E}+02$ \\
$5.438 \mathrm{E}+01$ \\
$9.092 \mathrm{E}+01$ \\
$7.111 \mathrm{E}+02$ \\
$7.915 \mathrm{E}+02$ \\
$1.730 \mathrm{E}+02$ \\
$6.697 \mathrm{E}+03$ \\
$1.167 \mathrm{E}+03$ \\
$3.682 \mathrm{E}+03$ \\
$2.810 \mathrm{E}+01$ \\
$4.658 \mathrm{E}+02$ \\
$3.182 \mathrm{E}+00$
\end{tabular}

Waste Type: Organic Liquids (CH-Non-alpha)

$6.677 E-01$

Total Mass $(\mathrm{kg} / \mathrm{yr})$ :

9. $741 E+02$

\begin{tabular}{c}
$\begin{array}{c}\text { Activity } \\
\text { (Ci/yr) }\end{array}$ \\
\hline $1.429 \mathrm{E}-06$ \\
$2.403 \mathrm{E}-07$ \\
$1.816 \mathrm{E}-07$ \\
$1.764 \mathrm{E}-03$ \\
$2.403 \mathrm{E}-07$ \\
$1.549 \mathrm{E}-07$ \\
$2.403 \mathrm{E}-07$ \\
$2.403 \mathrm{E}-07$ \\
$1.429 \mathrm{E}-06$ \\
$2.657 \mathrm{E}-05$ \\
$2.403 \mathrm{E}-07$ \\
$1.378 \mathrm{E}-06$ \\
$1.450 \mathrm{E}-05$ \\
$1.764 \mathrm{E}-03$ \\
$9.081 \mathrm{E}-08$ \\
$1.372 \mathrm{E}-06$ \\
$1.764 \mathrm{E}-03$
\end{tabular}

\begin{tabular}{c}
$\begin{array}{c}\text { Amount } \\
(\mathrm{kg} / \mathrm{Yr})\end{array}$ \\
\hline $1.527 \mathrm{E}+00$ \\
$3.769 \mathrm{E}-04$ \\
$1.615 \mathrm{E}-03$ \\
$1.071 \mathrm{E}+00$ \\
$8.108 \mathrm{E}-01$ \\
$1.123 \mathrm{E}+02$ \\
$2.505 \mathrm{E}+01$ \\
$4.625 \mathrm{E}-02$ \\
$9.331 \mathrm{E}-01$ \\
$2.184 \mathrm{E}+01$ \\
$1.277 \mathrm{E}+01$ \\
$6.122 \mathrm{E}-03$ \\
$2.045 \mathrm{E}-01$
\end{tabular}




\begin{tabular}{l} 
Shipping Site: RMI \\
Total Volume (m3/yr): \\
RadionucIide \\
\hline Ac-228 \\
Bi-212 \\
Pa-234 \\
Pa-234m \\
Pb-212 \\
Po-212 \\
Po-216 \\
Ra-224 \\
Ra-228 \\
Tc-99 \\
$\mathrm{Th}-228$ \\
$\mathrm{Th}-231$ \\
$\mathrm{Th}-232$ \\
$\mathrm{Th}-234$ \\
$\mathrm{~T} 1-208$ \\
$\mathrm{U}-235$ \\
$\mathrm{U}-238$
\end{tabular}

Shipping Site: SNLA

Total Volume (m3/yr):

Radionuclide

\begin{tabular}{l}
\hline $\mathrm{Ba}-137 \mathrm{~m}$ \\
$\mathrm{Co}-60$ \\
$\mathrm{Cs}-137$ \\
$\mathrm{Eu}-154$ \\
$\mathrm{Eu}-155$ \\
$\mathrm{Fe}-55$ \\
$\mathrm{H}-3$ \\
$\mathrm{Nb}-94$ \\
$\mathrm{Ni}-59$ \\
$\mathrm{Ni}-63$ \\
$\mathrm{~Pa}-234 \mathrm{~m}$ \\
$\mathrm{Pu}-238$ \\
$\mathrm{Pu}-240$ \\
$\mathrm{Pu}-241$ \\
$\mathrm{Sm}-151$ \\
$\mathrm{Sr}-90$ \\
$\mathrm{TC}-99$ \\
$\mathrm{Th}-234$ \\
$\mathrm{U}-238$ \\
$\mathrm{Y}-90$
\end{tabular}

Shipping Site: SNLA

Total volume (m3/yr):

Radionuclide

$\mathrm{Ba}-137 \mathrm{~m}$

Co-60

Cs -137

Eu-154

$\mathrm{Eu}-155$

$\mathrm{Fe}-55$

$\mathrm{H}-3$

$\mathrm{Nb}-94$

$\mathrm{Ni}-59$

$\mathrm{Ni}-63$

$\mathrm{Pa}-234 \mathrm{~m}$

Pu-238

$\mathrm{Pu}-240$

Pu-241

$\mathrm{Sm}-151$

$\mathrm{Sr}-90$

TC-99

Th -234

$\mathrm{U}-238$

$\mathrm{Y}-90$
Waste Type: Solids (CH-Non-alpha)

$1.091 E+00$

Total Mass $(\mathrm{kg} / \mathrm{Yr})$ :

$8.323 E+02$

\begin{tabular}{c}
$\begin{array}{c}\text { Activity } \\
\text { (Ci/yr) }\end{array}$ \\
\hline $2.370 \mathrm{E}-06$ \\
$3.988 \mathrm{E}-07$ \\
$3.013 \mathrm{E}-07$ \\
$2.927 \mathrm{E}-03$ \\
$3.988 \mathrm{E}-07$ \\
$2.570 \mathrm{E}-07$ \\
$3.988 \mathrm{E}-07$ \\
$3.815 \mathrm{E}-07$ \\
$2.267 \mathrm{E}-06$ \\
$4.218 \mathrm{E}-05$ \\
$3.988 \mathrm{E}-07$ \\
$2.286 \mathrm{E}-06$ \\
$2.407 \mathrm{E}-05$ \\
$2.927 \mathrm{E}-03$ \\
$1.506 \mathrm{E}-07$ \\
$2.277 \mathrm{E}-06$ \\
$2.927 \mathrm{E}-03$
\end{tabular}

\begin{tabular}{l} 
Chemica \\
\hline $57-12-5$ \\
$75-09-2$ \\
$\mathrm{Ag}$ \\
$\mathrm{As}$ \\
$\mathrm{Ba}$ \\
$\mathrm{Cd}$ \\
$\mathrm{Cl}-2-\mathrm{x}$ \\
$\mathrm{Cl}-3-\mathrm{x}$ \\
$\mathrm{Cl}-4-\mathrm{x}$ \\
$\mathrm{Cl}-\mathrm{F}-\mathrm{x}$ \\
$\mathrm{Cr}$ \\
$\mathrm{HC}-$ insol \\
$\mathrm{HC}-\mathrm{solub}$ \\
$\mathrm{Hg}$ \\
$\mathrm{Pb}$ \\
$\mathrm{Se}$
\end{tabular}

\begin{tabular}{c}
$\begin{array}{c}\text { Amount } \\
(\mathrm{kg} / \mathrm{yr})\end{array}$ \\
\hline $4.647 \mathrm{E}-03$ \\
$2.315 \mathrm{E}-01$ \\
$6.651 \mathrm{E}-03$ \\
$1.974 \mathrm{E}-03$ \\
$5.734 \mathrm{E}-01$ \\
$1.835 \mathrm{E}-02$ \\
$2.862 \mathrm{E}-01$ \\
$2.561 \mathrm{E}+00$ \\
$7.371 \mathrm{E}-01$ \\
$3.452 \mathrm{E}-01$ \\
$2.638 \mathrm{E}-01$ \\
$8.888 \mathrm{E}+00$ \\
$2.825 \mathrm{E}+00$ \\
$2.678 \mathrm{E}-02$ \\
$8.498 \mathrm{E}-02$ \\
$6.070 \mathrm{E}-03$
\end{tabular}

Waste Type: Organic Liquids (CH-Non-alpha)

5.487E-04 Total Mass (kg/Yr): 5.793E-01

\begin{tabular}{c}
$\begin{array}{c}\text { Activity } \\
(\mathrm{Ci} / \mathrm{Y})\end{array}$ \\
\hline $6.627 \mathrm{E}-05$ \\
$9.630 \mathrm{E}-05$ \\
$7.006 \mathrm{E}-05$ \\
$3.516 \mathrm{E}-07$ \\
$8.115 \mathrm{E}-08$ \\
$1.920 \mathrm{E}-06$ \\
$1.223 \mathrm{E}-03$ \\
$4.598 \mathrm{E}-07$ \\
$9.089 \mathrm{E}-06$ \\
$1.099 \mathrm{E}-03$ \\
$3.246 \mathrm{E}-07$ \\
$2.380 \mathrm{E}-06$ \\
$1.893 \mathrm{E}-06$ \\
$1.893 \mathrm{E}-06$ \\
$7.574 \mathrm{E}-07$ \\
$6.519 \mathrm{E}-05$ \\
$2.705 \mathrm{E}-08$ \\
$3.246 \mathrm{E}-07$ \\
$3.246 \mathrm{E}-07$ \\
$6.519 \mathrm{E}-05$
\end{tabular}

\begin{tabular}{l} 
Chemical \\
\hline $57-12-5$ \\
$75-09-2$ \\
Ag \\
As \\
Ba \\
Cd \\
C1-2-x \\
CI-3-x \\
C1-4-x \\
CI-F-x \\
Cr \\
HC-insol \\
HC-solub \\
Hg \\
Pb \\
Se
\end{tabular}

\begin{tabular}{c}
$\begin{array}{c}\text { Amount } \\
(\mathrm{kg} / \mathrm{yr})\end{array}$ \\
\hline $5.085 \mathrm{E}-05$ \\
$3.859 \mathrm{E}-05$ \\
$2.549 \mathrm{E}-05$ \\
$3.265 \mathrm{E}-06$ \\
$2.226 \mathrm{E}-05$ \\
$1.384 \mathrm{E}-06$ \\
$1.098 \mathrm{E}-04$ \\
$4.322 \mathrm{E}-03$ \\
$2.442 \mathrm{E}-04$ \\
$8.905 \mathrm{E}-05$ \\
$5.933 \mathrm{E}-05$ \\
$3.553 \mathrm{E}-03$ \\
$1.018 \mathrm{E}-01$ \\
$1.444 \mathrm{E}-04$ \\
$2.214 \mathrm{E}-05$ \\
$2.228 \mathrm{E}-05$
\end{tabular}

Waste Type: Solids (CH-Non-alpha)

$2.849 \mathrm{E}+00$

Total Mass (kg/yr): $2.300 \mathrm{E}+03$

\begin{tabular}{r}
$\begin{array}{r}\text { Activity } \\
\text { (Ci/yr) }\end{array}$ \\
\hline $3.441 \mathrm{E}-01$ \\
$5.000 \mathrm{E}-01$ \\
$3.637 \mathrm{E}-01$ \\
$1.825 \mathrm{E}-03$ \\
$4.213 \mathrm{E}-04$ \\
$9.972 \mathrm{E}-03$ \\
$6.351 \mathrm{E}+00$ \\
$2.387 \mathrm{E}-03$ \\
$4.719 \mathrm{E}-02$ \\
$5.706 \mathrm{E}+00$ \\
$1.685 \mathrm{E}-03$ \\
$1.236 \mathrm{E}-02$ \\
$9.832 \mathrm{E}-03$ \\
$9.832 \mathrm{E}-03$ \\
$3.932 \mathrm{E}-03$ \\
$3.385 \mathrm{E}-01$ \\
$1.404 \mathrm{E}-04$ \\
$1.685 \mathrm{E}-03$ \\
$1.685 \mathrm{E}-03$ \\
$3.385 \mathrm{E}-01$
\end{tabular}

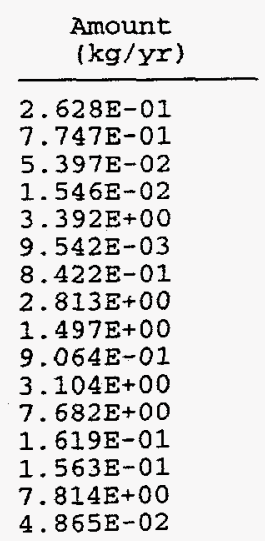


Shipping Site: SNLL

Total Volume (m3/yr) :

Radionuclide

Ba $-137 \mathrm{~m}$

$\mathrm{Cs}-137$

$\mathrm{H}-3$

Pa-234m

Pu-241

Sr -90

Th-234

$\mathrm{U}-238$

$Y-90$

Shipping site: SNLL

Total Volume (m3/yr):

Radionuclide

$\mathrm{Ba}-137 \mathrm{~m}$

Cs -137

$\mathrm{H}-3$

$\mathrm{Pa}-234 \mathrm{~m}$

Pu-241

Sr -90

Th -234

$\mathrm{U}-238$

$Y-90$

Shipping site:

SRS

Total Volume (m3/yr):

Radionuclide

$\mathrm{Ba}-137 \mathrm{~m}$

Co- 60

$\mathrm{Cs}-137$

Eu-154

Eu-155

Fe- 55

$\mathrm{H}-3$

$\mathrm{Nb}-94$

$\mathrm{Ni}-59$

$\mathrm{Ni}-63$

$\mathrm{Pa}-234 \mathrm{~m}$

Pu -238

Pu-240

Pu-241

Sm-151

Sr -90

TC-99

Th-234

$\mathrm{U}-238$

$\mathrm{Y}-90$

Waste Type: Organic Liquids (CH-Non-alpha)

Total Mass (kg/Yr): $7.356 \mathrm{E}+03$

$.662 \mathrm{E}+00$

\begin{tabular}{l} 
Chemical \\
\hline $75-09-2$ \\
As \\
$\mathrm{Ba}$ \\
$\mathrm{Cd}$ \\
$\mathrm{Cl}-2-\mathrm{x}$ \\
$\mathrm{Cl}-3-\mathrm{x}$ \\
$\mathrm{Cl}-4-\mathrm{x}$ \\
$\mathrm{Cr}$ \\
$\mathrm{HC}-$ insol \\
$\mathrm{HC}-$ solub \\
$\mathrm{Hg}$ \\
$\mathrm{Pb}$
\end{tabular}

\begin{tabular}{c}
$\begin{array}{c}\text { Amount } \\
(\mathrm{kg} / \mathrm{yr})\end{array}$ \\
\hline $7.061 \mathrm{E}+00$ \\
$5.664 \mathrm{E}-02$ \\
$2.427 \mathrm{E}-01$ \\
$8.091 \mathrm{E}+00$ \\
$3.678 \mathrm{E}+00$ \\
$5.517 \mathrm{E}+01$ \\
$1.176 \mathrm{E}+01$ \\
$6.767 \mathrm{E}+00$ \\
$3.236 \mathrm{E}+03$ \\
$1.029 \mathrm{E}+03$ \\
$4.413 \mathrm{E}-02$ \\
$1.544 \mathrm{E}+00$
\end{tabular}

Waste Type: Solids (CH-Non-alpha)

Tota1 Mass $(\mathrm{kg} / \mathrm{yr}): 1.193 \mathrm{E}+03$

$7.770 \mathrm{E}-01$

\begin{tabular}{l}
$\begin{array}{c}\text { Activity } \\
\text { (Ci/yr) }\end{array}$ \\
\hline $1.027 E-02$ \\
$1.076 E-02$ \\
$4.890 E+01$ \\
$2.496 E-03$ \\
$2.447 E-03$ \\
$1.027 E-02$ \\
$2.496 E-03$ \\
$2.496 E-03$ \\
$1.027 E-02$
\end{tabular}

\begin{tabular}{l} 
Chemical \\
\hline $57-12-5$ \\
$75-09-2$ \\
Ag \\
As \\
$\mathrm{Ba}$ \\
$\mathrm{Cd}$ \\
$\mathrm{Cl}-2-\mathrm{x}$ \\
$\mathrm{Cl}-3-\mathrm{x}$ \\
$\mathrm{Cl}-4-\mathrm{x}$ \\
$\mathrm{Cl}-\mathrm{F}-\mathrm{x}$ \\
$\mathrm{Cr}$ \\
$\mathrm{HC}-\mathrm{insol}$ \\
$\mathrm{HC}-\mathrm{solub}$ \\
$\mathrm{Hg}$ \\
$\mathrm{Pb}$ \\
$\mathrm{Se}$
\end{tabular}

\begin{tabular}{c}
$\begin{array}{c}\text { Amount } \\
(\mathrm{kg} / \mathrm{y})\end{array}$ \\
\hline $6.924 \mathrm{E}-03$ \\
$7.497 \mathrm{E}+00$ \\
$1.014 \mathrm{E}-02$ \\
$6.737 \mathrm{E}-03$ \\
$3.347 \mathrm{E}-01$ \\
$8.243 \mathrm{E}-02$ \\
$3.816 \mathrm{E}+00$ \\
$4.480 \mathrm{E}+01$ \\
$3.090 \mathrm{E}+01$ \\
$6.827 \mathrm{E}-02$ \\
$6.765 \mathrm{E}-01$ \\
$1.187 \mathrm{E}+02$ \\
$5.754 \mathrm{E}+01$ \\
$1.257 \mathrm{E}-01$ \\
$2.595 \mathrm{E}-01$ \\
$3.934 \mathrm{E}-02$
\end{tabular}

Waste Type: Organic Liquids (CH-Non-alpha)
$4.691 E+02$

Total Mass ( $\mathrm{kg} / \mathrm{yr})$ :

$5.814 \mathrm{E}+05$

\begin{tabular}{l} 
Chemical \\
\hline $57-12-5$ \\
$75-09-2$ \\
Ag \\
As \\
$\mathrm{Ba}$ \\
Cd \\
Cl-2-x \\
Cl-3-x \\
Cl-4-x \\
Cl-F-x \\
Cr \\
$\mathrm{HC}-$ insol \\
$\mathrm{HC}-\mathrm{solub}$ \\
$\mathrm{Hg}$ \\
$\mathrm{Pb}$ \\
$\mathrm{Se}$
\end{tabular}


Shipping Site: SRS

Waste Type: Solids (CH-Non-alpha)

Total volume $(\mathrm{m} 3 / \mathrm{yr})$ :

$1.868 \mathrm{E}+02$

Total Mass (kg/yr): $2.329 \mathrm{E}+05$

Radionuclide

$\mathrm{Ba}-137 \mathrm{~m}$

Co-60

Cs-137

Eu-154

$\mathrm{Eu}-155$

$\mathrm{Fe}-55$

Hi 3

$\mathrm{Nb}-94$

$\mathrm{Ni}-59$

Ni-63

$\mathrm{Pa}-234 \mathrm{~m}$

Pu -238

$\mathrm{Pu}-240$

Pu-241

$\mathrm{Sm}-151$

S $r-90$

TC-99

Th -234

$\mathrm{U}-238$

$Y-90$

Shipping Site: SRS

Total volume (m3/yr):

Radionuclide

$\mathrm{Ba}-137 \mathrm{~m}$

Co-60

Cs-137

Eu-154

Eu-155

$\mathrm{Fe}-55$

$\mathrm{Nb}-94$

$\mathrm{Ni}-59$

$\mathrm{Ni}-63$

$\mathrm{Pa}-234 \mathrm{~m}$

Pu-238

Pu-239

Pu-240

Pu-241

$S m-151$

Sr-90

Th-234

$\mathrm{U}-238$

$\mathrm{Y}-90$

Shipping site:

SRS

Total volume $(\mathrm{m} 3 / \mathrm{yr})$ :

Radionuclide

$\mathrm{Ba}-137 \mathrm{~m}$

Co-60

Cs -137

Eu-154

Eu-155

$\mathrm{Fe}-55$

$\mathrm{H}-3$

$\mathrm{Nb}-94$

$\mathrm{Ni}-59$

$\mathrm{Ni}-63$

$\mathrm{Pa}-234 \mathrm{~m}$

Pu-238

Pu-239

Pu-240

Pu-241

Sm-151

$\mathrm{Sr}-90$

$\mathrm{Th}-234$

$\mathrm{U}-238$

$\mathrm{Y}-90$

\begin{tabular}{c}
$\begin{array}{c}\text { Activity } \\
\text { (Ci/yr) }\end{array}$ \\
\hline $2.257 \mathrm{E}+01$ \\
$3.280 \mathrm{E}+01$ \\
$2.386 \mathrm{E}+01$ \\
$1.197 \mathrm{E}-01$ \\
$2.764 \mathrm{E}-02$ \\
$6.542 \mathrm{E}-01$ \\
$4.166 \mathrm{E}+02$ \\
$1.566 \mathrm{E}-01$ \\
$3.096 \mathrm{E}+00$ \\
$3.743 \mathrm{E}+02$ \\
$1.105 \mathrm{E}-01$ \\
$8.109 \mathrm{E}-01$ \\
$6.450 \mathrm{E}-01$ \\
$6.450 \mathrm{E}-01$ \\
$2.580 \mathrm{E}-01$ \\
$2.220 \mathrm{E}+01$ \\
$9.212 \mathrm{E}-03$ \\
$1.105 \mathrm{E}-01$ \\
$1.105 \mathrm{E}-01$ \\
$2.220 \mathrm{E}+01$
\end{tabular}

\begin{tabular}{l} 
Chemical \\
\hline $57-12-5$ \\
$75-09-2$ \\
$\mathrm{Ag}$ \\
$\mathrm{As}$ \\
$\mathrm{Ba}$ \\
$\mathrm{Cd}$ \\
$\mathrm{Cl}-2-\mathrm{x}$ \\
$\mathrm{Cl}-3-\mathrm{x}$ \\
$\mathrm{C} 1-4-\mathrm{x}$ \\
$\mathrm{C} 1-\mathrm{F}-\mathrm{x}$ \\
$\mathrm{Cr}$ \\
$\mathrm{HC}-$ insol \\
$\mathrm{HC}-$ Solub \\
$\mathrm{Hg}$ \\
$\mathrm{Pb}$ \\
$\mathrm{Se}$
\end{tabular}

\begin{tabular}{|c|}
\hline $\begin{array}{l}\text { Amount } \\
(\mathrm{kg} / \mathrm{yr})\end{array}$ \\
\hline $\begin{array}{l}2.631 \mathrm{E}+01 \\
4.659 \mathrm{E}+01 \\
1.180 \mathrm{E}+01 \\
4.217 \mathrm{E}+00 \\
3.783 \mathrm{E}+02 \\
1.304 \mathrm{E}+01 \\
3.100 \mathrm{E}+01 \\
4.540 \mathrm{E}+02 \\
4.891 \mathrm{E}+01 \\
3.618 \mathrm{E}+01 \\
4.287 \mathrm{E}+02 \\
1.802 \mathrm{E}+03 \\
8.514 \mathrm{E}+02 \\
1.397 \mathrm{E}+02 \\
7.059 \mathrm{E}+02 \\
5.588 \mathrm{E}+01\end{array}$ \\
\hline
\end{tabular}

Waste Type: Organic Liquids (CH-Alpha)

$6.450 \mathrm{E}+00$

Total Mass (kg/yr):

$6.811 \mathrm{E}+03$

\begin{tabular}{c}
$\begin{array}{c}\text { Activity } \\
\text { (Ci/YY) }\end{array}$ \\
\hline $6.249 \mathrm{E}-01$ \\
$6.697 \mathrm{E}-01$ \\
$6.598 \mathrm{E}-01$ \\
$2.738 \mathrm{E}-03$ \\
$4.979 \mathrm{E}-04$ \\
$1.269 \mathrm{E}-02$ \\
$9.864 \mathrm{E}+00$ \\
$4.979 \mathrm{E}-03$ \\
$1.063 \mathrm{E}-01$ \\
$1.153 \mathrm{E}+01$ \\
$3.485 \mathrm{E}-03$ \\
$3.510 \mathrm{E}-02$ \\
$9.959 \mathrm{E}-04$ \\
$3.734 \mathrm{E}-03$ \\
$1.429 \mathrm{E}-01$ \\
$7.718 \mathrm{E}-03$ \\
$6.075 \mathrm{E}-01$ \\
$3.485 \mathrm{E}-03$ \\
$3.485 \mathrm{E}-03$ \\
$6.075 \mathrm{E}-01$
\end{tabular}

\begin{tabular}{l} 
Chemical \\
\hline $57-12-5$ \\
$75-09-2$ \\
$\mathrm{Ag}$ \\
$\mathrm{As}$ \\
$\mathrm{Ba}$ \\
$\mathrm{Cd}$ \\
$\mathrm{CI}-2-\mathrm{x}$ \\
$\mathrm{CI}-3-\mathrm{x}$ \\
$\mathrm{CI}-4-\mathrm{x}$ \\
$\mathrm{CI}-\mathrm{F}-\mathrm{x}$ \\
$\mathrm{Cr}$ \\
$\mathrm{HC}-\mathrm{insol}$ \\
$\mathrm{HC}-\mathrm{Solub}$ \\
$\mathrm{Hg}$ \\
$\mathrm{Pb}$ \\
$\mathrm{Se}$
\end{tabular}

\begin{tabular}{c}
$\begin{array}{c}\text { Amount } \\
(\mathrm{kg} / \mathrm{Yr})\end{array}$ \\
\hline $5.978 \mathrm{E}-01$ \\
$4.536 \mathrm{E}-01$ \\
$2.996 \mathrm{E}-01$ \\
$3.838 \mathrm{E}-02$ \\
$2.617 \mathrm{E}-01$ \\
$1.627 \mathrm{E}-02$ \\
$1.291 \mathrm{E}+00$ \\
$5.081 \mathrm{E}+01$ \\
$2.871 \mathrm{E}+00$ \\
$1.046 \mathrm{E}+00$ \\
$6.974 \mathrm{E}-01$ \\
$4.176 \mathrm{E}+01$ \\
$1.197 \mathrm{E}+03$ \\
$1.697 \mathrm{E}+00$ \\
$2.602 \mathrm{E}-01$ \\
$2.619 \mathrm{E}-01$
\end{tabular}

Waste TYpe: Solids (CH-Alpha)

$1.209 \mathrm{E}+02$

Total Mass $(\mathrm{kg} / \mathrm{yr}): \quad 1.823 \mathrm{E}+05$

\begin{tabular}{c}
$\begin{array}{r}\text { Activity } \\
\text { (Ci/yr) }\end{array}$ \\
\hline $1.171 \mathrm{E}+01$ \\
$1.255 \mathrm{E}+01$ \\
$1.237 \mathrm{E}+01$ \\
$5.135 \mathrm{E}-02$ \\
$9.338 \mathrm{E}-03$ \\
$2.381 \mathrm{E}-01$ \\
$1.849 \mathrm{E}+02$ \\
$9.338 \mathrm{E}-02$ \\
$1.993 \mathrm{E}+00$ \\
$2.163 \mathrm{E}+02$ \\
$6.536 \mathrm{E}-02$ \\
$6.583 \mathrm{E}-01$ \\
$1.867 \mathrm{E}-02$ \\
$7.003 \mathrm{E}-02$ \\
$2.680 \mathrm{E}+00$ \\
$1.447 \mathrm{E}-01$ \\
$1.139 \mathrm{E}+01$ \\
$6.536 \mathrm{E}-02$ \\
$6.536 \mathrm{E}-02$ \\
$1.139 \mathrm{E}+01$
\end{tabular}

\begin{tabular}{l} 
Chemical \\
\hline $57-12-5$ \\
$75-09-2$ \\
$\mathrm{Ag}$ \\
$\mathrm{As}$ \\
$\mathrm{Ba}$ \\
$\mathrm{Cd}$ \\
$\mathrm{Cl}-2-\mathrm{x}$ \\
$\mathrm{Cl}-3-\mathrm{x}$ \\
$\mathrm{Cl}-4-\mathrm{x}$ \\
$\mathrm{Cl}-\mathrm{F}-\mathrm{x}$ \\
$\mathrm{Cr}$ \\
$\mathrm{HC}-$ insol \\
$\mathrm{HC}-$ Solub \\
$\mathrm{Hg}$ \\
$\mathrm{Pb}$ \\
$\mathrm{Se}$
\end{tabular}

\begin{tabular}{c}
$\begin{array}{c}\text { Amount } \\
(\mathrm{kg} / \mathrm{Y})\end{array}$ \\
\hline $2.345 \mathrm{E}+01$ \\
$3.624 \mathrm{E}+01$ \\
$4.582 \mathrm{E}+00$ \\
$1.744 \mathrm{E}+00$ \\
$2.871 \mathrm{E}+02$ \\
$5.887 \mathrm{E}+00$ \\
$3.624 \mathrm{E}+01$ \\
$5.966 \mathrm{E}+02$ \\
$1.435 \mathrm{E}+02$ \\
$1.769 \mathrm{E}+01$ \\
$2.954 \mathrm{E}+02$ \\
$1.869 \mathrm{E}+03$ \\
$8.725 \mathrm{E}+02$ \\
$1.964 \mathrm{E}+01$ \\
$6.984 \mathrm{E}+02$ \\
$5.697 \mathrm{E}+00$
\end{tabular}


Shipping Site: UMC Waste Type: Solids (CH-Alpha)

Total Volume $(\mathrm{m} 3 / \mathrm{yr})$ :

$3.944 \mathrm{E}-02$

Total Mass (kg/yr): $5.809 \mathrm{E}+01$

Radionuclide

Am-241

$\mathrm{cm}-244$

$\mathrm{Pa}-234 \mathrm{~m}$

$\mathrm{Pu}-238$

Pu-239

Pu-240

Pu-241

Th-234

U -238
Shipping site: WVDE

Total Volume (m3/yr):

Radionuclide

Am-241

$\mathrm{Ba}-137 \mathrm{~m}$

Co-60

Cs -137

Eu-154

$\mathrm{H}-3$

$\mathrm{Nb}-94$

$\mathrm{Ni}-59$

$\mathrm{Ni}-63$

$\mathrm{Pa}-234 \mathrm{~m}$

Pu-238

Pu-239

Pu-2 40

Pu-241

Sm-151

Sr -90

TC -99

Th-234

$\mathrm{u}-238$

$Y-90$

Shipping site: WVDP

Total Volume $(\mathrm{m} 3 / \mathrm{yr})$ :

Radionuclide

Am-241

$\mathrm{Ba}-137 \mathrm{~m}$

Co- 60

Cs-137

Eu-154

$\mathrm{H}-3$

$\mathrm{Nb}-94$

$\mathrm{Ni}-59$

Ni-63

$\mathrm{Pa}-234 \mathrm{~m}$

Pu-238

Pu-239

Pu-240

Pu-241

$\mathrm{Sm}-151$

Sr -90

Tc-99

Th-234

$\mathrm{U}-238$

$Y-90$

\begin{tabular}{r}
$\begin{array}{c}\text { Activity } \\
\text { (Ci/yr) }\end{array}$ \\
\hline $1.743 \mathrm{E}-07$ \\
$2.358 \mathrm{E}-07$ \\
$8.511 \mathrm{E}-07$ \\
$9.108 \mathrm{E}-05$ \\
$9.331 \mathrm{E}-06$ \\
$3.260 \mathrm{E}-05$ \\
$8.894 \mathrm{E}-04$ \\
$8.511 \mathrm{E}-07$ \\
$8.511 \mathrm{E}-07$
\end{tabular}

Chemica

57-12-5

75-09-2

Ag

As

$\mathrm{Ba}$

Cd

c $1-2-x$

Cl $-3-x$

Cl-4-x

$\mathrm{Cl}-\mathrm{F}-\mathrm{X}$

$\mathrm{Cr}$

HC-insol

HC-solub

$\mathrm{Hg}$

$\mathrm{Pb}$

se
Waste Type: Organic Liquids (CH-Alpha)

Total Mass (kg/Yr): $1.897 \mathrm{E}+03$

$.773 \mathrm{E}+00$

\begin{tabular}{l} 
Chemical \\
\hline $57-12-5$ \\
$75-09-2$ \\
Ag \\
As \\
$\mathrm{Ba}$ \\
$\mathrm{Cd}$ \\
$\mathrm{Cl}-2-\mathrm{x}$ \\
$\mathrm{Cl}-3-\mathrm{x}$ \\
$\mathrm{Cl}-4-\mathrm{x}$ \\
$\mathrm{Cl}-\mathrm{F}-\mathrm{x}$ \\
$\mathrm{Cr}$ \\
$\mathrm{HC}-\mathrm{insol}$ \\
$\mathrm{HC}-\mathrm{solvb}$ \\
$\mathrm{Hg}$ \\
$\mathrm{Pb}$ \\
$\mathrm{Se}$
\end{tabular}

\begin{tabular}{c}
$\begin{array}{c}\text { Amount } \\
(\mathrm{kg} / \mathrm{Yr})\end{array}$ \\
\hline $1.600 \mathrm{E}-01$ \\
$2.840 \mathrm{E}-01$ \\
$7.866 \mathrm{E}-02$ \\
$9.986 \mathrm{E}-03$ \\
$6.783 \mathrm{E}-02$ \\
$1.250 \mathrm{E}-01$ \\
$4.212 \mathrm{E}-01$ \\
$2.491 \mathrm{E}+01$ \\
$3.373 \mathrm{E}+00$ \\
$2.744 \mathrm{E}-01$ \\
$2.914 \mathrm{E}-01$ \\
$1.686 \mathrm{E}+01$ \\
$3.228 \mathrm{E}+02$ \\
$4.473 \mathrm{E}-01$ \\
$9.175 \mathrm{E}-02$ \\
$6.881 \mathrm{E}-02$
\end{tabular}

$1.541 \mathrm{E}+00$

7. $694 \mathrm{E}-04$

$9.529 \mathrm{E}-04$

$9.529 E-04$

1. $541 \mathrm{E}+00$
Amount

9.876E-03

1. $161 \overrightarrow{\mathrm{a}}-02$

$1.742 E-03$

$5.809 \mathrm{E}-04$

1. $151 \mathrm{E}-01$

2. $904 E-04$

1. $161 \mathrm{E}-02$

3. $253 \mathrm{E}-02$

$2.033 \mathrm{E}-02$

$5.809 \mathrm{E}-03$

$1.161 \mathrm{E}-01$

$5.809 E-02$

$2.904 \mathrm{E}-03$

5. 809E-03

2. $904 \mathrm{E}-01$

1. $742 \mathrm{E}-03$ 
$B-1$

APPENDIX B:

DETAILED RADIOLOGICAL RISK ASSESSMENT RESULTS 
B-2 


\section{APPENDIX B: \\ DETAILED RADIOLOGICAL RISK ASSESSMENT RESULTS}

Detailed results of the collective population radiological risk assessment for transportation of low-level mixed waste (LLMW) are presented in this appendix for the Waste Management (WM) LLMW alternatives. For each WM alternative, results are presented separately for the following categories of LLMW:

- Contact-handled $(\mathrm{CH})$ organic liquids, nonalpha - untreated;

- $\mathrm{CH}$ organic liquids, alpha - untreated;

- CH heterogeneous solids, nonalpha - untreated;

- $\mathrm{CH}$ heterogeneous solids, alpha - untreated;

- CH heterogeneous solids, nonalpha - treated; and

- $\mathrm{CH}$ heterogeneous solids, alpha - treated.

Note that some cases do not involve the transportation of all six categories of LLMW listed previously. The total transportation impacts for an alternative can be found by summing the quantities for the six categories.

Similar to the WASTE_MGMT files presented in Appendix A of this report, the collective risk results are presented on an annual basis. As stated previously, the Waste Management Programmatic Environmental Impact Statement (WM PEIS) considers a 20-year time frame for WM LLMW. For the WM alternatives considered in the WM PEIS, the assumption was that shipments would occur uniformly over a 10-year period, which would allow an initial 10-year period to build treatment, storage, and disposal facilities. Sites would essentially store their own waste for the first 10 years and ship all waste over the subsequent 10 years.

Because of the differences in the time frames noted and the uncertainties in the actual timing of future waste shipments, the transportation impacts for the various cases are best compared by examining the total impacts of transporting a specific inventory of waste, independent of the annual shipping rates. The total impacts of LLMW transported for each alternative can be calculated by multiplying the annual shipment impacts by 10 years for the WM alternatives. The total impacts are provided in Section 5 of the main body of this report. Detailed chemical-specific calculations for the maximally exposed individual (MEI) are provided in Tables B.1 through B.5. 
TABLE B.1 MEI Potential Increased Cancer Risk Associated with the Most Severe Chemical Accident ${ }^{\mathrm{a}, \mathrm{b}}$

\begin{tabular}{|c|c|c|c|c|c|c|c|c|c|c|c|c|}
\hline $\begin{array}{c}\text { LlMW } \\
\text { Type }\end{array}$ & $\begin{array}{c}\text { Transportation } \\
\text { Mode }\end{array}$ & $\begin{array}{c}\text { Release } \\
\text { Mode }\end{array}$ & $\begin{array}{c}\text { Chemical } \\
\text { Code }\end{array}$ & Chemical Name & $\begin{array}{c}\text { ALOHA } \\
\text { Concentration } \\
\text { (ppm) }\end{array}$ & $\begin{array}{c}\text { Molecular } \\
\text { Weight }\end{array}$ & $\begin{array}{c}\text { ALOHA } \\
\text { Concentration } \\
\left(\mathrm{mg} / \mathrm{m}^{3}\right)\end{array}$ & $\begin{array}{c}\text { Exposure } \\
\text { Time } \\
\text { (min/d) }\end{array}$ & $\begin{array}{c}\text { Inhalation } \\
\text { Air Intake } \\
\text { (mg/kg/d) }\end{array}$ & $\begin{array}{l}\text { Inhalation } \\
\text { Unit Risk } \\
\left(\mu \mathrm{g} / \mathbf{m}^{3}\right)^{-1} \\
\end{array}$ & $\begin{array}{c}\text { Slope } \\
\text { Factor } \\
\left(m g / \mathrm{kg}^{\prime} / \mathbf{d}\right)^{-1}\end{array}$ & $\begin{array}{c}\text { Carcinogenic } \\
\text { MEI Kisk }\end{array}$ \\
\hline \multirow{5}{*}{$\begin{array}{l}\text { WM } \\
\text { (neutral) }\end{array}$} & \multirow[t]{5}{*}{ Highway } & Liquid & $75-09-2$ & Dichloromethane & 3.73E-02 & 85 & $1.29 \mathrm{E}-01$ & 60 & $6.08 \mathrm{E}-08$ & $4.70 \mathrm{E}-07$ & $1.65 \mathrm{E}-03$ & $1.00 \mathrm{E}-10$ \\
\hline & & aerosol & Cl.2-x & Dichloroethane & $9.26 \mathrm{E}-02$ & 99 & $3.74 \mathrm{E}-01$ & 60 & $1.76 \mathrm{E}-07$ & $2.60 \mathrm{E}-05$ & $9.10 \mathrm{E}-02$ & $1.60 \mathrm{E}-08$ \\
\hline & & (direct) & Cl-4-x & Tetrachloroethene & $1.48 \mathrm{E}-01$ & 166 & $1.00 E+00$ & 60 & $4.71 \mathrm{E}-07$ & $1.70 \mathrm{E}-06$ & $5.95 \mathrm{E}-03$ & $2.80 \mathrm{E}-09$ \\
\hline & & & HC-solublec & Benzene & $1.09 E+03$ & $\begin{array}{l}78.1 \\
d\end{array}$ & $3.47 E+03$ & 60 & $1.63 E-03$ & $8.30 \mathrm{E}-06$ & $2.91 \mathrm{E}-02$ & $4.74 \mathrm{E}-05$ \\
\hline & & & & Subtotal & $\frac{1.09 E+03}{2.04}$ & $\therefore$ & $\frac{3.48 E+03}{80}$ & & & & - & $4.74 E-05$ \\
\hline \multirow{5}{*}{$\begin{array}{l}\text { WM } \\
\text { (stable) }\end{array}$} & \multirow[t]{5}{*}{ Highway } & Liqquid & $75-09-2$ & Dichloromethane & $\overline{5.34 \mathrm{E}-01}$ & 85 & $\overline{1.85 \mathrm{E}+00}$ & 60 & $8.70 \mathrm{E}-07$ & $4.70 \mathrm{E}-07$ & $1.65 \mathrm{E}-0.3$ & $\overline{1.43 \mathrm{E}-09}$ \\
\hline & & aerosol & $\mathrm{Cl}-2-\mathrm{x}$ & Dichloroethane & $2.02 \mathrm{E}+00$ & 99 & $8.16 \mathrm{E}+00$ & 60 & $3.83 \mathrm{E}-06$ & $2.60 \mathrm{E}-05$ & $9.10 \mathrm{~K}-02$ & $3.49 \mathrm{E}-07$ \\
\hline & & (direct) & Cl-4-x & Tetrachloroothene & $1.10 \mathrm{E}+00$ & 166 & $7.45 E+00$ & 60 & $3.50 \mathrm{E}-06$ & $1.70 \mathrm{E}-06$ & $5.95 \mathrm{E}-03$ & $2.08 \mathrm{E}-08$ \\
\hline & & & HC-soluble & Benzene & $2.57 E+03$ & 78.1 & $8.19 E+03$ & 60 & $3.85 \mathrm{E}-03$ & 8.30E-06 & $2.91 \mathrm{E}-02$ & $1.12 \mathrm{E}-04$ \\
\hline & & & & Sublotal & $\underline{2.67 E+03}$ & & $8.21 E+03$ & & & & 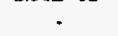 & $1.12 E-04$ \\
\hline \multirow{5}{*}{$\begin{array}{l}\text { WM } \\
\text { (neutral) }\end{array}$} & \multirow[t]{5}{*}{ Railroad } & Liquid & $75-09-2$ & Dichloromethane & $\overline{2.99 E-01}$ & 85 & $\overline{1.04 \mathrm{E}+00}$ & 60 & 4.87E-07 & 4.70E-07 & $1.65 \mathrm{E}-03$ & $\overline{8.01 \mathrm{E}-10}$ \\
\hline & & aerosol & $\mathrm{Cl} \cdot 2 \cdot \mathrm{x}$ & Dichloroethane & $1.39 \mathrm{E}-01$ & 99 & $5.62 \mathrm{E}-01$ & 60 & $2.64 \mathrm{E}-07$ & $2.60 \mathrm{E}-05$ & $9.10 E-02$ & $2.40 E-08$ \\
\hline & & (direct) & Cl-4-x & Tetrachloroethene & $2.22 \mathrm{E}-01$ & 166 & $1.50 \mathrm{E}+00$ & 60 & $7.06 \mathrm{E}-07$ & $1.70 \mathrm{E}-06$ & $5.95 \mathrm{E}-03$ & $4.20 \mathrm{E}-09$ \\
\hline & & & HC-soluble & Benzane & $1.46 \mathrm{E}+03$ & 78.1 & $4.65 E+03$ & 60 & $2.19 E-03$ & $8.30 E-06$ & $2.91 E-02$ & $6.35 E-05$ \\
\hline & & & & $\frac{\text { Sublotal }}{D \text { Dibhom }}$ & $\frac{1.46 E+03}{408 \pi .00}$ & & $4.66 E+03$ & & & & & $6.35 E-05$ \\
\hline \multirow{4}{*}{$\begin{array}{l}\text { WM } \\
\text { (stable) }\end{array}$} & \multirow[t]{4}{*}{ Railroad } & Liquid & $75 \cdot 09 \cdot 2$ & Dichloromethane & $\overline{4.88 E+00}$ & 85 & $\overline{1.69 \mathrm{E}+01}$ & 60 & $7.95 E-06$ & $4.70 \mathrm{E}-07$ & $1,65 \mathrm{E}-03$ & $\overline{1.31 E-08}$ \\
\hline & & aerosol & $\mathrm{Cl}-2 \cdot \mathrm{x}$ & Dichloroethane & $2.41 \mathrm{E}+00$ & 99 & $9.74 \mathrm{E}+00$ & 60 & $4.57 \mathrm{E}-06$ & $2.60 \mathrm{E}-05$ & $9.10 \mathrm{E}-02$ & $4.16 \mathrm{E}-07$ \\
\hline & & (direet) & Cl-4-x & Tetrachloroethene & $1.53 E+00$ & 166 & $1.04 E+01$ & 60 & $4.87 \mathrm{E}-06$ & $1.70 \mathrm{E}-06$ & $5.95 \mathrm{E}-03$ & $2.90 \mathrm{E}-08$ \\
\hline & & & HC-soluble & $\begin{array}{l}\text { Benzene } \\
\text { Subtatal } \\
\end{array}$ & $\begin{array}{r}3.99 E+03 \\
4.00 E+03 \\
\end{array}$ & 78.1 & $\begin{array}{r}1.27 E+04 \\
1.28 E+04 \\
\end{array}$ & 60 & $5.97 \mathrm{E}-03$ & $\begin{array}{c}8.30 \mathrm{E}-06 \\
\cdot\end{array}$ & $2.91 \mathrm{E}-02$ & $\begin{array}{r}1.74 E-04 \\
1.74 E-04 \\
\end{array}$ \\
\hline
\end{tabular}

- Neutral atmospheric conditions were based on Pasquill stability Class D with a wind speed of $4 \mathrm{~m} / \mathrm{s}(9 \mathrm{mph})$.

b Stable atmospheric conditions were based on Pasquill stability Class $F$ with a wind speed of $1 \mathrm{~m} / \mathrm{s}$ (2.2 $\mathrm{mph}$ ).

- $\mathrm{HC}=$ hydrocarbon

d Not applicable. 
TABLE B.2 MEI Hazard Quotient for PAE Risk Associated with the Most Severe Chemical Accident ${ }^{\mathrm{a}, \mathrm{b}}$

\begin{tabular}{|c|c|c|c|c|c|c|c|c|c|c|c|c|}
\hline $\begin{array}{c}\text { LLMW } \\
\text { Typo } \\
\end{array}$ & $\begin{array}{c}\text { Transportation } \\
\text { Mole }\end{array}$ & $\begin{array}{l}\text { Release } \\
\text { Mode }\end{array}$ & $\begin{array}{c}\text { Chemical } \\
\text { Code }\end{array}$ & Chemical Name & $\begin{array}{c}\text { ALOHA } \\
\begin{array}{c}\text { Concentration } \\
\text { (ppm) }\end{array}\end{array}$ & $\begin{array}{c}\text { Molecular } \\
\text { Weight }\end{array}$ & $\begin{array}{c}\text { ALOHA } \\
\text { Concentration } \\
\left(\mathrm{mg} / \mathrm{m}^{3}\right)\end{array}$ & $\begin{array}{c}\text { Exposure } \\
\text { Time } \\
\text { (min/d) }\end{array}$ & $\begin{array}{l}\text { Inhalation } \\
\text { Air Intake } \\
\text { (mg/kg/d) }\end{array}$ & $\underset{\left(\mathrm{mg} / \mathrm{m}^{3}\right)}{\mathrm{ReC}^{\mathrm{c}}}$ & $\underset{(\mathrm{mg} / \mathrm{kg} / \mathrm{d})}{\mathrm{RfD}^{d}}$ & $\begin{array}{l}\text { Hazard } \\
\text { Quotient }\end{array}$ \\
\hline \multirow{5}{*}{$\begin{array}{l}\text { WM } \\
\text { (neutral) }\end{array}$} & Highway & Liquid & $75-09-2$ & Dichloromethane & $3.73 E-02$ & 85 & $1.29 \mathrm{E}-01$ & 60 & 8.72E-04 & 3 & $8.57 \mathrm{E}-01$ & $1.02 \mathrm{E}-0 \mathrm{~s}$ \\
\hline & & aerosol & Cl-3-3-x & 1,1,1-Trichlonethane & $7.69 \mathrm{E}+00$ & 133.4 & $4.19 \mathrm{E}+01$ & 60 & $2.82 \mathrm{E}-01$ & 1 & $2.86 \mathrm{E}-01$ & $9.87 \mathrm{E}-01$ \\
\hline & & (direct) & Cl-F-x & Freon 113 & $3.88 \mathrm{E}-02$ & 187 & $2.96 \mathrm{E}-01$ & 60 & $1.99 \mathrm{E}-03$ & 30 & $8.57 \mathrm{E}+00$ & $2.33 \mathrm{E}-04$ \\
\hline & & & HC-soluble $0^{\circ}$ & Toluene & $9.16 \mathrm{E}+02$ & 92.14 & $3.44 \mathrm{E}+03$ & 60 & $2.32 \mathrm{E}+01$ & 0.4 & $1.14 \mathrm{E}-01$ & $2.03 \mathrm{E}+02$ \\
\hline & & & & Subtotal & $9.23 E+02$ & $t$ & $3.48 E+03$ & 0 & & & & $2.04 E+(12$ \\
\hline \multirow{5}{*}{$\begin{array}{l}\text { WM } \\
\text { (stables) }\end{array}$} & Highway & Liquid & $75-09-2$ & Dichloromethane & $5.34 \mathrm{E}-01$ & 85 & $1.85 \mathrm{E}+00$ & 60 & $1.25 \mathrm{E}-02$ & 3 & $8.57 \mathrm{E}-01$ & $\overline{1.46 \mathrm{E}-02}$ \\
\hline & & aerusol & Cl-3-x & 1,1,1-Trichloroethane & $2.28 \mathrm{E}+01$ & 133,4 & $1.24 \mathrm{E}+02$ & 60 & $8.36 \mathrm{E}-01$ & 1 & $2.86 \mathrm{E}-01$ & $2.93 \mathrm{E}+00$ \\
\hline & & (direct) & $\mathbf{C l} \cdot \mathbf{F} \cdot \mathbf{x}$ & Frean 113 & 3.58E-01 & 187 & $2.73 \mathrm{E}+00$ & 60 & $1.84 \mathrm{E}-02$ & 30 & $8.57 \mathrm{E}+00$ & $2.15 \mathrm{E}-03$ \\
\hline & & & HC-soluble & Toluene & $2.18 E+03$ & 92.14 & $8.20 \mathrm{E}+03$ & 60 & $5.52 E+01$ & 0.4 & $1.14 \mathrm{E}-01$ & $4.83 E+02$ \\
\hline & & & & Subtotal & $2.20 E+03$ & & $8.33 E+03$ & & & & & $4.86 E+02$ \\
\hline \multirow{4}{*}{$\begin{array}{l}\text { WM } \\
\text { (neutral) }\end{array}$} & Railroad & Liquid & $75.09-2$ & Dichloromethane & $2.99 \mathrm{E}-01$ & 85 & $\overline{1.04 \mathrm{E}+00}$ & 60 & $6.99 \mathrm{E}-03$ & 3 & $8.57 E-01$ & $8.15 E-03$ \\
\hline & & aerosol & Cl-3-x & 1,1,1-Trichloroethane & $4.98 \mathrm{E}+01$ & 133.4 & $2.71 \mathrm{E}+02$ & 60 & $1.83 \mathrm{E}+00$ & 1 & $2.86 \mathrm{E}-01$ & $6.39 \mathrm{E}+00$ \\
\hline & & (direct) & Cl-F.x & Freon 113 & $7.46 \mathrm{E}-01$ & 187 & $5.69 \mathrm{E}+00$ & 60 & 3.83E-02 & 30 & $8.67 \mathrm{E}+00$ & $4.47 \mathrm{E}-03$ \\
\hline & & & HC-soluble & $\begin{array}{l}\text { Toluene } \\
\text { Subtotol }\end{array}$ & $1.20 \mathrm{E}+03$ & 92.14 & $4.51 \mathrm{E}+03$ & 60 & $3.04 E+01$ & 0.4 & $1.14 \mathrm{E}-01$ & $2.66 \mathrm{E}+02$ \\
\hline \multirow{4}{*}{$\begin{array}{l}\text { WM } \\
\text { (stable) }\end{array}$} & Railroad & Liquid & $76-09-2$ & $\frac{\text { Dhorbloromethane }}{\text { Dis }}$ & $\frac{1.25 E+03}{4.88 E+00}$ & 85 & $\frac{4.79 E+03}{1.69 E+01}$ & 60 & $1.14 \mathrm{E}-01$ & 3 & $8.57 \mathrm{E}-01$ & $\frac{2.72 E+02}{1.33 \mathrm{E}-01}$ \\
\hline & & aerosol & Cl-3-x & 1,1,1-Trichlornethane & $1.07 \mathrm{E}+02$ & 133.4 & $5.83 \mathrm{E}+02$ & 60 & $3.92 \mathrm{E}+00$ & 1 & 2.86E-01 & $1.37 E+01$ \\
\hline & & (direct) & $C l \cdot F \cdot \mathbf{x}$ & Freon 113 & 5.14E+00 & 187 & $3.92 \mathrm{E}+01$ & 60 & $2.64 E-01$ & 30 & $8.57 E+00$ & $3.08 \mathrm{E}-02$ \\
\hline & & & HC-soluble & Toluene & $3.40 \mathrm{E}+03$ & 92.14 & $1.28 \mathrm{E}+04$ & 60 & $8.61 \mathrm{E}+01$ & 0.4 & $1.14 \mathrm{E}-01$ & $7.54 \mathrm{E}+02$ \\
\hline
\end{tabular}

a Neutral atmospheric conditions were based on Pasquill stability Class D with a wind speed of $4 \mathrm{~m} / \mathrm{s}(9 \mathrm{mph})$. PAE $=$ potential adverse effect.

b Stable atmospheric conditions were based on Pasquill stability Class F with a wind speed of $1 \mathrm{~m} / \mathrm{s}(2.2 \mathrm{mph})$.

- $\mathrm{RFC}=$ reference concentration .

d RID = reference dose.

- $\mathrm{HC}=$ hydrocarbon.

f Not applicable. 
TABLE B.3 MEI Potential Increased Cancer Risk Associated with the Most Severe Radiological Accident ${ }^{\mathrm{a}, \mathrm{b}}$

\begin{tabular}{|c|c|c|c|c|c|c|c|c|c|c|c|c|}
\hline $\begin{array}{c}\text { LLMW } \\
\text { Type }\end{array}$ & $\begin{array}{l}\text { Transportation } \\
\text { Mode }\end{array}$ & $\begin{array}{l}\text { Modeling } \\
\text { Approach }\end{array}$ & $\begin{array}{c}\text { Chemical } \\
\text { Code }\end{array}$ & Chemical Name & $\begin{array}{c}\text { ALOHA } \\
\text { Concentration } \\
\text { (ppm) }\end{array}$ & $\begin{array}{l}\text { Molecular } \\
\text { Weight }\end{array}$ & $\begin{array}{c}\text { ALOHA } \\
\text { Concentration } \\
\left(\mathrm{mg} / \mathrm{m}^{3}\right)\end{array}$ & $\begin{array}{c}\text { Exposure } \\
\text { Time } \\
\text { (min/d) }\end{array}$ & $\begin{array}{l}\text { Inhalation } \\
\text { Air Intake } \\
\text { (mg/kg/d) }\end{array}$ & $\begin{array}{l}\text { Inhalation } \\
\text { Unit Risk } \\
\left(\mu \mathrm{g} / \mathrm{m}^{3}\right)^{-1}\end{array}$ & $\begin{array}{c}\text { Slope } \\
\text { Factor } \\
(\mathbf{m g} / \mathbf{k g} / \mathbf{d})^{-1}\end{array}$ & $\begin{array}{l}\text { Carcinogenic } \\
\text { MEI Risk }\end{array}$ \\
\hline \multirow{5}{*}{$\begin{array}{l}\text { WM } \\
\text { (neutral) }\end{array}$} & \multirow[t]{5}{*}{ Highway } & Particulate & $75-09-2$ & Dichloromethane & $3.22 \mathrm{E}-02$ & 85 & $1.12 \mathrm{E}-01$ & 60 & $5.25 \mathrm{E}-08$ & $4.70 \mathrm{E}-07$ & $1.65 \mathrm{E}-03$ & $8.63 \mathrm{E}-11$ \\
\hline & & (severity & Cl-2-x & Dichloroethane & $1.20 \mathrm{E}-02$ & 99 & $4.85 \mathrm{E}-02$ & 60 & $2.28 \mathrm{E}-08$ & 2.60E-05 & $9.10 \mathrm{E}-02$ & $2.07 \mathrm{E}-09$ \\
\hline & & Categoriey & Cl-4-x & Totrachloroethene & $2.21 E-02$ & 166 & $1.60 \mathrm{E}-01$ & 60 & $7.03 E-08$ & $1.70 \mathrm{E} \sim 06$ & $5.95 E-03$ & $4.18 E-10$ \\
\hline & & IV-VIII) & HC-soluble ${ }^{\mathrm{e}}$ & Benzene & $3.51 \mathrm{E}-01$ & 78.1 & $1.12 \mathrm{E}+00$ & 60 & $5.26 \mathrm{E}-07$ & $8.30 \mathrm{E}-06$ & $2.91 \mathrm{E}-02$ & $1.53 E-08$ \\
\hline & & & & Subtotal & $4.17 E-01$ & $d$ & $1.43 E+00$ & & & & & $1.78 E-08$ \\
\hline \multirow{5}{*}{$\begin{array}{l}\text { WM } \\
\text { (stuble) }\end{array}$} & \multirow{5}{*}{ Highway } & Particulate & $75-09-2$ & Dichloromethane & $\overline{8.66 \mathrm{E}-02}$ & 85 & $\overline{3.00 E-01}$ & 60 & $1.41 \mathrm{E}-07$ & $4.70 \mathrm{E}-07$ & $1.65 \mathrm{E}-0.3$ & $\frac{2.32 E-10}{2.30}$ \\
\hline & & (severity & Cl-2-x & Dichloroethane & $6.19 \mathrm{E}-02$ & 99 & $2.50 \mathrm{E}-01$ & 60 & $1.17 \mathrm{E}-07$ & $2.60 \mathrm{E}-05$ & $9.10 \mathrm{E}-02$ & $1.07 \mathrm{E}-08$ \\
\hline & & Categories & Cl-4-x & Tetrachloroethene & $1.29 \mathrm{E}-01$ & 166 & $8.74 E-01$ & 60 & $4.11 E-07$ & $1.70 \mathrm{E}-06$ & $5.95 \mathrm{E}-03$ & $2.44 \mathrm{E}-09$ \\
\hline & & IV-VIII) & HC-soluble & Benzene & $9.76 \mathrm{E}-01$ & 78.1 & $3.11 \mathrm{E}+00$ & 60 & $1.46 \mathrm{E}-06$ & $8.30 \mathrm{E} \sim 06$ & $2.91 \mathrm{E}-02$ & $\begin{array}{l}2.44 \mathrm{E}-0 \mathrm{~J} \\
4.24 \mathrm{E}-08\end{array}$ \\
\hline & & & & Subtotal & $1.25 E+00$ & $\therefore$ & $4.54 E+00$ & & & & & $5.58 E-08$ \\
\hline \multirow{5}{*}{$\begin{array}{l}\text { WM } \\
\text { (neutral) }\end{array}$} & \multirow[t]{5}{*}{ Railroad } & Particulate & $75-09-2$ & Dichloromethane & $\overline{3.22 \mathrm{E}-02}$ & 85 & $\overline{1.12 \mathrm{E}-01}$ & 60 & $5.25 \mathrm{E}-08$ & $4.70 \mathrm{E}-07$ & $1.65 \mathrm{E}-03$ & $\overline{8.63 \mathrm{E}-11}$ \\
\hline & & (severity & $\mathrm{Cl} \cdot 2-\mathrm{x}$ & Dichloreethane & $1.20 \mathrm{E}-02$ & 99 & $4.85 \mathrm{E}-02$ & 60 & $2.28 \mathrm{E}-08$ & $2.60 \mathrm{E}-05$ & $9.10 \mathrm{E}-02$ & $2.07 \mathrm{E}-09$ \\
\hline & & Categories & Cl-4-x & Tetrachloroethene & $2.21 \mathrm{E}-02$ & 166 & $1.50 \mathrm{E}-01$ & 60 & $7.03 \mathrm{E}-08$ & $1.70 \mathrm{E}-06$ & $5.95 \mathrm{E}-03$ & $4.18 \mathrm{E}-10$ \\
\hline & & IV-VIII) & HC-soluble & Benzene & $3.51 \mathrm{E}-01$ & 78.1 & $1.12 \mathrm{E}+00$ & 60 & $5.26 \mathrm{E}-07$ & $8.30 \mathrm{E}-06$ & $2.91 \mathrm{E}-02$ & $1.53 \mathrm{E}-08$ \\
\hline & & & & Subtotal & $4.17 \varepsilon-01$ & & $1.43 E+00$ & 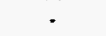 & 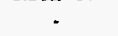 & (1) & (20) & $1.78 E-08$ \\
\hline \multirow{5}{*}{$\begin{array}{l}\text { WM } \\
\text { (stable) }\end{array}$} & \multirow[t]{5}{*}{ Railroad } & Particulate & $75-09-2$ & Dichloromethane & $\overline{8.66 \mathrm{E}-02}$ & 85 & $\overline{3.00 \mathrm{E}-01}$ & 60 & $1.41 \mathrm{E}-07$ & $4.70 \mathrm{E}-07$ & $1.65 \mathrm{E}-03$ & $2.32 \mathrm{E}-10$ \\
\hline & & (severity & $\mathrm{Cl}-2-\mathbf{x}$ & Dichloroethane & $6.19 \mathrm{E}-02$ & 99 & $2.50 \mathrm{E}-01$ & 60 & $1.17 \mathrm{E}-07$ & $2.60 \mathrm{E}-05$ & $9.10 \mathrm{E}-02$ & $1.07 \mathrm{E}-08$ \\
\hline & & Categories & Cl-4-x & Tetrachloroethene & $1.29 \mathrm{E}-01$ & 166 & $8.74 \mathrm{E}-01$ & 60 & $4.11 \mathrm{E}-07$ & $1.70 \mathrm{E}-06$ & $5.95 \mathrm{E}-03$ & $2.44 \mathrm{E}-09$ \\
\hline & & IV-VIII) & HC-soluble & Benzene & $9.76 \mathrm{E}-01$ & 78.1 & $3.11 \mathrm{E}+00$ & 60 & $1.46 \mathrm{E}-06$ & 8.30E -06 & $2.91 \mathrm{E}-02$ & $4.24 \mathrm{E}-08$ \\
\hline & & & & Subtotal & $1.25 E+00$ & $x_{0}$ & $4.54 E+00$ & $\omega^{2}+2$ & 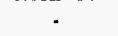 & - & & $5.58 E-08$ \\
\hline
\end{tabular}

a Neutral atmospheric conditions were based on Pasquill stability Class D with a wind speod of $4 \mathrm{~m} / \mathrm{s}(9 \mathrm{mph})$.

b Stable atmospheric conditions were based on Pasquill stability Class $F$ with a wind speed of $1 \mathrm{~m} / \mathrm{s}(2.2 \mathrm{mph})$.

- $\mathrm{HC}=$ hydrocarbon.

d Not applicable. 
TABLE B.4 MEI Hazard Quotient for PAE Risk Associated with the Most Severe Radiological Accident ${ }^{\mathrm{a}, \mathrm{b}}$

\begin{tabular}{|c|c|c|c|c|c|c|c|c|c|c|c|c|}
\hline $\begin{array}{l}\text { LLMW } \\
\text { Type }\end{array}$ & $\begin{array}{l}\text { Transportation } \\
\text { Mode }\end{array}$ & $\begin{array}{l}\text { Modeling } \\
\text { Approach }\end{array}$ & $\begin{array}{c}\text { Chemical } \\
\text { Code }\end{array}$ & Chemical Name & $\begin{array}{c}\text { ALOHA } \\
\text { Concentration } \\
\text { (ppm) }\end{array}$ & $\begin{array}{c}\text { Molecular } \\
\text { Weight }\end{array}$ & $\begin{array}{c}\text { ALOHA } \\
\text { Concentration } \\
\left(\mathrm{mg} / \mathrm{m}^{3}\right)\end{array}$ & $\begin{array}{c}\text { Exposure } \\
\text { Time } \\
\text { (min/d) }\end{array}$ & $\begin{array}{l}\text { Inhalation } \\
\text { Air Intake } \\
\text { (mg/kg/d) }\end{array}$ & $\underset{\left(\mathrm{mg} / \mathrm{m}^{3}\right)}{\mathrm{Re}^{\mathrm{c}}}$ & $\begin{array}{c}\operatorname{Rrf} D^{d} \\
(m g / k g / d)\end{array}$ & $\begin{array}{l}\text { Hazard } \\
\text { Quotien }\end{array}$ \\
\hline \multirow{5}{*}{$\begin{array}{l}\text { WM } \\
\text { (neutral) }\end{array}$} & \multirow[t]{5}{*}{ Highway } & Particulate & $75 \cdot 09 \cdot 2$ & Dichloromethane & $3.22 \mathrm{E}-02$ & 85 & $1.12 \mathrm{E}-01$ & 60 & $7.52 \mathrm{E}-04$ & 3 & $8.57 E-01$ & $8.78 \mathrm{E}-0$ \\
\hline & & (severity & Cl-3.x & 1,1,1-Trichloroethane & $1.54 \mathrm{E}-01$ & 133.4 & $8.39 \mathrm{E}-01$ & 60 & $5.65 \mathrm{E}-03$ & 1 & $2.86 \mathrm{E}-01$ & $1.98 \mathrm{E}-0$ \\
\hline & & Categories & Cl.F-x & Freon 113 & $5.82 \mathrm{E}-03$ & 187 & $4.44 \mathrm{E}-02$ & 60 & $2.99 \mathrm{E}-04$ & 30 & $8.57 E+00$ & $3.49 \mathrm{E}-0$ \\
\hline & & IV-VIII) & HC-soluble* & Toluene & $2.76 \mathrm{E}-01$ & 92.14 & $1.04 E+00$ & 60 & $6.99 \mathrm{E}-03$ & 0.4 & $1.14 E-01$ & $6.12 \mathrm{E}-0$ \\
\hline & & & & Subtotal & $4.68 E-01$ & $\stackrel{f}{ }$ & $2.03 E+00$ & . & & & & $8,18 E-0$ \\
\hline \multirow{4}{*}{$\begin{array}{l}\text { WM } \\
\text { (stable) }\end{array}$} & \multirow[t]{5}{*}{ Highway } & Particulate & $75-09-2$ & Dichloromethane & $\overline{8.66 \mathrm{E}-02}$ & 85 & $\overline{3.00 E-01}$ & 60 & $2.02 \mathrm{E}-03$ & 3 & $8.57 \mathrm{E}-01$ & $2.36 \mathrm{E}-0$ \\
\hline & & (severity & Cl-3.x & 1,1,1-Trichloroethane & $3.86 \mathrm{E}-01$ & 133.4 & $2.10 \mathrm{E}+00$ & 60 & $1.42 \mathrm{E}-02$ & 1 & $2.86 \mathrm{E}-01$ & $4.95 \mathrm{E}-0$ \\
\hline & & Categories & Cl-F-x & Froon 113 & $1.54 \mathrm{E}-02$ & 187 & $1.18 \mathrm{E}-01$ & 60 & $7.92 \mathrm{E}-04$ & 30 & $8.57 \mathrm{E}+00$ & $9.24 \mathrm{E}-0$ \\
\hline & & [V-vili) & HC-soluble & $\begin{array}{l}\text { Toluene } \\
\text { Subtotal }\end{array}$ & $7.83 \mathrm{E}-01$ & 92.14 & $2.94 \mathrm{E}+00$ & 60 & $1.98 E-02$ & 0.4 & $1.14 \mathrm{E}-01$ & $1.74 E-0$ \\
\hline \multirow{5}{*}{$\begin{array}{l}\text { WM } \\
\text { (neutral) }\end{array}$} & & Particulate & $75 \cdot 09 \cdot 2$ & $\frac{\text { Subtotal }}{\text { Dichloromethane }}$ & $\frac{1.27 E+00}{3.22 E-02}$ & 85 & $\frac{5.46 E+00}{1.12 E-01}$ & . 60 & $7.52 \mathrm{E}-04$ & 3 & $8.57 E-01$ & $\frac{2.26 E-0}{8.78 E-0}$ \\
\hline & \multirow{4}{*}{ Railroad } & (Boverity & Cl-3-x & 1,1,1-Trichloroethane & $1.54 \mathrm{E}-01$ & 133.4 & $8.39 \mathrm{E}-01$ & 60 & $5.65 \mathrm{E}-03$ & 1 & $2.86 \mathrm{E}-01$ & $1.98 \mathrm{E}-0$ \\
\hline & & Categories & Cl-F.x & Freon 113 & $5.82 \mathrm{E}-03$ & 187 & $4.44 \mathrm{E}-02$ & 60 & $2.99 \mathrm{E}-04$ & 30 & $8.57 E+00$ & $3.49 \mathrm{E}-0$ \\
\hline & & IV-VIII) & HC-soluble & Toluene & $2.76 \mathrm{E}-01$ & 92.14 & $1.04 \mathrm{E}+00$ & 60 & $6.99 \mathrm{E}-03$ & 0.4 & $1.14 \mathrm{E}-01$ & $6.12 \mathrm{E}-0$ \\
\hline & & & & Subtotal & $4.68 E-01$ & & $2.03 E+00$ & & & & & $8.18 E-0$ \\
\hline \multirow{4}{*}{$\begin{array}{l}\text { WM } \\
\text { (stable) }\end{array}$} & \multirow{4}{*}{ Railroad } & Particulate & $75-09-2$ & $\overline{\text { Dichloromethane }}$ & $\overline{8,66 \bar{E}-02}$ & 85 & $\overline{3.00 \mathrm{E}-01}$ & 60 & $2.02 \mathrm{E}-03$ & 3 & $8.57 \mathrm{E}-01$ & $2.36 \mathrm{E}-0$ \\
\hline & & (severity & Cl-3.3 & 1,1,1-Trichloroethane & $3.86 \mathrm{E}-01$ & 133.4 & $2.10 E+00$ & 60 & $1.42 \mathrm{E}-02$ & 1 & $2.86 \mathrm{E}-01$ & $4.95 \mathrm{E}-0$ \\
\hline & & Categories & Cl-F-x & Freon 113 & $1.54 E-02$ & 187 & $1.18 E-01$ & 60 & $7.92 \mathrm{E}-04$ & 30 & $8.57 \mathrm{E}+00$ & $9.24 E-0$ \\
\hline & & IV.VIII) & HC-soluble & $\begin{array}{l}\text { Toluene } \\
\text { Subtotal }\end{array}$ & $\begin{array}{l}7.83 E-01 \\
127 E+00\end{array}$ & 92.14 & $\begin{array}{l}2.94 E+00 \\
546 E+00\end{array}$ & 60 & $1.98 \mathrm{E}-02$ & 0.4 & $1.14 \mathrm{E}-01$ & $1.74 \mathrm{E}-0$ \\
\hline
\end{tabular}

A Neutral atmospheric conditions were based on Pasquill stability Class D with a wind speed of $4 \mathrm{~m} / \mathrm{s}(9 \mathrm{mph})$. PAE $=$ potential adverse effect.

b Stable atmospheric conditions were based on Pasquill stabjility Class F with a wind speed of $1 \mathrm{~m} / \mathrm{s}(2.2 \mathrm{mph})$.

c $\mathrm{RTC}=$ reference concentration.

d $\mathrm{Rm}=$ reference dose

- $\mathrm{HC}=$ hydrocarbon

c Not applicable. 
TABLE B.5 Comparisons of Plume Footprint/Impact Areas between Worst Radiological and Worst Hazardous-Chemical Accident Scenarios

\begin{tabular}{|c|c|c|c|c|c|c|c|c|c|c|c|c|c|c|c|c|}
\hline \multirow[b]{3}{*}{$\begin{array}{c}\text { LLMW } \\
\text { Typo }\end{array}$} & \multirow[b]{3}{*}{$\begin{array}{c}\text { Transpor- } \\
\text { tation Mode }\end{array}$} & \multirow[b]{3}{*}{ Case } & \multirow[b]{3}{*}{$\begin{array}{c}\text { Origin } \\
\text { Site } \\
\end{array}$} & \multirow[b]{3}{*}{$\begin{array}{c}\text { Treatment } \\
\text { Site }\end{array}$} & \multirow[b]{3}{*}{ State } & \multirow{3}{*}{$\begin{array}{c}\text { Waste } \\
\text { Quantity } \\
\text { (kg/yr) }\end{array}$} & \multirow{3}{*}{$\begin{array}{c}\text { No. of } \\
\text { Ship- } \\
\text { ments }\end{array}$} & \multirow{3}{*}{$\begin{array}{c}\text { Total } \\
\text { Mass per } \\
\text { Shipment } \\
\text { (kg) } \\
\end{array}$} & \multirow{2}{*}{\multicolumn{4}{|c|}{ Chemical Mass per Shipment $(\mathrm{kg})$}} & \multicolumn{4}{|c|}{ Plume Footprint Exposure Area } \\
\hline & & & & & & & & & & & & & \multicolumn{2}{|c|}{ Neutral Conditions ${ }^{4}$} & \multicolumn{2}{|c|}{ Stable Conditions ${ }^{b}$} \\
\hline & & & & & & & & & $75 \cdot 09 \cdot 2$ & Cl-2-x & Cl-4.x & $\begin{array}{c}\text { HC. } \\
\text { solublea }\end{array}$ & $\begin{array}{l}\text { Length } \\
\text { (yd) }\end{array}$ & $\begin{array}{c}\text { Area } \\
\left(\mathbf{n}^{2}\right)\end{array}$ & $\begin{array}{l}\text { Length } \\
\text { (yd) }\end{array}$ & $\begin{array}{l}\text { Area } \\
\left(\Omega^{2}\right)\end{array}$ \\
\hline \multicolumn{17}{|c|}{ Transportation Routes for Estimating Potential Increased Cancer Risks to Population } \\
\hline \multicolumn{17}{|c|}{ Worst radiological accident consequences ${ }^{c}$} \\
\hline wM & Highway & $7,10,15,17$ & PGDP & $\begin{array}{c}\text { ORR, } \\
\text { Hanford }\end{array}$ & Solid & 6,808 & 1 & 6,808 & $1.7 \mathrm{E}+00$ & $1.3 \mathrm{E}+01$ & $6.7 \mathrm{E}-01$ & $1.7 \mathrm{E}+01$ & $<33$ & 0 & $<33$ & 0 \\
\hline wM & Railroad & $7,10,15,17$ & PGDP & $\begin{array}{l}\text { ORR, } \\
\text { Hanford }\end{array}$ & Solid & 6,808 & 1 & 6,808 & $1.7 \mathrm{E}+00$ & $1.3 \mathrm{E}+01$ & $6.7 \mathrm{E}-01$ & $1.7 \mathrm{E}+01$ & $<33$ & 0 & $<33$ & 0 \\
\hline \multicolumn{17}{|c|}{ Worst hazardous-chemical accident consequences ${ }^{d}$} \\
\hline wM & Highway & 15,17 & PORTS & $\begin{array}{c}\text { ORR, } \\
\text { Hanford }\end{array}$ & Liquid & 47,788 & 3 & 15,929 & $3.4 \mathrm{E}-01$ & $9.6 \mathrm{E}-01$ & $2.6 \mathrm{E}+00$ & $4.8 \mathrm{E}+03$ & 268 & 83,400 & 518 & 257,600 \\
\hline WM & Railroad & 15,17 & PORTS & $\begin{array}{l}\text { ORR, } \\
\text { Hanford }\end{array}$ & Liquid & 47,788 & 2 & 23,894 & $5.1 \mathrm{E}-01$ & $1.4 \mathrm{E}+00$ & $3.9 \mathrm{E}+00$ & $7.2 \mathrm{E}+03$ & 393 & 120,190 & 630 & 364,100 \\
\hline \multicolumn{17}{|c|}{ Transportation Routes for Estimating Potential Adverse Effects to Population } \\
\hline \multicolumn{17}{|c|}{ Worst radiological accident consequences ${ }^{e}$} \\
\hline WM & Highway & $7,10,15,17$ & PGDP & $\begin{array}{c}\text { ORR, } \\
\text { Hanford }\end{array}$ & Solid & 6,808 & 1 & 6,808 & $1.7 \mathrm{E}+00$ & $1.3 \mathrm{E}+01$ & 6.7E-01 & $1.7 \mathrm{E}+01$ & $<33$ & 0 & $<33$ & 0 \\
\hline WM & Railroad & $7,10,15,17$ & PGDP & $\begin{array}{c}\text { ORR, } \\
\text { Hanford }\end{array}$ & Solid & 6,808 & 1 & 6,808 & $1.7 \mathrm{E}+00$ & $1.3 \mathrm{E}+01$ & $6.7 \mathrm{E}-01$ & $1.7 \mathrm{E}+01$ & $<33$ & 0 & $<33$ & 0 \\
\hline \multicolumn{17}{|c|}{ Worst hazardous-chemical accident consequences ${ }^{\mathrm{d}}$} \\
\hline wм & Highway & 15,17 & PORTS & $\begin{array}{c}\text { ORR, } \\
\text { Hanford }\end{array}$ & Liquid & 47,788 & 3 & 15,929 & $3,4 \mathrm{E}-01$ & $4.6 \mathrm{E}+01$ & $7.8 \mathrm{E}-01$ & $4.8 \mathrm{E}+03$ & 596 & 329,960 & 1,366 & $1,494,100$ \\
\hline wM & Railroad & $7,10,15,17$ & LLNL & Hanford & Liquid & 81,697 & 2 & 40,799 & $2.7 \mathrm{E}+00$ & $3.0 \mathrm{E}+02$ & $6.3 \mathrm{E}+00$ & $7.2 \mathrm{E}+03$ & 739 & 484,550 & 1,675 & $2,188,300$ \\
\hline
\end{tabular}

a $\mathrm{HC}=$ hydrocarbon.

b Neutral conditions were based on Pasquill stability Class D with a wind speed of $4 \mathrm{~m} / \mathrm{s}(9 \mathrm{mph})$, and stable conditions were based on Pasquill stability Class F with a wind speed of $1 \mathrm{~m} / \mathrm{s}$ (2.2 $\mathrm{mph}$ ).

- Worst radiological accident consequences ara caused by solid WM LLMW originating from PGDP.

d Worst hazardeus-chemical accident eonsequences are caused by liquid aerosol releases for WM cases. 


\section{B.1 WM LLMW DECENTRALIZED ALTERNATIVE (CASE 2): CONTACT-HANDLED ORGANIC LIQUIDS: ALPHA - UNTREATED}

Table 1. Summary of Route Information

\begin{tabular}{|c|c|c|c|c|c|}
\hline & \multirow[b]{2}{*}{ Route } & \multirow{2}{*}{$\begin{array}{c}\text { Distance } \\
\text { (miles) }\end{array}$} & \multicolumn{3}{|c|}{ Percentage in Zone } \\
\hline & & & Rural & Suburban & Urban \\
\hline \multicolumn{6}{|l|}{ Truck } \\
\hline Mound Plant & to Savannah River & 656 & 66.5 & 32.7 & 0.8 \\
\hline $\begin{array}{l}\text { West Valley DP } \\
\text { Rail }\end{array}$ & to Savannah River & 883 & 70.3 & 28.5 & 1.2 \\
\hline Mound Plant & to Savannah River & 744 & 67.4 & 29.2 & 3.3 \\
\hline West Valley DP & to Savannah River & 1217 & 62.8 & 32.4 & 4.9 \\
\hline
\end{tabular}

Table 2. Annual Number of Shipments and One-Way Shipment Distances

\begin{tabular}{|c|c|c|c|c|c|}
\hline \multirow[b]{2}{*}{ Route } & & \multicolumn{2}{|c|}{ \# of Shipments } & \multicolumn{2}{|c|}{ Total Mileage (mi) } \\
\hline & & Truck & Rail & Truck & $\operatorname{Rail}$ \\
\hline $\begin{array}{l}\text { Mound Plant } \\
\text { West valley DP }\end{array}$ & $\begin{array}{l}\text { to Savannah River } \\
\text { to Savannah River }\end{array}$ & $\begin{array}{l}1 \\
1\end{array}$ & $\begin{array}{l}1 \\
1\end{array}$ & $\begin{array}{l}6.56 \mathrm{E}+02 \\
8.83 \mathrm{E}+02\end{array}$ & $\begin{array}{l}7.44 \mathrm{E}+02 \\
1.22 \mathrm{E}+03\end{array}$ \\
\hline Totals & & 2 & 2 & $1.54 \mathrm{E}+03$ & $1.96 \mathrm{E}+03$ \\
\hline
\end{tabular}

Table 3. Incident-Free Dose per Shipment (person-rem/shipment)

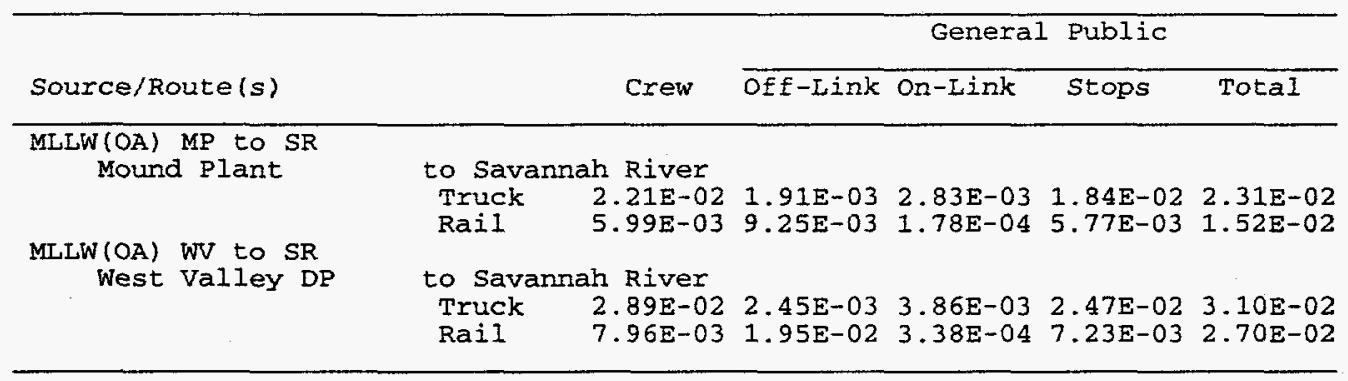

Table 4. Accident Dose Risk per Shipment (person-rem/shipment)

\begin{tabular}{llll}
\hline Source/Route & & Truck & Rail \\
\hline $\begin{array}{c}\text { MLIW(OA) MP to SR } \\
\text { Mound Plant }\end{array}$ & to Savannah River & $4.15 \mathrm{E}-03$ & $7.63 \mathrm{E}-04$ \\
$\begin{array}{c}\text { MLIW(OA) WV to SR } \\
\text { West Valley DP }\end{array}$ & to Savannah River & $4.27 \mathrm{E}-02$ & $1.37 \mathrm{E}-02$ \\
\hline
\end{tabular}

Table 5. Nonradiological Risk Factors per Shipment (fatalities/shipment)

\begin{tabular}{|c|c|c|c|}
\hline Mode & Route & Emission & Accident \\
\hline Truck & & & \\
\hline $\begin{array}{l}\text { Mound Plant } \\
\text { West Valley DP }\end{array}$ & $\begin{array}{l}\text { to Savannah River } \\
\text { to Savannah River }\end{array}$ & $\begin{array}{l}1.67 E-06 \\
3.28 E-06\end{array}$ & $\begin{array}{l}5.26 \mathrm{E}-05 \\
6.60 \mathrm{E}-05\end{array}$ \\
\hline $\begin{array}{l}\text { Rail } \\
\text { Mound Plant } \\
\text { West Valley DP }\end{array}$ & $\begin{array}{l}\text { to Savannah River } \\
\text { to Savannah River }\end{array}$ & $\begin{array}{l}1.03 E-05 \\
2.49 \mathrm{E}-05\end{array}$ & $\begin{array}{l}1.56 \mathrm{E}-06 \\
2.55 \mathrm{E}-06\end{array}$ \\
\hline
\end{tabular}




\section{B-10}

Table 6. Annual Incident-Free Dose for the Shipping Campaign (person-rem/yr)

\begin{tabular}{llcccc}
\hline \multirow{2}{*}{ Route } & & \multicolumn{2}{c}{ Truck } & & Rail \\
\cline { 5 - 6 } & & Crew & Public & Crew & Public \\
\hline Mound Plant & to Savannah River & $2.21 \mathrm{E}-02$ & $2.31 \mathrm{E}-02$ & $5.99 \mathrm{E}-03$ & $1.52 \mathrm{E}-02$ \\
West Valley DP & to Savannah River & $2.89 \mathrm{E}-02$ & $3.10 \mathrm{E}-02$ & $7.96 \mathrm{E}-03$ & $2.70 \mathrm{E}-02$ \\
\hline Totals & & $5.09 \mathrm{E}-02$ & $5.41 \mathrm{E}-02$ & $1.39 \mathrm{E}-02$ & $4.22 \mathrm{E}-02$
\end{tabular}

Table 7. Annual Accident Dose Risk for the Shipping Campaign (pexson-rem/yr)

\begin{tabular}{llcc}
\hline Route & & Truck & Rail \\
\hline Mound Plant & to Savannah River & $4.15 \mathrm{E}-03$ & $7.63 \mathrm{E}-04$ \\
West Valley DP & to Savannah River & $4.27 \mathrm{E}-02$ & $1.37 \mathrm{E}-02$ \\
\hline Totals & & $4.68 \mathrm{E}-02$ & $1.45 \mathrm{E}-02$ \\
\hline
\end{tabular}

Table 8. Expected Annual Fatalities for the Shipping Campaign

\begin{tabular}{llc}
\hline Exposure Group & Truck & Rail \\
\hline Radiological & & \\
Normal Crew & $2.0 E-05$ & $5.6 E-06$ \\
Normal Public & $2.7 E-05$ & $2.1 E-05$ \\
Accident Public & $2.3 E-05$ & $7.2 E-06$ \\
Nonradiological & & \\
Emission & $5.0 E-06$ & $3.5 E-05$ \\
Accident & $1.2 E-04$ & $4.1 E-06$ \\
\hline
\end{tabular}

Table 9. Expected Annual Cancer Incidence for the Shipping Campaign

\begin{tabular}{lll}
\hline Exposure Group & Truck & Rail \\
\hline Radiological & & \\
Normal Crew & $7.1 \mathrm{E}-05$ & $2.0 \mathrm{E}-05$ \\
Normal Public & $9.2 \mathrm{E}-05$ & $7.2 \mathrm{E}-05$ \\
Accident Public & $8.0 \mathrm{E}-05$ & $2.5 \mathrm{E}-05$ \\
Nonradiological & $5.0 \mathrm{E}-06$ & $3.5 \mathrm{E}-05$ \\
Emission & NA & NA \\
Accident & & \\
\hline
\end{tabular}

Table 10. Expected Annual Genetic Effects for the Shipping Campaign

\begin{tabular}{cll}
\hline Exposure Group & Truck & Rail \\
\hline Radiological & $3.1 \mathrm{E}-06$ & $8.4 \mathrm{E}-07$ \\
Normal Crew & $5.4 \mathrm{E}-06$ & $4.2 \mathrm{E}-06$ \\
Normal Public & $1.4 \mathrm{E}-06$ & $4.4 \mathrm{E}-07$ \\
Accident Public & & \\
Nonradiological & NA & NA \\
Emission & NA & NA \\
Accident & & \\
\hline
\end{tabular}




\section{B.2 WM LLMW DECENTRALIZED ALTERNATIVE (CASE 2): CONTACT-HANDLED HETEROGENEOUS SOLIDS: ALPHA - UNTREATED}

Table 1. Summary of Route Information

\begin{tabular}{|c|c|c|c|c|c|c|}
\hline & \multirow{2}{*}{\multicolumn{2}{|c|}{ Route }} & \multirow{2}{*}{$\begin{array}{l}\text { Distance } \\
\text { (miles) }\end{array}$} & \multicolumn{3}{|c|}{ Percentage in Zone } \\
\hline & & & & Rural & Suburban & Urban \\
\hline \multicolumn{7}{|l|}{ Truck } \\
\hline Bettis Atomic & to Savannah & River & 656 & 71.7 & 26.4 & 1.9 \\
\hline Mound Plant & to Savannah & River & 656 & 66.5 & 32.7 & 0.8 \\
\hline U of MO - Columbia & to Savannah & River & 858 & 70.6 & 27.0 & 2.3 \\
\hline West Valley DP & to Savannah & River & 883 & 70.3 & 28.5 & 1.2 \\
\hline \multicolumn{7}{|l|}{ Rail } \\
\hline Bettis Atomic & to Savannah & River & 947 & 68.5 & 27.1 & 4.4 \\
\hline Mound Plant & to Savannah & River & 744 & 67.4 & 29.2 & 3.3 \\
\hline U of MO - Columbia & to Savannah & River & 1011 & 66.6 & 29.5 & 4.0 \\
\hline West Valley DP & to Savannah & River & 1217 & 62.8 & 32.4 & 4.9 \\
\hline
\end{tabular}

Table 2. Annual Number of Shipments and One-Way Shipment Distances

\begin{tabular}{|c|c|c|c|c|c|}
\hline \multirow[b]{2}{*}{ Route } & & \multicolumn{2}{|c|}{ \# of Shipments } & \multicolumn{2}{|c|}{ Total Mileage (mi) } \\
\hline & & Truck & Rail & Truck & Rail \\
\hline $\begin{array}{l}\text { Bettis Atomic } \\
\text { Mound Plant } \\
U \text { of MO - Columbia } \\
\text { West Valley DP }\end{array}$ & $\begin{array}{l}\text { to Savannah River } \\
\text { to Savannah River } \\
\text { to Savannah River } \\
\text { to Savannah River }\end{array}$ & $\begin{array}{l}1 \\
1 \\
1 \\
1\end{array}$ & $\begin{array}{l}1 \\
1 \\
1 \\
1\end{array}$ & $\begin{array}{l}6.56 \mathrm{E}+02 \\
6.56 \mathrm{E}+02 \\
8.58 \mathrm{E}+02 \\
8.83 \mathrm{E}+02\end{array}$ & $\begin{array}{l}9.47 \mathrm{E}+02 \\
7.44 \mathrm{E}+02 \\
1.01 \mathrm{E}+03 \\
1.22 \mathrm{E}+03\end{array}$ \\
\hline Totals & & 4 & 4 & $3.05 E+03$ & $3.92 \mathrm{E}+03$ \\
\hline
\end{tabular}

Table 3. Incident-Free Dose per Shipment (person-rem/shipment)

\begin{tabular}{|c|c|c|c|c|c|}
\hline \multirow[b]{2}{*}{ Source/Route (s) } & \multirow[b]{2}{*}{ Crew } & \multicolumn{4}{|c|}{ General Public } \\
\hline & & off-Link & On-Link & Stops & Total \\
\hline \multirow[t]{2}{*}{$\begin{array}{r}\text { MLLW(CA) BA to SR } \\
\text { Bettis Atomic }\end{array}$} & to Savannah River & & & & \\
\hline & $\begin{array}{ll}\text { Truck } & 2.14 \mathrm{E}-02 \\
\text { Rail } & 6.83 \mathrm{E}-03\end{array}$ & $\begin{array}{l}1.96 \mathrm{E}-03 \\
1.32 \mathrm{E}-02\end{array}$ & $\begin{array}{l}3.14 \mathrm{E}-03 \\
2.31 \mathrm{E}-04\end{array}$ & $\begin{array}{l}1.84 \mathrm{E}-02 \\
6.39 \mathrm{E}-03\end{array}$ & $\begin{array}{l}2.35 \mathrm{E}-02 \\
1.99 \mathrm{E}-02\end{array}$ \\
\hline \multirow[t]{2}{*}{$\begin{array}{l}\text { MLLW(CA) MP to SR } \\
\text { Mound Plant }\end{array}$} & to Savannah River & & & & \\
\hline & $\begin{array}{ll}\text { Truck } & 2.21 \mathrm{E}-02 \\
\text { Rail } & 5.99 \mathrm{E}-03\end{array}$ & $\begin{array}{l}1.91 E-03 \\
9.25 E-03\end{array}$ & $\begin{array}{l}2.83 \mathrm{E}-03 \\
1.78 \mathrm{E}-04\end{array}$ & $\begin{array}{l}1.84 E-02 \\
5.77 E-03\end{array}$ & $\begin{array}{l}2.31 \mathrm{E}-02 \\
1.52 \mathrm{E}-02\end{array}$ \\
\hline \multirow[t]{2}{*}{$\begin{aligned} & M L L W(C A) \text { MC to SR } \\
& \text { U of MO - Columbia }\end{aligned}$} & to Savannah River & & & & \\
\hline & $\begin{array}{ll}\text { Truck } & 2.83 \mathrm{E}-02 \\
\text { Rail } & 7.10 \mathrm{E}-03\end{array}$ & $\begin{array}{l}2.79 E-03 \\
1.39 E-02\end{array}$ & $\begin{array}{l}4.37 E-03 \\
2.52 E-04\end{array}$ & $\begin{array}{l}2.40 \mathrm{E}-02 \\
6.59 \mathrm{E}-03\end{array}$ & $\begin{array}{l}3.12 \mathrm{E}-02 \\
2.07 \mathrm{E}-02\end{array}$ \\
\hline $\begin{array}{l}\text { MLLW (CA) WV to SR } \\
\text { West Valley DP }\end{array}$ & $\begin{array}{lr}\text { to Savannah River } \\
\text { Truck } & 2.89 \mathrm{E}-02 \\
\text { Rail } & 7.96 \mathrm{E}-03\end{array}$ & $\begin{array}{l}2.45 E-03 \\
1.95 E-02\end{array}$ & $\begin{array}{l}3.86 \mathrm{E}-03 \\
3.38 \mathrm{E}-04\end{array}$ & $\begin{array}{l}2.47 E-02 \\
7.23 E-03\end{array}$ & $\begin{array}{l}3.10 \mathrm{E}-02 \\
2.70 \mathrm{E}-02\end{array}$ \\
\hline
\end{tabular}

Table 4. Accident Dose Risk per Shipment (person-rem/shipment)

\begin{tabular}{llll}
\hline Source/Route & & Truck & Rail \\
\hline $\begin{array}{l}\text { MLLW(CA) BA to SR } \\
\text { Bettis Atomic }\end{array}$ & to Savannah River & $2.11 \mathrm{E}-04$ & $7.45 \mathrm{E}-05$ \\
$\begin{array}{c}\text { MLLW (CA) MP to SR } \\
\text { Mound Plant }\end{array}$ & to Savannah River & $9.48 \mathrm{E}-08$ & $1.85 \mathrm{E}-08$ \\
$\begin{array}{c}\text { MLLW(CA) MC to SR } \\
\text { U of MO - Columbia to Savannah River }\end{array}$ & $6.78 \mathrm{E}-07$ & $2.05 \mathrm{E}-07$ \\
$\begin{array}{c}\text { MLLW (CA) WV to SR } \\
\text { West Valley DP }\end{array}$ & to Savannah River & $3.36 \mathrm{E}-04$ & $1.07 \mathrm{E}-04$ \\
\hline
\end{tabular}


Table 5. Nonradiological Risk Factors per Shipment (fatalities/shipment)

\begin{tabular}{llll}
\hline Mode & Route & Emission & Accident \\
\hline Truck & & & \\
Bettis Atomic & to Savannah River & $3.99 \mathrm{E}-06$ & $4.90 \mathrm{E}-05$ \\
Mound Plant & to Savannah River & $1.67 \mathrm{E}-06$ & $5.26 \mathrm{E}-05$ \\
U of MO - Columbia to Savannah River & $6.41 \mathrm{E}-06$ & $6.09 \mathrm{E}-05$ \\
$\begin{array}{l}\text { West Valley DP } \\
\text { Rail to Savannah River }\end{array}$ & $3.28 \mathrm{E}-06$ & $6.60 \mathrm{E}-05$ \\
Bettis Atomic & to Savannah River & $1.74 \mathrm{E}-05$ & $1.98 \mathrm{E}-06$ \\
Mound Plant & to Savannah River & $1.03 \mathrm{E}-05$ & $1.56 \mathrm{E}-06$ \\
U of MO - Columbia to Savannah River & $1.69 \mathrm{E}-05$ & $2.12 \mathrm{E}-06$ \\
West Valley DP & to Savannah River & $2.49 \mathrm{E}-05$ & $2.55 \mathrm{E}-06$ \\
\hline
\end{tabular}

Table 6. Annual Incident-Free Dose for the Shipping Campaign (person-rem/yr)

\begin{tabular}{|c|c|c|c|c|c|c|}
\hline \multirow[b]{2}{*}{ Route } & & & \multicolumn{2}{|c|}{ Truck } & \multicolumn{2}{|c|}{ Rai1 } \\
\hline & & & Crew & Public & Crew & Public \\
\hline $\begin{array}{l}\text { Bettis Atomic } \\
\text { Mound Plant } \\
\text { U of MO - Columbia } \\
\text { West Valley DP }\end{array}$ & $\begin{array}{l}\text { to } \\
\text { to } \\
\text { to } \\
\text { to }\end{array}$ & $\begin{array}{l}\text { Savannah River } \\
\text { Savannah River } \\
\text { Savannah River } \\
\text { Savannah River }\end{array}$ & $\begin{array}{l}2.14 E-02 \\
2.21 E-02 \\
2.83 E-02 \\
2.89 E-02\end{array}$ & $\begin{array}{l}2.35 \mathrm{E}-02 \\
2.31 \mathrm{E}-02 \\
3.12 \mathrm{E}-02 \\
3.10 \mathrm{E}-02\end{array}$ & $\begin{array}{l}6.83 E-03 \\
5.99 E-03 \\
7.10 E-03 \\
7.96 E-03\end{array}$ & $\begin{array}{l}1.99 E-02 \\
1.52 E-02 \\
2.07 E-02 \\
2.70 E-02\end{array}$ \\
\hline Totals & & & $1.01 \mathrm{E}-01$ & $1.09 E-01$ & $2.79 E-02$ & $8.28 \mathrm{E}-02$ \\
\hline
\end{tabular}

Table 7. Annual Accident Dose Risk for the Shipping Campaign (person-rem/yr)

\begin{tabular}{llll}
\hline Route & & Truck & Rail \\
\hline Bettis Atomic & to Savannah River & $2.11 \mathrm{E}-04$ & $7.45 \mathrm{E}-05$ \\
Mound Plant & to Savannah River & $9.48 \mathrm{E}-08$ & $1.85 \mathrm{E}-08$ \\
U of MO - Columbia to Savannah River & $6.78 \mathrm{E}-07$ & $2.05 \mathrm{E}-07$ \\
West Valley DP & to Savannah River & $3.36 \mathrm{E}-04$ & $1.07 \mathrm{E}-04$ \\
\hline Totals & & $5.47 \mathrm{E}-04$ & $1.82 \mathrm{E}-04$ \\
\hline
\end{tabular}

Table 8. Expected Annual Fatalities for the Shipping Campaign

\begin{tabular}{ccc}
\hline Exposure Group & Truck & Rail \\
\hline Radiological & & \\
Normal Crew & $4.0 \mathrm{E}-05$ & $1.1 \mathrm{E}-05$ \\
Normal Public & $5.4 \mathrm{E}-05$ & $4.1 \mathrm{E}-05$ \\
Accident Public & $2.7 \mathrm{E}-07$ & $9.1 \mathrm{E}-08$ \\
Nonradiological & & \\
Emission & $1.5 \mathrm{E}-05$ & $6.9 \mathrm{E}-05$ \\
Accident & $2.3 \mathrm{E}-04$ & $8.2 \mathrm{E}-06$ \\
\hline
\end{tabular}

Table 9. Expected Annual Cancer Incidence for the Shipping Campaign

\begin{tabular}{lll}
\hline Exposure Group & Truck & Rail \\
\hline Radiological & & \\
Normal Crew & $1.4 E-04$ & $3.9 E-05$ \\
Normal Public & $1.8 E-04$ & $1.4 E-04$ \\
Accident Public & $9.3 E-07$ & $3.1 \mathrm{E}-07$ \\
Nonradiological & $1.5 \mathrm{E}-05$ & $6.9 \mathrm{E}-05$ \\
Emission & $\mathrm{NA}$ & $\mathrm{NA}$ \\
Accident & & \\
\hline
\end{tabular}




\section{$B-13$}

Table 10. Expected Annual Genetic Effects

for the Shipping Campaign

\begin{tabular}{lll}
\hline Exposure Group & Truck & Rail \\
\hline Radiological & & \\
Normal Crew & $6.0 \mathrm{E}-06$ & $1.7 \mathrm{E}-06$ \\
Normal Public & $1.1 \mathrm{E}-05$ & $8.3 \mathrm{E}-06$ \\
Accident Public & $4.1 \mathrm{E}-08$ & $1.4 \mathrm{E}-08$ \\
Nonradiological & NA & NA \\
Emission & NA & NA \\
Accident & &
\end{tabular}




\section{B.3 WM LLMW DECENTRALIZED ALTERNATIVE (CASE 2): CONTACT-HANDLED HETEROGENEOUS SOLIDS: NONALPHA — TREATED}

Table 1. Summary of Route Information

\begin{tabular}{|c|c|c|c|c|c|c|}
\hline & & & & Perc & entage in & Zone \\
\hline & Rot & & (miles) & Rural & Suburban & Urban \\
\hline Truck & & & & & & \\
\hline Ames Laboratory & & Argonne - East & 351 & 85.3 & 14.4 & 0.3 \\
\hline Bettis Atomic & & Portsmouth GDP & 265 & 58.8 & 37.4 & 3.8 \\
\hline Battelle columbus & & Portsmouth GDP & 84 & 68.4 & 28.3 & 3.3 \\
\hline Charleston, SC & & Savannah River & 200 & 75.7 & 23.8 & 0.5 \\
\hline Colonie & & Portsmouth GDP & 588 & 57.3 & 40.1 & 2.5 \\
\hline ETEC & & Lawrence Livermore & 406 & 77.9 & 10.0 & 12.1 \\
\hline General Atomic & 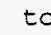 & Lawrence Livermore & 500 & 69.2 & 15.1 & 15.6 \\
\hline Grand Junction PO & to & Rocky Flats Plant & 273 & 79.9 & 16.7 & 3.3 \\
\hline Knolis Atomic & to $-(-1) \cdot(-1)$ & Portsmouth GDP & 688 & 57.3 & 40.1 & 2.5 \\
\hline Kansas City Plant & 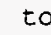 & Paducah GDP & 441 & 77.5 & 19.6 & 2.9 \\
\hline $\mathrm{kKS}$ & & Portsmouth GDP & 688 & 57.3 & 40.1 & 2.5 \\
\hline KWS & to & Portsmouth GDP & 688 & 57.3 & 40.1 & 2.5 \\
\hline Lawrence Berkeley & to & Lawrence Livermore & 45 & 38.4 & 33.3 & 28.2 \\
\hline LERHR & & Lawrence Livermore & 97 & 58.7 & 24.7 & 16.6 \\
\hline Mare Island & & Lawrence Livermore & 45 & 38.4 & 33.3 & 28.2 \\
\hline Norfolk Nav Shipyd & & Savannah River & 498 & 72.8 & 26.2 & 1.0 \\
\hline Pearl Harbor NS & to & Hanford Site & 226 & 76.8 & 20.9 & 2.3 \\
\hline Portsmouth NS & & Portsmouth GDP & 959 & 46.4 & 45.3 & 8.2 \\
\hline Princeton PPL & & Portsmouth GDP & 588 & 55.1 & 38.8 & 6.1 \\
\hline Puget Sound NS & to & Hanford Site & 226 & 76.8 & 20.9 & $2 . \overline{3}$ \\
\hline Reactive Metals & to & Portsmouth GDP & 276 & 59.5 & 37.8 & 2.8 \\
\hline Rail & & & & & & \\
\hline Ames Laboratory & & Argonne - East & 328 & 71.7 & 21.4 & 6.9 \\
\hline Bettis Atomic & & Portsmouth GDP & 429 & 52.6 & 39.6 & 7.8 \\
\hline Battelle Columbus & to & Portsmouth GDP & 91 & 63.4 & 31.8 & 4.8 \\
\hline Charleston, SC & & Savannah River & 140 & 83.9 & 13.6 & 2.5 \\
\hline Colonie & & Portsmouth GDP & 681 & 52.1 & 40.3 & 7.5 \\
\hline ETEC & & Lawrence Livermore & 474 & 60.0 & 24.8 & 15.2 \\
\hline General Atomic & & Lawrence Livermore & 579 & 57.1 & 24.9 & 18.0 \\
\hline Grand Junction Po & to & Rocky flats Plant & 239 & 95.5 & 4.3 & 0.3 \\
\hline Knolls Atomic & & Portsmouth GDP & 681 & 52.1 & 40.3 & 7.5 \\
\hline Kansas City Plant & & Paducah GDP & 482 & 75.0 & 20.3 & 4.8 \\
\hline KKS & & Portsmouth GDP & 681 & 52.1 & 40.3 & 7.5 \\
\hline KWS & & Portsmouth GDP & 681 & 52.1 & 40.3 & 7.5 \\
\hline Lawrence Berkeley & to & Lawrence Livermore & 46 & 39.0 & 34.5 & 26.5 \\
\hline LERHR & & Lawrence Livermore & 93 & 45.5 & 31.3 & 23.2 \\
\hline Mare Island & & Lawrence Livermore & 46 & 39.0 & 34.5 & 26.5 \\
\hline Norfolk Nav Shipyd & & Savannah River & 529 & 74.3 & 24.1 & 1.6 \\
\hline Pearl Harbor NS & to & Hanford Site & 416 & 73.7 & 20.1 & 6.2 \\
\hline Portsmouth NS & & Portsmouth GDP & 962 & 49.0 & 44.3 & 6.7 \\
\hline Princeton PPL & & Portsmouth GDP & 838 & 58.3 & 32.6 & 9.1 \\
\hline Puget Sound NS & to & Hanford site & 416 & 73.7 & 20.1 & 6.2 \\
\hline Reactive Metals & & Portsmouth GDP & 279 & 48.4 & 41.8 & 9.8 \\
\hline
\end{tabular}

Table 2. Annual Number of Shipments and Total One-Way Shipment Distances

\begin{tabular}{|c|c|c|c|c|c|c|}
\hline \multirow[b]{2}{*}{ Route } & & & \multicolumn{2}{|c|}{ \# of Shipments } & \multicolumn{2}{|c|}{ Total Mileage (mi) } \\
\hline & & & Truck & Rail & Truck & Rail \\
\hline Ames Laboratory & & Argonne - East & 1 & 1 & $3.51 E+02$ & $3.28 \mathrm{E}+02$ \\
\hline Bettis Atomic & & Portsmouth GDP & 1 & 1 & $2.65 \mathrm{E}+02$ & $4.29 E+02$ \\
\hline Battelle Columbus & & Portsmouth GDP & 1 & 1 & $8.41 E+01$ & $9.09 \mathrm{E}+01$ \\
\hline Charleston, SC & to & Savannah River & 1 & 1 & $2.00 \mathrm{E}+02$ & $1.40 \mathrm{E}+02$ \\
\hline Colonie & to & Portsmouth GDP & 1 & 1 & $6.88 \mathrm{E}+02$ & $6.81 E+02$ \\
\hline ETEC & to & Lawrence Livermore & 11 & 4 & $4.46 \mathrm{E}+03$ & $1.90 \mathrm{E}+03$ \\
\hline General Atomic & to & Lawrence Livermore & 1 & 1 & $5.00 \mathrm{E}+02$ & $5.79 E+02$ \\
\hline Grand Junction PO & to & Rocky Flats Plant & 1 & 1 & $2.73 \mathrm{E}+02$ & $2.39 E+02$ \\
\hline Knolls Atomic & to & Portsmouth GDP & 1 & 1 & $6.88 \mathrm{E}+02$ & $6.81 E+02$ \\
\hline Kansas City Plant & to & Paducah GDP & $\bar{I}$ & $\overline{1}$ & $4.41 E+02$ & $4.82 E+02$ \\
\hline KKS & & Portsmouth GDP & 1 & 1 & $6.88 E+02$ & $6.81 E+02$ \\
\hline KWS & 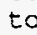 & Portsmouth GDP & $\overline{1}$ & 1 & $6.88 \mathrm{E}+02$ & $6.81 E+02$ \\
\hline Lawrence Berkeley & & Lawrence Iivermore & 1 & 1 & $4.50 E+01$ & $4.61 E+01$ \\
\hline LERHR & to & Lawrence Livermore & 1 & 1 & $9.72 \mathrm{E}+01$ & $9.30 E+01$ \\
\hline
\end{tabular}


Table 2. Annual Number of Shipments and Total One-Way Shipment Distances (Cont.)

\begin{tabular}{|c|c|c|c|c|c|}
\hline \multirow[b]{2}{*}{ Route } & & \multicolumn{2}{|c|}{ \# of Shipments } & \multicolumn{2}{|c|}{ Total Mileage (mi) } \\
\hline & & Truck & Rail & Truck & Rail \\
\hline Mare Island & to Lawrence Livermore & 1 & 1 & $4.50 E+01$ & $4.61 E+01$ \\
\hline Norfolk Nav Shipyd & to Savannah River & $\vec{I}$ & $\overline{1}$ & $4.98 \mathrm{E}+02$ & $5.29 \mathrm{E}+02$ \\
\hline Pearl Harbor NS & to Hanford Site & 1 & 1 & $2.26 \mathrm{E}+02$ & $4.16 \mathrm{E}+02$ \\
\hline Portsmouth NS & to Portsmouth GDP & 1 & $i$ & $9.59 \mathrm{E}+02$ & $9.62 \mathrm{E}+02$ \\
\hline Princeton PPL & to Portsmouth GDP & 1 & 1 & $5.88 \mathrm{E}+02$ & $8.38 \mathrm{E}+02$ \\
\hline Puget sound NS & to Hanford Site & 1 & 1 & $2.26 E+02$ & $4.16 \mathrm{E}+02$ \\
\hline Reactive Metals & to Portsmouth GDP & 1 & 1 & $2.76 \mathrm{E}+02$ & $2.79 E+02$ \\
\hline Totals & & 31 & 24 & $1.23 \mathrm{E}+04$ & $1.05 \mathrm{E}+04$ \\
\hline
\end{tabular}

Table 3. Incident-Free Dose per Shipment (person-rem/shipment)

\begin{tabular}{|c|c|c|c|c|c|}
\hline \multirow[b]{2}{*}{ Source/Route (s) } & \multirow[b]{2}{*}{ Crew } & \multicolumn{4}{|c|}{ General Public } \\
\hline & & off-Link & on-Link & Stops & Total \\
\hline \multicolumn{6}{|l|}{ MLLW(CT) AL to AN } \\
\hline Ames Laboratory & to Argonne - East & & & & \\
\hline & $\begin{array}{ll}\text { Truck } & 9.87 \mathrm{E}-03 \\
\text { Rail } & 4.25 \mathrm{E}-03\end{array}$ & $\begin{array}{l}4.50 \mathrm{E}-04 \\
5.78 \mathrm{E}-03\end{array}$ & $\begin{array}{l}1.11 \mathrm{E}-03 \\
8.30 \mathrm{E}-05\end{array}$ & $\begin{array}{l}9.83 \mathrm{E}-03 \\
4.49 \mathrm{E}-03\end{array}$ & $\begin{array}{l}1.14 \mathrm{E}-02 \\
1.04 \mathrm{E}-02\end{array}$ \\
\hline \multirow{2}{*}{\multicolumn{6}{|c|}{$\begin{array}{l}\text { MLLW(CT) BA to WV } \\
\text { Bettis Atomic }\end{array}$}} \\
\hline & & & & & \\
\hline & $9.78 \mathrm{E}-03$ & $1.26 \mathrm{E}-03$ & $1.73 \mathrm{E}-03$ & $7.42 \mathrm{E}-03$ & $1.04 E-02$ \\
\hline \multirow{2}{*}{\multicolumn{6}{|c|}{$\begin{array}{l}\text { MLLW(CT) BC to PO } \\
\text { Battelle Columbus }\end{array}$}} \\
\hline Battelle Columbus & to Portsmouth GDP & & & & \\
\hline & $\begin{array}{ll}\text { Truck } & 2.86 \mathrm{E}-03 \\
\text { Rail } & 3.26 \mathrm{E}-03\end{array}$ & $\begin{array}{l}3.24 \mathrm{E}-04 \\
1.44 \mathrm{E}-03\end{array}$ & $\begin{array}{l}4.91 \mathrm{E}-04 \\
2.49 \mathrm{E}-05\end{array}$ & $\begin{array}{l}2.35 \mathrm{E}-03 \\
3.76 \mathrm{E}-03\end{array}$ & $\begin{array}{l}3.17 \mathrm{E}-03 \\
5.22 \mathrm{E}-03\end{array}$ \\
\hline \multicolumn{6}{|l|}{$\mathrm{MLLW}(\mathrm{CT}) \mathrm{CH}$ to $\mathrm{SR}$} \\
\hline \multirow{2}{*}{ Charleston, SC } & to Savannah River & & & & \\
\hline & $\begin{array}{l}6.19 \mathrm{E}-03 \\
3.47 \mathrm{E}-03\end{array}$ & $\begin{array}{l}4.20 E-04 \\
1.07 E-03\end{array}$ & $\begin{array}{l}7.45 \mathrm{E}-04 \\
2.07 \mathrm{E}-05\end{array}$ & $\begin{array}{l}5.60 E-03 \\
3.91 E-03\end{array}$ & $\begin{array}{l}6.77 \mathrm{E}-03 \\
5.00 \mathrm{E}-03\end{array}$ \\
\hline \multicolumn{6}{|l|}{$\begin{array}{c}M L L W(C T) C L \text { to } W V \\
\text { Colonie }\end{array}$} \\
\hline & $\begin{array}{l}\text { Lo Portsmouth GDP } \\
\text { Truck } 2.54 \mathrm{E}-02\end{array}$ & $2.99 \pm-03$ & $4.01 \mathrm{E}-03$ & $1.93 \mathrm{E}-02$ & $2.63 \mathrm{E}-02$ \\
\hline & $5.72 E-03$ & $1.54 \mathrm{E}-02$ & $2.43 E-04$ & -03 & $2.13 \mathrm{E}-02$ \\
\hline \multicolumn{6}{|l|}{$\operatorname{MLLW(CT)~LC~to~LL~}$} \\
\hline ETEC & $\begin{array}{ll}\text { to Lawrence Livermo } & \text { Liver } \\
\text { Truck } & 1.39 \mathrm{E}-02 \\
\text { Rail } & 4.86 \mathrm{E}-03\end{array}$ & $\begin{array}{l}\text { ore } \\
2.80 \mathrm{E}-03 \\
1.60 \mathrm{E}-02\end{array}$ & $\begin{array}{l}4.47 \mathrm{E}-03 \\
1.86 \mathrm{E}-04\end{array}$ & $\begin{array}{l}1.14 \mathrm{E}-02 \\
4.94 \mathrm{E}-03\end{array}$ & $\begin{array}{l}1.86 \mathrm{E}-02 \\
2.11 \mathrm{E}-02\end{array}$ \\
\hline \multirow{2}{*}{\multicolumn{6}{|c|}{ MLLW(CT) so to LI }} \\
\hline & & & & & \\
\hline & $\begin{array}{l}1.90 \mathrm{E}-02 \\
5.30 \mathrm{E}-03\end{array}$ & $\begin{array}{l}4.51 E-03 \\
2.26 E-02\end{array}$ & $\begin{array}{l}6.81 \mathrm{E}-03 \\
2.50 \mathrm{E}-04\end{array}$ & $\begin{array}{l}1.40 \mathrm{E}-02 \\
5.26 \mathrm{E}-03\end{array}$ & $\begin{array}{l}2.53 \mathrm{E}-02 \\
2.81 \mathrm{E}-02\end{array}$ \\
\hline \multicolumn{6}{|l|}{ MLLW (CT) GJ to RF } \\
\hline Grand Junction PO & $\begin{array}{cc}\text { to Rocky } & \text { Flats Plan } \\
\text { Truck } & 8.39 \mathrm{E}-03 \\
\text { Rail } & 3.88 \mathrm{E}-03\end{array}$ & $\begin{array}{l}8.15 \mathrm{E}-04 \\
3.51 \mathrm{E}-04\end{array}$ & $\begin{array}{l}1.46 \mathrm{E}-03 \\
1.62 \mathrm{E}-05\end{array}$ & $\begin{array}{l}7.65 E-03 \\
4.21 E-03\end{array}$ & $\begin{array}{l}9.92 \mathrm{E}-03 \\
4.58 \mathrm{E}-03\end{array}$ \\
\hline$M L L W(C T)$ KA to $W V$ & & & & & \\
\hline Knolls Atomic & to Portsmouth GDP & & & & \\
\hline & $2.54 \mathrm{E}-02$ & $2.99 \mathrm{E}-03$ & $4.01 \mathrm{E}-03$ & $1.93 \mathrm{E}-02$ & $2.63 \mathrm{E}-02$ \\
\hline \multirow{2}{*}{\multicolumn{6}{|c|}{$\begin{array}{l}\text { MLLW(CT) KC to PA } \\
\text { Kansas City Plant }\end{array}$}} \\
\hline Kansas City Plant & & & & & \\
\hline & $\begin{array}{ll}\text { Truck } & 1.38 \mathrm{E}-02 \\
& 4.89 \mathrm{E}-03\end{array}$ & $\begin{array}{l}1.32 \mathrm{E}-03 \\
6.38 \mathrm{E}-03\end{array}$ & $2.28 \mathrm{E}-03$ & $\begin{array}{l}1.23 \mathrm{E}-02 \\
4.96 \mathrm{E}-03\end{array}$ & $\begin{array}{l}1.59 E-02 \\
1.4 F-02\end{array}$ \\
\hline $\mathrm{MLLW}(\mathrm{CT}) \mathrm{KK}$ to $\mathrm{WV}$ & & & & & \\
\hline KKS & to Portsmouth GDP & & & & \\
\hline & $\begin{array}{ll}\text { Truck } & 2.54 \mathrm{E}-02 \\
\text { Rail } & 5.72 \mathrm{E}-03\end{array}$ & $\begin{array}{l}2.99 E-03 \\
1.54 E-02\end{array}$ & $\begin{array}{l}4.01 \mathrm{E}-03 \\
2.43 \mathrm{E}-04\end{array}$ & $\begin{array}{l}1.93 \mathrm{E}-02 \\
5.58 \mathrm{E}-03\end{array}$ & $\begin{array}{l}2.63 \mathrm{E}-02 \\
2.13 \mathrm{E}-02\end{array}$ \\
\hline \multirow{2}{*}{\multicolumn{6}{|c|}{$\begin{array}{c}\text { MLLW(CT) KW to WV } \\
\text { KWS }\end{array}$}} \\
\hline & & & & & \\
\hline & $\begin{array}{ll}\text { Truck } & 2.54 \mathrm{E}-02 \\
\text { Rail } & 5.72 \mathrm{E}-03\end{array}$ & $\begin{array}{l}2.99 E-03 \\
1.54 E-02\end{array}$ & $\begin{array}{l}4.01 E-03 \\
2.43 E-04\end{array}$ & $\begin{array}{l}1.93 \mathrm{E}-02 \\
5.58 \mathrm{E}-03\end{array}$ & $\begin{array}{l}2.63 \mathrm{E}-02 \\
2.13 \mathrm{E}-02\end{array}$ \\
\hline MLLW (CT) LB to LL & & & & & \\
\hline ence Ber & $\begin{array}{l}\text { to Lawrence Livermo } \\
\text { Truck } 2.31 \mathrm{E}-03\end{array}$ & $\begin{array}{l}\text { ore } \\
7.51 E-04\end{array}$ & 1.0 & 03 & 03 \\
\hline & $3.07 \pm-03$ & $2.62 \mathrm{E}-03$ & $2.79 \pm-05$ & $3.62 \mathrm{E}-03$ & $6.27 E-03$ \\
\hline
\end{tabular}


Table 3. Incident-Free Dose per Shipment (person-rem/shipment) (Cont.)

\begin{tabular}{|c|c|c|c|c|c|}
\hline \multirow[b]{2}{*}{ Source/Route (s) } & \multirow[b]{2}{*}{ Crew } & \multicolumn{4}{|c|}{ General Public } \\
\hline & & off-Link & On-Link & Stops & Total \\
\hline \multicolumn{6}{|l|}{ MLLW(CT) SM to LL } \\
\hline & $\begin{array}{ll}\text { Truck } & 4.02 \mathrm{E}-03 \\
\text { Rail } & 3.27 \mathrm{E}-03\end{array}$ & $\begin{array}{l}9.92 E-04 \\
4.67 E-03\end{array}$ & $\begin{array}{l}1.43 E-03 \\
5.04 E-05\end{array}$ & $\begin{array}{l}2.72 E-03 \\
3.77 E-03\end{array}$ & $\begin{array}{l}5.14 E-03 \\
8.48 E-03\end{array}$ \\
\hline \multicolumn{6}{|l|}{$\begin{array}{l}\text { MLLW (CT) MZ to LL } \\
\text { Mare Island }\end{array}$} \\
\hline Mare Island & $\begin{array}{ll}\text { to Lawrence Livermo } & \text { Liver } \\
\text { Truck } & 2.31 \mathrm{E}-03 \\
\text { Rail } & 3.07 \mathrm{E}-03\end{array}$ & $\begin{array}{l}7.51 E-04 \\
2.62 E-03\end{array}$ & $\begin{array}{l}1.03 E-03 \\
2.79 E-05\end{array}$ & $\begin{array}{l}1.26 \mathrm{E}-03 \\
3.62 \mathrm{E}-03\end{array}$ & $\begin{array}{l}3.04 E-03 \\
6.27 E-03\end{array}$ \\
\hline \multicolumn{6}{|c|}{$\begin{array}{l}\text { MLLW(CT) US to SR } \\
\text { Norfolk Nav Shipyd to Savannah River }\end{array}$} \\
\hline & $\begin{array}{ll}\text { Truck } & 1.59 \mathrm{E}-02 \\
\text { Rail } & 5.09 \mathrm{E}-03\end{array}$ & $\begin{array}{l}1.26 \mathrm{E}-03 \\
4.35 \mathrm{E}-03\end{array}$ & $\begin{array}{l}2.07 E-03 \\
9.95 E-05\end{array}$ & $\begin{array}{l}1.39 E-02 \\
5.11 E-03\end{array}$ & $\begin{array}{l}1.73 \mathrm{E}-02 \\
9.55 \mathrm{E}-03\end{array}$ \\
\hline \multirow{2}{*}{$\begin{array}{l}\text { MLLW(CT) SW to HS } \\
\text { Pear1 Harbor NS }\end{array}$} & ford site & & & & \\
\hline & $\begin{array}{ll}\text { Truck } & 7.06 \mathrm{E}-03 \\
\text { Rail } & 4.62 \mathrm{E}-03\end{array}$ & $\begin{array}{l}6.28 \mathrm{E}-04 \\
6.63 \mathrm{E}-03\end{array}$ & $\begin{array}{l}1.09 \mathrm{E}-03 \\
9.79 \mathrm{E}-05\end{array}$ & $\begin{array}{l}6.33 \mathrm{E}-03 \\
4.76 \mathrm{E}-03\end{array}$ & $\begin{array}{l}8.04 \mathrm{E}-03 \\
1.15 \mathrm{E}-02\end{array}$ \\
\hline \multirow{2}{*}{$\begin{array}{l}\text { MLLW(CT) PT to WV } \\
\text { Portsmouth NS }\end{array}$} & & & & & \\
\hline & $\begin{array}{ll}\text { to Portsmouth GDP } \\
\text { Truck } & 4.03 \mathrm{E}-02 \\
\text { Rail } & 6.89 \mathrm{E}-03\end{array}$ & $\begin{array}{l}7.28 \mathrm{E}-03 \\
2.10 \mathrm{E}-02\end{array}$ & $\begin{array}{l}9.52 \mathrm{E}-03 \\
3.50 \mathrm{E}-04\end{array}$ & $\begin{array}{l}2.68 \mathrm{E}-02 \\
6.44 \mathrm{E}-03\end{array}$ & $\begin{array}{l}4.36 \mathrm{E}-02 \\
2.78 \mathrm{E}-02\end{array}$ \\
\hline \multirow{2}{*}{$\begin{array}{l}\text { MLLW(CT) PR to WV } \\
\text { Princeton PPL }\end{array}$} & & & & & \\
\hline & $\begin{array}{ll}\text { to Portsmouth GDP } \\
\text { Truck } & 2.28 \mathrm{E}-02 \\
\text { Rail } & 6.38 \mathrm{E}-03\end{array}$ & $\begin{array}{l}3.55 \mathrm{E}-03 \\
2.00 \mathrm{E}-02\end{array}$ & $\begin{array}{l}4.81 \mathrm{E}-03 \\
2.85 \mathrm{E}-04\end{array}$ & $\begin{array}{l}1.65 E-02 \\
6.06 E-03\end{array}$ & $\begin{array}{l}2.48 \mathrm{E}-02 \\
2.64 \mathrm{E}-02\end{array}$ \\
\hline \multirow{3}{*}{$\begin{array}{l}\text { MLLW(CT) PN to HS } \\
\text { Puget Sound NS }\end{array}$} & & & & & \\
\hline & to Hanford Site & & & & \\
\hline & $\begin{array}{ll}\text { Truck } & 7.06 \mathrm{E}-03 \\
\text { Rail } & 4.62 \mathrm{E}-03\end{array}$ & $\begin{array}{l}6.28 \mathrm{E}-04 \\
6.63 \mathrm{E}-03\end{array}$ & $\begin{array}{l}1.09 \mathrm{E}-03 \\
9.79 \mathrm{E}-05\end{array}$ & $\begin{array}{l}6.33 \mathrm{E}-03 \\
4.76 \mathrm{E}-03\end{array}$ & $\begin{array}{l}8.04 \mathrm{E}-03 \\
1.15 \mathrm{E}-02\end{array}$ \\
\hline \multirow[t]{2}{*}{$\begin{array}{l}\text { MLLW(CT) RM to PO } \\
\text { Reactive Metals }\end{array}$} & to Portsmouth GDP. & & & & \\
\hline & $\begin{array}{ll}\text { Truck } & 1.00 \mathrm{E}-02 \\
\text { Rail } & 4.05 \mathrm{E}-03\end{array}$ & $\begin{array}{l}1.18 \mathrm{E}-03 \\
7.58 \mathrm{E}-03\end{array}$ & $\begin{array}{l}1.62 \mathrm{E}-03 \\
1.11 \mathrm{E}-04\end{array}$ & $\begin{array}{l}7.73 E-03 \\
4.34 E-03\end{array}$ & $\begin{array}{l}1.05 E-02 \\
1.20 E-02\end{array}$ \\
\hline
\end{tabular}

Table 4. Accident Dose Risk per Shipment (person-rem/shipment)

\begin{tabular}{|c|c|c|c|}
\hline \multicolumn{2}{|l|}{ Source/Route } & \multirow{2}{*}{$\frac{\text { Truck }}{2.41 \mathrm{E}-08}$} & \multirow{3}{*}{$\frac{\text { Rail }}{1.38 \mathrm{E}-08}$} \\
\hline $\begin{array}{l}\text { MLLW (CT) AI to AN } \\
\text { Ames Laboratory }\end{array}$ & to Argonne - East & & \\
\hline$M L L W(C T)$ BA to WV & & & \\
\hline $\begin{array}{l}\text { Bettis Atomic } \\
\text { MLLW (CT) BC to PO }\end{array}$ & to Portsmouth GDP & $1.11 \mathrm{E}-04$ & $4.47 \mathrm{E}-05$ \\
\hline $\begin{array}{l}\text { Battelle columbus } \\
\text { MLLW(CT) CH to SR }\end{array}$ & to Portsmouth GDP & $2.49 \mathrm{E}-10$ & $6.63 E-11$ \\
\hline $\begin{array}{l}\text { Charleston, SC } \\
\text { MLLW(CT) CL to WV }\end{array}$ & to Savannah River & $6.91 E-04$ & $8.02 \mathrm{E}-05$ \\
\hline $\begin{array}{l}\text { Colonie } \\
\text { MLLW (CT) LC to LL }\end{array}$ & to Portsmouth GDP & $2.17 \mathrm{E}-06$ & $5.04 \mathrm{E}-07$ \\
\hline $\begin{array}{c}\text { ETEC } \\
\text { MLLW(CT) So to LL }\end{array}$ & to Lawrence Livermore & $5.06 \mathrm{E}-05$ & $1.78 \mathrm{E}-04$ \\
\hline $\begin{array}{l}\text { General Atomic } \\
\text { MLLW(CT) GJ to RF }\end{array}$ & to Lawrence Livermore & $1.17 E-06$ & $1.28 \mathrm{E}-06$ \\
\hline $\begin{array}{l}\text { Grand Junction PO } \\
\text { MLLW(CT) KA to WV }\end{array}$ & to Rocky Flats Plant & $5.76 \mathrm{E}-08$ & $6.35 \mathrm{E}-10$ \\
\hline $\begin{array}{l}\text { Knolls Atomic } \\
\text { MLLW(CT) KC to PA }\end{array}$ & to Portsmouth GDP & 2 & $5.43 E-03$ \\
\hline $\begin{array}{l}\text { Ransas City Plant } \\
\text { MLLW(CT) KK to WV }\end{array}$ & to Paducah GDP & $1.11 E-04$ & $2.52 \mathrm{E}-05$ \\
\hline KKS & to Portsmouth GDP & $2.44 E-02$ & $5.72 \mathrm{E}-03$ \\
\hline$\underset{\text { KWS }}{\text { MLLW (CT) }}$ KW to $W V$ & to Portsmouth GDP & 7.34 & $1.72 \mathrm{E}-03$ \\
\hline MLLW(CT) LB to LL & & & \\
\hline $\begin{array}{l}\text { Lawrence Berkeley } \\
\text { MLLW(CT) SM to LI }\end{array}$ & to Lawrence Livermore & $8.41 E-07$ & $5.96 \mathrm{E}-07$ \\
\hline $\begin{array}{l}\text { IERHR } \\
\text { MLLW(CT) MZ to LL }\end{array}$ & to Lawrence Livermore & $1.28 \mathrm{E}-06$ & $1.05 E-06$ \\
\hline $\begin{array}{l}\text { Mare Island } \\
\text { MLLW(CT) US to SR }\end{array}$ & to Lawrence Livermore & $6.50 \mathrm{E}-04$ & $4.68 \mathrm{E}-04$ \\
\hline $\begin{array}{l}\text { Norfolk Nav Shipyd } \\
\text { MLLW(CT) SW to HS }\end{array}$ & to Savannah River & $8.05 E-04$ & $1.41 \mathrm{E}-04$ \\
\hline Pearl Harbor NS & to Hanford Site & $1.12 \mathrm{E}-04$ & $8.26 \mathrm{E}-05$ \\
\hline
\end{tabular}


Table 4. Accident Dose Risk per Shipment (person-rem/shipment) (Cont.)

\begin{tabular}{llcc}
\hline Source/Route & & Truck & Rail \\
\hline $\begin{array}{c}\text { MLLW (CT) PT to WV } \\
\text { Portsmouth NS }\end{array}$ & to Portsmouth GDP & $3.37 E-04$ & $1.53 E-04$ \\
$\begin{array}{c}\text { MLLW (CT) PR to WV } \\
\text { Princeton PPL }\end{array}$ & to Portsmouth GDP & $2.71 \mathrm{E}-09$ & $1.28 E-09$ \\
$\begin{array}{c}\text { MLLW (CT) PN to HS } \\
\text { Puget Sound NS }\end{array}$ & to Hanford Site & $2.49 E-03$ & $1.84 \mathrm{E}-03$ \\
$\begin{array}{c}\text { MLLW (CT) RM to PO } \\
\text { Reactive Metals }\end{array}$ & to Portsmouth GDP & $1.47 \mathrm{E}-06$ & $4.24 \mathrm{E}-07$ \\
\hline
\end{tabular}

Table 5. Nonradiological Risk Factors per Shipment (fatalities/shipment)

\begin{tabular}{|c|c|c|c|}
\hline Mode & Route & Emission & Accident \\
\hline $\begin{array}{l}\text { Truck } \\
\text { Ames Laboratory } \\
\text { Bettis Atomic } \\
\text { Battelle Columbus } \\
\text { Charleston, SC } \\
\text { Colonie } \\
\text { ETEC } \\
\text { General Atomic } \\
\text { Grand Junction Po } \\
\text { Knolls Atomic } \\
\text { Kansas City Plant } \\
\text { KKS } \\
\text { KWS } \\
\text { Lawrence Berkeley } \\
\text { LERHR } \\
\text { Mare Island } \\
\text { Norfolk Nav Shipyd } \\
\text { Pearl Harbor NS } \\
\text { Portsmouth NS } \\
\text { Princeton PPL } \\
\text { Puget Sound NS } \\
\text { Reactive Metals }\end{array}$ & $\begin{array}{l}\text { to Argonne - East } \\
\text { to Portsmouth GDP } \\
\text { to Portsmouth GDP } \\
\text { to Savannah River } \\
\text { to Portsmouth GDP } \\
\text { to Lawrence Livermore } \\
\text { to Lawrence Livermore } \\
\text { to Rocky Flats Plant } \\
\text { to Portsmouth GDP } \\
\text { to Paducah GDP } \\
\text { to Portsmouth GDP } \\
\text { to Portsmouth GDP } \\
\text { to Lawrence Livermore } \\
\text { to Lawrence Livermore } \\
\text { to Lawrence Livermore } \\
\text { to Savannah River } \\
\text { to Hanford Site } \\
\text { to Portsmouth GDP } \\
\text { to Portsmouth GDP } \\
\text { to Hanford Site } \\
\text { to Portsmouth GDP }\end{array}$ & $\begin{array}{l}3.22 \mathrm{E}-07 \\
3.22 \mathrm{E}-06 \\
9.01 \mathrm{E}-07 \\
3.22 \mathrm{E}-07 \\
5.63 \mathrm{E}-06 \\
1.59 \mathrm{E}-05 \\
2.52 \mathrm{E}-05 \\
2.93 \mathrm{E}-06 \\
5.63 \mathrm{E}-06 \\
4.12 \mathrm{E}-06 \\
5.63 \mathrm{E}-06 \\
5.63 \mathrm{E}-06 \\
4.09 \mathrm{E}-06 \\
5.18 \mathrm{E}-06 \\
4.09 \mathrm{E}-06 \\
1.58 \mathrm{E}-06 \\
1.67 \mathrm{E}-06 \\
2.55 \mathrm{E}-05 \\
1.16 \mathrm{E}-05 \\
1.67 \mathrm{E}-06 \\
2.45 \mathrm{E}-06\end{array}$ & $\begin{array}{l}1.87 \mathrm{E}-05 \\
1.33 \mathrm{E}-05 \\
3.65 \mathrm{E}-06 \\
1.70 \mathrm{E}-05 \\
3.56 \mathrm{E}-05 \\
3.01 \mathrm{E}-05 \\
3.54 \mathrm{E}-05 \\
2.32 \mathrm{E}-05 \\
3.56 \mathrm{E}-05 \\
2.89 \mathrm{E}-05 \\
3.56 \mathrm{E}-05 \\
3.56 \mathrm{E}-05 \\
2.56 \mathrm{E}-06 \\
6.50 \mathrm{E}-06 \\
2.56 \mathrm{E}-06 \\
4.45 \mathrm{E}-05 \\
9.56 \mathrm{E}-06 \\
6.65 \mathrm{E}-05 \\
3.99 \mathrm{E}-05 \\
9.56 \mathrm{E}-06 \\
1.21 \mathrm{E}-05\end{array}$ \\
\hline $\begin{array}{l}\text { Rail } \\
\text { Ames Laboratory } \\
\text { Bettis Atomic } \\
\text { Battelle Columbus } \\
\text { Charleston, SC } \\
\text { Colonie } \\
\text { ETEC } \\
\text { General Atomic } \\
\text { Grand Junction Po } \\
\text { Knolls Atomic } \\
\text { Kansas City Plant } \\
\text { KRS } \\
\text { KWS } \\
\text { Lawrence Berkeley } \\
\text { LERHR } \\
\text { Mare Island } \\
\text { Norfolk Nav Shipyd } \\
\text { Pearl Harbor NS } \\
\text { Portsmouth NS } \\
\text { Princeton PPL } \\
\text { Puget Sound NS } \\
\text { Reactive Metals }\end{array}$ & $\begin{array}{l}\text { to Argonne - East } \\
\text { to Portsmouth GDP } \\
\text { to Portsmouth GDP } \\
\text { to Savannah River } \\
\text { to Portsmouth GDP } \\
\text { to Lawrence Livermore } \\
\text { to Lawrence Livermore } \\
\text { to Rocky Flats Plant } \\
\text { to Portsmouth GDP } \\
\text { to Paducah GDP } \\
\text { to Portsmouth GDP } \\
\text { to Portsmouth GDP } \\
\text { to Lawrence Livermore } \\
\text { to Lawrence Livermore } \\
\text { to Lawrence Livermore } \\
\text { to Savannah River } \\
\text { to Hanford Site } \\
\text { to Portsmouth GDP } \\
\text { to Portsmouth GDP } \\
\text { to Hanford Site } \\
\text { to Portsmouth GDP }\end{array}$ & $\begin{array}{l}9.54 \mathrm{E}-06 \\
1.40 \mathrm{E}-05 \\
1.84 \mathrm{E}-06 \\
1.46 \mathrm{E}-06 \\
2.15 \mathrm{E}-05 \\
3.01 \mathrm{E}-05 \\
4.35 \mathrm{E}-05 \\
2.51 \mathrm{E}-07 \\
2.15 \mathrm{E}-05 \\
9.58 \mathrm{E}-06 \\
2.15 \mathrm{E}-05 \\
2.15 \mathrm{E}-05 \\
5.10 \mathrm{E}-06 \\
9.04 \mathrm{E}-06 \\
5.10 \mathrm{E}-06 \\
3.64 \mathrm{E}-06 \\
1.08 \mathrm{E}-05 \\
2.69 \mathrm{E}-05 \\
3.18 \mathrm{E}-05 \\
1.08 \mathrm{E}-05 \\
1.14 \mathrm{E}-05\end{array}$ & $\begin{array}{l}6.87 \mathrm{E}-07 \\
8.98 \mathrm{E}-07 \\
1.90 \mathrm{E}-07 \\
2.93 \mathrm{E}-07 \\
1.43 \mathrm{E}-06 \\
9.92 \mathrm{E}-07 \\
1.21 \mathrm{E}-06 \\
4.99 \mathrm{E}-07 \\
1.43 \mathrm{E}-06 \\
1.01 \mathrm{E}-06 \\
1.43 \mathrm{E}-06 \\
1.43 \mathrm{E}-06 \\
9.64 \mathrm{E}-08 \\
1.95 \mathrm{E}-07 \\
9.64 \mathrm{E}-08 \\
1.11 \mathrm{E}-06 \\
8.70 \mathrm{E}-07 \\
2.01 \mathrm{E}-06 \\
1.75 \mathrm{E}-06 \\
8.70 \mathrm{E}-07 \\
5.84 \mathrm{E}-07\end{array}$ \\
\hline
\end{tabular}


Table 6. Annual Incident-Free Dose for the Shipping Campaign (person-rem/yr)

\begin{tabular}{|c|c|c|c|c|c|c|}
\hline \multirow[b]{2}{*}{ Route } & & & \multicolumn{2}{|c|}{ Truck } & \multicolumn{2}{|c|}{ Rail } \\
\hline & & & Crew & Public & Crew & Public \\
\hline $\begin{array}{l}\text { Ames Laboratory } \\
\text { Bettis Atomic } \\
\text { Battelle Columbus } \\
\text { Charleston, SC } \\
\text { Colonie } \\
\text { ETEC } \\
\text { General Atomic } \\
\text { Grand Junction PO } \\
\text { Knolls Atomic } \\
\text { Kansas City Plant } \\
\text { KKS } \\
\text { KWS } \\
\text { Lawrence Berkeley } \\
\text { LERHR } \\
\text { Mare Island } \\
\text { Norfolk Nav Shipyd } \\
\text { Pearl Harbor NS } \\
\text { Portsmouth NS } \\
\text { Princeton PPL } \\
\text { Puget Sound NS } \\
\text { Reactive Metals }\end{array}$ & $\begin{array}{l}\text { to } \\
\text { to } \\
\text { to } \\
\text { to } \\
\text { to } \\
\text { to } \\
\text { to } \\
\text { to } \\
\text { to } \\
\text { to } \\
\text { to } \\
\text { to } \\
\text { to } \\
\text { to } \\
\text { to } \\
\text { to } \\
\text { to } \\
\text { to } \\
\text { to } \\
\text { to } \\
\text { to }\end{array}$ & $\begin{array}{l}\text { Argonne - East } \\
\text { Portsmouth GDP } \\
\text { Portsmouth GDP } \\
\text { Savannah River } \\
\text { Portsmouth GDP } \\
\text { Lawrence Livermore } \\
\text { Lawrence Livermore } \\
\text { Rocky Flats Plant } \\
\text { Portsmouth GDP } \\
\text { Paducah GDP } \\
\text { Portsmouth GDP } \\
\text { Portsmouth GDP } \\
\text { Lawrence Livermore } \\
\text { Lawrence Livermore } \\
\text { Lawrence Livermore } \\
\text { Savannah River } \\
\text { Hanford Site } \\
\text { Portsmouth GDP } \\
\text { Portsmouth GDP } \\
\text { Hanford Site } \\
\text { Portsmouth GDP }\end{array}$ & $\begin{array}{l}9.87 \mathrm{E}-03 \\
9.78 \mathrm{E}-03 \\
2.86 \mathrm{E}-03 \\
6.19 \mathrm{E}-03 \\
2.54 \mathrm{E}-02 \\
1.53 \mathrm{E}-01 \\
1.90 \mathrm{E}-02 \\
8.39 \mathrm{E}-03 \\
2.54 \mathrm{E}-02 \\
1.38 \mathrm{E}-02 \\
2.54 \mathrm{E}-02 \\
2.54 \mathrm{E}-02 \\
2.31 \mathrm{E}-03 \\
4.02 \mathrm{E}-03 \\
2.31 \mathrm{E}-03 \\
1.59 \mathrm{E}-02 \\
7.06 \mathrm{E}-03 \\
4.03 \mathrm{E}-02 \\
2.28 \mathrm{E}-02 \\
7.06 \mathrm{E}-03 \\
1.00 \mathrm{E}-02\end{array}$ & $\begin{array}{l}1.14 \mathrm{E}-02 \\
1.04 \mathrm{E}-02 \\
3.17 \mathrm{E}-03 \\
6.77 \mathrm{E}-03 \\
2.63 \mathrm{E}-02 \\
2.05 \mathrm{E}-01 \\
2.53 \mathrm{E}-02 \\
9.92 \mathrm{E}-03 \\
2.63 \mathrm{E}-02 \\
1.59 \mathrm{E}-02 \\
2.63 \mathrm{E}-02 \\
2.63 \mathrm{E}-02 \\
3.04 \mathrm{E}-03 \\
5.14 \mathrm{E}-03 \\
3.04 \mathrm{E}-03 \\
1.73 \mathrm{E}-02 \\
8.04 \mathrm{E}-03 \\
4.36 \mathrm{E}-02 \\
2.48 \mathrm{E}-02 \\
8.04 \mathrm{E}-03 \\
1.05 \mathrm{E}-02\end{array}$ & $\begin{array}{l}4.25 \mathrm{E}-03 \\
4.67 \mathrm{E}-03 \\
3.26 \mathrm{E}-03 \\
3.47 \mathrm{E}-03 \\
5.72 \mathrm{E}-03 \\
1.94 \mathrm{E}-02 \\
5.30 \mathrm{E}-03 \\
3.88 \mathrm{E}-03 \\
5.72 \mathrm{E}-03 \\
4.89 \mathrm{E}-03 \\
5.72 \mathrm{E}-03 \\
5.72 \mathrm{E}-03 \\
3.07 \mathrm{E}-03 \\
3.27 \mathrm{E}-03 \\
3.07 \mathrm{E}-03 \\
5.09 \mathrm{E}-03 \\
4.62 \mathrm{E}-03 \\
6.89 \mathrm{E}-03 \\
6.38 \mathrm{E}-03 \\
4.62 \mathrm{E}-03 \\
4.05 \mathrm{E}-03\end{array}$ & $\begin{array}{l}1.04 \mathrm{E}-02 \\
1.48 \mathrm{E}-02 \\
5.22 \mathrm{E}-03 \\
5.00 \mathrm{E}-03 \\
2.13 \mathrm{E}-02 \\
8.45 \mathrm{E}-02 \\
2.81 \mathrm{E}-02 \\
4.58 \mathrm{E}-03 \\
2.13 \mathrm{E}-02 \\
1.14 \mathrm{E}-02 \\
2.13 \mathrm{E}-02 \\
2.13 \mathrm{E}-02 \\
6.27 \mathrm{E}-03 \\
8.48 \mathrm{E}-03 \\
6.27 \mathrm{E}-03 \\
9.55 \mathrm{E}-03 \\
1.15 \mathrm{E}-02 \\
2.78 \mathrm{E}-02 \\
2.64 \mathrm{E}-02 \\
1.15 \mathrm{E}-02 \\
1.20 \mathrm{E}-02\end{array}$ \\
\hline
\end{tabular}

Table 7. Annual Accident Dose Risk for the Shipping Campaign (person-rem/yr)

\begin{tabular}{llcc}
\hline Route & & Truck & Rail \\
\hline Ames Laboratory & to Argonne - East & $2.41 \mathrm{E}-08$ & $1.38 \mathrm{E}-08$ \\
Bettis Atomic & to Portsmouth GDP & $1.11 \mathrm{E}-04$ & $4.47 \mathrm{E}-05$ \\
Battelle Columbus & to Portsmouth GDP & $2.49 \mathrm{E}-10$ & $5.63 \mathrm{E}-11$ \\
Charleston, SC & to Savannah River & $6.91 \mathrm{E}-04$ & $8.02 \mathrm{E}-05$ \\
Colonie & to Portsmouth GDP & $2.17 \mathrm{E}-06$ & $5.04 \mathrm{E}-07$ \\
ETEC & to Lawrence Livermore & $5.57 \mathrm{E}-04$ & $7.12 \mathrm{E}-04$ \\
General Atomic & to Lawrence Livermore & $1.17 \mathrm{E}-06$ & $1.28 \mathrm{E}-06$ \\
Grand Junction Po to Rocky Flats Plant & $5.76 \mathrm{E}-08$ & $6.35 \mathrm{E}-10$ \\
Knolls Atonic & to Portsmouth GDP & $2.32 \mathrm{E}-02$ & $5.43 \mathrm{E}-03$ \\
Ransas City Plant & to Paducah GDP & $1.11 \mathrm{E}-04$ & $2.52 \mathrm{E}-05$ \\
KKS & to Portsmouth GDP & $2.44 \mathrm{E}-02$ & $5.72 \mathrm{E}-03$ \\
KWS & to Portsmouth GDP & $7.34 \mathrm{E}-03$ & $1.72 \mathrm{E}-03$ \\
Lawrence Berkeley & to Lawrence Livermore & $8.41 \mathrm{E}-07$ & $5.96 \mathrm{E}-07$ \\
LERHR & to Lawrence Livermore & $1.28 \mathrm{E}-06$ & $1.05 \mathrm{E}-06$ \\
Mare Island & to Lawrence Livermore & $6.50 \mathrm{E}-04$ & $4.68 \mathrm{E}-04$ \\
Norfolk Nav Shipyd to Savannah River & $8.05 \mathrm{E}-04$ & $1.41 \mathrm{E}-04$ \\
Pearl Harbor NS & to Hanford Site & $1.12 \mathrm{E}-04$ & $8.26 \mathrm{E}-05$ \\
Portsmouth NS & to Portsmouth GDP & $3.37 \mathrm{E}-04$ & $1.53 \mathrm{E}-04$ \\
Princeton PPL & to Portsmouth GDP & $2.71 \mathrm{E}-09$ & $1.28 \mathrm{E}-09$ \\
Puget Sound NS & to Hanford Site & $2.49 \mathrm{E}-03$ & $1.84 \mathrm{E}-03$ \\
Reactive Metals & to Portsmouth GDP & $1.47 \mathrm{E}-06$ & $4.24 \mathrm{E}-07$ \\
& & & \\
\hline Totals & & $6.08 \mathrm{E}-02$ & $1.64 \mathrm{E}-02$ \\
& & & \\
\hline
\end{tabular}

Table 8. Expected Annual Fatalities for the Shipping Campaign

\begin{tabular}{lll}
\hline Exposure Group & Truck & Rail \\
\hline Radiological & & \\
Normal Crew & $1.7 E-04$ & $4.5 E-05$ \\
$\quad$ Normal Public & $2.6 E-04$ & $1.8 \mathrm{E}-04$ \\
$\quad$ Accident Public & $3.0 \mathrm{E}-05$ & $8.2 \mathrm{E}-06$ \\
Nonradiological & & \\
$\quad$ Emission & $2.9 \mathrm{E}-04$ & $4.0 \mathrm{E}-04$ \\
Accident & $8.1 \mathrm{E}-04$ & $2.2 \mathrm{E}-05$ \\
\hline
\end{tabular}


Table 9. Expected Annual Cancer Incidence for the Shipping Campaign

\begin{tabular}{lll}
\hline Exposure Group & Truck & Rail \\
\hline Radiological & & \\
$\quad$ Normal Crew & $6.1 \mathrm{E}-04$ & $1.6 \mathrm{E}-04$ \\
$\quad$ Normal Public & $8.8 \mathrm{E}-04$ & $6.3 \mathrm{E}-04$ \\
$\quad$ Accident Public & $1.0 \mathrm{E}-04$ & $2.8 \mathrm{E}-05$ \\
Nonradiological & $2.9 \mathrm{E}-04$ & $4.0 \mathrm{E}-04$ \\
$\quad$ Emission & $\mathrm{NA}$ & $\mathrm{NA}$ \\
Accident & & \\
\hline
\end{tabular}

Table 10. Expected Annual Genetic Effects for the Shipping Campaign

\begin{tabular}{lll}
\hline Exposure Group & Truck & Rail \\
\hline Radiological & & \\
Normal Crew & $2.6 E-05$ & $6.8 E-06$ \\
Normal Public & $5.2 E-05$ & $3.7 E-05$ \\
Accident Public & $6.0 E-06$ & $1.6 E-06$ \\
Nonradiological & NA & NA \\
Emission & NA & NA \\
Accident & & \\
\hline
\end{tabular}




\section{B.4 WM LLMW DECENTRALIZED ALTERNATIVE (CASE 2): CONTACT-HANDLED HETEROGENEOUS SOLIDS: ALPHA — TREATED}

Table 1. Summary of Route Information

\begin{tabular}{|c|c|c|c|c|c|}
\hline & \multirow[b]{2}{*}{ Route } & \multirow{2}{*}{$\begin{array}{l}\text { Distance } \\
\text { (miles) }\end{array}$} & \multicolumn{3}{|c|}{ Percentage in Zone } \\
\hline & & & Rural & Suburban & Urban \\
\hline \multicolumn{6}{|l|}{ Truck } \\
\hline $\begin{array}{l}\text { Lawrence Livermore } \\
\text { West Valley DP } \\
\text { Rail }\end{array}$ & $\begin{array}{l}\text { to Nevada Test Site } \\
\text { to Savannah River }\end{array}$ & $\begin{array}{l}678 \\
883\end{array}$ & $\begin{array}{l}84.7 \\
70.3\end{array}$ & $\begin{array}{r}9.2 \\
28.5\end{array}$ & $\begin{array}{l}6 \cdot 1 \\
1.2\end{array}$ \\
\hline $\begin{array}{l}\text { Lawrence Livermore } \\
\text { West Valley DP }\end{array}$ & $\begin{array}{l}\text { to Nevada Test Site } \\
\text { to Savannah River }\end{array}$ & $\begin{array}{l}1370 \\
1217\end{array}$ & $\begin{array}{l}94.5 \\
62.8\end{array}$ & $\begin{array}{r}3.8 \\
32.4\end{array}$ & $\begin{array}{l}1.8 \\
4.9\end{array}$ \\
\hline
\end{tabular}

Table 2. Annual Number of Shipments and One-Way Shipment Distances

\begin{tabular}{|c|c|c|c|c|c|}
\hline \multirow[b]{2}{*}{ Route } & & \multicolumn{2}{|c|}{ \# of Shipments } & \multicolumn{2}{|c|}{ Total Mileage(mi) } \\
\hline & & Truck & Rail & Truck & Rail \\
\hline $\begin{array}{l}\text { Lawrence Livermore } \\
\text { West Valley DP }\end{array}$ & $\begin{array}{l}\text { to Nevada Test Site } \\
\text { to Savannah River }\end{array}$ & $\begin{array}{r}10 \\
1\end{array}$ & $\begin{array}{l}4 \\
1\end{array}$ & $\begin{array}{l}6.78 \mathrm{E}+03 \\
8.83 \mathrm{E}+02\end{array}$ & $\begin{array}{l}5.48 E+03 \\
1.22 E+03\end{array}$ \\
\hline Totals & & 11 & 5 & $7.66 \mathrm{E}+03$ & $6.70 E+03$ \\
\hline
\end{tabular}

Table 3. Incident-Free Dose per Shipment (person-rem/shipment)

\begin{tabular}{|c|c|c|c|c|c|c|}
\hline \multirow[b]{2}{*}{ Source/Route (s) } & & \multirow[b]{2}{*}{ Crew } & \multicolumn{4}{|c|}{ General Public } \\
\hline & & & $\overline{\text { off-Link }}$ & On-Link & Stops & Total \\
\hline $\begin{array}{l}\text { MLLW(T) LI to NT } \\
\text { Lawrence Livermore }\end{array}$ & $\begin{array}{l}\text { to Nevada } \\
\text { Truck } \\
\text { Rail }\end{array}$ & $\begin{array}{l}\text { Test Site } \\
2.05 \mathrm{E}-02 \\
8.60 \mathrm{E}-03\end{array}$ & $\begin{array}{l}\mathrm{e} \\
2.57 \mathrm{E}-03 \\
5.75 \mathrm{E}-03\end{array}$ & $\begin{array}{l}4.65 E-03 \\
1.20 E-04\end{array}$ & $\begin{array}{l}1.90 \mathrm{E}-02 \\
7.70 \mathrm{E}-03\end{array}$ & $\begin{array}{l}2.62 \mathrm{E}-02 \\
1.36 \mathrm{E}-02\end{array}$ \\
\hline $\begin{array}{l}M L L W(T) \text { WV to } S R \\
\text { West Valley DP }\end{array}$ & $\begin{array}{l}\text { to Savanna } \\
\text { Truck } \\
\text { Rail }\end{array}$ & $\begin{array}{l}\text { River } \\
2.89 \mathrm{E}-02 \\
7.96 \mathrm{E}-03\end{array}$ & $\begin{array}{l}2.45 \mathrm{E}-03 \\
1.95 \mathrm{E}-02\end{array}$ & $\begin{array}{l}3.86 \mathrm{E}-03 \\
3.38 \mathrm{E}-04\end{array}$ & $\begin{array}{l}2.47 \mathrm{E}-02 \\
7.23 \mathrm{E}-03\end{array}$ & $\begin{array}{l}3.10 \mathrm{E}-02 \\
2.70 \mathrm{E}-02\end{array}$ \\
\hline
\end{tabular}

Table 4. Accident Dose Risk per Shipment (person-rem/shipment)

\begin{tabular}{llll}
\hline Source/Route & Truck & Rail \\
\hline MLLW (T) LL to NT \\
$\begin{array}{c}\text { Lawrence Livermore to Nevada Test Site } \\
\text { MLLW) WV to SR } \\
\text { West Valley DP }\end{array}$ & to Savannah River & $4.80 \mathrm{E}-05$ & $3.53 \mathrm{E}-05$ \\
\hline
\end{tabular}

Table 5. Nonradiological Risk Factors per Shipment (fatalities/shipment)

\begin{tabular}{llll}
\hline Mode & Route & Emission & Accident \\
\hline $\begin{array}{l}\text { Truck } \\
\text { Lawrence Livermore }\end{array}$ & to Nevada Test Site & $1.32 \mathrm{E}-05$ & $5.15 \mathrm{E}-05$ \\
West Valley DP & to Savannah River & $3.28 \mathrm{E}-06$ & $6.60 \mathrm{E}-05$ \\
Rail & & $1.01 \mathrm{E}-05$ & $2.87 \mathrm{E}-06$ \\
Lawrence Livermore & to Nevada Test Site & $2.49 \mathrm{E}-05$ & $2.55 \mathrm{E}-06$ \\
\hline
\end{tabular}


Table 6. Annual Incident-Free Dose for the Shipping Campaign (person-rem/yr)

\begin{tabular}{|c|c|c|}
\hline \multirow[b]{2}{*}{ Route } & Truek & Rail \\
\hline & Public & Public \\
\hline $\begin{array}{ll}\text { Lawrence Livermore to Nevada Test Site } \\
\text { West Valley DP } & \text { to Savannah River }\end{array}$ & $\begin{array}{ll}2.05 \mathrm{E}-01 & 2.62 \mathrm{E}-01 \\
2.89 \mathrm{E}-02 & 3.10 \mathrm{E}-02\end{array}$ & $\begin{array}{ll}3.44 \mathrm{E}-02 & 5.43 \mathrm{E}-02 \\
7.96 \mathrm{E}-03 & 2.70 \mathrm{E}-02\end{array}$ \\
\hline Totals & $2.34 \mathrm{E}-01 \quad 2.93 \mathrm{E}-01$ & $4.23 \mathrm{E}-028.13 \mathrm{E}-02$ \\
\hline
\end{tabular}

Table 7. Annual Accident Dose Risk for the Shipping Campaign (person-rem/yr)

\begin{tabular}{llcc}
\hline Route & & Truck & Rail \\
\hline $\begin{array}{l}\text { Lawrence Livermore } \\
\text { West Valley DP }\end{array}$ & to Savana Test Site & $4.80 \mathrm{E}-04$ & $1.41 \mathrm{E}-04$ \\
& & $4.43 \mathrm{E}-05$ & $1.42 \mathrm{E}-05$ \\
\hline Totals & $5.25 \mathrm{E}-04$ & $1.55 \mathrm{E}-04$ \\
\hline
\end{tabular}

Table 8. Expected Annual Fatalities for the Shipping Campaign

\begin{tabular}{lll}
\hline Exposure Group & Truck & Rail \\
\hline Radiological & & \\
$\quad$ Normal Crew & $9.4 \mathrm{E}-05$ & $1.7 \mathrm{E}-05$ \\
$\quad$ Normal Public & $1.5 \mathrm{E}-04$ & $4.1 \mathrm{E}-05$ \\
$\quad$ Accident Public & $2.6 \mathrm{E}-07$ & $7.8 \mathrm{E}-08$ \\
Nonradiological & & \\
$\quad$ Emission & $1.4 \mathrm{E}-04$ & $6.5 \mathrm{E}-05$ \\
Accident & $5.8 \mathrm{E}-04$ & $1.4 \mathrm{E}-05$ \\
\hline
\end{tabular}

Table 9. Expected Annual Cancer Incidence for the Shipping Campaign

\begin{tabular}{lll}
\hline Exposure Group & Truck & Rail \\
\hline Radiological & $3.3 \mathrm{E}-04$ & $5.9 \mathrm{E}-05$ \\
Normal Crew & $5.0 \mathrm{E}-04$ & $1.4 \mathrm{E}-04$ \\
Normal Public & $8.9 \mathrm{E}-07$ & $2.6 \mathrm{E}-07$ \\
Accident Public & $1.4 \mathrm{E}-04$ & $6.5 \mathrm{E}-05$ \\
Nonradiological & NA & NA \\
Emission & & \\
Accident & &
\end{tabular}

Table 10. Expected Annual Genetic Effects for the Shipping Campaign

\begin{tabular}{lll}
\hline Exposure Group & Truck & Rail \\
\hline Radiological & & \\
Normal Crew & $1.4 \mathrm{E}-05$ & $2.5 \mathrm{E}-06$ \\
Normal Public & $2.9 \mathrm{E}-05$ & $8.1 \mathrm{E}-06$ \\
Accident Public & $2.0 \mathrm{E}-08$ & $6.2 \mathrm{E}-09$ \\
Nonradiological & & NA \\
Emission & NA & NA \\
Accident & NA & \\
\hline
\end{tabular}




\section{B.5 WM LLMW REGIONALIZED 1 ALTERNATTVE (CASE 4): CONTACT-HANDLED ORGANIC LIQUIDS: NONALPHA - UNTREATED}

Table 1. Sumnary of Route Information

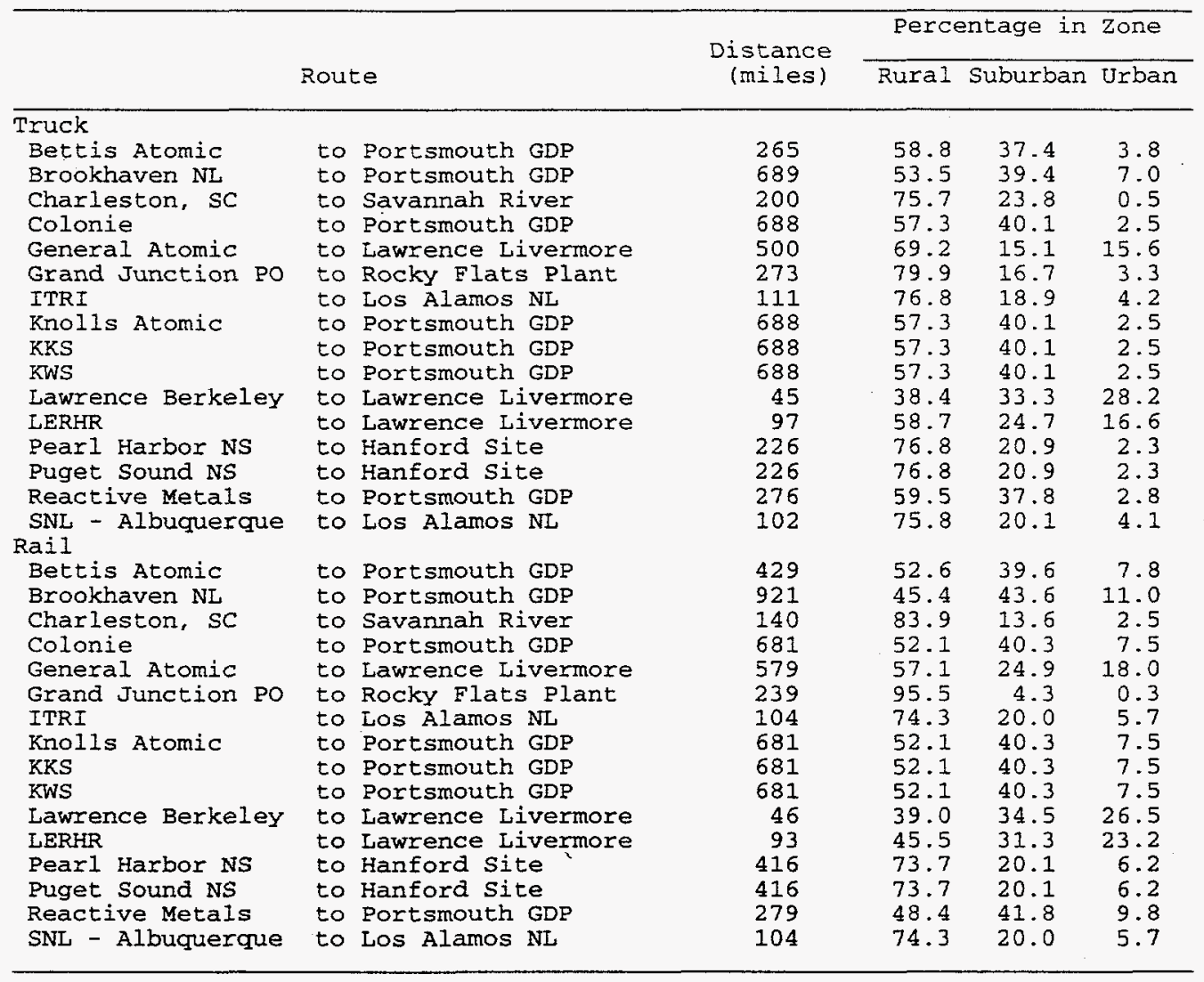

Table 2. Annual Number of Shipments and One-Way Shipment Distances

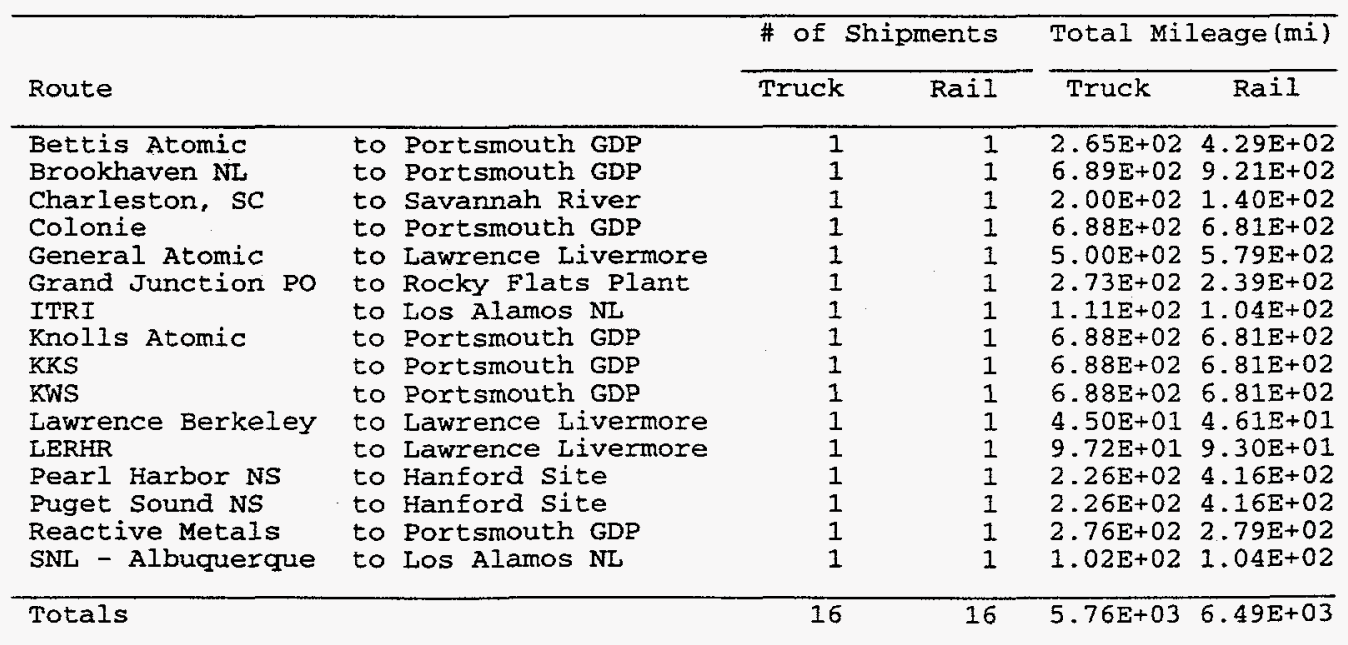


Table 3. Incident-Free Dose per Shipment (person-rem/shipment)

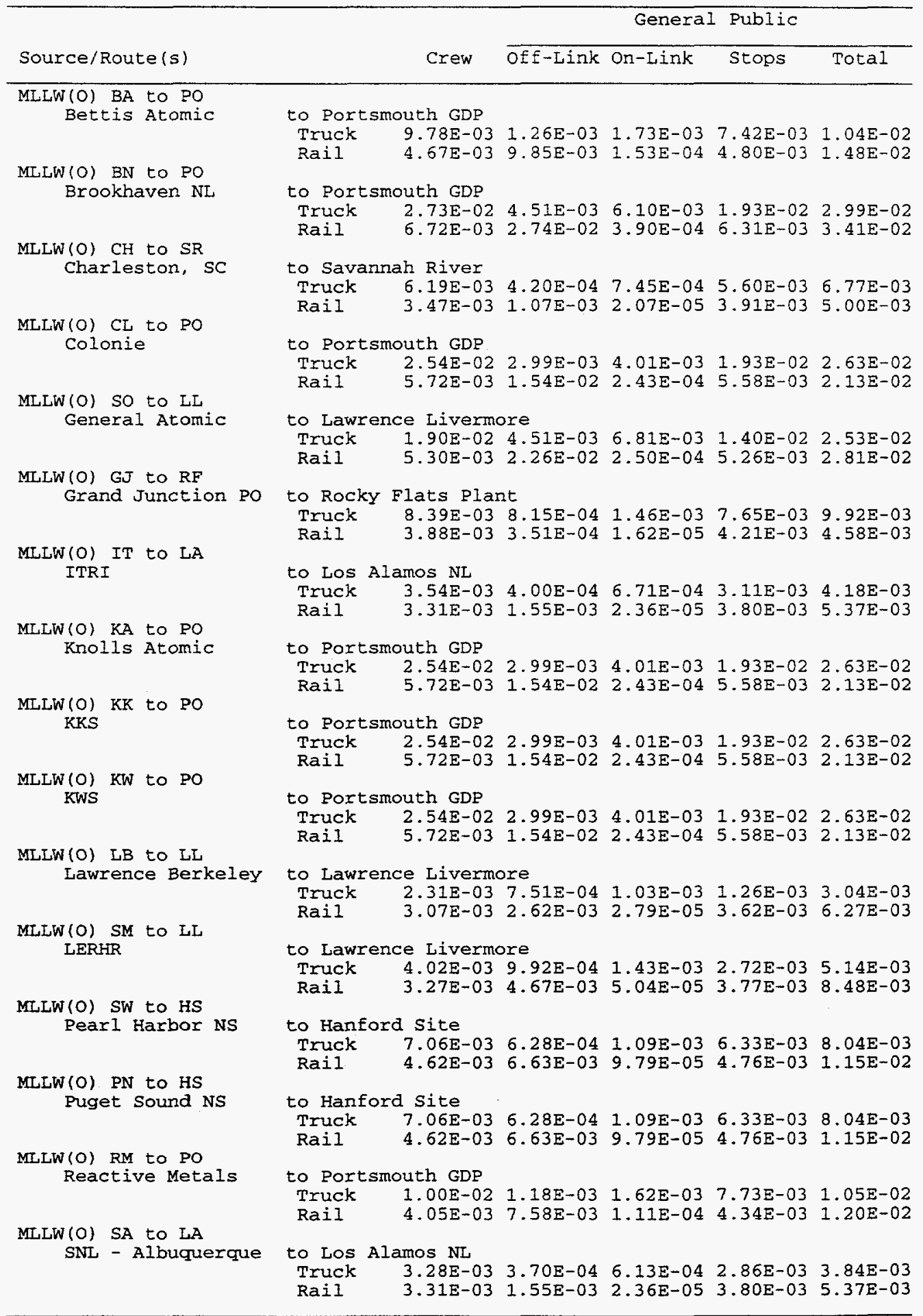


Table 4. Accident Dose Risk per Shipment (person-rem/shipment)

\begin{tabular}{|c|c|c|c|}
\hline \multicolumn{2}{|l|}{ Source/Route } & \multirow{3}{*}{$\begin{array}{c}\text { Truck } \\
5.04 E-04\end{array}$} & \multirow{3}{*}{$\frac{\operatorname{Rail}}{2.16 \mathrm{E}-04}$} \\
\hline MLLW(O) BA to PO & & & \\
\hline Bettis Atomic & to Portsmouth GDP & & \\
\hline $\begin{array}{l}\text { Brookhaven NL } \\
\text { MLLW(O) CH to SR }\end{array}$ & to Portsmouth GDP & $4.87 \mathrm{E}-03$ & $2.63 E-03$ \\
\hline $\begin{array}{l}\text { Charleston, SC } \\
\text { MLLW(O) CL to PO }\end{array}$ & to Savannah River & $7.75 \mathrm{E}-03$ & $8.99 \mathrm{E}-04$ \\
\hline $\begin{array}{l}\text { Colonie } \\
\text { Col }\end{array}$ & to Portsmouth GDP & $3.74 \mathrm{E}-04$ & $8.80 \mathrm{E}-05$ \\
\hline $\begin{array}{l}\text { MLLW (0) So to LL } \\
\text { General Atomic } \\
\text { MLLW(O) GJ to RF }\end{array}$ & to Lawrence Livermore & $5.23 \mathrm{E}-06$ & $6.00 \mathrm{E}-06$ \\
\hline $\begin{array}{l}\text { Grand Junction PO } \\
\text { MLLW(O) IT to LA }\end{array}$ & to Rocky Flats Plant & $1.33 \mathrm{E}-06$ & $1.13 \mathrm{E}-08$ \\
\hline $\begin{array}{l}\text { ITRI } \\
\text { MLIW (O) KA to PO }\end{array}$ & to Los Alamos NL & $8.49 E-06$ & $1.55 E-07$ \\
\hline Knolls Atomic & to Portsmouth GDP & $4.50 \mathrm{E}-02$ & $1.06 \mathrm{E}-02$ \\
\hline $\begin{array}{l}\text { KKS } \\
\text { MLLW(O) KW to PO }\end{array}$ & to Portsmouth GDP & $3.45 \mathrm{E}-02$ & $8.095-03$ \\
\hline $\begin{array}{l}\text { KWS } \\
\text { MLLW(O) LB to LL }\end{array}$ & to Portsmouth GDP & $4.71 \mathrm{E}-03$ & $1.10 \mathrm{E}-03$ \\
\hline $\begin{array}{l}\text { Lawrence Berkeley } \\
\text { MT.W (O) }\end{array}$ & to Lawrence Livermore & $5.87 \mathrm{E}-06$ & $4.23 E-06$ \\
\hline LERHR & to Lawrence Livermore & $9.63 \mathrm{E}-07$ & $8.20 E-07$ \\
\hline $\begin{array}{l}\text { MLLW(O) SW to HS } \\
\text { Pearl Harbor NS } \\
\text { MLLW(O) PN to HS }\end{array}$ & to Hanford Site & $8.47 E-06$ & $6.32 E-06$ \\
\hline $\begin{array}{l}\text { Puget Sound NS } \\
\text { MLLW(O) RM to PO }\end{array}$ & to Hanford Site & $1.95 E-04$ & $1.46 \mathrm{E}-04$ \\
\hline $\begin{array}{l}\text { Reactive Metals } \\
\text { MLLW(O) SA to LA }\end{array}$ & to Portsmouth GDP & $7.40 \mathrm{E}-05$ & $2.29 \mathrm{E}-05$ \\
\hline SNL - Albuquerque & to Los Alamos $\mathrm{NL}$ & $5.82 E-07$ & $1.10 \mathrm{E}-08$ \\
\hline
\end{tabular}

Table 5. Nonradiological Risk Factors per Shipment (fatalities/shipment)

\begin{tabular}{|c|c|c|c|}
\hline \multirow{2}{*}{$\begin{array}{l}\text { Mode } \\
\text { Truck }\end{array}$} & Route & \multirow[t]{2}{*}{ Emission } & \multirow[t]{2}{*}{ Accident } \\
\hline & & & \\
\hline Bettis Atomic & to Portsmouth GDP & $3.22 \mathrm{E}-06$ & $1.33 E-05$ \\
\hline Brookhaven NL & to Portsmouth GDP & $1.56 \bar{E}-05$ & $4.90 E-05$ \\
\hline Charleston, SC & to Savannah River & $3.22 E-07$ & $1.70 E-05$ \\
\hline Colonie & to Portsmouth GDP & $5.63 E-06$ & $3.56 \mathrm{E}-05$ \\
\hline General Atomic & to Lawrence Livermore & $2.52 E-05$ & $3.54 \mathrm{E}-05$ \\
\hline Grand Junction PO & to Rocky Flats Plant & $2.93 \mathrm{E}-06$ & $2.32 E-05$ \\
\hline ITRI & to Los Alamos NL & $1.51 \mathrm{E}-06$ & $1.28 \mathrm{E}-05$ \\
\hline Knolis Atomic & to Portsmouth GDP & $5.63 \mathrm{E}-06$ & $3.56 \mathrm{E}-05$ \\
\hline KKS & to Portsmouth GDP & $5.63 E-06$ & $3.56 \mathrm{E}-05$ \\
\hline KWS & to Portsmouth GDP & $5.63 E-06$ & $3.56 \mathrm{E}-05$ \\
\hline Lawrence Berkeley & to Lawrence Livermore & $4.09 E-06$ & $2.66 \mathrm{E}-06$ \\
\hline LERHR & to Lawrence Livermore & $5.18 E-06$ & $6.50 E-06$ \\
\hline Pearl Harbor NS & to Hanford site & $1.67 \mathrm{E}-06$ & $9.56 \mathrm{E}-06$ \\
\hline Puget Sound NS & to Hanford site & $1.67 \mathrm{E}-06$ & $9.56 \mathrm{E}-06$ \\
\hline Reactive Metals & to Portsmouth GDP & $2.45 \mathrm{E}-06$ & $1.21 \mathrm{E}-05$ \\
\hline SNL - Albuquerque & to Los Alamos NL & $1.35 E-06$ & $1.20 \mathrm{E}-05$ \\
\hline \multicolumn{4}{|l|}{ Rail } \\
\hline Bettis Atomic & to Portsmouth GDP & $1.40 \mathrm{E}-05$ & $8.98 E-07$ \\
\hline Brookhaven NL & to Portsmouth GDP & $4.22 \mathrm{E}-05$ & $1.93 E-06$ \\
\hline Charleston, SC & to Savannah River & $1.46 \mathrm{E}-06$ & $2.93 \mathrm{E}-07$ \\
\hline Colonie & to Portsmouth GDP & $2.15 \mathrm{E}-05$ & $1.43 \mathrm{E}-06$ \\
\hline General Atomic & to Lawrence Livermore & $4.35 E-05$ & $1.21 E-06$ \\
\hline Grand Junction Po & to Rocky Flats Plant & $2.51 \mathrm{E}-07$ & $4.99 E-07$ \\
\hline ITRI & to Los Alamos NL & $2.47 E-06$ & $2.18 \mathrm{E}-07$ \\
\hline Knolls Atomic & to Portsmouth GDP & $2.15 \mathrm{E}-05$ & $1.43 \mathrm{E}-06$ \\
\hline KKS & to Portsmouth GDP & $2.15 \mathrm{E}-05$ & $1.43 \mathrm{E}-06$ \\
\hline KWS & to Portsmouth GDP & $2.15 \mathrm{E}-05$ & $1.43 \mathrm{E}-06$ \\
\hline Lawrence Berkeley & to Lawrence Livermore & $5.10 E-06$ & $9.64 E-08$ \\
\hline LERHR & to Lawrence Livermore & $9.04 \mathrm{E}-06$ & $1.95 E-07$ \\
\hline Pearl Harbor NS & to Hanford Site & $1.08 \mathrm{E}-05$ & $8.70 E-07$ \\
\hline Puget Sound NS & to Hanford Site & $1.08 \mathrm{E}-05$ & $8.70 E-07$ \\
\hline Reactive Metals & to Portsmouth GDP & $1.14 E-05$ & $5.84 \mathrm{E}-07$ \\
\hline SNL - Albuquerque & to Los Alamos NL & $2.47 \mathrm{E}-06$ & $2.18 \mathrm{E}-07$ \\
\hline
\end{tabular}


Table 6. Annual Incident-Free Dose for the Shipping Campaign (person-rem/yr)

\begin{tabular}{|c|c|c|c|c|c|c|}
\hline \multirow[b]{2}{*}{ Route } & & & \multicolumn{2}{|c|}{ Truck } & \multicolumn{2}{|c|}{ Rail } \\
\hline & & & Crew & Public & Crew & Public \\
\hline $\begin{array}{l}\text { Bettis Atomic } \\
\text { Brookhaven NL } \\
\text { Charleston, SC } \\
\text { Colonie } \\
\text { General Atomic } \\
\text { Grand Junction Po } \\
\text { ITRI } \\
\text { Knolls Atomic } \\
\text { KKS } \\
\text { KWS } \\
\text { Lawrence Berkeley } \\
\text { LERHR } \\
\text { Pearl Harbor NS } \\
\text { Puget Sound NS } \\
\text { Reactive Metals } \\
\text { SNL - Albuquerque }\end{array}$ & $\begin{array}{l}\text { to } \\
\text { to } \\
\text { to } \\
\text { to } \\
\text { to } \\
\text { to } \\
\text { to } \\
\text { to } \\
\text { to } \\
\text { to } \\
\text { to } \\
\text { to } \\
\text { to } \\
\text { to } \\
\text { to } \\
\text { to }\end{array}$ & $\begin{array}{l}\text { Portsmouth GDP } \\
\text { Portsmouth GDP } \\
\text { Savannah River } \\
\text { Portsmouth GDP } \\
\text { Lawrence Livermore } \\
\text { Rocky Flats Plant } \\
\text { Los Alamos NL } \\
\text { Portsmouth GDP } \\
\text { Portsmouth GDP } \\
\text { Portsmouth GDP } \\
\text { Lawrence Livermore } \\
\text { Lawrence Livermore } \\
\text { Hanford Site } \\
\text { Hanford Site } \\
\text { Portsmouth GDP } \\
\text { Los Alamos NL }\end{array}$ & $\begin{array}{l}9.78 \mathrm{E}-03 \\
2.73 \mathrm{E}-02 \\
6.19 \mathrm{E}-03 \\
2.54 \mathrm{E}-02 \\
1.90 \mathrm{E}-02 \\
8.39 \mathrm{E}-03 \\
3.54 \mathrm{E}-03 \\
2.54 \mathrm{E}-02 \\
2.54 \mathrm{E}-02 \\
2.54 \mathrm{E}-02 \\
2.31 \mathrm{E}-03 \\
4.02 \mathrm{E}-03 \\
7.06 \mathrm{E}-03 \\
7.06 \mathrm{E}-03 \\
1.00 \mathrm{E}-02 \\
3.28 \mathrm{E}-03\end{array}$ & $\begin{array}{l}1.04 \mathrm{E}-02 \\
2.99 \mathrm{E}-02 \\
6.77 \mathrm{E}-03 \\
2.63 \mathrm{E}-02 \\
2.53 \mathrm{E}-02 \\
9.92 \mathrm{E}-03 \\
4.18 \mathrm{E}-03 \\
2.63 \mathrm{E}-02 \\
2.63 \mathrm{E}-02 \\
2.63 \mathrm{E}-02 \\
3.04 \mathrm{E}-03 \\
5.14 \mathrm{E}-03 \\
8.04 \mathrm{E}-03 \\
8.04 \mathrm{E}-03 \\
1.05 \mathrm{E}-02 \\
3.84 \mathrm{E}-03\end{array}$ & $\begin{array}{l}4.67 \mathrm{E}-03 \\
6.72 \mathrm{E}-03 \\
3.47 \mathrm{E}-03 \\
5.72 \mathrm{E}-03 \\
5.30 \mathrm{E}-03 \\
3.88 \mathrm{E}-03 \\
3.31 \mathrm{E}-03 \\
5.72 \mathrm{E}-03 \\
5.72 \mathrm{E}-03 \\
5.72 \mathrm{E}-03 \\
3.07 \mathrm{E}-03 \\
3.27 \mathrm{E}-03 \\
4.62 \mathrm{E}-03 \\
4.62 \mathrm{E}-03 \\
4.05 \mathrm{E}-03 \\
3.31 \mathrm{E}-03\end{array}$ & $\begin{array}{l}1.48 \mathrm{E}-02 \\
3.41 \mathrm{E}-02 \\
5.00 \mathrm{E}-03 \\
2.13 \mathrm{E}-02 \\
2.81 \mathrm{E}-02 \\
4.58 \mathrm{E}-03 \\
5.37 \mathrm{E}-03 \\
2.13 \mathrm{E}-02 \\
2.13 \mathrm{E}-02 \\
2.13 \mathrm{E}-02 \\
6.27 \mathrm{E}-03 \\
8.48 \mathrm{E}-03 \\
1.15 \mathrm{E}-02 \\
1.15 \mathrm{E}-02 \\
1.20 \mathrm{E}-02 \\
5.37 \mathrm{E}-03\end{array}$ \\
\hline Totals & & & 2.03 & $2.30 \mathrm{E}-01$ & $7.32 \mathrm{E}-02$ & $2.32 \mathrm{E}-01$ \\
\hline
\end{tabular}

Table 7. Annual Accident Dose Risk for the Shipping Campaign (person-rem/yr)

\begin{tabular}{llcc}
\hline Route & & Truck & Rail \\
\hline Bettis Atomic & to Portsmouth GDP & $5.04 \mathrm{E}-04$ & $2.16 \mathrm{E}-04$ \\
Brookhaven NL & to Portsmouth GDP & $4.87 \mathrm{E}-03$ & $2.63 \mathrm{E}-03$ \\
Charleston, SC & to Savannah River & $7.75 \mathrm{E}-03$ & $8.99 \mathrm{E}-04$ \\
Colonie & to Portsmouth GDP & $3.74 \mathrm{E}-04$ & $8.80 \mathrm{E}-05$ \\
General Atomic & to Lawrence Iivermore & $5.23 \mathrm{E}-06$ & $6.00 \mathrm{E}-06$ \\
Grand Junction PO & to Rocky Flats Plant & $1.33 \mathrm{E}-06$ & $1.13 \mathrm{E}-08$ \\
ITRI & to Los Alamos NL & $8.49 \mathrm{E}-06$ & $1.55 \mathrm{E}-07$ \\
Knolls Atomic & to Portsmouth GDP & $4.50 \mathrm{E}-02$ & $1.06 \mathrm{E}-02$ \\
KKS & to Portsmouth GDP & $3.45 \mathrm{E}-02$ & $8.09 \mathrm{E}-03$ \\
KWS & to Portsmouth GDP & $4.71 \mathrm{E}-03$ & $1.10 \mathrm{E}-03$ \\
Lawrence Berkeley & to Lawrence Iivermore & $5.87 \mathrm{E}-06$ & $4.23 \mathrm{E}-06$ \\
LERHR & to Lawrence Livermore & $9.63 \mathrm{E}-07$ & $8.20 \mathrm{E}-07$ \\
Pearl Harbor NS & to Hanford Site & $8.47 \mathrm{E}-06$ & $6.32 \mathrm{E}-06$ \\
Puget Sound NS & to Hanford Site & $1.95 \mathrm{E}-04$ & $1.46 \mathrm{E}-04$ \\
Reactive Metals & to Portsmouth GDP & $7.40 \mathrm{E}-05$ & $2.29 \mathrm{E}-05$ \\
SNL - Albuquerque & to Los Alamos NL & $5.82 \mathrm{E}-07$ & $1.10 \mathrm{E}-08$ \\
& & & \\
\hline
\end{tabular}

Table 8. Expected Annual Fatalities for the Shipping Campaign

\begin{tabular}{|c|c|c|}
\hline Exposure Group & Truck & Rail \\
\hline \multicolumn{3}{|l|}{ Radiological } \\
\hline Normal Crew & $8.4 E-05$ & $2.9 E-05$ \\
\hline Normal public & $1.2 \mathrm{E}-04$ & 1. $2 \mathrm{E}-04$ \\
\hline Accident Public & $4.9 E-05$ & 1. $2 \mathrm{E}-05$ \\
\hline \multicolumn{3}{|l|}{ Nonradiological } \\
\hline Emission & $8.8 \mathrm{E}-05$ & $2.4 \mathrm{E}-04$ \\
\hline Accident & $3.5 \mathrm{E}-04$ & $1.4 \mathrm{E}-05$ \\
\hline
\end{tabular}

Table 9. Expected Annual Cancer Incidence for the Shipping Campaign

\begin{tabular}{lll}
\hline Exposure Group & Truck & \multicolumn{1}{c}{ Rail } \\
\hline Radiological & & \\
Normal Crew & $2.9 \mathrm{E}-04$ & $1.0 \mathrm{E}-04$ \\
Normal Public & $3.9 \mathrm{E}-04$ & $3.9 \mathrm{E}-04$ \\
Accident Public & $1.7 \mathrm{E}-04$ & $4.0 \mathrm{E}-05$ \\
Nonradiological & & \\
Emission & $8.8 \mathrm{E}-05$ & $2.4 \mathrm{E}-04$ \\
Accident & $\mathrm{NA}$ & $\mathrm{NA}$ \\
\hline
\end{tabular}


Table 10. Expected Annual Genetic Effects for the Shipping Campaign

\begin{tabular}{lll}
\hline Exposure Group & Truck & Rail \\
\hline Radiological & & \\
Normal Crew & $1.3 \mathrm{E}-05$ & $4.4 \mathrm{E}-06$ \\
Normal Public & $2.3 \mathrm{E}-05$ & $2.3 \mathrm{E}-05$ \\
Accident Public & $7.5 \mathrm{E}-06$ & $1.7 \mathrm{E}-06$ \\
Nonradiological & & \\
Emission & NA & NA \\
Accident & NA & NA \\
\hline
\end{tabular}




\section{B.6 WM LLMW REGIONALIZED 1 ALTERNATIVE (CASE 4): CONTACT-HANDLED ORGANIC LIQUIDS: ALPHA - UNTREATED}

Table 1. Summary of Route Information

\begin{tabular}{|c|c|c|c|c|c|}
\hline & & & Perce & entage in & Zone \\
\hline & Route & $\begin{array}{l}\text { Discance } \\
\text { (miles) }\end{array}$ & Rural & Suburban & Urban \\
\hline Truck & & & & & \\
\hline Lawrence Livermore & to Nevada Test Site & 678 & 84.7 & 9.2 & 6.1 \\
\hline Mound Plant & to Savannah River & 656 & 66.5 & 32.7 & 0.8 \\
\hline West valley DP & to Savannah River & 883 & 70.3 & 28.5 & 1.2 \\
\hline Rail & & & & & \\
\hline Lawrence Livermore & to Nevada Test Site & 1370 & 94.5 & 3.8 & 1.8 \\
\hline Mound Plant & to Savannah River & 744 & 67.4 & 29.2 & 3.3 \\
\hline West valley DP & to Savannah River & 1217 & 62.8 & 32.4 & 4.9 \\
\hline
\end{tabular}

Table 2. Annual Number of Shipments and One-Way Shipment Distances

\begin{tabular}{|c|c|c|c|c|c|}
\hline \multirow[b]{2}{*}{ Route } & & \multicolumn{2}{|c|}{ \# of Shipments } & \multicolumn{2}{|c|}{ Total Mileage (mi) } \\
\hline & & Truck & Rail & Truck & Rail \\
\hline $\begin{array}{l}\text { Lawrence Livermore } \\
\text { Mound Plant } \\
\text { West Valley DP }\end{array}$ & $\begin{array}{l}\text { to Nevada Test Site } \\
\text { to Savannah River } \\
\text { to Savannah River }\end{array}$ & $\begin{array}{l}1 \\
1 \\
1\end{array}$ & $\begin{array}{l}1 \\
1 \\
1\end{array}$ & $\begin{array}{l}6.78 E+02 \\
6.56 E+02 \\
8.83 E+02\end{array}$ & $\begin{array}{l}1.37 E+03 \\
7.44 E+02 \\
1.22 E+03\end{array}$ \\
\hline Totals & & 3 & 3 & $2.22 \mathrm{E}+03$ & $3.33 E+03$ \\
\hline
\end{tabular}

Table 3. Incident-Free Dose per Shipment (person-rem/shipment)

\begin{tabular}{|c|c|c|c|c|c|c|}
\hline \multirow[b]{2}{*}{ Source/Route (s) } & & \multirow[b]{2}{*}{ Crew } & \multicolumn{4}{|c|}{ General Public } \\
\hline & & & $\overline{\text { off-Link }}$ & on-Link & Stops & Total \\
\hline $\begin{array}{l}\text { MLLW(OA) LL to NT } \\
\text { Lawrence Livermore }\end{array}$ & $\begin{array}{l}\text { to Nevada } \\
\text { Truck } \\
\text { Rail }\end{array}$ & $\begin{array}{l}\text { Test Site } \\
2.05 \mathrm{E}-02 \\
8.60 \mathrm{E}-03\end{array}$ & $\begin{array}{l}2.57 \mathrm{E}-03 \\
5.75 \mathrm{E}-03\end{array}$ & $\begin{array}{l}4.65 \mathrm{E}-03 \\
1.20 \mathrm{E}-04\end{array}$ & $\begin{array}{l}1.90 \mathrm{E}-02 \\
7.70 \mathrm{E}-03\end{array}$ & $\begin{array}{l}2.62 E-02 \\
1.36 E-02\end{array}$ \\
\hline $\begin{array}{l}\text { MLLW (OA) MP to SR } \\
\text { Mound Plant }\end{array}$ & $\begin{array}{l}\text { to Savannal } \\
\text { Truck } \\
\text { Rail }\end{array}$ & $\begin{array}{l}\text { hiver } \\
2.21 \mathrm{E}-02 \\
5.99 \mathrm{E}-03\end{array}$ & $\begin{array}{l}1.91 \mathrm{E}-03 \\
9.25 \mathrm{E}-03\end{array}$ & $\begin{array}{l}2.83 \mathrm{E}-03 \\
1.78 \mathrm{E}-04\end{array}$ & $\begin{array}{l}1.84 \mathrm{E}-02 \\
5.77 \mathrm{E}-03\end{array}$ & $\begin{array}{l}2.31 \mathrm{E}-02 \\
1.52 \mathrm{E}-02\end{array}$ \\
\hline $\begin{aligned} & \text { MLLW }(O A) W \text { to } S R \\
& \text { West Valley } D P\end{aligned}$ & $\begin{array}{l}\text { to Savannal } \\
\text { Truck } \\
\text { Rail }\end{array}$ & $\begin{array}{l}\text { River } \\
2.89 \mathrm{E}-02 \\
7.96 \mathrm{E}-03\end{array}$ & $\begin{array}{l}2.45 E-03 \\
1.95 E-02\end{array}$ & $\begin{array}{l}3.86 \mathrm{E}-03 \\
3.38 \mathrm{E}-04\end{array}$ & $\begin{array}{l}2.47 \mathrm{E}-02 \\
7.23 \mathrm{E}-03\end{array}$ & $\begin{array}{l}3.10 E-02 \\
2.70 E-02\end{array}$ \\
\hline
\end{tabular}

Table 4. Accident Dose Risk per Shipment (person-rem/shipment)

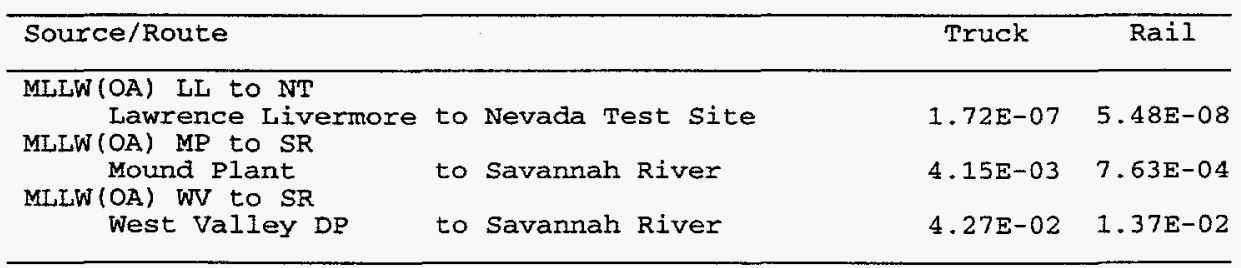

Table 5. Nonradiological Risk Factors per Shipment (fatalities/shipment)

\begin{tabular}{llll}
\hline Mode & Route & Enission & Accident \\
\hline $\begin{array}{l}\text { Truck } \\
\text { Lawrence Livermore }\end{array}$ & to Nevada Test Site & $1.32 \mathrm{E}-05$ & $5.15 \mathrm{E}-05$ \\
Mound Plant & to Savannah River & $1.67 \mathrm{E}-06$ & $5.26 \mathrm{E}-05$ \\
West Valley DP & to Savannah River & $3.28 \mathrm{E}-06$ & $6.60 \mathrm{E}-05$ \\
Rail & Lawrence Livermore & & \\
Mound Plant & to Nevada Test Site & $1.01 \mathrm{E}-05$ & $2.87 \mathrm{E}-06$ \\
West Valley DP & to Savannah River & $1.03 \mathrm{E}-05$ & $1.56 \mathrm{E}-06$ \\
& & $2.49 \mathrm{E}-05$ & $2.55 \mathrm{E}-06$ \\
\hline
\end{tabular}


Table 6. Annual Incident-Free Dose for the Shipping Campaign (person-rem/yr)

\begin{tabular}{|c|c|c|c|c|c|}
\hline \multirow[b]{2}{*}{ Route } & & \multicolumn{2}{|c|}{ Truck } & \multicolumn{2}{|c|}{ Rail } \\
\hline & & Crew & Public & Crew & Public \\
\hline $\begin{array}{l}\text { Lawrence Livermore } \\
\text { Mound Plant } \\
\text { West Valley DP }\end{array}$ & $\begin{array}{l}\text { to Nevada Test Site } \\
\text { to Savannah River } \\
\text { to Savannah River }\end{array}$ & $\begin{array}{l}2.05 \mathrm{E}-02 \\
2.21 \mathrm{E}-02 \\
2.89 \mathrm{E}-02\end{array}$ & $\begin{array}{l}2.62 \mathrm{E}-02 \\
2.31 \mathrm{E}-02 \\
3.10 \mathrm{E}-02\end{array}$ & $\begin{array}{l}8.60 \mathrm{E}-03 \\
5.99 \mathrm{E}-03 \\
7.96 \mathrm{E}-03\end{array}$ & $\begin{array}{l}1.36 \mathrm{E}-02 \\
1.52 \mathrm{E}-02 \\
2.70 \mathrm{E}-02\end{array}$ \\
\hline Totals & & $7.15 E-02$ & $8.03 E-02$ & $2.25 \mathrm{E}-02$ & $5.58 \mathrm{E}-02$ \\
\hline
\end{tabular}

Table 7. Annual Accident Dose Risk for the Shipping Campaign (person-rem/yr)

\begin{tabular}{llcc}
\hline Route & & Truck & Rail \\
\hline Lawrence Livermore to Nevada Test Site & $1.72 \mathrm{E}-07$ & $5.48 \mathrm{E}-08$ \\
Mound Plant & to Savannah River & $4.15 \mathrm{E}-03$ & $7.63 \mathrm{E}-04$ \\
West Valley DP & to Savannah River & $4.27 \mathrm{E}-02$ & $1.37 \mathrm{E}-02$ \\
\hline Totals & & $4.68 \mathrm{E}-02$ & $1.45 \mathrm{E}-02$ \\
\hline
\end{tabular}

Table 8. Expected Annual Fatalities for the Shipping Campaign

\begin{tabular}{|c|c|c|}
\hline Exposure Group & Truck & Rail \\
\hline \multicolumn{3}{|l|}{ Radiological } \\
\hline Normal Crew & $2.9 E-05$ & $9.0 E-06$ \\
\hline Normal Public & $4.0 \mathrm{E}-05$ & $2.8 E-05$ \\
\hline Accident Public & $2.3 \mathrm{E}-05$ & $7.2 \mathrm{E}-06$ \\
\hline \multicolumn{3}{|l|}{ Nonradiological } \\
\hline Emission & $1.8 E-05$ & $4.5 \mathrm{E}-05$ \\
\hline Accident & $1.7 \mathrm{E}-04$ & $7.0 \mathrm{E}-06$ \\
\hline
\end{tabular}

Table 9. Expected Annual Cancer Incidence for the Shipping Campaign

\begin{tabular}{lll}
\hline Exposure Group & Truck & Rail \\
\hline Radiological & & \\
Normal Crew & $1.0 \mathrm{E}-04$ & $3.2 \mathrm{E}-05$ \\
Normal Public & $1.4 \mathrm{E}-04$ & $9.5 \mathrm{E}-05$ \\
$\quad$ Accident Public & $8.0 \mathrm{E}-05$ & $2.5 \mathrm{E}-05$ \\
$\begin{array}{c}\text { Nonradiological } \\
\text { Emission }\end{array}$ & $1.8 \mathrm{E}-05$ & $4.5 \mathrm{E}-05$ \\
Accident & NA & NA \\
\hline
\end{tabular}

Table 10. Expected Annual Genetic Effects for the Shipping Campaign

\begin{tabular}{lll}
\hline Exposure Group & Truck & Rail \\
\hline Radiological & & \\
Normal Crew & $4.3 E-06$ & $1.4 E-06$ \\
Normal Public & $8.0 E-06$ & $5.6 \pm-06$ \\
Accident Public & $1.4 E-06$ & $4.4 \mathrm{E}-07$ \\
Nonradiological & & \\
Emission & NA & NA \\
Accident & NA & NA \\
\hline
\end{tabular}




\section{B.7 WM LLMW REGIONALIZED 1 ALTERNATIVE (CASE 4): CONTACT-HANDLED HETEROGENEOUS SOLIDS: NONALPHA - UNTREATED}

Table 1. Summary of Route Information

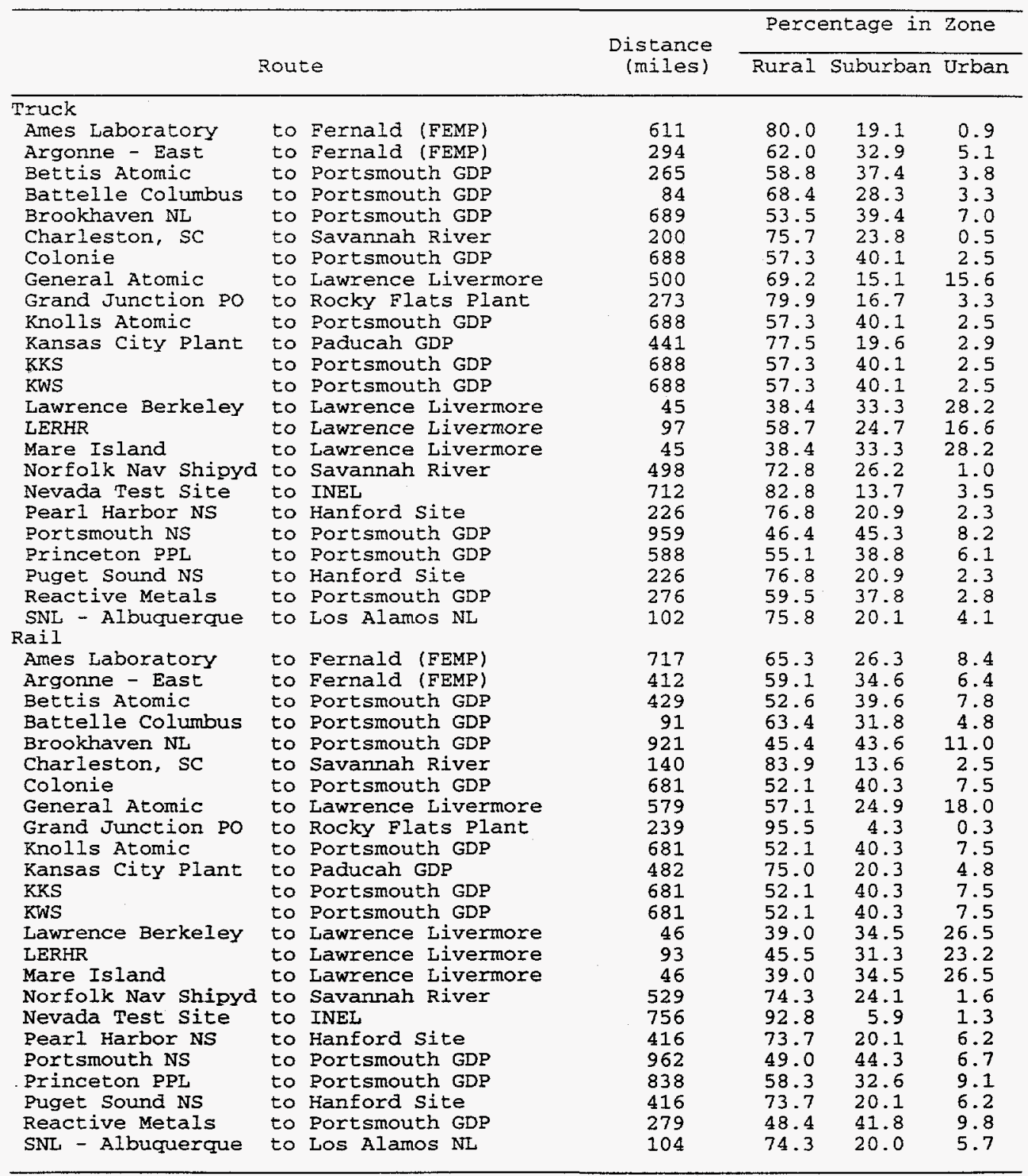


Table 2. Annual Number of Shipments and One-Way Shipment Distances

\begin{tabular}{|c|c|c|c|c|c|c|}
\hline \multirow[b]{2}{*}{ Route } & & & \multicolumn{2}{|c|}{ \# of Shipments } & \multicolumn{2}{|c|}{ Total Mileage (mi) } \\
\hline & & & Truck & Rail & Truck & Rail \\
\hline Ames Laboratory & to & Fernald (FEMP) & 1 & 1 & $6.11 E+02$ & $7.17 E+02$ \\
\hline Argonne - East & to & Fermald (FEMP) & 44 & 17 & $1.29 E+04$ & $7.018+03$ \\
\hline Bettis Atomic & to & Portsmouth GDP & 1 & 1 & $2.65 \mathrm{E}+02$ & $4.29 E+02$ \\
\hline Battelie Columbus & to & Portsmouth GDP & 1 & 1 & $8.41 E+01$ & $9.09 \mathrm{E}+01$ \\
\hline Brookhaven NL & to & Portsmouth GDP & 1 & 1 & $6.89 E+02$ & $9.21 \mathrm{E}+02$ \\
\hline Charleston, SC & to & Savannah River & 1 & 1 & $2.00 \mathrm{E}+02$ & $1.40 \mathrm{E}+02$ \\
\hline Colonie & to & Portsmouth GDP & 1 & 1 & $6.88 E+02$ & $6.81 E+02$ \\
\hline General Atomic & to & Lawrence Livermore & 1 & 1 & $5.00 \mathrm{E}+02$ & $5.79 \mathrm{E}+02$ \\
\hline Grand Junction PO & to & Rocky Flats Plant & 1 & 1 & $2.73 E+02$ & $2.39 E+02$ \\
\hline Knolls Atomic & to & Portsmouth GDP & 1 & 1 & $6.88 E+02$ & $6.81 E+02$ \\
\hline Kansas City Plant & to & Paducah GDP & 1 & 1 & $4.41 \mathrm{E}+02$ & $4.82 E+02$ \\
\hline KKS & to & Portsmouth GDP & 1 & 1 & $6.88 E+02$ & $6.81 \mathrm{E}+02$ \\
\hline KWS & to & Portsmouth GDP & 1 & 1 & $6.88 \mathrm{E}+02$ & $6.81 E+02$ \\
\hline Lawrence Berkeley & to & Lawrence Livermore & 1 & 1 & $4.50 \mathrm{E}+01$ & $4.61 E+01$ \\
\hline LERHR & to & Lawrence Livermore & 1 & 1 & $9.72 \mathrm{E}+01$ & $9.30 E+01$ \\
\hline Mare Island & to & Lawrence Livermore & 1 & 1 & $4.50 E+01$ & $4.61 E+0 I$ \\
\hline Norfolk Nav Shipyd & to & Savannah River & 1 & 1 & $4.98 E+02$ & $5.29 \mathrm{E}+02$ \\
\hline Nevada Test Site & to & INEL & 1 & 1 & $7.12 \mathrm{E}+02$ & $7.56 \mathrm{E}+02$ \\
\hline Pearl Harbor NS & to & Hanford site & 1 & 1 & $2.26 \mathrm{E}+02$ & $4.16 \mathrm{E}+02$ \\
\hline Portsmouth NS & & Portsmouth GDP & 1 & 1 & $9.59 E+02$ & $9.62 \mathrm{E}+02$ \\
\hline Princeton PRL & & Portsmouth GDP & 1 & 1 & $5.88 \mathrm{E}+02$ & $8.38 E+02$ \\
\hline Puget Sound NS & to & Hanford Site & 1 & $\overline{1}$ & $2.26 \mathrm{E}+02$ & $4.16 E+02$ \\
\hline Reactive Metals & & Portsmouth GDP & 1 & $\overline{1}$ & $2.76 \Xi+02$ & $2.79 \mathrm{E}+02$ \\
\hline SNL - Albuquerque & to & Los Alamos NL & 1 & 1 & $1.02 \mathrm{E}+02$ & $1.04 \mathrm{E}+02$ \\
\hline Totals & & & 67 & 40 & $2.25 \mathrm{E}+04$ & $1.78 \mathrm{E}+04$ \\
\hline
\end{tabular}

Table 3. Incident-Free Dose per Shipment (person-rem/shipment)

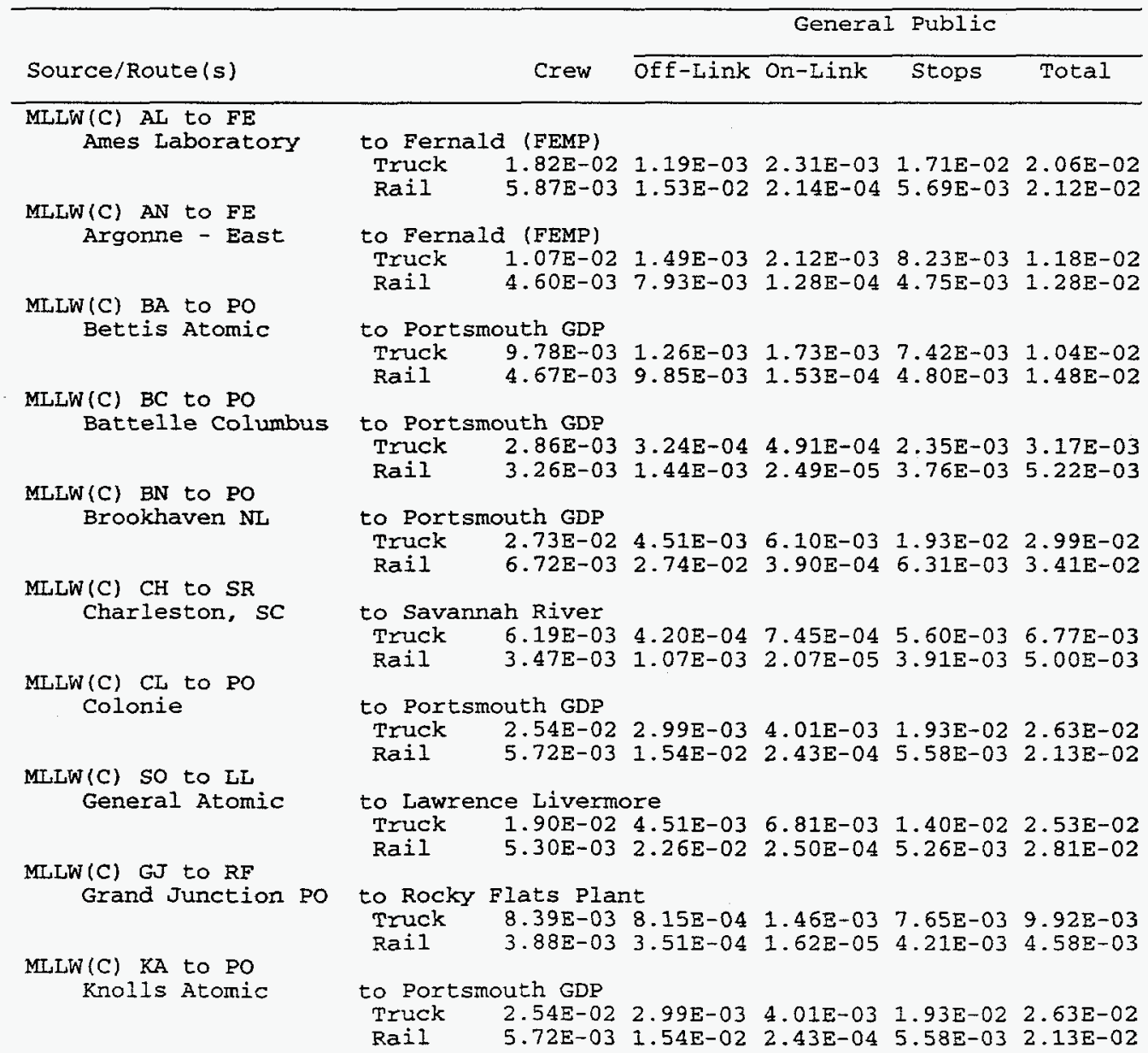


Table 3. Incident-Free Dose per Shipment (person-rem/shipment) (Cont.)

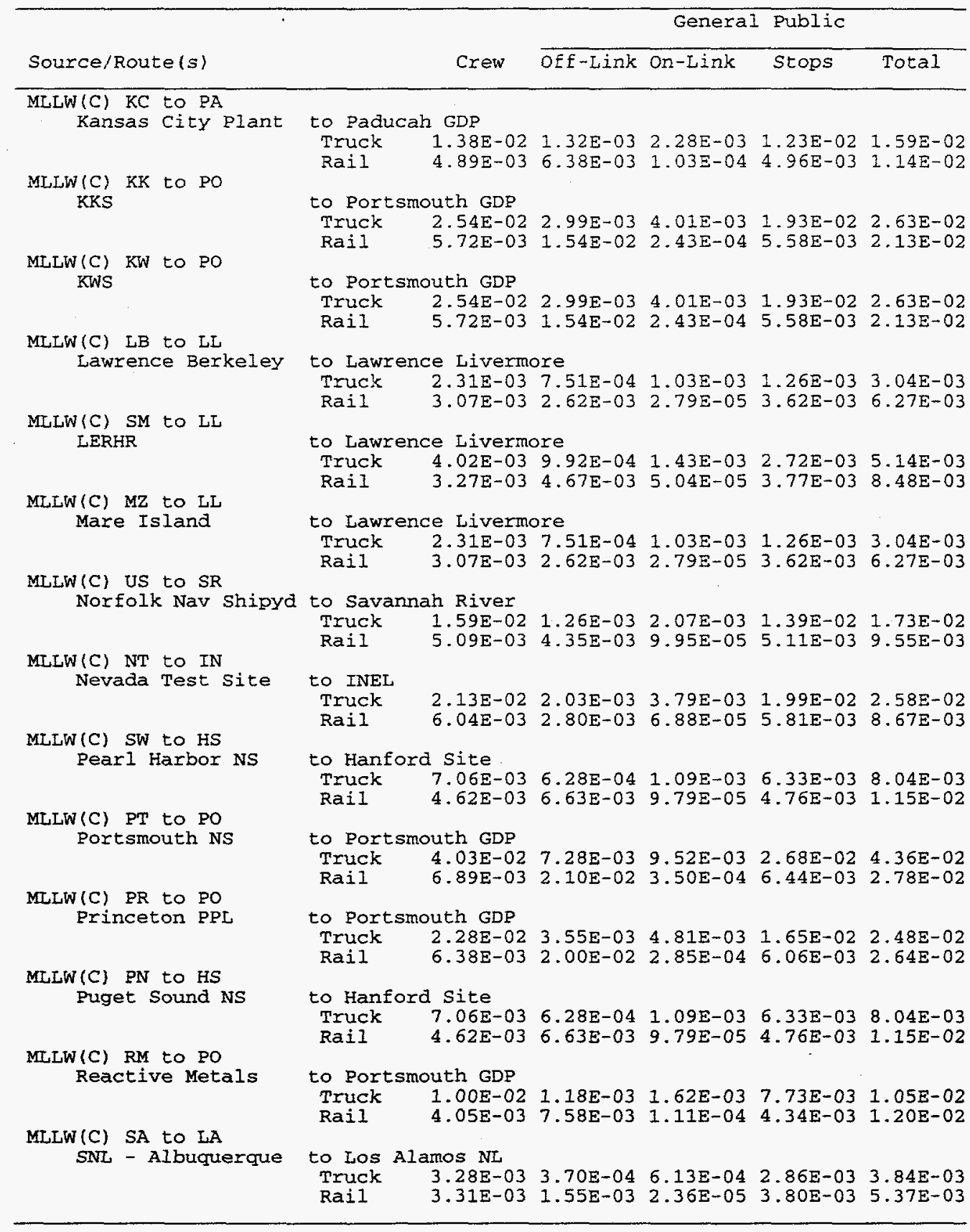


Table 4. Accident Dose Risk per Shipment (person-rem/shipment)

\begin{tabular}{|c|c|c|c|}
\hline \multicolumn{2}{|l|}{ Source/Route } & \multirow{3}{*}{$\frac{\text { Truck }}{2.21 \mathrm{E}-06}$} & \multirow{3}{*}{$\frac{\text { Rail }}{1.07 \mathrm{E}-06}$} \\
\hline MLLW (C) AL to $\mathrm{FE}$ & & & \\
\hline $\begin{array}{l}\text { Ames Laboratory } \\
\text { MLLW(C) AN to FE }\end{array}$ & to Fernald (FEMP) & & \\
\hline $\begin{array}{l}\text { Argonne - East } \\
\text { MLLW(C) BA to po }\end{array}$ & to Fernald (FEMP) & $3.87 E-04$ & $2.70 \mathrm{E}-04$ \\
\hline $\begin{array}{l}\text { Bettis Atomic } \\
\text { MLLW(C) BC to PO }\end{array}$ & to Portsmouth GDP & $1.00 E-04$ & $4.05 \mathrm{E}-05$ \\
\hline $\begin{array}{l}\text { Battelle Columbus } \\
\text { MLLW(C) BN to PO }\end{array}$ & to Portsmouth GDP & $2.49 E-10$ & $6.63 \mathrm{E}-11$ \\
\hline $\begin{array}{l}\text { Brookhaven NL } \\
\text { MLLW(C) } \mathrm{CH} \text { to } \mathrm{SR}\end{array}$ & to Portsmouth GDP & $4.93 \mathrm{E}-04$ & $2.53 \mathrm{E}-04$ \\
\hline $\begin{array}{l}\text { Charleston, SC } \\
\text { MLLW(C) CL to PO }\end{array}$ & to Savannah River & $5.98 \mathrm{E}-05$ & $6.95 \mathrm{E}-06$ \\
\hline $\begin{array}{l}\text { Colonie } \\
\text { MLLW(C) So to LL }\end{array}$ & to Portsmouth GDP & $3.28 \mathrm{E}-08$ & $7.61 E-09$ \\
\hline $\begin{array}{l}\text { General Atomic } \\
\text { MLLW(C) GJ to RF }\end{array}$ & to Lawrence Livermore & $2.24 \mathrm{E}-07$ & $2.45 E-07$ \\
\hline $\begin{array}{l}\text { Grand Junction PO } \\
\text { MLLW(C) KA to PO }\end{array}$ & to Rocky Flats Plant & $1.60 \mathrm{E}-08$ & $1.77 \mathrm{E}-10$ \\
\hline $\begin{array}{l}\text { Knolls Atomic } \\
\text { MLLW(C) KC to PA }\end{array}$ & to Portsmouth GDP & $1.98 \mathrm{E}-02$ & $4.64 \mathrm{E}-03$ \\
\hline $\begin{array}{l}\text { Ransas City Plant } \\
\text { MLLW(C) KK to PO }\end{array}$ & to Paducah GDP & $1.11 \mathrm{E}-04$ & $2.52 \mathrm{E}-0.5$ \\
\hline $\begin{array}{l}\text { KKS } \\
\text { MLLW(C) KW to PO }\end{array}$ & to Portsmouth GDP & $2.17 \mathrm{E}-02$ & $5.10 E-03$ \\
\hline $\begin{array}{l}\text { KWS } \\
\text { MLLW(C) LB to LL }\end{array}$ & to Portsmouth GDP & $6.98 E-03$ & $1.64 \mathrm{E}-03$ \\
\hline $\begin{array}{l}\text { Lawrence Berkeley } \\
\text { MLLW(C) SM to LL }\end{array}$ & to Lawrence Livermore & $7.64 \mathrm{E}-07$ & $5.42 \mathrm{E}-07$ \\
\hline LERHR & to Lawrence Liverm & $1.12 \mathrm{E}-06$ & $9.15 E-07$ \\
\hline $\begin{array}{l}\text { MLLW (C) MZ to LL } \\
\text { Mare Island } \\
\text { MLLW (C) US to SR }\end{array}$ & to Lawrence Livermore & $6.50 E-04$ & $4.68 \mathrm{E}-04$ \\
\hline $\begin{array}{l}\text { Norfolk Nav Shipyd } \\
\text { MLLW (C) NT to IN }\end{array}$ & to Savannah River & $8.05 E-04$ & $1.41 \mathrm{E}-04$ \\
\hline $\begin{array}{l}\text { Nevada Test Site } \\
\text { MLLW (C) SW to HS }\end{array}$ & to INEL & $1.30 \mathrm{E}-08$ & $2.10 \mathrm{E}-09$ \\
\hline $\begin{array}{l}\text { Pearl Harbor NS } \\
\text { MLLW (C) PT to PO }\end{array}$ & to Hanford Site & $1.11 \mathrm{E}-04$ & $8.21 E-05$ \\
\hline $\begin{array}{l}\text { Portsmouth NS } \\
\text { MLLW(C) PR to Po }\end{array}$ & to Portsmouth GDP & $3.37 E-04$ & $1.53 \mathrm{E}-04$ \\
\hline $\begin{array}{l}\text { Princeton PPL } \\
\text { MLLW(C) PN to HS }\end{array}$ & to Portsmouth GDP & $2.72 \mathrm{E}-09$ & $1.28 \mathrm{E}-09$ \\
\hline $\begin{array}{l}\text { Puget Sound NS } \\
\text { MLLW(C) RM to PO }\end{array}$ & to Hanford Site & $2.47 \mathrm{E}-03$ & $1.83 \mathrm{E}-03$ \\
\hline $\begin{array}{l}\text { Reactive Metals } \\
\text { MLIW(C) SA to LA }\end{array}$ & to Portsmouth GDP & $7.25 E-07$ & $2.09 E-07$ \\
\hline SNL - Albuquerque & to Los Alamos NL & $8.43 \mathrm{E}-05$ & $1.60 \mathrm{E}-06$ \\
\hline
\end{tabular}


Table 5. Nonradiological Risk Factors per Shipment (fatalities/shipment)

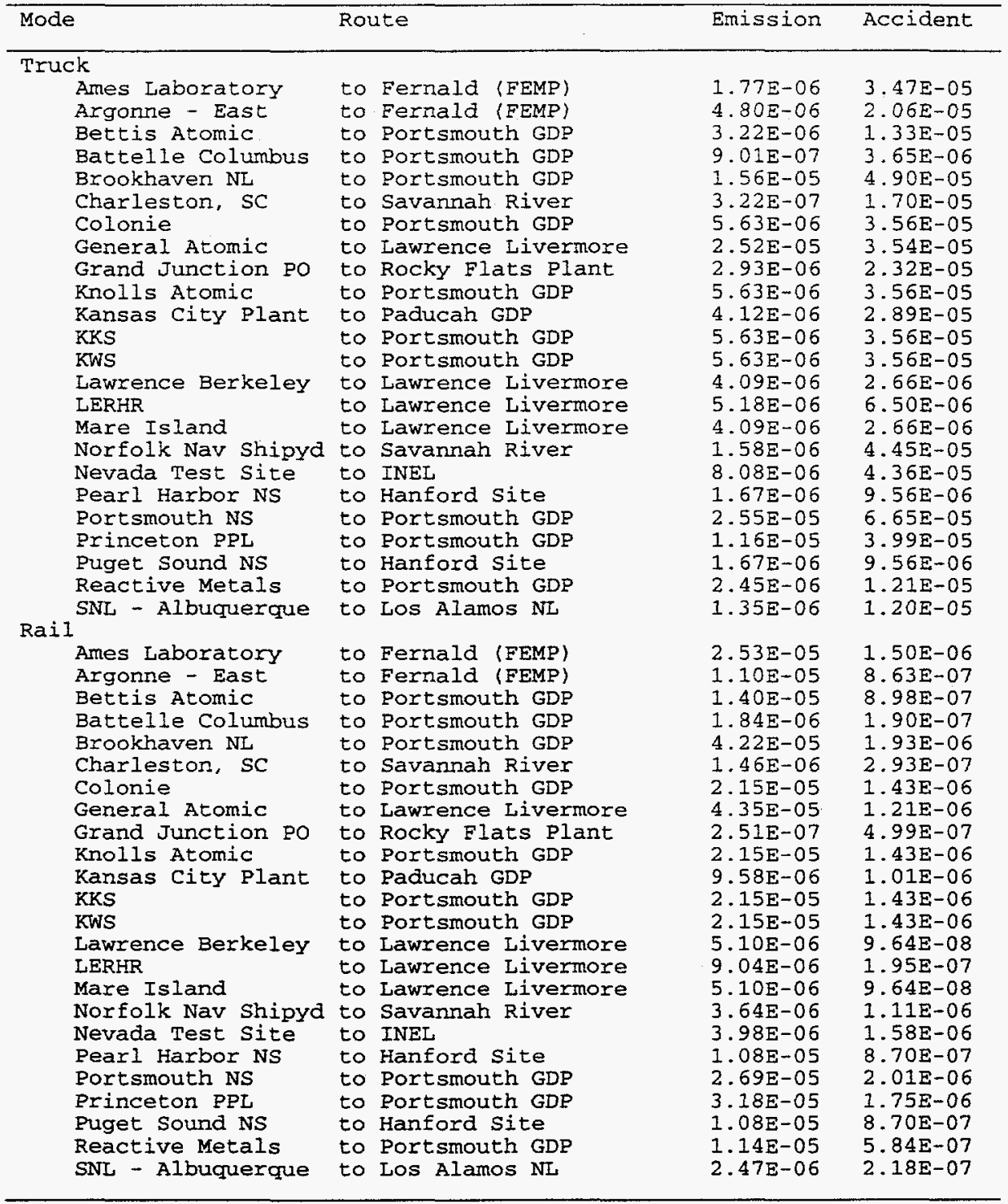


Table 6. Annual Incident-Free Dose for the Shipping Campaign (person-rem/yr)

\begin{tabular}{|c|c|c|c|c|c|c|}
\hline \multirow{2}{*}{ Route } & & & \multicolumn{2}{|c|}{ Truck } & \multicolumn{2}{|c|}{ Rail } \\
\hline & & & Crew & Public & Crew & Public \\
\hline Ames Laboratory & & Fernald (FEMP) & $1.82 \mathrm{E}-02$ & $2.06 \Xi-02$ & $.87 E-03$ & $2.12 \mathrm{E}-02$ \\
\hline Argonne - East & & Fernald (FEMP) & $4.71 \mathrm{E}-01$ & $5.21 E-01$ & $7.82 \mathrm{E}-02$ & $18 \mathrm{E}-01$ \\
\hline Bettis Atomic & to & Portsmouth GDP & $9.78 \mathrm{E}-03$ & $04 \mathrm{E}-02$ & $67 \mathrm{E}-03$ & $48 \mathrm{E}-02$ \\
\hline Battelle Columbus & to & Portsmouth GDP & $2.86 \varepsilon-03$ & $17 E-03$ & $3.26 \mathrm{E}-03$ & $5.22 E-03$ \\
\hline Brookhaven NL & to & Portsmouth GDP & $2.73 \mathrm{E}-02$ & $2.99 \pm-02$ & $6.72 \mathrm{E}-03$ & $1 E-02$ \\
\hline Charleston, SC & & Savannah River & $6.19 E-03$ & $6.77 E-03$ & $3.47 \mathrm{E}-03$ & $5.00 \mathrm{E}-03$ \\
\hline Colonie & to & Portsmouth GDP & $2.54 \mathrm{E}-02$ & $2.63 \mathrm{E}-02$ & $5.72 \mathrm{E}-03$ & $13 E-02$ \\
\hline General Atomic & to & Lawrence Livermore & $1.90 \mathrm{E}-02$ & $2.53 \mathrm{E}-02$ & $5.30 \mathrm{E}-03$ & $2.81 E-02$ \\
\hline Grand Junction Po & & Rocky Flats plant & $8.39 \mathrm{E}-03$ & $9.92 \mathrm{E}-03$ & $3.88 \mathrm{E}-03$ & $8 E-03$ \\
\hline Knolls Atomic & to & Portsmouth GDP & $2.54 \mathrm{E}-02$ & $3 E-02$ & $5.72 \mathrm{E}-03$ & $3 E-0$ \\
\hline Kansas City Plant & to & Paducah GDP & $8 \mathrm{E}-02$ & $9 E-02$ & $4.89 \mathrm{E}-03$ & $4 E-02$ \\
\hline KKS & to & Portsmouth GDP & $2.54 \mathrm{E}-02$ & $3 E-02$ & -03 & $3 E-0$ \\
\hline KWS & to & Portsmouth GDP & $4 E-02$ & $2.63 E-02$ & $5.72 \mathrm{E}-03$ & $3 E-02$ \\
\hline Lawrence Berkeley & to & Lawrence Livermore & $2.31 E-03$ & $3.04 \mathrm{E}-03$ & $3.07 \mathrm{E}-03$ & $7 E-0$ \\
\hline LERHR & to & Lawrence Livermore & $4.02 \mathrm{E}-03$ & $4 \mathrm{E}-03$ & $3.27 E-03$ & $8 E-03$ \\
\hline Mare Island & & Lawrence Livermore & $2.31 \mathrm{E}-03$ & $4 E-03$ & $3.07 E-03$ & $7 E-0$ \\
\hline Norfolk Nav Shipyd & to & Savannah River & $1.59 \mathrm{E}-02$ & $3 E-02$ & $5.09 E-03$ & $5 E-0$ \\
\hline Nevada Test Site & to & INEL & $2.13 \mathrm{E}-02$ & $2.58 \mathrm{E}-02$ & -03 & $7 E-0$ \\
\hline Pearl Harbor NS & to & Hanford Site & $7.06 \mathrm{E}-03$ & $8.04 \mathrm{E}-03$ & $4.62 E-03$ & $1.15 \mathrm{E}-02$ \\
\hline Portsmouth NS & & Portsmouth GDP & $4.03 \mathrm{E}-02$ & $4.36 E-02$ & $6.89 E-03$ & $2.78 \mathrm{E}-02$ \\
\hline Princeton PPI & & Portsmouth GDP & $3 E-02$ & $2.48 \mathrm{E}-02$ & $6.38 \mathrm{E}-03$ & $2.64 \mathrm{E}-02$ \\
\hline Puget Sound NS & to & Hanford Site & $7.06 \mathrm{E}-03$ & $8.04 E-03$ & $4.62 \mathrm{E}-03$ & $1.15 \mathrm{E}-02$ \\
\hline Reactive Metals & & Portsmouth GDP & $1.00 E-02$ & $1.05 E-02$ & $4.05 E-03$ & $1.20 \mathrm{E}-02$ \\
\hline Albuqu & to & Los Alamos NL & $3.28 \mathrm{E}-03$ & $3.84 \mathrm{E}-03$ & $3.31 E-03$ & $5.37 \mathrm{E}-03$ \\
\hline
\end{tabular}

Table 7. Annual Accident Dose Risk for the Shipping Campaign (person-rem/yr)

\begin{tabular}{lllc}
\hline Route & & Truck & Rail \\
\hline Ames Laboratory & to Fernald (FEMP) & $2.21 \mathrm{E}-06$ & $1.07 \mathrm{E}-06$ \\
Argonne - East & to Fernald (FEMP) & $1.70 \mathrm{E}-02$ & $4.59 \mathrm{E}-03$ \\
Bettis Atomic & to Portsmouth GDP & $1.00 \mathrm{E}-04$ & $4.05 \mathrm{E}-05$ \\
Battelle Columbus & to Portsmouth GDP & $2.49 \mathrm{E}-10$ & $6.63 \mathrm{E}-11$ \\
Brookhaven NL & to Portsmouth GDP & $4.93 \mathrm{E}-04$ & $2.53 \mathrm{E}-04$ \\
Charleston, SC & to Savannah River & $5.98 \mathrm{E}-05$ & $6.95 \mathrm{E}-06$ \\
Colonie & to Portsmouth GDP & $3.28 \mathrm{E}-08$ & $7.61 \mathrm{E}-09$ \\
General Atomic & to Lawrence Livermore & $2.24 \mathrm{E}-07$ & $2.45 \mathrm{E}-07$ \\
Grand Junction PO & to RockY Flats Plant & $1.60 \mathrm{E}-08$ & $1.77 \mathrm{E}-10$ \\
Knolis Atomic & to Portsmouth GDP & $1.98 \mathrm{E}-02$ & $4.64 \mathrm{E}-03$ \\
Kansas City Plant & to Paducah GDP & $1.11 \mathrm{E}-04$ & $2.52 \mathrm{E}-05$ \\
KKS & to Portsmouth GDP & $2.17 \mathrm{E}-02$ & $5.10 \mathrm{E}-03$ \\
KWS & to Portsmouth GDP & $6.98 \mathrm{E}-03$ & $1.64 \mathrm{E}-03$ \\
Lawrence Berkeley & to Lawrence Livermore & $7.64 \mathrm{E}-07$ & $5.42 \mathrm{E}-07$ \\
LERHR & to Lawrence Livermore & $1.12 \mathrm{E}-06$ & $9.15 \mathrm{E}-07$ \\
Mare Island & to Lawrence Livermore & $6.50 \mathrm{E}-04$ & $4.68 \mathrm{E}-04$ \\
Norfolk Nav Shipyd to Savannah River & $8.05 \mathrm{E}-04$ & $1.41 \mathrm{E}-04$ \\
Nevada Test Site & to INEL & $1.30 \mathrm{E}-08$ & $2.10 \mathrm{E}-09$ \\
Pearl Harbor NS & to Hanford Site & $1.11 \mathrm{E}-04$ & $8.21 \mathrm{E}-05$ \\
Portsmouth NS & to Portsmouth GDP & $3.37 \mathrm{E}-04$ & $1.53 \mathrm{E}-04$ \\
Princeton PPL & to Portsmouth GDP & $2.72 \mathrm{E}-09$ & $1.28 \mathrm{E}-09$ \\
Puget Sound NS & to Hanford Site & $2.47 \mathrm{E}-03$ & $1.83 \mathrm{E}-03$ \\
Reactive Metals & to Portsmouth GDP & $7.25 \mathrm{E}-07$ & $2.09 \mathrm{E}-07$ \\
SNL - Albuquerque & to Los Alamos NL & $8.43 \mathrm{E}-05$ & $1.60 \mathrm{E}-06$ \\
& & & \\
\hline Totals & & $7.08 \mathrm{E}-02$ & $1.90 \mathrm{E}-02$ \\
& & & \\
\hline
\end{tabular}

Table 8. Expected Annual Fatalities for the Shipping Campaign

\begin{tabular}{llc}
\hline Exposure Group & Truck & Rail \\
\hline Radiological & & \\
Normal Crew & $3.3 E-04$ & $7.6 \mathrm{E}-05$ \\
Normal Public & $4.5 \mathrm{E}-04$ & $2.8 \mathrm{E}-04$ \\
Accident Public & $3.5 \mathrm{E}-05$ & $9.5 \mathrm{E}-06$ \\
Nonradiological & & \\
Emission & $3.5 \mathrm{E}-04$ & $5.3 \mathrm{E}-04$ \\
Accident & $1.5 \mathrm{E}-03$ & $3.7 \mathrm{E}-05$ \\
\hline
\end{tabular}


Table 9. Expected Annual Cancer Incidence for the Shipping Campaign

\begin{tabular}{lll}
\hline Exposure Group & Truck & \multicolumn{1}{c}{ Rail } \\
\hline Radiological & $1.1 \mathrm{E}-03$ & $2.7 \mathrm{E}-04$ \\
Normal Crew & $1.5 \mathrm{E}-03$ & $9.5 \mathrm{E}-04$ \\
Normal Public & $1.2 \mathrm{E}-04$ & $3.2 \mathrm{E}-05$ \\
$\quad$ Accident Public & $3.5 \mathrm{E}-04$ & $5.3 \mathrm{E}-04$ \\
Nonradiological & NA & NA \\
Emission & Accident & \\
\hline
\end{tabular}

Table 10. Expected Annual Genetic Effects for the Shipping Campaign

\begin{tabular}{lll}
\hline Exposure Group & Truck & Rail \\
\hline Radiological & & \\
Normal Crew & $4.9 \mathrm{E}-05$ & $1.1 \mathrm{E}-05$ \\
Normal Public & $9.0 \mathrm{E}-05$ & $5.6 \mathrm{E}-05$ \\
Accident Public & $6.4 \mathrm{E}-06$ & $1.7 \mathrm{E}-06$ \\
Nonradiological & $\mathrm{NA}$ & $\mathrm{NA}$ \\
Emission & NA & NA \\
Accident & & \\
\hline
\end{tabular}




\section{B.8 WM LLMW REGIONALIZED 1 ALTERNATIVE (CASE 4): CONTACT-HANDLED HETEROGENEOUS SOLIDS: ALPHA - UNTREATED}

Table 1. Summary of Route Information

\begin{tabular}{|c|c|c|c|c|c|}
\hline & \multirow[b]{2}{*}{ Route } & \multirow{2}{*}{$\begin{array}{c}\text { Distance } \\
\text { (miles) }\end{array}$} & \multicolumn{3}{|c|}{ Percentage in Zone } \\
\hline & & & Rural & Suburban & Urban \\
\hline \multicolumn{6}{|l|}{ Truck } \\
\hline Bettis Atomic & to Savannah River & 656 & 71.7 & 26.4 & 1.9 \\
\hline Mound Plant & to Savannah River & 656 & 66.5 & 32.7 & 0.8 \\
\hline U of MO - Columbia & to Savannah River & 858 & 70.6 & 27.0 & 2.3 \\
\hline West Valley DR & to Savannah River & 883 & 70.3 & 28.5 & 1.2 \\
\hline \multicolumn{6}{|l|}{ Rail } \\
\hline Bettis Atomic & to Savannah River & 947 & 68.5 & 27.1 & 4.4 \\
\hline Mound Plant & to Savannah River & 744 & 57.4 & 29.2 & 3.3 \\
\hline U of Mo - Columbia & to Savannah River & 1011 & 66.6 & 29.5 & 4.0 \\
\hline West VaIley $D P$ & to Savannah River & 1217 & 62.8 & 32.4 & 4.9 \\
\hline
\end{tabular}

Table 2. Annual Number of Shipments and One-Way Shipment Distances

\begin{tabular}{|c|c|c|c|c|c|}
\hline \multirow[b]{2}{*}{ Route } & & \multicolumn{2}{|c|}{$\#$ of shipments } & \multicolumn{2}{|c|}{ Total Mileage (mi) } \\
\hline & & Truck & Rail & Truck & Rail \\
\hline $\begin{array}{l}\text { Bettis Atomic } \\
\text { Mound Plant } \\
\text { U of Mo - Columbia } \\
\text { West Valley DP }\end{array}$ & $\begin{array}{l}\text { to Savannah River } \\
\text { to Savannah River } \\
\text { to Savannah River } \\
\text { to Savannah River }\end{array}$ & $\begin{array}{l}1 \\
1 \\
1 \\
1\end{array}$ & $\begin{array}{l}1 \\
1 \\
1 \\
1\end{array}$ & $\begin{array}{l}6.56 \mathrm{E}+02 \\
6.56 \mathrm{E}+02 \\
8.58 \mathrm{E}+02 \\
8.83 \mathrm{E}+02\end{array}$ & $\begin{array}{l}9.47 \mathrm{E}+02 \\
7.44 \mathrm{E}+02 \\
1.01 \mathrm{E}+03 \\
1.22 \mathrm{E}+03\end{array}$ \\
\hline Totals & & 4 & 4 & $3.05 E+03$ & $3.92 E+03$ \\
\hline
\end{tabular}

Table 3. Incident-Free Dose per Shipment (person-rem/shipment)

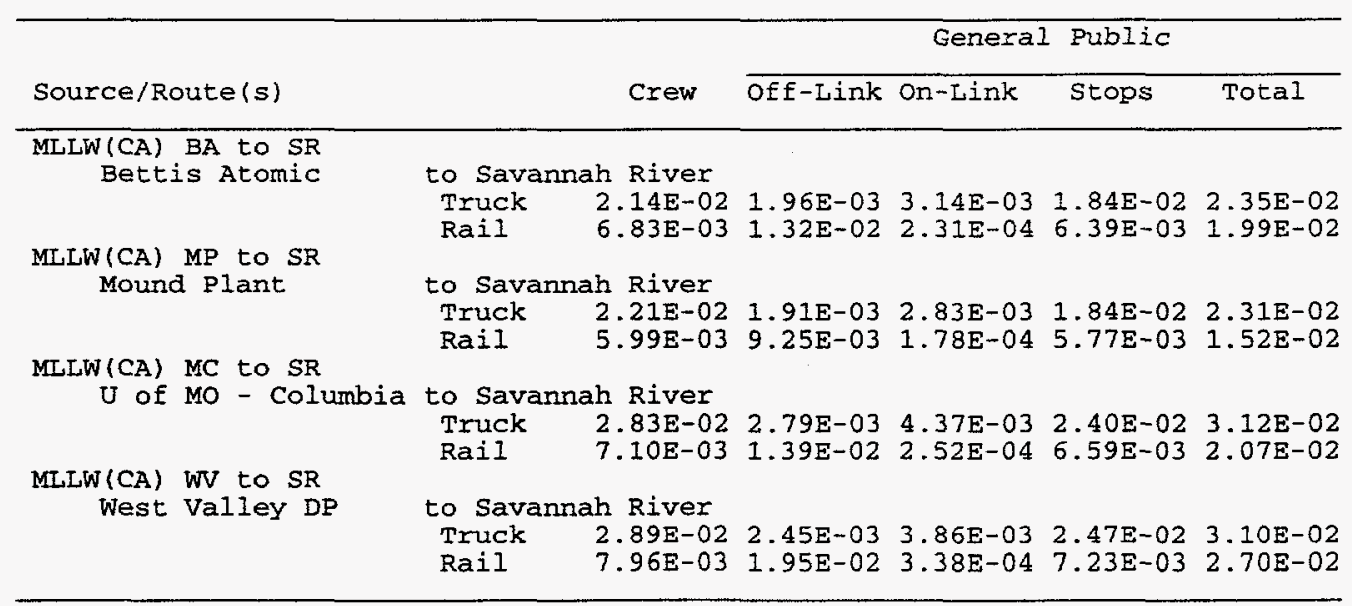

Table 4. Accident Dose Risk per Shipment (person-rem/shipment)

\begin{tabular}{llll}
\hline Source/Route & & Truck & Rail \\
\hline $\begin{array}{c}\text { MLLW (CA) BA to SR } \\
\text { Bettis Atomic }\end{array}$ & to Savannah River & $2.11 \mathrm{E}-04$ & $7.45 \mathrm{E}-05$ \\
$\begin{array}{c}\text { MLLW (CA) MP to SR } \\
\text { Mound Plant }\end{array}$ & $\begin{array}{c}\text { to Savannah River } \\
\text { MLLW (CA) MC to SR } \\
\text { U of MO - Columbia }\end{array}$ & $9.48 \mathrm{E}-08$ & $1.85 \mathrm{E}-08$ \\
$\begin{array}{c}\text { MLLW(CA) WV to SR } \\
\text { West Valley DP }\end{array}$ & to Savannah River & $6.78 \mathrm{E}-07$ & $2.05 \mathrm{E}-07$ \\
\end{tabular}


Table 5. Nonradiological Risk Factors per Shipment (Eatalities/shipment)

\begin{tabular}{|c|c|c|c|}
\hline Mode & Route & Emission & Accident \\
\hline \multicolumn{4}{|l|}{ Truck } \\
\hline Bettis Atomic & to Savannah River & $3.99 E-06$ & $4.90 E-05$ \\
\hline Mound Plant & to Savannah River & $1.67 \mathrm{E}-06$ & $5.26 E-05$ \\
\hline U of MO - Columbia & to Savannah River & $6.41 E-06$ & $6.09 E-05$ \\
\hline West Valley DP & to Savannah River & $3.28 E-06$ & $6.50 E-05$ \\
\hline \multicolumn{4}{|l|}{ Rail } \\
\hline Bettis Atomic & to Savannah River & $1.74 \mathrm{E}-05$ & I. $98 \mathrm{E}-06$ \\
\hline Mound Plant & to Savannah River & $1.03 E-05$ & $1.56 \mathrm{E}-06$ \\
\hline U of MO - Columbia & to Savannah River & $1.69 E-05$ & $2.12 E-06$ \\
\hline West Valley DP & to Savannah River & $2.49 E-05$ & $2.55 E-06$ \\
\hline
\end{tabular}

Table 6. Annual Incident-Free Dose for the Shipping Campaign (person-rem/yr)

\begin{tabular}{|c|c|c|c|c|c|c|}
\hline \multirow[b]{2}{*}{ Route } & & & \multicolumn{2}{|c|}{ Truck } & \multicolumn{2}{|c|}{ Rail } \\
\hline & & & Crew & Public & Crew & Public \\
\hline $\begin{array}{l}\text { Bettis Atomic } \\
\text { Mound Plant } \\
\text { U of MO - Columbia } \\
\text { West Valley DP }\end{array}$ & $\begin{array}{l}\text { to } \\
\text { to } \\
\text { to } \\
\text { to }\end{array}$ & $\begin{array}{l}\text { Savannah River } \\
\text { Savannah River } \\
\text { Savannah River } \\
\text { Savannah River }\end{array}$ & $\begin{array}{l}2.14 \mathrm{E}-02 \\
2.21 \mathrm{E}-02 \\
2.83 \mathrm{E}-02 \\
2.89 \mathrm{E}-02\end{array}$ & $\begin{array}{l}2.35 E-02 \\
2.31 E-02 \\
3.12 E-02 \\
3.10 E-02\end{array}$ & $\begin{array}{l}6.83 E-03 \\
5.99 E-03 \\
7.10 E-03 \\
7.96 E-03\end{array}$ & $\begin{array}{l}1.99 \mathrm{E}-02 \\
1.52 \mathrm{E}-02 \\
2.07 \mathrm{E}-02 \\
2.70 \mathrm{E}-02\end{array}$ \\
\hline Totals & & & $1.01 \mathrm{E}-01$ & $1.09 \mathrm{E}-01$ & $2.79 \mathrm{E}-02$ & $8.28 \mathrm{E}-02$ \\
\hline
\end{tabular}

Table 7. Annual Accident Dose Risk for the Shipping Campaign (person-rem/yr)

\begin{tabular}{|c|c|c|c|}
\hline Route & & Truck & Rail \\
\hline $\begin{array}{l}\text { Bettis Atomic } \\
\text { Mound Plant } \\
\text { U of MO - Columbia } \\
\text { West Valley DP }\end{array}$ & $\begin{array}{l}\text { to Savannah River } \\
\text { to Savannah River } \\
\text { to Savannah River } \\
\text { to Savannah River }\end{array}$ & $\begin{array}{l}2.11 \mathrm{E}-04 \\
9.48 \mathrm{E}-08 \\
6.78 \mathrm{E}-07 \\
3.36 \mathrm{E}-04\end{array}$ & $\begin{array}{l}7.45 \mathrm{E}-05 \\
1.85 \mathrm{E}-08 \\
2.05 \mathrm{E}-07 \\
1.07 \mathrm{E}-04\end{array}$ \\
\hline Totals & & $5.47 \mathrm{E}-04$ & $1.82 \mathrm{E}-04$ \\
\hline
\end{tabular}

Table 8. Expected Annual Fatalities for the Shipping Campaign

\begin{tabular}{lll}
\hline Exposure Group & Truck & Rail \\
\hline Radiological & $4.0 \mathrm{E}-05$ & $1.1 \mathrm{E}-05$ \\
Normal Crew & $5.4 \mathrm{E}-05$ & $4.1 \mathrm{E}-05$ \\
Normal Public & $2.7 \mathrm{E}-07$ & $9.1 \mathrm{E}-08$ \\
Accident Public & & \\
Nonradiological & $1.5 \mathrm{E}-05$ & $6.9 \mathrm{E}-05$ \\
Emission & $2.3 \mathrm{E}-04$ & $8.2 \mathrm{E}-06$ \\
Accident & \\
\hline
\end{tabular}

Table 9. Expected Annual Cancer Incidence for the shipping Campaign

\begin{tabular}{lll}
\hline Exposure Group & Truck & Rail \\
\hline Radiological & & \\
Normal Crew & $1.4 \mathrm{E}-04$ & $3.9 \mathrm{E}-05$ \\
Normal Public & $1.8 \mathrm{E}-04$ & $1.4 \mathrm{E}-04$ \\
Accident Public & $9.3 \mathrm{E}-07$ & $3.1 \mathrm{E}-07$ \\
Nonradiological & $1.5 \mathrm{E}-05$ & $6.9 \mathrm{E}-05$ \\
Emission & $\mathrm{NA}$ & $\mathrm{NA}$ \\
Accident & & \\
\hline
\end{tabular}


Table 10. Expected Annual Genetic Effects for the Shipping Campaign

\begin{tabular}{lll}
\hline Exposure Group & Truck & Rail \\
\hline Radiological & & \\
Normal Crew & $6.0 \mathrm{E}-06$ & $1.7 \mathrm{E}-06$ \\
$\quad$ Normal Public & $1.1 \mathrm{E}-05$ & $8.3 \mathrm{E}-06$ \\
Accident Public & $4.1 \mathrm{E}-08$ & $1.4 \mathrm{E}-08$ \\
Nonradiological & & \\
$\quad$ Emission & NA & NA \\
Accident & NA & NA \\
\hline
\end{tabular}




\section{B.9 WM LLMW REGIONALIZED 1 ALTERNATIVE (CASE 4): CONTACT-HANDLED HETEROGENEOUS SOLIDS: NONALPHA - TREATED}

Table 1. Summary of Route Information

\begin{tabular}{|c|c|c|c|c|c|c|}
\hline & \multirow{2}{*}{\multicolumn{2}{|c|}{ Route }} & \multirow{2}{*}{$\begin{array}{c}\text { Distance } \\
\text { (miles) }\end{array}$} & \multicolumn{3}{|c|}{ Percentage in Zone } \\
\hline & & & & Rural & Suburban & Urban \\
\hline \multicolumn{7}{|l|}{ Truck } \\
\hline Ames Laboratory & to & Portsmouth GDP & 755 & 77.4 & 21.0 & 1.7 \\
\hline Argonne - East & to & Portsmouth GDP & 428 & 64.6 & 30.5 & 4.9 \\
\hline Charleston, SC & to & Savannah River & 200 & 75.7 & 23.8 & 0.5 \\
\hline Colonie & & Portsmouth GDP & 688 & 57.3 & 40.1 & 2.5 \\
\hline ETEC & to & Lawrence Livermore & 406 & 77.9 & 10.0 & 12.1 \\
\hline Fernald (FEMP) & to & Portsmouth GDP & 173 & 68.0 & 31.6 & 0.3 \\
\hline General Atomic & to & Lawrence Iivermore & 500 & 69.2 & 15.1 & 15.6 \\
\hline Grand Junction Po & to & Rocky Flats Plant & 273 & 79.9 & 16.7 & 3.3 \\
\hline LERHR & to & Lawrence Livermore & 97 & 58.7 & 24.7 & 16.6 \\
\hline Reactive Metals & to & Portsmouth GDP & 276 & 59.5 & 37.8 & 2.8 \\
\hline \multicolumn{7}{|l|}{ Rail } \\
\hline Ames Laboratory & to & Portsmouth GDP & 727 & 68.6 & 23.8 & 7.7 \\
\hline Argonne - East & to & Portsmouth GDP & 422 & 64.8 & 30.0 & 5.1 \\
\hline Charleston, SC & to & Savannah River & 140 & 83.9 & 13.6 & 2.5 \\
\hline Colonie & to & Portsmouth GDP & 681 & 52.1 & 40.3 & 7.5 \\
\hline ETEC & to & Lawrence Livermore & 474 & 60.0 & 24.8 & 15.2 \\
\hline Fernald (FEMP) & to & Portsmouth GDP & 207 & 69.6 & 26.5 & 4.0 \\
\hline General Atomic & to & Lawrence Livermore & 579 & 57.1 & 24.9 & 18.0 \\
\hline Grand Junction PO & to & Rocky Flats Plant & 239 & 95.5 & 4.3 & 0.3 \\
\hline LERHR & to & Iawrence Iivermore & 93 & 45.5 & 31.3 & 23.2 \\
\hline Reactive Metals & to & Portsmouth GDP & 279 & 48.4 & 41.8 & 9.8 \\
\hline
\end{tabular}

Table 2. Annual Number of Shipments and One-Way Shipment Distances

\begin{tabular}{|c|c|c|c|c|c|}
\hline \multirow[b]{2}{*}{ Route } & & \multicolumn{2}{|c|}{ \# of shipments } & \multicolumn{2}{|c|}{ Total Mileage (mi) } \\
\hline & & Truck & Rail & Truck & Rail \\
\hline $\begin{array}{l}\text { Ames Laboratory } \\
\text { Argonne - East } \\
\text { Charleston, SC } \\
\text { Colonie } \\
\text { ETEC } \\
\text { Fernald (FEMP) } \\
\text { General Atomic } \\
\text { Grand Junction PO } \\
\text { LERHR } \\
\text { Reactive Metals }\end{array}$ & $\begin{array}{l}\text { to Portsmouth GDP } \\
\text { to Portsmouth GDP } \\
\text { to Savanmah River } \\
\text { to Portsmouth GDP } \\
\text { to Lawrence Livermore } \\
\text { to Portsmouth GDP } \\
\text { to Lawrence Iivermore } \\
\text { to Rocky Flats Plant } \\
\text { to Lawrence Livermore } \\
\text { to Portsmouth GDP }\end{array}$ & $\begin{array}{r}1 \\
1 \\
1 \\
1 \\
11 \\
61 \\
1 \\
1 \\
1 \\
1\end{array}$ & $\begin{array}{r}1 \\
1 \\
1 \\
1 \\
4 \\
23 \\
1 \\
1 \\
1 \\
1\end{array}$ & $\begin{array}{l}7.55 \mathrm{E}+02 \\
4.28 \mathrm{E}+02 \\
2.00 \mathrm{E}+02 \\
6.88 \mathrm{E}+02 \\
4.46 \mathrm{E}+03 \\
1.05 \mathrm{E}+04 \\
5.00 \mathrm{E}+02 \\
2.73 \mathrm{E}+02 \\
9.72 \mathrm{E}+01 \\
2.76 \mathrm{E}+02\end{array}$ & $\begin{array}{l}7.27 \mathrm{E}+02 \\
4.22 \mathrm{E}+02 \\
1.40 \mathrm{E}+02 \\
6.81 \mathrm{E}+02 \\
1.90 \mathrm{E}+03 \\
4.77 \mathrm{E}+03 \\
5.79 \mathrm{E}+02 \\
2.39 \mathrm{E}+02 \\
9.30 \mathrm{E}+01 \\
2.79 \mathrm{E}+02\end{array}$ \\
\hline Totals & & 80 & 35 & $1.82 \mathrm{E}+04$ & $9.83 \mathrm{E}+03$ \\
\hline
\end{tabular}


Table 3. Incident-Free Dose per Shipment (person-rem/shipment)

\begin{tabular}{|c|c|c|c|c|c|c|}
\hline \multirow[b]{2}{*}{ Source/Route (s) } & & \multirow[b]{2}{*}{ Crew } & \multicolumn{4}{|c|}{ General Public } \\
\hline & & & off-Link & On-Link & Stops & Total \\
\hline \multirow{2}{*}{\multicolumn{7}{|c|}{$\begin{aligned} \text { MLLW(CT) AL to PO } \\
\text { Ames Laboratory }\end{aligned}$}} \\
\hline & to Portsn & nouth GDP & & & & \\
\hline & $\begin{array}{l}\text { Truck } \\
\text { Rail }\end{array}$ & $\begin{array}{l}2.33 \mathrm{E}-02 \\
5.91 \mathrm{E}-03\end{array}$ & $\begin{array}{l}1.86 \mathrm{E}-03 \\
1.42 \mathrm{E}-02\end{array}$ & $\begin{array}{l}3.31 \mathrm{E}-03 \\
2.00 \mathrm{E}-04\end{array}$ & $\begin{array}{l}2.11 E-02 \\
5.72 E-03\end{array}$ & $\begin{array}{l}2.63 \mathrm{E}-02 \\
2.01 \mathrm{E}-02\end{array}$ \\
\hline \multirow{2}{*}{\multicolumn{7}{|c|}{$\begin{array}{l}\text { MLLW(CT) AN to PO } \\
\text { Argonne - East }\end{array}$}} \\
\hline & & & & & & \\
\hline & $\begin{array}{l}\text { Truck } \\
\text { Rail }\end{array}$ & $\begin{array}{l}1.53 \mathrm{E}-02 \\
4.64 \mathrm{E}-03\end{array}$ & $\begin{array}{l}2.05 \mathrm{E}-03 \\
6.76 \mathrm{E}-03\end{array}$ & $\begin{array}{l}2.99 \mathrm{E}-03 \\
1.14 \mathrm{E}-04\end{array}$ & $\begin{array}{l}1.20 \mathrm{E}-02 \\
4.78 \mathrm{E}-03\end{array}$ & $\begin{array}{l}1.70 \mathrm{E}-02 \\
1.17 \mathrm{E}-02\end{array}$ \\
\hline \multicolumn{7}{|l|}{$\mathrm{MLLW}(\mathrm{CT}) \mathrm{CH}$ to $\mathrm{SR}$} \\
\hline Charleston, SC & to Savanr & nah River & & & & \\
\hline & $\begin{array}{l}\text { Truck. } \\
\text { Rail }\end{array}$ & $\begin{array}{l}6.19 \mathrm{E}-03 \\
3.47 \mathrm{E}-03\end{array}$ & $\begin{array}{l}4.20 \mathrm{E}-04 \\
1.07 \mathrm{E}-03\end{array}$ & $\begin{array}{l}7.45 \mathrm{E}-04 \\
2.07 \mathrm{E}-05\end{array}$ & $\begin{array}{l}5.60 E-03 \\
3.91 E-03\end{array}$ & $\begin{array}{l}6.77 \mathrm{E}-03 \\
5.00 \mathrm{E}-03\end{array}$ \\
\hline \multirow{2}{*}{\multicolumn{7}{|c|}{ MLLW (CT) CL to PO }} \\
\hline & & & & & & \\
\hline & Truck & $2.54 \mathrm{E}-02$ & $2.99 E-03$ & $4.01 E-03$ & $1.93 E-02$ & $2.63 \Xi-02$ \\
\hline \multirow{2}{*}{\multicolumn{7}{|c|}{ ce Livermore }} \\
\hline & & & & & & \\
\hline & Truck & $\begin{array}{l}1.39 \mathrm{E}-02 \\
4.86 \mathrm{E}-03\end{array}$ & $\begin{array}{l}2.80 \mathrm{E}-03 \\
1.60 \mathrm{E}-02\end{array}$ & $\begin{array}{l}4.47 E-03 \\
86 E-04\end{array}$ & $\begin{array}{l}1.14 E-02 \\
4.94 E-03\end{array}$ & $\begin{array}{l}1.86 \mathrm{E}-02 \\
2.11 \mathrm{E}-02\end{array}$ \\
\hline \multirow{2}{*}{\multicolumn{7}{|c|}{$\begin{array}{l}\text { MLIW (CT) FE to PO } \\
\text { Fernald (FEMP) }\end{array}$}} \\
\hline & & & & & & \\
\hline & Truck & $5.72 \mathrm{E}-03$ & $4.51 E-04$ & $6.85 E-04$ & $4.84 E-03$ & $5.98 \mathrm{E}-03$ \\
\hline & Rail & $3.75 \mathrm{E}-03$ & $2.71 \mathrm{E}-03$ & $4.86 \mathrm{E}-05$ & $4.12 \mathrm{E}-03$ & $6.87 \mathrm{E}-03$ \\
\hline \multirow{2}{*}{\multicolumn{7}{|c|}{$\begin{array}{l}\text { MLIW(CT) So to LL } \\
\text { General Atomic }\end{array}$}} \\
\hline & & & & & & \\
\hline & Truck & $1.90 \mathrm{E}-02$ & $4.51 E-03$ & $6.81 E-03$ & $1.40 \mathrm{E}-02$ & $2.53 E-02$ \\
\hline \multirow{2}{*}{\multicolumn{7}{|c|}{ MLLW (CT) GJ to RF }} \\
\hline & & & & & & \\
\hline Grand Junclion Fu & Truck & $8.39 \mathrm{E}-03$ & $8.15 \mathrm{E}-04$ & $1.46 \mathrm{E}-03$ & $7.65 E-03$ & .9 \\
\hline & & $3.88 \mathrm{E}-03$ & $3.51 \mathrm{E}-04$ & $1.62 \mathrm{E}-05$ & $4.21 E-03$ & $4.58 \mathrm{E}-\mathrm{C}$ \\
\hline \multirow{2}{*}{\multicolumn{7}{|c|}{$\underset{\text { LERHR }}{M L I W(C T)}$ SM to LL }} \\
\hline LERHR & to Lawren & ace Livermo & ore & & & \\
\hline & Truck & $4.02 \mathrm{E}-03$ & $9.92 \mathrm{E}-04$ & $1.43 E-03$ & $2.72 \mathrm{E}-03$ & $5.14 \mathrm{E}-03$ \\
\hline \multirow{3}{*}{$\begin{array}{l}\text { MLLW(CT) RM to PO } \\
\text { Reactive Metals }\end{array}$} & $\operatorname{Ra} 11$ & 3 & 4 & 5 & 03 & $8.48 \mathrm{E}-03$ \\
\hline & to Portsm & nouth GDP & & & & \\
\hline & $\begin{array}{l}\text { Truck } \\
\text { Rail }\end{array}$ & $\begin{array}{l}1.00 \mathrm{E}-02 \\
4.05 \mathrm{E}-03\end{array}$ & $\begin{array}{l}1.18 \mathrm{E}-03 \\
7.58 \mathrm{E}-03\end{array}$ & $\begin{array}{l}1.62 \mathrm{E}-03 \\
1.11 \mathrm{E}-04\end{array}$ & $\begin{array}{l}7.73 E-03 \\
4.34 E-03\end{array}$ & $\begin{array}{l}1.05 \mathrm{E}-02 \\
1.20 \mathrm{E}-02\end{array}$ \\
\hline
\end{tabular}

Table 4. Accident Dose Risk per Shipment (person-rem/shipment)

\begin{tabular}{|c|c|c|c|}
\hline \multicolumn{2}{|l|}{ Source/Route } & \multirow{3}{*}{$\frac{\text { Truck }}{3.14 \mathrm{E}-08}$} & \multirow{3}{*}{$\frac{\text { Rail }}{1.28 \mathrm{E}-08}$} \\
\hline MLLW(CT) AL to PO & & & \\
\hline Ames Laboratory & to Portsmouth GDP & & \\
\hline $\begin{array}{l}\text { MLLW(CT) AN to PO } \\
\text { Argonne - East }\end{array}$ & to Portsmouth GDP & .05 & $2.13 E-06$ \\
\hline $\mathrm{MLLW}(\mathrm{CT}) \mathrm{CH}$ to $\mathrm{SR}$ & & & \\
\hline $\begin{array}{l}\text { Charleston, SC } \\
\text { MLLW(CT) CL to PO }\end{array}$ & to Savannah River & $4.40 \mathrm{E}-05$ & $5.11 E-06$ \\
\hline $\begin{array}{l}\text { Colonie } \\
\text { MLLW(CT) LC to LL }\end{array}$ & to Portsmouth GDP & $1.48 E-07$ & $3.44 \mathrm{E}-08$ \\
\hline $\begin{array}{l}\text { ETEC } \\
\text { MLLW(CT) FE to Po }\end{array}$ & to Lawrence Livermore & $5.06 E-05$ & $1.78 \mathrm{E}-04$ \\
\hline $\begin{array}{l}\text { Fernald (FEMP) } \\
\operatorname{MLLW}(\mathrm{CT}) \text { So to LL }\end{array}$ & to Portsmouth GDP & $9.35 E-05$ & $5.55 \mathrm{E}-05$ \\
\hline $\begin{array}{l}\text { General Atomic } \\
\text { MLLW (CT) GJ to RF }\end{array}$ & to Lawrence Livermore & $9.14 \mathrm{E}-07$ & $9.99 E-07$ \\
\hline $\begin{array}{l}\text { Grand Junction PO } \\
\text { MLLW(CT) SM to LI }\end{array}$ & to Rocky Flats Plant & $3.47 E-08$ & $3.83 \mathrm{E}-10$ \\
\hline LERHR & to Lawrence Livermore & $1.50 \mathrm{E}-07$ & $1.22 \mathrm{E}-07$ \\
\hline $\begin{array}{l}\text { MLLW (CT) RM to PO } \\
\text { Reactive Metals }\end{array}$ & to Portsmouth GDP & $3.05 \mathrm{E}-07$ & $8.81 E-08$ \\
\hline
\end{tabular}


Table 5. Nonradiological Risk Factors per Shipment (fatalities/shipment)

\begin{tabular}{llll}
\hline Mode & Route & Emission & Accident \\
\hline Truck & & & \\
Ames Laboratory & to Portsmouth GDP & $4.02 \mathrm{E}-06$ & $4.11 \mathrm{E}-05$ \\
Argonne - East & to Portsmouth GDP & $6.73 \mathrm{E}-06$ & $2.60 \mathrm{E}-05$ \\
Charleston, SC & to Savannah River & $3.22 \mathrm{E}-07$ & $1.70 \mathrm{E}-05$ \\
Colonie & to Portsmouth GDP & $5.53 \mathrm{E}-06$ & $3.56 \mathrm{E}-05$ \\
ETEC & to Lawrence Livermore & $1.59 \mathrm{E}-05$ & $3.01 \mathrm{E}-05$ \\
Fernald (FEMP) & to Portsmouth GDP & $1.93 \mathrm{E}-07$ & $7.51 \mathrm{E}-06$ \\
General Atomic & to Lawrence Livermore & $2.52 \mathrm{E}-05$ & $3.54 \mathrm{E}-05$ \\
Grand Junction Po & to Rocky Flats Plant & $2.93 \mathrm{E}-06$ & $2.32 \mathrm{E}-05$ \\
LERHR & to Lawrence Livermore & $5.18 \mathrm{E}-06$ & $6.50 \mathrm{E}-06$ \\
Reactive Metals & to Portsmouth GDP & $2.45 \mathrm{E}-06$ & $1.21 \mathrm{E}-05$ \\
Rail & & $2.34 \mathrm{E}-05$ & $1.52 \mathrm{E}-06$ \\
Ames Laboratory & to Portsmouth GDP & $9.08 \mathrm{E}-06$ & $8.84 \mathrm{E}-07$ \\
Argonne - East & to Portsmouth GDP & $1.46 \mathrm{E}-06$ & $2.93 \mathrm{E}-07$ \\
Charleston, SC & to Savannah River & $2.15 \mathrm{E}-05$ & $1.43 \mathrm{E}-06$ \\
Colonie & to Portsmouth GDP & $3.01 \mathrm{E}-05$ & $9.92 \mathrm{E}-07$ \\
ETEC & to Lawrence Livernore & $3.43 \mathrm{E}-06$ & $4.34 \mathrm{E}-07$ \\
Fernald (FEMP) & to Portsmouth GDP & $4.35 \mathrm{E}-05$ & $1.21 \mathrm{E}-06$ \\
General Atomic & to Lawrence Livermore & $2.51 \mathrm{E}-07$ & $4.99 \mathrm{E}-07$ \\
Grand Junction PO to Rocky Flats Plant & $9.04 \mathrm{E}-06$ & $1.95 \mathrm{E}-07$ \\
LERHR & to Lawrence Livermore & $1.14 \mathrm{E}-05$ & $5.84 \mathrm{E}-07$ \\
Reactive Metals & to Portsmouth GDP & & \\
\hline
\end{tabular}

Table 6. Annual Incident-Free Dose for the Shipping Campaign (person-rem/yr)

\begin{tabular}{|c|c|c|c|c|c|c|}
\hline \multirow[b]{2}{*}{ Route } & & & \multicolumn{2}{|c|}{ Truck } & \multicolumn{2}{|c|}{$\operatorname{Rai1}$} \\
\hline & & & Crew & Public & Crew & Public \\
\hline $\begin{array}{l}\text { Ames Laboratory } \\
\text { Argonne - East } \\
\text { Charleston, SC } \\
\text { Colonie } \\
\text { ETEC } \\
\text { Fernald (FEMP) } \\
\text { General Atomic } \\
\text { Grand Junction Po } \\
\text { LERHR } \\
\text { Reactive Metals }\end{array}$ & $\begin{array}{l}\text { to } \\
\text { to } \\
\text { to } \\
\text { to } \\
\text { to } \\
\text { to } \\
\text { to } \\
\text { to } \\
\text { to } \\
\text { to }\end{array}$ & $\begin{array}{l}\text { Portsmouth GDP } \\
\text { Portsmouth GDP } \\
\text { Savannah River } \\
\text { Portsmouth GDP } \\
\text { Lawrence Livermore } \\
\text { Portsmouth GDP } \\
\text { Lawrence Livermore } \\
\text { Rocky Flats Plant } \\
\text { Lawrence Livermore } \\
\text { Portsmouth GDP }\end{array}$ & $\begin{array}{l}2.33 \mathrm{E}-02 \\
1.53 \mathrm{E}-02 \\
6.19 \mathrm{E}-03 \\
2.54 \mathrm{E}-02 \\
1.53 \mathrm{E}-01 \\
3.49 \mathrm{E}-01 \\
1.90 \mathrm{E}-02 \\
8.39 \mathrm{E}-03 \\
4.02 \mathrm{E}-03 \\
1.00 \mathrm{E}-02\end{array}$ & $\begin{array}{l}2.63 E-02 \\
1.70 E-02 \\
6.77 E-03 \\
2.63 E-02 \\
2.05 E-01 \\
3.65 E-01 \\
2.53 E-02 \\
9.92 E-03 \\
5.14 E-03 \\
1.05 E-02\end{array}$ & $\begin{array}{l}5.91 \mathrm{E}-03 \\
4.64 \mathrm{E}-03 \\
3.47 \mathrm{E}-03 \\
5.72 \mathrm{E}-03 \\
1.94 \mathrm{E}-02 \\
8.62 \mathrm{E}-02 \\
5.30 \mathrm{E}-03 \\
3.88 \mathrm{E}-03 \\
3.27 \mathrm{E}-03 \\
4.05 \mathrm{E}-03\end{array}$ & $\begin{array}{l}2.01 E-02 \\
1.17 E-02 \\
5.00 E-03 \\
2.13 E-02 \\
8.45 E-02 \\
1.58 E-01 \\
2.81 E-02 \\
4.58 E-03 \\
8.48 E-03 \\
1.20 E-02\end{array}$ \\
\hline Totals & & & $0.14 E-U 1$ & $6.97 \mathrm{E}-01$ & $1.42 E-U 1$ & 3.541 \\
\hline
\end{tabular}

Table 7. Annual Accident Dose Risk for the Shipping Campaign (person-rem/yr)

\begin{tabular}{|c|c|c|c|}
\hline Route & & Truck & Rail \\
\hline $\begin{array}{l}\text { Ames Laboratory } \\
\text { Argonne - East } \\
\text { Charleston, SC } \\
\text { Colonie } \\
\text { ETEC } \\
\text { Fernald (FEMP) } \\
\text { General Atomic } \\
\text { Grand Junction Po } \\
\text { LERHR } \\
\text { Reactive Metals }\end{array}$ & $\begin{array}{l}\text { to Portsmouth GDP } \\
\text { to Portsmouth GDP } \\
\text { to Savannah River } \\
\text { to Portsmouth GDP } \\
\text { to Lawrence Livermore } \\
\text { to Portsmouth GDP } \\
\text { to Lawrence Livermore } \\
\text { to Rocky Flats Plant } \\
\text { to Lawrence Livermore } \\
\text { to Portsmouth GDP }\end{array}$ & $\begin{array}{l}3.14 \mathrm{E}-08 \\
1.04 \mathrm{E}-05 \\
4.40 \mathrm{E}-05 \\
1.48 \mathrm{E}-07 \\
5.57 \mathrm{E}-04 \\
5.70 \mathrm{E}-03 \\
9.14 \mathrm{E}-07 \\
3.47 \mathrm{E}-08 \\
1.50 \mathrm{E}-07 \\
3.05 \mathrm{E}-07\end{array}$ & $\begin{array}{l}1.28 \mathrm{E}-08 \\
2.13 \mathrm{E}-06 \\
5.11 \mathrm{E}-06 \\
3.44 \mathrm{E}-08 \\
7.12 \mathrm{E}-04 \\
1.28 \mathrm{E}-03 \\
9.99 \mathrm{E}-07 \\
3.83 \mathrm{E}-10 \\
1.22 \mathrm{E}-07 \\
8.81 \mathrm{E}-08\end{array}$ \\
\hline Totals & & $6.32 \mathrm{E}-03$ & $2.00 \mathrm{E}-03$ \\
\hline
\end{tabular}


Table 8. Expected Annual Fatalities for the Shipping Campaign

\begin{tabular}{lll}
\hline Exposure Group & Truck & Rail \\
\hline Radiological & & \\
Normal Crew & $2.5 E-04$ & $5.7 \pm-05$ \\
Normal Public & $3.5 E-04$ & $1.8 E-04$ \\
Accident Public & $3.2 E-06$ & $1.0 E-06$ \\
Nonradiological & & \\
Emission & $2.4 E-04$ & $3.2 E-04$ \\
Accident & $9.9 E-04$ & $2.1 E-05$ \\
\hline
\end{tabular}

Table 9. Expected Annual Cancer Incidence for the Shipping Campaign

\begin{tabular}{lll}
\hline Exposure Group & Truck & Rail \\
\hline Radiological & & \\
Normal Crew & $8.6 E-04$ & $2.0 E-04$ \\
Normal Public & $1.2 E-03$ & $6.0 E-04$ \\
Accident Public & $1.1 E-05$ & $3.4 E-06$ \\
Nonradiological & & \\
Emission & $2.4 E-04$ & $3.2 E-04$ \\
Accident & NA & NA \\
\hline
\end{tabular}

Table 10. Expected Annual Genetic Effects for the Shipping Campaign

\begin{tabular}{lll}
\hline Exposure Group & Truck & Rail \\
\hline Radiological & & \\
Normal Crew & $3.7 E-05$ & $8.5 E-06$ \\
Normal Public & $7.0 E-05$ & $3.5 E-05$ \\
Accident Public & $4.4 E-07$ & $1.5 E-07$ \\
Nonradiological & & \\
Emission & NA & NA \\
Accident & NA & NA \\
\hline
\end{tabular}




\section{B.10 WM LLMW REGIONALIZED 1 ALTERNATIVE (CASE 4): CONTACT-HANDLED HETEROGENEOUS SOLIDS: ALPHA - TREATED}

Table 1. Summary of Route Information

\begin{tabular}{|c|c|c|c|c|c|}
\hline & \multirow[b]{2}{*}{ Route } & \multirow{2}{*}{$\begin{array}{c}\text { Distance } \\
\text { (miles) }\end{array}$} & \multicolumn{3}{|c|}{ Percentage in Zone } \\
\hline & & & Rural & Suburban & Urban \\
\hline \multicolumn{6}{|l|}{ Truck } \\
\hline Lawrence Livermore & to Nevada Test Site & 678 & 84.7 & 9.2 & 6.1 \\
\hline \multicolumn{6}{|c|}{ Rail } \\
\hline Lawrence Livermore & to Nevada Test Site & 1370 & 94.5 & 3.8 & 1.8 \\
\hline West Valley DP & to Savannah River & 1217 & 62.8 & 32.4 & 4.9 \\
\hline
\end{tabular}

Table 2. Annual Number of Shipments and One-Way shipment Distances

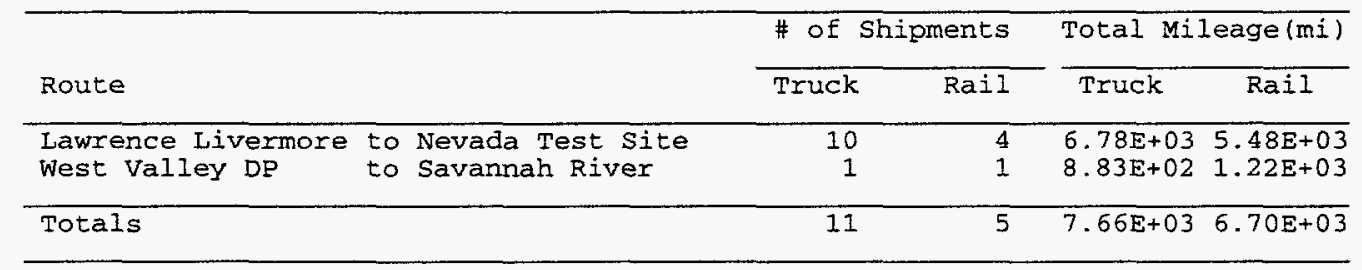

Table 3. Incident-Free Dose per Shipment (person-rem/shipment)

\begin{tabular}{|c|c|c|c|c|c|c|}
\hline \multirow[b]{2}{*}{ Source/Route (s) } & & \multirow[b]{2}{*}{ Crew } & \multicolumn{4}{|c|}{ General Public } \\
\hline & & & off-Link & on-Link & Stops & Total \\
\hline $\begin{array}{l}\text { MLLW(T) LL to NT } \\
\text { Lawrence Livermore }\end{array}$ & $\begin{array}{l}\text { to Nevada } \\
\text { Truck } \\
\text { Rail }\end{array}$ & $\begin{array}{l}\text { Test site } \\
2.05 \mathrm{E}-02 \\
8.60 \mathrm{E}-03\end{array}$ & $\begin{array}{l}\text { e } \\
2.57 E-03 \\
5.75 E-03\end{array}$ & $\begin{array}{l}4.65 \mathrm{E}-03 \\
1.20 \mathrm{E}-04\end{array}$ & $\begin{array}{l}1.90 \mathrm{E}-02 \\
7.70 \mathrm{E}-03\end{array}$ & $\begin{array}{l}2.62 \mathrm{E}-02 \\
1.36 \mathrm{E}-02\end{array}$ \\
\hline $\begin{array}{l}\text { MLLW (T) WV to SR } \\
\text { West Valley DP }\end{array}$ & $\begin{array}{l}\text { to Savanna } \\
\text { Truck } \\
\text { Rail }\end{array}$ & $\begin{array}{l}\text { ah River } \\
2.89 \mathrm{E}-02 \\
7.96 \mathrm{E}-03\end{array}$ & $\begin{array}{l}2.45 \mathrm{E}-03 \\
1.95 \mathrm{E}-02\end{array}$ & $\begin{array}{l}3.86 \mathrm{E}-03 \\
3.38 \mathrm{E}-04\end{array}$ & $\begin{array}{l}2.47 \mathrm{E}-02 \\
7.23 \mathrm{E}-03\end{array}$ & $\begin{array}{l}3.10 \mathrm{E}-02 \\
2.70 \mathrm{E}-02\end{array}$ \\
\hline
\end{tabular}

Table 4. Accident Dose Risk per Shipment (person-rem/shipment)

\begin{tabular}{llll}
\hline Source/Route & Truck & Rail \\
\hline $\begin{array}{c}\text { MLLW(T) LL to NT } \\
\text { Lawrence Livermore to Nevada Test Site }\end{array}$ & $4.80 \mathrm{E}-05$ & $3.53 \mathrm{E}-05$ \\
$\begin{array}{c}\text { MLLW (T) WV to SR } \\
\text { West Valley DP }\end{array}$ & to Savannah River & $4.43 \mathrm{E}-05$ & $\mathbf{1 . 4 2 E - 0 5}$ \\
\hline
\end{tabular}

Table 5. Nonradiological Risk Factors per Shipment (fatalities/shipment)

\begin{tabular}{llll}
\hline Mode & Route & Emission & Accident \\
\hline Truck & & $1.32 \mathrm{E}-05$ & $5.15 \mathrm{E}-05$ \\
$\begin{array}{c}\text { Lawrence Livermore } \\
\text { West Valley DP }\end{array}$ & to Nevada Test Site & $3.28 \mathrm{E}-06$ & $6.60 \mathrm{E}-05$ \\
Rail & & $1.01 \mathrm{E}-05$ & $2.87 \mathrm{E}-06$ \\
$\begin{array}{l}\text { Lawrence Livermore } \\
\text { West Valley DP }\end{array}$ & to Sevada Test Site & $2.49 \mathrm{E}-05$ & $2.55 \mathrm{E}-06$ \\
\hline
\end{tabular}


Table 6. Annual Incident-Free Dose for the Shipping Campaign (person-rem/yr)

\begin{tabular}{|c|c|c|c|c|c|}
\hline \multirow[b]{2}{*}{ Route } & & \multicolumn{2}{|c|}{ Truck } & \multicolumn{2}{|c|}{ Rail } \\
\hline & & Crew & Public & Crew & Public \\
\hline $\begin{array}{l}\text { Lawrence Livermore } \\
\text { West Valley DP }\end{array}$ & $\begin{array}{l}\text { to Nevada Test Site } \\
\text { to Savannah River }\end{array}$ & $\begin{array}{l}2.05 \mathrm{E}-01 \\
2.89 \mathrm{E}-02\end{array}$ & $\begin{array}{l}2.62 E-01 \\
3.10 \mathrm{E}-02\end{array}$ & $\begin{array}{l}3.44 \mathrm{E}-02 \\
7.96 \mathrm{E}-03\end{array}$ & $\begin{array}{l}5.43 E-02 \\
2.70 E-02\end{array}$ \\
\hline Totals & & $2.34 \mathrm{E}-01$ & $2.93 \mathrm{E}-01$ & $4.23 \mathrm{E}-02$ & $8.13 \mathrm{E}-02$ \\
\hline
\end{tabular}

Table 7. Annual Accident Dose Risk for the Shipping Campaign (person-rem/yr)

\begin{tabular}{lccc}
\hline Route & Truck & Rail \\
\hline Lawrence Livermore to Nevada Test Site & $4.80 \mathrm{E}-04$ & $1.41 \mathrm{E}-04$ \\
West Valley DP & to Savannah River & $4.43 \mathrm{E}-05$ & $1.42 \mathrm{E}-05$ \\
\hline Totals & & $5.25 \mathrm{E}-04$ & $1.55 \mathrm{E}-04$ \\
\hline
\end{tabular}

Table 8. Expected Annual Fatalities for the Shipping Campaign

\begin{tabular}{lll}
\hline Exposure Group & Truck & Rail \\
\hline Radiological & & \\
Normal Crew & $9.4 \mathrm{E}-05$ & $1.7 \mathrm{E}-05$ \\
Normal Public & $1.5 \mathrm{E}-04$ & $4.1 \mathrm{E}-05$ \\
Accident Public & $2.6 \mathrm{E}-07$ & $7.8 \mathrm{E}-08$ \\
Nonradiological & & \\
Emission & $1.4 \mathrm{E}-04$ & $6.5 \mathrm{E}-05$ \\
Accident & $5.8 \mathrm{E}-04$ & $1.4 \mathrm{E}-05$ \\
\hline
\end{tabular}

Table 9. Expected Annual Cancer Incidence for the Shipping Campaign

\begin{tabular}{lll}
\hline Exposure Group & Truck & \multicolumn{1}{c}{ Rail } \\
\hline Radiological & & \\
Normal Crew & $3.3 \mathrm{E}-04$ & $5.9 \mathrm{E}-05$ \\
Normal Public & $5.0 \mathrm{E}-04$ & $1.4 \mathrm{E}-04$ \\
Accident Public & $8.9 \mathrm{E}-07$ & $2.6 \mathrm{E}-07$ \\
Nonradiological & $1.4 \mathrm{E}-04$ & $6.5 \mathrm{E}-05$ \\
Emission & $\mathrm{NA}$ & $\mathrm{NA}$ \\
Accident & & \\
\hline
\end{tabular}

Table 10. Expected Annual Genetic Effects for the Shipping Campaign

\begin{tabular}{lll}
\hline Exposure Group & Truck & Rail \\
\hline Radiological & & \\
Normal Crew & $1.4 E-05$ & $2.5 \mathrm{E}-06$ \\
Normal Public & $2.9 \mathrm{E}-05$ & $8.1 \mathrm{E}-06$ \\
Accident Public & $2.0 \mathrm{E}-08$ & $6.2 \mathrm{E}-09$ \\
Nonradiological & & NA \\
Emission & NA & NA \\
Accident & NA & \\
\hline
\end{tabular}


B.11 WM LLMW REGIONALIZED 2 ALTERNATIVE (CASE 7): CONTACT-HANDLED ORGANIC LIQUIDS: NONALPHA - UNTREATED

Table 1. Summary of Route Information

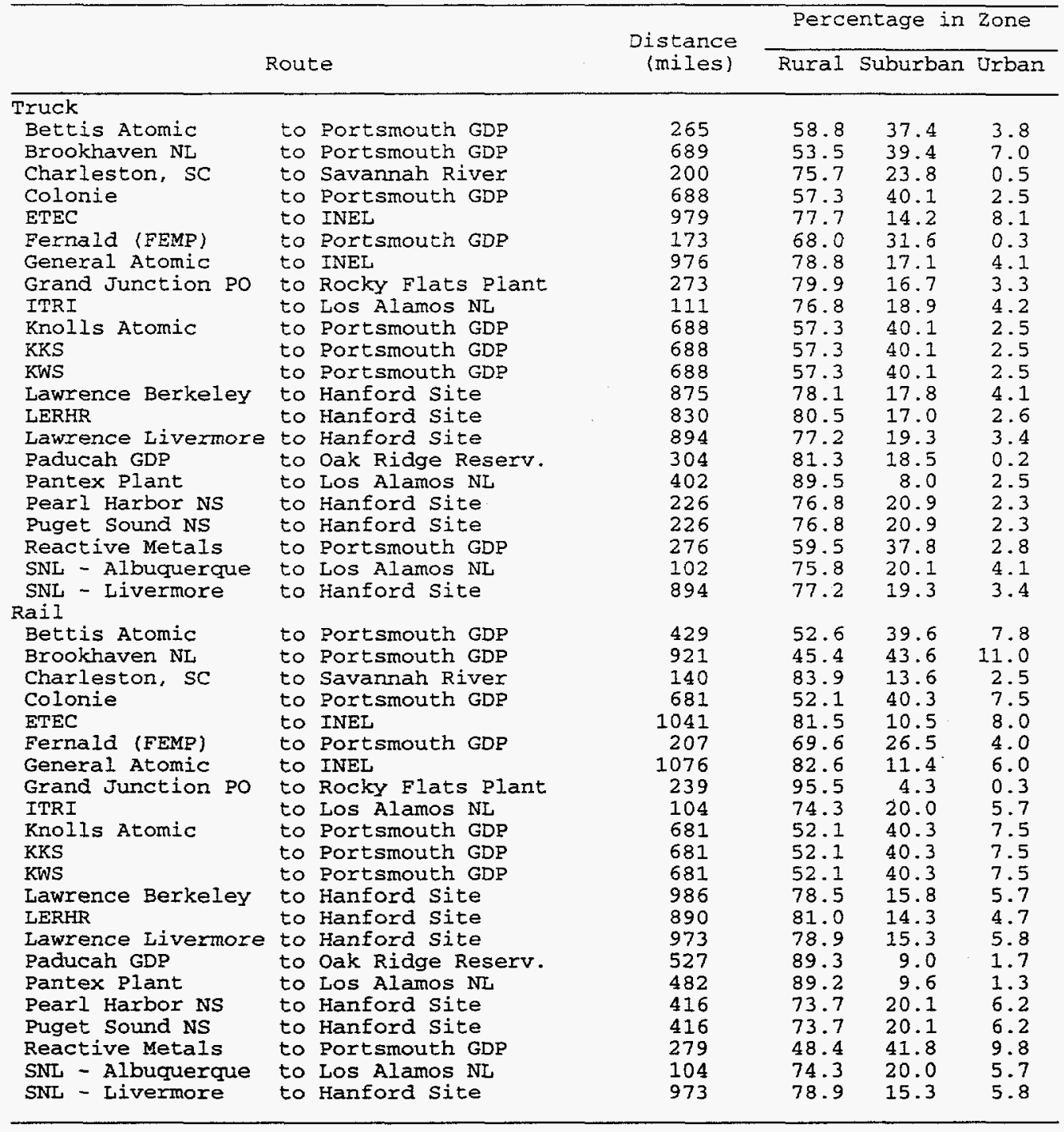


Table 2. Annual Number of Shipments and One-Way Shipment Distances

\begin{tabular}{|c|c|c|c|c|c|c|}
\hline \multirow[b]{2}{*}{ Route } & & & \multicolumn{2}{|c|}{ \# of Shipments } & \multicolumn{2}{|c|}{ Total Mileage (mi) } \\
\hline & & & Truck & Rail & Truck & Rail \\
\hline Bettis Atomic & & Portsmouth GDP & 1 & 1 & $2.65 E+02$ & $4.29 E+02$ \\
\hline Brookhaven NL & to & Portsmouth GDP & 1 & 1 & $6.89 E+02$ & $9.21 \mathrm{E}+02$ \\
\hline Charleston, SC & to & Savannah River & 1 & 1 & $2.00 \mathrm{E}+02$ & $1.40 \mathrm{E}+02$ \\
\hline Colonie & & Portsmouth GDP & $\overline{1}$ & 1 & $6.88 \mathrm{E}+02$ & $6.81 E+02$ \\
\hline ETEC & to & INEL & 1 & 1 & $9.79 \mathrm{E}+02$ & $1.04 \mathrm{E}+03$ \\
\hline Fernald (FEMP) & to & Portsmouth GDP & 2 & 1 & $3.46 \mathrm{E}+02$ & $2.07 \mathrm{E}+02$ \\
\hline General Atomic & to & INEL & 1 & 1 & $9.76 \mathrm{E}+02$ & $1.08 E+03$ \\
\hline Grand Junction PO & to & Rocky Flats Plant & 1 & 1 & $2.73 E+02$ & $2.39 \mathrm{E}+02$ \\
\hline ITRI & to & Los Alamos NL & $\vec{I}$ & 1 & $1.11 \Xi+02$ & $1.04 \bar{E}+02$ \\
\hline Knolls Atomic & to & Portsmouth GDP & 1 & $i$ & $6.88 \mathrm{E}+02$ & $6.81 E+02$ \\
\hline KKS & to & Portsmouth GDP & 1 & 1 & $5.88 \mathrm{E}+02$ & $6.81 E+02$ \\
\hline KWS & to & Portsmouth GDP & 1 & 1 & $6.88 \mathrm{E}+02$ & $6.81 E+02$ \\
\hline Iawrence Berkeley & to & Hanford Site & 1 & 1 & $8.75 \mathrm{E}+02$ & $9.86 \mathrm{E}+02$ \\
\hline LERHR & to & Hanford Site & 1 & 1 & $8.30 \mathrm{E}+02$ & $8.90 \mathrm{E}+02$ \\
\hline Lawrence Livermore & to & Hanford Site & 5 & 2 & $4.47 E+03$ & $1.95 E+03$ \\
\hline Paducah GDP & to & Oak Ridge Reserv. & 3 & $\mathrm{I}$ & $9.12 \mathrm{E}+02$ & $5.27 E+02$ \\
\hline Pantex Plant & to & Los Alamos NL & 1 & 1 & $4.02 \mathrm{E}+02$ & $4.82 E+02$ \\
\hline Pearl Harbor NS & to & Hanford Site & 1 & 2 & $2.26 E+02$ & $4.16 \mathrm{E}+02$ \\
\hline Puget Sound NS & to & Hanford Site & 1 & 1 & $2.26 \mathrm{E}+02$ & $4.16 \mathrm{E}+02$ \\
\hline Reactive Metals & to & Portsmouth GDP & 1 & 1 & $2.76 \mathrm{E}+02$ & $2.79 E+02$ \\
\hline SNL - Albuquerque & to & Los Alamos NL & 1 & 1 & $1.02 E+02$ & $1.04 \mathrm{E}+02$ \\
\hline SNL - Livermore & to & Hanford Site & 1 & 1 & $8.94 E+02$ & $9.73 E+02$ \\
\hline Totals & & & 29 & 23 & $1.58 \mathrm{E}+04$ & $1.39 \mathrm{E}+04$ \\
\hline
\end{tabular}

Tabie 3. Incident-Free Dose per Shipment (person-rem/shipment)

\begin{tabular}{|c|c|c|c|c|c|c|}
\hline \multirow[b]{2}{*}{ Source/Route (s) } & & \multirow[b]{2}{*}{ Crew } & \multicolumn{4}{|c|}{ General Public } \\
\hline & & & off-Iink & On-Link & Stops & Total \\
\hline \multirow[t]{2}{*}{$\begin{array}{l}\text { MLLW(O) BA to PO } \\
\text { Bettis Atomic }\end{array}$} & to Portsn & mouth GDP & & & & \\
\hline & $\begin{array}{l}\text { Truck } \\
\text { Rail }\end{array}$ & $\begin{array}{l}9.78 \mathrm{E}-03 \\
4.67 \mathrm{E}-03\end{array}$ & $\begin{array}{l}1.26 \mathrm{E}-03 \\
9.85 \mathrm{E}-03\end{array}$ & $\begin{array}{l}1.73 \mathrm{E}-03 \\
1.53 \mathrm{E}-04\end{array}$ & $\begin{array}{l}7.42 \mathrm{E}-03 \\
4.80 \mathrm{E}-03\end{array}$ & $\begin{array}{l}1.04 \mathrm{E}-02 \\
1.48 \mathrm{E}-02\end{array}$ \\
\hline \multirow{2}{*}{$\begin{array}{l}\text { MLLW(O) BN to PO } \\
\text { Brookhaven NL }\end{array}$} & & & & & & \\
\hline & $\begin{array}{l}\text { to Portsn } \\
\text { Truck } \\
\text { Rai1 }\end{array}$ & $\begin{array}{l}\text { mouth GDP } \\
2.73 \mathrm{E}-02 \\
6.72 \mathrm{E}-03\end{array}$ & $\begin{array}{l}4.51 E-03 \\
2.74 E-02\end{array}$ & $\begin{array}{l}6.10 E-03 \\
3.90 E-04\end{array}$ & $\begin{array}{l}1.93 E-02 \\
6.31 E-03\end{array}$ & $\begin{array}{l}2.99 E-02 \\
3.41 E-02\end{array}$ \\
\hline \multirow{2}{*}{$\begin{array}{l}\text { MLLW(O) CH to SR } \\
\text { Charleston, SC }\end{array}$} & & & & & & \\
\hline & $\begin{array}{l}\text { to Savanr } \\
\text { Truck } \\
\text { Rail }\end{array}$ & $\begin{array}{r}\text { nah River } \\
6.19 \mathrm{E}-03 \\
3.47 \mathrm{E}-03\end{array}$ & $\begin{array}{l}4.20 \mathrm{E}-04 \\
1.07 \mathrm{E}-03\end{array}$ & $\begin{array}{l}7.45 \mathrm{E}-04 \\
2.07 \mathrm{E}-05\end{array}$ & $\begin{array}{l}5.60 \mathrm{E}-03 \\
3.91 \mathrm{E}-03\end{array}$ & $\begin{array}{l}6.77 \mathrm{E}-03 \\
5.00 \mathrm{E}-03\end{array}$ \\
\hline \multirow[t]{2}{*}{$\begin{array}{l}\text { MLLW(O) CL to PO } \\
\text { Colonie }\end{array}$} & to Portsn & mouth GDP. & & & & \\
\hline & $\begin{array}{l}\text { Truck } \\
\text { Rail }\end{array}$ & $\begin{array}{l}2.54 \mathrm{E}-02 \\
5.72 \mathrm{E}-03\end{array}$ & $\begin{array}{l}2.99 \mathrm{E}-03 \\
1.54 \mathrm{E}-02\end{array}$ & $\begin{array}{l}4.01 E-03 \\
2.43 E-04\end{array}$ & $\begin{array}{l}1.93 E-02 \\
5.58 E-03\end{array}$ & $\begin{array}{l}2.63 \mathrm{E}-02 \\
2.13 \mathrm{E}-02\end{array}$ \\
\hline \multicolumn{7}{|l|}{$M L L W(O)$ LC to IN } \\
\hline & $\begin{array}{l}\text { Truck } \\
\text { Rail }\end{array}$ & $\begin{array}{l}3.23 \mathrm{E}-02 \\
7.22 \mathrm{E}-03\end{array}$ & $\begin{array}{l}5.06 \mathrm{E}-03 \\
1.80 \mathrm{E}-02\end{array}$ & $\begin{array}{l}8.27 E-03 \\
2.22 E-04\end{array}$ & $\begin{array}{l}2.74 \mathrm{E}-02 \\
6.68 \mathrm{E}-03\end{array}$ & $\begin{array}{l}4.07 E-02 \\
2.49 E-02\end{array}$ \\
\hline \multirow{2}{*}{$\begin{array}{l}\text { MLLW(O) FE to PO } \\
\text { Fernald (FEMP) }\end{array}$} & & & & & & \\
\hline & $\begin{array}{l}\text { to Portsn } \\
\text { Truck } \\
\text { Rail }\end{array}$ & $\begin{array}{r}\text { mouth GDP } \\
5.72 \mathrm{E}-03 \\
3.75 \mathrm{E}-03\end{array}$ & $\begin{array}{l}4.51 E-04 \\
2.71 E-03\end{array}$ & $\begin{array}{l}6.85 \mathrm{E}-04 \\
4.86 \mathrm{E}-05\end{array}$ & $\begin{array}{l}4.84 E-03 \\
4.12 E-03\end{array}$ & $\begin{array}{l}5.98 E-03 \\
6.87 E-03\end{array}$ \\
\hline \multirow{2}{*}{$\begin{array}{l}\text { MLLW(O) So to IN } \\
\text { General Atomic }\end{array}$} & & & & & & \\
\hline & $\begin{array}{l}\text { Truck } \\
\text { Rail }\end{array}$ & $\begin{array}{l}3.06 \mathrm{E}-02 \\
7.37 \mathrm{E}-03\end{array}$ & $\begin{array}{l}3.33 \mathrm{E}-03 \\
1.48 \mathrm{E}-02\end{array}$ & $\begin{array}{l}5.75 \mathrm{E}-03 \\
2.03 \mathrm{E}-04\end{array}$ & $\begin{array}{l}2.73 E-02 \\
6.79 E-03\end{array}$ & $\begin{array}{l}3.64 \mathrm{E}-02 \\
2.18 \mathrm{E}-02\end{array}$ \\
\hline $\begin{array}{l}\text { MLLW(O) GJ to RF } \\
\text { Grand Junction PO }\end{array}$ & $\begin{array}{l}\text { to Rocky } \\
\text { Truck } \\
\text { Rail }\end{array}$ & $\begin{array}{c}\text { Flats Plar } \\
8.39 \mathrm{E}-03 \\
3.88 \mathrm{E}-03\end{array}$ & $\begin{array}{l}8.15 E-04 \\
3.51 E-04\end{array}$ & $\begin{array}{l}1.46 \mathrm{E}-03 \\
1.62 \mathrm{E}-05\end{array}$ & $\begin{array}{l}7.65 E-03 \\
4.21 E-03\end{array}$ & $\begin{array}{l}9.92 E-03 \\
4.58 E-03\end{array}$ \\
\hline $\begin{array}{l}\text { MLLW(O) IT to LA } \\
\text { ITRI }\end{array}$ & $\begin{array}{l}\text { to Los A] } \\
\text { Truck } \\
\text { Rail }\end{array}$ & $\begin{array}{l}\text { Iamos NL } \\
3.54 \mathrm{E}-03 \\
3.31 \mathrm{E}-03\end{array}$ & $\begin{array}{l}4.00 \mathrm{E}-04 \\
1.55 \mathrm{E}-03\end{array}$ & $\begin{array}{l}5.71 E-04 \\
2.36 E-05\end{array}$ & $\begin{array}{l}3.11 \mathrm{E}-03 \\
3.80 \mathrm{E}-03\end{array}$ & $\begin{array}{l}4.18 E-03 \\
5.37 E-03\end{array}$ \\
\hline $\begin{array}{l}\text { MLLW(O) KA to PO } \\
\text { Knolis Atomic }\end{array}$ & $\begin{array}{l}\text { to Portsm } \\
\text { Truck. } \\
\text { Rail }\end{array}$ & $\begin{array}{r}\text { mouth GDP } \\
2.54 \mathrm{E}-02 \\
5.72 \mathrm{E}-03\end{array}$ & $\begin{array}{l}2.99 E-03 \\
1.54 E-02\end{array}$ & $\begin{array}{l}4.01 \Xi-03 \\
2.43 \Sigma-04\end{array}$ & $\begin{array}{l}1.93 \mathrm{E}-02 \\
5.58 \mathrm{E}-03\end{array}$ & $\begin{array}{l}2.63 E-02 \\
2.13 E-02\end{array}$ \\
\hline
\end{tabular}


Table 3. Incident-Free Dose per Shipment (person-rem/shipment) (Cont.)

\begin{tabular}{|c|c|c|c|c|c|}
\hline \multirow[b]{2}{*}{ Source/Route (s) } & \multirow[b]{2}{*}{ Crew } & \multicolumn{4}{|c|}{ General Public } \\
\hline & & off-Link & on-Link & Stops & Total \\
\hline \multicolumn{6}{|l|}{ MLLW(O) KK to PO } \\
\hline & $\begin{array}{ll}\text { Truck } & 2.54 \mathrm{E}-02 \\
\text { Rail } & 5.72 \mathrm{E}-03\end{array}$ & $\begin{array}{l}2.99 \mathrm{E}-03 \\
1.54 \mathrm{E}-02\end{array}$ & $\begin{array}{l}4.01 \mathrm{E}-03 \\
2.43 \mathrm{E}-04\end{array}$ & $\begin{array}{l}1.93 \mathrm{E}-02 \\
5.58 \mathrm{E}-03\end{array}$ & $\begin{array}{l}2.63 \mathrm{E}-02 \\
2.13 \mathrm{E}-02\end{array}$ \\
\hline \multicolumn{6}{|l|}{$\begin{array}{c}\text { MLLW(O) KW to PO } \\
\text { KWS }\end{array}$} \\
\hline & $\begin{array}{ll}\text { Truck } & 2.54 \mathrm{E}-02 \\
\text { Rail } & 5.72 \mathrm{E}-03\end{array}$ & $\begin{array}{l}2.99 \mathrm{E}-03 \\
1.54 \mathrm{E}-02\end{array}$ & $\begin{array}{l}4.01 E-03 \\
2.43 E-04\end{array}$ & $\begin{array}{l}1.93 E-02 \\
5.58 E-03\end{array}$ & $\begin{array}{l}2.63 \mathrm{E}-02 \\
2.13 \mathrm{E}-02\end{array}$ \\
\hline \multicolumn{6}{|c|}{$\begin{array}{l}\text { MLLW (O) LB to HS } \\
\text { Lawrence Berkeley to Hanford Site }\end{array}$} \\
\hline & $\begin{array}{ll}\text { Truck } & 2.76 \mathrm{E}-02 \\
\text { Rail } & 6.99 \mathrm{E}-03\end{array}$ & $\begin{array}{l}3.02 \mathrm{E}-03 \\
1.38 \mathrm{E}-02\end{array}$ & $\begin{array}{l}5.17 E-03 \\
2.03 E-04\end{array}$ & $\begin{array}{l}2.45 \mathrm{E}-02 \\
6.51 \mathrm{E}-03\end{array}$ & $\begin{array}{l}3.27 E-02 \\
2.05 E-02\end{array}$ \\
\hline \multicolumn{6}{|c|}{ to Hanford site } \\
\hline LERHR & $\begin{array}{l}\text { to Hanford Site } \\
\text { Truck } \\
\text { Rail } \\
2.51 \mathrm{E}-02 \\
6.59 \mathrm{E}-03\end{array}$ & $\begin{array}{l}2.17 \mathrm{E}-03 \\
1.07 \mathrm{E}-02\end{array}$ & $\begin{array}{l}4.00 \mathrm{E}-03 \\
1.64 \mathrm{E}-04\end{array}$ & $\begin{array}{l}2.32 \mathrm{E}-02 \\
6.22 \mathrm{E}-03\end{array}$ & $\begin{array}{l}2.94 \mathrm{E}-02 \\
1.70 \mathrm{E}-02\end{array}$ \\
\hline \multicolumn{6}{|c|}{$\begin{array}{l}\text { MLLW(O) LL to HS } \\
\text { Lawrence Livermore to Hanford site }\end{array}$} \\
\hline & $\begin{array}{ll}\text { Truck } & 2.82 \mathrm{E}-02 \\
\text { Rail } & 6.94 \mathrm{E}-03\end{array}$ & $\begin{array}{l}2.88 \mathrm{E}-03 \\
1.38 \mathrm{E}-02\end{array}$ & $\begin{array}{l}4.92 \mathrm{E}-03 \\
2.00 \mathrm{E}-04\end{array}$ & $\begin{array}{l}2.50 \mathrm{E}-02 \\
6.48 \mathrm{E}-03\end{array}$ & $\begin{array}{l}3.28 \mathrm{E}-02 \\
2.05 \mathrm{E}-02\end{array}$ \\
\hline \multicolumn{6}{|l|}{$M L L W(O)$ PA to OR } \\
\hline Fathe Git G5 & $\begin{array}{ll}\text { Truck } & 8.88 \mathrm{E}-03 \\
\text { Rail } & 5.08 \mathrm{E}-03\end{array}$ & $\begin{array}{l}4.70 \mathrm{E}-04 \\
2.75 \mathrm{E}-03\end{array}$ & $\begin{array}{l}9.98 \mathrm{E}-04 \\
5.97 \mathrm{E}-05\end{array}$ & $\begin{array}{l}8.51 E-03 \\
5.10 E-03\end{array}$ & $\begin{array}{l}9.98 E-03 \\
7.91 E-03\end{array}$ \\
\hline \multicolumn{6}{|l|}{$\begin{array}{l}\text { MLLW(O) PP to LA } \\
\text { Pantex Plant }\end{array}$} \\
\hline & $\begin{array}{l}\text { to Los Alamos NL } \\
\text { Truck } 1.11 \mathrm{E}-02\end{array}$ & 7.7 & -03 & -02 & $-02+2>>>$ \\
\hline & $4.89 E-03$ & $2.18 E-03$ & $5.29 E-05$ & $4.96 E-03$ & $7.19 E-03$ \\
\hline \multicolumn{5}{|l|}{$\begin{array}{l}\text { MLLW(O) SW to HS } \\
\text { Pearl Harbor NS }\end{array}$} & \\
\hline & $\begin{array}{ll}\text { Truck } & 7.06 \mathrm{E}-03 \\
\text { Rail } & 4.62 \mathrm{E}-03\end{array}$ & $\begin{array}{l}6.28 \mathrm{E}-04 \\
6.63 \mathrm{E}-03\end{array}$ & $\begin{array}{l}1.09 E-03 \\
9.79 E-05\end{array}$ & $\begin{array}{l}6.33 \mathrm{E}-03 \\
4.76 \mathrm{E}-03\end{array}$ & $\begin{array}{l}8.04 \mathrm{E}-03 \\
1.15 \mathrm{E}-02\end{array}$ \\
\hline \multirow{2}{*}{\multicolumn{6}{|c|}{$\begin{array}{l}\text { MLLW (O) PN to HS } \\
\text { Puget Sound NS }\end{array}$}} \\
\hline & to Hanford Site & & & & \\
\hline & $\begin{array}{l}7.06 \mathrm{E}-03 \\
4.62 \mathrm{E}-03\end{array}$ & $\begin{array}{l}6.28 \mathrm{E}-04 \\
6.63 \mathrm{E}-03\end{array}$ & $\begin{array}{l}1.09 \mathrm{E}-03 \\
9.79 \mathrm{E}-05\end{array}$ & $\begin{array}{l}6.33 \mathrm{E}-03 \\
4.76 \mathrm{E}-03\end{array}$ & $\begin{array}{l}8.04 E-03 \\
1.15 E-02\end{array}$ \\
\hline \multirow{2}{*}{\multicolumn{6}{|c|}{$\begin{array}{l}\text { MLLW (O) RM to PO } \\
\text { Reactive Metals }\end{array}$}} \\
\hline & to Portsmouth GDP & & & & \\
\hline & $\begin{array}{l}1.00 \mathrm{E}-02 \\
4.05 \mathrm{E}-03\end{array}$ & $\begin{array}{l}1.18 \mathrm{E}-03 \\
7.58 \mathrm{E}-03\end{array}$ & $\begin{array}{l}1.62 \mathrm{E}-03 \\
1.11 \mathrm{E}-04\end{array}$ & $\begin{array}{l}7.73 \mathrm{E}-03 \\
4.34 \mathrm{E}-03\end{array}$ & $\begin{array}{l}1.05 \mathrm{E}-02 \\
1.20 \mathrm{E}-02\end{array}$ \\
\hline \multicolumn{6}{|c|}{$\begin{array}{l}\text { MLLW(O) SA to LA } \\
\text { SNL - Albuquerque to Los Alamos NL }\end{array}$} \\
\hline SNL - Albuquerque & $\begin{array}{lr}\text { to Los } & \text { Alamos NL } \\
\text { Truck } & 3.28 \mathrm{E}-03 \\
\text { Rail } & 3.31 \mathrm{E}-03\end{array}$ & $\begin{array}{l}3.70 \mathrm{E}-04 \\
1.55 \mathrm{E}-03\end{array}$ & $\begin{array}{l}6.13 E-04 \\
2.36 \mathrm{E}-05\end{array}$ & $\begin{array}{l}2.86 \mathrm{E}-03 \\
3.80 \mathrm{E}-03\end{array}$ & $\begin{array}{l}3.84 \mathrm{E}-03 \\
5.37 \mathrm{E}-03\end{array}$ \\
\hline \multirow{2}{*}{$\begin{array}{l}\text { MLLW(O) SL to HS } \\
\text { SNL - Livermore }\end{array}$} & & & & & \\
\hline & $\begin{array}{lr}\text { to Hanford Site } \\
\text { Truck } & 2.82 \mathrm{E}-02 \\
\text { Rail } & 6.94 \mathrm{E}-03\end{array}$ & $\begin{array}{l}2.88 \mathrm{E}-03 \\
1.38 \mathrm{E}-02\end{array}$ & $\begin{array}{l}4.92 \mathrm{E}-03 \\
2.00 \mathrm{E}-04\end{array}$ & $\begin{array}{l}2.50 \mathrm{E}-02 \\
6.48 \mathrm{E}-03\end{array}$ & $\begin{array}{l}3.28 \mathrm{E}-02 \\
2.05 \mathrm{E}-02\end{array}$ \\
\hline
\end{tabular}


Table 4. Accident Dose Risk per Shipment (person-rem/shipment)

\begin{tabular}{|c|c|c|c|}
\hline Source/Route & & Truck & Rail \\
\hline MLLW(O) BA to PO & & & \\
\hline $\begin{array}{l}\text { Bettis Atomic } \\
\text { MLLW(0) BN to PO }\end{array}$ & to Portsmouth GDP & $5.04 E-04$ & $2.16 E-04$ \\
\hline $\begin{array}{l}\text { Brookhaven NL } \\
\text { MLLW(O) CH to SR }\end{array}$ & to Portsmouth GDP & $4.87 E-03$ & $2.63 E-03$ \\
\hline $\begin{array}{l}\text { Charleston, SC } \\
\text { MLLW(O) CL to PO }\end{array}$ & to Savannah River & $7.75 E-03$ & $8.99 \mathrm{E}-04$ \\
\hline $\begin{array}{l}\text { Colonie } \\
\text { MLLW(O) LC to IN }\end{array}$ & to Portsmouth GDP & $3.74 \mathrm{E}-04$ & $8.80 E-05$ \\
\hline $\begin{array}{c}\text { ETEC } \\
M L L W(O) F E \text { to PO }\end{array}$ & to INEL & $9.54 \mathrm{E}-06$ & $4.35 \mathrm{E}-06$ \\
\hline $\begin{array}{l}\text { Fernald (FEMP) } \\
\text { MLLW (O) So to IN }\end{array}$ & to Portsmouth GDP & $5.76 \mathrm{E}-04$ & $2.78 \mathrm{E}-04$ \\
\hline $\begin{array}{l}\text { General Atomic } \\
\text { MLLW(O) GJ to RF }\end{array}$ & to INEL & $1.08 \mathrm{E}-05$ & $4.28 \mathrm{E}-06$ \\
\hline $\begin{array}{l}\text { Grand Junction PO } \\
\text { MLLW(O) IT to LA }\end{array}$ & to Rocky Flats Plant & $1.33 E-06$ & $1.13 E-08$ \\
\hline $\begin{array}{l}\text { ITRI } \\
\text { MLLW(O) KA to PO }\end{array}$ & to Los Alamos NL & $8.49 \mathrm{E}-06$ & $1.55 \mathrm{E}-07$ \\
\hline $\begin{array}{l}\text { Knolls Atomic } \\
\text { MLLW(O) KK to PO }\end{array}$ & to Portsmouth GDP & $4.50 E-02$ & $1.06 \mathrm{E}-02$ \\
\hline $\begin{array}{l}\text { KKS } \\
\text { MLLW (O) KW to Po }\end{array}$ & to Portsmouth GDP & $3.45 \mathrm{E}-02$ & $8.09 E-03$ \\
\hline $\begin{array}{l}\text { KWS } \\
\text { MLLW(O) LB to HS }\end{array}$ & to Portsmouth GDP & $4.71 E-03$ & $1.10 \mathrm{E}-03$ \\
\hline $\begin{array}{l}\text { Lawrence Berkeley } \\
\text { MLLW(0) SM to HS }\end{array}$ & to Hanford Site & $7.79 \mathrm{E}-05$ & $4.38 \mathrm{E}-05$ \\
\hline $\begin{array}{l}\text { LERHR } \\
\text { MLLW(O) LL to HS }\end{array}$ & to Hanford Site & $7.78 \mathrm{E}-06$ & $4.14 \mathrm{E}-06$ \\
\hline $\begin{array}{l}\text { Lawrence Iivermore } \\
\text { MLLW(O) PA to OR }\end{array}$ & to Hanford site & $3.93 \mathrm{E}-03$ & $5.23 E-03$ \\
\hline $\begin{array}{l}\text { Paducah GDP } \\
\text { MLLW(O) PP to LA }\end{array}$ & to Oak Ridge Reserv. & $3.89 \mathrm{E}-01$ & $1.21 E-01$ \\
\hline $\begin{array}{l}\text { Pantex Plant } \\
\text { MLLW(O) SW to HS }\end{array}$ & to Los Alamos NL & $1.60 \mathrm{E}-04$ & $9.37 E-06$ \\
\hline $\begin{array}{l}\text { Pearl Harbor NS } \\
\text { MLLW(0) PN to HS }\end{array}$ & to Hanford Site & $8.47 E-06$ & $6.32 \mathrm{E}-06$ \\
\hline $\begin{array}{l}\text { Puget sound NS } \\
\text { MLLW(O) RM to PO }\end{array}$ & to Hanford Site & $1.95 E-04$ & $1.46 \mathrm{E}-04$ \\
\hline $\begin{array}{l}\text { Reactive Metals } \\
\text { MLLW(0) SA to LA }\end{array}$ & to Portsmouth GDP & $7.40 E-05$ & $2.29 \mathrm{E}-05$ \\
\hline $\begin{array}{l}\text { SNL - Albuquerque } \\
\text { MLLW(O) SL to HS }\end{array}$ & to Los Alamos NI & $5.82 \mathrm{E}-07$ & $1.10 E-08$ \\
\hline SNL - Livermore & to Hanford Site & $1.95 \mathrm{E}-03$ & $1.04 \mathrm{E}-03$ \\
\hline
\end{tabular}


Table 5. Nonradiological Risk Factors per Shipment fatalities/shipment)

\begin{tabular}{|c|c|c|c|}
\hline Mode & Route & Emission & Accident \\
\hline $\begin{array}{l}\text { Truck } \\
\text { Bettis Atomic } \\
\text { Brookhaven NL } \\
\text { Charleston, SC } \\
\text { Colonie } \\
\text { ETEC } \\
\text { Fernald (FEMP) } \\
\text { General Atomic } \\
\text { Grand Junction Po } \\
\text { ITRI } \\
\text { Knolls Atomic } \\
\text { KKS } \\
\text { KWS } \\
\text { Lawrence Berkeley } \\
\text { LERHR } \\
\text { Lawrence Livermore } \\
\text { Paducah GDP } \\
\text { Pantex Plant } \\
\text { Pearl Harbor NS } \\
\text { Puget Sound NS } \\
\text { Reactive Metals } \\
\text { SNL - Albuquerque } \\
\text { SNL - Livermore }\end{array}$ & $\begin{array}{l}\text { to Portsmouth GDP } \\
\text { to Portsmouth GDP } \\
\text { to Savannah River } \\
\text { to Portsmouth GDP } \\
\text { to INEL } \\
\text { to Portsmouth GDP } \\
\text { to INEL } \\
\text { to Rocky Elats Plant } \\
\text { to Los Alamos NL } \\
\text { to Portsmouth GDP } \\
\text { to Portsmouth GDP } \\
\text { to Portsmouth GDP } \\
\text { to Hanford Site } \\
\text { to Hanford Site } \\
\text { to Hanford Site } \\
\text { to Oak Ridge Reserv. } \\
\text { to Los Alamos NL } \\
\text { to Hanford Site } \\
\text { to Hanford Site } \\
\text { to Portsmouth GDP } \\
\text { to Los Alamos NL } \\
\text { to Hanford Site }\end{array}$ & $\begin{array}{l}3.22 \mathrm{E}-06 \\
1.56 \mathrm{E}-05 \\
3.22 \mathrm{E}-07 \\
5.63 \mathrm{E}-06 \\
2.55 \mathrm{E}-05 \\
1.93 \mathrm{E}-07 \\
1.30 \mathrm{E}-05 \\
2.93 \mathrm{E}-06 \\
1.51 \mathrm{E}-06 \\
5.63 \mathrm{E}-06 \\
5.63 \mathrm{E}-06 \\
5.63 \mathrm{E}-06 \\
1.15 \mathrm{E}-05 \\
6.82 \mathrm{E}-06 \\
9.85 \mathrm{E}-06 \\
1.93 \mathrm{E}-07 \\
3.28 \mathrm{E}-06 \\
1.67 \mathrm{E}-06 \\
1.67 \mathrm{E}-06 \\
2.45 \mathrm{E}-06 \\
1.35 \mathrm{E}-06 \\
9.85 \mathrm{E}-06\end{array}$ & $\begin{array}{l}1.33 E-05 \\
4.90 \mathrm{E}-05 \\
1.70 \mathrm{E}-05 \\
3.56 \mathrm{E}-05 \\
6.21 \mathrm{E}-05 \\
7.51 \mathrm{E}-06 \\
6.23 \mathrm{E}-05 \\
2.32 \mathrm{E}-05 \\
1.28 \mathrm{E}-05 \\
3.56 \mathrm{E}-05 \\
3.56 \mathrm{E}-05 \\
3.56 \mathrm{E}-05 \\
4.91 \mathrm{E}-05 \\
4.56 \mathrm{E}-05 \\
5.03 \mathrm{E}-05 \\
2.14 \mathrm{E}-05 \\
3.21 \mathrm{E}-05 \\
9.56 \mathrm{E}-06 \\
9.56 \mathrm{E}-06 \\
1.21 \mathrm{E}-05 \\
1.20 \mathrm{E}-05 \\
5.03 \mathrm{E}-05\end{array}$ \\
\hline $\begin{array}{l}\text { Rail } \\
\text { Bettis Atomic } \\
\text { Brookhaven NL } \\
\text { Charleston, SC } \\
\text { Colonie } \\
\text { ETEC } \\
\text { Fernald (FEMP) } \\
\text { General Atomic } \\
\text { Grand Junction PO } \\
\text { ITRI } \\
\text { Knol1s Atomic } \\
\text { KKS } \\
\text { KWS } \\
\text { Lawrence Berkeley } \\
\text { LERHR } \\
\text { Lawrence Livermore } \\
\text { Paducah GDP } \\
\text { Pantex Plant } \\
\text { Pearl Harbor NS } \\
\text { Puget Sound NS } \\
\text { Reactive Metals } \\
\text { SNL - Albuguerque } \\
\text { SNL - Livermore }\end{array}$ & $\begin{array}{l}\text { to Portsmouth GDP } \\
\text { to Portsmouth GDP } \\
\text { to Savannah River } \\
\text { to Portsmouth GDP } \\
\text { to INEL } \\
\text { to Portsmouth GDP } \\
\text { to INEL } \\
\text { to Rocky Flats PIant } \\
\text { to Los Alamos NL } \\
\text { to Portsmouth GDP } \\
\text { to Portsmouth GDP } \\
\text { to Portsmouth GDP } \\
\text { to Hanford Site } \\
\text { to Hanford Site } \\
\text { to Hanford Site } \\
\text { to Oak Ridge Reserv. } \\
\text { to Los Alamos NL } \\
\text { to Hanford Site } \\
\text { to Hanford Site } \\
\text { to Portsmouth GDP } \\
\text { to Los Alamos NL } \\
\text { to Hanford Site }\end{array}$ & $\begin{array}{l}1.40 \mathrm{E}-05 \\
4.22 \mathrm{E}-05 \\
1.46 \mathrm{E}-06 \\
2.15 \mathrm{E}-05 \\
3.48 \mathrm{E}-05 \\
3.43 \mathrm{E}-06 \\
2.72 \mathrm{E}-05 \\
2.51 \mathrm{E}-07 \\
2.47 \mathrm{E}-06 \\
2.15 \mathrm{E}-05 \\
2.15 \mathrm{E}-05 \\
2.15 \mathrm{E}-05 \\
2.33 \mathrm{E}-05 \\
1.77 \mathrm{E}-05 \\
2.36 \mathrm{E}-05 \\
3.81 \mathrm{E}-06 \\
2.59 \mathrm{E}-06 \\
1.08 \mathrm{E}-05 \\
1.08 \mathrm{E}-05 \\
1.14 \mathrm{E}-05 \\
2.47 \mathrm{E}-06 \\
2.36 \mathrm{E}-05\end{array}$ & $\begin{array}{l}8.98 E-07 \\
1.93 E-06 \\
2.93 E-07 \\
1.43 E-06 \\
2.18 E-06 \\
4.34 E-07 \\
2.25 E-06 \\
4.99 E-07 \\
2.18 E-07 \\
1.43 E-06 \\
1.43 E-06 \\
1.43 E-06 \\
2.06 E-06 \\
1.86 E-06 \\
2.04 E-06 \\
1.10 E-06 \\
1.01 E-06 \\
8.70 E-07 \\
8.70 E-07 \\
5.84 E-07 \\
2.18 E-07 \\
2.04 E-06\end{array}$ \\
\hline
\end{tabular}

Table 6. Annual Incident-Free Dose for the Shipping Campaign (person-rem/yr)

\begin{tabular}{|c|c|c|c|c|c|c|}
\hline \multirow[b]{2}{*}{ Route } & & & \multicolumn{2}{|c|}{ Truck } & \multicolumn{2}{|c|}{ Rail } \\
\hline & & & Crew & Public & Crew & Public \\
\hline $\begin{array}{l}\text { Bettis Atomic } \\
\text { Brookhaven NL } \\
\text { Charleston, SC } \\
\text { Colonie } \\
\text { ETEC } \\
\text { Fernald (FEMP) } \\
\text { General Atomic } \\
\text { Grand Junction Po } \\
\text { ITRI } \\
\text { Knolls Atomic } \\
\text { KKS } \\
\text { KWS } \\
\text { Lawrence Berkeley } \\
\text { LERHR } \\
\text { Lawrence Livermore } \\
\text { Paducah GDP } \\
\text { Pantex Plant } \\
\text { Pearl Harbor NS } \\
\text { Puget Sound NS } \\
\text { Reactive Metals } \\
\text { SNL - Albuquerque } \\
\text { SNL - Livermore }\end{array}$ & $\begin{array}{l}\text { to } \\
\text { to } \\
\text { to } \\
\text { to } \\
\text { to } \\
\text { to } \\
\text { to } \\
\text { to } \\
\text { to } \\
\text { to } \\
\text { to } \\
\text { to } \\
\text { to } \\
\text { to } \\
\text { to } \\
\text { to } \\
\text { to } \\
\text { to } \\
\text { to } \\
\text { to } \\
\text { to } \\
\text { to }\end{array}$ & $\begin{array}{l}\text { Portsmouth GDP } \\
\text { Portsmouth GDP } \\
\text { Savannah River } \\
\text { Portsmouth GDP } \\
\text { INEL } \\
\text { Portsmouth GDP } \\
\text { INEL } \\
\text { Rocky Flats Plant } \\
\text { Los Alamos NI } \\
\text { Portsmouth GDP } \\
\text { Portsmouth GDP } \\
\text { Portsmouth GDP } \\
\text { Hanford Site } \\
\text { Hanford Site } \\
\text { Hanford Site } \\
\text { Oak Ridge Reserv. } \\
\text { Los Alamos NL } \\
\text { Hanford Site } \\
\text { Hanford Site } \\
\text { Portsmouth GDP } \\
\text { Los Alamos NL } \\
\text { Hanford Site }\end{array}$ & $\begin{array}{l}9.78 \mathrm{E}-03 \\
2.73 \mathrm{E}-02 \\
6.19 \mathrm{E}-03 \\
2.54 \mathrm{E}-02 \\
3.23 \mathrm{E}-02 \\
1.14 \mathrm{E}-02 \\
3.06 \mathrm{E}-02 \\
8.39 \mathrm{E}-03 \\
3.54 \mathrm{E}-03 \\
2.54 \mathrm{E}-02 \\
2.54 \mathrm{E}-02 \\
2.54 \mathrm{E}-02 \\
2.76 \mathrm{E}-02 \\
2.51 \mathrm{E}-02 \\
1.41 \mathrm{E}-01 \\
2.67 \mathrm{E}-02 \\
1.11 \mathrm{E}-02 \\
7.06 \mathrm{E}-03 \\
7.06 \mathrm{E}-03 \\
1.00 \mathrm{E}-02 \\
3.28 \mathrm{E}-03 \\
2.82 \mathrm{E}-02\end{array}$ & $\begin{array}{l}1.04 \mathrm{E}-02 \\
2.99 \mathrm{E}-02 \\
6.77 \mathrm{E}-03 \\
2.63 \mathrm{E}-02 \\
4.07 \mathrm{E}-02 \\
1.20 \mathrm{E}-02 \\
3.64 \mathrm{E}-02 \\
9.92 \mathrm{E}-03 \\
4.18 \mathrm{E}-03 \\
2.63 \mathrm{E}-02 \\
2.63 \mathrm{E}-02 \\
2.63 \mathrm{E}-02 \\
3.27 \mathrm{E}-02 \\
2.94 \mathrm{E}-02 \\
1.64 \mathrm{E}-01 \\
2.99 \mathrm{E}-02 \\
1.38 \mathrm{E}-02 \\
8.04 \mathrm{E}-03 \\
8.04 \mathrm{E}-03 \\
1.05 \mathrm{E}-02 \\
3.84 \mathrm{E}-03 \\
3.28 \mathrm{E}-02\end{array}$ & $\begin{array}{l}4.67 \mathrm{E}-03 \\
6.72 \mathrm{E}-03 \\
3.47 \mathrm{E}-03 \\
5.72 \mathrm{E}-03 \\
7.22 \mathrm{E}-03 \\
3.75 \mathrm{E}-03 \\
7.37 \mathrm{E}-03 \\
3.88 \mathrm{E}-03 \\
3.31 \mathrm{E}-03 \\
5.72 \mathrm{E}-03 \\
5.72 \mathrm{E}-03 \\
5.72 \mathrm{E}-03 \\
6.99 \mathrm{E}-03 \\
6.59 \mathrm{E}-03 \\
1.39 \mathrm{E}-02 \\
5.08 \mathrm{E}-03 \\
4.89 \mathrm{E}-03 \\
4.62 \mathrm{E}-03 \\
4.62 \mathrm{E}-03 \\
4.05 \mathrm{E}-03 \\
3.31 \mathrm{E}-03 \\
6.94 \mathrm{E}-03\end{array}$ & $\begin{array}{l}1.48 \mathrm{E}-02 \\
3.41 \mathrm{E}-02 \\
5.00 \mathrm{E}-03 \\
2.13 \mathrm{E}-02 \\
2.49 \mathrm{E}-02 \\
6.87 \mathrm{E}-03 \\
2.18 \mathrm{E}-02 \\
4.58 \mathrm{E}-03 \\
5.37 \mathrm{E}-03 \\
2.13 \mathrm{E}-02 \\
2.13 \mathrm{E}-02 \\
2.13 \mathrm{E}-02 \\
2.05 \mathrm{E}-02 \\
1.70 \mathrm{E}-02 \\
4.10 \mathrm{E}-02 \\
7.91 \mathrm{E}-03 \\
7.19 \mathrm{E}-03 \\
1.15 \mathrm{E}-02 \\
1.15 \mathrm{E}-02 \\
1.20 \mathrm{E}-02 \\
5.37 \mathrm{E}-03 \\
2.05 \mathrm{E}-02\end{array}$ \\
\hline
\end{tabular}


Table 7. Annual Accident Dose Risk for the Shipping Campaign person-rem/yr)

\begin{tabular}{|c|c|c|c|}
\hline \multicolumn{2}{|l|}{ Route } & \multirow{2}{*}{$\begin{array}{c}\text { Truck } \\
5.04 \mathrm{E}-04\end{array}$} & \multirow{2}{*}{$\begin{array}{c}\text { Rail } \\
2.16 \mathrm{E}-04\end{array}$} \\
\hline Bettis Atomic & to Portsmouth GDP & & \\
\hline Brookhaven NL & to Portsmouth GDP & $4.87 \mathrm{E}-03$ & \\
\hline Charleston, SC & to Savannah River & $7.75 \mathrm{E}-03$ & $8.99 E-04$ \\
\hline Colonie & to Portsmouth GDP & $3.74 \mathrm{E}-04$ & $8.80 E-05$ \\
\hline ETEC & to INEL & $9.54 \mathrm{E}-06$ & $4.35 \mathrm{E}-06$ \\
\hline Fernald (FEMP) & to Portsmouth GDP & $1.15 E-03$ & $2.78 E-04$ \\
\hline General Atomic & to INEL & $1.08 \mathrm{E}-05$ & $4.28 \mathrm{E}-06$ \\
\hline Grand Junction PO & to Rocky Flats Plant & $1.33 \mathrm{E}-06$ & $1.13 E-08$ \\
\hline ITRI & to Los Alamos NL & $8.49 \mathrm{E}-06$ & $1.55 E-07$ \\
\hline Knolis Atomic & to Portsmouth GDP & $4.50 E-02$ & $1.06 \mathrm{E}-02$ \\
\hline KKS & to Portsmouth GDP & $3.45 \mathrm{E}-02$ & $8.09 E-03$ \\
\hline KWS & to Portsmouth GDP & $4.71 \mathrm{E}-03$ & $1.10 \mathrm{E}-03$ \\
\hline Lawrence Berkeley & to Hanford Site & $7.79 \mathrm{E}-05$ & $4.38 E-05$ \\
\hline LERHR & to Hanford Site & $7.78 \mathrm{E}-06$ & $4.14 \mathrm{E} \rightarrow 06$ \\
\hline Lawrence Livermore & to Hanford Site & $1.97 E-02$ & $1.05 E-02$ \\
\hline Paducah GDP & to Oak Ridge Reserv. & $1.17 E+00$ & $1.21 E-01$ \\
\hline Pantex Plant & to Los Alamos NL & $1.60 \mathrm{E}-04$ & $9.37 E-06$ \\
\hline Pearl Harbor NS & to Hanford Site & $8.47 \mathrm{E}-06$ & $6.32 \mathrm{E}-06$ \\
\hline Puget Sound NS & to Hanford Site & $1.95 \mathrm{E}-04$ & $1.46 \mathrm{E}-04$ \\
\hline Reactive Metals & to Portsmouth GDP & $7.40 \mathrm{E}-05$ & $2.29 \mathrm{E}-05$ \\
\hline SNL - Albuquerque & to Los Alamos NL & $5.82 \mathrm{E}-07$ & $1.10 \mathrm{E}-08$ \\
\hline SNL - Livermore & to Hanford Site & $1.95 \mathrm{E}-03$ & $1.04 \mathrm{E}-03$ \\
\hline & & $1.29 \mathrm{E}+00$ & $1.56 \mathrm{E}-01$ \\
\hline
\end{tabular}

Table 8. Expected Annual Fatalities for the Shipping Campaign

\begin{tabular}{llc}
\hline Exposure Group & Truck & Rail \\
\hline Radiological & & \\
Normal Crew & $2.1 \mathrm{E}-04$ & $5.0 \mathrm{E}-05$ \\
$\quad$ Normal Public & $2.9 \mathrm{E}-04$ & $1.8 \mathrm{E}-04$ \\
$\quad$ Accident Public & $6.4 \mathrm{E}-04$ & $7.8 \mathrm{E}-05$ \\
Nonradiological & & \\
$\quad$ Emission & $1.7 \mathrm{E}-04$ & $3.7 \mathrm{E}-04$ \\
Accident & $9.3 \mathrm{E}-04$ & $2.9 \mathrm{E}-05$
\end{tabular}

Table 9. Expected Annual Cancer Incidence for the Shipping Campaign

\begin{tabular}{lll}
\hline Exposure Group & Truck & \multicolumn{1}{c}{ Rail } \\
\hline Radiological & & \\
Normal Crew & $7.2 \mathrm{E}-04$ & $1.7 \mathrm{E}-04$ \\
$\quad$ Normal Public & $1.0 \mathrm{E}-03$ & $6.1 \mathrm{E}-04$ \\
$\quad$ Accident Public & $2.2 \mathrm{E}-03$ & $2.7 \mathrm{E}-04$ \\
$\begin{array}{c}\text { Nonradiological } \\
\text { Emission }\end{array}$ & $1.7 \mathrm{E}-04$ & $3.7 \mathrm{E}-04$ \\
Accident & NA & NA \\
\hline
\end{tabular}

Table 10. Expected Annual Genetic Effects for the Shipping Campaign

\begin{tabular}{lll}
\hline Exposure Group & Truck & Rail \\
\hline Radiological & $3.1 \mathrm{E}-05$ & $7.5 \mathrm{E}-06$ \\
Normal Crew & $5.9 \mathrm{E}-05$ & $3.6 \mathrm{E}-05$ \\
Normal Public & $1.2 \mathrm{E}-05$ & $2.5 \mathrm{E}-06$ \\
Accident Public & & \\
Nonradiological & NA & NA \\
Emission & NA & NA \\
Accident & & \\
\hline
\end{tabular}




\section{B.12 WM LLMW REGIONALIZED 2 ALTERNATIVE (CASE 7): CONTACT-HANDLED ORGANIC LIQUIDS: ALPHA - UNTREATED}

Table 1. Summary of Route Information

\begin{tabular}{|c|c|c|c|c|c|}
\hline & \multirow[b]{2}{*}{ Route } & \multirow{2}{*}{$\begin{array}{c}\text { Distance } \\
\text { (miles) }\end{array}$} & \multicolumn{3}{|c|}{ Percentage in Zone } \\
\hline & & & Rura1 & Suburban & Urban \\
\hline \multicolumn{6}{|l|}{ Truck } \\
\hline Lawrence Livermore & to INEL & 972 & 85.7 & 10.4 & 4.0 \\
\hline Mound Plant & to Savannah River & 656 & 66.5 & 32.7 & 0.8 \\
\hline West Valley DP & to Savannah River & 883 & 70.3 & 28.5 & 1.2 \\
\hline \multicolumn{6}{|l|}{ Rail } \\
\hline Lawrence Livermore & to INEL & 1100 & 89.5 & 7.4 & 3.1 \\
\hline Mound Plant & to Savannah River & 744 & 67.4 & 29.2 & 3.3 \\
\hline West valley DP & to Savannah River & 1217 & 62.8 & 32.4 & 4.9 \\
\hline
\end{tabular}

Table 2. Annual Number of Shipments and One-Way Shipment Distances

\begin{tabular}{|c|c|c|c|c|c|}
\hline \multirow[b]{2}{*}{ Route } & & \multicolumn{2}{|c|}{ \# of Shipments } & \multicolumn{2}{|c|}{ Total Mileage (mi) } \\
\hline & & Truck & Rail & Truck & Rail \\
\hline $\begin{array}{l}\text { Lawrence Livermore } \\
\text { Mound Plant } \\
\text { West Valley DP }\end{array}$ & $\begin{array}{l}\text { to INEL } \\
\text { to Savannah River } \\
\text { to Savannah River }\end{array}$ & $\begin{array}{l}4 \\
1 \\
1\end{array}$ & $\begin{array}{l}2 \\
1 \\
1\end{array}$ & $\begin{array}{l}3.89 E+03 \\
6.56 E+02 \\
8.83 E+02\end{array}$ & $\begin{array}{l}2.20 \mathrm{E}+03 \\
7.44 \mathrm{E}+02 \\
1.22 \mathrm{E}+03\end{array}$ \\
\hline Totals & & 6 & 4 & $5.43 E+03$ & $4.16 \mathrm{E}+03$ \\
\hline
\end{tabular}

Table 3. Incident-Free Dose per Shipment (person-rem/shipment)

\begin{tabular}{|c|c|c|c|c|c|c|}
\hline \multirow[b]{2}{*}{ Source/Route (s) } & & \multirow[b]{2}{*}{ Crew } & \multicolumn{4}{|c|}{ General Public } \\
\hline & & & $\overline{\text { OEE-Link }}$ & On-Link & Stops & Total \\
\hline $\begin{array}{l}\text { MIIW }(O A) \text { LI to IN } \\
\text { Lawrence Livermore }\end{array}$ & $\begin{array}{l}\text { to INEL } \\
\text { Truck } \\
\text { Rail }\end{array}$ & $\begin{array}{l}2.85 \mathrm{E}-02 \\
7.47 \mathrm{E}-03\end{array}$ & $\begin{array}{l}2.74 \mathrm{E}-03 \\
8.12 \mathrm{E}-03\end{array}$ & $\begin{array}{l}5.33 \mathrm{E}-03 \\
1.38 \mathrm{E}-04\end{array}$ & $\begin{array}{l}2.72 \mathrm{E}-02 \\
6.87 \mathrm{E}-03\end{array}$ & $\begin{array}{l}3.53 \mathrm{E}-02 \\
1.51 \mathrm{E}-02\end{array}$ \\
\hline $\begin{array}{l}\text { MLLW(OA) MP to SR } \\
\text { Mound Plant }\end{array}$ & $\begin{array}{l}\text { to Savann } \\
\text { Truck } \\
\text { Rail }\end{array}$ & $\begin{array}{l}\text { ah River } \\
2.21 \mathrm{E}-02 \\
5.99 \mathrm{E}-03\end{array}$ & $\begin{array}{l}1.91 \mathrm{E}-03 \\
9.25 \mathrm{E}-03\end{array}$ & $\begin{array}{l}2.83 \mathrm{E}-03 \\
1.78 \mathrm{E}-04\end{array}$ & $\begin{array}{l}1.84 \mathrm{E}-02 \\
5.77 \mathrm{E}-03\end{array}$ & $\begin{array}{l}2.31 \mathrm{E}-02 \\
1.52 \mathrm{E}-02\end{array}$ \\
\hline $\begin{aligned} \text { MLIW (OA) WV to SR } \\
\text { West Valley DP }\end{aligned}$ & $\begin{array}{l}\text { to Savann } \\
\text { Trúck } \\
\text { Rail }\end{array}$ & $\begin{array}{l}\text { ah River } \\
2.89 \mathrm{E}-02 \\
7.96 \mathrm{E}-03\end{array}$ & $\begin{array}{l}2.45 \mathrm{E}-03 \\
1.95 \mathrm{E}-02\end{array}$ & $\begin{array}{l}3.86 E-03 \\
3.38 E-04\end{array}$ & $\begin{array}{l}2.47 \mathrm{E}-02 \\
7.23 \mathrm{E}-03\end{array}$ & $\begin{array}{l}3.10 \mathrm{E}-02 \\
2.70 \mathrm{E}-02\end{array}$ \\
\hline
\end{tabular}

Table 4. Accident Dose Risk per Shipment (person-rem/shipment)

\begin{tabular}{llcc}
\hline Source/Route & Truck & Rail \\
\hline $\begin{array}{c}\text { MLLW (OA) LL to IN } \\
\text { Lawrence Livermore to INEI }\end{array}$ & $1.16 \mathrm{E}-02$ & $7.55 \mathrm{E}-03$ \\
$\begin{array}{c}\text { MLLW (OA) MP to SR } \\
\begin{array}{c}\text { Mound Plant } \\
\text { MLLW (OA) WV to SR } \\
\text { West Valley DP }\end{array}\end{array}$ & $\begin{array}{c}\text { to Savannah River } \\
\text { to Savannah River }\end{array}$ & $4.15 \mathrm{E}-03$ & $7.63 \mathrm{E}-04$ \\
\hline
\end{tabular}

Table 5. Nonradiological Risk Factors per Shipment

(fatalities/shipment)

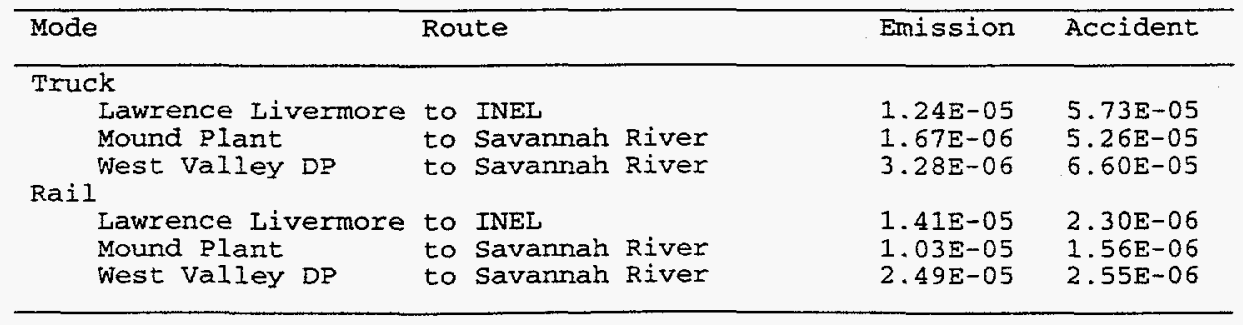


Table 6. Annual Incident-Eree Dose for the Shipping Campaign (person-rem/yr)

\begin{tabular}{|c|c|c|c|c|c|}
\hline \multirow[b]{2}{*}{ Route } & & \multicolumn{2}{|c|}{ Truck } & \multicolumn{2}{|c|}{ Rail } \\
\hline & & Crew & Public & Crew & Public \\
\hline $\begin{array}{l}\text { Lawrence Iivermore } \\
\text { Mound Plant } \\
\text { West valley DR }\end{array}$ & $\begin{array}{l}\text { to INEL } \\
\text { to Savannah River } \\
\text { to Savannah River }\end{array}$ & $\begin{array}{l}1.14 \mathrm{E}-01 \\
2.21 \mathrm{E}-02 \\
2.89 \mathrm{E}-02\end{array}$ & $\begin{array}{l}1.41 E-01 \\
2.31 E-02 \\
3.10 E-02\end{array}$ & $\begin{array}{l}1.49 \mathrm{E}-02 \\
5.99 \mathrm{E}-03 \\
7.96 \mathrm{E}-03\end{array}$ & $\begin{array}{l}3.03 \mathrm{E}-02 \\
1.52 \mathrm{E}-02 \\
2.70 \mathrm{E}-02\end{array}$ \\
\hline Totals & & $1.65 \mathrm{E}-01$ & $1.95 \mathrm{E}-01$ & $2.89 \mathrm{E}-02$ & $7.25 \mathrm{E}-02$ \\
\hline
\end{tabular}

Table 7. Annual Accident Dose Risk for the Shipping Campaign (person-rem/yr)

\begin{tabular}{lllc}
\hline Route & & Truck & Rail \\
\hline Lawrence Livermore to INEI & $4.63 \mathrm{E}-02$ & $1.51 \mathrm{E}-02$ \\
Mound Plant & to Savannah River & $4.15 \mathrm{E}-03$ & $7.63 \mathrm{E}-04$ \\
West Valley DP & to Savannah River & $4.27 \mathrm{E}-02$ & $1.37 \mathrm{E}-02$ \\
\hline Totals & & $9.31 \mathrm{E}-02$ & $2.96 \mathrm{E}-02$ \\
\hline
\end{tabular}

Table 8. Expected Annual Fatalities for the Shipping Campaign

\begin{tabular}{llc}
\hline Exposure Group & Truck & Rail \\
\hline Radiological & & \\
$\quad$ Normal Crew & $6.6 \mathrm{E}-05$ & $1.2 \mathrm{E}-05$ \\
$\quad$ Normal Public & $9.8 \mathrm{E}-05$ & $3.6 \mathrm{E}-05$ \\
$\quad \begin{array}{cc}\text { Accident Public } \\
\text { Nonradiological }\end{array}$ & $4.7 \mathrm{E}-05$ & $1.5 \mathrm{E}-05$ \\
$\quad$ Emission & $5.5 \mathrm{E}-05$ & $6.3 \mathrm{E}-05$ \\
Accident & $3.5 \mathrm{E}-04$ & $8.7 \mathrm{E}-06$ \\
\hline
\end{tabular}

Table 9. Expected Annual Cancer Incidence for the shipping Campaign

\begin{tabular}{lll}
\hline Exposure Group & Truck & Rail \\
\hline Radiological & & \\
Normal Crew & $2.3 \mathrm{E}-04$ & $4.0 \mathrm{E}-05$ \\
Normal Public & $3.3 \mathrm{E}-04$ & $1.2 \mathrm{E}-04$ \\
Accident Public & $1.5 \mathrm{E}-04$ & $5.0 \mathrm{E}-05$ \\
Nonradiological & & \\
$\quad$ Emission & $5.5 \mathrm{E}-05$ & $6.3 \mathrm{E}-05$ \\
Accident & NA & NA \\
\hline
\end{tabular}

Table 10. Expected Annual Genetic Effects for the Shipping Campaign

\begin{tabular}{lll}
\hline Exposure Group & Truck & Rail \\
\hline Radiological & & \\
Normal Crew & $9.9 \mathrm{E}-06$ & $1.7 \mathrm{E}-06$ \\
Normal Public & $2.0 \mathrm{E}-05$ & $7.2 \mathrm{E}-06$ \\
Accident Public & $2.3 \mathrm{E}-06$ & $7.3 \mathrm{E}-07$ \\
Nonradiological & & \\
Emission & NA & NA \\
Accident & NA & NA \\
\hline
\end{tabular}




\section{B.13 WM LLMW REGIONALIZED 2 ALTERNATIVE (CASE 7): CONTACT-HANDLED HETEROGENEOUS SOLIDS: NONALPHA — UNTREATED}

Table 1. Summary of Route Information

\begin{tabular}{|c|c|c|c|c|c|c|}
\hline & & & & Perce & entage in & Zone \\
\hline & Rout & & (miles) & Rural & Suburban & Urbari \\
\hline Truck & & & & & & \\
\hline Ames Laboratory & & Portsmouth GDP & 755 & 77.4 & 21.0 & 1.7 \\
\hline Argonne - East & & Portsmouth GDP & 428 & 64.6 & 30.5 & 4.9 \\
\hline Bettis Atomic & & Portsmouth GDP & 265 & 58.8 & 37.4 & 3.8 \\
\hline Battelle Columbus & & Portsmouth GDP & 84 & 68.4 & 28.3 & 3.3 \\
\hline Brookhaven NL & & Portsmouth GDP & 689 & 53.5 & 39.4 & 7.0 \\
\hline Charleston, SC & & Savannah River & 200 & 75.7 & 23.8 & 0.5 \\
\hline Colonie & & Portsmouth GDP & 688 & 57.3 & 40.1 & 2.5 \\
\hline ETEC & to & INEL & 979 & 77.7 & 14.2 & 8.1 \\
\hline Fernald (FEMP) & to & Portsmouth GDP & 173 & 68.0 & 31.6 & $0 . \overline{3}$ \\
\hline General Atomic & to & INEI & 976 & 78.8 & 17.1 & 4.1 \\
\hline Grand Junction PO & to & Rocky Flats Plant & 273 & 79.9 & 16.7 & 3.3 \\
\hline Knolis Atomic & & Portsmouth GDP & 688 & 57.3 & 40.1 & 2.5 \\
\hline Kansas City Plant & to & Rocky Flats Plant & 631 & 89.0 & 9.4 & 1.6 \\
\hline KKS & to & Portsmouth GDP & 688 & 57.3 & 40.1 & 2.5 \\
\hline KWS & to & Portsmouth GDP & 688 & 57.3 & 40.1 & 2.5 \\
\hline Lawrence Berkeley & to & Hanford Site & 875 & 78.1 & 17.8 & 4.1 \\
\hline LERHR & to & Hanford Site & 830 & 80.5 & 17.0 & $2 . \overline{6}$ \\
\hline Lawrence Iivermore & to & Hanford Site & 894 & 77.2 & 19.3 & 3.4 \\
\hline Mare Island & to & Hanford Site & 875 & 78.1 & 17.8 & 4.1 \\
\hline Norfolk Nav Shipyd & to & Savannah River & 498 & 72.8 & 26.2 & 1.0 \\
\hline Nevada Test Site & to & INEL & 712 & 82.8 & 13.7 & 3.5 \\
\hline Paducah GDP & to & Dak Ridge Reserv. & 304 & 81.3 & 18.5 & 0.2 \\
\hline Pantex Plant & to & Los Alamos NL & 402 & 89.5 & 8.0 & 2.5 \\
\hline Pearl Harbor NS & to & Hanford Site & 226 & 76.8 & 20.9 & 2.3 \\
\hline Portsmouth NS & to & Portsmouth GDP & 959 & 46.4 & 45.3 & 8.2 \\
\hline Princeton PPI & to & Portsmouth GDP & 588 & 55.1 & 38.8 & 6.1 \\
\hline Puget Sound NS & to & Hanford Site & 226 & 76.8 & 20.9 & 2.3 \\
\hline Reactive Metals & to & Portsmouth GDP & 276 & 59.5 & 37.8 & 2.8 \\
\hline SNL - Albuquerque & to & Los Alamos NL & 102 & 75.8 & 20.1 & 4.1 \\
\hline SNL - Livermore & to & Hanford Site & 894 & 77.2 & 19.3 & 3.4 \\
\hline Rail & & & & & & \\
\hline Ames Laboratory & to & Portsmouth GDP & 727 & 68.6 & 23.8 & 7.7 \\
\hline Argonne - East & & Portsmouth GDP & 422 & 64.8 & 30.0 & 5.1 \\
\hline Bettis Atomic & & Portsmouth GDP & 429 & 52.6 & 39.6 & 7.8 \\
\hline Battelle columbus & to & Portsmouth GDP & 91 & 63.4 & 31.8 & 4.8 \\
\hline Brookhaven NL & & Portsmouth GDP & 921 & 45.4 & 43.6 & 11.0 \\
\hline Charleston, SC & & Savannah River & 140 & 83.9 & 13.6 & 2.5 \\
\hline Colonie & & Portsmouth GDP & 681 & 52.1 & 40.3 & 7.5 \\
\hline ETEC & to & INEL & 1041 & 81.5 & 10.5 & 8.0 \\
\hline Fernald (FEMP) & to & Portsmouth GDP & 207 & 69.6 & 26.5 & 4.0 \\
\hline General Atomic & to & INEL & 1076 & 82.6 & 11.4 & 6.0 \\
\hline Grand Junction Po & to & Rocky Flats Plant & 239 & 95.5 & 4.3 & 0.3 \\
\hline Krolls Atomic & to & Portsmouth GDP & 681 & 52.1 & 40.3 & 7.5 \\
\hline Kansas City Plant & & Rocky Flats Plant & 778 & 89.8 & 7.8 & 2.3 \\
\hline $\mathrm{KKS}$ & & Portsmouth GDP & 681 & 52.1 & 40.3 & 7.5 \\
\hline KWS & to & Portsmouth GDP & 681 & 52.1 & 40.3 & 7.5 \\
\hline Lawrence Berkeley & to & Hanford site & 986 & 78.5 & 15.8 & 5.7 \\
\hline LERHR & to & Hanford Site & 890 & 81.0 & 14.3 & 4.7 \\
\hline Lawrence Livermore & to & Hanford site & 973 & 78.9 & 15.3 & 5.8 \\
\hline Mare Island & & Hanford site & 986 & 78.5 & 15.8 & 5.7 \\
\hline Norfolk Nav Shipyd & to & Savannah River & 529 & 74.3 & 24.1 & 1.6 \\
\hline Nevada Test Site & to & INEL & 756 & 92.8 & 5.9 & 1.3 \\
\hline Paducah GDP & to & Oak Ridge Reserv. & 527 & 89.3 & 9.0 & 1.7 \\
\hline Pantex Plant & & Los Alamos NL & 482 & 89.2 & 9.6 & 1.3 \\
\hline Pearl Harbor NS & to & Hanford Site & 416 & 73.7 & 20.1 & 6.2 \\
\hline Portsmouth NS & to & Portsmouth GDP & 962 & 49.0 & 44.3 & 6.7 \\
\hline Princeton PPL & to & Portsmouth GDP & 838 & 58.3 & 32.6 & 9.1 \\
\hline Puget Sound NS & to & Hanford Site & 416 & 73.7 & 20.1 & 6.2 \\
\hline Reactive Metals & to & Portsmouth GDP & 279 & 48.4 & 41.8 & 9.8 \\
\hline SNL - Albuquerque & to & Los Alamos NL & 104 & 74.3 & 20.0 & 5.7 \\
\hline SNL - Livermore & to & Hanford Site & 973 & 78.9 & 15.3 & 5.8 \\
\hline
\end{tabular}


Table 2. Annual Number of Shipments and One-Way shipment Distances

\begin{tabular}{|c|c|c|c|c|c|c|}
\hline \multirow[b]{2}{*}{ Route } & & & \multicolumn{2}{|c|}{ \# of Shipments } & \multicolumn{2}{|c|}{ Total Mileage (mi) } \\
\hline & & & Truck & Rail & Truck & $\operatorname{Rail}$ \\
\hline Ames Laboratory & to & Portsmouth GDP & 1 & 1 & $7.55 E+02$ & $7.27 E+02$ \\
\hline Argonne - East & to & Portsmouth GDP & 44 & 17 & $1.88 \mathrm{E}+04$ & $7.18 E+03$ \\
\hline Bettis Atomic & to & Portsmouth GDP & 1 & 1 & $2.65 \mathrm{E}+02$ & $4.29 E+02$ \\
\hline Battelle Columbus & to & Portsmouth GDP & 1 & 1 & $8.41 \mathrm{E}+01$ & $9.09 E+01$ \\
\hline Brookhaven NL & to & Portsmouth GDP & 1 & 1 & $6.89 E+02$ & $9.21 E+02$ \\
\hline Charleston, SC & to & Savannah River & 1 & 1 & $2.00 E+02$ & 1. $40 \mathrm{E}+02$ \\
\hline Colonie & to & Portsmouth GDP & 1 & 1 & $6.88 \mathrm{E}+02$ & $6.81 E+02$ \\
\hline ETEC & to & INEL & 8 & 3 & $7.83 E+03$ & $3.12 \mathrm{E}+03$ \\
\hline Fernald (FEMP) & to & Portsmouth GDP & 7 & 3 & 1. $21 \mathrm{E}+03$ & $6.22 E+02$ \\
\hline General Atomic & to & INEL & 1 & 1 & $9.76 \mathrm{E}+02$ & $1.08 E+03$ \\
\hline Grand Junction PO & to & Rocky Flats Plant & 1 & 1 & $2.73 \mathrm{E}+02$ & $2.39 E+02$ \\
\hline Knolis Atomic & to & Portsmouth GDP & 1 & $\overline{1}$ & $6.88 \mathrm{E}+02$ & $6.81 E+02$ \\
\hline Kansas City Plant & to & Rocky Flats Plant & 1 & 1 & $6.31 E+02$ & $7.78 \mathrm{E}+02$ \\
\hline $\mathrm{KKS}$ & to & Portsmouth GDP & 1 & 1 & $6.88 E+02$ & $6.81 E+02$ \\
\hline KWS & to & Portsmouth GDP & 1 & 1 & $6.88 E+02$ & $6.81 \mathrm{E}+02$ \\
\hline Lawrence Berkeley & to & Hanford site & 1 & 1 & $8.75 \mathrm{E}+02$ & $9.86 E+02$ \\
\hline LERHR & to & Hanford site & 1 & 1 & 8. $30 E+02$ & $8.90 \mathrm{E}+02$ \\
\hline Lawrence Livermore & to & Hanford Site & 4 & $\overline{2}$ & $3.58 \mathrm{E}+03$ & $1.95 \mathrm{E}+03$ \\
\hline Mare Island & to & Hanford Site & 1 & 1 & $8.75 E+02$ & $9.86 \mathrm{E}+02$ \\
\hline Norfolk Nav Shipyd & to & Savannah River & 1 & 1 & $4.98 E+02$ & $5.29 E+02$ \\
\hline Nevada Test Site & to & INEL & 1 & 1 & $7.12 E+02$ & $7.56 \mathrm{E}+02$ \\
\hline Paducah GDP & to & Oak Ridge Reserv. & 1 & 1 & $3.04 E+02$ & $5.27 E+02$ \\
\hline Pantex Plant & & Los Alamos NL & 2 & 1 & $8.04 E+02$ & $4.82 \mathrm{E}+02$ \\
\hline Pearl Harbor NS & to & Hanford Site & 1 & $\bar{I}$ & $2.26 \mathrm{E}+02$ & $4.16 \mathrm{E}+02$ \\
\hline Portsmouth NS & to & Portsmouth GDP & 1 & 1 & $9.59 \mathrm{E}+02$ & $9.62 \mathrm{E}+02$ \\
\hline Princeton PPI & to & Portsmouth GDP & 1 & $\overline{1}$ & $5.88 \mathrm{E}+02$ & $8.38 E+02$ \\
\hline Puget Sound NS & to & Hanford Site & 1 & 1 & $2.26 \bar{E}+02$ & $4.16 E+02$ \\
\hline Reactive Metals & to & Portsmouth GDP & 1 & 1 & $2.76 \mathrm{E}+02$ & $2.79 E+02$ \\
\hline SNL - Albuquerque & to & Los Alamos NL & 1 & 1 & $1.02 E+02$ & $1.04 E+02$ \\
\hline SNL - Livermore & to & Hanford site & 1 & 1 & $8.94 E+02$ & $9.73 E+02$ \\
\hline Totals & & & 90 & 5 & 104 & $2.91 E+04$ \\
\hline
\end{tabular}


Table 3. Incident-Free Dose per Shipment (person-rem/shipment)

\begin{tabular}{|c|c|c|c|c|c|c|}
\hline \multirow[b]{2}{*}{ Source/Route (s) } & & \multirow[b]{2}{*}{ Crew } & \multicolumn{4}{|c|}{ General Public } \\
\hline & & & off-Link & On-Link & Stops & Total \\
\hline \multicolumn{7}{|l|}{ MLLW (C) AL to PO } \\
\hline Ames Laboracory & to Portsm & nouth GDP & & & & \\
\hline & $\begin{array}{l}\text { Truck } \\
\text { Rail }\end{array}$ & $\begin{array}{l}2.33 \mathrm{E}-02 \\
5.91 \mathrm{E}-03\end{array}$ & $\begin{array}{l}1.86 \mathrm{E}-03 \\
1.42 \mathrm{E}-02\end{array}$ & $\begin{array}{l}3.31 \pm-03 \\
2.00 E-04\end{array}$ & $\begin{array}{l}2.11 \mathrm{E}-02 \\
5.72 \mathrm{E}-03\end{array}$ & $\begin{array}{l}2.63 \mathrm{E}-02 \\
2.01 \mathrm{E}-02\end{array}$ \\
\hline \multicolumn{7}{|l|}{ MLLW (C) AN to PO } \\
\hline Argonne - East & to Portsm & nouth GDP & & & & \\
\hline & Truck & $1.53 E-02$ & $2.05 \mathrm{E}-03$ & $2.99 \mathrm{E}-03$ & $1.20 \mathrm{E}-02$ & $1.70 \mathrm{E}-02$ \\
\hline $\operatorname{MLLW}(C)$ BA to $\mathrm{PO}$ & Rail & $4.64 \mathrm{E}-03$ & $6.76 \mathrm{E}-03$ & $1.14 \mathrm{E}-04$ & $4.78 \mathrm{E}-03$ & $1.17 \mathrm{E}-02$ \\
\hline \multicolumn{7}{|l|}{$\begin{array}{l}\text { MLIW(C) BA to PO } \\
\text { Bettis Atomic }\end{array}$} \\
\hline & Truck & $9.78 E-03$ & $1.26 \mathrm{E}-03$ & $1.73 \mathrm{E}-03$ & $7.42 \mathrm{E}-03$ & $1.04 \mathrm{E}-02$ \\
\hline & Rail & $4.67 E-03$ & $9.85 E-03$ & $1.53 \mathrm{E}-04$ & $4.80 \mathrm{E}-03$ & $1.48 \mathrm{E}-02$ \\
\hline \multirow{2}{*}{\multicolumn{7}{|c|}{$\begin{array}{l}\text { MLIW(C) BC to PO } \\
\text { Battelle Columbus }\end{array}$}} \\
\hline & & & & & & \\
\hline & Truck & $2.86 \mathrm{E}-03$ & $3.24 \mathrm{E}-04$ & $4.91 E-04$ & $2.35 \mathrm{E}-03$ & $3.17 \mathrm{E}-03$ \\
\hline & Rail & $3.26 \mathrm{E}-03$ & $1.44 E-03$ & $2.49 E-05$ & $3.76 \mathrm{E}-03$ & $5.22 \mathrm{E}-03$ \\
\hline \multirow{2}{*}{\multicolumn{7}{|c|}{$\begin{array}{l}\text { MLLW (C) BN to PO } \\
\text { Brookhaven NL }\end{array}$}} \\
\hline & & & & & & \\
\hline & $\begin{array}{l}\text { Truck } \\
\text { Rail }\end{array}$ & $\begin{array}{l}2.73 \mathrm{E}-02 \\
6.72 \mathrm{E}-03\end{array}$ & $\begin{array}{l}4.51 E-03 \\
2.74 E-02\end{array}$ & $\begin{array}{l}6.10 E-03 \\
3.90 E-04\end{array}$ & $\begin{array}{l}1.93 \mathrm{E}-02 \\
6.31 \mathrm{E}-03\end{array}$ & $\begin{array}{l}2.99 \mathrm{E}-02 \\
3.41 \mathrm{E}-02\end{array}$ \\
\hline \multirow{2}{*}{\multicolumn{7}{|c|}{$\begin{array}{l}\text { MLIW(C) CH to SR } \\
\text { Charleston, SC }\end{array}$}} \\
\hline & & & & & & \\
\hline & Truck & $6.19 E-03$ & $4.20 E-04$ & $7.45 E-04$ & $5.60 E-03$ & $6.77 E-03$ \\
\hline \multirow{2}{*}{\multicolumn{7}{|c|}{$\begin{array}{l}\text { MLLW(C) CL to PO } \\
\text { Colonie }\end{array}$}} \\
\hline & & & & & & \\
\hline & $\begin{array}{l}\text { Truck } \\
\text { Rail }\end{array}$ & $\begin{array}{l}2.54 E-02 \\
5.72 E-03\end{array}$ & $\begin{array}{l}2.99 \mathrm{E}-03 \\
1.54 \mathrm{E}-02\end{array}$ & $\begin{array}{l}4.01 \mathrm{E}-03 \\
2.43 \mathrm{E}-04\end{array}$ & $\begin{array}{l}1.93 \mathrm{E}-02 \\
5.58 \mathrm{E}-03\end{array}$ & $\begin{array}{l}2.63 \mathrm{E}-02 \\
2.13 \mathrm{E}-02\end{array}$ \\
\hline \multirow{2}{*}{\multicolumn{7}{|c|}{$\begin{array}{c}\text { MLLW(C) LC to IN } \\
\text { ETEC }\end{array}$}} \\
\hline ETEC & to INEL & & & & & \\
\hline & $\begin{array}{l}\text { Truck } \\
\text { Rail }\end{array}$ & $\begin{array}{l}3.23 E-02 \\
7.22 E-03\end{array}$ & $\begin{array}{l}5.06 E-03 \\
1.80 E-02\end{array}$ & $\begin{array}{l}8.27 \mathrm{E}-03 \\
2.22 \mathrm{E}-04\end{array}$ & $\begin{array}{l}2.74 E-02 \\
6.68 \mathrm{E}-03\end{array}$ & $\begin{array}{l}4.07 E-02 \\
2.49 E-02\end{array}$ \\
\hline \multirow{2}{*}{\multicolumn{7}{|c|}{$\begin{array}{l}\text { MLLW(C) FE to PO } \\
\text { Fernald (FEMP) }\end{array}$}} \\
\hline & & & & & & \\
\hline & $\begin{array}{l}\text { Truck } \\
\text { Rail }\end{array}$ & $\begin{array}{l}5.72 E-03 \\
3.75 E-03\end{array}$ & $\begin{array}{l}4.51 E-04 \\
2.71 E-03\end{array}$ & $\begin{array}{l}6.85 E-04 \\
4.86 \mathrm{E}-05\end{array}$ & $\begin{array}{l}4.84 E-03 \\
4.12 \mathrm{E}-03\end{array}$ & $\begin{array}{l}5.98 E-03 \\
6.87 E-03\end{array}$ \\
\hline \multirow{2}{*}{\multicolumn{7}{|c|}{$\begin{array}{l}\text { MLLW (C) So to IN } \\
\text { General Atomic }\end{array}$}} \\
\hline General Atomic & to INEI & & & & & \\
\hline & $\begin{array}{l}\text { Truck } \\
\text { Rail }\end{array}$ & $\begin{array}{l}3.06 \mathrm{E}-02 \\
7.37 \mathrm{E}-03\end{array}$ & $\begin{array}{l}3.33 E-03 \\
1.48 E-02\end{array}$ & $\begin{array}{l}5.75 \mathrm{E}-03 \\
2.03 \mathrm{E}-04\end{array}$ & $\begin{array}{l}2.73 E-02 \\
6.79 E-03\end{array}$ & $\begin{array}{l}3.64 \mathrm{E}-02 \\
2.18 \mathrm{E}-02\end{array}$ \\
\hline \multicolumn{7}{|l|}{ MLLW(C) GJ to RF } \\
\hline Grand Junction PO & $\begin{array}{l}\text { to Rocky } \\
\text { Truck } \\
\text { Rail }\end{array}$ & $\begin{array}{c}\text { Flats Plan } \\
8.39 \mathrm{E}-03 \\
3.88 \mathrm{E}-03\end{array}$ & $\begin{array}{l}8.15 E-04 \\
3.51 E-04\end{array}$ & $\begin{array}{l}1.46 \mathrm{E}-03 \\
1.62 \mathrm{E}-05\end{array}$ & $\begin{array}{l}7.65 E-03 \\
4.21 E-03\end{array}$ & $\begin{array}{l}9.92 \mathrm{E}-03 \\
4.58 \mathrm{E}-03\end{array}$ \\
\hline \multicolumn{7}{|l|}{$\begin{array}{l}\text { MLLW(C) KA to PO } \\
\text { Knolis Atomic }\end{array}$} \\
\hline & $\begin{array}{l}\text { Truck } \\
\text { Rail }\end{array}$ & $\begin{array}{l}2.54 \mathrm{E}-02 \\
5.72 \mathrm{E}-03\end{array}$ & $\begin{array}{l}2.99 \mathrm{E}-03 \\
1.54 \mathrm{E}-02\end{array}$ & $\begin{array}{l}4.01 \mathrm{E}-03 \\
2.43 \mathrm{E}-04\end{array}$ & $\begin{array}{l}1.93 \mathrm{E}-02 \\
5.58 \mathrm{E}-03\end{array}$ & $\begin{array}{l}2.63 \mathrm{E}-02 \\
2.13 \mathrm{E}-02\end{array}$ \\
\hline \multirow{2}{*}{\multicolumn{7}{|c|}{$\begin{array}{l}\text { MLLW(C) KC to RF } \\
\text { Kansas C ty }\end{array}$}} \\
\hline Kansas City Plant & to Rocky & Flats Plan & & & & \\
\hline & Rail & $\begin{array}{l}1.14 \mathrm{E}-02 \\
6.13 \mathrm{E}-03\end{array}$ & $\begin{array}{l}9.83 E-04 \\
4.77 E-03\end{array}$ & $\begin{array}{l}2.41 \mathrm{E}-03 \\
9.07 \mathrm{E}-05\end{array}$ & $5.87 E-03$ & $1.07 E-02$ \\
\hline$\underset{\text { KKS }}{M L L W(C)} \mathrm{KK}$ to Po & & & & & & \\
\hline & to Portsm & $\begin{array}{l}\text { nouth GDP } \\
2.54 \mathrm{E}-02\end{array}$ & 2.991 & & & \\
\hline & Rail & $5.72 \mathrm{E}-03$ & $1.54 \mathrm{~F}$ & 2.43 & $5.58 E-03$ & $2.13 \mathrm{E}-02$ \\
\hline$M L L W(C) \quad K W$ to $P O$ & & & & & & \\
\hline KWS & to Portsm & nouth GDP & & & & \\
\hline & Truck & $2.54 \mathrm{E}-02$ & $2.99 E-03$ & $4.01 \mathrm{E}-03$ & $1.93 \mathrm{E}-02$ & $2.63 E-02$ \\
\hline MLLW (C) LB to HS & Rail & -03 & 1.5 & 04 & E-03 & 2.3 \\
\hline Lawrence Berkeley & to Hanfor & d site & & & & \\
\hline & Truck & $2.76 \mathrm{E}-02$ & $3.02 \mathrm{E}-03$ & $5.17 \mathrm{E}-03$ & $2.45 \mathrm{E}-02$ & $3.27 E-02$ \\
\hline MLLW (C) SM to HS & R & $9 \mathrm{E}-03$ & 1.3 & 2.0 & $E-03$ & 2.0 \\
\hline LERHR & to Hanfor & d site & & & & \\
\hline & Truck & $2.51 \mathrm{E}-02$ & $2.17 E-03$ & $4.00 E-03$ & $2.32 \mathrm{E}-02$ & $2.94 \mathrm{E}-02$ \\
\hline MLLW(C) II to HS & 8 & 03 & 2 & 04 & $E-03$ & 1. \\
\hline Lawrence Livermore & to Hanfor & d site & & & & \\
\hline & Truck & -02 & $2.88 \mathrm{E}-03$ & $4.92 E-03$ & $2.50 E-02$ & $3.28 \mathrm{E}-02$ \\
\hline MLLW(C) MZ to HS & & & & & & \\
\hline Mare Island & to Hanfor & d Site & & & & \\
\hline & $\begin{array}{l}\text { Truck } \\
\text { Rail }\end{array}$ & $\begin{array}{l}2.76 \mathrm{E}-02 \\
6.99 \mathrm{E}-03\end{array}$ & $\begin{array}{l}3.02 \mathrm{E}-03 \\
1.38 \mathrm{E}-02\end{array}$ & $\begin{array}{l}5.17 E-03 \\
2.03 E-04\end{array}$ & $\begin{array}{l}2.45 \mathrm{E}-02 \\
6.51 \mathrm{E}-03\end{array}$ & $\begin{array}{l}3.27 E-02 \\
2.05 E-02\end{array}$ \\
\hline
\end{tabular}


Table 3. Incident-Free Dose per Shipment (person-rem/shipment) (Cont.)

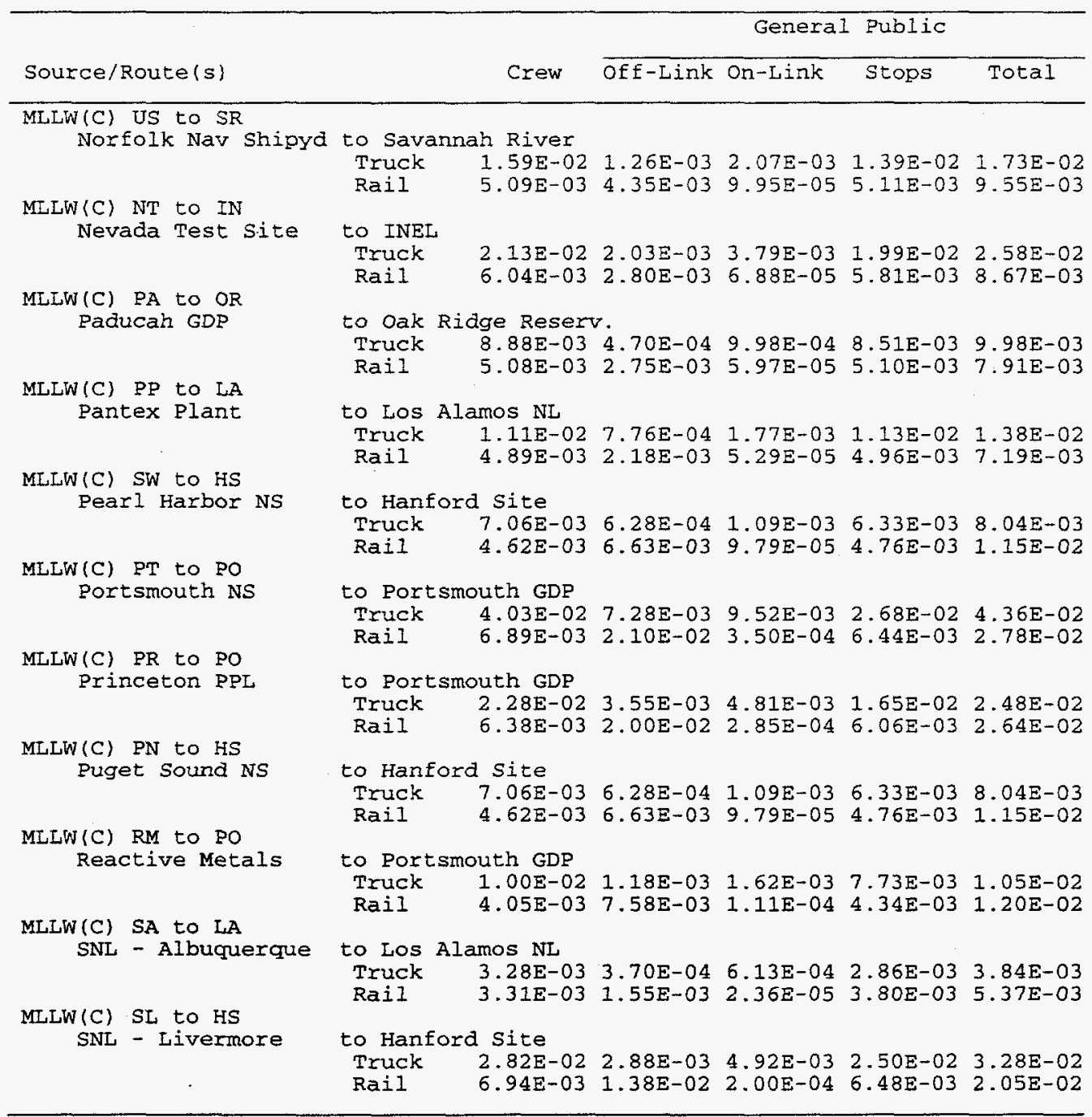


Table 4. Accident Dose Risk per Shipment (person-rem/shipment)

\begin{tabular}{|c|c|c|c|}
\hline Source/Route & & Truck & Rail \\
\hline MLLW(C) AL to PO & & & \\
\hline $\begin{array}{l}\text { Ames Laboratory } \\
\text { MLLW(C) AN to PO }\end{array}$ & to Portsmouth GDP & $1.84 \mathrm{E}-08$ & $7.49 \mathrm{E}-09$ \\
\hline $\begin{array}{l}\text { Argonne - East } \\
\text { MLLW(C) BA to PO }\end{array}$ & to Portsmouth GDP & $4.72 E-04$ & $2.52 \mathrm{E}-04$ \\
\hline Bettis Atomic & to Portsmouth GDP & $1.00 \mathrm{E}-04$ & $4.05 E-05$ \\
\hline MLLW (C) BC to PO & to Portsmouth GDP & $2.49 E-10$ & $6.63 \mathrm{E}-11$ \\
\hline MLLW(C) BN to PO & & & \\
\hline $\begin{array}{l}\text { Brookhaven NL } \\
\text { MLLW.(C) CH to SR }\end{array}$ & to Portsmouth GDP & $4.93 E-04$ & $2.53 \mathrm{E}-04$ \\
\hline $\begin{array}{l}\text { Charleston, SC } \\
\text { MLLW(C) CL to PO }\end{array}$ & to Savannah River & $5.98 E-05$ & $6.95 E-06$ \\
\hline $\begin{array}{l}\text { Colonie } \\
\text { MLLW(C) LC to IN }\end{array}$ & to Portsmouth GDP & $3.28 \mathrm{E}-08$ & $7.61 \Xi-09$ \\
\hline $\begin{array}{c}\text { ETEC } \\
\text { MLLW (C) FE to Po }\end{array}$ & to INEL & $2.18 \mathrm{E}-04$ & $2.61 E-04$ \\
\hline $\begin{array}{l}\text { Fernald (FEMP) } \\
\text { MLLW(C) So to IN }\end{array}$ & to Portsmouth GDP & $4.03 E-06$ & $2.15 E-06$ \\
\hline $\begin{array}{l}\text { General Atomic } \\
\text { MLLW(C) GJ to RE }\end{array}$ & to INEL & $4.51 E-07$ & $1.77 \mathrm{E}-07$ \\
\hline $\begin{array}{l}\text { Grand Junction PO } \\
\text { MLLW(C) KA to PO }\end{array}$ & to Rocky Flats Plant & $1.60 \mathrm{E}-08$ & $1.77 \mathrm{E}-10$ \\
\hline $\begin{array}{l}\text { Knolis Atomic } \\
\text { MLEW(C) KC to RF }\end{array}$ & to Portsmouth GDP & $1.98 E-02$ & $4.64 \mathrm{E}-03$ \\
\hline $\begin{array}{l}\text { Ransas City Plant } \\
\text { MLLW(C) KK to PO }\end{array}$ & to Rocky Flats Plant & $6.95 E-05$ & $1.08 \mathrm{E}-05$ \\
\hline $\begin{array}{l}\text { KKS } \\
\text { MLLW(C) KW to Po }\end{array}$ & to Portsmouth GDP & $2.17 E-02$ & $5.10 E-03$ \\
\hline $\begin{array}{l}\text { KWS } \\
\text { MLLW (C) LB to HS }\end{array}$ & to Portsmouth GDP & $6.98 \mathrm{E}-03$ & $1.64 \mathrm{E}-03$ \\
\hline $\begin{array}{l}\text { Lawrence Berkeley } \\
\text { MLIW(C) SM to }\end{array}$ & to Hanford Site & $1.05 E-05$ & $5.80 \mathrm{E}-06$ \\
\hline $\begin{array}{l}\text { LERHR } \\
\text { MLLW(C) LI to HS }\end{array}$ & to Hanford Site & $9.03 E-06$ & $4.74 E-06$ \\
\hline $\begin{array}{l}\text { Lawrence Livermore } \\
\text { MLLW(C) MZ to HS }\end{array}$ & to Hanford Site & $4.37 \mathrm{E}-05$ & $4.58 E-05$ \\
\hline $\begin{array}{l}\text { Mare Is land } \\
\text { MLIW(C) US to SR }\end{array}$ & to Hanford Site & $8.65 E-03$ & $4.86 E-03$ \\
\hline $\begin{array}{l}\text { Norfolk Nav Shipyd } \\
\text { MLLW(C) NT to IN }\end{array}$ & to Savannah River & $8.05 E-04$ & $1.41 \mathrm{E}-04$ \\
\hline $\begin{array}{l}\text { Nevada Test site } \\
\text { MLIW(C) PA to OR }\end{array}$ & to INEL & $1.30 \mathrm{E}-08$ & $2.10 \mathrm{E}-09$ \\
\hline $\begin{array}{l}\text { Paducah GDP } \\
\text { MLLW (C) PP to LA }\end{array}$ & to Oak Ridge Reserv. & $9.75 E-04$ & $1.25 \mathrm{E}-04$ \\
\hline $\begin{array}{l}\text { Pantex Plant } \\
\text { MLLW(C) SW to HS }\end{array}$ & to Los Alamos NL & $7.12 \mathrm{E}-05$ & $8.96 \mathrm{E}-06$ \\
\hline $\begin{array}{l}\text { Pearl Harbor NS } \\
\text { MLLW(C) PT to PO }\end{array}$ & to Hanford Site & $1.11 \mathrm{E}-04$ & $8.21 E-05$ \\
\hline $\begin{array}{l}\text { Portsmouth NS } \\
\text { MLLW (C) PR to Po }\end{array}$ & to Portsmouth GDP & $3.37 \mathrm{E}-04$ & $1.53 \mathrm{E}-04$ \\
\hline $\begin{array}{l}\text { Princeton PPL } \\
\text { MLIW(C) PN to HS }\end{array}$ & to Portsmouth GDP & $2.72 E-09$ & $1.28 \mathrm{E}-09$ \\
\hline $\begin{array}{l}\text { Puget Sound NS } \\
\text { MLLW(C) RM to Po }\end{array}$ & to Hanford Site & $2.47 E-03$ & $1.83 \mathrm{E}-03$ \\
\hline $\begin{array}{l}\text { Reactive Metals } \\
\text { MLLW(C) SA to SA }\end{array}$ & to Portsmouth GDP & $7.25 \mathrm{E}-07$ & $2.09 E-07$ \\
\hline $\begin{array}{l}\text { SNL - Albuquerque } \\
\text { MLLW(C) SL to HS }\end{array}$ & to Los Alamos NL & $8.43 E-05$ & $1.60 \mathrm{E}-06$ \\
\hline SNL - Livermore & to Hanford Site & $2.72 E-06$ & I. $43 \mathrm{E}-06$ \\
\hline
\end{tabular}


Table 5. Nonradiological Risk Factors per Shipment (fatalities/shipment)

\begin{tabular}{|c|c|c|c|}
\hline Mode & Route & Emission & Accident \\
\hline $\begin{array}{l}\text { Truck } \\
\text { Ames Laboratory } \\
\text { Argonne - East } \\
\text { Bettis Atomic } \\
\text { Battelle Columbus } \\
\text { Brookhaven NL } \\
\text { Charleston, SC } \\
\text { Colonie } \\
\text { ETEC } \\
\text { Fernald (FEMP) } \\
\text { General Atomic } \\
\text { Grand Junction Po } \\
\text { Knolls Atomic } \\
\text { Kansas City Plant } \\
\text { KKS } \\
\text { KwS } \\
\text { Lawrence Berkeley } \\
\text { LERHR } \\
\text { Lawrence Livermore } \\
\text { Mare Island } \\
\text { Norfolk Nav Shipyd } \\
\text { Nevada Test Site } \\
\text { Paducah GDP } \\
\text { Pantex Plant } \\
\text { Pearl Harbor NS } \\
\text { Portsmouth NS } \\
\text { Princeton PPL } \\
\text { Puget Sound NS } \\
\text { Reactive Metals } \\
\text { SNL - Albuquerque } \\
\text { SNL - Livermore }\end{array}$ & $\begin{array}{l}\text { to Portsmouth GDP } \\
\text { to Portsmouth GDP } \\
\text { to Portsmouth GDP } \\
\text { to Portsmouth GDP } \\
\text { to Portsmouth GDP } \\
\text { to Savannah River } \\
\text { to Portsmouth GDP } \\
\text { to INEL } \\
\text { to Portsmouth GDP } \\
\text { to INEL } \\
\text { to Rocky Flats Plant } \\
\text { to Portsmouth GDP } \\
\text { to Rocky Flats Plant } \\
\text { to Portsmouth GDP } \\
\text { to Portsmouth GDP } \\
\text { to Hanford Site } \\
\text { to Hanford Site } \\
\text { to Hanford Site } \\
\text { to Hanford Site } \\
\text { to Savannah River } \\
\text { to INEL } \\
\text { to Oak Ridge Reserv. } \\
\text { to Ios Alamos NL } \\
\text { to Hanford Site } \\
\text { to Portsmouth GDP } \\
\text { to Portsmouth GDP } \\
\text { to Hanford Site } \\
\text { to Portsmouth GDP } \\
\text { to Los Alamos NL } \\
\text { to Hanford Site }\end{array}$ & $\begin{array}{l}4.02 \mathrm{E}-06 \\
5.73 \mathrm{E}-06 \\
3.22 \mathrm{E}-06 \\
9.01 \mathrm{E}-07 \\
1.56 \mathrm{E}-05 \\
3.22 \mathrm{E}-07 \\
5.63 \mathrm{E}-06 \\
2.55 \mathrm{E}-05 \\
1.93 \mathrm{E}-07 \\
1.30 \mathrm{E}-05 \\
2.93 \mathrm{E}-06 \\
5.63 \mathrm{E}-06 \\
3.22 \mathrm{E}-06 \\
5.63 \mathrm{E}-06 \\
5.63 \mathrm{E}-06 \\
1.16 \mathrm{E}-05 \\
6.82 \mathrm{E}-06 \\
9.85 \mathrm{E}-06 \\
1.16 \mathrm{E}-05 \\
1.58 \mathrm{E}-06 \\
8.08 \mathrm{E}-06 \\
1.93 \mathrm{E}-07 \\
3.28 \mathrm{E}-06 \\
1.67 \mathrm{E}-06 \\
2.55 \mathrm{E}-05 \\
1.16 \mathrm{E}-05 \\
1.67 \mathrm{E}-06 \\
2.45 \mathrm{E}-06 \\
1.35 \mathrm{E}-06 \\
9.85 \mathrm{E}-06\end{array}$ & $\begin{array}{l}4.11 E-05 \\
2.60 \mathrm{E}-05 \\
1.33 \mathrm{E}-05 \\
3.65 \mathrm{E}-06 \\
4.90 \mathrm{E}-05 \\
1.70 \mathrm{E}-05 \\
3.56 \mathrm{E}-05 \\
6.21 \mathrm{E}-05 \\
7.51 \mathrm{E}-06 \\
6.23 \mathrm{E}-05 \\
2.32 \mathrm{E}-05 \\
3.56 \mathrm{E}-05 \\
4.74 \mathrm{E}-05 \\
3.56 \mathrm{E}-05 \\
3.56 \mathrm{E}-05 \\
4.91 \mathrm{E}-05 \\
4.66 \mathrm{E}-05 \\
5.03 \mathrm{E}-05 \\
4.91 \mathrm{E}-05 \\
4.45 \mathrm{E}-05 \\
4.36 \mathrm{E}-05 \\
2.14 \mathrm{E}-05 \\
3.21 \mathrm{E}-05 \\
9.56 \mathrm{E}-05 \\
6.65 \mathrm{E}-05 \\
3.99 \mathrm{E}-05 \\
9.56 \mathrm{E}-06 \\
1.21 \mathrm{E}-05 \\
1.20 \mathrm{E}-05 \\
5.03 \mathrm{E}-05\end{array}$ \\
\hline $\begin{array}{l}\text { Rail } \\
\text { Ames Laboratory } \\
\text { Argonne - East } \\
\text { Bettis Atomic } \\
\text { Battelle Columbus } \\
\text { Brookhaven NL } \\
\text { Charleston, SC } \\
\text { Colonie } \\
\text { ETEC } \\
\text { Fernald (FEMP) } \\
\text { General Atomic } \\
\text { Grand Junction Po } \\
\text { Krolls Atomic } \\
\text { Kansas City Plant } \\
\text { KKS } \\
\text { KWS } \\
\text { Iawrence Berkeley } \\
\text { LERHR } \\
\text { Lawrence Livermore } \\
\text { Mare Island } \\
\text { Norfolk Nav Shipyd } \\
\text { Nevada Test Site } \\
\text { Paducah GDP } \\
\text { Pantex Plant } \\
\text { Pearl Harbor NS } \\
\text { Portsmouth NS } \\
\text { Princeton PPL } \\
\text { Puget Sound NS } \\
\text { Reactive Metals } \\
\text { SNL - Albuquerque } \\
\text { SNL - Livermore }\end{array}$ & $\begin{array}{l}\text { to Portsmouth GDP } \\
\text { to Portsmouth GDP } \\
\text { to Portsmouth GDP } \\
\text { to Portsmouth GDP } \\
\text { to Portsmouth GDP } \\
\text { to Savannah River } \\
\text { to Portsmouth GDP } \\
\text { to INEL } \\
\text { to Portsmouth GDP } \\
\text { to INEL } \\
\text { to Rocky Flats Plant } \\
\text { to Portsmouth GDP } \\
\text { to Rocky Flats Plant } \\
\text { to Portsmouth GDP } \\
\text { to Portsmouth GDP } \\
\text { to Hanford Site } \\
\text { to Hanford Site } \\
\text { to Hanford Site } \\
\text { to Hanford Site } \\
\text { to Savannah River } \\
\text { to INEL } \\
\text { to Oak Ridge Reserv. } \\
\text { to Los Alamos NL } \\
\text { to Hanford Site } \\
\text { to Portsmouth GDP } \\
\text { to Portsmouth GDP } \\
\text { to Hanford Site } \\
\text { to Portsmouth GDP } \\
\text { to Los Alamos NL } \\
\text { to Hanford Site }\end{array}$ & $\begin{array}{l}2.34 \mathrm{E}-05 \\
9.08 \mathrm{E}-06 \\
1.40 \mathrm{E}-05 \\
1.84 \mathrm{E}-06 \\
4.22 \mathrm{E}-05 \\
1.46 \mathrm{E}-06 \\
2.15 \mathrm{E}-05 \\
3.48 \mathrm{E}-05 \\
3.43 \mathrm{E}-06 \\
2.72 \mathrm{E}-05 \\
2.51 \mathrm{E}-07 \\
2.15 \mathrm{E}-05 \\
7.62 \mathrm{E}-06 \\
2.15 \mathrm{E}-05 \\
2.15 \mathrm{E}-05 \\
2.33 \mathrm{E}-05 \\
1.77 \mathrm{E}-05 \\
2.36 \mathrm{E}-05 \\
2.33 \mathrm{E}-05 \\
3.64 \mathrm{E}-06 \\
3.98 \mathrm{E}-06 \\
3.81 \mathrm{E}-06 \\
2.59 \mathrm{E}-06 \\
1.08 \mathrm{E}-05 \\
2.69 \mathrm{E}-05 \\
3.18 \mathrm{E}-05 \\
1.08 \mathrm{E}-05 \\
1.14 \mathrm{E}-05 \\
2.47 \mathrm{E}-06 \\
2.36 \mathrm{E}-05\end{array}$ & $\begin{array}{l}1.52 E-06 \\
8.84 E-07 \\
8.98 E-07 \\
1.90 E-07 \\
1.93 E-06 \\
2.93 E-07 \\
1.43 E-06 \\
2.18 E-06 \\
4.34 E-07 \\
2.25 E-06 \\
4.99 E-07 \\
1.43 E-06 \\
1.63 E-06 \\
1.43 E-06 \\
1.43 E-06 \\
2.06 E-06 \\
1.86 E-06 \\
2.04 E-06 \\
2.06 E-06 \\
1.11 E-06 \\
1.58 E-06 \\
1.10 E-06 \\
1.01 E-06 \\
8.70 E-07 \\
2.01 E-06 \\
1.75 E-06 \\
8.70 E-07 \\
5.84 E-07 \\
2.18 E-07 \\
2.04 E-06\end{array}$ \\
\hline
\end{tabular}


Table 6. Annual Incident-Free Dose for the Shipping Campaign (person-rem/yr)

\begin{tabular}{|c|c|c|c|c|c|c|}
\hline \multirow{2}{*}{ Route } & & & \multicolumn{2}{|c|}{ Truck } & \multicolumn{2}{|c|}{ Rail } \\
\hline & & & Crew & Public & Crew & Public \\
\hline $\begin{array}{l}\text { Ames Laboratory } \\
\text { Argonne - East } \\
\text { Bettis Atomic } \\
\text { Battelle Columbus } \\
\text { Brookhaven NL } \\
\text { Charleston, SC } \\
\text { Colonie } \\
\text { ETEC } \\
\text { Fernald (FEMP) } \\
\text { General Atomic } \\
\text { Grand Junction Po } \\
\text { Knolis Atomic } \\
\text { Kansas City Plant } \\
\text { KKS } \\
\text { KWS } \\
\text { Lawrence Berkeley } \\
\text { LERHR } \\
\text { Lawrence Livermore } \\
\text { Mare Island } \\
\text { Norfolk Nav Shipyd } \\
\text { Nevada Test Site } \\
\text { Paducah GDP } \\
\text { Pantex Plant } \\
\text { Pearl Harbor NS } \\
\text { Portsmouth NS } \\
\text { Princeton PPL } \\
\text { Puget Sound NS } \\
\text { Reactive Metals } \\
\text { SNL - Albuquerque } \\
\text { SNL - Iivermore }\end{array}$ & $\begin{array}{l}\text { to } \\
\text { to } \\
\text { to } \\
\text { to } \\
\text { to } \\
\text { to } \\
\text { to } \\
\text { to } \\
\text { to } \\
\text { to } \\
\text { to } \\
\text { to } \\
\text { to } \\
\text { to } \\
\text { to } \\
\text { to } \\
\text { to } \\
\text { to } \\
\text { to } \\
\text { to } \\
\text { to } \\
\text { to } \\
\text { to } \\
\text { to } \\
\text { to } \\
\text { to } \\
\text { to } \\
\text { to } \\
\text { to } \\
\text { to }\end{array}$ & $\begin{array}{l}\text { Portsmouth GDP } \\
\text { Portsmouth GDP } \\
\text { Portsmouth GDP } \\
\text { Portsmouth GDP } \\
\text { Portsmouth GDP } \\
\text { Savannah River } \\
\text { Portsmouth GDP } \\
\text { INEL } \\
\text { Portsmouth GDP } \\
\text { INEL } \\
\text { RockY Flats Plant } \\
\text { Portsmouth GDP } \\
\text { Rocky Flats Plant } \\
\text { Portsmouth GDP } \\
\text { Portsmouth GDP } \\
\text { Hanford Site } \\
\text { Hanford Site } \\
\text { Hanford Site } \\
\text { Hanford Site } \\
\text { Savannah River } \\
\text { INEL } \\
\text { Oak Ridge Reserv. } \\
\text { Los Alamos NL } \\
\text { Hanford Site } \\
\text { Portsmouth GDP } \\
\text { Portsmouth GDP } \\
\text { Hanford Site } \\
\text { Portsmouth GDP } \\
\text { Los Alamos NL } \\
\text { Hanford Site }\end{array}$ & $\begin{array}{l}2.33 \mathrm{E}-02 \\
6.71 \mathrm{E}-01 \\
9.78 \mathrm{E}-03 \\
2.86 \mathrm{E}-03 \\
2.73 \mathrm{E}-02 \\
6.19 \mathrm{E}-03 \\
2.54 \mathrm{E}-02 \\
2.58 \mathrm{E}-01 \\
4.00 \mathrm{E}-02 \\
3.06 \mathrm{E}-02 \\
8.39 \mathrm{E}-03 \\
2.54 \mathrm{E}-02 \\
1.74 \mathrm{E}-02 \\
2.54 \mathrm{E}-02 \\
2.54 \mathrm{E}-02 \\
2.76 \mathrm{E}-02 \\
2.51 \mathrm{E}-02 \\
1.13 \mathrm{E}-01 \\
2.76 \mathrm{E}-02 \\
1.59 \mathrm{E}-02 \\
2.13 \mathrm{E}-02 \\
8.88 \mathrm{E}-03 \\
2.23 \mathrm{E}-02 \\
7.06 \mathrm{E}-03 \\
4.03 \mathrm{E}-02 \\
2.28 \mathrm{E}-02 \\
7.06 \mathrm{E}-03 \\
1.00 \mathrm{E}-02 \\
3.28 \mathrm{E}-03 \\
2.82 \mathrm{E}-02\end{array}$ & $\begin{array}{l}2.63 \mathrm{E}-02 \\
7.49 \mathrm{E}-01 \\
1.04 \mathrm{E}-02 \\
3.17 \mathrm{E}-03 \\
2.99 \mathrm{E}-02 \\
6.77 \mathrm{E}-03 \\
2.63 \mathrm{E}-02 \\
3.26 \mathrm{E}-01 \\
4.18 \mathrm{E}-02 \\
3.64 \mathrm{E}-02 \\
9.92 \mathrm{E}-03 \\
2.63 \mathrm{E}-02 \\
2.11 \mathrm{E}-02 \\
2.63 \mathrm{E}-02 \\
2.63 \mathrm{E}-02 \\
3.27 \mathrm{E}-02 \\
2.94 \mathrm{E}-02 \\
1.31 \mathrm{E}-01 \\
3.27 \mathrm{E}-02 \\
1.73 \mathrm{E}-02 \\
2.58 \mathrm{E}-02 \\
9.98 \mathrm{E}-03 \\
2.76 \mathrm{E}-02 \\
8.04 \mathrm{E}-03 \\
4.36 \mathrm{E}-02 \\
2.48 \mathrm{E}-02 \\
8.04 \mathrm{E}-03 \\
1.05 \mathrm{E}-02 \\
3.84 \mathrm{E}-03 \\
3.28 \mathrm{E}-02\end{array}$ & $\begin{array}{l}5.91 \mathrm{E}-03 \\
7.89 \mathrm{E}-02 \\
4.67 \mathrm{E}-03 \\
3.26 \mathrm{E}-03 \\
5.72 \mathrm{E}-03 \\
3.47 \mathrm{E}-03 \\
5.72 \mathrm{E}-03 \\
2.17 \mathrm{E}-02 \\
1.12 \mathrm{E}-02 \\
7.37 \mathrm{E}-03 \\
3.88 \mathrm{E}-03 \\
5.72 \mathrm{E}-03 \\
6.13 \mathrm{E}-03 \\
5.72 \mathrm{E}-03 \\
5.72 \mathrm{E}-03 \\
6.99 \mathrm{E}-03 \\
6.59 \mathrm{E}-03 \\
1.39 \mathrm{E}-02 \\
6.99 \mathrm{E}-03 \\
5.09 \mathrm{E}-03 \\
6.04 \mathrm{E}-03 \\
5.08 \mathrm{E}-03 \\
4.89 \mathrm{E}-03 \\
4.62 \mathrm{E}-03 \\
6.89 \mathrm{E}-03 \\
6.38 \mathrm{E}-03 \\
4.62 \mathrm{E}-03 \\
4.05 \mathrm{E}-03 \\
3.31 \mathrm{E}-03 \\
6.94 \mathrm{E}-03\end{array}$ & $\begin{array}{l}2.01 E-02 \\
1.98 E-01 \\
1.48 E-02 \\
5.22 E-03 \\
3.41 E-02 \\
5.00 E-03 \\
2.13 E-02 \\
7.47 E-02 \\
2.06 E-02 \\
2.18 E-02 \\
4.58 E-03 \\
2.13 E-02 \\
1.07 E-02 \\
2.13 E-02 \\
2.13 E-02 \\
2.05 E-02 \\
1.70 E-02 \\
4.10 \mathrm{E}-02 \\
2.05 \mathrm{E}-02 \\
9.55 \mathrm{E}-03 \\
8.67 \mathrm{E}-03 \\
7.91 \mathrm{E}-03 \\
7.19 \mathrm{E}-03 \\
1.15 \mathrm{E}-02 \\
2.78 \mathrm{E}-02 \\
2.64 \mathrm{E}-02 \\
1.15 \mathrm{E}-02 \\
1.20 \mathrm{E}-02 \\
5.37 \mathrm{E}-03 \\
2.05 \mathrm{E}-02\end{array}$ \\
\hline lotals & & & 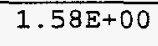 & $+\infty 0$ & 01 & 7.4 \\
\hline
\end{tabular}

Table 7. Annual Accident Dose Risk for the Shipping Campaign (person-rem/yr)

\begin{tabular}{|c|c|c|c|}
\hline Route & & Truck & Rail \\
\hline $\begin{array}{l}\text { Ames Laboratory } \\
\text { Argonne - East } \\
\text { Bettis Atomic } \\
\text { Battelle Columbus } \\
\text { Brookhaven NL } \\
\text { Charleston, SC } \\
\text { Colonie } \\
\text { ETEC } \\
\text { Fernald (FEMP) } \\
\text { General Atomic } \\
\text { Grand Junction Po } \\
\text { Knolls Atomic } \\
\text { Kansas City Plant } \\
\text { KRS } \\
\text { KWS } \\
\text { Lawrence Berkeley } \\
\text { LERHR } \\
\text { Lawrence Livermore } \\
\text { Mare Island } \\
\text { Norfolk Nav Shipyd } \\
\text { Nevada Test Site } \\
\text { Paducah GDP } \\
\text { Pantex Plant } \\
\text { Pearl Harbor NS } \\
\text { Portsmouth NS } \\
\text { Princeton PPL } \\
\text { Puget Sound NS } \\
\text { Reactive Metals } \\
\text { SNL - Albuquerque } \\
\text { SNL - Livermore }\end{array}$ & $\begin{array}{l}\text { to Portsmouth GDP } \\
\text { to Portsmouth GDP } \\
\text { to Portsmouth GDP } \\
\text { to Portsmouth GDP } \\
\text { to Portsmouth GDP } \\
\text { to Savannah River } \\
\text { to Portsmouth GDP } \\
\text { to INEL } \\
\text { to Portsmouth GDP } \\
\text { to INEL } \\
\text { to Rocky Flats Plant } \\
\text { to Portsmouth GDP } \\
\text { to Rocky Flats Plant } \\
\text { to Portsmouth GDP } \\
\text { to Portsmouth GDP } \\
\text { to Hanford Site } \\
\text { to Hanford Site } \\
\text { to Hanford Site } \\
\text { to Hanford Site } \\
\text { to Savannah River } \\
\text { to INEL } \\
\text { to Oak Ridge Reserv. } \\
\text { to Los Alamos NL } \\
\text { to Hanford Site } \\
\text { to Portsmouth GDP } \\
\text { to Portsmouth GDP } \\
\text { to Hanford Site } \\
\text { to Portsmouth GDP } \\
\text { to Los Alamos NL } \\
\text { to Hanford Site }\end{array}$ & $\begin{array}{l}1.84 \mathrm{E}-08 \\
2.08 \mathrm{E}-02 \\
1.00 \mathrm{E}-04 \\
2.49 \mathrm{E}-10 \\
4.93 \mathrm{E}-04 \\
5.98 \mathrm{E}-05 \\
3.28 \mathrm{E}-08 \\
1.75 \mathrm{E}-03 \\
2.82 \mathrm{E}-05 \\
4.51 \mathrm{E}-07 \\
1.60 \mathrm{E}-08 \\
1.98 \mathrm{E}-02 \\
6.95 \mathrm{E}-05 \\
2.17 \mathrm{E}-02 \\
6.98 \mathrm{E}-03 \\
1.05 \mathrm{E}-05 \\
9.03 \mathrm{E}-06 \\
1.75 \mathrm{E}-04 \\
8.65 \mathrm{E}-03 \\
8.05 \mathrm{E}-04 \\
1.30 \mathrm{E}-08 \\
9.75 \mathrm{E}-04 \\
1.42 \mathrm{E}-04 \\
1.11 \mathrm{E}-04 \\
3.37 \mathrm{E}-04 \\
2.72 \mathrm{E}-09 \\
2.47 \mathrm{E}-03 \\
7.25 \mathrm{E}-07 \\
8.43 \mathrm{E}-05 \\
2.72 \mathrm{E}-06\end{array}$ & $\begin{array}{l}7.49 \mathrm{E}-09 \\
4.28 \mathrm{E}-03 \\
4.05 \mathrm{E}-05 \\
6.53 \mathrm{E}-11 \\
2.53 \mathrm{E}-04 \\
6.95 \mathrm{E}-06 \\
7.61 \mathrm{E}-09 \\
7.83 \mathrm{E}-04 \\
6.44 \mathrm{E}-06 \\
1.77 \mathrm{E}-07 \\
1.77 \mathrm{E}-10 \\
4.64 \mathrm{E}-03 \\
1.08 \mathrm{E}-05 \\
5.10 \mathrm{E}-03 \\
1.64 \mathrm{E}-03 \\
5.80 \mathrm{E}-06 \\
4.74 \mathrm{E}-06 \\
9.17 \mathrm{E}-05 \\
4.86 \mathrm{E}-03 \\
1.41 \mathrm{E}-04 \\
2.10 \mathrm{E}-09 \\
1.25 \mathrm{E}-04 \\
8.96 \mathrm{E}-06 \\
8.21 \mathrm{E}-05 \\
1.53 \mathrm{E}-04 \\
1.28 \mathrm{E}-09 \\
1.83 \mathrm{E}-03 \\
2.09 \mathrm{E}-07 \\
1.60 \mathrm{E}-06 \\
1.43 \mathrm{E}-06\end{array}$ \\
\hline Totals & & $8.56 E-02$ & -02 \\
\hline
\end{tabular}


Table 8. Expected Annual Fatalities for the Shipping Campaign

\begin{tabular}{llc}
\hline Exposure Group & Truck & Rail \\
\hline Radiological & & \\
Normal Crew & $6.3 \mathrm{E}-04$ & $1.1 \mathrm{E}-04$ \\
$\quad$ Normal Public & $9.0 \mathrm{E}-04$ & $3.7 \mathrm{E}-04$ \\
$\quad$ Accident Public & $4.3 \mathrm{E}-05$ & $1.2 \mathrm{E}-05$ \\
Nonradiological & & \\
$\quad$ Emission & $7.1 \mathrm{E}-04$ & $7.2 \mathrm{E}-04$ \\
Accident & $2.8 \mathrm{E}-03$ & $6.1 \mathrm{E}-05$ \\
\hline
\end{tabular}

Table 9. Expected Annual Cancer Incidence for the shipping Campaign

\begin{tabular}{lll}
\hline Exposure Group & Truck & \multicolumn{1}{c}{ Rail } \\
\hline Radiological & $2.2 \mathrm{E}-03$ & $3.8 \mathrm{E}-04$ \\
Normal Crew & $3.1 \mathrm{E}-03$ & $1.3 \mathrm{E}-03$ \\
Normal Public & $1.5 \mathrm{E}-04$ & $4.1 \mathrm{E}-05$ \\
Accident Public & $7.1 \mathrm{E}-04$ & $7.2 \mathrm{E}-04$ \\
Nonradiological & NA & NA \\
Emission & & \\
Accident & &
\end{tabular}

Table 10. Expected Annual Genetic Effects for the shipping Campaign

\begin{tabular}{lll}
\hline Exposure Group & Truck & Rail \\
\hline Radiological & & \\
Normal Crew & $9.5 \mathrm{E}-05$ & $1.6 \mathrm{E}-05$ \\
Normal Public & $1.8 \mathrm{E}-04$ & $7.4 \mathrm{E}-05$ \\
Accident Public & $7.6 \mathrm{E}-06$ & $2.2 \mathrm{E}-06$ \\
Nonradiological & & NA \\
Emission & NA & NA \\
Accident & NA & \\
\hline
\end{tabular}




\section{B.14 WM LLMW REGIONALIZED 2 ALTERNATIVE (CASE 7): CONTACT-HANDLED HETEROGENEOUS SOLIDS: ALPHA - UNTREATED}

Tabie 1. Summary of Route Information

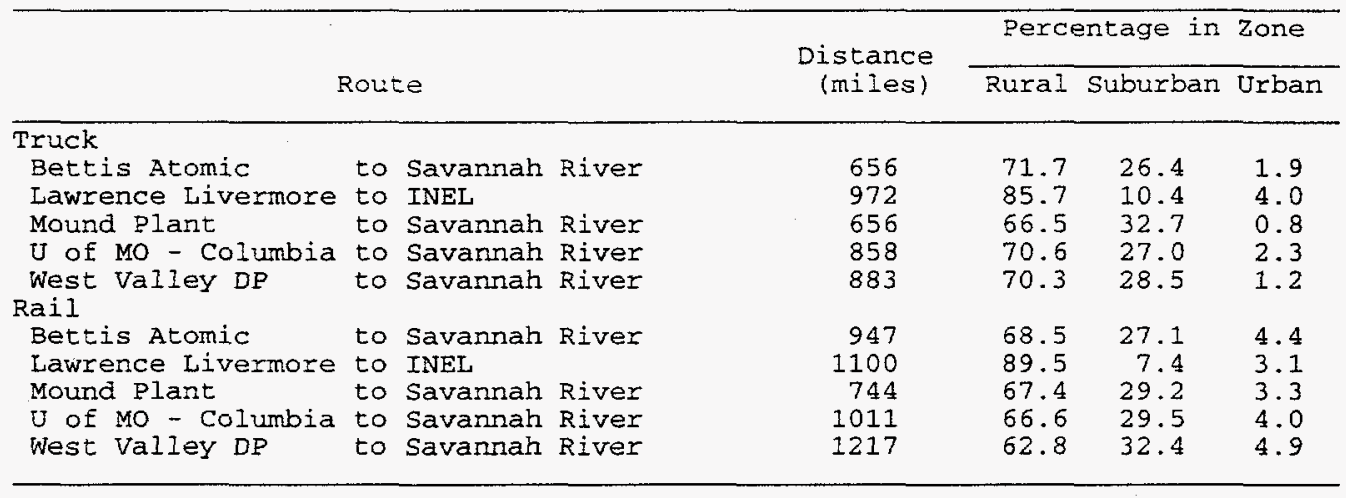

Table 2. Annual Number of Shipments and one-way Shipment Distances

\begin{tabular}{|c|c|c|c|c|c|}
\hline \multirow[b]{2}{*}{ Route } & & \multicolumn{2}{|c|}{ \# of shipments } & \multicolumn{2}{|c|}{ Total Mileage (mi) } \\
\hline & & Truck & Rail & Truck & Rail \\
\hline $\begin{array}{l}\text { Bettis Atomic } \\
\text { Lawrence Livermore } \\
\text { Mound Plant } \\
\text { U of MO - Columbia } \\
\text { West Valley DP }\end{array}$ & $\begin{array}{l}\text { to Savannah River } \\
\text { to INEL } \\
\text { to Savannah River } \\
\text { to Savannah River } \\
\text { to Savannah River }\end{array}$ & $\begin{array}{l}1 \\
6 \\
1 \\
1 \\
1\end{array}$ & $\begin{array}{l}1 \\
3 \\
1 \\
1 \\
1\end{array}$ & $\begin{array}{l}6.56 \mathrm{E}+02 \\
5.83 \mathrm{E}+03 \\
6.56 \mathrm{E}+02 \\
8.58 \mathrm{E}+02 \\
8.83 \mathrm{E}+02\end{array}$ & $\begin{array}{l}9.47 \mathrm{E}+02 \\
3.30 \mathrm{E}+03 \\
7.44 \mathrm{E}+02 \\
1.01 \mathrm{E}+03 \\
1.22 \mathrm{E}+03\end{array}$ \\
\hline Totals & & 10 & 7 & $8.88 E+03$ & $7.22 \mathrm{E}+03$ \\
\hline
\end{tabular}

Table 3. Incident-Free Dose per Shipment (person-rem/shipment)

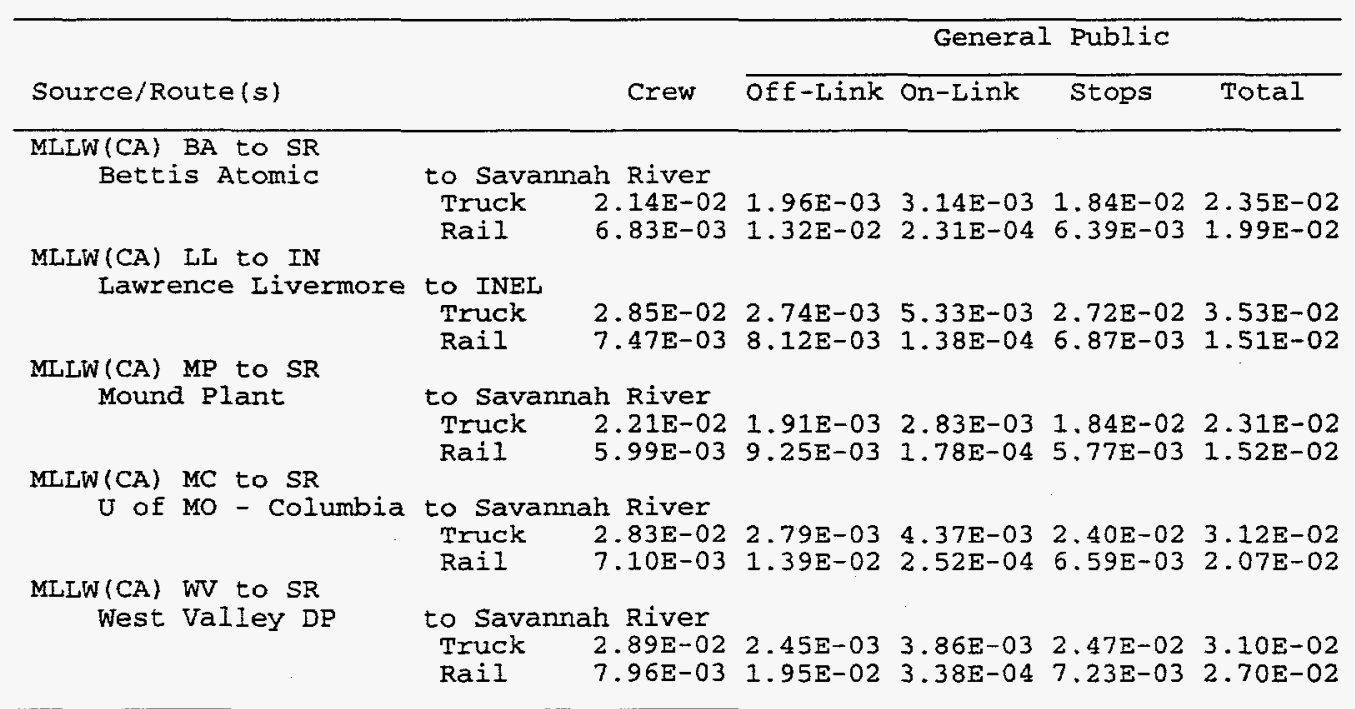


Table 4. Accident Dose Risk per Shipment (person-rem/shipment)

\begin{tabular}{|c|c|c|c|}
\hline \multicolumn{2}{|l|}{ Source/Route } & Truck & Rail \\
\hline$M L L W(C A)$ BA to $S R$ & & & \\
\hline $\begin{array}{l}\text { Bettis Atomic } \\
\text { MLLW(CA) LL to IN }\end{array}$ & to Savannah River & $2.11 \mathrm{E}-04$ & $7.45 \mathrm{E}-05$ \\
\hline Lawrence Livermore & to INEL & $5.14 E-05$ & $3.46 \mathrm{E}-05$ \\
\hline $\begin{array}{c}\text { MLLW (CA) MP to SR } \\
\text { Mound Plant }\end{array}$ & to Savannah River & $9.48 \mathrm{E}-08$ & $1.85 \mathrm{E}-08$ \\
\hline $\begin{array}{c}\text { MLLW (CA) MC to SR } \\
\text { U of MO - Columbia } \\
\text { MLLW(CA) WV to SR }\end{array}$ & to Savannah River & $6.78 \mathrm{E}-07$ & $2.05 E-07$ \\
\hline West valley $D ?$ & to Savannah River & $3.36 \mathrm{E}-04$ & $1.07 \mathrm{E}-04$ \\
\hline
\end{tabular}

Table 5. Nonradiological Risk Factors per Shipment (Eatalities/shipment)

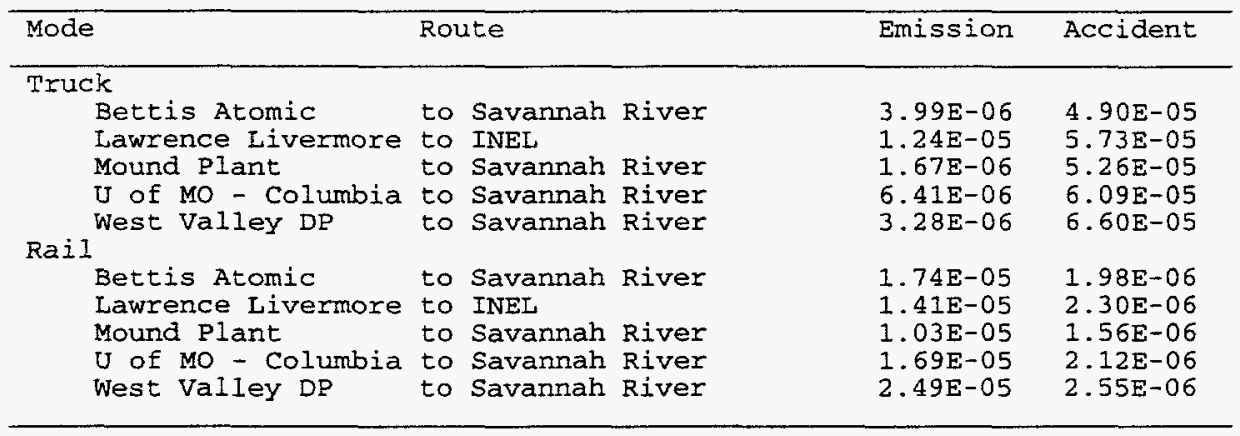

Table 6. Annual Incident-Free Dose for the Shipping Campaign (person-rem/yr)

\begin{tabular}{|c|c|c|c|c|c|}
\hline \multirow[b]{2}{*}{ Route } & & \multicolumn{2}{|c|}{ Truck } & \multicolumn{2}{|c|}{ Rail } \\
\hline & & Crew & Public & Crew & Public \\
\hline $\begin{array}{l}\text { Bettis Atomic } \\
\text { Lawrence Livermore } \\
\text { Mound Plant } \\
\text { U of MO - Columbia } \\
\text { West Valley DP }\end{array}$ & $\begin{array}{l}\text { to Savannah River } \\
\text { to INEL } \\
\text { to Savannah River } \\
\text { to Savannah River } \\
\text { to Savannah River }\end{array}$ & $\begin{array}{l}2.14 \mathrm{E}-02 \\
1.71 \mathrm{E}-01 \\
2.21 \mathrm{E}-02 \\
2.83 \mathrm{E}-02 \\
2.89 \mathrm{E}-02\end{array}$ & $\begin{array}{l}2.35 \mathrm{E}-02 \\
2.12 \mathrm{E}-01 \\
2.31 \mathrm{E}-02 \\
3.12 \mathrm{E}-02 \\
3.10 \mathrm{E}-02\end{array}$ & $\begin{array}{l}6.83 \mathrm{E}-03 \\
2.24 \mathrm{E}-02 \\
5.99 \mathrm{E}-03 \\
7.10 \mathrm{E}-03 \\
7.96 \mathrm{E}-03\end{array}$ & $\begin{array}{l}1.99 \mathrm{E}-02 \\
4.54 \mathrm{E}-02 \\
1.52 \mathrm{E}-02 \\
2.07 \mathrm{E}-02 \\
2.70 \mathrm{E}-02\end{array}$ \\
\hline Totals & & $2.71 \mathrm{E}-01$ & $3.20 \mathrm{E}-01$ & $5.03 E-02$ & $1.28 E-01$ \\
\hline
\end{tabular}

Table 7. Annual Accident Dose Risk for the Shipping Campaign (person-rem/yr)

\begin{tabular}{llcc}
\hline Route & & Truck & Rail \\
\hline Bettis Atomic & to Savannah River & $2.11 \mathrm{E}-04$ & $7.45 \mathrm{E}-05$ \\
Lawrence Livermore to INEL & $3.09 \mathrm{E}-04$ & $1.04 \mathrm{E}-04$ \\
Mound Plant & to Savannah River & $9.48 \mathrm{E}-08$ & $1.85 \mathrm{E}-08$ \\
U of MO - Columbia to Savannah River & $6.78 \mathrm{E}-07$ & $2.05 \mathrm{E}-07$ \\
West Valley DP & to Savannah River & $3.36 \mathrm{E}-04$ & $1.07 \mathrm{E}-04$ \\
\hline Totals & & $8.56 \mathrm{E}-04$ & $2.86 \mathrm{E}-04$ \\
\hline
\end{tabular}

Table 8. Expected Annual Fatalities for the Shipping Campaign

\begin{tabular}{llc}
\hline Exposure Group & Truck & Rail \\
\hline Radiological & $1.1 \mathrm{E}-04$ & $2.0 \mathrm{E}-05$ \\
$\quad$ Normal Crew & $1.6 \mathrm{E}-04$ & $6.4 \mathrm{E}-05$ \\
$\quad$ Normal Public & $4.3 \mathrm{E}-07$ & $1.4 \mathrm{E}-07$ \\
$\quad \begin{array}{cc}\text { Accident Public } \\
\text { Nonradiological } \\
\text { Emission }\end{array}$ & $9.0 \mathrm{E}-05$ & $1.1 \mathrm{E}-04$ \\
Accident & $5.7 \mathrm{E}-04$ & $1.5 \mathrm{E}-05$ \\
\hline
\end{tabular}


Table 9. Expected Annual Cancer Incidence for the Shipping Campaign

\begin{tabular}{|c|c|c|}
\hline Exposure Group & Truck & Rail \\
\hline \multicolumn{3}{|l|}{ Radiological } \\
\hline Normal Crew & $3.8 \pm-04$ & $7.0 \mathrm{E}-05$ \\
\hline Normal Public & $5.4 E-04$ & $2.2 \mathrm{E}-04$ \\
\hline Accident Public & $1.5 E-06$ & $4.9 \mathrm{E}-07$ \\
\hline \multicolumn{3}{|l|}{ Nonradiological } \\
\hline Enission & $9.0 \pm-05$ & $1.1 E-04$ \\
\hline Accident & $\mathrm{NA}$ & $\mathrm{NA}$ \\
\hline
\end{tabular}

Table 10. Expected Annual Genetic Effects for the Shipping Campaign

\begin{tabular}{lll}
\hline Exposure Group & Truck & Rail \\
\hline Radiological & & \\
Normal Crew & $1.6 \mathrm{E}-05$ & $3.0 \mathrm{E}-06$ \\
Normal Public & $3.2 \mathrm{E}-05$ & $1.3 \mathrm{E}-05$ \\
Accident Public & $5.2 \mathrm{E}-08$ & $1.7 \mathrm{E}-08$ \\
Nonradiological & & \\
Emission & NA & NA \\
Accident & NA & NA \\
\hline
\end{tabular}




\section{B.15 WM LLMW REGIONALIZED 2 ALTERNATIVE (CASE 7): CONTACT-HANDLED HETEROGENEOUS SOLIDS: NONALPHA - TREATED}

Table 1. Summary of Route Information

\begin{tabular}{|c|c|c|c|c|c|c|}
\hline & \multirow{2}{*}{\multicolumn{2}{|c|}{ Route }} & \multirow{2}{*}{$\begin{array}{c}\text { Distance } \\
\text { (miles) }\end{array}$} & \multicolumn{3}{|c|}{ Percentage in Zone } \\
\hline & & & & Rura1 & Suburban & Urban \\
\hline \multicolumn{7}{|l|}{ Truck } \\
\hline Ames Laboratory & to & Oak Ridge Reserv. & 900 & 75.2 & 23.4 & 1.5 \\
\hline Argonne - East & & Oak Ridge Reserv. & 584 & 67.0 & 30.1 & 2.9 \\
\hline Charleston, SC & & Savannah River & 200 & 75.7 & 23.8 & 0.5 \\
\hline Colonie & & Oak Ridge Reserv. & 2193 & 84.1 & 13.8 & 2.1 \\
\hline Fernald (FEMP) & & Oak Ridge Reserv. & 312 & 69.9 & 29.5 & 0.6 \\
\hline General Atomic & & Nevada Test Site & 398 & 73.9 & 19.8 & 6.3 \\
\hline Grand Junction PO & to & Los Alamos NL. & 685 & 84.2 & 13.7 & 2.2 \\
\hline LERHR & & Nevada Test Site & 735 & 81.1 & 11.2 & 7.6 \\
\hline Paducah GDP & & Oak Ridge Reserv. & 304 & 81.3 & 18.5 & 0.2 \\
\hline Pantex Plant & to & Los Alamos NL & 402 & 89.5 & 8.0 & 2.5 \\
\hline Portsmouth GDP & & Dak Ridge Reserv. & 371 & 74.5 & 24.6 & 0.9 \\
\hline Rocky Flats Plant & to & Los Alamos NL & $45 \overline{2}$ & 82.2 & 14.9 & 2.9 \\
\hline Reactive Metals & to & Oak Ridge Reserv. & 608 & 64.1 & 34.1 & 1.8 \\
\hline \multicolumn{7}{|l|}{ Rail } \\
\hline Ames Laboratory & to & Oak Ridge Reserv. & 954 & 71.7 & 22.0 & 6.3 \\
\hline Argonne - East & & Oak Ridge Reserv. & 548 & 70.7 & 25.3 & 4.0 \\
\hline Charleston, SC & & Savannah River & 140 & 83.9 & 13.6 & 2.5 \\
\hline Colonie & & Oak Ridge Reserv. & 2709 & 86.8 & 10.0 & 3.1 \\
\hline Fernald (FEMP) & & Oak Ridge Reserv. & 358 & 75.7 & 21.8 & 2.5 \\
\hline General Atomic & & Nevada Test Site & 518 & 73.4 & 15.9 & 10.7 \\
\hline Grand Junction PO & to & Los Alamos NL & 682 & 95.6 & 4.1 & 0.2 \\
\hline LERHR & to & Nevada Test Site & 827 & 75.4 & 17.7 & 6.9 \\
\hline Paducah GDP & to & Oak Ridge Reserv. & 527 & 89.3 & 9.0 & 1.7 \\
\hline Pantex Plant & to & Los Alamos NL & 482 & 89.2 & 9.6 & 1.3 \\
\hline Portsmouth GDP & to & Oak Ridge Reserv. & 442 & 66.8 & 31.5 & 1.7 \\
\hline Rocky Flats Plant & to & Los Alamos NL & 504 & 85.7 & 11.1 & 3.2 \\
\hline Reactive Metals & & Oak Ridge Reserv. & 600 & 59.4 & 33.2 & 7.4 \\
\hline
\end{tabular}

Table 2. Annual Number of Shipments and One-Way Shipment Distances

\begin{tabular}{|c|c|c|c|c|c|c|}
\hline \multirow[b]{2}{*}{ Route } & & & \multicolumn{2}{|c|}{ \# of shipments } & \multicolumn{2}{|c|}{ Total Mileage(mi) } \\
\hline & & & Truck & Rail & Truck & Rail \\
\hline $\begin{array}{l}\text { Ames Laboratory } \\
\text { Argonne - East } \\
\text { Charleston, SC } \\
\text { Colonie } \\
\text { Fernald (FEMP) } \\
\text { General Atomic } \\
\text { Grand Junction PO } \\
\text { LERHR } \\
\text { Paducah GDP } \\
\text { Pantex Plant } \\
\text { Portsmouth GDP } \\
\text { Rocky Flats Plant } \\
\text { Reactive Metals }\end{array}$ & $\begin{array}{l}\text { to } \\
\text { to } \\
\text { to } \\
\text { to } \\
\text { to } \\
\text { to } \\
\text { to } \\
\text { to } \\
\text { to } \\
\text { to } \\
\text { to } \\
\text { to } \\
\text { to }\end{array}$ & $\begin{array}{l}\text { Oak Ridge Reserv. } \\
\text { Oak Ridge Reserv. } \\
\text { Savannah River } \\
\text { Oak Ridge Reserv. } \\
\text { Oak Ridge Reserv. } \\
\text { Nevada Test Site } \\
\text { Los Alamos NL } \\
\text { Nevada Test Site } \\
\text { Oak Ridge Reserv. } \\
\text { Los Alamos NL } \\
\text { Oak Ridge Reserv. } \\
\text { Los Alamos NL } \\
\text { Oak Ridge Reserv. }\end{array}$ & $\begin{array}{r}1 \\
1 \\
1 \\
1 \\
2 \\
1 \\
1 \\
1 \\
1 \\
1 \\
155 \\
1 \\
1\end{array}$ & $\begin{array}{l}1 \\
1 \\
1 \\
1 \\
1 \\
1 \\
1 \\
1 \\
1 \\
1 \\
57 \\
1 \\
1\end{array}$ & $\begin{array}{l}9.00 E+02 \\
5.84 E+02 \\
2.00 E+02 \\
2.19 E+03 \\
6.24 E+02 \\
3.98 E+02 \\
6.85 E+02 \\
7.35 E+02 \\
3.04 E+02 \\
4.02 E+02 \\
5.75 E+04 \\
4.52 E+02 \\
6.08 E+02\end{array}$ & $\begin{array}{l}9.54 \mathrm{E}+02 \\
6.48 \mathrm{E}+02 \\
1.40 \mathrm{E}+02 \\
2.71 \mathrm{E}+03 \\
3.58 \mathrm{E}+02 \\
5.18 \mathrm{E}+02 \\
6.82 \mathrm{E}+02 \\
8.27 \mathrm{E}+02 \\
5.27 \mathrm{E}+02 \\
4.82 \mathrm{E}+02 \\
2.52 \mathrm{E}+04 \\
5.04 \mathrm{E}+02 \\
6.00 \mathrm{E}+02\end{array}$ \\
\hline Totals & & & 168 & 69 & $6.55 E+04$ & $3.41 E+04$ \\
\hline
\end{tabular}


Table 3. Incident-Free Dose per Shipment (person-rem/shipment)

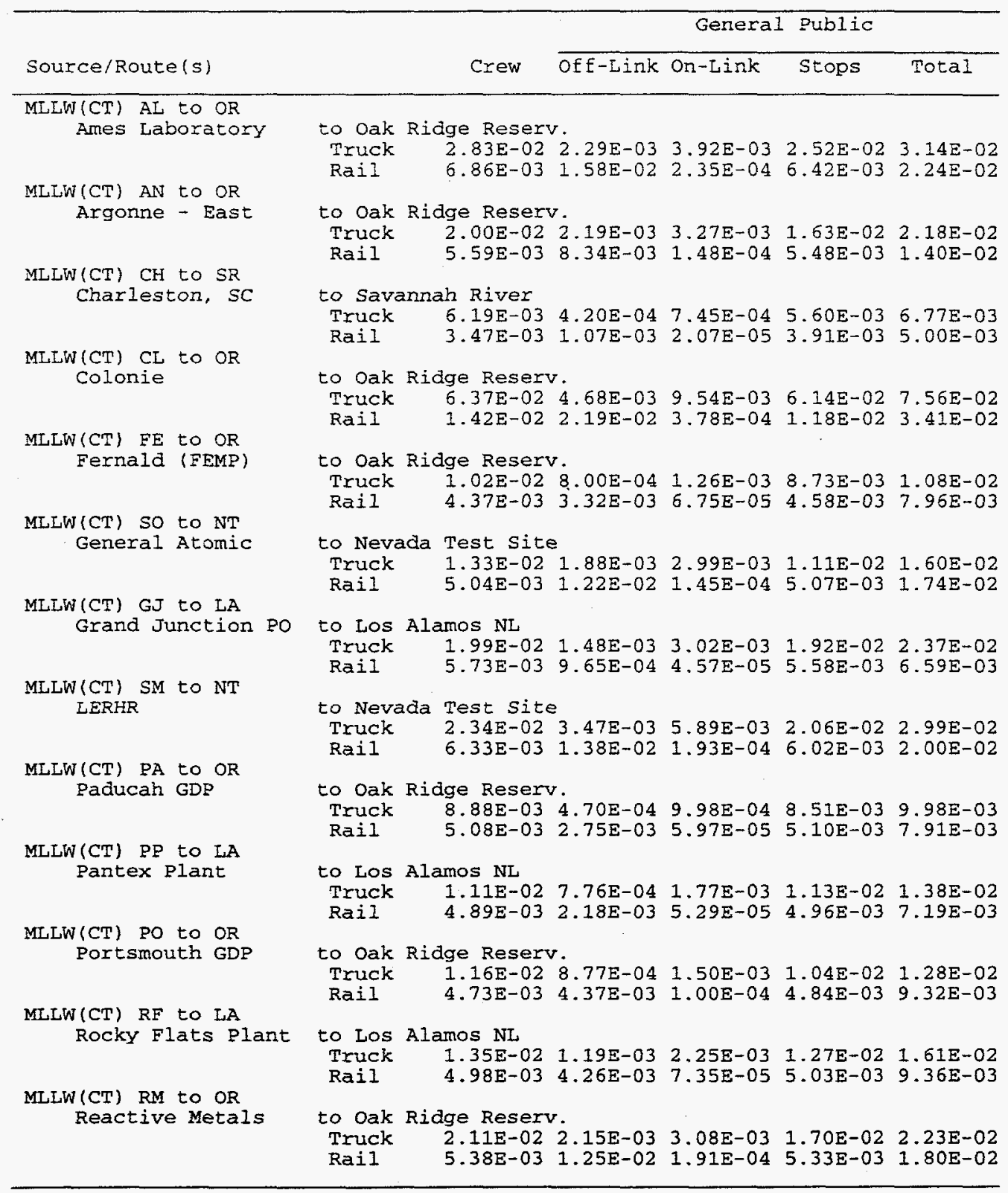


Table 4. Accident Dose Risk per Shipment (person-rem/shipment)

\begin{tabular}{|c|c|c|c|}
\hline Source/Route & & Truck & Rail \\
\hline $\begin{array}{l}\text { MLLW(CT) AI to OR } \\
\text { Ames Laboratory }\end{array}$ & to Oak Ridge Reserv. & $4.17 \mathrm{E}-08$ & $1.36 \Xi-08$ \\
\hline $\begin{array}{l}\text { MLLW(CT) AN to OR } \\
\text { Argonne - East } \\
\text { MLLW(CT) CH to SR }\end{array}$ & to Oak Ridge Reserv. & $1.48 \mathrm{E}-05$ & $2.57 \mathrm{E}-06$ \\
\hline $\begin{array}{l}\text { Charleston, SC } \\
\text { MLLW(CT) CL to OR }\end{array}$ & to Savannah River & $4.40 E-05$ & $5.11 E-06$ \\
\hline $\begin{array}{l}\text { Colonie } \\
\text { MLLW(CT) FE to OR }\end{array}$ & to Oak Ridge Reserv. & $1.66 \mathrm{E}-07$ & $4.81 E-08$ \\
\hline $\begin{array}{l}\text { Fernald (FEMP) } \\
M L L W(C T) \text { So to NT }\end{array}$ & to Oak Riage Reserv. & $3.14 \mathrm{E}-05$ & $7.218-06$ \\
\hline $\begin{array}{l}\text { General Atomic } \\
\text { MLLW(CT) GJ to LA }\end{array}$ & to Nevada Test Site & $9.22 \mathrm{E}-07$ & $5.27 \mathrm{E}-07$ \\
\hline $\begin{array}{l}\text { Grand Junction PO } \\
\text { MLLW(CT) SM to NT }\end{array}$ & to Los Alamos NL & $7.50 \mathrm{E}-08$ & $9.34 \mathrm{E}-10$ \\
\hline $\begin{array}{l}\text { LERHR } \\
\text { MLLW(CT) PA to OR }\end{array}$ & to Nevada Test Site & $7.12 \mathrm{E}-07$ & $4.22 E-07$ \\
\hline $\begin{array}{l}\text { Paducah GDP } \\
\text { MLLW(CT) PP to LA }\end{array}$ & to Oak Ridge Reserv. & $2.33 E-04$ & $2.98 \mathrm{E}-05$ \\
\hline $\begin{array}{l}\text { Pantex Plant } \\
\text { MLLW(CT) Po to OR }\end{array}$ & to Los Alamos NL & $1.02 \mathrm{E}-05$ & $6.41 E-07$ \\
\hline $\begin{array}{l}\text { Portsmouth GDP } \\
\text { MLLW (CT) RF to LA }\end{array}$ & to Oak Ridge Reserv. & $2.50 E-04$ & $1.29 \mathrm{E}-04$ \\
\hline $\begin{array}{l}\text { Rocky Flats Plant } \\
\text { MLLW(CT) RM to OR }\end{array}$ & to Los Alamos NL & $9.40 \mathrm{E}-05$ & $3.72 \mathrm{E}-06$ \\
\hline Reactive Metals & to Oak Ridge Reserv. & $7.69 \mathrm{E}-07$ & $1.45 \mathrm{E}-07$ \\
\hline
\end{tabular}

Table 5. Nonradiological Risk Factors per Shipment (fatalities/shipment)

\begin{tabular}{llll}
\hline Mode & Route & Emission & Accident \\
\hline Truck & & & \\
Ames Laboratory & to Oak Ridge Reserv. & $4.25 \mathrm{E}-06$ & $5.79 \mathrm{E}-05$ \\
Argonne - East & to Oak Ridge Reserv. & $5.38 \mathrm{E}-06$ & $4.21 \mathrm{E}-05$ \\
Charleston, SC & to Savannah River & $3.22 \mathrm{E}-07$ & $1.70 \mathrm{E}-05$ \\
Colonie & to Oak Ridge Reserv. & $1.46 \mathrm{E}-05$ & $1.61 \mathrm{E}-04$ \\
Fernald (FEMP) & to Oak Ridge Reserv. & $5.79 \mathrm{E}-07$ & $2.25 \mathrm{E}-05$ \\
General Atomic & to Nevada Test Site & $8.11 \mathrm{E}-06$ & $2.85 \mathrm{E}-05$ \\
Grand Junction Po & to Los Alamos NL & $4.76 \mathrm{E}-06$ & $5.68 \mathrm{E}-05$ \\
LERHR & to Nevada Test Site & $1.81 \mathrm{E}-05$ & $5.49 \mathrm{E}-05$ \\
Paducah GDP & to Oak Ridge Reserv. & $1.93 \mathrm{E}-07$ & $2.14 \mathrm{E}-05$ \\
Pantex Plant & to Los Alamos NL & $3.28 \mathrm{E}-06$ & $3.21 \mathrm{E}-05$ \\
Portsmouth GDP & to Oak Ridge Reserv. & $1.09 \mathrm{E}-06$ & $2.56 \mathrm{E}-05$ \\
Rocky Flats Plant & to Los Alamos NL & $4.25 \mathrm{E}-06$ & $3.76 \mathrm{E}-05$ \\
Reactive Metals & to Oak Ridge Reserv. & $3.57 \mathrm{E}-06$ & $3.56 \mathrm{E}-05$ \\
Rail & & $2.52 \mathrm{E}-05$ & $2.00 \mathrm{E}-06$ \\
Ames Laboratory & to Oak Ridge Reserv. & $1.08 \mathrm{E}-05$ & $1.36 \mathrm{E}-06$ \\
Argonne - East & to Oak Ridge Reserv. & $1.46 \mathrm{E}-06$ & $2.93 \mathrm{E}-07$ \\
Charleston. SC & to Savannah River & $3.56 \mathrm{E}-05$ & $5.67 \mathrm{E}-06$ \\
Colonie & to Oak Ridge Reserv. & $3.68 \mathrm{E}-06$ & $7.48 \mathrm{E}-07$ \\
Fernald (FEMP) & to Oak Ridge Reserv. & $2.32 \mathrm{E}-05$ & $1.08 \mathrm{E}-06$ \\
General Atomic & to Nevada Test Site & $6.69 \mathrm{E}-07$ & $1.43 \mathrm{E}-06$ \\
Grand Junction PO to Los Alamos NL & $2.38 \mathrm{E}-05$ & $1.73 \mathrm{E}-06$ \\
LERHR & to Nevada Test Site & $3.81 \mathrm{E}-06$ & $1.10 \mathrm{E}-06$ \\
Paducah GDP & to Oak Ridge Reserv. & $2.59 \mathrm{E}-06$ & $1.01 \mathrm{E}-06$ \\
Pantex Plant & to Los Alamos NL & $\mathbf{3} .18 \mathrm{E}-06$ & $9.25 \mathrm{E}-07$ \\
Portsmouth GDP & to Oak Ridge Reserv. & $1.85 \mathrm{E}-05$ & $1.25 \mathrm{E}-06$ \\
Rocky Flats Plant & to Los Alamos NL & \\
Reactive Metals & to Oak Ridge Reserv. & & \\
\hline
\end{tabular}


Table 6. Annual Incident-Free Dose for the Shipping Campaign (person-rem/yr)

\begin{tabular}{|c|c|c|c|c|c|c|}
\hline \multirow[b]{2}{*}{ Route } & & & \multicolumn{2}{|c|}{ Truck } & \multicolumn{2}{|c|}{ Rail } \\
\hline & & & Crew & Public & Crew & Public \\
\hline $\begin{array}{l}\text { Ames Laboratory } \\
\text { Argonne - East } \\
\text { Charleston, SC } \\
\text { Colonie } \\
\text { Fernald (IEMP) } \\
\text { General Atomic } \\
\text { Grand Junction Po } \\
\text { LERHR } \\
\text { Paducah GDP } \\
\text { Pantex Plant } \\
\text { Portsmouth GDP } \\
\text { Rocky Flats Plant } \\
\text { Reactive Metals }\end{array}$ & $\begin{array}{l}\text { to } \\
\text { to } \\
\text { to } \\
\text { to } \\
\text { to } \\
\text { to } \\
\text { to } \\
\text { to } \\
\text { to } \\
\text { to } \\
\text { to } \\
\text { to } \\
\text { to }\end{array}$ & $\begin{array}{l}\text { Oak Ridge Reserv. } \\
\text { Oak Ridge Reserv. } \\
\text { Savannah River } \\
\text { Oak Ridge Reserv. } \\
\text { Oak Ridge Reserv. } \\
\text { Nevada Test Site } \\
\text { Los Alamos NL } \\
\text { Nevada Test Site } \\
\text { Oak Ridge Reserv. } \\
\text { Los Alamos NL } \\
\text { Oak Ridge Reserv. } \\
\text { Los Alamos NL } \\
\text { Oak Ridge Reserv. }\end{array}$ & $\begin{array}{l}2.83 \mathrm{E}-02 \\
2.00 \mathrm{E}-02 \\
6.19 \mathrm{E}-03 \\
6.37 \mathrm{E}-02 \\
2.03 \mathrm{E}-02 \\
1.33 \mathrm{E}-02 \\
1.99 \mathrm{E}-02 \\
2.34 \mathrm{E}-02 \\
8.88 \mathrm{E}-03 \\
1.11 \mathrm{E}-02 \\
1.81 \mathrm{E}+00 \\
1.35 \mathrm{E}-02 \\
2.11 \mathrm{E}-02\end{array}$ & $\begin{array}{l}3.14 \mathrm{E}-02 \\
2.18 \mathrm{E}-02 \\
6.77 \mathrm{E}-03 \\
7.56 \mathrm{E}-02 \\
2.16 \mathrm{E}-02 \\
1.60 \mathrm{E}-02 \\
2.37 \mathrm{E}-02 \\
2.99 \mathrm{E}-02 \\
9.98 \mathrm{E}-03 \\
1.38 \mathrm{E}-02 \\
1.98 \mathrm{E}+00 \\
1.61 \mathrm{E}-02 \\
2.23 \mathrm{E}-02\end{array}$ & $\begin{array}{l}6.86 \mathrm{E}-03 \\
5.59 \mathrm{E}-03 \\
3.47 \mathrm{E}-03 \\
1.42 \mathrm{E}-02 \\
4.37 \mathrm{E}-03 \\
5.04 \mathrm{E}-03 \\
5.73 \mathrm{E}-03 \\
5.33 \mathrm{E}-03 \\
5.08 \mathrm{E}-03 \\
4.89 \mathrm{E}-03 \\
2.69 \mathrm{E}-01 \\
4.98 \mathrm{E}-03 \\
5.38 \mathrm{E}-03\end{array}$ & $\begin{array}{l}2.24 \mathrm{E}-02 \\
1.40 \mathrm{E}-02 \\
5.00 \mathrm{E}-03 \\
3.41 \mathrm{E}-02 \\
7.96 \mathrm{E}-03 \\
1.74 \mathrm{E}-02 \\
6.59 \mathrm{E}-03 \\
2.00 \mathrm{E}-02 \\
7.91 \mathrm{E}-03 \\
7.19 \mathrm{E}-03 \\
5.31 \mathrm{E}-01 \\
9.36 \mathrm{E}-03 \\
1.80 \mathrm{E}-02\end{array}$ \\
\hline Total & & & $06 E+00$ & $2.278+00$ & $3.41 E-01$ & 1.0 \\
\hline
\end{tabular}

Table 7. Annual Accident Dose Risk for the Shipping Campaign (person-rem/yr)

\begin{tabular}{llcc}
\hline Route & & Truck & Rail \\
\hline Ames Laboratory & to Oak Ridge Reserv. & $4.17 \mathrm{E}-08$ & $1.36 \mathrm{E}-08$ \\
Argonne - East & to Oak Ridge Reserv. & $1.48 \mathrm{E}-05$ & $2.57 \mathrm{E}-06$ \\
Charleston, SC & to Savannah River & $4.40 \mathrm{E}-05$ & $5.11 \mathrm{E}-06$ \\
Colonie & to Oak Ridge Reserv. & $1.66 \mathrm{E}-07$ & $4.81 \mathrm{E}-08$ \\
Fernald (FEMP) & to Oak Ridge Reserv. & $6.28 \mathrm{E}-05$ & $7.21 \mathrm{E}-06$ \\
General Atomic & to Nevada Test Site & $9.22 \mathrm{E}-07$ & $5.27 \mathrm{E}-07$ \\
Grand Junction PO to Los Alamos NL & $7.50 \mathrm{E}-08$ & $9.34 \mathrm{E}-10$ \\
LERHR & to Nevada Test Site & $7.12 \mathrm{E}-07$ & $4.22 \mathrm{E}-07$ \\
Paducah GDP & to Oak Ridge Reserv. & $2.33 \mathrm{E}-04$ & $2.98 \mathrm{E}-05$ \\
Pantex Plant & to Los Alamos NL & $1.02 \mathrm{E}-05$ & $6.41 \mathrm{E}-07$ \\
Portsmouth GDP & to Oak Ridge Reserv. & $3.88 \mathrm{E}-02$ & $7.34 \mathrm{E}-03$ \\
Rocky Flats Plant & to Los Alamos NL & $9.40 \mathrm{E}-05$ & $3.72 \mathrm{E}-06$ \\
Reactive Metals & to Oak Ridge Reserv. & $7.69 \mathrm{E}-07$ & $1.45 \mathrm{E}-07$ \\
\hline Totals & & & \\
& & & \\
\hline
\end{tabular}

Table 8. Expected Annual Fatalities for the Shipping Campaign

\begin{tabular}{llc}
\hline Exposure Group & Truck & Rail \\
\hline Radiological & & \\
$\quad$ Normal Crew & $8.2 \mathrm{E}-04$ & $1.4 \mathrm{E}-04$ \\
Normal Public & $1.1 \mathrm{E}-03$ & $3.5 \mathrm{E}-04$ \\
$\quad$ Accident Public & $2.0 \mathrm{E}-05$ & $3.7 \mathrm{E}-06$ \\
Nonradiological & & \\
Emission & $2.4 \mathrm{E}-04$ & $3.4 \mathrm{E}-04$ \\
Accident & $4.6 \mathrm{E}-03$ & $7.1 \mathrm{E}-05$ \\
\hline
\end{tabular}

Table 9. Expected Annual Cancer Incidence for the shipping Campaign

\begin{tabular}{lll}
\hline Exposure Group & Truck & Rail \\
\hline Radiological & & \\
$\quad$ Normal Crew & $2.9 \mathrm{E}-03$ & $4.8 \mathrm{E}-04$ \\
$\quad$ Normal Public & $3.9 \mathrm{E}-03$ & $1.2 \mathrm{E}-03$ \\
$\quad$ Accident Public & $6.7 \mathrm{E}-05$ & $1.3 \mathrm{E}-05$ \\
Nonradiological & & \\
$\quad$ Emission & $2.4 \mathrm{E}-04$ & $3.4 \mathrm{E}-04$ \\
Accident & NA & NA \\
\hline
\end{tabular}


Table 10. Expected Annual Genetic Effects for the Shipping Campaign

\begin{tabular}{lll}
\hline Exposure Group & Truck & Rail \\
\hline Radiological & & \\
Normal Crew & $1.2 E-04$ & $2.0 \mathrm{E}-05$ \\
Normal Public & $2.3 \mathrm{E}-04$ & $7.0 \mathrm{E}-05$ \\
Accident Public & $3.5 \mathrm{E}-06$ & $6.6 \mathrm{E}-07$ \\
Nonradiological & & \\
Emission & NA & NA \\
Accident & NA & NA \\
\hline
\end{tabular}




\section{B.16 WM LLMW REGIONALIZED 2 ALTERNATIVE (CASE 7): CONTACT-HANDLED HETEROGENEOUS SOLIDS: ALPHA — TREATED}

Table 1. Summary of Route Information

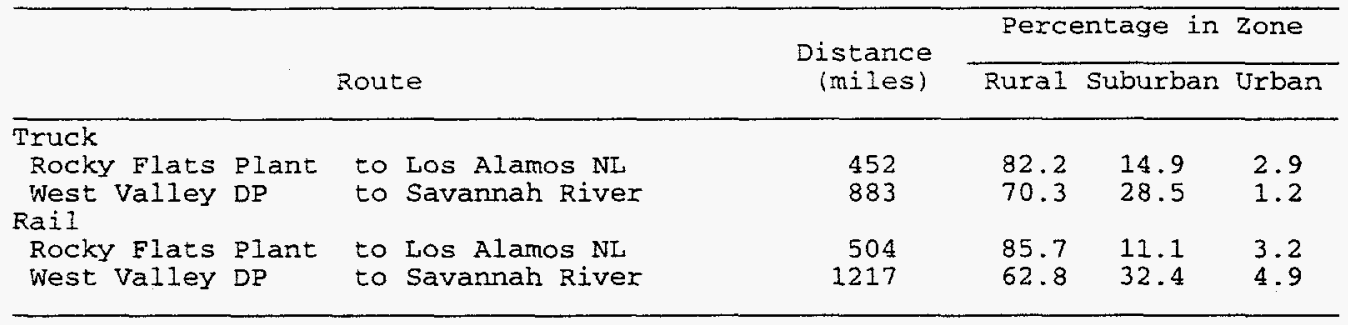

Table 2. Annual Number of Shipments and One-Way Shipment Distances

\begin{tabular}{|c|c|c|c|c|c|}
\hline \multirow[b]{2}{*}{ Route } & & \multicolumn{2}{|c|}{ \# of Shipments } & \multicolumn{2}{|c|}{ Total Mileage (mi) } \\
\hline & & Truck & Rail & Truck & Rail \\
\hline $\begin{array}{l}\text { Rocky Flats Plant } \\
\text { West Valley } \mathrm{DP}\end{array}$ & $\begin{array}{l}\text { to Los Alamos NL } \\
\text { to Savannah River }\end{array}$ & $\begin{array}{r}252 \\
1\end{array}$ & $\begin{array}{r}94 \\
1\end{array}$ & $\begin{array}{l}1.14 \mathrm{E}+05 \\
8.83 \mathrm{E}+02\end{array}$ & $\begin{array}{l}4.73 \mathrm{E}+04 \\
1.22 \mathrm{E}+03\end{array}$ \\
\hline Totals & & 253 & 95 & $1.15 \mathrm{E}+05$ & $4.86 \mathrm{E}+04$ \\
\hline
\end{tabular}

Table 3. Incident-Free Dose per Shipment (person-rem/shipment)

\begin{tabular}{|c|c|c|c|c|c|}
\hline \multirow[b]{2}{*}{ Source/Route (s) } & \multirow[b]{2}{*}{ Crew } & \multicolumn{4}{|c|}{ General Public } \\
\hline & & off-Link & On-Link & Stops & Total \\
\hline $\begin{array}{l}\text { MLLW(T) RF to LA } \\
\text { Rocky Flats Plant }\end{array}$ & $\begin{array}{cr}\text { to Los Alamos NL } \\
\text { Truck } & 1.35 \mathrm{E}-02 \\
\text { Rail } & 4.98 \mathrm{E}-03\end{array}$ & $\begin{array}{l}1.19 E-03 \\
4.26 E-03\end{array}$ & $\begin{array}{l}2.25 \mathrm{E}-03 \\
7.35 \mathrm{E}-05\end{array}$ & $\begin{array}{l}1.27 \mathrm{E}-02 \\
5.03 \mathrm{E}-03\end{array}$ & $\begin{array}{l}1.61 \mathrm{E}-02 \\
9.36 \mathrm{E}-03\end{array}$ \\
\hline $\begin{array}{l}\text { MLLW(T) WV to SR } \\
\text { West Valley DP }\end{array}$ & $\begin{array}{lr}\text { to Savannah River } \\
\text { Truck } 2.89 \mathrm{E}-02 \\
\text { Rail } & 7.96 \mathrm{E}-03\end{array}$ & $\begin{array}{l}2.45 \mathrm{E}-03 \\
1.95 \mathrm{E}-02\end{array}$ & $\begin{array}{l}3.86 \mathrm{E}-03 \\
3.38 \mathrm{E}-04\end{array}$ & $\begin{array}{l}2.47 \mathrm{E}-02 \\
7.23 \mathrm{E}-03\end{array}$ & $\begin{array}{l}3.10 \mathrm{E}-02 \\
2.70 \mathrm{E}-02\end{array}$ \\
\hline
\end{tabular}

Table 4. Accident Dose Risk per Shipment (person-rem/shipment)

\begin{tabular}{|c|c|c|c|}
\hline Source/Route & & Truck & Rail \\
\hline $\begin{array}{l}\text { MLLW(T) RF to LA } \\
\text { Rocky Flats Plant } \\
\text { MLLW(T) WV to SR } \\
\text { West Valley DP }\end{array}$ & $\begin{array}{l}\text { to Los Alamos NL } \\
\text { to Savannah River }\end{array}$ & $\begin{array}{l}1.04 E-04 \\
4.43 E-05\end{array}$ & $\begin{array}{l}1.20 \mathrm{E}-05 \\
1.42 \mathrm{E}-05\end{array}$ \\
\hline
\end{tabular}

Table 5. Nonradiological Risk Factors per Shipment (fatalities/shipment)

\begin{tabular}{|c|c|c|c|}
\hline Mode & Route & Emission & Accident \\
\hline \multicolumn{4}{|l|}{ Truck } \\
\hline $\begin{array}{l}\text { Rocky Flats Plant } \\
\text { West Valley DP }\end{array}$ & $\begin{array}{l}\text { to Los Alamos NL } \\
\text { to Savannah River }\end{array}$ & $\begin{array}{l}4.25 \mathrm{E}-06 \\
3.28 \mathrm{E}-06\end{array}$ & $\begin{array}{l}3.76 \mathrm{E}-05 \\
6.60 \mathrm{E}-05\end{array}$ \\
\hline \multicolumn{4}{|c|}{ - } \\
\hline $\begin{array}{l}\text { Rocky Flats Plant } \\
\text { West Valley DP }\end{array}$ & $\begin{array}{l}\text { to Los Alamos NL } \\
\text { to Savannah River }\end{array}$ & $\begin{array}{l}6.78 \mathrm{E}-06 \\
2.49 \mathrm{E}-05\end{array}$ & $\begin{array}{l}1.05 \mathrm{E}-06 \\
2.55 \mathrm{E}-06\end{array}$ \\
\hline
\end{tabular}


Table 6. Annual Incident-Free Dose for the Shipping Campaign (person-rem/yr)

\begin{tabular}{|c|c|c|c|c|c|}
\hline \multirow[b]{2}{*}{ Route } & & \multicolumn{2}{|c|}{ Truck } & \multicolumn{2}{|c|}{ Rail } \\
\hline & & Crew & Public & Crew & Public \\
\hline $\begin{array}{l}\text { Rocky Flats Plant } \\
\text { West valley } \mathrm{DP}\end{array}$ & $\begin{array}{l}\text { to Los Alamos NI } \\
\text { to Savannah River }\end{array}$ & $\begin{array}{l}3.41 \mathrm{E}+00 \\
2.89 \mathrm{E}-02\end{array}$ & $\begin{array}{l}4.05 E+00 \\
3.10 E-02\end{array}$ & $\begin{array}{l}4.68 \mathrm{E}-01 \\
7.96 \mathrm{E}-03\end{array}$ & $\begin{array}{l}8.80 E-01 \\
2.70 E-02\end{array}$ \\
\hline Totals & & $3.44 \mathrm{E}+00$ & $4.09 \mathrm{E}+00$ & $4.76 \mathrm{E}-01$ & .0 \\
\hline
\end{tabular}

Table 7. Annual Accident Dose Risk for the Shipping Campaign (person-rem/yr)

\begin{tabular}{llrc}
\hline Route & & Truck & Rail \\
\hline $\begin{array}{l}\text { Rocky Flats Plant } \\
\text { West Valley DP }\end{array}$ & to Sos Alamos NL & $2.62 \mathrm{E}-02$ & $1.13 \mathrm{E}-03$ \\
& & $4.43 \mathrm{E}-05$ & $1.42 \mathrm{E}-05$ \\
\hline Totals & & $2.63 \mathrm{E}-02$ & $1.15 \mathrm{E}-03$ \\
\hline
\end{tabular}

Table 8. Expected Annual Fatalities for the Shipping Campaign

\begin{tabular}{lll}
\hline Exposure Group & Truck & Rail \\
\hline Radiological & & \\
$\quad$ Normal Crew & $1.4 \mathrm{E}-03$ & $1.9 \mathrm{E}-04$ \\
Normal Public & $2.0 \mathrm{E}-03$ & $4.5 \mathrm{E}-04$ \\
Accident Public & $1.3 \mathrm{E}-05$ & $5.7 \mathrm{E}-07$ \\
Nonradiological & & \\
$\quad$ Emission & $1.1 \mathrm{E}-03$ & $6.6 \mathrm{E}-04$ \\
Accident & $9.6 \mathrm{E}-03$ & $1.0 \mathrm{E}-04$ \\
\hline
\end{tabular}

Table 9. Expected Annual Cancer Incidence for the Shipping Campaign

\begin{tabular}{cll}
\hline Exposure Group & Truck & Rail \\
\hline Radiological & & \\
Normal Crew & $4.8 \mathrm{E}-03$ & $6.7 \mathrm{E}-04$ \\
Normal Public & $6.9 \mathrm{E}-03$ & $1.5 \mathrm{E}-03$ \\
Accident Public & $4.5 \mathrm{E}-05$ & $1.9 \mathrm{E}-06$ \\
Nonradiological & & \\
Emission & $1.1 \mathrm{E}-03$ & $6.6 \mathrm{E}-04$ \\
Accident & $\mathrm{NA}$ & $\mathrm{NA}$ \\
\hline
\end{tabular}

Table 10. Expected Annual Genetic Effects for the Shipping Campaign

\begin{tabular}{lll}
\hline Exposure Group & Truck & Rail \\
\hline Radiological & & \\
Normal Crew & $2.1 \mathrm{E}-04$ & $2.9 \mathrm{E}-05$ \\
Nornal Public & $4.1 \mathrm{E}-04$ & $9.1 \mathrm{E}-05$ \\
Accident Public & $7.8 \mathrm{E}-07$ & $3.7 \mathrm{E}-08$ \\
Nonradiological & $\mathrm{NA}$ & $\mathrm{NA}$ \\
Emission & $\mathrm{NA}$ & $\mathrm{NA}$ \\
Accident & & \\
\hline
\end{tabular}




\section{B.17 WM LLMW REGIONALIZED 3 ALTERNATIVE (CASE 10a): CONTACT-HANDLED ORGANIC LIQUIDS: NONALPHA - UNTREATED}

Table 1. Summary of Route Information

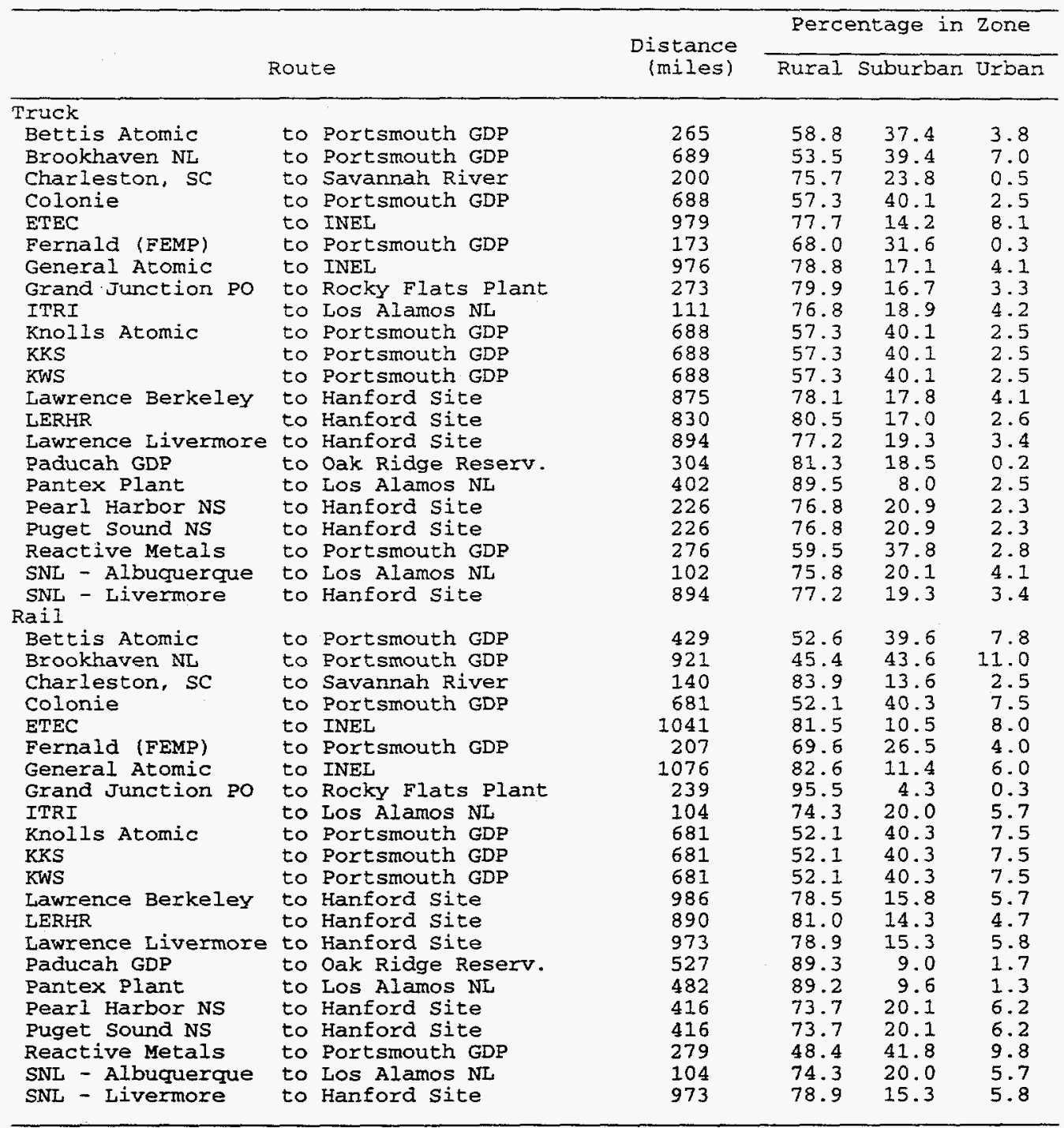


Table 2. Annual Number of Shipments and One-Way Shipment Distances

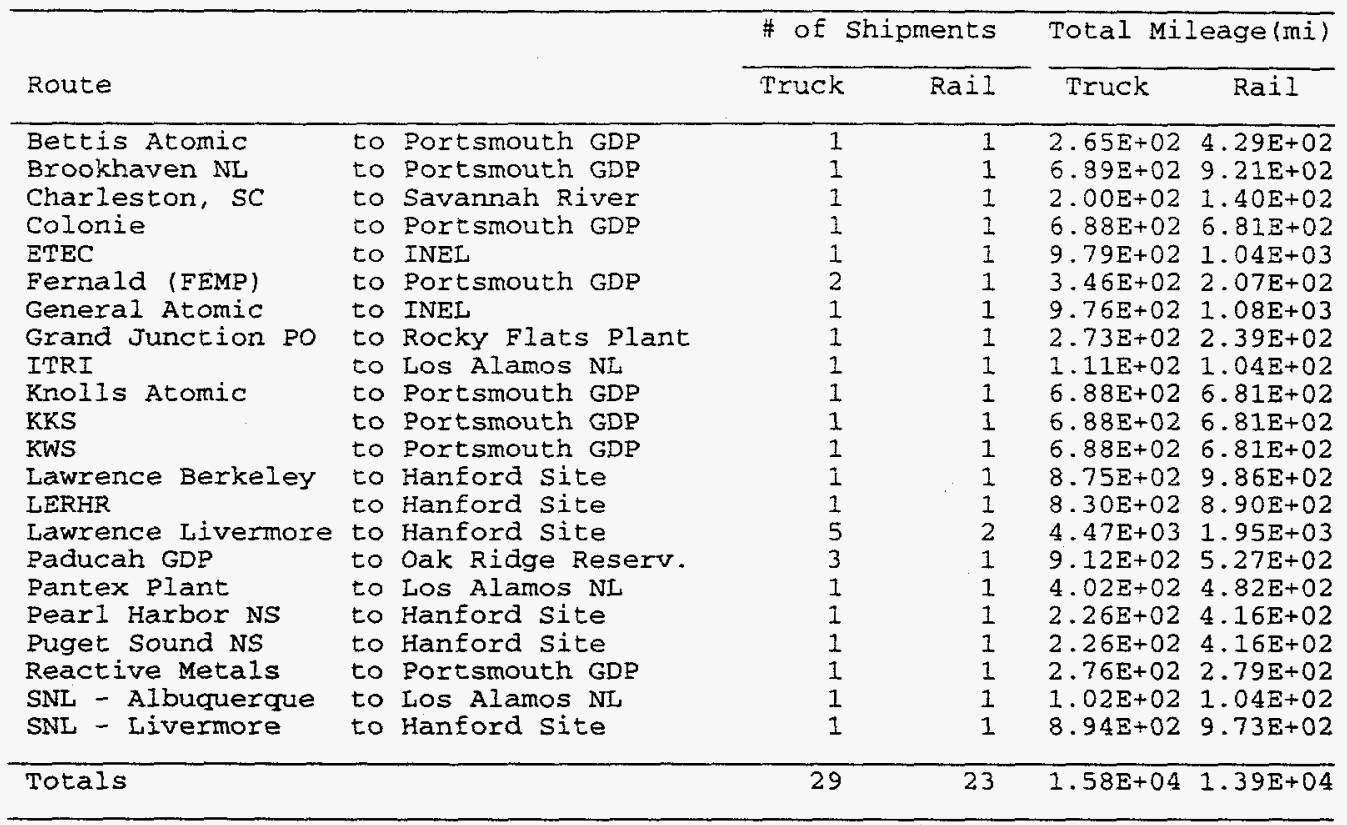

Table 3. Incident-Free Dose per Shipment (person-rem/shipment)

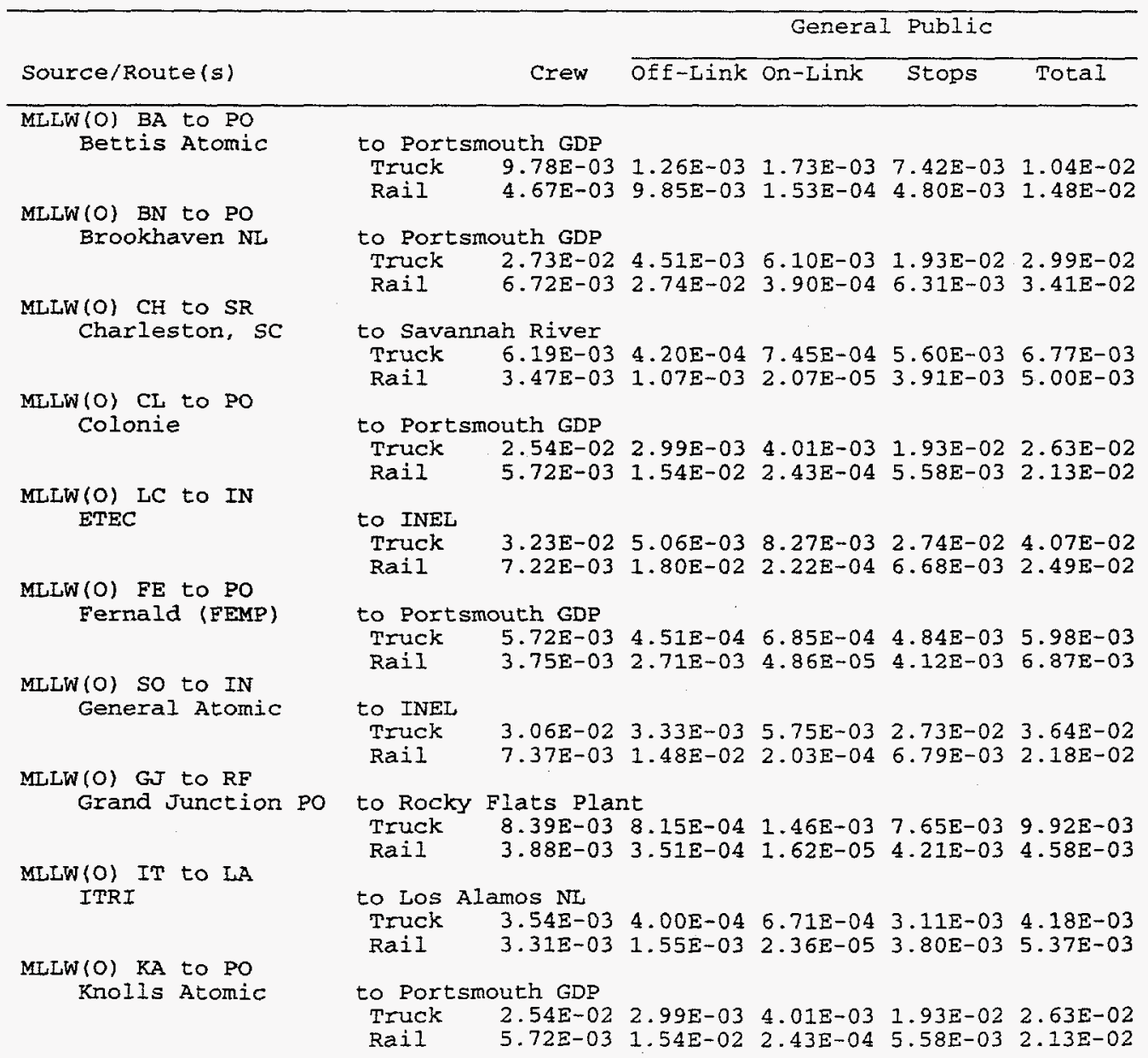


Table 3. Incident-Free Dose per Shipment (person-rem/shipment) (Cont.)

\begin{tabular}{|c|c|c|c|c|c|}
\hline \multirow[b]{2}{*}{ Source/Route (s) } & \multirow[b]{2}{*}{ Crew } & \multicolumn{4}{|c|}{ General Public } \\
\hline & & Off-Link & On-Link & Stops & Total \\
\hline \multicolumn{6}{|l|}{$\begin{array}{c}\text { MLLW }(0) \\
\text { KKS }\end{array}$} \\
\hline & $\begin{array}{ll}\text { Truck } & 2.54 \mathrm{E}-02 \\
\text { Rail } & 5.72 \mathrm{E}-03\end{array}$ & $\begin{array}{l}2.99 \mathrm{E}-03 \\
1.54 \mathrm{E}-02\end{array}$ & $\begin{array}{l}4.01 E-03 \\
2.43 E-04\end{array}$ & $\begin{array}{l}1.93 E-02 \\
5.58 E-03\end{array}$ & $\begin{array}{l}2.63 \mathrm{E}-02 \\
2.13 \mathrm{E}-02\end{array}$ \\
\hline \multicolumn{6}{|l|}{$\begin{array}{c}M L L W(O) \\
K W W\end{array}$} \\
\hline & $\begin{array}{ll}\text { Truck } & 2.54 \mathrm{E}-02 \\
\text { Rail } & 5.72 \mathrm{E}-03\end{array}$ & $\begin{array}{l}2.99 \mathrm{E}-03 \\
1.54 \mathrm{E}-02\end{array}$ & $\begin{array}{l}4.01 E-03 \\
2.43 E-04\end{array}$ & $\begin{array}{l}1.93 \mathrm{E}-02 \\
5.58 \mathrm{E}-03\end{array}$ & $\begin{array}{l}2.63 \mathrm{E}-02 \\
2.13 \mathrm{E}-02\end{array}$ \\
\hline \multicolumn{6}{|c|}{ MLLW(O) LB to HS } \\
\hline Lawrence Berkeley & $\begin{array}{lr}\text { to Hanford Site } \\
\text { Truck } & 2.76 \mathrm{E}-02 \\
\text { Rail } & 6.99 \mathrm{E}-03\end{array}$ & $\begin{array}{l}3.02 \mathrm{E}-03 \\
1.38 \mathrm{E}-02\end{array}$ & $\begin{array}{l}5.17 E-03 \\
2.03 E-04\end{array}$ & $\begin{array}{l}2.45 \mathrm{E}-02 \\
6.51 \mathrm{E}-03\end{array}$ & $\begin{array}{l}3.27 \mathrm{E}-02 \\
2.05 \mathrm{E}-02\end{array}$ \\
\hline \multirow{2}{*}{\multicolumn{6}{|c|}{$\begin{array}{l}\text { MLLW(O) SM to HS } \\
\text { IERHR }\end{array}$}} \\
\hline & & & & & \\
\hline & $\begin{array}{ll}\text { Truck } & 2.51 \mathrm{E}-02 \\
\text { Rail } & 6.59 \mathrm{E}-03\end{array}$ & $\begin{array}{l}2.17 \mathrm{E}-03 \\
1.07 \mathrm{E}-02\end{array}$ & $\begin{array}{l}4.00 \mathrm{E}-03 \\
1.64 \mathrm{E}-04\end{array}$ & $\begin{array}{l}2.32 \mathrm{E}-02 \\
6.22 \mathrm{E}-03\end{array}$ & $\begin{array}{l}2.94 E-02 \\
1.70 E-02\end{array}$ \\
\hline \multicolumn{6}{|l|}{ MLLW(O) LL to HS } \\
\hline & $\begin{array}{ll}\text { Truck } & 2.82 \mathrm{E}-02 \\
\text { Rail } & 6.94 \mathrm{E}-03\end{array}$ & $\begin{array}{l}2.88 \mathrm{E}-03 \\
1.38 \mathrm{E}-02\end{array}$ & $\begin{array}{l}4.92 E-03 \\
2.00 E-04\end{array}$ & $\begin{array}{l}2.50 \mathrm{E}-02 \\
6.48 \mathrm{E}-03\end{array}$ & $\begin{array}{l}3.28 \mathrm{E}-02 \\
2.05 \mathrm{E}-02\end{array}$ \\
\hline \multicolumn{6}{|c|}{ (2) } \\
\hline Paducah GDP & $\begin{array}{cc}\text { to Oak Ridge Reserv } \\
\text { Truck } & 8.88 \mathrm{E}-03 \\
\text { Rail } & 5.08 \mathrm{E}-03\end{array}$ & $\begin{array}{l}4.70 \mathrm{E}-04 \\
2.75 \mathrm{E}-03\end{array}$ & $\begin{array}{l}9.98 \mathrm{E}-04 \\
5.97 \mathrm{E}-05\end{array}$ & $\begin{array}{l}8.51 \mathrm{E}-03 \\
5.10 \mathrm{E}-03\end{array}$ & $\begin{array}{l}9.98 \mathrm{E}-03 \\
7.91 \mathrm{E}-03\end{array}$ \\
\hline \multicolumn{6}{|l|}{$M L L W(O)$ PP to LA } \\
\hline Pantex Plant & $\begin{array}{cr}\text { to Los } & \text { Alamos NL } \\
\text { Truck } & 1.11 \mathrm{E}-02 \\
\text { Rail } & 4.89 \mathrm{E}-03\end{array}$ & $\begin{array}{l}7.76 \mathrm{E}-04 \\
2.18 \mathrm{E}-03\end{array}$ & $\begin{array}{l}1.77 E-03 \\
5.29 E-05\end{array}$ & $\begin{array}{l}1.13 \mathrm{E}-02 \\
4.96 \mathrm{E}-03\end{array}$ & $\begin{array}{l}1.38 \mathrm{E}-02 \\
7.19 \mathrm{E}-03\end{array}$ \\
\hline \multirow{2}{*}{\multicolumn{6}{|c|}{$\begin{array}{l}\text { MLLW(O) SW to HS } \\
\text { Pearl Harbor NS }\end{array}$}} \\
\hline & to Hanford Site & & & & \\
\hline & $\begin{array}{ll}\text { Truck } & 7.06 \mathrm{E}-03 \\
\text { Rail } & 4.62 \mathrm{E}-03\end{array}$ & $\begin{array}{l}6.28 \mathrm{E}-04 \\
6.63 \mathrm{E}-03\end{array}$ & $\begin{array}{l}1.09 \mathrm{E}-03 \\
9.79 \mathrm{E}-05\end{array}$ & $\begin{array}{l}6.33 \mathrm{E}-03 \\
4.76 \mathrm{E}-03\end{array}$ & $\begin{array}{l}8.04 \mathrm{E}-03 \\
1.15 \mathrm{E}-02\end{array}$ \\
\hline \multicolumn{6}{|l|}{$\begin{array}{l}\text { MLLW(O) PN to HS } \\
\text { Puget Sound NS }\end{array}$} \\
\hline & to Hanford Site & $5-04$ & 1.0 & $5 \quad 33 E-03$ & \\
\hline & $4.62 \mathrm{E}-03$ & $6.63 E-03$ & $9.79 \mathrm{E}-05$ & $4.76 \mathrm{E}-03$ & $\begin{array}{l}0.042-03 \\
1.15 \mathrm{E}-02\end{array}$ \\
\hline \multirow{2}{*}{$\begin{array}{l}\text { MLIW(O) RM to PO } \\
\text { Reactive Metals }\end{array}$} & to Partamouth GDP & & & & \\
\hline & $\begin{array}{ll}\text { Truck } & 1.00 \mathrm{E}-02 \\
\text { Rail } & 4.05 \mathrm{E}-03\end{array}$ & $\begin{array}{l}1.18 \mathrm{E}-03 \\
7.58 \mathrm{E}-03\end{array}$ & $\begin{array}{l}1.62 E-03 \\
1.11 E-04\end{array}$ & $\begin{array}{l}7.73 \mathrm{E}-03 \\
4.34 \mathrm{E}-03\end{array}$ & $\begin{array}{l}1.05 \mathrm{E}-02 \\
1.20 \mathrm{E}-02\end{array}$ \\
\hline \multirow{2}{*}{$\begin{array}{l}\text { MLLW(O) SA to LA } \\
\text { SNL - AIbuquerque }\end{array}$} & & & & & \\
\hline & $\begin{array}{lr}\text { to Los Alamos NL } \\
\text { Truck } & 3.28 \mathrm{E}-03 \\
\text { Rail } & 3.31 \mathrm{E}-03\end{array}$ & $\begin{array}{l}3.70 \mathrm{E}-04 \\
1.55 \mathrm{E}-03\end{array}$ & $\begin{array}{l}6.13 E-04 \\
2.36 E-05\end{array}$ & $\begin{array}{l}2.86 \mathrm{E}-03 \\
3.80 \mathrm{E}-03\end{array}$ & $\begin{array}{l}3.84 \mathrm{E}-03 \\
5.37 \mathrm{E}-03\end{array}$ \\
\hline \multirow{2}{*}{$\begin{aligned} \text { MLLW (O) SL to HS } \\
\text { SNL - Livermore }\end{aligned}$} & & & & & \\
\hline & $\begin{array}{ll}\text { to Hanford Site } \\
\text { Truck } & 2.82 \mathrm{E}-02 \\
\text { Rail } & 6.94 \mathrm{E}-03\end{array}$ & $\begin{array}{l}2.88 \mathrm{E}-03 \\
1.38 \mathrm{E}-02\end{array}$ & $\begin{array}{l}4.92 \mathrm{E}-03 \\
2.00 \mathrm{E}-04\end{array}$ & $\begin{array}{l}2.50 \mathrm{E}-02 \\
6.48 \mathrm{E}-03\end{array}$ & $\begin{array}{l}3.28 \mathrm{E}-02 \\
2.05 \mathrm{E}-02\end{array}$ \\
\hline
\end{tabular}


Table 4. Accident Dose Risk per Shipment (person-rem/shipment)

\begin{tabular}{|c|c|c|c|}
\hline Source/Route & & Truck & $\operatorname{Rail}$ \\
\hline$M L L W(O) B A$ to $P O$ & & & \\
\hline $\begin{array}{l}\text { Bettis Atomic } \\
\text { MLLW(O) BN to PO }\end{array}$ & to Portsmouth GDP & $5.04 E-04$ & $2.16 \mathrm{E}-04$ \\
\hline $\begin{array}{l}\text { Brookhaven NL } \\
\text { MLLW(O) CH to } \mathrm{SR}\end{array}$ & to Portsmouth GDP & $4.87 E-03$ & $2.63 E-03$ \\
\hline $\begin{array}{l}\text { Charleston, SC } \\
\text { MLLW(O) CL to PO }\end{array}$ & to Savannah River & $7.75 \mathrm{E}-03$ & $8.99 E-04$ \\
\hline $\begin{array}{l}\text { Colonie } \\
\text { MLLW(O) LC to IN }\end{array}$ & to Portsmouth GDP & $3.74 E-04$ & $8.80 E-05$ \\
\hline ETEC & to INEL & $9.54 \mathrm{E}-06$ & $4.35 \mathrm{E}-06$ \\
\hline $\begin{array}{l}\text { MLLW (O) FE to PO } \\
\text { FernaId (FEMP) } \\
\text { MLLW (O) so to IN }\end{array}$ & to Portsmouth GDP & $5.76 \mathrm{E}-04$ & $2.78 \mathrm{E}-04$ \\
\hline $\begin{array}{l}\text { MLLW (Oeneral Atomic } \\
\text { MLLW (O) GJ to RF }\end{array}$ & to INEL & $1.08 E-05$ & $4.28 \mathrm{E}-06$ \\
\hline $\begin{array}{l}\text { Grand Junction PO } \\
\text { MLLW(O) IT to LA }\end{array}$ & to Rocky Flats Plant & $1.33 \mathrm{E}-06$ & $1.13 \mathrm{E}-08$ \\
\hline $\begin{array}{l}\text { ITRI } \\
\text { MLLW(O) KA to PO }\end{array}$ & to Los Alamos NL & $8.49 \mathrm{E}-06$ & $1.55 \mathrm{E}-07$ \\
\hline $\begin{array}{l}\text { Knolls Atomic } \\
\text { MLLW(O) KK to PO }\end{array}$ & to Portsmouth GDP & $4.50 E-02$ & $1.06 \mathrm{E}-02$ \\
\hline $\begin{array}{l}\text { KKS } \\
\text { MLLW (O) KW to PO }\end{array}$ & to Portsmouth GDP & $3.45 \mathrm{E}-02$ & $8.09 \mathrm{E}-03$ \\
\hline $\begin{array}{l}\text { KWS } \\
\text { MLLW (O) LB to HS }\end{array}$ & to Portsmouth GDP & $4.71 E-03$ & $1.10 \mathrm{E}-03$ \\
\hline $\begin{array}{l}\text { Lawrence Berkeley } \\
\text { MLLW(O) SM to HS }\end{array}$ & to Hanford Site & $7.79 E-05$ & $4.38 \mathrm{E}-05$ \\
\hline $\begin{array}{l}\text { LERHR } \\
\text { MLLW(O) LL to HS }\end{array}$ & to Hanford Site & $7.78 \mathrm{E}-06$ & $4.14 \mathrm{E}-06$ \\
\hline $\begin{array}{l}\text { Lawrence Livermore } \\
\text { MLLW(O) PA to OR }\end{array}$ & to Hanford Site & $3.93 E-03$ & $5.23 E-03$ \\
\hline $\begin{array}{l}\text { Paducah GDP } \\
\text { MLLW (O) PP to IA }\end{array}$ & to Oak Ridge Reserv. & $3.89 \mathrm{E}-01$ & $1.21 \mathrm{E}-01$ \\
\hline $\begin{array}{l}\text { Pantex Plant } \\
\text { MLLW(O) SW to HS }\end{array}$ & to Los Alamos NL & $1.60 \mathrm{E}-04$ & $9.37 \mathrm{E}-06$ \\
\hline $\begin{array}{l}\text { Pearl Harbor NS } \\
\text { MLLW(O) PN to HS }\end{array}$ & to Hanford Site & $8.47 \mathrm{E}-06$ & $6.32 E-06$ \\
\hline $\begin{array}{l}\text { Puget Sound NS } \\
\text { MLLW(O) RM to RO }\end{array}$ & to Hanford Site & $1.95 \mathrm{E}-04$ & $1.46 \mathrm{E}-04$ \\
\hline $\begin{array}{l}\text { Reactive Metals } \\
\text { MLLW(O) SA to LA }\end{array}$ & to Portsmouth GDP & $7.40 E-05$ & $2.29 \mathrm{E}-05$ \\
\hline $\begin{array}{l}\text { SNL - Albuquerque } \\
\text { MLLW(O) SL to HS }\end{array}$ & to Los Alamos NL & $5.82 \mathrm{E}-07$ & $1.10 \mathrm{E}-08$ \\
\hline SNL - Livermore & to Hanford Site & $1.95 \mathrm{E}-03$ & $1.04 E-03$ \\
\hline
\end{tabular}


Table 5. Nonradiological Risk Factors per Shipment (fatalities/shipment)

\begin{tabular}{|c|c|c|c|}
\hline Mode & Route & Emission & Accident \\
\hline $\begin{array}{l}\text { Truck } \\
\text { Bettis Atomic } \\
\text { Brookhaven NL } \\
\text { Charleston, SC } \\
\text { Colonie } \\
\text { ETEC } \\
\text { Fernald (FEMP) } \\
\text { General Atomic } \\
\text { Grand Junction Po } \\
\text { ITRI } \\
\text { Knolls Atomic } \\
\text { KKS } \\
\text { KWS } \\
\text { Lawrence Berkeley } \\
\text { LERHR } \\
\text { Lawrence Livermore } \\
\text { Paducah GDP } \\
\text { Pantex Plant } \\
\text { Pearl Harbor NS } \\
\text { Puget Sound NS } \\
\text { Reactive Metals } \\
\text { SNL - Albuquerque } \\
\text { SNL - Livermore }\end{array}$ & $\begin{array}{l}\text { to Portsmouth GDP } \\
\text { to Portsmouth GDP } \\
\text { to Savannah River } \\
\text { to Portsmouth GDP } \\
\text { to INEL } \\
\text { to Portsmouth GDP } \\
\text { to INEL } \\
\text { to Rocky Flats Plant } \\
\text { to Los Alamos NI } \\
\text { to Portsmouth GDP } \\
\text { to Portsmouth GDP } \\
\text { to Portsmouth GDP } \\
\text { to Hanford Site } \\
\text { to Hanford Site } \\
\text { to Hanford Site } \\
\text { to Oak Ridge Reserv. } \\
\text { to Los Alamos NL } \\
\text { to Hanford Site } \\
\text { to Hanford Site } \\
\text { to Portsmouth GDP } \\
\text { to Los Alamos NI } \\
\text { to Hanford Site }\end{array}$ & $\begin{array}{l}3.22 \mathrm{E}-06 \\
1.56 \mathrm{E}-05 \\
3.22 \mathrm{E}-07 \\
5.63 \mathrm{E}-06 \\
2.55 \mathrm{E}-05 \\
1.93 \mathrm{E}-07 \\
1.30 \mathrm{E}-05 \\
2.93 \mathrm{E}-06 \\
1.51 \mathrm{E}-06 \\
5.63 \mathrm{E}-06 \\
5.63 \mathrm{E}-06 \\
5.63 \mathrm{E}-06 \\
1.16 \mathrm{E}-05 \\
6.82 \mathrm{E}-06 \\
9.85 \mathrm{E}-06 \\
1.93 \mathrm{E}-07 \\
3.28 \mathrm{E}-06 \\
1.67 \mathrm{E}-06 \\
1.67 \mathrm{E}-06 \\
2.45 \mathrm{E}-06 \\
1.35 \mathrm{E}-06 \\
9.85 \mathrm{E}-06\end{array}$ & $\begin{array}{l}1.33 \mathrm{E}-05 \\
4.90 \mathrm{E}-05 \\
1.70 \mathrm{E}-05 \\
3.56 \mathrm{E}-05 \\
6.21 \mathrm{E}-05 \\
7.51 \mathrm{E}-06 \\
5.23 \mathrm{E}-05 \\
2.32 \mathrm{E}-05 \\
1.28 \mathrm{E}-05 \\
3.56 \mathrm{E}-05 \\
3.56 \mathrm{E}-05 \\
3.56 \mathrm{E}-05 \\
4.91 \mathrm{E}-05 \\
4.66 \mathrm{E}-05 \\
5.03 \mathrm{E}-05 \\
2.14 \mathrm{E}-05 \\
3.21 \mathrm{E}-05 \\
9.56 \mathrm{E}-06 \\
9.56 \mathrm{E}-06 \\
1.21 \mathrm{E}-05 \\
1.20 \mathrm{E}-05 \\
5.03 \mathrm{E}-05\end{array}$ \\
\hline $\begin{array}{l}\text { Rail } \\
\text { Bettis Atomic } \\
\text { Brookhaven NI } \\
\text { Charleston, SC } \\
\text { Colonie } \\
\text { ETEC } \\
\text { Fernald (FEMP) } \\
\text { General Atomic } \\
\text { Grand Junction PO } \\
\text { IrRI } \\
\text { Knolls Atomic } \\
\text { KKS } \\
\text { KWS } \\
\text { Lawrence Berkeley } \\
\text { LERHR } \\
\text { Lawrence Livermore } \\
\text { Paducah GDP } \\
\text { Pantex Plant } \\
\text { Pearl Harbor NS } \\
\text { Puget Sound NS } \\
\text { Reactive Metals } \\
\text { SNL - Albuquerque } \\
\text { SNL - Livermore }\end{array}$ & $\begin{array}{l}\text { to Portsmouth GDP } \\
\text { to Portsmouth GDP } \\
\text { to Savannah River } \\
\text { to Portsmouth GDP } \\
\text { to INEL } \\
\text { to Portsmouth GDP } \\
\text { to INEL } \\
\text { to Rocky Flats Plant } \\
\text { to Los Alamos NL } \\
\text { to Portsmouth GDP } \\
\text { to Portsmouth GDP } \\
\text { to Portsmouth GDP } \\
\text { to Hanford Site } \\
\text { to Hanford Site } \\
\text { to Hanford Site } \\
\text { to Oak Ridge Reserv. } \\
\text { to Los Alamos NL } \\
\text { to Hanford Site } \\
\text { to Hanford Site } \\
\text { to Portsmouth GDP } \\
\text { to Los Alamos NL } \\
\text { to Hanford Site }\end{array}$ & $\begin{array}{l}1.40 \mathrm{E}-05 \\
4.22 \mathrm{E}-05 \\
1.46 \mathrm{E}-06 \\
2.15 \mathrm{E}-05 \\
3.48 \mathrm{E}-05 \\
3.43 \mathrm{E}-06 \\
2.72 \mathrm{E}-05 \\
2.51 \mathrm{E}-07 \\
2.47 \mathrm{E}-06 \\
2.15 \mathrm{E}-05 \\
2.15 \mathrm{E}-05 \\
2.15 \mathrm{E}-05 \\
2.33 \mathrm{E}-05 \\
1.77 \mathrm{E}-05 \\
2.36 \mathrm{E}-05 \\
3.81 \mathrm{E}-06 \\
2.59 \mathrm{E}-06 \\
1.08 \mathrm{E}-05 \\
1.08 \mathrm{E}-05 \\
1.14 \mathrm{E}-05 \\
2.47 \mathrm{E}-06 \\
2.36 \mathrm{E}-05\end{array}$ & $\begin{array}{l}8.98 E-07 \\
1.93 E-06 \\
2.93 E-07 \\
1.43 E-06 \\
2.18 E-06 \\
4.34 E-07 \\
2.25 E-06 \\
4.99 E-07 \\
2.18 E-07 \\
1.43 E-06 \\
1.43 E-06 \\
1.43 E-06 \\
2.06 E-06 \\
1.86 E-06 \\
2.04 E-06 \\
1.10 E-06 \\
1.01 E-06 \\
8.70 E-07 \\
8.70 E-07 \\
5.84 E-07 \\
2.18 E-07 \\
2.04 E-06\end{array}$ \\
\hline
\end{tabular}


Table 6. Annual Incident-Free Dose for the Shipping Campaign (person-rem/yr)

\begin{tabular}{|c|c|c|c|c|c|c|}
\hline \multirow[b]{2}{*}{ Route } & & & \multicolumn{2}{|c|}{ Truck } & \multicolumn{2}{|c|}{ Rail } \\
\hline & & & Crew & Public & Crew & Public \\
\hline Bettis Atomic & & Portsmouth GDP & $78 E-03$ & $1.04 \mathrm{E}-02$ & $4.67 \mathrm{E}-03$ & $1.48 \mathrm{E}$ \\
\hline$n \mathrm{NL}$ & & Portsmouth GDP & $73 E-02$ & $99 E-02$ & $6.72 \mathrm{E}-03$ & $1 E-6$ \\
\hline Charleston, SC & & Savannah River & $.19 \mathrm{E}-03$ & $6.77 \mathrm{E}-03$ & $3.47 E-03$ & $0 \mathrm{E}-03$ \\
\hline Colonie & & Portsmouth GDP & $54 \mathrm{E}-02$ & 53E-02 & $5.72 \mathrm{E}-03$ & $3 E-C$ \\
\hline ETEC & & INEL & $e-02$ & $4.07 E-02$ & $7.22 \mathrm{E}-03$ & $9 E-02$ \\
\hline Fernald (FEMP) & to & Portsmouth GDP & $1.14 \mathrm{E}-02$ & $0 E-02$ & $3.75 \mathrm{E}-03$ & $6.87 \mathrm{E}-\mathrm{C}$ \\
\hline General Atomic & to & INEL & $z-02$ & $4 E-02$ & -03 & \\
\hline Grand Junction PO & to & Rocky Flats Pla & $E-03$ & $9.92 \mathrm{E}-03$ & $3.88 \mathrm{E}-03$ & $8-03$ \\
\hline ITRI & to & Los Alamos NL & -03 & $8 E-03$ & -03 & $E-03$ \\
\hline Knolls Atomic & & Portsmouth GDP & -02 & $3 E-02$ & $5.72 \mathrm{E}-03$ & $3 E-02$ \\
\hline KKS & & Portsmouth GDP & $E-02$ & $2.63 \mathrm{E}-02$ & $5.72 \mathrm{E}-03$ & $3 E-02$ \\
\hline KWS & to & Portsmouth GDP & 02 & $2.63 \mathrm{E}-02$ & $5.72 \mathrm{E}-03$ & $\mathrm{E}-02$ \\
\hline Lawrence Berkeley & to & Hanford Site & $2.76 \mathrm{E}-02$ & $3.27 E-02$ & $6.99 E-03$ & $5 E-02$ \\
\hline LERHR & to & Hanford site & 02 & E-02 & & \\
\hline Lawrence Livermore & to & Hanford Site & $1.41 E-01$ & $=1.64 \mathrm{E}-01$ & $1.39 \mathrm{E}-02$ & $4.10 E-02$ \\
\hline Paducah GDP & to & Oak Ridge Reserv. & $2.67 E-02$ & $E-02$ & $8-03$ & $E-03$ \\
\hline Pantex Plant & to & Los Alamos NL & $1.11 \mathrm{E}-02$ & $1.38 \mathrm{E}-02$ & $4.89 \mathrm{E}-03$ & $7.19 \mathrm{E}-03$ \\
\hline Pearl Harbor NS & to & Hanford site & $7.06 \mathrm{E}-03$ & $8.04 E-03$ & $4.62 E-03$ & $5 E-02$ \\
\hline Puget sound NS & to & Hanford site & $7.06 E-03$ & $8.04 E-03$ & $4.62 \mathrm{E}-03$ & $1.15 \mathrm{E}-02$ \\
\hline Reactive Metals & to & Portsmouth GDP & 1. $00 \mathrm{E}-02$ & $1.05 E-02$ & $4.05 \mathrm{E}-03$ & $\Xi-02$ \\
\hline SNL - Albuquerque & to & Los Alamos NL & $3.28 \mathrm{E}-03$ & $3.84 \mathrm{E}-03$ & $3.31 E-03$ & $5.37 E-03$ \\
\hline SNL - L & to & Hanford Site & $2.82 E-02$ & $3.28 \mathrm{E}-02$ & $6.94 \mathrm{E}-03$ & $2.05 \mathrm{E}-02$ \\
\hline
\end{tabular}

Table 7. Annual Accident Dose Risk for the Shipping Campaign (person-rem/yr)

\begin{tabular}{llcc}
\hline Route & & Truck & Rail \\
\hline Bettis Atomic & to Portsmouth GDP & $5.04 \mathrm{E}-04$ & $2.16 \mathrm{E}-04$ \\
Brookhaven NL & to Portsmouth GDP & $4.87 \mathrm{E}-03$ & $2.63 \mathrm{E}-03$ \\
Charleston, SC & to Savannah River & $7.75 \mathrm{E}-03$ & $8.99 \mathrm{E}-04$ \\
Colonie & to Portsmouth GDP & $3.74 \mathrm{E}-04$ & $8.80 \mathrm{E}-05$ \\
ETEC & to INEL & $9.54 \mathrm{E}-06$ & $4.35 \mathrm{E}-06$ \\
Fernald (FEMP) & to Portsmouth GDP & $1.15 \mathrm{E}-03$ & $2.78 \mathrm{E}-04$ \\
General Atomic & to INEL & $1.08 \mathrm{E}-05$ & $4.28 \mathrm{E}-06$ \\
Grand Junction Po & to Rocky Flats Plant & $1.33 \mathrm{E}-06$ & $1.13 \mathrm{E}-08$ \\
ITRI & to Los Alamos NL & $8.49 \mathrm{E}-06$ & $1.55 \mathrm{E}-07$ \\
Knol1s Atomic & to Portsmouth GDP & $4.50 \mathrm{E}-02$ & $1.06 \mathrm{E}-02$ \\
KKS & to Portsmouth GDP & $3.45 \mathrm{E}-02$ & $8.09 \mathrm{E}-03$ \\
KWS & to Portsmouth GDP & $4.71 \mathrm{E}-03$ & $1.10 \mathrm{E}-03$ \\
Lawrence Berkeley & to Hanford Site & $7.79 \mathrm{E}-05$ & $4.38 \mathrm{E}-05$ \\
LERHR & to Hanford Site & $7.78 \mathrm{E}-06$ & $4.14 \mathrm{E}-06$ \\
Lawrence Livermore & to Hanford Site & $1.97 \mathrm{E}-02$ & $1.05 \mathrm{E}-02$ \\
Paducah GDP & to Oak Ridge Reserv. & $1.17 \mathrm{E}+00$ & $1.21 \mathrm{E}-01$ \\
Pantex Plant & to Los Alamos NL & $1.60 \mathrm{E}-04$ & $9.37 \mathrm{E}-06$ \\
Pearl Harbor NS & to Hanford Site & $8.47 \mathrm{E}-06$ & $6.32 \mathrm{E}-06$ \\
Puget Sound NS & to Hanford Site & $1.95 \mathrm{E}-04$ & $1.46 \mathrm{E}-04$ \\
Reactive Metals & to Portsmouth GDP & $7.40 \mathrm{E}-05$ & $2.29 \mathrm{E}-05$ \\
SNL - Albuquerque & to Los Alamos NL & $5.82 \mathrm{E}-07$ & $1.10 \mathrm{E}-08$ \\
SNL - Livermore & to Hanford Site & $1.95 \mathrm{E}-03$ & $1.04 \mathrm{E}-03$ \\
\hline Totals & & & \\
\hline
\end{tabular}

Table 8. Expected Annual Fatalities for the Shipping Campaign

\begin{tabular}{llc}
\hline Exposure Group & Truck & Rail \\
\hline Radiological & & \\
Normal Crew & $2.1 \mathrm{E}-04$ & $5.0 \mathrm{E}-05$ \\
Normal Public & $2.9 \mathrm{E}-04$ & $1.8 \mathrm{E}-04$ \\
Accident Public & $5.4 \mathrm{E}-04$ & $7.8 \mathrm{E}-05$ \\
Nonradiological & & \\
Emission & $1.7 \mathrm{E}-04$ & $3.7 \mathrm{E}-04$ \\
Accident & $9.3 \mathrm{E}-04$ & $2.9 \mathrm{E}-05$ \\
\hline
\end{tabular}


Table 9. Expected Annual Cancer Incidence for the Shipping Campaign

\begin{tabular}{lll}
\hline Exposure Group & Truck & Rail \\
\hline Radiological & & \\
$\quad$ Normal Crew & $7.2 \mathrm{E}-04$ & $1.7 \mathrm{E}-04$ \\
$\quad$ Normal Public & $1.0 \mathrm{E}-03$ & $6.1 \mathrm{E}-04$ \\
$\quad$ Accident Public & $2.2 \mathrm{E}-03$ & $2.7 \mathrm{E}-04$ \\
$\begin{array}{c}\text { Nonradiological } \\
\text { Emission }\end{array}$ & $1.7 \mathrm{E}-04$ & $3.7 \mathrm{E}-04$ \\
Accident & NA & NA \\
\hline
\end{tabular}

Table 10. Expected Annual Genetic Effects for the Shipping Campaign

\begin{tabular}{lll}
\hline Exposure Group & Truck & Rail \\
\hline Radiological & $3.1 \mathrm{E}-05$ & $7.5 \mathrm{E}-06$ \\
Normal Crew & $5.9 \mathrm{E}-05$ & $3.6 \mathrm{E}-05$ \\
Normal Public & $1.2 \mathrm{E}-05$ & $2.5 \mathrm{E}-06$ \\
Accident Public & & \\
Nonradiological & NA & NA \\
Emission & NA & NA \\
Accident & & \\
\hline
\end{tabular}




\section{B.18 WM LLMW REGIONALIZED 3 ALTERNATIVE (CASE 10a); CONTACT-HANDLED ORGANIC LIQUIDS: ALPHA - UNTREATED}

Table 1. Summary of Route Information

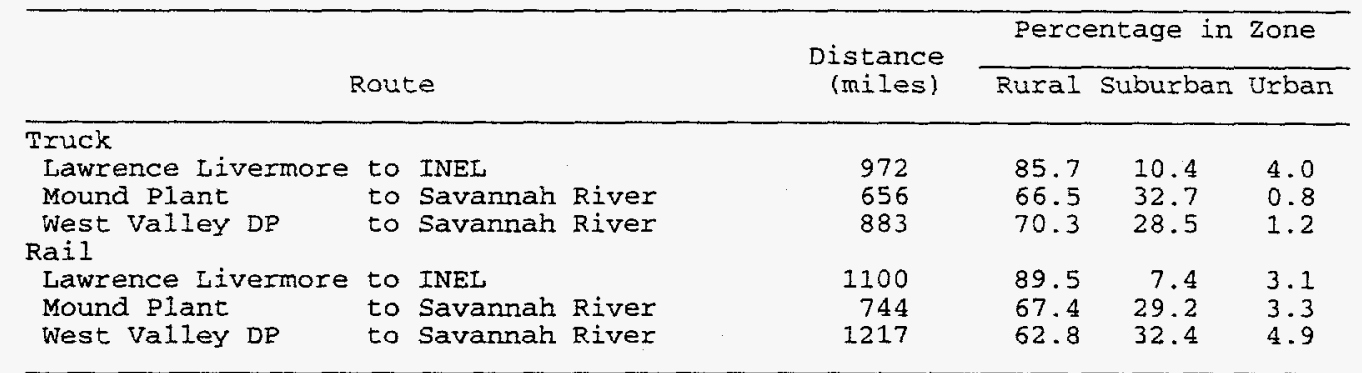

Table 2. Annual Number of Shipments and one-Way Shipment Distances

\begin{tabular}{|c|c|c|c|c|c|}
\hline \multirow[b]{2}{*}{ Route } & & \multicolumn{2}{|c|}{ \# of shipments } & \multicolumn{2}{|c|}{ Total Mileage (mi) } \\
\hline & & Truck & Rail & Truck & Rail \\
\hline $\begin{array}{l}\text { Lawrence Livermore } \\
\text { Mound Plant } \\
\text { West Valley DP }\end{array}$ & $\begin{array}{l}\text { to INEL } \\
\text { to Savannah River } \\
\text { to Savannah River }\end{array}$ & $\begin{array}{l}4 \\
1 \\
1\end{array}$ & $\begin{array}{l}2 \\
1 \\
1\end{array}$ & $\begin{array}{l}3.89 E+03 \\
6.56 E+02 \\
8.83 E+02\end{array}$ & $\begin{array}{l}2.20 \mathrm{E}+03 \\
7.44 \mathrm{E}+02 \\
1.22 \mathrm{E}+03\end{array}$ \\
\hline Totals & & 6 & 4 & $5.43 E+03$ & $\overline{4.16 \mathrm{E}+03}$ \\
\hline
\end{tabular}

Table 3. Incident-Free Dose per Shipment (person-rem/shipment)

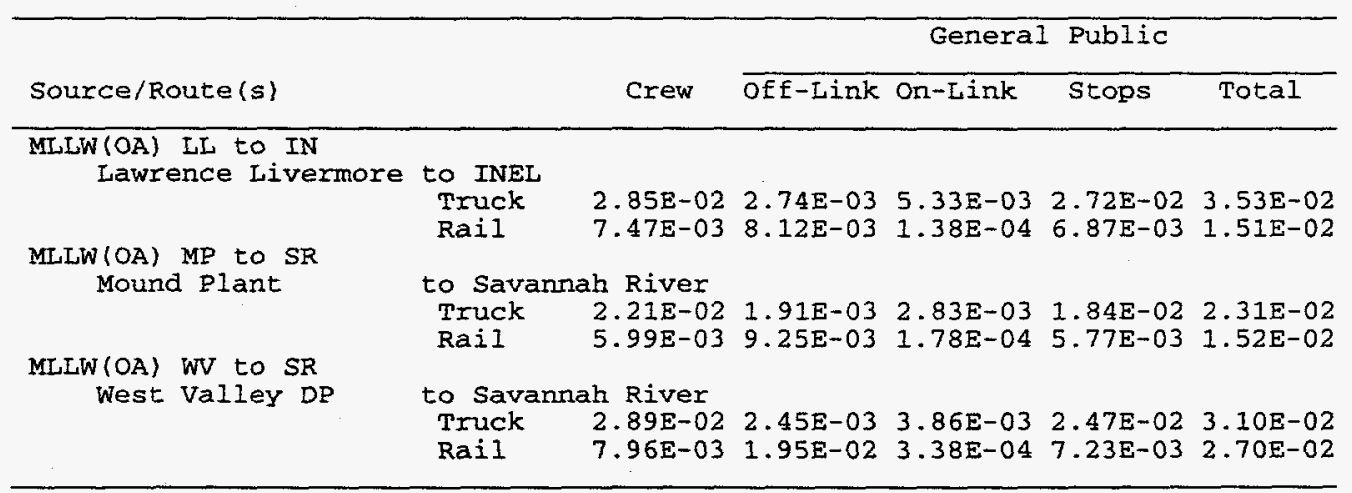

Table 4. Accident Dose Risk per Shipment (person-rem/shipment)

\begin{tabular}{llcc}
\hline Source/Route & Truck & Rail \\
\hline $\begin{array}{c}\text { MLLW(OA) LI to IN } \\
\text { Lawrence Livermore to INEL }\end{array}$ & $1.16 \mathrm{E}-02$ & $7.55 \mathrm{E}-03$ \\
$\begin{array}{c}\text { MLLW(OA) MP to SR } \\
\text { Mound Plant }\end{array}$ & to Savannah River & $4.15 \mathrm{E}-03$ & $7.63 \mathrm{E}-04$ \\
$\begin{array}{c}\text { MLLW (OA) WV to SR } \\
\text { West Valley DP }\end{array}$ & to Savannah River & $4.27 \mathrm{E}-02$ & $1.37 \mathrm{E}-02$ \\
\hline
\end{tabular}




\section{$B-79$}

Table 5. Nonradiological Risk Factors per Shipment (facalities/shipment)

\begin{tabular}{|c|c|c|c|}
\hline Mode & Route & Emission & Accident \\
\hline \multicolumn{4}{|l|}{ Truck } \\
\hline Lawrence Livermore & to INEL & $1.24 E-05$ & $5.73 E-05$ \\
\hline Mound Plant & to Savannah River & $1.67 \mathrm{E}-06$ & $5.26 E-05$ \\
\hline West valley DE & to Savannah River & $3.28 \mathrm{E}-06$ & $6.60 \pm-05$ \\
\hline \multicolumn{4}{|c|}{ 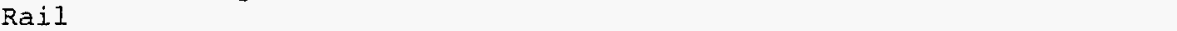 } \\
\hline Lawrence Livermore & to INEL & $1.41 E-05$ & $2.30 E-06$ \\
\hline Mound Plant & to Savannah River & $1.03 E-05$ & $1.56 \mathrm{E}-06$ \\
\hline West valley DP & to Savannah River & $2.49 \mathrm{E}-05$ & $2.55 \mathrm{E}-06$ \\
\hline
\end{tabular}

Table 6. Annual Incident-Free Dose for the Shipping Campaign (person-rem/yr)

\begin{tabular}{|c|c|c|c|c|c|}
\hline \multirow[b]{2}{*}{ Route } & & \multicolumn{2}{|c|}{ Truck } & \multicolumn{2}{|c|}{ Rail } \\
\hline & & Crew & Public & Crew & Public \\
\hline $\begin{array}{l}\text { Lawrence Livermore } \\
\text { Mound Plant } \\
\text { West valley DP }\end{array}$ & $\begin{array}{l}\text { to INEL } \\
\text { to Savannah River } \\
\text { to Savannah River }\end{array}$ & $\begin{array}{l}1.14 \mathrm{E}-01 \\
2.21 \mathrm{E}-02 \\
2.89 \mathrm{E}-02\end{array}$ & $\begin{array}{l}1.41 \mathrm{E}-01 \\
2.31 \mathrm{E}-02 \\
3.10 \mathrm{E}-02\end{array}$ & $\begin{array}{l}1.49 \mathrm{E}-02 \\
5.99 \mathrm{E}-03 \\
7.96 \mathrm{E}-03\end{array}$ & $\begin{array}{l}3.03 E-02 \\
1.52 E-02 \\
2.70 E-02\end{array}$ \\
\hline Totals & & $1.65 \mathrm{E}-01$ & $1.95 \mathrm{E}-01$ & $2.89 \mathrm{E}-02$ & $7.25 \mathrm{E}-02$ \\
\hline
\end{tabular}

Table 7. Annual Accident Dose Risk for the Shipping Campaign (person-rem/yr)

\begin{tabular}{|c|c|c|c|}
\hline Route & & Truck & Rail \\
\hline $\begin{array}{l}\text { Lawrence Livermore } \\
\text { Mound Plant } \\
\text { West Valley DP }\end{array}$ & $\begin{array}{l}\text { to INEL } \\
\text { to Savannah River } \\
\text { to Savannah River }\end{array}$ & $\begin{array}{l}4.63 \mathrm{E}-02 \\
4.15 \mathrm{E}-03 \\
4.27 \mathrm{E}-02\end{array}$ & $\begin{array}{l}1.51 \Xi-02 \\
7.63 E-04 \\
1.37 E-02\end{array}$ \\
\hline Totals & & $9.31 \mathrm{E}-02$ & $2.96 \mathrm{E}-02$ \\
\hline
\end{tabular}

Table 8. Expected Annual Fatalities for the Shipping Campaign

\begin{tabular}{llc}
\hline Exposure Group & Truck & Rail \\
\hline Radiological & & \\
Normal Crew & $6.6 \mathrm{E}-05$ & $1.2 \mathrm{E}-05$ \\
Normal Public & $9.8 \mathrm{E}-05$ & $3.6 \mathrm{E}-05$ \\
Accident Public & $4.7 \mathrm{E}-05$ & $1.5 \mathrm{E}-05$ \\
Nonradiological & $5.5 \mathrm{E}-05$ & $6.3 \mathrm{E}-05$ \\
$\quad$ Emission & $3.5 \mathrm{E}-04$ & $8.7 \mathrm{E}-06$ \\
\hline
\end{tabular}

Table 9. Expected Annual Cancer Incidence for the Shipping Campaign

\begin{tabular}{lll}
\hline Exposure Group & Truck & Rail \\
\hline Radiological & & \\
Normal Crew & $2.3 \mathrm{E}-04$ & $4.0 \mathrm{E}-05$ \\
Normal Public & $3.3 \mathrm{E}-04$ & $1.2 \mathrm{E}-04$ \\
$\quad$ Accident Public & $1.6 \mathrm{E}-04$ & $5.0 \mathrm{E}-05$ \\
Nonradiological & $5.5 \mathrm{E}-05$ & $6.3 \mathrm{E}-05$ \\
$\quad$ Emission & $\mathrm{NA}$ & $\mathrm{NA}$ \\
Accident & &
\end{tabular}


Table 10. Expected Annual Genetic Effects for the Shipping Campaign

\begin{tabular}{lll}
\hline Exposure Group & Truck & Rail \\
\hline Radiological & & \\
$\quad$ Normal Crew & $9.9 E-06$ & $1.7 E-06$ \\
Normal Public & $2.0 \mathrm{E}-05$ & $7.2 \mathrm{E}-06$ \\
Accident Public & $2.3 \mathrm{E}-06$ & $7.3 \mathrm{E}-07$ \\
$\begin{array}{l}\text { Nonradiological } \\
\text { Emission }\end{array}$ & NA & NA \\
Accident & NA & NA \\
\hline
\end{tabular}




\section{B.19 WM LLMW REGIONALIZED 3 ALTERNATIVE (CASE 10a): CONTACT-HANDLED HETEROGENEOUS SOLIDS: NONALPHA - UNTREATED}

Table 1. Sumnary of Route Information

\begin{tabular}{|c|c|c|c|c|c|c|}
\hline & & & & Perce & entage in & Zone \\
\hline & Rout & te & (miles) & Rural & Suburban & Urban \\
\hline Truck & & & & & & \\
\hline Ames Laboratory & to & Portsmouth GDP & 755 & 77.4 & 21.0 & 1.7 \\
\hline Argonne - East & to & Portsmouth GDP & 428 & 64.6 & 30.5 & 4.9 \\
\hline Bettis Atomic & & Portsmouth GDP & 265 & 58.8 & 37.4 & 3.8 \\
\hline Battelle Columbus & & Portsmouth GDP & 84 & 68.4 & 28.3 & 3.3 \\
\hline Brookhaven NL & & Portsmouth GDP & 689 & 53.5 & 39.4 & 7.0 \\
\hline Charleston, SC & & Savannah River & 200 & 75.7 & 23.8 & 0.5 \\
\hline Colonie & & Portsmouth GDP & 688 & 57.3 & 40.1 & 2.5 \\
\hline ETEC & to & INEL & 979 & 77.7 & 14.2 & 8.1 \\
\hline Fernald (FEMP) & to & Portsmouth GDP & 173 & 68.0 & 31.6 & 0.3 \\
\hline General Atomic & to & INEL & 976 & 78.8 & 17.1 & 4.1 \\
\hline Grand Junction PO & to & Rocky Flats Plant & 273 & 79.9 & 16.7 & 3.3 \\
\hline Knolls Atomic & to & Portsmouth GDP & 688 & 57.3 & 40.1 & 2.5 \\
\hline Kansas City Plant & to & Rocky Flats Plant & 631 & 89.0 & 9.4 & 1.6 \\
\hline KKS & to & Portsmouth GDP & 688 & 57.3 & 40.1 & 2.5 \\
\hline KWS & to & Portsmouth GDP & 688 & 57.3 & 40.1 & 2.5 \\
\hline Lawrence Berkeley & to & Hanford Site & 875 & 78.1 & 17.8 & 4.1 \\
\hline LERHR & to & Hanford Site & 830 & 80.5 & 17.0 & 2.6 \\
\hline Lawrence Iivermore & to & Hanford Site & 894 & 77.2 & 19.3 & 3.4 \\
\hline Mare Island & to & Hanford Site & 875 & 78.1 & 17.8 & 4.1 \\
\hline Norfolk Nav Shipya & to & Savannah River & 498 & 72.8 & 26.2 & 1.0 \\
\hline Nevada Test Site & to & INEL & 712 & 82.8 & 13.7 & 3.5 \\
\hline Paducah GDP & & Oak Riage Reserv. & 304 & 81.3 & 18.5 & 0.2 \\
\hline Pantex Plant & to & Los Alamos NL & 402 & 89.5 & 8.0 & $2 . \overline{5}$ \\
\hline Pearl Harbor NS & to & Hanford Site & 226 & 76.8 & 20.9 & 2.3 \\
\hline Portsmouth NS & to & Portsmouth GDP & 959 & 46.4 & 45.3 & 8.2 \\
\hline Princeton PPL & to & Portsmouth GDP & 588 & 55.1 & 38.8 & 6.1 \\
\hline Puget Sound NS & to & Hanford Site & 226 & 76.8 & 20.9 & 2.3 \\
\hline Reactive Metals & to & Portsmouth GDP & 276 & 59.5 & 37.8 & 2.8 \\
\hline SNL - Albuquerque & to & Los Alamos NL & 102 & 75.8 & 20.1 & 4.1 \\
\hline SNL - Livermore & to & Hanford Site & 894 & 77.2 & 19.3 & 3.4 \\
\hline Rail & & & & & & \\
\hline Ames Laboratory & to & Portsmouth GDP & 727 & 68.6 & 23.8 & 7.7 \\
\hline Argonne - East & to & Portsmouth GDP & 422 & 64.8 & 30.0 & 5.1 \\
\hline Bettis Atomic & to & Portsmouth GDP & 429 & 52.6 & 39.6 & 7.8 \\
\hline Battelle columbus & to & Portsmouth GDP & 91 & 63.4 & 31.8 & 4.8 \\
\hline Brookhaven NL & to & Portsmouth GDP & 921 & 45.4 & 43.6 & 11.0 \\
\hline Charleston, SC & to & Savannah River & 140 & 83.9 & 13.6 & 2.5 \\
\hline Colonie & to & Portsmouth GDP & 681 & 52.1 & 40.3 & 7.5 \\
\hline ETEC & to & INEL & 1041 & 81.5 & 10.5 & 8.0 \\
\hline Fernald (FEMP) & to & Portsmouth GDP & 207 & 69.6 & 26.5 & 4.0 \\
\hline General Atomic & to & INEL & 1076 & 82.6 & 11.4 & 6.0 \\
\hline Grand Junction PO & to & Rocky Flats Plant & 239 & 95.5 & 4.3 & 0.3 \\
\hline Krolls Atomic & to & Portsmouth GDP & 681 & 52.1 & 40.3 & 7.5 \\
\hline Kansas City Plant & to & Rocky Flats Plant & 778 & 89.8 & 7.8 & 2.3 \\
\hline KKS & to & Portsmouth GDP & 681 & 52.1 & 40.3 & 7.5 \\
\hline KWS & to & Portsmouth GDP & 681 & 52.1 & 40.3 & 7.5 \\
\hline Lawrence Berkeley & to & Hanford Site & 986 & 78.5 & 15.8 & 5.7 \\
\hline LERHR & to & Hanford Site & 890 & 81.0 & 14.3 & 4.7 \\
\hline Lawrence Livermore & to & Hanford site & 973 & 78.9 & 15.3 & 5.8 \\
\hline Mare Island & to & Hanford site & 986 & 78.5 & 15.8 & 5.7 \\
\hline Norfolk Nav Shipyd & to & Savannah River & 529 & 74.3 & 24.1 & 1.6 \\
\hline Nevada Test Site & to & INEL & 756 & 92.8 & 5.9 & 1.3 \\
\hline Paducah GDP & to & Oak Ridge Reserv. & 527 & 89.3 & 9.0 & 1.7 \\
\hline Pantex Plant & to & Los Alamos NL & 482 & 89.2 & 9.6 & 1.3 \\
\hline Pearl Harbor NS & to & Hanford Site & 416 & 73.7 & 20.1 & 6.2 \\
\hline Portsmouth NS & to & Portsmouth GDP & 962 & 49.0 & 44.3 & 6.7 \\
\hline Princeton PPL & to & Portsmouth GDP & 838 & 58.3 & 32.6 & 9.1 \\
\hline Puget Sound NS & to & Hanford Site & 416 & 73.7 & 20.1 & 6.2 \\
\hline Reactive Metals & to & Portsmouth GDP & 279 & 48.4 & 41.8 & 9.8 \\
\hline SNI - Albuquerque & to & Los Alamos NL & 104 & 74.3 & 20.0 & 5.7 \\
\hline SNI - Iivermore & to & Hanford Site & 973 & 78.9 & 15.3 & 5.8 \\
\hline
\end{tabular}


Table 2. Annual Number of Shipments and One-Way Shipment Distances

\begin{tabular}{|c|c|c|c|c|c|c|}
\hline \multirow[b]{2}{*}{ Route } & & & \multicolumn{2}{|c|}{ \# of Shipments } & \multicolumn{2}{|c|}{ Total Mileage (mi) } \\
\hline & & & Truck & Rail & Truck & Rail \\
\hline Ames Laboratory & & Portsmouth GDP & 1 & 1 & $7.55 E+02$ & $7.27 E+02$ \\
\hline Argonne - East & & Portsmouth GDP & 44 & 17 & $1.88 E+04$ & $7.18 \mathrm{E}+03$ \\
\hline Bettis Atomic & & Portsmouth GDP & 1 & 1 & $2.65 E+02$ & $4.29 \mathrm{E}+02$ \\
\hline Battelle Columbus & to & Portsmouth GDP & 1 & 1 & $8.41 E+01$ & $9.09 \mathrm{E}+0 \mathrm{I}$ \\
\hline Brookhaven NL & & Portsmouth GDP & 1 & 1 & $6.89 E+02$ & $9.21 E+02$ \\
\hline Charleston, SC & & Savannah River & 1 & 1 & $2.00 \mathrm{E}+02$ & $1.40 \mathrm{E}+02$ \\
\hline Colonie & to & Portsmouth GDP & 1 & 1 & $6.88 \mathrm{E}+02$ & $6.81 E+02$ \\
\hline ETEC & to & INEL & 8 & 3 & $7.83 E+03$ & $3.12 E+03$ \\
\hline Fernald (EEMP) & to & Portsmouth GDP & 7 & 3 & $1.21 E+03$ & $6.22 \mathrm{E}+02$ \\
\hline General Atomic & to & INEL & 1 & 1 & $9.76 \mathrm{E}+02$ & $1.08 E+03$ \\
\hline Grand Junction PO & to & Rocky Flats Plant & 1 & 1 & $2.73 E+02$ & $2.39 E+02$ \\
\hline Knolls Atomic & & Portsmouth GDP & 1 & 1 & $6.88 \mathrm{E}+02$ & $6.81 E+02$ \\
\hline Kansas City Plant & to & Rocky Flats Plant & 1 & 1 & $6.31 E+02$ & $7.78 \mathrm{E}+02$ \\
\hline KKS & to & Portsmouth GDP & 1 & 1 & $6.88 \mathrm{E}+02$ & $6.81 E+02$ \\
\hline KWS & to & Portsmouth GDP & 1 & 1 & $6.88 \mathrm{E}+02$ & $6.81 \Xi+02$ \\
\hline Lawrence Berkeley & to & Hanford Site & 1 & 1 & $8.75 E+02$ & $9.86 \mathrm{E}+02$ \\
\hline LERHR & to & Hanford Site & 1 & 1 & $8.30 E+02$ & $8.90 E+02$ \\
\hline Lawrence Livermore & to & Hanford Site & 4 & 2 & $3.58 E+03$ & $1.95 E+03$ \\
\hline Mare Island & to & Hanford Site & 1 & 1 & $8.75 E+02$ & $9.86 E+02$ \\
\hline Norfolk Nav Shipyd & to & Savannah River & 1 & 1 & $4.98 \mathrm{E}+02$ & $5.29 E+02$ \\
\hline Nevada Test Site & to & INEL & 1 & 1 & $7.12 \mathrm{E}+02$ & $7.56 \mathrm{E}+02$ \\
\hline Paducah GDP & to & Oak Ridge Reserv. & 1 & 1 & $3.04 E+02$ & $5.27 E+02$ \\
\hline Pantex Plant & & Los Alamos NL & 2 & 1 & $8.04 \mathrm{E}+02$ & $4.82 E+02$ \\
\hline Pearl Harbor NS & to & Hanford Site & 1 & 1 & $2.26 \mathrm{E}+02$ & $4.16 \mathrm{E}+02$ \\
\hline Portsmouth NS & & Portsmouth GDP & 1 & 1 & $9.59 E+02$ & $9.62 E+02$ \\
\hline Princeton PPL & to & Portsmouth GDP & 1 & 1 & $5.88 \mathrm{E}+02$ & $8.38 \varepsilon+02$ \\
\hline Puget Sound NS & to & Hanford Site & 1 & 1 & $2.26 \mathrm{E}+02$ & $4.16 E+02$ \\
\hline Reactive Metals & to & Portsmouth GDP & 1 & 1 & $2.76 \mathrm{E}+02$ & $2.79 E+02$ \\
\hline SNL - Albuquerque & to & Los Alamos NL & 1 & 1 & $1.02 E+02$ & $1.04 E+02$ \\
\hline SNL - Livermore & to & Hanford Site & 1 & 1 & $8.94 \mathrm{E}+02$ & $9.73 E+02$ \\
\hline 20 & & & 90 & 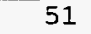 & $4.62 \mathrm{E}+04$ & $2.91 E+04$ \\
\hline
\end{tabular}


Table 3. Incident-Free Dose per Shipment (person-rem/shipment)

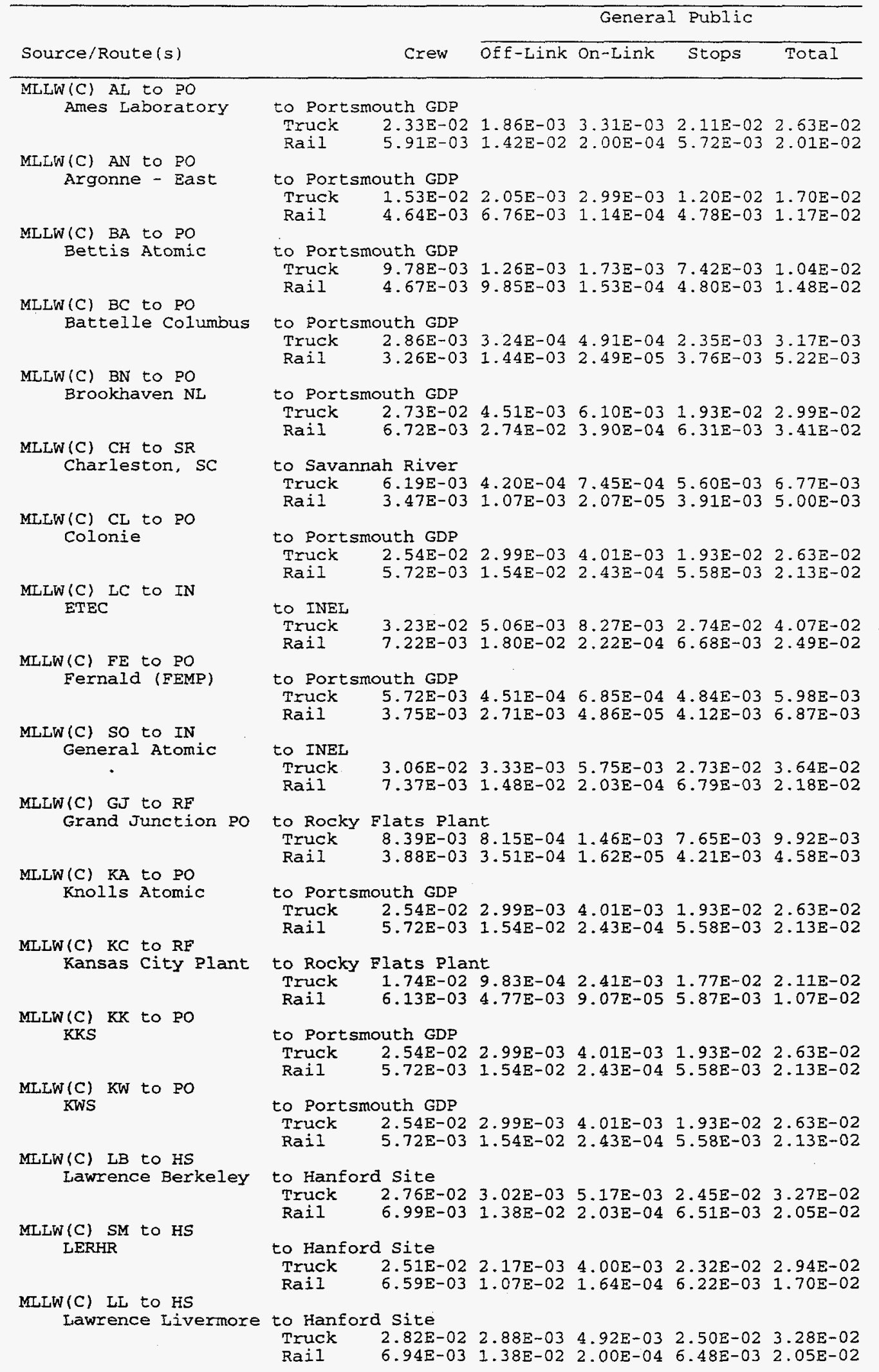




\section{B-84}

Table 3. Incident-Free Dose per Shipment (person-rem/shipment) (Cont.)

\begin{tabular}{|c|c|c|c|c|c|}
\hline \multirow[b]{2}{*}{ Source/Route(s) } & \multirow[b]{2}{*}{ Crew } & \multicolumn{4}{|c|}{ General Public } \\
\hline & & off-Link & On-Link & Stops & Total \\
\hline \multicolumn{6}{|l|}{ MLLW(C) MZ to HS } \\
\hline & $\begin{array}{ll}\text { Eo hantord site } \\
\text { Truck } 2.76 \mathrm{E}-02\end{array}$ & $3.02 E-03$ & $5.17 \mathrm{E}-03$ & $2.45 E-02$ & $3.27 \mathrm{E}-02$ \\
\hline & $6.99 \mathrm{E}-03$ & $1.38 E-02$ & 2.03 & $6.51 E-03$ & $2.05 E-02$ \\
\hline \multirow{2}{*}{\multicolumn{6}{|c|}{$\begin{array}{l}\text { MLLW(C) US to SR } \\
\text { Norfolk Nav Shipyd to Savannah River }\end{array}$}} \\
\hline & & & & & \\
\hline & $\begin{array}{ll}\text { Truck } & 1.59 \mathrm{E}-02 \\
\text { Rail } & 5.09 \mathrm{E}-03\end{array}$ & $\begin{array}{l}1.26 \mathrm{E}-03 \\
4.35 \mathrm{E}-03\end{array}$ & $2.07 \mathrm{E}-03$ & $1.39 \mathrm{E}-02$ & 1. $73 \mathrm{E}-02$ \\
\hline \multirow{2}{*}{\multicolumn{6}{|c|}{$\begin{array}{r}\text { MLLW(C) NT to IN } \\
\text { Nevada Test Site }\end{array}$}} \\
\hline Nevada Test Site & & & & & \\
\hline & $\begin{array}{l}2.13 \mathrm{E}-02 \\
6.04 \mathrm{E}-03\end{array}$ & $\begin{array}{l}2.03 E-03 \\
2.80 E-03\end{array}$ & $\begin{array}{l}3.79 E-03 \\
6.88 \mathrm{E}-05\end{array}$ & $\begin{array}{l}1.99 \mathrm{E}-02 \\
5.81 \mathrm{E}-03\end{array}$ & $2.58 \mathrm{E}-02$ \\
\hline \multicolumn{6}{|l|}{ MLLW (C) PA to OR } \\
\hline Paducah GDP & to Oak Ridge Reser & & & & \\
\hline & $\begin{array}{ll}\text { Truck } & 8.88 \mathrm{E}-03 \\
\text { Rail } & 5.08 \mathrm{E}-03\end{array}$ & $\begin{array}{l}4.70 \mathrm{E}-04 \\
2.75 \mathrm{E}-03\end{array}$ & $\begin{array}{l}9.98 \mathrm{E}-04 \\
5.97 \mathrm{E}-05\end{array}$ & $\begin{array}{l}8.51 \mathrm{E}-03 \\
5.10 \mathrm{E}-03\end{array}$ & $\begin{array}{l}9.98 E-03 \\
7.91 E-03\end{array}$ \\
\hline \multirow{2}{*}{\multicolumn{6}{|c|}{$\begin{array}{l}\text { MLLW (C) PP to LA } \\
\text { Pantex Plant }\end{array}$}} \\
\hline & to Los Alamos NL & & & & \\
\hline & $\begin{array}{ll}\text { Truck } & 1.11 \mathrm{E}-02 \\
\text { Rail } & 4.89 \mathrm{E}-03\end{array}$ & $\begin{array}{l}7.76 \mathrm{E}-04 \\
2.18 \mathrm{E}-03\end{array}$ & $\begin{array}{l}1.77 \mathrm{E}-03 \\
5.29 \mathrm{E}-05\end{array}$ & $\begin{array}{l}1.13 \mathrm{E}-02 \\
4.96 \mathrm{E}-03\end{array}$ & $\begin{array}{l}1.38 \mathrm{E}-02 \\
7.19 \mathrm{E}-03\end{array}$ \\
\hline \multirow{2}{*}{\multicolumn{6}{|c|}{$\begin{array}{l}\text { MLLW(C) SW to HS } \\
\text { Pearl Harbor NS }\end{array}$}} \\
\hline & & & & & \\
\hline & $7.06 \mathrm{E}-03$ & $6.28 \mathrm{E}-04$ & $1.09 \mathrm{E}-03$ & $6.33 E-03$ & $8.04 \mathrm{E}-03$ \\
\hline \multirow{2}{*}{\multicolumn{6}{|c|}{$\begin{array}{l}\text { MLLW(C) PT to PO } \\
\text { Portsmouth NS }\end{array}$}} \\
\hline & & & & & \\
\hline & $4.03 \mathrm{E}-02$ & $7.28 \mathrm{E}-03$ & $9.52 \mathrm{E}-03$ & $2.68 \mathrm{E}-02$ & $4.36 \mathrm{E}-02$ \\
\hline \multirow{2}{*}{\multicolumn{6}{|c|}{$\begin{array}{l}\text { MLLW (C) PR to PO } \\
\text { Princeton PPL }\end{array}$}} \\
\hline & & & & & \\
\hline & $2.28 \mathrm{E}-02$ & $3.55 E-03$ & $4.81 \mathrm{E}-03$ & $1.65 \mathrm{E}-02$ & $2.48 \mathrm{E}-02$ \\
\hline \multirow{2}{*}{\multicolumn{6}{|c|}{$\begin{array}{l}\text { MLLW (C) PN to HS } \\
\text { Puget Sound NS }\end{array}$}} \\
\hline & & & & & \\
\hline & $7.06 \mathrm{E}-03$ & $6.28 \mathrm{E}-04$ & $1.09 E-03$ & $6.33 E-03$ & $8.04 E-03$ \\
\hline \multirow{3}{*}{$\begin{array}{l}\text { MLLW(C) RM to PO } \\
\text { Reactive Metals }\end{array}$} & 4.6 & 0.0. & & 4 & \\
\hline & to Portsmouth GDP & & & & \\
\hline & $\begin{array}{ll}\text { Truck } & 1.00 \mathrm{E}-02 \\
\text { Rail } & 4.05 \mathrm{E}-03\end{array}$ & $\begin{array}{l}1.18 \mathrm{E}-03 \\
7.58 \mathrm{E}-03\end{array}$ & $\begin{array}{l}1.62 \mathrm{E}-03 \\
1.11 \mathrm{E}-04\end{array}$ & $\begin{array}{l}7.73 \mathrm{E}-03 \\
4.34 \mathrm{E}-03\end{array}$ & $\begin{array}{l}1.05 \mathrm{E}-02 \\
1.20 \mathrm{E}-02\end{array}$ \\
\hline \multirow{3}{*}{$\begin{array}{l}\text { MLLW(C) SA to LA } \\
\text { SNL - Albuquerque }\end{array}$} & & & & & \\
\hline & to Los Alamos NL & & & & \\
\hline & $\begin{array}{ll}\text { Truck } & 3.28 \mathrm{E}-03 \\
\text { Rail } & 3.31 \mathrm{E}-03\end{array}$ & $\begin{array}{l}3.70 E-04 \\
1.55 E-03\end{array}$ & $\begin{array}{l}6.13 \mathrm{E}-04 \\
2.36 \mathrm{E}-05\end{array}$ & $\begin{array}{l}2.86 \mathrm{E}-03 \\
3.80 \mathrm{E}-03\end{array}$ & $\begin{array}{l}3.84 E-03 \\
5.37 E-03\end{array}$ \\
\hline \multirow{3}{*}{$\begin{array}{l}\text { MLLW(C) SL to HS } \\
\text { SNL - Livermore }\end{array}$} & & & & & \\
\hline & to Hanford Site & & & & \\
\hline & $\begin{array}{l}2.82 \mathrm{E}-02 \\
6.94 \mathrm{E}-03\end{array}$ & $\begin{array}{l}2.88 \mathrm{E}-03 \\
1.38 \mathrm{E}-02\end{array}$ & $\begin{array}{l}4.92 \mathrm{E}-03 \\
2.00 \mathrm{E}-04\end{array}$ & $\begin{array}{l}2.50 \mathrm{E}-02 \\
6.48 \mathrm{E}-03\end{array}$ & $\begin{array}{l}3.28 \mathrm{E}-02 \\
2.05 \mathrm{E}-02\end{array}$ \\
\hline
\end{tabular}


Table 4. Accident Dose Risk per Shipment (person-rem/shipment)

\begin{tabular}{|c|c|c|c|c|}
\hline \multicolumn{3}{|l|}{ Source/Route } & \multirow[t]{2}{*}{ Truck } & \multirow[t]{2}{*}{ Rail } \\
\hline MLIW(C) AL to PO & & & & \\
\hline Ames Laboratory & to & Portsmouth GDP & $1.84 E-08$ & $7.49 \Xi-09$ \\
\hline \multicolumn{5}{|l|}{ MLLW (C) AN to PO } \\
\hline Argonne - East & to & Portsmouth GDP & $4.72 E-04$ & $2.52 E-04$ \\
\hline \multicolumn{5}{|l|}{ MLLW (C) BA to PO } \\
\hline Bettis Atomic & to & Portsmouth GDP & $1.00 \Xi-04$ & $4.05 E-05$ \\
\hline \multicolumn{5}{|l|}{ MLLW $(C)$ BC to PO } \\
\hline Battelle Columbus & to & Portsmouth GDP & $2.49 E-10$ & $6.63 E-11$ \\
\hline \multicolumn{5}{|l|}{ MLLW (C) BN to PO } \\
\hline \multicolumn{3}{|l|}{ MLLW(C) CH to SR } & $4.93 E-04$ & $2.53 E-04$ \\
\hline Charleston, SC & to & Savannah River & $5.98 E-05$ & $6.95 \mathrm{E}-06$ \\
\hline \multicolumn{5}{|l|}{$\operatorname{MLIN}(\mathrm{C})$ CL to po } \\
\hline Colonie & to & Portsmouth GDP & $3.28 E-08$ & $7.61 E-09$ \\
\hline \multicolumn{5}{|l|}{ MLIW (C) LC to IN } \\
\hline ETEC & to & INEL & $2.18 E-04$ & $2.61 E-04$ \\
\hline \multicolumn{5}{|l|}{ MLLW (C) FE to PO } \\
\hline Fernald (FEMP) & to & Portsmouth GDP & $4.03 E-06$ & $2.15 \mathrm{E}-06$ \\
\hline \multicolumn{5}{|l|}{ MLLW (C) So to IN } \\
\hline General Atomic & to & INEL & $4.51 E-07$ & $1.77 \mathrm{E}-07$ \\
\hline \multicolumn{5}{|l|}{ MLLW (C) GJ to RF } \\
\hline Grand Junction PO & to & Rocky Flats Plant & $1.60 \mathrm{E}-08$ & $1.77 E-10$ \\
\hline \multicolumn{5}{|l|}{$M L L W(C) \quad K A$ to $P O$} \\
\hline Knolis Atomic & to & Portsmouth GDP & $1.98 E-02$ & $4.64 \mathrm{E}-03$ \\
\hline \multicolumn{5}{|l|}{ MLLW $(C) \mathrm{KC}$ to $\mathrm{RF}$} \\
\hline Kansas City Plant & to & Rocky Flats Plant & $6.95 z-05$ & $1.08 \mathrm{E}-05$ \\
\hline \multicolumn{5}{|l|}{ MLLW(C) KK to PO } \\
\hline KKS & to & Portsmouth GDP & $2.17 \mathrm{E}-02$ & $5.10 \mathrm{E}-03$ \\
\hline \multicolumn{5}{|l|}{$\operatorname{MLLW}(\mathrm{C}) \mathrm{KW}$ to $\mathrm{PO}$} \\
\hline KWS & to & Portsmouth GDP & $6.98 \mathrm{E}-03$ & $1.64 E-03$ \\
\hline \multicolumn{5}{|l|}{ MLLW (C) LB to HS } \\
\hline Lawrence Berkeley & to & Hanford Site & $1.05 \mathrm{E}-05$ & $5.80 \mathrm{E}-06$ \\
\hline MLLW (C) SM to HS & & & & \\
\hline LERHR & to & Hanford Site & $9.03 E-06$ & $4.74 E-06$ \\
\hline MLLW (C) LL to HS & & & & \\
\hline Lawrence Livermore & to & Hanford Site & $4.37 E-05$ & $4.58 \mathrm{E}-05$ \\
\hline MLLW(C) MZ to HS & & & & \\
\hline Mare Island & to & Hanford Site & $8.65 \mathrm{E}-03$ & $4.86 \mathrm{E}-03$ \\
\hline MLLW(C) US to SR & & & & \\
\hline Norfolk Nav Shipyd & to & Savannah River & $8.05 E-04$ & $1.41 E-04$ \\
\hline MLLW (C) NT to IN & & & & \\
\hline Nevada Test Site & to & INEL & $1.30 \mathrm{E}-08$ & $2.10 \mathrm{E}-09$ \\
\hline MLLW (C) PA to OR & & & & \\
\hline $\begin{array}{l}\text { Paducah GDP } \\
\text { MLLW (C) PP to IA }\end{array}$ & to & Oak Ridge Reserv. & $9.75 \mathrm{E}-04$ & $1.25 \mathrm{E}-04$ \\
\hline Pantex Plant & to & Los Alamos NL & $7.12 \mathrm{E}-05$ & $8.96 \mathrm{E}-06$ \\
\hline MLLW(C) SW to HS & & & & \\
\hline Pearl Harbor NS & to & Hanford Site & $1.11 \mathrm{E}-04$ & $8.21 E-05$ \\
\hline MLLW (C) PT to PO & & & & \\
\hline $\begin{array}{l}\text { Portsmouth NS } \\
\text { MLLW(C) PR to PO }\end{array}$ & to & Portsmouth GDP & $3.37 \mathrm{E}-04$ & 1. $53 E-04$ \\
\hline Princeton PPL & to & Portsmouth GDP & $2.72 \mathrm{E}-09$ & $1.28 \mathrm{E}-09$ \\
\hline MULW (C) PN to HS & & & & \\
\hline Puget Sound NS & to & Hanford site & $2.47 \mathrm{E}-03$ & $1.83 \mathrm{E}-03$ \\
\hline$M L L W(C)$ RM to PO & & & & \\
\hline Reactive Metals & to & Portsmouth GDP & $7.25 \mathrm{E}-07$ & $2.09 E-07$ \\
\hline MLLW (C) SA to LA & & & & \\
\hline SNL - Albuquerque & to & Los Alamos NL & $8.43 E-05$. & $1.60 E-06$ \\
\hline $\begin{array}{l}\text { MLLW (C) SL to HS } \\
\text { SNL - Livermore }\end{array}$ & to & Hanford $s$ & $2.72 E-06$ & $1.43 \mathrm{E}$ \\
\hline 3IVD LLVERU & & & & $1.43 E-06$ \\
\hline
\end{tabular}


Table 5. Nonradiological Risk Factors per Shipment (fatalities/shipment)

\begin{tabular}{|c|c|c|c|}
\hline Mode & Route & Emission & Accident \\
\hline Truck & & & \\
\hline Ames Laboratory & to Portsmouth GDP & $4.02 \mathrm{E}-06$ & $4.11 \mathrm{E}-05$ \\
\hline Argonne - East & to Portsmouth GDP & $6.73 E-06$ & $2.60 \mathrm{E}-05$ \\
\hline Bettis Atomic & to Portsmouth GDP & $3.22 \mathrm{E}-06$ & $1.33 E-05$ \\
\hline Battelle Columbus & to Portsmouth GDP & $9.01 \mathrm{E}-07$ & $3.65 E-06$ \\
\hline Brookhaven NL & to Portsmouth GDP & $1.56 \mathrm{E}-05$ & $4.90 \mathrm{E}-05$ \\
\hline Charleston, SC & to Savannah River & $3.22 \mathrm{E}-07$ & $1.70 \mathrm{E}-05$ \\
\hline Colonie & to Portsmouth GDP & $5.63 \mathrm{E}-06$ & $3.56 \mathrm{E}-05$ \\
\hline ETEC & to INEL & $2.55 E-05$ & $6.21 E-05$ \\
\hline Fernald (FEMP) & to Portsmouth GDP & $1.93 \mathrm{E}-07$ & $7.51 \varepsilon-06$ \\
\hline General Atomic & to INEL & 1.30E-05 & $6.23 E-05$ \\
\hline Grand Junction PO & to Rocky Flats Plant & $2.93 E-06$ & $2.32 \mathrm{E}-05$ \\
\hline Knolls Atomic & to Portsmouth GDP & $5.63 E-06$ & $3.56 \mathrm{E}-05$ \\
\hline Kansas City Plant & to Rocky Flats Plant & $3.22 \mathrm{E}-06$ & $4.74 \mathrm{E}-05$ \\
\hline KKS & to Portsmouth GDP & $5.63 E-06$ & $3.56 \mathrm{E}-05$ \\
\hline KWS & to Portsmouth GDP & $5.63 E-06$ & $3.56 \mathrm{E}-05$ \\
\hline Lawrence Berkeley & to Hanford Site & $1.16 \mathrm{E}-05$ & $4.91 \mathrm{E}-05$ \\
\hline LERHR & to Hanford Site & $6.82 \mathrm{E}-06$ & $4.66 \mathrm{E}-05$ \\
\hline Lawrence Livermore & to Hanford Site & $9.85 \mathrm{E}-06$ & $5.03 E-05$ \\
\hline Mare Island & to Hanford Site & $1.16 \mathrm{E}-05$ & $4.91 \mathrm{E}-05$ \\
\hline Norfolk Nav Shipyd & to Savannah River & $1.58 \mathrm{E}-06$ & $4.45 E-05$ \\
\hline Nevada Test Site & to INEI & $8.08 \mathrm{E}-06$ & $4.36 \mathrm{E}-05$ \\
\hline Paducah GDP & to Oak Ridge Reserv. & $1.93 \mathrm{E}-07$ & $2.14 \mathrm{E}-05$ \\
\hline Pantex Plant & to Los Alamos NL & $3.28 E-06$ & $3.21 \mathrm{E}-05$ \\
\hline Pearl Harbor NS & to Hanford Site & $1.67 E-06$ & $9.56 \mathrm{E}-06$ \\
\hline Portsmouth NS & to Portsmouth GDP & $2.55 E-05$ & $6.65 \mathrm{E}-05$ \\
\hline Princeton PPL & to Portsmouth GDP & $1.16 \mathrm{E}-05$ & $3.99 \mathrm{E}-05$ \\
\hline Puget Sound NS & to Hanford Site & $1.67 E-06$ & $9.56 \mathrm{E}-06$ \\
\hline Reactive Metals & to Portsmouth GDP & $2.45 \mathrm{E}-06$ & $1.21 \mathrm{E}-05$ \\
\hline SNL - Albuquerque & to Los Alamos NL & $1.35 \mathrm{E}-06$ & $1.20 \mathrm{E}-05$ \\
\hline SNL - Livermore & to Hanford site & $9.85 E-06$ & $5.03 \mathrm{E}-05$ \\
\hline Rail & & & \\
\hline Ames Laboratory & to Portsmouth GDP & $2.34 E-05$ & $1.52 E-06$ \\
\hline Argonne - East & to Portsmouth GDP & $9.08 \mathrm{E}-06$ & $8.84 \mathrm{E}-07$ \\
\hline Bettis Atomic & to Portsmouth GDP & $1.40 \mathrm{E}-05$ & $8.98 \mathrm{E}-07$ \\
\hline Battelle columbus & to Portsmouth GDP & $1.84 \mathrm{E}-06$ & $1.90 \mathrm{E}-07$ \\
\hline Brookhaven NL & to Portsmouth GDP & $4.22 \mathrm{E}-05$ & $1.93 E-06$ \\
\hline Charleston, SC & to Savannah River & $1.46 \mathrm{E}-06$ & $2.93 E-07$ \\
\hline Colonie & to Portsmouth GDP & $2.15 \mathrm{E}-05$ & $1.43 \mathrm{E}-06$ \\
\hline ETEC & to INEL & $3.48 \mathrm{E}-05$ & $2.18 \mathrm{E}-06$ \\
\hline Fernald (FEMP) & to Portsmouth GDP & $3.43 \mathrm{E}-06$ & $4.34 E-07$ \\
\hline General Atomic & to INEL & $2.72 \mathrm{E}-05$ & $2.25 E-06$ \\
\hline Grand Junction Po & to Rocky Flats Plant & $2.51 \mathrm{E}-07$ & $4.99 \mathrm{E}-07$ \\
\hline Knolls Atomic & to Portsmouth GDP & $2.15 \mathrm{E}-05$ & $1.43 \mathrm{E}-06$ \\
\hline Kansas City Plant & to Rocky Flats Plant & $7.62 \mathrm{E}-06$ & $1.63 \mathrm{E}-06$ \\
\hline KKS & to Portsmouth GDP & $2.15 \mathrm{E}-05$ & $1.43 \mathrm{E}-06$ \\
\hline KWS & to Portsmouth GDP & $2.15 E-05$ & $1.43 \mathrm{E}-06$ \\
\hline Lawrence Berkeley & to Hanford Site & $2.33 E-05$ & $2.06 \mathrm{E}-06$ \\
\hline LERHR & to Hanford site & $1.77 \mathrm{E}-05$ & $1.86 \mathrm{E}-06$ \\
\hline Lawrence Livermore & to Hanford Site & $2.36 \mathrm{E}-05$ & $2.04 E-06$ \\
\hline Mare Island & to Hanford Site & $2.33 E-05$ & $2.06 E-06$ \\
\hline Norfolk Nav Shipyd & to Savannah River & $3.64 \bar{E}-06$ & $1.11 \mathrm{E}-06$ \\
\hline Nevada Test Site & to INEL & $3.98 E-06$ & $1.58 \mathrm{E}-06$ \\
\hline Paducah GDP & to Oak Ridge Reserv. & $3.81 E-06$ & $1.10 \mathrm{E}-06$ \\
\hline Pantex Plant & to Los Alamos NL & $2.59 \mathrm{E}-06$ & $1.01 \mathrm{E}-06$ \\
\hline Pearl Harbor NS & to Hanford Site & $1.08 \mathrm{E}-05$ & $8.70 \mathrm{E}-07$ \\
\hline Portsmouth NS & to Portsmouth GDP & $2.69 E-05$ & $2.01 E-06$ \\
\hline Princeton PPI & to Portsmouth GDP & $3.18 \mathrm{E}-05$ & $1.75 E-06$ \\
\hline Puget Sound NS & to Hanford Site & $1.08 \mathrm{E}-05$ & $8.70 \mathrm{E}-07$ \\
\hline Reactive Metals & to Portsmouth GDP & $1.14 E-05$ & $5.84 E-07$ \\
\hline SNL - Albuquerque & to Los Alamos NL & $2.47 E-06$ & $2.18 E-07$ \\
\hline SNL - Livermore & to Hanford Site & $2.36 \mathrm{E}-05$ & $2.04 \mathrm{E}-06$ \\
\hline
\end{tabular}


Table 6. Annual Incident-Free Dose for the Shipping Campaign (person-rem/yr)

\begin{tabular}{|c|c|c|c|c|c|c|}
\hline \multirow{2}{*}{ Route } & & & \multicolumn{2}{|c|}{ Truck } & \multicolumn{2}{|c|}{ Rail } \\
\hline & & & Crew & Public & Crew & Public \\
\hline Ames Laboratory & to & Portsmouth GDP & $2.33 E-02$ & $2.63 \mathrm{E}-02$ & $5.91 \mathrm{E}-03$ & $2.01 \mathrm{E}-02$ \\
\hline Argonne - East & & Portsmouth GDP & $6.71 \mathrm{E}-01$ & $7.49 \mathrm{E}-01$ & $7.89 E-02$ & $1.98 \mathrm{E}-01$ \\
\hline Bettis Atomic & & Portsmouth GDP & $9.78 \mathrm{E}-03$ & $1.04 \mathrm{E}-02$ & $4.67 \varepsilon-03$ & $48 \mathrm{~F}-02$ \\
\hline Battelle columbus & to & Portsmouth GDP & $2.86 E-03$ & $3.17 E-03$ & $3.26 E-03$ & $22 E-0$ \\
\hline Brookhaven NL & to & Portsmouth GDP & $2.73 E-02$ & $2.99 \mathrm{E}-02$ & $6.72 \mathrm{E}-03$ & \\
\hline Charleston, SC & to & Savannah River & $6.19 \mathrm{E}-03$ & $6.77 \mathrm{E}-03$ & $3.47 \mathrm{E}-03$ & $5.00 E-03$ \\
\hline Colonie & & Portsmouth GDP & $2.54 \mathrm{E}-02$ & $2.63 \mathrm{E}-02$ & $5.72 \mathrm{E}-03$ & $13 E-02$ \\
\hline ETEC & to & INEL & $2.58 E-01$ & $3.26 E-01$ & $2.17 \mathrm{E}-02$ & $7.47 \mathrm{E}-02$ \\
\hline Fernald (FEMP) & to & Portsmouth GDP & $4.00 \mathrm{E}-02$ & $18 \mathrm{E}-02$ & $1.12 \mathrm{E}-02$ & $2.06 \mathrm{E}-02$ \\
\hline General Atomic & to & INEL & $3.06 \mathrm{E}-02$ & $3.64 \mathrm{E}-02$ & $7.37 \mathrm{E}-03$ & $18 \mathrm{E}-0$ \\
\hline Grand Junction PO & to & Rocky Flats Pla & $8.39 E-03$ & $9.92 \mathrm{E}-03$ & $3.88 E-03$ & \\
\hline Knolls Atomic & to & Portsmouth GDP & $2.54 \mathrm{E}-02$ & $2.63 \mathrm{E}-02$ & $5.72 E-03$ & $2.13 E-C$ \\
\hline Kansas City Plant & to & Rocky Flats Pla & $1.74 \mathrm{E}-02$ & $2.11 \mathrm{E}-02$ & $6.13 \mathrm{E}-03$ & 1. $07 \mathrm{E}-0$ \\
\hline KKS & to & Portsmouth GDP & $2.54 E-02$ & $2.63 E-02$ & $5.72 E-03$ & 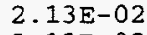 \\
\hline KWS & to & Portsmouth GDP & $2.54 E-02$ & $2.63 E-02$ & $5.72 E-03$ & $E-02$ \\
\hline Lawrence Berkeley & to & Fanford Site & $2.76 \mathrm{E}-02$ & $3.27 E-02$ & $5.99 E-03$ & $2.05 E-0$ \\
\hline LERHR & to & Hanford site & $2.51 E-02$ & $2.94 E-02$ & $6.59 E-03$ & $1.70 \mathrm{E}-02$ \\
\hline Lawrence Livermore & to & Hanford Site & $1.13 \mathrm{E}-01$ & $1.31 \mathrm{E}-01$ & I. $39 \mathrm{E}-02$ & $4.10 \mathrm{E}-$ \\
\hline Mare Island & to & Hanford site & $2.76 \mathrm{E}-02$ & $3.27 \mathrm{E}-02$ & $6.99 \mathrm{E}-03$ & $2.05 E-0$ \\
\hline Norfolk Nav Shipyd & to & Savannah River & $1.59 \mathrm{E}-02$ & $1.73 \mathrm{E}-02$ & $5.09 \mathrm{E}-03$ & $9.55 \mathrm{E}-\mathrm{C}$ \\
\hline Nevada Test Site & to & INEL & $2.13 E-02$ & $2.58 E-02$ & $5.04 \mathrm{E}-03$ & $8.67 \mathrm{E}-0$ \\
\hline Paducah GDP & to & Oak Ridge Reserv. & $8.88 E-03$ & $9.98 \mathrm{E}-03$ & $5.08 \mathrm{E}-03$ & $7.91 \mathrm{E}-$ \\
\hline Pantex Plant & to & Los Alamos NL & $2.23 \mathrm{E}-02$ & $2.76 E-02$ & $4.898-03$ & $7.19 E-C$ \\
\hline Pearl Harbor NS & to & Hanford Site & $7.06 \mathrm{E}-03$ & $8.04 \mathrm{E}-03$ & $4.62 \mathrm{E}-03$ & $1.15 \mathrm{E}-$ \\
\hline Portsmouth NS & to & Portsmouth GDP & $4.03 E-02$ & $4.36 \mathrm{E}-02$ & $6.89 \mathrm{E}-03$ & $2.78 \mathrm{E}-0$ \\
\hline Princeton PPL & to & Portsmouth GDP & $2.28 \mathrm{E}-02$ & $2.48 \mathrm{E}-02$ & $6.38 \mathrm{E}-03$ & $2.64 E-C$ \\
\hline Puget Sound NS & to & Hanford site & $7.06 \mathrm{E}-03$ & $8.04 \mathrm{E}-03$ & 4. $62 \mathrm{E}-03$ & 1. $15 \mathrm{E}-0$ \\
\hline Reactive Metals & to & Portsmouth GDP & $1.00 \mathrm{E}-02$ & $1.05 E-02$ & $4.05 E-03$ & 1.20E-02 \\
\hline SNL - Albuquerque & to & Los Alamos NL & $3.28 \mathrm{E}-03$ & $3.84 \mathrm{E}-03$ & $3.31 \mathrm{E}-03$ & $5.37 E-03$ \\
\hline SNL - Livermore & to & Hanford Site & $2.82 \mathrm{E}-02$ & $3.28 \mathrm{E}-02$ & $6.94 \mathrm{E}-03$ & $2.05 E-02$ \\
\hline & & & & & & \\
\hline
\end{tabular}

Table 7. Annual Accident Dose Risk for the Shipping Campaign (person-rem/yr)

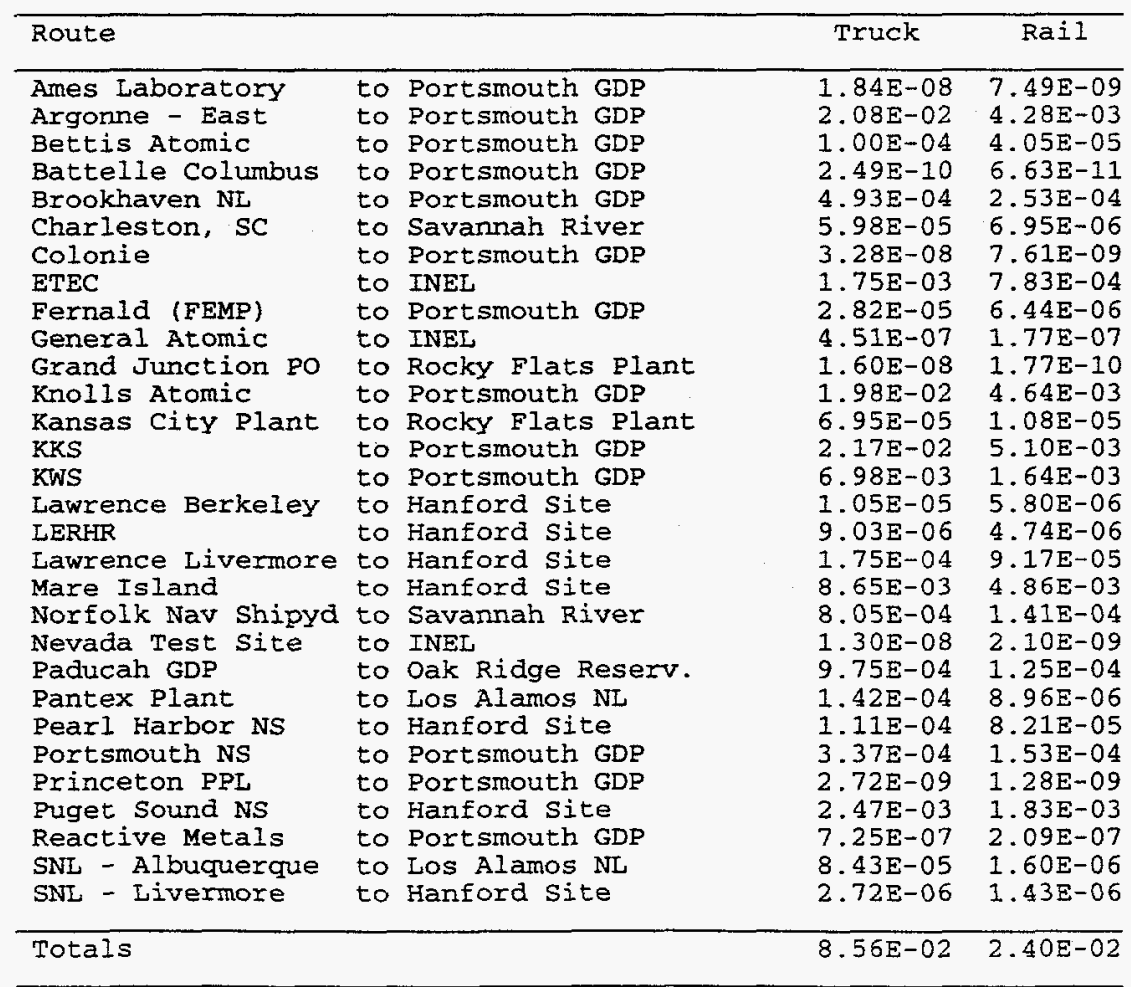


Table 8. Expected Annual Fatalities for the Shipping Campaign

\begin{tabular}{llc}
\hline Exposure Group & Truck & Rail \\
\hline Radiological & & \\
Normal Crew & $6.3 \mathrm{E}-04$ & $1.1 \mathrm{E}-04$ \\
$\quad$ Normal Public & $9.0 \mathrm{E}-04$ & $3.7 \mathrm{E}-04$ \\
Accident Public & $4.3 \mathrm{E}-05$ & $1.2 \mathrm{E}-05$ \\
Nonradiological & $7.1 \mathrm{E}-04$ & $7.2 \mathrm{E}-04$ \\
$\quad$ Emission & $2.8 \mathrm{E}-03$ & $6.1 \mathrm{E}-05$ \\
Accident & \\
\hline
\end{tabular}

Table 9. Expected Annual Cancer Incidence for the Shipping Campaign

\begin{tabular}{lll}
\hline Exposure Group & Truck & Rail \\
\hline Radiological & $2.2 \mathrm{E}-03$ & $3.8 \mathrm{E}-04$ \\
Normal Crew & $3.1 \mathrm{E}-03$ & $1.3 \mathrm{E}-03$ \\
Normal Public & $1.5 \mathrm{E}-04$ & $4.1 \mathrm{E}-05$ \\
Accident Public & $7.1 \mathrm{E}-04$ & $7.2 \mathrm{E}-04$ \\
Nonradiological & NA & NA \\
Emission & & \\
Accident & & \\
\hline
\end{tabular}

Table 10. Expected Annual Genetic Effects for the Shipping Campaign

\begin{tabular}{lll}
\hline Exposure Group & Truck & Rail \\
\hline Radiological & & \\
Normal Crew & $9.5 \mathrm{E}-05$ & $1.6 \mathrm{E}-05$ \\
Normal Public & $1.8 \mathrm{E}-04$ & $7.4 \mathrm{E}-05$ \\
Accident Public & $7.6 \mathrm{E}-06$ & $2.2 \mathrm{E}-06$ \\
Nonradiological & & \\
Emission & NA & NA \\
Accident & NA & NA \\
\hline
\end{tabular}




\section{B.20 WM LLMW REGIONALIZED 3 ALTERNATIVE (CASE 10a): CONTACT-HANDLED HETEROGENEOUS SOLIDS: ALPHA - UNTREATED}

Table 1. Sumary of Route Information

\begin{tabular}{|c|c|c|c|c|c|}
\hline & \multirow[b]{2}{*}{ Route } & \multirow{2}{*}{$\begin{array}{c}\text { Distance } \\
\text { (miles) }\end{array}$} & \multicolumn{3}{|c|}{ Percentage in Zone } \\
\hline & & & Rural & Suburban & Urban \\
\hline \multicolumn{6}{|l|}{ Truck } \\
\hline Bettis Atomic & to Savannah River & 656 & 71.7 & 26.4 & 1.9 \\
\hline Lawrence Livermore & to INEL & 972 & 85.7 & 10.4 & 4.0 \\
\hline Mound Plant & to Savannah River & 656 & 66.5 & 32.7 & 0.8 \\
\hline U of MO - Columbia & to Savannah River & 858 & 70.6 & 27.0 & 2.3 \\
\hline West Valley DP & to Savannah River & 883 & 70.3 & 28.5 & 1.2 \\
\hline \multicolumn{6}{|l|}{ Rail } \\
\hline Bettis Atomic & to Savannah River & 947 & 68.5 & 27.1 & 4.4 \\
\hline Lawrence Livermore & to INEI & 1100 & 89.5 & 7.4 & 3.1 \\
\hline Mound Plant & to Savannah River & 744 & 67.4 & 29.2 & 3.3 \\
\hline U of MO - Columbia & to Savannah River & 1011 & 66.6 & 29.5 & 4.0 \\
\hline West Valley DP & to Savannah River & 1217 & 62.8 & 32.4 & 4.9 \\
\hline
\end{tabular}

Table 2. Annual Number of Shipments and One-Way Shipment Distances

\begin{tabular}{|c|c|c|c|c|c|}
\hline \multirow[b]{2}{*}{ Route } & & \multicolumn{2}{|c|}{ \# of Shipments } & \multicolumn{2}{|c|}{ Total Mileage (mi) } \\
\hline & & Truck & Rail & Truck & Rail \\
\hline $\begin{array}{l}\text { Bettis Atomic } \\
\text { Lawrence Livermore } \\
\text { Mound Plant } \\
\text { U of MO - Columbia } \\
\text { West Valley DP }\end{array}$ & $\begin{array}{ll}\text { to Savannah River } \\
\text { to INEL } \\
\text { to Savannah River } \\
\text { to Savannah River } \\
\text { to Savannah River }\end{array}$ & $\begin{array}{l}1 \\
6 \\
1 \\
1 \\
1\end{array}$ & $\begin{array}{l}1 \\
3 \\
1 \\
1 \\
1\end{array}$ & $\begin{array}{l}6.56 \mathrm{E}+02 \\
5.83 \mathrm{E}+03 \\
6.56 \mathrm{E}+02 \\
8.58 \mathrm{E}+02 \\
8.83 \mathrm{E}+02\end{array}$ & $\begin{array}{l}9.47 \mathrm{E}+02 \\
3.30 \mathrm{E}+03 \\
7.44 \mathrm{E}+02 \\
1.01 \mathrm{E}+03 \\
1.22 \mathrm{E}+03\end{array}$ \\
\hline Totals & & 10 & 7 & $8.88 \mathrm{E}+03$ & $7.22 \mathrm{E}+03$ \\
\hline
\end{tabular}

Table 3. Incident-Free Dose per Shipment (person-rem/shipment)

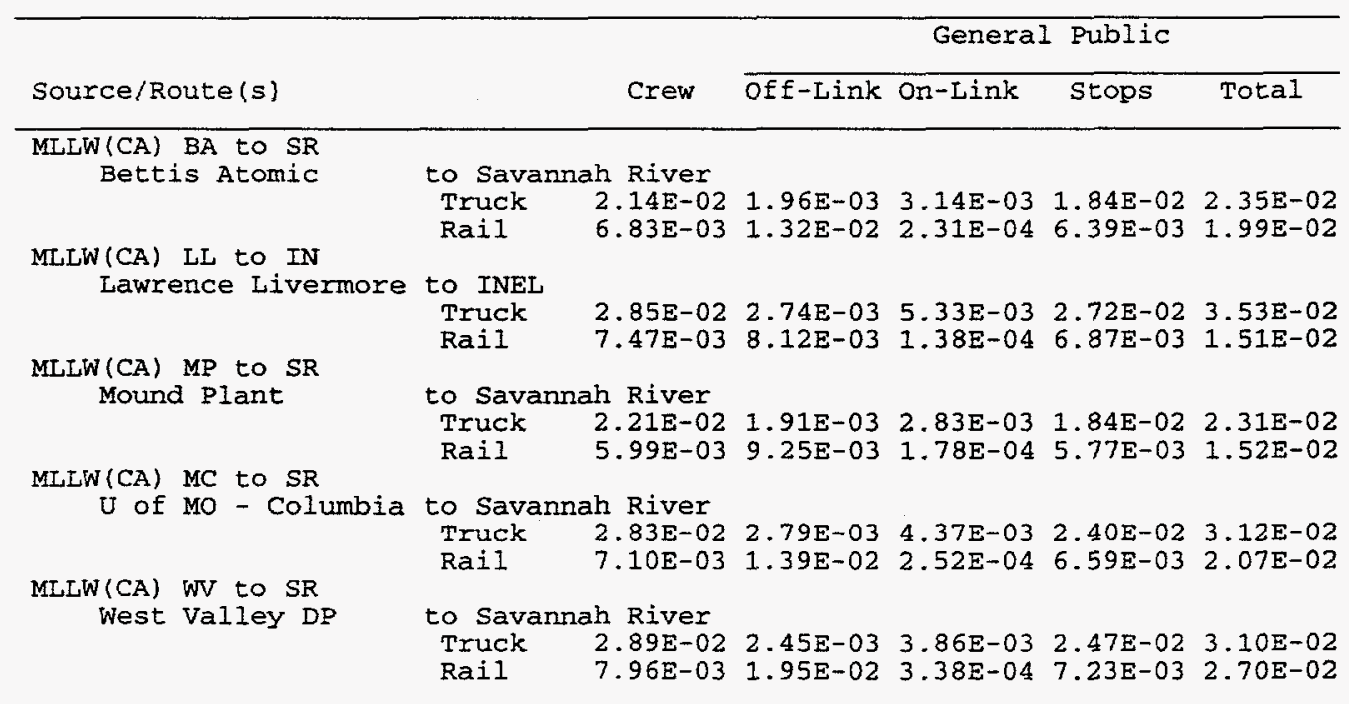


Table 4. Accident Dose Risk per Shipment (person-rem/shipment)

\begin{tabular}{llll}
\hline Source/Route & & Truck & Rail \\
\hline $\begin{array}{c}\text { MLLW(CA) BA to SR } \\
\text { Bettis Atomic }\end{array}$ & to Savannah River & $2.11 \mathrm{E}-04$ & $7.45 \mathrm{E}-05$ \\
$\begin{array}{c}\text { MLLW(CA) LL to IN } \\
\text { Lawrence Livermore to INEL }\end{array}$ & $5.14 \mathrm{E}-05$ & $3.46 \mathrm{E}-05$ \\
$\begin{array}{l}\text { MLLW(CA) MP to SR } \\
\text { Mound Plant }\end{array}$ & to Savannah River & $9.48 \mathrm{E}-08$ & $1.85 \mathrm{E}-08$ \\
$\begin{array}{c}\text { MLLW (CA) MC to SR } \\
\text { U Of MO - Columbia to Savannah River }\end{array}$ & $5.78 \mathrm{E}-07$ & $2.05 \mathrm{E}-07$ \\
$\begin{array}{c}\text { MLLW (CA) WV to SR } \\
\text { West Valley DP }\end{array}$ & to Savannah River & $3.36 \mathrm{E}-04$ & $1.07 \mathrm{E}-04$ \\
\hline
\end{tabular}

Table 5. Nonradiological Risk Factors per Shipment (fatalities/shipment)

\begin{tabular}{llll}
\hline Mode & Route & Emission & Accident \\
\hline Truck & & & \\
Bettis Atomic & to Savannah River & $3.99 \mathrm{E}-06$ & $4.90 \mathrm{E}-05$ \\
Lawrence Livermore & to INEL & $1.24 \mathrm{E}-05$ & $5.73 \mathrm{E}-05$ \\
Mound Plant & to Savannah River & $1.67 \mathrm{E}-06$ & $5.26 \mathrm{E}-05$ \\
U of MO - Columbia to Savannah River & $6.41 \mathrm{E}-06$ & $6.09 \mathrm{E}-05$ \\
West Valley DP & to Savannah River & $3.28 \mathrm{E}-06$ & $6.60 \mathrm{E}-05$ \\
Rail & & & \\
Bettis Atomic & to Savannah River & $1.74 \mathrm{E}-05$ & $1.98 \mathrm{E}-06$ \\
Lawrence Iivermore to INEL & $1.41 \mathrm{E}-05$ & $2.30 \mathrm{E}-06$ \\
Mound Plant & to Savannah River & $1.03 \mathrm{E}-05$ & $1.56 \mathrm{E}-06$ \\
U of MO - Columbia to Savannah River & $1.69 \mathrm{E}-05$ & $2.12 \mathrm{E}-06$ \\
West Valley DP & to Savannah River & $2.49 \mathrm{E}-05$ & $2.55 \mathrm{E}-06$ \\
\hline
\end{tabular}

Table 6. Annual Incident-Free Dose for the Shipping Campaign (person-rem/yr)

\begin{tabular}{|c|c|c|c|c|c|}
\hline \multirow[b]{2}{*}{ Route } & & \multicolumn{2}{|c|}{ Truck } & \multicolumn{2}{|c|}{ Rail } \\
\hline & & Crew & Public & Crew & Public \\
\hline $\begin{array}{l}\text { Bettis Atomic } \\
\text { Lawrence Livermore } \\
\text { Mound Plant } \\
U \text { of Mo-Columbia } \\
\text { West Valley DP }\end{array}$ & $\begin{array}{l}\text { to Savannah River } \\
\text { to INEI } \\
\text { to Savannah River } \\
\text { to Savannah River } \\
\text { to Savannah River }\end{array}$ & $\begin{array}{l}2.14 \mathrm{E}-02 \\
1.71 \mathrm{E}-01 \\
2.21 \mathrm{E}-02 \\
2.83 \mathrm{E}-02 \\
2.89 \mathrm{E}-02\end{array}$ & $\begin{array}{l}2.35 \mathrm{E}-02 \\
2.12 \mathrm{E}-01 \\
2.31 \mathrm{E}-02 \\
3.12 \mathrm{E}-02 \\
3.10 \mathrm{E}-02\end{array}$ & $\begin{array}{l}6.83 \mathrm{E}-03 \\
2.24 \mathrm{E}-02 \\
5.99 \mathrm{E}-03 \\
7.10 \mathrm{E}-03 \\
7.96 \mathrm{E}-03\end{array}$ & $\begin{array}{l}1.99 \mathrm{E}-02 \\
4.54 \mathrm{E}-02 \\
1.52 \mathrm{E}-02 \\
2.07 \mathrm{E}-02 \\
2.70 \mathrm{E}-02\end{array}$ \\
\hline Tot & & 01 & .01 & $5.03 E-02$ & 1.2 \\
\hline
\end{tabular}

Table 7. Annual Accident Dose Risk for the Shipping Campaign (person-rem/yr)

\begin{tabular}{llcc}
\hline Route & & Truck & Rail \\
\hline Bettis Atomic & to Savannah River & $2.11 \mathrm{E}-04$ & $7.45 \mathrm{E}-05$ \\
Lawrence Livermore & to INEL & $3.09 \mathrm{E}-04$ & $1.04 \mathrm{E}-04$ \\
Mound Plant & to Savannah River & $9.48 \mathrm{E}-08$ & $1.85 \mathrm{E}-08$ \\
U of MO - Columbia & to Savannah River & $6.78 \mathrm{E}-07$ & $2.05 \mathrm{E}-07$ \\
West Valley DP & to Savannah River & $3.36 \mathrm{E}-04$ & $1.07 \mathrm{E}-04$ \\
\hline Totals & & $8.56 \mathrm{E}-04$ & $2.86 \mathrm{E}-04$ \\
\hline
\end{tabular}

Table 8. Expected Annual Fatalities for the Shipping Campaign

\begin{tabular}{lll}
\hline Exposure Group & Truck & Rail \\
\hline Radiological & & \\
Normal Crew & $1.1 \mathrm{E}-04$ & $2.0 \mathrm{E}-05$ \\
Normal Public & $1.6 \mathrm{E}-04$ & $6.4 \mathrm{E}-05$ \\
Accident Public & $4.3 \mathrm{E}-07$ & $1.4 \mathrm{E}-07$ \\
Nonradiological & $9.0 \mathrm{E}-05$ & $1.1 \mathrm{E}-04$ \\
Enission & $5.7 \mathrm{E}-04$ & $1.5 \mathrm{E}-05$ \\
Accident & \\
\hline
\end{tabular}


Table 9. Expected Annual Cancer Incidence for the Shipping Campaign

\begin{tabular}{lll}
\hline Exposure Group & Truck & Rail \\
\hline Radiological & & \\
Normal Crew & $3.8 E-04$ & $7.0 \mathrm{E}-05$ \\
Normal Public & $5.4 \mathrm{E}-04$ & $2.2 \mathrm{E}-04$ \\
Accident Public & $1.5 \mathrm{E}-06$ & $4.9 \mathrm{E}-07$ \\
Nonradiological & $9.0 \mathrm{E}-05$ & $1.1 \mathrm{E}-04$ \\
Emission & $\mathrm{NA}$ & $\mathrm{NA}$ \\
\hline Accident & & \\
\hline
\end{tabular}

Table 10. Expected Annual Genetic Effects for the Shipping Campaign

\begin{tabular}{lll}
\hline Exposure Group & Truck & Rail \\
\hline Radiological & & \\
Normal Crew & $1.6 \mathrm{E}-05$ & $3.0 \mathrm{E}-06$ \\
Normal Public & $3.2 \mathrm{E}-05$ & $1.3 \mathrm{E}-05$ \\
Accident Public & $5.2 \mathrm{E}-08$ & $1.7 \mathrm{E}-08$ \\
Nonradiological & $\mathrm{NA}$ & $\mathrm{NA}$ \\
Emission & $\mathrm{NA}$ & $\mathrm{NA}$ \\
Accident & & \\
\hline
\end{tabular}


B.21 WM LLMW REGIONALIZED 3 ALTERNATIVE (CASE 10a): CONTACT-HANDLED HETEROGENEOUS SOLIDS: NONALPHA - TREATED

Table 1. Summary of Route Information

\begin{tabular}{|c|c|c|c|c|c|c|c|c|}
\hline & \multirow{2}{*}{\multicolumn{2}{|c|}{ Route }} & & & \multirow{2}{*}{$\begin{array}{c}\text { Distance } \\
\text { (miles) }\end{array}$} & \multicolumn{3}{|c|}{ Percentage in Zone } \\
\hline & & & & & & Rura 1 & Suburban & Urban \\
\hline \multicolumn{9}{|l|}{ Truck } \\
\hline Ames Laboratory & to & Nevada & Test & Site & 1520 & 92.0 & 6.8 & 1.2 \\
\hline Argonne - East & to & Nevada & Test & Site & 1815 & 91.0 & 8.0 & 1.0 \\
\hline Charleston, SC & to & Nevada & Test & Site & 2545 & 84.4 & 14.0 & 1.6 \\
\hline Colonie & to & Nevada & Test & Site & 407 & 76.9 & 18.3 & 4.8 \\
\hline Fernald (FEMP) & to & Nevada & Test & Site & 2014 & 86.9 & 11.5 & 1.6 \\
\hline General Atomic & to & Nevada & Test & Site & 398 & 73.9 & 19.8 & 6.3 \\
\hline Grand Junction Po & to & Nevada & Test & Site & 573 & 93.1 & 5.3 & 1.7 \\
\hline Hanford Site & to & Nevada & Test & Site & 1128 & 86.5 & 10.9 & 2.6 \\
\hline INEL & to & Nevada & Test & Site & 712 & 82.8 & 13.7 & 3.5 \\
\hline Los Alamos NL & to & Nevada & Test & Site & 997 & 93.2 & 5.7 & 1.1 \\
\hline LERHR & to & Nevada & Test & Site & 735 & 81.1 & 11.2 & 7.6 \\
\hline Oak Ridge Reserv. & to & Nevada & Test & Site & 2151 & 86.9 & 11.5 & 1.6 \\
\hline Paducah GDP & to & Nevada & Test & site & 1864 & 87.6 & 10.7 & 1.8 \\
\hline Pantex Plant & to & Nevada & Test & Site & 1209 & 93.9 & 4.6 & 1.6 \\
\hline Portsmouth GDP & to & Nevada & Test & Site & 2158 & 85.5 & 12.6 & 1.9 \\
\hline Rocky Flats plant & to & Nevada & Test & site & 836 & 89.5 & 8.5 & 2.0 \\
\hline Reactive Metals & to & Nevada & Test & Site & 2214 & 85.8 & 12.2 & 2.0 \\
\hline Savannah River & to & Nevada & Test & Site & 2414 & 83.1 & 15.1 & 1.8 \\
\hline \multicolumn{9}{|l|}{ Rail } \\
\hline Ames Laboratory & to & Nevada & Test & Site & 1674 & 94.0 & 5.1 & 0.9 \\
\hline Argonne - East & to & Nevada & Test & Site & 2348 & 92.8 & 6.4 & 0.8 \\
\hline Charleston, SC & to & Nevada & Test & Site & 2945 & 84.3 & 13.7 & 2.0 \\
\hline Colonie & to & Nevada & Test & Site & 518 & 73.4 & 15.9 & 10.7 \\
\hline Fernald (FEMP) & to & Nevada & Test & Site & 2391 & 85.4 & 11.4 & 3.1 \\
\hline General Atomic & to & Nevada & Test & Site & 518 & 73.4 & 15.9 & 10.7 \\
\hline Grand Junction po & to & Nevada & Test & Site & 749 & 96.0 & 3.8 & 0.2 \\
\hline Hanford Site & to & Nevada & Test & site & 1302 & 93.0 & 5.9 & 1.1 \\
\hline INEL & to & Nevada & Test & Site & 756 & 92.8 & 5.9 & $1 . \overline{3}$ \\
\hline Los Alamos NL & to & Nevada & Test & Site & 1169 & 92.8 & 5.9 & 1.3 \\
\hline LERHR & to & Nevada & Test & Site & 827 & 75.4 & 17.7 & 6.9 \\
\hline Oak Ridge Reserv. & to & Nevada & Test & Site & 2487 & 91.4 & 7.2 & 1.5 \\
\hline Paducah GDP & to & Nevada & Test & Site & 2131 & 90.9 & 7.6 & 1.5 \\
\hline Pantex Plant & to & Nevada & Test & Site & 1376 & 94.9 & 4.4 & 0.7 \\
\hline Portsmouth GDP & to & Nevada & Test & Site & 2401 & 86.3 & 10.7 & 2.9 \\
\hline Rocky Flats Plant & to & Nevada & Test & Site & 987 & 95.9 & 3.9 & 0.2 \\
\hline Reactive Metals & to & Nevada & Test & Site & 2391 & 83.1 & 13.1 & 3.7 \\
\hline Savannah River & to & Nevada & Test & site & 2839 & 84.5 & 13.5 & 1.9 \\
\hline
\end{tabular}

Table 2. Annual Number of Shipments and One-way Shipment Distances

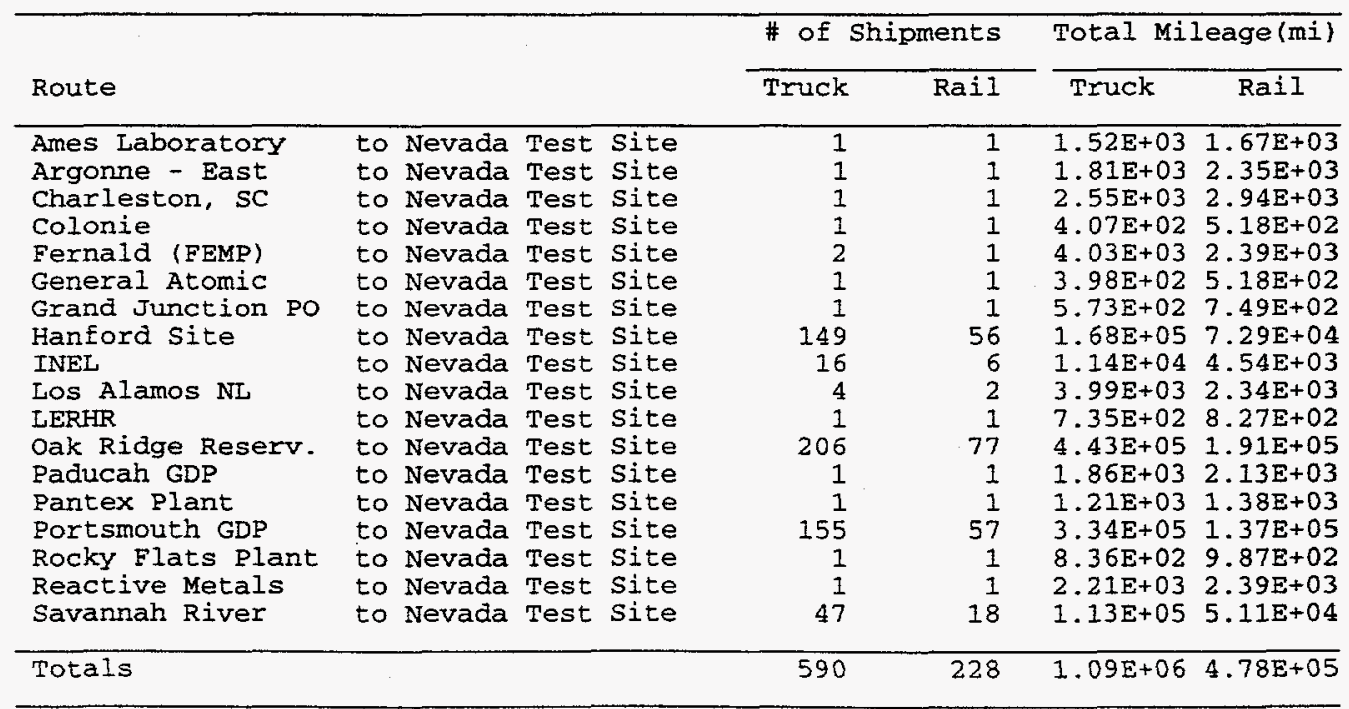


Table 3. Incident-Free Dose per Shipment (person-rem/shipment)

\begin{tabular}{|c|c|c|c|c|c|c|}
\hline \multirow[b]{2}{*}{ Source/Route (s) } & & \multirow[b]{2}{*}{ Crew } & \multicolumn{4}{|c|}{ General Public } \\
\hline & & & off-tink & on-Link & Stops & Total \\
\hline \multicolumn{7}{|l|}{ MLLW (CT) AL to NT } \\
\hline \multirow[t]{3}{*}{ Ames Laboratory } & to Nevada & \multicolumn{5}{|l|}{ Test site } \\
\hline & Truck & $\begin{array}{l}4.03 \mathrm{E}-02 \\
9.87 \mathrm{E}-03\end{array}$ & $\begin{array}{l}1.78 \mathrm{E}-03 \\
4.68 \mathrm{E}-03\end{array}$ & $\begin{array}{l}5.25 \mathrm{E}-03 \\
1.36 \mathrm{E}-04\end{array}$ & $\begin{array}{l}4.25 E-02 \\
8.63 E-03\end{array}$ & $\begin{array}{l}4.96 \mathrm{E}-02 \\
1.34 \mathrm{E}-02\end{array}$ \\
\hline & \multicolumn{6}{|c|}{ MLLW(CT) AN to NT } \\
\hline \multirow{2}{*}{ Argonne - East } & to Nevada & \multicolumn{2}{|l|}{ Test Site } & & & \\
\hline & $\begin{array}{l}\text { Truck } \\
\text { Rail }\end{array}$ & $\begin{array}{l}4.86 \mathrm{E}-02 \\
1.27 \mathrm{E}-02\end{array}$ & $\begin{array}{l}2.13 E-03 \\
6.96 E-03\end{array}$ & $\begin{array}{l}6.15 E-03 \\
2.04 E-04\end{array}$ & $\begin{array}{l}5.08 E-02 \\
1.07 E-02\end{array}$ & $\begin{array}{l}5.91 E-02 \\
1.79 E-02\end{array}$ \\
\hline \multirow{2}{*}{\multicolumn{2}{|c|}{$\begin{array}{l}\text { MLLW(CT) CH to NT } \\
\text { Charleston, SC }\end{array}$}} & \multirow{2}{*}{\multicolumn{2}{|c|}{ Test Site }} & & & \\
\hline & & & & & & \\
\hline & Truck & $7.34 \mathrm{E}-02$ & $4.87 E-03$ & $1.03 \mathrm{E}-02$ & $7.13 \mathrm{E}-02$ & $8.64 \mathrm{E}-02$ \\
\hline & Rail & 1. $52 \mathrm{E}-02$ & 1. $96 \mathrm{E}-02$ & $4.14 \mathrm{E}-04$ & $1.25 \mathrm{E}-02$ & $3.25 \mathrm{E}-02$ \\
\hline \multicolumn{7}{|l|}{ MLLW (CT) CL to NT } \\
\hline & $\begin{array}{l}\text { to Nevada } \\
\text { Truck }\end{array}$ & 1.31E-02 & $1.56 \mathrm{E}-03$ & $2.61 E-03$ & $1.14 \mathrm{E}-02$ & $1.56 \mathrm{E}-02$ \\
\hline & Rail & $5.04 \mathrm{E}-03$ & $1.22 \mathrm{E}-02$ & 1.451 & $5.07 \mathrm{E}-03$ & 1.7 \\
\hline \multirow{2}{*}{\multicolumn{7}{|c|}{$\begin{array}{rll}M L L W(C T) & F E & \text { to } N T \\
\text { Fernald } & \text { (FEMP) }\end{array}$}} \\
\hline & & & & & & \\
\hline & Truck & $5.67 \mathrm{E}-02$ & $3.50 E-03$ & $7.96 \mathrm{E}-03$ & $5.64 E-02$ & $6.78 \mathrm{E}-02$ \\
\hline \multirow{2}{*}{\multicolumn{7}{|c|}{ 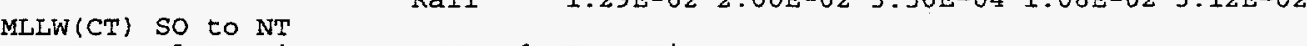 }} \\
\hline \multirow{4}{*}{ General Atomic } & to Nevada & Test site & & & & \\
\hline & Truck & $1.33 \mathrm{E}-02$ & $1.88 \mathrm{E}-03$ & $2.99 \mathrm{E}-03$ & $1.11 \mathrm{E}-02$ & $1.60 \mathrm{E}$ \\
\hline & Rail & $5.04 \mathrm{E}-03$ & $1.22 \mathrm{E}-02$ & 1.4 & $5.07 \mathrm{E}-03$ & $1.74 E-02$ \\
\hline & & & & & & \\
\hline Grand Junction PO & to Nevada & Test Site & & & & \\
\hline & $\begin{array}{l}\text { Truck } \\
\text { Rail }\end{array}$ & $\begin{array}{l}1.51 \mathrm{E}-02 \\
6.01 \mathrm{E}-03\end{array}$ & $\begin{array}{l}7.35 \mathrm{E}-04 \\
9.43 \mathrm{E}-04\end{array}$ & $\begin{array}{l}2.12 \mathrm{E}-03 \\
4.85 \mathrm{E}-05\end{array}$ & $\begin{array}{l}1.60 \mathrm{E}-02 \\
5.78 \mathrm{E}-03\end{array}$ & $\begin{array}{l}1.89 \mathrm{E}-02 \\
6.78 \mathrm{E}-03\end{array}$ \\
\hline MLLW(CT) HS to NT & & & & & & \\
\hline Hanford Site & to Nevada & Test Site & & & & \\
\hline & Truck & $3.22 \mathrm{E}-02$ & $2.46 E-03$ & $5.18 \mathrm{E}-03$ & $3.16 \mathrm{E}-02$ & $3.92 \mathrm{E}-02$ \\
\hline & Rail & $8.31 \mathrm{E}-03$ & $4.39 E-03$ & $1.15 \mathrm{E}-04$ & $7.49 \mathrm{E}-03$ & $1.20 \mathrm{E}-02$ \\
\hline $\begin{array}{c}\text { MLLW(CT) IN to NT } \\
\text { INEL }\end{array}$ & & & & & & \\
\hline INEL & to Nevada & Test Site & & & & \\
\hline & Rail & $6.04 \mathrm{E}-03$ & $2.80 \pm-03$ & $6.88 \mathrm{E}-05$ & $5.81 \mathrm{E}-03$ & $8.67 \mathrm{E}-03$ \\
\hline MLLW (CT) LA to NT & & & & & & \\
\hline Los Alamos NL & to Nevada & Test Site & & & & \\
\hline & Truck & $2.61 \mathrm{E}-02$ & $1.04 \mathrm{E}-03$ & $3.33 E-03$ & $2.79 E-02$ & $3.23 \mathrm{E}-02$ \\
\hline & Rail & $7.76 \mathrm{E}-03$ & $4.40 E-03$ & 1.07 & $7.08 \mathrm{E}-03$ & $1.16 \mathrm{E}-02$ \\
\hline SM to NT & & & & & & \\
\hline & $\begin{array}{l}\text { to Nevada } \\
\text { Truck }\end{array}$ & Test Site & & & & \\
\hline & Rail & $6.33 \mathrm{E}-03$ & $1.38 \mathrm{E}-02$ & $1.93 \mathrm{E}-04$ & $6.02 \mathrm{E}-03$ & $2.00 \mathrm{E}-02$ \\
\hline $\operatorname{MLLW}(C T)$ OR to NT & & & & & & \\
\hline Oak Ridge Reserv. & to Nevada & Test Site & & & & \\
\hline & Truck & $\begin{array}{l}6.04 \mathrm{E}-02 \\
1.33 \mathrm{E}-02\end{array}$ & $\begin{array}{l}3.66 \mathrm{E}-03 \\
1.08 \mathrm{E}-02\end{array}$ & $\begin{array}{l}8.38 \mathrm{E}-03 \\
2.50 \mathrm{E}-04\end{array}$ & $\begin{array}{l}6.02 E-02 \\
1.11 E-02\end{array}$ & $\begin{array}{l}7.22 \mathrm{E}-02 \\
2.22 \mathrm{E}-02\end{array}$ \\
\hline$M L L W(C T)$ PA to NT & & & & & & \\
\hline Paducah GDP & to Nevada & Test Site & & & & \\
\hline & Tru & $5.22 \mathrm{E}-02$ & $3.26 \mathrm{E}-03$ & $7.47 \mathrm{E}-03$ & $5.22 \mathrm{E}-02$ & $6.29 \mathrm{E}-02$ \\
\hline & & 1.18 & $9.42 \mathrm{E}-03$ & $2.18 \mathrm{E}-04$ & $1.00 \mathrm{E}-02$ & $1.97 \mathrm{E}-02$ \\
\hline$M L L W(C T)$ PP to NT & & & & & & \\
\hline Pantex Plant & to Nevada & Test Site & & & & \\
\hline & $\begin{array}{l}\text { Truck } \\
\text { Rail }\end{array}$ & $\begin{array}{l}3.16 \mathrm{E}-02 \\
8.62 \mathrm{E}-03\end{array}$ & $\begin{array}{l}1.43 \mathrm{E}-03 \\
3.19 \mathrm{E}-03\end{array}$ & $\begin{array}{l}4.34 \mathrm{E}-03 \\
1.03 \mathrm{E}-04\end{array}$ & $\begin{array}{l}3.38 \mathrm{E}-02 \\
7.72 \mathrm{E}-03\end{array}$ & $\begin{array}{l}3.96 \mathrm{E}-02 \\
1.10 \mathrm{E}-02\end{array}$ \\
\hline MLLW (CT) PO to NT & & & & & & \\
\hline Portsmouth GDP & to Nevada & Test Site & & & & \\
\hline & Truck & $6.17 \mathrm{E}-02$ & $4.18 \mathrm{E}-03$ & $8.96 \mathrm{E}-03$ & $6.04 \mathrm{E}-02$ & $7.36 \mathrm{E}-02$ \\
\hline$M L L W(C T) \quad R F$ to $\mathrm{NT}$ & & & 1. & 3 & 1 & 3 \\
\hline Rocky Flats Plant & to Nevada & Test Site & & & & \\
\hline & $\operatorname{Tr}$ & $2.30 \mathrm{E}-02$ & $1.44 \mathrm{E}-03$ & $3.43 \mathrm{E}-03$ & $2.34 \mathrm{E}-02$ & $2.83 E-02$ \\
\hline & & -03 & $1.29 E-03$ & $6.47 \mathrm{E}-05$ & $6.52 E-03$ & $7.88 \mathrm{E}-03$ \\
\hline $\begin{array}{l}\text { MLLW(CT) RM to NT } \\
\text { Reactive Metals }\end{array}$ & & & & & & \\
\hline Lals & to Nevada & 6 T38-0? & & & & \\
\hline & Rail & $1.29 \mathrm{E}-02$ & $\begin{array}{l}9.39 E-03 \\
2.35 \mathrm{E}-02\end{array}$ & $3.91 E-04$ & $1.08 \mathrm{E}-02$ & $\begin{array}{l}1.58 \mathrm{E}-02 \\
3.47 \mathrm{E}-02\end{array}$ \\
\hline MLLW(CT) SR to NT & & & & & & \\
\hline Savannah River & to Nevada & Test Site & & & & \\
\hline & $\begin{array}{l}\text { Truck } \\
\text { Rail }\end{array}$ & $\begin{array}{l}7.07 \mathrm{E}-02 \\
1.47 \mathrm{E}-02\end{array}$ & $\begin{array}{l}5.10 E-03 \\
1.85 E-02\end{array}$ & $\begin{array}{l}1.03 E-02 \\
3.95 E-04\end{array}$ & $\begin{array}{l}6.76 \mathrm{E}-02 \\
1.22 \mathrm{E}-02\end{array}$ & $\begin{array}{l}8.30 E-02 \\
3.11 E-02\end{array}$ \\
\hline
\end{tabular}


Table 4. Accident Dose Risk per Shipment (person-rem/shipment)

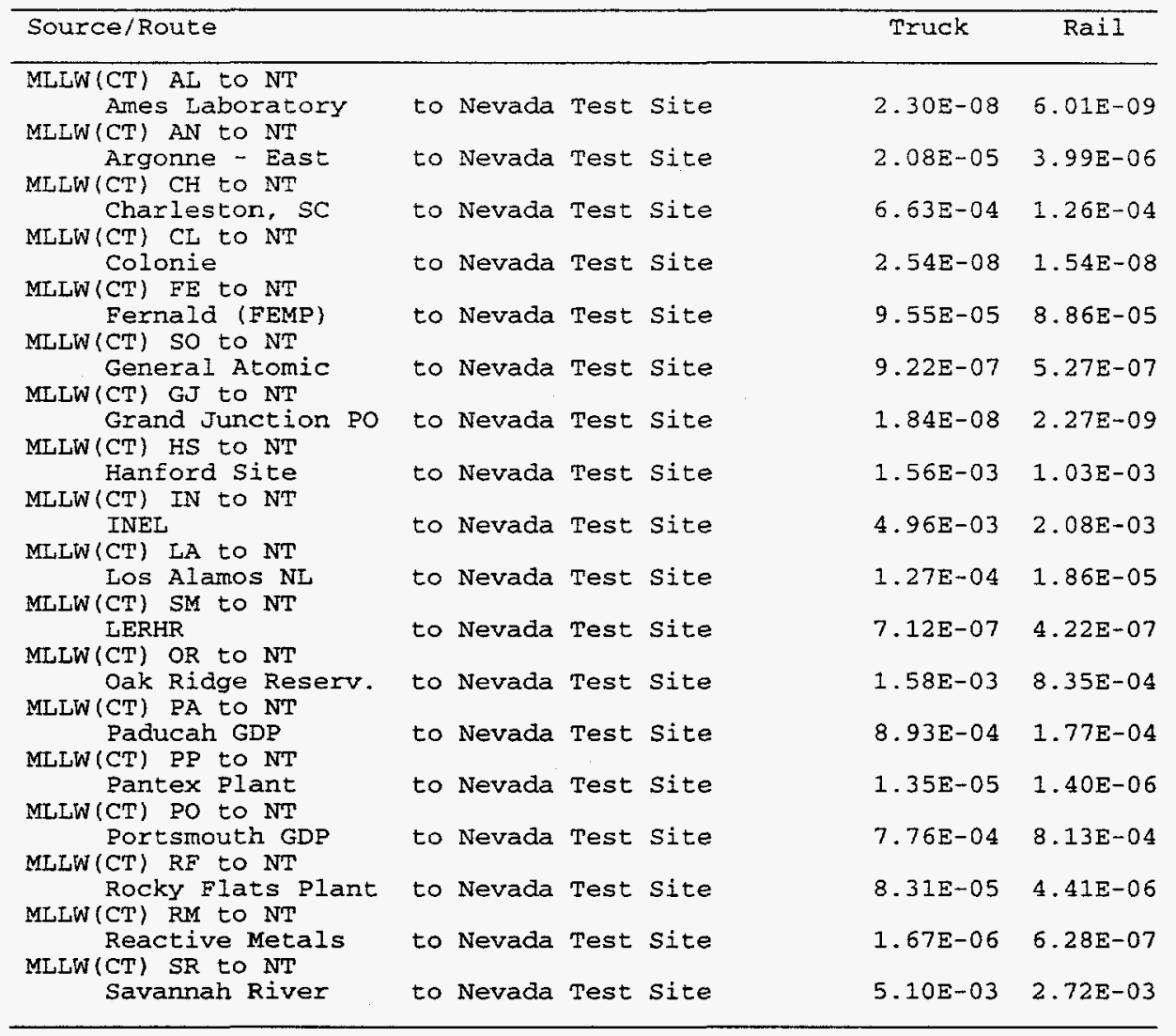


Table 5. Nonradiological Risk Factors per shipment (fatalities/shipment)

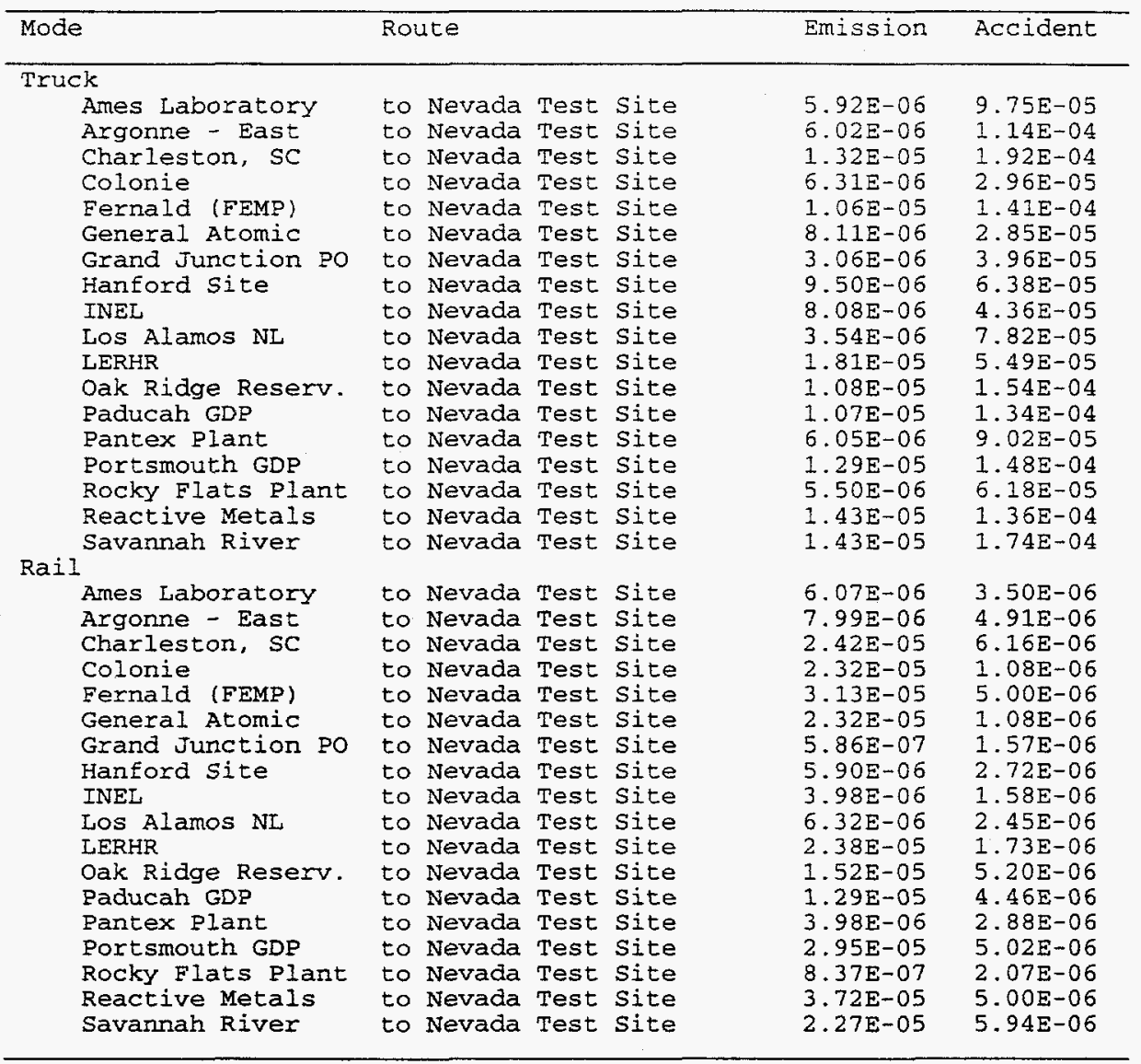

Table 6. Annual Incident-Free Dose for the Shipping Campaign (person-rem/Yr)

\begin{tabular}{|c|c|c|c|c|c|c|c|}
\hline \multirow[b]{2}{*}{ Route } & & & & \multicolumn{2}{|c|}{ Truck } & \multicolumn{2}{|c|}{ Rail } \\
\hline & & & & Crew & Public & Crew & Public \\
\hline $\begin{array}{l}\text { Ames Laboratory } \\
\text { Argonne - East } \\
\text { Charleston, SC } \\
\text { Colonie } \\
\text { Fernald (FEMP) } \\
\text { General Atomic } \\
\text { Grand Junction Po } \\
\text { Hanford Site } \\
\text { INEL } \\
\text { Los Alamos NL } \\
\text { LERHR } \\
\text { Oak Ridge Reserv. } \\
\text { Paducah GDP } \\
\text { Pantex Plant } \\
\text { Portsmouth GDP } \\
\text { Rocky Flats Plant } \\
\text { Reactive Metals } \\
\text { Savannah River }\end{array}$ & $\begin{array}{l}\text { to } \\
\text { to } \\
\text { to } \\
\text { to } \\
\text { to } \\
\text { to } \\
\text { to } \\
\text { to } \\
\text { to } \\
\text { to } \\
\text { to } \\
\text { to } \\
\text { to } \\
\text { to } \\
\text { to } \\
\text { to } \\
\text { to } \\
\text { to }\end{array}$ & $\begin{array}{l}\text { Nevada } \\
\text { Nevada } \\
\text { Nevada } \\
\text { Nevada } \\
\text { Nevada } \\
\text { Nevada } \\
\text { Nevada } \\
\text { Nevada } \\
\text { Nevada } \\
\text { Nevada } \\
\text { Nevada } \\
\text { Nevada } \\
\text { Nevada } \\
\text { Nevada } \\
\text { Nevada } \\
\text { Nevada } \\
\text { Nevada } \\
\text { Nevada }\end{array}$ & $\begin{array}{l}\text { Test Site } \\
\text { Test Site } \\
\text { Test Site } \\
\text { Test Site } \\
\text { Test Site } \\
\text { Test Site } \\
\text { Test Site } \\
\text { Test Site } \\
\text { Test Site } \\
\text { Test Site } \\
\text { Test Site } \\
\text { Test Site } \\
\text { Test Site } \\
\text { Test Site } \\
\text { Test Site } \\
\text { Test Site } \\
\text { Test Site } \\
\text { Test Site }\end{array}$ & $\begin{array}{l}4.03 E-02 \\
4.86 E-02 \\
7.34 E-02 \\
1.31 E-02 \\
1.13 E-01 \\
1.33 E-02 \\
1.51 E-02 \\
4.80 E+00 \\
3.41 E-01 \\
1.04 E-01 \\
2.34 E-02 \\
1.24 E+01 \\
5.22 E-02 \\
3.16 E-02 \\
9.57 E+00 \\
2.30 E-02 \\
6.33 E-02 \\
3.32 E+00\end{array}$ & $\begin{array}{l}4.96 \mathrm{E}-02 \\
5.91 \mathrm{E}-02 \\
8.64 \mathrm{E}-02 \\
1.56 \mathrm{E}-02 \\
1.36 \mathrm{E}-01 \\
1.60 \mathrm{E}-02 \\
1.89 \mathrm{E}-02 \\
5.84 \mathrm{E}+00 \\
4.12 \mathrm{E}-01 \\
1.29 \mathrm{E}-01 \\
2.99 \mathrm{E}-02 \\
1.49 \mathrm{E}+01 \\
6.29 \mathrm{E}-02 \\
3.96 \mathrm{E}-02 \\
1.14 \mathrm{E}+01 \\
2.83 \mathrm{E}-02 \\
7.58 \mathrm{E}-02 \\
3.90 \mathrm{E}+00\end{array}$ & $\begin{array}{l}9.87 \mathrm{E}-03 \\
1.27 \mathrm{E}-02 \\
1.52 \mathrm{E}-02 \\
5.04 \mathrm{E}-03 \\
1.29 \mathrm{E}-02 \\
5.04 \mathrm{E}-03 \\
6.01 \mathrm{E}-03 \\
4.66 \mathrm{E}-01 \\
3.62 \mathrm{E}-02 \\
1.55 \mathrm{E}-02 \\
6.33 \mathrm{E}-03 \\
1.02 \mathrm{E}-00 \\
1.18 \mathrm{E}-02 \\
8.62 \mathrm{E}-03 \\
7.35 \mathrm{E}-01 \\
7.00 \mathrm{E}-03 \\
1.29 \mathrm{E}-02 \\
2.65 \mathrm{E}-01\end{array}$ & $\begin{array}{l}1.34 \mathrm{E}-02 \\
1.79 \mathrm{E}-02 \\
3.25 \mathrm{E}-02 \\
1.74 \mathrm{E}-02 \\
3.12 \mathrm{E}-02 \\
1.74 \mathrm{E}-02 \\
6.78 \mathrm{E}-03 \\
6.72 \mathrm{E}-01 \\
5.20 \mathrm{E}-02 \\
2.32 \mathrm{E}-02 \\
2.00 \mathrm{E}-02 \\
1.71 \mathrm{E}+00 \\
1.97 \mathrm{E}-02 \\
1.10 \mathrm{E}-02 \\
1.71 \mathrm{E}+00 \\
7.88 \mathrm{E}-03 \\
3.47 \mathrm{E}-02 \\
5.60 \mathrm{E}-01\end{array}$ \\
\hline
\end{tabular}


Table 7. Annual Accident Dose Risk for the Shipping Campaign (person-rem/yr)

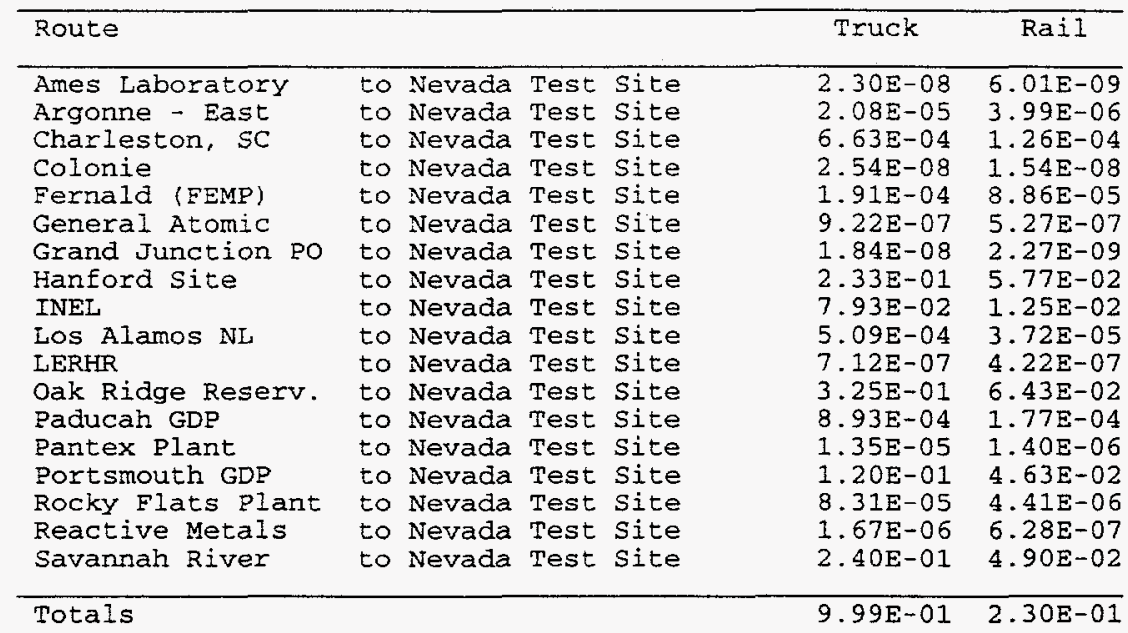

Table 8. Expected Annual Fatalities for the Shipping Campaign

\begin{tabular}{llc}
\hline Exposure Group & Truck & Rail \\
\hline Radiological & & \\
Normal Crew & $1.2 \mathrm{E}-02$ & $1.1 \mathrm{E}-03$ \\
Normal Public & $1.9 \mathrm{E}-02$ & $2.5 \mathrm{E}-03$ \\
Accident Public & $5.0 \mathrm{E}-04$ & $1.2 \mathrm{E}-04$ \\
Nonradiological & & \\
Emission & $6.6 \mathrm{E}-03$ & $3.8 \mathrm{E}-03$ \\
Accident & $7.5 \mathrm{E}-02$ & $1.0 \mathrm{E}-03$ \\
\hline
\end{tabular}

Table 9. Expected Annual Cancer Incidence for the Shipping Campaign

\begin{tabular}{lll}
\hline Exposure Group & Truck & Rail \\
\hline Radiological & & \\
$\quad$ Normal Crew & $4.4 \mathrm{E}-02$ & $3.7 \mathrm{E}-03$ \\
Normal Public & $6.3 \mathrm{E}-02$ & $8.4 \mathrm{E}-03$ \\
Accident Fublic & $1.7 \mathrm{E}-03$ & $3.9 \mathrm{E}-04$ \\
Nonradiological & $6.6 \mathrm{E}-03$ & $3.8 \mathrm{E}-03$ \\
$\quad$ Emission & $\mathrm{NA}$ & $\mathrm{NA}$ \\
Accident & & \\
\hline
\end{tabular}

Table 10. Expected Annual Genetic Effects for the Shipping Campaign

\begin{tabular}{lll}
\hline Exposure Group & Truck & Rail \\
\hline Radiological & & \\
Normal Crew & $1.9 \mathrm{E}-03$ & $1.6 \mathrm{E}-04$ \\
Normal Public & $3.7 \mathrm{E}-03$ & $5.0 \mathrm{E}-04$ \\
Accident Public & $7.7 \mathrm{E}-05$ & $1.6 \mathrm{E}-05$ \\
Nonradiological & NA & NA \\
Emission & NA & NA \\
Accident & & \\
\hline
\end{tabular}




\section{B.22 WM LLMW REGIONALIZED 3 ALTERNATIVE (CASE 10a): CONTACT-HANDLED HETEROGENEOUS SOLIDS: ALPHA - TREATED}

Table 1. Summary of Route Information

\begin{tabular}{|c|c|c|c|c|c|c|c|c|}
\hline & \multirow{2}{*}{\multicolumn{2}{|c|}{ Route }} & & & \multirow{2}{*}{$\begin{array}{c}\text { Distance } \\
\text { (miles) }\end{array}$} & \multicolumn{3}{|c|}{ Percentage in Zone } \\
\hline & & & & & & Rural & Suburban & Urban \\
\hline \multicolumn{9}{|l|}{ Truck } \\
\hline INEI & to & Nevada & Test & Site & 712 & 82.8 & 13.7 & 3.5 \\
\hline Los Alamos NL & to & Nevada & Test & Site & 997 & 93.2 & 5.7 & 1.1 \\
\hline Rocky Flats Plant & to & Nevada & Test & site & 836 & 89.5 & 8.5 & 2.0 \\
\hline Savannah River & to & Nevada & Test & site & 2414 & 83.1 & 15.1 & 1.8 \\
\hline West Valley $\mathrm{DP}$ & to & Nevada & Test & Site & 2373 & 83.7 & 14.3 & 2.0 \\
\hline \multicolumn{9}{|l|}{$\operatorname{Rail}$} \\
\hline INEL & to & Nevada & Test & Site & 756 & 92.8 & 5.9 & 1.3 \\
\hline Los Alamos NL & to & Nevada & Test & site & 1169 & 92.8 & 5.9 & 1.3 \\
\hline Rocky Flats Plant & to & Nevada & Test & Site & 987 & 95.9 & 3.9 & 0.2 \\
\hline Savannah River & to & Nevada & Test & Site & 2839 & 84.5 & 13.5 & 1.9 \\
\hline West Valley DP & to & Nevada & Test & site & 2554 & 80.8 & 15.1 & 4.0 \\
\hline
\end{tabular}

Table 2. Annual Number of Shipments and One-Way Shipment Distances

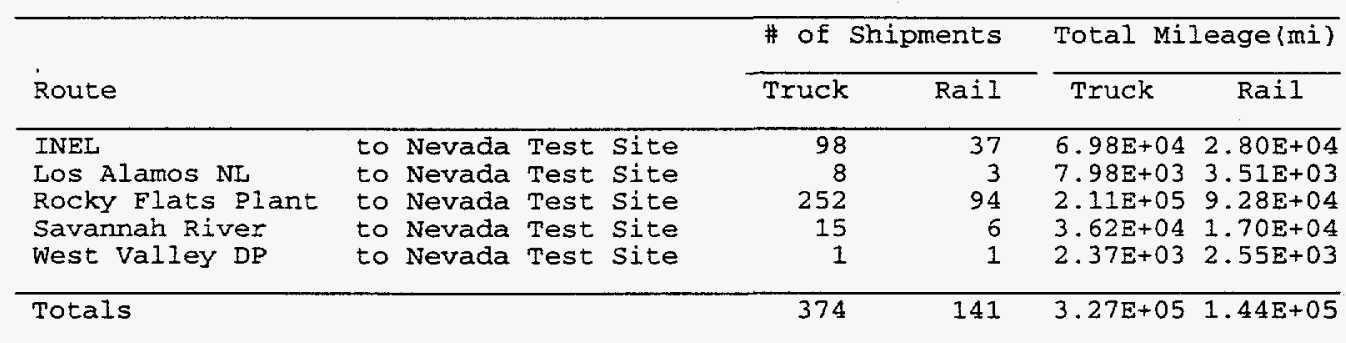

Table 3. Incident-Free Dose per Shipment (person-rem/shipment)

\begin{tabular}{|c|c|c|c|c|c|c|}
\hline \multirow[b]{2}{*}{ Source/Route (s) } & & \multirow[b]{2}{*}{ Crew } & \multicolumn{4}{|c|}{ General Public } \\
\hline & & & off-Link & on-Iink & Stops & Total \\
\hline $\begin{array}{l}\text { MLLW(T) IN to NT } \\
\text { INEL }\end{array}$ & $\begin{array}{l}\text { to Nevada } \\
\text { Truck } \\
\text { Rail }\end{array}$ & $\begin{array}{l}\text { Test Site } \\
2.13 \mathrm{E}-02 \\
6.04 \mathrm{E}-03\end{array}$ & $\begin{array}{l}2.03 E-03 \\
2.80 E-03\end{array}$ & $\begin{array}{l}3.79 \mathrm{E}-03 \\
6.88 \mathrm{E}-05\end{array}$ & $\begin{array}{l}1.99 \mathrm{E}-02 \\
5.81 \mathrm{E}-03\end{array}$ & $\begin{array}{l}2.58 E-02 \\
8.67 E-03\end{array}$ \\
\hline $\begin{aligned} \text { MLLW(T) LA to NT } \\
\text { LOS Alamos NL }\end{aligned}$ & $\begin{array}{l}\text { to Nevada } \\
\text { Truck } \\
\text { Rail }\end{array}$ & $\begin{array}{l}\text { Test Site } \\
2.61 \mathrm{E}-02 \\
7.76 \mathrm{E}-03\end{array}$ & $\begin{array}{l}1.04 \mathrm{E}-03 \\
4.40 \mathrm{E}-03\end{array}$ & $\begin{array}{l}3.33 \mathrm{E}-03 \\
1.07 \mathrm{E}-04\end{array}$ & $\begin{array}{l}2.79 \mathrm{E}-02 \\
7.08 \mathrm{E}-03\end{array}$ & $\begin{array}{l}3.23 \mathrm{E}-02 \\
1.16 \mathrm{E}-02\end{array}$ \\
\hline $\begin{array}{l}\text { MLLW (T) RF to NT } \\
\text { Rocky Elats Plant }\end{array}$ & $\begin{array}{l}\text { to Nevada } \\
\text { Truck } \\
\text { Rail }\end{array}$ & $\begin{array}{l}\text { Test Site } \\
2.30 \mathrm{E}-02 \\
7.00 \mathrm{E}-03\end{array}$ & $\begin{array}{l}1.44 E-03 \\
1.29 E-03\end{array}$ & $\begin{array}{l}3.43 E-03 \\
6.47 E-05\end{array}$ & $\begin{array}{l}2.34 \mathrm{E}-02 \\
6.52 \mathrm{E}-03\end{array}$ & $\begin{array}{l}2.83 E-02 \\
7.88 E-03\end{array}$ \\
\hline $\begin{array}{l}\text { MLLW (T) SR to NT } \\
\text { Savannah River }\end{array}$ & $\begin{array}{l}\text { to Nevada } \\
\text { Truck } \\
\text { Rail }\end{array}$ & $\begin{array}{l}\text { Test site } \\
7.07 \mathrm{E}-02 \\
1.47 \mathrm{E}-02\end{array}$ & $\begin{array}{l}5.10 \mathrm{E}-03 \\
1.85 \mathrm{E}-02\end{array}$ & $\begin{array}{l}1.03 E-02 \\
3.95 E-04\end{array}$ & $\begin{array}{l}6.76 \mathrm{E}-02 \\
1.22 \mathrm{E}-02\end{array}$ & $\begin{array}{l}8.30 E-02 \\
3.11 E-02\end{array}$ \\
\hline $\begin{array}{l}\text { MLLW(T) WV to NT } \\
\text { West Valley DP. }\end{array}$ & $\begin{array}{l}\text { to Nevada } \\
\text { Truck } \\
\text { Rail }\end{array}$ & $\begin{array}{l}\text { Test Site } \\
6.92 \mathrm{E}-02 \\
1.35 \mathrm{E}-02\end{array}$ & $\begin{array}{l}5.06 E-03 \\
2.77 E-02\end{array}$ & $\begin{array}{l}1.02 \mathrm{E}-02 \\
4.56 \mathrm{E}-04\end{array}$ & $\begin{array}{l}6.64 \mathrm{E}-02 \\
1.13 \mathrm{E}-02\end{array}$ & $\begin{array}{l}8.17 E-02 \\
3.95 E-02\end{array}$ \\
\hline
\end{tabular}


Table 4. Accident Dose Risk per Shipment (person-rem/shipment)

\begin{tabular}{llll}
\hline Source/Route & & Truck & Rail \\
\hline $\begin{array}{c}\text { MLLW(T) IN to NT } \\
\text { INEL }\end{array}$ & to Nevada Test Site & $7.53 \mathrm{E}-04$ & $3.19 \mathrm{E}-04$ \\
$\begin{array}{c}\text { MLLW (T) LA to NT } \\
\text { Los Alamos NL }\end{array}$ & to Nevada Test Site & $1.16 \mathrm{E}-04$ & $2.50 \mathrm{E}-05$ \\
$\begin{array}{c}\text { MLLW (T) RF to NT } \\
\text { Rocky Flats Plant }\end{array}$ & to Nevada Test Site & $9.61 \mathrm{E}-05$ & $1.45 \mathrm{E}-05$ \\
$\begin{array}{c}\text { MLLW(T) SR to NT } \\
\text { Savannah River }\end{array}$ & to Nevada Test Site & $2.41 \mathrm{E}-03$ & $1.27 \mathrm{E}-03$ \\
$\begin{array}{c}\text { MLLW(T) WV to NT } \\
\text { West Valley DP }\end{array}$ & to Nevada Test Site & $1.42 \mathrm{E}-04$ & $5.58 \mathrm{E}-05$ \\
\hline
\end{tabular}

Table 5. Nonradiological Risk Factors per Shipment (fatalities/shipment)

\begin{tabular}{lllll}
\hline Mode & Route & & Emission & Accident \\
\hline Truck & to Nevada Test Site & $8.08 \mathrm{E}-06$ & $4.36 \mathrm{E}-05$ \\
INEL & to Nevada Test Site & $3.54 \mathrm{E}-06$ & $7.82 \mathrm{E}-05$ \\
Los Alamos NL & to Nevada Test Site & $5.50 \mathrm{E}-06$ & $5.18 \mathrm{E}-05$ \\
Rocky Flats Plant & to Nevada Test Site & $1.43 \mathrm{E}-05$ & $1.74 \mathrm{E}-04$ \\
Savannah River & to & $1.52 \mathrm{E}-05$ & $1.46 \mathrm{E}-04$ \\
West Valley DP & to Nevada Test Site & & \\
Rail & & & $3.98 \mathrm{E}-06$ & $1.58 \mathrm{E}-06$ \\
INEL & to Nevada Test Site & $5.32 \mathrm{E}-06$ & $2.45 \mathrm{E}-06$ \\
Los Alanos NL & to Nevada Test Site & $8.37 \mathrm{E}-07$ & $2.07 \mathrm{E}-06$ \\
Rocky Flats Plant & to Nevada Test Site & $2.27 \mathrm{E}-05$ & $5.94 \mathrm{E}-06$ \\
Savannah River & to Nevada Test Site & $4.33 \mathrm{E}-05$ & $5.34 \mathrm{E}-06$ \\
West Valley DP & to Nevada Test Site & & \\
\hline
\end{tabular}

Table 6. Annual Incident-Free Dose for the Shipping Campaign (person-rem/yr)

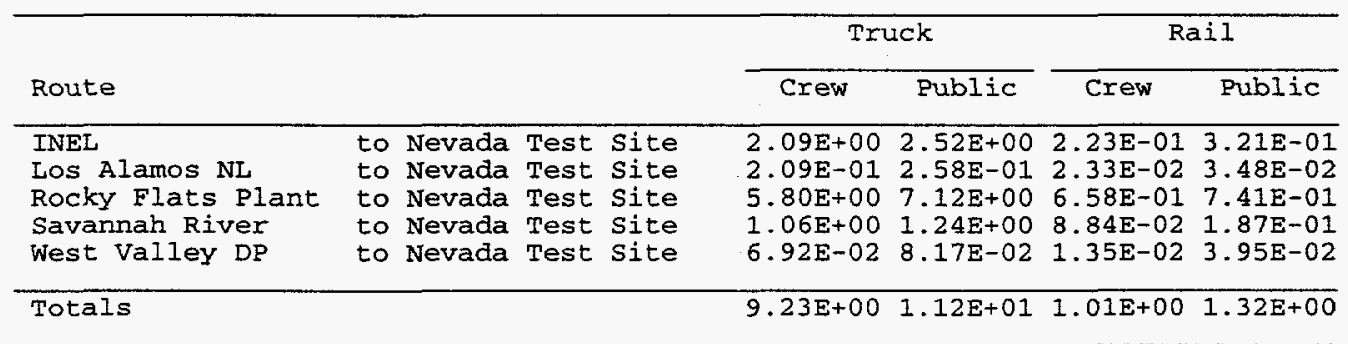

Table 7. Annual Accident Dose Risk for the Shipping Campaign (person-rem/yr)

\begin{tabular}{llllc}
\hline Route & & Truck & Rail \\
\hline INEI & to Nevada Test Site & $7.38 \mathrm{E}-02$ & $1.18 \mathrm{E}-02$ \\
Los Alamos NL & to Nevada Test Site & $9.27 \mathrm{E}-04$ & $7.51 \mathrm{E}-05$ \\
Rocky Flats Plant & to Nevada Test Site & $2.42 \mathrm{E}-02$ & $1.36 \mathrm{E}-03$ \\
Savannah River & to Nevada Test Site & $3.61 \mathrm{E}-02$ & $7.62 \mathrm{E}-03$ \\
West Valley DP & to Nevada Test Site & $1.42 \mathrm{E}-04$ & $5.58 \mathrm{E}-05$ \\
\hline Totals & & $1.35 \mathrm{E}-01$ & $2.09 \mathrm{E}-02$ \\
\end{tabular}

Table 8. Expected Annual Fatalities for the Shipping Campaign

\begin{tabular}{lll}
\hline Exposure Group & Truck & Rail \\
\hline Radiological & $3.7 \mathrm{E}-03$ & $4.0 \mathrm{E}-04$ \\
$\quad$ Normal Crew & $5.6 \mathrm{E}-03$ & $6.6 \mathrm{E}-04$ \\
Normal Public & $6.8 \mathrm{E}-05$ & $1.0 \mathrm{E}-05$ \\
$\quad$ Accident Public & $2.4 \mathrm{E}-03$ & $4.2 \mathrm{E}-04$ \\
Nonradiological & $2.3 \mathrm{E}-02$ & $3.0 \mathrm{E}-04$ \\
$\quad$ Emission & & \\
Accident & & \\
\hline
\end{tabular}


Table 9. Expected Annual Cancer Incidence for the Shipping Campaign

\begin{tabular}{lll}
\hline Exposure Group & Truck & \multicolumn{1}{c}{ Rail } \\
\hline Radiological & & \\
$\quad$ Normal Crew & $1.3 \mathrm{E}-02$ & $1.4 \mathrm{E}-03$ \\
Normal Public & $1.9 \mathrm{E}-02$ & $2.2 \mathrm{E}-03$ \\
Accident Public & $2.3 \mathrm{E}-04$ & $3.6 \mathrm{E}-05$ \\
Nonradiological & $2.4 \mathrm{E}-03$ & $4.2 \mathrm{E}-04$ \\
Emission & $\mathrm{NA}$ & $\mathrm{NA}$ \\
Accident & & \\
\hline
\end{tabular}

Table 10. Expected Annual Genetic Effects for the Shipping Campaign

\begin{tabular}{lll}
\hline Exposure Group & Truck & Rail \\
\hline Radiological & & \\
Normal Crew & $5.5 E-04$ & $6.0 \mathrm{E}-05$ \\
Normal Public & $1.1 \mathrm{E}-03$ & $1.3 \mathrm{E}-04$ \\
Accident Public & $1.0 \mathrm{E}-05$ & $1.6 \mathrm{E}-06$ \\
Nonradiological & NA & NA \\
Emission & NA & NA \\
Accident & & \\
\hline
\end{tabular}




\section{B.23 WM LLMW REGIONALIZED 4 ALTERNATIVE (CASE 15): CONTACT-HANDLED ORGANIC LIQUIDS: NONALPHA - UNTREATED}

Table 1. Summary of Route Information

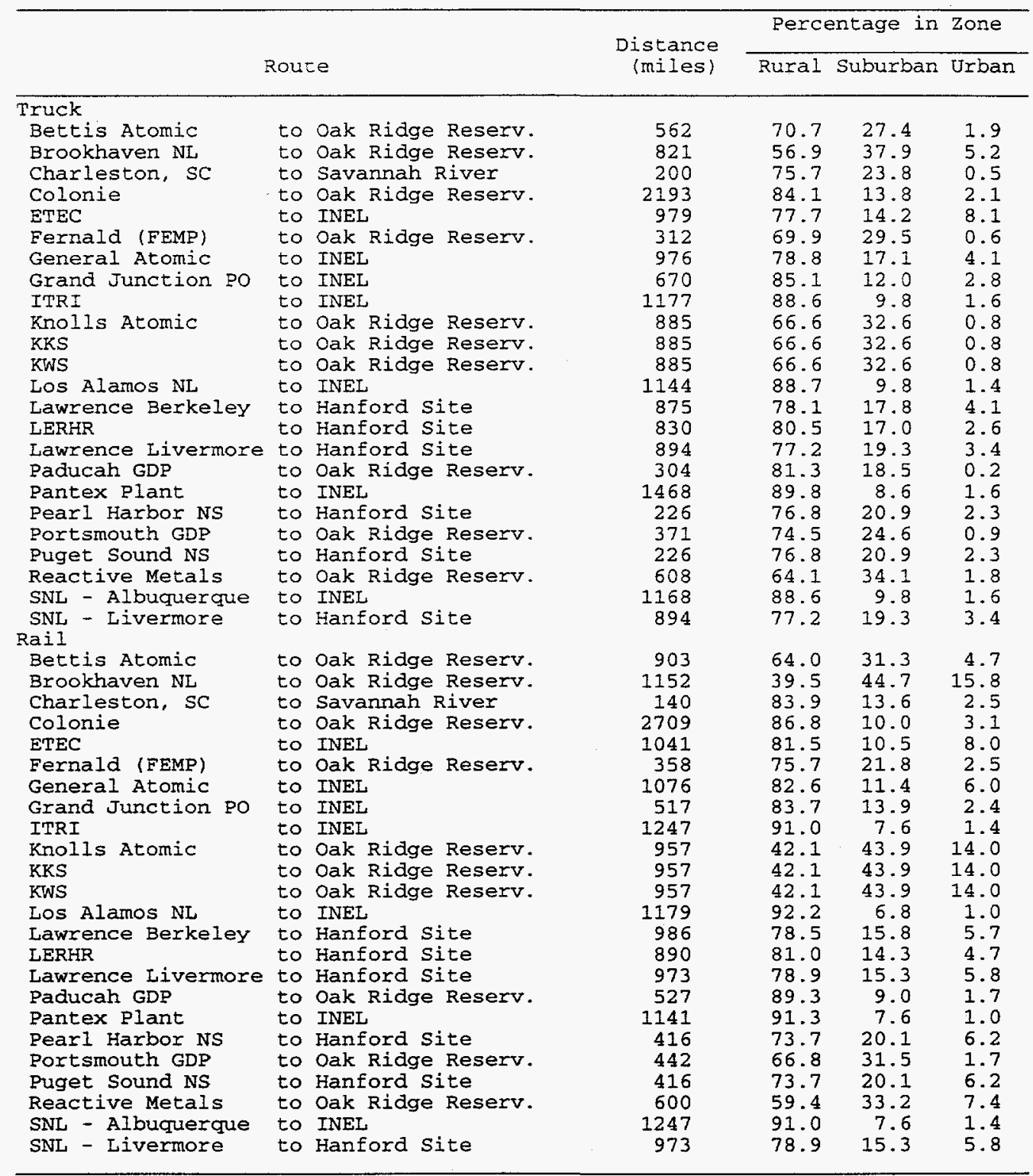


Table 2. Annual Number of Shipments and One-Way Shipment Distances

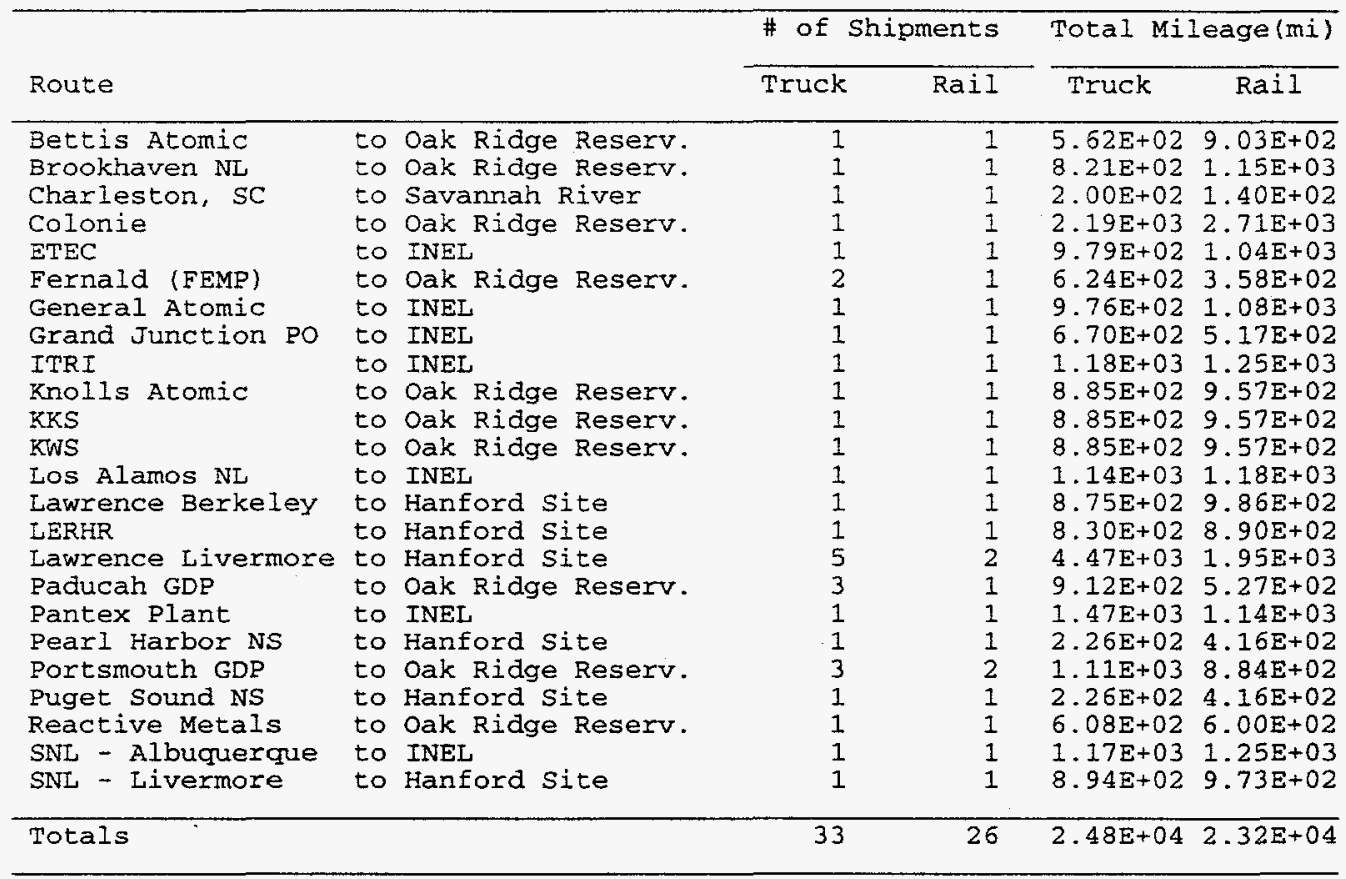


Table 3. Incident-Free Dose per Shipment (person-rem/shipment)

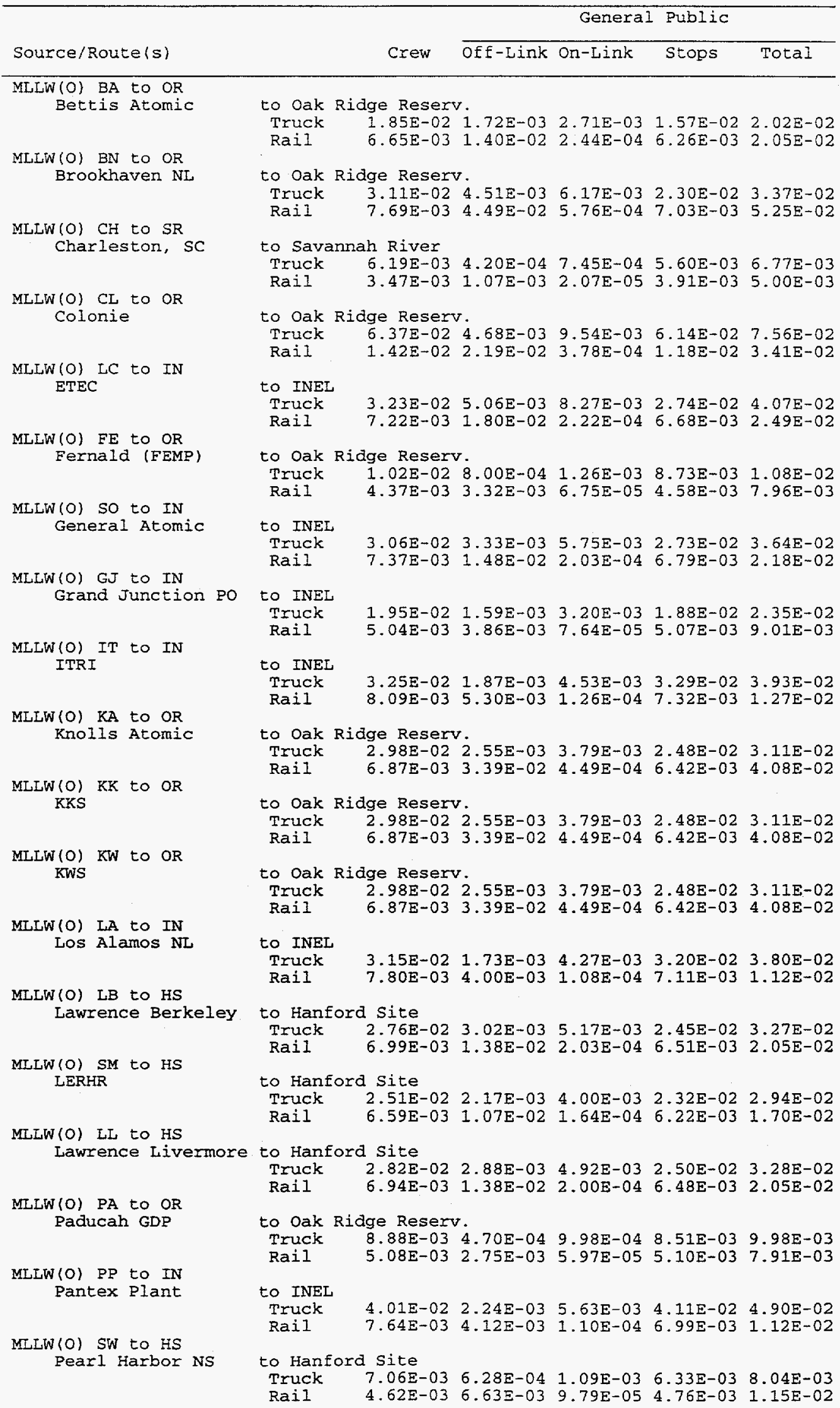


Table 3. Incident-Free Dose per Shipment (person-rem/shipment) (Cont.)

\begin{tabular}{|c|c|c|c|c|c|}
\hline \multirow[b]{2}{*}{ Source/Route (s) } & \multirow[b]{2}{*}{ Crew } & \multicolumn{4}{|c|}{ General Public } \\
\hline & & off-Iink & on-Link & Stops & Total \\
\hline $\begin{array}{l}\text { MLLW (O) PO to OR } \\
\text { Portsmouth GDP }\end{array}$ & $\begin{array}{cr}\text { to Oak Ridge Reserv } \\
\text { Truck } & 1.16 \mathrm{E}-02 \\
\text { Rail } & 4.73 \mathrm{E}-03\end{array}$ & $\begin{array}{l}8.77 E-04 \\
4.37 E-03\end{array}$ & $\begin{array}{l}1.50 \mathrm{E}-03 \\
1.00 \mathrm{E}-04\end{array}$ & $\begin{array}{l}1.04 \mathrm{E}-02 \\
4.84 \mathrm{E}-03\end{array}$ & $\begin{array}{l}1.28 \mathrm{E}-02 \\
9.32 \mathrm{E}-03\end{array}$ \\
\hline $\begin{array}{l}\text { MLLW(O) PN to HS } \\
\text { Puget Sound NS }\end{array}$ & $\begin{array}{ll}\text { to Hanford Site } \\
\text { Truck } & 7.06 \mathrm{E}-03 \\
\text { Rail } & 4.62 \mathrm{E}-03\end{array}$ & $\begin{array}{l}6.28 E-04 \\
6.63 E-03\end{array}$ & $\begin{array}{l}1.09 E-03 \\
9.79 E-05\end{array}$ & $\begin{array}{l}6.33 \mathrm{E}-03 \\
4.76 \mathrm{E}-03\end{array}$ & $\begin{array}{l}8.04 E-03 \\
1.15 E-02\end{array}$ \\
\hline $\begin{array}{l}\text { MLLW(O) RM to OR } \\
\text { Reactive Metals }\end{array}$ & $\begin{array}{cr}\text { to oak Ridge Reser } \\
\text { Truck } & 2.11 \mathrm{E}-02 \\
\text { Rail } & 5.38 \mathrm{E}-03\end{array}$ & $\begin{array}{l}\text { 2. } \\
1.25 E-03 \\
1.25 E-02\end{array}$ & $\begin{array}{l}3.08 \mathrm{E}-03 \\
1.91 \mathrm{E}-04\end{array}$ & $\begin{array}{l}1.70 \mathrm{E}-02 \\
5.33 \mathrm{E}-03\end{array}$ & $\begin{array}{l}2.23 \mathrm{E}-02 \\
1.80 \mathrm{E}-02\end{array}$ \\
\hline $\begin{aligned} \text { MLLW(O) SA to IN } \\
\text { SNL }- \text { Albuquerque }\end{aligned}$ & $\begin{array}{l}\text { to INEL } \\
\text { Truck } \\
\text { Rail }\end{array}$ & $\begin{array}{l}1.83 E-03 \\
5.30 E-03\end{array}$ & $\begin{array}{l}4.46 \mathrm{E}-03 \\
1.26 \mathrm{E}-04\end{array}$ & $\begin{array}{l}3.27 E-02 \\
7.32 E-03\end{array}$ & $\begin{array}{l}3.90 \mathrm{E}-02 \\
1.27 \mathrm{E}-02\end{array}$ \\
\hline $\begin{aligned} & \text { MLLW (O) } \text { SL to HS } \\
& \text { SNL - Livermore }\end{aligned}$ & $\begin{array}{l}\text { to Hanford Site } \\
\text { Truck } \\
\text { Rail }\end{array}$ & $\begin{array}{l}2.88 E-03 \\
1.38 E-02\end{array}$ & $\begin{array}{l}4.92 \mathrm{E}-03 \\
2.00 \mathrm{E}-04\end{array}$ & $\begin{array}{l}2.50 \mathrm{E}-02 \\
6.48 \mathrm{E}-03\end{array}$ & $\begin{array}{l}3.28 E-02 \\
2.05 E-02\end{array}$ \\
\hline
\end{tabular}

Table 4. Accident Dose Risk per Shipment (person-rem/shipment)

\begin{tabular}{|c|c|c|c|}
\hline Source/Route & & Truck & Rail \\
\hline $\mathrm{LLW}(0) \mathrm{BA}$ to $\mathrm{OR}$ & & & \\
\hline $\begin{array}{l}\text { Bettis Atomic } \\
\text { MTLin (a) BN to OR }\end{array}$ & to Oak Ridge Reserv. & $9.86 \mathrm{E}-04$ & $3.35 \mathrm{E}-04$ \\
\hline $\begin{array}{l}\text { Brookhaven NL } \\
\text { MLLW (a) Cr to SR }\end{array}$ & to Oak Ridge Reserv. & $6.04 \mathrm{E}-03$ & $4.26 \mathrm{E}-03$ \\
\hline $\begin{array}{l}\text { Charleston, SC } \\
\text { MLLW (O) CL to OR }\end{array}$ & to Savannah River & $7.75 E-03$ & $8.99 \mathrm{E}-04$ \\
\hline $\begin{array}{l}\text { Colonie } \\
\text { MLLW(O) LC to IN }\end{array}$ & to Oak Ridge Reserv. & $4.25 E-04$ & $9.32 \mathrm{E}-05$ \\
\hline $\begin{array}{l}\text { ETEC } \\
\text { MLLW(O) FE to OR }\end{array}$ & to INEL & $9.54 E-06$ & $4.35 \mathrm{E}-06$ \\
\hline $\begin{array}{l}\text { MLLW (O) FE to OR } \\
\text { Fernald (FEMP) } \\
\text { MLLW (O) so to IN }\end{array}$ & to Oak Ridge Reserv. & $1.80 \mathrm{E}-03$ & $3.68 \mathrm{E}-04$ \\
\hline $\begin{array}{l}\text { General Atomic } \\
\text { MLLw(o) GJ to IN }\end{array}$ & to INEL & $1.08 \mathrm{E}-05$ & $4.28 \mathrm{E}-06$ \\
\hline $\begin{array}{l}\text { Grand Junction PO } \\
\text { MLLW(O) IT to IN }\end{array}$ & to INEL & $1.05 E-06$ & $3.06 \mathrm{E}-07$ \\
\hline ITRI & to INEL & $2.64 \mathrm{E}-05$ & $1.59 \mathrm{E}-06$ \\
\hline $\begin{array}{l}\text { MLLW (O) KA to OR } \\
\text { Knolis Atomic } \\
\text { MLLW (O) KR to OR }\end{array}$ & to Oak Ridge Reserv. & $.05 E-02$ & $2.38 \mathrm{E}-02$ \\
\hline KKS & to Oak Ridge Reserv. & $3.11 \mathrm{E}-02$ & $1.83 \mathrm{E}-02$ \\
\hline $\begin{array}{l}\text { KWS } \\
\text { MLLW (O) LA to IN }\end{array}$ & to Oak Ridge Reserv. & $4.24 E-03$ & $2.49 \mathrm{E}-03$ \\
\hline $\begin{array}{l}\text { LOS Alamos NL } \\
\text { MLLW(O) LB to HS }\end{array}$ & to INEL & $1.71 E-03$ & $1.01 E-04$ \\
\hline $\begin{array}{l}\text { Lawrence Berkeley } \\
\text { MLLW(O) SM to HS }\end{array}$ & to Hanford Site & $7.79 \mathrm{E}-05$ & $4.38 \mathrm{E}-05$ \\
\hline $\begin{array}{l}\text { LERHR } \\
\text { MLLW (O) IL to HS }\end{array}$ & to Hanford Site & $7.78 E-06$ & $4.14 \mathrm{E}-06$ \\
\hline $\begin{array}{l}\text { Lawrence Iivermore } \\
\text { MLLW (O) PA to OR }\end{array}$ & to Hanford Site & $3.93 E-03$ & $5.23 E-03$ \\
\hline $\begin{array}{l}\text { Paducah GDP } \\
\text { MLLW (O) PP to IN }\end{array}$ & to Oak Ridge Reserv. & $3.89 E-01$ & $1.21 \mathrm{E}-01$ \\
\hline $\begin{array}{l}\text { Pantex Plant } \\
\text { MLLW (O) SW to HS }\end{array}$ & to INEL & $4.15 \mathrm{E}-04$ & $2.58 E-05$ \\
\hline $\begin{array}{l}\text { Pearl Harbor NS } \\
\text { MLLW (O) PO to OR }\end{array}$ & to Hanford Site & $8.47 \mathrm{E}-06$ & $6.32 E-06$ \\
\hline $\begin{array}{l}\text { Portsmouth GDP } \\
\text { MLLW (O) PN to HS }\end{array}$ & to Oak Ridge Reserv. & $6.55 \mathrm{E}-04$ & $1.86 \mathrm{E}-04$ \\
\hline $\begin{array}{l}\text { Puget Sound NS } \\
\text { MLLW (O) RM to OR }\end{array}$ & to Hanford Site & $1.95 \mathrm{E}-04$ & $1.46 \mathrm{E}-04$ \\
\hline $\begin{array}{l}\text { Reactive Metals } \\
\text { MLLW(0) SA to IN }\end{array}$ & to Oak Ridge Reserv. & $2.01 E-04$ & $3.81 E-05$ \\
\hline $\begin{array}{l}\text { SNL - Albuquerque } \\
\text { MLLW(O) SL to HS }\end{array}$ & to INEL & $1.85 E-06$ & $1.12 \mathrm{E}-07$ \\
\hline SNL - Livermore & to Hanford Site & $1.95 E-03$ & $1.04 E-03$ \\
\hline
\end{tabular}


Table 5. Nonradiological Risk Factors per Shipment (fatalities/shipment)

\begin{tabular}{|c|c|c|c|}
\hline Mode & Route & Emission & Accident \\
\hline $\begin{array}{l}\text { Truck } \\
\text { Bettis Atonic } \\
\text { Brookhaven NL } \\
\text { Charleston, SC } \\
\text { Colonie } \\
\text { ETEC } \\
\text { Fernald (FEMP) } \\
\text { General Atomic } \\
\text { Grand Junction PO } \\
\text { ITRI } \\
\text { Knolis Atomic } \\
\text { KKS } \\
\text { KWS } \\
\text { LOS Alamos NL } \\
\text { Lawrence Berkeley } \\
\text { LERHR } \\
\text { Lawrence Livermore } \\
\text { Paducah GDP } \\
\text { Pantex Plant } \\
\text { Pearl Harbor NS } \\
\text { Portsmouth GDP } \\
\text { Puget Sound NS } \\
\text { Reactive Metals } \\
\text { SNL - Albuquerque } \\
\text { SNL - Livermore }\end{array}$ & $\begin{array}{l}\text { to Oak Ridge Reserv. } \\
\text { to Oak Ridge Reserv. } \\
\text { to Savannah River } \\
\text { to Oak Ridge Reserv. } \\
\text { to INEL } \\
\text { to Oak Ridge Reserv. } \\
\text { to INEL } \\
\text { to INEL } \\
\text { to INEI } \\
\text { to Oak Ridge Reserv. } \\
\text { to Oak Ridge Reserv. } \\
\text { to Oak Ridge Reserv. } \\
\text { to INEL } \\
\text { to Hanford Site } \\
\text { to Hanford Site } \\
\text { to Hanford Site } \\
\text { to Oak Ridge Reserv. } \\
\text { to INEL } \\
\text { to Hanford Site } \\
\text { to Oak Ridge Reserv. } \\
\text { to Hanford Site } \\
\text { to Oak Ridge Reserv. } \\
\text { to INEL } \\
\text { to Hanford Site }\end{array}$ & $\begin{array}{l}3.41 \mathrm{E}-06 \\
1.37 \mathrm{E}-05 \\
3.22 \mathrm{E}-07 \\
1.46 \mathrm{E}-05 \\
2.55 \mathrm{E}-05 \\
5.79 \mathrm{E}-07 \\
1.30 \mathrm{E}-05 \\
6.08 \mathrm{E}-06 \\
5.02 \mathrm{E}-06 \\
2.16 \mathrm{E}-06 \\
2.16 \mathrm{E}-06 \\
2.16 \mathrm{E}-06 \\
5.25 \mathrm{E}-06 \\
1.16 \mathrm{E}-05 \\
6.82 \mathrm{E}-06 \\
9.85 \mathrm{E}-06 \\
1.93 \mathrm{E}-07 \\
7.79 \mathrm{E}-06 \\
1.67 \mathrm{E}-06 \\
1.09 \mathrm{E}-06 \\
1.67 \mathrm{E}-06 \\
3.57 \mathrm{E}-06 \\
5.83 \mathrm{E}-06 \\
9.85 \mathrm{E}-06\end{array}$ & $\begin{array}{l}3.71 \mathrm{E}-05 \\
5.92 \mathrm{E}-05 \\
1.70 \mathrm{E}-05 \\
1.61 \mathrm{E}-04 \\
6.21 \mathrm{E}-05 \\
2.25 \mathrm{E}-05 \\
6.23 \mathrm{E}-05 \\
4.24 \mathrm{E}-05 \\
8.53 \mathrm{E}-05 \\
6.08 \mathrm{E}-05 \\
6.08 \mathrm{E}-05 \\
6.08 \mathrm{E}-05 \\
8.21 \mathrm{E}-05 \\
4.91 \mathrm{E}-05 \\
4.66 \mathrm{E}-05 \\
5.03 \mathrm{E}-05 \\
2.14 \mathrm{E}-05 \\
1.05 \mathrm{E}-04 \\
9.56 \mathrm{E}-06 \\
2.56 \mathrm{E}-05 \\
9.56 \mathrm{E}-06 \\
3.56 \mathrm{E}-05 \\
8.45 \mathrm{E}-05 \\
5.03 \mathrm{E}-05\end{array}$ \\
\hline $\begin{array}{l}\text { Rail } \\
\text { Bettis Atomic } \\
\text { Brookhaven NL } \\
\text { Charleston, SC } \\
\text { Colonie } \\
\text { ETEC } \\
\text { Fernald (FEMP) } \\
\text { General Atomic } \\
\text { Grand Junction Po } \\
\text { ITRI } \\
\text { Knolls Atomic } \\
\text { KKS } \\
\text { KWS } \\
\text { Los Alamos NL } \\
\text { Lawrence Berkeley } \\
\text { LERHR } \\
\text { Lawrence Livermore } \\
\text { Paducah GDP } \\
\text { Pantex Plant } \\
\text { Pearl Harbor NS } \\
\text { Portsmouth GDP } \\
\text { Puget Sound NS } \\
\text { Reactive Metals } \\
\text { SNI - Albuquerque } \\
\text { SNL - Livermore }\end{array}$ & $\begin{array}{l}\text { to Oak Ridge Reserv. } \\
\text { to Oak Ridge Reserv. } \\
\text { to Savannah River } \\
\text { to Oak Ridge Reserv. } \\
\text { to INEL } \\
\text { to Oak Ridge Reserv. } \\
\text { to INEL } \\
\text { to INEL } \\
\text { to INEL } \\
\text { to Oak Ridge Reserv. } \\
\text { to Oak Ridge Reserv. } \\
\text { to Oak Ridge Reserv. } \\
\text { to INEL } \\
\text { to Hanford Site } \\
\text { to Hanford Site } \\
\text { to Hanford Site } \\
\text { to Oak Ridge Reserv. } \\
\text { to INEL } \\
\text { to Hanford Site } \\
\text { to Oak Ridge Reserv. } \\
\text { to Hanford Site } \\
\text { to Oak Ridge Reserv. } \\
\text { to INEL } \\
\text { to Hanford Site }\end{array}$ & $\begin{array}{l}1.78 \mathrm{E}-05 \\
7.59 \mathrm{E}-05 \\
1.46 \mathrm{E}-06 \\
3.56 \mathrm{E}-05 \\
3.48 \mathrm{E}-05 \\
3.68 \mathrm{E}-06 \\
2.72 \mathrm{E}-05 \\
5.15 \mathrm{E}-06 \\
7.11 \mathrm{E}-06 \\
5.59 \mathrm{E}-05 \\
5.59 \mathrm{E}-05 \\
5.59 \mathrm{E}-05 \\
4.90 \mathrm{E}-06 \\
2.33 \mathrm{E}-05 \\
1.77 \mathrm{E}-05 \\
2.36 \mathrm{E}-05 \\
3.81 \mathrm{E}-06 \\
4.85 \mathrm{E}-06 \\
1.08 \mathrm{E}-05 \\
3.18 \mathrm{E}-06 \\
1.08 \mathrm{E}-05 \\
1.85 \mathrm{E}-05 \\
7.11 \mathrm{E}-06 \\
2.36 \mathrm{E}-05\end{array}$ & $\begin{array}{l}1.89 \mathrm{E}-06 \\
2.41 \mathrm{E}-06 \\
2.93 \mathrm{E}-07 \\
5.67 \mathrm{E}-06 \\
2.18 \mathrm{E}-06 \\
7.48 \mathrm{E}-07 \\
2.25 \mathrm{E}-06 \\
1.08 \mathrm{E}-06 \\
2.51 \mathrm{E}-06 \\
2.00 \mathrm{E}-06 \\
2.00 \mathrm{E}-06 \\
2.00 \mathrm{E}-06 \\
2.47 \mathrm{E}-06 \\
2.06 \mathrm{E}-06 \\
1.85 \mathrm{E}-06 \\
2.04 \mathrm{E}-06 \\
1.10 \mathrm{E}-06 \\
2.39 \mathrm{E}-06 \\
8.70 \mathrm{E}-07 \\
9.25 \mathrm{E}-07 \\
8.70 \mathrm{E}-07 \\
1.25 \mathrm{E}-06 \\
2.61 \mathrm{E}-06 \\
2.04 \mathrm{E}-06\end{array}$ \\
\hline
\end{tabular}


Table 6. Annual Incident-Free Dose for the Shipping Campaign (person-rem/yr)

\begin{tabular}{|c|c|c|c|c|c|c|}
\hline \multirow[b]{2}{*}{ Route } & & & \multicolumn{2}{|c|}{ Truck } & \multicolumn{2}{|c|}{ Rail } \\
\hline & & & Crew & Public & Crew & Public \\
\hline $\begin{array}{l}\text { Bettis Atomic } \\
\text { Brookhaven NL } \\
\text { Charleston, SC } \\
\text { Colonie } \\
\text { ETEC } \\
\text { Fernald (FEMP) } \\
\text { General Atomic } \\
\text { Grand Junction po } \\
\text { ITRI } \\
\text { Knolls Atomic } \\
\text { KKS } \\
\text { KWS } \\
\text { Los Alamos NL } \\
\text { Lawrence Berkeley } \\
\text { LERHR } \\
\text { Lawrence Livermore } \\
\text { Paducah GDP } \\
\text { Pantex Plant } \\
\text { Pearl Harbor NS } \\
\text { Portsmouth GDP } \\
\text { Puget Sound NS } \\
\text { Reactive Metals } \\
\text { SNL - Albuquerque } \\
\text { SNL - Livermore }\end{array}$ & $\begin{array}{l}\text { to } \\
\text { to } \\
\text { to } \\
\text { to } \\
\text { to } \\
\text { to } \\
\text { to } \\
\text { to } \\
\text { to } \\
\text { to } \\
\text { to } \\
\text { to } \\
\text { to } \\
\text { to } \\
\text { to } \\
\text { to } \\
\text { to } \\
\text { to } \\
\text { to } \\
\text { to } \\
\text { to } \\
\text { to } \\
\text { to } \\
\text { to }\end{array}$ & $\begin{array}{l}\text { Oak Ridge Reserv. } \\
\text { Oak Ridge Reserv. } \\
\text { Savannah River } \\
\text { Oak Ridge Reserv. } \\
\text { INEL } \\
\text { Oak Ridge Reserv. } \\
\text { INEL } \\
\text { INEL } \\
\text { INEL } \\
\text { Oak Ridge Reserv. } \\
\text { Oak Ridge Reserv. } \\
\text { Oak Ridge Reserv. } \\
\text { INEL } \\
\text { Hanford Site } \\
\text { Hanford Site } \\
\text { Hanford Site } \\
\text { Oak Ridge Reserv. } \\
\text { INEL } \\
\text { Hanford Site } \\
\text { Oak Ridge Reserv. } \\
\text { Hanford Site } \\
\text { Oak Ridge Reserv. } \\
\text { INEL } \\
\text { Hanford Site }\end{array}$ & $\begin{array}{l}1.35 \mathrm{E}-02 \\
3.11 \mathrm{E}-02 \\
6.19 \mathrm{E}-03 \\
6.37 \mathrm{E}-02 \\
3.23 \mathrm{E}-02 \\
2.03 \mathrm{E}-02 \\
3.06 \mathrm{E}-02 \\
1.95 \mathrm{E}-02 \\
3.25 \mathrm{E}-02 \\
2.98 \mathrm{E}-02 \\
2.98 \mathrm{E}-02 \\
2.98 \mathrm{E}-02 \\
3.15 \mathrm{E}-02 \\
2.76 \mathrm{E}-02 \\
2.51 \mathrm{E}-02 \\
1.41 \mathrm{E}-01 \\
2.67 \mathrm{E}-02 \\
4.01 \mathrm{E}-02 \\
7.06 \mathrm{E}-03 \\
3.49 \mathrm{E}-02 \\
7.06 \mathrm{E}-03 \\
2.11 \mathrm{E}-02 \\
3.22 \mathrm{E}-02 \\
2.82 \mathrm{E}-02\end{array}$ & $\begin{array}{l}2.02 \mathrm{E}-02 \\
3.37 \mathrm{E}-02 \\
6.77 \mathrm{E}-03 \\
7.56 \mathrm{E}-02 \\
4.07 \mathrm{E}-02 \\
2.16 \mathrm{E}-02 \\
3.64 \mathrm{E}-02 \\
2.35 \mathrm{E}-02 \\
3.93 \mathrm{E}-02 \\
3.11 \mathrm{E}-02 \\
3.11 \mathrm{E}-02 \\
3.11 \mathrm{E}-02 \\
3.80 \mathrm{E}-02 \\
3.27 \mathrm{E}-02 \\
2.94 \mathrm{E}-02 \\
1.64 \mathrm{E}-01 \\
2.99 \mathrm{E}-02 \\
4.90 \mathrm{E}-02 \\
8.04 \mathrm{E}-03 \\
3.83 \mathrm{E}-02 \\
8.04 \mathrm{E}-03 \\
2.23 \mathrm{E}-02 \\
3.90 \mathrm{E}-02 \\
3.28 \mathrm{E}-02\end{array}$ & $\begin{array}{l}6.65 \mathrm{E}-03 \\
7.69 \mathrm{E}-03 \\
3.47 \mathrm{E}-03 \\
1.42 \mathrm{E}-02 \\
7.22 \mathrm{E}-03 \\
4.37 \mathrm{E}-03 \\
7.37 \mathrm{E}-03 \\
5.04 \mathrm{E}-03 \\
8.09 \mathrm{E}-03 \\
6.87 \mathrm{E}-03 \\
6.87 \mathrm{E}-03 \\
6.87 \mathrm{E}-03 \\
7.80 \mathrm{E}-03 \\
6.99 \mathrm{E}-03 \\
6.59 \mathrm{E}-03 \\
1.39 \mathrm{E}-02 \\
5.08 \mathrm{E}-03 \\
7.64 \mathrm{E}-03 \\
4.62 \mathrm{E}-03 \\
9.45 \mathrm{E}-03 \\
4.62 \mathrm{E}-03 \\
5.38 \mathrm{E}-03 \\
8.09 \mathrm{E}-03 \\
6.94 \mathrm{E}-03\end{array}$ & $\begin{array}{l}2.05 \mathrm{E}-02 \\
5.25 \mathrm{E}-02 \\
5.00 \mathrm{E}-03 \\
3.41 \mathrm{E}-02 \\
2.49 \mathrm{E}-02 \\
7.96 \mathrm{E}-03 \\
2.18 \mathrm{E}-02 \\
9.01 \mathrm{E}-03 \\
1.27 \mathrm{E}-02 \\
4.08 \mathrm{E}-02 \\
4.08 \mathrm{E}-02 \\
4.08 \mathrm{E}-02 \\
1.12 \mathrm{E}-02 \\
2.05 \mathrm{E}-02 \\
1.70 \mathrm{E}-02 \\
4.10 \mathrm{E}-02 \\
7.91 \mathrm{E}-03 \\
1.12 \mathrm{E}-02 \\
1.15 \mathrm{E}-02 \\
1.86 \mathrm{E}-02 \\
1.15 \mathrm{E}-02 \\
1.80 \mathrm{E}-02 \\
1.27 \mathrm{E}-02 \\
2.05 \mathrm{E}-02\end{array}$ \\
\hline & & & & & & \\
\hline
\end{tabular}

Table 7. Annual Accident Dose Risk for the Shipping Campaign (person-rem/yr)

\begin{tabular}{llcc}
\hline Route & & Truck & Rail \\
\hline Bettis Atomic & to Oak Ridge Reserv. & $9.86 \mathrm{E}-04$ & $3.35 \mathrm{E}-04$ \\
Brookhaven NL & to Oak Ridge Reserv. & $6.04 \mathrm{E}-03$ & $4.26 \mathrm{E}-03$ \\
Charleston, SC & to Savannah River & $7.75 \mathrm{E}-03$ & $8.99 \mathrm{E}-04$ \\
Colonie & to Oak Ridge Reserv. & $4.25 \mathrm{E}-04$ & $9.32 \mathrm{E}-05$ \\
ETEC & to INEL & $9.54 \mathrm{E}-06$ & $4.35 \mathrm{E}-06$ \\
Fernald (FEMP) & to Oak Ridge Reserv. & $3.60 \mathrm{E}-03$ & $3.68 \mathrm{E}-04$ \\
General Atomic & to INEL & $1.08 \mathrm{E}-05$ & $4.28 \mathrm{E}-06$ \\
Grand Junction PO & to INEL & $1.05 \mathrm{E}-06$ & $3.06 \mathrm{E}-07$ \\
ITRI & to INEL & $2.64 \mathrm{E}-05$ & $1.59 \mathrm{E}-06$ \\
Knolls Atomic & to Oak Ridge Reserv. & $4.06 \mathrm{E}-02$ & $2.38 \mathrm{E}-02$ \\
KRS & to Oak Ridge Reserv. & $3.11 \mathrm{E}-02$ & $1.83 \mathrm{E}-02$ \\
KWS & to Oak Ridge Reserv. & $4.24 \mathrm{E}-03$ & $2.49 \mathrm{E}-03$ \\
Los Alamos NL & to INEL & $1.71 \mathrm{E}-03$ & $1.01 \mathrm{E}-04$ \\
Lawrence Berkeley & to Hanford Site & $7.79 \mathrm{E}-05$ & $4.38 \mathrm{E}-05$ \\
LERHR & to Hanford Site & $7.78 \mathrm{E}-06$ & $4.14 \mathrm{E}-06$ \\
Lawrence Livermore & to Hanford Site & $1.97 \mathrm{E}-02$ & $1.05 \mathrm{E}-02$ \\
Paducah GDP & to Oak Ridge Reserv. & $1.17 \mathrm{E}+00$ & $1.21 \mathrm{E}-01$ \\
Pantex Plant & to INEL & $4.15 \mathrm{E}-04$ & $2.58 \mathrm{E}-05$ \\
Pearl Harbor NS & to Hanford Site & $8.47 \mathrm{E}-06$ & $6.32 \mathrm{E}-06$ \\
Portsmouth GDP & to Oak Ridge Reserv. & $1.96 \mathrm{E}-03$ & $3.72 \mathrm{E}-04$ \\
Puget Sound NS & to Hanford Site & $1.95 \mathrm{E}-04$ & $1.46 \mathrm{E}-04$ \\
Reactive Metals & to Oak Ridge Reserv. & $2.01 \mathrm{E}-04$ & $3.81 \mathrm{E}-05$ \\
SNL - Albuquerque & to INEL & $1.85 \mathrm{E}-06$ & $1.12 \mathrm{E}-07$ \\
SNL - Livermore & to Hanford Site & $1.95 \mathrm{E}-03$ & $1.04 \mathrm{E}-03$ \\
& & & \\
\hline Totals & & & \\
\hline
\end{tabular}

Table 8. Expected Annual Fatalities for the Shipping Campaign

\begin{tabular}{lll}
\hline Exposure Group & Truck & Rail \\
\hline Radiological & $3.1 \mathrm{E}-04$ & $6.9 \mathrm{E}-05$ \\
Normal Crew & $4.4 \mathrm{E}-04$ & $2.6 \mathrm{E}-04$ \\
Normal Public & $6.4 \mathrm{E}-04$ & $9.2 \mathrm{E}-05$ \\
$\quad \begin{array}{cc}\text { Accident Public } \\
\text { Nonradiological }\end{array}$ & $2.0 \mathrm{E}-04$ & $5.6 \mathrm{E}-04$ \\
$\quad$ Emission & $1.6 \mathrm{E}-03$ & $4.9 \mathrm{E}-05$ \\
Accident & & \\
\hline
\end{tabular}


Table 9. Expected Annual Cancer Incidence for the Shipping Campaign

\begin{tabular}{lll}
\hline Exposure Group & Truck & \multicolumn{1}{c}{ Rail } \\
\hline Radiological & $1.1 \mathrm{E}-03$ & $2.4 \mathrm{E}-04$ \\
$\quad$ Normal Crew & $1.5 \mathrm{E}-03$ & $8.7 \mathrm{E}-04$ \\
Normal Public & $2.2 \mathrm{E}-03$ & $3.1 \mathrm{E}-04$ \\
Accident Public & & \\
Nonradiological & $2.0 \mathrm{E}-04$ & $5.6 \mathrm{E}-04$ \\
Emission & $\mathrm{NA}$ & $\mathrm{NA}$ \\
Accident & &
\end{tabular}

Table 10. Expected Annual Genetic Effects for the Shipping Campaign

\begin{tabular}{lll}
\hline Exposure Group & Truck & Rail \\
\hline Radiological & & \\
Normal Crew & $4.6 \mathrm{E}-05$ & $1.0 \mathrm{E}-05$ \\
Normal Public & $8.8 \mathrm{E}-05$ & $5.1 \mathrm{E}-05$ \\
Accident Public & $1.1 \mathrm{E}-05$ & $4.5 \mathrm{E}-06$ \\
Nonradiological & & \\
Emission & NA & NA \\
Accident & NA & NA \\
\hline
\end{tabular}




\section{B.24 WM LLMW REGIONALIZED 4 ALTERNATIVE (CASE 15): CONTACT-HANDLED ORGANIC LIQUIDS: ALPHA - UNTREATED}

Table 1. Summary of Route Information

\begin{tabular}{|c|c|c|c|c|c|c|}
\hline & \multirow{2}{*}{\multicolumn{2}{|c|}{ Route }} & \multirow{2}{*}{$\begin{array}{l}\text { Distance } \\
\text { (miles) }\end{array}$} & \multicolumn{3}{|c|}{ Percentage in Zone } \\
\hline & & & & Rural & Suburban & Urban \\
\hline \multicolumn{7}{|l|}{ Truck } \\
\hline Los Alamos NL & to INEL & & 1144 & 88.7 & 9.8 & 1.4 \\
\hline Lawrence Livermore & to INEL & & 972 & 85.7 & 10.4 & 4.0 \\
\hline Mound Plant & to Savannah & River & 656 & 66.5 & 32.7 & 0.8 \\
\hline Rocky Flats Plant & to INEL & & 716 & 91.3 & 8.1 & 0.5 \\
\hline West Valley DP & to Savannah & River & 883 & 70.3 & 28.5 & 1.2 \\
\hline \multicolumn{7}{|l|}{ Rail } \\
\hline Los Alamos NL & to INEL & & 1179 & 92.2 & 6.8 & 1.0 \\
\hline Lawrence Livermore & to INEL & & 1100 & 89.5 & 7.4 & 3.1 \\
\hline Mound Plant & to Savannah & River & 744 & 67.4 & 29.2 & 3.3 \\
\hline Rocky Flats Plant & to INEL & & 738 & 93.0 & 5.2 & 1.8 \\
\hline West valley DP & to Savannah & River & 1217 & 62.8 & 32.4 & 4.9 \\
\hline
\end{tabular}

Table 2. Annual Number of Shipments and One-Way Shipment Distances

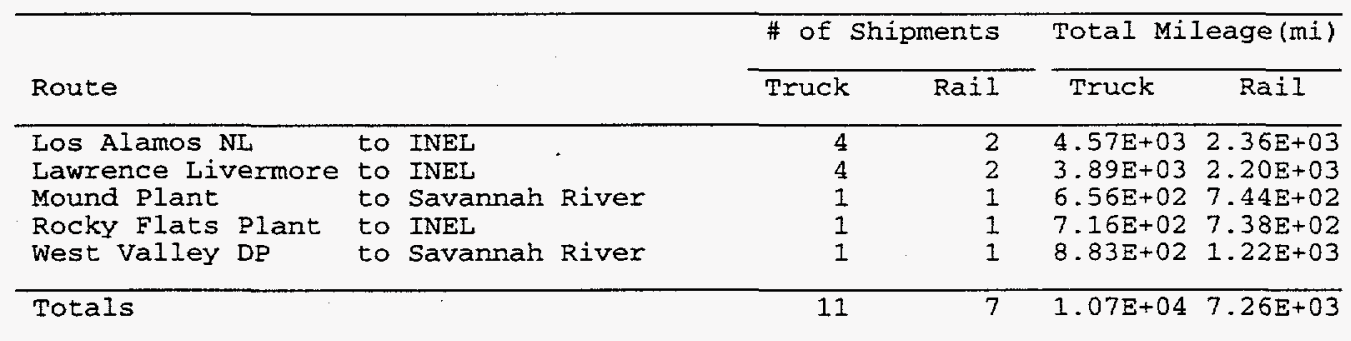

Table 3. Incident-Free Dose per Shipment (person-rem/shipment)

\begin{tabular}{|c|c|c|c|c|c|c|}
\hline \multirow[b]{2}{*}{ Source/Route (s) } & & \multirow[b]{2}{*}{ Crew } & \multicolumn{4}{|c|}{ General Public } \\
\hline & & & off-Link & On-Link & Stops & Total \\
\hline \multicolumn{7}{|l|}{ MLLW(OA) LA to IN } \\
\hline Los Alamos NL & $\begin{array}{l}\text { to INEL } \\
\text { Truck } \\
\text { Rail }\end{array}$ & $\begin{array}{l}3.15 \mathrm{E}-02 \\
7.80 \mathrm{E}-03\end{array}$ & $\begin{array}{l}1.73 E-03 \\
4.00 \mathrm{E}-03\end{array}$ & $\begin{array}{l}4.27 \mathrm{E}-03 \\
1.08 \mathrm{E}-04\end{array}$ & $\begin{array}{l}3.20 \mathrm{E}-02 \\
7.11 \mathrm{E}-03\end{array}$ & $\begin{array}{l}3.80 \mathrm{E}-02 \\
1.12 \mathrm{E}-02\end{array}$ \\
\hline \multicolumn{7}{|l|}{$\operatorname{MLLW}(O A)$ LL to IN } \\
\hline Lawrence Livermore & $\begin{array}{l}\text { to INEL } \\
\text { Truck } \\
\text { Rail }\end{array}$ & $\begin{array}{l}2.85 \mathrm{E}-02 \\
7.47 \mathrm{E}-03\end{array}$ & $\begin{array}{l}2.74 \mathrm{E}-03 \\
8.12 \mathrm{E}-03\end{array}$ & $\begin{array}{l}5.33 \mathrm{E}-03 \\
1.38 \mathrm{E}-04\end{array}$ & $\begin{array}{l}2.72 E-02 \\
6.87 E-03\end{array}$ & $\begin{array}{l}3.53 \mathrm{E}-02 \\
1.51 \mathrm{E}-02\end{array}$ \\
\hline \multicolumn{7}{|c|}{ thes } \\
\hline Mound Plant & $\begin{array}{l}\text { to Savan } \\
\text { Truck } \\
\text { Rail }\end{array}$ & $\begin{array}{l}\text { ah River } \\
2.21 \mathrm{E}-02 \\
5.99 \mathrm{E}-03\end{array}$ & $\begin{array}{l}1.91 \mathrm{E}-03 \\
9.25 \mathrm{E}-03\end{array}$ & $\begin{array}{l}2.83 \mathrm{E}-03 \\
1.78 \mathrm{E}-04\end{array}$ & $\begin{array}{l}1.84 \mathrm{E}-02 \\
5.77 \mathrm{E}-03\end{array}$ & $\begin{array}{l}2.31 \mathrm{E}-02 \\
1.52 \mathrm{E}-02\end{array}$ \\
\hline \\
\hline Rocky Flats Plant & $\begin{array}{l}\text { to INEL } \\
\text { Truck } \\
\text { Rail }\end{array}$ & $\begin{array}{l}1.90 \mathrm{E}-02 \\
5.96 \mathrm{E}-03\end{array}$ & $\begin{array}{l}6.95 \mathrm{E}-04 \\
3.32 \mathrm{E}-03\end{array}$ & $\begin{array}{l}2.22 E-03 \\
7.00 E-05\end{array}$ & $\begin{array}{l}2.00 \mathrm{E}-02 \\
5.75 \mathrm{E}-03\end{array}$ & $\begin{array}{l}2.30 \mathrm{E}-02 \\
9.14 \mathrm{E}-03\end{array}$ \\
\hline$M L L W(O A)$ WV to $S R$ & & & & & & \\
\hline West Valley DP & $\begin{array}{l}\text { to Savanr } \\
\text { Truck } \\
\text { Rail }\end{array}$ & $\begin{array}{l}\text { ah River } \\
2.89 \mathrm{E}-02 \\
7.96 \mathrm{E}-03\end{array}$ & $\begin{array}{l}2.45 \mathrm{E}-03 \\
1.95 \mathrm{E}-02\end{array}$ & $\begin{array}{l}3.86 \mathrm{E}-03 \\
3.38 \mathrm{E}-04\end{array}$ & $\begin{array}{l}2.47 \mathrm{E}-02 \\
7.23 \mathrm{E}-03\end{array}$ & $\begin{array}{l}3.10 \mathrm{E}-02 \\
2.70 \mathrm{E}-02\end{array}$ \\
\hline
\end{tabular}


Table 4. Accident Dose Risk per Shipment (person-rem/shipment)

\begin{tabular}{|c|c|c|c|}
\hline Source/Route & & Truck & Rail \\
\hline MLLW (OA) LA to IN & & & \\
\hline $\begin{array}{l}\text { LOS Alamos NL } \\
\text { MLIW(OA) LI to IN }\end{array}$ & to INEL & $2.33 E-02$ & $2.71 \mathrm{E}-03$ \\
\hline $\begin{array}{l}\text { Lawrence Livermore } \\
\text { MLIW(OA) MP to SR }\end{array}$ & to INEL & $1.16 \mathrm{E}-02$ & $7.55 \mathrm{E}-03$ \\
\hline $\begin{array}{l}\text { Mound Plant } \\
\text { MLLW(OA) RF to IN }\end{array}$ & to Savannah River & $4.15 \mathrm{E}-03$ & $7.63 \mathrm{E}-04$ \\
\hline $\begin{array}{l}\text { Rocky Flats Plant } \\
\text { MLLW(OA) WV to SR }\end{array}$ & to INEL & $3.75 E-03$ & $5.26 \mathrm{E}-04$ \\
\hline West valley DP & to Savannah River & $4.27 E-02$ & $1.37 \mathrm{E}-02$ \\
\hline
\end{tabular}

Table 5. Nonradiological Risk Factors per Shipment (fatalities/shipment)

\begin{tabular}{llll}
\hline Mode & Route & Emission & Accident \\
\hline Truck & & & \\
Los Alamos NL & to INEL & $5.25 \mathrm{E}-06$ & $8.21 \mathrm{E}-05$ \\
Lawrence Livermore & to INEL & $1.24 \mathrm{E}-05$ & $5.73 \mathrm{E}-05$ \\
$\begin{array}{l}\text { Mound Plant } \\
\text { Rocky Flats Plant }\end{array}$ & to Savannah River & $1.67 \mathrm{E}-06$ & $5.26 \mathrm{E}-05$ \\
West Valley DP & to SNEL & $1.42 \mathrm{E}-06$ & $4.58 \mathrm{E}-05$ \\
Rail & & $3.28 \mathrm{E}-06$ & $6.60 \mathrm{E}-05$ \\
Los Alamos NL & to INEL & $4.90 \mathrm{E}-06$ & $2.47 \mathrm{E}-06$ \\
Lawrence Livermore & to INEL & $1.41 \mathrm{E}-05$ & $2.30 \mathrm{E}-06$ \\
Mound Plant & to Savannah River & $1.03 \mathrm{E}-05$ & $1.56 \mathrm{E}-06$ \\
Rocky Flats Plant & to INEL & $5.44 \mathrm{E}-06$ & $1.54 \mathrm{E}-06$ \\
West Valley DP & to Savannah River & $2.49 \mathrm{E}-05$ & $2.55 \mathrm{E}-06$ \\
\hline
\end{tabular}

Table 6. Annual Incident-Free Dose for the Shipping Campaign (person-rem/yr)

\begin{tabular}{|c|c|c|c|c|c|}
\hline \multirow[b]{2}{*}{ Route } & & \multicolumn{2}{|c|}{ Truck } & \multicolumn{2}{|c|}{ Rail } \\
\hline & & Crew & Public & Crew & Public \\
\hline $\begin{array}{l}\text { LOs Alamos NL } \\
\text { Lawrence Livermore } \\
\text { Mound Plant } \\
\text { Rocky Flats Plant } \\
\text { West Valley DP }\end{array}$ & $\begin{array}{l}\text { to INEL } \\
\text { to INEL } \\
\text { to Savannah River } \\
\text { to INEL } \\
\text { to Savannah River }\end{array}$ & $\begin{array}{l}1.26 \mathrm{E}-01 \\
1.14 \mathrm{E}-01 \\
2.21 \mathrm{E}-02 \\
1.90 \mathrm{E}-02 \\
2.89 \mathrm{E}-02\end{array}$ & $\begin{array}{l}1.52 \mathrm{E}-01 \\
1.41 \mathrm{E}-01 \\
2.31 \mathrm{E}-02 \\
2.30 \mathrm{E}-02 \\
3.10 \mathrm{E}-02\end{array}$ & $\begin{array}{l}1.56 \mathrm{E}-02 \\
1.49 \mathrm{E}-02 \\
5.99 \mathrm{E}-03 \\
5.96 \mathrm{E}-03 \\
7.96 \mathrm{E}-03\end{array}$ & $\begin{array}{l}2.24 \mathrm{E}-02 \\
3.03 \mathrm{E}-02 \\
1.52 \mathrm{E}-02 \\
9.14 \mathrm{E}-03 \\
2.70 \mathrm{E}-02\end{array}$ \\
\hline
\end{tabular}

Table 7. Annual Accident Dose Risk for the Shipping Campaign (person-rem/yr)

\begin{tabular}{llcc}
\hline Route & & Truck & Rail \\
\hline Los Alamos NL & to INEL & $9.32 \mathrm{E}-02$ & $5.42 \mathrm{E}-03$ \\
Lawrence Livermore & to INEL & $4.63 \mathrm{E}-02$ & $1.51 \mathrm{E}-02$ \\
Mound Plant & to Savannah River & $4.15 \mathrm{E}-03$ & $7.63 \mathrm{E}-04$ \\
Rocky Flats Plant & to INEL & $3.75 \mathrm{E}-03$ & $5.26 \mathrm{E}-04$ \\
West Valley DP & to Savannah River & $4.27 \mathrm{E}-02$ & $1.37 \mathrm{E}-02$ \\
\hline Totals & & $1.90 \mathrm{E}-01$ & $3.55 \mathrm{E}-02$ \\
\hline
\end{tabular}

Table 8. Expected Annual Fatalities for the Shipping Campaign

\begin{tabular}{lll}
\hline Exposure Group & Truck & Rail \\
\hline Radiological & $1.2 \mathrm{E}-04$ & $2.0 \mathrm{E}-05$ \\
Normal Crew & $1.9 \mathrm{E}-04$ & $5.2 \mathrm{E}-05$ \\
Normal Public & $9.5 \mathrm{E}-05$ & $1.8 \mathrm{E}-05$ \\
Accident Public & $7.7 \mathrm{E}-05$ & $7.9 \mathrm{E}-05$ \\
Nonradiological & $7.2 \mathrm{E}-04$ & $1.5 \mathrm{E}-05$ \\
Enission & & \\
Accident &
\end{tabular}




\begin{tabular}{lll}
$\begin{array}{l}\text { Table 9. Expected Annual Cancer } \\
\text { Eor the Shipping Campaign }\end{array}$ & \\
\hline & Truck & Rail \\
\hline Exposure Group & & \\
\hline Radiological & $4.3 \mathrm{E}-04$ & $7.1 \mathrm{E}-05$ \\
$\quad$ Normal Crew & $6.3 \mathrm{E}-04$ & $1.8 \mathrm{E}-04$ \\
$\quad$ Normal Public & $3.2 \mathrm{E}-04$ & $6.0 \mathrm{E}-05$ \\
Accident Public & $7.7 \mathrm{E}-05$ & $7.9 \mathrm{E}-05$ \\
Nonradiological & $\mathrm{NA}$ & $\mathrm{NA}$ \\
$\quad$ Emission & & \\
Accident & & \\
\hline
\end{tabular}

Table 10. Expected Annual Genetic Effects for the Shipping Campaign

\begin{tabular}{lll}
\hline Exposure Group & Truck & Rail \\
\hline Radiological & & \\
Normal Crew & $1.9 E-05$ & $3.0 \mathrm{E}-06$ \\
Normal Public & $3.7 \mathrm{E}-05$ & $1.0 \mathrm{E}-05$ \\
Accident Public & $4.9 \mathrm{E}-06$ & $9.0 \mathrm{E}-07$ \\
Nonradiological & & \\
Emission & NA & NA \\
Accident & NA & NA \\
\hline
\end{tabular}




\section{B.25 WM LLMW REGIONALIZED 4 ALTERNATIVE (CASE 15): CONTACT-HANDLED HETEROGENEOUS SOLIDS: NONALPHA - UNTREATED}

Table 1. Summary of Route Information

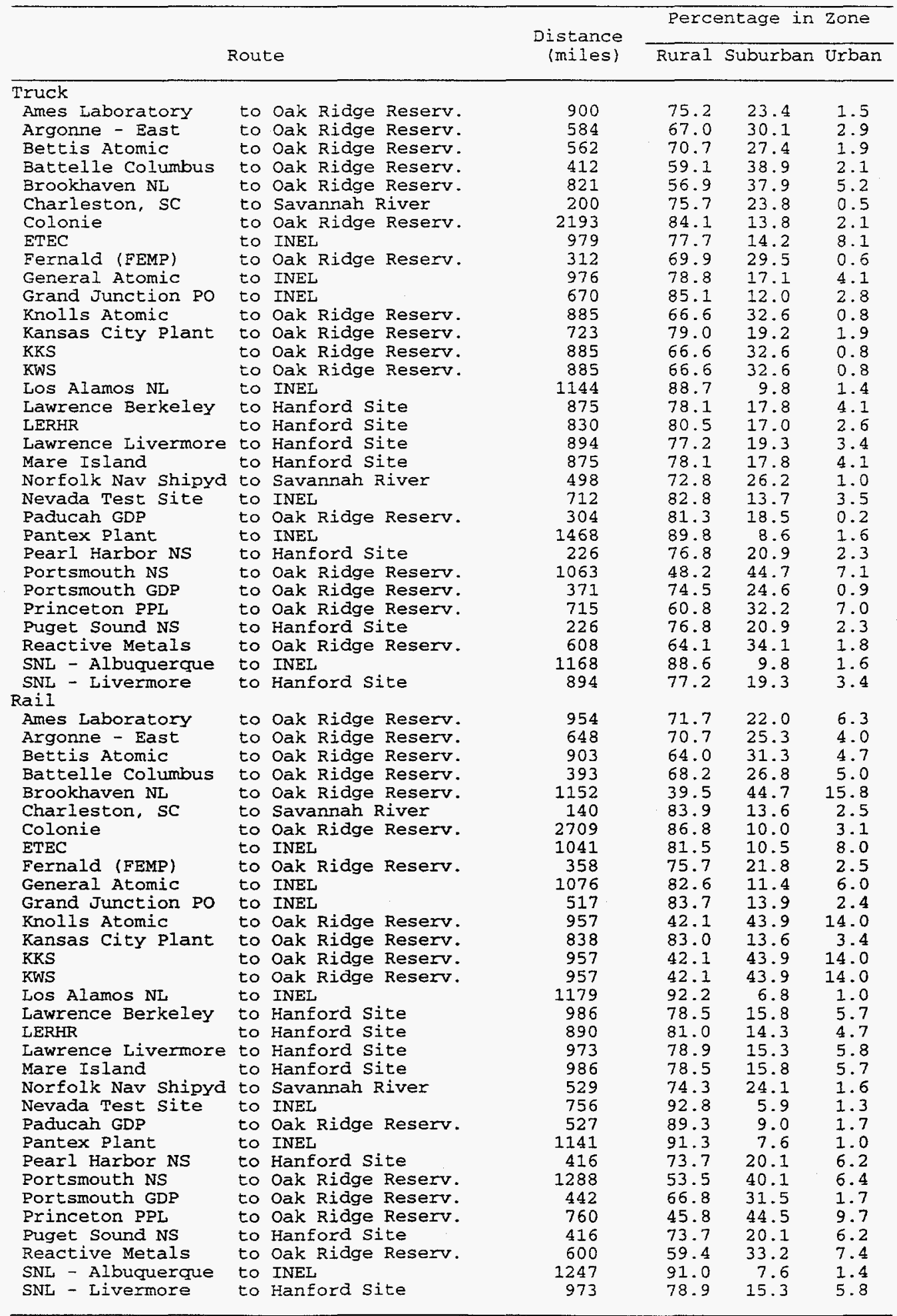


Table 2. Annual Number of Shipments and One-Way Shipment Distances

\begin{tabular}{|c|c|c|c|c|c|c|}
\hline \multirow[b]{2}{*}{ Route } & & & \multicolumn{2}{|c|}{ \# of Shipments } & \multicolumn{2}{|c|}{ Total Mileage (mi) } \\
\hline & & & Truck & Rail & Truck & Rail \\
\hline Ames Laboratory & to & Oak Ridge Reserv. & 1 & 1 & $9.00 E+02$ & $9.54 E+02$ \\
\hline Argonne - East & & Oak Ridge Reserv. & 44 & 17 & $2.57 \mathrm{E}+04$ & $1.10 \mathrm{E}+04$ \\
\hline Bettis Atomic & to & Oak Ridge Reserv. & 1 & 1 & $5.62 \mathrm{E}+02$ & $9.03 E+02$ \\
\hline Battelle columbus & to & Oak Ridge Reserv. & $I$ & 1 & $4.12 \mathrm{E}+02$ & $3.93 \mathrm{E}+02$ \\
\hline Brookhaven NL & to & Oak Ridge Reserv. & 1 & 1 & $8.21 E+02$ & $1.15 \mathrm{E}+03$ \\
\hline Charleston, SC & to & Savannah River & $\bar{\imath}$ & 1 & $2.00 E+02$ & $1.40 \mathrm{E}+02$ \\
\hline Colonie & to & Oak Ridge Reserv. & 1 & 1 & $2.19 E+03$ & $2.71 E+03$ \\
\hline ETEC & to & INEL & 8 & 3 & $7.83 E+03$ & $3.12 \mathrm{E}+03$ \\
\hline Fernald (FEMP) & to & Oak Ridge Reserv. & 7 & 3 & $2.18 \mathrm{E}+03$ & $1.07 E+03$ \\
\hline General Atomic & to & INEL & 1 & 1 & $9.76 \mathrm{E}+02$ & $1.08 \mathrm{E}+03$ \\
\hline Grand Junction PO & to & INEL & 1 & 1 & $6.70 \mathrm{E}+02$ & $5.17 \mathrm{E}+02$ \\
\hline Knolis Atomic & to & Oak Ridge Reserv. & 1 & 1 & $8.85 E+02$ & $9.57 \mathrm{E}+02$ \\
\hline Kansas City Plant & to & Oak Ridge Reserv. & 1 & 1 & $7.23 \mathrm{E}+02$ & $8.38 \mathrm{E}+02$ \\
\hline KKS & to & Oak Ridge Reserv. & 1 & 1 & $8.85 E+02$ & $9.57 \mathrm{E}+02$ \\
\hline KWS & to & Oak Ridge Reserv. & 1 & 1 & $8.85 E+02$ & $9.57 E+02$ \\
\hline Los Alamos NL & to & INEL & 2 & 1 & $2.29 E+03$ & $1.18 \mathrm{E}+03$ \\
\hline Lawrence Berkeley & to & Hanford Site & 1 & 1 & $8.75 E+02$ & $9.86 \mathrm{E}+02$ \\
\hline LERHR & to & Hanford Site & 1 & 1 & $8.30 E+02$ & $8.90 E+02$ \\
\hline Lawrence Livermore & to & Hanford Site & 4 & 2 & $3.58 E+03$ & $1.95 E+03$ \\
\hline Mare Island & to & Hanford Site & 1 & 1 & $8.75 E+02$ & $9.86 \mathrm{E}+02$ \\
\hline Norfolk Nav Shipyd & to & Savannah River & 1 & 1 & $4.98 \mathrm{E}+02$ & $5.29 \mathrm{E}+02$ \\
\hline Nevada Test Site & to & INEL & $\overline{1}$ & 1 & $7.12 \mathrm{E}+02$ & $7.56 E+02$ \\
\hline Paducah GDP & to & Oak Ridge Reserv. & 1 & 1 & $3.04 E+02$ & $5.27 \mathrm{E}+02$ \\
\hline Pantex Plant & to & INEL & 2 & 1 & $2.94 \mathrm{E}+03$ & $1.14 \mathrm{E}+03$ \\
\hline Pearl Harbor NS & & Hanford Site & 1 & 1 & $2.26 \mathrm{E}+02$ & $4.16 \mathrm{E}+02$ \\
\hline Portsmouth NS & to & Oak Ridge Reserv. & 1 & 1 & $1.06 \mathrm{E}+03$ & $1.29 \mathrm{E}+03$ \\
\hline Portsmouth GDP & to & Oak Ridge Reserv. & 60 & 23 & $2.22 \mathrm{E}+04$ & $1.02 \mathrm{E}+04$ \\
\hline Princeton PPL & to & Oak Ridge Reserv. & 1 & 1 & $7.15 E+02$ & $7.60 \mathrm{E}+02$ \\
\hline Puget Sound NS & & Hanford Site & 1 & 1 & $2.26 E+02$ & $4.16 \mathrm{E}+02$ \\
\hline Reactive Metals & & Oak Ridge Reserv. & 1 & 1 & $6.08 \mathrm{E}+02$ & $6.00 \mathrm{E}+02$ \\
\hline SNL - Albuquerque & to & INEL & 1 & 1 & $1.17 \mathrm{E}+03$ & $1.25 E+03$ \\
\hline SNL - Livermore & to & Hanford Site & 1 & 1 & $8.94 E+02$ & $9.73 E+02$ \\
\hline Totals & & & 152 & 75 & $8.59 \mathrm{E}+04$ & $5.16 \mathrm{E}+04$ \\
\hline
\end{tabular}


Table 3. Incident-Free Dose per Shipment (person-rem/shipment)

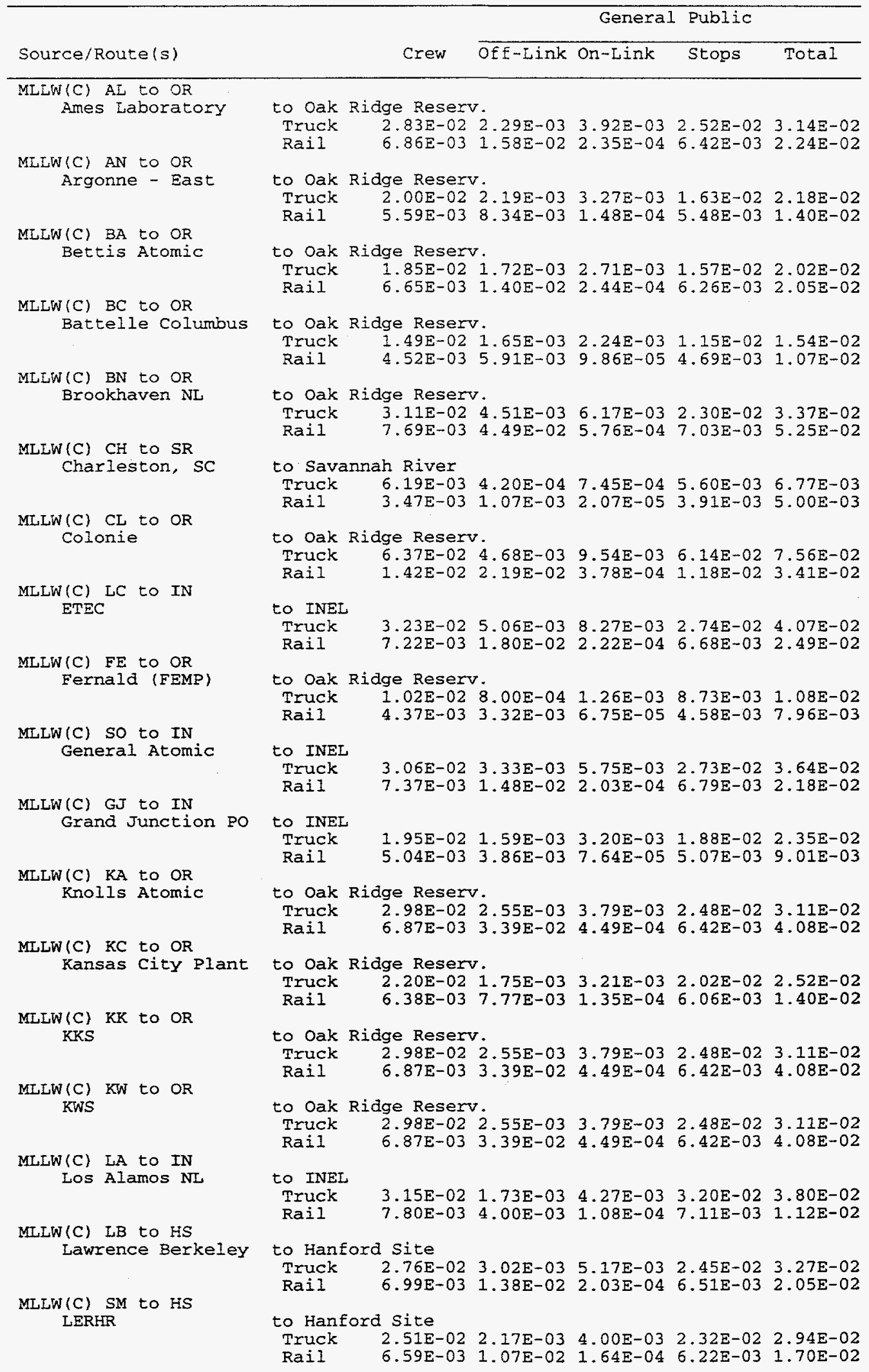


Table 3. Incident-Free Dose per Shipment (person-rem/shipment) (Cont.)

\begin{tabular}{|c|c|c|c|c|c|}
\hline \multirow[b]{2}{*}{ Source/Route (s) } & \multirow[b]{2}{*}{ Crew } & \multicolumn{4}{|c|}{ General Public } \\
\hline & & off-Link & on-Iink & Stops & Total \\
\hline \multicolumn{6}{|l|}{$\begin{array}{l}\text { MLLW(C) LL to HS } \\
\text { Lawrence Liver }\end{array}$} \\
\hline & $\begin{array}{ll}\text { Truck } & 2.82 \mathrm{E}-02 \\
\text { Rail } & 6.94 \mathrm{E}-03\end{array}$ & $\begin{array}{l}2.88 \mathrm{E}-03 \\
1.38 \mathrm{E}-02\end{array}$ & $\begin{array}{l}4.92 \mathrm{E}-03 \\
2.00 \mathrm{E}-04\end{array}$ & $\begin{array}{l}2.50 \mathrm{E}-02 \\
6.48 \mathrm{E}-03\end{array}$ & $\begin{array}{l}3.28 \mathrm{E}-02 \\
2.05 \mathrm{E}-02\end{array}$ \\
\hline \multirow{2}{*}{\multicolumn{6}{|c|}{$\begin{array}{c}\text { MLLW(C) MZ to HS } \\
\text { Mare Island }\end{array}$}} \\
\hline & & & & & \\
\hline & $\begin{array}{ll}\text { Truck } & 2.76 \mathrm{E}-02 \\
\text { Rail } & 6.99 \mathrm{E}-03\end{array}$ & $\begin{array}{l}3.02 \mathrm{E}-03 \\
1.38 \mathrm{E}-02\end{array}$ & $\begin{array}{l}5.17 \mathrm{E}-03 \\
2.03 \mathrm{E}-04\end{array}$ & $\begin{array}{l}2.45 \mathrm{E}-02 \\
6.51 \mathrm{E}-03\end{array}$ & $\begin{array}{l}3.27 \mathrm{E}-02 \\
2.05 \mathrm{E}-02\end{array}$ \\
\hline \multicolumn{6}{|c|}{$\begin{array}{l}\text { MLLW(C) US to SR } \\
\text { Norfolk Nav Shipyd to Savannah River }\end{array}$} \\
\hline & Truck $\quad 1.59 \mathrm{E}-02$ & $1.26 \mathrm{E}-03$ & $2.07 E-03$ & $1.39 \mathrm{E}-02$ & 1. $73 \mathrm{E}-02$ \\
\hline \multirow{2}{*}{\multicolumn{6}{|c|}{$\begin{array}{l}\text { MLLW(C) NT to IN } \\
\text { Nevada Test site }\end{array}$}} \\
\hline & & & & & \\
\hline & $\begin{array}{l}2.13 E-02 \\
6.04 E-03\end{array}$ & $\begin{array}{l}2.03 \mathrm{E}-03 \\
2.80 \mathrm{E}-03\end{array}$ & $\begin{array}{l}3.79 \mathrm{E}-03 \\
6.88 \mathrm{E}-05\end{array}$ & $\begin{array}{l}1.99 \mathrm{E}-02 \\
5.81 \mathrm{E}-03\end{array}$ & $\begin{array}{l}2.58 E-02 \\
8.67 E-03\end{array}$ \\
\hline \multirow{2}{*}{\multicolumn{6}{|c|}{$\begin{array}{c}\text { MLLW(C) PA to OR } \\
\text { Paducah GDP }\end{array}$}} \\
\hline & to Oak Ridge Reser & & & & \\
\hline & $\begin{array}{l}8.88 \mathrm{E}-03 \\
5.08 \mathrm{E}-03\end{array}$ & $4.70 \mathrm{E}-04$ & $9.98 \mathrm{E}-04$ & $8.51 \mathrm{E}-03$ & $9.98 \mathrm{E}-03$ \\
\hline \multirow{2}{*}{\multicolumn{6}{|c|}{$\begin{array}{l}\text { MLLW(C) PP to IN } \\
\text { Pantex Plant }\end{array}$}} \\
\hline Pantex Plant & to INEL & & & & \\
\hline & $\begin{array}{l}4.01 \mathrm{E}-02 \\
7.64 \mathrm{E}-03\end{array}$ & $\begin{array}{l}2.24 \mathrm{E}-03 \\
4.12 \mathrm{E}-03\end{array}$ & $\begin{array}{l}5.63 E-03 \\
1.10 E-04\end{array}$ & $\begin{array}{l}4.11 \mathrm{E}-02 \\
6.99 \mathrm{E}-03\end{array}$ & $\begin{array}{l}4.90 \mathrm{E}-02 \\
1.12 \mathrm{E}-02\end{array}$ \\
\hline \multicolumn{6}{|l|}{$\begin{array}{l}\text { MLLW(C) SW to HS } \\
\text { PearI Harbor NS }\end{array}$} \\
\hline & $7.06 \mathrm{E}-03$ & $6.28 \mathrm{E}-04$ & $1.09 E-03$ & $6.33 E-03$ & $8.04 \mathrm{E}-03$ \\
\hline & \multicolumn{4}{|c|}{ MLLW (C) PT to OR } & $1.15 \mathrm{E}-02$ \\
\hline Portsmouth NS & to Oak Ridge Reser & & & & \\
\hline & $\begin{array}{l}4.37 E-02 \\
8.26 E-03\end{array}$ & $\begin{array}{l}7.41 \mathrm{E}-03 \\
2.64 \mathrm{E}-02\end{array}$ & $\begin{array}{l}9.70 \mathrm{E}-03 \\
4.36 \mathrm{E}-04\end{array}$ & $\begin{array}{l}2.98 \mathrm{E}-02 \\
7.45 \mathrm{E}-03\end{array}$ & $\begin{array}{l}4.69 \mathrm{E}-02 \\
3.42 \mathrm{E}-02\end{array}$ \\
\hline \multicolumn{6}{|l|}{$\begin{array}{l}\text { MLLW(C) Po to OR } \\
\text { Portsmouth GDP }\end{array}$} \\
\hline Portsmouth GDP & $\begin{array}{lr}\text { to Oak } & \text { Ridge Reser } \\
\text { Truck } & 1.16 \mathrm{E}-02 \\
\text { Rail } & 4.73 \mathrm{E}-03\end{array}$ & $\begin{array}{l}8.77 \mathrm{E}-04 \\
4.37 \mathrm{E}-03\end{array}$ & $\begin{array}{l}1.50 \mathrm{E}-03 \\
1.00 \mathrm{E}-04\end{array}$ & $\begin{array}{l}1.04 \mathrm{E}-02 \\
4.84 \mathrm{E}-03\end{array}$ & $\begin{array}{l}1.28 E-02 \\
9.32 E-03\end{array}$ \\
\hline \\
\hline Princeton PPL & $\begin{array}{cr}\text { to Oak Ridge Reser } \\
\text { Truck } & 2.68 \mathrm{E}-02 \\
\text { Rail } & 6.05 \mathrm{E}-03\end{array}$ & $\begin{array}{r}4.27 E-03 \\
2.10 \mathrm{E}-02\end{array}$ & $\begin{array}{l}6.06 \mathrm{E}-03 \\
3.11 \mathrm{E}-04\end{array}$ & $\begin{array}{l}2.00 \mathrm{E}-02 \\
5.82 \mathrm{E}-03\end{array}$ & $\begin{array}{l}3.04 \mathrm{E}-02 \\
2.71 \mathrm{E}-02\end{array}$ \\
\hline \multicolumn{6}{|l|}{ MLLW (C) PN to HS } \\
\hline Puget Sound NS & to Hanford Site & & & & \\
\hline & $\begin{array}{l}7.06 \mathrm{E}-03 \\
4.62 \mathrm{E}-03\end{array}$ & $\begin{array}{l}6.28 \mathrm{E}-04 \\
6.63 \mathrm{E}-03\end{array}$ & $\begin{array}{l}1.09 \mathrm{E}-03 \\
9.79 \mathrm{E}-05\end{array}$ & $\begin{array}{l}6.33 \mathrm{E}-03 \\
4.76 \mathrm{E}-03\end{array}$ & $\begin{array}{l}8.04 \mathrm{E}-03 \\
1.15 \mathrm{E}-02\end{array}$ \\
\hline \multicolumn{6}{|l|}{$\begin{array}{l}\text { MLIW(C) RM to OR } \\
\text { Reactive Metals }\end{array}$} \\
\hline Reactive Metals & $\begin{array}{cr}\text { to Oak } & \text { Ridge Reser } \\
\text { Truck } & 2.11 \mathrm{E}-02 \\
\text { Rail } & 5.38 \mathrm{E}-03\end{array}$ & $\begin{array}{l}\text { v. } \\
2.15 E-03 \\
1.25 E-02\end{array}$ & $\begin{array}{l}3.08 \mathrm{E}-03 \\
1.91 \mathrm{E}-04\end{array}$ & $\begin{array}{l}1.70 \mathrm{E}-02 \\
5.33 \mathrm{E}-03\end{array}$ & $\begin{array}{l}2.23 \mathrm{E}-02 \\
1.80 \mathrm{E}-02\end{array}$ \\
\hline \\
\hline SNL - Albuquerque & $\begin{array}{l}\text { to INEL } \\
\text { Truck } \\
\text { Rail }\end{array}$ & $\begin{array}{l}1.83 E-03 \\
5.30 E-03\end{array}$ & $\begin{array}{l}4.46 \mathrm{E}-03 \\
1.26 \mathrm{E}-04\end{array}$ & $\begin{array}{l}3.27 \mathrm{E}-02 \\
7.32 \mathrm{E}-03\end{array}$ & $\begin{array}{l}3.90 \mathrm{E}-02 \\
1.27 \mathrm{E}-02\end{array}$ \\
\hline \multirow{3}{*}{$\begin{array}{r}\text { MLIW(C) SL to HS } \\
\text { SNL - Livermore }\end{array}$} & & & & & \\
\hline & to Hanford Site & & & & \\
\hline & $\begin{array}{ll}\text { Truck } & 2.82 \mathrm{E}-02 \\
\text { Rail } & 6.94 \mathrm{E}-03\end{array}$ & $\begin{array}{l}2.88 \mathrm{E}-03 \\
1.38 \mathrm{E}-02\end{array}$ & $\begin{array}{l}4.92 \mathrm{E}-03 \\
2.00 \mathrm{E}-04\end{array}$ & $\begin{array}{l}2.50 \mathrm{E}-02 \\
6.48 \mathrm{E}-03\end{array}$ & $\begin{array}{l}3.28 \mathrm{E}-02 \\
2.05 \mathrm{E}-02\end{array}$ \\
\hline
\end{tabular}


Table 4. Accident Dose Risk per Shipment (person-rem/shipment)

\begin{tabular}{|c|c|c|c|}
\hline Source/Route & & Truck & Rail \\
\hline MLLW(C) AL to OR & & & \\
\hline $\begin{array}{l}\text { Ames Laboratory } \\
\text { MLLW(C) AN to OR }\end{array}$ & to Oak Ridge Reserv. & $2.44 \mathrm{E}-08$ & $7.95 E-09$ \\
\hline $\begin{array}{l}\text { Argonne - East } \\
\text { MLIW(C) BA to OR }\end{array}$ & to Oak Ridge Reserv. & $6.72 \mathrm{E}-04$ & $3.03 E-04$ \\
\hline $\begin{array}{l}\text { Bettis Atomic } \\
\text { MLIW(C) BC to OR }\end{array}$ & to Oak Ridge Reserv. & $1.56 E-04$ & $6.60 E-05$ \\
\hline $\begin{array}{l}\text { Battelle Columbus } \\
\text { MLIW (C) BN to OR }\end{array}$ & to Oak Ridge Reserv. & $1.96 \mathrm{E}-09$ & $2.32 \mathrm{E}-10$ \\
\hline $\begin{array}{l}\text { Brookhaven NL } \\
\text { MLLW(C) CH to SR }\end{array}$ & to Oak Ridge Reserv. & $5.76 \Xi-04$ & $3.98 E-04$ \\
\hline $\begin{array}{l}\text { Charleston, SC } \\
\text { MLLW (C) CL to OR }\end{array}$ & to Savannah River & $5.98 E-05$ & $6.95 E-06$ \\
\hline $\begin{array}{l}\text { Colonie } \\
\text { MLLW(C) LC to IN }\end{array}$ & to Oak Ridge Reserv. & $3.67 \Xi-08$ & $1.06 \mathrm{E}-08$ \\
\hline $\begin{array}{l}\text { ETEC } \\
\text { MLLW (C) FE to OR }\end{array}$ & to INEL & $2.18 \Xi-04$ & $2.61 E-04$ \\
\hline $\begin{array}{l}\text { Fernald (FEMP) } \\
\text { MLLW(C) So to IN }\end{array}$ & to Oak Ridge Reserv. & $1.04 \Xi-05$ & $2.79 E-06$ \\
\hline $\begin{array}{l}\text { General Atomic } \\
\text { MLLW(C) GJ to IN }\end{array}$ & to INEL & $4.51 E-07$ & $1.77 E-07$ \\
\hline $\begin{array}{l}\text { Grand Junction PO } \\
\text { MLLW(C) KA to OR }\end{array}$ & to INEL & $1.26 \mathrm{E}-08$ & $3.64 E-09$ \\
\hline $\begin{array}{l}\text { Knolls Atomic } \\
\text { MLLW(C) KC to OR }\end{array}$ & to Oak Ridge Reserv. & $1.78 \mathrm{E}-02$ & $1.05 E-02$ \\
\hline $\begin{array}{l}\text { Kansas City Plant } \\
\text { MLIW(C) KK to OR }\end{array}$ & to Oak Ridge Reserv. & $1.79 E-04$ & $3.07 E-05$ \\
\hline $\begin{array}{l}\text { KKS } \\
\text { MLLW(C) KW to OR }\end{array}$ & to Oak Ridge Reserv. & $1.96 \mathrm{E}-02$ & $1.15 E-02$ \\
\hline $\begin{array}{l}\text { KWS } \\
\text { MLIW(C) LA to IN }\end{array}$ & to Oak Ridge Reserv. & $6.28 \mathrm{E}-03$ & $3.68 \mathrm{E}-03$ \\
\hline $\begin{array}{l}\text { Los Alamos NL } \\
\text { MLLW(C) LB to HS }\end{array}$ & to INEL & $5.25 E-05$ & $6.56 \mathrm{E}-06$ \\
\hline $\begin{array}{l}\text { Lawrence Berkeley } \\
\text { MIIW(C) SM to HS }\end{array}$ & to Hanford Site & $1.05 E-05$ & $5.80 E-06$ \\
\hline LERHR & to Hanford Site & $9.03 E-06$ & $4.74 E-06$ \\
\hline $\begin{array}{l}\text { MLLW (C) LL to HS } \\
\text { Lawrence Livermore } \\
\text { MLLW(C) MZ to HS }\end{array}$ & to Hanford Site & $4.37 E-05$ & $4.58 \mathrm{E}-05$ \\
\hline $\begin{array}{l}\text { Mare Island } \\
\text { MLIW(C) US to SR }\end{array}$ & to Hanford Site & $8.65 \mathrm{E}-03$ & $4.86 \mathrm{E}-03$ \\
\hline $\begin{array}{l}\text { Norfolk Nav Shipyd } \\
\text { MLIW(C) NT to IN }\end{array}$ & to Savannah River & $8.05 E-04$ & $1.41 E-04$ \\
\hline $\begin{array}{l}\text { Nevada Test Site } \\
\text { MLIW(C) PA to OR }\end{array}$ & to INEI. & 1.301 & $2.10 \mathrm{E}-09$ \\
\hline $\begin{array}{l}\text { Paducah GDP } \\
\text { MLLW(C) PP to IN }\end{array}$ & to Oak Ridge Reserv. & $9.758-04$ & $1.25 \mathrm{E}-04$ \\
\hline $\begin{array}{l}\text { Pantex Plant } \\
\text { MLLW(C) SW to HS }\end{array}$ & to INEL & $1.88 E-04$ & $2.55 E-05$ \\
\hline $\begin{array}{l}\text { Pearl Harbor NS } \\
\text { MLIW(C) PT to OR }\end{array}$ & to Hanford Site & $1.11 E-04$ & $8.21 E-05$ \\
\hline $\begin{array}{l}\text { Portsmouth NS } \\
\text { MLLW(C) PO to OR }\end{array}$ & to Oak Ridge Reserv. & $3.89 \varepsilon-04$ & $1.70 E-04$ \\
\hline $\begin{array}{l}\text { Portsmouth GDP } \\
\text { MLIW(C) PR to OR }\end{array}$ & to Oak Ridge Reserv. & $2.80 \pm-06$ & $1.38 \mathrm{E}-06$ \\
\hline $\begin{array}{l}\text { Princeton PPL } \\
\text { MLLW(C) PN to HS }\end{array}$ & to Oak Ridge Reserv. & $3.07 \pm-09$ & $1.20 E-09$ \\
\hline $\begin{array}{l}\text { Puget Sound NS } \\
\text { MLIW (C) RM to oR }\end{array}$ & to Hanford Site & $2.47 \mathrm{E}-03$ & $1.83 \mathrm{E}-03$ \\
\hline $\begin{array}{l}\text { Reactive Metals } \\
\text { MLLW(C) SA to IN }\end{array}$ & to Oak Ridge Reserv. & $1.83 E-06$ & $3.43 E-07$ \\
\hline $\begin{array}{l}\text { SNL - Albuquerque } \\
\text { MLLW(C) SL to HS }\end{array}$ & to INEL & $2.77 E-04$ & $1.78 E-05$ \\
\hline SNI - Livermore & to Hanford Site & $2.72 E-06$ & $1.43 E-06$ \\
\hline
\end{tabular}


Table 5. Nonradiological Risk Factors per Shipment (fatalities/shipment)

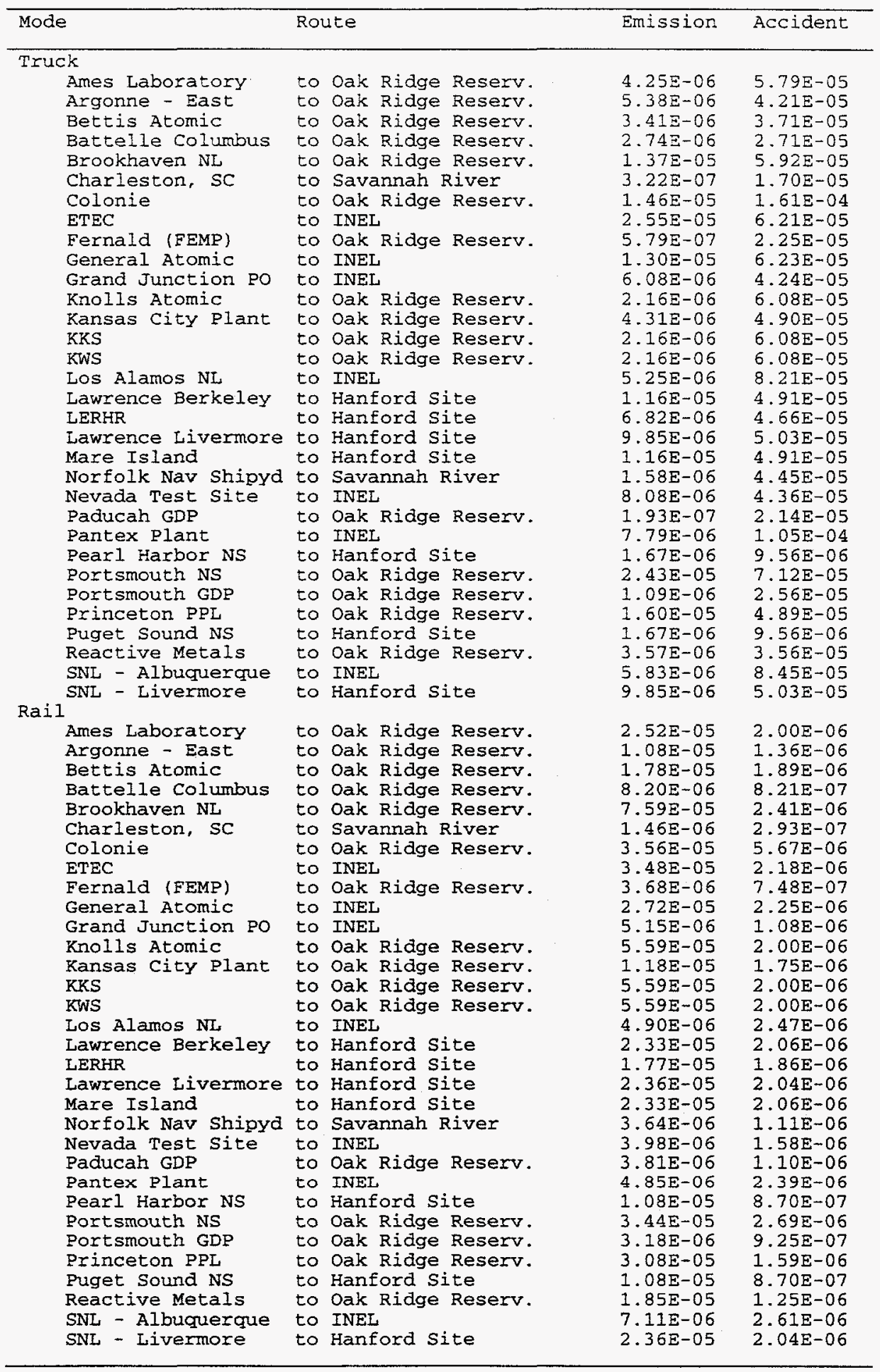


Table 6. Annual Incident-Free Dose for the Shipping Campaign (person-rem/yr)

\begin{tabular}{|c|c|c|c|c|c|c|}
\hline \multirow[b]{2}{*}{ Route } & & & \multicolumn{2}{|c|}{ Truck } & \multicolumn{2}{|c|}{ Rail } \\
\hline & & & Crew & Public & Crew & Public \\
\hline $\begin{array}{l}\text { Ames Laboratory } \\
\text { Argonne - East } \\
\text { Bettis Atomic } \\
\text { Battelle Columbus } \\
\text { Brookhaven NL } \\
\text { Charleston, SC } \\
\text { Colonie } \\
\text { ETEC } \\
\text { Fernald (FEMP) } \\
\text { General Atomic } \\
\text { Grand Junction Po } \\
\text { Knolls Atomic } \\
\text { Kansas City Plant } \\
\text { KKS } \\
\text { KWS } \\
\text { Los Alamos NL } \\
\text { Lawrence Berkeley } \\
\text { LERHR } \\
\text { Lawrence Livermore } \\
\text { Mare Island } \\
\text { Norfolk Nav Shipyd } \\
\text { Nevada Test Site } \\
\text { Paducah GDP } \\
\text { Pantex Plant } \\
\text { Pearl Harbor NS } \\
\text { Portsmouth NS } \\
\text { Portsmouth GDP } \\
\text { Princeton PPL } \\
\text { Puget Sound NS } \\
\text { Reactive Metals } \\
\text { SNL - Albuquerque } \\
\text { SNL - Livermore }\end{array}$ & $\begin{array}{l}\text { to } \\
\text { to } \\
\text { to } \\
\text { to } \\
\text { to } \\
\text { to } \\
\text { to } \\
\text { to } \\
\text { to } \\
\text { to } \\
\text { to } \\
\text { to } \\
\text { to } \\
\text { to } \\
\text { to } \\
\text { to } \\
\text { to } \\
\text { to } \\
\text { to } \\
\text { to } \\
\text { to } \\
\text { to } \\
\text { to } \\
\text { to } \\
\text { to } \\
\text { to } \\
\text { to } \\
\text { to } \\
\text { to } \\
\text { to } \\
\text { to }\end{array}$ & $\begin{array}{l}\text { Oak Ridge Reserv. } \\
\text { Oak Ridge Reserv. } \\
\text { Oak Ridge Reserv. } \\
\text { Oak Ridge Reserv. } \\
\text { Oak Ridge Reserv. } \\
\text { Savannah River } \\
\text { Oak Ridge Reserv. } \\
\text { INEL } \\
\text { Oak Ridge Reserv. } \\
\text { INEL } \\
\text { INEL } \\
\text { Oak Ridge Reserv. } \\
\text { Oak Ridge Reserv. } \\
\text { Oak Ridge Reserv. } \\
\text { Oak Ridge Reserv. } \\
\text { INEL } \\
\text { Hanford Site } \\
\text { Hanford Site } \\
\text { Hanford Site } \\
\text { Hanford Site } \\
\text { Savannah River } \\
\text { INEL } \\
\text { Oak Ridge Reserv. } \\
\text { INEL } \\
\text { Hanford Site } \\
\text { Oak Ridge Reserv. } \\
\text { Oak Ridge Reserv. } \\
\text { Oak Ridge Reserv. } \\
\text { Hanford Site } \\
\text { Oak Ridge Reserv. } \\
\text { INEL } \\
\text { Hanford Site }\end{array}$ & $\begin{array}{l}2.83 \mathrm{E}-02 \\
8.79 \mathrm{E}-01 \\
1.85 \mathrm{E}-02 \\
1.49 \mathrm{E}-02 \\
3.11 \mathrm{E}-02 \\
6.19 \mathrm{E}-03 \\
6.37 \mathrm{E}-02 \\
2.58 \mathrm{E}-01 \\
7.12 \mathrm{E}-02 \\
3.06 \mathrm{E}-02 \\
1.95 \mathrm{E}-02 \\
2.98 \mathrm{E}-02 \\
2.20 \mathrm{E}-02 \\
2.98 \mathrm{E}-02 \\
2.98 \mathrm{E}-02 \\
6.30 \mathrm{E}-02 \\
2.76 \mathrm{E}-02 \\
2.51 \mathrm{E}-02 \\
1.13 \mathrm{E}-01 \\
2.76 \mathrm{E}-02 \\
1.59 \mathrm{E}-02 \\
2.13 \mathrm{E}-02 \\
8.88 \mathrm{E}-03 \\
8.02 \mathrm{E}-02 \\
7.06 \mathrm{E}-03 \\
4.37 \mathrm{E}-02 \\
6.99 \mathrm{E}-01 \\
2.68 \mathrm{E}-02 \\
7.06 \mathrm{E}-03 \\
2.11 \mathrm{E}-02 \\
3.22 \mathrm{E}-02 \\
2.82 \mathrm{E}-02\end{array}$ & $\begin{array}{l}3.14 \mathrm{E}-02 \\
9.59 \mathrm{E}-01 \\
2.02 \mathrm{E}-02 \\
1.54 \mathrm{E}-02 \\
3.37 \mathrm{E}-02 \\
6.77 \mathrm{E}-03 \\
7.56 \mathrm{E}-02 \\
3.26 \mathrm{E}-01 \\
7.55 \mathrm{E}-02 \\
3.64 \mathrm{E}-02 \\
2.35 \mathrm{E}-02 \\
3.11 \mathrm{E}-02 \\
2.52 \mathrm{E}-02 \\
3.11 \mathrm{E}-02 \\
3.11 \mathrm{E}-02 \\
7.60 \mathrm{E}-02 \\
3.27 \mathrm{E}-02 \\
2.94 \mathrm{E}-02 \\
1.31 \mathrm{E}-01 \\
3.27 \mathrm{E}-02 \\
1.73 \mathrm{E}-02 \\
2.58 \mathrm{E}-02 \\
9.98 \mathrm{E}-03 \\
9.79 \mathrm{E}-02 \\
8.04 \mathrm{E}-03 \\
4.69 \mathrm{E}-02 \\
7.65 \mathrm{E}-01 \\
3.04 \mathrm{E}-02 \\
8.04 \mathrm{E}-03 \\
2.23 \mathrm{E}-02 \\
3.90 \mathrm{E}-02 \\
3.28 \mathrm{E}-02\end{array}$ & $\begin{array}{l}6.86 \mathrm{E}-03 \\
9.50 \mathrm{E}-02 \\
6.65 \mathrm{E}-03 \\
4.52 \mathrm{E}-03 \\
7.69 \mathrm{E}-03 \\
3.47 \mathrm{E}-03 \\
1.42 \mathrm{E}-02 \\
2.17 \mathrm{E}-02 \\
1.31 \mathrm{E}-02 \\
7.37 \mathrm{E}-03 \\
5.04 \mathrm{E}-03 \\
6.87 \mathrm{E}-03 \\
6.38 \mathrm{E}-03 \\
6.87 \mathrm{E}-03 \\
6.87 \mathrm{E}-03 \\
7.80 \mathrm{E}-03 \\
6.99 \mathrm{E}-03 \\
6.59 \mathrm{E}-03 \\
1.39 \mathrm{E}-02 \\
6.99 \mathrm{E}-03 \\
5.09 \mathrm{E}-03 \\
6.04 \mathrm{E}-03 \\
5.08 \mathrm{E}-03 \\
7.64 \mathrm{E}-03 \\
4.62 \mathrm{E}-03 \\
8.26 \mathrm{E}-03 \\
1.09 \mathrm{E}-01 \\
6.05 \mathrm{E}-03 \\
4.62 \mathrm{E}-03 \\
5.38 \mathrm{E}-03 \\
8.09 \mathrm{E}-03 \\
6.94 \mathrm{E}-03\end{array}$ & $\begin{array}{l}2.24 \mathrm{E}-02 \\
2.37 \mathrm{E}-01 \\
2.05 \mathrm{E}-02 \\
1.07 \mathrm{E}-02 \\
5.25 \mathrm{E}-02 \\
5.00 \mathrm{E}-03 \\
3.41 \mathrm{E}-02 \\
7.47 \mathrm{E}-02 \\
2.39 \mathrm{E}-02 \\
2.18 \mathrm{E}-02 \\
9.01 \mathrm{E}-03 \\
4.08 \mathrm{E}-02 \\
1.40 \mathrm{E}-02 \\
4.08 \mathrm{E}-02 \\
4.08 \mathrm{E}-02 \\
1.12 \mathrm{E}-02 \\
2.05 \mathrm{E}-02 \\
1.70 \mathrm{E}-02 \\
4.10 \mathrm{E}-02 \\
2.05 \mathrm{E}-02 \\
9.55 \mathrm{E}-03 \\
8.67 \mathrm{E}-03 \\
7.91 \mathrm{E}-03 \\
1.12 \mathrm{E}-02 \\
1.15 \mathrm{E}-02 \\
3.42 \mathrm{E}-02 \\
2.14 \mathrm{E}-01 \\
2.71 \mathrm{E}-02 \\
1.15 \mathrm{E}-02 \\
1.80 \mathrm{E}-02 \\
1.27 \mathrm{E}-02 \\
2.05 \mathrm{E}-02\end{array}$ \\
\hline Totals & & & $2.78 E+00$ & $3.13 \mathrm{E}+00$ & $4.31 \mathrm{E}-01$ & $1.15 E+00$ \\
\hline
\end{tabular}

Table 7. Annual Accident Dose Risk for the Shipping Campaign (person-rem/yr)

\begin{tabular}{llcc}
\hline Route & & Truck & Rail \\
\hline Ames Laboratory & to Oak Ridge Reserv. & $2.44 \mathrm{E}-08$ & $7.95 \mathrm{E}-09$ \\
Argonne - East & to Oak Ridge Reserv. & $2.96 \mathrm{E}-02$ & $5.16 \mathrm{E}-03$ \\
Bettis Atomic & to Oak Ridge Reserv. & $1.56 \mathrm{E}-04$ & $6.60 \mathrm{E}-05$ \\
Battelle Columbus & to Oak Ridge Reserv. & $1.96 \mathrm{E}-09$ & $2.32 \mathrm{E}-10$ \\
Brookhaven NL & to Oak Ridge Reserv. & $5.76 \mathrm{E}-04$ & $3.98 \mathrm{E}-04$ \\
Charleston, SC & to Savannah River & $5.98 \mathrm{E}-05$ & $6.95 \mathrm{E}-06$ \\
Colonie & to Oak Ridge Reserv. & $3.67 \mathrm{E}-08$ & $1.06 \mathrm{E}-08$ \\
ETEC & to INEL & $1.75 \mathrm{E}-03$ & $7.83 \mathrm{E}-04$ \\
Fernald (FEMP) & to Oak Ridge Reserv. & $7.30 \mathrm{E}-05$ & $8.37 \mathrm{E}-06$ \\
General Atomic & to INEL & $4.51 \mathrm{E}-07$ & $1.77 \mathrm{E}-07$ \\
Grand Junction PO & to INEL & $1.26 \mathrm{E}-08$ & $3.64 \mathrm{E}-09$ \\
Knolls Atomic & to Oak Ridge Reserv. & $1.78 \mathrm{E}-02$ & $1.05 \mathrm{E}-02$ \\
Kansas City Plant to Oak Ridge Reserv. & $1.79 \mathrm{E}-04$ & $3.07 \mathrm{E}-05$ \\
KKS & to Oak Ridge Reserv. & $1.96 \mathrm{E}-02$ & $1.15 \mathrm{E}-02$ \\
KWS & to Oak Ridge Reserv. & $6.28 \mathrm{E}-03$ & $3.68 \mathrm{E}-03$ \\
Los Alamos NL & to INEL & $1.05 \mathrm{E}-04$ & $6.56 \mathrm{E}-06$ \\
Lawrence Berkeley to Hanford Site & $1.05 \mathrm{E}-05$ & $5.80 \mathrm{E}-06$ \\
LERHR & to Hanford Site & $9.03 \mathrm{E}-06$ & $4.74 \mathrm{E}-06$ \\
Lawrence Livermore to Hanford Site & $1.75 \mathrm{E}-04$ & $9.17 \mathrm{E}-05$ \\
Mare Island & to Hanford Site & $8.65 \mathrm{E}-03$ & $4.86 \mathrm{E}-03$ \\
Norfolk Nav Shipyd to Savannah River & $8.05 \mathrm{E}-04$ & $1.41 \mathrm{E}-04$ \\
Nevada Test Site & to INEL & $1.30 \mathrm{E}-08$ & $2.10 \mathrm{E}-09$ \\
Paducah GDP & to Oak Ridge Reserv. & $9.75 \mathrm{E}-04$ & $1.25 \mathrm{E}-04$ \\
Pantex Plant & to INEL & $3.76 \mathrm{E}-04$ & $2.55 \mathrm{E}-05$ \\
Pearl Harbor NS & to Hanford Site & $1.11 \mathrm{E}-04$ & $8.21 \mathrm{E}-05$ \\
Portsmouth NS & to Oak Ridge Reserv. & $3.89 \mathrm{E}-04$ & $1.70 \mathrm{E}-04$ \\
Portsmouth GDP & to Oak Ridge Reserv. & $1.68 \mathrm{E}-04$ & $3.18 \mathrm{E}-05$ \\
Princeton PPL & to Oak Ridge Reserv. & $3.07 \mathrm{E}-09$ & $1.20 \mathrm{E}-09$ \\
Puget Sound NS & to Hanford Site & $2.47 \mathrm{E}-03$ & $1.83 \mathrm{E}-03$ \\
Reactive Metals & to Oak Ridge Reserv. & $1.83 \mathrm{E}-06$ & $3.43 \mathrm{E}-07$ \\
SNL - Albuquerque & to INEL & $2.77 \mathrm{E}-04$ & $1.78 \mathrm{E}-05$ \\
SNL - Livermore & to Hanford Site & $2.72 \mathrm{E}-06$ & $1.43 \mathrm{E}-06$ \\
& & & \\
\hline Totals & & $9.06 \mathrm{E}-02$ & $3.95 \mathrm{E}-02$ \\
& & & \\
& & &
\end{tabular}


Table 8. Expected Annual Fatalities for the Shipping Campaign

\begin{tabular}{llc}
\hline Exposure Group & Truck & Rail \\
\hline Radiological & $1.1 \mathrm{E}-03$ & $1.7 \mathrm{E}-04$ \\
Normal Crew & $1.6 \mathrm{E}-03$ & $5.7 \mathrm{E}-04$ \\
Normal Public & $4.5 \mathrm{E}-05$ & $2.0 \mathrm{E}-05$ \\
Accident Public & & \\
Nonradiological & $7.5 \mathrm{E}-04$ & $1.0 \mathrm{E}-03$ \\
Emission & $5.9 \mathrm{E}-03$ & $1.1 \mathrm{E}-04$ \\
Accident & & \\
\hline
\end{tabular}

Table 9. Expected Annual Cancer Incidence for the Shipping Campaign

\begin{tabular}{lll}
\hline Exposure Group & Truck & Rail \\
\hline Radiological & $3.9 E-03$ & $6.0 \mathrm{E}-04$ \\
Normal Crew & $5.3 \mathrm{E}-03$ & $1.9 \mathrm{E}-03$ \\
Normal Public & $1.5 \mathrm{E}-04$ & $6.7 \mathrm{E}-05$ \\
$\quad$ Accident Public & $7.5 \mathrm{E}-04$ & $1.0 \mathrm{E}-03$ \\
$\begin{array}{c}\text { Nonradiological } \\
\text { Emission }\end{array}$ & $\mathrm{NA}$ & $\mathrm{NA}$ \\
Accident & & \\
\hline
\end{tabular}

Table 10. Expected Annual Genetic Effects for the Shipping Campaign

\begin{tabular}{lll}
\hline Exposure Group & Truck & Rail \\
\hline Radiological & & \\
Normal Crew & $1.7 E-04$ & $2.6 \mathrm{E}-05$ \\
Normal Public & $3.1 \mathrm{E}-04$ & $1.1 \mathrm{E}-04$ \\
Accident Public & $7.9 \mathrm{E}-06$ & $3.7 \mathrm{E}-06$ \\
Nonradiological & & \\
Emission & NA & NA \\
Accident & NA & NA \\
\hline
\end{tabular}




\section{B.26 WM LLMW REGIONALIZED 4 ALTERNATIVE (CASE 15): CONTACT-HANDLED HETEROGENEOUS SOLIDS: ALPHA - UNTREATED}

Table 1. Summary of Route Information

\begin{tabular}{|c|c|c|c|c|c|c|}
\hline & \multirow{2}{*}{\multicolumn{2}{|c|}{ Route }} & \multirow{2}{*}{$\begin{array}{c}\text { Distance } \\
\text { (miles) }\end{array}$} & \multicolumn{3}{|c|}{ Percentage in Zone } \\
\hline & & & & Rural & Suburban & Urban \\
\hline \multicolumn{7}{|l|}{ Truck } \\
\hline Bettis Atomic & & Savannah River & 656 & 71.7 & 26.4 & 1.9 \\
\hline Los Alamos NL & to & INEI & 1144 & 88.7 & 9.8 & 1.4 \\
\hline Lawrence Livermore & & INEL & 972 & 85.7 & 10.4 & 4.0 \\
\hline Mound Plant & & Savannah River & $65 \overline{6}$ & 66.5 & 32.7 & 0.8 \\
\hline Rocky Flats Plant & to & INEL & 716 & 91.3 & 8.1 & 0.6 \\
\hline U of MO - Columbia & & Savannah River & 858 & 70.6 & 27.0 & 2.3 \\
\hline West Valley DP & to & Savannah River & 883 & 70.3 & 28.5 & 1.2 \\
\hline \multicolumn{7}{|l|}{ Rail } \\
\hline Bettis Atomic & & Savannah River & 947 & 68.5 & 27.1 & 4.4 \\
\hline Los Alamos NL & & INEL & 1179 & 92.2 & 6.8 & 1.0 \\
\hline Lawrence Livermore & to & INEL & 1100 & 89.5 & 7.4 & 3.1 \\
\hline Mound Plant & to & Savannah River & 744 & 67.4 & 29.2 & 3.3 \\
\hline Rocky Flats Plant & to & INEL & 738 & 93.0 & 5.2 & 1.8 \\
\hline U of MO - Columbia & to & Savannah River & 1011 & 66.6 & 29.5 & 4.0 \\
\hline West Valley DP & to & Savannah River & 1217 & 62.8 & 32.4 & 4.9 \\
\hline
\end{tabular}

Table 2. Annual Number of Shipments and One-Way Shipment Distances

\begin{tabular}{|c|c|c|c|c|c|}
\hline \multirow[b]{2}{*}{ Route } & & \multicolumn{2}{|c|}{ \# of Shipments } & \multicolumn{2}{|c|}{ Total Mileage(mi) } \\
\hline & & Truck & Rail & Truck & Rail \\
\hline $\begin{array}{l}\text { Bettis Atomic } \\
\text { Los Alamos NL } \\
\text { Lawrence Iivermore } \\
\text { Mound Plant } \\
\text { Rocky Flats Plant } \\
\text { U of MO - Columbia } \\
\text { West Valley DP }\end{array}$ & $\begin{array}{l}\text { to Savannah River } \\
\text { to INEL } \\
\text { to INEI } \\
\text { to Savannah River } \\
\text { to INEL } \\
\text { to Savannah River } \\
\text { to Savannah River }\end{array}$ & $\begin{array}{r}1 \\
4 \\
6 \\
1 \\
130 \\
1 \\
1\end{array}$ & $\begin{array}{r}1 \\
2 \\
3 \\
1 \\
48 \\
1 \\
1\end{array}$ & $\begin{array}{l}6.56 \mathrm{E}+02 \\
4.57 \mathrm{E}+03 \\
5.83 \mathrm{E}+03 \\
6.56 \mathrm{E}+02 \\
9.30 \mathrm{E}+04 \\
8.58 \mathrm{E}+02 \\
8.83 \mathrm{E}+02\end{array}$ & $\begin{array}{l}9.47 \mathrm{E}+02 \\
2.36 \mathrm{E}+03 \\
3.30 \mathrm{E}+03 \\
7.44 \mathrm{E}+02 \\
3.54 \mathrm{E}+04 \\
1.01 \mathrm{E}+03 \\
1.22 \mathrm{E}+03\end{array}$ \\
\hline Totals & & 144 & 57 & $1.06 \mathrm{E}+05$ & $4.50 \mathrm{E}+04$ \\
\hline
\end{tabular}

Table 3. Incident-Free Dose per Shipment (person-rem/shipment)

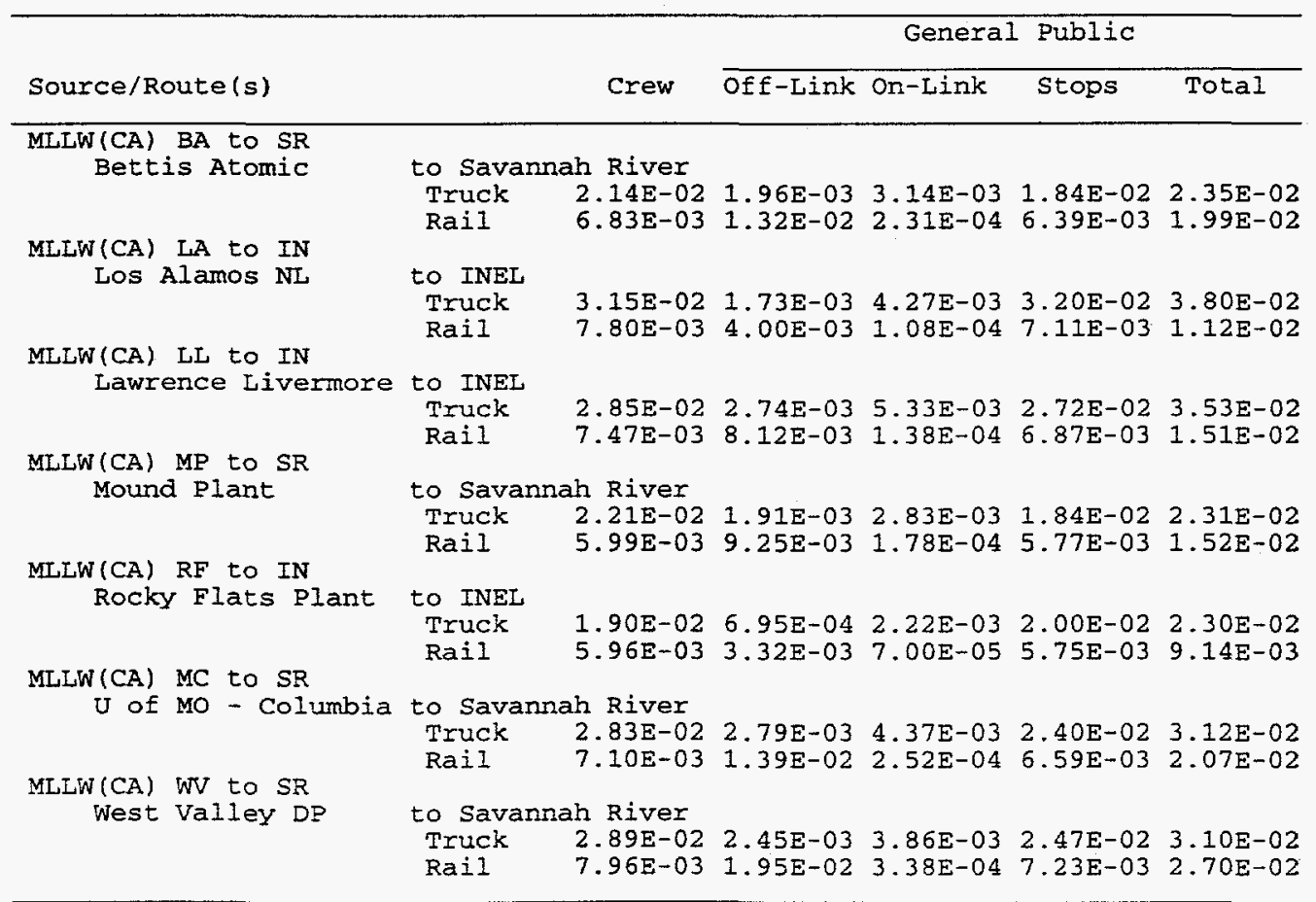


Table 4. Accident Dose Risk per Shipment (person-rem/shipment)

\begin{tabular}{|c|c|c|c|}
\hline \multicolumn{2}{|l|}{ Source/Route } & Truck & Rail \\
\hline $\begin{array}{l}\text { MLLW (CA) BA to SR } \\
\text { Bettis Atomic }\end{array}$ & to Savannah River & $2.11 E-04$ & $7.45 \mathrm{E}-05$ \\
\hline MLLW (CA) IA to IN & & & \\
\hline $\begin{array}{l}\text { Los Alamos NL } \\
\text { MLLW(CA) LL to IN }\end{array}$ & to INEL & $1.13 \mathrm{E}-04$ & 1. $54 \mathrm{E}-05$ \\
\hline Lawrence Iivermore & to INEL & $5.14 \Xi-05$ & $3.46 \mathrm{E}-05$ \\
\hline $\begin{array}{c}\text { Mound Plant } \\
\text { MLLW(CA) RF to IN }\end{array}$ & to Savannah River & $9.48 \mathrm{E}-08$ & $1.85 E-08$ \\
\hline Rocky Flats plant & to INEL & $2.17 \mathrm{E}-05$ & $9.38 E-06$ \\
\hline $\begin{array}{l}\text { U of MO - Columbia } \\
\text { MLLW (CA) WV to SR }\end{array}$ & to Savannah River & $6.78 \mathrm{E}-07$ & $2.05 E-07$ \\
\hline West Valley DP & to Savannah River & $3.36 \mathrm{E}-04$ & $1.07 E-04$ \\
\hline
\end{tabular}

Table 5. Nonradiological Risk Factors per Shipment (fatalities/shipment)

\begin{tabular}{llll}
\hline Mode & Route & Emission & Accident \\
\hline Truck & & & \\
Bettis Atomic & to Savannah River & $3.99 E-06$ & $4.90 \mathrm{E}-05$ \\
Los Alamos NL & to INEL & $5.25 \mathrm{E}-06$ & $8.21 \mathrm{E}-05$ \\
Lawrence Livermore & to INEL & $1.24 \mathrm{E}-05$ & $5.73 \mathrm{E}-05$ \\
Mound Plant & to Savannah River & $1.67 \mathrm{E}-06$ & $5.25 \mathrm{E}-05$ \\
Rocky Flats Plant & to INEL & $1.42 \mathrm{E}-06$ & $4.68 \mathrm{E}-05$ \\
U of MO - Columbia & to Savannah River & $6.41 \mathrm{E}-06$ & $6.09 \mathrm{E}-05$ \\
West Valley DP & to Savannah River & $3.28 \mathrm{E}-06$ & $6.60 \mathrm{E}-05$ \\
Rail & & & \\
Bettis Atomic & to Savannah River & $1.74 \mathrm{E}-05$ & $1.98 \mathrm{E}-06$ \\
Los Alamos NL & to INEL & $1.90 \mathrm{E}-06$ & $2.47 \mathrm{E}-06$ \\
Lawrence Livermore & to INEL & $1.41 \mathrm{E}-05$ & $2.30 \mathrm{E}-06$ \\
Mound Plant & to Savannah River & $5.44 \mathrm{E}-05$ & $1.56 \mathrm{E}-06$ \\
RockY Flats Plant & to INEL & $1.54 \mathrm{E}-06$ \\
Uof MO - Columbia to Savannah River & $1.69 \mathrm{E}-05$ & $2.12 \mathrm{E}-06$ \\
West Valley DP & to Savannah River & $2.49 \mathrm{E}-05$ & $2.55 \mathrm{E}-06$ \\
\hline
\end{tabular}

Table 6. Annual Incident-Free Dose for the Shipping Campaign (person-rem/yx)

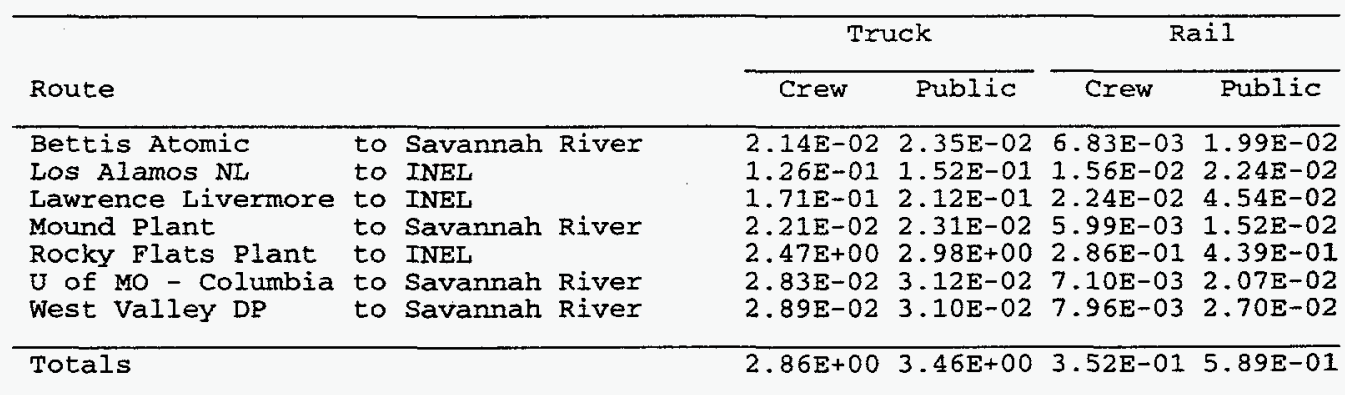

Table 7. Annual Accident Dose Risk for the Shipping Campaign (person-rem/yr)

\begin{tabular}{llcc}
\hline Route & & Truck & Rail \\
\hline Bettis Atomic & to Savannah River & $2.11 E-04$ & $7.45 E-05$ \\
Los Alamos NL & to INEL & $4.51 E-04$ & $3.07 E-05$ \\
Lawrence Iivermore to INEL & $3.09 E-04$ & $1.04 E-04$ \\
Mound Plant & to Savannah River & $9.48 E-08$ & $1.85 E-08$ \\
Rocky Flats Plant to INEL & $2.82 E-03$ & $4.50 E-04$ \\
U of MO - Columbia to Savannah River & $6.78 E-07$ & $2.05 E-07$ \\
West Valley DP & to Savannah River & $3.36 \mathrm{E}-04$ & $1.07 \mathrm{E}-04$ \\
& & & \\
\hline Totals & & $4.12 \mathrm{E}-03$ & $7.67 \mathrm{E}-04$ \\
\hline
\end{tabular}


Table 8. Expected Annual Fatalities for the Shipping Campaign

\begin{tabular}{llc}
\hline Exposure Group & Truck & Rail \\
\hline Radiological & & \\
$\quad$ Normal Crew & $1.1 \mathrm{E}-03$ & $1.4 \mathrm{E}-04$ \\
Normal Public & $1.7 \mathrm{E}-03$ & $2.9 \mathrm{E}-04$ \\
$\quad$ Accident Public & $2.1 \mathrm{E}-05$ & $3.8 \mathrm{E}-07$ \\
Nonradiological & & \\
$\quad$ Emission & $2.9 \mathrm{E}-04$ & $3.8 \mathrm{E}-04$ \\
Accident & $7.0 \mathrm{E}-03$ & $9.4 \mathrm{E}-05$ \\
\hline
\end{tabular}

Table 9. Expected Annual Cancer Incidence for the Shipping Campaign

\begin{tabular}{lll}
\hline Exposure Group & Truck & Rail \\
\hline Radiological & & \\
Normal Crew & $4.0 E-03$ & $4.9 \mathrm{E}-04$ \\
Normal Public & $5.9 \mathrm{E}-03$ & $1.0 \mathrm{E}-03$ \\
Accident Public & $7.0 \mathrm{E}-05$ & $1.3 \mathrm{E}-06$ \\
Nonradiological & $2.9 \mathrm{E}-04$ & $3.8 \mathrm{E}-04$ \\
$\quad$ Enission & $\mathrm{NA}$ & $\mathrm{NA}$ \\
Accident & & \\
\hline
\end{tabular}

Table 10. Expected Annual Genetic Effects for the Shipping Campaign

\begin{tabular}{lll}
\hline Exposure Group & Truck & Rail \\
\hline Radiological & & \\
Normal Crew & $1.7 \mathrm{E}-04$ & $2.1 \mathrm{E}-05$ \\
Normal Public & $3.5 \mathrm{E}-04$ & $5.9 \mathrm{E}-05$ \\
Accident Public & $1.7 \mathrm{E}-07$ & $3.5 \mathrm{E}-08$ \\
Nonradiological & NA & NA \\
Emission & NA & NA \\
Accident & & \\
\hline
\end{tabular}




\section{B.27 WM LLMW REGIONALIZED 4 ALTERNATIVE (CASE 15): CONTACT-HANDLED HETEROGENEOUS SOLIDS: NONALPHA — TREATED}

Table 1. Summary of Route Information

\begin{tabular}{|c|c|c|c|c|c|c|}
\hline & \multirow{2}{*}{\multicolumn{2}{|c|}{ Route }} & \multirow{2}{*}{$\begin{array}{c}\text { Distance } \\
\text { (miles) }\end{array}$} & \multicolumn{3}{|c|}{ Percentage in Zone } \\
\hline & & & & Rural & Suburban & Urban \\
\hline \multicolumn{7}{|l|}{ Truck } \\
\hline Ames Laboratory & to & Oak Ridge Reserv. & 900 & 75.2 & 23.4 & 1.5 \\
\hline Argonne - East & to & Oak Ridge Reserv. & 584 & 67.0 & 30.1 & 2.9 \\
\hline Charleston, SC & & Savannah River & 200 & 75.7 & 23.8 & 0.5 \\
\hline Colonie & & Oak Ridge Reserv. & 2193 & 84.1 & 13.8 & 2.1 \\
\hline Fernald (FEMP) & to & Oak Ridge Reserv. & 312 & 69.9 & 29.5 & $0 . \overline{6}$ \\
\hline Genera1 Atomic & & INEL & 976 & 78.8 & 17.1 & 4.1 \\
\hline Grand Junction Po & to & INEI & 670 & 85.1 & 12.0 & 2.8 \\
\hline Los Alamos NL & to & INEL & 1144 & 88.7 & 9.8 & 1.4 \\
\hline LERHR & & Hanford site & 830 & 80.5 & 17.0 & 2.6 \\
\hline Paducah GDP & & Oak Ridge Reserv. & 304 & 81.3 & 18.5 & 0.2 \\
\hline Pantex Plant & to & INEI & 1468 & 89.8 & 8.6 & 1.5 \\
\hline Portsmouth GDP & to & Oak Ridge Reserv. & 371 & 74.5 & 24.6 & 0.9 \\
\hline \multirow{2}{*}{\multicolumn{7}{|c|}{ Rail }} \\
\hline & & & & & & \\
\hline Ames Laboratory & to & Oak Ridge Reserv. & 954 & 71.7 & 22.0 & 6.3 \\
\hline Argonne - East & to & Oak Ridge Reserv. & 648 & 70.7 & 25.3 & 4.0 \\
\hline Charleston, SC & & Savannah River & $140^{\circ}$ & 83.9 & 13.6 & 2.5 \\
\hline Colonie & & Oak Ridge Reserv. & 957 & 42.1 & 43.9 & 14.0 \\
\hline Fernald (FEMP) & & oak Ridge Reserv. & 358 & 75.7 & 21.8 & 2.5 \\
\hline General Atomic & & INEL & 1076 & 82.6 & 11.4 & 6.0 \\
\hline Grand Junction PO & to & INEL & 517 & 83.7 & 13.9 & 2.4 \\
\hline Los Alamos NL & to & INEL & 1179 & 92.2 & 6.8 & 1.0 \\
\hline LERHR & to & Hanford site & 890 & 81.0 & 14.3 & 4.7 \\
\hline Paducah GDP & to & Oak Ridge Reserv. & 527 & 89.3 & 9.0 & 1.7 \\
\hline Pantex Plant & to & INEL & 1141 & 91.3 & 7.6 & 1.0 \\
\hline Portsmouth GDP & to & Oak Ridge Reserv. & 442 & 66.8 & 31.5 & 1.7 \\
\hline Reactive Metals & to & Oak Ridge Reserv. & 600 & 59.4 & 33.2 & 7.4 \\
\hline
\end{tabular}

Table 2. Annual Number of Shipments and Total One-Way Shipment Distances

\begin{tabular}{|c|c|c|c|c|c|c|}
\hline \multirow[b]{2}{*}{ Route } & & & \multicolumn{2}{|c|}{ \# of Shipments } & \multicolumn{2}{|c|}{ Total Mileage (mi) } \\
\hline & & & Truck & Rail & Truck & Rail \\
\hline $\begin{array}{l}\text { Ames Laboratory } \\
\text { Argonne - East } \\
\text { Charleston, SC } \\
\text { Colonie } \\
\text { Fernald (FEMP) } \\
\text { General Atomic } \\
\text { Grand Junction PO } \\
\text { Los Alamos NL } \\
\text { LERHR } \\
\text { Paducah GDP } \\
\text { Pantex Plant } \\
\text { Portsmouth GDP } \\
\text { Reactive Metals }\end{array}$ & $\begin{array}{l}\text { to } \\
\text { to } \\
\text { to } \\
\text { to } \\
\text { to } \\
\text { to } \\
\text { to } \\
\text { to } \\
\text { to } \\
\text { to } \\
\text { to } \\
\text { to } \\
\text { to }\end{array}$ & $\begin{array}{l}\text { Oak Ridge Reserv. } \\
\text { Oak Ridge Reserv. } \\
\text { Savannah River } \\
\text { Oak Ridge Reserv. } \\
\text { Oak Ridge Reserv. } \\
\text { INEL } \\
\text { INEI } \\
\text { INEL } \\
\text { Hanford Site } \\
\text { Oak Ridge Reserv. } \\
\text { INEL } \\
\text { Oak Ridge Reserv. } \\
\text { Oak Ridge Reserv. }\end{array}$ & $\begin{array}{l}1 \\
1 \\
1 \\
1 \\
2 \\
1 \\
1 \\
1 \\
1 \\
1 \\
1 \\
2 \\
1\end{array}$ & $\begin{array}{l}1 \\
1 \\
1 \\
1 \\
1 \\
1 \\
1 \\
1 \\
1 \\
1 \\
1 \\
1 \\
1\end{array}$ & $\begin{array}{l}9.00 \mathrm{E}+02 \\
5.84 \mathrm{E}+02 \\
2.00 \mathrm{E}+02 \\
2.19 \mathrm{E}+03 \\
6.24 \mathrm{E}+02 \\
9.76 \mathrm{E}+02 \\
6.70 \mathrm{E}+02 \\
1.14 \mathrm{E}+03 \\
8.30 \mathrm{E}+02 \\
3.04 \mathrm{E}+02 \\
1.47 \mathrm{E}+03 \\
7.41 \mathrm{E}+02 \\
6.08 \mathrm{E}+02\end{array}$ & $\begin{array}{l}9.54 \mathrm{E}+02 \\
6.48 \mathrm{E}+02 \\
1.40 \mathrm{E}+02 \\
9.57 \mathrm{E}+02 \\
3.58 \mathrm{E}+02 \\
1.08 \mathrm{E}+03 \\
5.17 \mathrm{E}+02 \\
1.18 \mathrm{E}+03 \\
8.90 \mathrm{E}+02 \\
5.27 \mathrm{E}+02 \\
1.14 \mathrm{E}+03 \\
4.42 \mathrm{E}+02 \\
6.00 \mathrm{E}+02\end{array}$ \\
\hline Totals & & & 15 & 13 & $1.12 \mathrm{E}+04$ & $9.43 E+03$ \\
\hline
\end{tabular}


Table 3. Incident-Free Dose per Shipment (person-rem/shipment)

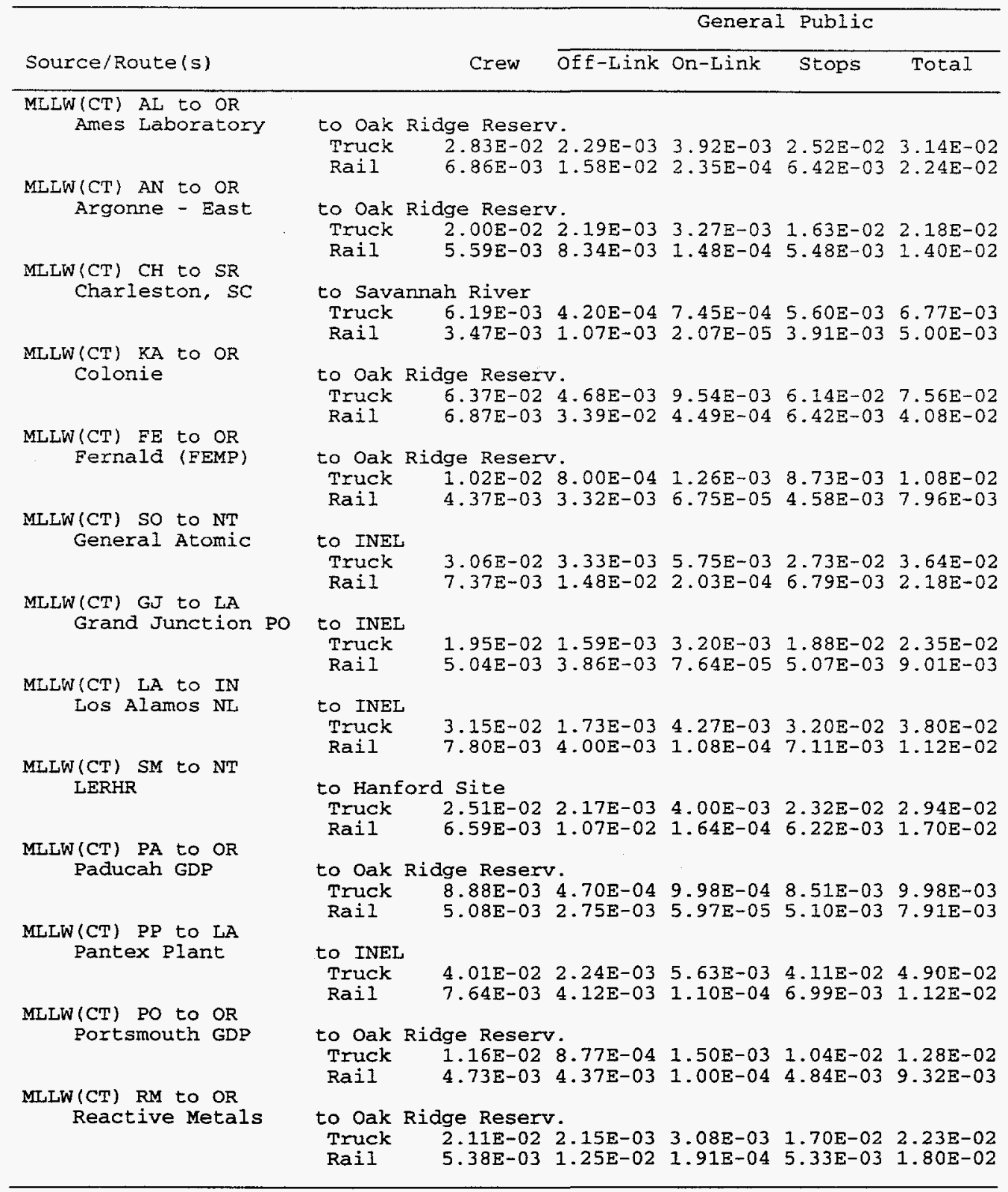


Table 4. Accident Dose Risk per Shipment (person-rem/shipment)

\begin{tabular}{|c|c|c|c|}
\hline \multicolumn{2}{|l|}{ Source/Route } & \multirow{4}{*}{$\begin{array}{l}\text { Truck } \\
4.17 \mathrm{E}-08\end{array}$} & \multirow{4}{*}{$\frac{\text { Rail }}{1.36 \mathrm{E}-08}$} \\
\hline MLLW (CT) AL to OR & & & \\
\hline Ames Laboratory & to Oak Ridge Reserv. & & \\
\hline MLLW(CT) AN to OR & & & \\
\hline $\begin{array}{l}\text { Argonne - East } \\
\text { MLLW (CT) CF to SR }\end{array}$ & to Oak Ridge Reserv. & $1.48 E-05$ & $2.57 E-06$ \\
\hline Charleston, SC & to Savannah River & $4.40 \mathrm{E}-05$ & $5.11 \mathrm{E}-06$ \\
\hline $\begin{array}{c}\text { MLLW(CT) KA to OR } \\
\text { Colonie }\end{array}$ & to Oak Ridge Reserv. & $1.66 \mathrm{E}-07$ & $7.54 \mathrm{E}-08$ \\
\hline$M L L W(C T) \quad F E$ to $O R$ & & & \\
\hline $\begin{array}{l}\text { Fernald (FEMP) } \\
\text { MLLW(CT) So to NT }\end{array}$ & to Oak Ridge Reserv. & $3.14 \mathrm{E}-05$ & $7.21 E-06$ \\
\hline $\begin{array}{l}\text { General Atomic } \\
\text { MLLW(CT) GJ to LA }\end{array}$ & to INEL & $1.84 \mathrm{E}-06$ & $7.22 E-07$ \\
\hline $\begin{array}{l}\text { Grand Junction Po } \\
\text { MLLW(CT) LA to IN }\end{array}$ & to INEL & $2.73 \mathrm{E}-08$ & $7.88 E-09$ \\
\hline $\begin{array}{l}\text { Los Alamos NL } \\
\text { MLLW(CT) SM to NT }\end{array}$ & to INEL & $3.14 \mathrm{E}-05$ & $1.96 \mathrm{E}-06$ \\
\hline $\begin{array}{l}\text { LERHR } \\
\text { MLIW (CT) } \mathrm{PA} \text { to OR }\end{array}$ & to Hanford Site & $1.21 \mathrm{E}-06$ & $6.33 \mathrm{E}-07$ \\
\hline $\begin{array}{c}\text { MLLW (CT) PA to OR } \\
\text { Paducah GDP } \\
\text { MLLW(CT) PP to LA }\end{array}$ & to Oak Ridge Reserv. & $2.33 E-04$ & $2.98 \mathrm{E}-05$ \\
\hline $\begin{array}{l}\text { Pantex Plant } \\
\text { MLLW(CT) PO to oR }\end{array}$ & to INEL & $2.69 \mathrm{E}-05$ & $1.83 E-06$ \\
\hline $\begin{array}{l}\text { Portsmouth GDP } \\
\text { MLLW (CT) RM to OR }\end{array}$ & to Oak Ridge Reserv. & $9.59 \mathrm{E}-06$ & $3.63 \mathrm{E}-06$ \\
\hline Reactive Metals & to Oak Ridge Reserv. & $7.69 E-07$ & $1.45 \mathrm{E}-07$ \\
\hline
\end{tabular}

Table 5. Nonradiological Risk Factors per Shipment (fatalities/shipment)

\begin{tabular}{llll}
\hline Mode & Route & Emission & Accident \\
\hline Truck & & & \\
Ames Laboratory & to Oak Ridge Reserv. & $4.25 \mathrm{E}-06$ & $5.79 \mathrm{E}-05$ \\
Argonne - East & to Oak Ridge Reserv. & $5.38 \mathrm{E}-06$ & $4.21 \mathrm{E}-05$ \\
Charleston, SC & to Savannah River & $3.22 \mathrm{E}-07$ & $1.70 \mathrm{E}-05$ \\
Colonie & to Oak Ridge Reserv. & $1.46 \mathrm{E}-05$ & $1.61 \mathrm{E}-04$ \\
Fernald (FEMP) & to Oak Ridge Reserv. & $5.79 \mathrm{E}-07$ & $2.25 \mathrm{E}-05$ \\
General Atomic & to INEL & $1.30 \mathrm{E}-05$ & $6.23 \mathrm{E}-05$ \\
Grand Junction PO PO & to INEL & $6.08 \mathrm{E}-06$ & $4.24 \mathrm{E}-05$ \\
Los Alamos NL & to INEL & $5.25 \mathrm{E}-06$ & $8.21 \mathrm{E}-05$ \\
LERHR & to Hanford Site & $6.82 \mathrm{E}-06$ & $4.66 \mathrm{E}-05$ \\
Paducah GDP & to Oak Ridge Reserv. & $1.93 \mathrm{E}-07$ & $2.14 \mathrm{E}-05$ \\
Pantex Plant & to INEL & $7.79 \mathrm{E}-06$ & $1.05 \mathrm{E}-04$ \\
Portsmouth GDP & to Oak Ridge Reserv. & $1.09 \mathrm{E}-06$ & $2.56 \mathrm{E}-05$ \\
Reactive Metals & to Oak Ridge Reserv. & $3.57 \mathrm{E}-06$ & $3.56 \mathrm{E}-05$ \\
Rail & & & \\
Ames Laboratory & to Oak Ridge Reserv. & $2.52 \mathrm{E}-05$ & $2.00 \mathrm{E}-06$ \\
Argonne - East & to Oak Ridge Reserv. & $1.08 \mathrm{E}-05$ & $1.36 \mathrm{E}-06$ \\
Charleston, SC & to Savannah River & $1.46 \mathrm{E}-06$ & $2.93 \mathrm{E}-07$ \\
Colonie & to Oak Ridge Reserv. & $5.59 \mathrm{E}-05$ & $2.00 \mathrm{E}-06$ \\
Fernald (FEMP) & to Oak Ridge Reserv. & $3.68 \mathrm{E}-06$ & $7.48 \mathrm{E}-07$ \\
General Atomic & to INEL & $2.72 \mathrm{E}-05$ & $2.25 \mathrm{E}-06$ \\
Grand Junction PO & to INEL & $5.15 \mathrm{E}-06$ & $1.08 \mathrm{E}-06$ \\
Los Alamos NL & to INEL & $4.90 \mathrm{E}-06$ & $2.47 \mathrm{E}-06$ \\
LERHR & to Hanford Site & $1.77 \mathrm{E}-05$ & $1.86 \mathrm{E}-06$ \\
Paducah GDP & to Oak Ridge Reserv. & $3.81 \mathrm{E}-06$ & $1.10 \mathrm{E}-06$ \\
Pantex Plant & to INEL & $4.85 \mathrm{E}-06$ & $2.39 \mathrm{E}-06$ \\
PortSmouth GDP & to Oak Ridge Reserv. & $3.18 \mathrm{E}-06$ & $9.25 \mathrm{E}-07$ \\
Reactive Metals & to Oak Ridge Reserv. & $1.85 \mathrm{E}-05$ & $1.25 \mathrm{E}-06$ \\
\hline
\end{tabular}


Table 6. Annual Incident-Free Dose for the Shipping Campaign (person-rem/yr)

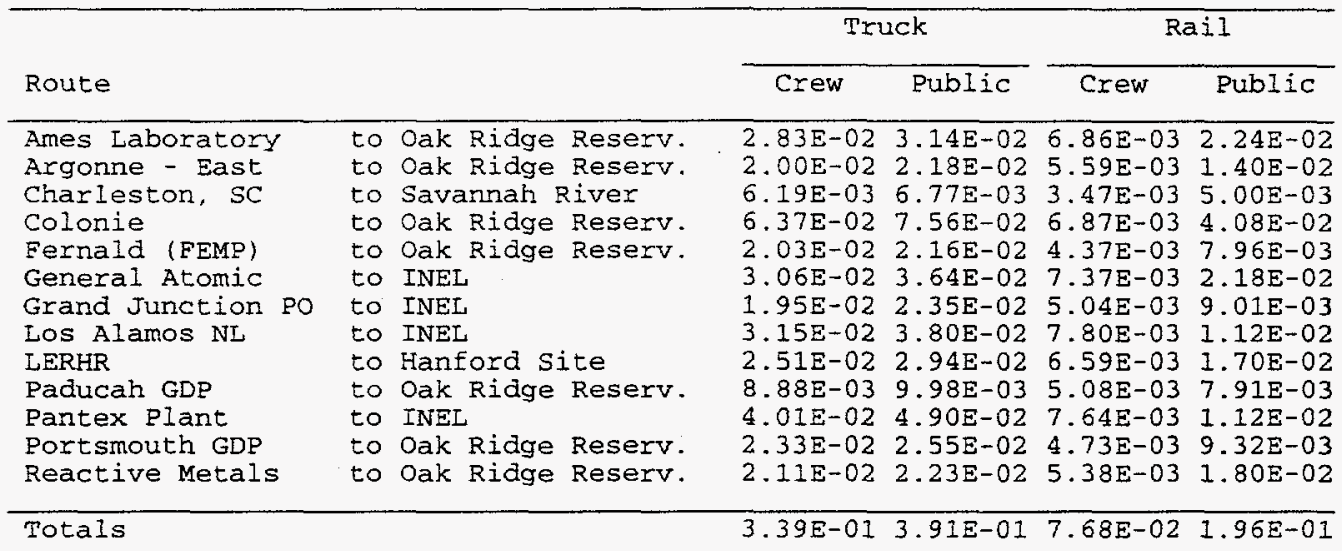

Table 7. Annual Accident Dose Risk for the Shipping Campaign (person-rem/yr)

\begin{tabular}{llcc}
\hline Route & & Truck & Rail \\
\hline Ames Laboratory & to Oak Ridge Reserv. & $4.17 \mathrm{E}-08$ & $1.36 \mathrm{E}-08$ \\
Argonne - East & to Oak Ridge Reserv. & $1.48 \mathrm{E}-05$ & $2.57 \mathrm{E}-06$ \\
Charleston, SC & to Savannah River & $4.40 \mathrm{E}-05$ & $5.11 \mathrm{E}-06$ \\
Colonie & to Oak Ridge Reserv. & $1.66 \mathrm{E}-07$ & $7.54 \mathrm{E}-08$ \\
Fernald (FEMP) & to Oak Ridge Reserv. & $6.28 \mathrm{E}-05$ & $7.21 \mathrm{E}-06$ \\
General Atomic & to INEL & $1.84 \mathrm{E}-06$ & $7.22 \mathrm{E}-07$ \\
Grand Junction PO & to INEL & $2.73 \mathrm{E}-08$ & $7.88 \mathrm{E}-09$ \\
Los Alamos NL & to INEL & $3.14 \mathrm{E}-05$ & $1.96 \mathrm{E}-06$ \\
LERHR & to Hanford Site & $1.21 \mathrm{E}-06$ & $6.33 \mathrm{E}-07$ \\
Paducah GDP & to Oak Ridge Reserv. & $2.33 \mathrm{E}-04$ & $2.98 \mathrm{E}-05$ \\
Pantex Plant & to INEL & $2.69 \mathrm{E}-05$ & $1.83 \mathrm{E}-06$ \\
Portsmouth GDP & to Oak Ridge Reserv. & $1.92 \mathrm{E}-05$ & $3.63 \mathrm{E}-06$ \\
Reactive Metals & to Oak Ridge Reserv. & $7.69 \mathrm{E}-07$ & $1.45 \mathrm{E}-07$ \\
& & & \\
\hline Totals & & & \\
\end{tabular}

Table 8. Expected Annual Fatalities for the Shipping Campaign

\begin{tabular}{llc}
\hline Exposure Group & Truck & Rail \\
\hline Radiological & & \\
Normal Crew & $1.4 \mathrm{E}-04$ & $3.1 \mathrm{E}-05$ \\
Normal Public & $2.0 \mathrm{E}-04$ & $9.8 \mathrm{E}-05$ \\
Accident Public & $2.2 \mathrm{E}-07$ & $2.7 \mathrm{E}-08$ \\
Nonradiological & $7.1 \mathrm{E}-05$ & $1.8 \mathrm{E}-04$ \\
Emission & $7.7 \mathrm{E}-04$ & $2.0 \mathrm{E}-05$ \\
Accident & & \\
\hline
\end{tabular}

Table 9. Expected Annual Cancer Incidence for the Shipping campaign

\begin{tabular}{lll}
\hline Exposure Group & Truck & Rail \\
\hline Radiological & & \\
Normal Crew & $4.7 \mathrm{E}-04$ & $1.1 \mathrm{E}-04$ \\
Normal Public & $6.7 \mathrm{E}-04$ & $3.3 \mathrm{E}-04$ \\
Accident Public & $7.4 \mathrm{E}-07$ & $9.1 \mathrm{E}-08$ \\
Nonradiological & $7.1 \mathrm{E}-05$ & $1.8 \mathrm{E}-04$ \\
Emission & NA & NA \\
Accident & & \\
\hline
\end{tabular}


Table 10. Expected Annual Genetic Effects for the Shipping Campaign

\begin{tabular}{lll}
\hline Exposure Group & Truck & Rail \\
\hline Radiological & & \\
Normal Crew & $2.0 \mathrm{E}-05$ & $4.6 \mathrm{E}-06$ \\
Normal Public & $3.9 \mathrm{E}-05$ & $2.0 \mathrm{E}-05$ \\
Accident Public & $1.2 \mathrm{E}-08$ & $1.2 \mathrm{E}-09$ \\
Nonradiological & & \\
Emission & NA & NA \\
Accident & NA & NA \\
\hline
\end{tabular}




\section{B.28 WM LLMW REGIONALIZED 4 ALTERNATIVE (CASE 15): CONTACT-HANDLED HETEROGENEOUS SOLIDS: ALPHA - TREATED}

Table 1. Summary of Route Information

\begin{tabular}{|c|c|c|c|c|c|}
\hline & \multirow[b]{2}{*}{ Route } & \multirow{2}{*}{$\begin{array}{c}\text { Distance } \\
\text { (miles) }\end{array}$} & \multicolumn{3}{|c|}{ Percentage in Zone } \\
\hline & & & Rura1 & Suburban & Urban \\
\hline \multicolumn{6}{|l|}{ Truck } \\
\hline Los Alamos NL & to INEL & 1144 & 88.7 & 9.8 & 1.4 \\
\hline Rocky Flats Plant & to INEL & 716 & 91.3 & 8.1 & 0.6 \\
\hline West Valley DP & to Savannah River & 883 & 70.3 & 28.5 & 1.2 \\
\hline \multicolumn{6}{|l|}{ Rail } \\
\hline Los Alamos NL & to INEL & 1179 & 92.2 & 6.8 & 1.0 \\
\hline Rocky Flats Plant & to INEL & 738 & 93.0 & 5.2 & 1.8 \\
\hline West valley $\mathrm{DP}$ & to Savannah River & 1217 & 62.8 & 32.4 & 4.9 \\
\hline
\end{tabular}

Table 2. Annual Number of Shipments and Total One-Way Shipment Distances

\begin{tabular}{llrrrrr}
\hline & & \# of Shipments & \multicolumn{2}{c}{ Total Mileage(mi) } \\
Route & & Truck & Rail & Truck & Rail \\
\hline Los Alamos NL & to INEL & 1 & 1 & $1.14 \mathrm{E}+03$ & $1.18 \mathrm{E}+03$ \\
Rocky Flats Plant & to INEL & 68 & 25 & $4.87 \mathrm{E}+04$ & $1.85 \mathrm{E}+04$ \\
West Valley DP & to Savannah River & 1 & 1 & $8.83 \mathrm{E}+02$ & $1.22 \mathrm{E}+03$ \\
\hline Totals & & 70 & 27 & $5.07 \mathrm{E}+04$ & $2.09 \mathrm{E}+04$ \\
\hline
\end{tabular}

Table 3. Incident-Free Dose per Shipment (person-rem/shipment)

\begin{tabular}{|c|c|c|c|c|c|c|}
\hline \multirow[b]{2}{*}{ Source/Route (s) } & & \multirow[b]{2}{*}{ Crew } & \multicolumn{4}{|c|}{ General Public } \\
\hline & & & off-Link & On-Link & Stops & Total \\
\hline $\begin{aligned} & \text { MLLW(T) } \text { LA to IN } \\
& \text { LOS Alamos NL }\end{aligned}$ & $\begin{array}{l}\text { to INEL } \\
\text { Truck } \\
\text { Rail }\end{array}$ & $\begin{array}{l}3.15 \mathrm{E}-02 \\
7.80 \mathrm{E}-03\end{array}$ & $\begin{array}{l}1.73 E-03 \\
4.00 E-03\end{array}$ & $\begin{array}{l}4.27 E-03 \\
1.08 E-04\end{array}$ & $\begin{array}{l}3.20 \mathrm{E}-02 \\
7.11 \mathrm{E}-03\end{array}$ & $\begin{array}{l}3.80 E-02 \\
1.12 E-02\end{array}$ \\
\hline $\begin{array}{l}\text { MLLW(T) RF to LA } \\
\text { Rocky Flats Plant }\end{array}$ & $\begin{array}{l}\text { to INEL } \\
\text { Truck } \\
\text { Rail }\end{array}$ & $\begin{array}{l}1.90 \mathrm{E}-02 \\
5.96 \mathrm{E}-03\end{array}$ & $\begin{array}{l}6.95 \mathrm{E}-04 \\
3.32 \mathrm{E}-03\end{array}$ & $\begin{array}{l}2.22 \mathrm{E}-03 \\
7.00 \mathrm{E}-05\end{array}$ & $\begin{array}{l}2.00 \mathrm{E}-02 \\
5.75 \mathrm{E}-03\end{array}$ & $\begin{array}{l}2.30 \mathrm{E}-02 \\
9.14 \mathrm{E}-03\end{array}$ \\
\hline $\begin{array}{l}\text { MLLW(T) WV to SR } \\
\text { West Valley DP }\end{array}$ & $\begin{array}{l}\text { to Savan } \\
\text { Truck } \\
\text { Rail }\end{array}$ & $\begin{array}{l}\text { River } \\
2.89 \mathrm{E}-02 \\
7.96 \mathrm{E}-03\end{array}$ & $\begin{array}{l}2.45 \mathrm{E}-03 \\
1.95 \mathrm{E}-02\end{array}$ & $\begin{array}{l}3.86 \mathrm{E}-03 \\
3.38 \mathrm{E}-04\end{array}$ & $\begin{array}{l}2.47 \mathrm{E}-02 \\
7.23 \mathrm{E}-03\end{array}$ & $\begin{array}{l}3.10 \mathrm{E}-02 \\
2.70 \mathrm{E}-02\end{array}$ \\
\hline
\end{tabular}

Table 4. Accident Dose Risk per Shipment (person-rem/shipment)

\begin{tabular}{llll}
\hline Source/Route & & Truck & Rail \\
\hline $\begin{array}{c}\text { MLLW(T) LA to IN } \\
\text { Los Alamos NL }\end{array}$ & to INEL & $2.67 \mathrm{E}-05$ & $1.82 \mathrm{E}-06$ \\
$\begin{array}{c}\text { MLLW (T) RF to LA } \\
\text { Rocky Flats Plant }\end{array}$ & to INEL & $1.57 \mathrm{E}-04$ & $6.82 \mathrm{E}-05$ \\
$\begin{array}{c}\text { MLLW (T) WV to SR } \\
\text { West valley DP }\end{array}$ & to Savannah River & $4.43 \mathrm{E}-05$ & $1.42 \mathrm{E}-05$ \\
\hline
\end{tabular}


Table 5. Nonradiological Risk Factors per Shipment (fatalities/shipment)

\begin{tabular}{llll}
\hline Mode & Route & Emission & Accident \\
\hline Truck & & & \\
Los Alamos NL & to INEI & $5.25 E-06$ & $8.21 E-05$ \\
RockY Flats Plant & to INEL & $1.42 E-06$ & $4.68 \mathrm{E}-05$ \\
West Valley DP & to Savannah River & $3.28 \mathrm{E}-06$ & $6.60 \mathrm{E}-05$ \\
Rail & & & \\
Los Alamos NL & to INEL & $5.90 \mathrm{E}-06$ & $2.47 \mathrm{E}-06$ \\
RockY Flats PIant & to INEL & $2.44 \mathrm{E}-06$ & $1.54 \mathrm{E}-06$ \\
West Valley DP & to Savannah River & & $2.55 \mathrm{E}-06$ \\
\hline
\end{tabular}

Table 6. Annual Incident-Free Dose for the Shipping Campaign (person-rem/yr)

\begin{tabular}{|c|c|c|c|c|c|}
\hline \multirow[b]{2}{*}{ Route } & & \multicolumn{2}{|c|}{ Truck } & \multicolumn{2}{|c|}{ Rail } \\
\hline & & Crew & Public & Crew & Public \\
\hline $\begin{array}{l}\text { Los Alamos NL } \\
\text { Rocky Flats Plant } \\
\text { West Valley DP }\end{array}$ & $\begin{array}{l}\text { to INEL } \\
\text { to INEL } \\
\text { to Savannah River }\end{array}$ & $\begin{array}{l}3.15 \mathrm{E}-02 \\
1.29 \mathrm{E}+00 \\
2.89 \mathrm{E}-02\end{array}$ & $\begin{array}{l}3.80 E-02 \\
1.56 E+00 \\
3.10 E-02\end{array}$ & $\begin{array}{l}7.80 E-03 \\
1.49 E-01 \\
7.96 E-03\end{array}$ & $\begin{array}{l}1.12 E-02 \\
2.29 E-01 \\
2.70 E-02\end{array}$ \\
\hline Totals & & $1.35 E+00$ & $1.63 \mathrm{E}+00$ & $1.65 \mathrm{E}-01$ & $2.67 \mathrm{E}-01$ \\
\hline
\end{tabular}

Table 7. Annual Accident Dose Risk for the Shipping Campaign (person-rem/yr)

\begin{tabular}{llcc}
\hline Route & & Truck & Rail \\
\hline Los Alamos NL & to INEL & $2.67 \mathrm{E}-05$ & $1.82 \mathrm{E}-06$ \\
Rocky Flats Plant & to INEL & $1.07 \mathrm{E}-02$ & $1.71 \mathrm{E}-03$ \\
West Valley DP & to Savannah River & $4.43 \mathrm{E}-05$ & $1.42 \mathrm{E}-05$ \\
\hline Totals & & $1.07 \mathrm{E}-02$ & $1.72 \mathrm{E}-03$ \\
\hline
\end{tabular}

Table 8. Expected Annual Fatalities for the Shipping Campaign

\begin{tabular}{|c|c|c|}
\hline Exposure Group & Truck & Rail \\
\hline $\begin{array}{l}\text { Radiological } \\
\text { Normal Crew } \\
\text { Normal Public } \\
\text { Accident Public } \\
\text { Nonradiological }\end{array}$ & $\begin{array}{l}5.4 \mathrm{E}-04 \\
8.1 \mathrm{E}-04 \\
5.4 \mathrm{E}-06\end{array}$ & $\begin{array}{l}6.6 \mathrm{E}-05 \\
1.3 \mathrm{E}-04 \\
8.6 \mathrm{E}-07\end{array}$ \\
\hline $\begin{array}{l}\text { Emission } \\
\text { Accident }\end{array}$ & $\begin{array}{l}1.0 E-04 \\
3.3 E-03\end{array}$ & $\begin{array}{l}1.7 \mathrm{E}-04 \\
4.4 \mathrm{E}-05\end{array}$ \\
\hline
\end{tabular}

Table 9. Expected Annual Cancer Incidence for the Shipping Campaign

\begin{tabular}{lll}
\hline Exposure Group & Truck & Rail \\
\hline Radiological & $1.9 \mathrm{E}-03$ & $2.3 \mathrm{E}-04$ \\
$\quad$ Normal Crew & $2.8 \mathrm{E}-03$ & $4.5 \mathrm{E}-04$ \\
$\quad$ Normal Public & $1.8 \mathrm{E}-05$ & $2.9 \mathrm{E}-06$ \\
$\quad$ Accident Public & $1.0 \mathrm{E}-04$ & $1.7 \mathrm{E}-04$ \\
Nonradiological & NA & NA \\
Emission & & \\
\hline
\end{tabular}

Table 10. Expected Annual Genetic Effects for the Shipping Campaign

\begin{tabular}{lll}
\hline Exposure Group & Truck & Rail \\
\hline Radiological & & \\
Normal Crew & $8.1 E-05$ & $9.9 E-05$ \\
Normal Public & $1.6 \mathrm{E}-04$ & $2.7 \mathrm{E}-05$ \\
Accident Public & $3.6 \mathrm{E}-07$ & $6.1 \mathrm{E}-08$ \\
$\begin{array}{c}\text { Nonradiological } \\
\text { Emission }\end{array}$ & NA & NA \\
Accident & NA & NA \\
\hline
\end{tabular}




\section{B.29 WM LLMW CENTRALIZED ALTERNATIVE (CASE 17): CONTACT-HANDLED ORGANIC LIQUIDS: NONALPHA - UNTREATED}

Table 1. Summary of Rouce Information

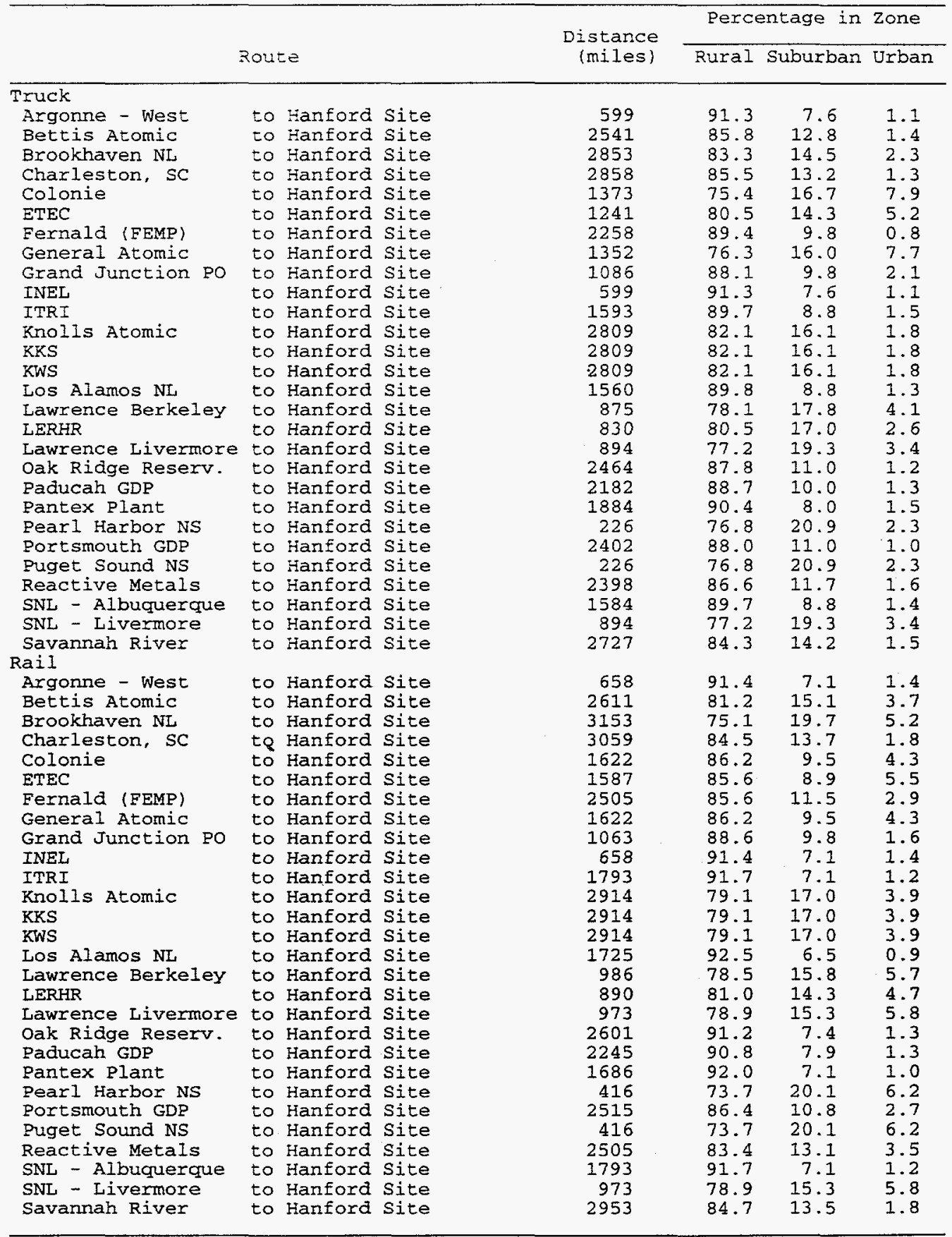


Table 2. Annual Number of Shipments and one-Way Shipment Distances

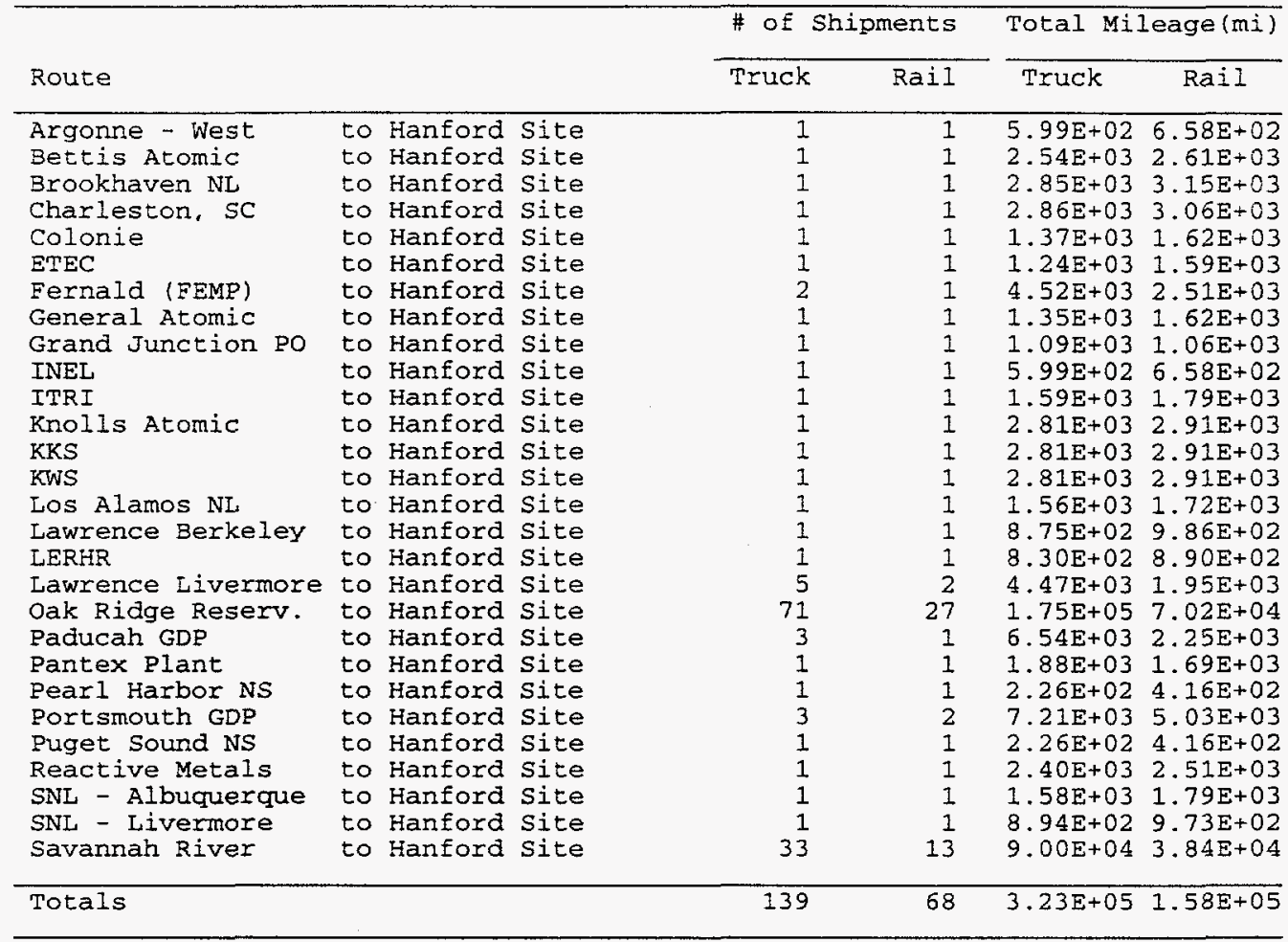

Table 3. Incident-Free Dose per Shipment (person-rem/shipment)

\begin{tabular}{|c|c|c|c|c|c|}
\hline \multirow[b]{2}{*}{ Source/Route (s) } & \multirow[b]{2}{*}{ Crew } & \multicolumn{4}{|c|}{ General Public } \\
\hline & & off-Link & On-Link & Stops & Total \\
\hline $\begin{array}{l}\text { MLLW (O) AW to HS } \\
\text { Argonne - West }\end{array}$ & 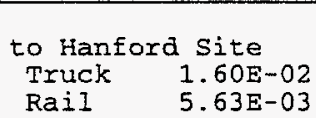 & $\begin{array}{l}7.18 E-04 \\
2.83 E-03\end{array}$ & $\begin{array}{l}2.06 E-03 \\
6.58 E-05\end{array}$ & $\begin{array}{l}1.68 E-02 \\
5.51 E-03\end{array}$ & $\begin{array}{l}1.95 E-02 \\
8.40 E-03\end{array}$ \\
\hline $\begin{array}{l}\text { MLLW(O) BA to HS } \\
\text { Bettis Atomic }\end{array}$ & $\begin{array}{ll}\text { to Hanford site } \\
\text { Truck } & 7.21 \mathrm{E}-02 \\
\text { Rail } & 1.38 \mathrm{E}-02\end{array}$ & $\begin{array}{l}4.36 E-03 \\
2.67 E-02\end{array}$ & $\begin{array}{l}9.77 E-03 \\
4.53 E-04\end{array}$ & $\begin{array}{l}7.11 \mathrm{E}-02 \\
1.15 \mathrm{E}-02\end{array}$ & $\begin{array}{l}8.53 E-02 \\
3.87 E-02\end{array}$ \\
\hline $\begin{array}{l}\text { MLIW (O) BN to HS } \\
\text { Brookhaven NL }\end{array}$ & $\begin{array}{ll}\text { to Hanford site } \\
\text { Truck } & 8.38 \mathrm{E}-02 \\
\text { Rail } & 1.60 \mathrm{E}-02\end{array}$ & $\begin{array}{l}6.48 \mathrm{E}-03 \\
4.38 \mathrm{E}-02\end{array}$ & $\begin{array}{l}1.28 E-02 \\
6.87 E-04\end{array}$ & $\begin{array}{l}7.99 \mathrm{E}-02 \\
1.32 \mathrm{E}-02\end{array}$ & $\begin{array}{l}9.92 \mathrm{E}-02 \\
5.76 \mathrm{E}-02\end{array}$ \\
\hline $\begin{array}{l}\text { MLIW (O) CH to HS } \\
\text { Charleston, SC }\end{array}$ & $\begin{array}{lr}\text { to Hanford site } \\
\text { Truck } & 8.12 \mathrm{E}-02 \\
\text { Rail } & 1.56 \mathrm{E}-02\end{array}$ & $\begin{array}{l}4.87 \mathrm{E}-03 \\
1.96 \mathrm{E}-02\end{array}$ & $\begin{array}{l}1.09 E-02 \\
4.24 E-04\end{array}$ & $\begin{array}{l}\text { 8.00E-02 } \\
1.29 \mathrm{E}-02\end{array}$ & $\begin{array}{l}9.58 \mathrm{E}-02 \\
3.29 \mathrm{E}-02\end{array}$ \\
\hline $\begin{array}{l}\text { MLLW(O) CL to HS } \\
\text { Colonie }\end{array}$ & $\begin{array}{ll}\text { to Hanford site } \\
\text { Truck } & 4.61 \mathrm{E}-02 \\
\text { Rail } & 9.65 \mathrm{E}-03\end{array}$ & $\begin{array}{l}7.22 \mathrm{E}-03 \\
1.64 \mathrm{E}-02\end{array}$ & $\begin{array}{l}1 \cdot 16 \mathrm{E}-02 \\
2 \cdot 49 \mathrm{E}-04\end{array}$ & $\begin{array}{l}3.84 \mathrm{E}-02 \\
8.47 \mathrm{E}-03\end{array}$ & $\begin{array}{l}5.72 \mathrm{E}-02 \\
2.52 \mathrm{E}-02\end{array}$ \\
\hline $\begin{array}{l}\text { MLLW(O) LC to HS } \\
\text { ETEC }\end{array}$ & 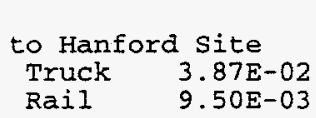 & $\begin{array}{l}4.63 \mathrm{E}-03 \\
1.96 \mathrm{E}-02\end{array}$ & $\begin{array}{l}8.05 \mathrm{E}-03 \\
2.68 \mathrm{E}-04\end{array}$ & $\begin{array}{l}3.47 E-02 \\
8.37 E-03\end{array}$ & $\begin{array}{l}4.74 E-02 \\
2.82 E-02\end{array}$ \\
\hline $\begin{array}{l}\text { MLLW(O) FE to HS } \\
\text { Fernald (FEMP) }\end{array}$ & 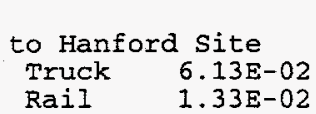 & $\begin{array}{l}2.71 \mathrm{E}-03 \\
2.00 \mathrm{E}-02\end{array}$ & $\begin{array}{l}7.49 E-03 \\
3.60 E-04\end{array}$ & $\begin{array}{l}6.32 \mathrm{E}-02 \\
1.12 \mathrm{E}-02\end{array}$ & $\begin{array}{l}7.34 E-02 \\
3.16 E-02\end{array}$ \\
\hline $\begin{array}{l}\text { MLLW(O) so to HS } \\
\text { General Atomic }\end{array}$ & 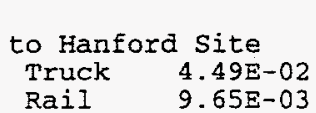 & $\begin{array}{l}6.88 \mathrm{E}-03 \\
1.64 \mathrm{E}-02\end{array}$ & $\begin{array}{l}1.11 E-02 \\
2.49 E-04\end{array}$ & $\begin{array}{l}3.78 E-02 \\
8.47 E-03\end{array}$ & $\begin{array}{l}5.58 E-02 \\
2.52 E-02\end{array}$ \\
\hline $\begin{array}{l}\text { MLIW (O) GJ to HS } \\
\text { Grand Junction PO }\end{array}$ & 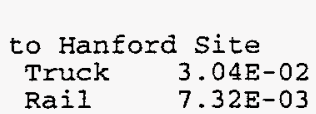 & $\begin{array}{l}2.03 \mathrm{E}-03 \\
5.46 \mathrm{E}-03\end{array}$ & $\begin{array}{l}\text { 4. } 58 E-03 \\
1.23 E-04\end{array}$ & $\begin{array}{l}3.04 E-02 \\
6.75 E-03\end{array}$ & $\begin{array}{l}3.70 \mathrm{E}-02 \\
1.23 \mathrm{E}-02\end{array}$ \\
\hline
\end{tabular}


Table 3. Incident-Free Dose per Shipment (person-rem/shipment) (Cont.)

\begin{tabular}{|c|c|c|c|c|c|}
\hline & & & General & 1 Public & \\
\hline Source/Route (s) & Crew & off-Link & On-Iink & Stops & Total \\
\hline MLLW $(O)$ IN to HS & & & & & \\
\hline INEL & $\begin{array}{ll}\text { to Hanford Site } \\
\text { Truck } & 1.60 \mathrm{E}-02 \\
\text { Rail } & 5.63 \mathrm{E}-03\end{array}$ & $\begin{array}{l}7.18 E-04 \\
2.83 E-03\end{array}$ & $\begin{array}{l}2.06 \mathrm{E}-03 \\
6.58 \mathrm{E}-05\end{array}$ & $\begin{array}{l}1.68 E-02 \\
5.51 E-03\end{array}$ & $\begin{array}{l}1.95 E-02 \\
8.40 E-03\end{array}$ \\
\hline MLLW(O) IT to HS & & & & & \\
\hline ITRI & $\begin{array}{lr}\text { to Hanford site } \\
\text { Truck } & 4.34 \mathrm{E}-02 \\
\text { Rail } & 1.04 \mathrm{E}-02\end{array}$ & $\begin{array}{l}2.30 \mathrm{E}-03 \\
6.90 \mathrm{E}-03\end{array}$ & $\begin{array}{l}5.91 \mathrm{E}-03 \\
1.72 \mathrm{E}-04\end{array}$ & $\begin{array}{l}4.46 \mathrm{E}-02 \\
9.00 \mathrm{E}-03\end{array}$ & $\begin{array}{l}5.28 \mathrm{E}-02 \\
1.61 \mathrm{E}-02\end{array}$ \\
\hline MLLW(O) KA to HS & & & & & \\
\hline Knolls Atomic & $\begin{array}{l}\text { to Hanford Site } \\
\text { Truck } 8.30 \mathrm{E}-02 \\
\text { Rail } 1.50 \mathrm{E}-02\end{array}$ & $\begin{array}{l}6.03 E-03 \\
3.18 \mathrm{E}-02\end{array}$ & $\begin{array}{l}1.19 \mathrm{E}-02 \\
5.40 \mathrm{E}-04\end{array}$ & $\begin{array}{l}7.87 E-02 \\
1.25 E-02\end{array}$ & $\begin{array}{l}9.66 \mathrm{E}-02 \\
4.48 \mathrm{E}-02\end{array}$ \\
\hline$\underset{\text { KKS }}{M L L W(O)} \mathrm{KK}$ to $\mathrm{HS}$ & to Hanford Site & & & & \\
\hline & $\begin{array}{ll}\text { Truck } & 8.30 \mathrm{E}-02 \\
\text { Rail } & 1.50 \mathrm{E}-02\end{array}$ & $\begin{array}{l}6.03 \mathrm{E}-03 \\
3.18 \mathrm{E}-02\end{array}$ & $\begin{array}{l}1.19 \mathrm{E}-02 \\
5.40 \mathrm{E}-04\end{array}$ & $\begin{array}{l}7.87 \mathrm{E}-02 \\
1.25 \mathrm{E}-02\end{array}$ & $\begin{array}{l}9.66 \mathrm{E}-02 \\
4.48 \mathrm{E}-02\end{array}$ \\
\hline$\underset{\text { KWS }}{M L L W(O)}$ KW to $H S$ & to Hanford Site & & & & \\
\hline & $\begin{array}{ll}\text { Truck } & 8.30 \mathrm{E}-02 \\
\text { Rail } & 1.50 \mathrm{E}-02\end{array}$ & $\begin{array}{l}6.03 E-03 \\
3.18 \mathrm{E}-02\end{array}$ & $\begin{array}{l}1.19 \mathrm{E}-02 \\
5.40 \mathrm{E}-04\end{array}$ & $\begin{array}{l}7.87 \mathrm{E}-02 \\
1.25 \mathrm{E}-02\end{array}$ & $\begin{array}{l}9.66 \mathrm{E}-02 \\
4.48 \mathrm{E}-02\end{array}$ \\
\hline MLLW(O) LA to HS & & & & & \\
\hline Los Alamos NL & to Hanford Site & & & & \\
\hline & $\begin{array}{ll}\text { Truck } & 4.24 \mathrm{E}-02 \\
\text { Rail } & 1.01 \mathrm{E}-02\end{array}$ & $\begin{array}{l}2.16 \mathrm{E}-03 \\
5.60 \mathrm{E}-03\end{array}$ & $\begin{array}{l}5.65 \mathrm{E}-03 \\
1.54 \mathrm{E}-04\end{array}$ & $\begin{array}{l}4.37 E-02 \\
8.79 E-03\end{array}$ & $\begin{array}{l}5.15 \mathrm{E}-02 \\
1.45 \mathrm{E}-02\end{array}$ \\
\hline MLLW(O) LB to HS & 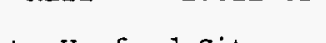 & & & & \\
\hline Lawrence Berkeley & to Hanford Site & & & & \\
\hline & $\begin{array}{ll}\text { Truck } & 2.76 \mathrm{E}-02 \\
\text { Rail } & 6.99 \mathrm{E}-03\end{array}$ & $\begin{array}{l}3.02 \mathrm{E}-03 \\
1.38 \mathrm{E}-02\end{array}$ & $\begin{array}{l}5.17 \mathrm{E}-03 \\
2.03 \mathrm{E}-04\end{array}$ & $\begin{array}{l}2.45 E-02 \\
6.51 \mathrm{E}-03\end{array}$ & $\begin{array}{l}3.27 E-02 \\
2.05 E-02\end{array}$ \\
\hline MLLW(O) SM to HS & & & & & \\
\hline LERHR & $\begin{array}{l}\text { to Hanford Site } \\
\text { Truck } 2.51 \mathrm{E}-02\end{array}$ & $2.17 \mathrm{E}-03$ & $4.00 E-03$ & $2.32 \mathrm{E}-02$ & $2.94 \mathrm{E}-02$ \\
\hline & Rail $\quad 6.59 \mathrm{E}-03$ & $1.07 \mathrm{E}-02$ & $1.64 \mathrm{E}-04$ & $6.22 \mathrm{E}-03$ & $1.70 \mathrm{E}-02$ \\
\hline MLLW(O) LL to HS & & & & & \\
\hline Lawrence Live & to Hanford Site & & & & \\
\hline & $\begin{array}{ll}\text { Truck } & 2.82 \mathrm{E}-02 \\
\text { Rail } & 6.94 \mathrm{E}-03\end{array}$ & $\begin{array}{l}2.88 \mathrm{E}-03 \\
1.38 \mathrm{E}-02\end{array}$ & $\begin{array}{l}4.92 \mathrm{E}-03 \\
2.00 \mathrm{E}-04\end{array}$ & $\begin{array}{l}2.50 E-02 \\
6.48 E-03\end{array}$ & $\begin{array}{l}3.28 \mathrm{E}-02 \\
2.05 \mathrm{E}-02\end{array}$ \\
\hline MLLW(O) OR to HS & & & & & \\
\hline Oak Ridge Reserv. & to Hanford Site & & & & \\
\hline & $\begin{array}{ll}\text { Truck } & 6.83 \mathrm{E}-02 \\
\text { Rail } & 1.37 \mathrm{E}-02\end{array}$ & $\begin{array}{l}3.65 \mathrm{E}-03 \\
1.08 \mathrm{E}-02\end{array}$ & $\begin{array}{l}8.95 \mathrm{E}-03 \\
2.59 \mathrm{E}-04\end{array}$ & $\begin{array}{l}6.90 \mathrm{E}-02 \\
1.15 \mathrm{E}-02\end{array}$ & $\begin{array}{l}8.16 \mathrm{E}-02 \\
2.26 \mathrm{E}-02\end{array}$ \\
\hline MLLW(O) PA to HS & & & & & \\
\hline Paducah GDP & to Hanford Site & & & & \\
\hline & $\begin{array}{ll}\text { Truck } & 6.00 \mathrm{E}-02 \\
\text { Rail } & 1.22 \mathrm{E}-02\end{array}$ & $\begin{array}{l}3.22 \mathrm{E}-03 \\
9.43 \mathrm{E}-03\end{array}$ & $\begin{array}{l}8.02 \mathrm{E}-03 \\
2.28 \mathrm{E}-04\end{array}$ & $\begin{array}{l}6.11 \mathrm{E}-02 \\
1.04 \mathrm{E}-02\end{array}$ & $\begin{array}{l}7.23 \mathrm{E}-02 \\
2.01 \mathrm{E}-02\end{array}$ \\
\hline MLLW(O) PP to HS & & & & & \\
\hline Pantex Plant & to Hanford Site & & & & \\
\hline & $\begin{array}{ll}\text { Truck } & 5.10 \mathrm{E}-02 \\
\text { Rail } & 9.92 \mathrm{E}-03\end{array}$ & $\begin{array}{l}2.68 \mathrm{E}-03 \\
5.71 \mathrm{E}-03\end{array}$ & $\begin{array}{l}7.01 \mathrm{E}-03 \\
1.56 \mathrm{E}-04\end{array}$ & $\begin{array}{l}5.27 E-02 \\
8.67 E-03\end{array}$ & $\begin{array}{l}6.24 \mathrm{E}-02 \\
1.45 \mathrm{E}-02\end{array}$ \\
\hline MLLW(O) SW to HS & & & & & \\
\hline Pearl Harbor NS & to Hanford Site & & & & \\
\hline & $\begin{array}{ll}\text { Truck } & 7.06 \mathrm{E}-03 \\
\text { Rail } & 4.62 \mathrm{E}-03\end{array}$ & $\begin{array}{l}6.28 \mathrm{E}-04 \\
6.63 \mathrm{E}-03\end{array}$ & $\begin{array}{l}1.09 \mathrm{E}-03 \\
9.79 \mathrm{E}-05\end{array}$ & $\begin{array}{l}6.33 E-03 \\
4.76 E-03\end{array}$ & $\begin{array}{l}8.04 \mathrm{E}-03 \\
1.15 \mathrm{E}-02\end{array}$ \\
\hline MLLW(O) PO to HS & & & & & \\
\hline Portsmouth GDP & to Hanford Site & & & & \\
\hline & $\begin{array}{ll}\text { Truck } & 6.63 \mathrm{E}-02 \\
\text { Rail } & 1.34 \mathrm{E}-02\end{array}$ & $\begin{array}{l}3.38 \mathrm{E}-03 \\
1.89 \mathrm{E}-02\end{array}$ & $\begin{array}{l}8.48 \mathrm{E}-03 \\
3.46 \mathrm{E}-04\end{array}$ & $\begin{array}{l}6.72 \mathrm{E}-02 \\
1.12 \mathrm{E}-02\end{array}$ & $\begin{array}{l}7.91 \mathrm{E}-02 \\
3.04 \mathrm{E}-02\end{array}$ \\
\hline MLLW(O) PN to HS & & & & & \\
\hline Puget Sound NS & to Hanford Site & & & & \\
\hline & $\begin{array}{ll}\text { Rruck } & 4.62 \mathrm{E}-03 \\
\text { Rail } & 4.62 \mathrm{E}-03\end{array}$ & $\begin{array}{l}0.28 \mathrm{E}-04 \\
6.63 \mathrm{E}-03\end{array}$ & $\begin{array}{l}1.09 \mathrm{E}-03 \\
9.79 \mathrm{E}-05\end{array}$ & $\begin{array}{l}6.33 E-03 \\
4.76 E-03\end{array}$ & $\begin{array}{l}\text { 8. } \\
1.15 \mathrm{E}-02\end{array}$ \\
\hline$M L L W(0) R M$ to HS & & & & & \\
\hline Reactive Metals & to Hanford Site & & & & \\
\hline & $\begin{array}{ll}\text { Truck } & 6.76 \mathrm{E}-02 \\
\text { Rail } & 1.33 \mathrm{E}-02\end{array}$ & $\begin{array}{l}4.22 \mathrm{E}-03 \\
2.35 \mathrm{E}-02\end{array}$ & $\begin{array}{l}9.51 \mathrm{E}-03 \\
4.01 \mathrm{E}-04\end{array}$ & $\begin{array}{l}6.71 \mathrm{E}-02 \\
1.12 \mathrm{E}-02\end{array}$ & $\begin{array}{l}8.09 \mathrm{E}-02 \\
3.51 \mathrm{E}-02\end{array}$ \\
\hline MLLW (O) SA to HS & & & & & \\
\hline SNL - Albuquerque & to Hanford Site & & & & \\
\hline & $\begin{array}{ll}\text { Truck } & 4.31 \mathrm{E}-02 \\
\text { Rail } & 1.04 \mathrm{E}-02\end{array}$ & $\begin{array}{l}2.27 \mathrm{E}-03 \\
6.90 \mathrm{E}-03\end{array}$ & $\begin{array}{l}5.84 \mathrm{E}-03 \\
1.72 \mathrm{E}-04\end{array}$ & $\begin{array}{l}4.43 \mathrm{E}-02 \\
9.00 \mathrm{E}-03\end{array}$ & $\begin{array}{l}5.24 \mathrm{E}-02 \\
1.61 \mathrm{E}-02\end{array}$ \\
\hline MLLW (O) SL to HS & & & & & \\
\hline SNL - Iivermore & to Hanford Site & & & & \\
\hline & $\begin{array}{ll}\text { Truck } & 2.82 \mathrm{E}-02 \\
\text { Rail } & 6.94 \mathrm{E}-03\end{array}$ & $\begin{array}{l}2.88 \mathrm{E}-03 \\
1.38 \mathrm{E}-02\end{array}$ & $\begin{array}{l}4.92 \mathrm{E}-03 \\
2.00 \mathrm{E}-04\end{array}$ & $\begin{array}{l}2.50 \mathrm{E}-02 \\
6.48 \mathrm{E}-03\end{array}$ & $\begin{array}{l}3.28 \mathrm{E}-02 \\
2.05 \mathrm{E}-02\end{array}$ \\
\hline MLLW $(0)$ SR to HS & & & & & \\
\hline Savannah River & to Hanford site & & & & \\
\hline & $\begin{array}{ll}\text { Truck } & 7.86 \mathrm{E}-02 \\
\text { Rail } & 1.52 \mathrm{E}-02\end{array}$ & $\begin{array}{l}5.10 \mathrm{E}-03 \\
1.85 \mathrm{E}-02\end{array}$ & $\begin{array}{l}1.08 \mathrm{E}-02 \\
4.05 \mathrm{E}-04\end{array}$ & $\begin{array}{l}7.64 \mathrm{E}-02 \\
1.26 \mathrm{E}-02\end{array}$ & $\begin{array}{l}9.23 E-02 \\
3.15 E-02\end{array}$ \\
\hline
\end{tabular}


Table 4. Accident Dose Risk per Shipment (person-rem/shipment)

\begin{tabular}{|c|c|c|c|}
\hline \multicolumn{2}{|l|}{ Source/Route } & \multirow[t]{2}{*}{ Truck } & \multirow[t]{2}{*}{ Rail } \\
\hline MLLW $(0)$ AW to HS & & & \\
\hline $\begin{array}{l}\text { Argonne - West } \\
\text { MLLW (O) BA to HS }\end{array}$ & to Hanford Site & $1.00 \mathrm{E}-05$ & $5.37 \mathrm{E}-06$ \\
\hline $\begin{array}{l}\text { Bettis Atomic } \\
\text { MLLW(0) BN to HS }\end{array}$ & to Hanford Site & $2.62 \mathrm{E}-03$ & $9.99 \mathrm{E}-04$ \\
\hline $\begin{array}{l}\text { Brookhaven NL } \\
\text { MLLW(O) CH to HS }\end{array}$ & to Hanford Site & $9.79 \mathrm{E}-03$ & $4.90 \mathrm{E}-03$ \\
\hline $\begin{array}{l}\text { Charleston, SC } \\
\text { MLLW(O) CL to HS }\end{array}$ & to Hanford Site & $1.12 \mathrm{E}-01$ & $2.27 \mathrm{E}-02$ \\
\hline $\begin{array}{l}\text { Colonie } \\
\text { MLLW(O) LC to HS }\end{array}$ & to Hanford Site & $2.25 \mathrm{E}-04$ & $6.67 E-05$ \\
\hline $\begin{array}{l}\text { ETEC } \\
\text { MLLW (O) FE to HS }\end{array}$ & to Hanford Site & $1.30 \mathrm{E}-05$ & $5.22 \mathrm{E}-06$ \\
\hline $\begin{array}{l}\text { Fernald (FEMP) } \\
\text { MLLW(O) so to HS }\end{array}$ & to Hanford Site & $3.88 \mathrm{E}-03$ & $3.18 \mathrm{E}-03$ \\
\hline $\begin{array}{l}\text { General Atomic } \\
\text { MLLW(O) GJ to HS }\end{array}$ & to Hanford Site & $1.69 \mathrm{E}-05$ & $5.22 E-06$ \\
\hline $\begin{array}{l}\text { Grand Junction Po } \\
\text { MLLW(O) IN to HS }\end{array}$ & to Hanford Site & $1.38 E-06$ & $4.77 \mathrm{E}-07$ \\
\hline $\begin{array}{l}\text { INEL } \\
\text { MLLW (O) IT to HS }\end{array}$ & to Hanford Site & $2.25 \mathrm{E}-02$ & $1.21 \mathrm{E}-02$ \\
\hline $\begin{array}{l}\text { ITRI } \\
\text { MLLW(O) KA to HS }\end{array}$ & to Hanford Site & $2.96 \mathrm{E}-05$ & $3.23 \mathrm{E}-06$ \\
\hline $\begin{array}{l}\text { Krolls Atomic } \\
\text { MLLW(O) KK to HS }\end{array}$ & to Hanford Site & $8.53 \mathrm{E}-02$ & $2.73 \mathrm{E}-02$ \\
\hline $\begin{array}{c}\text { KKS } \\
\text { MLLW (O) KW to HS }\end{array}$ & to Hanford Site & $6.55 \mathrm{E}-02$ & $2.10 \mathrm{E}-02$ \\
\hline $\begin{array}{l}\text { KWS } \\
\text { MLLW (O) LA to HS }\end{array}$ & to Hanford Site & $8.93 E-03$ & $2.86 E-03$ \\
\hline $\begin{array}{l}\text { Los Alamos NL } \\
\text { MLLW (O) LB to HS }\end{array}$ & to Hanford Site & $1.93 \mathrm{E}-03$ & $2.13 \mathrm{E}-04$ \\
\hline $\begin{array}{l}\text { Lawrence Berkeley } \\
\text { MLLW(O) SM to HS }\end{array}$ & to Hanford Site & $7.79 \mathrm{E}-05$ & $4.38 E-05$ \\
\hline $\begin{array}{l}\text { LERHR } \\
\text { MLLW (O) LL to HS }\end{array}$ & to Hanford Site & $7.78 \mathrm{E}-06$ & $4.14 \mathrm{E}-06$ \\
\hline $\begin{array}{l}\text { Lawrence Livermore } \\
\text { MLLW(O) OR to HS }\end{array}$ & to Hanford Site & $3.93 \mathrm{E}-03$ & $5.23 \mathrm{E}-03$ \\
\hline $\begin{array}{l}\text { Oak Ridge Reserv. } \\
\text { MLLW(0) PA to HS }\end{array}$ & to Hanford Site & $7.41 \mathrm{E}-02$ & $3.07 E-02$ \\
\hline $\begin{array}{l}\text { Paducah GDP } \\
\text { MLLW(O) PP to HS }\end{array}$ & to Hanford Site & $1.14 \mathrm{E}+00$ & $5.74 E-01$ \\
\hline $\begin{array}{l}\text { Pantex Plant } \\
\text { MLLW(O) SW to HS }\end{array}$ & to Hanford Site & $4.59 E-04$ & $4.90 E-05$ \\
\hline $\begin{array}{l}\text { Pear1 Harbor NS } \\
\text { MLLW(O) PO to HS }\end{array}$ & to Hanford Site & $8.47 E-06$ & $6.32 \mathrm{E}-06$ \\
\hline $\begin{array}{l}\text { Portsmouth GDP } \\
\text { MLLW (0) PN to HS }\end{array}$ & to Hanford Site & $1.56 \mathrm{E}-03$ & $8.20 \mathrm{E}-04$ \\
\hline $\begin{array}{l}\text { Puget Sound NS } \\
\text { MLLW(O) RM to HS }\end{array}$ & to Hanford site & $1.95 \mathrm{E}-04$ & $1.46 E-04$ \\
\hline $\begin{array}{l}\text { Reactive Metals } \\
\text { MLIW(O) SA to HS }\end{array}$ & to Hanford Site & $2.99 E-04$ & $1.12 \mathrm{E}-04$ \\
\hline $\begin{array}{l}\text { SNL - Albuquerque } \\
\text { MLLW(O) SL to HS }\end{array}$ & to Hanford Site & $2.07 E-06$ & $2.27 E-07$ \\
\hline $\begin{array}{l}\text { SNL - Livermore } \\
\text { MLIW(O) SR to HS }\end{array}$ & to Hanford Site & $1.95 E-03$ & $1.04 E-03$ \\
\hline Savannah River & to Hanford Site & $1.53 \mathrm{E}-01$ & $7.69 \mathrm{E}-02$ \\
\hline
\end{tabular}


Table 5. Nonradiological Risk Factors per Shipment (fatalities/shipment)

\begin{tabular}{|c|c|c|c|}
\hline Mode & Route & Emission & Accident \\
\hline Truck & & & \\
\hline Argonne - West & to Hanford site & $2.19 E-06$ & $3.07 E-05$ \\
\hline Bettis Atomic & to Hanford Site & $1.14 \mathrm{E}-05$ & 1. $36 E-04$ \\
\hline Brookhaven NL & to Hanford Site & $2.07 E-05$ & $1.68 \mathrm{E}-04$ \\
\hline Charleston, SC & to Hanford Site & $1.20 E-05$ & $1.99 \mathrm{E}-04$ \\
\hline Colonie & to Hanford Site & $3.48 \mathrm{E}-05$ & $8.47 E-05$ \\
\hline ETEC & to Hanford Site & $2.08 \mathrm{E}-05$ & $7.74 \mathrm{E}-05$ \\
\hline Fernald (FEMP) & to Hanford site & $5.86 \mathrm{E}-06$ & $1.22 \mathrm{E}-04$ \\
\hline General Atomic & to Hanford site & $3.33 E-05$ & $8.36 \mathrm{E}-05$ \\
\hline Grand Junction PO & to Hanford Site & $7.50 E-06$ & $6.25 \mathrm{E}-05$ \\
\hline INEL & to Hanford Site & $2.19 E-06$ & $3.07 \mathrm{E}-05$ \\
\hline ITRI & to Hanford Site & $7.44 E-06$ & $1.05 \mathrm{E}-04$ \\
\hline Knolis Atomic & to Hanfora site & $1.59 \mathrm{E}-05$ & 1. $51 \mathrm{E}-04$ \\
\hline KKS & to Hanford Site & $1.59 \mathrm{E}-05$ & $1.51 \mathrm{E}-04$ \\
\hline KWS & to Hanford Site & $1.59 \mathrm{E}-05$ & 1. $51 \mathrm{E}-04$ \\
\hline Los Alamos NL & to Hanford Site & $6.66 \mathrm{E}-06$ & $1.02 \mathrm{E}-04$ \\
\hline Lawrence Berkeley & to Hanford Site & $1.16 \mathrm{E}-05$ & $4.91 E-05$ \\
\hline LERHR & to Hanford Site & $6.82 \mathrm{E}-06$ & $4.66 \mathrm{E}-05$ \\
\hline Lawrence Livermore & to Hanford Site & $9.85 \mathrm{E}-06$ & $5.03 E-05$ \\
\hline Oak Ridge Reserv. & to Hanford site & $9.50 E-06$ & $1.61 E-04$ \\
\hline Paducah GDP & to Hanford Site & $9.30 \mathrm{E}-06$ & $1.41 E-04$ \\
\hline Pantex Plant & to Hanford Site & $9.21 E-06$ & $1.25 E-04$ \\
\hline Pearl Harbor NS & to Hanford site & $1.67 \mathrm{E}-06$ & $9.56 E-05$ \\
\hline Portsmouth GDP & to Hanford Site & $8.11 \mathrm{E}-06$ & 1. $28 E-04$ \\
\hline Puget Sound NS & to Hanford Site & $1.67 \mathrm{E}-06$ & $9.56 \mathrm{E}-06$ \\
\hline Reactive Metals & to Hanford site & $1.27 \mathrm{E}-05$ & $1.28 \mathrm{E}-04$ \\
\hline SNL - Albuquerque & to Hanford Site & $7.24 \mathrm{E}-06$ & $1.05 \mathrm{E}-04$ \\
\hline SNL - Livermore & to Hanford Site & $9.85 \mathrm{E}-06$ & $5.03 E-05$ \\
\hline Savannah River & to Hanford Site & $1.31 \mathrm{E}-05$ & $1.81 E-04$ \\
\hline Rail & & & \\
\hline Argonne - West & to Hanford Site & $3.98 E-06$ & $1.38 \mathrm{E}-06$ \\
\hline Bettis Atomic & to Hanford Site & $4.07 E-05$ & $5.46 \mathrm{E}-06$ \\
\hline Brookhaven NL & to Hanford Site & $6.80 E-05$ & $6.60 E-06$ \\
\hline Charleston, SC & to Hanford Site & $2.35 \mathrm{E}-05$ & $6.40 E-06$ \\
\hline Colonie & to Hanford Site & $2.91 E-05$ & $3.39 E-06$ \\
\hline ETEC & to Hanford site & $3.67 E-05$ & $3.32 \mathrm{E}-06$ \\
\hline Fernald (FEMP) & to Hanford Site & $3.07 E-05$ & $5.24 E-06$ \\
\hline General Atomic & to Hanford Site & $2.91 \mathrm{E}-05$ & $3.39 \mathrm{E}-06$ \\
\hline Grand Junction Po & to Hanford Site & $7.07 \mathrm{E}-06$ & $2.22 \mathrm{E}-06$ \\
\hline INEL & to Hanford Site & $3.98 E-06$ & $1.38 \mathrm{E}-06$ \\
\hline ITRI & to Hanford Site & $9.04 \mathrm{E}-06$ & $3.75 \mathrm{E}-06$ \\
\hline Knolls Atomic & to Hanford Site & $4.72 \mathrm{E}-05$ & $6.10 \mathrm{E}-06$ \\
\hline KKS & to Hanford Site & $4.72 \mathrm{E}-05$ & $6.10 \mathrm{E}-06$ \\
\hline KWS & to Hanford Site & $4.72 \mathrm{E}-05$ & $6.10 \mathrm{E}-06$ \\
\hline Los Alamos NL & to Hanford Site & $6.82 \mathrm{E}-06$ & $3.61 E-06$ \\
\hline Lawrence Berkeley & to Hanford Site & $2.33 E-05$ & $2.06 \mathrm{E}-06$ \\
\hline LERHR & to Hanford Site & $1.77 \mathrm{E}-05$ & $1.86 \mathrm{E}-06$ \\
\hline Lawrence Livermore & to Hanford Site & $2.36 \mathrm{E}-05$ & $2.04 \mathrm{E}-06$ \\
\hline Oak Ridge Reserv. & to Hanford Site & $1.45 \mathrm{E}-05$ & $5.44 \mathrm{E}-06$ \\
\hline Paducah GDP & to Hanford Site & $1.23 \mathrm{E}-05$ & $4.70 E-06$ \\
\hline Pantex Plant & to Hanford Site & $6.78 \mathrm{E}-06$ & $3.53 \mathrm{E}-06$ \\
\hline Pearl Harbor NS & to Hanford Site & $1.08 \mathrm{E}-05$ & $8.70 \mathrm{E}-07$ \\
\hline Portsmouth GDP & to Hanford site & $2.88 \mathrm{E}-05$ & $5.26 \mathrm{E}-06$ \\
\hline Puget Sound NS & to Hanford site & $1.08 \mathrm{E}-05$ & $8.70 E-07$ \\
\hline Reactive Metals & to Hanford Site & $3.65 E-05$ & $5.24 E-06$ \\
\hline SNL - Albuquerque & to Hanford site & $9.04 \mathrm{E}-06$ & $3.75 \mathrm{E}-06$ \\
\hline SNL - Livermore & to Hanford Site & $2.36 \mathrm{E}-05$ & $2.04 E-06$ \\
\hline Savannah River & to Hanford Site & $2.21 \mathrm{E}-05$ & $6.18 \mathrm{E}-06$ \\
\hline
\end{tabular}


Table 6. Annual Incident-Free Dose for the Shipping Campaign (person-rem/yr)

\begin{tabular}{|c|c|c|c|c|c|c|}
\hline \multirow[b]{2}{*}{ Route } & & & \multicolumn{2}{|c|}{ Truck } & \multicolumn{2}{|c|}{ Rail } \\
\hline & & & Crew & Public & Crew & Public \\
\hline Argonne - West & & Hanford Site & $1.60 \mathrm{E}-02$ & $1.95 E-02$ & $5.63 E-03$ & $8.40 E-03$ \\
\hline Bettis Atomic & & Hanford Site & $7.21 \mathrm{E}-02$ & $8.53 E-02$ & $1.38 \mathrm{E}-02$ & $3.87 E-02$ \\
\hline Brookhaven NL & 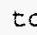 & Hanford Site & $8.38 \mathrm{E}-02$ & $9.92 E-02$ & $1.60 \mathrm{E}-02$ & $5.76 E-02$ \\
\hline Charleston, SC & 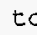 & Hanford Site & $8.12 \mathrm{E}-02$ & $9.58 \mathrm{E}-02$ & $1.56 \mathrm{E}-02$ & $3.29 E-02$ \\
\hline Colonie & 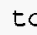 & Hanford Site & $4.61 E-02$ & $5.72 E-02$ & $9.65 E-03$ & $2.52 E-02$ \\
\hline ETEC & to & Hanford Site & $3.87 \varepsilon-02$ & $4.74 E-02$ & $9.50 \mathrm{E}-03$ & $2.82 E-02$ \\
\hline Fernald (FEMP) & 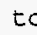 & Hanford Site & $1.23 E-01$ & $1.47 \mathrm{E}-01$ & $1.33 E-02$ & $3.16 \mathrm{E}-02$ \\
\hline General Atomic & 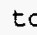 & Hanford Site & $4.49 E-02$ & $5.58 E-02$ & $9.65 E-03$ & $2.52 E-02$ \\
\hline Grand Junction PO & to & Hanford Site & $3.04 \mathrm{E}-02$ & $3.70 \mathrm{E}-02$ & $7.32 \mathrm{E}-03$ & $1.23 \mathrm{E}-02$ \\
\hline INEL & 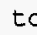 & Hanford Site & $1.60 \mathrm{E}-02$ & $1.95 E-02$ & $5.63 E-03$ & $8.40 E-03$ \\
\hline ITRI & 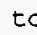 & Hanford Site & $4.34 E-02$ & $5.28 \mathrm{E}-02$ & $1.04 \mathrm{E}-02$ & $1.61 E-02$ \\
\hline Knolls Atomic & 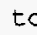 & Hanford Site & $30 E-02$ & $9.66 \mathrm{E}-02$ & $1.50 \mathrm{E}-02$ & $4.48 \mathrm{E}-02$ \\
\hline KKS & to & Hanford Site & $8.30 \mathrm{E}-02$ & $9.66 \mathrm{E}-02$ & $1.50 \mathrm{E}-02$ & $4.48 \mathrm{E}-02$ \\
\hline KWS & to & Hanford Site & $8.30 \mathrm{E}-02$ & $9.66 E-02$ & $1.50 E-02$ & $4.48 \mathrm{E}-02$ \\
\hline Los Alamos NL & tc & Hanford Site & $4.24 \mathrm{E}-02$ & $5.15 E-02$ & $1.01 E-02$ & $1.45 E-02$ \\
\hline Lawrence Berkeley & to & Hanford Site & $2.76 \mathrm{E}-02$ & $3.27 E-02$ & $6.99 E-03$ & $2.05 E-02$ \\
\hline LERHR & tc & Hanford Site & $2.51 E-02$ & $2.94 E-02$ & $6.59 E-03$ & $1.70 \mathrm{E}-02$ \\
\hline Lawrence Livermore & to & Hanford Site & $1.41 \mathrm{E}-01$ & $1.64 E-01$ & $1.39 E-02$ & $4.10 \mathrm{E}-02$ \\
\hline Oak Ridge Reserv. & tc & Hanford Site & $4.85 E+00$ & $5.79 \mathrm{E}+00$ & $3.71 E-01$ & $6.09 E-01$ \\
\hline Paducah GDP & tc & Hanford Site & $1.80 E-01$ & $2.17 E-01$ & $1.22 \mathrm{E}-02$ & $2.01 E-02$ \\
\hline Pantex Plant & to & Hanford Site & $5.10 \mathrm{E}-02$ & $6.24 E-02$ & $9.92 E-03$ & $1.45 E-02$ \\
\hline Pearl Harbor NS & to & Hanford Site & $7.06 \mathrm{E}-03$ & $8.04 E-03$ & $4.62 \mathrm{E}-03$ & $1.15 \mathrm{E}-02$ \\
\hline Portsmouth GDP & 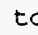 & Hanford Site & $1.99 \mathrm{E}-01$ & $2.37 E-01$ & $2.68 \mathrm{E}-02$ & $6.09 \pm-02$ \\
\hline Puget Sound NS & to & Hanford Site & $7.06 \mathrm{E}-03$ & $8.04 E-03$ & $4.62 \mathrm{E}-03$ & $1.15 E-02$ \\
\hline Reactive Metals & to $1-3 \cdot(-1)$ & Hanford Site & $6.76 \mathrm{E}-02$ & $8.09 \mathrm{E}-02$ & $1.33 E-02$ & $3.51 \mathrm{E}-02$ \\
\hline SNL - Albuquerque & to & Hanford Site & $4.31 E-02$ & $5.24 \mathrm{E}-02$ & $1.04 \mathrm{E}-02$ & $1.61 \mathrm{E}-02$ \\
\hline SNL - Livermore & & Hanford Site & $2.82 \mathrm{E}-02$ & $3.28 E-02$ & $6.94 \mathrm{E}-03$ & $2.05 E-02$ \\
\hline Savannah River & to & Hanford Site & $2.59 \mathrm{E}+00$ & $3.05 E+00$ & $1.98 \mathrm{E}-01$ & $4.10 E-01$ \\
\hline
\end{tabular}

Table 7. Annual Accident Dose Risk for the Shipping Campaign (person-rem/yr)

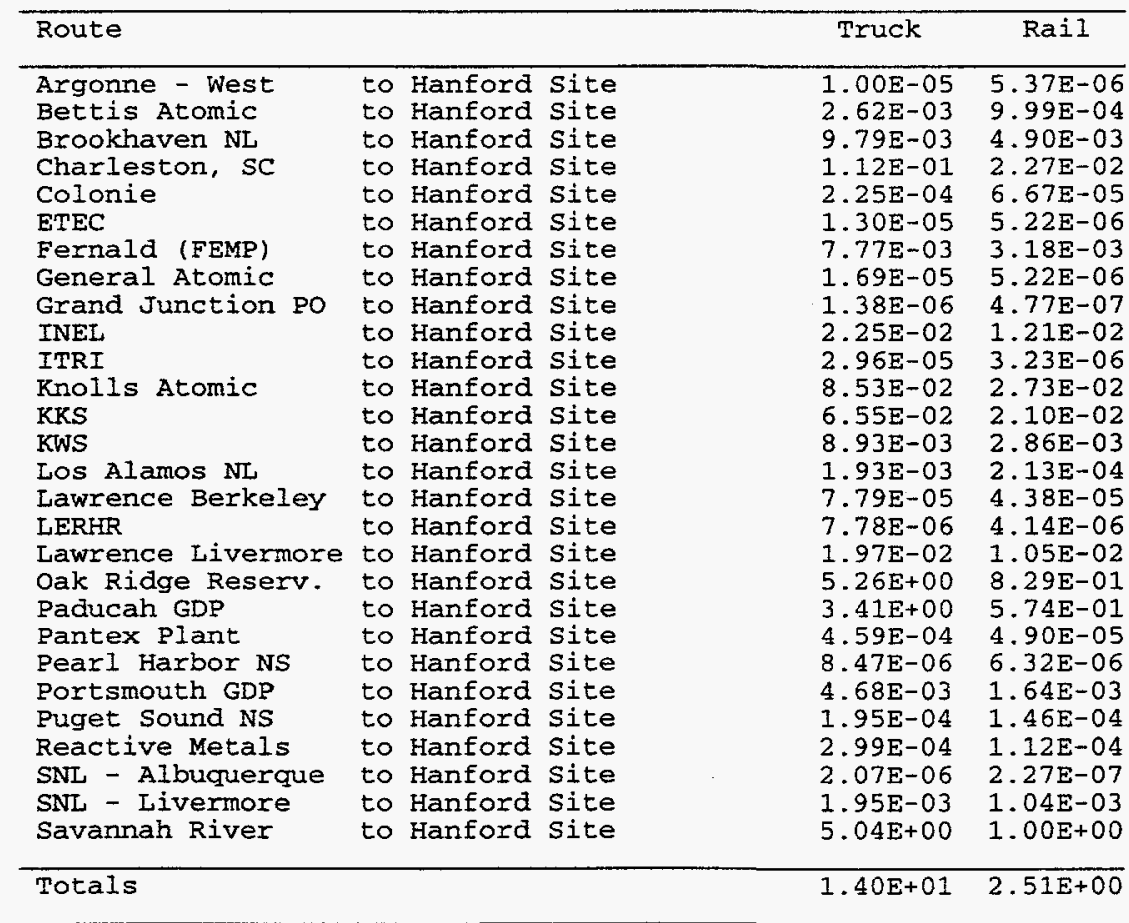


Table 8. Expected Annual Fatalities for the Shipping Campaign

\begin{tabular}{lll}
\hline Exposure Group & Truck & Rail \\
\hline Radiological & & \\
Normal Crew & $3.6 \mathrm{E}-03$ & $3.4 \mathrm{E}-04$ \\
Normal Public & $5.4 \mathrm{E}-03$ & $8.6 \mathrm{E}-04$ \\
Accident Public & $7.0 \mathrm{E}-03$ & $1.3 \mathrm{E}-03$ \\
Nonradiological & & \\
Emission & $1.5 \mathrm{E}-03$ & $1.4 \mathrm{E}-03$ \\
Accident & $2.1 \mathrm{E}-02$ & $3.3 \mathrm{E}-04$ \\
\hline
\end{tabular}

Table 9. Expected Annual Cancer Incidence for the Shipping Campaign

\begin{tabular}{lll}
\hline Exposure Group & Truck & Rail \\
\hline Radiological & & \\
Normal Crew & $1.3 \mathrm{E}-02$ & $1.2 \mathrm{E}-03$ \\
Normal Public & $1.8 \mathrm{E}-02$ & $2.9 \mathrm{E}-03$ \\
Accident Public & $2.4 \mathrm{E}-02$ & $4.3 \mathrm{E}-03$ \\
Nonradiological & $1.5 \mathrm{E}-03$ & $1.4 \mathrm{E}-03$ \\
Emission & $\mathrm{NA}$ & $\mathrm{NA}$ \\
Accident & & \\
\hline
\end{tabular}

Table 10. Expected Annual Genetic Effects for the Shipping Campaign

\begin{tabular}{lll}
\hline Exposure Group & Truck & Rail \\
\hline Radiological & & \\
Normal Crew & $5.5 E-04$ & $5.1 E-05$ \\
Normal Public & $1.1 E-03$ & $1.7 E-04$ \\
Accident Public & $4.0 \mathrm{E}-04$ & $7.6 \mathrm{E}-05$ \\
Nonradiological & & $\mathrm{NA}$ \\
Emission & $\mathrm{NA}$ & $\mathrm{NA}$ \\
Accident & $\mathrm{NA}$ & \\
\hline
\end{tabular}




\section{B.30 WM LLMW CENTRALIZED ALTERNATIVE (CASE 17): CONTACT-HANDLED ORGANIC LIQUIDS: ALPHA - UNTREATED}

Table 1. Summary of Route Information

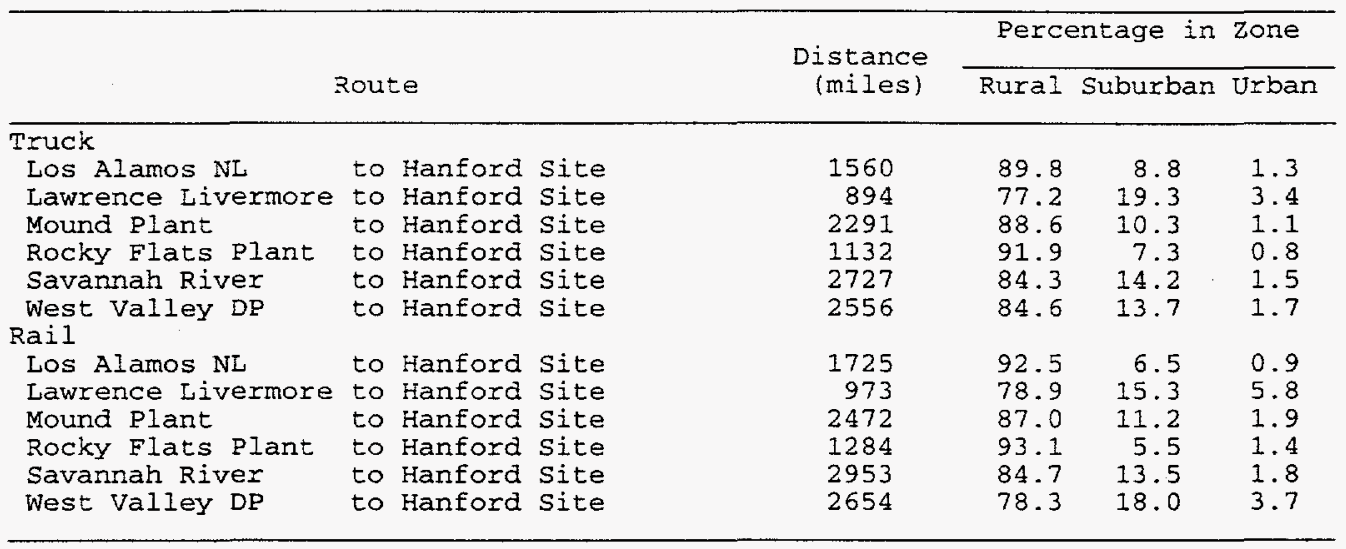

Table 2. Annual Number of Shipments and one-Way. Shipment Distances

\begin{tabular}{|c|c|c|c|c|c|}
\hline \multirow[b]{2}{*}{ Route } & & \multicolumn{2}{|c|}{ \# of Shipments } & \multicolumn{2}{|c|}{ Total Mileage (mi) } \\
\hline & & Truck & Rail & Truck & Rail \\
\hline $\begin{array}{l}\text { Los Alamos NL } \\
\text { Lawrence Livermore } \\
\text { Mound Plant } \\
\text { Rocky Flats Plant } \\
\text { Savannah River } \\
\text { West Valley DP }\end{array}$ & $\begin{array}{l}\text { to Hanford Site } \\
\text { to Hanford Site } \\
\text { to Hanford Site } \\
\text { to Hanford Site } \\
\text { to Hanford Site } \\
\text { to Hanford Site }\end{array}$ & $\begin{array}{l}4 \\
4 \\
1 \\
1 \\
1 \\
1\end{array}$ & $\begin{array}{l}2 \\
2 \\
1 \\
1 \\
1 \\
1\end{array}$ & $\begin{array}{l}6.24 \mathrm{E}+03 \\
3.58 \mathrm{E}+03 \\
2.29 \mathrm{E}+03 \\
1.13 \mathrm{E}+03 \\
2.73 \mathrm{E}+03 \\
2.56 \mathrm{E}+03\end{array}$ & $\begin{array}{l}3.45 \mathrm{E}+03 \\
1.95 \mathrm{E}+03 \\
2.47 \mathrm{E}+03 \\
1.28 \mathrm{E}+03 \\
2.95 \mathrm{E}+03 \\
2.65 \mathrm{E}+03\end{array}$ \\
\hline Totals & & 12 & 8 & $1.85 E+04$ & $1.48 \mathrm{E}+04$ \\
\hline
\end{tabular}

Table 3. Incident-Free Dose per Shipment (person-rem/shipment)

\begin{tabular}{|c|c|c|c|c|c|}
\hline \multirow[b]{2}{*}{ Source/Route (s) } & \multirow[b]{2}{*}{ Crew } & \multicolumn{4}{|c|}{ General Public } \\
\hline & & off-Iink & On-Link & Stops & Total \\
\hline \multicolumn{6}{|l|}{ MLLW(OA) I.A to HS } \\
\hline & $\begin{array}{lr}\text { Truck } & 4.24 \mathrm{E}-02 \\
\text { Rail } & 1.01 \mathrm{E}-02\end{array}$ & $\begin{array}{l}2.16 E-03 \\
5.60 E-03\end{array}$ & $\begin{array}{l}5.65 \mathrm{E}-03 \\
1.54 \mathrm{E}-04\end{array}$ & $\begin{array}{l}4.37 E-02 \\
8.79 E-03\end{array}$ & $\begin{array}{l}5.15 \mathrm{E}-02 \\
1.45 \mathrm{E}-02\end{array}$ \\
\hline \multicolumn{6}{|c|}{$\begin{array}{l}\text { MLLW(OA) LL to HS } \\
\text { Lawrence Livermore to Hanford site }\end{array}$} \\
\hline Lawrence Livermore & $\begin{array}{ll}\text { to Hanford Site } \\
\text { Truck } & 2.82 \mathrm{E}-02 \\
\text { Rail } & 6.94 \mathrm{E}-03\end{array}$ & $\begin{array}{l}2.88 \mathrm{E}-03 \\
1.38 \mathrm{E}-02\end{array}$ & $\begin{array}{l}4.92 \mathrm{E}-03 \\
2.00 \mathrm{E}-04\end{array}$ & $\begin{array}{l}2.50 \mathrm{E}-02 \\
6.48 \mathrm{E}-03\end{array}$ & $\begin{array}{l}3.28 \mathrm{E}-02 \\
2.05 \mathrm{E}-02\end{array}$ \\
\hline \multicolumn{6}{|c|}{ to Hanford site } \\
\hline Mound Plant & $\begin{array}{lr}\text { to Hanford site } \\
\text { Truck } & 6.29 \mathrm{E}-02 \\
\text { Rail } & 1.32 \mathrm{E}-02\end{array}$ & $\begin{array}{l}3.13 \mathrm{E}-03 \\
1.47 \mathrm{E}-02\end{array}$ & $\begin{array}{l}8.05 \mathrm{E}-03 \\
3.13 \mathrm{E}-04\end{array}$ & $\begin{array}{l}6.41 \mathrm{E}-02 \\
1.11 \mathrm{E}-02\end{array}$ & $\begin{array}{l}7.53 E-02 \\
2.61 E-02\end{array}$ \\
\hline \\
\hline Rocky Flats Plant & $\begin{array}{l}\text { to Hanford site } \\
\text { Truck } 2.99 \mathrm{E}-02 \\
\text { Rai1 } \\
8.24 \mathrm{E}-03\end{array}$ & $\begin{array}{l}1.13 E-03 \\
4.91 E-03\end{array}$ & $\begin{array}{l}3.61 \mathrm{E}-03 \\
1.16 \mathrm{E}-04\end{array}$ & $\begin{array}{l}3.77 \mathrm{E}-02 \\
7.43 \mathrm{E}-03\end{array}$ & $\begin{array}{l}3.64 \mathrm{E}-02 \\
1.25 \mathrm{E}-02\end{array}$ \\
\hline \multicolumn{6}{|l|}{ MLLW (OA) SR to HS } \\
\hline Savannah River & $\begin{array}{l}\text { to Hanford Site } \\
\text { Truck } 7.86 \mathrm{E}-02 \\
\text { Rail } 1.52 \mathrm{E}-02\end{array}$ & $\begin{array}{l}5.10 \mathrm{E}-03 \\
1.85 \mathrm{E}-02\end{array}$ & $\begin{array}{l}1.08 \mathrm{E}-02 \\
4.05 \mathrm{E}-04\end{array}$ & $\begin{array}{l}7.64 \mathrm{E}-02 \\
1.26 \mathrm{E}-02\end{array}$ & $\begin{array}{l}9.23 \mathrm{E}-02 \\
3.15 \mathrm{E}-02\end{array}$ \\
\hline \multirow{2}{*}{$\begin{array}{l}\text { MLLW(OA) WV to HS } \\
\text { West Valley DP }\end{array}$} & & & & & \\
\hline & $\begin{array}{l}\text { to Hanford site } \\
\text { Truck } 7.36 \mathrm{E}-02 \\
\text { Rail } 1.40 \mathrm{E}-02\end{array}$ & $\begin{array}{l}4.90 \mathrm{E}-03 \\
2.88 \mathrm{E}-02\end{array}$ & $\begin{array}{l}1.04 \mathrm{E}-02 \\
5.00 \mathrm{E}-04\end{array}$ & $\begin{array}{l}7.16 \mathrm{E}-02 \\
1.17 \mathrm{E}-02\end{array}$ & $\begin{array}{l}8.68 \mathrm{E}-02 \\
4.09 \mathrm{E}-02\end{array}$ \\
\hline
\end{tabular}


Table 4. Accident Dose Risk per Shipment (person-rem/shipment)

\begin{tabular}{|c|c|c|c|}
\hline Source/Route & & Truck & Rail \\
\hline MLLW(OA) LA to HS & & & \\
\hline Los Alamos NL & to Hanford Site & $2.62 \pm-02$ & $5.71 E-03$ \\
\hline $\begin{array}{l}\text { MLLW (OA) LL to HS } \\
\text { Lawrence Livermore }\end{array}$ & to Hanford Site & $1.89 E-02$ & $2.02 \mathrm{E}-02$ \\
\hline MLLW (OA) MP to HS & & & \\
\hline $\begin{array}{l}\text { Mound Plant } \\
\text { MLLW(OA) RF to HS }\end{array}$ & to Hanford Site & $5.43 E-03$ & $1.22 \mathrm{E}-03$ \\
\hline $\begin{array}{l}\text { ROCkY Flats Plant } \\
\text { MLLW (OA) SR to HS }\end{array}$ & to Hanford Site & $5.14 \varepsilon-03$ & $1.25 E-03$ \\
\hline $\begin{array}{l}\text { Savannah River } \\
\text { MLIW(OA) WV to HS }\end{array}$ & to Hanford Site & $5.47 E-02$ & $1.09 \mathrm{E}-02$ \\
\hline West Valley DP & to Hanford Site & $8.22 E-02$ & $3.21 \mathrm{E}-02$ \\
\hline
\end{tabular}

Table 5. Nonradiological Risk Factors per Shipment (fatalities/shipment)

\begin{tabular}{llll}
\hline Mode & Route & Emission & Accident \\
\hline $\begin{array}{c}\text { Truck } \\
\text { Los Alamos NL }\end{array}$ & to Hanford Site & $6.66 \mathrm{E}-06$ & $1.02 \mathrm{E}-04$ \\
Lawrence Livermore to Hanford Site & $9.85 \mathrm{E}-06$ & $5.03 \mathrm{E}-05$ \\
Mound Plant & to Hanford Site & $7.89 \mathrm{E}-06$ & $1.23 \mathrm{E}-04$ \\
Rocky Flats Plant to Hanford Site & $2.83 \mathrm{E}-06$ & $6.69 \mathrm{E}-05$ \\
Savannah River & to Hanford Site & $1.31 \mathrm{E}-05$ & $1.81 \mathrm{E}-04$ \\
West Valley DP & to Hanford Site & $1.36 \mathrm{E}-05$ & $1.38 \mathrm{E}-04$ \\
Rail & & & \\
Los Alamos NL & to Hanford Site & $6.82 \mathrm{E}-06$ & $3.61 \mathrm{E}-06$ \\
Lawrence Livermore to Hanford Site & $2.36 \mathrm{E}-05$ & $2.04 \mathrm{E}-06$ \\
Mound Plant & to Hanford Site & $1.94 \mathrm{E}-05$ & $5.17 \mathrm{E}-06$ \\
Rocky Flats Plant & to Hanford Site & $7.36 \mathrm{E}-06$ & $2.69 \mathrm{E}-06$ \\
Savannah River & to Hanford Site & $2.21 \mathrm{E}-05$ & $6.18 \mathrm{E}-06$ \\
West Valley DP & to Hanford Site & $4.14 \mathrm{E}-05$ & $5.55 \mathrm{E}-06$ \\
\hline
\end{tabular}

Table 6. Annual Incident-Free Dose for the Shipping Campaign (person-rem/yr)

\begin{tabular}{|c|c|c|c|c|c|}
\hline \multirow[b]{2}{*}{ Route } & & \multicolumn{2}{|c|}{ Truck } & \multicolumn{2}{|c|}{ Rail } \\
\hline & & Crew & Public & Crew & Public \\
\hline $\begin{array}{l}\text { Los Alamos NL } \\
\text { Lawrence Livermore } \\
\text { Mound Plant } \\
\text { Rocky Flats Plant } \\
\text { Savannah River } \\
\text { West Valley DP }\end{array}$ & $\begin{array}{l}\text { to Hanford Site } \\
\text { to Hanford site } \\
\text { to Hanford site } \\
\text { to Hanford site } \\
\text { to Hanford site } \\
\text { to Hanford site }\end{array}$ & $\begin{array}{l}1.70 \mathrm{E}-01 \\
1.13 \mathrm{E}-01 \\
6.29 \mathrm{E}-02 \\
2.99 \mathrm{E}-02 \\
7.86 \mathrm{E}-02 \\
7.36 \mathrm{E}-02\end{array}$ & $\begin{array}{l}2.06 \mathrm{E}-01 \\
1.31 \mathrm{E}-01 \\
7.53 \mathrm{E}-02 \\
3.64 \mathrm{E}-02 \\
9.23 \mathrm{E}-02 \\
8.68 \mathrm{E}-02\end{array}$ & $\begin{array}{l}2.02 \mathrm{E}-02 \\
1.39 \mathrm{E}-02 \\
1.32 \mathrm{E}-02 \\
8.24 \mathrm{E}-03 \\
1.52 \mathrm{E}-02 \\
1.40 \mathrm{E}-02\end{array}$ & $\begin{array}{l}2.91 \mathrm{E}-02 \\
4.10 \mathrm{E}-02 \\
2.61 \mathrm{E}-02 \\
1.25 \mathrm{E}-02 \\
3.15 \mathrm{E}-02 \\
4.09 \mathrm{E}-02\end{array}$ \\
\hline Totals & & $5.27 E-01$ & $6.28 \mathrm{E}-01$ & $8.46 \mathrm{E}-02$ & $1.81 \mathrm{E}-01$ \\
\hline
\end{tabular}

Table 7. Annual Accident Dose Risk for the Shipping Campaign (person-rem/yr)

\begin{tabular}{llcc}
\hline Route & & Truck & Rail \\
\hline Los Alamos NL & to Hanford Site & $1.05 \mathrm{E}-01$ & $1.14 \mathrm{E}-02$ \\
Lawrence Livermore to Hanford Site & $7.58 \mathrm{E}-02$ & $4.03 \mathrm{E}-02$ \\
Mound Plant & to Hanford Site & $5.43 \mathrm{E}-03$ & $1.22 \mathrm{E}-03$ \\
Rocky Flats Plant & to Hanford Site & $5.14 \mathrm{E}-03$ & $1.25 \mathrm{E}-03$ \\
Savannah River & to Hanford Site & $5.47 \mathrm{E}-02$ & $1.09 \mathrm{E}-02$ \\
West Valley DP & to Hanford Site & $8.22 \mathrm{E}-02$ & $3.21 \mathrm{E}-02$ \\
\hline Totals & & $3.28 \mathrm{E}-01$ & $9.72 \mathrm{E}-02$ \\
\hline
\end{tabular}


Table 8. Expected Annual Fatalities for the Shipping Campaign

\begin{tabular}{llc}
\hline Exposure Group & Truck & Rail \\
\hline Radiological & & \\
Normal Crew & $2.1 \mathrm{E}-04$ & $3.4 \mathrm{E}-05$ \\
Normal PubIic & $3.1 \mathrm{E}-04$ & $9.1 \mathrm{E}-05$ \\
$\quad \begin{array}{cc}\text { Acident Public } \\
\text { Nonradiological }\end{array}$ & $1.6 \mathrm{E}-04$ & $4.9 \mathrm{E}-05$ \\
$\quad \begin{array}{l}\text { Emission } \\
\text { Accident }\end{array}$ & $1.0 \mathrm{E}-04$ & $1.5 \mathrm{E}-04$ \\
& $1.1 \mathrm{E}-03$ & $3.1 \mathrm{E}-05$ \\
\hline
\end{tabular}

Table 9. Expected Annual Cancer Incidence for the Shipping Campaign

\begin{tabular}{lll}
\hline Exposure Group & Truck & \multicolumn{1}{c}{ Rail } \\
\hline Radiological & & \\
$\quad$ Normal Crew & $7.4 \mathrm{E}-04$ & $1.2 \mathrm{E}-04$ \\
Normal Public & $1.1 \mathrm{E}-03$ & $3.1 \mathrm{E}-04$ \\
Accident Public & $5.6 \mathrm{E}-04$ & $1.7 \mathrm{E}-04$ \\
$\begin{array}{c}\text { Nonradiological } \\
\text { Emission }\end{array}$ & $1.0 \mathrm{E}-04$ & $1.5 \mathrm{E}-04$ \\
Accident & NA & NA \\
\hline
\end{tabular}

Table 10. Expected Annual Genetic Effects for the Shipping Campaign

\begin{tabular}{lll}
\hline Exposure Group & Truck & Rail \\
\hline Radiological & & \\
Normal Crew & $3.2 \mathrm{E}-05$ & $5.1 \mathrm{E}-06$ \\
Normal Public & $6.3 \mathrm{E}-05$ & $1.8 \mathrm{E}-05$ \\
Accident Public & $9.2 \mathrm{E}-06$ & $2.6 \mathrm{E}-06$ \\
$\begin{array}{c}\text { Nonradiological } \\
\text { Emission }\end{array}$ & NA & NA \\
Accident & NA & NA \\
\hline
\end{tabular}




\section{B.31 WM LLMW CENTRALIZED ALTERNATIVE (CASE 17): CONTACT-HANDLED HETEROGENEOUS SOLIDS: NONALPHA - UNTREATED}

Table 1. Summary of Route Information

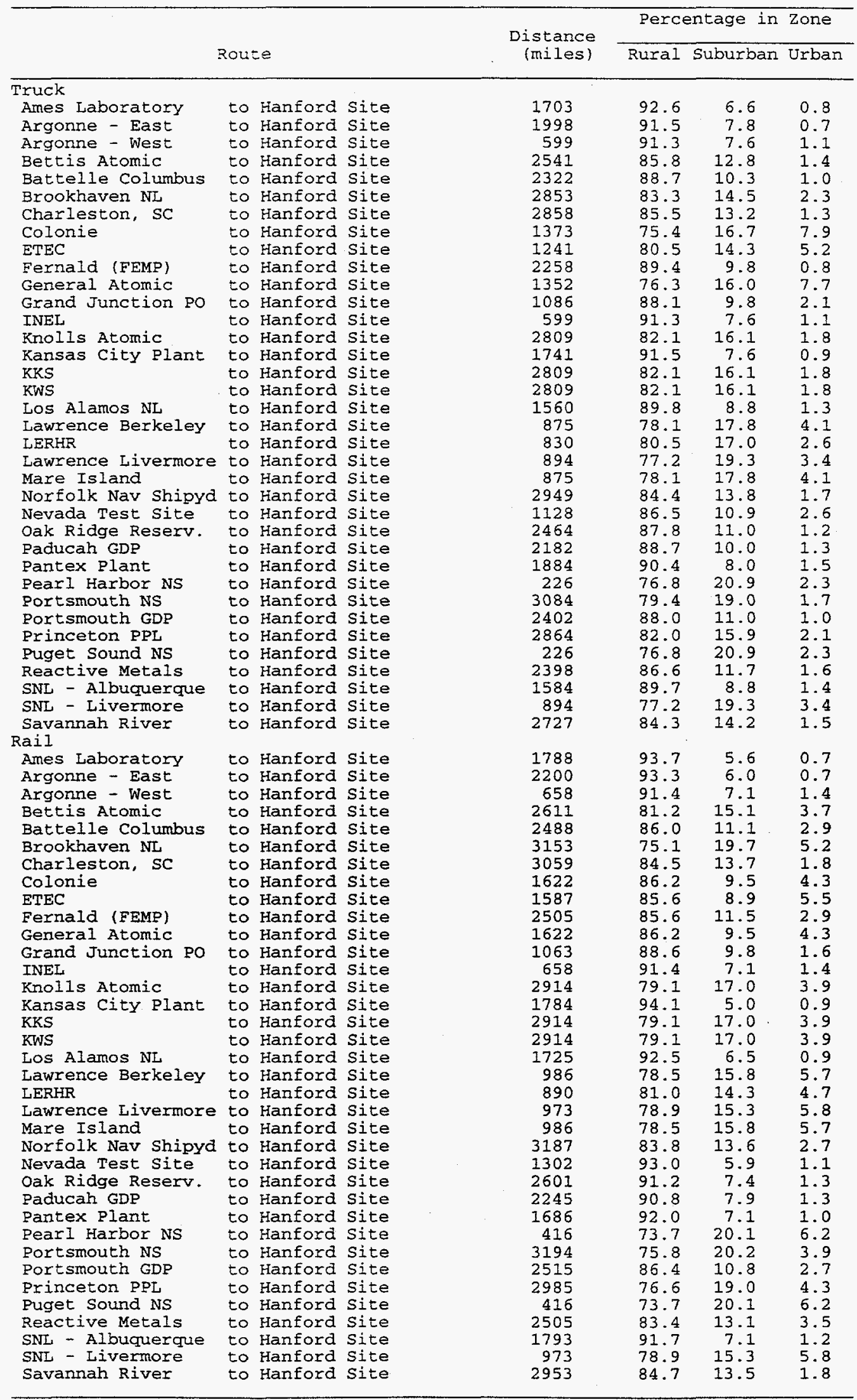


Table 2. Annual Number of Shipments and one-Way Shipment Distances

\begin{tabular}{|c|c|c|c|c|c|c|}
\hline \multirow[b]{2}{*}{ Route } & & & \multicolumn{2}{|c|}{ \# of Shipments } & \multicolumn{2}{|c|}{ Total Mileage (mi) } \\
\hline & & & Truck & Rail & Truck & Rail \\
\hline Ames Laboratory & to & Hanford Site & 1 & $I$ & $1.70 \mathrm{E}+03$ & $1.79 \mathrm{E}+03$ \\
\hline Argonne - East & to & Hanford Site & 44 & 17 & $8.79 E+04$ & $3.74 \mathrm{E}+04$ \\
\hline Argonne - West & to & Hanford Site & 1 & 1 & $5.99 \mathrm{E}+02$ & $6.58 \mathrm{E}+02$ \\
\hline Bettis Atomic & to & Hanford Site & 1 & 1 & $2.54 \mathrm{E}+03$ & $2.61 \mathrm{E}+03$ \\
\hline Battelle columbus & to & Hanford Site & 1 & 1 & $2.32 \mathrm{E}+03$ & $2.49 \mathrm{E}+03$ \\
\hline Brookhaven NL & to & Hanford Site & 1 & $i$ & $2.85 E+03$ & $3.15 \mathrm{E}+03$ \\
\hline Charleston, SC & to & Hanford Site & 1 & 1 & $2.86 E+03$ & $3.06 \mathrm{E}+03$ \\
\hline Colonie & to & Hanford Site & $\vec{I}$ & 1 & $1.37 \mathrm{E}+03$ & $1.62 \mathrm{E}+03$ \\
\hline ETEC & to & Hanford Site & 8 & 3 & $9.93 E+03$ & $4.76 E+03$ \\
\hline Fernald (FEMP) & to & Hanford Site & 7 & 3 & $1.58 \mathrm{E}+04$ & $7.52 E+03$ \\
\hline General Atomic & to & Hanford Site & 1 & 1 & 1. $35 E+03$ & $1.62 E+03$ \\
\hline Grand Junction po & to & Hanford Site & 1 & $\hat{1}$ & $1.09 E+03$ & $1.06 \mathrm{E}+03$ \\
\hline INEL & to & Hanford Site & 4 & 2 & $2.40 E+03$ & $1.32 E+03$ \\
\hline Knolls Atomic & to & Hanford Site & 1 & 1 & $2.81 E+03$ & $2.91 \mathrm{E}+03$ \\
\hline Kansas City Plant & to & Hanford Site & $\vec{I}$ & 1 & $1.74 \mathrm{E}+03$ & $1.78 \mathrm{E}+03$ \\
\hline KKS & to & Hanford Site & 1 & 1 & $2.81 E+03$ & $2.91 \mathrm{E}+03$ \\
\hline KWS & to & Hanford Site & 1 & 1 & $2.81 E+03$ & $2.91 E+03$ \\
\hline Los Alamos NL & to & Hanford Site & 2 & 1 & $3.12 \mathrm{E}+03$ & $1.72 E+03$ \\
\hline Lawrence Berkeley & to & Hanford Site & 1 & 1 & $8.75 E+02$ & $9.86 \mathrm{E}+02$ \\
\hline LERHR & to & Hanford Site & 1 & 1 & $8.30 \mathrm{E}+02$ & $8.90 E+02$ \\
\hline Lawrence Livermore & to & Hanford Site & 4 & 2 & $3.58 \mathrm{E}+03$ & $1.95 E+03$ \\
\hline Mare Island & to & Hanford Site & $i$ & 1 & $8.75 E+02$ & $9.86 \mathrm{E}+02$ \\
\hline Norfolk Nav Shipyd & to $-(x)+h e$ & Hanford Site & 1 & 1 & $2.95 E+03$ & $3.19 E+03$ \\
\hline Nevada Test Site & to & Hanford Site & $\bar{I}$ & 1 & $1.13 \mathrm{E}+03$ & $1.30 \mathrm{E}+03$ \\
\hline Oak Ridge Reserv. & to & Hanford Site & $12 \overline{4}$ & $4 \overline{5}$ & $3.05 \mathrm{E}+05$ & $1.20 E+05$ \\
\hline Paducah GDP & to & Hanford Site & 1 & 1 & $2.18 E+03$ & $2.25 E+03$ \\
\hline Pantex Plant & to & Hanford Site & 2 & 1 & $3.77 \mathrm{E}+03$ & $1.69 \mathrm{E}+03$ \\
\hline Pearl Harbor NS & to & Hanford Site & 1 & 1 & $2.26 \mathrm{E}+02$ & $4.16 E+02$ \\
\hline Portsmouth NS & to & Hanford Site & $\overline{1}$ & 1 & $3.08 \mathrm{E}+03$ & $3.19 \mathrm{E}+03$ \\
\hline Portsmouth GDP & to & Hanford Site & $6 \overline{0}$ & $2 \overline{3}$ & $1.44 \mathrm{E}+05$ & $5.78 \mathrm{E}+04$ \\
\hline Princeton PPL & to & Hanford Site & 1 & 1 & $2.86 \mathrm{E}+03$ & $2.98 E+03$ \\
\hline Puget Sound NS & to & Hanford site & 1 & 1 & $2.26 \mathrm{E}+02$ & $4.16 E+02$ \\
\hline Reactive Metals & to & Hanford Site & $\overline{1}$ & $\vec{I}$ & $2.40 E+03$ & $2.51 E+03$ \\
\hline SNL - Albuquerque & to & Hanford Site & 1 & 1 & $1.58 \mathrm{E}+03$ & $1.79 E+03$ \\
\hline SNL - Livermore & & Hanford Site & $\overline{1}$ & $\bar{I}$ & $8.94 E+02$ & $9.73 E+02$ \\
\hline Savannah River & to & Hanford Site & 13 & 5 & $3.55 \mathrm{E}+04$ & $1.48 E+04$ \\
\hline Totals & & & 294 & 129 & $6.58 \mathrm{E}+05$ & $2.99 E+05$ \\
\hline
\end{tabular}


Table 3. Incident-Free Dose per Shipment (person-rem/shipment)

\begin{tabular}{|c|c|c|c|c|c|}
\hline & & & General & 1 Public & \\
\hline Source/Route(s) & Crew & off-Link & on-Link & Stops & Total \\
\hline MLLW(C) AL to HS & & & & & \\
\hline Ames Laboratory & to Hanford Site & & & & \\
\hline & $\begin{array}{ll}\text { Truck } & 4.46 \mathrm{E}-02 \\
\text { Rail } & 1.03 \mathrm{E}-02\end{array}$ & $\begin{array}{l}1.62 \mathrm{E}-03 \\
4.69 \mathrm{E}-03\end{array}$ & $\begin{array}{l}5.39 \mathrm{E}-03 \\
1.46 \mathrm{E}-04\end{array}$ & $\begin{array}{l}4.77 \mathrm{E}-02 \\
8.98 \mathrm{E}-03\end{array}$ & $\begin{array}{l}5.47 \mathrm{E}-02 \\
1.38 \mathrm{E}-02\end{array}$ \\
\hline MLLW (C) AN to HS & & & & & \\
\hline Argonne - East & to Hanford Site & & & & \\
\hline & $\begin{array}{ll}\text { Truck } & 5.29 \mathrm{E}-02 \\
\text { Rail } & 1.21 \mathrm{E}-02\end{array}$ & $\begin{array}{l}1.97 \mathrm{E}-03 \\
5.75 \mathrm{E}-03\end{array}$ & $\begin{array}{l}6.28 \mathrm{E}-03 \\
1.82 \mathrm{E}-04\end{array}$ & $\begin{array}{l}5.59 \mathrm{E}-02 \\
1.03 \mathrm{E}-02\end{array}$ & $\begin{array}{l}6.42 \mathrm{E}-02 \\
1.62 \mathrm{E}-02\end{array}$ \\
\hline MLLW (C) AW to HS & & & & & \\
\hline Argonne - West & to Hanford Site & & & & \\
\hline & $\begin{array}{ll}\text { Truck } & 1.60 \mathrm{E}-02 \\
\text { Rail } & 5.63 \mathrm{E}-03\end{array}$ & $\begin{array}{l}7.18 \mathrm{E}-04 \\
2.83 \mathrm{E}-03\end{array}$ & $\begin{array}{l}2.06 \mathrm{E}-03 \\
6.58 \mathrm{E}-05\end{array}$ & $\begin{array}{l}1.68 \mathrm{E}-02 \\
5.51 \mathrm{E}-03\end{array}$ & $\begin{array}{l}1.95 E-02 \\
8.40 E-03\end{array}$ \\
\hline MLLW (C) BA to HS & & & & & \\
\hline Bettis Atomic & to Hanford Site & & & & \\
\hline & $\begin{array}{ll}\text { Truck } & 7.21 \mathrm{E}-02 \\
\text { Rail } & 1.38 \mathrm{E}-02\end{array}$ & $\begin{array}{l}4.36 \mathrm{E}-03 \\
2.67 \mathrm{E}-02\end{array}$ & $\begin{array}{l}9.77 E-03 \\
4.53 E-04\end{array}$ & $\begin{array}{l}\text { 7. } 11 \mathrm{E}-02 \\
1.15 \mathrm{E}-02\end{array}$ & $\begin{array}{l}8.53 E-02 \\
3.87 E-02\end{array}$ \\
\hline $\begin{array}{l}\text { MLLW(C) BC to HS } \\
\text { Battelle Columbus }\end{array}$ & & & & & \\
\hline 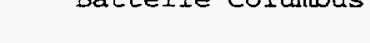 & $6 \quad 6.36 \mathrm{E}-02$ & $3.06 \mathrm{E}-03$ & $8.01 E-03$ & $6.50 \mathrm{E}-02$ & $7.61 E-02$ \\
\hline & $1.33 \mathrm{E}-02$ & $1.95 \mathrm{E}-02$ & $3.51 \mathrm{E}-04$ & $1.11 E-02$ & $3.10 E-02$ \\
\hline MLLW (C) BN to HS & & & & & \\
\hline Brookhaven NL & to Hanford Site & & & & \\
\hline & $\begin{array}{ll}\text { Truck } & 8.38 \mathrm{E}-02 \\
\text { Rail } & 1.60 \mathrm{E}-02\end{array}$ & $\begin{array}{l}5.48 \mathrm{E}-03 \\
4.38 \mathrm{E}-02\end{array}$ & $\begin{array}{l}1.28 \mathrm{E}-02 \\
6.87 \mathrm{E}-04\end{array}$ & $\begin{array}{l}7.99 \mathrm{E}-02 \\
1.32 \mathrm{E}-02\end{array}$ & $\begin{array}{l}9.92 \mathrm{E}-02 \\
5.76 \mathrm{E}-02\end{array}$ \\
\hline MLLW(C) $\mathrm{CH}$ to $\mathrm{HS}$ & & & & & \\
\hline Charleston, SC & to Hanford Site & & & & \\
\hline & $\begin{array}{l}8.12 \mathrm{E}-02 \\
1.56 \mathrm{E}-02\end{array}$ & $\begin{array}{l}4.87 \mathrm{E}-03 \\
1.96 \mathrm{E}-02\end{array}$ & $\begin{array}{l}1.09 \mathrm{E}-02 \\
4.24 \mathrm{E}-04\end{array}$ & $\begin{array}{l}8.00 \mathrm{E}-02 \\
1.29 \mathrm{E}-02\end{array}$ & $\begin{array}{l}9.58 \mathrm{E}-02 \\
3.29 \mathrm{E}-02\end{array}$ \\
\hline MLLW(C) CL to HS & & & & & \\
\hline & to Hanford Site & 7 & & -02 & \\
\hline & $9.65 \mathrm{E}-03$ & $1.64 \mathrm{E}-02$ & 2.4 & $8.47 \mathrm{E}-03$ & $2.52 \mathrm{E}-02$ \\
\hline MLLW(C) LC to HS & & & & & \\
\hline ETEC & to Hanford Site & & & & \\
\hline & $\begin{array}{ll}\text { Truck } & 3.87 \mathrm{E}-02 \\
\text { Rail } & 9.50 \mathrm{E}-03\end{array}$ & $\begin{array}{l}4.63 \mathrm{E}-03 \\
1.96 \mathrm{E}-02\end{array}$ & $\begin{array}{l}8.05 E-03 \\
2.68 E-04\end{array}$ & $\begin{array}{l}3.47 E-02 \\
8.37 E-03\end{array}$ & $\begin{array}{l}4.74 \mathrm{E}-02 \\
2.82 \mathrm{E}-02\end{array}$ \\
\hline MLLW(C) FE to HS & & & & & \\
\hline Fernald (FEMP) & to Hanford Site & & & & \\
\hline & $\begin{array}{ll}\text { Truck } & 6.13 \mathrm{E}-02 \\
\text { Rail } & 1.33 \mathrm{E}-02\end{array}$ & $\begin{array}{l}2.71 E-03 \\
2.00 E-02\end{array}$ & $\begin{array}{l}7.49 E-03 \\
3.60 E-04\end{array}$ & $\begin{array}{l}6.32 \mathrm{E}-02 \\
1.12 \mathrm{E}-02\end{array}$ & $\begin{array}{l}7.34 \mathrm{E}-02 \\
3.16 \mathrm{E}-02\end{array}$ \\
\hline MLLW (C) so to HS & & & & & \\
\hline General Atomic & to Hanford Site & & & & \\
\hline & $\begin{array}{ll}\text { Truck } & 4.49 \mathrm{E}-02 \\
\text { Rail } & 9.65 \mathrm{E}-03\end{array}$ & $\begin{array}{l}6.88 \mathrm{E}-03 \\
1.64 \mathrm{E}-02\end{array}$ & $\begin{array}{l}1.11 \mathrm{E}-02 \\
2.49 \mathrm{E}-04\end{array}$ & $\begin{array}{l}3.78 E-02 \\
8.47 E-03\end{array}$ & $\begin{array}{l}5.58 \mathrm{E}-02 \\
2.52 \mathrm{E}-02\end{array}$ \\
\hline MLLW(C) GJ to HS & & & & & \\
\hline Grand Junction PO & to Hanford site & & & & \\
\hline & $\begin{array}{ll}\text { Truck } & 3.04 \mathrm{E}-02 \\
\text { Rail } & 7.32 \mathrm{E}-03\end{array}$ & $\begin{array}{l}2.03 \mathrm{E}-03 \\
5.46 \mathrm{E}-03\end{array}$ & $\begin{array}{l}4.58 \mathrm{E}-03 \\
1.23 \mathrm{E}-04\end{array}$ & $\begin{array}{l}3.04 \mathrm{E}-02 \\
6.75 \mathrm{E}-03\end{array}$ & $\begin{array}{l}3.70 \mathrm{E}-02 \\
1.23 \mathrm{E}-02\end{array}$ \\
\hline $\begin{array}{c}\text { MLLW(C) IN to HS } \\
\text { INEL }\end{array}$ & to Hanford sito & & & & \\
\hline & $\begin{array}{ll}\text { Truck } & 1.60 \mathrm{E}-02 \\
\text { Rail } & 5.63 \mathrm{E}-03\end{array}$ & $\begin{array}{l}7.18 \mathrm{E}-04 \\
2.83 \mathrm{E}-03\end{array}$ & $\begin{array}{l}2.06 \mathrm{E}-03 \\
6.58 \mathrm{E}-05\end{array}$ & $\begin{array}{l}1.68 E-02 \\
5.51 E-03\end{array}$ & $\begin{array}{l}1.95 \mathrm{E}-02 \\
8.40 \mathrm{E}-03\end{array}$ \\
\hline MLLW(C) $\mathrm{KA}$ to $\mathrm{HS}$ & & & & & \\
\hline Knolls Atomic & to Hanford Site & & & & \\
\hline & Truck $\quad 8.3$ & $6.03 E-03$ & $1.19 \mathrm{E}-02$ & $7.87 E-02$ & $9.66 \mathrm{E}-02$ \\
\hline MLLW (C) KC to HS & 02 & & & $1.25 \mathrm{E}-02$ & 4 . \\
\hline Kansas City Plant & to Hanford site & & & & \\
\hline & ck $\quad 4.62 \mathrm{E}-02$ & $1.90 \mathrm{E}-03$ & $5.74 \mathrm{E}-03$ & $4.87 \mathrm{E}-02$ & $5.64 E-02$ \\
\hline MLIW (C) $\mathrm{KK}$ to $\mathrm{HS}$ & & & & & \\
\hline KKS & to Hanford Site & & & & \\
\hline & $\begin{array}{ll}\text { Truck } & 8.30 \mathrm{E}-02 \\
\text { Rail } & 1.50 \mathrm{E}-02\end{array}$ & $\begin{array}{l}6.03 \mathrm{E}-03 \\
3.18 \mathrm{E}-02\end{array}$ & $\begin{array}{l}1.19 \mathrm{E}-02 \\
5.40 \mathrm{E}-04\end{array}$ & $\begin{array}{l}7.87 \mathrm{E}-02 \\
1.25 \mathrm{E}-02\end{array}$ & $\begin{array}{l}9.66 \mathrm{E}-02 \\
4.48 \mathrm{E}-02\end{array}$ \\
\hline MLIW (C) KW to HS & & & & & \\
\hline KWS & to Hanford Site & & & & \\
\hline & $\begin{array}{ll}\text { Truck } & 8.30 \mathrm{E}-02 \\
\text { Rail } & 1.50 \mathrm{E}-02\end{array}$ & $\begin{array}{l}6.03 \mathrm{E}-03 \\
3.18 \mathrm{E}-02\end{array}$ & $\begin{array}{l}1.19 \mathrm{E}-02 \\
5.40 \mathrm{E}-04\end{array}$ & $\begin{array}{l}7.87 E-02 \\
1.25 E-02\end{array}$ & $\begin{array}{l}9.66 E-02 \\
4.48 E-02\end{array}$ \\
\hline MIIW(C) LA to HS & & & & & \\
\hline Los Alamos NL & to Hanford Site & & & & \\
\hline & $\begin{array}{ll}\text { Truck } & 4.24 \mathrm{E}-02 \\
\text { R } & 1.01 \mathrm{E}-02\end{array}$ & $\begin{array}{l}2.16 \mathrm{E}-03 \\
5.60 \mathrm{E}-03\end{array}$ & $\begin{array}{l}5.65 E-03 \\
1.54 E-04\end{array}$ & $\begin{array}{l}4.37 \mathrm{E}-02 \\
8.79 \mathrm{E}-03\end{array}$ & $\begin{array}{l}5.15 E-02 \\
1.45 E-02\end{array}$ \\
\hline
\end{tabular}


Table 3. Incident-Free Dose per Shipment (person-rem/shipment) (Cont.)

\begin{tabular}{|c|c|c|c|c|c|}
\hline & & & General & I Public & \\
\hline Source/Route (s) & Crew & of $f$-Link & On-Link & Stops & Total \\
\hline MLLW (C) LB to HS & & & & & \\
\hline Lawrence Berkeley & to Hanford Site & & & & \\
\hline & Truck $\quad 2.76 \mathrm{E}-02$ & $3.02 \mathrm{E}-03$ & $5.17 E-03$ & $2.45 \mathrm{E}-02$ & $3.27 \mathrm{E}-02$ \\
\hline MLLW(C) SM to HS & $6.99 \pm-03$ & $=-02$ & & $8-03$ & -02 \\
\hline LERHR & to Hanford Site & & & & \\
\hline & $2.51 E-02$ & $2.17 \mathrm{E}-03$ & $4.00 E-03$ & $2.32 \mathrm{E}-02$ & $2.94 \mathrm{E}-02$ \\
\hline & $6.59 \mathrm{E}-03$ & $1.07 \mathrm{E}-02$ & $1.64 \mathrm{E}-04$ & $6.22 \mathrm{E}-03$ & $1.70 \mathrm{E}-02$ \\
\hline MLLW(C) LL to HS & & & & & \\
\hline Lawrence Livermore & to Hanford Site & & & & \\
\hline & Truck $2.82 \mathrm{E}-02$ & $2.88 \mathrm{E}-03$ & $4.92 \mathrm{E}-03$ & $2.50 \mathrm{E}-02$ & $3.28 \mathrm{E}-02$ \\
\hline & $6.94 E-03$ & $1.38 \mathrm{E}-02$ & $2.00 \Xi-04$ & $6.48 \mathrm{E}-03$ & $2.05 E-02$ \\
\hline$M L L W(C) M Z$ to $\mathrm{HS}$ & & & & & \\
\hline Mare Island & to Hanford Site & & & & \\
\hline & Truck $\quad 2.76 \mathrm{E}-02$ & $3.02 \mathrm{E}-03$ & $5.17 E-03$ & $2.45 \mathrm{E}-02$ & $3.27 \mathrm{E}-02$ \\
\hline & $6.99 \mathrm{E}-03$ & $1.38 \mathrm{E}-02$ & $2.03 E-04$ & $6.51 E-03$ & $2.05 E-02$ \\
\hline $\begin{array}{l}\text { MLLW(C) US to HS } \\
\text { Norfolk Nay Shipyd }\end{array}$ & & & & & \\
\hline Norfolk Nav Shipyd & to Hanford Site & & & & \\
\hline & 8.5IE-02 & $5.79 E-03$ & 1.21E-02 & $8.26 E-02$ & $1.00 \mathrm{E}-0 \mathrm{I}$ \\
\hline MLLW(C) NT to HS & $1.62 \mathrm{E}-02$ & & 4.7 & $E-02$ & \\
\hline Nevada Tes $\tau$ Site & to Hanford Site & & & & \\
\hline & Truck $\quad 3.22 \mathrm{E}-02$ & $2.46 \mathrm{E}-03$ & $5.18 E-03$ & $3.16 \mathrm{E}-02$ & $3.92 \mathrm{E}-02$ \\
\hline & Rail $8.31 E-03$ & $4.39 E-03$ & $1.15 E-04$ & $7.49 E-03$ & $1.20 \mathrm{E}-02$ \\
\hline MLLW (C) OR to HS & & & & & \\
\hline Oak Ridge Reserv. & to Hanford Site & & & & \\
\hline & $6.83 E-02$ & $3.65 E-03$ & $8.95 E-03$ & $6.90 E-02$ & $8.16 E-02$ \\
\hline MLLW (C) PA to HS & $1.37 E-02$ & $1.08 \mathrm{E}-02$ & $2.59 E-04$ & $1.15 \mathrm{E}-02$ & $2.26 \mathrm{E}-02$ \\
\hline Paducah GDP & to Hanford Site & & & & \\
\hline & $\begin{array}{l}\text { to Hanford Site } \\
\text { Truck } 6.00 \mathrm{E}-02\end{array}$ & & & & \\
\hline & $1.22 \mathrm{E}-02$ & $9.43 \mathrm{E}-03$ & $2.28 E-04$ & $\begin{array}{l}0.11 \mathrm{E}-02 \\
1.04 \mathrm{E}-02\end{array}$ & $2.01 \mathrm{E}-02$ \\
\hline MLLW(C) PP to HS & & & & & \\
\hline Pantex Plant & to Hanford Site & & & & \\
\hline & Truck $\quad 5.10 \mathrm{E}-02$ & $2.68 \mathrm{E}-03$ & $7.01 E-03$ & $5.27 E-02$ & $6.24 \mathrm{E}-02$ \\
\hline & Rail $\quad 9.92 \mathrm{E}-03$ & $5.71 \mathrm{E}-03$ & 1.5 & $E-03$ & \\
\hline $\begin{array}{l}\text { MLLW(C) SW to HS } \\
\text { Pearl Harbor NS }\end{array}$ & & & & & \\
\hline Feath Maldot dis & $\begin{array}{l}\text { to hanrord } \\
\text { Truck } 7.06 \mathrm{E}-03\end{array}$ & $6.28 \mathrm{E}-04$ & $1.09 E-03$ & $6.33 E-03$ & -03 \\
\hline & Rail $4.62 \mathrm{E}-03$ & 6.63 & 9.7 & $E-03$ & -02 \\
\hline MLLW(C) PT to HS & & & & & \\
\hline Portsmouth NS & to Hanford Site & & & & \\
\hline & $9 \cdot 34 \mathrm{E}-02$ & $7.12 E-03$ & $1.32 \mathrm{E}-02$ & $8.64 \mathrm{E}-02$ & $1.07 \mathrm{E}-01$ \\
\hline & $1.62 \mathrm{E}-02$ & $3.74 \mathrm{E}-02$ & 6.47 & $1.33 \mathrm{E}-02$ & $5.14 \mathrm{E}-02$ \\
\hline MLLW (C) PO to HS & & & & & \\
\hline Portsmouth GDP & to Hanford Site & & & & \\
\hline & $6.63 E-02$ & $3.38 \mathrm{E}-03$ & $8.48 \mathrm{E}-03$ & $6.72 \mathrm{E}-02$ & $7.91 \mathrm{E}-02$ \\
\hline & $1.34 \mathrm{E}-02$ & $1.89 \mathrm{E}-02$ & $3.46 \mathrm{E}-04$ & $1.12 \mathrm{E}-02$ & $3.04 \mathrm{E}-02$ \\
\hline $\begin{array}{l}\text { MLLW(C) PR to HS } \\
\text { Princeton PPL }\end{array}$ & & & & & \\
\hline Princeton PPL & to Hanford Site & & & & \\
\hline & Rail $1.53 \mathrm{E}-02$ & $\begin{array}{l}0.03 \mathrm{E}-03 \\
3.63 \mathrm{E}-02\end{array}$ & $\begin{array}{l}1.28 \mathrm{E}-02 \\
6.03 \mathrm{E}-04\end{array}$ & $\begin{array}{l}8.02 \mathrm{E}-02 \\
1.27 \mathrm{E}-02\end{array}$ & $\begin{array}{l}9.97 \mathrm{E}-02 \\
4.96 \mathrm{E}-02\end{array}$ \\
\hline MLLW (C) PN to HS & & & & & \\
\hline Puget Sound NS & to Hanford Site & & & & \\
\hline & $\begin{array}{ll}\text { Truck } & 7.06 \mathrm{E}-03 \\
\text { Rail } & 4.62 \mathrm{E}-03\end{array}$ & $\begin{array}{l}5.28 \mathrm{E}-04 \\
6.63 \mathrm{E}-03\end{array}$ & $\begin{array}{l}1.09 \mathrm{E}-03 \\
9.79 \mathrm{E}-05\end{array}$ & $\begin{array}{l}6.33 \mathrm{E}-03 \\
4.76 \mathrm{E}-03\end{array}$ & $\begin{array}{l}8.04 \mathrm{E}-03 \\
1.15 \mathrm{E}-02\end{array}$ \\
\hline MLLW(C) RM to HS & & & & & \\
\hline Reactive Metals & to Hanford Site & & & & \\
\hline & 6.7 & $4.22 E-03$ & $9.51 \mathrm{E}-03$ & $6.71 \mathrm{E}-02$ & $8.09 \mathrm{E}-02$ \\
\hline MLLW (C) SA to HS & 02 & & & $E-02$ & \\
\hline SNL - Albuquerque & to Hanford Site & & & & \\
\hline & $4.31 \mathrm{E}-02$ & $2.27 \mathrm{E}-03$ & $5.84 E-03$ & $4.43 \mathrm{E}-02$ & $5.24 \mathrm{E}-02$ \\
\hline & 1.041 & 6. & 1.7 & $9.00 \mathrm{E}-03$ & -02 \\
\hline MLLW(C) SL to HS & & & & & \\
\hline SNL - Livermore & to Hanford Site & & & & \\
\hline & $k \quad 2.82 \mathrm{E}-02$ & $2.88 \mathrm{E}-03$ & $4.92 \mathrm{E}-03$ & $2.50 \mathrm{E}-02$ & $3.28 \mathrm{E}-02$ \\
\hline$M L L W(C)$ SR to $\mathrm{HS}$ & 3 & 02 & 2.0 & & \\
\hline Savannah River & to Hanford Site & & & & \\
\hline & Truck $\quad 7.86 \mathrm{E}-02$ & $5.10 \mathrm{E}-03$ & $1.08 \mathrm{E}-02$ & $7.64 \mathrm{E}-02$ & $\begin{array}{l}9.23 \mathrm{E}-02 \\
3.15 \mathrm{E}-02\end{array}$ \\
\hline & $1.52 \mathrm{E}-02$ & $1.85 \mathrm{E}-02$ & $4.05 \mathrm{E}-04$ & $1.26 \mathrm{E}-02$ & $3.15 \mathrm{E}-02$ \\
\hline
\end{tabular}


Table 4. Accident Dose Risk per Shipment (person-rem/shipment)

\begin{tabular}{|c|c|c|c|}
\hline \multicolumn{2}{|l|}{ Source/Route } & \multirow[t]{2}{*}{ Truck } & \multirow[t]{2}{*}{ Rail } \\
\hline MLLW(C) AL to $\mathrm{HS}$ & & & \\
\hline $\begin{array}{l}\text { Ames Laboratory } \\
\text { MLIW (C) AN to HS }\end{array}$ & to Hanford Site & $1.16 \mathrm{E}-08$ & $3.94 \mathrm{E}-09$ \\
\hline $\begin{array}{l}\text { Argonne - East } \\
\text { MLIW(C) AW to HS }\end{array}$ & to Hanford Site & $8.84 \mathrm{E}-04$ & $5.65 E-04$ \\
\hline $\begin{array}{l}\text { Argonne - West } \\
\text { ML }\end{array}$ & to Hanford Site & $3.17 E-04$ & $1.69 \pm-04$ \\
\hline $\begin{array}{l}\text { Bettis Atomic } \\
\text { MLLW(C) BC to HS }\end{array}$ & to Hanford Site & $7.32 \mathrm{E}-04$ & $3.03 E-04$ \\
\hline $\begin{array}{l}\text { Battelle Columbus } \\
\text { MLLW(C) BN to HS }\end{array}$ & to Hanford Site & $4.78 \mathrm{E}-09$ & $1.98 E-09$ \\
\hline $\begin{array}{l}\text { Brookhaven NL } \\
\text { MLLW(C) CH to HS }\end{array}$ & to Hanford Site & $1.31 \mathrm{E}-03$ & $6.47 E-04$ \\
\hline $\begin{array}{l}\text { Charleston, SC } \\
\text { MLLW(C) CL to HS }\end{array}$ & to Hanford Site & $8.69 \mathrm{E}-04$ & $1.79 E-04$ \\
\hline $\begin{array}{l}\text { Colonie } \\
\text { MLLW(C) LC to HS }\end{array}$ & to Hanford Site & $1.95 \mathrm{E}-08$ & $5.82 E-09$ \\
\hline $\begin{array}{l}\text { ETEC } \\
\text { MLLW(C) FE to HS }\end{array}$ & to Hanford Site & $3.06 \mathrm{E}-04$ & $3.23 E-04$ \\
\hline $\begin{array}{l}\text { Fernald (FEMP) } \\
\text { MLLW (C) So to HS }\end{array}$ & to Hanford Site & $3.46 \mathrm{E}-05$ & $3.56 \mathrm{E}-05$ \\
\hline $\begin{array}{l}\text { General Atomic } \\
\text { MLLW(C) GJ to HS }\end{array}$ & to Hanford Site & $7.14 \mathrm{E}-07$ & $2.22 \mathrm{E}-07$ \\
\hline $\begin{array}{l}\text { Grand Junction Po } \\
\text { MLLW(C) IN to HS }\end{array}$ & to Hanford Site & $1.71 E-08$ & $5.97 \mathrm{E}-09$ \\
\hline $\begin{array}{l}\text { INEL } \\
\text { MLLW (C) KA to HS }\end{array}$ & to Hanford Site & $5.25 \mathrm{E}-03$ & $5.61 \mathrm{E}-03$ \\
\hline $\begin{array}{l}\text { Knol1s Atomic } \\
\text { MLLW(C) KC to HS }\end{array}$ & to Hanford Site & $3.82 \mathrm{E}-02$ & $1.24 \mathrm{E}-02$ \\
\hline $\begin{array}{l}\text { Kansas City Plant } \\
\text { MLLW(C) KK to HS }\end{array}$ & to Hanford Site & $1.11 E-04$ & $2.02 E-05$ \\
\hline $\begin{array}{l}\text { KKS } \\
\text { MLLW(C) KW to HS }\end{array}$ & to Hanford Site & $4.20 \mathrm{E}-02$ & $1.36 \mathrm{E}-02$ \\
\hline $\begin{array}{l}\text { KWS } \\
\text { MLLW (C) LA to HS }\end{array}$ & to Hanford Site & $1.35 E-02$ & $4.37 E-03$ \\
\hline $\begin{array}{l}\text { Los Alamos NL } \\
\text { MLLW(C) LB to HS }\end{array}$ & to Hanford Site & $5.97 E-05$ & $1.41 \mathrm{E}-05$ \\
\hline $\begin{array}{l}\text { Lawrence Berkeley } \\
\text { MLLW(C) SM to HS }\end{array}$ & to Hanford Site & $1.05 \mathrm{E}-05$ & $5.80 \mathrm{E}-06$ \\
\hline IERHR & to Hanford Site & $9.03 E-06$ & $4.74 \mathrm{E}-06$ \\
\hline MLLW(C) LL to HS & to Hanford Site & $4.37 \mathrm{E}-05$ & $4.58 \mathrm{E}-05$ \\
\hline $\begin{array}{l}\text { MLLW(C) MZ to HS } \\
\text { Mare Island } \\
\text { MLLW(C) US to HS }\end{array}$ & to Hanford Site & -03 & $4.86 \mathrm{E}-03$ \\
\hline $\begin{array}{l}\text { Norfolk Nav Shipyd } \\
\text { MLLW(C) NT to HS }\end{array}$ & to Hanford site & $2.86 \mathrm{E}-03$ & $1.09 \mathrm{E}-03$ \\
\hline $\begin{array}{l}\text { Nevada Test Site } \\
\text { MLLW(C) OR to HS }\end{array}$ & to Hanford Site & $1.69 \mathrm{E}-08$ & $4.11 E-09$ \\
\hline $\begin{array}{l}\text { Oak Ridge Reserv. } \\
\text { MILLW(C) PA to HS }\end{array}$ & to Hanford Site & $1.34 \mathrm{E}-03$ & $8.03 E-04$ \\
\hline $\begin{array}{l}\text { Paducah GDP } \\
\text { MLLW(C) PP to HS }\end{array}$ & to Hanford Site & $3.55 \mathrm{E}-03$ & $8.14 E-04$ \\
\hline $\begin{array}{l}\text { Pantex Plant } \\
\text { MLLW(C) SW to HS }\end{array}$ & to Hanford Site & $2.10 \mathrm{E}-04$ & $4.85 \mathrm{E}-05$ \\
\hline $\begin{array}{l}\text { Pearl Harbor NS } \\
\text { MLLW(C) PT to HS }\end{array}$ & to Hanford Site & $1.11 E-04$ & $8.21 E-05$ \\
\hline $\begin{array}{l}\text { Portsmouth NS } \\
\text { MLLW(C) PO to HS }\end{array}$ & to Hanford Site & $5.84 E-04$ & $2.66 \mathrm{E}-04$ \\
\hline $\begin{array}{l}\text { Portsmouth GDP } \\
\text { MLLW(C) PR to HS }\end{array}$ & to Hanford Site & $1.00 \mathrm{E}-05$ & $1.04 \mathrm{E}-05$ \\
\hline $\begin{array}{l}\text { Princeton PPL } \\
\text { MLLW(C) PN to HS }\end{array}$ & to Hanford Site & $9.99 E-09$ & $3.93 E-09$ \\
\hline $\begin{array}{l}\text { Puget sound NS } \\
\text { MuLW(C) RM to HS }\end{array}$ & to Hanford Site & $2.47 E-03$ & $1.83 E-03$ \\
\hline $\begin{array}{l}\text { Reactive Metals } \\
\text { MLLW(C) SA to HS }\end{array}$ & to Hanford Site & $3.72 E-06$ & $1.55 \mathrm{E}-06$ \\
\hline $\begin{array}{l}\text { SNL - Albuquerque } \\
\text { MLLW(C) SL to HS }\end{array}$ & to Hanford Site & $3.14 \mathrm{E}-04$ & $3.68 \mathrm{E}-05$ \\
\hline $\begin{array}{l}\text { SNL - Livermore } \\
\text { MLLW(C) SR to HS }\end{array}$ & to Hanford Site & $2.72 E-06$ & $1.43 E-06$ \\
\hline Savannah River & to Hanford Site & $4.85 E-03$ & $2.82 \mathrm{E}-03$ \\
\hline
\end{tabular}


Table 5. Nonradiological Risk Factors per Shipment (fatalities/shipment)

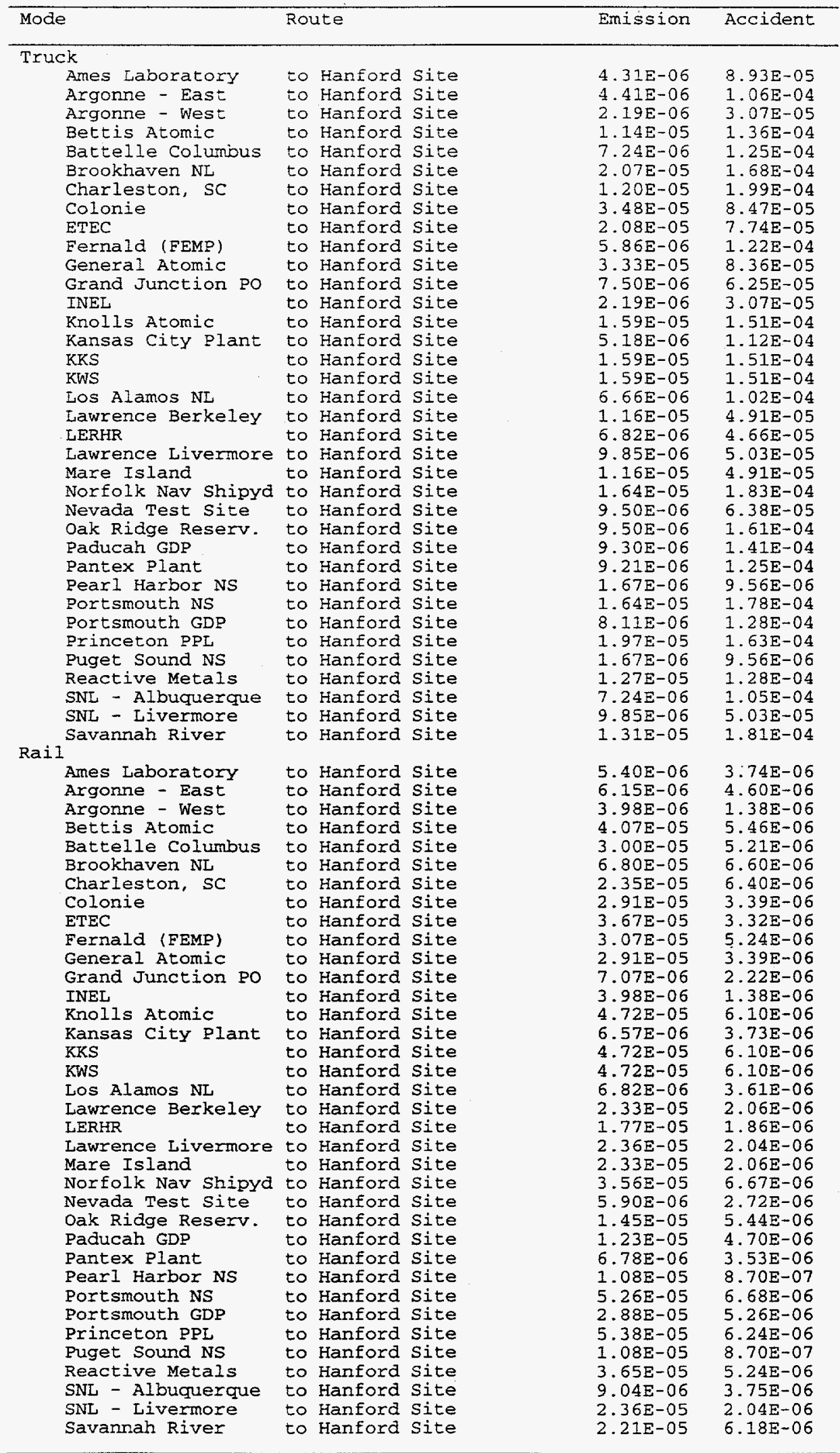




\section{$B-144$}

Table 6. Annual Incident-Free Dose for the Shipping Campaign (person-rem/yr)

\begin{tabular}{|c|c|c|c|c|c|c|}
\hline & & & $T r$ & uck & & ail \\
\hline Route & & & Crew & Public & Crew & Public \\
\hline Ames Laboratory & to & Hanford Site & $4.46 \mathrm{E}-02$ & $5.47 \mathrm{E}-02$ & $1.03 \mathrm{E}-02$ & $1.38 \mathrm{E}-02$ \\
\hline Argonne - East & to & Hanford Site & $2.33 E+00$ & $2.82 \mathrm{E}+00$ & $2.05 E-01$ & $2.75 \mathrm{E}-01$ \\
\hline Argonne - West & to & Hanford Site & $1.60 \mathrm{E}-02$ & $1.95 E-02$ & $5.63 \mathrm{E}-03$ & $8.40 E-03$ \\
\hline Bettis Atomic & to & Hanford site & $7.21 \mathrm{E}-02$ & $8.53 E-02$ & $1.38 \mathrm{E}-02$ & $3.87 E-02$ \\
\hline Battelle Columbus & to & Hanford site & $6.36 \mathrm{E}-02$ & $7.61 \mathrm{E}-02$ & $1.33 \mathrm{E}-02$ & $3.10 E-02$ \\
\hline Brookhaven NL & to & Hanford Site & $8.38 \mathrm{E}-02$ & $9.92 E-02$ & $1.60 \mathrm{E}-02$ & $5.76 \mathrm{E}-02$ \\
\hline Charleston, SC & to & Hanford site & $8.12 \mathrm{E}-02$ & $9.58 \mathrm{E}-02$ & $1.56 \mathrm{E}-02$ & $3.29 E-02$ \\
\hline Colonie & to & Hanford Site & $4.61 \mathrm{E}-02$ & $5.72 \mathrm{E}-02$ & $9.65 \mathrm{E}-03$ & $2.52 E-02$ \\
\hline ETEC & to & Hanford Site & $3.10 \mathrm{E}-01$ & $3.79 E-01$ & $2.85 \mathrm{E}-02$ & $8.47 E-02$ \\
\hline Fernald (FEMP) & to & Hanford Site & $4.29 \mathrm{E}-01$ & $5.14 \mathrm{E}-01$ & $4.00 \mathrm{E}-02$ & $9.47 \mathrm{E}-02$ \\
\hline General Atomic & to & Hanford Site & $4.49 \mathrm{E}-02$ & $5.58 \mathrm{E}-02$ & $9.65 E-03$ & $2.52 \mathrm{E}-02$ \\
\hline Grand Junction PO & to & Hanford Site & $3.04 \mathrm{E}-02$ & $3.70 E-02$ & $7.32 \mathrm{E}-03$ & $1.23 \mathrm{E}-02$ \\
\hline INEL & to & Hanford Site & $6.40 \mathrm{E}-02$ & $7.82 \mathrm{E}-02$ & $1.13 \mathrm{E}-02$ & $1.68 \mathrm{E}-02$ \\
\hline Knolls Atomic & to & Banford site & $8.30 \mathrm{E}-02$ & $9.66 \mathrm{E}-02$ & $1.50 \mathrm{E}-02$ & $4.48 \mathrm{E}-02$ \\
\hline Kansas City Plant & to & Hanford Site & 4. $62 \mathrm{E}-02$ & $5.64 \mathrm{E}-02$ & $1.03 \mathrm{E}-02$ & $1.41 \mathrm{E}-02$ \\
\hline KKS & to & Hanford site & $8.30 \mathrm{E}-02$ & $9.66 \mathrm{E}-02$ & $1.50 \mathrm{E}-02$ & $4.48 E-02$ \\
\hline KNS & to & Fanford Site & $8.30 \mathrm{E}-02$ & $9.66 \mathrm{E}-02$ & $1.50 \mathrm{E}-02$ & $4.48 E-02$ \\
\hline Los Alamos NL & to & Hanford Site & $8.48 \mathrm{E}-02$ & $1.03 E-01$ & $1.01 E-02$ & $1.45 E-02$ \\
\hline Lawrence Berkeley & to & Hanford Site & $2.76 \mathrm{E}-02$ & $3.27 \mathrm{E}-02$ & $6.99 E-03$ & $2.05 E-02$ \\
\hline LERHR & to & Hanford Site & $2.51 \mathrm{E}-02$ & $2.94 E-02$ & $6.59 \mathrm{E}-03$ & $1.70 E-02$ \\
\hline Lawrence Livermore & to & Hanford Site & $1.13 \mathrm{E}-01$ & $1.31 \mathrm{E}-01$ & $1.39 E-02$ & $4.10 E-02$ \\
\hline Mare Island & to & Hanford Site & $2.76 \mathrm{E}-02$ & $3.27 \mathrm{E}-02$ & $6.99 E-03$ & $2.05 E-02$ \\
\hline Norfolk Nav Shipyd & to & Hanford Site & $8.51 E-02$ & $1.00 E-01$ & $1.62 \mathrm{E}-02$ & $3.91 E-02$ \\
\hline Nevada Test Site & to & Hanford Site & $3.22 \mathrm{E}-02$ & $3.92 \mathrm{E}-02$ & $8.31 E-03$ & $1.20 E-02$ \\
\hline Oak Ridge Reserv. & to & Hanford Site & $8.47 E+00$ & $1.01 \mathrm{E}+01$ & $6.32 E-01$ & $1.04 E+00$ \\
\hline Paducah GDP & & Hanford Site & $6.00 \mathrm{E}-02$ & $7.23 E-02$ & $1.22 \mathrm{E}-02$ & $2.01 \mathrm{E}-02$ \\
\hline Pantex Plant & to & Hanford Site & $1.02 \mathrm{E}-01$ & $1.25 \mathrm{E}-01$ & $9.92 E-03$ & $1.45 \mathrm{E}-02$ \\
\hline Pearl Harbor NS & & Hanford Site & $7.06 \mathrm{E}-03$ & $8.04 \mathrm{E}-03$ & $4.62 \mathrm{E}-03$ & $1.15 \mathrm{E}-02$ \\
\hline Portsmouth NS & to & Hanford Site & $9.34 \mathrm{E}-02$ & $1.07 \mathrm{E}-01$ & $1.62 \mathrm{E}-02$ & $5.14 \mathrm{E}-02$ \\
\hline Portsmouth GDP & to & Hanford Site & $3.98 \mathrm{E}+00$ & $4.75 E+00$ & $3.08 \mathrm{E}-01$ & $7.00 E-01$ \\
\hline Princeton PPL & & Hanford Site & $8.51 E-02$ & $9.97 E-02$ & $1.53 \mathrm{E}-02$ & $4.96 \mathrm{E}-02$ \\
\hline Puget Sound NS & to & Hanford Site & $7.06 \mathrm{E}-03$ & $8.04 \mathrm{E}-03$ & $4.62 \mathrm{E}-03$ & $1.15 \mathrm{E}-02$ \\
\hline Reactive Metals & to & Hanford Site & $6.76 \mathrm{E}-02$ & $8.09 E-02$ & $1.33 \mathrm{E}-02$ & $3.51 \mathrm{E}-02$ \\
\hline SNL - Albuquerque & to & Hanford Site & $4.31 \mathrm{E}-02$ & $5.24 \mathrm{E}-02$ & $1.04 \mathrm{E}-02$ & $1.61 \mathrm{E}-02$ \\
\hline SNL - Livermore & to & Hanford Site & $2.82 \mathrm{E}-02$ & $3.28 \mathrm{E}-02$ & $6.94 \mathrm{E}-03$ & $2.05 \mathrm{E}-02$ \\
\hline Savannah River & to & Hanford Site & $1.02 \mathrm{E}+00$ & $1.20 \mathrm{E}+00$ & $7.60 \mathrm{E}-02$ & $1.58 \mathrm{E}-01$ \\
\hline Tot & & & .01 & 2.18 & $1.62 \mathrm{E}+00$ & 3.1 \\
\hline
\end{tabular}


Table 7. Annual Accident Dose Risk for the Shipping Campaign (person-rem/yr)

\begin{tabular}{|c|c|c|c|}
\hline Route & & Truck & Rail \\
\hline $\begin{array}{l}\text { Ames Laboratory } \\
\text { Argonne - East } \\
\text { Argonne - West } \\
\text { Bettis Atomic } \\
\text { Battelle Columbus } \\
\text { Brookhaven NL } \\
\text { Charleston, SC } \\
\text { Colonie } \\
\text { ETEC } \\
\text { Fernald (FEMP) } \\
\text { General Atomic } \\
\text { Grand Junction PO } \\
\text { INEL } \\
\text { Knolls Atomic } \\
\text { Kansas City Plant } \\
\text { KKS } \\
\text { KWS } \\
\text { Los Alamos NL } \\
\text { Lawrence Berkeley } \\
\text { LERHR } \\
\text { Lawrence Livermore } \\
\text { Mare Island } \\
\text { Norfolk Nav Shipyd } \\
\text { Nevada Test Site } \\
\text { Oak Ridge Reserv. } \\
\text { Paducah GDP } \\
\text { Pantex Plant } \\
\text { Pearl Harbor NS } \\
\text { Portsmouth NS } \\
\text { Portsmouth GDP } \\
\text { Princeton PPL } \\
\text { Puget Sound NS } \\
\text { Reactive Metals } \\
\text { SNL - Albuquerque } \\
\text { SNL - Livermore } \\
\text { Savannah River }\end{array}$ & $\begin{array}{l}\text { to Hanford Site } \\
\text { to Hanford Site } \\
\text { to Hanford Site } \\
\text { to Hanford Site } \\
\text { to Hanford Site } \\
\text { to Hanford Site } \\
\text { to Hanford Site } \\
\text { to Hanford Site } \\
\text { to Hanford Site } \\
\text { to Hanford Site } \\
\text { to Hanford Site } \\
\text { to Hanford Site } \\
\text { to Hanford Site } \\
\text { to Hanford Site } \\
\text { to Hanford Site } \\
\text { to Hanford Site } \\
\text { to Hanford Site } \\
\text { to Hanford Site } \\
\text { to Hanford Site } \\
\text { to Hanford Site } \\
\text { to Hanford Site } \\
\text { to Hanford Site } \\
\text { to Hanford Site } \\
\text { to Hanford Site } \\
\text { to Hanford Site } \\
\text { to Hanford Site } \\
\text { to Hanford Site } \\
\text { to Hanford Site } \\
\text { to Hanford Site } \\
\text { to Hanford Site } \\
\text { to Hanford Site } \\
\text { to Hanford Site } \\
\text { to Hanford Site } \\
\text { to Hanford Site } \\
\text { to Hanford Site } \\
\text { to Hanford Site }\end{array}$ & $\begin{array}{l}1.16 \mathrm{E}-08 \\
3.89 \mathrm{E}-02 \\
3.17 \mathrm{E}-04 \\
7.32 \mathrm{E}-04 \\
4.78 \mathrm{E}-09 \\
1.31 \mathrm{E}-03 \\
8.69 \mathrm{E}-04 \\
1.95 \mathrm{E}-08 \\
2.45 \mathrm{E}-03 \\
2.42 \mathrm{E}-04 \\
7.14 \mathrm{E}-07 \\
1.71 \mathrm{E}-08 \\
2.10 \mathrm{E}-02 \\
3.82 \mathrm{E}-02 \\
1.11 \mathrm{E}-04 \\
4.20 \mathrm{E}-02 \\
1.35 \mathrm{E}-02 \\
1.19 \mathrm{E}-04 \\
1.05 \mathrm{E}-05 \\
9.03 \mathrm{E}-06 \\
1.75 \mathrm{E}-04 \\
8.65 \mathrm{E}-03 \\
2.86 \mathrm{E}-03 \\
1.69 \mathrm{E}-08 \\
1.65 \mathrm{E}-01 \\
3.55 \mathrm{E}-03 \\
4.21 \mathrm{E}-04 \\
1.11 \mathrm{E}-04 \\
5.84 \mathrm{E}-04 \\
6.03 \mathrm{E}-04 \\
9.99 \mathrm{E}-09 \\
2.47 \mathrm{E}-03 \\
3.72 \mathrm{E}-06 \\
3.14 \mathrm{E}-04 \\
2.72 \mathrm{E}-06 \\
6.31 \mathrm{E}-02\end{array}$ & $\begin{array}{l}3.94 \mathrm{E}-09 \\
9.60 \mathrm{E}-03 \\
1.69 \mathrm{E}-04 \\
3.03 \mathrm{E}-04 \\
1.98 \mathrm{E}-09 \\
6.47 \mathrm{E}-04 \\
1.79 \mathrm{E}-04 \\
5.82 \mathrm{E}-09 \\
9.70 \mathrm{E}-04 \\
1.07 \mathrm{E}-04 \\
2.22 \mathrm{E}-07 \\
5.97 \mathrm{E}-09 \\
1.12 \mathrm{E}-02 \\
1.24 \mathrm{E}-02 \\
2.02 \mathrm{E}-05 \\
1.36 \mathrm{E}-02 \\
4.37 \mathrm{E}-03 \\
1.41 \mathrm{E}-05 \\
5.80 \mathrm{E}-06 \\
4.74 \mathrm{E}-06 \\
9.17 \mathrm{E}-05 \\
4.86 \mathrm{E}-03 \\
1.09 \mathrm{E}-03 \\
4.11 \mathrm{E}-09 \\
3.69 \mathrm{E}-02 \\
8.14 \mathrm{E}-04 \\
4.85 \mathrm{E}-05 \\
8.21 \mathrm{E}-05 \\
2.65 \mathrm{E}-04 \\
2.39 \mathrm{E}-04 \\
3.93 \mathrm{E}-09 \\
1.83 \mathrm{E}-03 \\
1.55 \mathrm{E}-06 \\
3.68 \mathrm{E}-05 \\
1.43 \mathrm{E}-06 \\
1.41 \mathrm{E}-02\end{array}$ \\
\hline Totals & & $4.08 \mathrm{E}-01$ & $1.14 \mathrm{E}-01$ \\
\hline
\end{tabular}

Table 8. Expected Annual Fatalities for the Shipping Campaign

\begin{tabular}{llc}
\hline Exposure Group & Truck & Rail \\
\hline Radiological & & \\
Normal Crew & $7.3 E-03$ & $6.5 E-04$ \\
Normal Public & $1.1 \mathrm{E}-02$ & $1.6 \mathrm{E}-03$ \\
Accident Public & $2.0 \mathrm{E}-04$ & $5.7 \mathrm{E}-05$ \\
Nonradiological & $2.6 \mathrm{E}-03$ & $2.5 \mathrm{E}-03$ \\
Enission & $4.0 \mathrm{E}-02$ & $6.3 \mathrm{E}-04$ \\
Accident & & \\
\hline
\end{tabular}

Table 9. Expected Annual Cancer Incidence for the Shipping Campaign

\begin{tabular}{lll}
\hline Exposure Group & Truck & Rail \\
\hline Radiological & & \\
Normal Crew & $2.6 \mathrm{E}-02$ & $2.3 \mathrm{E}-03$ \\
$\quad$ Normal Public & $3.7 \mathrm{E}-02$ & $5.4 \mathrm{E}-03$ \\
$\quad$ Accident Public & $6.9 \mathrm{E}-04$ & $1.9 \mathrm{E}-04$ \\
Nonradiological & $2.6 \mathrm{E}-03$ & $2.5 \mathrm{E}-03$ \\
$\quad$ Emission & $\mathrm{NA}$ & $\mathrm{NA}$ \\
Accident & & \\
\hline
\end{tabular}


Table 10. Expected Annual Genetic Effects for the Shipping Campaign

\begin{tabular}{lll}
\hline Exposure Group & Truck & Rail \\
\hline Radiological & & \\
Normal Crew & $1.1 E-03$ & $9.7 \mathrm{E}-05$ \\
Normal Public & $2.2 \mathrm{E}-03$ & $3.2 \mathrm{E}-04$ \\
Accident Public & $3.1 \mathrm{E}-05$ & $8.4 \mathrm{E}-06$ \\
Nonradiological & & \\
Emission & NA & NA \\
Accident & NA & NA \\
\hline
\end{tabular}




\section{B.32 WM LLMW CENTRALIZED ALTERNATIVE (CASE 17): CONTACT-HANDLED HETEROGENEOUS SOLIDS: ALPHA - UNTREATED}

Table 1. Summary of Route Information

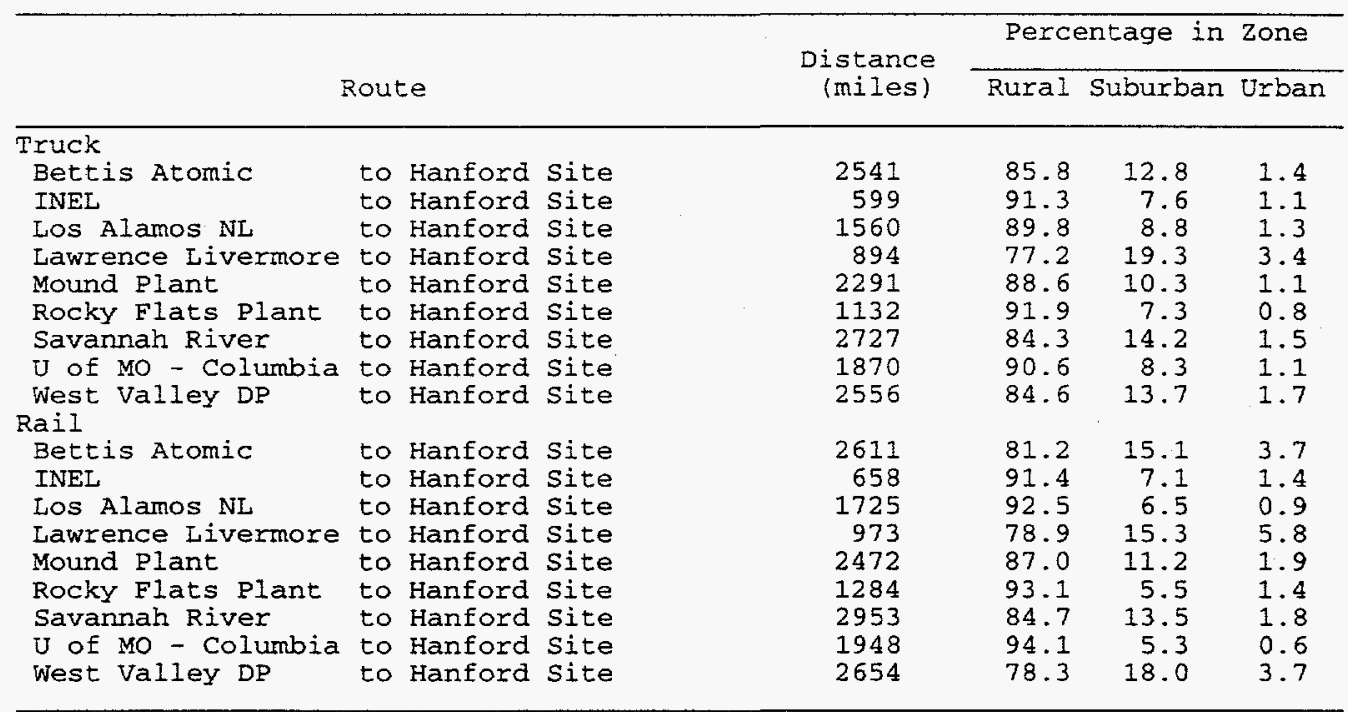

Table 2. Annual Number of Shipments and one-Way Shipment Distances

\begin{tabular}{|c|c|c|c|c|c|}
\hline \multirow[b]{2}{*}{ Route } & & \multicolumn{2}{|c|}{ \# of shipments } & \multicolumn{2}{|c|}{ Total Mileage (mi) } \\
\hline & & Truek & Rail & Truck & Rail \\
\hline $\begin{array}{l}\text { Bettis Atomic } \\
\text { INEL } \\
\text { Los Alamos NL } \\
\text { Lawrence Livermore } \\
\text { Mound Plant } \\
\text { Rocky Flats Plant } \\
\text { Savannah River } \\
\text { U of Mo-Columbia } \\
\text { West Valley DP }\end{array}$ & $\begin{array}{l}\text { to Hanford Site } \\
\text { to Hanford Site } \\
\text { to Hanford Site } \\
\text { to Hanford Site } \\
\text { to Hanford Site } \\
\text { to Hanford Site } \\
\text { to Hanford Site } \\
\text { to Hanford Site } \\
\text { to Hanford Site }\end{array}$ & $\begin{array}{r}1 \\
63 \\
4 \\
6 \\
1 \\
130 \\
10 \\
1 \\
1\end{array}$ & $\begin{array}{r}1 \\
24 \\
2 \\
3 \\
1 \\
48 \\
4 \\
1 \\
1\end{array}$ & $\begin{array}{l}2.54 \mathrm{E}+03 \\
3.77 \mathrm{E}+04 \\
6.24 \mathrm{E}+03 \\
5.36 \mathrm{E}+03 \\
2.29 \mathrm{E}+03 \\
1.47 \mathrm{E}+05 \\
2.73 \mathrm{E}+04 \\
1.87 \mathrm{E}+03 \\
2.56 \mathrm{E}+03\end{array}$ & $\begin{array}{l}2.61 \mathrm{E}+03 \\
1.58 \mathrm{E}+04 \\
3.45 \mathrm{E}+03 \\
2.92 \mathrm{E}+03 \\
2.47 \mathrm{E}+03 \\
6.16 \mathrm{E}+04 \\
1.18 \mathrm{E}+04 \\
1.95 \mathrm{E}+03 \\
2.65 \mathrm{E}+03\end{array}$ \\
\hline Totals & & 217 & 85 & $2.33 E+05$ & $1.05 \mathrm{E}+05$ \\
\hline
\end{tabular}


Table 3. Incident-Free Dose per Shipment (person-rem/shipment)

\begin{tabular}{|c|c|c|c|c|c|}
\hline \multirow[b]{2}{*}{ Source/Route (s) } & \multirow[b]{2}{*}{ Crew } & \multicolumn{4}{|c|}{ General Public } \\
\hline & & Off-Link & on-Link & stops & Total \\
\hline \multicolumn{6}{|l|}{ MLLW (CA) BA to HS } \\
\hline & $\begin{array}{ll}\text { Truck } & 7.21 \mathrm{E}-02 \\
\text { Rail } & 1.38 \mathrm{E}-02\end{array}$ & $\begin{array}{l}4.36 \mathrm{E}-03 \\
2.67 \mathrm{E}-02\end{array}$ & $\begin{array}{l}9.77 \mathrm{E}-03 \\
4.53 \mathrm{E}-04\end{array}$ & $\begin{array}{l}\text { 7.11E-02 } \\
1.15 \mathrm{E}-02\end{array}$ & $\begin{array}{l}8.53 \Xi-02 \\
3.87 \Xi-02\end{array}$ \\
\hline \multicolumn{6}{|l|}{ MLLW(CA) IN to HS } \\
\hline INEL & $\begin{array}{ll}\text { to Hanford Site } \\
\text { Truck } & 1.60 \mathrm{E}-02 \\
\text { Rail } & 5.63 \mathrm{E}-03\end{array}$ & $\begin{array}{l}7.18 \mathrm{E}-04 \\
2.83 \mathrm{E}-03\end{array}$ & $\begin{array}{l}2.06 \mathrm{E}-03 \\
6.58 \mathrm{E}-05\end{array}$ & $\begin{array}{l}1.68 \mathrm{E}-02 \\
5.51 \mathrm{E}-03\end{array}$ & $\begin{array}{l}1.95 E-02 \\
8.40 E-03\end{array}$ \\
\hline \multicolumn{6}{|l|}{ MLLW (CA) LA to HS } \\
\hline Los Alamos NL & $\begin{array}{lr}\text { to Hanford site } \\
\text { Truck } & 4.24 \mathrm{E}-02 \\
\text { Rail } & 1.01 \mathrm{E}-02\end{array}$ & $\begin{array}{l}2.16 \mathrm{E}-03 \\
5.60 \mathrm{E}-03\end{array}$ & $\begin{array}{l}5.65 \mathrm{E}-03 \\
1.54 \mathrm{E}-04\end{array}$ & $\begin{array}{l}4.37 E-02 \\
8.79 E-03\end{array}$ & $\begin{array}{l}5.15 \mathrm{E}-02 \\
1.45 \mathrm{E}-02\end{array}$ \\
\hline \multicolumn{6}{|c|}{$M L L W$ (CA) LL to HS } \\
\hline Lawrence Livermore & $\begin{array}{l}\text { to Hanford Site } \\
\text { Truck } 2.82 \mathrm{E}-02 \\
\text { Rail } \\
6.94 \mathrm{E}-03\end{array}$ & $\begin{array}{l}2.88 \mathrm{E}-03 \\
1.38 \mathrm{E}-02\end{array}$ & $\begin{array}{l}4.92 \mathrm{E}-03 \\
2.00 \mathrm{E}-04\end{array}$ & $\begin{array}{l}2.50 \mathrm{E}-02 \\
6.48 \mathrm{E}-03\end{array}$ & $\begin{array}{l}3.28 E-02 \\
2.05 E-02\end{array}$ \\
\hline MLLW(CA) MP to HS & & & & & \\
\hline Mound Plant & $\begin{array}{ll}\text { to Hanford Site } \\
\text { Truck } & 6.29 \mathrm{E}-02 \\
\text { Rail } & 1.32 \mathrm{E}-02\end{array}$ & $\begin{array}{l}3.13 \mathrm{E}-03 \\
1.47 \mathrm{E}-02\end{array}$ & $\begin{array}{l}8.05 E-03 \\
3.13 \mathrm{E}-04\end{array}$ & $\begin{array}{l}6.41 \mathrm{E}-02 \\
1.11 \mathrm{E}-02\end{array}$ & $\begin{array}{l}7.53 \mathrm{E}-02 \\
2.51 \mathrm{E}-02\end{array}$ \\
\hline MLLW (CA) RF to HS & & & & & \\
\hline Rocky Flats Plant & $\begin{array}{lr}\text { to Hanford Site } \\
\text { Truck } & 2.99 \mathrm{E}-02 \\
\text { Rail } & 8.24 \mathrm{E}-03\end{array}$ & $\begin{array}{l}1.13 \mathrm{E}-03 \\
4.91 \mathrm{E}-03\end{array}$ & $\begin{array}{l}3.61 \mathrm{E}-03 \\
1.16 \mathrm{E}-04\end{array}$ & $\begin{array}{l}3.17 \mathrm{E}-02 \\
7.43 \mathrm{E}-03\end{array}$ & $\begin{array}{l}3.64 E-02 \\
1.25 E-02\end{array}$ \\
\hline MLLW(CA) SR to HS & & & & & \\
\hline Savannah River & $\begin{array}{lr}\text { to Hanford Site } \\
\text { Truck } 7.86 \mathrm{E}-02 \\
\text { Rail } & 1.52 \mathrm{E}-02\end{array}$ & $\begin{array}{l}5.10 \mathrm{E}-03 \\
1.85 \mathrm{E}-02\end{array}$ & $\begin{array}{l}1.08 \mathrm{E}-02 \\
4.05 \mathrm{E}-04\end{array}$ & $\begin{array}{l}7.64 \mathrm{E}-02 \\
1.26 \mathrm{E}-02\end{array}$ & $\begin{array}{l}9.23 \mathrm{E}-02 \\
3.15 \mathrm{E}-02\end{array}$ \\
\hline MLLW(CA) MC to HS & & & & & \\
\hline U of MO - Columbia & $\begin{array}{lr}\text { to Hanford Site } \\
\text { Truck } 5.03 \mathrm{E}-02 \\
\text { Rail } & 1.10 \mathrm{E}-02\end{array}$ & $\begin{array}{l}2.31 \mathrm{E}-03 \\
4.60 \mathrm{E}-03\end{array}$ & $\begin{array}{l}6.45 \mathrm{E}-03 \\
1.53 \mathrm{E}-04\end{array}$ & $\begin{array}{l}5.23 \mathrm{E}-02 \\
9.48 \mathrm{E}-03\end{array}$ & $\begin{array}{l}6.11 \mathrm{E}-02 \\
1.42 \mathrm{E}-02\end{array}$ \\
\hline$M L L W(C A)$ WV to HS & & & & & \\
\hline West Valley DP & $\begin{array}{lr}\text { to Hanford Site } \\
\text { Truck } 7.36 \mathrm{E}-02 \\
\text { Rail } & 1.40 \mathrm{E}-02\end{array}$ & $\begin{array}{l}4.90 E-03 \\
2.88 E-02\end{array}$ & $\begin{array}{l}1.04 E-02 \\
5.00 E-04\end{array}$ & $\begin{array}{l}7.16 \mathrm{E}-02 \\
1.17 \mathrm{E}-02\end{array}$ & $\begin{array}{l}8.68 \mathrm{E}-02 \\
4.09 \mathrm{E}-02\end{array}$ \\
\hline
\end{tabular}

Table 4. Accident Dose Risk per Shipment (person-rem/shipment)

\begin{tabular}{|c|c|c|c|}
\hline Source/Route & & Truck & Rail \\
\hline $\begin{array}{l}\text { MLLW (CA) BA to HS } \\
\text { Bettis Atomic } \\
\text { MLLW (CA) IN to HS }\end{array}$ & to Hanford Site & $1.02 \mathrm{E}-03$ & $4.22 \mathrm{E}-04$ \\
\hline $\begin{array}{l}\text { INEL } \\
M L L W(C A) \text { LA to HS }\end{array}$ & to Hanford site & $4.98 E-04$ & $6.67 E-04$ \\
\hline $\begin{array}{l}\text { Los Alamos NL } \\
\text { MLLW(CA) LL to HS }\end{array}$ & to Hanford site & $1.30 \mathrm{E}-04$ & $3.35 \mathrm{E}-05$ \\
\hline $\begin{array}{l}\text { Lawrence Livermore } \\
\text { MLLW(CA) MP to HS }\end{array}$ & to Hanford Site & $8.38 \mathrm{E}-05$ & $8.72 \mathrm{E}-05$ \\
\hline $\begin{array}{l}\text { Mound Plant } \\
\text { MLLW (CA) RF to HS }\end{array}$ & to Hanford site & $2.93 E-07$ & $6.53 \mathrm{E}-08$ \\
\hline $\begin{array}{l}\text { Rocky Flats Plant } \\
\text { MLIW(CA) SR to HS }\end{array}$ & to Hanford Site & $3.19 \mathrm{E}-05$ & $2.35 \mathrm{E}-05$ \\
\hline $\begin{array}{l}\text { Savannah River } \\
\text { MLLW (CA) MC to HS }\end{array}$ & to Hanford site & $2.98 \mathrm{E}-03$ & $1.69 \mathrm{E}-03$ \\
\hline $\begin{array}{l}U \text { of MO - Columbia } \\
M L L W(C A) \text { WV to HS }\end{array}$ & to Hanford Site & $4.78 \mathrm{E}-07$ & $1.23 \mathrm{E}-07$ \\
\hline West Valley DP & to Hanford Site & $1.05 E-03$ & $4.44 \mathrm{E}-04$ \\
\hline
\end{tabular}


Table 5. Nonradiological Risk Factors per Shipment (fatalities/shipment)

\begin{tabular}{llll}
\hline Mode & Route & Emission & Accident \\
\hline Truck & & & \\
Bettis Atomic & to Hanford Site & $1.14 \mathrm{E}-05$ & $1.36 \mathrm{E}-04$ \\
INEL & to Hanford Site & $2.19 \mathrm{E}-06$ & $3.07 \mathrm{E}-05$ \\
Los Alamos NL & to Hanford Site & $6.66 \mathrm{E}-06$ & $1.02 \mathrm{E}-04$ \\
Lawrence Livermore & to Hanford Site & $9.85 \mathrm{E}-06$ & $5.03 \mathrm{E}-05$ \\
Mound Plant & to Hanford Site & $7.89 \mathrm{E}-06$ & $1.23 \mathrm{E}-04$ \\
Rocky Flats Plant & to Hanford Site & $2.83 \mathrm{E}-06$ & $6.69 \mathrm{E}-05$ \\
Savannah River & to Hanford Site & $1.31 \mathrm{E}-05$ & $1.81 \mathrm{E}-04$ \\
U of MO - Columbia & to Hanford Site & $6.66 \mathrm{E}-06$ & $1.20 \mathrm{E}-04$ \\
West Valley DP & to Hanford Site & $1.36 \mathrm{E}-05$ & $1.38 \mathrm{E}-04$ \\
Rail & & & \\
Bettis Atomic & to Hanford Site & $4.07 \mathrm{E}-05$ & $5.46 \mathrm{E}-06$ \\
INEL & to Hanford Site & $3.98 \mathrm{E}-06$ & $1.38 \mathrm{E}-06$ \\
Los Alamos NL & to Hanford Site & $6.82 \mathrm{E}-06$ & $3.61 \mathrm{E}-06$ \\
Lawrence Livermore to Hanford Site & $2.36 \mathrm{E}-05$ & $2.04 \mathrm{E}-06$ \\
Mound Plant & to Hanford Site & $1.94 \mathrm{E}-05$ & $5.17 \mathrm{E}-06$ \\
Rocky Flats Plant & to Hanford Site & $7.36 \mathrm{E}-06$ & $2.69 \mathrm{E}-06$ \\
Savannah River & to Hanford Site & $2.21 \mathrm{E}-05$ & $6.18 \mathrm{E}-06$ \\
U of MO Columbia to Hanford Site & $4.98 \mathrm{E}-06$ & $4.08 \mathrm{E}-06$ \\
West Valley DP & to Hanford Site & $4.14 \mathrm{E}-05$ & $5.55 \mathrm{E}-06$ \\
\hline
\end{tabular}

Table 6. Annuai Incident-Free Dose for the Shipping Campaign (person-rem/yr)

\begin{tabular}{|c|c|c|c|c|c|}
\hline \multirow[b]{2}{*}{ Route } & & \multicolumn{2}{|c|}{ Truck } & \multicolumn{2}{|c|}{ Rail } \\
\hline & & Crew & Public & Crew & Public \\
\hline $\begin{array}{l}\text { Bettis Atomic } \\
\text { INEL } \\
\text { LOS Alamos NL } \\
\text { Lawrence Livermore } \\
\text { Mound Plant } \\
\text { Rocky Flats Plant } \\
\text { Savannah River } \\
\text { U of MO - Columbia } \\
\text { West Valley DP }\end{array}$ & $\begin{array}{l}\text { to Hanford Site } \\
\text { to Hanford Site } \\
\text { to Hanford Site } \\
\text { to Hanford Site } \\
\text { to Hanford Site } \\
\text { to Hanford Site } \\
\text { to Hanford Site } \\
\text { to Hanford Site } \\
\text { to Hanford Site }\end{array}$ & $\begin{array}{l}7.21 \mathrm{E}-02 \\
1.01 \mathrm{E}+00 \\
1.70 \mathrm{E}-01 \\
1.69 \mathrm{E}-01 \\
6.29 \mathrm{E}-02 \\
3.88 \mathrm{E}+00 \\
7.86 \mathrm{E}-01 \\
5.03 \mathrm{E}-02 \\
7.36 \mathrm{E}-02\end{array}$ & $\begin{array}{l}8.53 \mathrm{E}-02 \\
1.23 \mathrm{E}+00 \\
2.06 \mathrm{E}-01 \\
1.97 \mathrm{E}-01 \\
7.53 \mathrm{E}-02 \\
4.73 \mathrm{E}+00 \\
9.23 \mathrm{E}-01 \\
6.11 \mathrm{E}-02 \\
8.68 \mathrm{E}-02\end{array}$ & $\begin{array}{l}1.38 \mathrm{E}-02 \\
1.35 \mathrm{E}-01 \\
2.02 \mathrm{E}-02 \\
2.08 \mathrm{E}-02 \\
1.32 \mathrm{E}-02 \\
3.96 \mathrm{E}-01 \\
6.08 \mathrm{E}-02 \\
1.10 \mathrm{E}-02 \\
1.40 \mathrm{E}-02\end{array}$ & $\begin{array}{l}3.87 \mathrm{E}-02 \\
2.02 \mathrm{E}-01 \\
2.91 \mathrm{E}-02 \\
6.15 \mathrm{E}-02 \\
2.61 \mathrm{E}-02 \\
5.98 \mathrm{E}-01 \\
1.26 \mathrm{E}-01 \\
1.42 \mathrm{E}-02 \\
4.09 \mathrm{E}-02\end{array}$ \\
\hline Totals & & $6.28 E+00$ & $7.60 E+00$ & $6.84 \mathrm{E}-0$ & $1.14 \mathrm{E}+00$ \\
\hline
\end{tabular}

Table 7. Annual Accident Dose Risk for the Shipping Campaign (person-rem/yr)

\begin{tabular}{llcc}
\hline Route & & Truck & Rail \\
\hline Bettis Atomic & to Hanford Site & $1.02 \mathrm{E}-03$ & $4.22 \mathrm{E}-04$ \\
INEL & to Hanford Site & $3.14 \mathrm{E}-02$ & $1.60 \mathrm{E}-02$ \\
Los Alamos NL & to Hanford Site & $5.21 \mathrm{E}-04$ & $6.70 \mathrm{E}-05$ \\
Lawrence Livermore to Hanford Site & $5.03 \mathrm{E}-04$ & $2.62 \mathrm{E}-04$ \\
Mound Plant & to Hanford Site & $2.93 \mathrm{E}-07$ & $6.53 \mathrm{E}-08$ \\
Rocky Flats Plant & to Hanford Site & $4.15 \mathrm{E}-03$ & $1.13 \mathrm{E}-03$ \\
Savannah River & to Hanford Site & $2.98 \mathrm{E}-02$ & $6.74 \mathrm{E}-03$ \\
U of MO Columbia to Hanford Site & $4.78 \mathrm{E}-07$ & $1.23 \mathrm{E}-07$ \\
West Valley DP & to Hanford Site & $1.05 \mathrm{E}-03$ & $4.44 \mathrm{E}-04$ \\
\hline Totals & & $6.85 \mathrm{E}-02$ & $2.51 \mathrm{E}-02$ \\
\end{tabular}

Table 8. Expected Annual Fatalities for the Shipping Campaign

\begin{tabular}{lll}
\hline Exposure Group & Truck & Rail \\
\hline Radiological & $2.5 \mathrm{E}-03$ & $2.7 \mathrm{E}-04$ \\
Normal Crew & $3.8 \mathrm{E}-03$ & $5.7 \mathrm{E}-04$ \\
Normal Public & $3.4 \mathrm{E}-05$ & $1.3 \mathrm{E}-05$ \\
Accident Public & & \\
Nonradiological & $7.6 \mathrm{E}-04$ & $7.3 \mathrm{E}-04$ \\
Emission & $1.4 \mathrm{E}-02$ & $2.2 \mathrm{E}-04$ \\
Accident & &
\end{tabular}


Table 9. Expected Annual Cancer Incidence for the Shipping Campaign

\begin{tabular}{lll}
\hline Exposure Group & Truck & \multicolumn{1}{c}{ Rail } \\
\hline Radiological & & \\
Normal Crew & $8.8 \mathrm{E}-03$ & $9.6 \mathrm{E}-04$ \\
Normal Public & $1.3 \mathrm{E}-02$ & $1.9 \mathrm{E}-03$ \\
Accident Public & $1.2 \mathrm{E}-04$ & $4.3 \mathrm{E}-05$ \\
Nonradiological & $7.6 \mathrm{E}-04$ & $7.3 \mathrm{E}-04$ \\
Emission & $\mathrm{NA}$ & $\mathrm{NA}$ \\
Accident & & \\
\hline
\end{tabular}

Table 10. Expected Annual Genetic Effects for the Shipping Campaign

\begin{tabular}{lll}
\hline Exposure Group & Truck & Rail \\
\hline Radiological & $3.8 \mathrm{E}-04$ & $4.1 \mathrm{E}-05$ \\
Normal Crew & $7.6 \mathrm{E}-04$ & $1.1 \mathrm{E}-04$ \\
Normal Public & $5.1 \mathrm{E}-06$ & $1.9 \mathrm{E}-06$ \\
Accident Public & & \\
Nonradiological & NA & NA \\
Emission & NA & NA \\
Accident & & \\
\hline
\end{tabular}




\section{B.33 WM LLMW CENTRALIZED ALTERNATIVE (CASE 17): CONTACT-HANDLED HETEROGENEOUS SOLIDS: NONALPHA — TREATED}

Table 1. Summary of Route Information

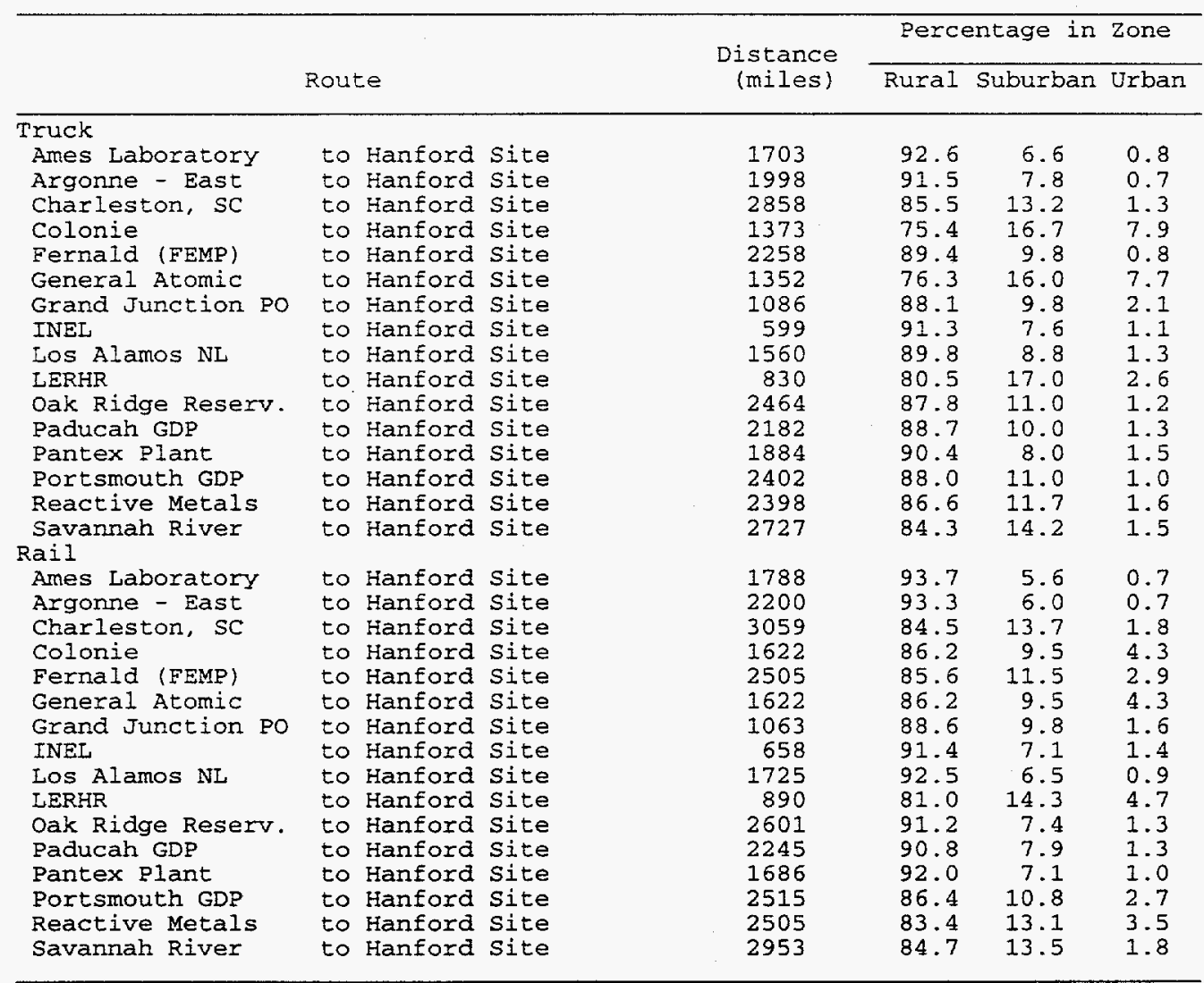

Table 2. Annual Number of Shipments and one-Way Shipment Distances

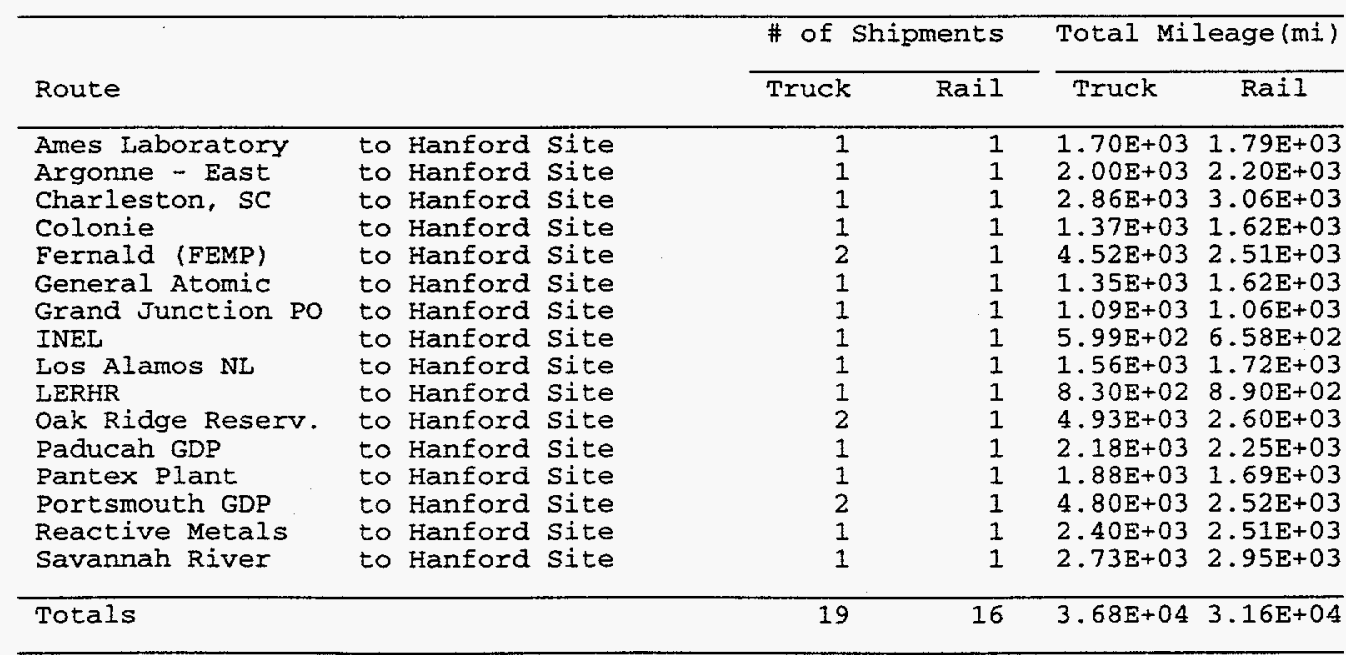


Table 3. Incident-Free Dose per Shipment (person-rem/shipment)

\begin{tabular}{|c|c|c|c|c|c|}
\hline \multirow[b]{2}{*}{ Source/Route (s) } & \multirow[b]{2}{*}{ Crew } & \multicolumn{4}{|c|}{ General Public } \\
\hline & & off-Link & on-Link & Stops & Total \\
\hline \multicolumn{6}{|l|}{ MLLW (CT) AL to HS } \\
\hline & $\begin{array}{ll}\text { Truek } & 4.46 \mathrm{E}-02 \\
\text { Rail } & 1.03 \mathrm{E}-02\end{array}$ & $\begin{array}{l}1.62 \mathrm{E}-03 \\
4.69 \mathrm{E}-03\end{array}$ & $\begin{array}{l}5.39 \mathrm{E}-03 \\
1.46 \mathrm{E}-04\end{array}$ & $\begin{array}{l}4.77 E-02 \\
8.98 E-03\end{array}$ & $\begin{array}{l}5.47 \mathrm{E}-02 \\
1.38 \mathrm{E}-02\end{array}$ \\
\hline \multicolumn{6}{|l|}{$\begin{array}{l}\text { MLLW (CT) AN to HS } \\
\text { Argonne - East }\end{array}$} \\
\hline Argonne - East & $\begin{array}{lr}\text { to Hanford Site } \\
\text { Truck } & 5.29 \mathrm{E}-02 \\
\text { Rail } & 1.21 \mathrm{E}-02\end{array}$ & $\begin{array}{l}1.97 E-03 \\
5.75 E-03\end{array}$ & $\begin{array}{l}6.28 \mathrm{E}-03 \\
1.82 \mathrm{E}-04\end{array}$ & $\begin{array}{l}5.59 \mathrm{E}-02 \\
1.03 \mathrm{E}-02\end{array}$ & $\begin{array}{l}6.42 \mathrm{E}-02 \\
1.62 \mathrm{E}-02\end{array}$ \\
\hline \multicolumn{6}{|l|}{$\begin{array}{l}\text { MLLW (CT) CH to HS } \\
\text { Charleston SC }\end{array}$} \\
\hline Charleston, SC & $\begin{array}{lr}\text { to Hanford Site } \\
\text { Truck } & 8.12 \mathrm{E}-02 \\
\text { Rail } & 1.56 \mathrm{E}-02\end{array}$ & $\begin{array}{l}4.87 \mathrm{E}-03 \\
1.96 \mathrm{E}-02\end{array}$ & $\begin{array}{l}1.09 \mathrm{E}-02 \\
4.24 \mathrm{E}-04\end{array}$ & $\begin{array}{l}8.00 \mathrm{E}-02 \\
1.29 \mathrm{E}-02\end{array}$ & $\begin{array}{l}9.58 \mathrm{E}-02 \\
3.29 \mathrm{E}-02\end{array}$ \\
\hline \multicolumn{6}{|l|}{$\begin{array}{l}\text { MLLW(CT) CL to HS } \\
\text { Colonie }\end{array}$} \\
\hline & $\begin{array}{ll}\text { Truck } & 4.61 \mathrm{E}-02 \\
\text { Rail } & 9.65 \mathrm{E}-03\end{array}$ & $\begin{array}{l}7.22 \mathrm{E}-03 \\
1.64 \mathrm{E}-02\end{array}$ & $\begin{array}{l}1.16 \Xi-02 \\
2.49 \Xi-04\end{array}$ & $\begin{array}{l}3.84 \mathrm{E}-02 \\
8.47 \mathrm{E}-03\end{array}$ & $\begin{array}{l}5.72 E-02 \\
2.52 E-02\end{array}$ \\
\hline \multicolumn{6}{|l|}{$\begin{array}{l}\text { MLLW (CT) FE to HS } \\
\text { Fernald (FEMP) }\end{array}$} \\
\hline & $6.13 \mathrm{E}-02$ & $2.71 E-03$ & $E-03$ & -02 & $7.34 \mathrm{E}-02$ \\
\hline & 1. $33 \mathrm{E}-02$ & 2.00 & 3.6 & 1. & 3. \\
\hline \multicolumn{6}{|l|}{$\begin{array}{l}\text { MLLW (CT) So to HS } \\
\text { General Atomic }\end{array}$} \\
\hline & $\begin{array}{ll}\text { Truck } & 4.49 \mathrm{E}-02 \\
\text { Rail } & 9.65 \mathrm{E}-03\end{array}$ & $\begin{array}{l}6.88 \mathrm{E}-03 \\
1.64 \mathrm{E}-02\end{array}$ & $\begin{array}{l}1.11 E-02 \\
2.49 E-04\end{array}$ & $\begin{array}{l}3.78 E-02 \\
8.47 E-03\end{array}$ & $\begin{array}{l}5.58 \mathrm{E}-02 \\
2.52 \mathrm{E}-02\end{array}$ \\
\hline \multicolumn{6}{|l|}{$M L I W(C T)$ GJ to HS } \\
\hline & $\begin{array}{ll}\text { Truck } & 3.04 \mathrm{E}-02 \\
\text { Rail } & 7.32 \mathrm{E}-03\end{array}$ & $\begin{array}{l}2.03 E-03 \\
5.46 \mathrm{E}-03\end{array}$ & $\begin{array}{l}4.58 E-03 \\
1.23 E-04\end{array}$ & $\begin{array}{l}3.04 \mathrm{E}-02 \\
6.75 \mathrm{E}-03\end{array}$ & $\begin{array}{l}3.70 \mathrm{E}-02 \\
1.23 \mathrm{E}-02\end{array}$ \\
\hline \multicolumn{6}{|l|}{$\begin{array}{c}\text { MLLW(CT) IN to HS } \\
\text { INEL }\end{array}$} \\
\hline & $\begin{array}{ll}\text { Truck } & 1.60 \mathrm{E}-02 \\
\text { Rail } & 5.63 \mathrm{E}-03\end{array}$ & $\begin{array}{l}7.18 \mathrm{E}-04 \\
2.83 \mathrm{E}-03\end{array}$ & $\begin{array}{l}2.06 \mathrm{E}-03 \\
6.58 \mathrm{E}-05\end{array}$ & $\begin{array}{l}1.68 \mathrm{E}-02 \\
5.51 \mathrm{E}-03\end{array}$ & $\begin{array}{l}1.95 \mathrm{E}-02 \\
8.40 \mathrm{E}-03\end{array}$ \\
\hline \multicolumn{6}{|l|}{$M L L W(C T)$ LA to HS } \\
\hline Los Alamos NL & $\begin{array}{l}\text { to Hanford Site } \\
\text { Truck } \\
\text { Rail } \\
4.24 \mathrm{E}-02 \\
1.01 \mathrm{E}-02\end{array}$ & $\begin{array}{l}2.16 \mathrm{E}-03 \\
5.60 \mathrm{E}-03\end{array}$ & $\begin{array}{l}5.65 \mathrm{E}-03 \\
1.54 \mathrm{E}-04\end{array}$ & $\begin{array}{l}4.37 \mathrm{E}-02 \\
8.79 \mathrm{E}-03\end{array}$ & $\begin{array}{l}5.15 E-02 \\
1.45 E-02\end{array}$ \\
\hline \multicolumn{6}{|l|}{$\begin{array}{l}\text { MLLW(CT) SM to HS } \\
\text { LERHR }\end{array}$} \\
\hline & $\begin{array}{ll}\text { Truck } & 2.51 \mathrm{E}-02 \\
\text { Rail } & 6.59 \mathrm{E}-03\end{array}$ & $\begin{array}{l}2.17 \Xi-03 \\
1.07 \mathrm{E}-02\end{array}$ & $\begin{array}{l}4.00 \mathrm{E}-03 \\
1.64 \mathrm{E}-04\end{array}$ & $\begin{array}{l}2.32 \mathrm{E}-02 \\
6.22 \mathrm{E}-03\end{array}$ & $\begin{array}{l}2.94 \mathrm{E}-02 \\
1.70 \mathrm{E}-02\end{array}$ \\
\hline \multicolumn{6}{|l|}{ MLLW(CT) OR to HS } \\
\hline idge Res & $\begin{array}{lr}\text { to Hanford Site } \\
\text { Truck } & 6.83 \mathrm{E}-02 \\
\text { Rail } & 1.37 \mathrm{E}-02\end{array}$ & $\begin{array}{l}3.65 \mathrm{E}-03 \\
1.08 \mathrm{E}-02\end{array}$ & $\begin{array}{l}8.95 E-03 \\
2.59 E-04\end{array}$ & $\begin{array}{l}6.90 \mathrm{E}-02 \\
1.15 \mathrm{E}-02\end{array}$ & $\begin{array}{l}8.16 \mathrm{E}-02 \\
2.26 \mathrm{E}-02\end{array}$ \\
\hline \multicolumn{6}{|l|}{$\begin{array}{c}\text { MLLW (CT) PA to HS } \\
\text { Paducah GDP }\end{array}$} \\
\hline & $\begin{array}{ll}\text { Truck } & 6.00 \mathrm{E}-02 \\
\text { Rail } & 1.22 \mathrm{E}-02\end{array}$ & $\begin{array}{l}3.22 \mathrm{E}-03 \\
9.43 \mathrm{E}-03\end{array}$ & $\begin{array}{l}8.02 \mathrm{E}-03 \\
2.28 \mathrm{E}-04\end{array}$ & $\begin{array}{l}6.11 \mathrm{E}-02 \\
1.04 \mathrm{E}-02\end{array}$ & $\begin{array}{l}7.23 \mathrm{E}-02 \\
2.01 \mathrm{E}-02\end{array}$ \\
\hline \multicolumn{6}{|l|}{$\begin{array}{c}\text { MLLW(CT) PP to HS } \\
\text { Pantex Plant }\end{array}$} \\
\hline & $\begin{array}{ll}\text { Truck } & 5.10 \mathrm{E}-02 \\
\text { Rai1 } & 9.92 \mathrm{E}-03\end{array}$ & $\begin{array}{l}2.68 \mathrm{E}-03 \\
5.71 \mathrm{E}-03\end{array}$ & $\begin{array}{l}7.01 \mathrm{E}-03 \\
1.56 \mathrm{E}-04\end{array}$ & $\begin{array}{l}5.27 \mathrm{E}-02 \\
8.67 \mathrm{E}-03\end{array}$ & $\begin{array}{l}6.24 \mathrm{E}-02 \\
1.45 \mathrm{E}-02\end{array}$ \\
\hline \multicolumn{6}{|l|}{$\begin{array}{l}\text { MLLW(CT) PO to HS } \\
\text { Portsmouth GDP }\end{array}$} \\
\hline & $\begin{array}{ll}\text { Truck } & 6.63 \mathrm{E}-02 \\
\text { Rail } & 1.34 \mathrm{E}-02\end{array}$ & $\begin{array}{l}3.38 \mathrm{E}-03 \\
1.89 \mathrm{E}-02\end{array}$ & $\begin{array}{l}8.48 \mathrm{E}-03 \\
3.46 \mathrm{E}-04\end{array}$ & $\begin{array}{l}6.72 \mathrm{E}-02 \\
1.12 \mathrm{E}-02\end{array}$ & $\begin{array}{l}7.91 E-02 \\
3.04 E-02\end{array}$ \\
\hline \multirow[t]{2}{*}{$\begin{array}{l}\text { MLLW(CT) RM to HS } \\
\text { Reactive Metals }\end{array}$} & to Hanford Site & & & & \\
\hline & $\begin{array}{ll}\text { Truck } & 6.76 \mathrm{E}-02 \\
\text { Rail } & 1.33 \mathrm{E}-02\end{array}$ & $\begin{array}{l}4.22 \mathrm{E}-03 \\
2.35 \mathrm{E}-02\end{array}$ & $\begin{array}{l}9.51 \mathrm{E}-03 \\
4.01 \mathrm{E}-04\end{array}$ & $\begin{array}{l}6.71 \mathrm{E}-02 \\
1.12 \mathrm{E}-02\end{array}$ & $\begin{array}{l}8.09 E-02 \\
3.51 E-02\end{array}$ \\
\hline $\begin{array}{l}\text { MLLW(CT) SR to HS } \\
\text { Savannah River }\end{array}$ & ford & & & & \\
\hline & $\begin{array}{l}7.86 \mathrm{E}-02 \\
1.52 \mathrm{E}-02\end{array}$ & $\begin{array}{l}5.10 \mathrm{E}-03 \\
1.85 \mathrm{E}-02\end{array}$ & $\begin{array}{l}1.08 \mathrm{E}-02 \\
4.05 \mathrm{E}-04\end{array}$ & $\begin{array}{l}7.64 \mathrm{E}-02 \\
1.26 \mathrm{E}-02\end{array}$ & $\begin{array}{l}9.23 \mathrm{E}-02 \\
3.15 \mathrm{E}-02\end{array}$ \\
\hline
\end{tabular}


Table 4. Accident Dose Risk per Shipment (person-rem/shipment)

\begin{tabular}{|c|c|c|c|}
\hline \multicolumn{2}{|l|}{ Source/Route } & \multirow{3}{*}{$\frac{\text { Truck }}{1.98 \mathrm{E}-08}$} & \multirow{3}{*}{$\frac{\text { Rail }}{6.73 E-09}$} \\
\hline MLLW(CT) AL to HS & & & \\
\hline $\begin{array}{l}\text { Ames Laboratory } \\
\text { MLLW(CT) AN to HS }\end{array}$ & to Hanford site & & \\
\hline $\begin{array}{c}\text { Argonne - East } \\
\text { MIN }\end{array}$ & to Hanford Site & $1.94 \mathrm{E}-05$ & $4.79 E-06$ \\
\hline $\begin{array}{l}\text { MLLW (CT) CH to HS } \\
\text { Charleston, SC }\end{array}$ & to Hanford Site & $6.40 E-04$ & $1.32 \mathrm{E}-04$ \\
\hline $\begin{array}{l}\text { MLLW(CT) CL to HS } \\
\text { Colonie }\end{array}$ & to Hanford Site & $8.81 E-08$ & $2.63 E-08$ \\
\hline $\begin{array}{l}\text { MLLW (CT) FE to HS } \\
\text { Fernald (FEMP) }\end{array}$ & to Hanford site & $1.04 \mathrm{E}-04$ & $9.19 E-05$ \\
\hline $\begin{array}{l}\text { MLLW (CT) so to HS } \\
\text { GeneraI Atomic } \\
\text { MLLW(CT) GJ to HS }\end{array}$ & to Hanford Site & $2.91 \mathrm{E}-06$ & $9.03 \mathrm{E}-07$ \\
\hline $\begin{array}{l}\text { Grand Junction PO } \\
\text { MLLW(CT) IN to HS }\end{array}$ & to Hanford Site & $3.70 \mathrm{E}-08$ & $1.29 \mathrm{E}-08$ \\
\hline $\begin{array}{l}\text { INEL } \\
\text { MLLW(CT) LA to HS }\end{array}$ & to Hanford Site & $6.55 E-03$ & $3.50 \mathrm{E}-03$ \\
\hline $\begin{array}{l}\text { LOS Alamos NL } \\
\text { MLLW(CT) SM to HS }\end{array}$ & to Hanford Site & $3.57 E-05$ & $4.20 E-06$ \\
\hline IERHR & to Hanford Site & $1.21 \mathrm{E}-06$ & $6.33 \mathrm{E}-07$ \\
\hline $\begin{aligned} & \text { MLLW(CT) } \text { OR to HS } \\
& \text { Oak Ridge Reserv. } \\
& \text { MLLW(CT) PA to HS }\end{aligned}$ & to Hanford Site & $5.39 \mathrm{E}-03$ & $2.40 \mathrm{E}-03$ \\
\hline $\begin{array}{c}\text { Paducah GDP } \\
\text { MLLW(CT) PP to HS }\end{array}$ & to Hanford Site & $8.48 \mathrm{E}-04$ & $1.95 E-04$ \\
\hline $\begin{array}{l}\text { Pantex Plant } \\
\text { MLLW(CT) PO to HS }\end{array}$ & to Hanford Site & $3.00 E-05$ & $3.48 \mathrm{E}-06$ \\
\hline $\begin{array}{l}\text { Portsmouth GDP } \\
\text { MLLW(CT) RM to HS }\end{array}$ & to Hanford Site & $3.44 E-05$ & $2.74 \mathrm{E}-05$ \\
\hline $\begin{array}{l}\text { Reactive Metals } \\
\text { MLLW(CT) SR to HS }\end{array}$ & to Hanford Site & $1.57 \mathrm{E}-06$ & $6.51 E-07$ \\
\hline Savannah River & to Hanford Site & $1.40 E-04$ & $3.13 E-05$ \\
\hline
\end{tabular}

Table 5. Nonradiological Risk Factors per Shipment (fatalities/shipment)

\begin{tabular}{|c|c|c|c|}
\hline Mode & Route & Emission & Accident \\
\hline \multicolumn{4}{|l|}{ Truck } \\
\hline Ames Laboratory & to Hanford Site & $4.31 E-06$ & $8.93 E-05$ \\
\hline Argonne - East & to Hanford Site & $4.41 E-06$ & $1.05 \mathrm{E}-04$ \\
\hline Charleston, SC & to Hanford Site & $1.20 \mathrm{E}-05$ & $1.99 \mathrm{E}-04$ \\
\hline Colonie & to Hanford Site & $3.48 \mathrm{E}-05$ & $8.47 \mathrm{E}-05$ \\
\hline Fernald (FEMP) & to Hanford Site & $5.86 \mathrm{E}-06$ & $1.22 \mathrm{E}-04$ \\
\hline General Atomic & to Hanford Site & $3.33 E-05$ & $8.36 E-05$ \\
\hline Grand Junction Po & to Hanford Site & $7.50 \mathrm{E}-06$ & $6.25 E-05$ \\
\hline INEL & to Hanford site & $2.19 E-06$ & $3.07 \mathrm{E}-05$ \\
\hline Los Alamos NL & to Hanford Site & $6.66 \mathrm{E}-06$ & $1.02 \mathrm{E}-04$ \\
\hline LERHR & to Hanford Site & $6.82 \mathrm{E}-06$ & $4.66 \mathrm{E}-05$ \\
\hline Oak Ridge Reserv. & to Hanford Site & $9.50 E-06$ & $1.61 \mathrm{E}-04$ \\
\hline Paducah GDP & to Hanford Site & $9.30 E-06$ & $1.41 \mathrm{E}-04$ \\
\hline Pantex Plant & to Hanford site & $9.21 E-06$ & $1.25 \mathrm{E}-04$ \\
\hline Portsmouth GDP & to Hanford Site & $8.11 E-06$ & $1.28 \mathrm{E}-04$ \\
\hline Reactive Metals & to Hanford Site & $1.27 E-05$ & $1.28 \mathrm{E}-04$ \\
\hline Savannah River & to Hanford Site & $1.31 \mathrm{E}-05$ & $1.81 \mathrm{E}-04$ \\
\hline \multicolumn{4}{|l|}{ Rail } \\
\hline Ames Laboratory & to Hanford site & $5.40 \mathrm{E}-06$ & $3.74 \mathrm{E}-06$ \\
\hline Argonne - East & to Hanford Site & $6.15 \mathrm{E}-06$ & $4.60 \mathrm{E}-06$ \\
\hline Charleston, SC & to Hanford Site & $2.35 E-05$ & $6.40 \mathrm{E}-06$ \\
\hline Colonie & to Hanford Site & $2.91 E-05$ & $3.39 E-06$ \\
\hline Fernald (FEMP) & to Hanford Site & $3.07 \mathrm{E}-05$ & $5.24 \mathrm{E}-06$ \\
\hline General Atomic & to Hanford Site & $2.91 E-05$ & $3.39 \mathrm{E}-06$ \\
\hline Grand Junction Po & to Hanford Site & $7.07 \mathrm{E}-06$ & $2.22 \mathrm{E}-06$ \\
\hline INEL & to Hanford Site & $3.98 E-06$ & $1.38 \mathrm{E}-06$ \\
\hline Los Alamos NL & to Hanford Site & $6.82 \mathrm{E}-06$ & $3.61 \mathrm{E}-06$ \\
\hline LERHR & to Hanford site & $1.77 \mathrm{E}-05$ & $1.86 \mathrm{E}-06$ \\
\hline Oak Ridge Reserv. & to Hanford site & $1.45 \mathrm{E}-05$ & $5.44 \mathrm{E}-06$ \\
\hline Paducah GDP & to Hanford Site & $1.23 E-05$ & $4.70 E-06$ \\
\hline Pantex Plant & to Hanford site & $6.78 \mathrm{E}-06$ & $3.53 E-06$ \\
\hline Portsmouth GDP & to Hanford Site & $2.88 \mathrm{E}-05$ & $5.26 E-06$ \\
\hline Reactive Metals & to Hanford site & $3.65 E-05$ & $5.24 E-06$ \\
\hline Savannah River & to Hanford Site & $2.21 \mathrm{E}-05$ & $6.18 E-06$ \\
\hline
\end{tabular}


Table 6. Annual Incident-Free Dose for the Shipping Campaign (person-rem/yr)

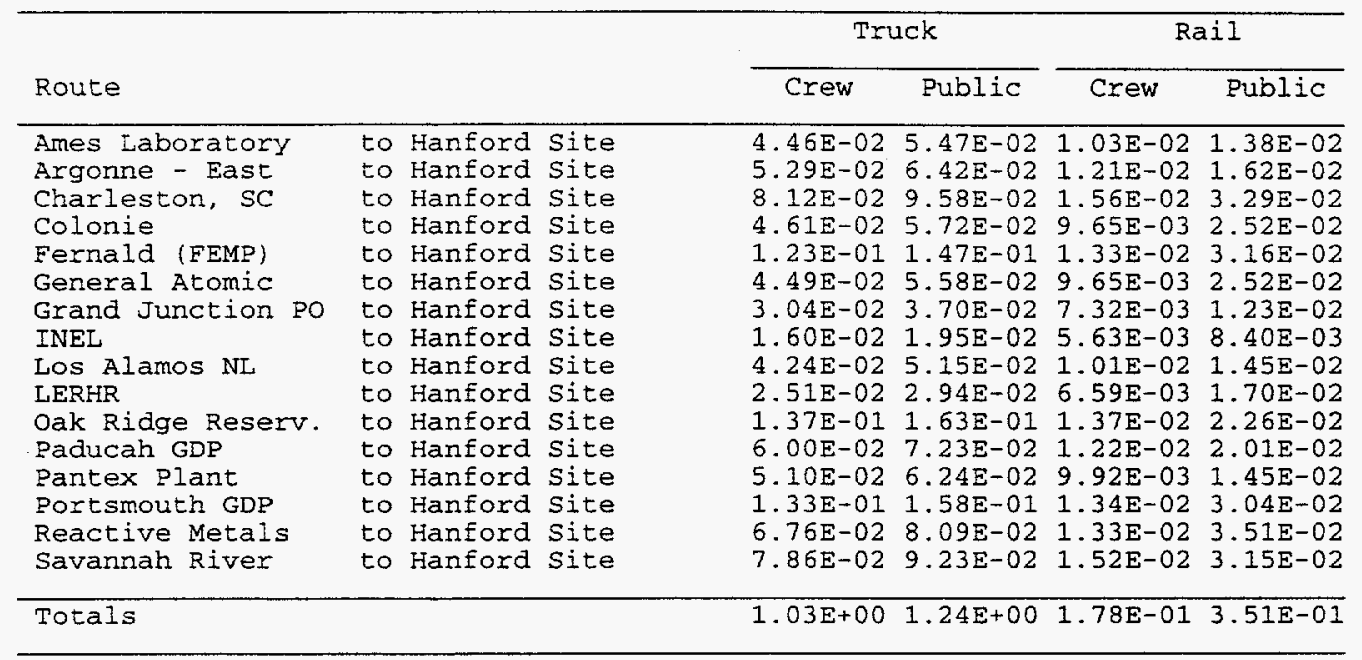

Table 7. Annual Accident Dose Risk for the Shipping Campaign (person-rem/yr)

\begin{tabular}{llcc}
\hline Route & & Truck & Rail \\
\hline Ames Laboratory & to Hanford Site & $1.98 \mathrm{E}-08$ & $6.73 \mathrm{E}-09$ \\
Argonne - East & to Hanford Site & $1.94 \mathrm{E}-05$ & $4.79 \mathrm{E}-06$ \\
Charleston, SC & to Hanford Site & $6.40 \mathrm{E}-04$ & $1.32 \mathrm{E}-04$ \\
Colonie & to Hanford Site & $8.81 \mathrm{E}-08$ & $2.63 \mathrm{E}-08$ \\
Fernald (FEMP) & to Hanford Site & $2.08 \mathrm{E}-04$ & $9.19 \mathrm{E}-05$ \\
General Atomic & to Hanford Site & $2.91 \mathrm{E}-06$ & $9.03 \mathrm{E}-07$ \\
Grand Junction PO & to Hanford Site & $3.70 \mathrm{E}-08$ & $1.29 \mathrm{E}-08$ \\
INEL & to Hanford Site & $6.55 \mathrm{E}-03$ & $3.50 \mathrm{E}-03$ \\
Los Alamos NL & to Hanford Site & $3.57 \mathrm{E}-05$ & $4.20 \mathrm{E}-06$ \\
LERHR & to Hanford Site & $1.21 \mathrm{E}-06$ & $6.33 \mathrm{E}-07$ \\
Oak Ridge Reserv. & to Hanford Site & $1.08 \mathrm{E}-02$ & $2.40 \mathrm{E}-03$ \\
Paducah GDP & to Hanford Site & $8.48 \mathrm{E}-04$ & $1.95 \mathrm{E}-04$ \\
Pantex Plant & to Hanford Site & $3.00 \mathrm{E}-05$ & $3.48 \mathrm{E}-06$ \\
Portsmouth GDP & to Hanford Site & $6.89 \mathrm{E}-05$ & $2.74 \mathrm{E}-05$ \\
Reactive Metals & to Hanford Site & $1.57 \mathrm{E}-06$ & $6.51 \mathrm{E}-07$ \\
Savannah River & to Hanford Site & $1.40 \mathrm{E}-04$ & $3.13 \mathrm{E}-05$ \\
& & & \\
\hline Totals & & $1.93 \mathrm{E}-02$ & $6.39 \mathrm{E}-03$ \\
\hline
\end{tabular}

Table 8. Expected Annual Fatalities for the Shipping Campaign

\begin{tabular}{llc}
\hline Exposure Group & Truck & Rail \\
\hline Radiological & $4.1 \mathrm{E}-04$ & $7.1 \mathrm{E}-05$ \\
Normal Crew & $6.2 \mathrm{E}-04$ & $1.8 \mathrm{E}-04$ \\
Normal Public & $9.7 \mathrm{E}-06$ & $3.2 \mathrm{E}-06$ \\
$\quad$ Accident Public & & \\
Nonradiological & $2.0 \mathrm{E}-04$ & $2.8 \mathrm{E}-04$ \\
Emission & $2.2 \mathrm{E}-03$ & $6.6 \mathrm{E}-05$ \\
Accident & \\
\hline
\end{tabular}

Table 9. Expected Annual Cancer Incidence for the Shipping Campaign

\begin{tabular}{lll}
\hline Exposure Group & Truck & Rail \\
\hline Radiological & & \\
Normal Crew & $1.4 \mathrm{E}-03$ & $2.5 \mathrm{E}-04$ \\
Normal Public & $2.1 \mathrm{E}-03$ & $6.0 \mathrm{E}-04$ \\
Accident Public & $3.3 \mathrm{E}-05$ & $1.1 \mathrm{E}-05$ \\
Nonradiological & $2.0 \mathrm{E}-04$ & $2.8 \mathrm{E}-04$ \\
Emission & $\mathrm{NA}$ & $\mathrm{NA}$ \\
Accident & & \\
\hline
\end{tabular}


Table 10. Expected Annual Genetic Effects for the Shipping Campaign

\begin{tabular}{lll}
\hline Exposure Group & Truck & Rail \\
\hline Radiological & & \\
Normal Crew & $6.2 E-05$ & $1.1 \mathrm{E}-05$ \\
Normal Public & $1.2 \mathrm{E}-04$ & $3.5 \mathrm{E}-05$ \\
Accident Public & $1.4 \mathrm{E}-06$ & $4.9 \mathrm{E}-07$ \\
Nonradiological & & \\
Emission & NA & NA \\
Accident & NA & NA \\
\hline
\end{tabular}




\section{B.34 WM LLMW CENTRALIZED ALTERNATIVE (CASE 17): CONTACT-HANDLED HETEROGENEOUS SOLIDS: ALPHA — TREATED}

Table 1. Summary of Route Information

\begin{tabular}{|c|c|c|c|c|c|}
\hline & \multirow[b]{2}{*}{ Route } & \multirow{2}{*}{$\begin{array}{c}\text { Distance } \\
\text { (miles) }\end{array}$} & \multicolumn{3}{|c|}{ Percentage in Zone } \\
\hline & & & Rural & Suburban & Urban \\
\hline \multicolumn{6}{|l|}{ Truck } \\
\hline INEL & to Hanford Site & 599 & 91.3 & 7.6 & 1.1 \\
\hline Los Alamos NL & to Hanford Site & 1560 & 89.8 & 8.8 & 1.3 \\
\hline Rocky Flats Plant & to Hanford Site & 1132 & 91.9 & 7.3 & 0.8 \\
\hline West valley $\mathrm{DP}$ & to Hanford Site & 2556 & 84.6 & 13.7 & 1.7 \\
\hline \multicolumn{6}{|l|}{ Rail } \\
\hline INEL & to Hanford Site & 658 & 91.4 & 7.1 & 1.4 \\
\hline Los Alamos NL & to Hanford Site & 1725 & 92.5 & 6.5 & 0.9 \\
\hline Rocky Flats Plant & to Hanford Site & 1284 & 93.1 & 5.5 & 1.4 \\
\hline West valley DP & to Hanford Site & 2654 & 78.3 & 18.0 & 3.7 \\
\hline
\end{tabular}

Table 2. Annual Number of Shipments and one-Way Shipment Distances

\begin{tabular}{|c|c|c|c|c|c|}
\hline \multirow[b]{2}{*}{ Route } & & \multicolumn{2}{|c|}{$\#$ of Shipments } & \multicolumn{2}{|c|}{ Total Mileage (mi) } \\
\hline & & Truck & Rail & Truck & Rail \\
\hline $\begin{array}{l}\text { INEL } \\
\text { Los Alamos NL } \\
\text { Rocky Flats Plant } \\
\text { West valley DP }\end{array}$ & $\begin{array}{l}\text { to Hanford Site } \\
\text { to Hanford Site } \\
\text { to Hanford Site } \\
\text { to Hanford Site }\end{array}$ & $\begin{array}{r}1 \\
68 \\
1\end{array}$ & $\begin{array}{r}1 \\
1 \\
25 \\
1\end{array}$ & $\begin{array}{l}5.99 \mathrm{E}+02 \\
1.56 \mathrm{E}+03 \\
7.69 \mathrm{E}+04 \\
2.56 \mathrm{E}+03\end{array}$ & $\begin{array}{l}6.58 \mathrm{E}+02 \\
1.72 \mathrm{E}+03 \\
3.21 \mathrm{E}+04 \\
2.65 \mathrm{E}+03\end{array}$ \\
\hline Totals & & 71 & 28 & $8.17 E+04$ & $3.71 \mathrm{E}+04$ \\
\hline
\end{tabular}

Table 3. Incident-Free Dose per Shipment (person-rem/shipment)

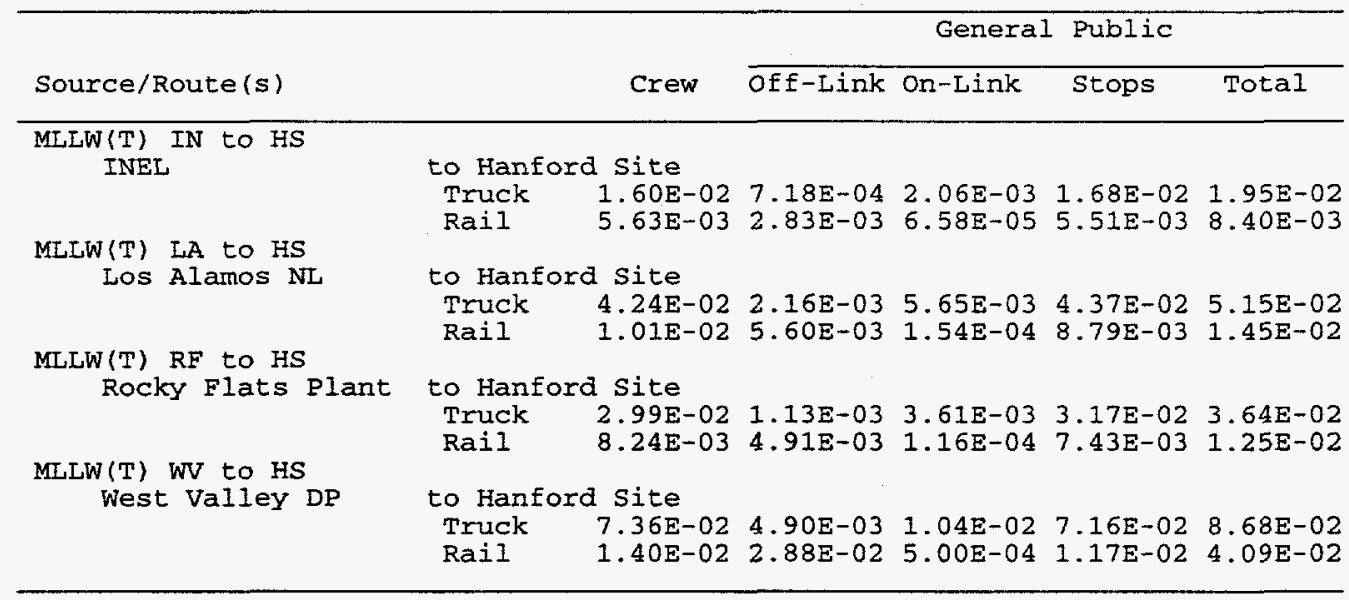

Table 4. Accident Dose Risk per Shipment (person-rem/shipment)

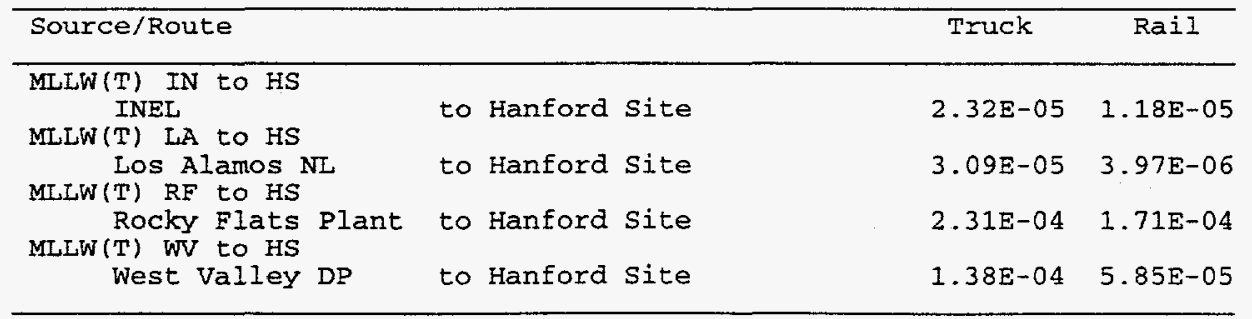


Table 5. Nonradiological Risk Factors per Shipment (fatalities/shipment)

\begin{tabular}{llll}
\hline Mode & Route & Enission & Accident \\
\hline Truck & to Hanford Site & $2.19 \mathrm{E}-06$ & $3.07 \mathrm{E}-05$ \\
INEL & to Hanford Site & $6.66 \mathrm{E}-06$ & $1.02 \mathrm{E}-04$ \\
Los Alamos NL & $2.83 \mathrm{E}-06$ & $5.69 \mathrm{E}-05$ \\
Rocky Flats Plant & to Hanford Site & $1.36 \mathrm{E}-05$ & $1.38 \mathrm{E}-04$ \\
West Valley DP & to Hanford Site & $3.98 \mathrm{E}-06$ & $1.38 \mathrm{E}-06$ \\
Rail & to Hanford Site & $6.82 \mathrm{E}-06$ & $3.61 \mathrm{E}-06$ \\
INEL & to Hanford Site & $7.36 \mathrm{E}-06$ & $2.69 \mathrm{E}-06$ \\
Los Alamos NL & to Hanford Site & $4.14 \mathrm{E}-05$ & $5.55 \mathrm{E}-06$ \\
Rocky Flats Plant & & & \\
West Valley DP & to Hanford Site & &
\end{tabular}

Table 6. Annual Incident-Free Dose for the Shipping Campaign (person-rem/yr)

\begin{tabular}{|c|c|c|c|c|c|}
\hline \multirow[b]{2}{*}{ Route } & & \multicolumn{2}{|c|}{ Truck } & \multicolumn{2}{|c|}{ Rail } \\
\hline & & Crew & Public & Crew & Public \\
\hline $\begin{array}{l}\text { INEL } \\
\text { Los Alamos NL } \\
\text { Rocky Flats Plant } \\
\text { West Valley DP }\end{array}$ & $\begin{array}{l}\text { to Hanford Site } \\
\text { to Hanford Site } \\
\text { to Hanford Site } \\
\text { to Hanford Site }\end{array}$ & $\begin{array}{l}1.60 \mathrm{E}-02 \\
4.24 \mathrm{E}-02 \\
2.03 \mathrm{E}+00 \\
7.36 \mathrm{E}-02\end{array}$ & $\begin{array}{l}1.95 E-02 \\
5.15 E-02 \\
2.48 E+00 \\
8.68 E-02\end{array}$ & $\begin{array}{l}5.63 \mathrm{E}-03 \\
1.01 \mathrm{E}-02 \\
2.06 \mathrm{E}-01 \\
1.40 \mathrm{E}-02\end{array}$ & $\begin{array}{l}8.40 \mathrm{E}-03 \\
1.45 \mathrm{E}-02 \\
3.12 \mathrm{E}-01 \\
4.09 \mathrm{E}-02\end{array}$ \\
\hline Totals & & $2.16 \mathrm{E}+00$ & $2.63 E+00$ & $2.36 \mathrm{E}-01$ & $3.75 \mathrm{E}-01$ \\
\hline
\end{tabular}

Table 7. Annual Accident Dose Risk for the Shipping Campaign (person-rem/yr)

\begin{tabular}{lllc}
\hline Route & & Truck & Rail \\
\hline INEL & to Hanford Site & $2.32 \mathrm{E}-05$ & $1.18 \mathrm{E}-05$ \\
Los Alamos NL & to Hanford Site & $3.09 \mathrm{E}-05$ & $3.97 \mathrm{E}-06$ \\
Rocky Flats Plant & to Hanford Site & $1.57 \mathrm{E}-02$ & $4.28 \mathrm{E}-03$ \\
West Valley DP & to Hanford Site & $1.38 \mathrm{E}-04$ & $5.85 \mathrm{E}-05$ \\
\hline Totals & & $1.59 \mathrm{E}-02$ & $4.35 \mathrm{E}-03$ \\
\hline
\end{tabular}

Table 8. Expected Annual Fatalities for the Shipping Campaign

\begin{tabular}{lll}
\hline Exposure Group & Truck & Rail \\
\hline Radiological & & \\
Normal Crew & $8.7 \mathrm{E}-04$ & $9.4 \mathrm{E}-05$ \\
Normal Public & $1.3 \mathrm{E}-03$ & $1.9 \mathrm{E}-04$ \\
Accident Public & $8.0 \mathrm{E}-06$ & $2.2 \mathrm{E}-06$ \\
Nonradiological & & \\
Emission & $2.2 \mathrm{E}-04$ & $2.4 \mathrm{E}-04$ \\
Accident & $4.8 \mathrm{E}-03$ & $7.8 \mathrm{E}-05$ \\
\hline
\end{tabular}

Table 9. Expected Annual Cancer Incidence for the shipping Campaign

\begin{tabular}{lll}
\hline Exposure Group & Truck & \multicolumn{1}{l}{ Rail } \\
\hline Radiological & $3.0 \mathrm{E}-03$ & $3.3 \mathrm{E}-04$ \\
Normal Crew & $4.5 \mathrm{E}-03$ & $6.4 \mathrm{E}-04$ \\
Normal Public & $2.7 \mathrm{E}-05$ & $7.4 \mathrm{E}-06$ \\
Accident Public & $2.2 \mathrm{E}-04$ & $2.4 \mathrm{E}-04$ \\
Nonradiological & $\mathrm{NA}$ & $\mathrm{NA}$ \\
Enission & & \\
Accident & & \\
\hline
\end{tabular}




\section{$B-158$}

Table 10. Expected Annual Genetic Effects

for the Shipping Campaign

\begin{tabular}{lll}
\hline Exposure Group & Truck & Rail \\
\hline Radiological & & \\
Normal Crew & $1.3 E-04$ & $1.4 \mathrm{E}-05$ \\
Normal Public & $2.6 \mathrm{E}-04$ & $3.8 \mathrm{E}-05$ \\
Accident Public & $5.5 E-07$ & $1.6 \mathrm{E}-07$ \\
Nonradiological & & \\
Emission & NA & NA \\
Accident & NA & NA \\
\hline
\end{tabular}

